\author{
Monograph \\ urn:1sid:zoobank.org:pub:FA508A12-9BDB-4A2B-9B0C-98FDD161443C
}

\title{
The bats of the Congo and of Rwanda and Burundi revisited (Mammalia: Chiroptera)
}

\author{
Victor VAN CAKENBERGHE ${ }^{1, *}$, Guy-Crispin GEMBU TUNGALUNA ${ }^{2}$, \\ Prescott MUSABA AKAWA ${ }^{3}$, Ernest SEAMARK ${ }^{4} \&$ Erik VERHEYEN $^{5}$ \\ ${ }^{1}$ University of Antwerp, Department of Biology, Functional Morphology, \\ Campus Drie Eiken, Universiteitsplein, 1, B-2610 Antwerpen (Wilrijk), Belgium. \\ ${ }^{1,4}$ AfricanBats NPC, 357 Botha Ave, Kloofsig, 0157, Republic of South Africa. \\ ${ }^{2,3}$ Faculté des Sciences, Université de Kisangani, Democratic Republic of the Congo. \\ ${ }^{4}$ Centre for Wildlife Management, University of Pretoria, Private Bag X20 Hatfield, \\ Pretoria 0028, Republic of South Africa. \\ ${ }^{5}$ Royal Belgian Institute of Natural Sciences - OD Taxonomy and Phylogeny, \\ Vautierstraat 29, 1000 Brussels, Belgium. \\ ${ }^{5}$ University of Antwerp, Department of Biology, Evolutionary Ecology, Campus Drie Eiken, \\ Universiteitsplein 1, B-2610 Antwerpen (Wilrijk), Belgium. \\ ${ }^{*}$ Corresponding author: Victor.VanCakenberghe@uantwerpen.be \\ ${ }^{2}$ Email: gembuguycrispin@gmail.com \\ ${ }^{3}$ Email: prescottmusaba@yahoo.fr \\ ${ }^{4}$ Email: ernest.seamark@africanbats.org \\ ${ }^{5}$ Email: erik.verheyen@naturalsciences.be

\footnotetext{
${ }^{1}$ urn:1sid:zoobank.org:author:9A0E1AF5-C248-4648-9D64-443112890346

${ }^{2}$ urn:lsid:zoobank.org:author:2AE2F96F-A138-419B-B650-6DB44D535D14

${ }^{3}$ urn:lsid:zoobank.org:author:4C40421C-1D84-4BD1-A444-360300ECBEEC

${ }^{4}$ urn:lsid:zoobank.org:author:B60CA9A1-D288-468E-AEEB-D0F136E96F5B

${ }^{5}$ urn:1sid:zoobank.org:author:86B40463-E3D9-4147-9ED3-D7302E0D64B6
}

\begin{abstract}
In 1966, Robert William Hayman, Xavier Misonne and Walter Verheyen published their listing of the Congolese, Rwandan and Burundian bat specimens in the collections in the museums of Tervuren, Brussels, Geneva, London and New York. In the fifty years that have passed since, some major changes have been introduced in the taxonomy of the Chiroptera: new species have been discovered, species have been split off, species have been moved to other genera, and additional material has been collected. We re-evaluated the data presented by Hayman et al., and supplemented this with specimen records found in the literature and in online catalogs. This resulted in 136 species, represented by 20231 specimens (compared to 113 species and 8567 specimens originally). When available, we also recorded additional information such as locality, sex and age, collector, collection date and preservation type of the voucher specimen. The distribution maps of the Congolese taxa are revised to represent the current taxonomy, and are presented in perspective against the taxon's Species Distribution Model to assess species distribution on the African continent. Additionally, an updated key to the various taxa is presented.
\end{abstract}

Keywords. Chiroptera, Democratic Republic of the Congo, Rwanda, Burundi, review. 
Van Cakenberghe V., Gembu Tungaluna G.-C., Musaba Akawa P., Seamark E., \& Verheyen E. 2017. The bats of the Congo and of Rwanda and Burundi revisited (Mammalia: Chiroptera). European Journal of Taxonomy 382: 1-327. https://doi.org/10.5852/ejt.2017.382

\section{Table of Contents}

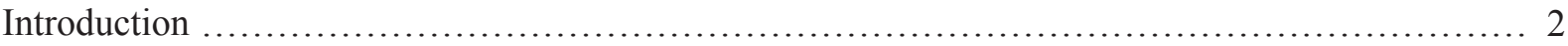

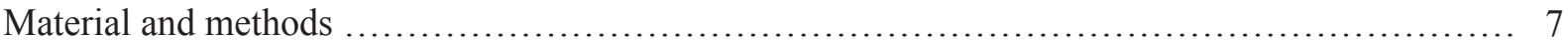

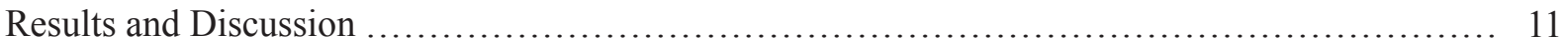

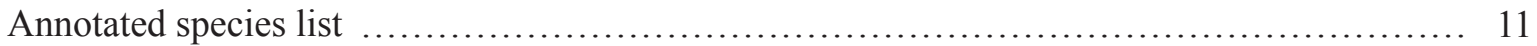

Nominal list of bats of the Congo, Rwanda and Burundi (CRB) region $\ldots \ldots \ldots \ldots \ldots \ldots \ldots \ldots . . \ldots 107$

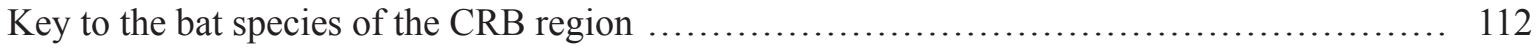

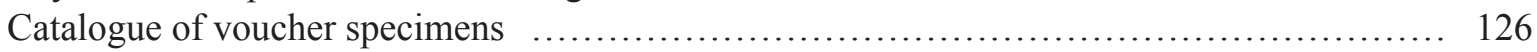

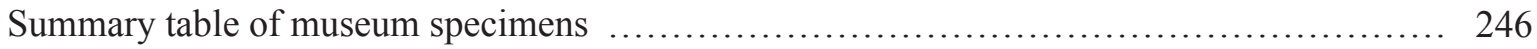

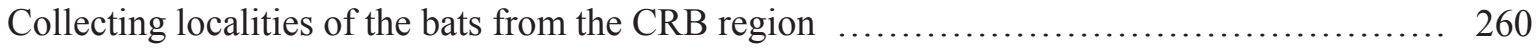

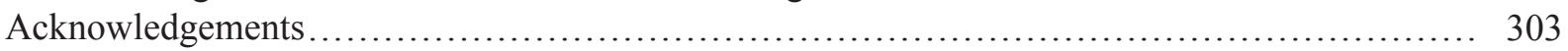

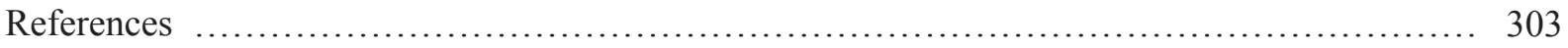

\section{Introduction}

In December 1966, issue number 154 of the "Koninklijk Museum voor Midden-Afrika - Annalen - Reeks In-8 - Zoologische Wetenschappen / Musée Royal de l'Afrique Central - Annales - Série In-8 - Sciences Zoologiques" appeared. In that issue, R.W. Hayman, X. Misonne and W. Verheyen reported on the bats occurring in the Congo, Rwanda and Burundi, primarily based on material in the collections of the three major Belgian natural history museums: the Royal Museum for Central Africa (RMCA) in Tervuren (also known by its Dutch and French names and acronyms: Koninklijk Museum voor Midden-Afrika (KMMA) / Musée Royal de l'Afrique Central (MRAC) and its historic name of "the Congo Museum"), the Royal Belgian Institute of Natural Sciences (RBINS) in Brussels (also: Koninklijk Belgisch Instituut voor Natuurwetenschappen (KBIN) / Institut Royal des Sciences Naturelles de Belgique (IRSN)), and the Institute of the National Parks of Belgian Congo (INPBC) (Instituut der Nationale Parken van Belgisch Congo (INPBC) / Institut des Parcs Nationaux du Congo (IPNC)), with additions from the British Museum (Natural History), London (BMNH) and the American Museum of Natural History, New York (AMNH) and an addenda section by V. Aellen, covering the bats in the collection of the Muséum d'Histoire Naturelle, Geneva (MHNG).

Today, 50 years after the appearance of "The Bats of the Congo and of Rwanda and Burundi" by Hayman et al. (1966), the current authors deemed it timely to revisit this publication and bring it up to date, as this last half a century has brought a lot of new techniques, which yielded important changes to our views into the systematics and the taxonomic status of bats (order Chiroptera). Ten of the species recognized in the current study were not known to science in 1966. Some taxa were recognized as subspecies or synonym and were raised to full species status. In a few other cases, the taxon's content remained the same, but the name needed to be changed due to priority rules.

As correctly remarked by Bates et al. (2013: 313), the title of Hayman et al. (1966) might have been a bit misleading as "The Congo" did not cover the territory of "The Republic of Congo" [= Congo (Brazzaville)], but only the area that used to be a Belgian colony or mandate area: The Democratic Republic of the Congo (DRC), Rwanda and Burundi. Further on in the text, the acronym CRB will be used to refer to this area.

As mentioned by Hayman et al. (1966: 1), the first records of bats from the CRB were reported by Johnston (1884) from the area along the Congo River from its mouth to Bolobo. The river formed (and 
still forms) an important route through Congo, as is also illustrated by more recent scientific expeditions, e.g., the 1974-1975 Zaire River Expedition (Gallagher \& Harrison 1977; 212 bats collected) and the 2009 and 2010 Congo River expeditions (in 2009 between Lisala and Kisangani and the "Boyekoli Ebale Congo" expedition in 2010 between Bumba and Kisangani; 594 bats; Gembu Tungaluna and Musaba, unpublished). The latter two were organized by the Congo2010 consortium, that consisted of the RMCA (Tervuren), the RBINS (Brussels), the Botanic Garden (Meise) and the University of Kisangani, with funding from the Belgian Federal Directorate general for Development and the Belgian Science Policy.

A second, enormously important contribution to the knowledge of bats in the CRB was provided by the "American Museum Congo Expedition", which started at Boma on 22 June 1909 and which took Herbert Lang and James P. Chapin to the northeastern part of the DRC, where they made collections in Poko (Bas-Uélé Province); Niangara, Faradje, Medje and Aba, in or near the Garamba National Park (HautUélé Province); Avakubi, near the Ituri River, Panga and Kisangani (Tshopo Province); and then back over Kinshasa, Boma and Matadi to Banana, where the expedition finally ended on 14 September 1915 (Osborn, 1919). They collected almost 800 bats and their data formed the basis for Allen et al. 's "The American Museum Congo Expedition collection of Bats" (Allen et al. 1917). Allen (1917) recognized 68 forms, covering 65 different species, which were reduced to 56 by Koopman (1965).

A further increase occurred during the 1930s to late 1950s, when major collections were made for the Institute of the National Parks of Belgian Congo in the National Parks of the eastern part of the CRB: Albert National Park (currently the Virunga National Park; ca 480 bats), the Garamba National Park (ca 600 bats), the Upemba National Park (ca 220 bats) and the Kagera National Park (currently the Akagera National Park in Rwanda; ca 170 bats). The results of these expeditions were reported by Schouteden (1934, 1935), Frechkop (1938, 1943, 1944, 1954) and Verschuren (1957). Henri Schouteden also published a number of papers on the mammals of the entire CRB area (Schouteden 1943, 1947, 1948).

Although being an entomologist, Narcisse Leleup collected over 260 bats between 1948 and 1958 in the DRC provinces of Katanga, Kongo Central, Maniema and Kivu, primarily for their insect parasites (Jobling 1954; Leleup 1956).

From the mid-1950s to the early 1960s, Anciaux de Faveaux made extensive collections in the Katanga area (currently provinces of Haut-Katanga, Haut-Lomami, Lualaba, Tanganyika; at least 1000 bats), which were followed in the mid-1960s by a collection from Rwanda (at least 50 bats). These resulted in a large number of publications, primarily on reproduction and parasites (Anciaux de Faveaux 1958, 1959 1960, 1960a, 1960b, 1964, 1965, 1972, 1977, 1978a, 1978b, 1983; Benoit 1958a, 1958b; Fain 1972; Krampitz \& Anciaux de Faveaux 1960).

In the 1960s, the independence of the Congo, Rwanda and Burundi led to a diminished presence of Belgian biologists in the area, or at least of Belgian led expeditions, although individual collectors still sent material to museums abroad (with number of bats in brackets), e.g., Toon De Roo (443), P. Lootens (664 - from the 1950s until the 1990s), M.J. Celis and M. Lejeune (70 combined, 300 Lejeune only), but other nationals also became involved, e.g., P. Kunkel (for the Senckenberg Museum Frankfurt [SMF]; 48). In the 1970s, major collections were made by Lynn and Brian Robbins (Carnegie Museum $[\mathrm{CM}$ ], United States National Museum [USNM]; 162), Michael Gallagher (Harrison Institute [formerly Harrison Zoological Museum - HZM]; 214), Frieder Meier (MHNG; 119), but also the first African names started to appear: Tshinyenye (RMCA; 118) and Betu Nsankulu (RMCA; 128). In the 1990s there were major collections by Julian Kerbis Peterhans (Field Museum of Natural History [FMNH]; 50) and Bob Swanepoel (Ditsong National Museum of Natural History [formerly Transvaal Museum - TM]; 515). In the first decade of the $21^{\text {st }}$ century, a large collection was made by Alexandre Hassanin and 
colleagues for the Muséum national d'Histoire naturelle (MNHN; 150 bats). Additionally, there was also the startup of the collection at the University of Kisangani (UNIKIS) by prof. Séraphin Ifuta (presently University of Kinshasa [UNIKIN]), which is currently still being expanded by material collected during field trips under the direction of the Congolese co-authors. At UNIKIS, a Centre de Surveillance de la Biodiversité (CSB, http://centresurveillancebiodiversite.org/) was created under the directorship of prof. Dudu Akaibe to ensure the long-term surveillance and management of how the flora and fauna of the Congo River rainforest evolves. As head of the department of terrestrial vertebrates, Prof. Gembu Tungaluna is responsible for the bat-related projects.

Of special interest are also the collections from Tandala and Yalosemba (1979; 215 bats - Breman et al. 1999) and Kikwit (1995; 538 bats - Van Cakenberghe et al. 2000) where, in the aftermath of the Ebola epidemics, expeditions were set up in search for the reservoir species for this virus, and of which the specimens were primarily deposited in the collections of the CM, MHNG, MSB (Museum of Southwestern Biology), RMCA and USNM. As indicated in Fig. 1, the material included in the current study covers almost the entire DRC, although there are still a few areas that are underrepresented, e.g.,

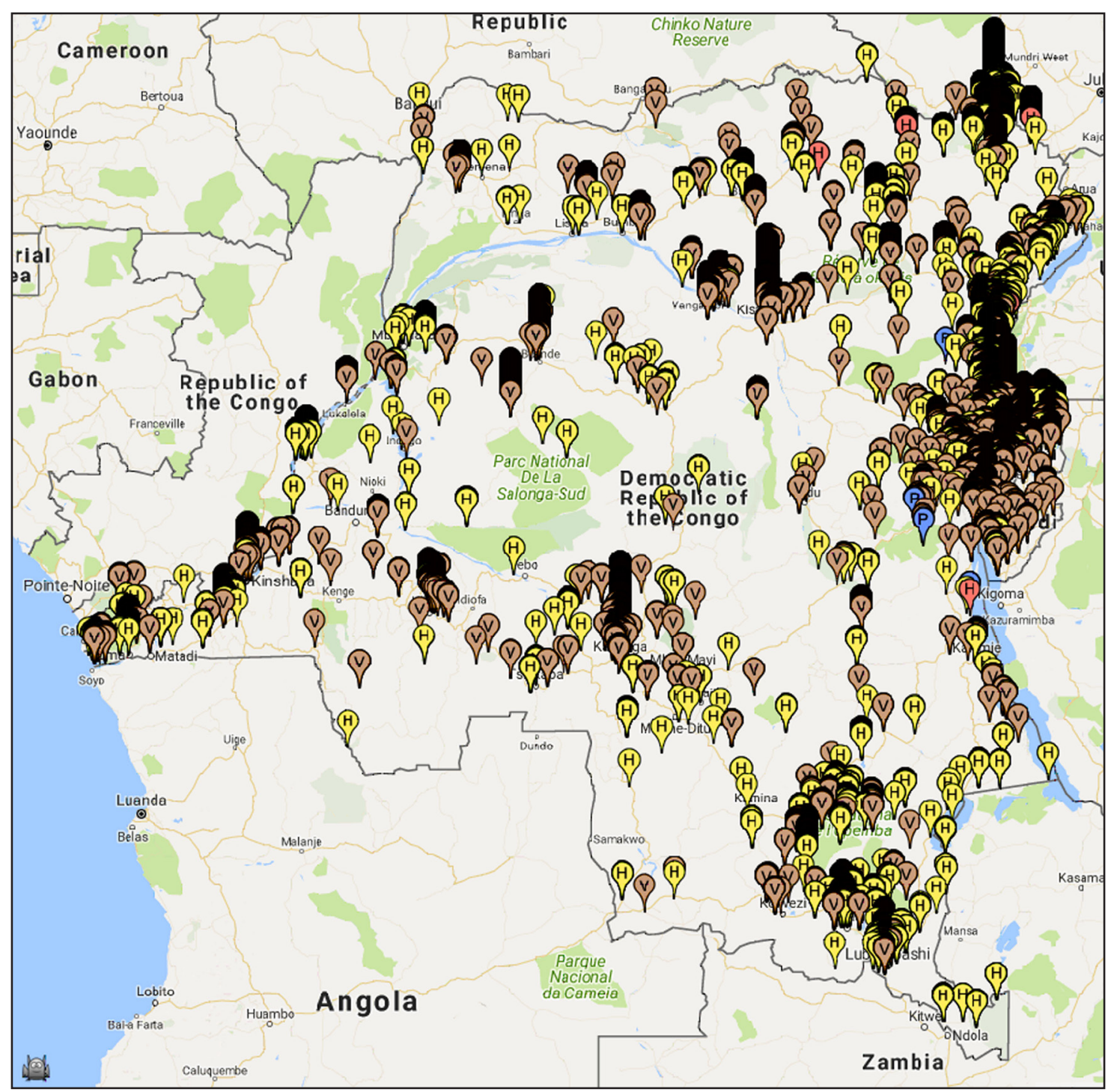

Fig. 1. Capture localities for all bats. The symbols represent: yellow $(\mathrm{H})$ : records included in Hayman et al. (1966), and brown (V): newly added records. Other coloured pins represent type specimens: red $(\mathrm{H})$ : holotype, blue (P): paratype or paralectotype (for further details, see text). 
the southern part of Kwango Province (former Bandundu Province), the central and northern part of Lualaba Province (western part of the former Katanga Province), the western part of Equateur Province, Lomami and Sankuru Provinces (former Kasai Oriental Province), and especially the central area covered by Tshuapa, Sankuru, Kasai and Mai Ndombe Provinces, where the Parc National de la Salonga (North and South) remains a blank area. This seems a bit strange as most of the other National Parks were already extensively surveyed from the 1930s to the 1950s (see above), but the Salonga National Park was only created in 1970 and is the only park situated in the Central African Rainforest, which is impenetrable and is only accessible along the rivers running through it. Its visibility of less than $100 \mathrm{~m}$ also makes it very difficult to assess any occurrence of wildlife in the park.

Jacques Verschuren collected 48 bats in Burundi in 1976 for the RBINS (Verschuren 1980). A major collection (327 bats) from the country was made by P.L. Niort in the late 1960s-early 1970s and deposited in the MHNG (Niort 1970). At the end of the 1970s Heinz Stephan collected 70 bats for the SMF, and at the beginning of the 1980s, Danny Meirte (RMCA) made another important collection of 156 bats. In the 1990s Julian Kerbis Peterhans (22), L. Davenport (33) and Jay L. Udelhoven (33) made collections for the FMNH. Besides the already mentioned Rwandese collection from the Akagera National Park made for the INPBC and the material collected by Anciaux de Faveaux, major contributions were provided by Alexandre Fain (late 1940s to mid 1950s; 108 bats), but primarily by a number of expeditions by the RMCA (Danny Meirte; 171 bats) and the University of Antwerp in the early 1980s (Baeten et al. 1984; 2102 bats).

Hayman et al. (1966: 77) reported on 8567 specimens belonging to 113 species. The additional material that has been traced for this study augmented these numbers to 20231 bats belonging to 136 species.

For the current study not all of the specimens were (re-)examined by the authors. This was only done for the material in the collection of UNIKIS, most of the specimens from Rwanda (Baeten et al. 1984), Kikwit (Van Cakenberghe et al. 2000) and for specific groups: Epomophorus (Claessen \& De Vree 1990, 1991), Nycteris (Van Cakenberghe \& De Vree 1985, 1993, 1999) and Pipistrellus /Eptesicus /Neoromicia/Hypsugo (Van Cakenberghe, unpublished).

The current study follows the structure used in Hayman et al. (1966), with the exception of two extra sections (Material and methods and Annotated species list) and the removal of the index section. The order in which the various taxa are presented follows the currently recognized view on chiropteran taxonomy. For an overview, we refer to the "Nominal list of bats of the Congo, Rwanda and Burundi (CRB) region" section. For each of the species, we provide the current name, and in the synonyms list we only include the original name and (prefixed by an asterisk) the names as used by Hayman et al. (1966). For a complete list of synonyms, we refer to ACR (2016).

The systematics follows Simmons (2005) and "Mammals of Africa volume IV" (Happold \& Happold 2013), unless explicitly mentioned in the annonated catalog section. However, due to the difficulties in assessing the subspecific boundaries of the various taxa, we did not distinguish any subspecies.

Since the days of Hayman et al. (1966), taxonomic techniques have evolved, leading to the discovery and/ or recognition of new species. Where morphological comparisions and univariate statistical tests were the state-of-the-art techniques, the rise of computers in the 1970s-1980s led to multivariate statistical analyses. Unfortunately, these were not always capable of distinguishing very similar or cryptic species (Baker \& Bradley 2006), and, therefore, other methods, looking at more fundamental building blocks of life (biochemical techniques), were investigated such as karyotypes, electrophoresis and in recent years DNA sequencing. Important new techniques, such as molecular phylogenetics and phylogeographic analyses, are also contributing to our current understanding of species and their distribution patterns. However, these biochemical techniques cannot stand on their own (Ruedas et al. 2000), and the collection 
of tissues should be backed by continued collecting of voucher specimens to substantiate their identity (Patterson 2002). Zachos et al. (2013: 1) discourage species splitting based on gene trees inferred from mitochondrial DNA only, as uncritical acceptance of such new species might create an unnecessary burden on the conservation of biodiversity.

As already pointed out by Hayman et al. (1966: 3), the central geographic position of the CRB region in Africa and its immense size makes it a crossroad for bat species from all over the continent. Konaté \& Linsenmaier (2010: 18) marked the eastern parts of the DRC provinces of Ituri, Nord-Kivu, Sud-Kivu and Tanganyika and the western parts of Rwanda and Burundi as one of the world's biodiversity hotspots: the Eastern Afromontane hotspot (see also Ceballos \& Ehrlich 2006). Some of the species occurring in the CRB area have an almost pan-African distribution (e.g., Nycteris thebaica, Pipistrellus rueppellii) whereas others were found in a very restricted area - in a number of cases only at the type locality, e.g., Rhinolophus hilli, $R$. kahuzi, $R$. ruwenzorii, $R$. willardi, Chaerephon gallagheri, Mops niangarae. With the exception of the two molossid species, all of these are only found in the eastern DRC endemic hotspot as defined by Herkt et al. (2015: figs 3-4). In between these extreme distribution ranges, there are species which are common in sub-Saharan Africa (e.g., Eidolon helvum, Hipposideros caffer, Taphozous mauritanus, Nycteris hispida, Chaerephon pumilus, Mops condylurus, Scotophilus dinganii, Neoromicia nana), others are found primarily in western Africa (e.g., Hipposideros abae, Rhinolophus alcyone, Myonycteris daubentonii), eastern Africa (e.g., Epomophorus minimus, Rhinolophus eloquens, Tadarida fulminans), Southern Africa (e.g., Plerotes anchietae, Rhinolophus swinnyi, Cloeotis percivali, Hypsugo anchietae, Laephotis botswanae) or Northern Africa (e.g., Taphozous nudiventris). Some of the species only occur in the African forest areas (e.g., Epomops franqueti, Hypsignathus monstrosus, Megaloglossus woermanni, Saccolaimus peli, Nycteris arge, Glauconycteris superba), and some even only in the Central African forest (e.g., Casinycteris argynnis). Hayman et al. (1966: 3) particularly pointed to the enormous extent of the tropical forest belt contributing to the importance of the CRB area. However, commercial logging and expansion of farming has made the Congo rainforest one of the most endangered ecosystems in the world (http://interesting-africa-facts.com/Africa-Landforms/CongoRainforest-Facts.html).

The apparent rarity of some of the species reported in this paper might be the result of small geographic ranges, limited habitat breadth, low local population density, or simply a sampling artefact linked to insufficient sampling effort, inadequate methodology (mist nets, canopy nets, harp traps, ...), or differential species detectability (Meyer et al. 2014: 114). In most cases, the exact reason for this is currently unknown, and might possibly only be resolved once more specimens are collected, which means that extensive field work is still a necessity, even in times where people think that everything can be solved from behind a computer screen.

Did we cover all of the known specimens/species? Probably not. Are all of the included specimens correctly identified? Unlikely. What we wanted to achieve was to provide an inventory of the CRB specimens that are available in collections worldwide that can be used for further research, and we certainly encourage other/local researchers to investigate these collections and verify the data provided here.

The bottom line, however, is: you cannot deliver good, reliable, reproducible science if you cannot confirm the identity of the animals you are (or have been) working on. This is especially important for bat-related studies, as these animals have recently been recognized as potential carriers of pathogenic and infectious agents that can pose threats to humans: e.g., rabies (Adedeji et al. 2010), ebola (Leirs et al. 1999; Leroy et al. 2005, 2009; Grard et al. 2011; Hayman et al. 2012; Leendertz 2016), see also Wibbelt et al. (2010), Newman et al. (2011), Schneeberger \& Voigt (2015), Ranjan et al. (2016). Moreover, Han et al. (2016: 6) illustrate that the northeastern part of the DRC forms an African hotspot related to the 
number of filovirus-positive bat species. To substantiate these studies, we stress the importance of the collection of voucher specimens and their safekeeping for future generations of scientists (Rocha et al. 2014; Tsang et al. 2015). However, as mentioned by Paul Racey (in Wibbelt et al. 2010: 440): “... every effort must be taken to avoid a reductionist approach to newly described infectious diseases and that all factors associated with the emergence of infectious diseases must be considered so that bats do not simply become targets for persecution and eradication." To which we could add that collecting bats for the sole purpose of increasing collection sizes isn't a good practice either, and hopefully this paper might contribute to a more efficient use of already established collections.

\section{Material and methods}

The base for the current publication was formed by the catalog data in Hayman et al. (1966), with additional information from the catalog records from the RMCA and RBINS. We also searched online catalogs (e.g., http://vertnet.org/, http://arctos.database.museum/SpecimenSearch.cfm and http://www. gbif.org/) and consulted the online catalogs of additional museums (for details see the "Summary table of museum specimens" section below). Further information was obtained from Ammerman et al. (2013), Anciaux de Faveaux (1972), Andersen (1912), Ansell (1960, 1974), Benda \& Vallo (2012), Bergmans (1988, 1989, 1991, 1994, 1997), Cotterill (2001b), Csorba et al. (2014), Dollman (1909, 1914), Eger \& Schlitter (2001), Fahr \& Ebigbo (2003), Fain (1972), Fain \& Aellen (1994), Freeman (1981), Gallagher \& Harrison (1977), Gregorin and Cirranello (2015), Gunnell et al. (2015a), Harrison (1957, 1959), Hassanin et al. (2015), Hayman (1937, 1946, 1960), Heller et al. (1994), Hill (1974a, 1977), Hill \& Harrison (1987), Hoofer \& Van Den Bussche (2003), Hoofer et al. (2006), Kerbis Peterhans et al. (2013), Kityo et al. (2009), Kock (1969, 1978, 1981a, 1981b, 1987), Kock \& Howell (1988), Kock et al. (2002), Koubínová et al. (2013), Lack et al. (2010), Laumanns et al. (2004), Monadjem et al. (2010b), Noack (1889), Omatsu et al. (2008), Ossa Gómez (2012), Rahm (1966), Ralph et al. (2015), Reeder et al. (2013), Robbins et al. (1985), Roehrs (2009), Roehrs et al. (2010), Soisook et al. (2015), Stanley et al. (1996), Thorn et al. (2007), Uchikawa (1985), Uchikawa \& Kock (1989), Van Cakenberghe \& De Vree (1985, 1993), Volleth \& Heller (1994) and Volleth et al. (2001).

The supplementary material included with this paper consists of a zip file which contains htm files and a separate directory, which contains pin symbols. The htm files need to be placed in a directory called "c: \CongoBatMaps" and the pins (*.png) in "c:lCongoBatMaps\pins". The zip file also contains some additional htm files for the specimens which were not identified to the species level.

The pins on these maps (as on Fig. 1) are coded as follows: yellow (H): records included in Hayman et al. (1966), and brown (V): newly added records. Other coloured pins represent type specimens: red $(\mathrm{H})$ : holotype, red (L): lectotype, blue (P): paratype or paralectotype, and green $(\mathrm{T})$ : topotype. To be able to separate multiple specimens from (almost) the same locality, the first specimen is plotted at the correct coordinates and subsequent specimens are moved slightly to the north.

For each of the pins, the following information is shown (when available): museum acronym and number, age and sex, sort of type and the original name, current name, country, locality, coordinates, altitude, collector and collection date. In the lower left hand corner of the map, there is a bat icon which contains the name of the species and the version number.

In the "Annotated Species List", the species name header is followed by a reference (Fig. xx) to the distribution maps. For each species, two maps are included: one ("A, C or E") shows the distribution within the CRB area. Each symbol on the map represents a locality where the species has been collected. The blue squares represent localities where the species was already reported from by Hayman et al. (1966), the red dots are new localities. 
To place the species occurring from the CRB in context, a Species Distribution Modelled (SDM) map ("B, D or F") was created using the point data from ACR (2016). MaxEnt was used to estimate the PanAfrican distribution of each species (Phillips et al. 2006), based on 19 climate variables from the World Clim (Hijmans et al. 2005). Each species output was clipped using a buffered minimum convex polygon of $500 \mathrm{~km}$ (Kremen et al. 2008) resulting in the solid red areas on the map. The final step was to draw subjectively a suggested range extent of the species combining the point localities, SDM clipped output and the Sayre et al. (2013) bioclimatic zones, resulting in the hatched area.

When examining the SDM output maps, we are aware that most make sense, while others might look doubtful. One of the major reasons for this is that one needs to have at least 10 separate localities to generate a reliable map, which for a number of species simply is not possible (e.g., Rhinolophus kahuzi: only known from the type locality). In a few cases, we also found that the various models we tested did not include areas where the species was actually collected by one of the authors. We, therefore, would like to highlight a flaw in some maps who use predicted species distribution models to show areas of high species richnesses (e.g., Herkt et al. 2015, 2017; Han et al. 2016). Areas may be shown as potentially
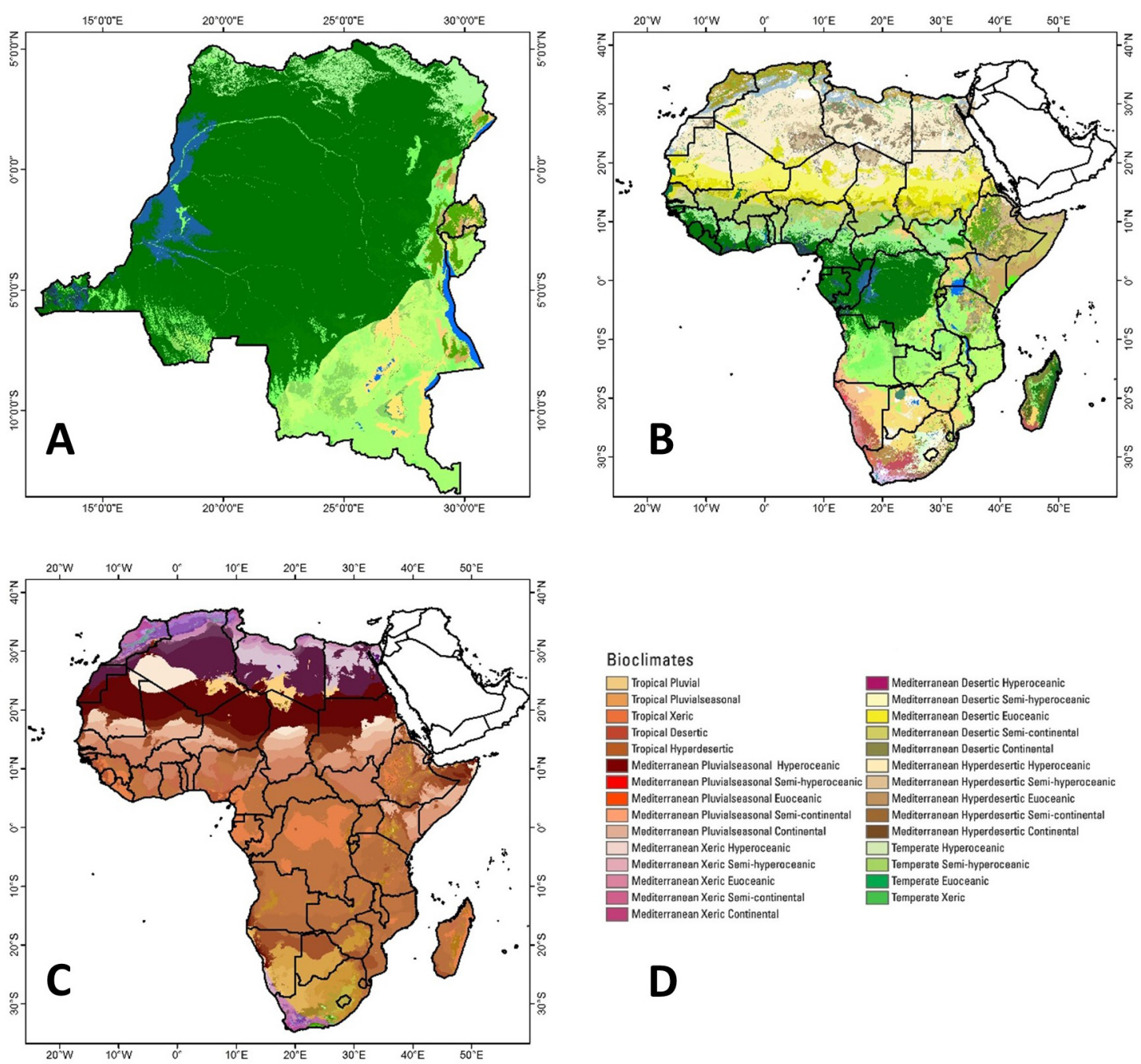

Fig. 2. A. Vegetation map of the CRB. B. Vegetation map of Africa (Blue $=$ Central Congo Basin Swamp Forest or Lakes, Dark green $=$ Forest to Open Woodland, Light green $=$ Shrubland or Grassland, Brown $=$ Desert or Semi-Desert). C. Bioclimates map. D. Colour codes used in Figure 2C (all modified after Sayre et al. 2013). 
containing areas of high species richness and important, but no evidence of any specimens exist. Likewise, areas of high species richness based on specimens, may similarly be underrepresented in the richness maps where these localities do not conform to the underlying fundamental niche assumptions for species distribution models. SDM and the species richness derived from these should be used to prioritize where field verification needs to take place (test the model). Any policies derived from such should only be created or implemented after verification has taken place.

Holt et al. (2013) identified 11 zoogeographic realms and 20 regions globally. Within Africa and its associated islands four zoogeographic realms - Saharo-Arabian, Afrotropical, Madagascan and Oceania [Reunion and islands eastwards] - were identified. The Afrotropical realm was further divided into two zoogreographical regions - Guineo-Congolian and Afrotropical. Africa contains five zoogeographical regions. The $\mathrm{CRB}$ area contains two of these zoogeographic regions, with the majority of the CRB containing a significant portion of the Guineo-Congolian zoogeographic region, and to a lesser extent Afrotropical region.

Figure 2 shows an overview of the various vegetation types occurring in the CRB region (A), in Africa (B), the African bioclimatic zones (C). Note that none of these maps include human land use change - if the true extent of forest is considered, approximately $1 / 3$ of the area is expected to be destroyed. These maps represent the status of about 100 to 150 years ago or a theoretical current status, if there were no humans. The current status most probably would include a much wider 'new' Savanna Grassland habitat, due to the removal of forest, either for agriculture or urbanization (charcoal industry). This might be the reason why we may be recording more Savanna species in the CRB. Furthermore, given the fact that we report on specimens that were collected over a timespan of more than a century, the vegetation type indicated on the map for any given locality might be different from the one at the actual time the specimen was collected or from the current situation. The fact that a species has occurred in a specific locality at a specific moment in time does not imply that this is currently still the case.

As the African vegetation zones have been shifting over the past century, we decided to use the Bioclimates data (Sayre et al. 2013: 6) as we suspect this to present a more stable background, although we cannot ignore the potential influence of global change.

\section{Collection acronyms}

\begin{tabular}{|c|c|c|}
\hline AMNH & $=$ & American Museum of Natural History, New York, USA \\
\hline BMNH & $=$ & The Natural History Museum (formerly British Museum of Natural History), London, UK \\
\hline CAS & $=$ & California Academy of Sciences, San Francisco, CA, USA \\
\hline $\mathrm{CM}$ & $=$ & Carnegie Museum, Pittsburgh, PA, USA \\
\hline DNSM & $=$ & Durban Natural Science Museum, Durban, South Africa \\
\hline FMNH & $=$ & Field Museum of Natural History, Chicago, IL, USA \\
\hline HNHM & $=$ & Hungarian Natural History Museum, Budapest, Hungary \\
\hline HZM & $=$ & Harrison Institute (formerly Harrison Zoological Museum), Sevenoaks, UK \\
\hline INPBC & $=$ & Institute of the National Parks of Belgian Congo, Brussels, Belgium \\
\hline KU & $=$ & University of Kansas, Natural History Museum, Lawrence, KS, USA \\
\hline MCZ & $=$ & Museum of Comparative Zoology, Harvard University, Cambridge, MA, USA \\
\hline MHNG & $=$ & Muséum d'Histoire Naturelle, Genève, Switzerland \\
\hline MNHN & $=$ & Muséum national d'Histoire naturelle, Paris, France \\
\hline MSB & $=$ & Museum of Southwestern Biology, Albuquerque, NM, USA \\
\hline NMBA & $=$ & Naturhistorisches Museum, Basel, Switzerland \\
\hline NMP & $=$ & National Museum (Natural History), Prague, Czech Republic \\
\hline NMW & $=$ & Naturhistorisches Museum, Wien, Austria \\
\hline NMZL & $=$ & National Museum of Zambia, Livingstone, Zambia \\
\hline
\end{tabular}




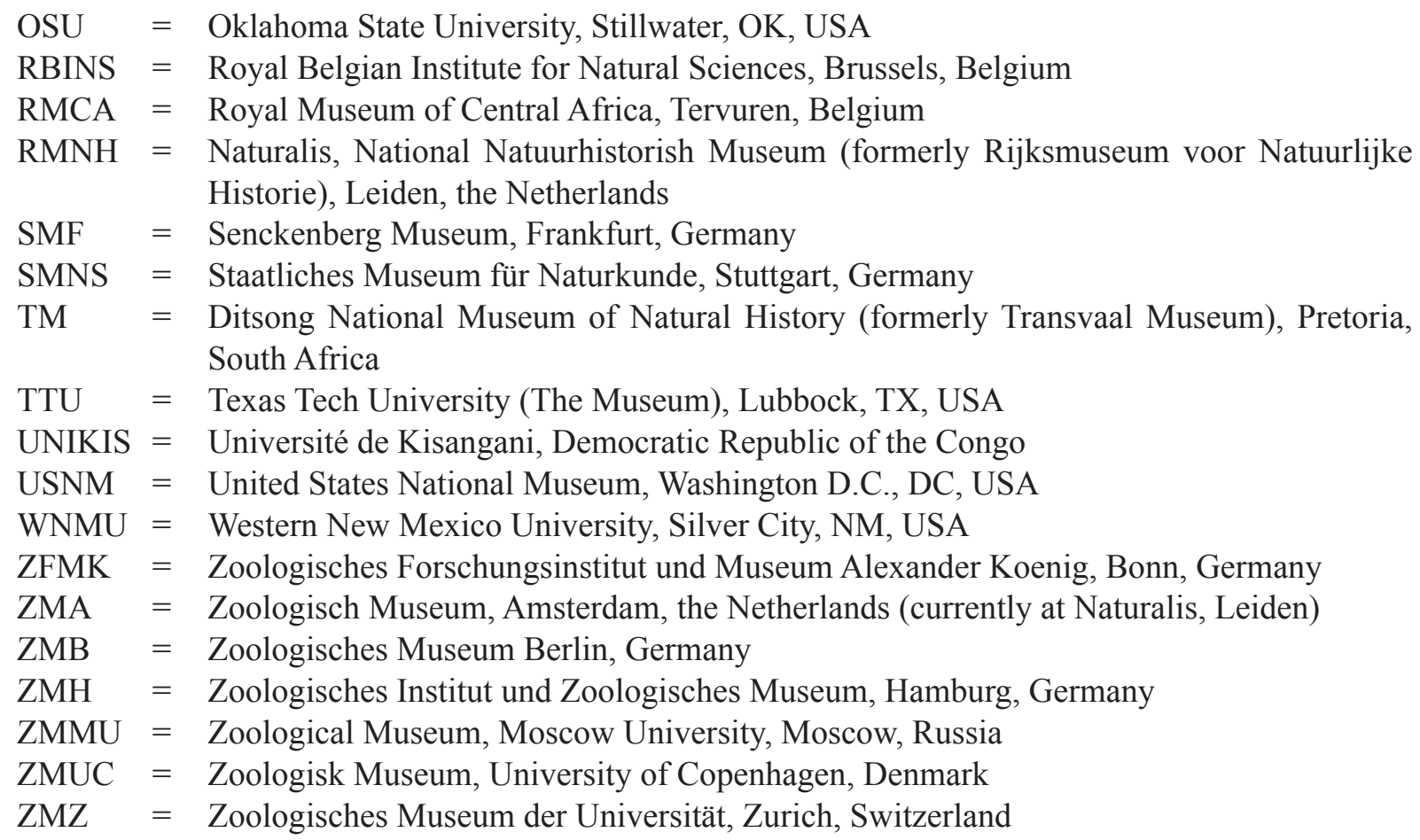

\section{Additional abbreviations and symbols}

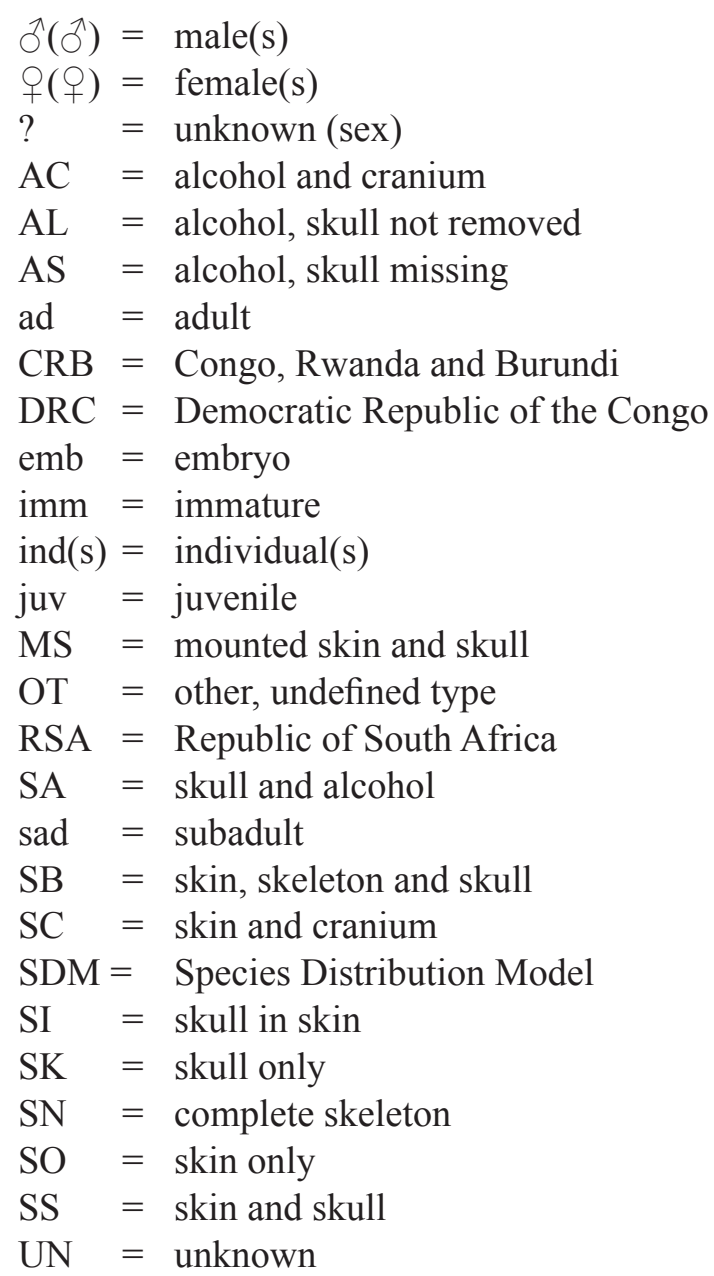




\section{Results and Discussion}

Annotated Species List

Class Mammalia Linnaeus, 1758

Order Chiroptera Blumenbach, 1779

Suborder Pteropodiformi Hutcheon \& Kirsch, 2006

Pteropodiformes Hutcheon \& Kirsch, 2006: 9.

The classical subdivision of the order Chiroptera into two suborders, Megachiroptera and Microchiroptera, has undergone quite an evolution over the last decennia, which resulted in a rearrangement into the suborders Yinpterochiroptera and Yangochiroptera (see ACR 2016: 11-13 for a discussion). As the content of these suborders has changed rather frequently, Hutcheon \& Kirsch (2006: 1) suggested two new subordinal names: Vespertilioniformes (for the group including Emballonuridae, Nycteridae, and the 'yangochiropterans') and Pteropodiformes (for the taxon comprised of Craseonycteridae, Hipposideridae, Megadermatidae, Rhinolophidae, Rhinopomatidae, and Pteropodidae). However, if the standardization of the nomenclature suggested by Alonso-Zarazaga (2005) is followed, '-formes' indicates ordinal level, while the subordinal suffix should be '-formi', leading to the suborder names Vespertilioniformi and Pteropodiformi.

Family Pteropodidae Gray, 1821

* Pteropidae Gray, 1821: 299.

For the correct formation on the family name, see Handley (1980).

The most recent suprageneric division of the Pteropodidae is the one presented by Almeida et al. (2016: 83), who recognize two subfamilies with representatives on the African continent: Rousettinae (subdivided into seven tribes: Rousettini: Rousettus; Eonycterini: Eonycteris; Scotonycterini: Scotonycteris, Casinycteris; Epomophorini: Epomophorus, Epomops, Hypsignathus, Nanonycteris, Micropteropus; Stenonycterini: Stenonycteris; Myonycterini: Myonycteris [including Lissonycteris], Megaloglossus; Plerotini: Plerotes), and Eidolinae (Eidolon).

Subfamily Eidolinae Almeida, Giannini \& Simmons, 2016

Genus Eidolon Rafinesque, 1815

Eidolon helvum (Kerr, 1792)

Fig. 3A-B

Vespertilio vampyrus helvus Kerr, 1792: 91.

* Eidolon helvum (Kerr, 1792).

Hayman et al. (1966: 74) reported a total of 347 specimens from almost all over the DRC. The 560 specimens we found do not lead to a large extension of the range, but we do have some records from Rwanda and Burundi and from the southern part of Haut Kantanga Province. On the overall distribution, Thomas \& Henry (2013a) show an almost uniform distribution south of the Sahara, with the exception of the drier areas of NE and SW Africa. Our assement of the distribution seems to suggest a split between a northern area and a southern/eastern area, which runs through the DRC provinces of Tanganyika, HautLomami and Lualaba. Whether this is a real gap or only a result of the lack of voucher specimens cannot be determined at this time. 
Subfamily Rousettinae K. Andersen, 1912

Tribe Epomophorini Gray, 1866

Genus Epomophorus Bennett, 1836

Epomophorus anselli Bergmans \& Van Strien, 2004

Fig. 3C-D

Epomophorus anselli Bergmans \& Van Strien, 2004: 258.

Nesi (2012: 179) reported a single specimen of this species from Kitombongo (Upemba National Park, Haut-Lomami Province). Bergmans (2013) mentions this species from two (possibly three) localities in Malawi and one in southwestern Tanzania. Based on these data, the SDM map suggests that $E$. anselli might occur from southeastern DRC over northeastern Zambia to southwestern Tanzania and northern Malawi.

Epomophorus crypturus Peters, 1852

Fig. 3E-F

* Epomophorus crypturus Peters, 1852: 26.

* Epomophorus gambianus (Ogilby, 1835): 100.

Some authors, e.g., Nesi et al. (2011: 547), consider E. crypturus and E. gambianus to differ at the subspecific level only. However, they admit that several recent classifications suggest they differ at the species level. Claessen \& De Vree (1990: 180) indicate that E. gambianus only occurs in the tropical savanna and woodland zones from Senegal to the Central African Republic, Sudan and Ethiopia. E. crypturus, on the other hand, only occurs in Zambia, southern Tanzania and southern DRC. Nesi et al. (2011: 547) furthermore add Mozambique and eastern RSA. As all of the E. gambianus specimens mentioned by Hayman et al. (1966: 23, map 6) are from the southernmost part of the DRC, these should be assigned to E. crypturus. The distribution map shows that all of the specimens currently assigned to E. crypturus are from the provinces of Haut-Katanga, Haut-Lomami and Lualaba, with one exception: RMCA 31557, a subadult female from Luluabourg (= Kananga) in Kasaï-Central Province. This specimen might need to be re-examined.

Epomophorus labiatus (Temminck, 1837)

Fig. 4A-B

Pteropus labiatus Temminck, 1837: 83.

* Epomophorus anurus Heuglin, 1864: 12.

Bergmans (1988) and Claessen \& De Vree (1991) considered E. anurus to be a synonym of E. labiatus. Bergmans (1997: 72), however, pointed out that some of the E. labiatus specimens identified by Claessen \& De Vree (1991) might be erroneously assigned to this species, and that their material included multiple species (e.g., E. anselli Bergmans \& Van Strien, 2004). See also Happold (2013a: 245), who indicated that even more species might be present.

The distribution map indicates that E. labiatus is primarily found along the eastern borders of the DRC and in Rwanda and Burundi, but also that there are some records from the more central parts of the DRC. Specimen RMCA 31127 from Léopoldville (= Kinshasa) probably needs reinvestigation. However, the SDM map indicates that other labiatus specimens have been found in Cameroon, Nigeria and Ghana, which might suggest that this species might also occur in the western part of the DRC.

Epomophorus minimus Claessen \& De Vree, 1991

Fig. 4C-D

Epomophorus minimus Claessen \& De Vree, 1991: 216. 

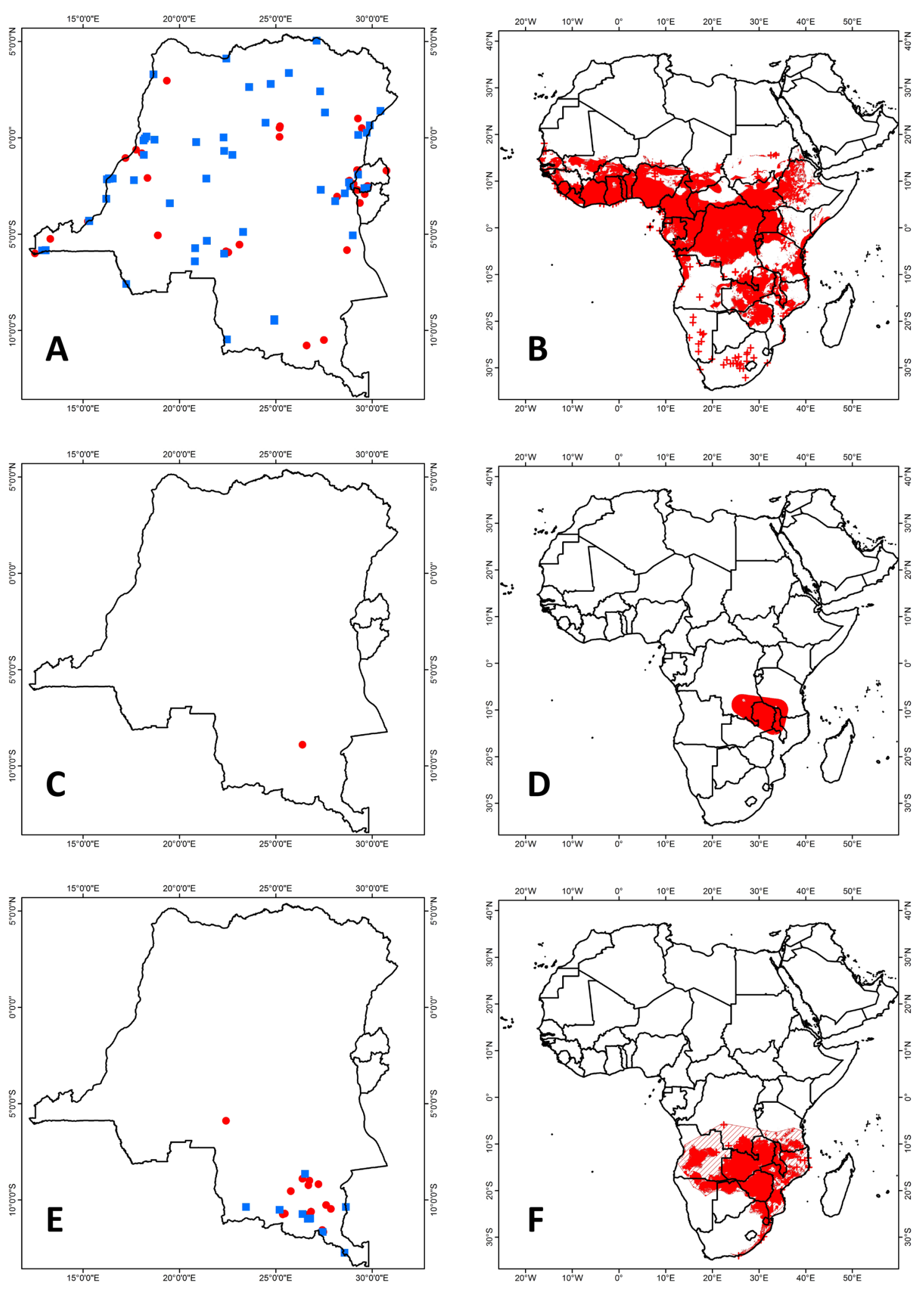

Fig. 3. Distribution maps. A-B. Eidolon helvum (Kerr, 1792). C-D. Epomophorus anselli Bergmans \& Van Strien, 2004. E-F. Epomophorus crypturus Peters, 1852. A, C, E. Distribution in the CRB area. B, D, F. Pan-African distribution. 
From the same locality as above (Kitombongo), Nesi (2012: 179) also reported the presence of E. minimus. He also reported it from a second locality near by: Luena. Happold (2013b: 248) indicated that the species is known from approximately 40 localities in northeastern Africa, in Ethiopia, Somalia, Kenya and Uganda, which are at quite some distance from these DRC localities. Re-investigation of the MNHN specimens might, therefore, be necessary to confirm their identity.

\section{Epomophorus minor Dobson, 1880}

Fig. 4E-F

Epomophorus minor Dobson, 1880: 715.

* Epomophorus labiatus minor (Dobson, 1880).

Happold (2013a) followed Claessen \& De Vree (1991) in considering minor to be a junior synonym of Epomophorus labiatus. She also pointed out that Bergmans (1997) was strongly opposed to this, as he pointed out a number errors concerning the E. labiatus specimens used by Claessen \& De Vree (1991).

The distribution map shows that the specimens from the eastern part from the DRC fit well with the major (eastern African) distribution area of the species. The specimen from Congo da Lemba (RMCA 408a, a juvenile male) does seem to fit with a potential distribution along the Angolan coast, which, however, is not yet supported by material from that country (ACR 2016).

Epomophorus wahlbergi (Sundevall, 1846)

Fig. 5A-B

Pteropus Wahlbergi Sundevall, 1846: 118.

* Epomophorus wahlbergi haldemani (Halowell, 1846): 52.

Bergmans (1988: 134) discussed the status of the two subspecies of E. wahlbergi and concluded that the size variations are of a clinal nature and the recognition of subspecies does not seem to be appropriate, a view which is also followed by Happold (2013c).

The records in Hayman et al. (1966: 24) were primarily from the southern half of the DRC, with two specimens from Rutshuru (Nord-Kivu Province - INPBC V1346 and V1636), and one specimen from Ibembo (Bas-Uélé Province - RMCA 20278). The additional material we report on, is also from the southern part of the DRC, but also from the Nord-Kivu and South-Kivu Provinces, as well as from Burundi and Rwanda. The locality of a further central Congolese specimen (RMCA 18844b from Bokungu in Tshuapa Province) is rather unexpected, as Bergmans (1988: 131) stated that E. wahlbergi is not found moist forests.

Genus Epomops Gray, 1870

Epomops dobsonii (Bocage, 1889)

Fig 5C-D

Epomophorus Dobsonii Bocage, 1889: 1.

* Epomops dobsoni (Bocage, 1889)

Bergmans (1989: 89) suggested that a number of cranial characters (e.g., the pterygoid wing morphology, the post dental palate and palatal ridges) of E. dobsonii are rather atypical for an Epomops and show more resemblance with Epomophorus, suggesting that the original generic assignment by Bocage might be correct. He nevertheless kept the species in Epomops. However, very recently a phylogenetic study 

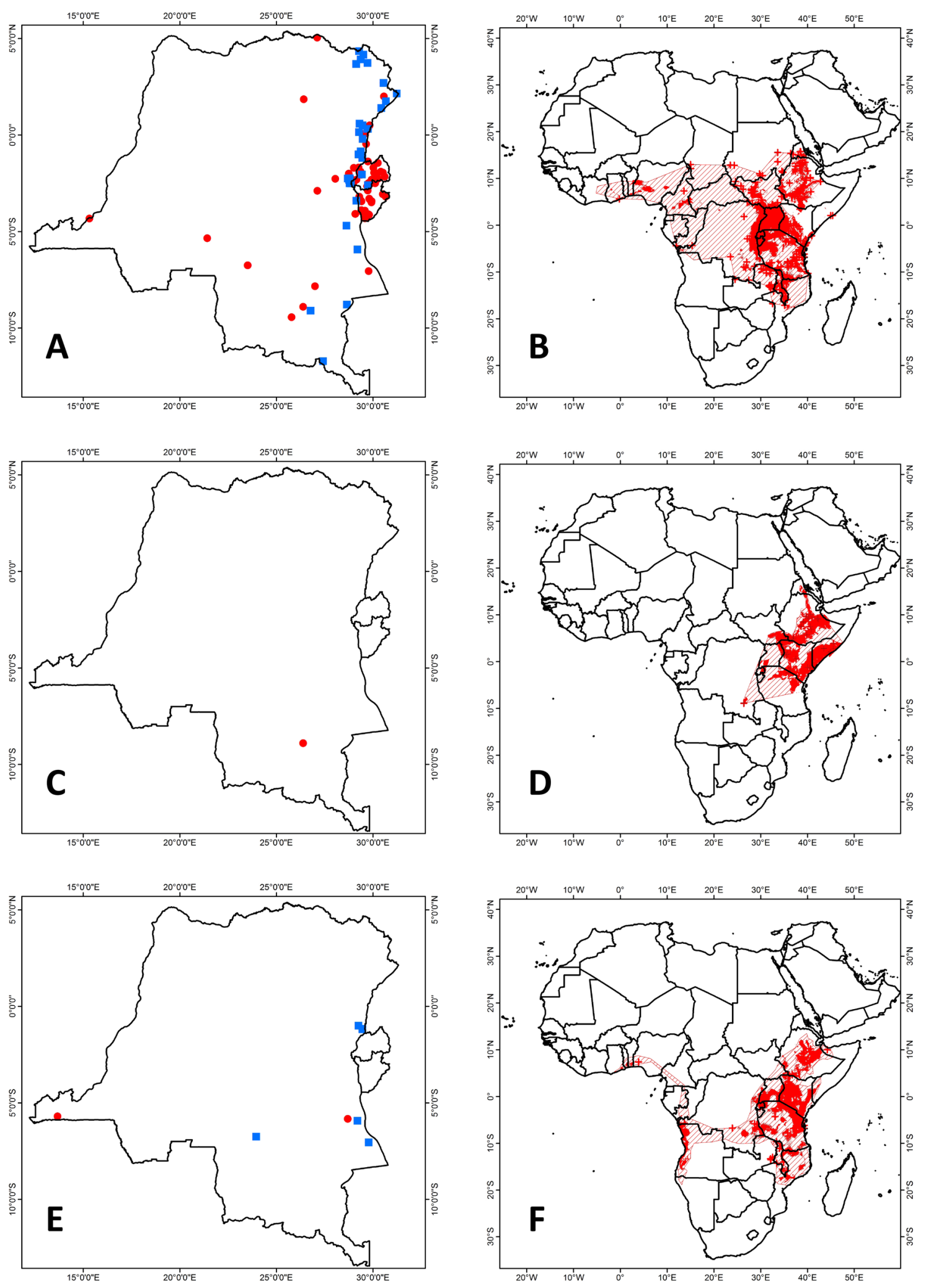

Fig. 4. Distribution maps. A-B. Epomophorus labiatus (Temminck, 1837). C-D. Epomophorus minimus Claessen \& De Vree, 1991. E-F. Epomophorus minor Dobson, 1880. A, C, E. Distribution in the CRB area. B, D, F. Pan-African distribution. 
by Almeida et al. (2016: 83) showed that dobsonii is most closely related to Epomophorus wahlbergi and these authors transferred the species to the genus Epomophorus.

Distributionwise, E. dobsonii was reported by Hayman et al. (1966: 24, map 9) from the extreme southern part of the DRC (provinces of Haut-Katanga and Lualaba). Further material from these provinces is reported in this paper, but also some records from Haut-Lomami Province (near the Upemba National Park), and from Kasende (MCZ 31738; near Kananga in Kasaï-Central Province). The specimens from Rwanda (Shangi Schad, RMCA 85.006-M-0463 and Rubona, RMCA 16037) extend the distribution range of the species. Bergmans (1979), who already reported on the latter specimen, indicated that this extension was not beyond the already known vegetation types: mopane and miombo woodlands, and the Central African rain forest block.

Epomops franqueti (Tomes, 1860)

Fig. 5E-F

Epomophorus franqueti Tomes, 1860: 54.

* Epomops franqueti franqueti (Tomes, 1860).

Bergmans (1989: 113) could not find any differences between E. f. franqueti and the West African E. f. strepitans K. Andersen, 1910 and considered the latter as a synonym of the nominate form.

Hayman et al. (1966: 24, map 10) provided records from all over the DRC, with the exception of the former Katanga Province. From that province, we report one specimen: RMCA 10514 from Mulongo, in the current Tanganyika Province. The Pan-African distribution of the species indicates that is primarily a forest species, but with some occurrences in savanna areas.

Genus Hypsignathus H. Allen, 1862

Hypsignathus monstrosus $\mathrm{H}$. Allen, 1862

Fig. 6A-B

H[ypsignathus] monstrosus H. Allen, 1862 (for 1861): 157.

* Hypsignathus monstrosus H. Allen, 1862.

The distribution of this large bat is very similar to that of Epomops franqueti. Also, this species occurs over almost the entire DRC, with the exception of the former Katanga Province. The additional specimens we were able to track fill in the spaces between the records reported by Hayman et al. (1966: 25, map 11), but do not expand the distribution area reported by these authors.

Genus Micropteropus Matschie, 1899

Micropteropus intermedius Hayman, 1963

Fig. 6C-D

* Micropteropus intermedius Hayman, 1963: 100.

Hayman et al. (1966: 27, map 13) reported two specimens belonging to this species, one from Thysville (= Mbanza-Ngungu) and one from Luluabourg (= Kananga). One additional specimen was reported by Bergmans (1989: 101) from "Banana/Netonna". Here, we add two further specimens from Luluabourg. All of these localities are relatively close to the Angolan border, where the holotype was collected.

As indicated by Bergmans (1989: 103) a number of these specimens were originally assigned to other, similar-sized epomophorine species (e.g., M. pusillus or Epomophorus grandis [not yet reported from 

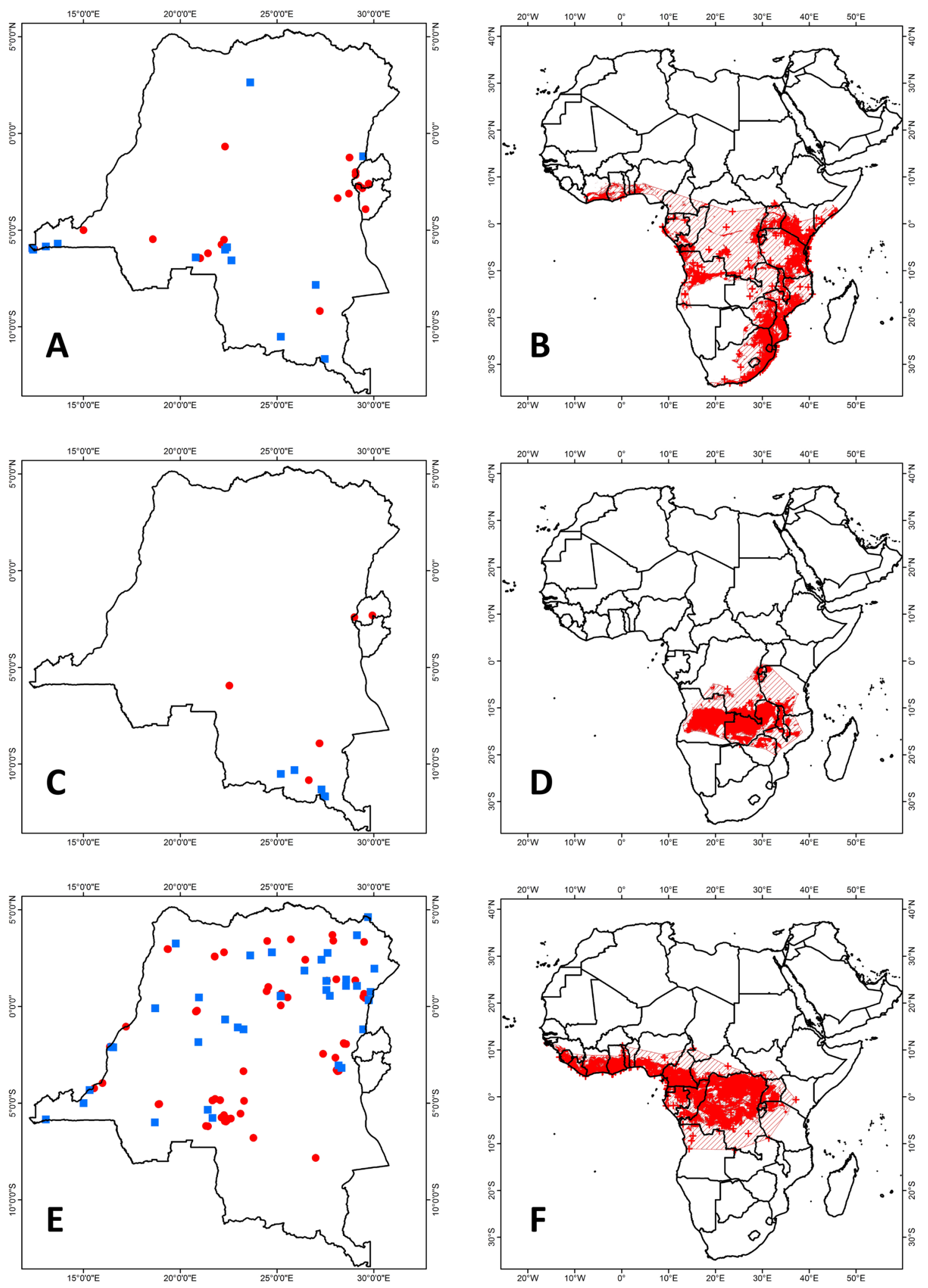

Fig. 5. Distribution maps. A-B. Epomophorus wahlbergi (Sundevall, 1846). C-D. Epomops dobsonii (Bocage, 1889). E-F. Epomops franqueti (Tomes, 1860). A, C, E. Distribution in the CRB area. B, D, F. Pan-African distribution. 
$\mathrm{CRB}])$ due to the careless preservation or examination of the soft palate. Therefore, we cannot exclude that some of the specimens reported here as M. pusillus might belong to the current species.

\section{Micropteropus pusillus (Peters, 1867)}

Fig. 6E-F

Epomophorus pusillus Peters, 1867: 870.

* Micropteropus pusillus (Peters, 1867).

Compared with Hayman et al. (1966: map 14), the distribution for this species has been extended in the eastern part of the DRC into Burundi, and in the southwestern part of the DRC, but in the northwestern part of the country it seems to be lacking. The specimens from Boende seem a bit out of place, as Thomas \& Henry (2013b: 271) mentioned that it avoids the Congolian rainforest except around its edges. Monfort (1992: 142) believes this species occurs in Rwanda, based on a reference to "RuandaUrundi" by Schouteden (1947) and the locality "Congo-Nil-Aka" mentioned by Hayman et al. (1966: 27 - RBINS 13522). Monfort claims this locality to refer to "Nil-Akagera", which is in Rwanda and not in Burundi, but Hayman et al. (1966: 81) provide coordinates for "Congo-Nil-Akka", 04³5' N, $29^{\circ} 45^{\prime}$ E, which put it in the Garamba National Park on the border with South Sudan.

Genus Nanonycteris Matschie, 1899

Nanonycteris veldkampii (Jentink, 1888)

Fig. 7B

Epomophorus veldkampii Jentink, 1887: 51.

Bergmans (1989: 147) referred to Frechkop (1954), who mentioned two N. veldkampii specimens from Lusinga (Upemba National Park, Haut Lomami Province). These immature specimens were re-identified by Hayman et al. (1966: 28) as Plerotes anchietae (Seabra, 1900).

The Paris museum, however, has three N. veldkampii specimens collected in the DRC by Nicolas Nesi, Blaise Kadjo and Christiane Denys in May 2010. Unfortunately, no specific locality for these specimens was available. The distribution map presented by Fahr (2013a: 278) indicates that this species primarily occurs in West Africa, from Guinea to western Cameroon, but there is also a record from Bangui in the Central African Republic. Based on this information, we can assume that $N$. veldkampii will occur in the northwestern part of the DRC.

Tribe Myonycterini Lawrence \& Novick, 1963

Genus Megaloglossus Pagenstecher, 1885

Megaloglossus woermanni Pagenstecher, 1885

Fig. 7C-D

* Megaloglossus woermanni Pagenstecher, 1885: 245.

* Megaloglossus woermanni prigoginei Hayman, 1966: 26.

In Hayman et al. (1966), Hayman described a separate subspecies for nine skins and skulls collected by Dr. A. Prigogine in the former Kivu Province and one additional specimen collected by Mr. Schepens in Lundjulu (currently in Nord-Kivu Province). This subspecies differed from the West African nominate subspecies in larger forearm length and skull size, and darker colour.

Bergmans \& Van Bree (1972) [in Bergmans 1997] found that the forearm length and the greatest length of the skull of the holotype of $M$. woermanni from Gabon fell within the range given by Hayman for 

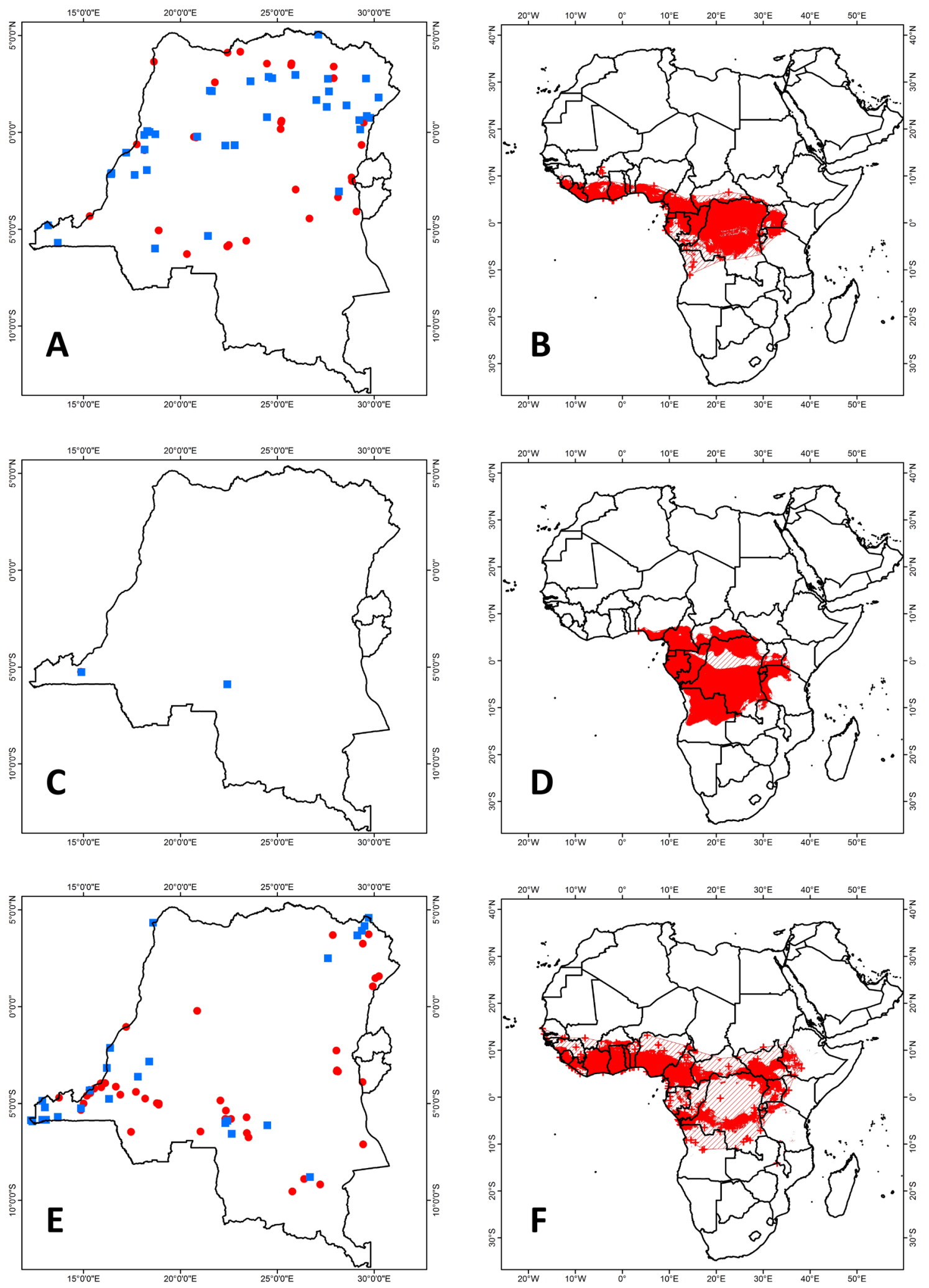

Fig. 6. Distribution maps. A-B. Hypsignathus monstrosus H. Allen, 1862. C-D. Micropteropus intermedius Hayman, 1963. E-F. Micropteropus pusillus (Peters, 1867). A, C, E. Distribution in the CRB area. B, D, F. Pan-African distribution. 
prigoginei, and should, therefore, be considered a synonym of the typical form, a view that has been accepted by almost all subsequent authors.

Distributionwise, Hayman et al. (1966: 26, map 12) reported this species from the northern part of the DRC. The additional records we report extend its presence in the southern part of the country, in the provinces Congo Central, Kwango, Kwilu, Kasaï and Kasaï-Oriental. The southernmost record is RMCA 33065 from the Kakanda Cave near Likasi (former Jadotville). Its occurrence so far from the modelled distribution makes it a bit doubtful, but the record was mentioned by Bergmans (1997: 60), who thereby confirmed its identification and locality information.

Genus Myonycteris Matschie, 1899

Myonycteris angolensis (Bocage, 1898)

Fig. 7E-F

Cynonycteris Angolensis Bocage, 1898: 133, 138.

* Rousettus (Lissonycteris) angolensis (Bocage, 1898).

The lack of acoustical orientation (as only species with Rousettus s.l.), their different mode of locomotion, and a number of characters which more closely resembles those of Eidolon and other pteropodids than of Rousettus, already made Novick (1958) [in Aellen \& Brosset 1968] and Lawrence \& Novick (1963) conclude that the subgeneric status of Lissonycteris should be raised to full generic status. Lawrence \& Novick (1963: 4) furthermore postulated from Myonycteris specimens stored in alcohol that the posture of their wings in life is like that of Lissonycteris. This character, together with the slender rostrum, made them propose to include the two genera in a myonycterine section. The narrowing of the anterior palate (which results in the upper tooth rows bending inwards) and the relative heaviness of $\mathrm{P}^{4}$ reported by Bergmans (1980: 179) confirmed this grouping, as did the very similar karyotype (only a different polymorphism in pair 1) reported by Haiduk et al. (1981:223). Genetic sequence analyses by Agnarsson et al. (2011) and Almeida et al. (2011) both confirmed the close relationship between Lissonycteris and Myonycteris, and led Nesi et al. (2012: 133) to conclude that Lissonycteris should be considered a synonym of Myonycteris.

The distribution of M. angolensis across CRB is split into three areas: one in Kongo Central Province in the extreme west, a second in the extreme southeastern part of the DRC (Haut-Katanga and Haut-Lomami Provinces) and a third along the eastern border of the DRC, extending into Rwanda and Burundi. More recently, the species has also been collected in the Kisangani area (Tshopo Province). RMCA 9904a, reported from Kabalo (Tanganyika Province) by Hayman et al. (1966: 30), was actually collected in the foothills of the Ruwenzori Mountains (Wim Wendelen, pers. comm.).

\section{Myonycteris relicta Bergmans, 1980}

Fig. 8A-B

Myonycteris relicta Bergmans, 1980: 171, 173.

One specimen, identified as belonging to this species by Norberto Giannini, was collected in the Kasai Province (Beyi - Kalonji). Taylor (2013: 274) reported it from the coastal forest mosaic from southeastern Kenya to southeastern Tanzania and from the Zambezian woodland in eastern Zimbabwe. The Durban Natural Science Museum also has a specimen from Mozambique (ACR 2016: 998). Based on these data, the SDM map shows that the species might potentially occur in southern Uganda and in the border area between the DRC and Tanzania, and that the currently reported locality might be too far to the west. 


\section{No data available}

A
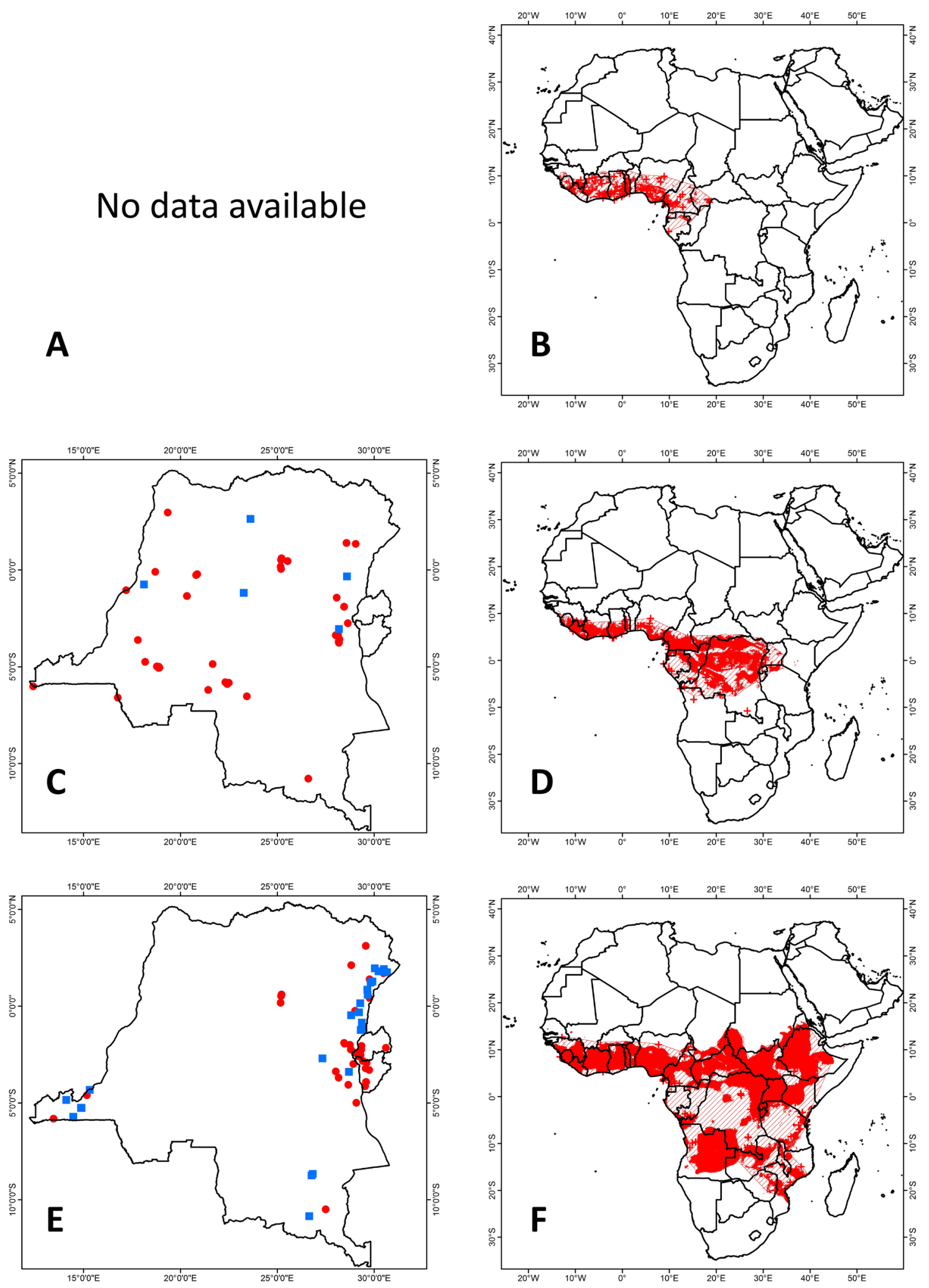

Fig. 7. Distribution maps. A-B. Nanonycteris veldkampii (Jentink, 1888). C-D. Megaloglossus woermanni Pagenstecher, 1885. E-F. Myonycteris angolensis (Bocage, 1898). A, C, E. Distribution in the CRB area. B, D, F. Pan-African distribution. 


\section{Myonycteris torquata (Dobson, 1878) \\ Fig. 8C-D}

Cynonycteris torquata Dobson, 1878: 71, 76.

* Myonycteris wroughtoni Andersen, 1908: 450.

Bergmans (1976) reviewed the genus Myonycteris and referred to Eisentraut (1963) and Brosset (1966a, b), who suggested that wroughtoni should be considered a subspecies of $M$. torquata, a view that was followed by Hayman \& Hill (1971), Jones (1971) and Koopman (1975, 1994). Bergmans (1976: 203-204) found that wroughtoni differs from torquata mainly in its somewhat larger greatest skull and forearms lengths and by a larger relative $\mathrm{M}^{2}$ length, and concluded that these differences are insufficient to warrant a subspecific division.

Hayman et al. (1966: 29, map 16) reported a total of nine specimens, all from the northeastern part of the DRC: provinces of Bas-Uélé, Haut-Uélé and Nord-Kivu. We were able to find data for 583 specimens from almost all over the DRC. The map provided by Thomas \& Henry (2013c: 275) is quite different as they do not mention M. torquata from the provinces of Tshopo and Tshuapa. The reason for this is probably the extremely recent nature of the collections: from 2010 onwards, although the species was previously reported from Kisangani (Tshopo Province) by Ifuta et al. (1987: 113).

Tribe Plerotini Bergmans, 1997

Genus Plerotes K. Andersen, 1910

Plerotes anchietae (Seabra, 1900)

Fig. 8E-F

Epomophorus anchietce Seabra, 1900: 116.

* Plerotes anchietae (Seabra, 1900).

Hayman et al. (1966: 29) mention three specimens from the DRC, two from Lusinga (RBINS 10685 and 10686) and one from Panda (RMCA 26210). The latter specimen, however, was examined by Bergmans (1989: 147), who found it to be an immature Epomophorus (probably minor). Bergmans (1989: 147) also referred to another specimen in the RMCA collection that was supposed to be from Likasi (former Jadotville), but indicated that this bat could not be traced. The information provided on the museum's holdings accessed through Wim Wendelen did not mention any specimen from Likasi either.

Tribe Rousettini K. Andersen, 1912

Genus Rousettus Gray, 1821

Rousettus aegyptiacus (E. Geoffroy St.-Hilaire, 1810)

Fig. 9A-B

Pteropus Egyptiacus E. Geoffroy Saint-Hilaire, 1810: 96.

* Rousettus aegyptiacus leachi (Smith, 1823): 433.

Happold (2013d: 290) distinguishes four African subspecies in R. aegyptiacus, of which leachii occurs from NE DRC to Ethiopia and southwards to South Africa, as well as on the islands of Pemba, Zanzibar and Mafia. A second subspecies, unicolor (Gray, 1870), occurs from Senegal to Cameroon, Bioko Island, and southwards to Angola. Bergmans (1994: 91) indicates that in leachii, a weaker skull and dentition, as compared to the typical subspecies, justifies the separation as a subspecies, but he also indicates that the variation within leachii is very large and that it would be worth examining a larger series from over its entire distribution area. Hayman \& Hill (1971: 12) furthermore mention that the morphological 

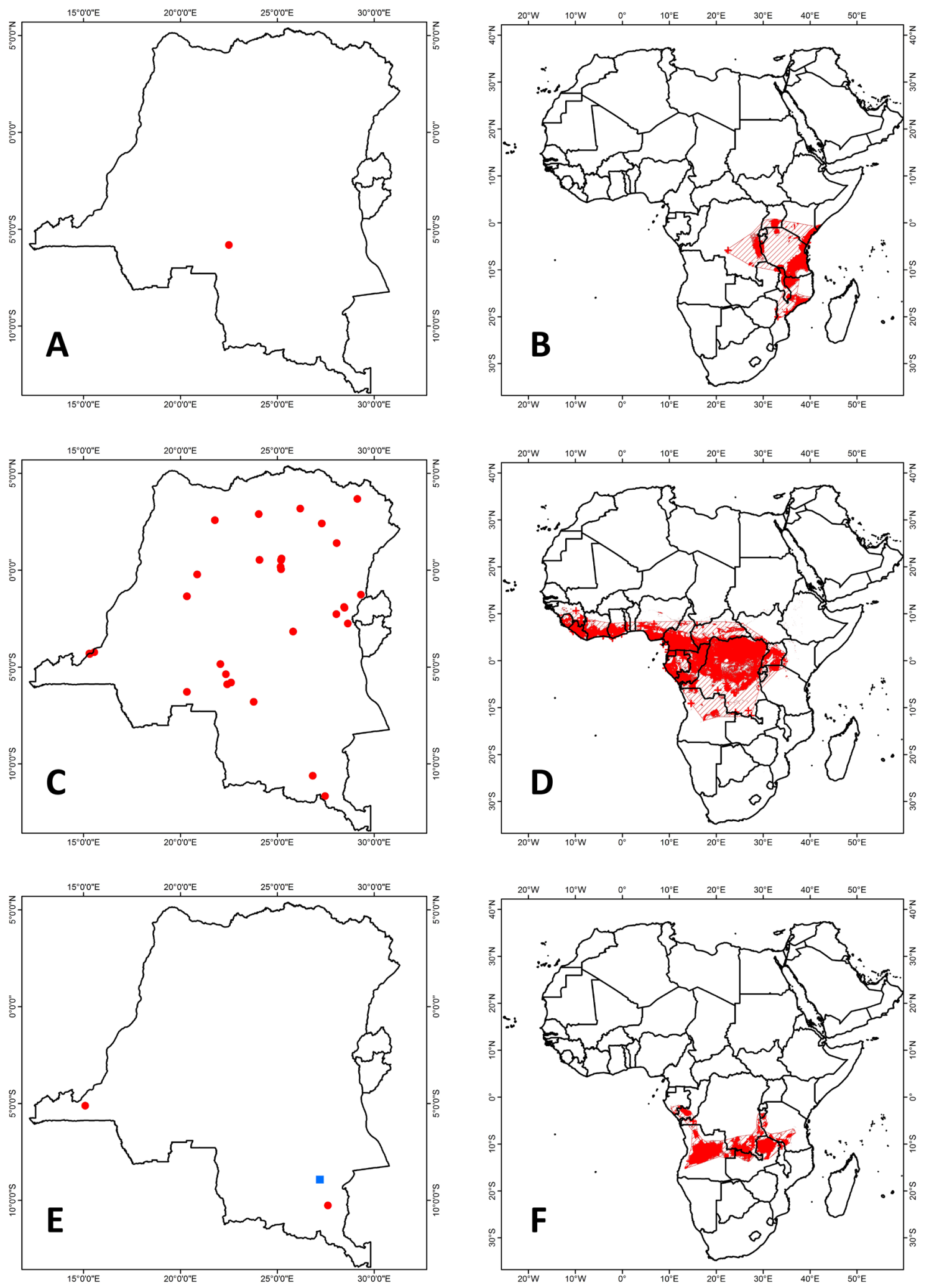

Fig. 8. Distribution maps. A-B. Myonycteris relicta Bergmans, 1980. C-D. Myonycteris torquata (Dobson, 1878). E-F. Plerotes anchietae (Seabra, 1900). A, C, E. Distribution in the CRB area. B, D, F. Pan-African distribution. 
and geographic limits of aegyptiacus and leachii are not yet clear, and intergrading may occur in the equatorial zone.

The distribution information provided by Hayman et al. (1966:30, map 17) suggested that $R$. aegyptiacus only occurred along the eastern border of the DRC (Ituri, Nord- and Sud-Kivu Provinces) and in the extreme south (Haut-Katanga Province) and the extreme west (Kongo Central Province). However, RBINS 12932 and 12933, reported by Hayman et al. (1966: 30) and Bergmans (1994: 90) from Kivu, were actually from Buta (Bas-Uélé Province; Tom Geerinckx, pers. comm.). More recently collected specimens further extend the occurrence of this species to the west of the DRC (Kisangani, Boende). These records indicate that two subspecies might occur in CRB: leachii in the eastern part of the DRC and in Rwanda and Burundi, and unicolor in the western part of the country. The subspecific assignment of the specimens from the intermediairy localities remains uncertain.

Tribe Scotonycterini Bergmans, 1997

Genus Casinycteris Thomas, 1910

Casinycteris argynnis Thomas, 1910

Fig. 9C-D

* Casinycteris argynnis Thomas, 1910: 111.

Hayman et al. (1966: 21, map 1) only mentioned three specimens from Irangi, Stanleyville and Medje in the northeastern part of the DRC and one further unnumbered and unmapped specimen from Luluabourg in the former Kasai Province. Meirte (1983) and Bergmans (1991: fig 4) already expanded its distribution in the DRC further to the west, and Hassanin et al. (2015: 198) indicated that $C$. argynnis is endemic to the Congo Basin, from southeastern Cameroon and eastern Gabon in the west, across southwestern Central African Republic, the Republic of the Congo and all provinces of the DRC, except Bas-Congo, to the Mitumba Mountains in eastern DRC. The latter, however, doesn't seem to be substantiated entirely by specimens we were able to trace.

Genus Scotonycteris Matschie, 1894

Scotonycteris bergmansi Hassanin et al., 2015

Fig. 9E-F

Scotonycteris bergmansi Hassanin, Khouider, Gembu, Goodman, Kadjo, Nesi, Pourrut, Nakouné \& Bonillo, 2015: 206.

* Scotonycteris zenkeri Matschie, 1894: 202.

Hassanin et al. (2015) reviewed the Scotonycterini tribe, which contained four species: Scotonycteris zenkeri Matschie, 1894, Casinycteris argynnis Thomas, 1910, C. campomaanensis Hassanin, 2014, and C. ophiodon (Pohle, 1943). Their analyses of cytochrome $b$ and nuclear DNA revealed that S. zenkeri should be split up into four clades representing three species: the extralimital (to CRB) S. zenkeri and S. occidentalis Hayman, 1947, and S. bergmansi Hassanin et al., 2015 occurring in the rainforests of Gabon, Equatorial Guinea, Republic of Congo, southern Central African Republic, and eastern Democratic Republic of the Congo. Hassanin et al. (2015) also found sufficient differences between the population from the western part of the distribution area (Gabon to Central African Republic) and the eastern part of the DRC to recognize two subspecies: the western $S . b$. bergmansi and the eastern S. b. congoensis Hassanin et al., 2015.

Hayman et al. (1966: 21) reported this species (as S. zenkeri) from Beni and Kiloboze (with the erroneous number RMCA 3145), in the extreme eastern part of the DRC. The new records extend the occurrence of 

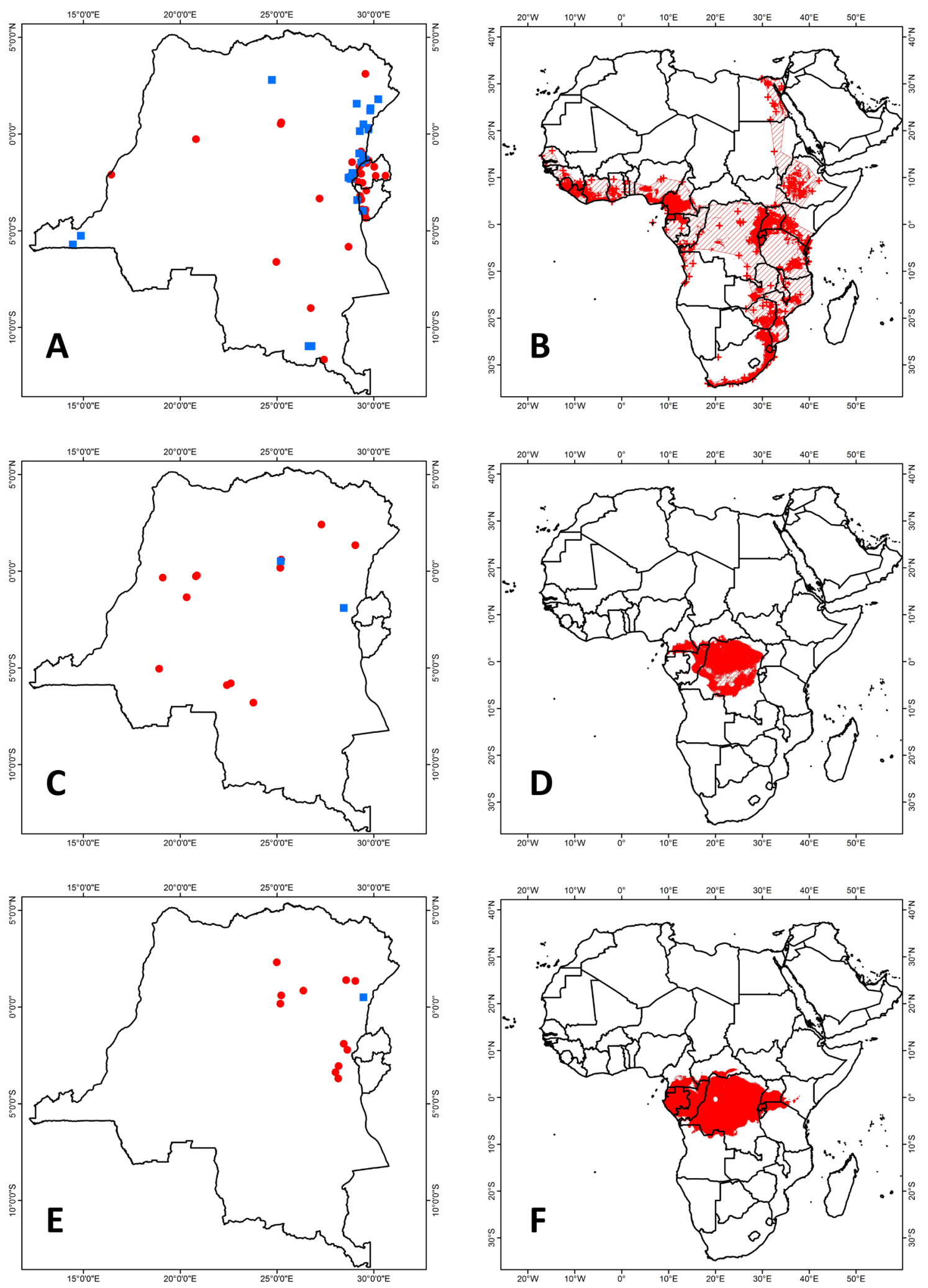

Fig. 9. Distribution maps. A-B. Rousettus aegyptiacus (E. Geoffroy St.-Hilaire, 1810). C-D. Casinycteris argynnis Thomas, 1910. E-F. Scotonycteris bergmansi Hassanin et al., 2015. A, C, E. Distribution in the CRB area. B, D, F. Pan-African distribution. 
the species much more to the west, up to the region around Boende in Tshuapa Province. Kipalu (2009) reported a specimen of $S$. zenkeri from near Mbanza-Ngungu (Kongo Central Province), but no details were given on voucher specimens.

Tribe Stenonycterini Nesi, Kadjo, Pourrut, Shongo, Cruaud \& Hassanin, 2012

Genus Stenonycteris Gray, 1870

Stenonycteris lanosus (Thomas, 1906)

Fig. 10A-B

Rousettus lanosus Thomas, 1906: 137.

*Rousettus (Stenonycteris) lanosus Thomas, 1906.

The separation of Stenonycteris from Rousettus was already suggested by Leche (1921) and Kingdon (1974) [in Bergmans (1994: 111)], but was only proven by genetic sequence analyses (Juste B. et al. 1999; Almeida et al. 2011; Nesi et al. 2012). Using cytochrome $b$ sequences, Nesi et al. (2012) found that the genus Rousettus was paraphyletic, and that Stenonycteris should be considered as a separate genus, even constituting its own tribe: Stenonycterini.

Hayman et al. (1966: 20, map 18) reported this species from the Kivu Province in the eastern part of the DRC. The additional specimens reported here are also mainly from the Nord- and Sud-Kivu Provinces, with extensions into Rwanda and Burundi. The only outlier is RMCA 36250 from Tshibasha, near Kananga (Kasaï-Central Province). The SDM map shows that this specimen is the westernmost representative of the species, but it also shows that the distribution is rather patchy to the east too, with a cluster of specimens in Kenya, and some dispersed records in Tanzania and Ethiopia.

Family Hipposideridae Lydekker, 1891

Genus Asellia Gray, 1838

Asellia tridens (E. Geoffroy St.-Hilaire, 1813)

Fig. 10C-D

Rhinolophus tridens E. Geoffroy Saint-Hilaire, 1813: 260.

Aulagnier (2013: 362) indicates that Asellia tridens is a desert species that occurs in the Sahara and Sahel savanna areas of northern Africa, which is quite some distance from the Kivu area in the DRC, where the 18 specimens reported here have been collected. However, the Paris museum also has a specimen from Zanzibar, which suggests that the species might occur more to the south than generally accepted.

\section{Genus Doryrhina Peters, 1871}

Very recently, Foley et al. (2017: 12) analysed the cytochrome $b$ sequences of the members of the genus Hipposideros and found this for " $\mathrm{H}$. cyclops" to be that different that they assigned this species to a separate genus: Doryrhina. This is also confirmed by the karyotype, which contains 36 chromosomes, whereas the members of the genus Hipposideros only have 32 .

Doryrhina cyclops (Temminck, 1853)

Fig. 10E-F

Phyllorrhina cyclops Temminck, 1853: 75.

* Hipposideros cyclops (Temminck, 1853).

Hayman et al. (1966: 44-45, map 51) only mentioned specimens from the northeastern part of the DRC (provinces of Nord-Kivu, Haut-Uélé, Bas-Uélé and Tshopo). Here, we report on additional specimens 

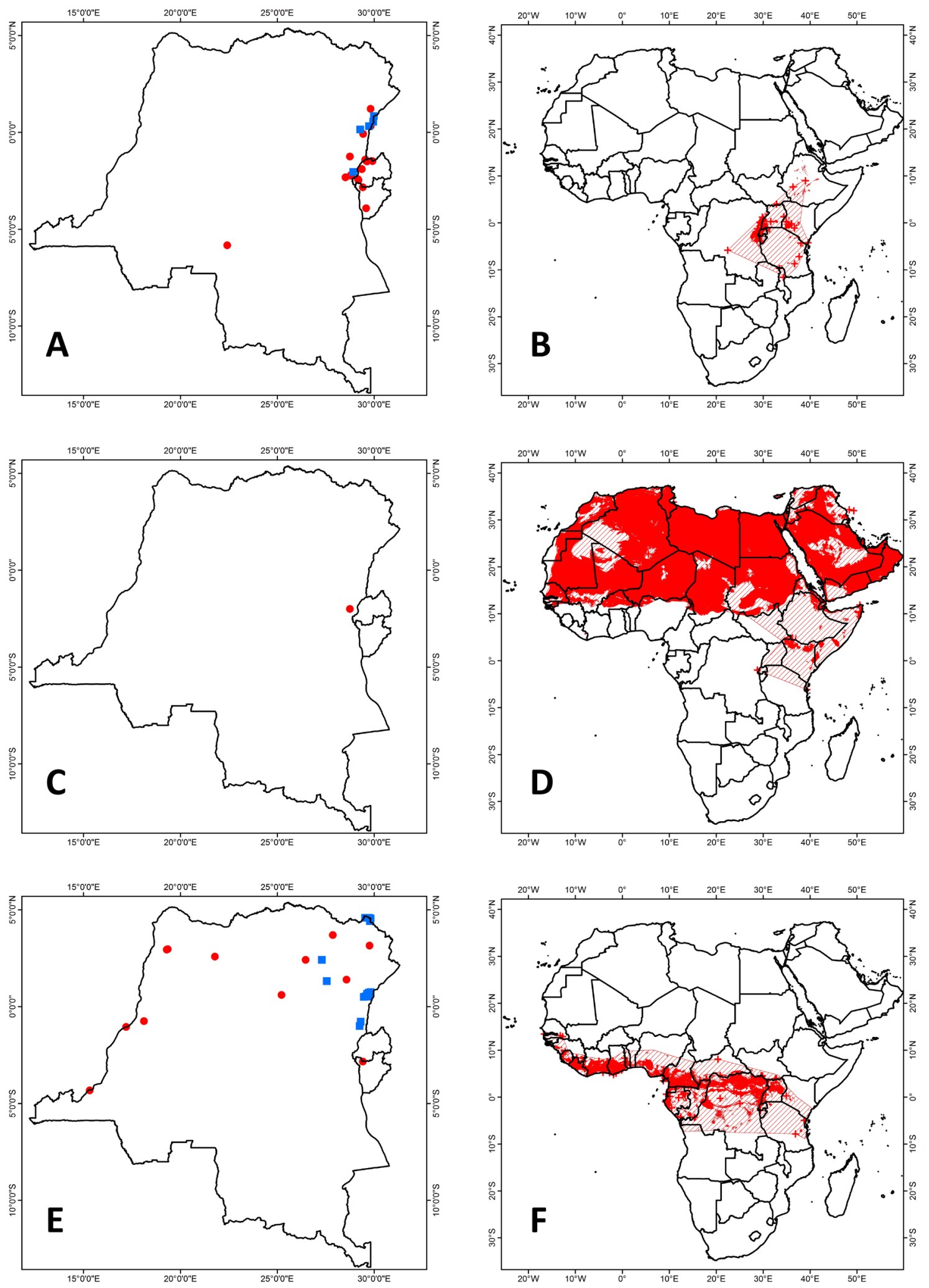

Fig. 10. Distribution maps. A-B. Stenonycteris lanosus Thomas, 1906. C-D. Asellia tridens (E. Geoffroy St.-Hilaire, 1813). E-F. Doryrhina cyclops (Temminck, 1853). A, C, E. Distribution in the CRB area. B, D, F. Pan-African distribution. 
from over almost the entire northern part of the DRC, and down along the Congo River to Kinshasa. The SDM map is very similar to the one presented by Fahr (2013d: 381), although we do believe that there is a connection between the populations from the DRC and those from Gabon and Cameroon (see also ACR 2016: 172).

Genus Hipposideros Gray, 1831

Hipposideros abae J.A. Allen, 1917

Fig. $11 \mathrm{~A}-\mathrm{B}$

Hipposideros abce J.A. Allen, 1917: 432.

* Hipposideros abae J.A. Allen, 1917.

Hipposideros abae is in the DRC only known from the extreme northeastern part of the country. Most records are from the Garamba National Park and from Aba (Haut-Uélé Province). There is one additional specimen from Digba (Bas-Uélé Province). The map provided by Happold (2013h: 372) shows a very patchy distribution from Guinea-Bissau in West Africa to northwestern Uganda. Based on climatic data, we suspect that the species might occur even further to the east through South Sudan to western Ethiopia.

Hipposideros beatus K. Andersen, 1906

Fig. 11C-D

Hipposiderus beatus K. Andersen, 1906: 275, 279.

* Hipposideros beatus Andersen, 1906.

* Hipposideros beatus maximus Verschuren, 1957: 365.

Hayman et al. (1966: 41, map 47) reported this species from the Garamba National Park and Faradje in Haut-Uélé Province, from Buta in Bas-Uélé Province, and from Ikengo in Equateur Province, all in the northern part of the DRC. Here, we report on additional specimens from the provinces of Ituri, Mongala, and Tshopo (also in the northern part of the DRC), Sud-Kivu, and from Thysville (=Mbanza-Ngungu) in the Kongo Central Province. Also for this species, Happold (2013i: 374) gives a patchy Pan-African distribution from Sierra Leone in West Africa to the eastern DRC. In this area it is found in closed forests, degraded areas within the lowland rainforest, coastal woodlands and coastal scrub, and mosaics of thickets and grasslands, usually (possibly always) near rivers or swamps.

Recently, Heller \& Volleth (2016: 3) reported on significant differences in echolocation calls for the H. beatus specimens in the DRC and those from West Africa. They do not explicitly separate the two as different species, but they suggest this should be looked at further. If this proves to be correct, beatus might be restricted to West Africa (possibly to the DRC border area) and maximus might need to be raised to species level.

Hipposideros caffer (Sundevall, 1846)

Fig. 11E-F

Rhinolophus caffer Sundevall, 1846: 118.

* Hipposideros caffer (Sundevall, 1846).

* Hipposideros caffer caffer (Sundevall, 1846).

* Hipposideros caffer tephrus Cabrera, 1906: 358.

* Hipposideros nanus J.A. Allen, 1917: 434.

The identification of a specimen from Nagero (southern edge of the Garamba National Park, Haut-Uélé Province; RBINS 14637) as H. caffer tephrus by Hayman et al. (1966: 44) is rather strange, as this subspecies (currently considered as a separate species) occurs in Morocco and the dry sub-Saharan belt 

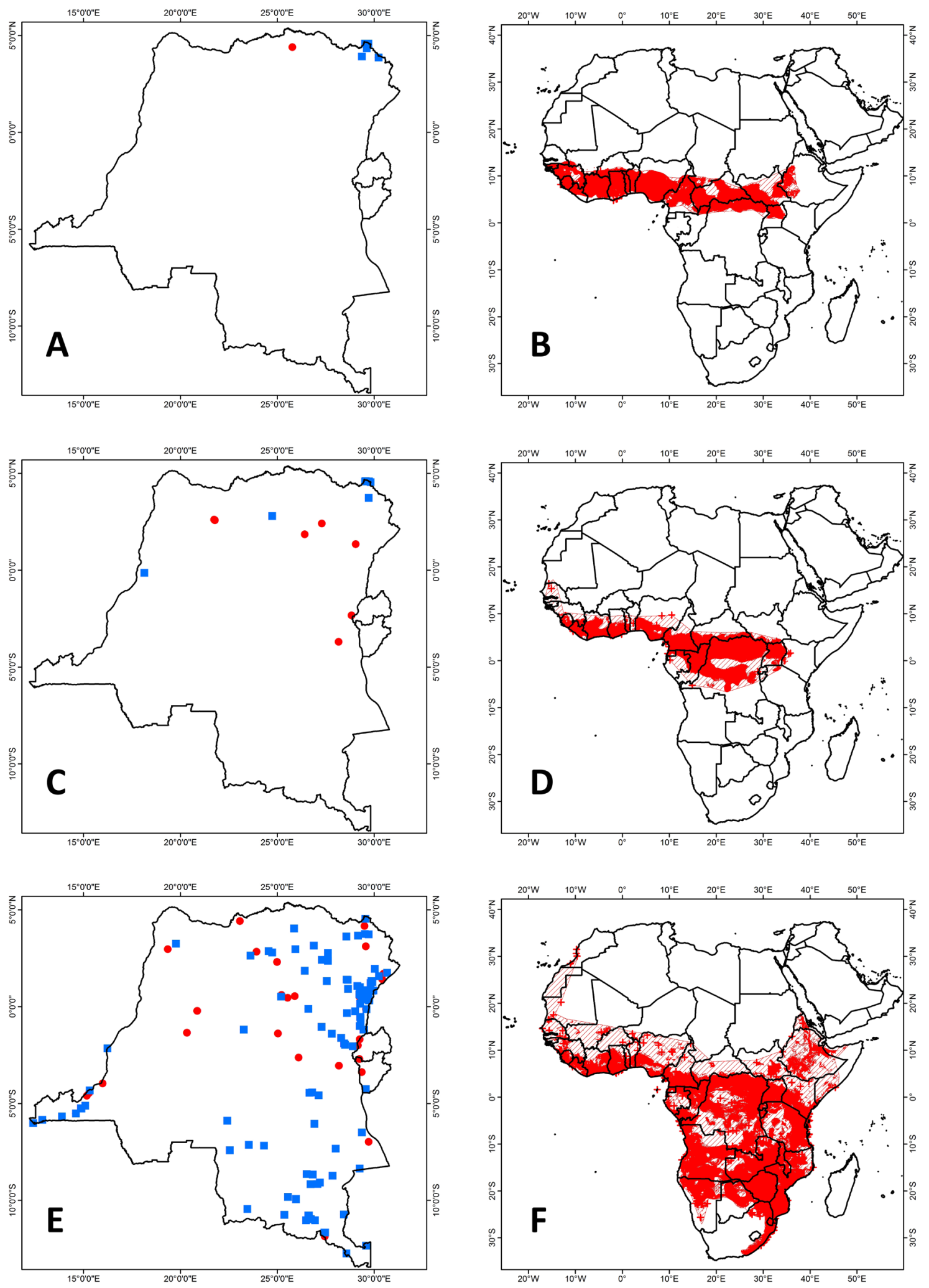

Fig. 11. Distribution maps. A-B. Hipposideros abae J.A. Allen, 1917. C-D. Hipposideros beatus (K. Anderson,1906). E-F. Hipposideros caffer (Sundevall, 1846). A, C, E. Distribution in the CRB area. B, D, F. Pan-African distribution. 
from Mauritania and Guinea to Sudan and Yemen at about $15^{\circ} \mathrm{N}$ (Vallo et al. 2009; Bernard \& Happold 2013b: 377). Bernard \& Happold (2013b: 376) assigned the specimens from the northeastern part of the DRC to H. c. nanus and those from the western part (Kongo Central Province) to H. c. angolensis (Seabra, 1898), leaving the remainder to H. c. caffer.

On the map presented by Bernard \& Happold (2013b: 377), the distribution of $H$. caffer only covers the area around the mouth of the Congo River and the eastern part of the DRC and the western frontiers of Rwanda and Burundi. Our data indicate that the species occurs much further to the west in the northern half of the DRC. We therefore believe the distribution is much more continuous, connecting the West African populations with those from Central and eastern Africa (see also ACR 2016: 164).

Hipposideros camerunensis Eisentraut, 1956

Fig. 12A-B

\section{* Hipposideros camerunensis Eisentraut, 1956: 526.}

Hipposideros camerunensis is a very rare species, with a very scattered distribution consisting of a few specimens from Cameroon, eastern DRC, Uganda and western Kenya. Happold (2013j: 379) suggests that this disjunct distribution might lead to the possibility that the various populations might be (sub-) specifically distinct.

\section{Hipposideros fuliginosus (Temminck, 1853)}

Fig. 12C-D

Phyllorrhina fuliginosa Temminck, 1853: 77.

* Hipposideros fuliginosus (Temminck, 1853).

Hayman et al. (1966: 45, map 52) mention only three specimens from two localities in the DRC in the collection of the BMNH: the Ituri River (Ituri Province) and Ngombe (Kasaï Province). The first one is probably BMNH 1930.11.11.144, but the second one could not be traced, and is, therefore, not plotted on the distribution map (Fahr 2013e: 384 also indicated that this specimen needs re-examination). We did find some additional specimens from Epulu (Ituri Province) and Yalosemba (Mongala Province), which resulted in the northern distribution of the species. Fahr (2013e: 384) plotted an additional locality near Yalosemba, but south of the Congo River, which could not be traced.

Hipposideros ruber (Noack, 1893)

Fig. 12E-F

Phyllorhina rubra Noack, 1893: 586.

* Hipposideros caffer ruber (Noack, 1893).

Although longtime considered to be a subspecies of $H$. caffer, ruber is currently generally accepted to represent a separate species. Vallo et al. (2009: 193) recognized a number of lineages in H. ruber, which in Central Africa include two sister clades, which become sympatric in Cameroon. This suggests that more than one species is involved, but the names for these taxa have not yet been determined.

The distribution map given by Happold (20131: 394) passes almost entirely around the DRC, except for the extreme northeastern and southeastern parts of the country. However, it does include both Rwanda and Burundi. Our data suggest that the distribution is wider in the DRC, where the species might only be missing in the central part of the country. 

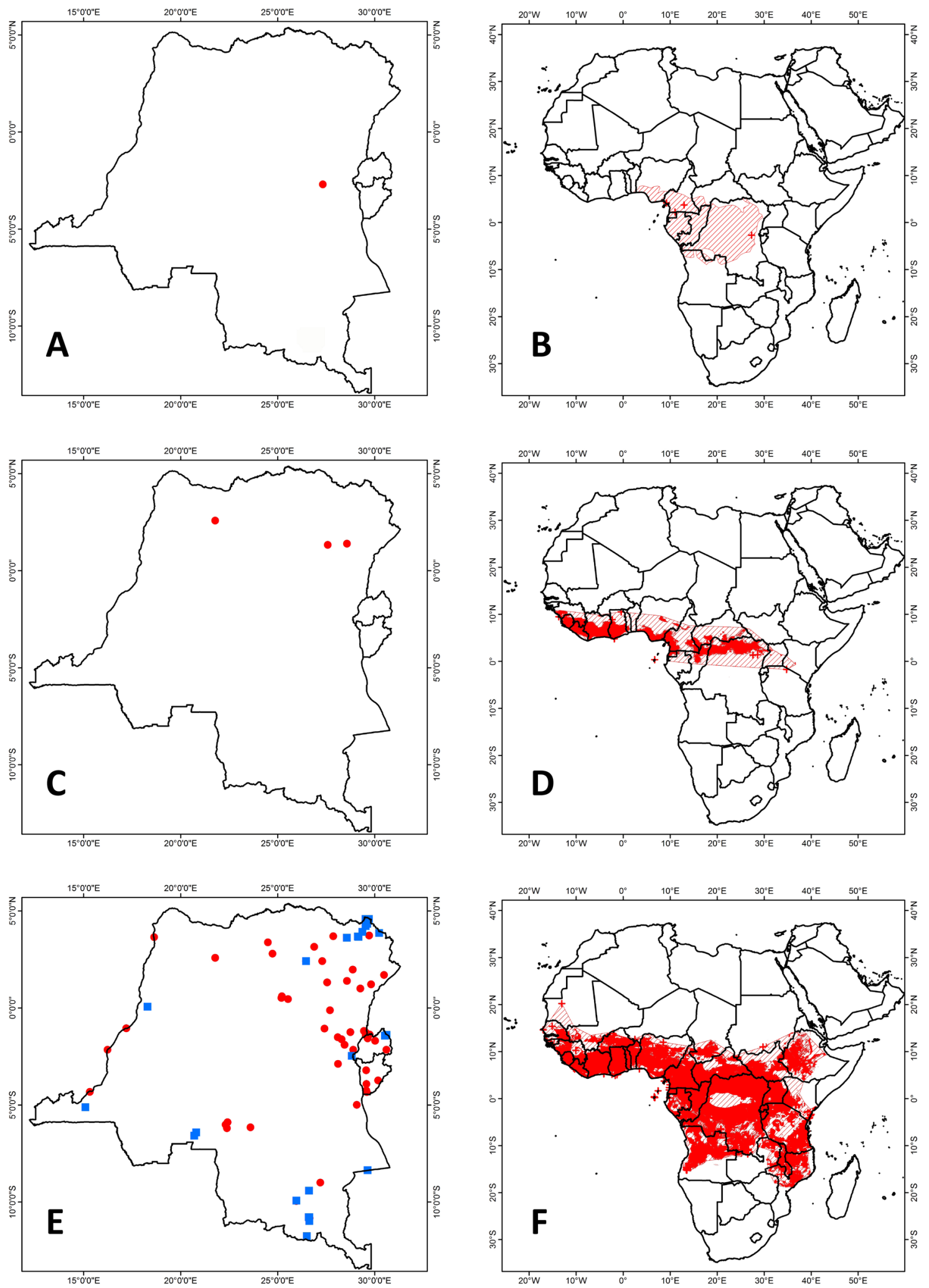

Fig. 12. Distribution maps. A-B. Hipposideros camerunensis Eisentraut, 1956. C-D. Hipposideros fuliginosus (Temminck, 1853). E-F. Hipposideros ruber (Noack, 1893). A, C, E. Distribution in the CRB area. B, D, F. Pan-African distribution. 
Genus Macronycteris Gray, 1866

In their very recently published study of the genus Hipposideros, Foley et al. (2017) found that the Cyt-b sequences for Hipposideros commersoni E. Geoffroy Saint-Hilaire, 1813, H. cryptovalorona Goodman et al., 2016, H. gigas (Wagner, 1845) and H. vittatus (Peters, 1852) are quite different from those of the other representatives of this genus, and therefore, they resurrected the genus Macronycteris Gray, 1866. This division is also supported by the deviant karyotype, which in Hipposideros counts 32 chromosomes and 52 in species assigned to Macronycteris.

\section{Macronycteris gigas (Wagner, 1845)}

Fig. 13A-B

Rhinolophus gigas Wagner, 1845: 148.

* Hipposideros commersoni gigas (Wagner, 1845).

For a long time "Hipposideros commersoni" was considered to occur across the African continent, but McWilliam (1982: 9) found gigas to be specifically different from commersoni, which ultimately led to the restriction of the usage of the name "Hipposideros commersoni" for populations from Madagascar only. Happold (2013k: 385) indicates that both gigas and vittatus (or its synonym marungensis) were reported to occur sympatrically, which makes them separate species as well (see also Simmons 2005: $372,377)$. Unfortunately, however, as mentioned by these two authors, this split probably makes it necessary to re-examine all material collected prior to 2005 to assess their identification.

Although Hayman et al. (1966: 44) report the two "subspecies", they only provided a map for $H$. commersoni gigas (map 50), which also contained the data for the other form. The distribution map provided by Happold (2013k: 386) indicates that "H. gigas" primarily occurs in western Africa to the Republic of the Congo, coastal Angola and in the coastal areas around the border between Kenya and Tanzania. Furthermore, she plots a few isolated records in the eastern part of the DRC. The SDM map is fairly similar, although we connect the western African populations with those from the DRC and believe the eastern African populations represent Macronycteris vittatus rather than M. gigas.

Macronycteris vittatus (Peters, 1852)

Fig. 13C-D

Phyllorrhina vittata Peters, 1852: 32.

* Hipposideros commersoni marungensis (Noack, 1887): 272.

The distribution map for "H. vittatus" given by Happold (2013m: 396) indicates that this species primarily occurs in southern Africa (northern Namibia, northern Botswana, Zimbabwe and southern Mozambique) and along the coast of Kenya and southern Somalia. Additionally, she plotted a few individual localities in northern Nigeria, northern Central African Republic, southwestern Angola, Tanzania and southwestern DRC. This leaves most of the territory of the DRC without any records. Based on the records we assigned to this species (see also ACR 2016) and the climatic data, we also found a strong indication that this species primarily will occur in southern Africa, but in a wider band extending to central Angola, Zambia and southern DRC. Furthermore, we expect it to occur in a narrow band along the northern frontier of the DRC, extending into southern Cameroon and coastal West Africa. However, as indicated above, the identification of these specimens might need re-assessment.

Family Megadermatidae H. Allen, 1864

See Handley (1980) regarding the correct formation of the family name. 

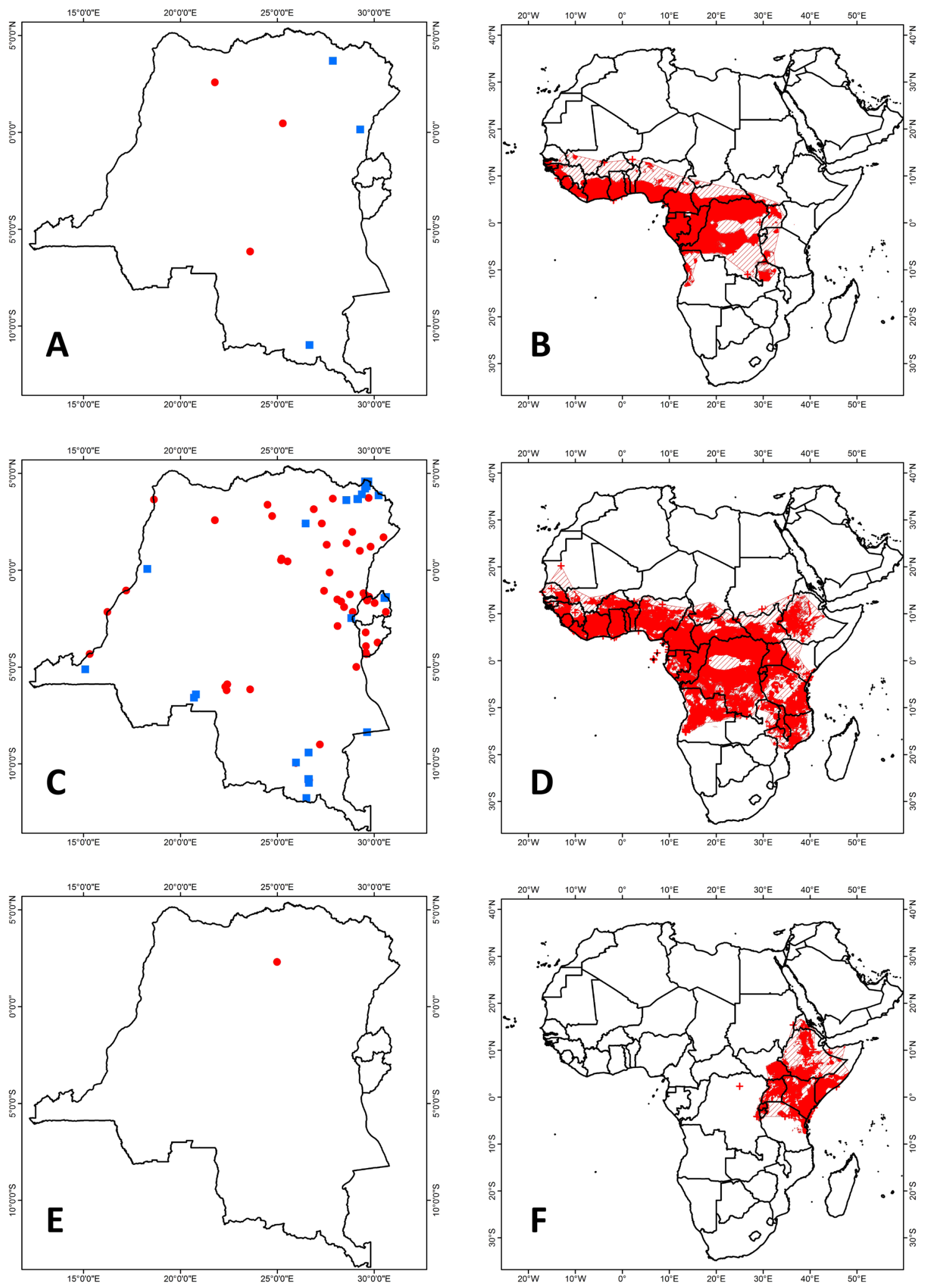

Fig. 13. Distribution maps. A-B. Macronycteris gigas (Wagner, 1845). C-D. Macronycteris vittatus (Peters, 1852). E-F. Cardioderma cor (Peters, 1872). A, C, E. Distribution in the CRB area. B, D, F. Pan-African distribution. 
Genus Cardioderma Peters, 1873

Cardioderma cor (Peters, 1872)

Fig. 13E-F

Megaderma cor Peters, 1872: 194.

The distribution area for Cardioderma cor reported by Happold (2013n: 404) covers part of northeastern Africa, from eastern Sudan at the border with Eritrea, over Eritrea and central Ethiopia and central Kenya to northern Tanzania, with an extention into western Somalia and some individual localities in eastern Ethiopia and easternmost Somalia. The westernmost occurrence of C. cor is northeastern Uganda and just across the border into South Sudan.

The DRC specimen reported here was collected by the Congolese authors at Rubi-Tele in Tshopo Province, which is a westward extension of about $800 \mathrm{~km}$ from the hereto closest known locality.

Genus Lavia Gray, 1838

Lavia frons (Geoffroy, 1810)

Fig $14 \mathrm{~A}-\mathrm{B}$

Megaderma frons E. Geoffroy Saint-Hilaire, 1810: 192.

* Lavia frons frons (E. Geoffroy St.-Hilaire, 1810).

In CRB, Lavia frons is widely distributed along the eastern part of the DRC, Rwanda and Burundi. The records from the western part of the DRC are more patchy and were all previously reported by Hayman et al. (1966: 37, map 35). Its Pan-African distribution stretches in a band between $15^{\circ} \mathrm{N}$ and $10^{\circ} \mathrm{S}$, where it occurs in the woodland savanna zones from West Africa to eastern Africa, in coastal mosaic, open degraded riverine forests in the Rainforest BZ of the central Congo Basin, East African Coastal Forest Mosaic and miombo woodland. In arid areas, it is found in bush country surrounding lakes, marshes and along rivers (Happold 2013o: 407).

Family Rhinolophidae Gray, 1825

Genus Rhinolophus Lacépède, 1799

Rhinolophus alcyone Temminck, 1852

Fig. $14 \mathrm{C}-\mathrm{D}$

Rhinolophus alcyone Temminck, 1852: 80.

* Rhinolophus alcyone alcyone Temminck, 1852.

Hayman et al. (1966: 38, map 37) only report two specimens belonging to this species, one from Buluku and one from the upper Makpe River, both in the Garamba National Park, Haut-Uélé Province. Two additional specimens are from Lukolela (Mai-Ndombe Province, on the border with the Republic of Congo) and Scierie Forest (Maniema Province). Although specimens were reported from the Republic of Congo by Dowsett et al. (1991: 256), these were from the coastal province of Kioulou at quite some distance from Lukolela. Happold (2013e: 311 ) mentions that this species primarily occurs in the lowland rainforests, but also in dense relict and riverine forests north of the rainforest.

Rhinolophus blasii Peters, 1867

Fig. 14E-F

Rhinolophus blasii Peters, 1867: 17. 

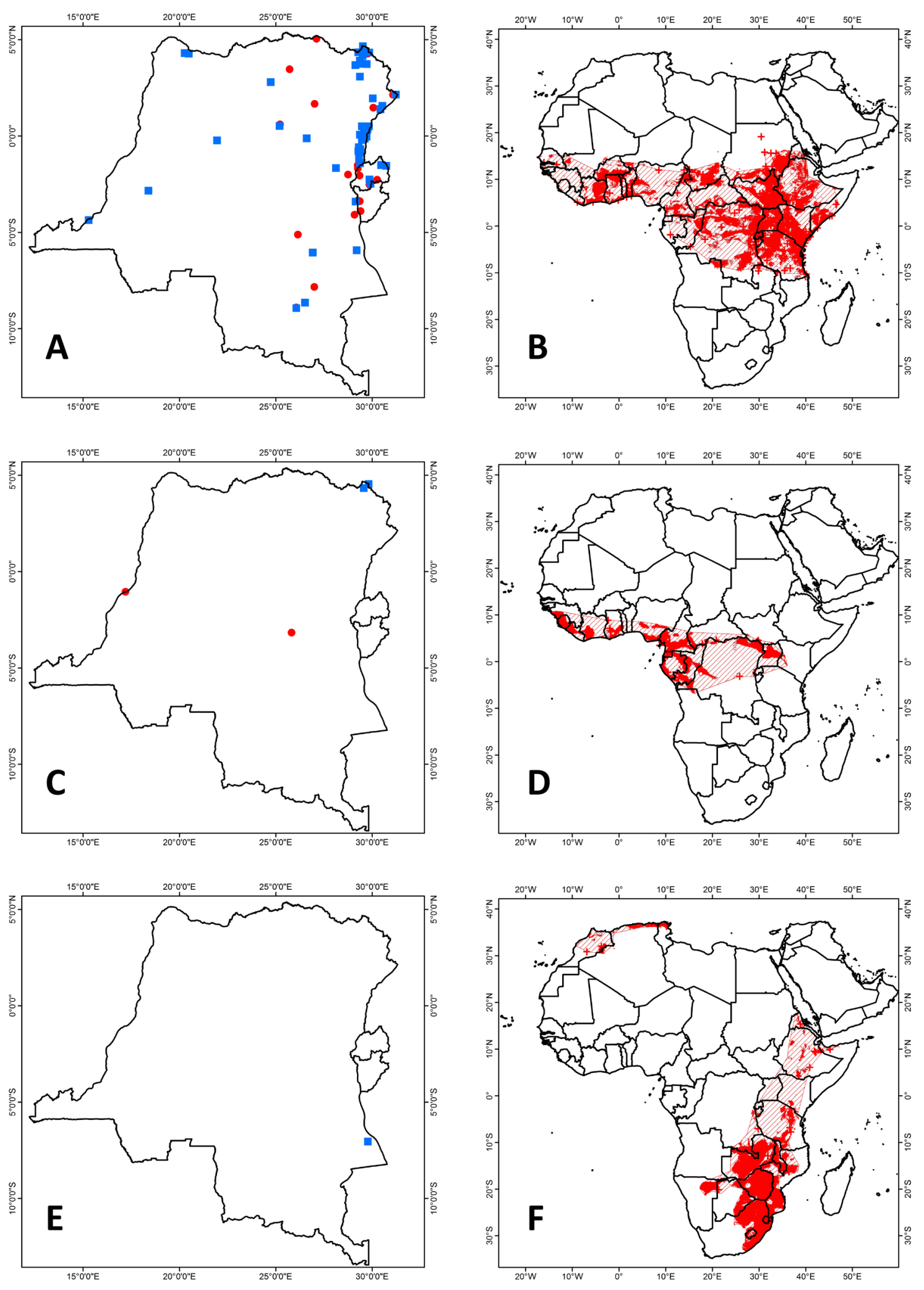

Fig. 14. Distribution maps. A-B. Lavia frons (E. Geoffroy St.-Hilaire, 1810). C-D. Rhinolophus alcyone Temminck, 1852. E-F. Rhinolophus blasii Peters, 1867. A, C, E. Distribution in the CRB area. B, D, F. Pan-African distribution. 
* Rhinolophus blasii empusa (Andersen, 1904: 378).

In the CRB region, this species is represented by a single specimen from Moba, Tanganyika Province. Happold (2013f: 312) mentions that $R$. blasii empusa occurs from the southern DRC to the former Transvaal (RSA). The map she presents also indicates that the Pan-African distribution for $R$. blasii contains three centres of gravity: one in the southern half of Africa, starting in the southern DRC and Tanzania to the northeastern part of the RSA; a second one in Ethiopia and a third north of the Sahara in Morocco, Algeria and Tunisia. Based on SDM data, we suspect that there might be a connection between the southern and the eastern areas, but the connection towards the Maghrabian populations doesn't seem to be straightforward. This might warrant further investigation to confirm the specific relation between these two separate areas.

Happold (2013f: 313) also plotted a second specimen in the neighbourhood of Likasi (Haut-Katanga Province), but that specimen could not be traced.

Rhinolophus clivosus Cretzschmar, 1826

Fig. $15 \mathrm{~A}-\mathrm{B}$

* Rhinolophus clivosus Cretzschmar, 1826: 47.

* Rhinolophus clivosus zuluensis (Andersen, 1904): 383.

Hayman et al. (1966: 38) mention two different taxa: $R$. clivosus and $R$. clivosus zuluensis, where the latter name is applied to specimens from the (south)western part of the DRC (Sud-Kivu, Haut-Lomami and Haut-Katanga). However, Bernard \& Happold (2013a: 316) mention coastal South Africa as region for the zuluensis subspecies. They do not provide any information on the identity of the subspecies occurring in the DRC. Based on the boundaries set by Bernard \& Happold (2013a: 316), the northern specimens (from Nord- and Sud-Kivu and from Rwanda and Burundi) might possibly be assigned to keniensis Hollister, 1916, and those from Haut-Lomami and Haut-Katanga to augur K. Andersen, 1904. The subspecific assignment of the westernmost specimen (RMCA 7718 from Kisantu, Kongo Central Province) cannot be performed as no other specimens from that area are known.

The Rwandan locality here attributed to Hayman et al. (1966) refers to three specimens from BugoyeKisenyi, Western Prefecture (RMCA 12265 to 12267), which were originally assigned to Rhinolophus fumigatus.

Recent research on nuclear introns by Dool et al. (2016: 202) indicates that $R$. clivosus is paraphyletic, which would suggest that some of the currently recognized subspecies might represent valid, separate species.

Stoffberg et al. (2012) already found that the mtDNA control region of the southern African R. clivosus specimens differed considerably from that of northern populations (as much as they differ from R. ferrumequinum). They suggest to call these $R$. geoffroyi Smith, 1829. However, they also point out that it "... is not currently recognized as a separate species because the name was unidentifiable and the type specimen apparently lost ...” This was already mentioned by Ellerman et al. (1953: 56), Hayman (1954: 284), and Csorba et al. (2003). Stoffberg et al. (2012) do not provide any information that substantiates the validity of geoffroyi, so we are reluctant to use this name. They also indicate that " $R$. geoffroyi" includes four subspecies: geoffroyi in western RSA, zuluensis in eastern RSA, augur in central RSA and Botswana and zambesiensis in Mozambique, Zimbabwe, Zambia and the southeastern-most part of the DRC (Sakania Territory, Haut-Katanga Province). The latter area is just southeast of the records from Haut-Katanga we report here, and seems to suggest that they do not belong to " $R$. geoffroyi". Stoffberg et al. (2012: 6) considered specimens from Kenya to belong to $R$. clivosus, which might suggest that the more northern CRB specimens (Sud- and Nord-Kivu, Burundi and Rwanda) could be assigned to 

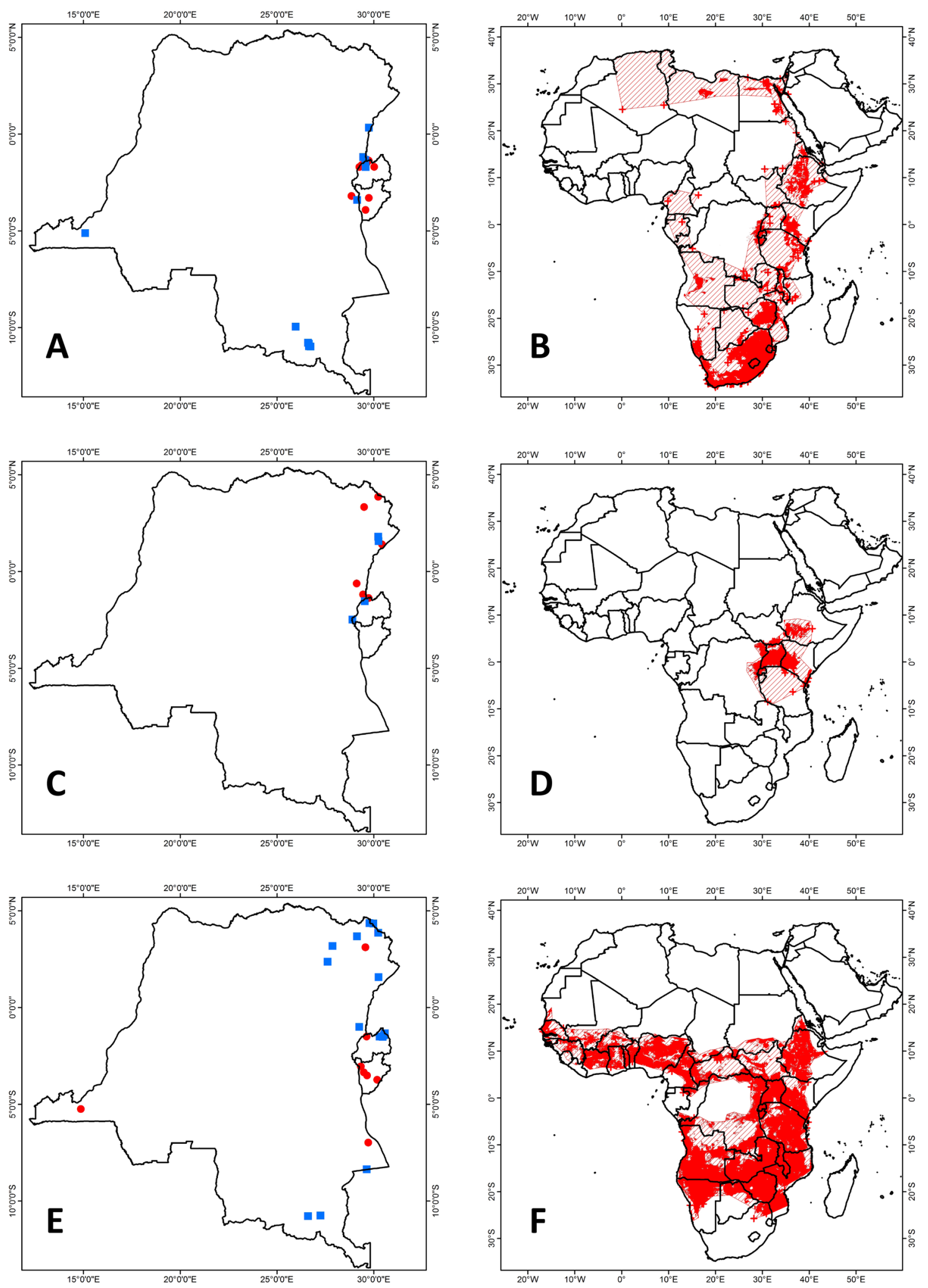

Fig. 15. Distribution maps. A-B. Rhinolophus clivosus Cretzschmar, 1826. C-D. Rhinolophus eloquens K. Andersen, 1905. E-F. Rhinolophus fumigatus Rüppell, 1842. A, C, E. Distribution in the CRB area. B, D, F. Pan-African distribution. 
R. clivosus. On the other hand, Benda \& Vallo (2012: 83) indicate that all populations occurring in the savanna belt from southeastern RSA to Kenya belong to one taxon, for which augur K. Andersen, 1904 is the prior available name. However, pending further research into the systematics of the R. clivosus complex, we tentatively retain the name $R$. clivosus for all the CRB specimens.

Cotterill (2002: 166) described a new closely related horseshoe bat (Rhinolophus sakejiensis) from Kavunda (11 $17^{\prime}$ S, $24^{\circ} 21^{\prime}$ E), between the Sakeji and Zambezi Rivers in Zambian Northwest Province. This locality is less than $10 \mathrm{~km}$ from the border with the DRC. It would, therefore, also be very likely that this species occurs in the DRC, but it has not yet been recovered from there.

Rhinolophus eloquens K. Andersen, 1905

Fig. 15C-D

Rhinolophus Hildebrandti eloquens K. Andersen, 1905: 74.

* Rhinolophus hildebrandti eloquens Andersen 1905.

Although described as a subspecies of hildebrandtii, eloquens was widely accepted to represent a separate species, already beginning with Andersen (1905: 660; 1907: 31, 34), and further Allen (1939), Koopman (1975: 389), Simmons (2005: 354) and Cotterill (2013a: 323). However, some authors, such as Hayman \& Hill (1971: 22), placed eloquens in R. fumigatus, whereas Kock (1969: 121) considered it a subspecies of $R$. aethiops (which is currently also considered a synonym of fumigatus). Cotterill (2013a: 324) indicates that eloquens can be distinguished from either fumigatus and hildebrandtii by its size, but the dimensions show a considerable overlap (see key).

The map presented by Cotterill (2013a: 324) shows a very restricted distribution in eastern Africa, with occurrences in Kenya, Uganda, South Sudan, Rwanda and extreme northeastern DRC. This agrees very well with the distribution we postulate, although we believe it might also occur in southern Ethiopia.

Rhinolophus fumigatus Rüppell, 1842

Fig. 15E-F

*Rhinolophus fumigatus Rüppell, 1842: 132.

In their synonymy list for R. fumigatus, Hayman et al. (1966: 38) include Rhinolophus abae J.A. Allen, 1917, and they refer to Koopman, who considered abae to be very close to fumigatus at the species level and indicated that abae might only be a subspecies of fumigatus. The records for these two taxa were grouped together in the text, but they still provided two separate maps: 36 for $R$. abae and 40 for R. fumigatus. These maps indicate that the two taxa occur in the northeastern RDC. Further fumigatus records were reported from Rwanda and the southern provinces of Tanganyika, Lualaba and HautKatanga. Hayman et al. (1966) also reported a series from $74 \mathrm{~km}$ on the Banana-Boma Road (RMCA 20649, 20651 to 20653), but these were re-identified as Rhinolophus lobatus ( $=$ R. landeri; Wim Wendelen, pers. comm.). However, two other R. fumigatus specimens have surfaced from the Kongo Central Province (Thysville: RBINS 22428 and 22429). Cotterill \& Happold (2013a: 330) indicate that $R$. fumigatus inhabits savanna areas (miombo and mopane woodlands in south-central Africa). Their distribution map shows a patchy covering of sub-Saharan Africa consisting of a number of red areas in West Africa, northeast Africa (including NE DRC, Rwanda, Uganda, Kenya and Ethiopia), and southwestern Africa (Angola and Namibia) as well as a number of individual records in southeastern Africa (Zambia, Zimbabwe, Mozambique). From the data in ACR (2016) we know that the distribution in southern Africa is more extensive than Cotterill \& Happold's map suggests, and this probably might also be the case in West Africa. As such, our distribution map is more extensive, indicating that R. fumigatus 
occurs over the major part of sub-Saharan Africa, with the exception of the coastal areas in West Africa, the Central African forest region and extreme southern Africa.

\section{Rhinolophus hildebrandtii Peters, 1878}

Fig. 16A-B

Rhinolophus Hildebrandtii Peters, 1878: 195.

* Rhinolophus hildebrandti Peters, 1878.

Hayman et al. (1966: 39) distinguished two forms in R. hildebrandtii; one they identified to subspecific level (see below) and one to specific level. However, they only included one distribution map (map 41) for these two forms. The distribution within CRB was restricted to the eastern part of the DRC and Rwanda, with specimens in the Congolese provinces of Nord-Kivu, Ituri and Haut-Uélé in the north on the one hand and in Tanganyika, Haut-Lomami and Haut-Katanga Provinces in the south on the other hand. Some of the specimens from Rwanda (Magachi, INPBC V1903; Lugarama, INPBC 3041) were re-identified as $R$. fumigatus, which might account for the remark made by Monfort (1992: 146) on the species being plotted on map 41 in Hayman et al. (1966), but being absent in their listing. Additional specimens of $R$. hildebrandtii are reported from Burundi and the southern Congolese provinces, but also from Gangala-na-Bodio (Haut-Uélé Province, RMCA 26664 and 26665) and Ibembo (Bas-Uélé Province, RMCA 89.004-M-0002). Based on their geographic location, the specimens from the Uélé Provinces might possibly need to be re-assigned to $R$. eloquens, but even for that species, the Ibembo specimen was found too far to the west.

Rhinolophus hilli Aellen, 1973

Fig. 16C-D

Rhinolophus hilli Aellen, 1973: 101.

Although the type specimen of Rhinolophus hilli was already collected in 1964, it was only described after the publication of Hayman et al. (1966). The species is only known from two specimens: the type specimen from Uwinka, at the top of a hill at $2300 \mathrm{~m}$, and another specimen from Ruta Bansugera (=Routabansougera) at the bottom of the same hill at $1750 \mathrm{~m}$ and $8 \mathrm{~km}$ to the northwest (Baeten et al. 1984: 194; Fahr 2013b: 335).

Rhinolophus kahuzi Fahr \& Kerbis Peterhans, 2013

Fig. 16E-F

Rhinolophus kahuzi Fahr \& Kerbis Peterhans, 2013: 192, 194.

This recently described species is only known from the type specimen (Kerbis Peterhans et al. 2013: 194).

Rhinolophus landeri Martin, 1838

Fig. 17A-B

Rhinolophus landeri Martin, 1838: 101.

* Rhinolophus landeri lobatus Peters, 1852: 41.

The form lobatus is currently still accepted to be a valid subspecies of $R$. landeri, occurring from Sudan and Ethiopia, southwards to the former Transvaal (RSA) (Happold 2013g: 340). The nominate subspecies (R. l. landeri) occurs from Gambia to Cameroon and further south to the mouth of the Congo River. This means that the specimens from Banana (RMCA 20746 and 20747) and from $74 \mathrm{~km}$ on the Banana-Boma Road (RMCA 20643 to 20653) might be assigned to $R$. landeri landeri and all other specimens to $R$. $l$. 

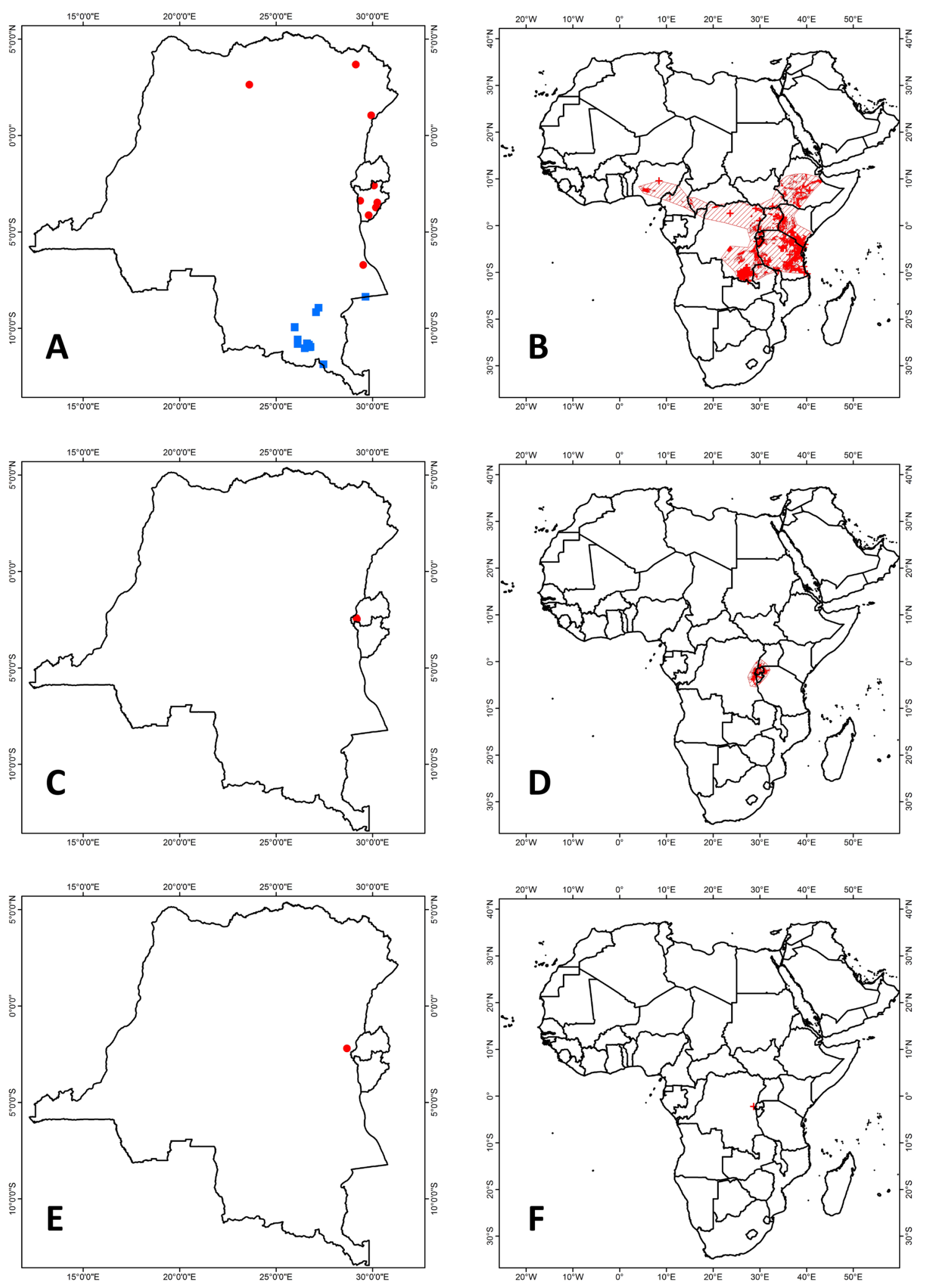

Fig. 16. Distribution maps. A-B. Rhinolophus hildebrandtii Peters, 1878. C-D. Rhinolophus hilli Aellen, 1973. E-F. Rhinolophus kahuzi Fahr \& Kerbis Peterhans, 2013. A, C, E. Distribution in the CRB area. B, D, F. Pan-African distribution. 
lobatus. The distribution map presented by Happold (2013g: 340) suggests there might be a connection between the eastern and western populations in the DRC, but that is not yet supported by evidence. Based on climatic data, the SDM map suggests that there probably might not be a direct connection between the eastern and western populations, but that the latter could be connected to the specimens from Angola, which implies that the specimens from the Kongo Central Province may possibly be assigned to R. l. angolensis Seabra, 1898. To resolve the subspecific status of these specimens, further material from western Gabon, Congo, Angola and DRC is required.

\section{Rhinolophus ruwenzorii Hill, 1942}

Fig. $17 \mathrm{C}-\mathrm{D}$

* Rhinolophus ruwenzorii J. Eric Hill, 1942: 1.

Rhinolophus ruwenzorii has a very restricted distribution of approximately $360 \mathrm{~km}^{2}$ in the DRC provinces of Nord-Kivu and Ituri (Fahr 2013c: 342). Some authors (e.g., Smith \& Hood 1980) consider ruwenzorii to be a subspecies of the west African $R$. maclaudi, which would result in an extremely disjunct distribution for this species (Baeten et al. 1984). However, Fahr et al. (2002) showed that both forms differ substantially and should be considered as separate species.

Rhinolophus simulator K. Andersen, 1912

Fig. 17E-F

Rhinolophus simulator K. Andersen, 1912: 384.

The 52 specimens of $R$. simulator reported here were already collected in the first half of the $1960 \mathrm{~s}$ at a number of localities in the provinces of Haut Katanga, Lualaba and Haut-Lomami (southeastern DRC). These localities form a westwards extension of the species in central Africa, but Cotterill \& Happold (2013b: 352) mention its presence in northern Zambia. Furthermore they plotted individual localities in Tanzania, Uganda and South Sudan, as well as in a few small areas in central Ethiopia and Malawi and in a larger area stretching from southern Zambia, over almost entire Zimbabwe, southwestern Mozambique and northern RSA. They also report its presence in a few localities in the border area between Guinea and Liberia and in central Nigeria and western Cameroon. ACR (2016: 1075) reported additional localities from Botswana and Namibia. Based on bioclimatic data, the species might also occur in central Angola, which would suggest that the southern African distribution area might connect to Cameroon along the coast.

Rhinolophus swinnyi Gough, 1908

Fig. 18A-B

* Rhinolophus swinnyi Gough, 1908: 71.

Hayman et al. (1966: 40) mention 25 specimens in the collection of the Tervuren Museum (RMCA) from Banana, Baudouinville, Kakontwe and Lubudi Cave, but contrary to all other taxa, no museum numbers were provided. A recent catalog list of the museum (Wim Wendelen, pers. comm.) doesn't mention any specimens belonging to this species either.

Based on the currently available data, $R$. swinnyi is restricted to the extreme southeastern part of the DRC (Haut-Lomami and Haut-Katanga Provinces). The Pan-African distribution of the species includes eastern (Tanzania) and southern Africa, where it occurs from the southern DRC, over Zambia, Zimbabwe, Malawi, southern Mozambique and extreme eastern RSA. 

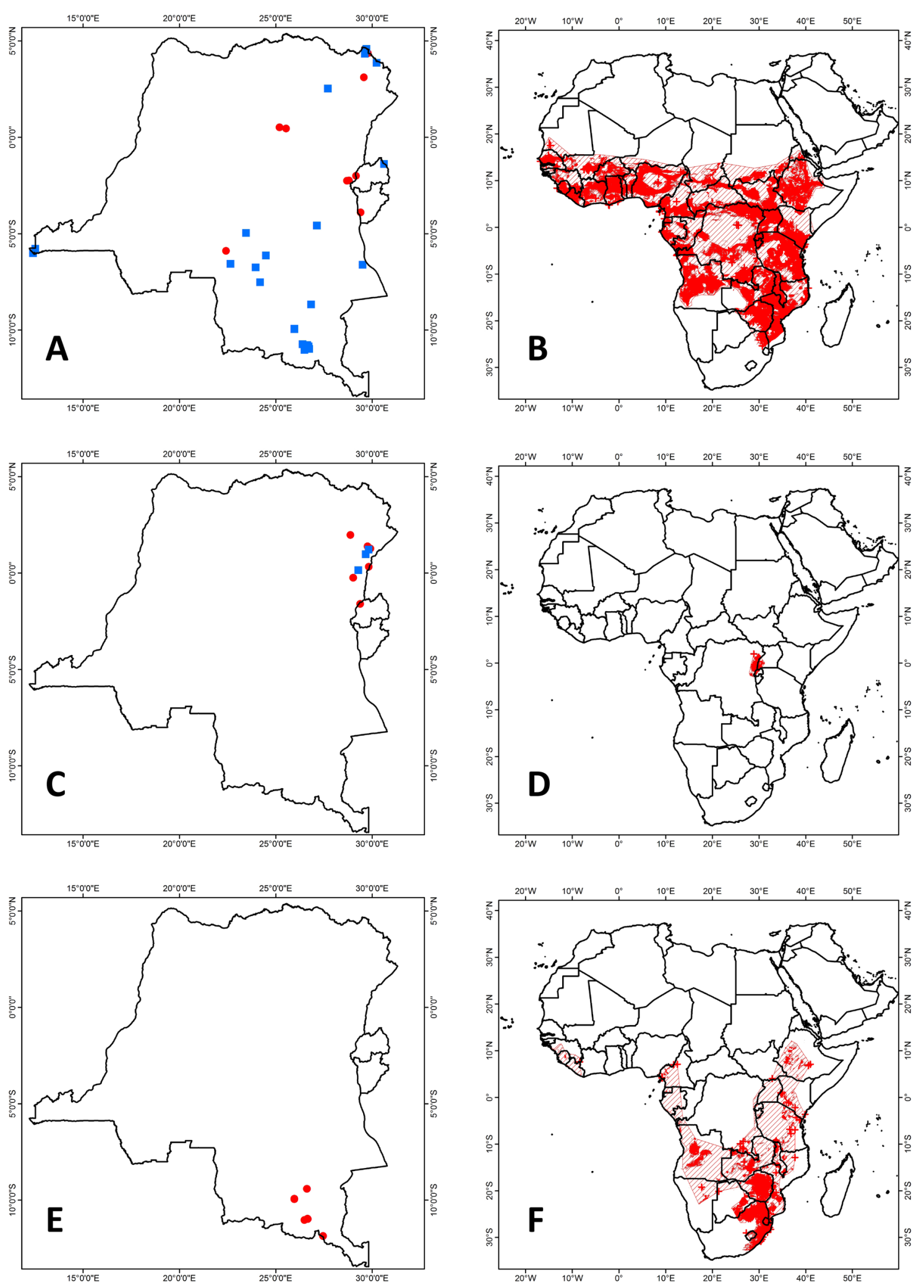

Fig. 17. Distribution maps. A-B. Rhinolophus landeri Martin, 1838. C-D. Rhinolophus ruwenzorii J. Eric Hill, 1942. E-F. Rhinolophus simulator K. Andersen, 1912. A, C, E. Distribution in the CRB area. B, D, F. Pan-African distribution. 

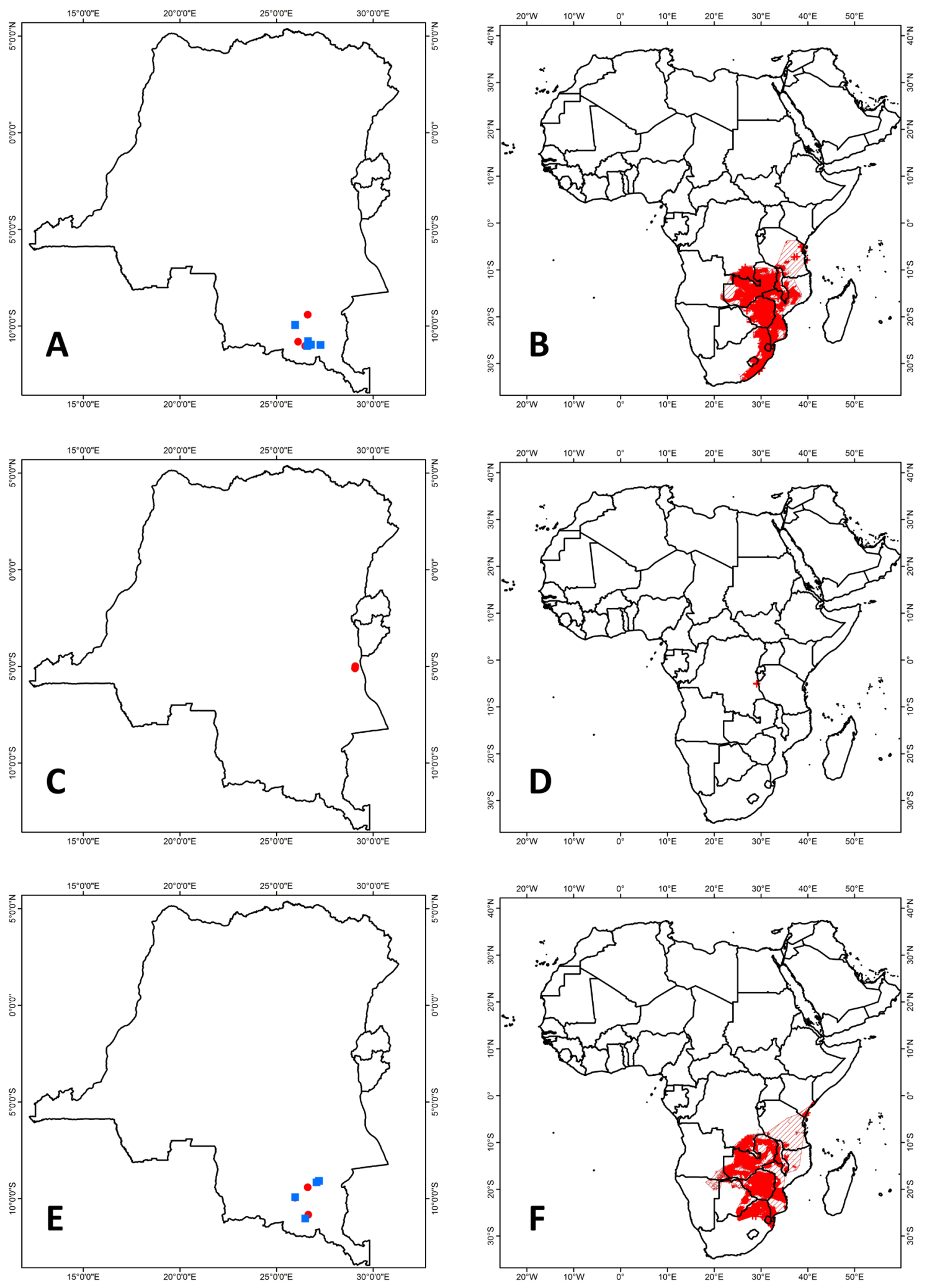

Fig. 18. Distribution maps. A-B. Rhinolophus swinnyi Gough, 1908. C-D. Rhinolophus willardi Kerbis Peterhans \& Fahr, 2013. E-F. Cloeotis percivali Thomas, 1901. A, C, E. Distribution in the CRB area. B, D, F. Pan-African distribution. 
Rhinolophus willardi Kerbis Peterhans \& Fahr, 2013

Fig. 18C-D

Rhinolophus willardi Kerbis Peterhans \& Fahr, 2013: 190.

Rhinolophus willardi is known from two localities in the Sud-Kivu Province, where the holotype and three paratypes were collected (Kerbis Peterhans et al. 2013: 191). These are the only known specimens of this species.

Family Rhinonycteridae Gray, 1866

From their study of 19 nuclear exon and intron gene fragments, Foley et al. (2014) demonstrated that the genera Cloeotis, Rhinonicteris, Paratriaenops and Triaenops differed significantly enough from the genera Asellia, Aselliscus, Coelops and Hipposideros to assign them to a separate family, the Rhinonycteridae.

Genus Cloeotis Thomas, 1901

Cloeotis percivali Thomas, 1901

Fig. 18E-F

Cloeotis Percivali Thomas, 1901: 28.

* Cloeotis percivali australis Roberts, 1917: 264.

In the CRB area, Cloeotis percivali is restricted to the southeastern Congolese provinces of Haut-Lomami and Haut-Katanga. Strangely enough, Jacobs (2013: 365) only refers to one locality: Shinkolobwe. Its PanAfrican distribution reaches from southern DRC over Zambia and Zimbabwe to northwestern RSA and Swaziland. All of these records are assigned to C. p. australis. The nominate subspecies is restricted to southeastern Kenya and northeastern Tanzania, where it is known from a few isolated specimens.

Suborder Vespertilioniformi Hutcheon \& Kirsch, 2006

Family Emballonuridae Gervais, 1855

Genus Coleura Peters, 1868

Coleura afra (Peters, 1852)

Fig. 19A-B

Emballonura afra Peters, 1852: 51.

* Coleura afra (Peters, 1852).

No further locality records for this species were found besides those mentioned by Hayman et al. (1966: 31 ), although we were able to track additional specimens from these localities. One locality (Kodja Hill, Haut-Uélé Province) is not indicated on our map as we could not trace the underlying material. Schwarz (1920: 1050) mentions this/these specimen(s) to be collected by the Alexander-Gosling expedition.

Genus Saccolaimus Temminck, 1838

Saccolaimus peli (Temminck, 1853)

Fig. 19C-D

Taphozous peli Temminck, 1853: 82.

* Taphozous (Saccolaimus) peli Temminck, 1853.

Saccolaimus has variably been assigned (sub)generic status by various authors, but Barghoorn (1977: 5) ranked it as a genus based on its skull proportions and ossification, and the morphology of the auditory bullae (see also fig. 88 in Happold 2013p). 

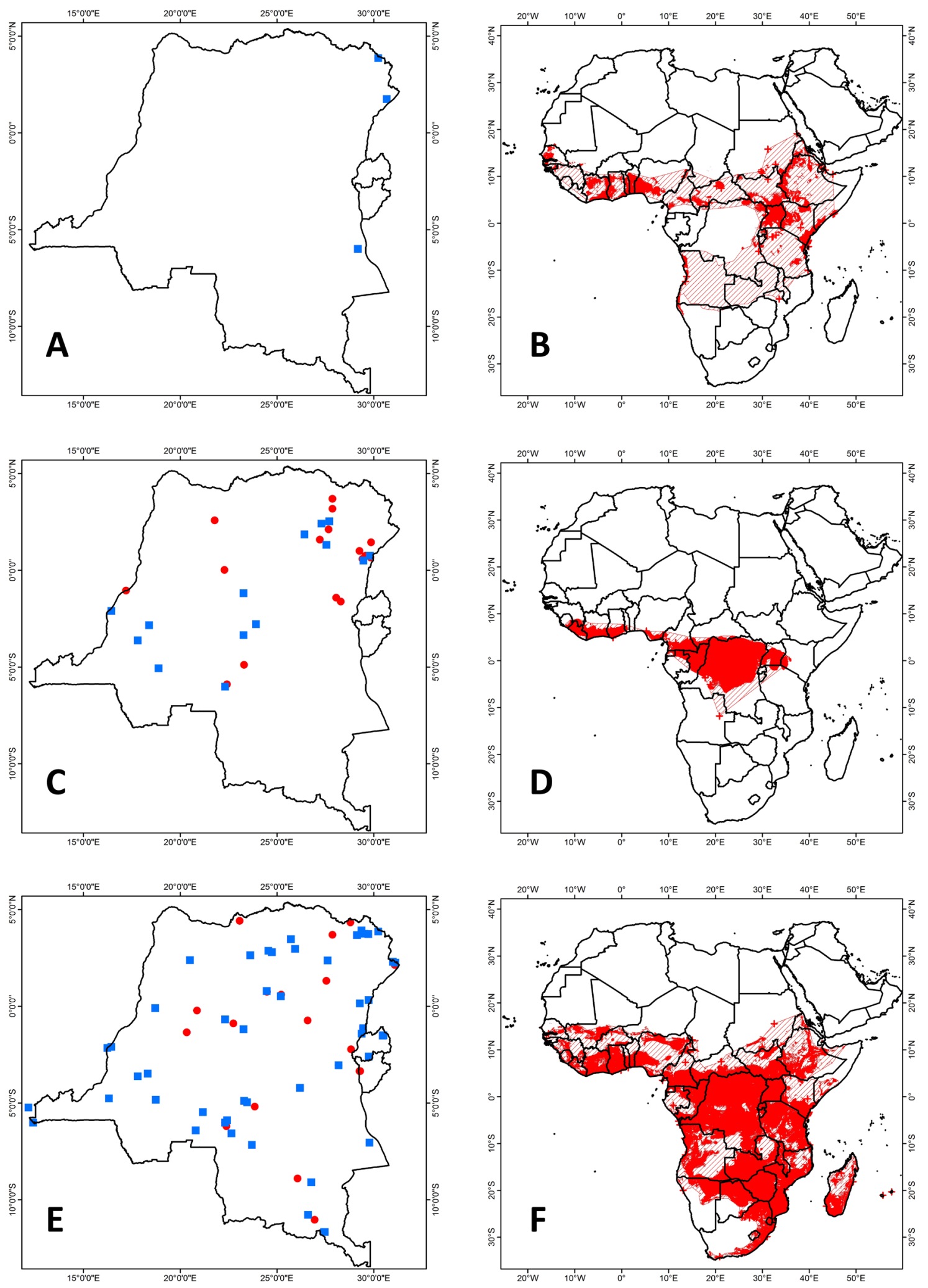

Fig. 19. Distribution maps. A-B. Coleura afra (Peters, 1852). C-D. Saccolaimus peli (Temminck, 1853). E-F. Taphozous mauritianus E. Geoffroy St.-Hilaire, 1818. A, C, E. Distribution in the CRB area. B, D, F. Pan-African distribution. 
Our distribution map hardly differs from the one presented by Hayman et al. (1966: map 24). We have a few extra records in the Nord-Kivu and Ituri Provinces in the northeastern part of the DRC, and in the Mongala and Tshuapa Provinces in the northwestern part of the country. The general map shows that $S$. peli is primarily a forest species.

Genus Taphozous E. Geoffroy Saint-Hilaire, 1818

Taphozous mauritianus E. Geoffroy St.-Hilaire, 1818

Fig. 19E-F

* Taphozous mauritianus E. Geoffroy Saint-Hilaire, 1818: 127.

This species was reported by Hayman et al. (1966: 31, map21) from all over CRB. The extra specimens we report here fill in a few gaps, but do not add any further information. The general distribution map indicates that T. mauritianus potentially occurs over the entire sub-Saharan part of Africa, with the exception of the Kalahari and a major part of the RSA.

Taphozous nudiventris Cretzschmar, 1830

Fig. 20A-B

Taphozous nudiventris Cretzschmar, 1830: 70.

* Taphozous (Liponycteris) nudiventris (Cretzschmar, 1830).

One single specimen from Cell II in the Garamba National Park (Haut-Uélé Province) was reported by Hayman et al. (1966: 32). No further specimens were found by us. The SDM map of the species indicates that its occurrence in the extreme northeastern corner of the DRC is not completely unexpected.

Taphozous perforatus E. Geoffroy St.-Hilaire, 1818

Fig. 20C-D

Taphozous perforatus E. Geoffroy Saint-Hilaire, 1818: 126.

* Taphozous sudani Thomas, 1915: 561.

Rosevear (1965: 150-151) compared material of both T. perforatus and T. sudani, and found that the only character distinguishing the two taxa is the darker pelage in sudani, generally combined with white wings. He also found a specimen with intermediate characters, which led him to retain sudani only as a mere subspecies of perforatus. This view was followed by most subsequent authors (e.g., Kock 1969: 74; Hayman \& Hill 1971: 16; Koopman 1975: 370).

With the exception of a few extra specimens from the same localities, all the material reported in this paper was already mentioned by Hayman et al. (1966: 32). The SDM map shows that T. perforatus primarily occurs in sub-Saharan Africa, above the equator, with a few records reported from northern Botswana and southern Zimbabwe.

Family Nycteridae Van der Hoeven, 1855

Genus Nycteris G. Cuvier \& E. Geoffroy Saint-Hilaire, 1795

Nycteris arge Thomas, 1903

Fig. 20E-F

* Nycteris arge Thomas, 1903: 633. 

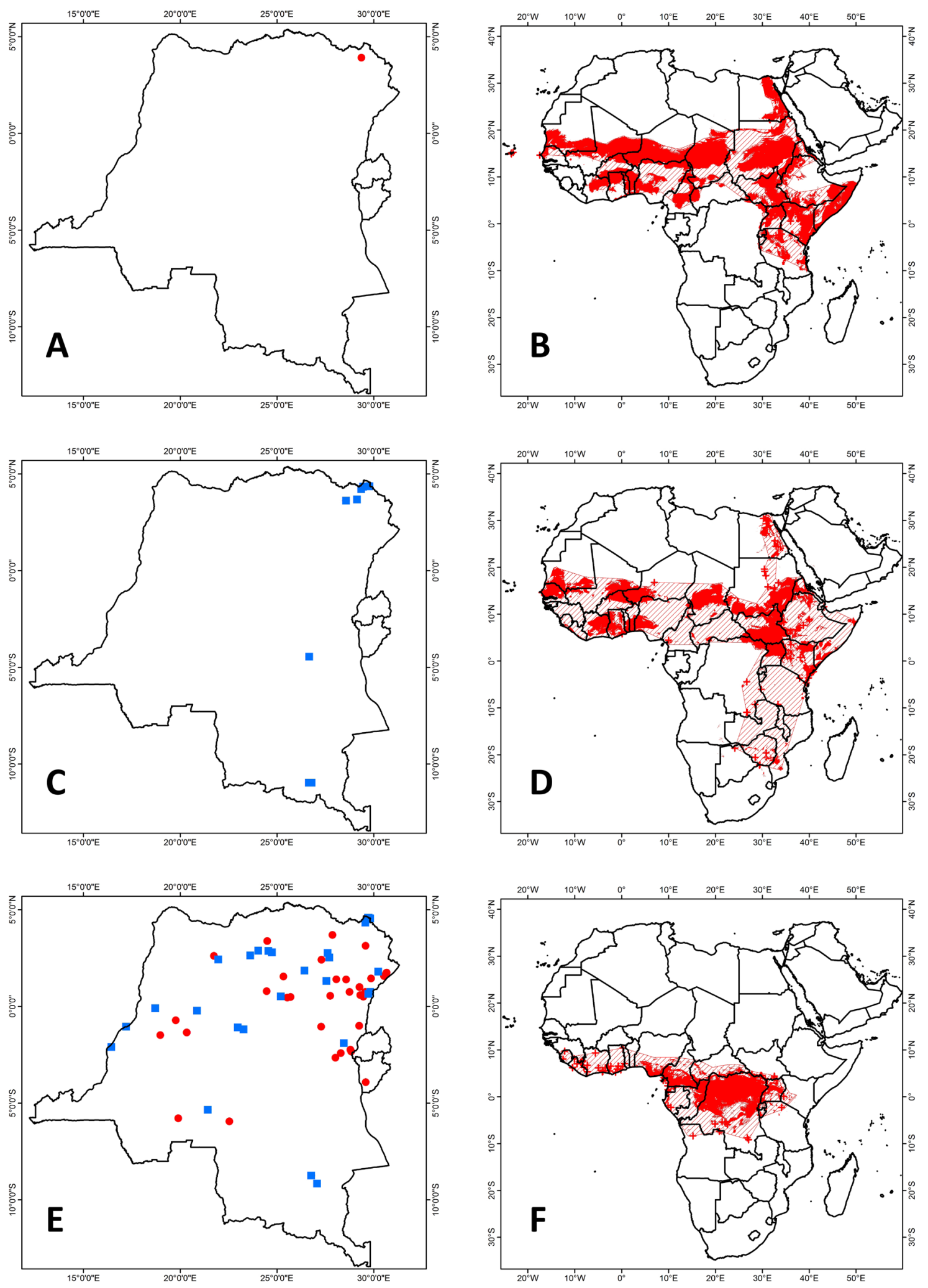

Fig. 20. Distribution maps. A-B. Taphozous nudiventris Cretzschmar, 1830. C-D. Taphozous perforatus E. Geoffroy St.-Hilaire, 1818. E-F. Nycteris arge Thomas, 1903. A, C, E. Distribution in the CRB area. B, D, F. Pan-African distribution. 
The distribution of this species is primarily restricted to the northern part of the DRC, with a few specimens from the Upemba National Park (Haut-Lomami Province) and Luebo (Kasaï Province), already reported by Hayman et al. (1966: 33, map 26), and some additional specimens from the Kananga (Kasaï-Central Province) and Kilembe (Kwilu Province) areas. In general, its distribution is mainly restricted to the rainforest zones and the rainforest-savanna mosaics (Fahr 2013f: 442).

\section{Nycteris grandis Peters, 1865}

Fig. 21A-B

* Nycteris grandis Peters, 1865: 351, 358.

Both the species distribution model and the distribution within CRB of Nycteris grandis are more or less similar to those of the previous species. For this species its core distribution within the DRC is in the northern part of the country. Although the SDM map also indicates that the species occurs in East Africa down to Zimbabwe and southern Mozambique, it has not been found in the southeastern provinces of the DRC.

Nycteris hispida (Schreber, 1774)

Fig. 21C-D

Vespertilio hispidus Schreber, 1774: 169, 188.

* Nycteris hispida (Schreber, 1774).

Nycteris hispida has a very wide distribution, both in CRB and Pan-African. In the DRC it is found over almost the entire country, and it is also known from both Rwanda and Burundi. The SDM map indicates that it occurs almost over the entire sub-Saharan African continent, with the exception of the extreme eastern part. It is also missing from Namibia, Botswana and Zimbabwe (except for the most northern parts of these latter two countries), and from most of the RSA, where it only occurs along the eastern coast line.

\section{Nycteris intermedia Aellen, 1959}

Fig. 21E-F

* Nycteris intermedia Aellen, 1959: 218.

In a note on page 35, Hayman et al. (1966) mention for $N$. intermedia: "A doubtfully valid species". However, the multivariate biometrical analyses by Van Cakenberghe \& De Vree (1985) showed that this species can clearly be distinguished from the larger N. arge and the smaller N. nana.

Contrary to the very patchy distribution map presented by Fahr (2013g: 450), we believe this species has a more extensive distribution over the African rainforest than all of the members of the Nycteris arge group, since representatives of this species might have been misidentified as $N$. arge. However, we do caution that in the DRC, the species has only been found in the northeastern part of the country (Ituri, Nord- and South-Kivu Provinces).

Nycteris macrotis Dobson, 1876

Fig. 22A-F

* Nycteris macrotis Dobson, 1876: 80.

* Nycteris aethiopica Dobson, 1878: 162, 165.

For a very long time, up to the early 1960 s and 1970s, $N$. aethiopia has been considered a separate species (from N. macrotis). Hayman et al. $(1966: 33,35,36)$ followed that view, but also referred to Koopman, who indicated that aethiopica and macrotis are conspecific. Hayman \& Hill (1971: 19) 

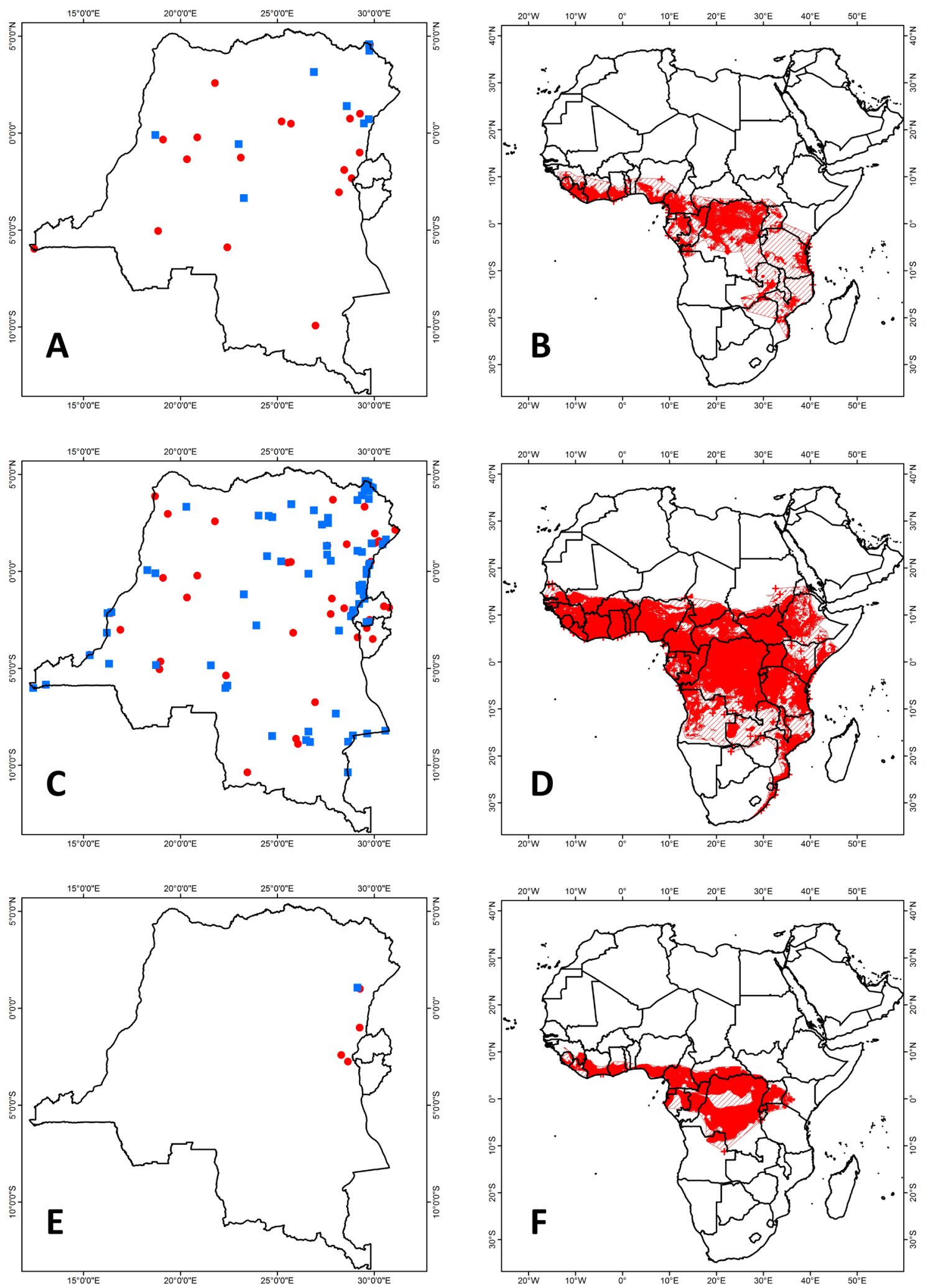

Fig. 21. Distribution maps. A-B. Nycteris grandis Peters, 1865. C-D. Nycteris hispida (Schreber, 1774). E-F. Nycteris intermedia Aellen, 1959. A, C, E. Distribution in the CRB area. B, D, F. Pan-African distribution. 
still mention the two as separate species, but note that their distinctness is by no means clear, as was suggested by Koopman (1965) and Kock (1969). The multivariate analyses by Van Cakenberghe \& De Vree (1985) confirmed that the two forms are conspecific.

Nycteris macrotis has a scattered distribution over CRB, where it occurs in almost all regions, except for the northwestern part of the DRC. The SDM map suggests a wide distribution across Africa south of the Sahara.

Nycteris major (K. Andersen, 1912)

Fig. 22C-D

Petalia major K. Andersen, 1912: 547.

* Nycteris major (Andersen, 1912).

Hayman et al. (1966: 36) only mention the type specimen of $N$. avakubia as representative of $N$. major in the DRC. However, on map 32, they have two dots, and neither of them refers to the type locality of avakubia, which is Avakubi (Tshopo Province). The two dots seem to refer to the two AMNH specimens reported by Allen (1917: 427) as belonging to N. major (49399 from the Garamba National Park and 49422 from Faradje), but of which Koopman (1965: 6) indicated that they actually belong to N. macrotis. Koopman (1965) furthermore refers to two additional specimens reported by Schouteden (1947): one from Macaco (Kasaï Province; reported for the first time by Kershaw 1923) and one from Boma (Kongo Central Province). These specimens were re-identified by Hayman et al. (1966: 35) and Van Cakenberghe \& De Vree (1985: 80) as N. macrotis.

Nycteris nana (K. Andersen, 1912)

Fig. 22E-F

Petalia nana K. Andersen, 1912: 547.

* Nycteris nana (Andersen, 1912).

Hayman et al. (1966: map 33) restricted the occurrence of $N$. nana in the DRC to the current provinces of Ituri and Haut-Uélé, with one record from Ibembo (Bas-Uélé Province). All of these are in the northeastern part of the DRC. Further specimens are recorded from as far west as Duma, on the border with the Central African Republic, and as far south as Kananga (Central-Kasaï Province). Due to the regular collection of specimens belonging to this species, Fahr (2013h: 455) doesn't believe the disjunct distribution he presented to be the result of insufficient sampling. However, he did not include the record from Duma (SMF 6390) nor the one from Wafanya (RMCA 89.020-M-0042), so the actual distribution of the species might be more wide than he suggested. Additionally, Fahr (2013h) also indicates that $N$. nana is recorded in drier types of forests than the other representatives of the $N$. arge group (arge, intermedia and major).

Nycteris parisii (de Beaux, 1924)

Fig. $23 \mathrm{~A}-\mathrm{B}$

Petalia parisii de Beaux, 1924 (for 1923): 254.

The systematic position of Nycteris parisii isn't completely clear yet. Van Cakenberghe \& De Vree (1985: 81) included it in $N$. woodi K. Andersen, 1914, but Cotterill (2013b: 456) considered it a separate species.

There is one specimen from Mount Né, near Blukwa (Ituri Province), which is in between the eastern African records from Ethiopia and Somalia on the one hand and the record from Cameroon on the other 

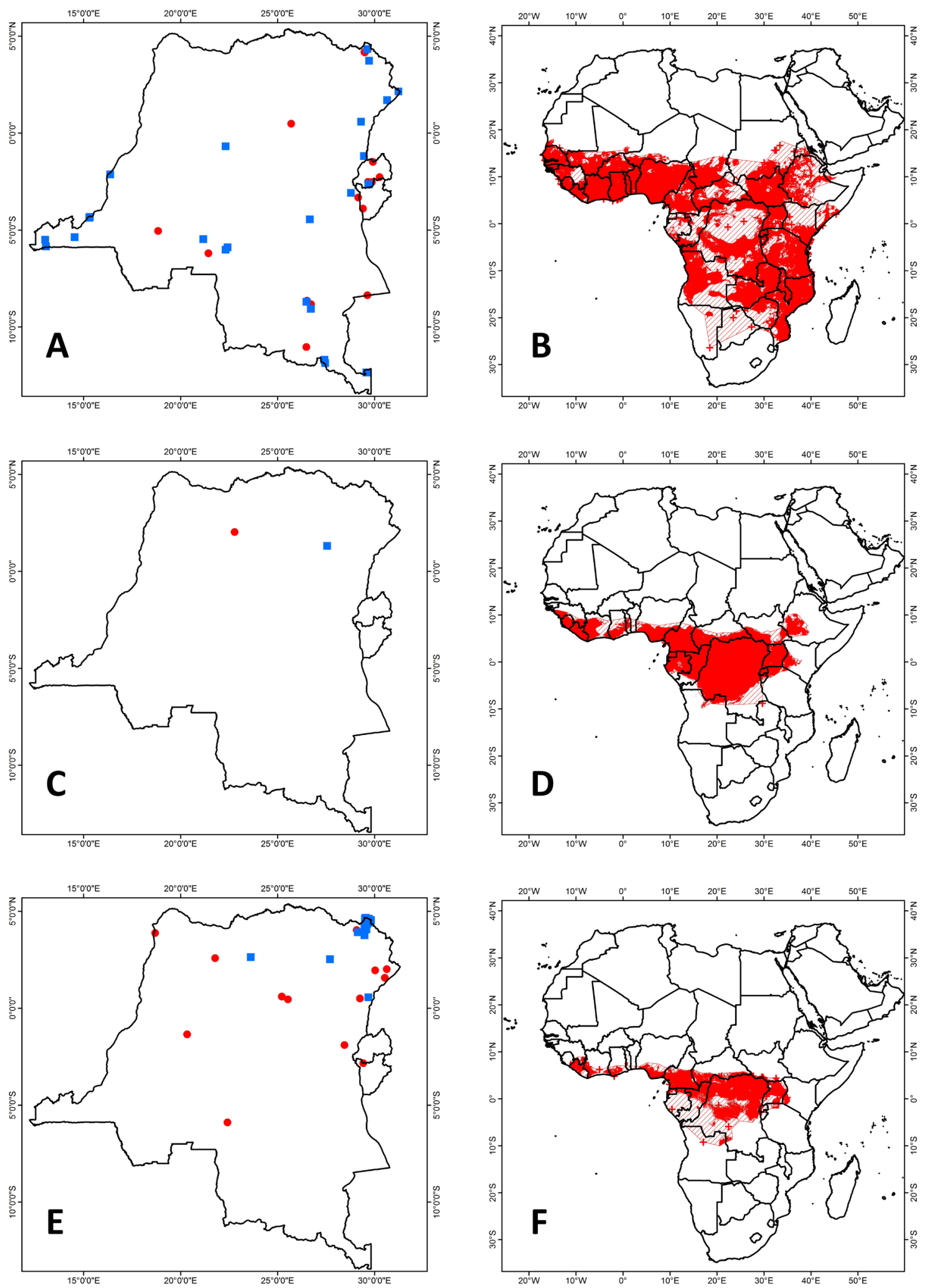

Fig. 22. Distribution maps. A-B. Nycteris macrotis Dobson, 1876. C-D. Nycteris major (K. Andersen, 1912). E-F. Nycteris nana (K. Andersen, 1912). A, C, E. Distribution in the CRB area. B, D, F. PanAfrican distribution. 
hand. Cotterill (2013b: 457) indicates that it occurs in the Sudan savanna, the Somalia-Masai bushland and the coastal forest mosaic biotic zones.

Nycteris thebaica Geoffroy, 1818

Fig. 23C-D

Nycteris Thebaicus E. Geoffroy Saint-Hilaire, 1818: 119.

* Nycteris capensis Smith, 1829: 434.

* Nycteris thebaica E. Geoffroy St.-Hilaire, 1818.

As all recent authors, Bernard \& Happold (2013c: 458) consider capensis to be a subspecies of $N$. thebaica, which is distributed over Tanzania, SE DRC and Zambia to Cape of Good Hope. As such, the records reported by Hayman et al. (1966: 34, map 27) from the northern provinces of Nord-Kivu and Ituri do not belong to this form. Furthermore, most of the specimens from Kateke River (RBINS 10813 to 10834) reported by Hayman et al. (1966: 34) as N. capensis were re-identified by Van Cakenberghe \& De Vree (1985: 80) as N. macrotis. Most of the records reported here are from the eastern part of CRB (provinces Ituri, Nord- and South-Kivu, Haut-Lomami and Haut-Katanga in the DRC and in Rwanda). The only exception is a series of five specimens, collected by Brian Robbins in Yalosemba in Mongala Province.

The distribution of the species is primarily sub-saharan Africa, but also along the Atlantic coast up to Morocco and along the River Nile and part of the coast of the Red Sea up to the Mediterranean.

Family Molossidae Gervais, 1856

At the time of Hayman et al. (1966), most of the African molossids were assigned to the genus Tadarida, which was split up in a number of subgenera. Freeman (1981:36), however, considered these subgenera to be valid genera.

Genus Chaerephon Dobson, 1874

Chaerephon aloysiisabaudiae (Festa, 1907)

Fig. 23E-F

Nyctinomus aloysii-sabaudice Festa, 1907: 1.

* Tadarida (Chaerephon) aloysii-sabaudiae (Festa, 1907).

* Tadarida (Tadarida) brunnea (Seabra, 1900): 83.

Hayman et al. (1966: 61) mentioned one DRC record, an immature specimen from Avakubi (AMNH 48934), which according to Koopman (1965: 23) represents T. (C.) aloysiisabaudiae. They also mentioned (p. 67) one specimen from Eala (RMCA 6565, Equateur Province) belonging to T. (T.) brunnea. According to Simmons (2005: 449), Nyctinomus brunneus is a synonym of Tadarida aegyptiaca. However, Fenton \& Peterson (1972: 19) re-examined this specimen and found that it represented T. aloysiisabaudiae. A third specimen was collected in Yalosemba (Mongala Province). A further specimen (RBINS 13826 from Iso.4, Garamba National Park) was identified as T. trevori by Hayman et al. (1966: 65), but was re-identified as T. aloysiisabaudiae by Peterson (1972: 2). All of these localities are in the northern part of the DRC. Fahr (2013k: 494) indicates that C. aloysiisabaudiae is mainly found in the rainforest and adjacent northern and eastern rainforest-savanna mosaics, one record very marginally in the Sudan savanna. Specimens were reported from Côte d'Ivoire, Ghana, Cameroon, Gabon, Central African Republic, DRC, South Sudan and Uganda. The SDM map suggests it might occur as far west as Liberia and as far east as central Ethiopia, western Kenya and northwestern Tanzania. Furthermore, it might extend southwards to southern DRC and northeastern Angola. 

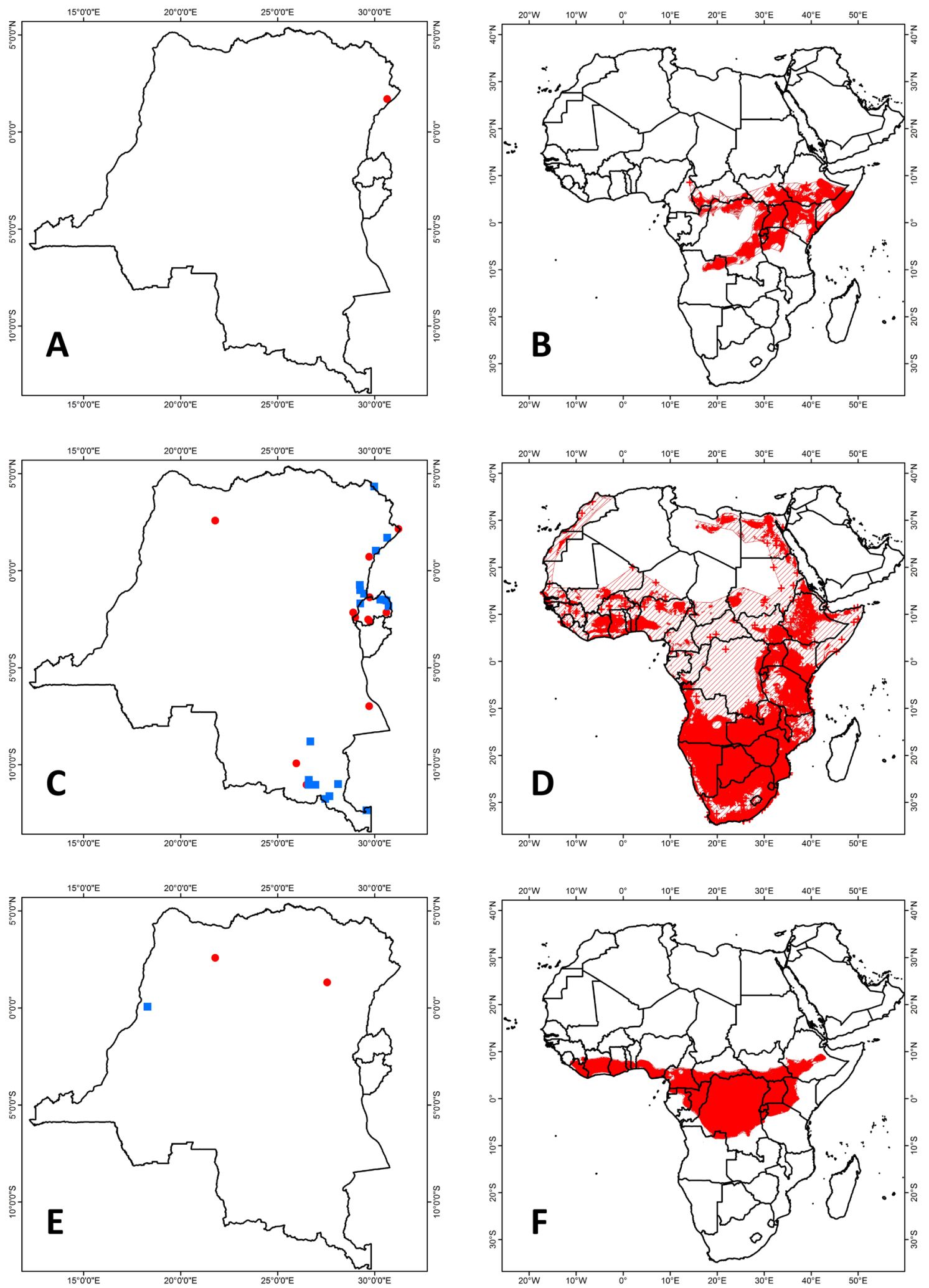

Fig. 23. Distribution maps. A-B. Nycteris parisii (de Beaux, 1924). C-D. Nycteris thebaica E. Geoffroy St.-Hilaire, 1818. E-F. Chaerephon aloysiisabaudiae (Festa, 1907). A, C, E. Distribution in the CRB area. B, D, F. Pan-African distribution. 


\section{Chaerephon ansorgei (Thomas, 1913)}

Fig. 24A-B

Nyctinomus ansorgei Thomas, 1919: 318.

* Tadarida (Tadarida) ansorgei (Thomas, 1913).

Hayman et al. (1966: 67, map 108) reported ansorgei from a number of localities in the eastern part of the DRC: the Garamba National Park and Faradje (Haut-Uélé Province) and from Jadotville (=Likasi) and Mwadingusha (Haut Katanga Province). The specimen from Vitshumbi (RMCA 17076) was reidentified by R.L. Peterson as belonging to Chaerephon bivittatus. Additional material was collected in Rwanda (Gabiro and Rukara) and in the DRC near Lake Kivu (Sud-Kivu Province) and in the Kikwit area (Kwilu Province). The latter is a major extention to the west of the DRC, but was overlooked by Cotterrill (2013b: 496). His map shows a scattered sub-Saharan distribution, composed of two larger areas: central Ethiopia and southwestern Zimbabwe, a few smaller areas in northeastern DRC, northeastern Zimbabwe, southwestern Kenya and the southern border between Nigeria and Cameroon, and some individual localities in eastern Côte d'Ivoire, northern Cameroon, northern Central African Republic, southern South Sudan, southwestern Tanzania, southeastern DRC, Zimbabwe and northern Angola. The SDM map suggests a potentially wider distribution in southern Africa, reaching further southwards in Angola towards northern Namibia.

\section{Chaerephon bemmeleni (Jentink, 1879)}

Fig. 24C-D

Nyctinomus Bemmeleni Jentink, 1879: 125.

* Tadarida (Tadarida) cistura (Thomas, 1903): 502.

Based on morphological comparisons, Peterson (1971b: 1347) found that cistura is only an eastern African subspecies of bemmeleni. Hayman et al. (1966: 67, map 110) reported the species from two localities in the Nord-Kivu Province, and no additional specimens were found.

Fahr (20131: 498) indicates that C. bemmeleni is known from five localities in West Africa (Sierra Leone, Liberia, Côte d'Ivoire and Cameroon) and from approximately 17 localities in eastern Africa, ranging from South Sudan, over Uganda and northeastern DRC into the border area between Kenya and Tanzania. Furthermore, he also plotted an additional locality in Maniema Province, but without providing details.

Chaerephon bivittatus (Heuglin, 1861)

Fig. 24E-F

Nyctinomus bivittatus Heuglin, 1861: 4, 13.

One immature specimen belonging to this species is known from the DRC. This specimen was originally identified as Tadarida ansorgei, but as indicated above, was re-identified by R. L. Peterson as belonging to the current species. Cotterill (2013d: 500) reported this species from a number of scattered areas in eastern Africa, reaching from northwestern Eritrea over central Ethiopia, western Kenya, the coastal area between Kenya and Tanzania, northern Zambia, southern Zambia, central Zimbabwe, the border between Zimbabwe and Mozambique, and also from a small area on the border between Uganda and South Sudan. This latter area is the closest record of C. bivittatus to the DRC.

Chaerephon chapini J.A. Allen, 1917

Fig. 25A-B

Choerephon (Lophomops) chapini J.A. Allen, 1917: 460, 461.

* Tadarida (Chaerephon) chapini J.A. Allen, 1917. 

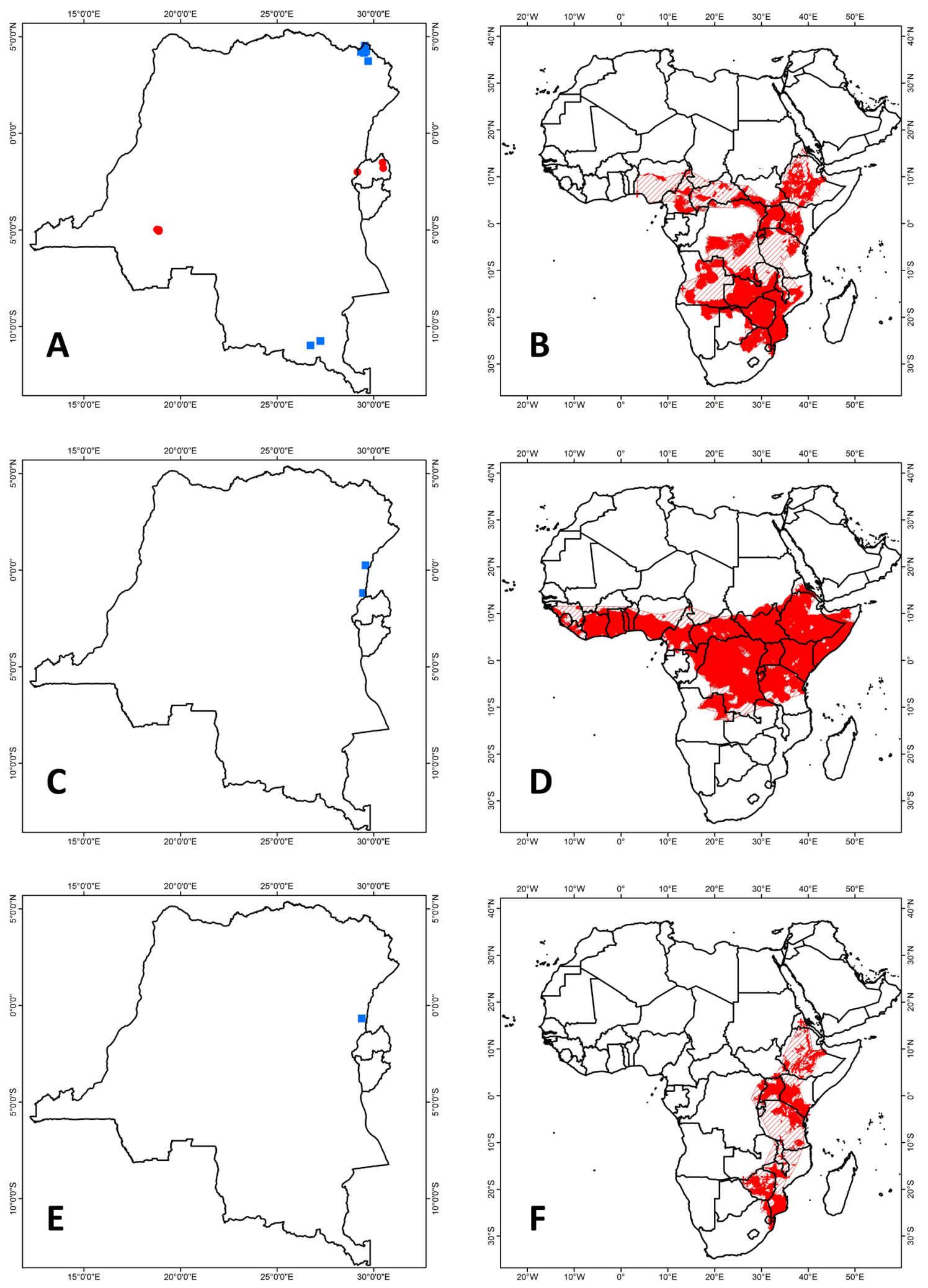

Fig. 24. Distribution maps. A-B. Chaerephon ansorgei (Thomas, 1913). C-D. Chaerephon bemmeleni (Jentink, 1879). E-F. Chaerephon bivittatus (Heuglin, 1861). A, C, E. Distribution in the CRB area. B, D, F. Pan-African distribution. 
Hayman et al. (1966: 61, map 92) reported chapini from three localities: Banana Creek (Kongo Central Province), Léopoldville (Kinshasa City Province) and Faradje (Haut-Uélé Province). The two former are in the western part of the DRC, whereas the latter is in the northeast. There is one additional specimen from Masha in northwestern Rwanda. Happold \& Cotterill (2013: 504) plotted two additional localities in southeastern DRC, but in their text they only refer to northeastern and southwestern DRC. Furthermore, they plotted individual localities in Côte d'Ivoire, Ghana, South Sudan, Ethiopia, Uganda, Zambia, Zimbabwe, and Namibia, as well as small distribution areas in western Kenya, northern Botswana and the western boundary between Namibia and Angola. The SDM map indicates that the species can occur in the countries between Ghana and South Sudan, although the Central African Republic doesn't seem to be that favorable. Furthermore, it might occur in a major part of Tanzania, Malawi and the western part of Mozambique.

Chaerephon gallagheri (Harrison, 1975)

Fig. 25C-D

Tadarida (Chaerephon) gallagheri Harrison, 1975: 313.

Also this species was described after the publication of Hayman et al. (1966) and is known from one specimen only (Harrison 1975: 313; Cotterill 2013f: 305).

Chaerephon leucogaster (A. Grandidier, 1869)

Fig. 25E-F

* Nyctinomus leucogaster A. Grandidier, 1869: 337.

In Mammals of Africa, Happold (2013v: 528) includes leucogaster in Tadarida pumila. However, we follow Peterson et al. (1995: 162) and Simmons (2005: 434) in retaining it as a separate species. Some of the forms we consider to be synonyms of leucogaster were treated as synonyms of pumilus by Hayman et al. (1966: 62): Chaerephon (Lophomops) cristatus J.A. Allen, 1917 and Chaerephon frater J.A. Allen, 1917. Simmons (2005) indicates that the distribution area of this species extends from Ethiopia to Ghana, including Nigeria, the DRC, Mali, and Madagascar. ACR (2016: 1130) mentions furthermore specimens from Cameroon, Central African Republic, Chad, Congo, Côte d'Ivoire, Mayotte, Sierra Leone, Tanzania and Zambia.

Chaerephon major (Trouessart, 1897)

Fig. 26A-B

[Nyctinomus (Nyctinomus) pumilus] var. Major Trouessart, 1897: 146.

* Tadarida (Chaerephon) major (Trouessart, 1897).

Hayman et al. (1966: 61, map 93) reported this species from the Garamba National Park and Faradje and Aba (Haut-Uélé Province). Additional specimens were found in Rwindi (Nord-Kivu Province), Tandala (Sud-Ubangi Province) and Mbandaka (Equateur Province). All of these localities are in the northern half of the DRC, with the latter two on the western side. These additional records are not mentioned by Happold (2013s: 517), who mapped the species from a number of distinct areas: one starting from the Garamba National Park along the River Nile up to Abu Hamad in Sudan, a second on the eastern side of Lake Victoria, a third along the coast of Kenya and Tanzania, and finally a larger area in West Africa, starting in eastern Guinea and reaching the western part of Nigeria. Based on the material in ACR (2016: 1131 ) and the climatological data, we believe the species can occur further to the west (to Senegal) and to the east (in western Ethiopia). Additionally ACR (2016: 1131) refers to material from Cameroon and the Central African Republic, bridging the gap between $10^{\circ} \mathrm{E}$ and $33^{\circ} \mathrm{E}$ referred to by Happold (2013s: 517). 

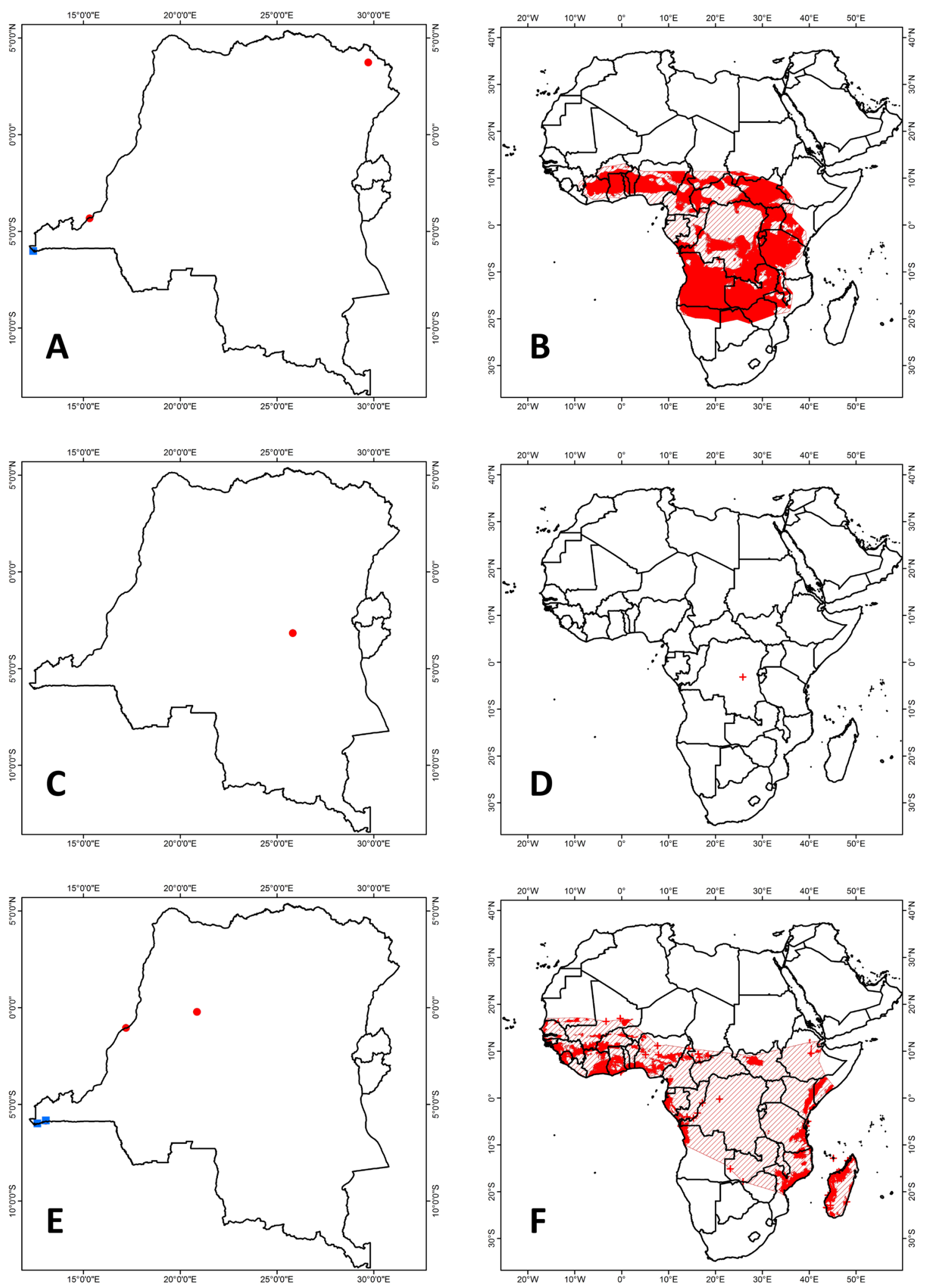

Fig. 25. Distribution maps. A-B. Chaerephon chapini J.A. Allen, 1917. C-D. Chaerephon gallagheri (Harrison, 1975). E-F. Chaerephon leucogaster (A. Grandidier, 1869). A, C, E. Distribution in the CRB area. B, D, F. Pan-African distribution. 
Chaerephon nigeriae Thomas, 1913

Fig. 26C-D

Chcerephon nigerice Thomas, 1913: 319.

* Tadarida (Chaerephon) nigeriae Thomas, 1913.

* Tadarida (Chaerephon) spillmanni (Monard, 1933): 51.

Contrary to most other changes proposed by Koopman, Hayman et al. (1966: 64) did not follow Koopman (1965: 21), who suggested that there is "no good reason to regard spillmanni as anything except a subspecies of $T$. (C.) nigeriae." They indicated (p. 62) that the different wing colour separates the two species. According to their maps (94 and 97), the dark-winged nigeriae occurs in the northeastern part of the DRC (Haut-Uélé Province) and the light-winged spillmanni in the southeastern part (Tanganyika, Haut-Katanga and Haut-Lomami Provinces). One additional record comes from Tandala (Equateur Province), in the northwestern part of the DRC. With the exception of the last record, Cotterill \& Happold (2013d: 524) plotted all of the DRC specimens. The southeastern records are part of an area also covering the north/western parts of Zambia. A further large area is found in Zimbabwe-Botswana, and they also drew smaller areas in Ghana, southern Nigeria, northern Cameroon, northern Central African Republic, central Ethiopia and south-central Angola, and individual localities in Guinea, Niger, South Sudan, Tanzania and Namibia. The SDM map suggests a much wider distribution in southern Africa (supported by material from northeastern RSA; ACR 2016: 1133).

Chaerephon pumilus (Cretzschmar, 1826)

Fig. 26E-F

Dysopes pumilus Cretzschmar, 1826: 69.

* Tadarida (Chaerephon) pumila (Cretzschmar, 1826).

Hayman et al. (1966: 62, map 95) reported this species from almost over the entire CRB area, with the exception of the northwestern and central parts of the DRC. The only more central locality is Stanleyville (= Kisangani, Tshopo Province), where a single specimen was collected in the $19^{\text {th }}$ century (RMCA 283). The additional records, we have found, seem to confirm this distribution. The distribution map given by Happold (2013v: 529) shows two main areas where C. pumilus occurs: one in West Africa, where it occurs from Senegal to Nigeria and northern Cameroon, although lacking in the coastal forest, and a second in eastern Africa, from the Red Sea coast in Eritrea over eastern Sudan, South Sudan and western Ethiopia, eastern DRC, Zambia, northern Zimbabwe, Malawi, Mozambique to northeastern RSA. It also occurs in southern Kenya, reaching the coast, going north into Somalia and south into Tanzania and Mozambique. Furthermore, she indicates a peculiar extension across the southern DRC to reach the Atlantic coast in Cabinda and Angola, and going south almost to the border with Namibia. ACR (2016: 387, 1135) mentions additional records from Cameroon, Gabon and Congo which narrow the gap between the western and eastern distribution ranges. However, there still seems to be a gap in the north between $20^{\circ}$ and $25^{\circ} \mathrm{E}$ in Chad, Sudan, Central African Republic and the DRC (with the exception of the single specimen from Kisangani), which might continue further in central and eastern Angola and Namibia towards the RSA. This would suggest that the western DRC specimens, as well as those from Angola, might be closer related to the specimens from western Africa than to those from the eastern part of the distribution area. A connection between the western and eastern populations might indeed be along the line suggested by Happold (2013v), but it might also be that the specimens from the Kasaï Provinces are the easternmost representatives of the western populations or the westernmost representatives of the eastern populations. 

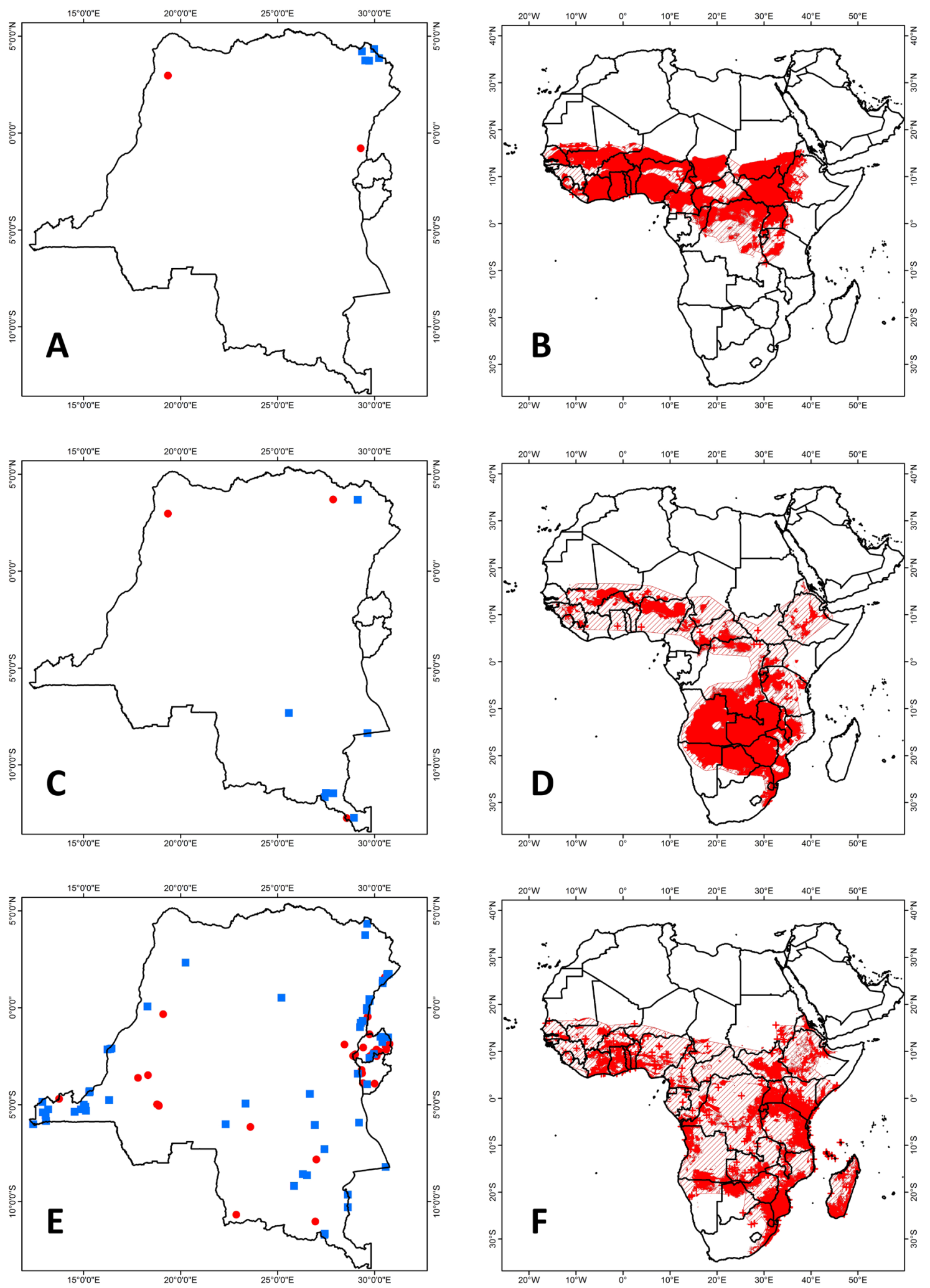

Fig. 26. Distribution maps. A-B. Chaerephon major (Trouessart, 1897). C-D. Chaerephon nigeriae Thomas, 1913. E-F. Chaerephon pumilus (Cretzschmar, 1826). A, C, E. Distribution in the CRB area. B, D, F. Pan-African distribution. 
Chaerephon russatus J.A. Allen, 1917

Fig. 27A-B

Chorephon russatus J.A. Allen, 1917: 458.

* Tadarida (Chaerephon) russata J.A. Allen, 1917.

The only specimens belonging to this species reported by Hayman et al. (1966: 64, map 96) were from the type locality in Haut-Uélé Province, northeastern DRC. There is one additional record from Tandala (Sud-Ubangi Province), northwestern DRC. The distribution map presented by Happold (2013w: 531) shows that the few known localities, where this species was found, are almost on a straight line reaching from southern Côte d'Ivoire, southern Ghana, Cameroon, northern DRC to southwestern Kenya.

Genus Mops Lesson, 1842

Subgenus Mops Lesson, 1842

Mops (Mops) condylurus (A. Smith, 1833)

Fig. 27C-D

Nyctinomus Condylurus A. Smith, 1833: 54.

* Tadarida (Mops) condylura (A. Smith, 1833).

* Tadarida (Mops) condylura condylura (A. Smith, 1833).

Hayman et al. (1966: 64) indicated that many of the specimens they reported under this name could actually belong to one of the next species (M. niveiventer). The major part of the specimens they mention are from the eastern part of the DRC (provinces Haut-Uélé, Ituri, Nord-Kivu, Sud-Kivu, Maniema, HautLomami, Haut-Katanga) and Rwanda. Their two remaining localities were from more western areas: Lubondai-Tshimbulu (Kasaï-Central Province) and Léopoldville (Kinshasa City Province). We found additional specimens from localities in Rwanda and Burundi, the Sud-Kivu, Tanganyika and KasaïCentral Provinces, and from further to the west (Kikwit, Kwilu Province). These localities were all in the general area already reported by Hayman et al. (1966). However, we also found two records from the northwestern part of the DRC (Duma and Tandala, both in Sud-Ubangi Province).

The map given by Happold (2013r: 505) shows a major distribution area in eastern Africa, ranging from southeastern Sudan, over South Sudan, eastern DRC, Uganda, Rwanda, Burundi, southern DRC, most of Angola, Zambia, Malawi, southern Mozambique to northeastern RSA. In Kenya, there is a small band along the border with Tanzania from Uganda to the coast, where it turns northwards to reach into Somalia. The absence of the species in almost entire Tanzania and northern Mozambique is rather strange, although a single locality is plotted on the coastal border between these two countries. In western Africa, there are three areas: one encompassing The Gambia, southern Senegal and Guinea-Bissau, a second in Sierra Leone, and a third covering eastern Côte d'Ivoire to central Nigeria. Happold (2013r) also marked a small area in Ethiopia. Our data (ACR 2016: 1153) suggest that $M$. condylurus might occur more extensively in Ethiopia, as well as in Tanzania and Mozambique, and also further to the north in West Africa. The distribution in southern DRC and Angola, however, might not be as extensive as proposed by Happold (2013r).

Mops (Mops) congicus J.A. Allen, 1917

Fig. 27E-F

Mops congicus J.A. Allen, 1917: 467.

* Tadarida (Mops) congica J.A. Allen, 1917. 

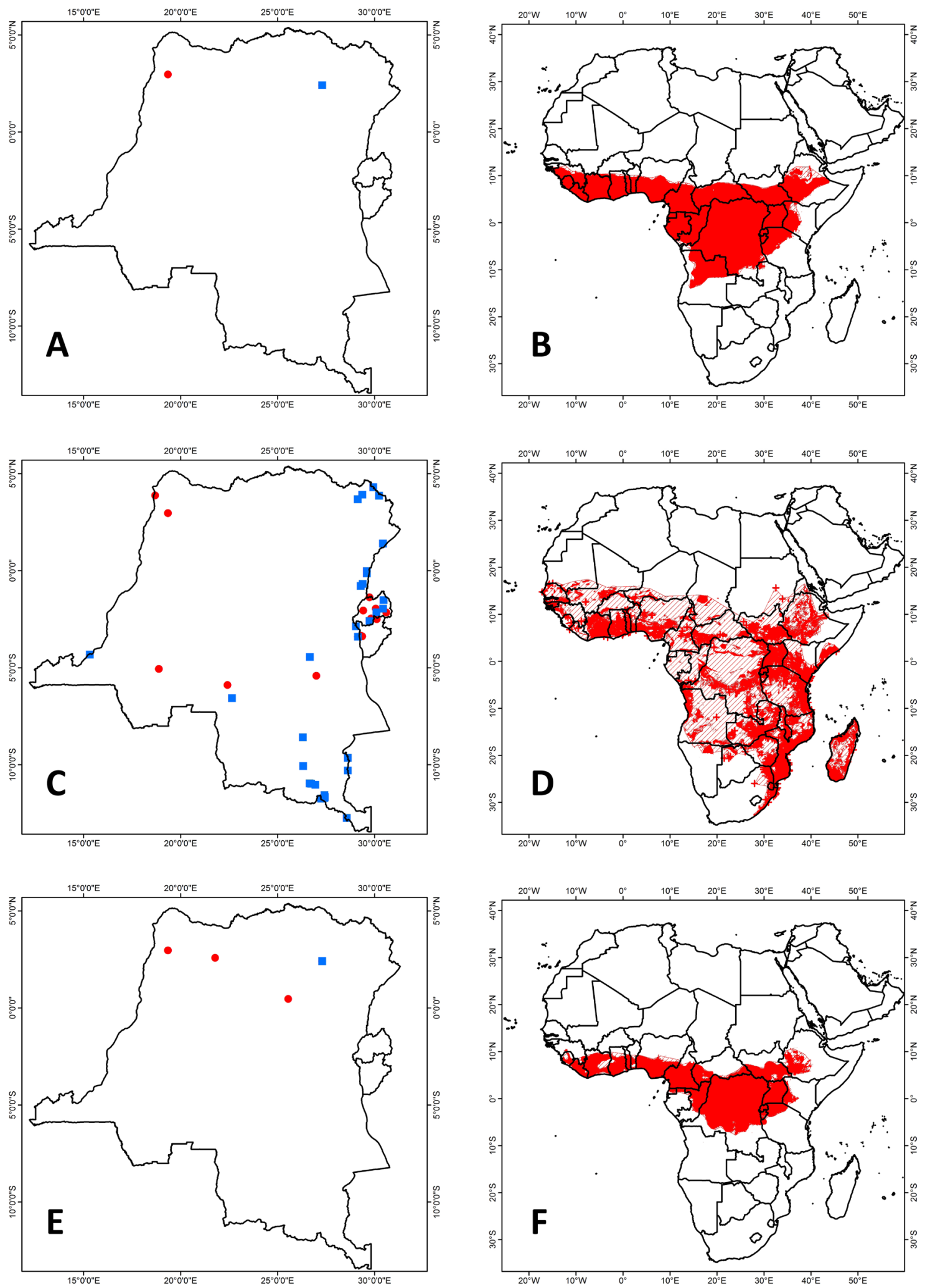

Fig. 27. Distribution maps. A-B. Chaerephon russatus J.A. Allen, 1917. C-D. Mops (Mops) condylurus (A. Smith, 1833). E-F. Mops (Mops) congicus J.A. Allen, 1917. A, C, E. Distribution in the CRB area. B, D, F. Pan-African distribution. 
Besides the type specimen, Hayman et al. (1966: 65) mention one extra locality: Medje. Additional occurrences of this species are reported from Tandala (Sud-Ubangi Province), Yalosemba (Mongala Province) and Tshopo. Fahr (2013m: 508) indicated that congica was found at a few isolated localities from southern Cameroon (south of the Sanaga River), northern DRC (north of the Congo River) and Uganda.

See also Mops (Mops) trevori J.A. Allen, 1917 below.

Mops (Mops) demonstrator (Thomas, 1903)

Fig. 28A-B

Nyctinomus demonstrator Thomas, 1903: 504.

* Tadarida (Mops) demonstrator (Thomas, 1903).

For this species, Hayman et al. (1966: 65) mention a number of localities in or near the Garamba National Park (Haut-Uélé Province). A number of specimens they mentioned as Tadarida (Mops) niveiventer from Keyberg (near Lubumbashi, Haut-Katanga Province) were re-identified by R.L. Peterson (Wim Wendelen, pers. comm.). This is rather strange as Fahr (2013n: 510) indicates that demonstrator only occurs in the northeastern part of the DRC, northwards along the River Nile to northern South Sudan and along the Blue Nile in Sudan. He also plotted a number of individual localities in Cameroon, Côte d'Ivoire, Ghana and Burkina Faso. All of these are north of the equator. However, there is one additional specimen from Mushe, Rwanda (RMCA 37983), which more or less bridges the gap between HautUélé and Haut-Katanga. Based on climatic data, the SDM map makes the extension towards Rwanda conceivable, but a further expansion to Haut-Katanga is still dubious.

Mops (Mops) midas (Sundevall, 1843)

Fig. 28C-D

Dysopes midas Sundevall, 1843 (for 1842): 207.

* Tadarida (Mops) midas (Sundevall, 1843).

Hayman et al. (1966: 66, map 103) reported specimens from in and near the Garamba National Park (Haut-Uélé Province), from Mwiga and Kamohohora (Nord-Kivu Province) and from Uvira (Sud-Kivu Province). There is one additional specimen from the Garamba Park, which apparently was overlooked by Hayman et al., as were two further specimens from Bwamanda (Sud-Ubangi Province). These two latter specimens are also not plotted by Cotterill \& Happold (2013c: 519) on their distribution map. This map indicates that $M$. midas occurs in a large number of isolated areas and individual localities, primarily in the eastern and southern part of Africa, and in two small areas in northern Cameroon and southern Burkina Faso, and one locality in northern Senegal. The SDM map also shows a patchy distribution, but nevertheless more extensive, especially in southern Africa, but also in the northern part of the distribution area, extending more northwards than suggested by Cotterill \& Happold (2013c: 519).

Mops (Mops) niangarae J.A. Allen, 1917

Fig. 28E-F

Mops niangarce J.A. Allen, 1917: 468.

* Tadarida (Mops) niangarae J.A. Allen, 1917.

As indicated by Happold (2013u: 522) the specific status of Mops niangarae remains uncertain. Hayman et al. (1966: 66) considered it a separate species, but noted that Koopman (1965: 25) already mentioned that it closely resembled M. congicus, except for the absence of a band connecting the ears. Hayman \& Hill (1971: 60) also consider it a valid species, but refer to Peterson (in litt.), who believed the missing 

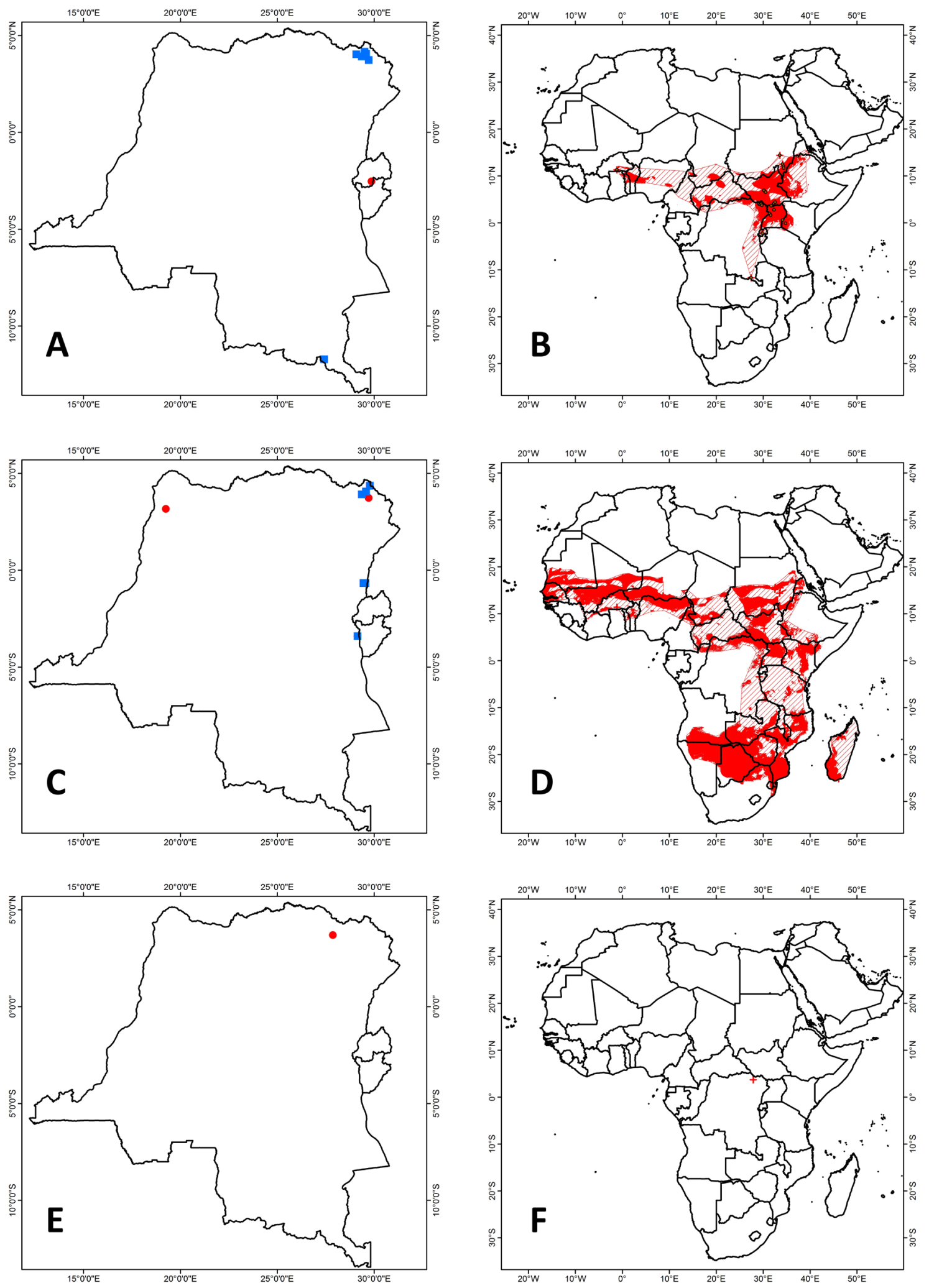

Fig. 28. Distribution maps. A-B. Mops (Mops) demonstrator (Thomas, 1903). C-D. Mops (Mops) midas (Sundevall, 1843). E-F. Mops (Mops) niangarae J.A. Allen, 1917. A, C, E. Distribution in the CRB area. B, D, F. Pan-African distribution. 
connection between the ears to be an artifact and thought that niangarae is identical to trevori, but not conspecific with congicus [contrary to Happold (2013u: 522) and Simmons (2005: 443), who indicated that Hayman \& Hill (1971) consider niangarae to be a subspecies of congicus]. Freeman (1981: 111) retained niangarae as a distinct species based on its more robust jaw and separated ears. This view is followed by Simmons (2005: 443), who suggested that a more formal revision of the trevori/congicus (and niangarae) complex is needed, but Thorn et al. (2009: 68) retain it as a synonym of M. trevori.

Due to the fact that the species is only known from the type specimen, the general distribution map only shows a single locality mark.

Mops (Mops) niveiventer Cabrera \& Ruxton, 1926

Fig. 29A-B

Mops angolensis niveiventer Cabrera \& Ruxton, 1926: 594.

* Tadarida (Mops) chitauensis J. Eric Hill, 1937: 2-3.

* Tadarida (Mops) niveiventer (Cabrera \& Ruxton, 1926).

Aellen (1966: 71, map 98) reported six specimens from Elisabethville (= Lubumbashi) and Jadotville (= Likasi) in southeastern DRC. Hayman \& Hill (1971: 60) considered chitauensis as a synonym of niveiventer, which was followed by Koopman (1975: 421), Freeman (1981: 160) and Simmons (2005: 443).

Hayman et al. (1966: 65) did not provide a separate map for this species, but probably included the specimen on the map for Tadarida (Mops) condylura (map 99). They reported the species from Astrida (= Butare, Rwanda), Samba (Maniema Province), a number of localities around Elisabethville (= Lubumbashi) and Jadoville (= Likasi) in Haut-Katanga Province, and from Luluabourg (= Kananga, Kasaï-Central Province). Additional records were found from Rwanda, Burundi, the area around Kananga and the area around Kikwit (Kwilu Province).

Cotterill (2013g: 526) mentioned that niveiventer is found in wetter savannas (miombo woodland). On his map, he marked an area covering southeastern DRC, western Zambia and extreme eastern Angola and a second area on the border between Zambia and northwestern Mozambique. Furthermore, he plotted a number of individual localities in Rwanda, the DRC, Zambia and Angola. Our map suggests a more uniform distribution from central Angola, the entire territory of Zambia and reaching the western part of Malawi, and a second area connecting the remaining DRC localities with those in Rwanda and Burundi.

Mops (Mops) trevori J.A. Allen, 1917

Fig. 29C-D

Mops trevori J.A. Allen, 1917: 469.

* Tadarida (Mops) congica J.A. Allen, 1917.

Hayman et al. (1966: 65) considered trevori to be a synonym (or - following Koopman 1965: 26 - at most as subspecies) of Tadarida (Mops) congica. However, based on size and subtle differences in the ear and facial features, Peterson (1972: 10) separated the smaller trevori from the larger congicus. This view was followed by Freeman $(1981: 159,160)$ and Simmons (2005: 444).

Besides the type specimen, Hayman et al. (1966: 65) mention one extra locality: Iso.4 (RBINS 13826). As indicated above, however, this specimen was re-identified as T. aloysiisabaudiae by Peterson (1972: 2), leaving the type specimen of M. trevori as the only representative of this species in the DRC. 

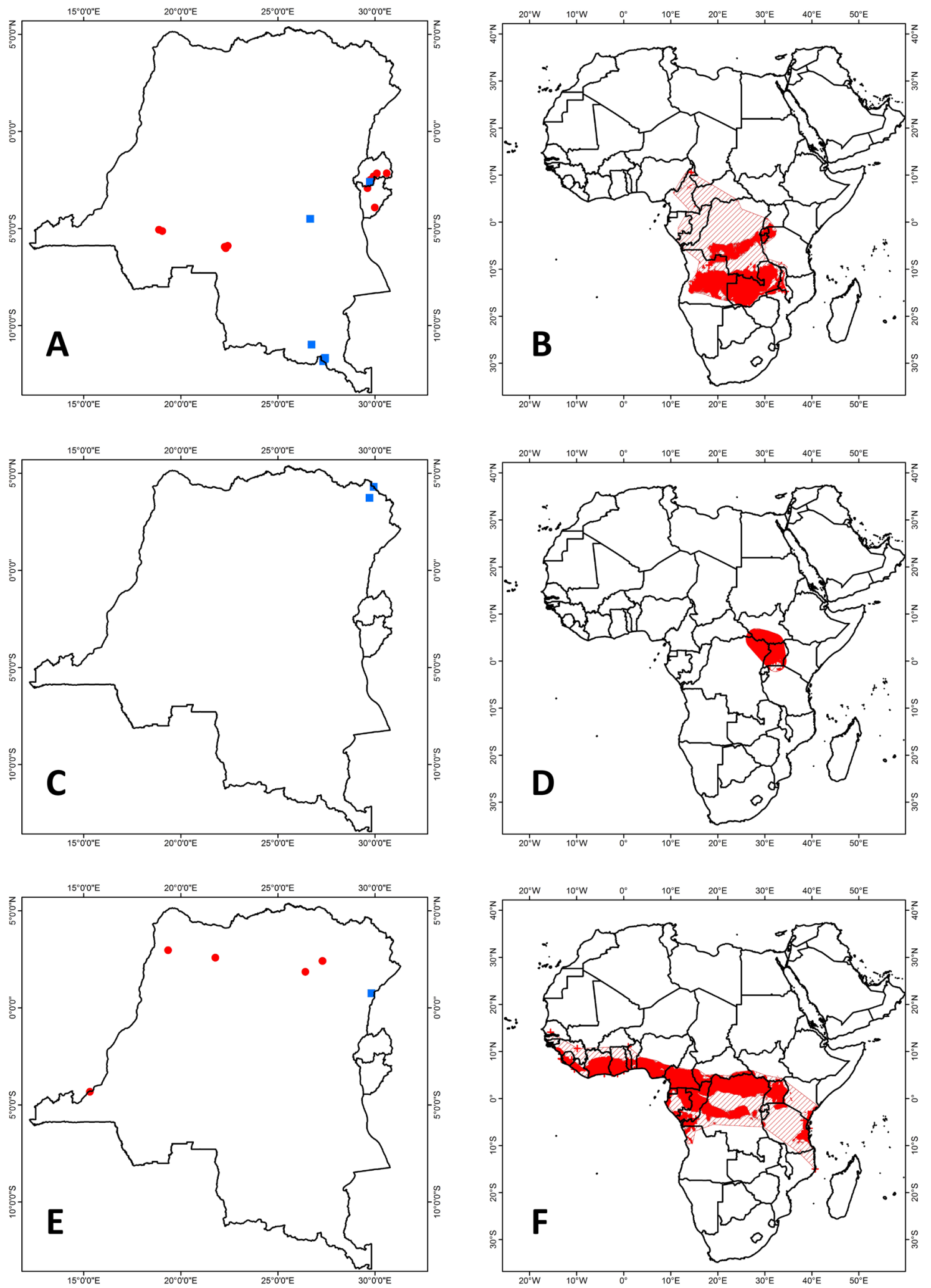

Fig. 29. Distribution maps. A-B. Mops (Mops) niveiventer Cabrera \& Ruxton, 1926. C-D. Mops (Mops) trevori J.A. Allen, 1917. E-F. Mops (Xiphonycteris) brachypterus (Peters, 1852). A, C, E. Distribution in the CRB area. B, D, F. Pan-African distribution. 
Happold (2013z: 538) marked the northeastern corner of the DRC and central Uganda as the core distribution area for trevori, with some further individual localities in Guinea, eastern Côte d'Ivoire, western Ghana, and western Nigeria. A record from the Central African Republic needs further investigation as it might represent either congica or trevori (Fahr 2013m; Happold 2013z). Concerning the habitat preferences of the two species, Peterson (1972: 17) stated that trevori is a forest edge or open forest dweller.

Subgenus Xiphonycteris Dollman, 1911

Mops (Xiphonycteris) brachypterus (Peters, 1852)

Fig. 29E-F

Dysopes brachypterus Peters, 1852: 59.

* Tadarida (Mops) leonis (Thomas, 1908): 373.

Following El-Rayah (1980, 1981), Simmons (2005: 442) and Happold (2013q: 501), we consider leonis to be a synonym of brachypterus.

Hayman et al. (1966: 66, map 102) report T. (M.) leonis from six localities, of which we were unable to trace the underlying BMNH specimens for the three southernmost records (Sud-Kivu Province). The three other localities are in the northeastern part of the DRC (Nord-Kivu, Haut-Uélé and Tshopo Provinces). The species was further reported from the northwestern part of the country (Yalosemba, Mongala Province and Tandala, Sud-Ubangi Province), but also from the southwestern part (Thysville [= Mbanza-Ngungu], Kongo Central Province and Léopoldville [= Kinshasa]). These last four localities were not plotted on the distribution map presented by Happold (2013q: 502). On her map, she painted four areas where this species occurs: northeastern DRC (with two individual localities in Uganda), the east African coast (from Malindi in Kenya to Mozambique Island), coastal West Africa (from Sierra Leone to western Togo, and from western Nigeria to Equatorial Guinea) expanding to the western part of the Central African Republic. The SDM map shows an almost similar image, although the western DCR records connect Happold's northeastern DRC area with the West African one.

Mops (Xiphonycteris) nanulus J.A. Allen, 1917

Fig. 30A-B

Mops (Allomops) nanulus J.A. Allen, 1917: 477.

* Tadarida (Mops) nanula (J.A. Allen, 1917).

This species was reported by Hayman et al. (1966: 66, map 104) from nine localities in the northeastern, southeastern and southwestern parts of the DRC. The specimens from Butembo (Nord-Kivu Province) were re-identified by Frits De Vree as belonging to $M$. (X.) spurrelli. Additional specimens were reported from the Kikwit area (Kwilu Province), Lulimbi (Nord-Kivu Province), the Isa River (Ituri Province), and Aba (Haut-Uélé Province). All of these are in the same general area. However, M. (X.) nanulus was also collected at Yalosemba (Mongala Province) and Tandala (Sud-Ubangi Province) in the northwestern part of the DRC. These two latter localities were not included on the map presented by Happold (2013t: 521). Her map shows a patchy distribution, with two major areas in the DRC: one in the southwestern part of the country and a second reaching from western South Sudan over northeastern DRC through Uganda to western Kenya. Furthermore, there are smaller areas in the border area of southern Nigeria and Cameroon, southern Ghana and Sierra Leone, as well as individual localities along the border of Liberia and Côte d'Ivoire and between Benin and Nigeria, in Cameroon, and western Ethiopia. Our Pan-African distribution map suggests that the species can occur from Sierra Leone to Togo and from southern Nigeria over Cameroon, northern Congo, southern Central African Republic, northern DRC, southern South Sudan and Uganda to western Kenya, and as well as from Cameroon, eastern Gabon, central Congo into southwestern and southcentral DRC. 

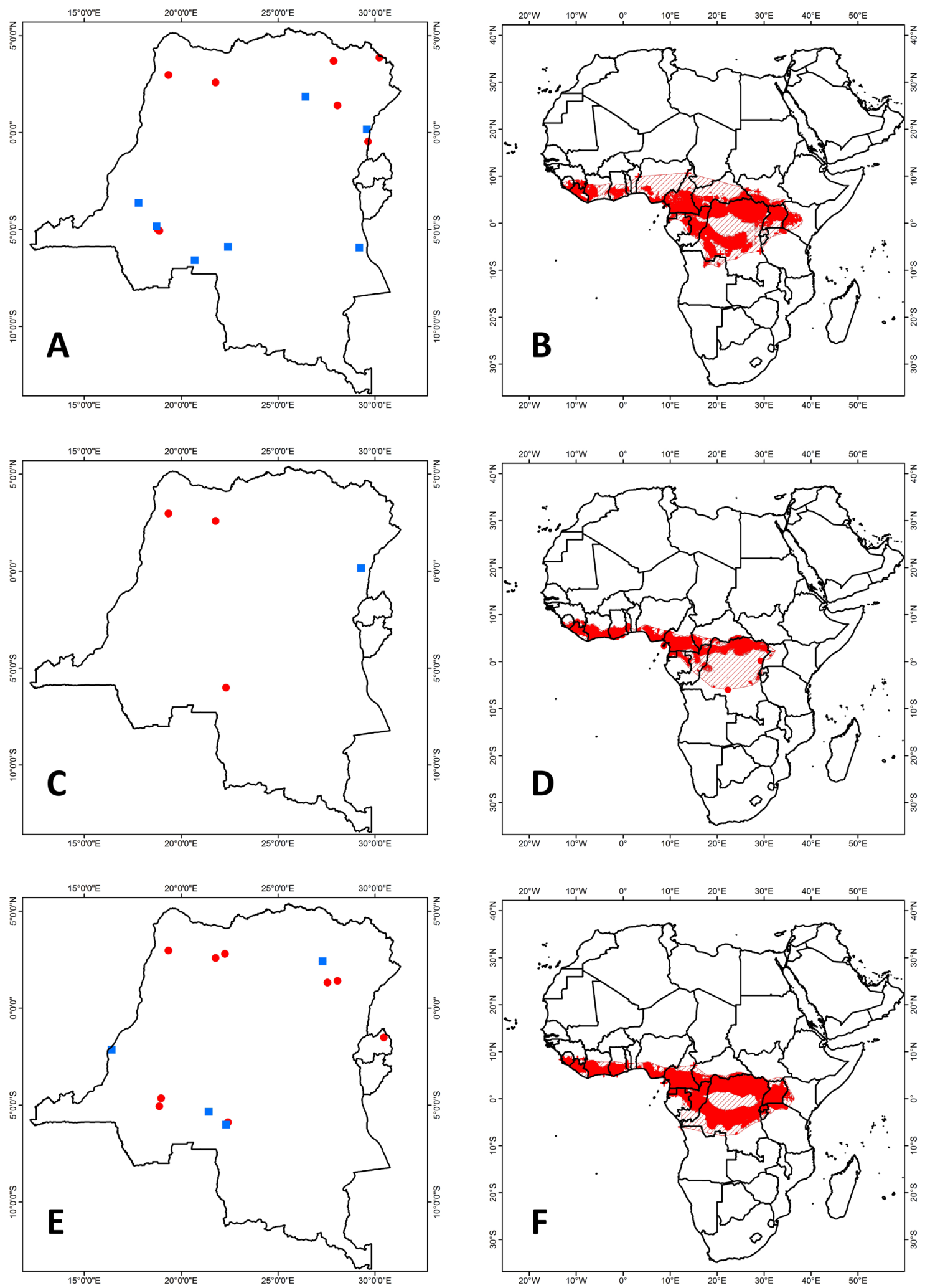

Fig. 30. Distribution maps. A-B. Mops (Xiphonycteris) nanulus J.A. Allen, 1917. C-D. Mops (Xiphonycteris) spurrelli (Dollman, 1911). E-F. Mops (Xiphonycteris) thersites (Thomas, 1903). A, C, E. Distribution in the CRB area. B, D, F. Pan-African distribution. 
Mops (Xiphonycteris) spurrelli (Dollman, 1911)

Fig. 30C-D

Xiphonycteris spurrelli Dollman, 1911: 211.

*? Xiphonycteris spurrelli Dollman, 1911.

Although Hayman et al. (1966: 8) did not mention this species explicitly in their 'Catalog of Species', they do include it with a question mark in their 'Nominal List of Congo Bats', and in their key, referring to Koopman (1965: 31), who tentatively identified a skin from Luluabourg (= Kananga, Kasaï Central Province) as belonging to this species. The specimens from Butembo (Nord-Kivu Province) were reported as Tadarida (Mops) nanula, but were re-identified as belonging to spurrelli (see above). Further specimens belonging to this species were collected in the northwestern part of the DRC: Yalosemba (Mongala Province) and Tandala (Sud-Ubangi Province). Happold (2013x: 533) ignored the record from Luluabourg and also missed out on the northwestern DRC records, mentioning it only from the northeastern part of the country. Furthermore, she also plotted individual localities from the Central African Republic, Equatorial Guinea, Togo, western Côte d'Ivoire and southern Guinea, and three small areas in Cameroon, southern Ghana and the border area between Sierra Leone and Liberia. The SDM map is very similar, but we suspect the areas to be more continuous north of the Central African rainforest and the record from Luluabourg suggests it also occurs south of the rainforest. Whether it occurs in the forest itself is not yet clear.

Mops (Xiphonycteris) thersites (Thomas, 1903)

Fig. 30E-F

Nyctinomus thersites Thomas, 1903: 634.

* Tadarida (Mops) thersites (Thomas, 1903).

Hayman et al. (1966: 66, map 106) mentioned this species from five localities: two in the northeast of the DRC (Avakubi, Tshopo Province and Medje, Haut-Uélé Province) and three in the southwestern part of the country (Luluabourg [= Kananga], Kasaï Central Province, Luebo, Kasaï Province and Ndwa, MaiNdombe Province). Additional specimens have been collected at a number of localities in Rwanda, in Kwilu Province (Mosenge and Kikwit), in Kongo Central Province (Banana), but also in Yalosemba and on the road between Bombanga and Bumba (Mongala Province) and Tandala (Sud-Ubangi Province), in the northwest of the DRC. Although the SDM map shows more red than the one presented by Happold (2013y: 536), it looks very similar, with a northern and southern-central branch in the DRC, passing around the Congolian rainforest. We, however, suspect that the two branches might reunite in the eastern part of the DRC.

Genus Myopterus E. Geoffroy Saint-Hilaire, 1818

Myopterus daubentonii Desmarest, 1820

Fig. 31A-B

Myopterus daubentonii Desmarest, 1820: 132.

* Myopterus albatus Thomas, 1915: 469.

Koopman (1989: 9) indicated that he believed that the larger forms of the genus Myopterus (daubentonii, senegalensis and albatus) are all the same species. Adam et al. (1993: 317) suggested that M. albatus might only be a mere subspecies of M. daubentonii. This view was also followed by Simmons (2005: 446) and Fahr (2013i: 476). Besides the specimens from Bunia (Ituri Province), Niangara (Haut-Uélé Province) and the Uélé River mentioned by Hayman et al. (1966: 68, map 113), no further representatives 

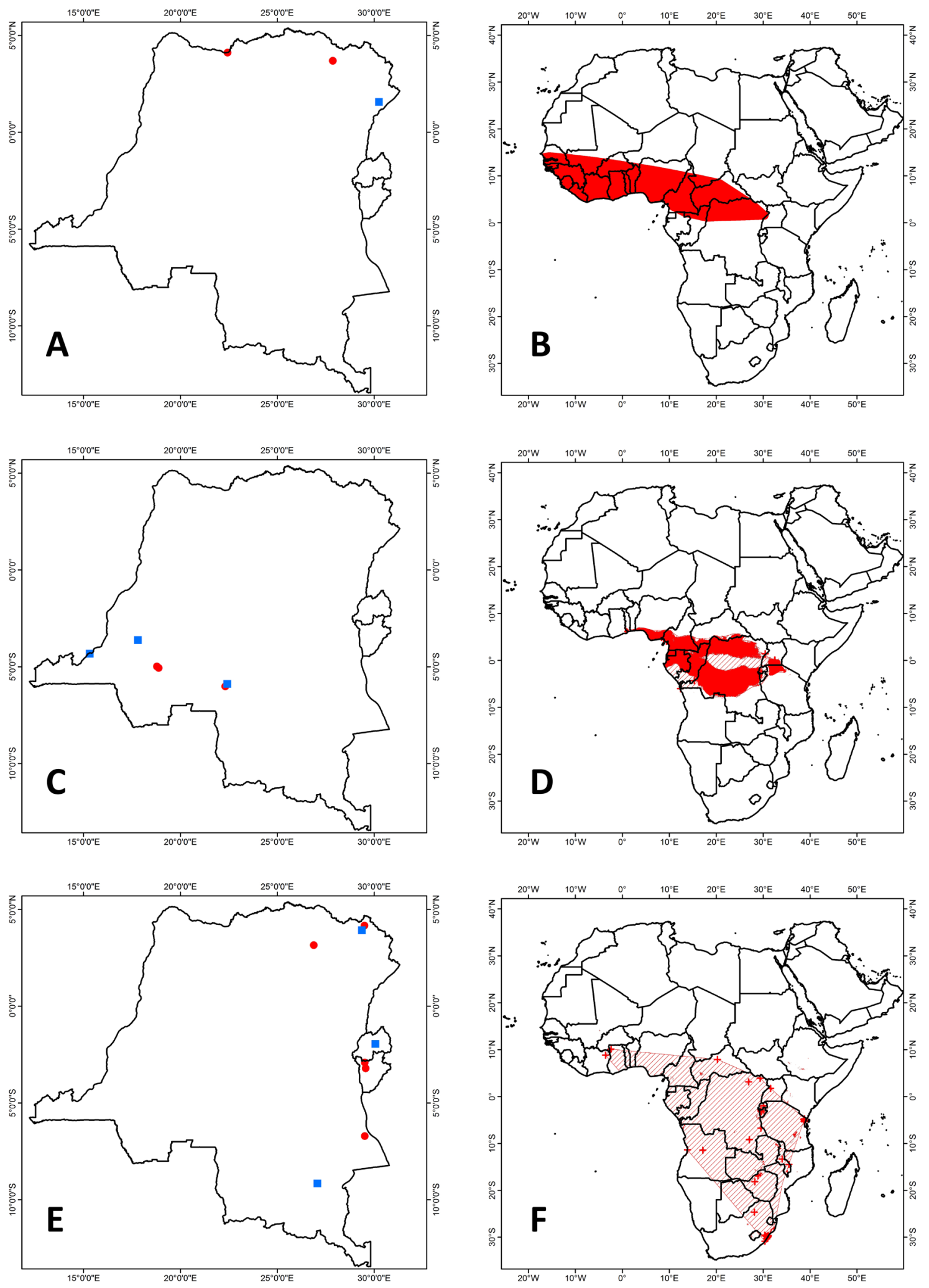

Fig. 31. Distribution maps. A-B. Myopterus daubentonii Desmarest, 1820. C-D. Myopterus whitleyi (Scharff, 1900). E-F. Otomops martiensseni (Matschie, 1897). A, C, E. Distribution in the CRB area. B, D, F. Pan-African distribution. 
of this species are known from the CRB area. The distribution map provided by Fahr (2013i: 477) includes additional specimens from northern Central African Republic, Côte d'Ivoire and Senegal.

Myopterus whitleyi (Scharff, 1900)

Fig. 31C-D

Mormopterus Whitleyi Scharff, 1900: 569.

* Myopterus whitleyi (Scharff, 1900).

Hayman et al. (1966: 68, map114) reported M. whitleyi from three localities: Léopoldville (= Kinshasa, Kinshasa City Province), Luluabourg (= Kananga, Kasaï Central Province) and Beno (Mai-Ndombe Province), all in the southwestern part of the DRC. Additional specimens were collected in the Kikwit area (Kwilu Province) and in Banana (Kongo Central Province), also in the southwestern part of the DRC. One further specimen, however, was collected in the northeastern part of the DRC: $10 \mathrm{~km} \mathrm{~N}$ of Kasenye (Ituri Province). Aellen (1952: 99) reported the species from Coquilhatville (= Mbandaka, Equateur Province), but that record could not be traced. The distribution map presented by Fahr (2013j: 478) shows two red areas: one encompassing the southwestern DRC records (except for Banana, which is plotted separately) and a second in coastal Nigeria/Cameroon (from the mouth of the Niger to the Mount Cameroon area). Furthermore, he plotted single localities in Ghana, the Central African Republic, Gabon, and Uganda. The SDM map looks very similar, although it doesn't reach Ghana, as we were unable to trace the specific locality for that country. The map also suggests that $M$. whitleyi does not occur in the Congolian rainforest, but encircles it both on the north and the south, and the two branches meet again in the Ugandan-Tanzanian border area.

Genus Otomops Thomas, 1913

Otomops martiensseni (Matschie, 1897)

Fig. 31E-F

Nyctinomus martiensseni Matschie, 1897: 84.

* Otomops martiensseni (Matschie, 1897).

Otomops martiensseni was reported by Hayman et al. (1966: 68, map 112) from three localities in the eastern part of the DRC (Poko in Bas-Uélé Province, the Garamba National Park in Haut-Uélé Province and the Upemba National Park in Haut-Lomami Province) and from one locality in Rwanda (Kigali). Additional specimens were collected in Burundi (Rwegura and the Kibira National Park) and in southeastern DRC (Marungu, Tanganyika Province). Yalden \& Happold (2013: 481) indicate that the distribution of this species in Africa is wide, but disjunct, with records from Côte d'Ivoire and Ghana in West Africa, the Central African Republic, DRC, Uganda, Rwanda and Angola in Central Africa, the RSA, Zimbabwe and Malawi in southern Africa, and a range along the border between Tanzania and Kenya, reaching to the border with Uganda. They also mark a few small areas in Ethiopia and Djibouti. However, the specimens from Ethiopia, Djibouti, Eritrea and Kenya have been found to represent a recently described species, Otomops harrisoni Ralph, Richards, Taylor, Napier \& Lamb, 2015. Ralph et al. (2015: 27) also indicate that the range of their new species might include additional localities in Uganda, and their MapEnt map on page 17 suggests that this species also has a high probability of occurring in the CRB area.

Genus Tadarida Rafinesque, 1814

Tadarida fulminans (Thomas, 1903)

Fig. 32A-B

Nyctinomus fulminans Thomas, 1903: 501.

* Tadarida (Tadarida) fulminans (Thomas, 1903). 

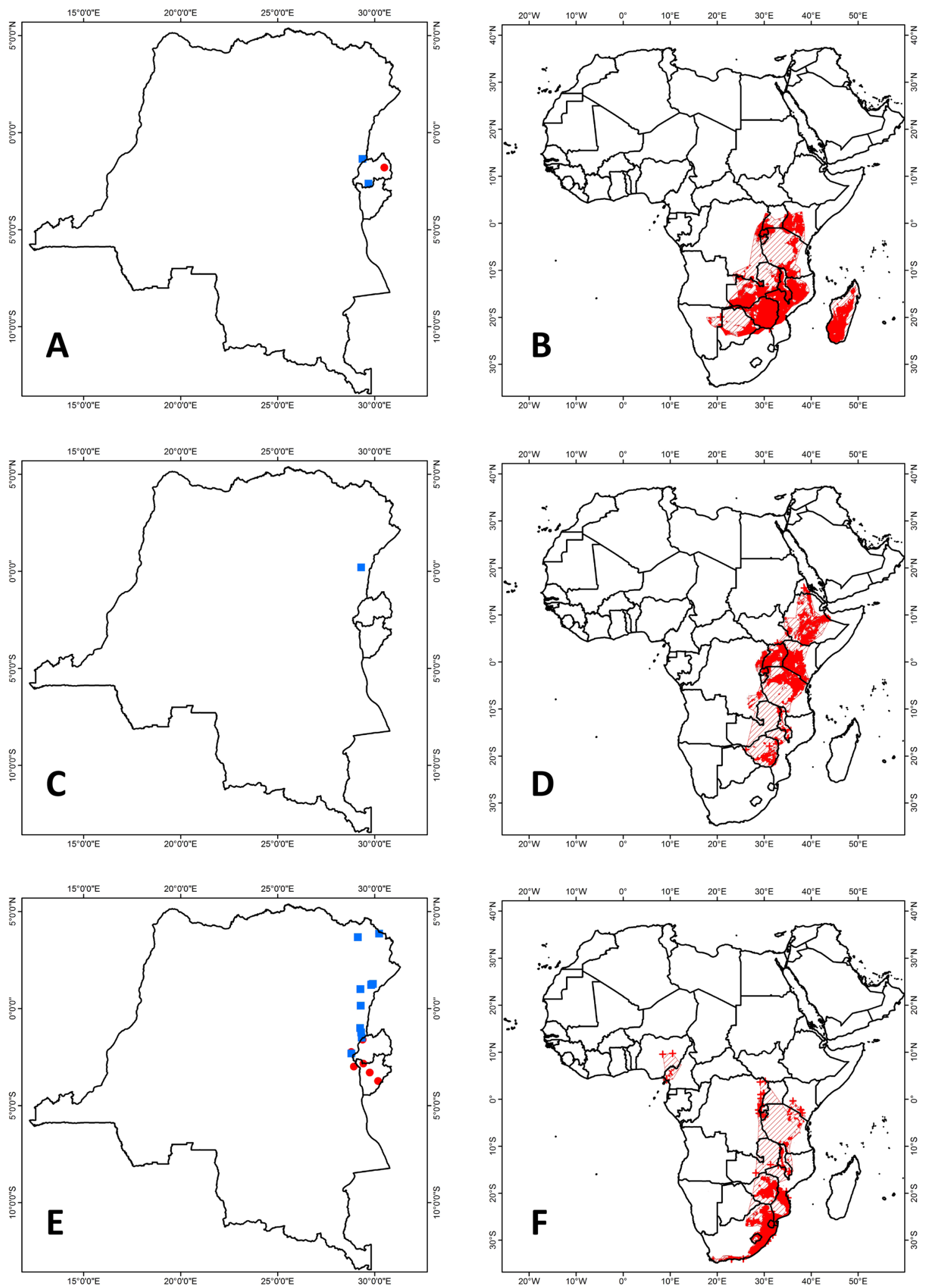

Fig. 32. Distribution maps. A-B. Tadarida fulminans (Thomas, 1903). C-D. Tadarida ventralis (Heuglin, 1861). E-F. Miniopterus fraterculus Thomas \& Schwann, 1906. A, C, E. Distribution in the $\mathrm{CRB}$ area. B, D, F. Pan-African distribution. 
Hayman et al. (1966: 68, map 111) reported T. fulminans from two localities: Nyakibanda (Rwanda) and Rumangabo (Nord-Kivu Province). Additional specimens were collected from two further localities in Rwanda: Nyanza and Rukara. On the African continent, T. fulminans is restricted to eastern Africa, from Kenya to extreme northern RSA. On his distribution map, Cotterill (2013e: 512) marked three areas: on the border between Kenya and Uganda, in northeastern Zimbabwe, reaching into Mozambique and in northwestern Zimbabwe, reaching into Zambia. All other occurrences are individual localities. Based on climatic data, the SDM map indicates a potential wider occurrence in southeastern Africa and two areas in the northern part: one in northeastern DRC, reaching into Uganda and Rwanda, and another in western Kenya, with a corridor-like connection between the latter and the southern range.

Tadarida ventralis (Heuglin, 1861)

Fig. 32C-D

Nyctinomus (Dysopes) ventralis Heuglin, 1861: 4, 11.

* Tadarida (Tadarida) africana (Dobson, 1876): 348.

Hayman et al. (1966: 67, map 107) reported that only one specimen was found in the CRB area: RMCA 29241 from Alima (Nord-Kivu Province). Until that time, T. africana was considered to be a valid species, occurring in southern Africa. Kock (1975: 6) discussed the features of an original specimen of Nyctinomus ventralis and concluded that africana is a synonym of ventralis. This view is followed by most recent authors.

No further specimens from the CRB area were found.

Cotterill (2001a: 201) indicates that $T$. ventralis has a wide and highly scattered distribution across eastern tropical Africa, which is confirmed by the distribution map presented by Cotterill (2013h: 539). The range covers a major part of western Kenya and the joining area in northern Tanzania. Furthermore, he marked individual localities in Eritrea, Ethiopa, South Sudan, DRC, Zambia, Malawi, northwestern Mozambique, Zimbabwe and possibly northeastern RSA. The SDM map looks very similar, although we excluded the RSA records, as we could not trace the specific specimens.

\section{Family Miniopteridae Dobson, 1875}

During a long period, the genus Miniopterus was considered to represent a separate subfamily within the Vespertilionidae. However, based on dental characters, the shape of the cranium, the double articulation of the humerus-scapulum, the length of the second phalanx of the third finger, the karyotype, the absence of a baculum and reproductive differences, Mein \& Tupinier (1977: 210) suggested that the distinction should be made at the family level. This was later confirmed with molecular data (Hoofer \& Van Den Bussche 2003: 1; Miller-Butterworth et al. 2007: 1553).

\section{Genus Miniopterus Bonaparte, 1837}

As mentioned by Happold (2013ap: 711), representatives of the genus Miniopterus show a remarkable degree of morphological uniformity, which makes it difficult to identify the various forms. In continental Africa, she recognizes six species, of which we believe four occur in the CRB region. However, we cannot exclude that some of the specimens were erroneously identified.

\section{Miniopterus fraterculus Thomas \& Schwann, 1906}

Fig. 32E-F

Miniopterus fraterculus Thomas \& Schwannn, 1906: 162.

* Miniopterus schreibersi villiersi Aellen, 1956: 890. 
Koopman (1965: 18) refers to Harrison (1953: 71), who revised the Miniopterus forms of southern Africa, where he distinguished two species: "the large natalensis and the small fraterculus". Unfortunately, however, this distinction doesn't seem to hold (see key).

Hayman et al. (1966: 59-60) assigned most of their material to Miniopterus schreibersi villiersi and only 15 specimens from two localities to M. s. vicinior. The latter are plotted on their map 90, but no separate map was presented for M. s. villiersi. Apparently, these specimens were included on map 88 (M. inflatus).

In "Mammals of Africa", Bernard \& Happold (2013d: 713; 2013e: 719) restrict M. fraterculus to the northwestern part of the DRC and M. natalensis to the southeastern part. We are following this division for the CRB material, with specimens from the DRC provinces of Haut-Uélé, Ituri, Nord-Kivu, and Sud-Kivu, as well as from Rwanda and Burundi being assigned to $M$. fraterculus and those from the provinces of Tanganyika, Haut-Lomami and Haut-Katanga to M. natalensis. However, we also assigned records from more southwestern localities to the latter species: Luluabourg (= Kananga, Kasaï-Central Province) and near Thysville (= Mbanza-Ngongo, Kongo Central Province).

See also Miniopterus natalensis (A. Smith, 1833) below.

Miniopterus inflatus Thomas, 1903

Fig. 33A-B

Miniopterus inflatus Thomas, 1903: 634.

* Miniopterus inflatus rufus Sanborn, 1936: 112.

With the exception of FMNH 152883 and 152884 from 32 km SE of Mbuji-Mayi (Kasaï Oriental Province), all other specimens are from the eastern part of the CRB area (DRC provinces of Haut-Uélé, Ituri, Tanganyika, Haut-Lomami and Haut-Katanga; Rwanda and Burundi).

Happold (2013aq: 715) indicated that M. inflatus occurs widespread south of the Sahara, but mostly in isolated localities. Contrary to her map, we excluded the specimens from Ethiopia, as we follow Peterson et al. (1995: 120), Lavrenchenko et al. (2004b: 144), Simmons (2005: 519) and Juste et al. (2007: 28) in considering africanus as a separate species. We also believe that the species occurs far further to the south, into the RSA.

Miniopterus minor Peters, 1867

Fig. 33C-D

* Miniopterus minor Peters, 1867 (for 1866): 870.

Hayman et al. (1966: 60) reported M. minor from one locality: Thysville (= Mbanza Ngungu, Kongo Central Province). Two specimens from caves near Thysville (RMCA 10061, 22501) were originally identified as $M$. schreibersi villiersi, but were re-identified as $M$. minor, and this was also the case for a specimen from Kisala (INPBC W1462). All these localities are in the extreme western part of the DRC.

Happold (2013ar: 717) indicates that M. minor is distributed over three areas in continental Africa: on São Tomé, in western DCR and southwestern Congo and in the coastal boundary area between Kenya and Tanzania. The species is furthermore also reported from the Central African Republic, the Comoro Islands and from Madagascar (ACR 2016: 1191).

Miniopterus natalensis (A. Smith, 1833)

Fig. 33E-F

Vespertilio Natalensis A. Smith, 1833: 59.

* Miniopterus natalensis arenarius Heller, 1912: 2. 

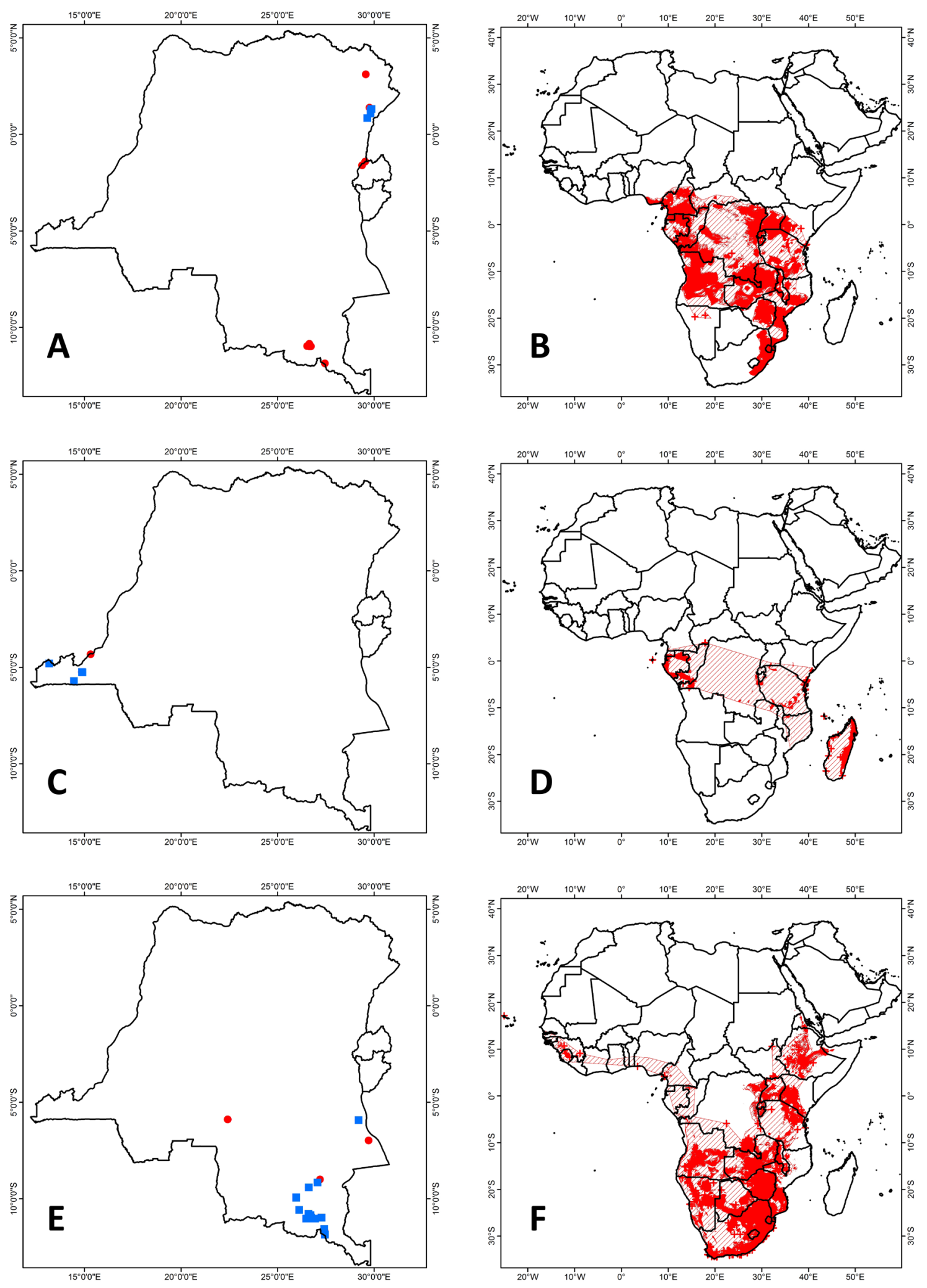

Fig. 33. Distribution maps. A-B. Miniopterus inflatus Thomas, 1903. C-D. Miniopterus minor Peters, 1867. E-F. Miniopterus natalensis (A. Smith, 1833). A, C, E. Distribution in the CRB area. B, D, F. Pan-African distribution. 
* Miniopterus schreibersi vicinior J.A. Allen, 1917: 450.

* Miniopterus schreibersi villiersi Aellen, 1956: 890.

Originally described as a subspecies of breyeri, vicinior was moved to M. natalensis by Sanborn (1936: 110). However, most later authors assigned the form to M. schreibersi, but we follow Simmons (2005: 521) and Monadjem et al. (2013: 138), who included it back in M. natalensis. On the other hand, Thorn et al. (2009: 49) included vicinior specimens from Uganda in M. fraterculus.

Aellen (1956) described villiersi as a subspecies of $M$. inflatus. Most recent authors, though, include it in M. schreibersii, but Fahr et al. (2006: 173), Decher et al. (2010: 170) and Schaer et al. (2013: 17416, suppl. 1) consider it a valid species, restricted to western Africa. Decher et al. (2016: 274, 276) are a bit dubious in using $M$. inflatus villiersi in their systematic review and $M$. villiersi in their discussion sections. Furthermore, Anciaux de Faveaux (1978a: 479) mentioned that he originally assigned all of his material to either Miniopterus inflatus villiersi or Miniopterus natalensis, but that - according to Felten and Aellen - actually almost all of his specimens belonged to Miniopterus schreibersi natalensis. Based on this, we consider the DRC villiersi specimens to represent $M$. natalensis.

See also Miniopterus fraterculus Thomas \& Schwann, 1906 above.

Family Vespertilionidae Gray, 1821

Simmons (2005) subdivided the African Vespertilionidae into four subfamilies: Kerivoulinae, Myotinae, Vespertilioninae and Miniopterinae. The latter has been raised to family level (see above), whereas Koubínová et al. (2013: 9) distinguished one additional subfamily: Scotophilinae.

Subfamily Kerivoulinae Miller, 1907

Genus Kerivoula Gray, 1842

Kerivoula argentata Tomes, 1861

Fig. 34A-B

* Kerivoula argentata Tomes, 1861: 31-32.

Eight specimens from three localities in the southern part of the DRC were mentioned by Hayman et al. (1966: 58, map 84). More recently, one additional specimen was collected much more to the north in Boende (Tshuapa Province). The map given by Cotterill (2013i: 726) indicates that the core distribution area for $K$. argentata covers western Mozambique, eastern Zimbabwe, southern Malawi, southeast to northwest Zambia and southeastern DRC. Furthermore, he plotted some individual localities in the RSA, Namibia, Tanzania, Kenya, Angola (?) and Luluabourg (= Kananga) in the DRC. Our distribution map (based on data from ACR 2016: 1199) suggests a distribution which is restricted to the southeastern part of the continent, reaching from the northeastern part of the RSA to southern Kenya, whereby the records from Luluabourg and Boende seem to be located too far to the northeast and should probably be re-examined.

Kerivoula cuprosa Thomas, 1912

Fig. 34C-D

Kerivoula cuprosa Thomas, 1912: 41.

In the DRC, Kerivoula cuprosa is known from three localities: Koteli (Bas-Uélé Province), Eala (Equateur Province) and Wafanya (Tshuapa). The specimens from Koteli and Eala were reported as K. smithii by Hayman et al. (1966: 59), but were re-identified by Jakob Fahr as K. cuprosa (Fahr 2013r: 728). Kityo et al. (2009: 135) indicate that four cuprosa specimens were reported by Schouteden (1947): 
Eala, Koteli, Bambesa and Lisala, but were not discussed by Hayman et al. (1966). However, three of these were explicitly included in K. smithii by Hayman et al. (1966: 59), and the remaining one (RMCA 14263) in reality came from Gumba in the Lisala Territory. RMCA 14263 actually includes two specimens, one of which was identified as a Nycteris sp. by Danny Meirte and one as a Kerivoula sp. by Jakob Fahr (Wim Wendelen, pers. comm.). Besides the three currently reported DRC localities, Fahr (2013r: 728) also mentions this species from Cameroon, Côte d'Ivoire and Guinea. The SDM map shows that cuprosa is restricted to the central African rain forest block, but this should probably be extended to the West African block as well (the records from Côte d'Ivoire and Guinea were not included when generating this map, as the underlying specimens could not be traced).

Kerivoula lanosa (A. Smith, 1847)

Fig. 34E-F

Vespertilio lanosus A. Smith, 1847: pl. 50 and text.

* Kerivoula harrisoni muscilla (Thomas, 1906): 294.

* Kerivoula harrisoni lucia (Hinton, 1920): 240.

Rosevear (1965: 307-308) doubted the validity of the characters separating harrisoni from lanosa, i.e., the number of cusps of the upper incisors (two in harrisoni and one in the other species) and the length of the rostrum (comparing $\mathrm{c}^{\mathrm{c}} \mathrm{m}^{3}$ with $\mathrm{m}^{3}-\mathrm{m}^{3}$, the first being greater than the second in harrisoni and smaller in the other species). This was further investigated by Hill (1977: 628), who considered harrisoni to be a subspecies of lanosa.

Hayman et al. (1966: 58, map 86) mention three specimens from the eastern part of the DRC: one from Pawa (Haut-Uélé Province) [considered to belong to K. harrisoni bellula Aellen, 1959 by Aellen 1959: 225], one from Lukulu (Tanganyika Province) and one from Funda Biabo (Lualaba Province). One additional specimen was collected along the road between Kamituga and Kampene in Sud-Kivu Province. The SDM map confirms the distribution suggested by Cotterill (2013j: 730), who mentions it is known from widespread, scattered records in the rain forest, the northern rain forest-savanna mosaic, Somalia-Masai bushland, coastal forest mosaic, Zambezian woodland, Afromontane-Afroalpine and south-west Cape biotic zones, but we suggest it might occur further to the west in northern Namibia and south-central to coastal Angola.

Kerivoula phalaena Thomas, 1912

Fig. 35A-B

Kerivoula phalcena Thomas, 1912: 281.

* Kerivoula phalaena Thomas, 1912.

Although not separately listed in their catalog part, Hayman et al. (1966: 57) refer to this species, as Koopman (1965:31) mentioned a specimen from Lukolela (Mai-Ndombe Province) in the AMNH. Two additional specimens have been collected since then: one from Wafanya (Tshuapa Province) and one from Irangi (Sud-Kivu Province). Fahr (2013t: 732) indicated that K. phalaena is mainly recorded from the rainforest zones in western and central Africa, with outlying records from the AfromontaneAfroalpine zones in northeastern DRC and western Uganda and from the Guinea Savanna BZ in Côte d'Ivoire. This is confirmed by our map, which is based on climatic data and suggests that $K$. phalaena might occur even further to the east.

Kerivoula smithii Thomas, 1880

Fig. 35C-D

Kerivoula Smithii Thomas, 1880: 166. 

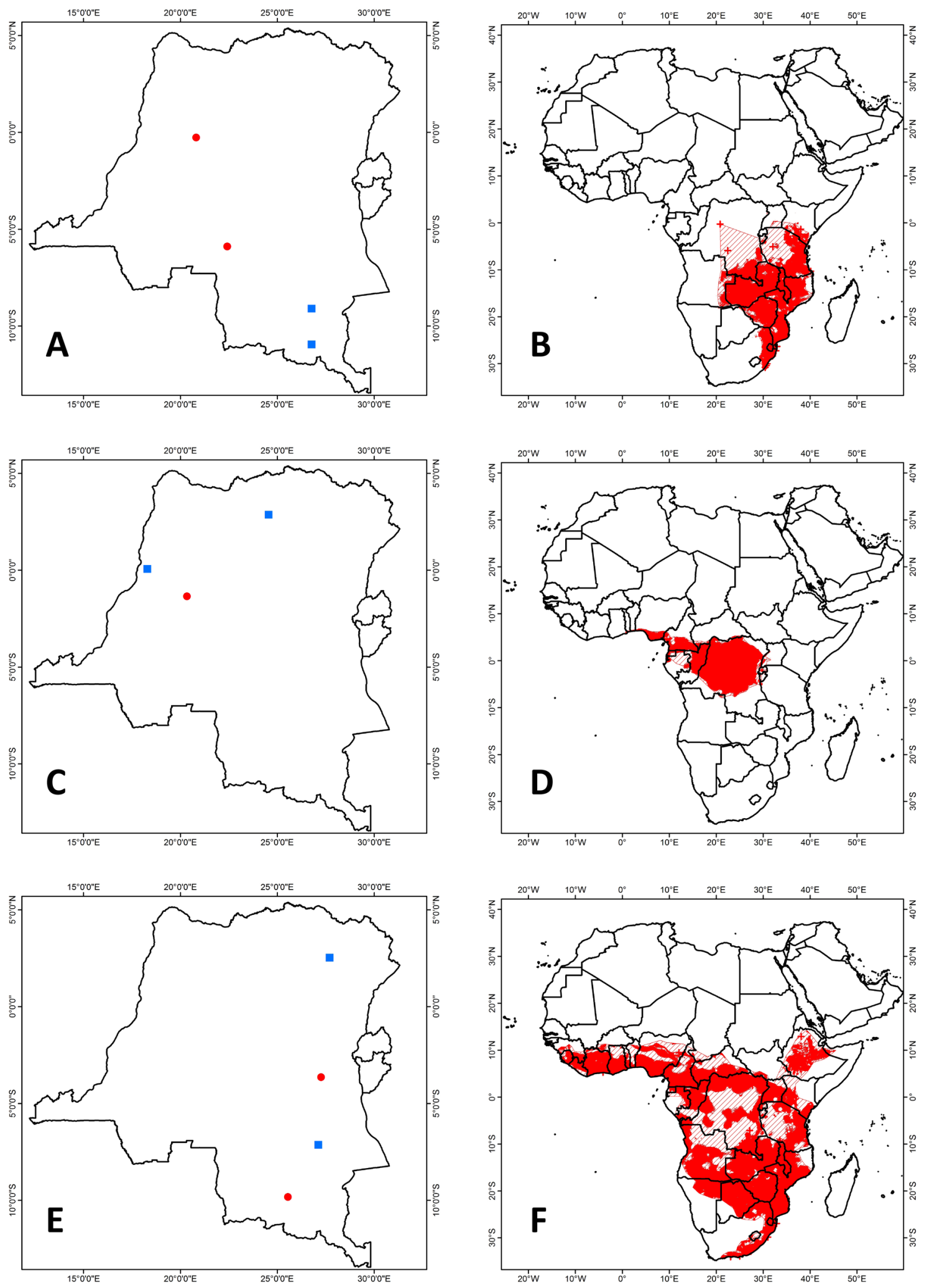

Fig. 34. Distribution maps. A-B. Kerivoula argentata Tomes, 1861. C-D. Kerivoula cuprosa Thomas, 1912. E-F. Kerivoula lanosa (A. Smith, 1847). A, C, E. Distribution in the CRB area. B, D, F. PanAfrican distribution. 
* Kerivoula smithi Thomas, 1880.

* Kerivoula ? aerosa Tomes, 1858: 333.

Hayman et al. (1966: 58) mention a specimen from Bukavu (RMCA 23172; Sud-Kivu Province) as possibly belonging to " $K$. aerosa". They also indicated that there is some doubt about the African origin of the types of this species. As aerosa is currently assigned to the mainly southeast Asian genus Phoniscus, it has been suggested that they actually came from the Malay Archipelago (Happold 2013as: 735). In the meantime, the Bukavu specimen has been re-identified by Jakob Fahr as belonging to K. smithii. He also re-identified three other animals: one from Koteli (RMCA 16190; Bas-Uélé Province), one from Eala (RMCA 16189; Equateur Province) and one from Gumba Island (RMCA 14263; Mongala Province). The two former represent $K$. cuprosa, whereas the latter could only be identified as Kerivoula sp. We also report four additional localities: Kalehe Territory (Sud-Kivu Province), Ukaika (Ituri Province), Aketi (Bas-Uélé Province) and Wafanya (Tshuapa Province), with the exception of the latter all in the northeastern part of the DRC. Fahr (2013u: 733) indicates that K. smithii is primarily found in the rain forest zones of southeastern Nigeria to the DRC and from the Afromontane-Afroalpine and the SomaliaMasai bushland zones in Kenya. The SDM map confirms this view.

Subfamily Myotinae Tate, 1942

Genus Myotis Kaup, 1829

Myotis bocagii (Peters, 1870)

Fig. 35E-F

Vespertilio Bocagii Peters, 1870: 125.

* Myotis bocagei (Peters, 1870).

* Myotis bocagei bocagei (Peters, 1870).

* Myotis bocagei hildegardeae Thomas, 1904: 209.

* Myotis bocagei cupreolus Thomas, 1904: 407.

Besides 40 specimens identified to the specific level, Hayman et al. (1966:51) recognized three subspecies, of which hildegardeae is currently included in the nominate subspecies. This agrees with a statement made by Hayman [in Fain 1953: 99]: “ ... since the more specimens [of M. b. hildegardeae] I see, the less I think it possible to maintain hildegardeae as a valid race”. Happold (2013an: 692) indicates that M. b. bocagii occurs in the woodland savannah from Ethiopia to Angola and the former Transvaal, and M. b. cupreolus in the rainforest from Liberia to the DRC. In the CRB area, Hayman et al. (1966, map 68) reported M. bocagii from the eastern part (provinces Bas-Uélé, Haut-Uélé, Ituri, Nord-Kivu, SudKivu, Tanganyika, Haut-Lomami and Haut-Katanga), Rwanda, and from the western part (provinces Kongo Central, Kinshasa and Kwilu) of the DRC. They did not report any specimens from the more central areas or from the northwestern part of the country. We were able to trace specimens from the latter area and from almost all of the northern half of the country, as well as from Rwanda and Burundi, but none from the south-central provinces (Kasaï-Central, Kasaï Oriental, Lualaba). The distribution map given by Happold (2013an: 693) doesn't include any of the additional northern specimens and, therefore, separates the eastern distribution patches from the western ones. Our map suggests that both are connected and that only the border zone between the DRC and Angola might not be an optimal habitat for M. bocagii.

Myotis tricolor (Temminck, 1832)

Fig. 36A-B

Vespertilio tricolor Temminck, 1832: 106.

* Myotis tricolor (Temminck, 1832). 

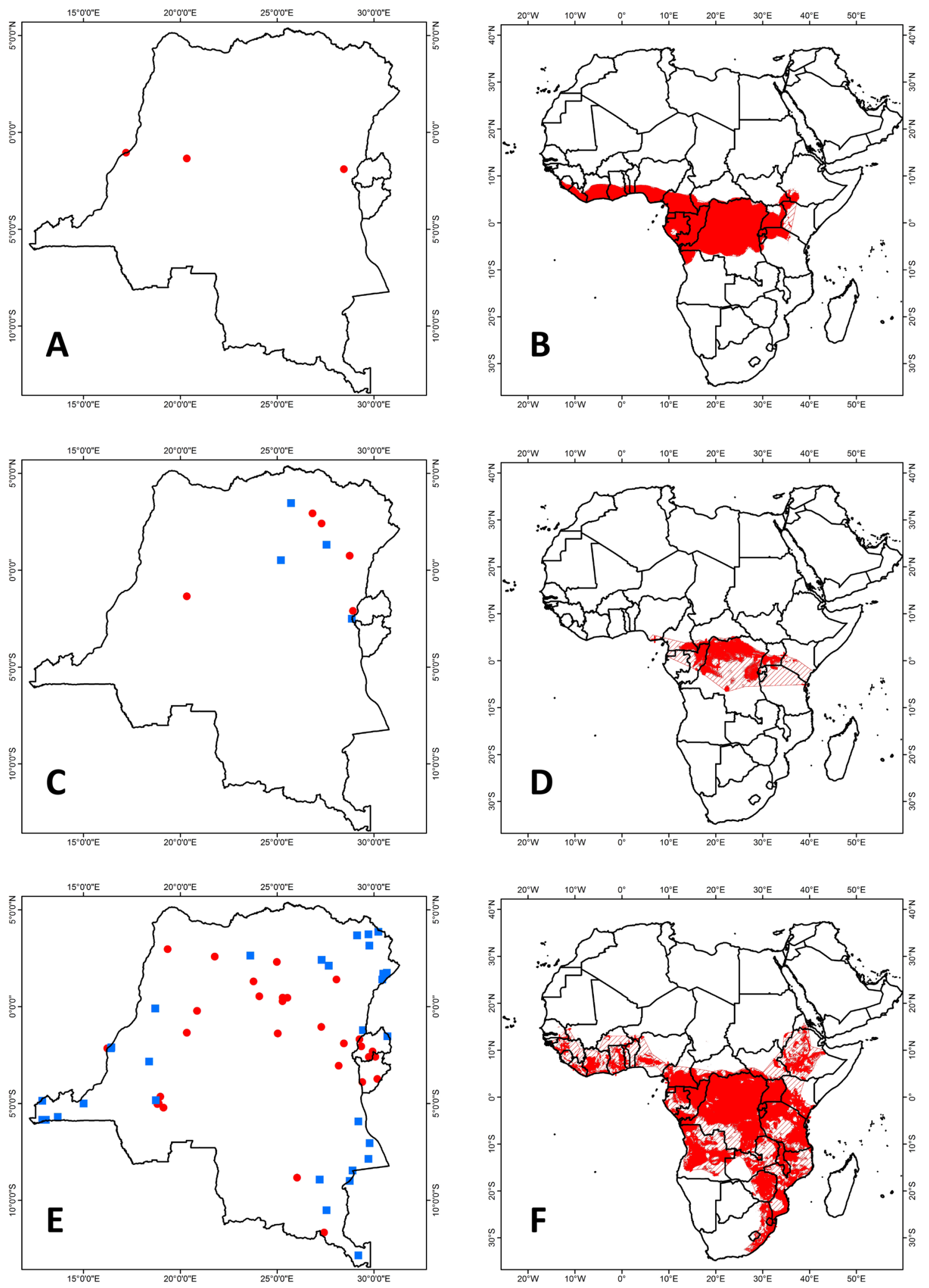

Fig. 35. Distribution maps. A-B. Kerivoula phalaena Thomas, 1912. C-D. Kerivoula smithii Thomas, 1880. E-F. Myotis bocagii (Peters, 1870). A, C, E. Distribution in the CRB area. B, D, F. Pan-African distribution. 
In general, M. tricolor primarily occurs in eastern and southern Africa, from Ethiopia to the RSA, only touching the eastern border of CRB (Bernard 2013: 707). The record from a cave near Thysville (RMCA 16450; Hayman et al. 1966: 52) is, therefore, already far to the west, but not impossible as Koopman (1989: 7) reported a specimen from Liberia, and ACR (2016: 1207) reported another from Nigeria.

Myotis welwitschii (Gray, 1866)

Fig. 36C-D

Scotophilus welwitschii Gray, 1866: 211.

* Myotis welwitschi venustus (Matschie, 1899).

The validity of the subspecies venustus was already doubted prior to Hayman et al. (1966), e.g., by Kershaw (1922: 181) and Frechkop (1954: 19), and this view was later confirmed by Kock (1967: 324).

In the DRC, Hayman et al. (1966: 52, map 71) reported this species from Nord-Kivu Province in the east and from the southeastern provinces of Haut-Lomami, Haut-Katanga and Lualaba. More recently, it has been found in Rwanda and Burundi. Happold (2013ao: 709) gives a patchy distribution in eastern and southern Africa, from Ethiopia to the RSA, with an extension into the southern part of the DRC and Angola. She also plotted two separate localities in Guinea and Cameroon.

Subfamily Scotophilinae Gray, 1866

Genus Scotophilus Leach, 1821

Scotophilus dinganii (A. Smith, 1833)

Fig. 36E-F

Vespertilio Dinganii A. Smith, 1833: 59.

* Scotophilus nigrita (Schreber, 1775): 58.

* Scotophilus nigrita herero Thomas, 1906: 174.

As indicated below, S. nigrita was erroneously used to identify the medium-sized African Scotophilus species. Robbins (1978: 212) showed that the correct name for this species should be $S$. dinganii.

Hayman et al. (1966: 57, map 83) reported "S. nigrita" from over almost the entire DRC and from northern Rwanda, with the exception of the northwestern part of the DRC and the most central part. Here, we include additional specimens from the northwestern, southwestern and southeastern parts of the country as well as from Rwanda and Burundi. This still leaves the central part of the DRC unoccupied by this species. The only central DRC record is a specimen from Boende (Tshuapa Province), but this is a juvenile specimen, which is probably too young to be identified with certainty.

Furthermore, we removed a number of localities from the northeastern part of the DRC as the underlying specimens belonged to the next species.

Happold (2013am: 674) based her distribution map on data from Robbins et al. (1985: 63), which resulted in a rather detailed map with a lot of finger-like extrusions and areas which almost touch one another. We believe that the species has a wider distribution, only lacking in eastern Somalia, southern Namibia and most of the RSA and probably also from the west and central African rainforest as mentioned by Happold. However, we need to point out that some of the east African records might need to be reassigned to a separate species, e.g., S. colias as was tentatively suggested by Vallo et al. (2011: 350). 

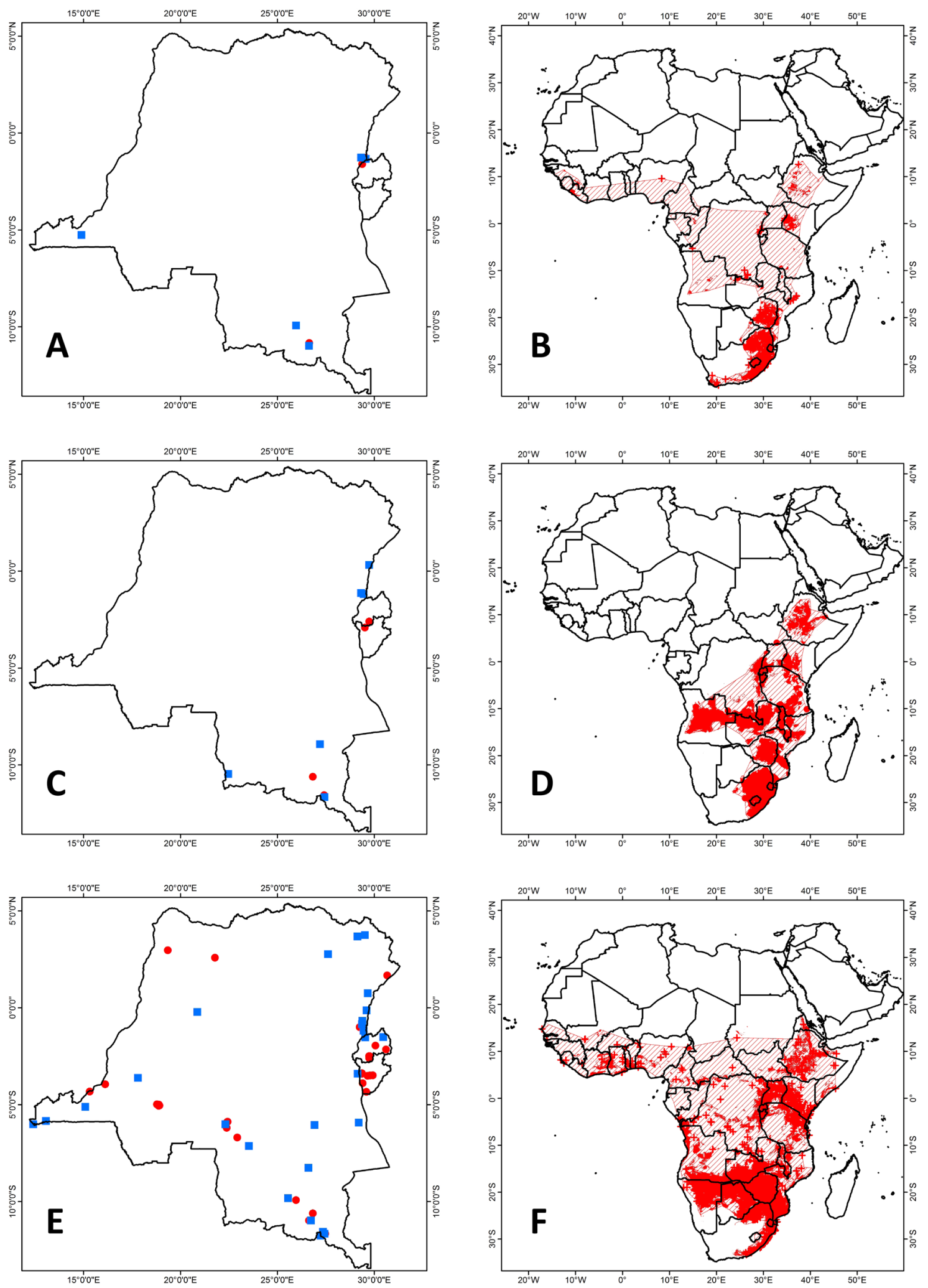

Fig. 36. Distribution maps. A-B. Myotis tricolor (Temminck, 1832). C-D. Myotis welwitschii (Gray, 1866). E-F. Scotophilus dinganii (A. Smith, 1833). A, C, E. Distribution in the CRB area. B, D, F. PanAfrican distribution. 
Scotophilus leucogaster (Cretzschmar, 1826)

Fig. 37A-B

Nycticejus leucogaster Cretzschmar, 1826: 71.

* Scotophilus leucogaster (Cretzschmar, 1826).

Hayman et al. (1966: 56-57, map 82) mention one locality in the DRC (Faradje, Haut-Uélé Province), and refer to Koopman (1965: 17) who indicated that Pachyotus altilis (the name under which Allen 1917: 447 reported these specimens) is a synonym of S. l. leucogaster. Allen (1917) and Hayman et al. (1966) report on two specimens, whereas the AMNH catalog mentions three. The third specimen (AMNH 49283, a foetal specimen) has the same original number (1865) as AMNH 49015 (an adult female).

Only one additional specimen has been reported since: MHNG 1896.016 from Mweya, Burundi.

The map presented by Van Cakenberghe \& Happold (2013i: 677) shows S. leucogaster to be present in a number of areas south of the Sahara: Senegal/Gambia; Guinea/Sierra Leone; Burkina Faso/northern Ghana to southwestern Chad; central Sudan; central Ethiopia; northeastern DRC; southwestern Zambia/northern Namibia/northern Botswana/western Zimbabwe. They also plotted individual records from Mauritania, Côte d'Ivoire, Central African Republic, Uganda, Kenya, western Angola and Namibia, and Mozambique. The SDM map suggests a sub-Saharan distribution, with a northward extention along the River Nile. The species probably will not occur in eastern Ethiopia, Eritrea or Somalia, in the central African rain forest or in southern Africa, except for the northeastern tip of the RSA and southern Mozambique.

Scotophilus nigrita (Schreber, 1774)

Fig. 37C-D

Vespertilio Nigrita Schreber, 1774: 171.

* Scotophilus gigas Dobson, 1875: 122.

Until Robbins (1978: 212), the largest representative of the genus Scotophilus was called S. gigas, whereas this should be $S$. nigrita, a name that had been used for the medium-sized African form for decades. Unfortunately, this led to confusion that left its mark on a lot of subsequent publications, where it wasn't always clear whether nigrita was used to designate the large or the medium-sized form.

Hayman et al. (1966: 56, map 81) reported "S. gigas" from one locality: Albertville (= Kalemie) in Tanganyika Province. The only other specimen from the CRB area was collected in Bujumbura, Burundi.

With the exception of two small areas in Ghana, Happold (2013am: 679) only plotted individual localities on her distribution map, which shows a major gap between the locality in western Nigeria (the easternmost for West Africa) and central Sudan. Further to the south, there are records from the DRC, coastal Kenya and Tanzania, and Mozambique. ACR (2016: 1120) provides some additional records from Niger, northern Cameroon, Sudan and South Sudan, which more or less bridge the gap between West Africa and Sudan, and which also may confirm the assignment of the Sudanese specimens to the nominate subspecies as mentioned by Happold (2013am: 678). The SDM map also suggests that such a connection might well be possible through the Central African Republic and central Cameroon or through central Chad and northern Cameroon. Although the species hasn't been confirmed from the central African rainforest, its occurrence in the West African forest block makes it plausible.

Scotophilus nux Thomas, 1904

Fig. 37E-F

* Scotophilus nigrita nux Thomas, 1904: 208. 

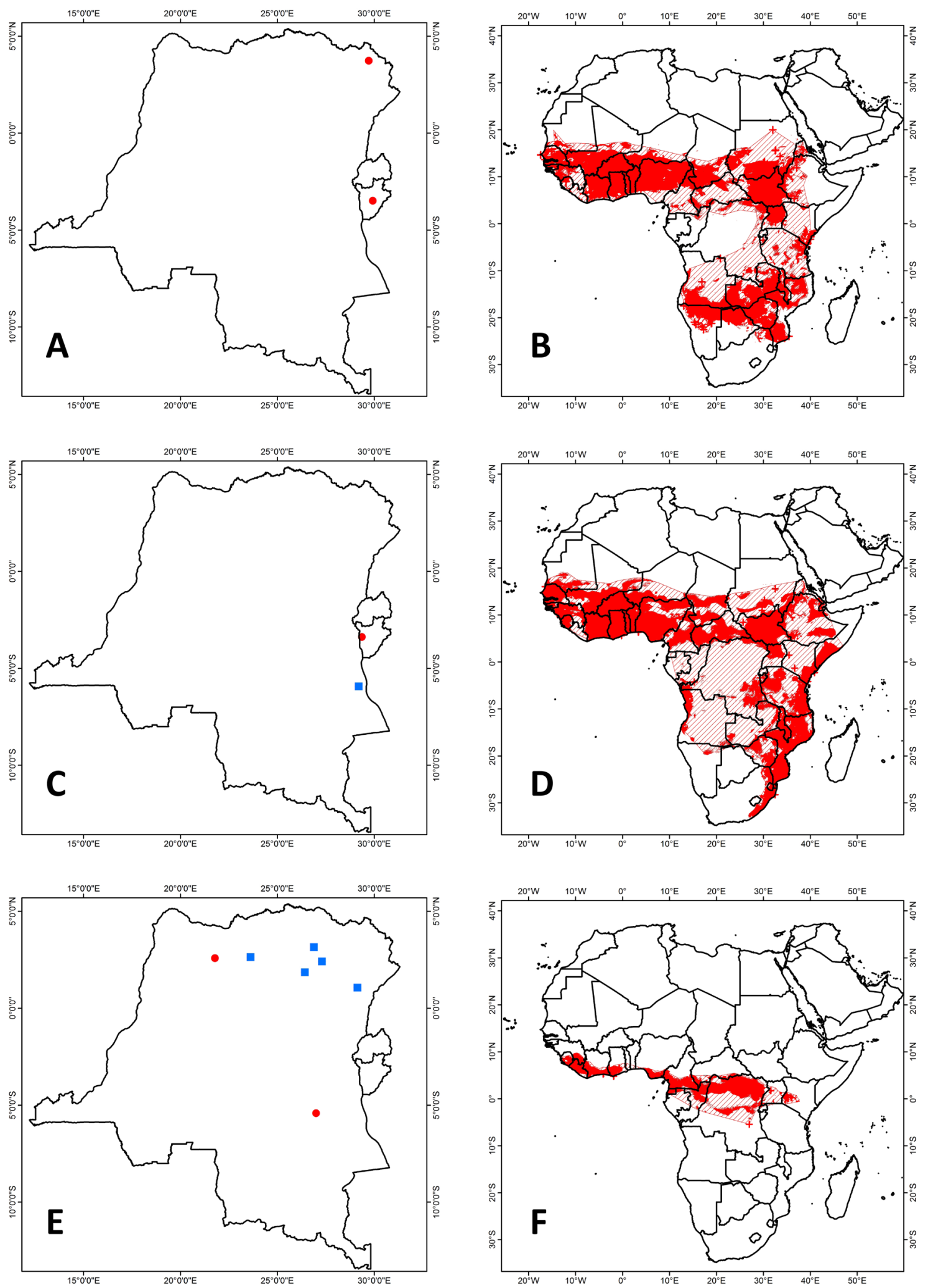

Fig. 37. Distribution maps. A-B. Scotophilus leucogaster (Cretzschmar, 1826). C-D. Scotophilus nigrita (Schreber, 1774). E-F. Scotophilus nux Thomas, 1904. A, C, E. Distribution in the CRB area. B, D, F. Pan-African distribution. 
Thomas (1915: 468) already mentioned "The conspicuous difference between the colour of S. nux and that of all forms of $S$. nigrita makes me now think that it should be recognized as a distinct species, and not merely a subspecies of the older known form." This view, however, was ignored by most subsequent authors, until it was resurrected by Robbins (1983: 19) and Robbins et al. $(1985: 53,71)$.

Hayman et al. (1966: 57) only mentioned specimens from the northeastern part of the DRC (BasUélé, Haut-Uélé and Ituri Provinces). One additional specimen was collected in Kongolo (Tanganyika Province) in southeastern DRC. Van Cakenberghe \& Happold (2013j: 681) indicate that the species is recorded from the rainforest and the northern and eastern rainforest-savanna mosaics from Sierra Leone in the west to Cameroon, and from northwestern DRC to western Kenya. We agree with this distribution, although it occurs further to the south in the DRC than previously accepted. The climatic data used for the SDM map confirm that the gap between Cameroon and the DRC is probably due to insufficient sampling, as suggested by Van Cakenberghe \& Happold. They also confirm the gap between southwestern Ghana and southern Nigeria, which might be linked to the "Dahomey Gap", but further investigation in that area is needed to confirm this.

Subfamily Vespertilioninae Gray, 1821
Genus Glauconycteris Dobson, 1875

Glauconycteris alboguttata J.A. Allen, 1917

Fig. 38A-B

* Glauconycteris alboguttatus J.A. Allen, 1917: 449.

The only known representatives of this species in the CRB area are the three specimens reported by Hayman et al. (48, 49, map 60) and one additional specimen from Yalosemba (Mongala Province; CM 86658) reported by Eger \& Schlitter (2001: 7) and Thorn et al. (2009: 61). Besides these four specimens, Happold (2013aa: 564) indicates that the species also occurs in the coastal area of Cameroon. Based on climatic data, we suspect that the species can occur over the central African forest belt from southern Nigeria to Rwanda and Burundi.

Glauconycteris argentata (Dobson, 1875)

Fig. 38C-D

Chalinolobus argentatus Dobson, 1875: 385.

* Glauconycteris argentata (Dobson, 1875).

Hayman et al. (1966: 49) mention 72 records for this species, of which at least one (RMCA 16213; Mukishi, Haut-Lomami Province) was re-identified as G. variegata (Wim Wendelen, pers. comm.). They also they mentioned the same museum numbers for the series from Boma and Butembo. These numbers apply for the Butembo specimens and the correct numbers for the Boma series are 6571 to 6576 and 7250). Their map 61 contains two localities we cannot identify from the specimens they listed. One of these is most probably Ibembo (Bas-Uélé Province), where they reported G. beatrix (see map 62), and another one is in Kasaï Province.

Within the CRB area, G. argentata occurs in three separate regions: one along the eastern border of the DRC (Ituri, Nord- and Sud-Kivu Provinces) and Rwanda and Burundi. A second region is at the mouth of the Congo River (Kongo Central Province), and a third runs from Mbandaka (Equateur Province) over Boende and Ikela (Tshuapa Province), Lodja (Sankuru Province) to Kananga (Kasaï-Central Province). This agrees very well with the map given by Happold (2013ab: 565), although she also cites the "Ibembo" record listed above. Extralimitally, the species occurs in the coastal areas of Cameroon and Equatorial Guinea, southwestern Kenya, northeastern Tanzania, northern Malawi and Angola. The SDM map 

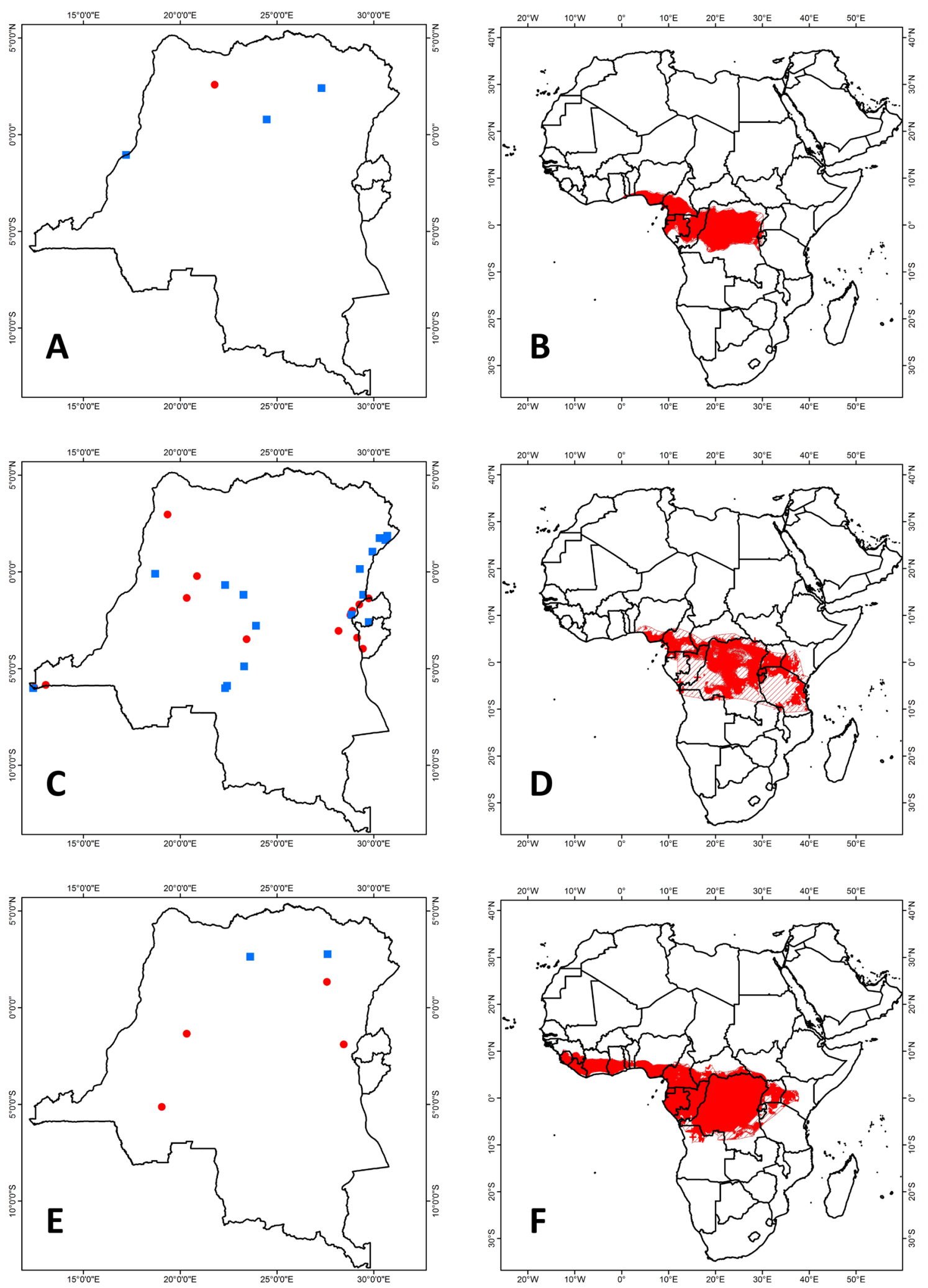

Fig. 38. Distribution maps. A-B. Glauconycteris alboguttata J.A. Allen, 1917. C-D. Glauconycteris argentata (Dobson, 1875). E-F. Glauconycteris beatrix Thomas, 1901. A, C, E. Distribution in the CRB area. B, D, F. Pan-African distribution. 
suggests that $G$. argentata might be more widespread over central and eastern Africa, from southern Nigeria in the west to Kenya and Tanzania in the east, between $5^{\circ} \mathrm{N}$ and $10^{\circ} \mathrm{S}$.

Glauconycteris beatrix Thomas, 1901

Fig. 38E-F

* Glauconycteris beatrix Thomas, 1901: 256.

Glauconycteris beatrix was reported by Hayman et al. (1966: 49, map 62) from two localities in northern DRC: Ibembo in Bas-Uélé Province and Paulis (= Isiro) in Haut-Uélé Province. Additional specimens are reported from Irangi (Sud-Kivu), Aketi (Bas-Uélé), Wafanya (Tshuapa) and Kimbinga, near Kikwit (Kwilu). The specimens from Kimbinga were erroneously reported as Miniopterus minor by Van Cakenberghe et al. (2000: 308). Happold (2013ac: 566) only mapped the specimens from Irangi, and except for the coastal areas of Cameroon and Equatorial Guinea, she only plotted individual records for Côte d'Ivoire, Ghana, southern Nigeria, Gabon and northern Angola. Our distribution map suggests that G. beatrix could occur in the forest belts of both West and Central Africa, ranging from Sierra Leone in the west to western Kenya in the east.

Glauconycteris curryae Eger \& Schlitter, 2001

Fig. 39A-B

Glauconycteris curryi Eger \& Schlitter, 2001: 2.

This species is known from only seven specimens from five rainforest localities in Cameroon and one in the DRC (Yalosemba, Mongala Province) (Eger 2013: 568).

Glauconycteris humeralis J.A. Allen, 1917

Fig. 39C-D

* Glauconycteris humeralis J.A. Allen, 1917: 448.

Glauconycteris humeralis was considered to be a valid species by Hayman et al. (1966: 49), but some later authors (e.g., Koopman 1971; Eger \& Schlitter 2001; Monadjem et al. 2010b) considered it to be a subspecies of G. beatrix. Heller et al. (1994: 6) state that humeralis could merely represent the spotted variety of beatrix, if it wasn't for the different form of the baculum in the two species, as was already illustrated by Hill \& Harrison (1987: Fig. 19).

Hayman et al. (1966: 49, map 63) mention six specimens from three localities, of which the BMNH specimen from Irangi could not be traced. However, the species has been confirmed from that locality by material in the collection of the SMF. All of these localities were in the northeastern part of the DRC. Other specimens were collected near Epulu (Ituri Province, northeastern DRC) and Yalosemba (Mongala, northwestern DRC) and in Kananga (Kasaï-Central Province, southern DRC). The latter specimen was apparently overlooked by Happold (2013ad: 571), as she only plotted the northern specimens. When the Kananga specimen is included, the distribution of the species covers the Congolese rain forest from eastern Cameroon over northern Congo and reaching into the rainforestsavanna mosaic in Uganda and western Kenya (see also Happold 2013ad: 571).

Glauconycteris poensis (Gray, 1842)

Fig. 39E-F

Kerivoula poensis Gray, 1842: 258.

* Glauconycteris poensis (Gray, 1842). 

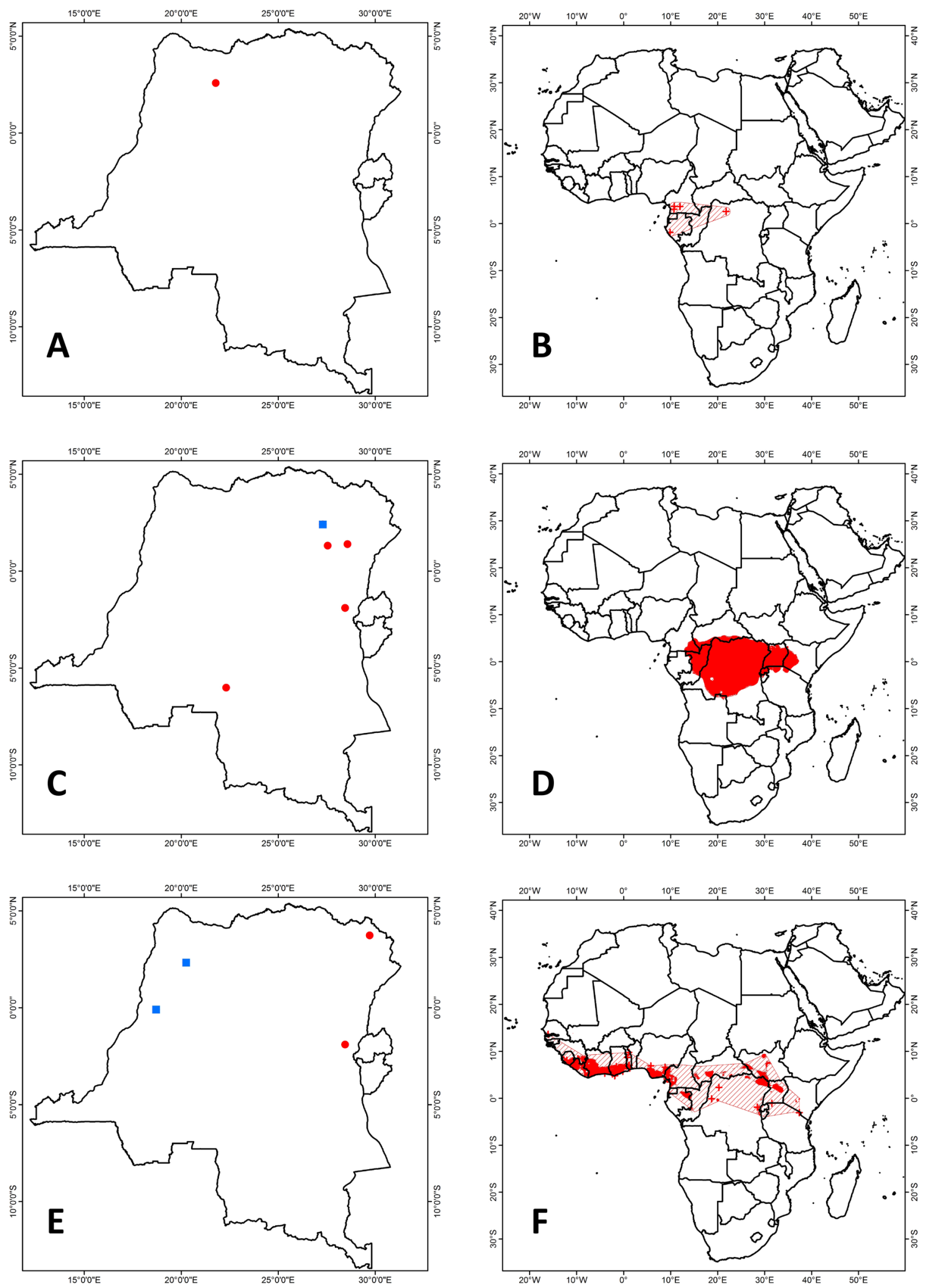

Fig. 39. Distribution maps. A-B. Glauconycteris curryae Eger \& Schlitter, 2001. C-D. Glauconycteris humeralis J.A. Allen, 1917. E-F. Glauconycteris poensis (Gray, 1842). A, C, E. Distribution in the CRB area. B, D, F. Pan-African distribution. 
In the DRC, G. poensis was collected at four localities in the northern half of the country, two in the northwestern part that were already reported by Hayman et al. (1966: 49, map 64), and two from the northeastern part. The one from Irangi (Nord-Kivu Province) is a relatively recent one, but the one from Faradje (Haut-Uélé) was already collected by Lang and Chapin. Allen (1917) doesn't report any G. poensis specimens in their collection, and the only Glauconycteris from Faradje that he mentions is G. papilio ( $=$ G. variegata). The original entry in the AMNH catalog mentions 15 G. papilio specimens from Niangara, Faradje and Aba, which were changed into Chalinolobus variegata (490569), Glauconycteris variegata variegata (49060-4), Chalinolobus (Glauconycteris) variegata papilio (49065) and Glauconycteris variegata variegata (49066-70). The online catalog, however, mentions the entire series, with the exception of 49066, as Glauconycteris variegata variegata. That specimen is identified as G. poensis. Happold (2013ae: 575) overlooked this record when drawing the map for this species. Her map indicates that $G$. poensis is primarily a West African species, with some outliers in the DRC. There is also a doubtful record from Marangu (Tanzania) of which J. Kingdon (in Grimshaw et al. 1995 ) suggests that it was misidentified, and most likely belongs to G. variegata. The online catalog still mentions the underlying specimens (219772-4) as G. poensis. The SDM map indicates that this locality is situated far to the east, but it is not impossible that poensis could occur over there.

Glauconycteris superba Hayman, 1939

Fig. 40A-B

Glauconycteris superba Hayman, 1939: 219.

* Glauconycteris superba superba Hayman, 1939.

At the time of Hayman et al. (1966) only two specimens of this remarkably coloured bat were known: the holotype of the species from the DRC and a specimen from Ghana, which was considered to belong to a separate subspecies, G. superba sheila Hayman, 1947. In 1972, a third specimen was collected in Côte d'Ivoire. In the next 40 years, not a single specimen of this species was found, but in 2012 two animals were collected: one in the Bangangai Game Reserve, South Sudan (Reeder et al. 2013), and one from Mbiye Island, near Kisangani, Tshopo Province, DRC (Gembu Tungaluna et al. 2013: 18). In 2015, another specimen was collected on Mbiye Island. This is the first time the species was collected at the same locality, suggesting that $G$. superba is not as rare as was assumed during the first 40 years after its initial discovery.

As if to confirm the previous statement, after the manuscript for this paper was submitted, Ing et al. (2016) and Hassanin et al. (2017) reported on ten additional specimens from Mbiye island and the Yoko Forest Reserve $\left(00^{\circ} 17^{\prime} \mathrm{N}, 25^{\circ} 17^{\prime} \mathrm{E}\right)$. Hassanin et al. (2017) also described a new species of Glauconycteris, which is not covered in the current paper.

Based on a number of morphological characters, Reeder et al. (2013) created a separate genus for superba: Niumbaha. These characters include the considerably larger skull; more striking body patterns; not excessively foreshortened (i.e., longer) rostrum; more robust muzzle; unreduced relative canine size; unelongated wing tips (second phalanxes); more robust, subquadrangular ears, with a larger free lobe at the inner margin; a more strongly curved tragus; position of the nostrils. Although these are quite important differences, we remain reluctant to follow Reeder et al. (2013) in separating superba from the genus Glauconycteris, due to the fact that the morphological characters were probably only examined on one specimen and some of them might be the result of the large size of superba. The rejection of Niumbaha as a separate genus is confirmed by the DNA analyses by Hassanin et al. (2017).

Given the fact that G. superba was rarely collected in the past, it is very difficult to assess its general distribution. The SDM map suggests that it may occur in the forest zones of West and Central Africa. 

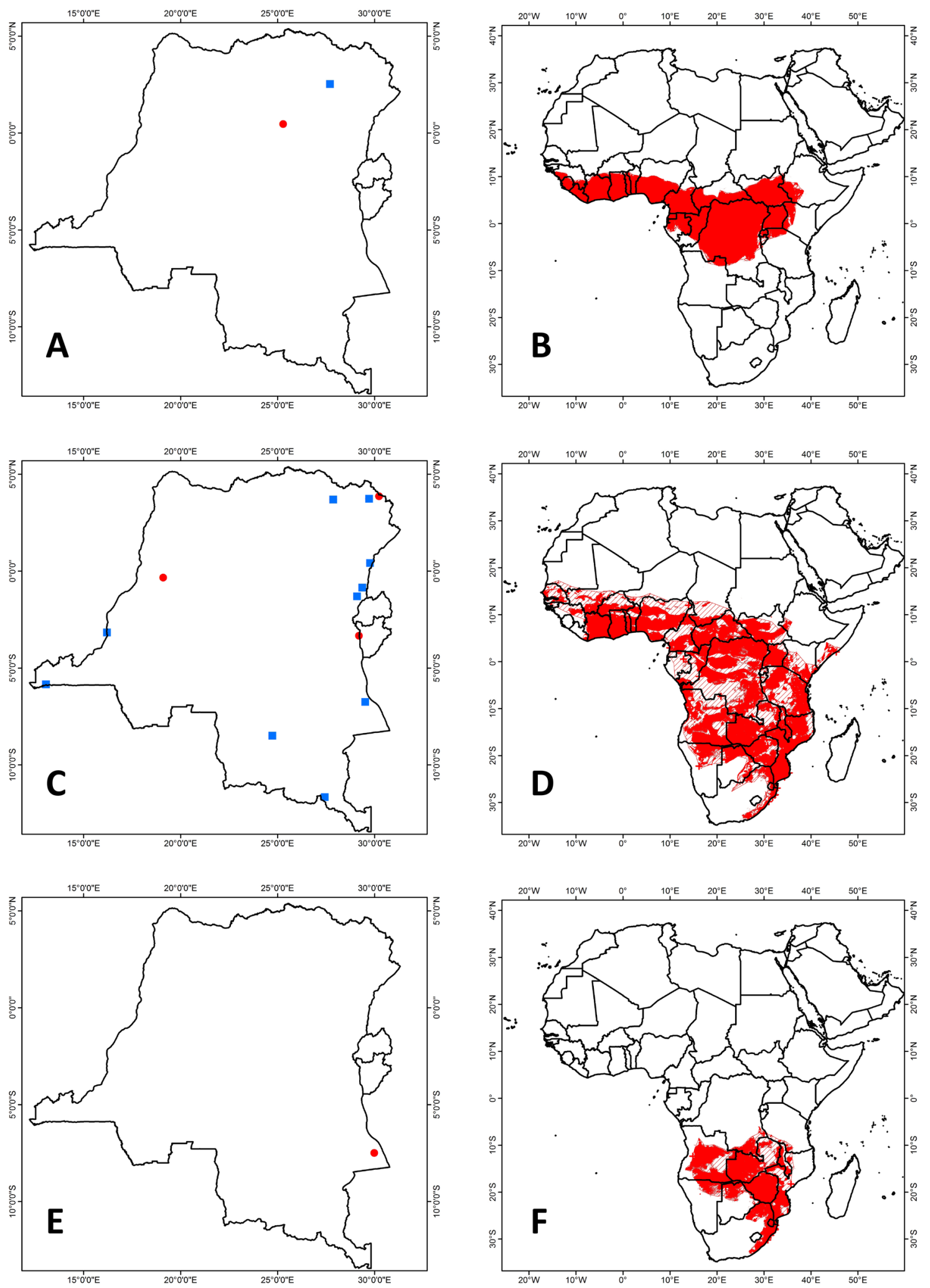

Fig. 40. Distribution maps. A-B. Glauconycteris superba Hayman, 1939. C-D. Glauconycteris variegata (Tomes, 1861). E-F. Hypsugo anchietae (Seabra, 1900). A, C, E. Distribution in the CRB area. B, D, F. Pan-African distribution. 
Glauconycteris variegata (Tomes, 1861)

Fig. $40 \mathrm{C}-\mathrm{D}$

Scotophilus variegatus Tomes, 1861: 31, 36 .

* Glauconycteris variegata papilio (Thomas, 1905): 77.

Although most recent authors consider papilio to be a synonym of G. variegata (e.g., Simmons 2005) or even G. v. variegata (e.g., Lanza et al. 2015), Monadjem et al. (2010b: 417) - referring to a statement made by Hayman (1963: 107) on the possible specific status of papilio - suggest that a detailed study of this species group is necessary to resolve this matter.

For the DRC, Hayman et al. (1966: 50, map 66) indicate that G. variegata primarily occurs in the northeastern part of the country (Haut-Uélé and Nord-Kivu Provinces) and in the southeast (HautLomami and Haut-Katanga Provinces). From the western part of the country, they report it from Boma (Kongo Central Province) and Kwamouth (Mai-Ndombe Province). The second record they mapped from Kongo Central Province could not be traced, but they also did not map Mbala in Tanganyika Province. We could only add two additional localities: one on the northwestern bank of Lake Tanganyika (Sud-Kivu Province) and at Boteka (Equateur Province). The map given by Happold (2013af: 577) shows a very patchy distribution area stretching from Senegal to Somalia and southwards to northeastern RSA. Based on specimen data from ACR (2016: 1230) and climatic data, our distribution map fills in most of the gaps between the patches.

Genus Hypsugo Kolenati, 1856

Hypsugo anchietae (Seabra, 1900)

Fig. 40E-F

Vesperugo Anchieta Seabra, 1900: 26.

Two specimens from Lubenge (Tanganyika Province) were re-identified by the first author as belonging to this species. Kearney (2013b: 611) indicates that the species occurs from southwestern to eastern Angola, and through southeastern DRC, western Zambia, Zimbabwe to eastern RSA and Swaziland, where it is found in riverine, coastal and scrub forests as well as bushveld biotopes.

Hypsugo crassulus (Thomas, 1904)

Fig. 41A-B

* Pipistrellus crassulus Thomas, 1904: 206.

As indicated by Fahr (2013q: 617) crassulus has been moved into various (sub)genera or "collective groups" over the past few years: Pipistrellus, P. (Vansonia), "Attalepharca", Hypsugo and Nycticeinops. The currently most accepted view is that it belongs to the genus Hypsugo.

Hayman et al. (1966: 52, map 72) reported it from two localities: Bokuma (Equateur Province) and Mulolo-Kisanga (Maniema Province), and more recently it was also found at Irangi (Sud-Kivu Province). The distribution map provided by Fahr (2013q: 618) only shows a very restricted red area covering the eastern parts of the Kivu Provinces reaching towards Maniema Province. Furthermore, there are single records from Guinea, in West Africa, over Côte d'Ivoire, southern Cameroon, northwestern DRC (the Bokuma record), northern Angola, southern South Sudan, southern Uganda and southwestern Kenya. Based on climatic data, the SDM map covers this area, but also suggests it might occur in Equatorial Guinea, Gabon, southern Congo, and southwestern and southeastern DRC. 

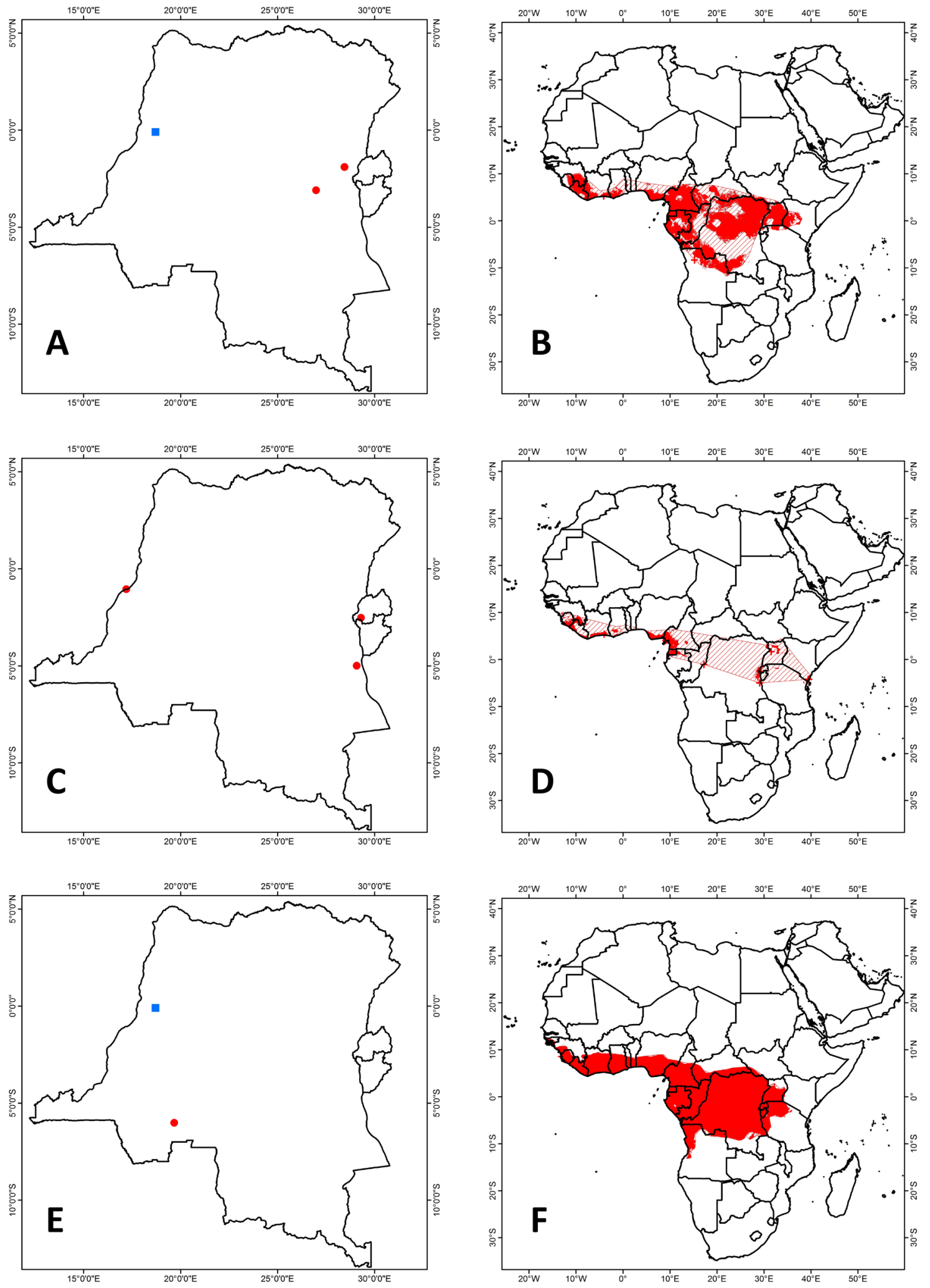

Fig. 41. Distribution maps. A-B. Hypsugo crassulus (Thomas, 1904). C-D. Hypsugo cf. eisentrauti (Hill, 1968). E-F. Hypsugo musciculus (Thomas, 1913). A, C, E. Distribution in the CRB area. B, D, F. Pan-African distribution. 
Hypsugo cf. eisentrauti (Hill, 1968)

Fig. $41 \mathrm{C}-\mathrm{D}$

Pipistrellus eisentrauti Hill, 1968: 45.

"Hypsugo eisentrauti" was collected at two localities in the DRC: Lukolela (Mai-Ndombe Province) and Kabobo Forest (Sud-Kivu Province) and in one locality in Rwanda: Nyungwe Forest Reserve. However, as already indicated by Van Cakenberghe \& Happold (2013b: 622), the specimens from Lukolela do not agree with the type specimens of eisentrauti from Cameroon. And this is also the case for the animal from Rwanda. Huhndorf \& Kaleme (2008: 26) and Kerbis Peterhans et al. (2013: 189) assigned the Kabobo Forest specimen to " $H$. cf. eisentrauti". Tentatively, we assign this name to all of the DRC and Rwandan specimens.

Hypsugo musciculus (Thomas, 1913)

Fig. 41E-F

* Pipistrellus musciculus Thomas, 1913: 316.

Similar to H. crassulus, H. musciculus has been assigned to various supra-specific units: Pipistrellus, P. (Hypsugo), "Attalepharca" and Hypsugo (see Van Cakenberghe \& Happold 2013e: 637). Here, we follow Simmons (2005: 491) in assigning it to the genus Hypsugo.

Hayman et al. (1966: map 74) have two localities plotted on their map: Bokuma in Equateur Province and Avakubi in Tshopo Province. For the latter, however, they refer (p. 53) to Koopman (1965: 13) who indicated that this specimen agreed better with the original description of $P$. nanulus than with that of P. musciculus. In the Paris museum, there is one other DRC specimen (from Bata, Kwilu Province) that had been identified as musciculus.

Hypsugo musciculus is primarily a West African species occurring from Sierra Leone in the west to Cameroon and Gabon in the east, with a few records extending into the DRC.

Genus Laephotis Thomas, 1901

Laephotis angolensis Monard, 1935

Fig. $42 \mathrm{~A}-\mathrm{B}$

Laephotis angolensis Monard, 1935: 45.

* Laephotis wintoni angolensis (Monard, 1935).

At the time Hayman et al. (1966) was written, the genus Laephotis was considered to be monotypic (Hill 1974: 1), with the nominate subspecies restricted to Kenya and L. w. angolensis occurring in Angola, Zambia, Botswana and the southern part of the DRC. However, based on cranial characteristics, Peterson (1971a: 888) found the two to be specifically different.

Laephotis angolensis was reported by Hayman et al. (1966: 50, map 67) with 13 specimens from two localities in Haut-Katanga, and no further localities have been added since. Of these 11 were found to represent L. botswanae (Kearney \& Seamark 2005: 73). Extralimitally, the species is further known from Angola, Zimbabwe and Zambia.

Laephotis botswanae Setzer, 1971

Fig. $42 \mathrm{C}-\mathrm{D}$

Laephotis botswanae Setzer, 1971: 260. 

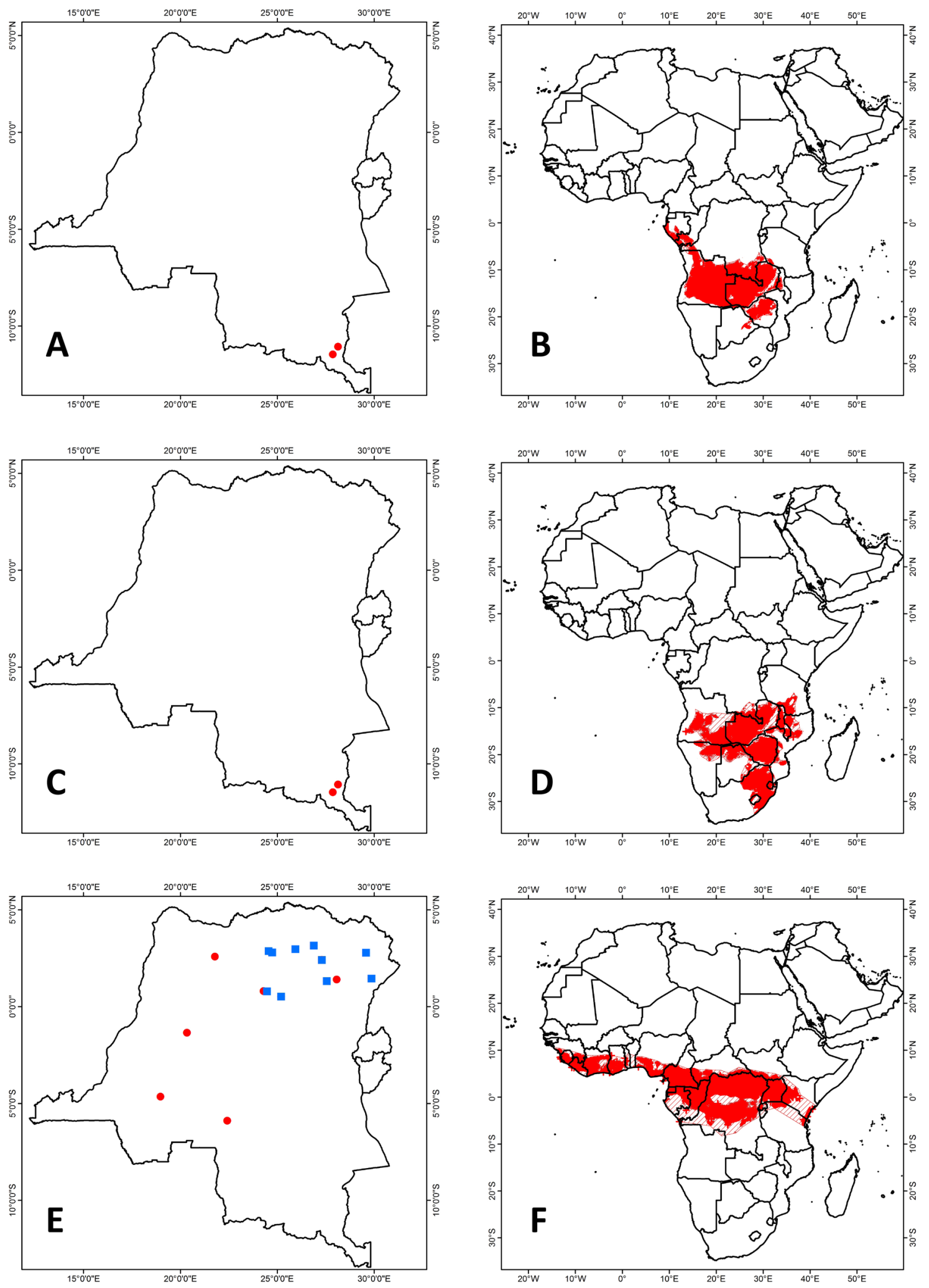

Fig. 42. Distribution maps. A-B. Laephotis angolensis Monard, 1935. C-D. Laephotis botswanae Setzer, 1971. E-F. Mimetillus moloneyi (Thomas, 1891). A, C, E. Distribution in the CRB area. B, D, F. Pan-African distribution. 
This species was reported from two localities near Lubumbashi (Haut-Katanga Province) in the extreme southeastern part of the DRC. The distribution map provided by Kearney (2013a: 580) indicates that the species occurs in a number of scattered areas in southern Africa: southeastern DRC and northwestern Zambia, central and southern Zimbabwe reaching into northern RSA, northwestern Botswana and northeastern Namibia, southern Malawi, and in some individual localities in Angola, southeastern RSA and northern Malawi. Kearney (2013a: 580), however, also pointed out that the relationship between angolensis and botswanae is obscure, because of the scarcity of the former, but she also refers to Setzer (1971: 262), who indicated that the two species might possibly occur sympatrically.

Genus Mimetillus Thomas, 1904

Mimetillus moloneyi (Thomas, 1891)

Fig. 42E-F

Vesperugo (Vesperus) Moloneyi Thomas, 1891: 528.

* Mimetillus moloneyi (Thomas, 1891).

Hayman et al. (1966: 50, map 68) mention this species from a number of localities in the northwestern part of the DRC (Bas- and Haut-Uélé, Ituri, Tshopo Provinces), but also from the southwestern Landana ("Lower Congo"). This last locality, however, is in Cabinda (Angola). The southern occurrence of M. moloneyi in the DRC is confirmed by records from Luluabourg (= Kananga) in Central Kasaï Province and Mosenge in Kwilu Province. These records were not included by Fahr (2013o: 587), who, therefore, plotted only a narrow band in the northern part of the DRC and individual localities in the border area between DRC, Uganda and Rwanda in the east, the border between the DRC and Zambia in the south and in Cabinda and the mouth of the Congo River in the west. He also stated that it is conspicuously absent from most of the central Congo Basin. Our additional records, however, suggest that it might be present in the fringes of the rain forest and other forest types and savannas reaching to the Kenyan and Tanzanian coast in the east and Sierra Leone and Guinea in the west.

Mimetillus thomasi Hinton, 1920

Fig. 43A-B

Mimetillus thomasi Hinton, 1920: 240.

Although Fahr (20130: 586) considers thomasi to be a subspecies of M. moloneyi, we follow Cotterill (2001b: 215), who proposed that the two are distinct evolutionary species, a view that was followed by Monadjem et al. (2010a: 390, 2010b: 444). The distribution map presented by Monadjem et al. (2010b: 444) indicates that M. thomasi occurs in the northern part of southern Africa, from Angola over southern DRC, Zambia and Zimbabwe to western and northern Mozambique. The DRC specimen from Elisabethville (= Lubumbashi, Haut-Katanga Province) reported here fits within this range.

Genus Neoromicia Roberts, 1926

Neoromicia brunnea (Thomas, 1880)

Fig. $43 \mathrm{C}-\mathrm{D}$

Vesperugo (Vesperus) brunneus Thomas, 1880: 165.

One specimen belonging to this species was collected at Lukolela (Mai-Ndombe Province, northwestern DRC). Fahr (2013p: 613) indicates that this species has a West African distribution, covering an area along the coast of Cameroon and Equatorial Guinea, and individual localities in Sierra Leone, Liberia, Côte d'Ivoire and Ghana. ACR (2016: 1237), furthermore, mentions specimens from Congo, Gabon and Nigeria. The Lukolela specimen is the easternmost record of this species. 

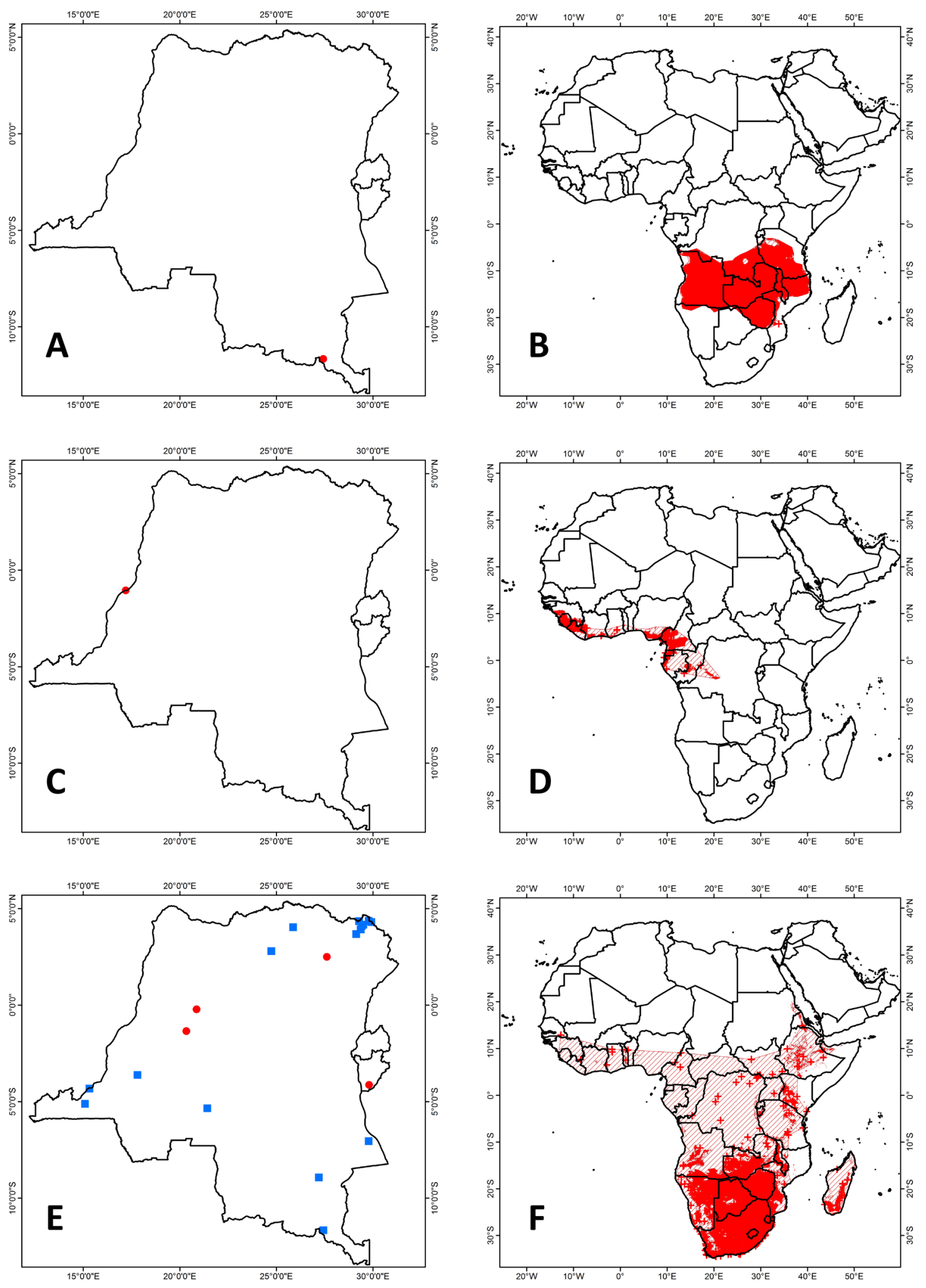

Fig. 43. Distribution maps. A-B. Mimetillus thomasi Hinton, 1920. C-D. Neoromicia brunnea (Thomas, 1880). E-F. Neoromicia capensis (A. Smith, 1829). A, C, E. Distribution in the CRB area. B, D, F. PanAfrican distribution. 
Neoromicia capensis (A. Smith, 1829)

Fig. 43E-F

Vespertilio Capensis A. Smith, 1829: 435.

* Eptesicus capensis (A. Smith, 1829).

* "Eptesicus garambae" J.A. Allen, 1917: 445.

* "Eptesicus minutus" (Temminck, 1835): 209.

* “Eptesicus grandidieri” (Dobson, 1876): 500.

Hayman et al. (1966: 46) already pointed to the confused status of the nomenclature of the small, darkwinged African members of the genus Eptesicus, where they indicated that E. capensis might include the following synonyms: garambae, minutus, grandidieri and possibly pusillus. Although the situation at the species level has been resolved, the supraspecific (generic) assignment of the African taxa once considered to belong to either Eptesicus or Pipistrellus still remains uncertain. Currently, these taxa are assigned to Eptesicus, Pipistrellus, Neoromicia and Hypsugo, of which the former does not have representatives in the CRB area. One of the major reasons for this confusion is that there are hardly any morphological characters which easily distinguish these genera (see Van Cakenberghe \& Happold 2013a: 600). Based on bacular morphology, Hill \& Harrison (1987: 261) suggested that capensis should be transferred from the genus Eptesicus to the genus Pipistrellus and more specifically to the subgenus Neoromicia. Based on dental characters, Menu (1987: 33) placed capensis in the genus Nycterikaupius. Karyological data led Volleth et al. (2001: 25) to elevate the subgenus Neoromicia to generic rank.

Distributionwise, Hayman et al. (1966: 46) provided data for four taxa: capensis, "garambae", "grandidieri" and "minutus", which were plotted on maps 54, 55, 56 and 57. The specimens identified as belonging to the latter two taxa were only reported from the area of the Garamba National Park (Haut Uélé Province). Specimens belonging to capensis were reported from two additional localities in the northern part of the DRC and also from a number of localities in the southern former provinces of Katanga and Kasai, as well as from Léopoldville (= Kinshasa). The SDM map indicates that the core area of distribution for $N$. capensis is southern Africa, south of the DRC, but also that specimens have been reported from as far north as Guinea, Ghana, Togo and Cameroon in western Africa and South Sudan and Ethiopia in the east. The map provided by Kearney (2013c: 615) shows a gap in NE (sic) Angola / SW DRC, and one from the central DRC over Tanzania to Mozambique, which might reflect insufficient sampling. However, she also left a gap between the DRC specimens and those from Cameroon, suggesting that the West African populations might be isolated from the remainder of the distribution area and represent another taxon. This might either be a different species or a form of capensis, which has not yet been named, as Kearney doesn't mention any subspecies from that part of the continent.

Neoromicia guineensis (Bocage, 1889)

Fig. 44A-B

Vesperus guineensis Bocage, 1889: 6.

The two specimens assigned to this species were collected by Herbert Lang and James Paul Chapin, and were reported by Allen (1917: 445) as belonging to Eptesicus minutus minutus (Temminck, 1835). Hayman et al. (1966: 47) refer to these specimens as "Eptesicus minutus", indicating that the name minutus was used in a tentative sense. However Rosevear (1965: 255) pointed out that minutus Temminck, 1835 is a synonym of Eptesicus capensis. Koopman (1965: 15) tentatively identified these two specimens as Eptesicus pusillus, but Koopman (1975: 407) furthermore pointed out that pusillus was based on two sources: Leconte (1858) and Noack (1889). The former was found to be unidentifiable and the latter to be a synonym of Pipistrellus nanus. Koopman's figure 39 indicates that the extralimital (to Sudan) specimen from northeastern DRC is considered to belong to Eptesicus guineensis. 

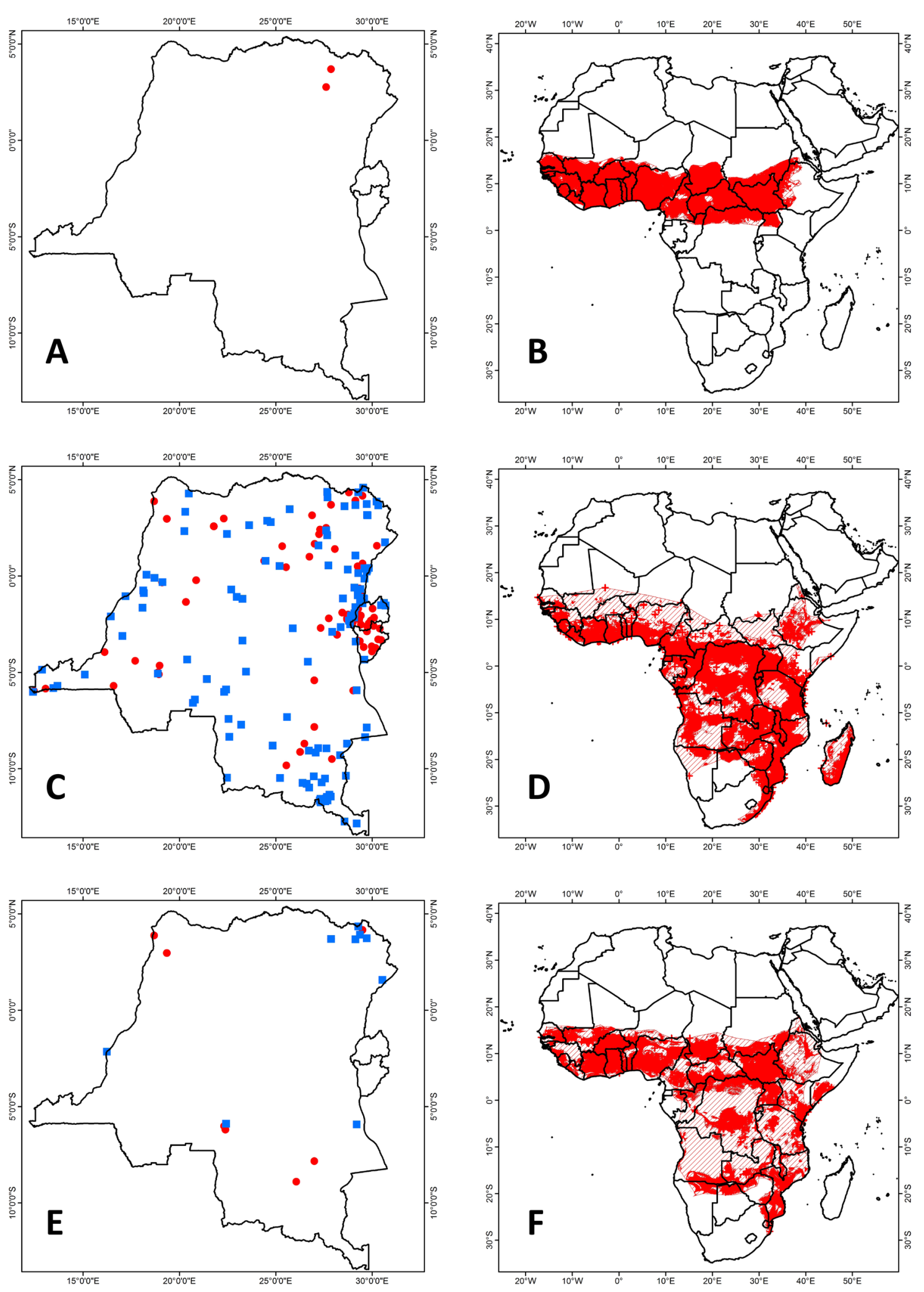

Fig. 44. Distribution maps. A-B. Neoromicia guineensis (Bocage, 1889). C-D. Neoromicia nana (Peters, 1852). E-F. Neoromicia rendalli (Thomas, 1889). A, C, E. Distribution in the CRB area. B, D, F. Pan-African distribution. 
Van Cakenberghe \& Happold (2013d: 625) indicate that guineensis primarily occurs disjunctly north of the equator in a band from Senegal, Guinea, northern Côte d'Ivoire, Ghana, Burkina Faso, northern Togo and Benin, and in a second band on the border between Nigeria and Cameroon, reaching to southern Chad. Furthermore, it occurs in a few smaller areas in central Sudan, the border between South Sudan and Uganda and in northwestern DRC. There are also some isolated records from Liberia, Nigeria, and western and eastern Ethiopia. The identifications of the specimens from Congo and southern DRC were found to be erroneous.

Neoromicia nana (Peters, 1852)

Fig. 44C-D

Vespertilio nanus Peters, 1852: 63.

* Pipistrellus culex Thomas, 1911: 458.

* Pipistrellus nanus nanus Peters, 1852.

Although they followed Koopman (1965) in many of his other conclusions, Hayman et al. (1966: 52) did not follow Koopman's view (1965: 12) on culex, which he tentatively considered a subspecies of P. stampflii (Jentink, 1888), but of which he also stated that it possibly only represents a small-sized population of $P$. nanus. Rosevear (1965: 268), Koopman (1966: 160) and Kock (1969: 170) considered culex to be a synonym of $P$. nanus. Over the past few decades Vespertilio nanus has changed genus a number of times (Happold 2013ah: 639): Pipistrellus, Neoromicia, Hypsugo. The current assignment to Neoromicia was based on karyotype data (Volleth et al. 2001: 44; Kearney et al. 2002: 55).

Hayman et al. (1966: 52, 53-55, maps 73, 76) reported $N$. nana from all over the CRB area, and some of the gaps have been filled in with additional specimens. The only exception seems to be the central part of the DRC (the area around the Salonga National Park), but that might possibly be attributed to the impenetrability of the region rather than the real absence of the species. The SDM map looks very similar to the one presented by Happold (2013ah: 640), although we show a number of hatched areas where the climatic circumstances might not be optimal for the species, e.g., the central forest area in the DRC (but see above), the border area between the DRC and Angola, eastern Angola and western Tanzania.

Neoromicia rendalli (Thomas, 1889)

Fig. 44E-F

Vesperugo (Vesperus) Rendalli Thomas, 1889: 362.

* Eptesicus rendalli (Thomas, 1889).

Hayman et al. (1966: 47, map 58) mention primarily specimens from the Garamba National Park area (Haut Uélé Province), and some single records from the provinces of Ituri, Tanganyika, Kasaï Central and Tshuapa. They also mention a specimen from Butembo (Nord-Kivu Province; RMCA 26585), but that specimen should be an Epomophorus anurus [= E. labiatus] (Wim Wendelen, pers. comm.). The patchy distribution map given by Van Cakenberghe \& Happold (2013g: 646) shows three red zones in the CBR area: one in the northeastern part of the DRC reaching northern Rwanda, a second in the northwestern part on the boundary between the DRC, Congo and the Central African Republic and a third in the province of Haut-Lomami towards Kasaï. Based on climatic data, our general map suggests a more continuous sub-Saharan distribution from about $15^{\circ} \mathrm{N}$ to $15^{\circ} \mathrm{S}$ (following the borders between Angola / Namibia and Zambia / Botswana + Zimbabwe) and further south to southern Mozambique and Swaziland, with possibly the exception of the central Congolese rain forest. 


\section{Neoromicia somalica (Thomas, 1901)}

Fig. 45A-B

Vespertilio minutus somalicus Thomas, 1901: 32.

Representatives of this species were collected at four localities, two in western DRC (Lukolela, MaiNdombe Province and Mbwambala, Kwilu Province), one in northeastern DRC (Mboga, Ituri Province), and one in Rwanda (Karama). The SMF has furthermore a specimen from a non-disclosed locality in the DRC. Van Cakenberghe \& Happold (2013h: 654) show that the distribution area of somalica forms a narrow band from southern Senegal over Guinea, Sierra Leone, northern Côte d'Ivoire, southern Burkina Faso, Togo, Benin, Nigeria, northern Cameroon, Central African Republic, South Sudan, splitting up in a branch towards Ethiopia and northern Somalia and a second branch to Uganda, eastern Rwanda, northern Tanzania, southern Kenya and reaching into southern Somalia. There are also scattered records from Congo and the DRC and some uncertain records from southern Tanzania, Mozambique, Zambia, Zimbabwe, Botswana, Angola and RSA. As indicated by Van Cakenberghe \& Happold, some of these latter records might represent the $N$. zuluensis.

\section{Neoromicia tenuipinnis (Peters, 1872)}

Fig. $45 \mathrm{C}-\mathrm{D}$

Vesperus tenuipinnis Peters, 1872: 236.

* Eptesicus tenuipinnis (Peters, 1872).

* Eptesicus tenuipinnis ater (J.A. Allen, 1917): 443.

Hayman et al. (1966: 47, 48, map 59) showed that tenuipinnis was frequently captured in the southwestern part of the DRC (from Banana in Kongo Central Province to the area of Mbandaka in Equateur Province) and in the northeastern part (provinces of Bas-Uélé, Haut-Uélé, Ituri and NordKivu). In the southeast they reach Kabongo (northern Haut-Lomami Province). The species seems to be missing in the southeastern provinces of Haut-Katanga and Tanganyika. More recently, specimens were collected in the northwestern part of the country (Tandala, Sud-Ubangi Province and Yalosemba, Mongala Province), and in northern Rwanda and western Burundi. Fahr (2013r: 656) reported that tenuipinnis is recorded from the lowland rainforest, swamp forest and coastal forests as well as from montane forest, mangroves, forest-savanna mosaic, Isoberlinia woodland, Acacia-Commiphora bushland and thicket and miombo woodland. Within the rainforest, it seems to prefer drier types of forest along the periphery.

Neoromicia zuluensis (Roberts, 1924)

Fig. 45E-F

Eptesicus zuluensis Roberts, 1924: 60.

As mentioned above, confusion with $N$. somalica might have led to some mis-identifications. However, one specimen in the collection of the Zürich Museum from Mimuli (Rwanda) was identified as belonging to this species. The distribution map presented by Happold et al. (2013: 658) indicates that the species seems to cover two major distribution areas, one covering the northeastern part of Uganda and the western part of Kenya, reaching into central Ethiopia, and a second reaching from the southeastern DRC over major parts of Zambia and Zimbabwe to northeastern and eastern Botswana and the northeastern RSA. They also indicate its presence at separate localities in eastern Kenya, Angola, Namibia, Malawi and central RSA. ACR (2016: 1264) furthermore mentions specimens from Mozambique, South Sudan and Swaziland. 

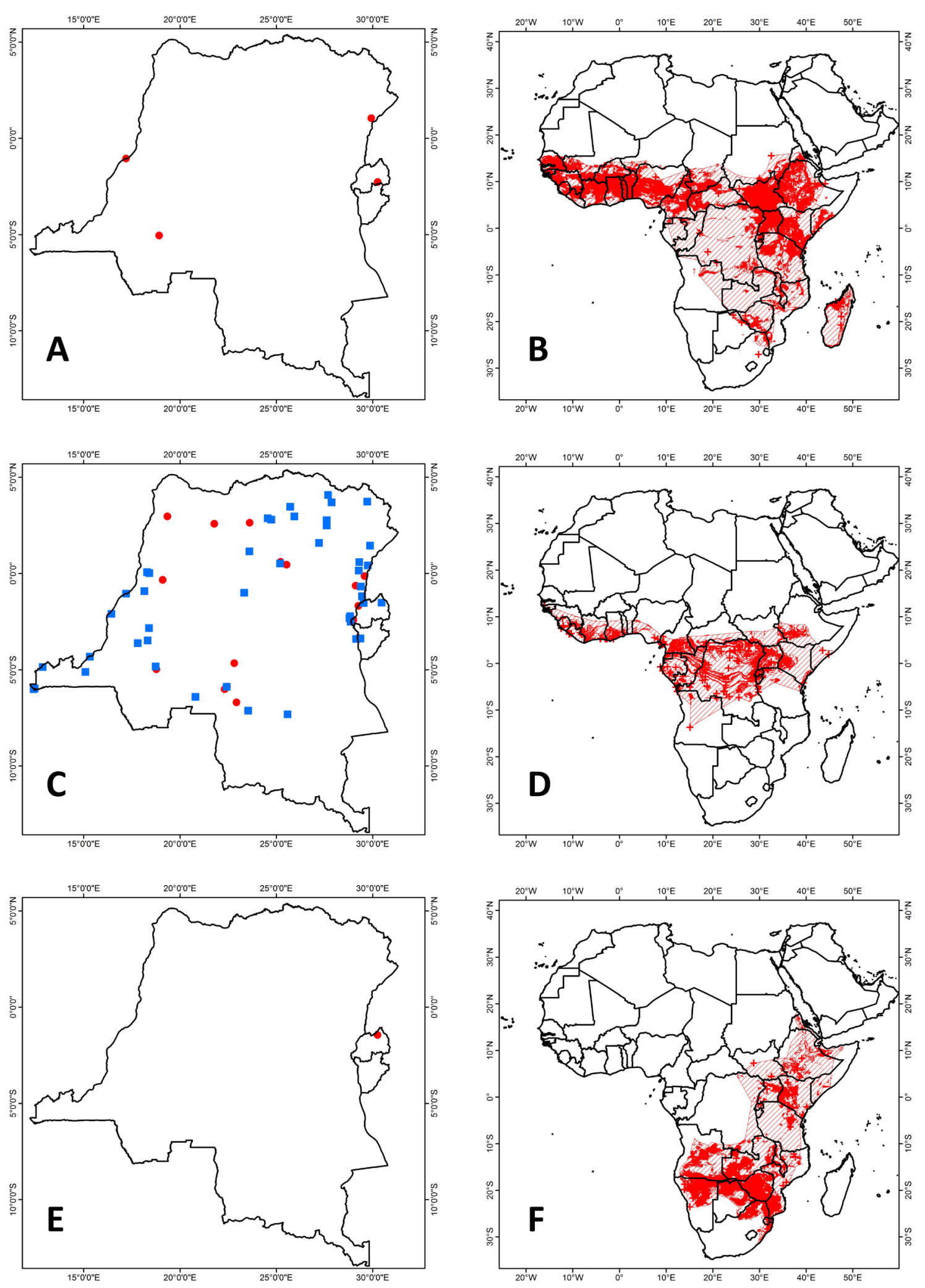

Fig. 45. Distribution maps. A-B. Neoromicia somalica (Thomas, 1901). C-D. Neoromicia tenuipinnis (Peters, 1872). E-F. Neoromicia zuluensis (Roberts, 1924). A, C, E. Distribution in the CRB area. B, D, F. Pan-African distribution. 
VAN CAKENBERGHE V. et al., The bats of Congo, Rwanda and Burundi revisited

Genus Nycticeinops Hill \& Harrison, 1987

Nycticeinops schlieffenii (Peters, 1860)

Fig. 46A-B

Nycticejus Schlieffenii Peters, 1860: 224.

* Nycticeius (Scoteinus) schlieffeni Peters, 1860.

* Nycticeius (Scoteinus) schlieffeni albiventer Thomas \& Wroughton, 1908: 540.

Based on the shape of the baculum, Hill \& Harrison (1987: 254) created a new genus (Nycticeinops), which contains schlieffenii as the only species. Happold (2013ag: 595) indicated that four subspecies were recognized by Hayman \& Hill (1971: 36), but these are of dubious validity.

Hayman et al. (1966: 55-56, map 78) report schlieffenii from the northeastern part of the DRC, where they plotted three localities. Of these, Niangara needs to be removed as they stated on page 56 that Koopman (1965: 17) reassigned this specimen to Scotoecus hirundo hindei. Furthermore, Hayman et al. (1966) mention specimens from the southeastern part of the DRC (Tanganyika, Haut-Katanga and HautLomami Provinces). The locality on Lake Tanganyika they mention is probably (Qua) Mpala, the type locality of Scotophilus minimus Noack, 1887, but this specimen could not be traced and is, therefore, not included here. More to the west, they also mentioned a specimen from Luluabourg. In the collection of the Tervuren museum, there is also an additional specimen from "Bas Congo" (RMCA 65b), which probably was overlooked by Hayman et al. (1966), as they report a Myotis bocagii from the same locality (RMCA 65). Recently, an additional specimen was collected by the team of the University of Kisangani at Aketi (Bas-Uélé Province).

The map presented by Happold (2013ag: 595) shows two major distribution areas: the Sudanian woodlands in West Africa (from the Mauritanian-Senegalese border in the west to Nigeria and northern Cameroon in the east). The second area covers a major part of eastern Africa (from the coastal area in Sudan and Eritrea, splitting in two narrow stretches in Ethiopia, combining again to cover central Kenya, passing through central Tanzania to the southeastern DRC, Zambia, extending to the border between Angola and Namibia, Malawi, northern Zimbabwe, central and eastern Mozambique to the extreme northeastern RSA), also including a separate, small area in the northeastern DRC. The specimens reported here confirm the species' presence in the two DRC regions, but also suggest that the species extends more to the west.

Genus Pipistrellus Kaup, 1829

Pipistrellus grandidieri (Dobson, 1876)

Fig $46 \mathrm{C}-\mathrm{D}$

Vesperugo (Vesperus) grandidieri Dobson, 1876: 500.

* Eptesicus grandidieri (Dobson, 1876).

Although Hayman et al. (1966: 46) report six "Eptesicus grandidieri" specimens from the DRC, none of these actually belong to that species, but rather to Neoromicia capensis. However, two additional specimens from Resha (Burundi; SMF 57586 and 57587) were found to belong to grandidieri, so the species does occur in the CRB area.

Thorn et al. (2007) re-evaluated the known material of grandidieri and concluded that this form should be assigned to a separate subgenus (Afropipistrellus) within the genus Pipistrellus. Van Cakenberghe \& Happold (2013c: 623) present an extremely patchy distribution range, which covers a number of isolated localities ranging from the Nigerian/Cameroonian border to southern Somalia, and southwards to central 

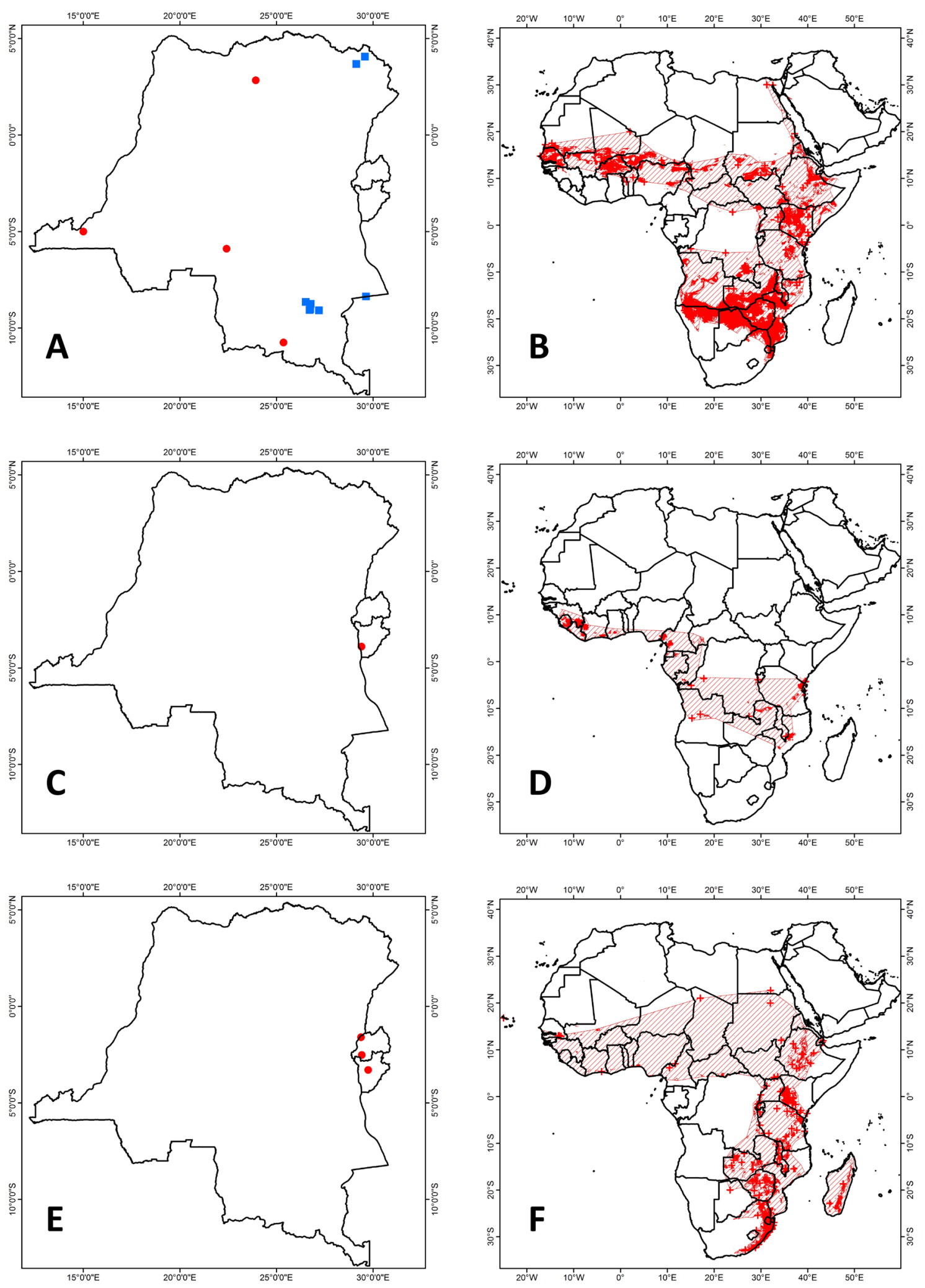

Fig. 46. Distribution maps. A-B. Nycticeinops schlieffenii (Peters, 1860). C-D. Pipistrellus grandidieri (Dobson, 1876). E-F. Pipistrellus hesperidus (Temminck, 1840). A, C, E. Distribution in the CRB area. B, D, F. Pan-African distribution. 
Angola and southern Mozambique. Here, we extend the distribution to Guinea and Liberia in West Africa, where "Pipistrellus cf. grandidieri" was reported by Decher et al. (2016: 273).

Pipistrellus hesperidus (Temminck, 1840)

Fig. 46E-F

Vespertilio hesperida Temminck, 1840: 211.

This species used to be included in Pipistrellus kuhlii, but as found by Göpfert et al. (1995: 68) and Volleth et al. (2001: 28), the sub-Saharan specimens have a different chromosome number than the specimens from Europe and northern Africa. For these sub-Saharan populations, Kock (2001: 277) reinstated the name hesperidus.

Specimens belonging to this species were collected at three localities in the CRB area: two in Rwanda (Mutura and Kitabi) and one in Burundi (Nyamugari Hill). Kearney (2013d: 630) indicates that $P$. hesperidus is distributed in a narrow band in eastern Africa, reaching from southwestern Eritrea, over western Ethiopia, eastern South Sudan to northern Uganda, where it splits into two branches, one along the Kenyan-Tanzanian border reaching the coast, and a second along the great lakes to eastern Zimbabwe and the eastern RSA, reaching just north of Port Elizabeth. Additionally, she marked the presence of the species in the Mount Cameroon area and in Djibouti, with some further individual localities in northeastern Somalia, western Zambia, Angola and southern RSA. Furthermore, she refers to Jakob Fahr, who indicated that some records from West Africa might be misidentified. The SDM map confirms the presence of the species in eastern Africa. The records from West Africa might need to be re-examined.

Pipistrellus nanulus Thomas, 1904

Fig. 47A-B

* Pipistrellus nanulus Thomas, 1904: 198.

Hayman et al. (1966: map 75) plotted one specimen from Avakubi, which is actually the same specimen they plotted as "Pipistrellus musciculus" (see above). One additional record is from from Mosenge in Kwilu Province.

The map given by Van Cakenberghe \& Happold (2013f: 639) shows that P. nanulus primarily occurs in the savannas of Sudan and Guinea, the northern rainforest-savanna mosaic in West Africa (from Guinea to Togo and from southern Nigeria to Gabon, and from north-central Benin to central Nigeria) and in the eastern rainforest-savanna mosaic in northeastern DRC, Uganda and western Kenya. Based on additional material mentioned in ACR (2016: 1276), our distribution map covers a more continuous area from Senegal to western Kenya, with a small gap at the Dahomey Gap in western Africa. The map also shows that it could occur over almost the entire northern half of the DRC, with the exception of the central part.

Pipistrellus rueppellii (Fischer, 1826)

Fig. 47C-D

Vespertilio Rüppelii J.B. Fischer, 1826: 109.

* Pipistrellus (Scotozous) rüppelli (Fischer, 1826).

* Pipistrellus (Scotozous) rüppelli fuscipes (Thomas, 1913): 315.

Happold (2013ai: 648) mentions that fuscipes is the subspecies of P. rueppellii occurring from Ethiopia to northern Angola, which would cover the CRB area. Hayman et al. (1966: 55, map 77) report the species 
from the northeastern part of the DRC (provinces Bas-Uélé, Tshopo, Ituri and Nord-Kivu), as well as from the southeast (Tanganyika and Haut-Katanga Provinces) and southwest parts (Kongo Central, Kasaï and Mai-Ndombe Provinces). The two additional records (RMCA 12247 from Mongbwalu and RMCA 993 from Lukula) were probably overlooked by Hayman et al. (1966), as these specimens were present in the Tervuren collection at the time.

Happold's (2013ai: 648) distribution map essentially shows the species following the River Nile southwards, along the great lakes to northern Zimbabwe and central Mozambique, speading in the southern part where it should occur over most of Zambia. Additionally, the species occurs in northern DRC, along the lower Congo River and up the Kasaï River; along the Moroccan-Algerian border crossing into the latter country; and along the Senegalese-Mauritanian border. Furthermore, there are some individual records from Egypt, Ethiopia, eastern Tanzania, Botswana, RSA, Angola, Gabon, Central African Republic, Chad, Nigeria, Libya, Tunisia and Morocco. The species seems to be lacking from West Africa, although climatic data used for the SDM map suggest that it might occur in that part of the continent.

Genus Scotoecus Thomas, 1901

Scotoecus albofuscus (Thomas, 1890)

Fig. 47E-F

Scotophilus albofuscus Thomas, 1890: 84.

* Nycticeius (Scotoecus) albofuscus woodi (Thomas, 1917): 280.

Although Hayman et al. (1966: 56) considered Scotoecus to be a subgenus of Nycticeius, its generic status was already restored by Rosevear (1965: 246) and Koopman (1965: 17), who were followed by Hill (1974b: 171), Koopman (1993: 226, 1994: 127) and Simmons (2005: 461, 464).

Only one specimen of this species has been reported from the CRB area up until now: RMCA 22412 from Baudouinville (= Moba), Tanganyika Province. It was first mentioned by Hayman (1957: 44) and subsequently confirmed by Fain (1959: 161), Hill (1974b: 177), Anciaux de Faveaux (1983: 29), Monadjem et al. (2010b: 506) and Happold (2013aj: 668).

The distribution map presented by Happold (2013aj: 668) clearly illustrates the rarity of this species in scientific collections, as only individual records are plotted, with the exception of small areas in Sierra Leone and The Gambia. The species occurs from the latter country in West Africa to Northern Cameroon and then continuing from northwestern Uganda, over Tanzania, southeastern DRC and Mozambique to the RSA. This leaves a wide gap between Cameroon and Uganda. ACR (2016: 1283) mentions three specimens from the Central African Republic, which narrow this gap a bit, but it nevertheless remains wide. We concur with Ansell \& Dowsett (1988: 43), who state that "this species is rarely collected, though it has a wide range from Senegal to southern Mozambique. It is no doubt more widespread in Malawi than the single recorded locality would indicate", and believe this is also the case for many other countries. The SDM map suggests that $S$. albofuscus potentially can occur along the northern border of the DRC, where it can bridge the gap between the two areas where it has been found up until now.

Scotoecus hindei Thomas, 1901

Fig. 48A-B

Scotoecus Hindei Thomas, 1901: 264.

* Nycticeius (Scotoecus) hirundo hindei (Thomas, 1901). 

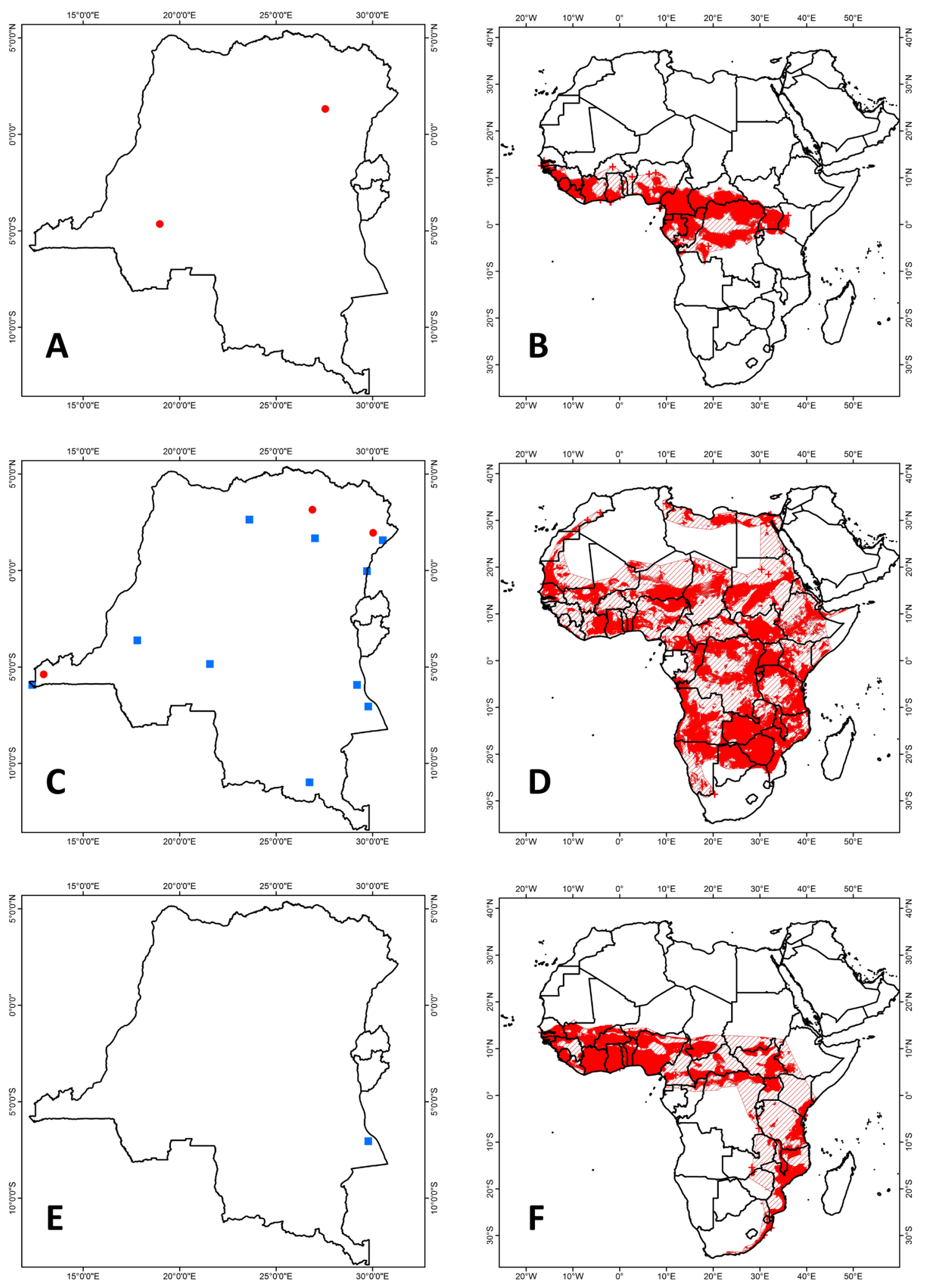

Fig. 47. Distribution maps. A-B. Pipistrellus nanulus Thomas, 1904. C-D. Pipistrellus rueppellii (Fischer, 1826). E-F. Scotoecus albofuscus (Thomas, 1890). A, C, E. Distribution in the CRB area. B, D, F. Pan-African distribution. 
The taxonomy of the dark-winged Scotoecus forms is not very clear. Pending a revision of this group, Happold (2013ak: 669) considers all the dark-winged Scotoecus to belong to one species: S. hirundo. However, we follow Ansell \& Dowsett (1988: 44), Taylor \& Van der Merwe (1998: 64), Cotterill (2001c: 219) and Simmons (2005: 464) in recognizing $S$. hindei as a separate species.

Scotoecus hindei has only been reported twice from the DRC: once from the southeast of the country (Kapolowe, Haut-Katanga Province) and once from the northeast (Niangara, Haut-Uélé Province). Both were already mentioned by Hayman et al. (1966: 56).

Since Happold (2013ak: 670) doesn't recognize hindei as a separate species, our distribution map cannot be compared with hers. Based on the data in ACR (2016: 1283). The SDM map suggests a primarily eastern African distribution, from Eritrea in the north to southern Mozambique and northern RSA in the south. It extends westwards across the northern part of the DRC, the Central African Republic, Cameroon and Nigeria.
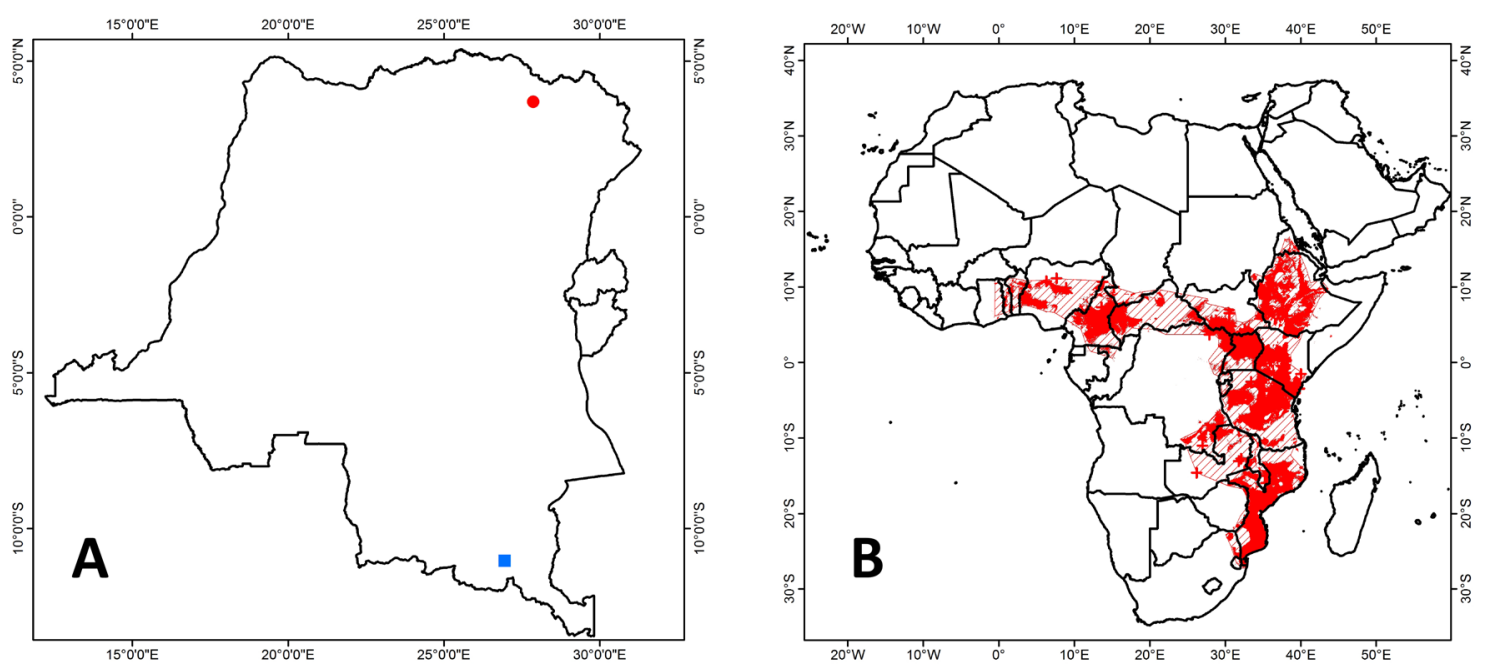

Fig. 48. Distribution maps. Scotoecus hindei Thomas, 1901. A. Distribution in the CRB area. B. PanAfrican distribution. 
VAN CAKENBERGHE V. et al., The bats of Congo, Rwanda and Burundi revisited Nominal list of bats of the Congo, Rwanda and Burundi (CRB) region

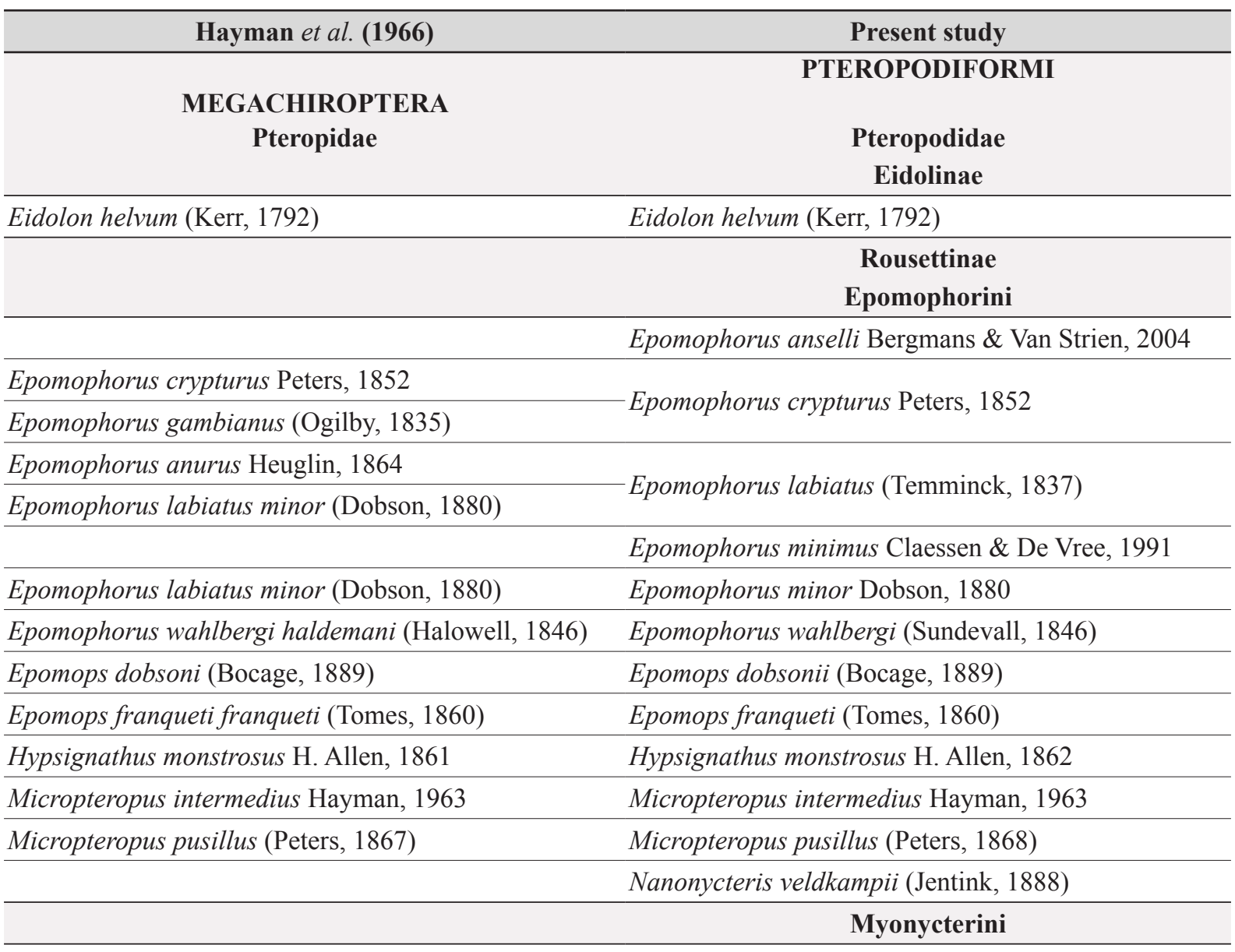

Megaloglossus woermanni Pagenstecher, 1885

Megaloglossus woermanni prigoginei Hayman, 1966

Rousettus (Lissonycteris) angolensis (Bocage, 1898) Myonycteris angolensis (Bocage, 1898)

\begin{tabular}{ll}
\hline Myonycteris wroughtoni Andersen, 1908 & Myonycteris relicta Bergmans, 1980 \\
\hline Plerotes anchietae (Seabra, 1900) & Myonycteris torquata (Dobson, 1878) \\
\hline \multicolumn{2}{l}{ Plerotini } \\
\hline Rousettus aegyptiacus leachi (Smith, 1823) & Plerotes anchietae (Seabra, 1900) \\
\hline Casinycteris argynnis Thomas, 1910 & Rousettus aegyptiacus (E. Geoffroy St.-Hilaire, 1810) \\
\hline Scotonycteris zenkeri Matschie, 1894 & Scotonycterini \\
\hline Rousettus (Stenonycteris) lanosus Thomas, 1906 & Casinycteris argynnis Thomas, 1910 \\
\hline MICROCHIROPTERA & Scotonycteris bergmansi Hassanin et al., 2015 \\
\hline Hipposideridae & Stenonycteris lanosus Thomas, 1906 \\
\hline Hipposideros cyclops (Temminck, 1853) & Hipposideridae \\
\hline
\end{tabular}


Hayman et al. (1966)

Present study

Hipposideros abae J.A. Allen, 1917

Hipposideros abae J.A. Allen, 1917

Hipposideros beatus Andersen, 1906

Hipposideros beatus maximus Verschuren, 1957

Hipposideros beatus K. Andersen, 1906

Hipposideros caffer (Sundevall, 1846)

Hipposideros caffer caffer (Sundevall, 1846)

Hipposideros caffer tephrus Cabrera, 1906

Hipposideros caffer (Sundevall, 1846)

Hipposideros nanus J.A. Allen, 1917

Hipposideros camerunensis Eisentraut, 1956

Hipposideros camerunensis Eisentraut, 1956

Hipposideros fuliginosus (Temminck, 1853)

Hipposideros fuliginosus (Temminck, 1853)

Hipposideros caffer ruber (Noack, 1893)

Hipposideros ruber (Noack, 1893)

Hipposideros commersoni gigas (Wagner, 1845)

Macronycteris gigas (Wagner, 1845)

Hipposideros commersoni marungensis (Noack, 1887)

Macronycteris vittatus (Peters, 1852)

\begin{tabular}{|c|c|}
\hline Megadermidae & Megadermatidae \\
\hline & Cardioderma cor (Peters, 1872) \\
\hline Lavia frons frons (Geoffroy, 1810) & Lavia frons (E. Geoffroy St.-Hilaire, 1810) \\
\hline \multicolumn{2}{|c|}{ Rhinolophidae } \\
\hline Rhinolophus alcyone alcyone Temminck, 1852 & Rhinolophus alcyone Temminck, 1852 \\
\hline Rhinolophus blasii empusa (Andersen, 1904) & Rhinolophus blasii Peters, 1867 \\
\hline Rhinolophus clivosus Cretzschmar, 1826 & \multirow{2}{*}{-Rhinolophus clivosus Cretzschmar, 1828} \\
\hline Rhinolophus clivosus zuluensis (Andersen, 1904) & \\
\hline Rhinolophus hildebrandti eloquens Andersen, 1905 & Rhinolophus eloquens K. Andersen, 1905 \\
\hline Rhinolophus fumigatus Rüppell, 1842 & Rhinolophus fumigatus Rüppell, 1842 \\
\hline \multirow[t]{3}{*}{ Rhinolophus hildebrandti Peters, 1878} & Rhinolophus hildebrandtii Peters, 1878 \\
\hline & Rhinolophus hilli Aellen, 1973 \\
\hline & Rhinolophus kahuzi Fahr \& Kerbis Peterhans, 2013 \\
\hline Rhinolophus landeri lobatus (Peters, 1852) & Rhinolophus landeri Martin, 1838 \\
\hline \multirow[t]{2}{*}{ Rhinolophus ruwenzorii Hill, 1942} & Rhinolophus ruwenzorii J. Eric Hill, 1942 \\
\hline & Rhinolophus simulator K. Andersen, 1904 \\
\hline \multirow[t]{2}{*}{ Rhinolophus swinnyi Gough, 1908} & Rhinolophus swinnyi Gough, 1908 \\
\hline & Rhinolophus willardi Kerbis Peterhans \& Fahr, 2013 \\
\hline Hipposideridae & Rhinonycteridae \\
\hline Cloeotis percivali australis Roberts, 1917 & Cloeotis percivali Thomas, 1901 \\
\hline \multicolumn{2}{|r|}{ VESPERTILIONIFORMI } \\
\hline Coleura afra (Peters, 1852) & Coleura afra (Peters, 1852) \\
\hline Taphozous (Saccolaimus) peli (Temminck, 1853) & Saccolaimus peli (Temminck, 1853) \\
\hline Taphozous mauritianus Geoffroy, 1818 & Taphozous mauritianus E. Geoffroy St.-Hilaire, 1818 \\
\hline Taphozous (Liponycteris) nudiventris (Cretzschmar, 1826) & Taphozous nudiventris Cretzschmar, 1830 \\
\hline Taphozous sudani Thomas, 1915 & Taphozous perforatus E. Geoffroy St.-Hilaire, 1818 \\
\hline \multicolumn{2}{|c|}{ Nycteridae } \\
\hline Nycteris arge Thomas, 1903 & Nycteris arge Thomas, 1903 \\
\hline
\end{tabular}


VAN CAKENBERGHE V. et al., The bats of Congo, Rwanda and Burundi revisited

Hayman et al. (1966)

Nycteris grandis Peters, 1865

Nycteris hispida (Schreber, 1775)

Nycteris intermedia Aellen, 1959

Nycteris aethiopica Dobson, 1878

Nycteris macrotis Dobson, 1876

Nycteris major (Andersen, 1912)

Nycteris nana (Andersen, 1912)

Nycteris capensis Smith, 1829

Nycteris thebaica Geoffroy, 1818

\section{Present study}

Nycteris grandis Peters, 1865

Nycteris hispida (Schreber, 1774)

Nycteris intermedia Aellen, 1959

Nycteris macrotis Dobson, 1876

Nycteris major (K. Andersen, 1912)

Nycteris nana (K. Andersen, 1912)

Nycteris parisii (de Beaux, 1924)

Nycteris thebaica E. Geoffroy St.-Hilaire, 1818

\section{Molossidae}

Tadarida (Chaerephon) aloysii-sabaudiae (Festa, 1907)

Tadarida (Tadarida) brunnea (Seabra, 1900)

Tadarida (Tadarida) ansorgei (Thomas, 1913)

Chaerephon aloysiisabaudiae (Festa, 1907)

Tadarida (Tadarida) cistura (Thomas, 1903)

Chaerephon ansorgei (Thomas, 1913)

Chaerephon bemmeleni (Jentink, 1879)

Chaerephon bivittatus (Heuglin, 1861)

Tadarida (Chaerephon) chapini J. A. Allen, 1917

Chaerephon chapini J.A. Allen, 1917

Chaerephon gallagheri (Harrison, 1975)

Chaerephon leucogaster (A. Grandidier, 1869)

Tadarida (Chaerephon) major (Trouessart, 1897) Chaerephon major (Trouessart, 1897)

Tadarida (Chaerephon) nigeriae Thomas, 1913

Tadarida (Chaerephon) spillmanni (Monard, 1932)

Tadarida (Chaerephon) pumila (Cretzschmar, 1826)

Chaerephon nigeriae Thomas, 1913

Tadarida (Chaerephon) russata J.A. Allen, 1917

Chaerephon pumilus (Cretzschmar, 1826)

Chaerephon russatus J.A. Allen, 1917

Tadarida (Mops) condylura (A. Smith, 1833)

Tadarida (Mops) condylura condylura (A. Smith, 1833)

Tadarida (Mops) congica J.A. Allen, 1917

Mops (Mops) condylurus (A. Smith, 1833)

Tadarida (Mops) demonstrator (Thomas, 1903)

Mops (Mops) congicus J.A. Allen, 1917

Tadarida (Mops) midas (Sundevall, 1843)

Mops (Mops) demonstrator (Thomas, 1903)

Tadarida (Mops) niangarae J.A. Allen, 1917

Mops (Mops) midas (Sundevall, 1843)

Mops (Mops) niangarae J.A. Allen, 1917

Tadarida (Mops) chitauensis Hill, 1937

Tadarida (Mops) niveiventer (Cabrera \& Ruxton, 1926)

Tadarida (Mops) congica J.A. Allen, 1917

Mops (Mops) niveiventer Cabrera \& Ruxton, 1926

Tadarida (Mops) leonis (Thomas, 1908)

Tadarida (Mops) nanula J.A. Allen, 1917

? Xiphonycteris spurrelli Dollman, 1911

Mops (Mops) trevori J.A. Allen, 1917

Mops (Xiphonycteris) brachypterus (Peters, 1852)

Mops (Xiphonycteris) nanulus J.A. Allen, 1917

Mops (Xiphonycteris) spurrelli (Dollman, 1911)

Tadarida (Mops) thersites (Thomas, 1903)

Myopterus albatus Thomas, 1915

Myopterus whitleyi (Scharff, 1900)

Mops (Xiphonycteris) thersites (Thomas, 1903)

Myopterus daubentonii Desmarest, 1820

Myopterus whitleyi (Scharff, 1900)

Otomops martiensseni (Matschie, 1897)

Otomops martiensseni (Matschie, 1897)

Tadarida (Tadarida) fulminans (Thomas, 1903)

Tadarida fulminans (Thomas, 1903)

Tadarida (Tadarida) africana (Dobson, 1876)

Tadarida ventralis (Heuglin, 1861) 
European Journal of Taxonomy 382: 1-327 (2017)

\begin{tabular}{|c|c|}
\hline Hayman et al. (1966) & Present study \\
\hline \multirow[t]{2}{*}{ Vespertilionidae } & Miniopteridae \\
\hline & Miniopterus fraterculus Thomas \& Schwann, 1906 \\
\hline Miniopterus inflatus rufus Sanborn, 1936 & Miniopterus inflatus Thomas, 1903 \\
\hline Miniopterus minor Peters, 1867 & Miniopterus minor Peters, 1867 \\
\hline Miniopterus natalensis arenarius Heller, 1912 & \multirow{3}{*}{ Miniopterus natalensis (A. Smith, 1833) } \\
\hline Miniopterus schreibersi vicinior (J.A. Allen, 1917) & \\
\hline Miniopterus schreibersi villiersi Aellen, 1956 & \\
\hline & Vespertilionidae \\
\hline & Kerivoulinae \\
\hline \multirow[t]{2}{*}{ Kerivoula argentata Tomes, 1861} & Kerivoula argentata Tomes, 1861 \\
\hline & Kerivoula cuprosa Thomas, 1912 \\
\hline Kerivoula harrisoni muscilla (Thomas, 1906) & \multirow{2}{*}{-Kerivoula lanosa (A. Smith, 1847) } \\
\hline Kerivoula harrisoni lucia (Hinton, 1920) & \\
\hline Kerivoula phalaena Thomas, 1912 & Kerivoula phalaena Thomas, 1912 \\
\hline Kerivoula smithi Thomas, 1880 & Kerivoula smithii Thomas, 1880 \\
\hline \multicolumn{2}{|l|}{ Kerivoula? aerosa Tomes, 1858} \\
\hline & Myotinae \\
\hline \multicolumn{2}{|l|}{ Myotis bocagei (Peters, 1870) } \\
\hline Myotis bocagei bocagei (Peters, 1870) & \multirow{3}{*}{-Myotis bocagii (Peters, 1870) } \\
\hline Myotis bocagei cupreolus Thomas, 1904 & \\
\hline Myotis bocagei hildegardeae Thomas, 1904 & \\
\hline Myotis tricolor (Temminck, 1832) & Myotis tricolor (Temminck, 1832) \\
\hline \multirow[t]{2}{*}{ Myotis welwitschi venustus (Matschie, 1899) } & Myotis welwitschii (Gray, 1866) \\
\hline & Scotophilinae \\
\hline Scotophilus nigrita (Schreber, 1775) & \multirow{2}{*}{-Scotophilus dinganii (A. Smith, 1833) } \\
\hline Scotophilus nigrita herero Thomas, 1906 & \\
\hline Scotophilus leucogaster (Cretzschmar, 1826) & Scotophilus leucogaster (Cretzschmar, 1826) \\
\hline Scotophilus gigas Dobson, 1875 & Scotophilus nigrita (Schreber, 1774) \\
\hline \multirow[t]{2}{*}{ Scotophilus nigrita nux Thomas, 1904} & Scotophilus nux Thomas, 1904 \\
\hline & Vespertilioninae \\
\hline Glauconycteris alboguttatus J.A. Allen, 1917 & Glauconycteris alboguttata J.A. Allen, 1917 \\
\hline Glauconycteris argentata (Dobson, 1875) & Glauconycteris argentata (Dobson, 1875) \\
\hline \multirow[t]{2}{*}{ Glauconycteris beatrix Thomas, 1901} & Glauconycteris beatrix Thomas, 1901 \\
\hline & Glauconycteris curryae Eger \& Schlitter, 2001 \\
\hline Glauconycteris humeralis J.A. Allen, 1917 & Glauconycteris humeralis J.A. Allen, 1917 \\
\hline Glauconycteris poensis (Gray, 1842) & Glauconycteris poensis (Gray, 1842) \\
\hline Glauconycteris superba superba Hayman, 1939 & Glauconycteris superba Hayman, 1939 \\
\hline \multirow[t]{2}{*}{ Glauconycteris variegata papilio (Thomas, 1905) } & Glauconycteris variegata (Tomes, 1861) \\
\hline & Hypsugo anchietae (Seabra, 1900) \\
\hline \multirow[t]{2}{*}{ Pipistrellus crassulus Thomas, 1904} & Hypsugo crassulus (Thomas, 1904) \\
\hline & Hypsugo cf. eisentrauti (Hill, 1968) \\
\hline
\end{tabular}


VAN CAKENBERGHE V. et al., The bats of Congo, Rwanda and Burundi revisited

\begin{tabular}{|c|c|}
\hline Hayman et al. (1966) & Present study \\
\hline Pipistrellus musciculus Thomas, 1913 & Hypsugo musciculus (Thomas, 1913) \\
\hline \multirow[t]{2}{*}{ Laephotis wintoni angolensis (Monard, 1935) } & Laephotis angolensis Monard, 1935 \\
\hline & Laephotis botswanae Setzer, 1971 \\
\hline \multirow[t]{3}{*}{ Mimetillus moloneyi (Thomas, 1891) } & Mimetillus moloneyi (Thomas, 1891) \\
\hline & Mimetillus thomasi Hinton, 1920 \\
\hline & Neoromicia brunnea (Thomas, 1880) \\
\hline \multicolumn{2}{|l|}{ Eptesicus capensis (Smith, 1829) } \\
\hline \multirow{2}{*}{\multicolumn{2}{|c|}{$\begin{array}{l}\text { "Eptesicus garambae" J.A. Allen, } 1917 \\
\text { "Eptesicus grandidieri" (Dobson, 1876) }\end{array}$}} \\
\hline & \\
\hline \multicolumn{2}{|l|}{ “Eptesicus minutus” (Temminck, 1835) } \\
\hline & Neoromicia guineensis (Bocage, 1889) \\
\hline \multirow{2}{*}{\multicolumn{2}{|c|}{$\begin{array}{l}\text { Pipistrellus culex Thomas, } 1911 \\
\text { Pipistrellus nanus nanus } \text { Peters, } 1852\end{array}$}} \\
\hline & \\
\hline \multirow{2}{*}{ Eptesicus rendalli (Thomas, 1889) } & Neoromicia rendalli (Thomas, 1889) \\
\hline & Neoromicia somalica (Thomas, 1901) \\
\hline \multirow{2}{*}{\multicolumn{2}{|c|}{$\begin{array}{l}\text { Eptesicus tenuipinnis (Peters, 1872) } \\
\text { Eptesicus tenuipinnis ater (J.A. Allen, }\end{array}$}} \\
\hline & \\
\hline & Neoromicia zuluensis (Roberts, 1924) \\
\hline \multicolumn{2}{|l|}{ Nycticeius (Scoteinus) schlieffeni Peters, 1860} \\
\hline $\begin{array}{l}\text { Nycticeius (Scoteinus) schlieffeni albiventer } \\
\text { Thomas \& Wroughton, } 1908\end{array}$ & Nycticeinops schlieffenii (Peters, 1859) \\
\hline \multirow[t]{2}{*}{ Eptesicus grandidieri (Dobson, 1876) } & Pipistrellus grandidieri (Dobson, 1876) \\
\hline & Pipistrellus hesperidus (Temminck, 1840) \\
\hline Pipistrellus nanulus Thomas, 1904 & Pipistrellus nanulus Thomas, 1904 \\
\hline \multicolumn{2}{|l|}{ Pipistrellus (Scotozous) rüppelli (Fischer, 1826) } \\
\hline $\begin{array}{l}\text { Pipistrellus (Scotozous) rüppelli fuscipes } \\
\text { (Thomas, 1913) }\end{array}$ & Pipistrellus rueppellii (Fischer, 1829) \\
\hline Nycticeius (Scotoecus) albofuscus woodi (Thomas, 1917) & Scotoecus albofuscus (Thomas, 1890) \\
\hline Nycticeius (Scotoecus) hirundo hindei (Thomas, 1901) & Scotoecus hindei Thomas, 1901 \\
\hline
\end{tabular}




\section{Key to the bat species of the CRB region}

This key is primarily modified from Hayman et al. (1966), with additional information from Hayman \& Hill (1971), Bergmans (1997), Cotterill (2001b), Happold \& Happold (2013), Kerbis Peterhans et al. (2013) and Foley et al. (2014).

1. Cheekteeth without W-pattern of cusps; second finger with claw; tail absent or rudimentary; interfemoral membrane reduced; ears very short, well separated, no tragus; margin of ear forming a complete ring (family Pteropidae)

- Cheekteeth with W-pattern of cusps; second finger entirely included in patagium and without claw; tail generally well developed; interfemoral membrane developed; ears very short to very long, widely separed or joined; tragus present in most cases (except for Rhinolophidae, Rhinonycteridae and Hipposideridae)...

2. Tail more or less completely included in uropatagium (in certain forms the last vertebra can be free)

- Tail not completely included in uropatagium ................................................................... 8

3. No nasal appendages; tragus conspicuous; ears very short to very long, widely separated or joined,

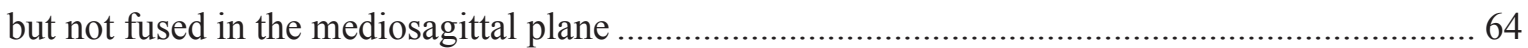

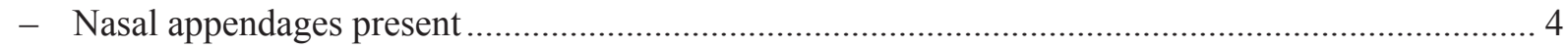

4. No tragus or tragus inconspicuous; ears very short to medium, not fused mediosagitally ............... 5

- Tragus present (sometimes small); ears fused or very close to each other mediosagitally ............... 6

5. Posterior leaflet of nose-leaf low, rounded or with three pointed projections (tridentate); two pedal

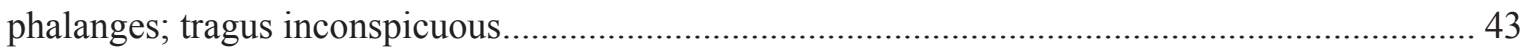

- Posterior leaflet of nose-leaf high, erect, pointed; three pedal phalanges; no tragus (family

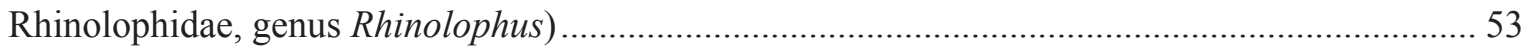

6. Tail shorter than uropatagium; no postorbital processes; no premaxillae present; noseleaf very large, comparatively simple; tragus very conspicuous, bifid (family Megadermatidae).

- Tail as long as uropatagium; last vertebra Y- or T-shaped; postorbital process and premaxillae present; noseleaf is formed by a longitudinal slit bordered by fleshy outgrowths; tragus conspicuous, not bifid (family Nycteridae)

7. Noseleaf less than $15 \mathrm{~mm}$; pelage grey without yellow tinge; frontal shield of skull strongly concave, minimum breadth of shield less than $6.5 \mathrm{~mm}$. Cardioderma cor (Peters, 1872)

- Noseleaf over $18 \mathrm{~mm}$; pelage bluish-grey, generally with yellowish tinge; frontal shield of skull comparatively flat, minimum breadth of shield over $6.5 \mathrm{~mm}$

Lavia frons (E. Geoffroy St.-Hilaire, 1810)

8. Basal part of tail included in uropatagium; distal part of tail resting free on the dorsal side of the uropatagium; ears very short, widely separated, not fused, backwards-pointing; tragus conspicuous (family Emballonuridae).

- Distal part of tail (ca half the width of the interfemoral membrane) not included in uropatagium and not resting on the dorsal side of the uropatagium; ears short, mostly united to each other by a cutaneous wall (family Molossidae). 108

9. Tongue simple, fixed to floor of mouth by posterior half, and without unfringed filiform papillae at tip; muzzle not elongated. 
- Tongue more extensible, fixed to floor of mouth by its posterior third, its terminal fourth or fifth covered above with unfringed filiform papillae ("brush-tipped"), muzzle elongated. Megaloglossus woermanni Pagenstecher, 1885

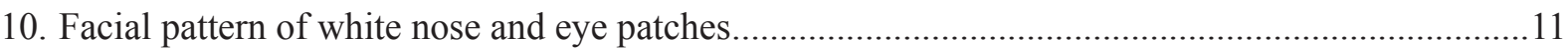

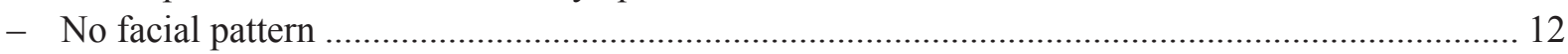

11. White tufts at base of ears inconspicuous or absent; bony palate extending well beyond teeth; rostrum, when viewed laterally, not upturned........... Scotonycteris bergmansi Hassanin et al., 2015

- White tufts at base of ears present, although sometimes inconspicuous; no postdental bony palate; rostrum, when viewed laterally, upturned. Casinycteris argynnis Thomas, 1910

12. No white tufts present at base of ears, or tufts inconspicuous 13

- White tufts at base of ears present, although sometimes inconspicuous 17

13. Facial axis of skull not noticeably deflected against basicranial axis; forearm $54-90 \mathrm{~mm}$, length second digit $71-81 \%$ of forearm; toes partially webbed; cheek teeth squarish or oblong; $6+2$ or $7+2$ palatal ridges (genus Myonycteris) 14

- Facial axis of skull more or less strongly deflected against basicranial axis; forearm $75-130 \mathrm{~mm}$, length of second digit $64-71 \%$ of forearm; toes not webbed; cheek teeth oblong; $7+1$ palatal ridges.

14. Tibia dorsally furred; $\mathrm{P}^{4}$ and $\mathrm{M}^{1}$ squarish in outline; two lower incisors; $\mathrm{M}_{3}$ reduced but present;

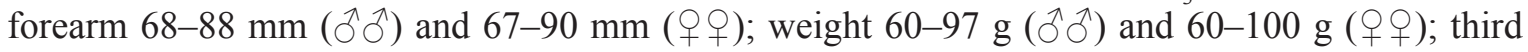
metacarpal $73-75 \%$ of forearm length; tibia $42-46 \%$ of forearm length

Myonycteris angolensis (Bocage, 1898)

- Tibia dorsally practically naked over distal quarter to third; $\mathrm{P}^{4}$ and $\mathrm{M}^{1}$ oblong in outline; one or two lower incisors; $M_{3}$ reduced or absent; forearm 54-76 mm; third metacarpal 67-73\% of forearm length; tibia $36-43 \%$ of forearm length.

15. Interfemoral membrane only furred near legs; palatal ridge pattern $6+2$ (sometimes $7+2$ ); two lower incisors; $\mathrm{M}_{3}$ absent; forearm $65-76 \mathrm{~mm}$.

Myonycteris relicta Bergmans, 1980

- Interfemoral membrane wholly furred; palatal ridge pattern $7+2$; one or two lower incisors; $\mathrm{M}_{3}$ reduced but normally present; forearm $54-68 \mathrm{~mm}$... Myonycteris torquata (Dobson, 1878)

16. Forearm 115-130 mm; $M_{1}$ equal in length to $M_{2}$ and $M_{3}$ combined; tympanic forming a short tubular bony auditory meatus; premaxillae spaced in front; colour generally tawny

Eidolon helvum (Kerr, 1792)

- Forearm 79-107 mm; $\mathrm{M}_{1}$ shorter than $\mathrm{M}_{2}$ and $\mathrm{M}_{3}$ combined; tympanic not forming a bony auditory meatus; premaxillae in contact or co-ossified in front; colour generally not tawny nor yellowish ....

17. Cheekteeth $4 / 5,4 / 6$ or $5 / 6$; unusual weak dentition; interfemoral membrane greatly reduced; calcar absent or rudimentary; forearm $47-53 \mathrm{~mm}$

Plerotes anchietae (Seabra, 1900)

- Cheekteeth $3 / 5$; interfemoral membrane well developed; calcar present; forearm generally larger (except for Micropteropus)

18. Size very large, forearm $118-133 \mathrm{~mm}$; rostrum very deep, end of muzzle truncated, ending in fleshy plate; no white shoulder tuft in 9

Hypsignathus monstrosus $\mathrm{H}$. Allen, 1862

- Size smaller, forearm 45-100 mm; rostrum shallow, end of muzzle simple; white shoulder tuft in $q$.... 


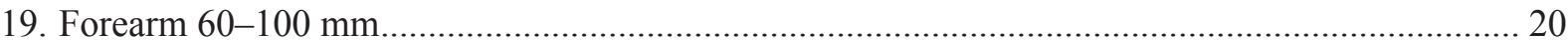

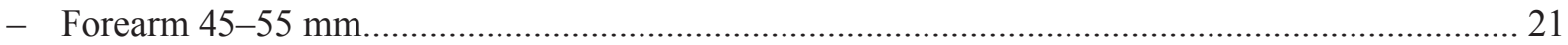

20. Six prominent palatal ridges, not more than two being post-dental; the latter not differentiated from inter-dental ridges; post-dental palate strongly concave posteriorly (genus Epomophorus)........... 24

- Five, or 9-11, palatal ridges, with sometimes from 5 to 7 post-dental; latter strongly differentiated from inter-dental ridges; post-dental palate flattened posteriorly (genus Epomops).

21. Rostrum broad; 5-6 palatal ridges, obviously divided by median groove (genus Micropteropus). 30

- Rostrum slender; 12-13 palatal ridges, only posterior group narrowly divided

Nanonycteris veldkampii (Jentink, 1888)

22. $\mathrm{Pm}_{1}$ subequal in bulk to a lower incisor; premaxillae co-ossified; braincase slightly deflected; wings from second toe; fur long and silky; forearm $79-83.5 \mathrm{~mm}$

Myonycteris angolensis (Bocage, 1898)

- $\mathrm{Pm}_{1}$ much larger in bulk than a lower incisor; premaxillae in contact; braincase conspicuously deflected; forearm at least $82.0 \mathrm{~mm}$; tibia furred or naked.

23. Braincase moderately deflected; width of last upper premolar about $1 / 3$ of palate between fronts of last upper premolars, clearly larger than half their lengths; wings from first toe (occasionally between first and second); antitragal lobe distinct; fur short; tibiae dorsally practically naked; predominantly

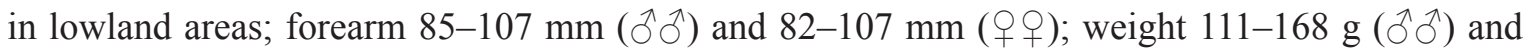
$100-155 \mathrm{~g}($ 우우)

Rousettus aegyptiacus (E. Geoffroy St.-Hilaire, 1810)

- Braincase strongly deflected; cheekteeth excessively narrow; width of last upper premolar about $1 / 5$ of that of the palate between fronts of last upper premolars, about half their lengths or slightly more; wings from second toe (occasionally between first and second); antitragal lobe obsolete; fur long and coarse; tibiae dorsally furred; mostly above $1000 \mathrm{~m}$; forearm 85-94 mm ( $え \precsim)$ and 85-95 (우); weight $102-140 \mathrm{~g}(\overbrace{}^{\Uparrow})$ and $94-162 \mathrm{~g}(+++)$

Stenonycteris lanosus Thomas, 1906

24. One conspicuous, thick postdental palatal ridge; total length of skull 44.4-57.3 mm (ठす đ), 41.0$55.5 \mathrm{~mm}($ (우); forearm 72-95 $\mathrm{mm}($ ふふ), 67-89 $\mathrm{mm}($ (우 $)$

Epomophorus.wahlbergi.(Sundevall, 1846).

- Two postdental palatal ridges

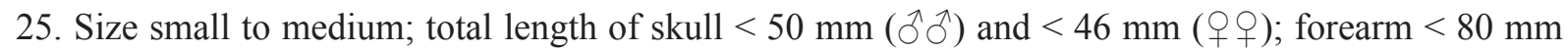

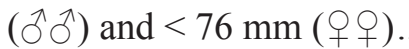

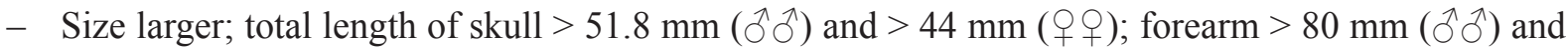
$>75 \mathrm{~mm}$ ( $(q)$ ); fourth palal ridge mid-way between third and fifth.

Epomophorus crypturus Peters, 1852

26. Two thick postdental palatal ridges in $\widehat{\partial} \widehat{\partial}$, one postdental and one partially postdental in $q Q+$; total length of skull $47.1 \mathrm{~mm}(\hat{)}), 38.7$ and $>41.2 \mathrm{~mm}$ (웅); forearm $77 \mathrm{~mm}(\hat{\jmath}), 68-74 \mathrm{~mm}$ (우우) (otherwise known from Malawi and SW Tanzania only)

Epomophorus anselli Bergmans \& Van Strien, 2004

- Two thick postdental palatal ridges in both sexes; total length of skull $<48 \mathrm{~mm}\left({ }_{\Im}^{\lambda}\right)$ and $<46 \mathrm{~mm}$

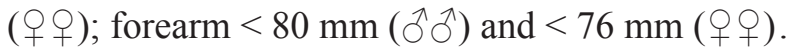

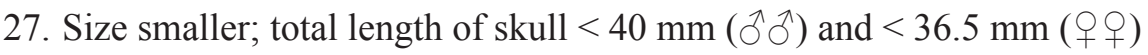

Epomophorus minimus Claessen \& De Vree, 1991

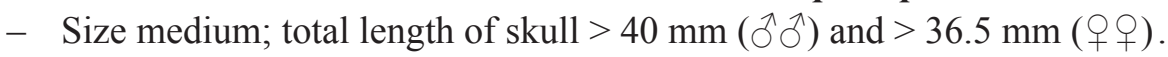




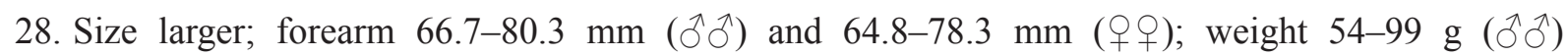
and 51-81 g (우); mainly woodlands. Distributed from NE Nigeria over S Sudan to Eritrea and to NE Tanzania; furthermore known from few localities in DRC, Malawi and SE Kenya

Epomophorus labiatus (Temminck, 1837)

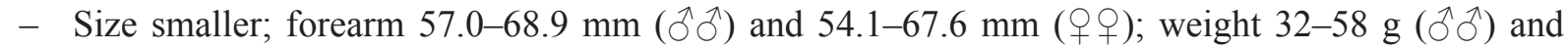
25-62 g (우); mainly woodlands. Distributed in E Sudan, Ethiopia, Somalia, lower parts of Kenya to $\mathrm{S}$ Malawi.

Epomophorus minor Dobson, 1880

29. Four thick, prominent interdental and about 5-7 thinner postdental palate ridges

Epomops franqueti (Tomes, 1860)

- Three thick and prominent interdental palate ridges; postdental palate with two pairs of prominent, triangular ridges at middle and one or a few thin and serrate ridges at palation border.

Epomops dobsonii (Bocage, 1889)

30. Forearm 50-53 mm; total skull length $29-29.8 \mathrm{~mm}$ Micropteropus pusillus (Peters, 1868)

- Forearm 59-63 mm; total skull length 33.2-33.4 mm ... Micropteropus intermedius Hayman, 1963

31. Six lower incisors; two or four upper incisors; forearm normally less than $52 \mathrm{~mm}$

Coleura afra (Peters, 1852)

- Four lower incisors; two upper incisors (very minute, often absent); forearm more than $57 \mathrm{~mm} . .32$

32. Forearm more than $80 \mathrm{~mm}(83-96 \mathrm{~mm})$; total skull length $30-33 \mathrm{~mm}$; skull with shallow frontal depression; no radio-metacarpal pouch; bullae complete; fur dark at base

Saccolaimus peli (Temminck, 1853)

- Forearm less than $80 \mathrm{~mm}$; total skull length 20-28 mm; skull with shallow (T. nudiventris) or deep frontal depression; radio-metacarpal pouch present; bullae incomplete; fur light at base

33. Forearm more than $70 \mathrm{~mm}$, posterior part of body naked above and below

Taphozous nudiventris Cretzschmar, 1830

- Forearm less than $70 \mathrm{~mm}$; body fully haired 34

34. Dorsally light brown, ventrally pale grey to dark greyish-brown; throat usually sepia-brown, darker than belly; no gular patch in either sex; forearm 60-67 mm

Taphozous perforatus E. Geoffroy St.-Hilaire, 1818

- Dorsally grizzled grey, ventrally pure white or stained yellowish; throat white; large gular sac in male; forearm 58-64 mm.

Taphozous mauritianus E. Geoffroy St.-Hilaire, 1818

35. Second premolar of lower jaw large (equal to or larger than half of the first lower premolar); $\mathrm{I}^{1-2}$ bifid ( $\mathrm{I}^{2}$ exceptionally trifid); marked constriction halfway along the height of the tragus (arge-group) 38

- Second lower premolar small (smaller than half of the first lower premolar); $\mathrm{I}^{1-2}$ bi- or trifid; no constriction in the tragus.....

36. $\mathrm{I}^{1-2}$ bifid 37

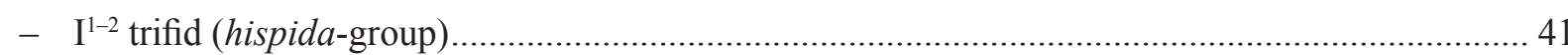

37. Tragus semilunate (macrotis-group).

- Tragus pyriform (free portion narrowest at base; its outer and inner margin evenly convex); forearm length 42-52 $\mathrm{mm}$; ear length 28-37 mm (thebaica-group)

... Nycteris thebaica E. Geoffroy St.-Hilaire, 1818

38. Forearm length more than $39 \mathrm{~mm}$; skull length more than $19 \mathrm{~mm}$

- Forearm length less than $38 \mathrm{~mm}$; skull length less than $19 \mathrm{~mm}$. 
39. Forearm length 47.3-50 mm; skull length 20.9-22.4 mm; ear length measuring 50-65 \% of forearm length Nycteris major (K. Andersen, 1912)

- Forearm length 39.5-46 mm; skull length 19.3-20.9 mm; ear length measuring 63-77\% of forearm length

Nycteris arge Thomas, 1903

40. Forearm length $36.5-37 \mathrm{~mm}$; skull length $18-18.7 \mathrm{~mm}$; tibia longer than half the forearm length ... Nycteris intermedia Aellen, 1959

- Forearm length 32-36.2 mm; skull length 15.9-16.6 mm; tibia shorter than half the forearm length Nycteris nana (K. Andersen, 1912)

41. Forearm length $39-43 \mathrm{~mm}$.

Nycteris hispida (Schreber, 1774)

- Forearm length 57-66 mm Nycteris grandis Peters, 1865

42. Forearm length $45-52 \mathrm{~mm}$; ear length $22-34 \mathrm{~mm}$ Nycteris macrotis Dobson, 1876

- Forearm length 37-42 mm; ear length 29-34 mm Nycteris parisii (de Beaux, 1924)

43. Posterior leaflet of noseleaf simple in outline, elliptical or rounded, not tridentate; ears either separate or united by low band; rostrum at least half as long as braincase; small upper premolar present; forearm over $40 \mathrm{~mm}$ (family Hipposideridae, genera Doryrhina, Hipposideros, Macronycteris).. 45

- Posterior leaflet of noseleaf tridentate; ears always separate; rostrum sometimes less than half as long as braincase; small upper premolar present or absent; forearm 31-54 mm 44

44. Rostrum less than half as long as braincase; ears low, $8 \mathrm{~mm}$, rounded, rim-like; tridentate nose-leaf; small, forearm 31-35 mm; tail not projecting beyond end of interfemoral membrane; two upper premolars on each side; $\mathrm{M}^{3}$ not reduced (family Rhinonycteridae) ...Cloeotis percivali Thomas, 1901

- Rostrum at least half as long as braincase; ears large, 18-20 mm with pointed tip; larger, forearm 44$54 \mathrm{~mm}$; tail projecting up to $7 \mathrm{~mm}$ beyond end of interfemoral membrane; one upper premolar on each side; $\mathrm{M}^{3}$ greatly reduced (family Hipposideridae)..... Asellia tridens (E. Geoffroy St.-Hilaire, 1813)

45. Ears long and narrow, pointed; cochleae greatly expanded, their width equal to at least 4 times the distance between them.

- Ears short, broad, rounded or triangular; cochleae not expanded, their width approximately equal to the distance between them

46. Ears rounded or broadly triangular; bluntly pointed upper incisors weak, the outer lobe obsolescent or obsolete; crown area of outer lower incisors equal to or only slightly greater than that of the inner lower incisors.

- Ears triangular; pointed upper incisors strong, usually retaining much of the outer lobe; crown area of the outer lower incisors much greater than that of the inner lower incisors...

47. Ears with a small projection at the antitragal fold; sphenoidal bridge narrow, not concealing lateral apertures; pterygoid wings underdeveloped; forearm 58-66 mm

Hipposideros abae J.A. Allen, 1917

- Ears without antitragal modification; sphenoidal bridge wide, partially concealing lateral apertures; pterygoid wings expanded; forearm $78-114 \mathrm{~mm}$

48. Number of lateral leaflets 3; smaller on average, forearm 93-106 mm (ふす) and 84-101 mm ( ( \&); pelage shorter, not slightly wooly; predominantly in savannas.....

Macronycteris vittatus (Peters, 1852)

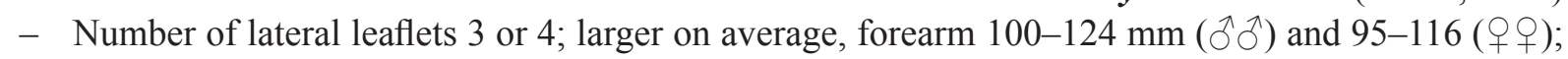
pelage longer, slightly wooly; predominantly in forests.

Macronycteris gigas Wagner, 1845 
49. Smaller, length of forearm less than $74.0 \mathrm{~mm}$; anteorbital foramen relatively large

Doryrhina cyclops (Temminck, 1853)

- Larger, length of forearm exceeding $74.0 \mathrm{~mm}$; anteorbital foramen relatively small

Hipposideros camerunensis Eisentraut, 1956

50. Posterior nose-leaf without a transverse supplementary structure developed from its posterior face or with such a structure low, undeveloped and lacking a serrated upper edge

Hipposideros fuliginosus (Temminck, 1853)

- Posterior nose-leaf having a transverse supplementary structure with a serrated upper edge developed from its posterior face

51. Anterior upper premolar small, slightly extruded from toothrow, or compressed between canine and second upper premolar. 52

- Anterior upper premolar minute, extruded from toothrow, canine and second upper premolar in contact or nearly so.

Hipposideros beatus (K. Andersen, 1906)

52. Frontal sac opening in both sexes; forearm generally less than $48 \mathrm{~mm}(42-52 \mathrm{~mm})$; tibia $18-22 \mathrm{~mm}$; dorsal pelage greyish or pale orange; median posterior narial compartiment of nasal swelling relatively narrow, with wide lateral inflations

Hipposideros caffer (Sundevall, 1846)

- Frontal sac opening in $\widehat{\jmath}$ only; forearm generally over $48 \mathrm{~mm}(47-55 \mathrm{~mm})$; tibia $18-24 \mathrm{~mm}$; dorsal pelage brownish or rufous; median posterior narial compartment of nasal swelling relatively wide, with narrow lateral inflations

Hipposideros ruber (Noack, 1893)

53. First upper premolar placed between the upper canine and second premolar or slightly reduced and partially displaced labially.....

- First upper premolar missing or very reduced and external to toothrow; the canine and second

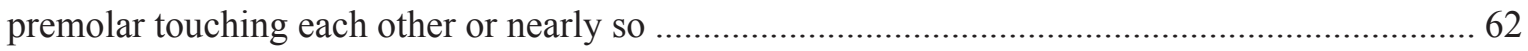

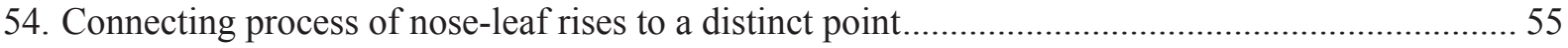

- Connecting process of nose-leaf low, subtriangular or obsolete .................................................... 56

55. First phalange of fourth finger long, more than half as long as second, $24-28 \%$ of metacarpal length; sella wedge-shaped, top narrow; lancet subtriangular or hastate, tip rounded

Rhinolophus blasii Peters, 1867

- First phalange of fourth finger shorter, 19-23\% of metacarpal length; sella sides slightly concave; lancet hastate, tip bluntly pointed

Rhinolophus landeri Martin, 1838

56. Connecting process of nose-leaf obsolete or poorly developed; ears large; forearm $50 \mathrm{~mm}$ or more.....

- Connecting process of nose-leaf low and rounded or subtriangular; forearm $55 \mathrm{~mm}$ or less ......... 60

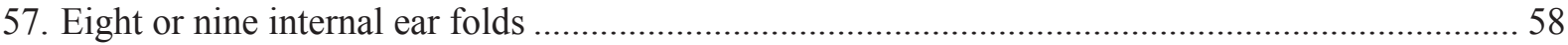

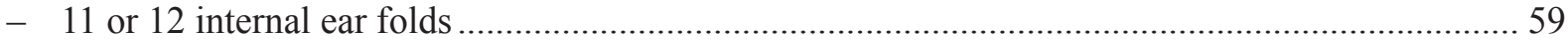

58. Lancet tip truncated, only moderately exceeding height of sella; ear with eight folds; larger, forearm 55-62 mm; skull with braincase conspicuously constricted behind the mastoid process; infraorbital bridge longer and more slender; lower molars comparatively small; ear length 32$40 \mathrm{~mm}$

Rhinolophus ruwenzorii J. Eric Hill, 1942

- Lancet tip bluntly pointed, conspicuously exceeding height of sella; ear with nine folds; smaller, forearm $54 \mathrm{~mm}$; skull with braincase not conspicuously constricted behind the mastoid process; infraorbital bridge shorter and stouter; lower molars comparatively large; ear length $28.5 \mathrm{~mm}$.

Rhinolophus hilli Aellen, 1973 
59. 11 internal ear folds; forearm length $54.5 \mathrm{~mm}$; ear length $34.5 \mathrm{~mm}$

. Rhinolophus kahuzi Fahr \& Kerbis Peterhans, 2013

- 12 internal ear folds; forearm length $49.7-51.5 \mathrm{~mm}$; ear length $24.2-29 \mathrm{~mm}$

Rhinolophus willardi Kerbis Peterhans \& Fahr, 2013

60. Forearm length 49-54 mm; skull length $22.4-23.3 \mathrm{~mm}$

Rhinolophus alcyone Temminck, 1853

- Forearm length 40-45 mm; skull length $17.6 \mathrm{~mm}$ or less

61. Sella comparatively narrow (1.2-1.3 mm prior to preservation); lancet subtriangular, almost hastate, sides concave, tip bluntly pointed; horseshoe 6.0-7.4 mm .........Rhinolophus swinnyi Gough, 1908

- Sella comparatively broad (1.5-1.7 mm after preservation); lancet subtriangular or hastate, tip somewhat rounded; horseshoe $6.7-9.0 \mathrm{~mm}$ Rhinolophus simulator K. Andersen, 1904

62. Face and edges of sella naked; forearm length $50-56 \mathrm{~mm}$; lancet hastate, tip rounded; horseshoe 6.6$9.6 \mathrm{~mm}$, narrower as muzzle; nasal swellings very low; frontal depression very shallow; supraorbital ridges weak; interpterygoid groove absent or very indistinct.

Rhinolophus clivosus Cretzschmar, 1828

- Face and edges of sella with long hairs .....

63. Forearm length $60-67 \mathrm{~mm}$; tibia $26-31 \mathrm{~mm}$; ear length $26-36 \mathrm{~mm}$; baculum $>3 \mathrm{~mm}$; anterior medial nasal swellings longer than broad.

Rhinolophus hildebrandtii Peters, 1878

- Forearm length 53-63 mm; tibia 22-25 mm; ear length 21-38 mm; baculum $<3 \mathrm{~mm}$.

Rhinolophus eloquens K. Andersen, 1905

- Forearm length 47-60 mm; tibia 19-24 mm; ear length 19-28 mm; anterior medial nasal swellings broader than long

.Rhinolophus fumigatus Rüppell, 1842

64. Second phalange of third digit nearly 3 times as long as the first (family Miniopteridae; genus Miniopterus)

- Second phalange of the third digit shorter (family Vespertilionidae). 65

65. Ear slightly but evidently funnel-formed; very high braincase (subfamily Kerivoulinae; genus Kerivoula).

- Ears largely open at their base and not funnel-formed; normal braincase...................................... 66

66. Six Pm and M in upper and lower jaw (subfamily Myotinae, genus Myotis) ................................. 80

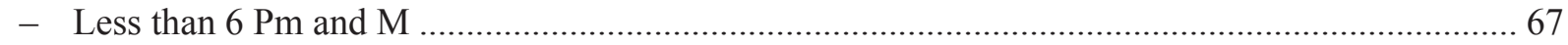

67. One upper incisor and $\mathrm{M}^{1}$ and $\mathrm{M}^{2}$ with $\mathrm{W}$-pattern distorted or nearly absent (subfamily Scotophilinae; genus Scotophilus)

- Two upper incisors or 1 upper incisor and $\mathrm{M}^{1}$ and $\mathrm{M}^{2}$ with W-pattern normal (subfamily

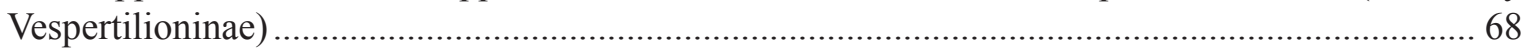

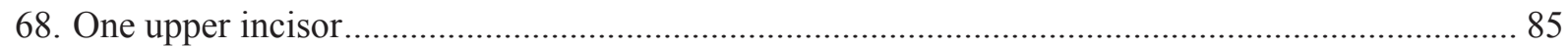

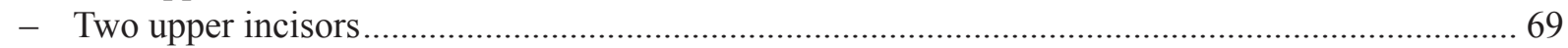

69. Five Pm and M in upper and lower jaw (genera Neoromicia / Pipistrellus / Hypsugo).................. 99

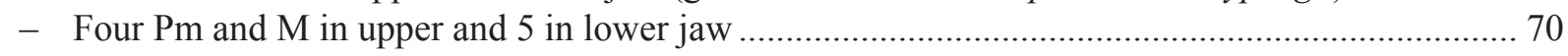

70. Ears very enlarged, about $15 \mathrm{~mm}$ and more (genus Laephotis).................................................... 74

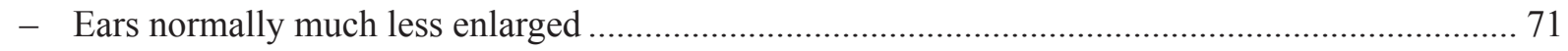

71. Very short and broad rostrum; the height of the braincase (with the bullae) more than $2 / 3$ of the condylo-basal length; lower lip with fleshy lobes at angle of mouth (genus Glauconycteris)........ 87 
- Height of the braincase (bullae included) much less than the distance from incisors to posterior zygoma root......

72. Very shortened wings; the third digit not longer than head and body length; skull much flattened (genus Mimetillus)

- Normal wings; skull not especially flattened (genus Neoromicia)

73. Pelage dark brown; wing membranes dark; occurring in forest habitats.

...Mimetillus moloneyi (Thomas, 1891)

- Pelage pale brown; wing membranes lighter; occurring in savanna habitats

Mimetillus thomasi Hinton, 1920

74. On average larger; greatest skull length $14.1-15.5 \mathrm{~mm}$; ear length $17-22 \mathrm{~mm}$; forearm length 30 $39 \mathrm{~mm}$; palatal length $6.2-7.3 \mathrm{~mm}$

Laephotis botswanae Setzer, 1971

- On average smaller; greatest skull length $13.7-13.8 \mathrm{~mm}$; ear length 15-18 mm; forearm length 32-36 mm; palatal length $6.1-6.2 \mathrm{~mm}$ Laephotis angolensis Monard, 1935

75. Forearm length $45-52 \mathrm{~mm}$; greatest skull length $16.4-17.8 \mathrm{~mm}$; hind foot (including claw) 7-11 mm; total length $92-128 \mathrm{~mm}$; weight $8-19 \mathrm{~g}$.

Miniopterus inflatus Thomas, 1903

- Forearm length 35-42 mm; greatest skull length 11.1-14.5 mm; hind foot (including claw) 5-10 mm; total length $82-97 \mathrm{~mm}$; weight $5-10 \mathrm{~g}$

Miniopterus minor Peters, 1867

- Forearm length 42-47 mm; greatest skull length 13.6-15.5 mm; hind foot (including claw) 6-11 mm; total length 104-115 mm; weight 6-11 g Miniopterus natalensis (A. Smith, 1833)

- Forearm length 41-45 mm; greatest skull length 13.3-15.5 mm; hind foot (including claw) 7-11 mm; total length 88-103 mm; weight 7-11 g ........... Miniopterus fraterculus Thomas \& Schwann, 1906

76. Comb-like fringe of hairs on interfemoral membrane well developed. 77

- Comb-like fringe of hairs on interfemoral membrane absent or scarcely developed....................... 78

77. Dorsal pelage bright reddish-brown with conspicuous frosting (highlighted tips); ventral pelage cream, dirty-white or white; size on average larger, forearm length $28-40 \mathrm{~mm}$; skull longer and more robust, greatest length 14.7-16.5 mm; outer lower incisors usually with three cusps, sometimes four; inner upper incisors uni-, bi- or tricuspid

Kerivoula argentata Tomes, 1861

- Dorsal pelage dark brown to pale grey with(in)conspicuous frosting, but not bright reddish-brown with conspicuous frosting; ventral pelage sepia brown, grey to whitish; size on average smaller, forearm length 26-34 mm; skull shorter and more delicate, greatest length $11.2-13.5 \mathrm{~mm}$; outer lower incisors with two or three cusps; inner upper incisors uni- or bicuspid.... Kerivoula lanosa (A. Smith, 1847)

78. Size smaller; forearm length $25-30 \mathrm{~mm}$; dorsal pelage pale reddish-brown, fawn-brown or greyishbrown, usually darker at base, without frosting; outer lower incisors unicuspid; inner upper incisors uni- or bicuspid...

Kerivoula phalaena Thomas, 1912

- Size larger; forearm length $30 \mathrm{~mm}$ or more; dorsal pelage dark, frosted; inner upper incisors bicuspid

79. Dorsal pelage blackish or dark brown; forearm length $32-36 \mathrm{~mm}$; lower incisors unicuspid.....

Kerivoula smithii Thomas, 1880

- Dorsal pelage dark brown with terminal third bright rusty-brown or chocolate brown; forearm length 30-33 mm; lower incisors tricuspid

Kerivoula cuprosa Thomas, 1912

80. With dichromatic (black and orange) wing membranes; forearm length 52-60 mm; sparse fringe of bristle-like hairs along margin of interfemoral membrane between tips of calcars and tip of tail

Myotis welwitschii (Gray, 1866) 
- Wing membranes uniformly dark; size smaller; no fringe of bristle-like hairs along margin of interfemoral membrane.

81. Larger, forearm length $47-53 \mathrm{~mm}$; wing membrane uniformly dark or inconspicuously particoloured, black on most of the membrane and orange to dark red along the fingerbones; ventral and dorsal hairs tricoloured.

Myotis tricolor (Temminck, 1832)

- Smaller, forearm length 33-42 mm; wing membrane uniformly blackish-brown; ventral hairs bicoloured; dorsal hairs bi- or tricoloured

Myotis bocagii (Peters, 1870)

82. Very large, forearm length $78-88 \mathrm{~mm}$ Scotophilus nigrita (Schreber, 1774)

- Smaller, forearm not over $61 \mathrm{~mm}$

83. Ventral pelage dark brown to reddish-orange; habitat rainforest.......Scotophilus nux Thomas, 1904

- Ventral pelage pure white, yellowish, yellow, orange-yellow; habitat savannas and rainforestsavanna mosaics.

84. Ventral pelage pure white to medium brown, never yellowish; dorsal pelage pale to medium brown, never tinged with yellow or green; forearm length $44-58 \mathrm{~mm}$; skull total length $16-$ $18 \mathrm{~mm}$

Scotophilus leucogaster (Cretzschmar, 1826)

- Ventral pelage pale yellow, bright yellow or orange-yellow, not tinged with brown; dorsal pelage sepia brown, greenish-brown, greyish-brown or reddish-brown; forearm 47-60 mm; skull total length $18-21 \mathrm{~mm}$

Scotophilus dinganii (A. Smith, 1833)

85. Penis short (5.6-6.2 $\mathrm{mm})$; upper canine with anterior surface rounded, not grooved; anterior lower premolar ca half height of posterior premolar

Nycticeinops schlieffenii (Peters, 1859)

- Penis extremely long (ca 9-16 mm); upper canine with anterior surface flat and grooved; anterior lower premolar at least two-thirds height of posterior premolar, usually subequal (genus Scotoecus)..... 86

86. Wing membranes dark-brown, slightly translucent

Scotoecus hindei Thomas, 1901

- Wing membranes light, semi-translucent, becoming brownish around edges and near body...

Scotoecus albofuscus (Thomas, 1890)

87. Wing and interfemoral membranes conspicuously veined; wings pale; colour of dorsal hairs creamybuff to yellowish-fawn (tricoloured or inconspicuously bicoloured); forearm length $38-45 \mathrm{~mm}$......

Glauconycteris variegata (Tomes, 1861)

- Wing and interfemoral membranes not conspicuously veined

.88

88. Dorsal body colour black and white; five to seven dorsal markings (spots, patches and stripes); ears subquadrangular; inner upper incisors unicuspid; forearm length $45-48 \mathrm{~mm}$

Glauconycteris superba Hayman, 1939

- Dorsal body colour not black and white; ears rounded

89. No body pattern; dorsal body colour umber-, sepia-, or reddish-brown; dorsal hairs tricoloured; inner upper incisors strongly bicuspid, with unequal cusps; forearm length $34-38 \mathrm{~mm}$

Glauconycteris curryae Eger \& Schlitter, 2001

- Markings restricted to flank stripes or shoulder spots; dorsal hairs bi- or tricoloured; inner upper incisors generally bicuspid (except for argentata), with subequal or unequal cusps; forearm length $44 \mathrm{~mm}$ or less.

90. Wing and interfemoral membranes pale brown; dorsal hairs pale golden-fawn or darker and greyer . Glauconycteris argentata (Dobson, 1875) 
- Wing and interfemoral membranes dark brown; dorsal hairs medium to dark greyish or dark sepia brown

91. Inner upper incisors strongly bicuspid, with subequal or unequal cusps; dorsal hairs tricoloured; no body patterns or at most a shoulder spot

- Inner upper incisors weakly bicuspid, with unequal cusps; dorsal hairs bicoloured; body pattern includes flank stripe and/or shoulder spot

92. No light lateral markings on body (perhaps rarely a white shoulder spot); forehead region of skull weakly concave.

Glauconycteris beatrix Thomas, 1901

- White shoulder spot is generally present; forehead region of skull moderately concave.

Glauconycteris humeralis J.A. Allen, 1917

93. Dorsal hairs sepia brown; wings not reticulated.

Glauconycteris alboguttata J.A. Allen, 1917

- Dorsal hairs medium to dark greyish or brownish; wings faintly reticulated.

Glauconycteris poensis (Gray, 1842)

94. Wings pale or white translucent; mid-ventral pelage cream, whitish or pale grey. 98

- Wings dark; mid-ventral pelage whitish or pale greyish-brown to medium brown

95. Mid-ventral pelage white, whitish, cream or buff; forehead region of skull straight to weakly concave; inner upper incisors weakly bicuspid (unicuspid when worn); forearm length 28-38 mm; occipital helmet usually well developed.

Neoromicia capensis (A. Smith, 1829)

- Mid-ventral pelage pale (greyish-)brown to (medium-)brown; forehead region of skull weakly to moderately concave (exceptionally almost straight); occipital helmet absent 96

96. Size large; forearm length 33-38 mm; greatest length of skull 13.0-14.1 mm; dorsal hairs unicoloured; mid-ventral pelage greyish-brown to medium brown; inner upper incisors unicuspid; no anterior upper premolar; forehead region of skull weakly concave.......Neoromicia brunnea (Thomas, 1880)

- Size smaller; forearm length less than $33 \mathrm{~mm}$; dorsal hairs not unicoloured; inner upper incisors usually unicuspid; anterior upper premolar extremely rarely present, in which case minute

97. Dorsal and ventral hairs faintly bicoloured; mid-ventral colour pale brown or brown; forehead region of skull weakly concave to almost straight; forearm length $22-32 \mathrm{~mm}$

Neoromicia somalica (Thomas, 1901)

- Dorsalhairs bi-or tricoloured; mid-ventral colourpale greyish-brown or brown; forehead region of skull weakly to moderately concave; forearm length $27-33 \mathrm{~mm}$....Neoromicia zuluensis (Roberts, 1924)

98. Dorsal colour dark brown, in strong contrast to pale wings; dorsal hairs usually unicoloured; ventral hairs bicoloured; inner upper incisors weakly bicuspid; forearm length $28-33 \mathrm{~mm}$

Neoromicia tenuipinnis (Peters, 1872)

- Dorsal colour light brown, not in strong contrast to pale wings; dorsal hairs bi- or tricoloured; ventral hairs bicoloured; inner upper incisors unicuspid; forearm length $29-38 \mathrm{~mm}$

.Neoromicia rendalli (Thomas, 1889)

99. Outer upper incisor greatly reduced; entire underside pure white; dorsal pelage grey to sepia brown [lower molars nyctalodont; forearm length 29-37 mm; anterior upper premolar medium sized, conspicuous, within toothrow or slightly displaced; dorsal and ventral hairs bicoloured]

Pipistrellus rueppellii (Fischer, 1829)

- Outer upper incisor not greatly reduced; underside light, dark or grey-tipped..... 
100. Inner upper incisors unicuspid; both dorsal and ventral hairs bicoloured; dorsal pelage greyishbrown, reddish-brown, dark brown or blackish; anterior upper premolar medium-size to minute, usually visible, within toothrow or fully displaced lingually; no white border on hind border of wing; forearm length 29-38 mm

Pipistrellus hesperidus (Temminck, 1840)

- Inner upper incisors bicuspid. 101

101. Dorsal and ventral hairs bicoloured 102

- Dorsal hairs unicoloured; ventral hairs usually unicoloured . 105

102. Outer edge of tragus angular (hatchet-shaped); dorsal pelage chocolate brown or sepia brown, sometimes with gold sheen; anterior upper premolar medium-sized, slightly displaced lingually; no white border on hind border of wing; forearm length 25-35 mm

Neoromicia nana (Peters, 1852)

- $\quad$ Outer edge of tragus curved (no sharp angle).

103. Skull larger, very weakly concave, greatest length 13.7-14.1 mm; wings without white hind border; forearm length 32-36 mm; mid-ventral pelage slightly reddish-brown tinged with beige; anterior upper premolar minute to medium-sized, usually visible, occasionally absent

Hypsugo eisentrauti (Hill, 1968)

- Skull smaller, weakly concave, greatest length less than $13.7 \mathrm{~mm}$; wings with or without white hind border.

104. Mid-ventral pelage pale brown, cream or white; anterior upper premolar minute, not visible above gum; greatest length of skull $11.2-13.7 \mathrm{~mm}$; forearm length $29-34 \mathrm{~mm}$

Hypsugo anchietae (Seabra, 1900)

- Mid-ventral pelage dark to medium rust-brown, sometimes speckled; anterior upper premolar absent; greatest length of skull $10.7-11.8 \mathrm{~mm}$; forearm length $23-35 \mathrm{~mm}$

Neoromicia guineensis (Bocage, 1889)

105. Size larger, forearm 33-38 mm; greatest length of skull $13.8-14.7 \mathrm{~mm}$; mid-ventral pelage pale brown, usually yellowish; anterior upper premolar minute, sometimes not visible, sometimes missing; forehead region of skull weakly concave, sometimes almost straight.

Pipistrellus grandidieri (Dobson, 1876)

- Size smaller, forearm $33 \mathrm{~mm}$ or less; greatest length of skull $13.6 \mathrm{~mm}$ or less 106

106. Anterior upper premolar medium-sized to well-developed, visible, in toothrow or slightly lingually displaced; dorsal pelage reddish-brown; white border on wing edge usually missing; forearm length 24-29 mm

Pipistrellus nanulus Thomas, 1904

- Anterior upper premolar minute, barely visible above gum or not at all

107. Size larger, forearm length $28-33 \mathrm{~mm}$; greatest length of skull $12.1-13.6 \mathrm{~mm}$; mid-ventral pelage pale to dark brown or reddish-brown.

Hypsugo crassulus (Thomas, 1904)

- Size smaller, forearm length 23-26 mm; greatest length of skull 10.3-11.4 mm; mid-ventral pelage dark umber-brown

Hypsugo musciculus (Thomas, 1913)

108. A prominent vertical projection on the zygomatic arch; ears very large, $38-40 \mathrm{~mm}$, conjoined on extended snout; size very large, forearm length $62-73 \mathrm{~mm}$; conspicuous bicolored dorsal pelage pattern, with pale collar separating dark anterior and posterior regions of body

Otomops martiensseni (Matschie, 1897)

- Not combining these characters..... 
109. Ears widely separated; ears and wing membranes translucent or whitish; underside of body entirely white; 4 post-canine upper cheek teeth, $\mathrm{M}^{3}$ much reduced; one lower incisor on each side, with concave crowns lengthened antero-posteriorly; upper incisors in contact with canines; no palatal emargination (genus Myopterus)

- Ears not widely separated, sometimes conjoined, never translucent; 4 or 5 post-canine upper cheek teeth (first upper premolar often minute, sometimes deciduous); one to three (generally two) lower incisors; upper incisors not in contact with canines

110. Inner margins of ears meeting or almost meeting to form a V-shaped valley; third ridge of posterior upper molar $\left(\mathrm{M}^{3}\right)$ more than half length of second or equal in length; two lower incisors on each side; anterior palatal emargination wide (genus Tadarida) 133

- Inner margins of ears generally joined by band of skin (uncertain in niangarae, where they might be well separated); anterior palatal emargination narrow or closed.....

111. Third ridge of posterior upper molar $\left(\mathrm{M}^{3}\right)$ more than half length of second or equal in length (genus Chaerephon)

- Third ridge of posterior upper molar $\left(\mathrm{M}^{3}\right)$ less than half length of second or absent (sometimes equal in condylurus) (genus Mops).....

112. Anterior palatal emargination closed (subgenus Mops)

- Anterior palatal emargination narrow (subgenus Xiphonycteris) 130

113. Size smaller, forearm length 33-37 mm; skull length $17-19 \mathrm{~mm}$; pelage dark brown above, without lateral bands Myopterus whitleyi (Scharff, 1900)

- Size larger, forearm length 48-56 mm; skull length 21.3-25.7 mm; dorsal pelage slaty-grey with two contrasting lateral bands of dirty white Myopterus daubentonii Desmarest, 1820

114. Ears separated by a lobe or lappet 115

- $\quad$ Ears joined by a band.

115. Ears long, separated by a large bulbous lobe projecting well over the forehead; ventral flank stripe absent; wing membrane greyish-black; basisphenoid pits deep and large; rostrum uniquely with prominent nasal swellings; forearm length $38 \mathrm{~mm}$

Chaerephon gallagheri (Harrison, 1975)

- Ears separated by a flat lappet over a small, backward-opening pocket; white or whitish ventral flank stripe present; wing membrane dark brown to blackish; depth of basisphenoid pits shallow to moderate, size small to medium; forearm length 39-46 $\mathrm{mm}$

Chaerephon major (Trouessart, 1897)

116. Size smaller; forearm length less than or equal to $41 \mathrm{~mm}$

- $\quad$ Size larger; forearm length larger than $41 \mathrm{~mm}$

117. Dorsal pelage pale grey, pale rusty-brown or medium greyish-brown; wing membrane usually white, sometimes pale brown or greyish-brown; $\widehat{\partial} \widehat{t}$ with long bicoloured interaural crest.

Chaerephon chapini J.A. Allen, 1917

- Dorsal pelage almost black, brown, greyish-brown or reddish-brown; wing membrane white or blackish-brown; $\widehat{\partial} \hat{\sigma}$ with short unicoloured interaural crest 118

118. Size smaller; forearm length 32.8-37.8 mm; condylobasal length $14.4-15.9 \mathrm{~mm}$; zygomatic width $9.3-10.3 \mathrm{~mm}$ Chaerephon leucogaster (A. Grandidier, 1869)

- Size larger; forearm length 38.3-41.0 mm; condylobasal length 15.3-16.4 mm; zygomatic width $10.4-10.9 \mathrm{~mm}$ Chaerephon pumilus (Cretzschmar, 1826) 


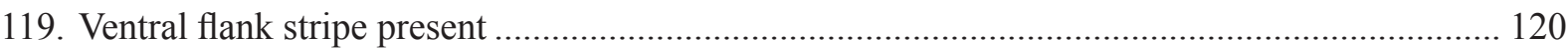

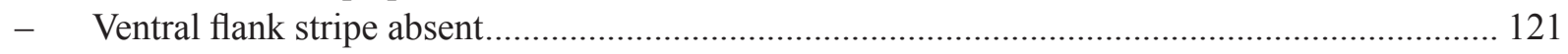

120. Flank stripe whitish; wing membranes pale grey; shallow basisphenoid pits of medium size; anterior palatal emargination narrow; forearm length 41-48 mm; uniquely with pair of tail-glands opening ventrally as a slit on each side of base of tail.......Chaerephon bemmeleni (Jentink, 1879)

- Flank stripe white; wing membranes dorsally whitish or blackish, ventrally white; shallow to medium basisphenoid pits of small to medium size; anterior palatal emargination closed; forearm length 41-51 mm; no such glands

Chaerephon nigeriae Thomas, 1913

121. Anterior palatal emargination closed; size of basisphenoid pits large.

- Anterior palatal emarganation narrow; size of basisphenoid pits small to medium.

122. Size larger; forearm length $48-53 \mathrm{~mm}$; basisphenoid pits deep; wing membrane blackish-brown; sometimes with greyish spots dorsally

Chaerephon aloysiisabaudiae (Festa, 1907)

- Size smaller; forearm length 42-46 mm; basisphenoid pits moderately deep; wing membrane mostly dark brown, lighter towards tip; no spots or stripes. Chaerephon russatus J.A. Allen, 1917

123. Wing membrane light reddish-brown or light grey; forearm length $43-48 \mathrm{~mm}$; dorsal pelage somewhat frosted, occasionally with white spots

Chaerephon ansorgei (Thomas, 1913)

- Wing membrane dark brown or light reddish-brown; forearm length 46-51 mm; white or whitish stripes and/or rows of spots on crown of most individuals, and sometimes on shoulders and flanks.

Chaerephon bivittatus (Heuglin, 1861)

124. Size smaller; forearm length $51 \mathrm{~mm}$ or less 125

- $\quad$ Size larger; forearm length $51 \mathrm{~mm}$ or more.

125. 3 o with paired glands between penis and anus; forearm length $41-46 \mathrm{~mm}$; phalanges of $3 \mathrm{rd}$ (16$19 \mathrm{~mm})$ and 4th (13-16 mm) fingers short; ventral pelage white or very pale; crown darker than back; whitish or pale grey flank stripe present, but faint; basisphenoid pits deep and large.

Mops (Mops) demonstrator (Thomas, 1903)

- $\lambda \hat{\sigma}$ without paired glands between penis and anus; phalanges of $3^{\text {rd }}$ and $4^{\text {th }}$ fingers medium to long 126

126. Forearm length $45-51 \mathrm{~mm}$; phalanges of $3 \mathrm{rd}(20-24 \mathrm{~mm})$ and 4 th $(17-20 \mathrm{~mm})$ fingers long; ventral pelage with little or no white; crown same colour as back; white ventral flank stripe present or absent; basisphenoid pits shallow and small

Mops (Mops) condylurus (A. Smith, 1833)

- Forearm length 44-48 mm; phalanges of 3rd (19-21 mm) and 4th (15-17 mm) fingers of medium length; ventral pelage predominantly white or cream; crown darker than back; ventral flank stripe absent; basisphenoid pits moderately deep and medium to large in size.

.Mops (Mops) niveiventer Cabrera \& Ruxton, 1926

127. Size largest; forearm length $59-67 \mathrm{~mm}$; white or pale ventral flank stripe present; dorsal pelage colour variable; frosted and flecked; basisphenoid pits moderately deep and medium to large in size Mops (Mops) midas (Sundevall, 1843)

- $\quad$ Size smaller; forearm $58 \mathrm{~mm}$ or less 128

128. Inner margin of ears uncertain, possibly well separated; ventral flank stripe probably absent; wing membrane dark brown; forearm length approximately $52 \mathrm{~mm}$.

Mops (Mops) niangarae J.A. Allen, 1917

- Inner margin of ears joined by a band 
129. Forearm length 51-55 mm; wing membrane dark brown; dorsal pelage medium sepia or yellowishbrown, or pale rusty-brown. .Mops (Mops) trevori J.A. Allen, 1917

- Forearm length 54-58 mm; wing membrane blackish-brown; dorsal pelage dark brown to almost black; not frosted or flecked Mops (Mops) congicus J.A. Allen, 1917

130. Size smaller, forearm length $31 \mathrm{~mm}$ or less

- Size larger, forearm length $34 \mathrm{~mm}$ or more

131. Two lower incisors on each side; lower canines without greatly enlarged cingula; upper incisors procumbent (inclined towards the lips); wing membrane blackish-brown, pale brown to whitish, ventral side paler; forearm length 27-31 mm.......Mops (Xiphonycteris) nanulus J.A. Allen, 1917

- Usually one lower incisor on each side, rarely two; lower canines with greatly enlarged cingula, especially in $\widehat{\partial} \widehat{\partial}$; wing membrane blackish; forearm length $27-30 \mathrm{~mm}$

Mops (Xiphonycteris) spurrelli (Dollman, 1911)

132. Ventral flank stripe present, but contrast weak; wing membrane blackish; ventral pelage dark; occipital helmet prominent; wing insertion higher on body; basisphenoid pits of moderately depth and small to medium in size; forearm length $35-42 \mathrm{~mm}$

Mops (Xiphonycteris) brachypterus (Peters, 1852)

- Ventral flank stripe absent; wing membrane various shades of brown; ventral pelage pale; occipital helmet moderately prominent; wing insertion lower on body; basisphenoid pits moderate or shallow deep and medium to large in size; forearm length $34-41 \mathrm{~mm}$.

Mops (Xiphonycteris) thersites (Thomas, 1903)

133. Little white on ventral surface; forearm length $60-67 \mathrm{~mm}$; both sides of wing membrane dark brown to almost black; basisphenoid pits medium in size; plantar pad absent

Tadarida ventralis (Heuglin, 1861)

- Clear white, cream or yellowish ventral flank band present; forearm length 56-61 mm; dorsal side of wing membrane brown, ventral side whitish; basisphenoid pits large in size; plantar pad present

Tadarida fulminans (Thomas, 1903) 


\section{Catalogue of voucher specimens}

This list gives an overview of all of the voucher specimens examined by the various contributors or for which information was found in the literature.

For the museum acronyms, see page 246.

For all of the specimens the following information is provided (as far as it was available):

Taxon name;

Country;

Locality;

The number of individuals (inds) of the species that were collected at that locality, with details on their sex $(\overbrace{}^{\lambda}\left({ }^{\lambda}\right)$ : male(s), $+(P)$ : female(s), ?(?): unknown), age ( $\mathrm{ad}=\mathrm{adult}$; emb = embryo; imm = immature; juv = juvenile; $\mathrm{sad}=$ subadult $)$, and conservation type $(\mathrm{AC}=$ alcohol and cranium; $\mathrm{AL}=$ alcohol, skull not removed; AS = alcohol, skull missing; $\mathrm{MS}=$ mounted skin and skull; OT = other, undefined type; $\mathrm{SA}=$ skull and alcohol; $\mathrm{SB}=$ skin, skeleton and skull; $\mathrm{SC}=$ skin and cranium; $\mathrm{SI}=$ skull in skin; $\mathrm{SK}=$ skull only; $\mathrm{SN}=$ complete skeleton; $\mathrm{SO}=$ skin only; $\mathrm{SS}=$ skin and skull), $\mathrm{UN}=$ unknown;

Museum acronym and registration number(s) in alphabetical and/or numeric order. If more than two consecutive number are present, the first and the last are connected by "to";

The collector(s) (leg. = legit) and the dates on which the specimens were collected. A question mark in the date field indicates that the specimens were collected on multiple dates of which some could not be determined.

\section{Chiroptera Blumenbach, 1779}

BURUNDI: 2 ?? [2 UN], Kinazi, between 5 Jan. 1953 and 8 Jan. 1953, leg. P. Basilewsky (RMCA: $21939,21940)$.

DEMOCRATIC REPUBLIC OF THE CONGO: 59 ?? [1 AL, $58 \mathrm{UN}$ ], unknown locality, unknown date, leg. Prescott Musaba, unknown date, leg. unknown collector(RMCA: a1.097-M-3221, UNIKIS: CH1085 to $\mathrm{CH} 1088$, CH1090 to CH1093, CH1095, CH1096, CH1098, CH2000 to CH2004, CH2006 to CH2017, CH2019, CH2020, CH2022 to CH2028, CH2030 to CH2032, CHYO106 to CHYO116, CR09-828, CR09-839, CR09-842, CR09-845, CR09-863, CRT813/G72, CRT815/GE67); 4 ?? [4 UN], AlbertvilleMoba (road?) [= Kalemie-Moba (road?)], 19 Nov. 1953, 26 Feb. 1954, between 1 Apr. 1954 and 30 Apr. 1954, leg. H. Bomans (RMCA: 23317 to 23319, 23321); 1 q [UN], Avakubi, 7 Jan. 1912, leg. Cuthbert Christy (RMCA: 1606a); 1 ? [UN], Bambesa, prior to 28 Jun. 1937, leg. J.M. Vrijdagh (RMCA: 13877); 2 ?? [2 UN], Banana, 12 Apr. 1952, leg. Is. Mesmaekers (RMCA: 20761, 20762); 7 ?? [7 UN], Beno, prior to 3 Jan. 1950, between 1 Jan. 1949 and 31 Dec. 1949, leg. P. Bayet (RMCA: 19834 to 19837 , 19854, 19855, 19474); 2 우, 1 ð [3 SS], Bitale, 12 Jun. 1970, 14 Jun. 1970, 21 Jun. 1970, leg. Alexandre Prigogine (RMCA: 36231, 36230, 36233); 1 ? [UN], Boende, between 1 Jan. 1966 and 31 Jan. 1966, leg. P. Lootens (RMCA: 34161); 1 ? [UN], Bokuma, prior to 5 Mar. 1953, leg. P. Lootens (RMCA: 21675); 1 đ [SO], Bokungu, 30 Jun. 1948, leg. L. Dupuis (RMCA: 18641); 1 q [SO], Bolobo, prior to 18 Jan. 1958, leg. Ngwe (RMCA: 26912); 2 ?? [2 UN], Boma, between 1 Feb. 1955 and 28 Feb. 1955, leg. Is. Mesmaekers (RMCA: 23310, 23311); 1 ? [UN], Bosobolo, prior to 23 Mar. 1951, leg. Vachaudez (RMCA: 20343); 1 ? [SK], Bukavu, between 1 Jan. 1952 and 31 Dec. 1952, leg. P.G. Vercammen (RMCA: 82.001-M-0020); 1 ? [UN], Buta, prior to 9 Nov. 1938, leg. Jozef Hutsebaut (RMCA: 14904); 2 đ̂̉ [2 SS], Byonga, 4 Jan. 1972, 16 Jan. 1973, leg. Alexandre Prigogine (RMCA: 38623, 38589); 1 ? [SS], Dilolo, 2 Feb. 1948, leg. Jozef Hutsebaut (RMCA: 18230); 1 ? [UN], Funda Biabo, between 1 Feb. 1914 and 28 Feb. 1914, leg. Charliers (RMCA: 5396); 5 ?? [5 UN], Ibembo, 20 Jul. 1950, between 1 Nov. 1949 and 30 Nov. 1949, leg. Jozef Hutsebaut (RMCA: 20093, 19858 to 19861); 1 [SS], Idjwi, Island, 16 Sep. 1969, leg. Alexandre Prigogine (RMCA: 36219); 1 ? [UN], Irumu, between 
1 Nov. 1951 and 30 Nov. 1951, leg. Alexandre Fain (RMCA: 21463); 1 § [SS], Kakanda Cave, 11 Sep. 1971, leg. Alexandre Prigogine (RMCA: 38566); 1 juv 우, 1 § [2 SS], Kamituga, 6 Apr. 1973, 26 Apr. 1973, leg. Alexandre Prigogine (RMCA: 38626, 38624); 1 q [SS], Kamusimbi, 11 Feb. 1973, leg. Alexandre Prigogine (RMCA: 38625); 4 우, 4 ô $\widehat{o}$ [8 SS], Kasebere, 14 Feb. 1970, 18 Jun. 1970, 25 Jun. 1970, 1 Dec. 1970, 2 Dec. 1970, 3 Dec. 1970, 24 Apr. 1971, leg. Alexandre Prigogine (RMCA: 38572, 36222, 36223, 36245, 38563, 38587, 36244, 38569); 1 ad ô, 1 ? [1 AL, 1 UN], Kibumba, 10 Aug. 1970, leg. M.J. Celis and M. Lejeune (RMCA: 36209, 36210); 1 ? [UN], Kibwe Lya Mikako Cave, 28 Dec. 1966, leg. M.J. Celis and M. Lejeune (RMCA: 35172); 2 ?? [1 AL, 1 UN], Kikwit, unknown date, leg. Prescott Musaba, Moïse Bipoo, Richard Tamaru, Pauline Isude and Joseph Kussa, between 10 Jun. 1995 and 28 Aug. 1995, leg. Herwig Leirs et al. (UNIKIS: KKT2845, RMCA: 97.021M-1175); 1 ? [UN], Kilo Mines [= Kilomines], prior to 27 Jun. 1913, leg. Thelie (RMCA: 1867); 1 त [SS], Kiloboze [= Kilobono], 12 Jan. 1973, leg. Alexandre Prigogine (RMCA: 38627); 3 $\widehat{\partial}$ [3 UN], Léopoldville [= Kinshasa], 13 May 1912, 17 May 1912, leg. Cuthbert Christy (RMCA: 1539e, 1539f, 1539d); 6 ?? [6 UN], Lubondai-Tshimbulu [= Lubondaie-Tshimbulu], prior to 9 Dec. 1955, leg. S. Marks (RMCA: 23238 to 23240, 23395 to 23397); 3 ?? [3 AL], Lukolela, 1 Feb. 1931, leg. James Paul Chapin (AMNH: 80958 to 80960); 1 juv ?, 1 O̊, 5 ?? [5 SK, 2 SO], Luluabourg [= Kananga], unknown date, 16 Nov. 1965, 28 Aug. 1969, leg. Antoon Emeric Marcel De Roo (RMCA: 34416 to 34420, 33982,

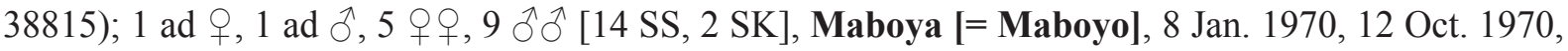
16 Nov. 1970, 1 Dec. 1970, 8 Dec. 1970, 25 Dec. 1970, 9 Jan. 1971, 21 Jan. 1971, 22 Jan. 1971, 23 Jan. 1971, 31 Jan. 1971, 2 Feb. 1971, 14 Feb. 1971, 10 Mar. 1971, leg. Alexandre Prigogine (RMCA: 38573, 38562, 36247, 38564, 38580, 36246, 38570, 38582, 38576, 38581, 38565, 38575, 38578, 36221, 38571, 38579); 1 ? [UN], Mahagi Port, 29 Mar. 1953, leg. Célestin Hecq (RMCA: 21899); 2 ðð [2 SS], Masibingi, 7 Mar. 1971, 20 Mar. 1971, leg. Alexandre Prigogine (RMCA: 38568, 38577); 2 ?? [2 UN], Moba, 19 Nov. 1953, leg. H. Bomans (RMCA: 22122, 22123); 2 우 [2 SS], Mule, 4 May 1970, 12 May 1970, leg. Alexandre Prigogine (RMCA: 36229, 36232); 1 q [SS], Mwenga, 29 Nov. 1971, leg. Alexandre Prigogine (RMCA: 38588); 2 $\widehat{\partial}$ [2 SS], Ndomo, 16 Mar. 1971, 18 Mar. 1971, leg. Alexandre

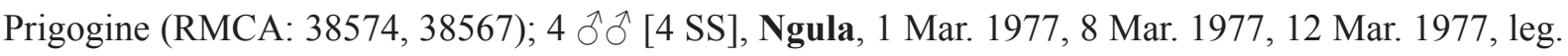
Tshinyenye (RMCA: 77.021-M-0019 to 77.021-M-0022); 1 ? [UN], Niambasha, prior to 25 Aug. 1959, leg. Uwe Rahm (RMCA: 28034b); 1 ? [UN], Pawa, prior to 23 Jan. 1939, leg. Degotte (RMCA: 14989); 1 ? [UN], Popokabaka, between 1 Nov. 1955 and 30 Nov. 1955, leg. René Close (RMCA: 23390); 1 ㅇ, 1 đ [2 UN], Rubi-Tele, 19 Aug. 2013, leg. Guy-Crispin Gembu Tungaluna, Prescott Musaba and André Malekani (UNIKIS: RBTL510, RBTL512); 1 ? [UN], Sombe, between 1 Jan. 1948 and 31 Dec. 1948, leg. Dewit (RMCA: 19074); 1 ð [SO], St. Jos. (Mission) [= Mikalaya], 9 Sep. 1924, leg. Richard Callewaert (AMNH: 86139); 10 우, 10 $\widehat{\jmath}$, 2 ?? [19 SK, 3 UN], St. Joseph de Luluabourg Mission [= Mikalaya], 15 Feb. 1923, 17 Feb. 1923, 19 Feb. 1923, 26 Feb. 1923, 11 Mar. 1923, 12 Mar. 1923, 27 Mar. 1923, 5 Apr. 1923, 11 Apr. 1923, 5 May 1923, 8 May 1923, 19 May 1923, 22 May 1923, 31 May 1923, 1 Jun. 1923, 2 Jun. 1923, 19 Jun. 1923, 27 Jun. 1923, 28 Jun. 1923, 15 Nov. 1923, leg. Richard Callewaert (RMCA: 7177, 7118, 7114, 7183, 7180, 7107, 7173, 7119, 7185, 7176, 7175, 7178, $7120,7179,7181,7170,7174,7113,7184,7117,7172,7171) ; 1 \lesssim$ [UN], Stanleyville [= Kisangani], 2 Jul. 1912, leg. Cuthbert Christy (RMCA: 1419); 1 ? [UN], Tshuapa district, between 1 Jan. 1960 and 31 Dec. 1964, leg. P. Lootens (RMCA: 34170); 3 ?? [3 UN], Yoko, unknown date, leg. unknown collector (UNIKIS: LK01, LK03, LK04).

RWANDA: 1 q [SS], Astrida [= Butare], 24 Dec. 1961, leg. unknown collector (RMCA: 38816). 


\author{
Pteropodiformi Hutcheon \& Kirsch, 2006 \\ Pteropodidae Gray, 1821 \\ Eidolinae Almeida, Giannini \& Simmons, 2016 \\ Eidolon Rafinesque, 1815
}

Eidolon helvum (Kerr, 1792)

BURUNDI: 8 q [ [4 AL, 4 SB], Buhoro, 21 Dec. 1970, leg. P.L. Niort (MHNG: 1498.003 to 1498.010); 5 ふึ઼,, 2 ?? [2 SS, 2 AL, 1 UN, 2 SB], Bujumbura, 1 Aug. 1976, leg. Jacques Verschuren, 3 Jun. 1971, 4 Jun. 1971, 12 Jul. 1973, leg. P.L. Niort, between 1 Jul. 1982 and 31 Jul. 1982, leg. Vissens (RBINS: 19908, MHNG: 1498.031, 1498.032, 1498.011, 1498.012, RMCA: 82.023-M-0001, 82.023-M-0002);

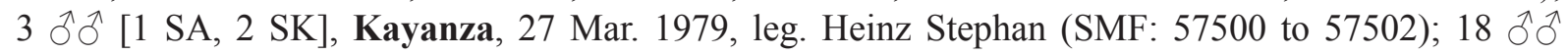
[18 SB], Mabaye [= Mabayi], 10 Dec. 1971, leg. P.L. Niort (MHNG: 1498.013 to 1498.030).

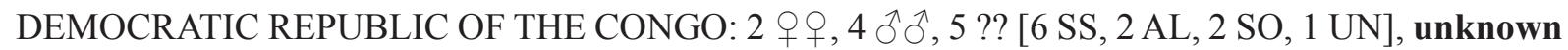
locality, unknown date, prior to 31 Dec. 1930, leg. unknown collector, prior to 17 Mar. 1953, leg. Leonet, prior to 28 Dec. 1955, leg. P. Dyleff, prior to 8 Dec. 1939, leg. Schwetz (RBINS: 181E, 6278, RMCA:

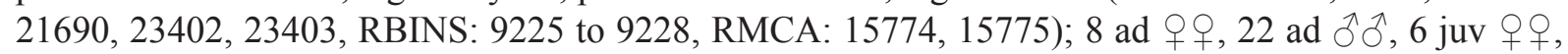
6 juv $\widehat{\partial} \hat{\jmath}, 3$ 우, 3 $\widehat{\partial}, 3$ ?? [46 SS, 5 AL], Avakubi, 20 Nov. 1909, 9 Sep. 1913, 1 Oct. 1913, 14 May 1914, 16 May 1914, 5 Jun. 1914, leg. Herbert Lang, James Paul Chapin and The American Museum Congo Expedition, 1 Oct. 1913, leg. J.A. Howard (AMNH: 48704, KU: 125194, AMNH: 48705, 48706, $48659,48660,48663$ to 48667,48669 to 48676,48678 to 48685,48687 to $48697,50363,51383,51384$, RMCA: 12371, 12372, 12375, AMNH: 48707 to 48711, 48702, 48703); 3 के $\sigma^{\lambda}$ [1 SS, 2 SO], Boende, 23 May 1939, 4 Jun. 1939, leg. R.P. Wynants, 6 Aug. 1947, leg. Huypens (RMCA: 15868, 15867, 18236); 2 की 0 [1 SS, 1 SO], Bokalakala, 8 May 1959, 13 Oct. 1959, leg. N'Kele (RMCA: 28541, 27946); 2 우, 1 § [3 SS], Bokuma, 16 Oct. 1950, leg. P. Lootens (RMCA: 20394 to 20396); 1 ? [SS], Bokungu, prior to 25 Jul. 1949, leg. L. Dupuis (RMCA: 18816); 1 juv ?, 2 우, 1 ? [3 SS, 1 SO], Bolobo, unknown date, prior to 20 Aug. 1958, leg. N'Kele (RMCA: 27416, 27276 to 27278); 1 ? [AL], Boma, between 1 Aug. 1928 and 31 Aug. 1928, leg. Gerard (RMCA: 16184); 1 ㅇ [UN], Bomane [= Bomaneh], 24 May 2010, leg. Guy-Crispin Gembu Tungaluna, Prescott Musaba and José Akaibe (UNIKIS: CRT2279); 3 ?? [3 SO], Bukavu, 16 Aug. 1952, leg. P.G. Vercammen (RMCA: 23174 to 23176); 13 ?? [9 SS, 4 SO], Buta, 14 Aug. 1938, 20 Jun. 1949, prior to 9 Jan. 1937, prior to 28 Dec. 1938, prior to 10 Feb. 1930, leg. Jozef Hutsebaut (RMCA: 14943, 18838 to 18841, 10127 to 10131, 14944,

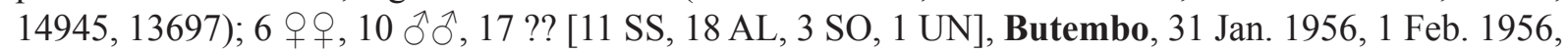
11 Feb. 1956, 3 May 1956, 22 Jul. 1958, 29 Jul. 1958, 27 Aug. 1958, prior to 28 Dec. 1955, prior to 26 Jan. 1955, prior to 25 Jan. 1956, prior to 23 Oct. 1959, prior to 17 Apr. 1956, between 1 Aug. 1955 and 31 Aug. 1955, between 1 Jul. 1955 and 31 Jul. 1955, between 1 Apr. 1957 and 30 Apr. 1957, between 1 Jan. 1956 and 31 Mar. 1956, leg. P. Dyleff (RMCA: 23554, 23555, 23677 to 23681, 23681b, 27494 , 27488, 27480, 23739, 23551, 23552, 23682 to 23689, 26421, 26422, 23313, 23135, 23556 to 23558, 28321, 23448, 22812, 23404); 1 ? [SS], Dilolo, between 1 Jan. 1932 and 31 Dec. 1932, leg. Delvaux (RMCA: 12024); 4 đิ $\widehat{\jmath}$ [4 SS], Dimbelenge, 23 Jun. 1980, 27 Jun. 1980, 28 Jun. 1980, leg. Betu Nsankulu (RMCA: 80.042-M-0001 to 80.042-M-0004); 1 ô [SS], Eala, 2 Jan. 1921, leg. Henri

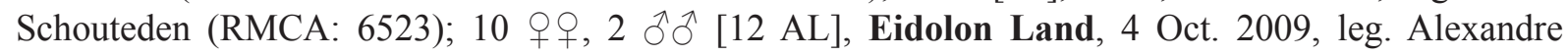
Hassanin, Nicolas Nesi, Célestin Pongombo, Peter Vallo and Didier Tshikung (MNHN: ZM-2011-714 to ZM-2011-725); 1 ad+foetus + [AL], Equateur Province, between 1 Jan. 1921 and 31 Dec. 1921, leg. Verlaine (RMCA: 38240); 1 ? [UN], Franz-Josef Falls, unknown date, leg. unknown collector (RMCA: 15074); 1 [UN], Goma, between 1 Dec. 1987 and 31 Dec. 1987, leg. J. Schoorl (ZMA: MAM.24139); $2 \mathrm{ad}+$ foetus $ㅇ+$, 3 우, 1 ภ, 4 ?? [6 SS, 2 SA, 1 SK, 1 UN], Ibembo, unknown date, 13 Aug. 1919, 30 Aug. 1919, 16 Sep. 1919, 22 Sep. 1919, leg. Jean Lebrun, 20 Feb. 1950, 21 Feb. 1950, 21 Dec. 1951, leg. Jozef Hutsebaut, prior to 17 Jul. 1924, leg. J. Rodhain (RMCA: 4941a, 4925, 4919, 19700 to 19702, 20660, 4936, 4930, 7641); $1 \hat{\jmath}$ [SS], Ikengo, 16 Feb. 1921, leg. Henri Schouteden (RMCA: 6512); 1 ㅇ, 
VAN CAKENBERGHE V. et al., The bats of Congo, Rwanda and Burundi revisited

$1 \precsim$ [2 SS], Inkongo [= Inkongo-Kakese], 27 Jan. 1923, leg. Wilson (RMCA: 6307, 6308); 1 ? [SN],

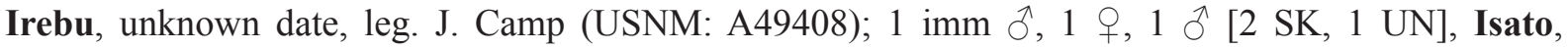
23 Dec. 1947, leg. unknown collector, prior to 25 Nov. 1947, leg. A. Henrion (RBINS: 7716, 7717, 13110); 1 § [SK], Ituri Forest, unknown date, leg. unknown collector (BMNH: 1907.1.2.8); 1 ? [SS], Iyonda, 2 May 1952, leg. Karl Michahelles (RMCA: 21773); 1 ㅇ, 1 § [1 SS, 1 SO], Kabambaie [River], 16 Oct. 1921, leg. Henri Schouteden (RMCA: 6507, 6508); 1 ๙ [SS], Kabobo, Mount, 18 Feb. 1957, leg. Alexandre Prigogine (RMCA: 27113); 2 우, 1 ○ [3 SS], Kakanda Cave, 22 Mar. 1966, leg. Alexandre Prigogine (RMCA: 34247 to 34249); 1 q [SS], Kakonda, 8 Oct. 1942, leg. F.L. Hendricks (AMNH: 118868); $1 \hat{\jmath}$ [SS], Kamituga, 17 Mar. 1965, leg. Alexandre Prigogine (RMCA: 33295); 1 [SS], Kasende, 6 Jul. 1934, leg. R.S. Pierrepont (MCZ: 31737); 1 ô [SS], Katana, 18 Mar. 1956, leg. James Paul Chapin (AMNH: 180892); 1 ? [AL], Katanga, prior to 31 Aug. 1900, leg. Charles Lemaire

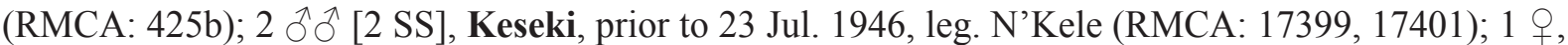
3 ठิ Robert (Bob) Swanepoel (TM: 46061, 46062, 46065 to 46072, 46075, 46077 to 46079, 45592, 45593, 45629, 45594); 1 đิ [UN], Kimvuadu, 19 Feb. 1939, leg. C. Vleeschouwers (RMCA: 16084); 1 , 1 ภ [1 SS, 1 SO], Kisanga, 27 Oct. 1948, leg. J. de Wilde (RBINS: 12778, 12779); 1 ㅇ, 1 ô, 2 ?? [2 AL, 2 UN], Kisangani, 7 Sep. 2012, leg. Prescott Musaba, Tembele and Marc Boketshu, 9 Jun. 2014, leg. Prescott Musaba, prior to 5 Aug. 1988, leg. Luc De Vos, Kimbembi and Alain Vandelanoote (UNIKIS: YK037CH, K13-591, RMCA: 88.025-M-0002, 88.025-M-0003); 1 ? [SO], Kiseny [= Kasenye], prior to 23 Jul. 1946, leg. R. Van Saceghem (RMCA: 17189); 8 ?? [8 SS], Kitundu Kinda, 22 Sep. 1914, leg. de Baillet Latour (RMCA: 4225, 4226, 4228, 4229, 4232 to 4235); 1 ô [SS], Kitutu, 14 Mar. 1960, leg. Alexandre Prigogine (RMCA: 28885); 1 ㅇ, 1 ○ [2 SS], Kitwabaluzi, 19 Apr. 1951, leg. Alexandre Prigogine (RMCA: 20689, 20690); 2 ad ?? [2 SS], Kivu [= Kivu region], 11 Sep. 1935, leg. unknown collector (MNHN: ZM-MO-1939-198, ZM-MO-1939-199); 1 đ [SK], Kongolo Island, between 1 Apr. 1982 and 30 Apr. 1982, leg. Marc Colyn (RMCA: 90.042-M-0271); 5 우, 1 § [5 SS, 1 SO], Kuleponge [= Kulepongi], 26 Jun. 1938, leg. J.M. Vrijdagh (RMCA: 14791 to 14795, 15322); 49 우, 17 ふふ, 2 ?? [62 SS, 1 SK, 4 SO, 1 UN], Kunungu, unknown date, prior to 6 Aug. 1925, prior to 25 Mar. 1929, prior to 22 Oct. 1930, between 1 Jan. 1922 and 31 Dec. 1922, leg. Ngwe, 9 Apr. 1921, leg. Henri Schouteden, prior to 22 Oct. 1930, leg. N'Kele (RMCA: 8176, 6484, 6485, 6487, 6491 to 6497,6516 to 6519,7741 to 7748,7756 to 7766,10562 to $10574,10618,10639$ to $10642,9706,9712$, 8152 to 8165); 3 우우 [3 SS], Kwamouth, 24 May 1921, leg. Henri Schouteden (RMCA: 6488 to 6490); 2 우, 1 तै [3 SS], Lake Tumba [= Tumba, Lake], 15 Nov. 1954, leg. James Paul Chapin (AMNH: 180894 to 180896); 2 오 [2 AL], Lake Tumba, Island in, 14 Jun. 1956, 17 Jun. 1965, leg. Alvin Novick (MCZ: 48181, 48182); 1 ? [SS], Lemba Plain, prior to 3 May 1930, leg. Van Delft (RMCA: 10262); 1 q [SS], Leopold 2nd, Lake [= Mai-Ndombe, Lake], unknown date, leg. unknown collector (BMNH: 1927.3.1.47); 2 우, 3 $\widehat{\partial}, 2$ ?? [2 SK, 3 SO, 2 UN], Léopoldville [= Kinshasa], unknown date, prior to 25 Nov. 1947, leg. A. Henrion, 12 Nov. 1945, leg. Jean Jacques Deheyn, 16 Jun. 1946, between 1 Jan. 1946 and 31 Dec. 1946, leg. M. Henrion, between 1 Feb. 1942 and 28 Feb. 1942, leg. Becquet (RBINS: 12271, RMCA: 17616a, 17797, 17955, 17956, 17356a, RBINS: 12771); 5 웅, 8 ๙ $\widehat{\partial}$ [12 SS, 1 SO], Luebo, 13 Dec. 1921, 15 Dec. 1921, leg. Henri Schouteden (RMCA: 6483a, 6483b, 6501 to 6506,6513 to $6515,6509,6510) ; 1$ \& [SS], Lufira River, upper, unknown date, leg. S.A. Neave (BMNH: 1909.1.3.1); 1 †, 3 ठิ $\widehat{~}$ [4 SS], Lukolela, 1 Dec. 1930, leg. James Paul Chapin, 13 Aug. 1930, 17 Aug. 1930, 18 Aug. 1930, leg. Franklin Edson (AMNH: 86879, 86758 to 86760); 3 $\precsim \widehat{\partial}$ [3 SS], Lulua [= Kananga], 30 Apr. 1976, leg. Betu Nsankulu (RMCA: 76.054-M-0001 to 76.054-M-0003); 1 \%, 2 ô ${ }^{\lambda}$ [3 SS], Luluabourg [= Kananga], unknown date, leg. unknown collector, unknown date, leg. Richard Callewaert (BMNH: 1926.11.1.23, 1926.7.6.45, 1926.7.6.46); 7 우, 4 ठ̊ Lusanga Mwanza, prior to 4 Aug. 1937, leg. Dartevelle (RMCA: 13936 to 13943, 13944a, 13944b, 13945); 2 우, 2 $\jmath^{\jmath}, 1$ ? [1 SS, 3 UN, 1 SB], Lwiro [= Luiro], unknown date, 11 May 1956, between 1 Aug. 1955 and 31 Aug. 1955, leg. J.J. Laarman, 20 Apr. 1955, leg. James Paul Chapin, 29 Jul. 2002, leg. Michael H. Huhndorf (RMNH: MAM.16356, MAM.16384, AMNH: 180893, FMNH: 173291, 
RMNH: MAM.16357); 1 q [AL], Mabali, between 1 May 1956 and 30 Jun. 1956, leg. Alvin Novick (MCZ: 48183); 1 \& [SK], Masako, 24 Nov. 1987, leg. Ndey B. Ifuta (RMCA: 93.079-M-0279); 1 ad $q$, 3 juv 우, 1 ? [1 SS, 4 UN], Medje, 12 May 1914, leg. Cuthbert Christy, 6 Jun. 1910, 20 May 1914, 22 May 1914, leg. Herbert Lang, James Paul Chapin and The American Museum Congo Expedition (RMCA: 4330, AMNH: 48699 to 48701, 48698); 1 ad $\widehat{~}$ [SS], Mondombe, 26 Dec. 1952, leg. L. Dupuis (RMCA: 21715); 2 우, 1 ㅈ [3 SS], Mongende, 11 Apr. 1921, leg. Henri Schouteden (RMCA: 6520 to 6522); 1 ad $\widehat{\partial}$ [SS], Mouth of Congo, between 1 Jan. 1892 and 31 Jan. 1892, leg. J.M. Lewis (BMNH: 1892.5.7.1); 1 ? [AL], Mpe, prior to 26 Jan. 1914, leg. Maes (RMCA: 2663); 8 ?? [8 SS], Muezi Lupungu, 1 Oct. 1914, leg. de Baillet Latour (RMCA: 4227, 4230, 4231, 4236a, 4236b, 4237 to 4239); 2 ?? [2 UN], Mulungu, unknown date, prior to 1 Feb. 1940, leg. F.L. Hendricks (RMCA: 15870, 15871); 1 ? [SS], Mulungu Miti [= Mulungu], 6 Jan. 1947, leg. F.L. Hendricks (RMCA: 19649); $1 \mathrm{imm}$ + [SS], Ndama, 21 Jul. 1954, leg. C.F. de Wilde (RBINS: 17295); 2 ?? [2 AL], New Beni [= Beni], between 1 Oct. 1926 and 31 Oct. 1926, leg. James Paul Chapin, Dewitt L. Sage, Frank P. Mathews and The Ruwenzori-Kivu Expedition (AMNH: 82360, 82361); 3 qㅜ, 3 $\widehat{\jmath}$ [6 SS], Ngerere Lepi, 5 Jan. 1943, 6 Jan. 1943, leg. J. de Wilde (RBINS: 12773, 12774, 12776, 12777, 12772, 12775); 4 ?? [4 UN], NiamNiam, unknown date, leg. unknown collector, 17 Jun. 1884, leg. Bohndorff (RBINS: 181c, 181, 181b, 181j); 2 우, 1 ○े [3 SO], Nyamunyunye, between 1 Jan. 1951 and 31 Dec. 1951, leg. F.L. Hendricks (RMCA: 74.044-M-0004 to 74.044-M-0006); 16 ?? [13 AL, 3 UN], Oshwe, prior to 12 Mar. 1914, leg. Maes (RMCA: 2773 to 2788); 3 ภึ कै [3 UN], Semmio [= Zemio], unknown date, leg. Bohndorff (ZMB: 10206, 7427, 7428); 5 ?? [5 AL], Shabunda, prior to 10 Jul. 1939, leg. Braun (RMCA: 15381 to 15385); 18 우, 6 ふึ, 1 ? [23 SS, 1 SK, 1 SB], St. Jos. (Misson) [= Mikalaya], unknown date, 19 Jun. 1924, 25 Jun. 1924, 3 Nov. 1924, leg. Richard Callewaert (AMNH: 86260, 86229 to 86250, 86253, 86254); 2 우, 1 ○ [2 SS, 1 UN], St. Joseph de Luluabourg Mission [= Mikalaya], 20 Jul. 1922, 3 Jun. 1923, 7 Jun. 1923, leg. Richard Callewaert (RMCA: 7494, 7123, 7116); 1 q [SS], Tandala, 18 Jul. 1979, leg. C. Brian Robbins (USNM: 537699); 1 $\curvearrowright$ [UN], TASOK (The American School of Kinshasa) [= Kinshasa, 10 km W], 15 Apr. 1980, leg. V. Wallach (ZMA: MAM.21145); 1 q [SS], Tondu, 23 Feb. 1921, leg. Henri Schouteden (RMCA: 6511); 4 우 [4 SS], Tshikapa, 26 Nov. 1921, leg. Henri

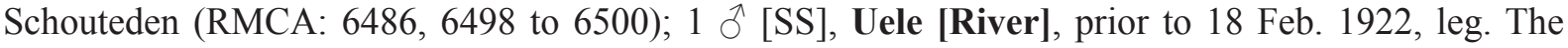
Antwerp Zoo (RMCA: 6065); 1 juv ?, 1 q, 4 के $\widehat{\partial}$ [3 SS, 1 SO, 2 UN], Umangi [Mission], prior to 31 Jul. 1899, leg. Charles de la Kethulle de Ryhove (RMCA: 182 to 187); 1 [UN], Yaekela, 3 May 2010, leg. Guy-Crispin Gembu Tungaluna, Prescott Musaba and José Akaibe (UNIKIS: CRT2387); 1 ๙ [SS], Yangambi, 28 Dec. 1947, leg. unknown collector (RBINS: 6985); 1 ? [SS], Yokamba Elongo, 28 Jun. 1954, leg. Stevenart (RMCA: 22411); 4 ?? [4 SS], Zambi, unknown date, leg. R. Van Saceghem (RMCA: 4861 to 4864).

RWANDA: 17 ?? [1 SS, 14 AL, 2 SB], Astrida [= Butare], 15 May 1962, leg. Van Parijs, 17 Apr. 1968, leg. P.L. Niort, 20 Jan. 1951, leg. Aureliaan (RMCA: 31176 to 31189, MHNG: 1498.001, 1498.002, RMCA: 20491); 1 ad +5 ad ${ }^{\wedge} \hat{~}$ [6 UN], Bugarura Island, between 27 Jun. 1953 and 29 Jun. 1953, leg. unknown collector (RBINS: 10537 to 10542); 1 ad $q$ [AL], Cyili, unknown date, leg. Benny Baeten

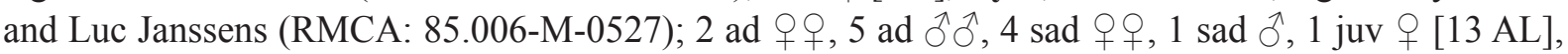
Gafunzo, unknown date, leg. Benny Baeten and Luc Janssens (RMCA: 85.006-M-0520, 85.006-M-0522 to 85.006-M-0525, 85.006-M-0528, 85.006-M-0530 to 85.006-M-0536); 1 q, 2 ?? [3 UN], Kissenji [= Gisenyi], unknown date, leg. Stegmann-Stein (ZMB: 67137 to 67139); 3 우, 1 ㅅ, 9 ?? [13 UN], Mugarura, unknown date, leg. Stegmann-Stein, unknown date, leg. H. Schubolz (ZMB: 66523, 67037 to $67041,67043,67146$ to $67149,67152,67153) ; 4$ 우, 1 ○ [4 SS, 1 SO], Nyarutovu, 19 Sep. 1954, 20 Sep. 1954, 10 Aug. 1955, 8 Oct. 1955, 10 Oct. 1955, leg. Alexandre Fain (RMCA: 23139, 23646,

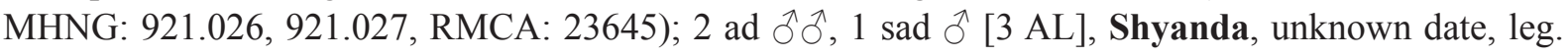
Benny Baeten and Luc Janssens (RMCA: 85.006-M-0521, 85.006-M-0526, 85.006-M-0529). 
VAN CAKENBERGHE V. et al., The bats of Congo, Rwanda and Burundi revisited

Rousettinae K. Andersen, 1912

BURUNDI: 2 juv ?? [1 AL, 1 UN], Bujumbura, 22 Apr. 1982, 26 Apr. 1982, leg. Danny Meirte (RMCA: 82.016-M-0020, 82.016-M-0082).

DEMOCRATIC REPUBLIC OF THE CONGO: 8 foetus ?? [1 AL, 5 SK, 2 SO], unknown locality, unknown date, leg. Tshinyenye, prior to 1 Aug. 1957, leg. Heinz Stephan, prior to 18 Aug. 1965, leg. Antoon Emeric Marcel De Roo (RMCA: 77.021-M-0017, 77.021-M-0018, 27420 to 27424, 33602); 1 o [SS], Bengale, 28 Dec. 1976, leg. Tshinyenye (RMCA: 77.012-M-0008); 1 [ [SS], Boma, 23 Nov. 1920, leg. Henri Schouteden (RMCA: 7294); 2 ?? [2 SS], Bukavu, prior to 30 Jun. 1999, leg. Camerman (RMCA: 99.011-M-0002, 99.011-M-0003); 2 juv ??, 2 ô ô, 1 ? [2 SS, 3 AL], Butembo, 17 Oct. 1971, between 21 May 1975 and 30 Jun. 1975, leg. M. Lejeune, 31 Dec. 1969, leg. M.J. Celis and M. Lejeune, prior to 18 Apr. 1958, leg. P. Dyleff (RMCA: 38242, 36196, 75.042-M-0007, 27042, 27043); 1 [SS], Byonga, 5 Apr. 1962, leg. Alexandre Prigogine (RMCA: 31242); 2 ?? [2 SS], Dilolo, 9 Feb. 1948, leg. Jozef Hutsebaut (RMCA: 18228, 18229); 2 ?? [2 AL], Epulu, unknown date, leg. Luc Bijnens (RMCA: 90.031-M-0005, 90.031-M-0006); 2 sad ô ô, 1 juv + , 1 ㅇ [4 SK], Idjwi Island, 19 Jul. 1965, 23 Aug. 1969, 26 Aug. 1969, between 25 Jul. 1969 and 25 Aug. 1969, leg. Alexandre Prigogine (RMCA: 33626, 36217, 36218, 36216); 1 ô [SS], Kakanda Cave, 21 Aug. 1964, leg. Alexandre Prigogine (RMCA: 33064); 1 o [SS], Kamueshi [= Kamuesha], 3 Apr. 1979, leg. Betu Nsankulu (RMCA: 79.032-M-0001); 1 đ [SS], Kasebere, 13 Jan. 1970, leg. Alexandre Prigogine (RMCA: 36220); 1 juv ? [AL], Kilinga, 15 Oct. 1954, leg. Gaston-François de Witte (RMCA: 37114);

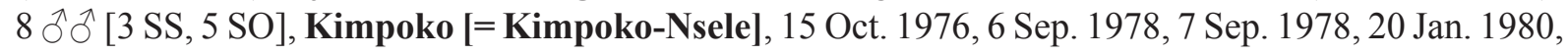
leg. Tshinyenye (RMCA: 77.012-M-0009, 80.038-M-0001, 80.038-M-0002, 79.003-M-0002 to 79.003M-0006); 1 \& [SS], Kingabwa, 31 Dec. 1975, leg. Tshinyenye (RMCA: 76.010-M-0001); 1 foetus ? [AL], Luluabourg [= Kananga], unknown date, leg. Jan Deheegher (RMCA: 31560); 1 \& [SS], Make, 30 Oct. 1992, leg. Tshinyenye (RMCA: 92.148-M-0002); 1 imm ? [AL], Moba, 26 Nov. 1953, leg. H. Bomans (RMCA: 22124); 1 +, 5 ?? [6 SO], Mudina Ndemba [= Ndemba], 18 Mar. 1975, leg. Betu Nsankulu (RMCA: 75.061-M-0001 to 75.061-M-0006); 1 § [SS], Mulungu, 8 Oct. 1965, leg. Alexandre Prigogine (RMCA: 33968); 1 juv ? [AL], Mutwanga, 8 Nov. 1954, leg. Gaston-François de Witte (RMCA: 37111); 1 †, 3 ठิ $\widehat{~}$ [4 SS], Ngula, 1 Mar. 1977, 5 Mar. 1977, 6 Mar. 1977, leg.

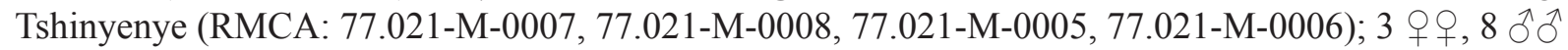
[11 SS], Nkiene, 28 Sep. 1980, 29 Sep. 1980, 30 Sep. 1980, leg. Tshinyenye (RMCA: 80.053-M-0002 to 80.053-M-0012); 1 ? [SS], Ogondjo, 24 Apr. 1942, leg. J.M. Vrijdagh (RMCA: 17758); 1 juv ? [AL], Uvira, unknown date, leg. Georges Marlier (RMCA: 79.011-M-0002); 1 ? [AL], Wafanya [= Wafania], 29 Aug. 1991, leg. P. Lootens (RMCA: 91.076-M-0060).

\section{Epomophorini Gray, 1866 \\ Epomophorus Bennett, 1836}

BURUNDI: 1 ad $ð[A L]$, unknown locality, 13 Nov. 1978, leg. F.C. Roest (RMNH: MAM.45841).

DEMOCRATIC REPUBLIC OF THE CONGO: 1 q [AL], unknown locality, 13 Nov. 1978, leg. F.C. Roest, 3 Dec. 1985, leg. unknown collector(RMNH: MAM.45692); 1 q [SA], Katwé, 1 Oct. 2009, leg. Alexandre Hassanin, Nicolas Nesi, Célestin Pongombo, Peter Vallo and Didier Tshikung (MNHN: ZM2011-788); 1 ? [UN], Kilinga, unknown date, leg. unknown collector (INPBC: V1257.1); 4 우 [4 SS], Kipangaribwe River, 18 Jul. 1947, 31 Dec. 1947, 6 May 1949, leg. unknown collector (RBINS: 10671 to 10673, 10676); 5 우 $ᄋ$, 1 § [3 SA, $3 \mathrm{AL}$ ], Kitombongo [= Kintobongo], 24 Sep. 2009, leg. Alexandre Hassanin, Nicolas Nesi, Célestin Pongombo, Peter Vallo and Didier Tshikung (MNHN: ZM-2011789 to ZM-2011-794); $1 \mathrm{emb}$ +, 1 emb ? [1 SA, 1 AL], Lubumbashi, 28 Sep. 2009, leg. Alexandre Hassanin, Nicolas Nesi, Célestin Pongombo, Peter Vallo and Didier Tshikung (MNHN: ZM-2011-795, 
ZM-2011-796); 2 우 [2 SA], Mukulakulu, 22 Sep. 2009, leg. Alexandre Hassanin, Nicolas Nesi, Célestin Pongombo, Peter Vallo and Didier Tshikung (MNHN: ZM-2011-797, ZM-2011-798); 1 ? [UN], Ndote, unknown date, leg. unknown collector (INPBC: W1282); 1 imm ? [SS], Panda [= Pande], 15 Dec. 1955, leg. De Smet (RMCA: 26210); 1 ? [UN], Rutshuru, unknown date, leg. unknown collector (INPBC: V1634).

RWANDA: 1 ? [AL], unknown locality, 27 Oct. 1979, leg. unknown collector (RMNH: MAM.45687).

Epomophorus anselli Bergmans \& Van Strien, 2004

DEMOCRATIC REPUBLIC OF THE CONGO: 1 ô [SA], Kitombongo [= Kintobongo], 24 Sep. 2009, leg. Alexandre Hassanin, Nicolas Nesi, Célestin Pongombo, Peter Vallo and Didier Tshikung (MNHN: ZM-2011-726).

\section{Epomophorus crypturus Peters, 1852}

DEMOCRATIC REPUBLIC OF THE CONGO: 1 ? [SK], unknown locality, prior to 8 Jan. 1940, leg. unknown collector (RBINS: 6283); 1 ad $q$ [SK], Dikulwe River, unknown date, leg. Neuve (BMNH: 1907.12.13.2); 3 ad $ㅇ+$, 1 ad $\hat{O}, 1$ juv $q$ [1 SS, 4 SA], Elisabethville [= Lubumbashi], 28 Dec. 1955, 29 Dec. 1955, leg. M. Lips, between 1 Apr. 1926 and 30 Apr. 1926, leg. Henri Schouteden, prior to 7 Jul. 1936, leg. Richard (RMCA: 26268 to 26270, 16208, 13316); 1 ? [UN], Gandu, unknown date, leg. Gaston Thierry (ZMB: 54663); 1 § [SS], Ganza, 28 Jun. 1949, leg. Gaston-François de Witte (RBINS:

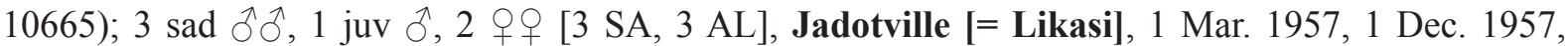
22 Jun. 1958, 15 Oct. 1958, 13 Feb. 1959, 6 Dec. 1959, leg. Félix / Michel Anciaux de Faveaux (RMCA: 26254, 27591, 27916 to 27918, RBINS: 14595); 1 ad $q$ [AL], Kakanda Cave, unknown date, leg. the Royal Museum for Central Africa (RMCA) (BMNH: 1955.136); 1 sad $\widehat{\jmath}, 1$ \& [1 SK, 1 SB], Kakanda Mutaka, 14 Jul. 1953, leg. petit Seminaire de St. Francois de Sales (RMCA: 21874, 21875); 1 ? [UN], Kakontwa [= Kakontwe], 17 Jan. 1961, leg. Félix / Michel Anciaux de Faveaux (RBINS: 14597); 1 ad đ, $1 \mathrm{sad}$ q, $1 \mathrm{sad}$ ô, 2 우 [1 SS, 3 SA, 1 SK], Kakontwe, 14 Nov. 1959, leg. William Frank Harding Ansell, 17 Jan. 1961, leg. unknown collector, 30 Jun. 1957, between 30 Jun. 1956 and 30 Jun. 1957, leg. Félix / Michel Anciaux de Faveaux (RMCA: 29246, RBINS: 14596, MHNG: 1046.035, RMCA: 26716,

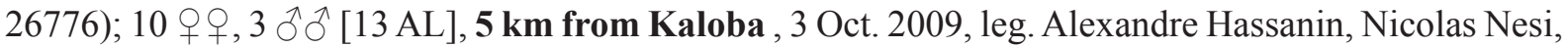
Célestin Pongombo, Peter Vallo and Didier Tshikung (MNHN: ZM-2011-743 to ZM-2011-755); 1 ad ô, $^{2}$ 1 juv ?, 2 imm 우, 1 ? [1 SS, 1 AL, 1 SK, 2 SO], Kanzenze Mission, 14 Jul. 1947, leg. R.P. Lefébure, 28 Nov. 1948, between 1 Dec. 1948 and 31 Dec. 1948, leg. Narcisse Leleup (RBINS: 13107, BMNH: 1954.786, RMCA: 22485, 22486, 22491); 2 ad 9 ㅇ [1 SA, 1 UN], Kasaji, unknown date, leg. W.S. Fisher,

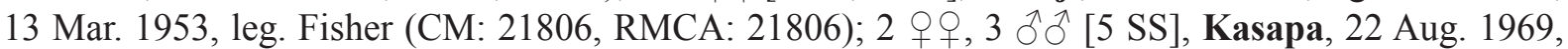
leg. J. Poelman, 22 Aug. 1969, leg. unknown collector (RBINS: 18779, 18780, 18841 to 18843); 1 ㅇ [AL], Kasenga Mission [= Kasenga], 28 Dec. 1956, leg. Van Iseghem (RMCA: 26273); 1 q [AL], Kasombo, 20 Sep. 2009, leg. Alexandre Hassanin, Nicolas Nesi, Célestin Pongombo, Peter Vallo and Didier Tshikung (MNHN: ZM-2011-756); 1 ad $\widehat{\partial}, 1$ sad $\odot$ [1 SA, 1 AL], Katanga s, unknown date, leg. André Pilette (RMCA: 16210, 16248); 2 우 [2 AL], Katwé, 1 Oct. 2009, 2 Oct. 2009, leg. Alexandre Hassanin, Nicolas Nesi, Célestin Pongombo, Peter Vallo and Didier Tshikung (MNHN: ZM-2011-757, ZM-2011-758); 1 ô [SN], Kipangaribwe River, 12 Jan. 1948, leg. unknown collector (RBINS: 10667); 6 우, $1 \widehat{\partial}$ [7 AL], Kitombongo [= Kintobongo], 24 Sep. 2009, 25 Sep. 2009, 26 Sep. 2009, leg. Alexandre Hassanin, Nicolas Nesi, Célestin Pongombo, Peter Vallo and Didier Tshikung (MNHN: ZM2011-759 to ZM-2011-765); 1 ad §̊ [SO], Kolwezi, 1 Mar. 1958, leg. unknown collector (OSU: 3817);

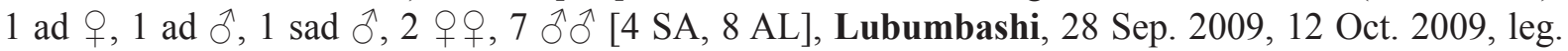
Alexandre Hassanin, Nicolas Nesi, Célestin Pongombo, Peter Vallo and Didier Tshikung, 24 Mar. 1966, leg. L. Poelman, 26 Mar. 1966, leg. Xavier Misonne (MNHN: ZM-2011-769 to ZM-2011-774, RBINS: 17207, RMCA: 34712, 34713, MNHN: ZM-2011-766 to ZM-2011-768); 1 sad $ᄋ$ [SA], Luluabourg 
VAN CAKENBERGHE V. et al., The bats of Congo, Rwanda and Burundi revisited

[= Kananga], 1 Jan. 1963, leg. Jan Deheegher (RMCA: 31557); 1 ô [SS], Mabwe, 7 Aug. 1947, leg. unknown collector (RBINS: 10670); 3 우 [3 AL], Mukulakulu, 22 Sep. 2009, leg. Alexandre Hassanin, Nicolas Nesi, Célestin Pongombo, Peter Vallo and Didier Tshikung (MNHN: ZM-2011-775 to ZM2011-777); 2 우 [1 SA, 1 AL], cave 4 km from Muvule , 8 Oct. 2009, 9 Oct. 2009, leg. Alexandre Hassanin, Nicolas Nesi, Célestin Pongombo, Peter Vallo and Didier Tshikung (MNHN: ZM-2011-778, ZM-2011-779); 1 ad $\widehat{\partial}$ [SA], Panda Camp [= Likasi], 1 Apr. 1958, leg. Félix / Michel Anciaux de

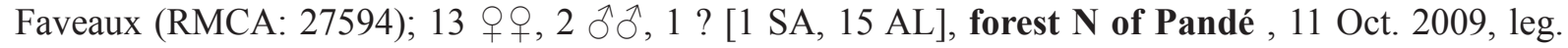
Alexandre Hassanin, Nicolas Nesi, Célestin Pongombo, Peter Vallo and Didier Tshikung (MNHN: ZM2011-727 to ZM-2011-742); 1 juv $\&$ [SA], Pempere Cave, 20 Oct. 1957, leg. Félix / Michel Anciaux de Faveaux (RMCA: 27607); 1 ? [SO], Sakania Mission [= Sakania], 19 Oct. 1930, leg. J. De Riemaecker (RMCA: 19418); 1 [AL], Upemba National Park, 25 Sep. 2009, leg. Alexandre Hassanin, Nicolas Nesi, Célestin Pongombo, Peter Vallo and Didier Tshikung (MNHN: ZM-2011-780).

\section{Epomophorus labiatus (Temminck, 1837)}

BURUNDI: $1 \widehat{\delta}[\mathrm{KB}$ ], Buhonga, 2 Jun. 1970, leg. P.L. Niort (MHNG: 1499.046); $6 \mathrm{ad} q q+, 5 \mathrm{sad} q$,

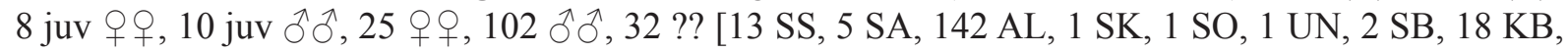
5 NS], Bujumbura, unknown date, between 1 Aug. 1976 and 31 Aug. 1976, leg. Jacques Verschuren, 25 Oct. 1967, 29 Oct. 1967, 6 Nov. 1967, 11 Nov. 1967, 22 Nov. 1967, 1 Dec. 1967, 5 Dec. 1967, 14 Dec. 1967, 1 Jan. 1968, 12 Jan. 1968, 13 Jan. 1968, 18 Oct. 1968, 1 Nov. 1968, 9 Nov. 1968, 5 Dec. 1968, 6 Dec. 1968, 8 Mar. 1969, 20 May 1969, 12 Nov. 1969, 14 Nov. 1969, 15 Nov. 1969, 31 Dec. 1969, 5 Jan. 1970, 6 Jan. 1970, 7 Jan. 1970, 9 Jan. 1970, 2 Feb. 1970, 26 Feb. 1970, 10 Mar. 1970, 26 Nov. 1970, 28 Jan. 1971, 29 Jan. 1971, 10 Mar. 1971, 1 Jan. 1972, 5 Jan. 1972, 24 Jan. 1972, 31 Jan. 1972, 30 Mar. 1972, 15 Aug. 1972, 17 Sep. 1972, 3 Feb. 1973, 14 Mar. 1973, 19 Jul. 1973, 10 Feb. 1974, 11 Feb. 1974, 13 Feb. 1974, 17 Feb. 1974, between 6 Jun. 1968 and 7 Jun. 1968, between 30 Mar. 1972 and 31 Mar. 1972, between 26 Oct. 1967 and 27 Oct. 1967, leg. P.L. Niort, 21 Apr. 1982, 22 Apr. 1982, 23 Apr. 1982, 24 Apr. 1982, 25 Apr. 1982, 26 Apr. 1982, 27 Apr. 1982, 28 Apr. 1982, 29 Apr. 1982, between 1 Apr. 1982 and 30 Apr. 1982, leg. Danny Meirte (RBINS: 19909, MHNG: $1498.054,1499.068,1498.065,1498.066,1498.049,1499.086,1499.026$ to $1499.028,1499.058$, $1499.087,1498.040$ to $1498.043,1498.055,1498.093$ to $1498.097,1498.056$ to $1498.058,1499.088$, $1499.081,1497.097$ to $1498.099,1498.052,1499.075,1498.100,1499.089,1499.076,1498.061$ to $1498.064,1499.085,1499.021,1499.023,1498.083,1498.084$, RMCA: 82.016-M-0003, 82.016M-0004, 82.016-M-0010 to 82.016-M-0019, 82.016-M-0021, 82.016-M-0022, MHNG: 1498.044 to 1498.048, RMCA: $82.016-\mathrm{M}-0023$ to $82.016-\mathrm{M}-0042,82.016-\mathrm{M}-0044$ to $82.016-\mathrm{M}-0048$, MHNG: 1499.071, RMCA: 82.016-M-0049 to 82.016-M-0067, 82.016-M-0070, 82.016-M-0071, 82.016-M0073 to 82.016-M-0079, MHNG: 1498.035, 1499.024, 1499.025, RMCA: 82.016-M-0080, 82.016M-0081, 82.016-M-0083 to 82.016-M-0093, 82.016-M-0125 to 82.016-M-0128, MHNG: 1499.050 , 1499.051, RMCA: 82.016-M-0130 to 82.016-M-0144, MHNG: 1499.054, RMCA: 82.016-M-0153, MHNG: 1499.057, RMCA: 82.016-M-0154, 82.016-M-0155, MHNG: 1498.037, 1498.038, 1497.098, $1499.072,1499.070,1499.005,1499.006,1499.069,1498.050,1498.051,1498.068$ to 1498.070 , 1499.008, 1498.039, 1498.071, 1499.007, 1498.075, 1499.009, 1498.067, RMCA: 82.016-M-0156, RBINS: 19909.1 to 19909.4, MHNG: 1498.036, 1499.073, 1498.059, 1498.060); 1 ○ [AL], Bukara, 17 Jun. 1972, leg. P.L. Niort (MHNG: 1499.074); 1 § [KB], Busiga, between 1 Jan. 1900 and 1 Jan. 1967, leg. P.L. Niort (MHNG: 1499.001); 1 q [SS], Cibitoke, 15 May 1978, leg. unknown collector (RBINS: 20272); 1 [ [SS], Gifuruzi [= Kifuruzi], 8 Apr. 1969, leg. P.L. Niort (MHNG: 1498.076); 1 ad $q$, 20 우우, 16 $\widehat{\jmath}, 1$ ? [3 SS, 23 AL, 2 SK, 1 SB, 1 SN, 7 KB, 1 AB], Gihanga, 18 Jan. 1970, 25 Jan. 1970, 8 Apr. 1970, 11 Apr. 1970, 21 May 1970, 1 Nov. 1970, 2 Nov. 1970, 15 Nov. 1970, 24 Jan. 1971, 29 Jan. 1971, 17 Feb. 1971, 23 Mar. 1971, 22 Nov. 1972, 18 May 1973, leg. P.L. Niort, 14 Mar. 1979, leg. Heinz Stephan (MHNG: 1499.047, 1499.038, 1499.039, SMF: 57512, MHNG: 1499.049, 1499.055, $1499.010,1499.011,1499.082$ to $1499.084,1499.048,1499.040$ to $1499.045,1499.077,1499.063$, 
1499.052, $1499.053,1497.099,1499.012$ to $1499.020,1499.056,1499.032$ to $1499.034,1499.036$,

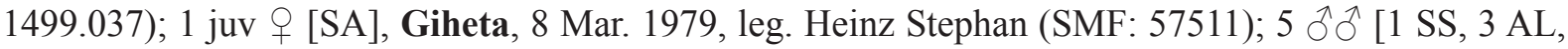
1 KB], Gitega, 29 Dec. 1967, 12 Mar. 1971, 14 Mar. 1971, 25 Aug. 1971, between 13 Mar. 1971 and 14 Mar. 1971, leg. P.L. Niort (MHNG: 1499.059 to 1499.061, 1498.053, 1499.062); 2 ๙ $\widehat{乛}$ [1 SS, 1 AL], Kibimba Mission, 3 Jan. 1969, leg. P.L. Niort (MHNG: 1498.073, 1498.074); 37 ?? [37 UN], Kigamba, 15 Jul. 1946, 15 Jul. 1976, leg. Jacques Verschuren (RBINS: 19900.01, 19902, 19900.02 to 19900.35, 19902.2); 2 우 [2 SK], Lake Cohoha [= Cohoha, Lake], 9 Mar. 1979, 11 Mar. 1979, leg. Heinz Stephan (SMF: 57510, 57509); 7 q 9 [2 SS, 2 AL, 3 KB], Makamba, 9 Apr. 1969, 10 Apr. 1969, 23 Mar. 1970, leg. P.L. Niort (MHNG: 1498.080, 1498.081, 1499.030, 1499.031, 1498.077 to 1498.079); 4 우우 [4 AL], Murore, 27 May 1971, leg. P.L. Niort (MHNG: 1499.064 to 1499.067); 2 ठぇ [1 SS,

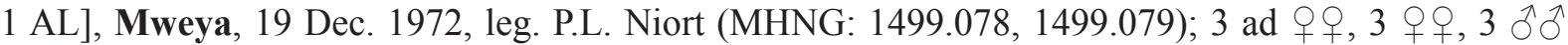
[2 SA, 6 SK, 1 CO], Resha, 28 Feb. 1979, 2 Mar. 1979, 3 Mar. 1979, 15 Mar. 1979, 17 Mar. 1979, 3 Apr. 1979, 16 Jun. 1979, leg. Heinz Stephan (SMF: 57504 to 57506, 57514, 57513, 57503, 57507, 57508, 57515); 1 q [SB], Ruhinga Hill, 12 Mar. 1993, leg. Jay L. Udelhoven (FMNH: 155941); 1 ad ${ }_{\text {}}$, 1 q [1 SS, 1 KB], Rumonge, 3 Nov. 1969, leg. P.L. Niort, between 1 Feb. 1955 and 28 Feb. 1955, leg.

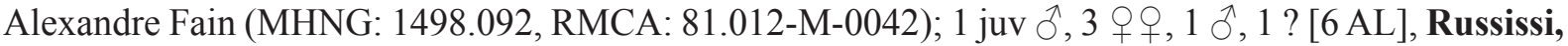
bank [= Rusizi Delta], 27 Apr. 1982, leg. Danny Meirte (RMCA: 82.016-M-0145, 82.016-M-0147, 82.016-M-0149 to 82.016-M-0152); 9 ㅇ [1 SS, 4 AL, 1 SB, 3 KB], Zimet Quarter, 13 Oct. 1969, 25 Oct. 1969, 29 Nov. 1969, 10 Dec. 1969, leg. P.L. Niort (MHNG: 1499.003, 1499.004, 1498.085, $1498.086,1498.088$ to $1498.091,1499.002)$.

DEMOCRATIC REPUBLIC OF THE CONGO: 1 ? [SS], unknown locality, prior to 5 Dec. 1957, leg. De Semeries (RMCA: 26682); 2 ?? [2 UN], Albert National Park [= Virunga National Park], unknown date, leg. unknown collector (INPBC: V1839.2.1, V1839.2.2); 2 ad के ô [1 SA, 1 AL], Albertville [= Kalemie], unknown date, leg. the Royal Museum for Central Africa (RMCA), prior to 12 Jul. 1955, leg. service hygiene publique albertville (BMNH: 1955.1191, RMCA: 22947); 1 ? [SK],

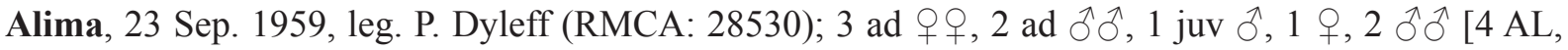
4 SK, 1 UN], Baraka, unknown date, 16 Apr. 1947, leg. the Belgian Hydrobiological Expedition to Tanganyika, 16 Apr. 1947, leg. unknown collector (BMNH: 1953.1, 1953.2, 1953.4, 1853.1, 1953.3, RBINS: 9061.1 to 9061.4); 1 ad ?, 2 juv ?? [3 SK], Beni, 2 Jun. 1914, leg. Bonnevie, between 20 Oct. 1932 and 20 Oct. 1933, between 1 Oct. 1932 and 31 Oct. 1932, leg. Henri Martin Hackars (RMCA: 3245, 12134, 12133); 1 juv $\widehat{~}[\mathrm{SA}]$, Bobandana, 10 Jul. 1933, leg. unknown collector (MNHN: ZMMO-1975-1066); 2 ?? [2 UN], Bongeya River, unknown date, leg. unknown collector (INPBC: V264.1, V264.3); 1 q [SS], Bushanganya, 20 Oct. 1953, leg. De Schrijver (RMCA: 27939); 3 ad $\widehat{\partial} \delta^{\lambda}, 1$ sad ${ }^{\lambda}$,

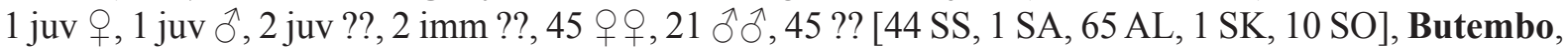
unknown date, 25 Apr. 1954, 22 Apr. 1956, 28 Apr. 1956, 27 Jul. 1956, 25 Jul. 1957, 20 Aug. 1957, 26 Aug. 1957, 8 Sep. 1957, 8 Jan. 1958, 10 Jan. 1958, 5 Feb. 1958, 27 Feb. 1958, 6 Mar. 1958, 18 Mar. 1958, 4 Apr. 1958, 16 Apr. 1958, 23 Apr. 1958, 24 Apr. 1958, 28 Apr. 1958, 5 May 1958, 14 May 1958, 16 May 1958, 2 Jun. 1958, 4 Jun. 1958, 9 Jun. 1958, 16 Jun. 1958, 8 Jul. 1958, 10 Jul. 1958, 15 Jul. 1958, 22 Jul. 1958, 12 Aug. 1958, 13 Aug. 1958, 14 Aug. 1958, 16 Aug. 1958, 20 Aug. 1958, 17 Sep. 1958, 1 Oct. 1958, 18 Jan. 1961, prior to 9 Oct. 1957, prior to 29 Jul. 1957, prior to 26 May 1959, prior to 26 Jan. 1955, prior to 23 Oct. 1959, prior to 18 Apr. 1958, prior to 17 Dec. 1956, between 7 Jun. 1958 and 8 Jul. 1958, between 1 Jul. 1956 and 31 Jul. 1956, between 1 Jun. 1956 and 30 Jun. 1956, between 1 Apr. 1957 and 30 Apr. 1957, between 1 Jan. 1931 and 31 Dec. 1931, leg. P. Dyleff, 31 Dec. 1969, 1 Jan. 1970, leg. M.J. Celis and M. Lejeune, 17 May 1975, 20 May 1975, 25 May 1975, 1 Jun. 1975, 7 Jun. 1975, leg. M. Lejeune, 13 Aug. 1956, leg. De Semeries, prior to 5 Jul. 1950, leg. Alexandre Prigogine (RMCA: 27651 to 27654, 36193, 36195, 36201, 36203, 75.042-M-0003, 27781, 27357, 27484, 27490, 26681, 27779, 27780, 27380, 27489, 27482, 27350, 27379, 27382, 27485, 27493, 75.042M-0004, 27491, 27492, 30809, 27356, 27383, 75.042-M-0005, 26650, 27486, 23741, 27487, 27355, 27351, 22722, 75.042-M-0006, 26652, 26651, 27352, 26198, 23740, 27349, 27353, 27376, 36197, 
VAN CAKENBERGHE V. et al., The bats of Congo, Rwanda and Burundi revisited

27481, 27385, 27358, 27354, 27381, 75.042-M-0001, 75.042-M-0002, 27386, 27483, 26894, 27377, $27387,26423,25848$ to 25851,24004 to $24013,27378,24014$ to $24018,27041,27044$ to 27050,28322 to 28339,22813 to 22815,27886 to $27890,26434,19922,26583$ to 26586 ); $1 \mathrm{sad}$ ㅇ, 3 웅 [ $3 \mathrm{SS}, 1 \mathrm{SK}$ ], Cel I [= Bagbele], 1 Feb. 1950, 24 Feb. 1950, 17 May 1950, leg. H. de Saeger et al., 24 Feb. 1950, leg.

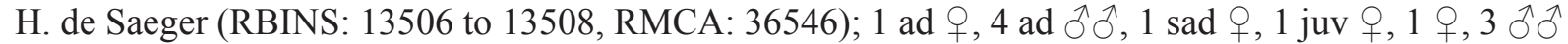
[1 SS, 2 SA, 4 SK, 4 UN], Cel II, 1 Feb. 1951, 1 Mar. 1951, 31 Mar. 1952, 2 Apr. 1952, leg. H. de Saeger et al., 1 Mar. 1951, 15 Jan. 1952, 31 Mar. 1952, 2 Apr. 1952, 3 Apr. 1952, leg. H. de Saeger (RBINS: 13511, 13510, RMCA: 36552, 36547, RBINS: 13516, RMCA: 36550, 36551, RBINS: 13513.1, 13513.2, RMCA: 36548, 36549); 1 juv ô, 1 ? [2 AL], Costermansville [= Bukavu], between 1 May 1950 and 31 May 1950, leg. Babilon, prior to 11 Jan. 1939, leg. Hautmann (RMCA: 20271, 14975); 6 ad $\odot$, 6

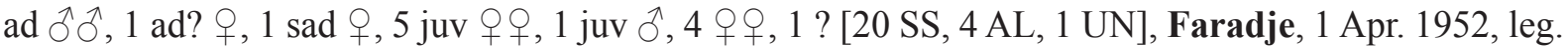
H. de Saeger et al., 7 Apr. 1911, 2 Jul. 1911, 6 Nov. 1911, 22 Nov. 1911, 23 Dec. 1911, 2 Jan. 1912, 23 Feb. 1912, 16 Sep. 1912, 22 Oct. 1912, 5 Nov. 1912, 2 Dec. 1912, 17 Dec. 1912, leg. Herbert Lang, James Paul Chapin and The American Museum Congo Expedition (RBINS: 13517, AMNH: 48747, 48750, 48743, 48745, 48746, RMCA: 12374, 12373, AMNH: 48749, 49204, 49200 to 49202, 48742, 48748, 48661, 48734 to 48741, 48732); 2 ठิ $\widehat{~}$ [2 UN], Fataki, between 1 Jan. 1948 and 6 Apr. 1964,

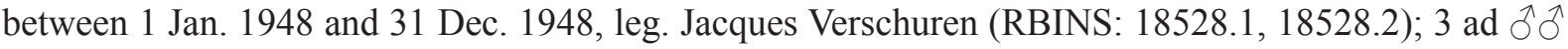
[3 SA], Fomulac-Katana, 29 Sep. 1963, 30 Sep. 1963, 3 Oct. 1963, leg. P. Kunkel (SMF: 31792, 31793, 31795); 2 ad 웅, 1 ㅇ, 2 수, 6 ?? [5 SS, 5 SK, 1 SO], Gangala-na-Bodio, 1 Dec. 1956, between 1 Dec. 1956 and 31 Dec. 1956, leg. Max Poll, 22 Aug. 1945, 22 Nov. 1947, 9 Apr. 1948, leg. unknown collector (RMCA: 25602, 25603, RBINS: 12936, 12934, 12935, 11658.1 to 11658.4, RMCA: 25604,

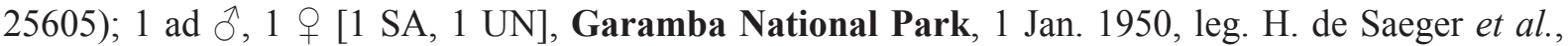
between 1 Jan. 1950 and 31 Dec. 1952, leg. H. de Saeger (RBINS: 13518, RMCA: 36553); 1 ad + [SS], Ibebero, 14 Jul. 1964, leg. Svepel (ZMZ: 15596); 1 ad? ? [SS], Kabale, between 10 Aug. 1939 and 5 Sep. 1939, leg. Schwetz (RMCA: 15833); 1 ad $\delta$ [SO], Kakondo, between 1 Dec. 1946 and 31 Dec. 1946, leg. F.L. Hendricks (RMCA: 19654); 1 ad $q, 1$ sad $\$, 3$ sad ?? [5 SK], Kaniki Kapangu, 12 Mar. 1966, 24 Dec. 1966, between 24 Mar. 1966 and 24 Dec. 1966, between 21 Dec. 1966 and 24 Dec. 1966, leg. Antoon Emeric Marcel De Roo (RMCA: 35276, 35282, 35284, 35285, 35283); 1 ? [SO], Kasai o, prior to 16 Oct. 1968, leg. Antoon Emeric Marcel De Roo (RMCA: 35286); 1 ad 9 [SA], Kasaka, prior to 7 Dec. 1971, leg. Vanderborght (RMCA: 38155); 1 juv 9,1 ? [1 AL, 1 UN], Katanda, unknown date, leg. unknown collector, 12 May 1954, leg. Gaston-François de Witte (INPBC: W976, RMCA: 37109); 2 ad $\hat{\partial}$, 1 sad ? [1 SS, 1 SK, 1 SO], Kere Kere [= Kerekere], between 1 Jan. 1950 and 27 Oct. 1950, leg. Turco (RMCA: 20094 to 20096); 1 \& [UN], Keyberg, prior to 5 Aug. 1952, leg. unknown collector (RBINS: 13106); 1 \% [SS], Kilwezi, 6 Aug. 1948, leg. Gaston-François de Witte (RBINS: 10677); 1 ad $\odot$ [SA], Kiseny [= Kasenye], between 1 Jan. 1926 and 31 Jan. 1926, leg. Henri Schouteden (RMCA: 16206); 1 ㅇ [SA], Kitombongo [= Kintobongo], 24 Sep. 2009, leg. Alexandre Hassanin, Nicolas Nesi, Célestin Pongombo, Peter Vallo and Didier Tshikung (MNHN: ZM-2011-781);

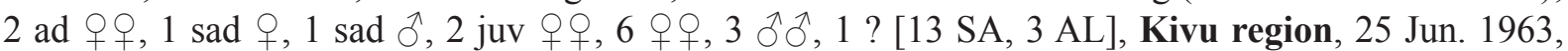
28 Jun. 1963, 22 Jul. 1963, 12 Sep. 1963, 24 Sep. 1963, 29 Sep. 1963, 30 Sep. 1963, 1 Oct. 1963, 2 Oct. 1963, 23 Nov. 1963, 4 Jun. 1964, 24 Dec. 1964, between 1 Jul. 1963 and 31 Dec. 1963, leg. P. Kunkel (SMF: 31798, 31804, 31808, 31806, 31814, 31813, 31805, 31802, 31815, 31794, 31796, 31799, 31797, 31807, 31800, 31801); 1 ? [AL], Kiyite, 7 Apr. 1969, leg. M.J. Celis and M. Lejeune (RMCA: 36199); 1 ㅇ [SS], Léopoldville [= Kinshasa], 16 Jul. 1942, leg. Colback (RMCA: 31127); 1 [ [AL], Luebo, 25 Jul. 1927, leg. James Paul Chapin (AMNH: 82375); 1 + [SA], Luena, 23 Sep. 2009, leg. Alexandre Hassanin, Nicolas Nesi, Célestin Pongombo, Peter Vallo and Didier Tshikung (MNHN: ZM-2011-782); 1 juv § [SA], Lukonzolwa, 20 Nov. 1911, leg. Louis Stappers (RMCA: 16490); 1 ad $\widehat{\partial}$ [SS], Lulimba, 9 Oct. 1953, leg. Alexandre Prigogine (RMCA: 22355); 1 ad $q$ [SA], Lulimbi, prior to 13 Jun. 1977, leg. M. Lejeune (RMCA: 77.029-M-0001); 1 ㅇ [SB], Lurhogole

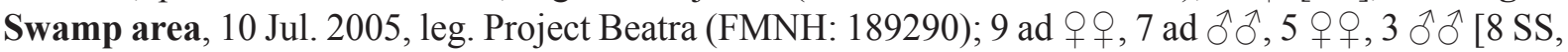
5 SA, 2 SK, 1 SO, 8 SB], Lwiro [= Luiro], 22 Jun. 1964, 10 Jul. 1964, 14 Jul. 1964, 15 Jul. 1964, 
17 Jul. 1964, 27 Nov. 1964, leg. Svepel, 9 Jul. 2001, 13 Jul. 2001, 29 Jul. 2002, leg. Michael H. Huhndorf, 14 Oct. 1963, 28 Nov. 1964, leg. Fritz Dieterlen, 24 Mar. 1963, 25 Sep. 1963, 27 Sep. 1963, 20 Dec. 1965, between 1 Aug. 1963 and 31 Aug. 1963, leg. P. Kunkel, 6 Apr. 1967, 28 Apr. 1967, leg. Alexandre Prigogine (ZMZ: 15528, FMNH: 171628, 171629, ZMZ: 15534, 16678, SMNS: 7289, ZMZ: 15594, 14140, SMF: 31810, ZMZ: 15535, SMF: 31803, 31812, 31811, ZMZ: 15581, RMCA: 35070, SMNS: 7290, FMNH: 173292 to 173294, RMCA: 35071, FMNH: 171625 to 171627, SMF: 31809); 1 ? [AL], Lwiro Katana [= Luiro], between 1 Aug. 1954 and 31 Oct. 1954, leg. Laurent (RMCA: 27505); 1 ad $\lesssim$ [SK], Mahagi Port, 5 May 1925, leg. Henri Schouteden (RMCA: 9004); 1 + [AL], Moba, unknown date, leg. H. Bomans (BMNH: 1957.431); 1 ad ô, 1 sad ?, 1 ? [2 SS, 1 SK], Mount Wago [= Wago, Mount], 18 Sep. 1952, leg. unknown collector, between 13 Sep. 1952 and 18 Sep. 1952, between 1 Jan. 1951 and 28 Mar. 1952, leg. Alexandre Fain (RBINS: 21667, RMCA: 20803, 23165); 1 ad [SA], Mukwande River, prior to 28 Dec. 1971, leg. Gaston-François de Witte (RMCA: 37108); 1 ? [UN], Mulala, unknown date, leg. unknown collector (INPBC: W332); 1 ad $\delta$ [SS], Mulongo, 30 Oct. 1974, leg. Michael D. Gallagher (HZM: 32.7869); 1 ad क, 1 §, 4 ?? [2 SS, 4 AL], Mulungu, 12 Feb. 1947, prior to 1 Feb. 1940, between 1 Jan. 1948 and 31 Dec. 1948, leg. F.L. Hendricks (RMCA: 19656, 19651, 15875 to 15878); 1 sad ? [SK], Mutembu Kaniki Kapangu [= Kaniki Kapangu],

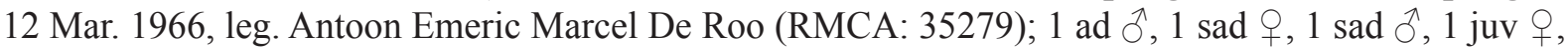
1 ô, 3 ?? [2 SA, 1 AL, 2 SO, 3 UN], Mutsora, unknown date, 14 May 1952, leg. unknown collector, 19 Nov. 1952, 1 Sep. 1954, 7 Jan. 1955, 2 Feb. 1955, leg. Gaston-François de Witte (INPBC: W1240, W1367, W1475, RMCA: 37110, RBINS: 17292, RMCA: 37106, 37113, 37112); 1 ? [UN], Ndotwe, unknown date, leg. unknown collector (INPBC: V1439); 1 juv $\widehat{\jmath}$ [SO], Ngokai, 21 Mar. 1953, leg. Gaston-François de Witte (RMCA: 37107); 1 ad $\widehat{\jmath}$ [SS], Nkene, 6 May 1959, leg. Uwe Rahm (RMCA: 28030); 1 juv ô [SS], Nyakaliba, 10 Feb. 1954, leg. Gaston-François de Witte (RBINS: 17293); 1 ㅇ [UN], Panga, between 1 Sep. 1925 and 30 Sep. 1925, leg. Henri Schouteden (RMCA: 9139a); 1 juv ô, 1 o, 1 ภ, 1 ? [4 SS], Parc National de Garamba [= Garamba National Park], 15 Jan. 1952, 4 Feb. 1952, 2 Apr. 1952, leg. H. de Saeger et al. (RBINS: 13512, 13519, 13514, 13515); 2 ad $\precsim \widehat{\partial}, 2$ sad 우우, 3 우우, 1 ㄱ, 15 ?? [3 SS, 1 SA, 2 AL, 2 SK, 15 UN], Rutshuru, unknown date, 8 Jan. 1938, 10 Jan. 1938, leg. unknown collector, 1 Mar. 1937, 30 Mar. 1937, leg. J. Ghesquière, 1 Aug. 1925, 3 Aug. 1925, leg. Edmund Heller, 23 May 1927, leg. James Paul Chapin, Dewitt L. Sage, Frank P. Mathews and The Ruwenzori-Kivu Expedition, between 1 Jun. 1935 and 30 Jun. 1935, leg. GastonFrançois de Witte (INPBC: V125, V1310, V1382.2, V1386.2, V1387, V1423, V1525.2, V1574, V1592, V1641, V397, V398, V408, V411, W618, RMCA: 13825, FMNH: 26497, RBINS: 4787, AMNH: 82378, FMNH: 30578, RMCA: 13867, RBINS: 4786, RMCA: 17082); 2 ad $q$ ㅇ, 1 ad $\lesssim$ [3 SK], Ruwenzori SE, unknown date, leg. R.E. Dent (BMNH: 1906.12.4.10, 1906.12.4.7, 1906.12.4.9); 1 ad $q$ [SS], Semmio [= Zemio], 1 Jan. 1884, leg. Bohndorff(RMNH: MAM.27276); 1 q [SS], Topetope, 9 Jul. 1966, leg. Alexandre Prigogine (RMCA: 34380); 1 ad $q$ [SO], Tshibinda, 16 Sep. 1950, leg. F.L. Hendricks

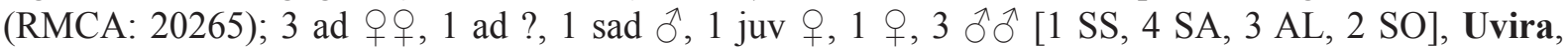
unknown date, 17 Jun. 1958, leg. Georges Marlier, unknown date, leg. Waldo LaSalle Schmitt and E. Baker, 1 Feb. 1910, leg. Graub, 12 Sep. 1951, leg. Narcisse Leleup, 23 Jan. 1962, 3 Feb. 1962, leg. Kiss, 4 May 1950, leg. Laurent (RMCA: 79.011-M-0001, USNM: 301713, 301714, NMW: 4880, RMCA: 22755, 22756, 27987, 31008, 31009, 79.010-M-0004); 1 ad $\widehat{\jmath}, 1$ sad ? [1 SS, 1 SK], Mount Wago, between 1 Jan. 1951 and 31 Dec. 1951, leg. Alexandre Fain (RMCA: 23149, 23150).

RWANDA: 3 ad $\widehat{\partial}, 2$ sad $\widehat{\partial}, 1$ juv ?, 7 ?? [7 SS, 2 SA, 4 AL], Astrida [= Butare], 3 May 1950, 10 Jan. 1951, leg. Aureliaan, 12 Oct. 1976, 14 Oct. 1976, leg. Frieder Meier, 14 Oct. 1955, between 1 May 1955 and 31 May 1955, leg. Alexandre Fain, 20 Feb. 1962, leg. Van Parijs, prior to 14 Apr. 1955, leg. P.G. Vercammen (RMCA: 20492, MHNG: 1872.016 to 1872.019, RMCA: 23140, MHNG: 1872.020,

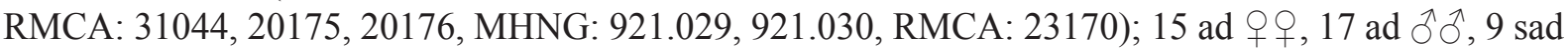

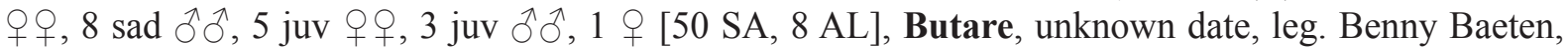
11 Dec. 1984, 20 Dec. 1984, 23 Dec. 1984, 24 Dec. 1984, 25 Dec. 1984, 31 Dec. 1984, 1 Jan. 1985, 
VAN CAKENBERGHE V. et al., The bats of Congo, Rwanda and Burundi revisited

2 Jan. 1985, 14 Jan. 1985, 15 Jan. 1985, leg. Benny Baeten and Luc Janssens, 7 Oct. 1981, 8 Oct. 1981, 9 Oct. 1981, 8 Nov. 1981, leg. Frits De Vree, Benny Baeten and Victor Van Cakenberghe (RMCA: 87.046-M-0350 to 87.046-M-0356, 87.046-M-0362, 87.046-M-0376 to 87.046-M-0378, 85.006$\mathrm{M}-0469,85.006-\mathrm{M}-0480,85.006-\mathrm{M}-0491$ to $85.006-\mathrm{M}-0494,85.006-\mathrm{M}-0490,85.006-\mathrm{M}-0470$ to 85.006-M-0475, 85.006-M-0479, 85.006-M-0483, 85.006-M-0484, 85.006-M-0500, 85.006-M-0501, 85.006-M-0508, 85.006-M-0509, 85.006-M-0478, 85.006-M-0481, 85.006-M-0482, 85.006-M-0495 to 85.006-M-0499, 85.006-M-0506, 85.006-M-0507, 85.006-M-0505, 85.006-M-0476, 85.006-M-0502, 85.006-M-0503, 85.006-M-0519, 85.006-M-0466, 85.006-M-0467, 85.006-M-0477, 85.006-M-0504, 85.006-M-0468, 82.006-M-1076 to 82.006-M-1078, 82.006-M-1031 to 82.006-M-1033, 82.006-M-

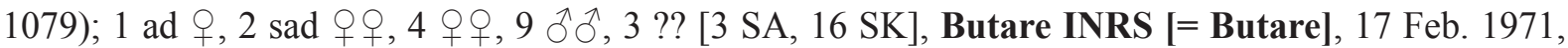
20 Feb. 1971, 22 Apr. 1971, leg. M. Lejeune, 23 Apr. 1971, leg. A. Elbl (RMCA: 74.020-M-0048 to 74.020-M-0054, 74.020-M-0056 to 74.020-M-0062, 74.020-M-0055, 74.020-M-0063, 35912 to 35914);

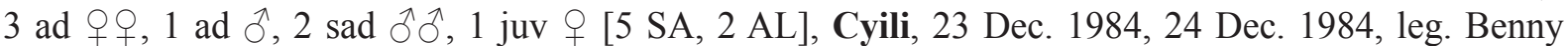
Baeten and Luc Janssens (RMCA: 85.006-M-0485, 85.006-M-0511, 85.006-M-0512, 85.006-M-0486 to 85.006-M-0488, 85.006-M-0510); 1 ad $\$, 1$ ad $\delta, 1$ sad $\delta$ [3 SA], Gafumba, 12 Jan. 1985, leg.

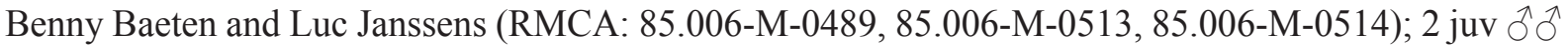
[2 SA], Gafunzo Kigaga, 5 Jan. 1985, 6 Jan. 1985, leg. Benny Baeten and Luc Janssens (RMCA: 85.006-M-0515, 85.006-M-0516); 4 ad 웅, 2 ad $\widehat{\jmath} \hat{\delta}$ [6 SS], Gakoma (Mission), unknown date, 20 Sep. 1964, 21 Sep. 1964, 27 Sep. 1964, 28 Sep. 1964, leg. Svepel (ZMZ: 15540, 15544, 16677,

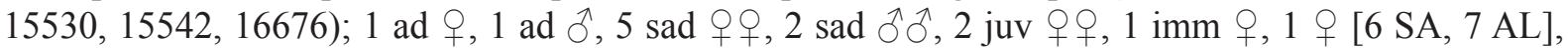
Gisenyi, 21 Oct. 1980, leg. Danny Meirte, 21 Oct. 1981, leg. Frits De Vree, Benny Baeten and Victor Van Cakenberghe, 28 Jun. 1980, leg. Hans H. de Iongh (RMCA: 80.055-M-0015 to 80.055-M-0017, 80.055-M-0025 to 80.055-M-0031, 82.006-M-1034, ZMA: MAM.21241, MAM.21242); 1 ad $\overbrace{}^{\uparrow}$ [SA], Gitovu, 28 Apr. 1971, leg. A. Elbl (RMCA: 35911); 1 sad + [AL], Karama, 13 Oct. 1981, leg. Frits

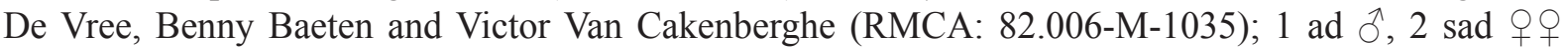
[2 SA, 1 AL], Kayonza, 6 Nov. 1980, leg. Danny Meirte (RMCA: 80.055-M-0049 to 80.055-M-0051);

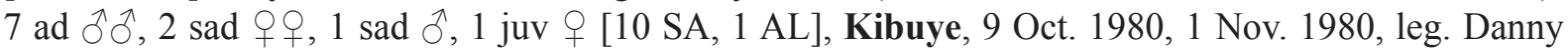
Meirte, 3 Dec. 1971, leg. Alena Vanderborght-Elbl, G. Mathys and A. Elbl (RMCA: 80.055-M-0019, 80.055-M-0020, 80.055-M-0042 to 80.055-M-0048, 73.058-M-0001, 80.055-M-0010); 3 ad $q$, 5 ad

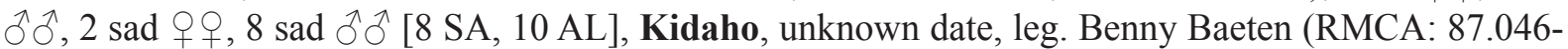

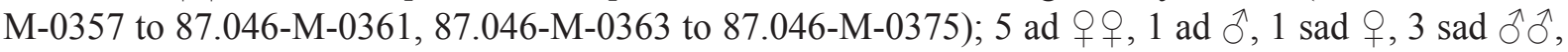
1 juv $\bigcirc$ [7 SA, 4 AL], Kigali, 19 Nov. 1971, leg. Alena Vanderborght-Elbl, G. Mathys and A. Elbl, 26 Oct. 1980, 27 Oct. 1980, leg. Danny Meirte (RMCA: 73.057-M-0011, 80.055-M-0033 to 80.055M-0035, 80.055-M-0018, 80.055-M-0036 to 80.055-M-0041); 1 , $1 \curvearrowright$ [2 AL], Kisenyi [= Gisenyi], 5 Jul. 1927, leg. James Paul Chapin, Dewitt L. Sage, Frank P. Mathews and The Ruwenzori-Kivu Expedition (AMNH: 82376, 82377); 1 ad $q, 1$ juv $\widehat{\partial}$ [2 SA], Mabanza, 10 Oct. 1980, leg. Danny Meirte

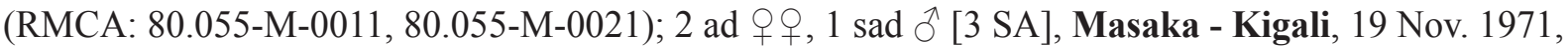
leg. Alena Vanderborght-Elbl, G. Mathys and A. Elbl (RMCA: 73.057-M-0003 to 73.057-M-0005); 4 ad

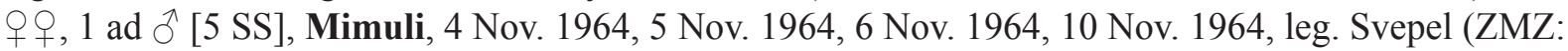
15539, 15541, 15536, 14141, 15527); 3 우 [3 SO], Mugusa, Mount, 12 Jun. 1968, leg. unknown collector (RBINS: 16031 to 16033); 3 ad $+q_{1}, 1$ ad $\partial$, 1 sad $\partial$, 2 emb ?? [5 SA, 2 AL], Muhasi [= Muhazi], 20 Aug. 1971, 23 Aug. 1971, 25 Aug. 1971, leg. Alena Vanderborght-Elbl, G. Mathys and A. Elbl (RMCA: 73.057-M-0006, 73.057-M-0001, 73.057-M-0007, 73.057-M-0002, 73.057-M-0008 to

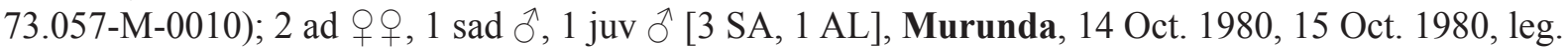
Danny Meirte (RMCA: 80.055-M-0022, 80.055-M-0012, 80.055-M-0023, 80.055-M-0024); 1 ๙ [SS], Musha, 25 Mar. 1968, leg. Félix / Michel Anciaux de Faveaux (RBINS: 16023); 4 ad $q$, 1 ad $§, 4$ sad

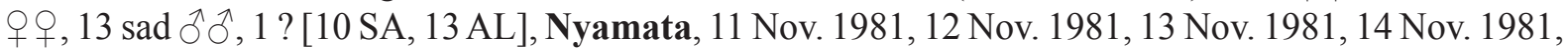
15 Nov. 1981, 16 Nov. 1981, leg. Frits De Vree, Benny Baeten and Victor Van Cakenberghe (RMCA: 82.006-M-1093, 82.006-M-1101, 82.006-M-1062, 82.006-M-1102 to 82.006-M-1104, 82.006-M-1058, 82.006-M-1105, 82.006-M-1059, 82.006-M-1094 to 82.006-M-1096, 82.006-M-1055, 82.006-M-1060, 
82.006-M-1097 to 82.006-M-1099, 82.006-M-1056, 82.006-M-1057, 82.006-M-1061, 82.006-M-1092,

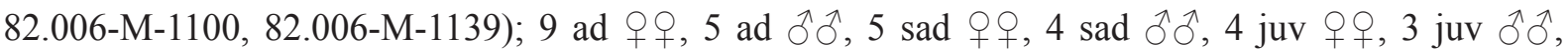
$1 \mathrm{imm} 9$ [15 SA, 16 AL], Nyumba Cave, 3 Oct. 1981, 6 Oct. 1981, 7 Oct. 1981, leg. Frits De Vree, Benny Baeten and Victor Van Cakenberghe (RMCA: 82.006-M-1037, 82.006-M-1039, 82.006-M-1040, 82.006-M-1042 to 82.006-M-1044, 82.006-M-1047, 82.006-M-1049, 82.006-M-1054, 82.006-M-1080 to $82.006-\mathrm{M}-1090,82.006-\mathrm{M}-1140,82.006-\mathrm{M}-1036,82.006-\mathrm{M}-1038,82.006-\mathrm{M}-1041,82.006-\mathrm{M}-$ 1045, 82.006-M-1048, 82.006-M-1050 to 82.006-M-1053, 82.006-M-1091); 1 ad $\curvearrowright, 1$ juv [3 SA], Nyundo, 17 Oct. 1980, 18 Oct. 1980, 23 Oct. 1980, leg. Danny Meirte (RMCA: 80.055-M0013, 80.055-M-0014, 80.055-M-0032); 1 sad $q$ [SA], Rubengera (Mission), between 1 Jan. 1931

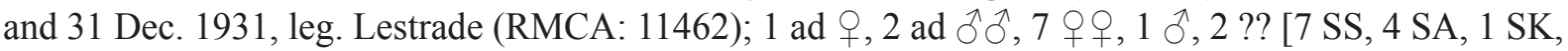
1 SO], Rubona, 18 Jan. 1966, 15 Oct. 1966, 22 Nov. 1966, 4 Dec. 1966, 10 Dec. 1966, 21 Dec. 1966, 27 Dec. 1966, 8 Jan. 1967, leg. Félix / Michel Anciaux de Faveaux, between 1 Jan. 1985 and 30 Jun. 1985, leg. Théoneste (RBINS: 16028 to 16030, 15481.1, 15481.2, 16035, 16027, 16026, 16024, 16025,

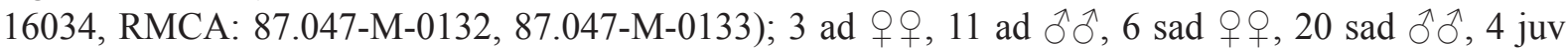

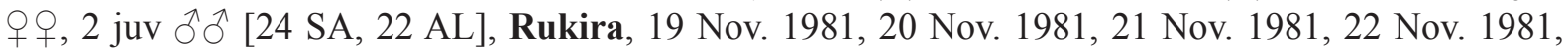
23 Nov. 1981, 24 Nov. 1981, 25 Nov. 1981, 26 Nov. 1981, leg. Frits De Vree, Benny Baeten and Victor Van Cakenberghe (RMCA: 82.006-M-1106, 82.006-M-1063, 82.006-M-1066, 82.006-M-1067, 82.006M-1107 to $82.006-\mathrm{M}-1111,82.006-\mathrm{M}-1117$ to $82.006-\mathrm{M}-1120,82.006-\mathrm{M}-1064,82.006-\mathrm{M}-1070$ to 82.006-M-1073, 82.006-M-1121 to 82.006-M-1125, 82.006-M-1065, 82.006-M-1074, 82.006-M-1112, 82.006-M-1113, 82.006-M-1126 to 82.006-M-1131, 82.006-M-1068, 82.006-M-1114, 82.006-M-1132 to $82.006-\mathrm{M}-1135,82.006-\mathrm{M}-1075,82.006-\mathrm{M}-1115,82.006-\mathrm{M}-1116,82.006-\mathrm{M}-1136,82.006-\mathrm{M}-1069$, 82.006-M-1137, 82.006-M-1138); 2 ad ठิ ठิ [1 SA, 1 AL], Shangi, 5 Jan. 1985, 6 Jan. 1985, leg. Benny Baeten and Luc Janssens (RMCA: 85.006-M-0517, 85.006-M-0518).

\section{Epomophorus minimus Claessen \& De Vree, 1991}

DEMOCRATIC REPUBLIC OF THE CONGO: 3 우, 1 đ [4 SA], Kitombongo [= Kintobongo], 24 Sep. 2009, 25 Sep. 2009, 26 Sep. 2009, leg. Alexandre Hassanin, Nicolas Nesi, Célestin Pongombo, Peter Vallo and Didier Tshikung (MNHN: ZM-2011-783 to ZM-2011-786); 1 q [SA], Luena, 21 Sep. 2009, leg. Alexandre Hassanin, Nicolas Nesi, Célestin Pongombo, Peter Vallo and Didier Tshikung (MNHN: ZM-2011-787).

\section{Epomophorus minor Dobson, 1880}

DEMOCRATIC REPUBLIC OF THE CONGO: 1 ? [UN], Albert National Park [= Virunga National Park], unknown date, leg. unknown collector (INPBC: V720); $1 \mathrm{imm} \hat{\jmath}, 1$ ㅇ [2 SK], Albertville [= Kalemie], 4 Mar. 1947, 5 Jun. 1947, leg. unknown collector (RBINS: 9065, 9066); 1 ? [UN], Bunyenzi, unknown date, leg. unknown collector (INPBC: W1515); 1 juv $\lesssim[A L]$, Congo da Lemba, between 13 Jan. 1900 and 22 Jan. 1900, leg. Paul Van Bellighen (RMCA: 408a); 1 q [SK], Gandajika, 10 Dec. 1951, leg. unknown collector (RBINS: 14599); 1 foetus ?, 1 ㅇ [1 SS, 1 AL], Kakonda, 8 Oct. 1942, leg. F.L. Hendricks (AMNH: 118866, 118867); 1 juv $q$, 1 juv $\precsim$ [2 AL], Moba, 19 Nov. 1953, 23 Apr. 1954, leg. H. Bomans (RMCA: 26271, 26272); 1 \& [SK], Rutshuru, 1 Jul. 1937, leg. unknown collector (RBINS: 4788).

\section{Epomophorus wahlbergi (Sundevall, 1846)}

BURUNDI: 1 [SB], Giserama, Colline, 31 Jul. 1991, leg. Julian C. Kerbis Peterhans (FMNH: 148972); $1 \mathrm{imm} \widehat{\partial}$ [SA], Kigamba, 15 Jul. 1946, leg. Jacques Verschuren (RBINS: 19928); 4 $\widehat{\partial}$ [3 AL, 1 SO], Mabaye [= Mabayi], 20 Dec. 1970, 10 Dec. 1971, leg. P.L. Niort (MHNG: 1499.092, 1499.093, 1499.090, 1499.091); 1 ㅇ [SB], Mumushwizi Valley, 23 Aug. 1993, leg. Jay L. Udelhoven (FMNH: 155942). 
DEMOCRATIC REPUBLIC OF THE CONGO: 1 ad $\widehat{\partial}$ [SA], unknown locality, 17 Oct. 1950, leg.

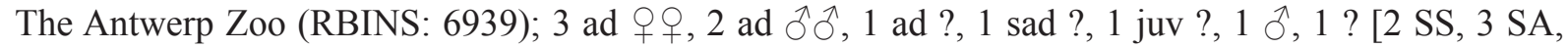
2 AL, 3 SK], Banana, 1 Jan. 1889, leg. P. Hesse, 25 Jul. 1920, between 26 Jul. 1920 and 26 Aug. 1920, leg. Henri Schouteden, 9 May 1910, leg. Abel Gruvel, prior to 3 Jun. 1936, leg. Schwetz (SMF: 2510, RMCA: 6539, 6540, MNHN: ZM-MO-1911-1565, ZM-MO-1911-1565A, ZM-MO-1911-1565B, ZMMO-1911-1565C, RMCA: 6541, 13303, 13304); 1 ad,+ 1 juv ô [2 SK], Banana or Netonna, unknown date, prior to 31 Dec. 1889, leg. P. Hesse (SMF: 2511, 2512); 1 \& [UN], Bas Congo (former) Province [= Kongo Central Province], prior to 31 Dec. 1897, leg. Alphonse Cabra (RMCA: 73); 1 sad ? [SK], Biakabomba, between 1 Jan. 1931 and 31 Dec. 1931, leg. Betu Nsankulu (RMCA: 79.032-M-0019); 1 ? [SK], Bokungu, between 1 Jan. 1949 and 9 Sep. 1949, leg. L. Dupuis (RMCA: 18844b); 1 ad ô, $1 \mathrm{sad}$ ㅇ, 3 우, 1 J , 30 ?? [7 SS, 1 SA, 21 AL, 1 SK, 6 SO], Boma, 5 Jul. 1920, between 1 Oct. 1920 and 31 Oct. 1920, leg. Henri Schouteden, 7 Oct. 1913, leg. Styczynski, between 1 Apr. 1936 and 30 Sep. 1936, leg. Schwetz, prior to 25 Nov. 1937, between 1 Aug. 1928 and 31 Aug. 1928, leg. Gerard, prior to 1 Dec. 1950, leg. Van Riel, prior to 26 May 1933, leg. Colsoulle, prior to 30 Mar. 1934, leg. Institute of Tropical Medicine "Prince Leopold", prior to 4 Aug. 1937, leg. Dartevelle (RMCA: 6532, 6538, 2484a, 2484b, 13705, 13706, 16261, 16204, 16205, 20177, 20178, 14182, 12125a to 12125d, $12127 \mathrm{j}$ to $12127 \mathrm{~m}, 12251$ to $12256,13946,13948$ to $13950,13952,13953,13955$ to 13958$) ; 1$ juv ㅇ [SK], Byonga, 22 Jan. 1965, leg. Alexandre Prigogine (RMCA: 33285); 1 ad $\lesssim$ [SS], Cape Lopez, 10 Feb. 1915, leg. Herbert Lang, James Paul Chapin and The American Museum Congo Expedition (AMNH: 48662); 1 ? [AL], Congo da Lemba, prior to 22 Jan. 1900, leg. Paul Van Bellighen (RMCA: 409); $1 \mathrm{imm}+$ [UN], Gandajika, between 1 Oct. 1948 and 31 Oct. 1948, leg. P. de Francquen (RBINS: 13108); 1 ? [UN], Ibembo, unknown date, leg. unknown collector (RMCA: 20278); 1 ad $\widehat{\diamond}$ [SK], Island of Idjwi , 19 Jul. 1965, leg. Alexandre Prigogine (RMCA: 33625); 1 § [SO], Kabanga, 12 Jan. 1952, leg. L. Spaute (RBINS: 13329); 1 sad + [SK], Kamueshi [= Kamuesha], between 1 Jan. 1931 and 31 Dec. 1931, leg. Betu Nsankulu (RMCA: 79.032-M-0018); 2 ?? [2 UN], Kananga, 31 Mar. 1924, leg. P. Callewaert (NMBA: 3800, 7695); 3 우 [3 SK], Kanzenze Mission, 1 Oct. 1948, leg. unknown collector (RBINS: 13280 to 13282); 2 sad 99 [1 SA, 1 AL], Lubondai-Tshimbulu [= Lubondaie-

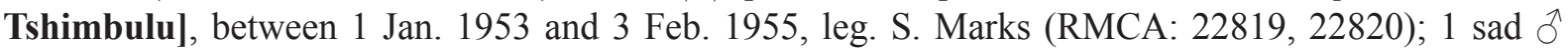

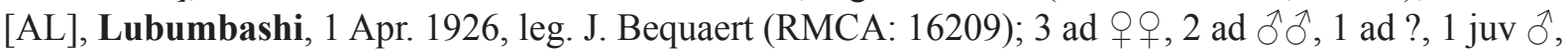
3 우, 11 ?? [14 SS, 3 SA, 1 AL, 2 SK, 1 SO], Luluabourg [= Kananga], 1 Jan. 1963, 18 Apr. 1963, leg. Jan Deheegher, 21 Sep. 1963, 22 Sep. 1963, 1 Feb. 1964, 14 Feb. 1964, 22 Feb. 1964, 2 Mar. 1964, 18 Apr. 1964, 6 May 1964, 10 May 1964, 14 Dec. 1964, 21 Feb. 1965, 27 Feb. 1965, 16 Mar. 1965, 22 Mar. 1965, 3 Aug. 1968, between 1 Jan. 1964 and 31 Dec. 1964, leg. Antoon Emeric Marcel De Roo, 26 Nov. 1922, leg. unknown collector (RMCA: 31554, AMNH: 207736, RMCA: 32651, AMNH: 207737, 206843, RMCA: 33409, 33644, 31550, 32387, 32378, 33407, 32661, AMNH: 207738, RMCA: 33410, 32662, MNHN: ZM-MO-1960-3859, ZM-MO-1960-3860, RMCA: 33408, 35609,

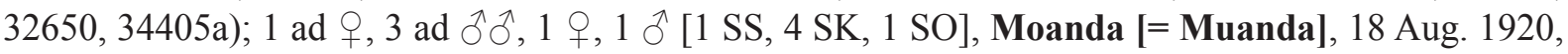
21 Aug. 1920, between 19 Aug. 1920 and 21 Aug. 1920, leg. Henri Schouteden (RMCA: 6534, 7290, 6533, 6535, 6537, 6536); 1 9, 1 ? [2 SO], Mulongo, unknown date, leg. Gerard (RMCA: 10515, 10517); $1 \widehat{\delta}[\mathrm{SS}]$, Mungamba, 17 Jan. 1982, leg. Betu Nsankulu (RMCA: 83.025-M-0005); 3 우, 1 ô, 1 ? [5 AL], 4 km from Muvule Cave, 8 Oct. 2009, 9 Oct. 2009, leg. Alexandre Hassanin, Nicolas Nesi, Célestin Pongombo, Peter Vallo and Didier Tshikung (MNHN: ZM-2011-799 to ZM-2011-803);

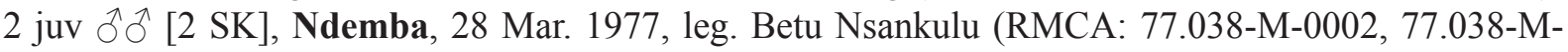
0003); 3 के $\delta$ [3 AL], Nyabiondo, 29 Dec. 1990, 30 Dec. 1990, leg. J.P. Lubula Bulambo and Ngenge Masumbuko Kamitongo (ZMA: MAM.24349 to MAM.24351); 2 ?? [2 UN], Rutshuru, unknown date,

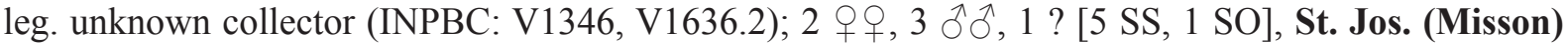
[= Mikalaya], 23 Nov. 1922, 22 Dec. 1923, 7 Aug. 1924, 30 Nov. 1924, leg. Richard Callewaert (AMNH: 86259, 86255, 86256, 86258, 86251, 86252); 4 ?? [4 SS], St. Joseph de Luluabourg Mission [= Mikalaya], 5 May 1922, leg. Richard Callewaert (RMCA: 7537 to 7540); $1 \mathrm{sad}$ ?, 2 우 , 2 ふぇ [2 SS, 1 SK, 2 SO], Tshibashi (River), 27 Feb. 1976, between 1 Feb. 1976 and 27 Feb. 1976, leg. 
Betu Nsankulu (RMCA: 76.015-M-0002 to 76.015-M-0006); 1 ? [AL], Tshikapa, between 1 Jan. 1921 and 31 Dec. 1921, leg. Henri Schouteden (RMCA: 16231); 2 우 [1 AL, 1 SB], Washiha Forest, 19 Jul. 2001, leg. Michael H. Huhndorf (FMNH: 171630, 171717).

RWANDA: 1 juv \& [AL], Astrida [= Butare], prior to 15 Jul. 1951, leg. The Antwerp Zoo (RMCA: 20496).

\section{Epomops Gray, 1866}

DEMOCRATIC REPUBLIC OF THE CONGO: 2 ㅇ $q$ [1 SA, 1 AL], Kikwit, 27 Jun. 2015, 29 Jun. 2015, leg. Prescott Musaba, Moïse Bipoo, Charle Andabhati, Benjamin Kirongozi and Kambale Karupao (UNIKIS: KKT2847, KKT2860); 2 ๙ึ [2 AL], Rethy, 20 Oct. 2015, leg. Anne Laudisoit et al. (UNIKIS: ITU703, ITU704).

Epomops dobsonii (Bocage, 1889)

DEMOCRATIC REPUBLIC OF THE CONGO: $1 \mathrm{imm}$ q, 2 우 [1 AL, $2 \mathrm{UN}]$, Elisabethville [= Lubumbashi], 1 Jan. 1930, leg. unknown collector, 12 Jan. 1956, leg. Félix / Michel Anciaux de Faveaux, between 1 Jan. 1930 and 31 Dec. 1930, leg. H.J. Bredo (RBINS: 6280, BMNH: 1957.432, ZMA: MAM.20163); 1 imm $ð$ [UN], Kambove, 6 Feb. 1907, leg. S.A. Neave (BMNH: 1907.12.13.1);

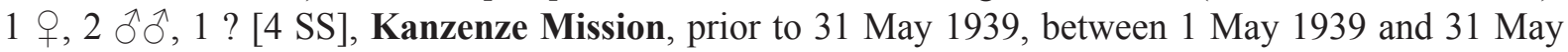
1939, leg. unknown collector, between 1 May 1939 and 31 May 1939, leg. R.P. Lefébure (RBINS: 1811a, 1811b, 1790, 1790B); 1 ठ [SS], Kasende, 26 Jun. 1934, leg. R.S. Pierrepont (MCZ: 31738); 1 ? [SS], Katentania, between 1 Jan. 1926 and 31 Jan. 1926, leg. R. Van Saceghem (RMCA: 8243); $1 \mathrm{imm}$ ?, 1 , 5 ठิ ठิ [5 SS, 2 SN], Kipangaribwe River, 17 Dec. 1947, 3 Jan. 1948, 12 Jan. 1948, 14 Jan. 1948, 19 Jan. 1948, leg. Gaston-François de Witte, between 1 Jan. 1941 and 31 Dec. 1943, leg. R. Grauvet (RBINS: 10668, 10666, 10664, 10669, 10674, 10675, 10678); 1 ? [AL], Mwera, prior to 26 Sep. 1956, leg. R.P. Th. de Caters (RMCA: 23945); 1 ? [AL], Vinamont Farm, 18 Nov. 1955, leg. Félix / Michel Anciaux de Faveaux (RMCA: 26267).

RWANDA: 1 ad $\widehat{\text { ô }}$ [SS], Rubona, 21 Dec. 1966, leg. Félix / Michel Anciaux de Faveaux (RBINS: 16037); 1 ad $\widehat{\partial}[\mathrm{SA}]$, Shangi Schad [= Shangi], 5 Jan. 1985, leg. Benny Baeten and Luc Janssens (RMCA: 85.006-M-0463).

\section{Epomops franqueti (Tomes, 1860)}

DEMOCRATIC REPUBLIC OF THE CONGO: 1 0 , 3 ?? [1 SS, 2 AL, 1 SO], unknown locality, unknown date, leg. Prescott Musaba, 12 Mar. 1956, leg. J.J. Laarman, between 1 Jan. 1985 and 31 Dec. 1986, leg. WHO and Marc Colyn, prior to 28 Aug. 1978, leg. Betu Nsankulu (RMCA: a1.097-M-3172, RMNH: MAM.16345, RMCA: 88.011-M-0010, 78.020-M-0002); 1 , 1 § [2 AL], Aketi, 23 Jun. 2015, 25 Jun. 2015, leg. Vlir/Aketi (UNIKIS: AKETI616, AKETI617); 1 ad $\widehat{\partial}$, 1 juv 우, 3 juv §ึ, 3 우, 2 ?? [7 SS, 1 AL, 1 SO, 1 UN], Avakubi, 30 Sep. 1912, 1 Oct. 1912, 30 Oct. 1912, 31 Oct. 1912, leg. Cuthbert Christy, 24 Sep. 1913, 27 Mar. 1914, 14 Apr. 1914, 28 Apr. 1914, 13 May 1914, 23 May 1914, leg. Herbert Lang, James Paul Chapin and The American Museum Congo Expedition (RMCA: 1421, AMNH: 48730, 48728, 48731, 48727, 48658, 48729, RMCA: 1611, 1599, 1600); 1 [ [UN], near Avakubi , between 8 Dec. 1974 and 15 Dec. 1974, leg. Michael D. Gallagher (HZM: 31.7858); 1 juv $\widehat{\jmath}, 1$ [2 SS], Bakwakenge [= Bakwa-Kenge], 20 Aug. 1979, 12 Oct. 1979,

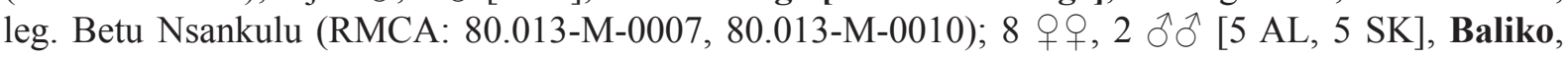
11 Jun. 2012, 12 Jun. 2012, 13 Jun. 2012, 15 Jun. 2012, leg. William ("Bill”) T. Stanley, prior to 31 Jan. 2007, leg. Guy-Crispin Gembu Tungaluna (FMNH: 219582 to 219586, RMCA: a1.097-M-0417 to a1.097-M-0421); 1 foetus ?, 1 क [1 UN, 1 SB], Bambesa, 3 Jun. 1938, leg. J.M. Vrijdagh (RMCA: 14797a, 14797b); 1 ? [SO], Bas Congo (former) Province [= Kongo Central Province], prior to 
VAN CAKENBERGHE V. et al., The bats of Congo, Rwanda and Burundi revisited

18 Apr. 1952, leg. Richard (RMCA: 22716); 1 [SS], Befale, 1 Sep. 1927, leg. J. Ghesquière (RMCA: 9342); 1 ठ [SS], Bena Mbala, 12 Jan. 1984, leg. Betu Nsankulu (RMCA: 84.035-M-0023); 1 juv ?, 1 ㅇ [2 SS], Bene Longo, 23 Aug. 1978, 12 Oct. 1979, leg. Betu Nsankulu (RMCA: 80.013-M-0011, 79.022M-0003); 1 imm $\lesssim$ [UN], Beni, unknown date, leg. Rudolf Grauer (NMW: 4879); 1 [SS], Beyi Kalonji, 22 Feb. 1980, leg. Betu Nsankulu (RMCA: 80.020-M-0002); 2 ?? [2 SS], Boangi, 17 Apr. 1954, leg. P. Verbuyt (RMCA: 22186, 22187); 6 ?? [5 AL, 1 SO], Boende, 25 Sep. 1939, leg. E.P. Wijnant, between 1 Jan. 1967 and 31 Dec. 1967, between 1 Jan. 1966 and 31 Jan. 1966, leg. P. Lootens (RMCA: 17335, 34163, 38649, 38654 to 38656); 1 ㅇ, 1 ô, 2 ?? [4 SS], Bokuma, 1 Oct. 1950, 2 Oct. 1950, 4 Oct. 1950, between 1 Oct. 1950 and 31 Oct. 1950, leg. P. Lootens (RMCA: 20220, 20219, 20221, 20393); 2 ?? [1 SS, 1 SO], Bokungu, prior to 20 Apr. 1950, between 1 Jan. 1949 and 9 Sep. 1949, leg. L. Dupuis (RMCA: 18844a, 19659); 1 ? [SS], Boma, unknown date, leg. J. Rodhain (RMCA: 7640); 10

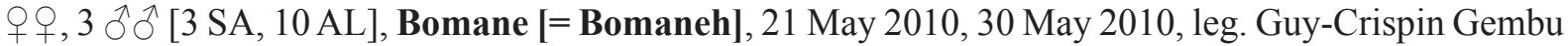
Tungaluna, Prescott Musaba and José Akaibe (UNIKIS: CRT2131 to CRT2133, CRT2142, CRT2173, CRT2176, CRT2180, CRT2183, CRT2198, CRT2199, CRT2063, CRT2065, CRT2066); 1 ô [AL], Bombanga - Bumba, 7 Aug. 1986, leg. WHO and Marc Colyn (RMCA: 88.011-M-0011); 2 ?? [2 SO], Bombo Lumene, 20 Aug. 1992, leg. Tshinyenye (RMCA: 92.119-M-0005, 92.119-M-0006); 1 ? [SO], Bongale, 22 Mar. 1951, leg. P. Lootens (RMCA: 20559); 1 ㅇ [AL], Bonzale, 13 Jul. 1979, leg. Frieder Meier (MHNG: 1870.042); 1 juv ?, 14 ?? [14 SS, 1 SO], Buta, 11 Mar. 1935, 13 Jan. 1936, 19 Jan. 1936, 12 Jan. 1944, 20 Jun. 1949, prior to 3 Oct. 1938, prior to 20 Dec. 1927, prior to 16 Jun. 1937, prior to 15 Jul. 1935, between 1 Jun. 1934 and 30 Jun. 1934, between 1 Jan. 1932 and 31 Dec. 1932, leg. Jozef Hutsebaut (RMCA: 12859, 17304, 13307, 13306, 18835, 11827, 12565 to 12569, 12858, 13854, 9388, 14841); 2 우, 5 ठึ $\widehat{~}[7 \mathrm{SS}$ ], Byonga, 24 Nov. 1962, 11 Jan. 1964, 12 Jan. 1964, 28 Jan. 1965, 22 Feb. 1965, 21 Sep. 1966, 23 Sep. 1966, leg. Alexandre Prigogine (RMCA: 32400, 32401, 34799, 33284, 34798, 31402, 33286); 1 Ô, 1 ? [2 SS], Dimbelenge, 27 Jun. 1980, 28 Jun. 1980, leg. Betu Nsankulu (RMCA: 80.042-M-0006, 80.042-M-0005); 8 우, 7 ठิ ठิ [4 SA, 11 AL], Djabir, 8 May 2011, 9 May 2011, 12 May 2011, 13 May 2011, leg. Prescott Musaba and Jonathan Kosele (UNIKIS: DJB148, DJB150, DJB151, DJB168, DJB002, DJB005, DJB021, DJB059, DJB066, DJB069, DJB072 to DJB075, DJB171); 8 우, 7 Љð [1 SS, 14 AL], Epulu, 16 Jun. 1958, leg. Station de domestication des okapis Epulu, 25 Aug. 2015, 26 Aug. 2015, 27 Aug. 2015, 29 Aug. 2015, 30 Aug. 2015, 31 Aug. 2015, leg. André Malekani (RMCA: 27751, UNIKIS: EPLU631, EPLU633, EPLU634, EPLU653, EPLU655 to EPLU657, EPLU661, EPLU678, EPLU692, EPLU693, EPLU697, EPLU698, EPLU701); 1 ? [SB], 2 km W of Epulu, 29 Jun. 1991, leg. Julian C. Kerbis Peterhans (FMNH: 149391); 18 ?? [1 AL, 17 UN], Faradje, unknown date, leg. unknown collector (UNIKIS: CRT801/G63, CRT802 to CRT805, CRT807, CRT808/G62, CRT809 to CRT812, CRT814, CRT816, CRT817, CRT818/G64, CRT819/G61, CRT820, CRT821/G70); 1 ad $\widehat{\partial}$ [SS], Fort Beni [= Vieux Beni], unknown date, leg. R.E. Dent (BMNH: 1906.12.4.59); $1 \mathrm{imm} \hat{\delta}[\mathrm{UN}]$, Gangala-na-Bodio, 31 May 1948, leg. unknown collector (RBINS: 11659); 1 ? [SS], Gemena, 26 Jun. 1959, leg. Jacobs (RMCA: 28087); 1 ? [SS], Ibembo, 17 Sep. 1950, leg. Jozef Hutsebaut (RMCA: 20204); 1 ad $\widehat{\partial}$ [AL], Ikela, prior to 4 Jan. 1958, leg. P. Lootens (RMCA: 27016); 2 우, $3 \widehat{\partial} \widehat{\partial}$ [5 UN], Île Mafi [= Mafi Island], 20 Feb. 2012, 29 Mar. 2012, 31 Mar. 2012, leg. Prescott Musaba, Tembele and Marc Boketshu (UNIKIS: CHIMA13, CHIMA31 to CHIMA33, CHIMA39); 6 우, 4 ふึ , 1 ? [4 AL, 7 UN], Île Mbiye [= Mbiye Island], 20 Feb. 2012, 14 Apr. 2012, 19 Apr. 2014, 20 Apr. 2014, between 31 Mar. 2012 and 13 Apr. 2012, leg. Prescott Musaba, 16 Jan. 2012, 18 Feb. 2012, 31 Mar. 2012, leg. Prescott Musaba, Tembele and Marc Boketshu (RMCA: a1.097-M-3178, UNIKIS: CHIM06, CHIM08, CHIM05/14, RMCA: a1.097-M-3176, a1.097-M-3177, UNIKIS: CHIM010/14, CHIM09/14, CHIM46, CHIM49, RMCA: a1.097-M-3179); 1 ठै [SS], Inkongo

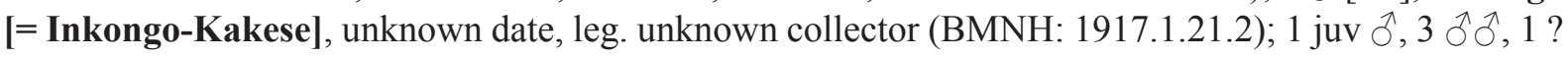
[2 SA, 3 AL], Irangi, 16 Mar. 1964, 17 Aug. 1964, leg. P. Kunkel, 16 Oct. 1990, 17 Oct. 1990, leg. Wim Bergmans (SMF: 31835, ZMA: MAM.24190, SMF: 31834, ZMA: MAM.24191, MAM.24192); 4 우 [4 UN], Isai River, between 16 Dec. 1974 and 23 Dec. 1974, leg. Michael D. Gallagher (HZM: 20.7833, 23.7836, 29.7856, 32.7859); 1 \& [SS], Isoga, 17 Jan. 1964, leg. Alexandre Prigogine (RMCA: 32402); 
3 우, 1 § [3 SS, $1 \mathrm{AL}$ ], Ituri River, unknown date, leg. unknown collector, unknown date, leg. Howard de Walden (BMNH: 1930.11.11.66 to 1930.11.11.69); 3 우우 [3 UN], Ituri River, Bridge, between 8 Dec. 1974 and 15 Dec. 1975, between 8 Dec. 1974 and 15 Dec. 1974, leg. Michael D. Gallagher (HZM: 16.7829, 25.7838, 22.7835); 1 đ [SS], Kagada, 20 Jul. 1952, leg. Alexandre Prigogine (RMCA:

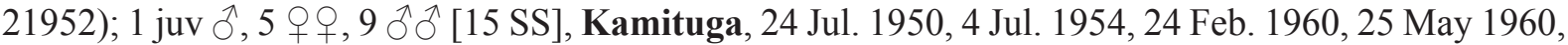
9 Nov. 1962, 26 Nov. 1962, 26 Jul. 1963, 15 Nov. 1963, 11 Mar. 1966, 21 Mar. 1967, 25 Mar. 1967, 26 Mar. 1967, 27 Mar. 1967, 11 Nov. 1968, leg. Alexandre Prigogine (RMCA: 34209, 35512, 31663, 35069, 28886, 20431, 35066, 35068, 30556, 35072, 31662, 31403, 35067, 23424, 31346); 1 ๙ [SS], Kamueshi [= Kamuesha], 3 Apr. 1979, leg. Betu Nsankulu (RMCA: 79.032-M-0016); 1 +, 5 § [8 SS, 1 SK], Kasai, 13 Dec. 1981, 17 Jan. 1982, 10 Feb. 1982, 17 Feb. 1982, prior to 12 Aug. 1983, leg. Betu Nsankulu (RMCA: 83.025-M-0012, 83.025-M-0017, 83.025-M-0018, 83.025-M-0014, 83.025-M0016, 83.025-M-0013，83.025-M-0011，83.025-M-0015，83.025-M-0024); 1 q [SS], Katabayi, 10 Apr. 1979, leg. Betu Nsankulu (RMCA: 79.032-M-0017); 1 ? [SS], Katende Falls, 2 Aug. 1957, leg. Vaillemans (RMCA: 26554); 2 juv ??, 1 q [3 AL], Katshungu, 30 Jan. 1950, leg. Laurent (RMCA: 79.010-M-0001 to 79.010-M-0003); 1 § [SS], Keseki, prior to 23 Jul. 1946, leg. N'Kele (RMCA: 17400); 3 우 [3 SA], Kikole-Pecheur, 17 May 1980, leg. M. Mutashia (SMF: 58070 to 58072); $1 \mathrm{imm}$ ? [UN], Kimpoko [= Kimpoko-Nsele], 22 Jun. 1979, leg. Tsh Mutashiya (NMW: 25774); 1 ๙

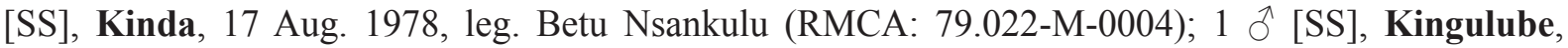
4 Dec. 1968, leg. Alexandre Prigogine (RMCA: 35506); 1 juv ô, 5 우의, 1 ? [1 SS, 1 AL, 1 SO, 4 UN], Kinshasa, unknown date, leg. Matashiya-Tshinyenye, 2 Jul. 1909, leg. Herbert Lang, James Paul Chapin and The American Museum Congo Expedition, 21 Apr. 1964, leg. Jo Van Orshoven, between 15 Jan. 1975 and 26 Jan. 1975, leg. Michael D. Gallagher (FMNH: 125133, AMNH: 48712, ZMA: MAM.11169, HZM: 26.7853, 27.7854, 33.7860, 8.7807); 2 ?? [2 UN], Kinshasa City Province, between 1 Apr. 1999 and 30 Apr. 1999, leg. unknown collector (SMF: 93241, 93242); 1 ad + [UN], Kinzambi Mission, 26 Jul. 1995, leg. Herwig Leirs et al. (MSB: 274829); 3 우, 3 §ో 22 May 1986, between 1 Apr. 1986 and 30 Apr. 1986, leg. Ndey B. Ifuta, between 1 Sep. 1985 and 30 Sep. 1985, leg. Marc Colyn, between 6 Jan. 1975 and 14 Jan. 1975, leg. Michael D. Gallagher (RMCA: 87.010-M-0001, 87.010-M-0011, 87.010-M-0012, 86.024-M-0003, 86.024-M-0004, HZM: 12.7811, 13.7812); 3 우우 [3 SS], Kitutu, 6 Feb. 1964, 14 Feb. 1964, 1 Mar. 1964, leg. Alexandre Prigogine (RMCA: 32403 to 32405); 1 ? [SA], Kivu region, 16 Mar. 1964, leg. P. Kunkel (SMF: 31833); 1 ? [SA], Koloka, between 1 Jun. 1911 and 30 Jun. 1911, leg. H. Schubolz (SMF: 6357); 4 q 9,7 $\widehat{\jmath}$, 1 ? [7 SA, 5 AL], Kona, 12 May 2010, 13 May 2010, 15 May 2010, 16 May 2010, 17 May 2010, leg. Guy-Crispin Gembu Tungaluna, Prescott Musaba and José Akaibe (UNIKIS: CRT1917, CRT1921, CRT1929, CRT1939, CRT1940, CRT1978, CRT1984, CRT1999, CRT2000, CRT2011, CRT2012, CRT2014); 1 ? [SK], Kongolo Island, between 1 Dec. 1977 and 31 Dec. 1977, leg. Marc Colyn (RMCA:

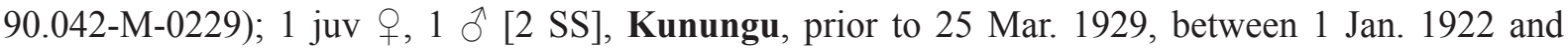
31 Dec. 1922, leg. Ngwe (RMCA: 7773, 9711); 1 ㅇ, 1 đ [2 AL], Lai Lay [= Lailai], 28 Apr. 1992, leg. Ngenge Masumbuko Kamitongo (ZMA: MAM.24562, MAM.24563); 6 $ぇ \hat{\jmath}, 1$ ? [1 SS, 1 AL, 2 SK, 2 SO, 1 UN], Léopoldville [= Kinshasa], unknown date, leg. Vandenbranden, 11 Apr. 1912, 16 Apr. 1912, leg. Cuthbert Christy, 12 Nov. 1945, leg. Jean Jacques Deheyn, 16 Jun. 1946, leg. M. Henrion, 18 May 1945, leg. Lepersonne, between 1 Feb. 1942 and 28 Feb. 1942, leg. Becquet (RMCA: 5381, 1538f, 17616b, 1538b, 17798, 17336, 17356b); 1 ㅇ [SA], Lieki, 1 May 2010, leg. Guy-Crispin Gembu Tungaluna, Prescott Musaba and José Akaibe (UNIKIS: CRT2036); 1 ad? Ô, 18 ?? [19 SS], Lovanium University [= Kinshasa], 1 Apr. 1962, 5 Apr. 1962, 7 May 1962, 26 May 1962, 5 Jun. 1962, 12 Jun. 1962, leg. De Bont, 10 Jan. 1962, 24 Sep. 1962, 24 Nov. 1962, 5 Jan. 1963, 3 May 1963, 7 May 1963, 16 Jul. 1963, 12 Apr. 1964, 19 Apr. 1964, 21 Apr. 1964, between 1 Apr. 1963 and 20 Mar. 1964, leg. De Bont and Jo Van Orshoven (RMCA: 31194, 32600, 32598, 31196, 32590, 32599, 32597, 32593, 32595, 31193, 32592, 32589, 31190, 31191, 31195, 31192, 32591, 32594, 32596); 1 ㅇ [SS], Luebo, 19 Dec. 1921, leg. Henri Schouteden (RMCA: 6646); 2 ડ̄ $\widehat{~[1 ~ S S, ~} 1$ AL], Lukolela, 25 Jul. 1930, leg. Franklin Edson, 25 Jul. 1930, leg. James Paul Chapin (AMNH: 86765, 86906); 1 juv ô, 1 juv ?, 2 q 0 
VAN CAKENBERGHE V. et al., The bats of Congo, Rwanda and Burundi revisited

[4 SS], Lukonga, 7 Oct. 1978, 2 Aug. 1979, 6 Aug. 1979, leg. Betu Nsankulu (RMCA: 80.013-M-0008, 80.013-M-0009, 80.013-M-0012, 79.022-M-0002); 1 ô, 1 ? [2 SS], Lulua [= Kananga], 2 May 1976,

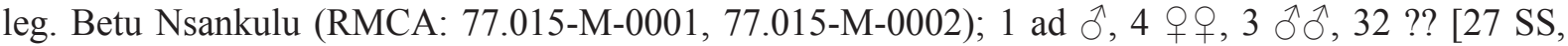
12 AL, 1 SO], Luluabourg [= Kananga], unknown date, leg. Richard Callewaert, unknown date, 18 Apr. 1963, leg. Jan Deheegher, 22 Sep. 1963, 11 Jan. 1964, 4 May 1964, 10 May 1964, 2 Sep. 1964, 14 Nov. 1964, 14 Feb. 1965, 20 Feb. 1965, 21 Feb. 1965, 22 Feb. 1965, 25 Feb. 1965, 27 Feb. 1965, 4 Mar. 1965, 6 Mar. 1965, 8 Mar. 1965, 19 Mar. 1965, 12 Apr. 1965, 30 Apr. 1965, 8 May 1965, 22 May 1965, 15 Sep. 1969, between 1 Nov. 1962 and 30 Nov. 1962, between 1 Jan. 1964 and 31 Dec. 1964, leg. Antoon Emeric Marcel De Roo (BMNH: 1926.7.6.55, 1926.7.6.56, RMCA: 31553, 31555, 31556, 31558, 31559, 32653, 32654, AMNH: 207739, RMCA: 33404, 33647, 33390, 33646, 35796, 31551, $31552,33403,32690,33391$ to $33393,33648,32663$ to 32665,33394 to $33396,33405,33397$ to 33399 , 32652, 33400 to 33402, 33406, 34405b, 31548); 1 q [AL], Lwana River, 12 Jun. 1992, leg. Ngenge

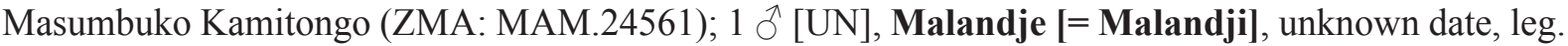
Mechow (ZMB: 10031); 4 우, 3 $\widehat{\partial} \widehat{\partial}$ [1 SS, 2 SK, 1 SO, 3 UN], Mambaka, unknown date, 17 Jan. 1913, 20 Jan. 1913, 21 Jan. 1913, 22 Jan. 1913, 2 Jan. 1914, 20 Jan. 1914, leg. Cuthbert Christy (BMNH:

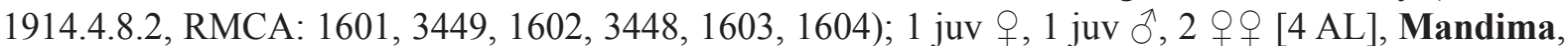
24 Jul. 1976, 29 Jul. 1976, 1 Aug. 1976, leg. Frieder Meier (MHNG: 1872.039, 1872.036 to 1872.038);

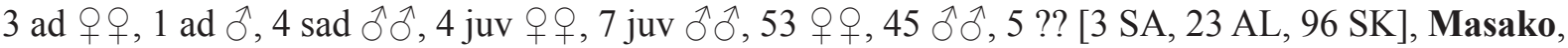
22 May 1986, 24 Nov. 1987, 25 Nov. 1987, 26 Nov. 1987, 27 Nov. 1987, 30 Apr. 1989, 25 Aug. 1989, 26 Aug. 1989, 27 Aug. 1989, 28 Aug. 1989, 30 Aug. 1989, 14 Sep. 1989, 21 Nov. 1989, 12 Dec. 1989, 17 Dec. 1989, 22 Dec. 1989, 28 Dec. 1989, 7 Jan. 1990, 21 Jan. 1990, 25 Jan. 1990, 26 Jan. 1990, 27 Jan. 1990, 28 Jan. 1990, 29 Jan. 1990, 10 Feb. 1990, 13 Feb. 1990, 14 Feb. 1990, 16 Feb. 1990, 17 Feb. 1990, 27 Feb. 1990, 28 Feb. 1990, 17 Mar. 1990, 23 Mar. 1990, 25 Mar. 1990, 27 Oct. 1990, 30 Oct. 1990, between 1 Oct. 1989 and 31 Oct. 1989, leg. Ndey B. Ifuta, 12 Jun. 2015, leg. Prescott Musaba, Moïse Bipoo, Charle Andabhati, Benjamin Kirongozi and Kambale Karupao (RMCA: 93.079M-0162 to 93.079-M-0164, UNIKIS: CHMS068, RMCA: 93.079-M-0136 to 93.079-M-0139, 93.079M-0190 to 93.079-M-0192, 93.079-M-0271, 93.079-M-0193, 93.079-M-0194, 93.079-M-0168, 93.079$\mathrm{M}-0169$, 93.079-M-0140, 93.079-M-0155 to 93.079-M-0161, 93.079-M-0129 to 93.079-M-0135, 87.010-M-0004, 87.010-M-0005, 93.079-M-0141 to 93.079-M-0144, 93.079-M-0262, 93.079-M-0001 to $93.079-\mathrm{M}-0008,93.079-\mathrm{M}-0200$ to $93.079-\mathrm{M}-0209$, 93.079-M-0263, 93.079-M-0314, 93.079-M0184, 93.079-M-0195, 93.079-M-0172 to 93.079-M-0174, 93.079-M-0009 to 93.079-M-0014, 93.079M-0210 to 93.079-M-0212, 93.079-M-0185, 93.079-M-0175, 93.079-M-0176, 93.079-M-0015 to 93.079-M-0020, 93.079-M-0213, 93.079-M-0264 to 93.079-M-0266, 93.079-M-0186, 93.079-M-0165, 93.079-M-0177 to 93.079-M-0180, 93.079-M-0196, 93.079-M-0021 to 93.079-M-0025, 93.079-M0187, 93.079-M-0188, 93.079-M-0154, 93.079-M-0166, 93.079-M-0167, 93.079-M-0199, 93.079-M0181, 93.079-M-0145 to 93.079-M-0150, 93.079-M-0189, 93.079-M-0171, 93.079-M-0182, 93.079M-0183, 93.079-M-0197, 93.079-M-0198, 93.079-M-0151 to 93.079-M-0153, 93.079-M-0126 to 93.079-M-0128); 1 ? [AL], Masako km 15 Buta, 22 May 1986, leg. Ndey B. Ifuta (RMCA: 87.010-M0003); 1 ? [SK], Mawambi, 8 Aug. 1913, leg. Cuthbert Christy (RMCA: 3450); 1 ad $\circ$ [SA], Mbwambala, 7 Jul. 1995, leg. Herwig Leirs et al. (RMCA: 97.021-M-0748); 3 ad $9+9,1$ ad $\widehat{\jmath}, 3$ juv

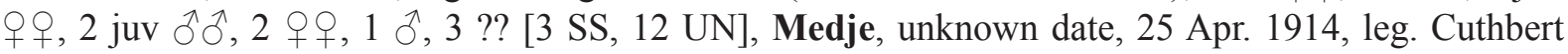
Christy, 21 Mar. 1910, 12 Sep. 1910, 14 Sep. 1910, 15 Sep. 1910, 2 Oct. 1910, 11 Oct. 1910, 11 Mar. 1914, 19 Mar. 1914, 22 Mar. 1914, 7 Apr. 1914, leg. Herbert Lang, James Paul Chapin and The American Museum Congo Expedition (BMNH: 1919.5.8.9, AMNH: 48726, 49205, RMCA: 12398, AMNH: 48713, 48719, 48721, 48722, 48716, 48714, 48715, 48723, RMCA: 4331, AMNH: 51309, RMCA: 12358); 1 embryo,+ 1 embryo $\widehat{\jmath}, 1$ embryo ? [2 SS, 1 AL], Mistandunga, 23 Jul. 1930, leg. Franklin Edson, 23 Jul. 1930, leg. James Paul Chapin (AMNH: 86766, 86767, 86907); 1 imm 우 [UN], Moera, unknown date, leg. Rudolf Grauer (NMW: 4878); 1 ? [UN], Mongbwalu, unknown date, leg. unknown collector (RMCA: 15566); 1 § [SO], Mulongo, unknown date, leg. Gerard (RMCA: 10514); 1 ㅇ, 1 ? [1 SS, 1 UN], Mutsora, unknown date, leg. unknown collector, 4 Apr. 1951, leg. R. Christiaens 
(INPBC: W703, RBINS: 13327); 1 imm +, 1 ? [1 SS, $1 \mathrm{UN}$ ], Mutwanga, unknown date, leg. unknown collector, 15 Jul. 1946, leg. J. de Wilde (INPBC: W1283, RBINS: 13326); 1 ? [SS], Nganza River, 27 May 1966, leg. Antoon Emeric Marcel De Roo (RMCA: 35277); 1 ad + [SS], Niangara, 7 Oct. 1910, leg. Herbert Lang, James Paul Chapin and The American Museum Congo Expedition (AMNH: 48717);

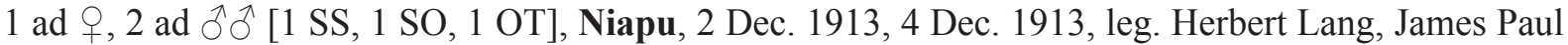
Chapin and The American Museum Congo Expedition (AMNH: 49196, 48724, 51308); 1 q [SO],

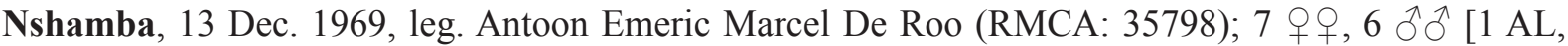
3 SK, 9 UN], Obenge, 4 Feb. 2013, 5 Feb. 2013, 7 Feb. 2013, leg. Prescott Musaba and André Malekani (UNIKIS: TLL351 to TLL353, TLL355, TLL360, TLL362, TLL372, TLL373, TLL378 to TLL380, TLL394, TLL416); 1 ? [AL], Omaniumdu, between 5 Jul. 1959 and 8 Jul. 1959, leg. Laurent (RMCA: 34353); 2 우 [2 SS], Panga, between 1 Sep. 1925 and 30 Sep. 1925, leg. Henri Schouteden (RMCA: 9139b, 9140); 2 ?? [2 SS], Paulis [= Isiro], between 1 Jan. 1947 and 31 Dec. 1947, leg. Abbeloos (RMCA: 17927, 17928); 1 ठ [UN], Pidigala Nord River, 23 Apr. 1952, leg. H. de Saeger et al. (RBINS: 13501); 2 우우 [1 SK, 1 UN], Pili Pili [= Pilipili], 19 Dec. 1912, 19 Dec. 1913, leg. Cuthbert Christy (RMCA: 1605, 3447); 2 우 [2 SK], Quatorze, 28 Jun. 2013, 29 Jun. 2013, leg. William («Bill»)

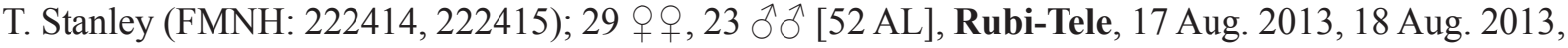
19 Aug. 2013, 20 Aug. 2013, 21 Aug. 2013, 22 Aug. 2013, 23 Aug. 2013, 24 Aug. 2013, 25 Aug. 2013, 26 Aug. 2013, 27 Aug. 2013, 28 Aug. 2013, leg. Guy-Crispin Gembu Tungaluna, Prescott Musaba and André Malekani (UNIKIS: RBTL483 to RBTL485, RBTL496, RBTL500, RBTL501, RBTL506 to RBTL509, RBTL511, RBTL513, RBTL517 to RBTL519, RBTL523 to RBTL527, RBTL531 to RBTL533, RBTL535 to RBTL539, RBTL543 to RBTL546, RBTL552, RBTL553, RBTL559 to RBTL561, RBTL565 to RBTL572, RBTL574, RBTL573, RBTL578 to RBTL582); 2 ?? [1 AL, 1 UN], Rutshuru, unknown date, leg. unknown collector, 27 May 1955, leg. Gaston-François de Witte (INPBC: V1626, RMCA: 37115); 1 ? [SS], Semliki River, between 1 Aug. 1932 and 15 Aug. 1932, leg. Henri Martin Hackars and Georges Marlier (RMCA: 12165); 1 imm ?, 1 foetus ?, 5 q 4 AL, 1 SK, 1 UN], Stanleyville [= Kisangani], unknown date, 26 May 1912, 26 Jun. 1912, 2 Jul. 1912, 20 Jul. 1912, prior to 12 Mar. 1913, leg. Cuthbert Christy, 4 May 1915, 1 Jul. 1915, leg. Herbert Lang, James Paul Chapin and The American Museum Congo Expedition (BMNH: 1914.4.8.1, AMNH: 49206, RMCA: 1414, 1417, 1415, 1411 to 1413, 1416, 1418, 1420, AMNH: 49203, RMCA: 1530a, 1531); 1 , 1 đ [2 AL], Tandala, 12 Jul. 1979, leg. Frieder Meier, 19 Jul. 1979, leg. C. Brian Robbins (MHNG: 1870.040, USNM: 463474); 1 juv +, 1 ㅇ, 1 o [2 SS, 1 UN], Teturi, 6 Feb. 1947, 30 Jun. 1947, 15 Mar. 1948, leg. unknown collector (RBINS: 7044, 7053, 7052); 1 § [UN], Tingasi, unknown date, leg. Emin Pascha / Pasha / Bey (BMNH: 1887.12.1.26); 1 ㅇ [SO], Tshibasha, 19 Sep. 1969, leg. Antoon Emeric Marcel De Roo (RMCA: 35797); 1 \& [SS], Tshibashi (River), 27 Feb. 1976, leg. Betu Nsankulu (RMCA: 76.015-M-0001); 1 \& [SS], Tshibungu, 27 Nov. 1978, leg. Betu Nsankulu (RMCA: 79.022M-0005); 2 ?? [2 SK], Tshopo, between 1 Jun. 1982 and 30 Jun. 1982, leg. Marc Colyn (RMCA: 90.042M-0230, 90.042-M-0231); 1 ? [AL], Tshoppo Falls [= Tshopo Falls], prior to 12 Mar. 1913, leg. Cuthbert Christy (RMCA: 1530b); 1 ? [AL], Tshuapa District, between 1 Jan. 1960 and 31 Dec. 1964, leg. P. Lootens (RMCA: 34184); 13 우, 14 ठิ ठิ [16 AL, 6 SK, 5 UN], Uma, 15 Aug. 2012, 16 Aug. 2012, 17 Aug. 2012, 18 Aug. 2012, 10 Apr. 2014, 11 Apr. 2014, 12 Apr. 2014, leg. Guy-Crispin Gembu Tungaluna, Prescott Musaba and André Malekani, 16 Aug. 2012, 18 Aug. 2012, leg. Prescott Musaba (UNIKIS: UMA535, UMA542, UMA570, UMA572, UMA574 to UMA576, UMA587, UMA606, UMA608, UMA613, UMA614, UMA631, UM001 to UM003, UM009, UM012, UM019, UM023, RMCA: a1.097-M-3173, a1.097-M-3175, UNIKIS: UM011, UM015, UM017, RMCA: a1.097-M-3174, UNIKIS: UM020); 1 juv + [SS], Vankerckhovenville [= Nzoro], 17 Apr. 1912, leg. Herbert Lang, James Paul Chapin and The American Museum Congo Expedition (AMNH: 48720); 12 우 [12 UN], Weko Forest, between 27 Dec. 1974 and 3 Jan. 1975, leg. Michael D. Gallagher (HZM: 10.7809, $11.7810,15.7828,17.7830$ to $19.7832,21.7834,30.7857,35.7862$ to $37.7864,9.7808) ; 2$ 우, 1 ㄱ [1 SK, 2 UN], Yaekela, 3 May 2010, 4 May 2010, 6 May 2010, leg. Guy-Crispin Gembu Tungaluna, Prescott Musaba and José Akaibe (UNIKIS: CRT2374, CRT2395, CRT1867); 3 우, 2 ふふ઼ [2 SS, 3 AL], 
VAN CAKENBERGHE V. et al., The bats of Congo, Rwanda and Burundi revisited

Yalosemba, 12 Jun. 1979, 13 Jun. 1979, 15 Jun. 1979, leg. C. Brian Robbins (USNM: 463471, 537696, 463472, 537697, 463473); 1 ad $\hat{\partial}, 1 \hat{\jmath}$ [1 SS, 1 AL], Yalusaka, 10 Mar. 1958, between 1 Mar. 1957 and 31 Mar. 1957, leg. F. Carbone (RMCA: 27174, 31717); 1 ô [AL], Yambombo, 20 Jun. 1979, leg. Frieder Meier (MHNG: 1870.041); 5 우, 1 ○ [6 UN], Yangambi, between 4 Jan. 1975 and 6 Jan. 1975, leg. Michael D. Gallagher (HZM: 14.7827, 24.7837, 34.7861, 38.7855, 38.7865, 7.7805); 12 우, 1 ऽ, 1 ? [14 UN], Yoko, unknown date, 18 May 2009, 25 May 2009, 26 May 2009, 23 Sep. 2009, 25 Sep. 2009, leg. unknown collector, 4 Sep. 2012, 5 Sep. 2012, 7 Sep. 2012, leg. Prescott Musaba, Tembele and Marc Boketshu, 5 Apr. 2014, leg. Prescott Musaba (UNIKIS: YK034CH, GE241/G16, GE244, GE279/G19, GE285/G17, GE289, GE290, YK007CH, YK008CH, YK023CH, YK026CH, YK027CH, YK031, YK033CH).

Hypsignathus H. Allen, 1862

Hypsignathus monstrosus $\mathrm{H}$. Allen, 1862

DEMOCRATIC REPUBLIC OF THE CONGO: 1 foetus ?, 1 ? [1 SK, 1 UN], Alipago, 29 May 1938, leg. J.M. Vrijdagh (RMCA: 14796a, 14796b); 1 \& [UN], Angu, near, 22 Apr. 1906, leg. unknown collector (BMNH: 1907.7.8.23); 2 juv ??, 2 q ㅇ [4 SS], Arebi Bondo Mabe, 19 Jul. 1925, leg. Henri

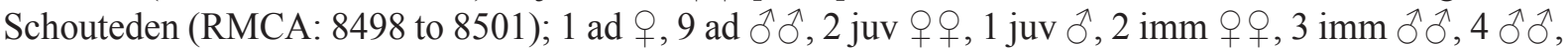
4 ?? [19 SS, 5 AL, 2 UN], Avakubi, unknown date, 3 Mar. 1914, 11 May 1914, 27 May 1914, leg. unknown collector, 6 May 1910, 23 Dec. 1913, 24 Feb. 1914, 26 Feb. 1914, 27 Feb. 1914, 28 Feb. 1914, 3 Mar. 1914, 5 Mar. 1914, 6 Mar. 1914, 7 May 1914, 11 May 1914, 12 May 1914, 15 May 1914, 18 May 1914, 27 May 1914, 28 May 1914, 28 Feb. 1915, leg. Herbert Lang, James Paul Chapin and The American Museum Congo Expedition, 6 Mar. 1914, leg. J.A. Howard (NMW: 18282, RMCA: 12.376, 12376, AMNH: 48646, 48645, 48647 to 48649, 48636, 49279, 48656, 48637, 48639, 48650, MCZ: 17363, AMNH: 48657, 48638, 48651, 48640, MCZ: 17362, AMNH: 48655, 48641, 48653, KU: 125195, AMNH: 49208, 48643); 1 त [SS], Bafwabaka, 2 May 1910, leg. Herbert Lang (FMNH: 43873); 1 ad

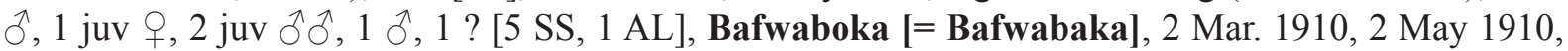
leg. Herbert Lang, James Paul Chapin and The American Museum Congo Expedition (AMNH: 48654, 48631, 48632, 48635, 49280, RMCA: 12360); 2 우, 1 ๙े [1 AL, 2 SK], Baliko, 10 Jun. 2012, 12 Jun. 2012, leg. William ("Bill”) T. Stanley, prior to 31 Jan. 2007, leg. Guy-Crispin Gembu Tungaluna (FMNH: 219587, 219588, RMCA: a1.097-M-0413); 1 q [SS], Bamanya, 16 Apr. 1952, leg. Leonet (RMCA: 21689); 1 juv ?, 2 우 [3 UN], Bambesa, 29 May 1938, 27 Aug. 1938, leg. J.M. Vrijdagh (RMCA: 14677, 14678, 14796); 1 ad के [SK], Bambu [= Kilomines], 1 Jan. 1948, leg. unknown collector (RBINS: 7037); 1 ? [UN], Baraka, unknown date, leg. Rudolf Grauer (ZMB: 67118); 1 ad $q$

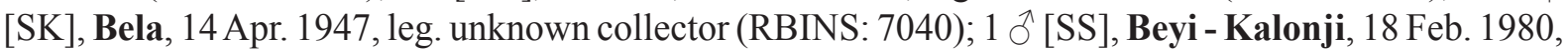
leg. Betu Nsankulu (RMCA: 80.020-M-0003); 1 ? [UN], Boende, prior to 16 Mar. 1940, leg. R.P. Wynants (RMCA: 15949); 1 ? [SS], Bokuma, 22 Oct. 1950, leg. P. Lootens (RMCA: 20397); 1 §ै, 2 ?? [3 SS], Bokungu, 2 Dec. 1948, prior to 25 Jul. 1949, leg. L. Dupuis (RMCA: 18693, 18817, 18818); 6 ?? [3 SS, 3 SO], Bolafa, between 1 May 1956 and 31 May 1956, leg. Stevenart (RMCA: 25841 to 25846); 1 ? [SS], Bongale, 15 Mar. 1951, leg. P. Lootens (RMCA: 20560); 1 ? [SS], Bukavu, prior to 30 Jun. 1999, leg. Camerman (RMCA: 99.011-M-0001); 9 ?? [7 SS, 2 UN], Buta, 12 Jun. 1936, 10 May 1940, 2 Aug. 1940, prior to 19 Jan. 1923, prior to 15 Jul. 1935, between 1 Dec. 1934 and 31 Dec. 1934, between 1 Jun. 1934 and 30 Jun. 1934, leg. Jozef Hutsebaut (RMCA: 17321, 13379, 13380, 17320, 12563, 12564 , 12671, 12857, 6273); 1 ? [AL], Butembo, between 1 Apr. 1956 and 30 Apr. 1956, leg. P. Dyleff (RMCA: 23956); 1 đ [SS], Byonga, 10 Jan. 1972, leg. Alexandre Prigogine (RMCA: 38586); 1 ? [AL], Congo

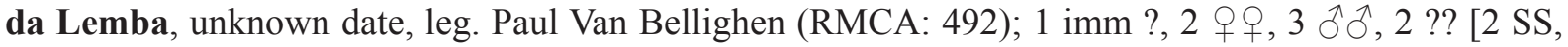
2 AL, 2 SO, 2 UN], Eala, unknown date, between 1 Jan. 1921 and 31 Dec. 1921, leg. Verlaine, 4 Jan. 1921, 5 Jan. 1921, leg. Henri Schouteden (RMCA: 16422, 6526 to 6528, 6531, 6524, 6524a, 16215); 7 우우, 1 ô, 9 ?? [7 SS, 6 AL, 1 SK, 3 SO], Epulu, unknown date, leg. Luc Bijnens, 4 Mar. 1957, 9 May 1957, 17 May 1957, 26 May 1958, leg. Station de domestication des okapis Epulu, 21 Sep. 1956, 24 Sep. 1956, 
between 1 Sep. 1956 and 30 Sep. 1956, leg. Max Poll, 4 Mar. 1957, leg. Heinz Stephan (RMCA: 90.031M-0002, 26610, 25597 to 25599, 25596, 27750, 26609, 27417, 27418, 26611, 26612, 27645 to 27649); 1 \ [AL], Epulu, 6 km S, 2 km W, 6 Jun. 1981, leg. John A. Hart (FMNH: 138136); 1 ? [SN], Equateur Province, between 1 Jan. 1921 and 31 Dec. 1921, leg. Verlaine (RMCA: 38239); 1 ad? $\&$ [SS], Eringete [= Eringite], 19 Sep. 1949, leg. unknown collector (RBINS: 12945); 1 juv ?, 1 ô, 3 ?? [3 SS, 1 AL, 1 SK], Ibembo, unknown date, leg. J. Rodhain, 10 Sep. 1949, 10 Feb. 1950, 20 Dec. 1951, between 1 Dec. 1952 and 31 Dec. 1952, leg. Jozef Hutsebaut (RMCA: 7642, 19620, 19527, 20744, 21714); 1 ? [SO], Ikengo, 15 Feb. 1921, leg. Henri Schouteden (RMCA: 6529); 1 ad ? [AL], Inongo, 1 Oct. 1945, leg. Gustave Gustin (RMCA: 18124); 1 ○े [AL], Irebu, unknown date, leg. J. Camp (USNM: 61684); 2 우 [2 SS], Kamituga, 2 Aug. 1954, 10 Jun. 1964, leg. Alexandre Prigogine (RMCA: 32855, 23422); 1 q [AL], Karambi, 3 Apr. 1992, leg. unknown collector (ZMA: MAM.24557); 4 ?? [4 SK], Kasai, prior to 11 Jul. 1905, leg. La Compagnie du Kassai (RMCA: 738, 738a to 738c); 1 ? [AL], Kasongo, between 1 Sep. 1959 and 31 Jan. 1960, leg. Benoit (RMCA: 31106); 1 $\delta$ [SK], Kasongo Baza [= Kasonga-Banze], between 1 Feb. 1960 and 28 Feb. 1960, leg. Faber (RMCA: 30820); 9 ?? [9 UN], Kikwit, unknown date, leg. unknown collector (TM: 45595, 45628, 45630, 46063, 46064, 46073, 46074, 46080, 46081); 1 ad+foetus $ᄋ$ [AL], Kilo mines [= Kilomines], prior to 15 Dec. 1930, leg. du Soleil (RMCA: 10775); 1 đ [SS], Kilomamensa, unknown date, leg. André Pilette (RMCA: 6143); 1 ? [UN], Kindu, unknown date, leg. Rudolf Grauer (ZMB: A.4809); 1 , 1 ○े [1 SS, 1 UN], Kisala [= Kisala-Kiama], 11 Mar. 1957, leg. J.J. Laarman, 16 Oct. 1920, leg. Henri Schouteden (RMNH: MAM.16346, RMCA: 6530); 1 q [SS], Kitangua, 13 Dec. 1981, leg. Betu Nsankulu (RMCA: 83.025-M-0007); 1 q [UN], Kona, 12 May 2010, leg. Guy-Crispin Gembu Tungaluna, Prescott Musaba and José Akaibe (UNIKIS: CRT1916); 1 q [SS], Koteli [= Kotili], 2 Jan. 1925, leg. Henri Schouteden (RMCA: 8784); 2 우 [2 SS], Kunungu, prior to 19 Nov. 1937, prior to 1 Aug. 1939, leg. N'Kele (RMCA: 15509, 14169); 2 ?? [2 SS], Lesse, 4 Nov. 1913, leg. Bonnevie (RMCA: 3236, 3237); 1 ? [UN], Libenge, 10 Apr. 1938, leg. Leontovitch (RMCA: 14686); 2 ?? [1 SS, 1 SK], Lisala, prior to 19 Jan. 1929, leg. Babilon (RMCA: 9574, 9575); $1 \widehat{\jmath}$ [UN], Lobi, 19 Feb. 1906, leg. unknown collector (BMNH: 1907.7.8.21); 1 ? [SS], Lovanium University [= Kinshasa], 9 May 1963, leg. De Bont and Jo Van Orshoven (RMCA: 32588); 1 q [SS], Luebo, 26 Aug. 1921, leg. Henri Schouteden (RMCA: 6525); $1 \mathrm{imm}$ ㅇ [UN], Lukoi River, unknown date, leg. D. Fraser (BMNH: 1927.3.1.17); 1 juv ON $_{2} 2$ 우우, 3 ठึô, 2 ?? [6 SS, 2 AL], Lukolela, 6 Aug. 1930, 28 Aug. 1930, 29 Aug. 1930, 31 Aug. 1930, leg. Franklin Edson, 31 Jul. 1931, leg. Lecocq, 6 Aug. 1930, leg. James Paul Chapin, between 1 Aug. 1923 and 31 Aug. 1923, leg. J. Ghesquière (AMNH: 86764, 86763, RMCA: 11736, AMNH: 86761, 86762, 86946, 86947, RMCA: 7038); 1 § [AL], Lukolela, 4 mi from, 31 Aug. 1930, leg. James Paul Chapin (AMNH: 80957); 1 क, 1 ? [1 SO, 1 UN], Luluabourg [= Kananga], unknown date, leg. Richard Callewaert, 3 Feb. 1966, leg. Antoon Emeric Marcel De Roo (BMNH: 1926.7.6.48, RMCA: 35275); 2 웅, 3 ठ지 [1 SS, 4 AL], Mabali, 9 Nov. 1954, leg. James Paul Chapin, between 1 May 1956 and 30 Jun. 1956, leg. Alvin Novick (AMNH: 185262 to 185264, MCZ: 48184, 48185); 2 우 [2 SK], Masako, 23 May 1989, leg. Ndey B. Ifuta (RMCA: 93.079-M-0367, 93.079-M-0368); 1 ? [AL], Mpe, prior to 26 Jan. 1914, leg. Maes (RMCA: 2662); 1 ㅇ, 1 ? [2 SS], Ndwa, prior to 1 Aug. 1939, between 1 Jan. 1938 and 31 Dec. 1939, leg. Ngwe (RMCA: 15421, 15422); 2 ?? [2 AL], Nepoko Bomili, prior to 12 Dec. 1946, leg. M. Henrion (RMCA: 18137, 18137a); 1 imm ô, 3 ?? [1 SS, 3 AL], New Beni [= Beni], 25 Oct. 1926, leg. James Paul Chapin, between 1 Oct. 1926 and 31 Oct. 1926, leg. James Paul Chapin, Dewitt L. Sage, Frank P. Mathews and The Ruwenzori-Kivu Expedition (AMNH: 82529, 82357 to 82359); 1 ? [SS], Paulis [= Isiro], 4 May 1951, leg. Abbeloos (RMCA: 20510); 1 ad $\widehat{\partial}, 1$ ภ [1 SS, 1 UN], Penge, 21 Apr. 1914, leg. Herbert Lang, James Paul Chapin and The American Museum Congo Expedition, 21 Apr. 1914, leg. unknown collector (AMNH: 48642, RMCA: 48642); 1 [ [AL], RubiTele, 19 Aug. 2013, leg. Guy-Crispin Gembu Tungaluna, Prescott Musaba and André Malekani (UNIKIS: RBTL514); 2 ad $ㅇ ㅜ, 2$ juv $ㅇ+$, 1 ㅇ [4 SS, 1 AL], Stanleyville [= Kisangani], 18 Aug. 1909, 16 Dec. 1914, leg. Herbert Lang, James Paul Chapin and The American Museum Congo Expedition, 18 Aug. 1909, leg. unknown collector (AMNH: 48652, 48629, 48630, 49199, MCZ: 17364); 2 우 
[2 UN], Tingasi, 29 Oct. 1883, leg. unknown collector (BMNH: 1887.12.1.24, 1887.12.1.25); 1 § [SK], Tondu, 19 Feb. 1921, leg. Henri Schouteden (RMCA: 7784); 1 ad $\circ$ [SK], Tschuapa [= Tshuapa], 18 Oct. 1966, leg. unknown collector (RBINS: 15188); 1 ㅇ [SS], Uele [River], between 1 Dec. 1927 and 31 Dec. 1927, leg. Jozef Hutsebaut (MCZ: 23935); 1 đ [UN], Yaekela, 3 May 2010, leg. GuyCrispin Gembu Tungaluna, Prescott Musaba and José Akaibe (UNIKIS: CRT2388); 2 ad $q$,, 2 ad $\widehat{\partial} \widehat{\partial}$,

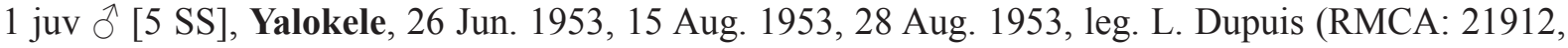
21913, 21889, 21890, 21911); $1 \mathrm{emb}$ ㅇ, 1 우 [1 SS, 1 AL], Yalosemba, 21 Jun. 1979, leg. C. Brian Robbins (USNM: 463475, 537698); 2 우우, 2 $\widehat{\jmath}$ [2 SS, 1 SO, 1 UN], Yangambi, 1 Jan. 1938, leg. unknown collector, 18 Sep. 1939, leg. J.M. Vrijdagh, 25 Oct. 1947, leg. Rodolphe Germain, 7 Aug. 1951, leg. L. Toussaint (RBINS: 5941, RMCA: 17238, RBINS: 6984, 12944); 2 우, 5 ๙ぇ [2 AL, 5 UN], Yoko, 24 Sep. 2009, leg. unknown collector, 4 Sep. 2012, 7 Sep. 2012, leg. Prescott Musaba, 4 Sep. 2012, 5 Sep. 2012, 7 Sep. 2012, leg. Prescott Musaba, Tembele and Marc Boketshu (UNIKIS: GE283, RMCA: a1.097-M-3164, UNIKIS: YK006, YK028CH, YK029CH, RMCA: a1.097-M-3165, UNIKIS: YK032CH); 1 [UN], Zemio, unknown date, leg. unknown collector (RBINS: 184); 1 ad $q$, 1 ad O, $_{1}$ ad? ?, 1 ? [4 SS], Zobia, prior to 18 Apr. 1946, leg. unknown collector (RBINS: 6963 to 6966).

Micropteropus Matschie, 1899

DEMOCRATIC REPUBLIC OF THE CONGO: 10 우, 9 ฮิ $\widehat{~}$ [1 SK, 18 UN], Kikwit, 27 Jun. 2015, 28 Jun. 2015, 29 Jun. 2015, leg. Prescott Musaba, Moïse Bipoo, Charle Andabhati, Benjamin Kirongozi and Kambale Karupao (UNIKIS: KKT2848 to KKT2859, KKT2861 to KKT2866, KKT2877); 3 우우, 1 ô [4 UN], Rethy, 22 Oct. 2015, 23 Oct. 2015, leg. Anne Laudisoit et al. (UNIKIS: ITU706, ITU710, ITU712, ITU714).

\section{Micropteropus intermedius Hayman, 1963}

DEMOCRATIC REPUBLIC OF THE CONGO: 1 \& [SA], Banana or Netonna, unknown date, leg. P. Hesse (SMF: 2509); 1 \& , 2 ?? [2 SS, 1 SA], Luluabourg [= Kananga], 15 Mar. 1964, 31 Dec. 1964, leg. Antoon Emeric Marcel De Roo (RMCA: 32380, 34406, 34409); 1 ते [SA], Thysville Cave b 13 a [= Banjan Caves], 13 Jul. 1949, leg. Narcisse Leleup (RMCA: 22661).

\section{Micropteropus pusillus (Peters, 1868)}

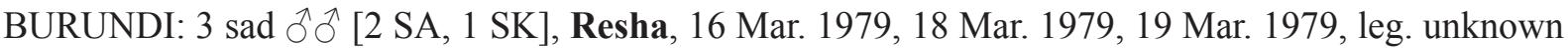
collector (SMF: 57493 to 57495 ).

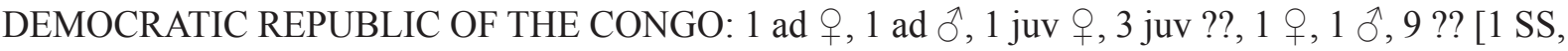
$2 \mathrm{SA}, 8 \mathrm{AL}, 4 \mathrm{SK}, 2 \mathrm{UN}$ ], unknown locality, unknown date, leg. unknown collector, unknown date, leg. Tshinyenye, unknown date, prior to 31 Dec. 1889, leg. P. Hesse, between 3 Feb. 1980 and 23 Mar. 1980, leg. Betu Nsankulu, prior to 18 Aug. 1965, leg. Antoon Emeric Marcel De Roo, prior to 4 Jul. 1958, leg. E. Jans (BMNH: 1990.223, MNHN: ZM-MO-1996-2470, ZM-MO-1996-2471, RMCA: 80.026-M0010, SMF: 11744, 11746, 11747, 2505 to 2508, RMCA: 80.020-M-0004, 33562, 33564, 33583, SMF: 11750, RMCA: 27375); 2 우, 1 ? [3 SS], Bakwakenge [= Bakwa-Kenge], 9 Sep. 1978, 12 Oct. 1979, leg. Betu Nsankulu (RMCA: 80.013-M-0004, 80.013-M-0005, 79.022-M-0001); 1 ? [UN], Bas Congo (former) Province [= Kongo Central Province], unknown date, leg. Alphonse Cabra (RMCA: 67); $1 \delta$ [SS], Bengale, 28 Dec. 1976, leg. Tshinyenye (RMCA: 77.012-M-0002); 3 우, 3 ?? [1 SA, 4 AL, $1 \mathrm{UN}$ ], Beno, unknown date, leg. the Royal Museum for Central Africa (RMCA), prior to 3 Jan. 1950, leg. P. Bayet (BMNH: 1954.784, 1954.785, RMCA: 19477a to 19477c, 19478); 3 ?? [3 AL], Benza Masola, between 1 May 1913 and 31 May 1913, leg. Mayné (RMCA: 2099a to 2099c); 1 đ [SS], Benzale, 28 Dec. 1976, leg. Tshinyenye (RMCA: 77.031-M-0008); 1,+ 3 $\lesssim$ [4 SS], Biakabomba,

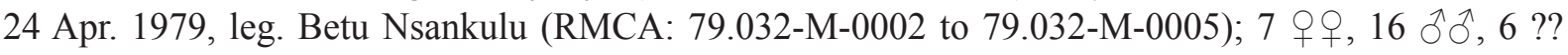


[29 AL], Boende, prior to 31 Jan. 1969, between 1 Jan. 1967 and 31 Dec. 1967, leg. P. Lootens (RMCA: 38657 to 38670,38677 to 38685,35629 to 35634); 1 क [SS], Bokalakala, between 1 Oct. 1949 and 30 Nov. 1949, leg. Ngwe (RMCA: 19544); 1 ? [AL], Bokoro, prior to 26 Aug. 1952, leg. E. Jans (RMCA: 21297); 9 우의 1 ㄱ, 30 ?? [11 SS, 1 SA, 25 AL, 1 SO, 2 UN], Boma, unknown date, leg. unknown collector, 20 Nov. 1920, 21 Nov. 1920, 24 Nov. 1920, 26 Nov. 1920, leg. Henri Schouteden, between 1 Aug. 1928 and 31 Aug. 1928, leg. Gerard, prior to 24 May 1912, leg. Barthélemy, prior to 26 May 1933, leg. Colsoulle, prior to 4 Aug. 1937, leg. Dartevelle (RMCA: 13851, 7292, 7293, 6542, 7295, $7297,6543,6545,6544,16181,16216,16219,16220,1051,12125$ e to $12125 \mathrm{~h}, 12126 \mathrm{a}$ to $12126 \mathrm{j}$, 12127a to 12127i, 13947, 13951, 13954); 1 § [SS], Byonga, 6 Oct. 1966, leg. Alexandre Prigogine (RMCA: 34800); 1 ㅇ [SK], Catchpole's Farm, 24 Oct. 1924, leg. Edmund Heller (FMNH: 35684); 2 우, 1 ? [1 AL, 2 UN], Cel II, 16 Nov. 1951, leg. H. de Saeger et al., 16 Nov. 1951, leg. H. de Saeger (RBINS: 13521.1, 13521.2, RMCA: 36555); 1 ㅇ, 1 ô [1 SS, 1 SO], Cinputu, 14 Sep. 1983, leg. Betu Nsankulu (RMCA: 84.035-M-0009, 84.035-M-0012); 2 ?? [2 AL], Congo da Lemba, between 13 Jan. 1900 and 22 Jan. 1900, leg. Paul Van Bellighen, prior to 13 Mar. 1913, leg. Mayné (RMCA: 408b, 1545); 1 q [SS], Dedegwa River, between 1 Jan. 1950 and 17 May 1952, leg. H. de Saeger (RMCA: 36554); 1 ? [AL], Faradje, 12 Sep. 2010, leg. Guy-Crispin Gembu Tungaluna (RMCA: a1.097-M-0553); 1 ? [SS], Fwa, Lake, 25 May 1984, leg. Betu Nsankulu (RMCA: 84.035-M0008); 3 ?? [3 AL], Ganda-Sundi, 20 Apr. 1931, leg. James Paul Chapin, 31 Mar. 1964, leg. Alexandre Fain (AMNH: 86860, 86861, RMCA: 33262); 4 우 [4 SO], Gangala [= Gangala-na-Bodio], 24 Mar. 1957, 25 Mar. 1957, 26 Mar. 1957, leg. Heinz Stephan (RMCA: 27426 to 27429); 1 ? [UN], Gangala-na-Bodio, 25 May 1948, leg. unknown collector (RBINS: 11660); 1 ? [AL], Kabinda, between 1 Jan. 1945 and 31 Dec. 1946, leg. Hautmann (RMCA: 18148); 2 ?? [2 SS], Kaniki Kapangu, 12 Mar. 1966, 13 Mar. 1966, leg. Antoon Emeric Marcel De Roo (RMCA: 35278, 35280); 3 q , 2 ?? [3 SS, 2 SK], Kasai, 24 Dec. 1981, 17 Jan. 1982, 24 Feb. 1982, prior to 12 Aug. 1983, leg. Betu Nsankulu (RMCA: 83.025-M-0009, 83.025-M-0010, 83.025-M-0008, 83.025-M-0020, 83.025-M-0022); 4 우우, 2 $ठ \widehat{~[5 ~ S S, ~} 1$ SO], Kaswabilenga, 6 Oct. 1947, 7 Oct. 1947, leg. unknown collector, 6 Oct. 1947, 7 Oct. 1947, leg. Gaston-François de Witte (RBINS: 10679 to 10684); 2 ?? [2 AL], Kavuaya, prior to 31 Dec. 2007, prior to 2 Feb. 2007, leg. A. Kimbembi-ma-Ibaka (RMCA: a7.006-M-0012, a7.006-M-0013); 6 우, 1 ô [7 SA], Kikole-Pecheur, 17 May 1980, leg. M. Mutashia (SMF: 58058 to 58064); 7 ad $q$ 9,4 ad $\widehat{\partial} \widehat{\partial}$ [5 SA, 6 UN], Kikwit ITPK [= Kikwit], 1 Aug. 1995, leg. Herwig Leirs et al. (MSB: 274975 to 274979, 274985, RMCA: 97.021-M-0805 to 97.021-M-0809); 1 ad 9 [UN],

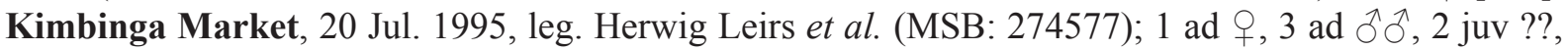
7 웅, 15 ภึ [20 SS, 8 SN], Kimpoko [= Kimpoko-Nsele], unknown date, leg. Tsh Mutashiya, 22 Aug. 1976, 27 Aug. 1976, 28 Aug. 1976, 30 Aug. 1976, 14 Oct. 1976, 15 Oct. 1976, 31 Oct. 1976, 1 Nov. 1976, 24 Dec. 1976, 25 Aug. 1979, 27 Apr. 1980, leg. Tshinyenye (MNHN: ZM-MO-1986-1059 to ZM-MO-1986-1061, ZM-MO-1986-299, RMCA: 77.012-M-0003, 77.031-M-0005, 77.031-M-0006, 77.031-M-0002, 77.031-M-0003, 76.046-M-0001, 77.031-M-0007, 80.026-M-0002 to 80.026-M-0009, 80.038-M-0003 to 80.038-M-0006, 76.046-M-0002 to 76.046-M-0004, 77.021-M-0011, 77.031-M0004); 1 ? [SS], Kingabwa, prior to 3 Mar. 1976, leg. Tshinyenye (RMCA: 76.010-M-0002); 3 ?? [3 UN], Kinkole, 15 Aug. 1980, leg. V. Wallach (ZMA: MAM.21146 to MAM.21148); 7 §ิ ô, 31 ?? [7 SO, 31 UN], Kinshasa, between 15 Jan. 1975 and 22 Jan. 1975, leg. Michael D. Gallagher, between 5 Feb. 1993 and 31 Dec. 1993, between 3 Feb. 1993 and 31 Dec. 1993, between 24 Jan. 1993 and 31 Dec. 1993, between 22 Feb. 1993 and 31 Dec. 1993, between 2 Jan. 1993 and 31 Dec. 1993, between 19 Feb. 1993 and 31 Dec. 1993, leg. Tshinyenye (HZM: 10.7795 to 37.7822, 7.7792 to 9.7794, RMCA: 93.097-M-0020, 93.097-M-0021, 93.097-M-0019, 93.097-M-0017, 93.097-M-0018, 93.097-M-0016, 93.097-M-0015); 45 ?? [45 UN], Kinshasa City Province, 12 Feb. 2001, 28 Feb. 2001, between 1 Apr. 1999 and 30 Apr. 1999, leg. unknown collector (SMF: 92974 to 92978, 92972 to 92973, 93243 to

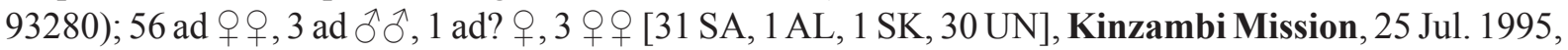
26 Jul. 1995, 27 Jul. 1995, 30 Jul. 1995, 31 Jul. 1995, leg. Herwig Leirs et al. (MSB: 274943 to 274945, RMCA: 97.021-M-0773 to 97.021-M-0775, MSB: 274946 to 274949, RMCA: 97.021-M-0776 to 
VAN CAKENBERGHE V. et al., The bats of Congo, Rwanda and Burundi revisited

97.021-M-0779, MSB: 274951 to 274962, 274964 to 274971, RMCA: 97.021-M-0780 to 97.021-M0801, 97.021-M-0849, MSB: 274972, RMCA: 97.021-M-0802, MSB: 274973, 274974, RMCA: 97.021-

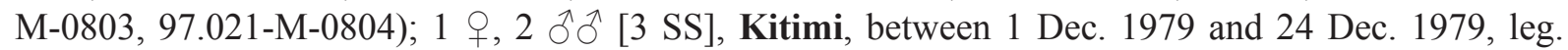
Tshinyenye (RMCA: 80.006-M-0001 to 80.006-M-0003); 3 q $ᄋ$ [ $3 \mathrm{AL}$ ], Kitombongo [= Kintobongo], 25 Sep. 2009, 26 Sep. 2009, leg. Alexandre Hassanin, Nicolas Nesi, Célestin Pongombo, Peter Vallo and Didier Tshikung (MNHN: ZM-2011-807 to ZM-2011-809); 1 ㅇ [SS], Kitutu, 15 Feb. 1964, leg. Alexandre Prigogine (RMCA: 32406); 2 ๙ $\widehat{\jmath}$ [2 SS], Kuili, 4 Jan. 1977, leg. Tshinyenye (RMCA: 77.031-M-0010, 77.031-M-0011); 3 के के [3 SS], Kuili Ngongo [= Kuili], 5 Jan. 1977, 6 Jan. 1977, leg. Tshinyenye (RMCA: 77.012-M-0005, 77.012-M-0006, 77.012-M-0001); 1 q [SS], Kwamouth, 29 May 1921, leg. Henri Schouteden (RMCA: 6546); 3 đิ $\widehat{~}$ [3 SS], Kwango, 29 Dec. 1976, leg. Tshinyenye (RMCA: 77.012-M-0004, 77.021-M-0015, 77.031-M-0009); 1 ㅇ, 1 § [1 SS, 1 SO], Léopoldville [= Kinshasa], 5 Apr. 1912, leg. Cuthbert Christy, 8 Jun. 1943, leg. R. Fiasse (RMCA: 1431, 17656); 13 ?? [12 SS, 1 UN], Lovanium University [= Kinshasa], 1 Nov. 1962, 24 Nov. 1962, 26 Nov. 1962, 24 Dec. 1962, 28 Dec. 1962, 5 Jan. 1963, 17 Jan. 1963, 2 Apr. 1963, 19 Apr. 1963, 23 Nov. 1963, between 1 Apr. 1963 and 1 Apr. 1964, leg. De Bont and Jo Van Orshoven, between 6 Apr. 1964 and 7 Apr. 1964, leg. Antoon Emeric Marcel De Roo (RMCA: 32618, 32617, 32622, 32619, 32613, 32623, 32614, 32615, 32620, 32621, 32612, 32616, 32783); 1 ? [AL], Lubondai-Tshimbulu [= Lubondaie-Tshimbulu], 12 Dec. 1955, leg. S. Marks (RMCA: 26274); 1 ㅇ, 1 ? [2 AL], Lukolela, 3 Nov. 1930, 28 Feb. 1931, leg. James Paul Chapin (AMNH: 86862, 86905); 1 juv ? [SS], Lukonga, 6 Aug. 1979, leg. Betu Nsankulu

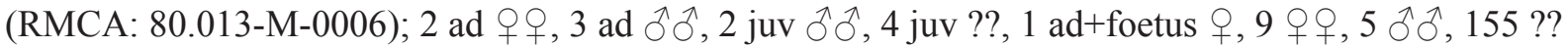
[111 SS, $58 \mathrm{AL}, 9 \mathrm{SO}, 3 \mathrm{UN}$ ], Luluabourg [= Kananga], unknown date, leg. unknown collector, unknown date, 4 Nov. 1963, 5 Nov. 1963, 22 Nov. 1963, 25 Nov. 1963, 26 Nov. 1963, 29 Nov. 1963, 19 Dec. 1963, 11 Jan. 1964, 11 Feb. 1964, 8 Mar. 1964, 9 Mar. 1964, 10 Mar. 1964, 11 Mar. 1964, 12 Mar. 1964, 14 Mar. 1964, 16 Mar. 1964, 17 Mar. 1964, 19 Mar. 1964, 16 Apr. 1964, 17 Apr. 1964, 18 Apr. 1964, 19 Apr. 1964, 22 Apr. 1964, 23 Apr. 1964, 24 Apr. 1964, 25 Apr. 1964, 3 May 1964, 4 May 1964, 10 May 1964, 28 May 1964, 30 May 1964, 2 Jun. 1964, 3 Jun. 1964, 6 Jun. 1964, 10 Jun. 1964, 13 Jun. 1964, 21 Sep. 1964, 16 Oct. 1964, 19 Oct. 1964, 21 Oct. 1964, 22 Oct. 1964, 24 Oct. 1964, 31 Oct. 1964, 1 Nov. 1964, 4 Nov. 1964, 7 Nov. 1964, 11 Nov. 1964, 25 Nov. 1964, 1 Dec. 1964, 31 Dec. 1964, 2 Feb. 1965, 14 Feb. 1965, 15 Feb. 1965, 18 Feb. 1965, 20 Feb. 1965, 22 Feb. 1965, 27 Feb. 1965, 28 Feb. 1965, 3 Mar. 1965, 4 Mar. 1965, 6 Mar. 1965, 8 Mar. 1965, 12 Mar. 1965, 19 Mar. 1965, 20 Mar. 1965, 22 Mar. 1965, 25 Mar. 1965, 1 Apr. 1965, 12 Apr. 1965, 28 Apr. 1965, 2 May 1965, 8 May 1965, 16 Jun. 1965, 26 Jun. 1965, 3 Jul. 1965, 16 Nov. 1965, 3 Mar. 1966, 27 May 1966, 14 Aug. 1968, 20 Aug. 1968, prior to 18 Aug. 1965, between 1 Jan. 1965 and 31 Dec. 1965, between 1 Jan. 1964 and 31 Dec. 1964, leg. Antoon Emeric Marcel De Roo (BMNH: 1926.7.6.60, 1926.7.6.61, RMCA: 32695,33329 to 33331,33431 to $33433,33671,33555,33677,33678,34408$, $34414,32375,32376,32642$ to $32649,32768,33579,33580$, AMNH: 206839 to 206842, KU: 125197 , RMCA: 32377, 33645, 32379, 33426, 33680, 33581, 33416, 32692, 32693, 35610 to 35612, 33323, 32381 to $32383,32673,32674,33681,33682,33653$ to $33655,34411,34415,32384,32385,32675$ to $32680,33417,32681,32386,33427,33428,32682$, 32683, 33656, 33657, AMNH: 206836 to 206838, RMCA: 33322,33434 to $33438,33575,33321,33327,33328,35613,32689,33658$ to 33663,33418 , $33429,32640,32641,33664,33665,32366,32684$ to 32687,33666 to $33668,33430,32688,32367$ to $32369,33675,33676,33683,32370,32371,33419,35281,33420,33556,33572,32372,32373,33421$ to $33423,34412,34413,32694,33574,33576,33557,33573,33669,33670,33415,33679,33324$, $32696,32697,32363,32364,33672$ to $33674,32365,33325,33326,33424,33558,33577,33578$, $33320,32691,33425,33439$ to $33448,32374,34407,34410,33559$ to $33561,33563,33565$ to 33567 , 33582, 33584); 2 우, 1 đ , 1 ? [1 SS, 3 SO], Make, 1 Jul. 1989, 12 Aug. 1992, 1 Nov. 1992, between 18 Apr. 1993 and 31 Dec. 1993, leg. Tshinyenye (RMCA: 89.057-M-0004, 92.148-M-0003, 92.119-M0004, 93.097-M-0014); 1 ? [AL], Makengo, 11 Dec. 1946, leg. Dartevelle (RMCA: 18160); 1 § [SS], Masaba, 27 Sep. 1976, leg. Tshinyenye (RMCA: 77.012-M-0007); 1 ? [AL], Mayumbe, prior to 31 Aug. 1899, leg. Alphonse Cabra (RMCA: 331); 1 ad + [SA], Mbalaka (Plantation), 23 Jul. 1995, 
leg. Herwig Leirs et al. (RMCA: 97.021-M-0772); 3 đð [3 SS], Mbawu, 27 May 1979, leg. Betu Nsankulu (RMCA: 79.032-M-0010 to 79.032-M-0012); 1 우 [SS], Mbelekelo, 24 Feb. 1965, leg. Alexandre Prigogine (RMCA: 33287); 1 +, 1 đ , 7 ?? [3 AL, 6 UN], Mboga [= Boga], unknown date, leg. unknown collector, unknown date, leg. Howard de Walden (BMNH: 1930.11.11.70 to 1930.11.11.78);

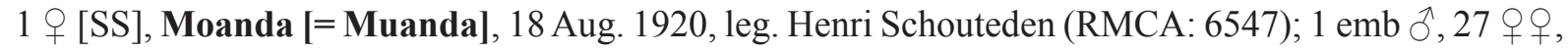
16 đô [44 AL], Mukulakulu, 22 Sep. 2009, leg. Alexandre Hassanin, Nicolas Nesi, Célestin Pongombo, Peter Vallo and Didier Tshikung (MNHN: ZM-2011-810 to ZM-2011-853); 2 qo+, 2 ô đo [4 SS], Mukwaya, 20 May 1979, 22 May 1979, leg. Betu Nsankulu (RMCA: 79.032-M-0006 to 79.032-M0009); 2 우 [2 AL], 4 km from Muvule Cave, 8 Oct. 2009, leg. Alexandre Hassanin, Nicolas Nesi, Célestin Pongombo, Peter Vallo and Didier Tshikung (MNHN: ZM-2011-854, ZM-2011-855); 4 q , 6 ठૈ $\widehat{o}$ [10 SO], Ndjili, between 4 Apr. 1993 and 31 Dec. 1993, between 22 Mar. 1993 and 31 Dec. 1993, between 19 Mar. 1993 and 31 Dec. 1993, between 15 Apr. 1993 and 31 Dec. 1993, leg. Tshinyenye (RMCA: 93.097-M-0013, 93.097-M-0011, 93.097-M-0012, 93.097-M-0004 to 93.097-M-0010); 1 §ै, 1 ? [2 SS], Nganza, 19 Sep. 1983, leg. Betu Nsankulu (RMCA: 84.035-M-0007, 84.035-M-0013); 1 ઈ [SS], Ngula, 6 Mar. 1977, leg. Tshinyenye (RMCA: 77.031-M-0012); $1 \mathrm{sad} \hat{\delta}[\mathrm{SS}]$, Niangara, 18 Nov. 1910, leg. Herbert Lang, James Paul Chapin and The American Museum Congo Expedition (AMNH: 48756); 1 \& [SS], Nkiene, 30 Sep. 1980, leg. Tshinyenye (RMCA: 80.053-M-0001); 1 ? [UN],

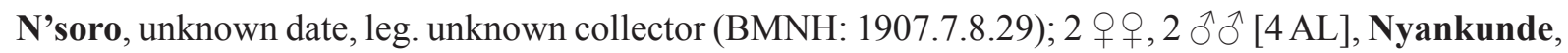
16 Aug. 1976, 18 Aug. 1976, leg. Frieder Meier (MHNG: 1872.055 to 1872.058); 1 ठ [UN], Parc National de Garamba [= Garamba National Park], 22 May 1952, leg. H. de Saeger et al. (RBINS: 13522); 1 § [UN], Rethy, 23 Oct. 2015, leg. Anne Laudisoit et al. (UNIKIS: ITU708); 1 § [AL], Sacred Heart Mission, 2 Aug. 1995, leg. Herwig Leirs et al. (RMCA: 97.021-M-1174); 2 ðぇ, 1 ? [1 SS, 1 SO, 1 UN], St. Jos. (Misson) [= Mikalaya], 23 Nov. 1922, 19 Oct. 1924, 29 Oct. 1924, leg. Richard Callewaert (AMNH: 86147, 86257, 86151); 2 우, 2 ?? [1 SS, 2 SK, 1 SO], St. Joseph de Luluabourg Mission [= Mikalaya], 5 May 1922, 23 Sep. 1922, 23 Feb. 1923, between 19 Feb. 1923 and 1 Jun. 1923, leg. Richard Callewaert (RMCA: 7115, 7547, 7548, 7109); 1 ? [SS], Sud-Kivu Province, 3 Nov. 1966, leg. A. Elbl (USNM: 535399); 1 q, 2 ?? [1 SA, 2 AL], Thysville [= Mbanza-

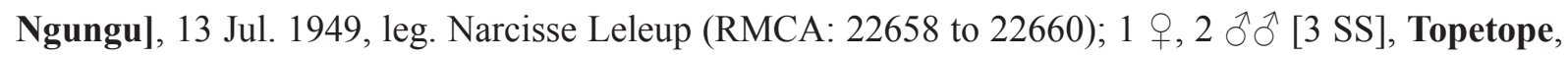

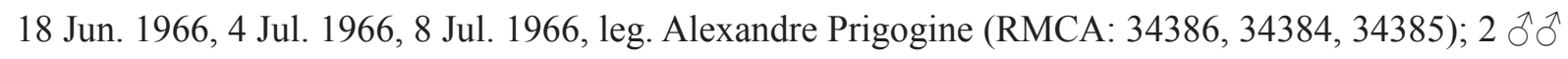
[2 SS], Tschinputu [= Cinputu], 19 Sep. 1983, 20 Sep. 1983, leg. Betu Nsankulu (RMCA: 84.035-M0011, 84.035-M-0010); 3 우, 1 đ [4 SS], Tshibasha, 18 Nov. 1970, leg. Betu Nsankulu (RMCA: 36251 to 36254); 3 ?? [3 SO], Tshimputu, 23 May 1975, leg. Betu Nsankulu (RMCA: 75.061-M-0008 to 75.061-M-0010); 1 q, 7 ?? [8 AL], Tshuapa District, between 1 Jan. 1960 and 31 Dec. 1964, leg. P. Lootens (RMCA: 34166, 34167, 34169, 34174, 34175, 34179, 34182, 34183); 1 ? [AL], Vista [= Nsiamfumu], between 1 Jan. 1933 and 31 Dec. 1933, leg. Dartevelle (RMCA: 12167); 1 ? [UN], Zambi, prior to 31 Jan. 1899, leg. J.M. Derscheid (RMCA: 149); 1 q [SK], Zongo, 2 Jul. 1948, leg. unknown collector (RBINS: 7715).

Nanonycteris Matschie, 1899

Nanonycteris veldkampii (Jentink, 1888)

DEMOCRATIC REPUBLIC OF THE CONGO: 3 ?? [3 SK], unknown locality, between 1 May 2010 and 31 May 2010, leg. Nicolas Nesi, Blaise Kadjo and Christiane Denys (MNHN: ZM-2014-1169, ZM2014-1171, ZM-2014-1173). 
VAN CAKENBERGHE V. et al., The bats of Congo, Rwanda and Burundi revisited

Myonycterini Lawrence \& Novick, 1963

Megaloglossus Pagenstecher, 1885

Megaloglossus woermanni Pagenstecher, 1885

DEMOCRATIC REPUBLIC OF THE CONGO: $1 \hat{\jmath}, 1$ ? [2 UN], unknown locality, unknown date,

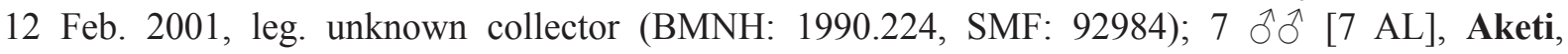
22 Jun. 2015, 23 Jun. 2015, leg. Vlir/Aketi (UNIKIS: AKETI608 to AKETI611, AKETI612 to AKETI614); 1 ? [SS], Ba Balaye, 24 Apr. 1978, leg. Betu Nsankulu (RMCA: 78.020-M-0001); 4 우으, 1 đ [2 AL, 3 SK], Baliko, 10 Jun. 2012, 11 Jun. 2012, 15 Jun. 2012, leg. William (“Bill”) T. Stanley, prior to 31 Jan. 2007, leg. Guy-Crispin Gembu Tungaluna (FMNH: 219589 to 219591, RMCA: a1.097-M-0410, a1.097-M-0411); 1 ○ [SK], Banana, unknown date, leg. P. Hesse (SMF: 2513); 1 q [SS], Bene Longo, 23 Aug. 1978, leg. Betu Nsankulu (RMCA: 79.022-M-0007); 1 q [AL], Beno, unknown date, leg. the Royal Museum for Central Africa (RMCA) (BMNH: 1954.787); 1 + [SS], Bikoro, 2 Mar. 1921, leg. Henri Schouteden (RMCA: 6548); 6 우, 12 ôึ, 25 ?? [43 AL], Boende, prior to 31 Jan. 1969, between 1 Dec. 1969 and 31 Dec. 1969, between 1 Nov. 1969 and 30 Nov. 1969, between 1 Feb. 1970 and 28 Feb. 1970, between 1 Jan. 1974 and 31 Dec. 1974, between 1 Jan. 1973 and 31 Dec. 1973, between 1 Jan. 1970 and 31 Jan. 1970, between 1 Jan. 1967 and 31 Dec. 1967, leg. P. Lootens (RMCA: 38686 to $38705,38817,38818,75.035-\mathrm{M}-0005$ to $75.035-\mathrm{M}-0019$, 75.035-M0001, 75.035-M-0004, 35635 to 35638); 1 ? [AL], Bokuma, 14 Dec. 1952, leg. P. Lootens (RMCA: 22031); 5 우우, 5 ふోळ [4 SK, 6 UN], Bomane [= Bomaneh], 21 May 2010, 30 May 2010, leg. GuyCrispin Gembu Tungaluna, Prescott Musaba and José Akaibe (UNIKIS: CRT2159, CRT2171, CRT2174, CRT2190, CRT2192, CRT2194, CRT2054 to CRT2056, CRT2076); 1 đ [SS], Byonga, 24 Jan. 1965, leg. Alexandre Prigogine (RMCA: 33288); 7 우, 16 ๙ $\widehat{\jmath}, 1$ ? [24 AL], Epulu, unknown date, leg. Luc Bijnens, 25 Aug. 2015, 27 Aug. 2015, 29 Aug. 2015, 30 Aug. 2015, 31 Aug. 2015, leg. André Malekani (RMCA: 90.031-M-0001, UNIKIS: EPLU630, EPLU632, EPLU654, EPLU658, EPLU659, EPLU662, EPLU665 to EPLU668, EPLU671 to EPLU675, EPLU681, EPLU685, EPLU694, EPLU699, EPLU700, EPLU703 to EPLU705); 10 ऽึ [3 AL, 2 SK, 3 SB, 2 SN], Epulu, 2 km W, 2 Jul. 1991, 3 Jul. 1991, 4 Jul. 1991, leg. Julian C. Kerbis Peterhans (FMNH: 149392 to 149401); 3 $\lesssim \widehat{~[3 ~ A L], ~ E q u a t e u r ~}$ (former) Province, prior to 7 Oct. 1996, leg. unknown collector (SMF: 84407 to 84409); 1 ? [AL], Ibembo, between 1 Nov. 1949 and 30 Nov. 1949, leg. Jozef Hutsebaut (RMCA: 19857); 1 ? [AL], Ikela, prior to 4 Jan. 1958, leg. P. Lootens (RMCA: 27017); 1 + [UN], Île Mafi [= Mafi Island], 15 Apr. 2012, leg. Prescott Musaba, Tembele and Marc Boketshu (UNIKIS: CHIMA54); 9 q $q$, 22 $\widehat{\jmath} \widehat{\jmath}, 7$ ?? [26 AL, 12 UN], Île Mbiye [= Mbiye Island], 15 Jan. 2012, 24 Feb. 2012, 25 Feb. 2012, 26 Jul. 2012, leg. Prescott Musaba, 15 Jan. 2012, 30 Mar. 2012, leg. Prescott Musaba, Tembele and Marc Boketshu, 19 Feb. 2012, 25 Feb. 2012, leg. Prescott Musaba et al., 19 Feb. 2012, 24 Feb. 2012, 25 Feb. 2012, 19 Apr. 2014, 22 Jun. 2014, 23 Jun. 2014, leg. Prescott Musaba, Moïse Bipoo, Richard Tamaru, Pauline Isude and Joseph Kussa, 8 Aug. 2015, 9 Aug. 2015, 25 Dec. 2015, 26 Dec. 2015, leg. Prescott Musaba, Moïse Bipoo, Charle Andabhati, Benjamin Kirongozi and Kambale Karupao (RMCA: a1.097-M-3206 to a1.097-M-3209, UNIKIS: CHIM01, RMCA: a1.097-M-3210, UNIKIS: CHIM09, CHIM04/14, CHIM018/14, CHIM021/14, CHIM022/14, CHIM023/14, RMCA: a1.097-M-3199 to a1.097-M-3201, UNIKIS: CHIM17, RMCA: a1.097-M-3202 to a1.097-M-3205, UNIKIS: CHIM24, CHIM25, ABCHIM03 to ABCHIM05, RMCA: a1.097-M-3211, UNIKIS: ABCHIM06, CHIM36, CHIM37,

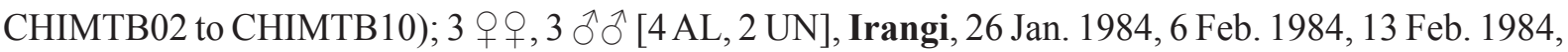
leg. Heinz Stephan, 17 Oct. 1990, leg. Wim Bergmans (SMF: 65004, ZMA: MAM.24195, MAM.24196, SMF: 65001 to 65003); 1 ? [SS], Kakanda Cave, 29 Aug. 1964, leg. Alexandre Prigogine (RMCA:

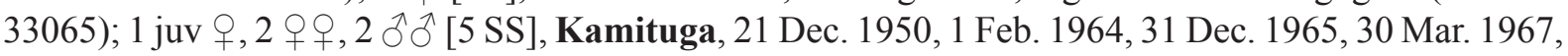
31 Mar. 1967, leg. Alexandre Prigogine (RMCA: 32394 [paratype Megaloglossus woermanni prigoginei Hayman, 1966], 20429 [paratype Megaloglossus woermanni prigoginei Hayman, 1966], 35073, 35074, 33291); $1 \widehat{\partial}$ [SS], Kamueshi [= Kamuesha], between 2 Apr. 1979 and 3 Apr. 1979, leg. 
Betu Nsankulu (RMCA: 79.032-M-0014); 1 ㅇ [AL], Kananga, 17 Feb. 1964, leg. Antoon Emeric Marcel De Roo (AMNH: 212578); 1 q, 1 § [2 UN], Karambi, 6 Apr. 1992, leg. Ngenge Masumbuko Kamitongo (ZMA: MAM.24564, MAM.24565); 2 우 [2 SA], Kikole-Pecheur, 17 May 1980, leg. M. Mutashia (SMF: 58067, 58068); 4 우, 1 ○ [5 SS], Kiliza [River], 24 May 1964, 25 May 1964, 26 May 1964, leg. Alexandre Prigogine (RMCA: 32582 [paratype Megaloglossus woermanni prigoginei Hayman, 1966], 32577 [holotype Megaloglossus woermanni prigoginei Hayman, 1966], 32579 [paratype Megaloglossus woermanni prigoginei Hayman, 1966], 32578, 32583 [paratype Megaloglossus woermanni prigoginei Hayman, 1966]); 21 ?? [21 UN], Kinshasa City Province, between 1 Apr. 1999 and 30 Apr. 1999, leg. unknown collector (SMF: 93297 to 93317); 4 ad 9 ㅇ, 2 ad

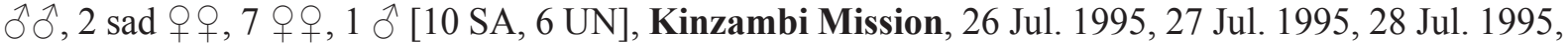
31 Jul. 1995, leg. Herwig Leirs et al. (MSB: 274831, 274832, 274834, 274835, RMCA: 97.021-M-0762 to 97.021-M-0765, MSB: 274929, RMCA: 97.021-M-0766 to 97.021-M-0769, MSB: 274930, RMCA: 97.021-M-0770, 97.021-M-0771); 1 ? [AL], Kisangani, between 1 Jun. 1982 and 30 Jun. 1982, leg. Marc Colyn (RMCA: 82.031-M-0011); 2 우우 [2 SS], Kitongo, 3 Jun. 1964, 4 Jun. 1964, leg. Alexandre Prigogine (RMCA: 32581 [paratype Megaloglossus woermanni prigoginei Hayman, 1966], 32580); 41 우, 8 ठึ [29 SA, 3 AL, 12 SK, 5 UN], Kona, 12 May 2010, 13 May 2010, 14 May 2010, 15 May 2010, 16 May 2010, 17 May 2010, 21 May 2010, between 13 May 2010 and 14 May 2010, leg. GuyCrispin Gembu Tungaluna, Prescott Musaba and José Akaibe (UNIKIS: CRT1908, CRT1912 to CRT1915, CRT1919, CRT1922 to CRT1924, CRT1927, CRT1928, CRT1930 to CRT1936, CRT1938, CRT1941, CRT1946, CRT1952, CRT1954 to CRT1957, CRT1961, CRT1962, CRT1964, CRT1965, CRT1985 to CRT1990, CRT1998, CRT2002, CRT2004 to CRT2010, CRT2013, CRT2191, CRT2193, CRT1951); 1 ? [AL], Konyola, prior to 5 Aug. 1988, leg. Luc De Vos, Kimbembi and Alain Vandelanoote (RMCA: 88.025-M-0004); 4 우, 3 ठૈ $\widehat{~[2 ~ S K, ~} 5$ UN], Lieki, 22 May 2010, 30 May 2010, 1 Jun. 2010, 3 Jun. 2010, leg. Guy-Crispin Gembu Tungaluna, Prescott Musaba and José Akaibe (UNIKIS: CRT2031, CRT2026, CRT2024, CRT2025, CRT2027, CRT2049, CRT2053); 2 qㅇ [2 SK], Luki Biosphere Reserve, 13 Jul. 2013, leg. William ("Bill”) T. Stanley (FMNH: 222419, 222420); 1 ㅇ [AL], Luki Biosphere Reserve, Forest NE of, 17 Sep. 2005, leg. P. Lukamba (ZMA: MAM.26275); 2 $\widehat{\jmath}$ [1 SS, 1 AL], Lukolela, 12 Aug. 1930, leg. Franklin Edson, 12 Aug. 1930, leg. James Paul Chapin (AMNH: 86771, 86908); 1 กै, 11 ?? [2 SS, 10 AL], Luluabourg [= Kananga], 26 Apr. 1964, 15 Feb. 1965, 16 Feb. 1965, 20 Feb. 1965, 25 Feb. 1965, 25 Mar. 1965, 2 May 1965, 30 Jun. 1965, prior to 18 Aug. 1965, leg. Antoon Emeric Marcel De Roo (RMCA: 33341 to 33343, 33371, 33344 to 33346, 33370, 33604, 33553, 33554, 33603); 1 ? [SS], Lundjulu, 14 Oct. 1952, leg. Schepens (RMCA: 21586 [paratype Megaloglossus woermanni prigoginei Hayman, 1966]); 1 ऽ [UN], Malembe, 20 Apr. 1992, leg. Ngenge Masumbuko Kamitongo (ZMA: MAM.24566); 1 \& [AL], Mandima, 7 Jul. 1976, leg. Frieder

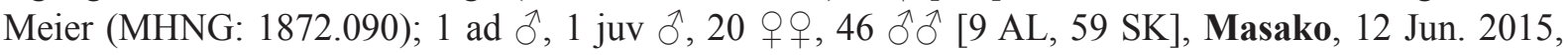
13 Jun. 2015, leg. Prescott Musaba, Moïse Bipoo, Charle Andabhati, Benjamin Kirongozi and Kambale Karupao, 17 Dec. 1987, 18 Dec. 1987, 19 Dec. 1987, 20 Dec. 1987, 21 Dec. 1987, 22 Dec. 1987, 13 Jan. 1988, 15 Jan. 1988, 20 Jan. 1988, 23 May 1989, 18 Sep. 1989, 28 Nov. 1989, 22 Mar. 1990, 29 Apr. 1990, leg. Ndey B. Ifuta (UNIKIS: CHMS067, RMCA: 93.079-M-0080, 93.079-M-0081, UNIKIS: CHMS069, CHMS073, CHMS074, RMCA: 93.079-M-0082, 93.079-M-0083, 93.079-M0269, 93.079-M-0229, 93.079-M-0257, 93.079-M-0060 to 93.079-M-0062, 93.079-M-0230 to 93.079M-0233, 93.079-M-0293 to 93.079-M-0297, 93.079-M-0063, 93.079-M-0064, 93.079-M-0298, 93.079M-0084 to 93.079-M-0091, 93.079-M-0065 to 93.079-M-0068, 93.079-M-0234 to 93.079-M-0239, 93.079-M-0069 to 93.079-M-0071, 93.079-M-0240, 93.079-M-0241, 93.079-M-0268, 93.079-M-0280, 93.079-M-0299, 93.079-M-0300, 93.079-M-0259, 93.079-M-0260, 93.079-M-0072 to 93.079-M-0079, 93.079-M-0242, 93.079-M-0301, 93.079-M-0369, 93.079-M-0258, 93.079-M-0261); 1 ad ${ }^{\lambda}, 1$ sad + [2 SA], Mbalaka (Plantation), 19 Jul. 1995, 23 Jul. 1995, leg. Herwig Leirs et al. (RMCA: 97.021-M-

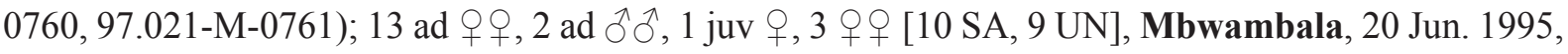
21 Jun. 1995, 23 Jun. 1995, 1 Jul. 1995, 2 Jul. 1995, 3 Jul. 1995, 4 Jul. 1995, 6 Jul. 1995, 7 Jul. 1995, 11 Jul. 1995, leg. Herwig Leirs et al. (RMCA: 97.021-M-0756, MSB: 274637, 274648, RMCA: 97.021- 
VAN CAKENBERGHE V. et al., The bats of Congo, Rwanda and Burundi revisited

M-0750, MSB: 274638, RMCA: 97.021-M-0751, MSB: 274643, 274644, 274646, 274647, RMCA: 97.021-M-0753 to 97.021-M-0755, 97.021-M-0752, 97.021-M-0757, MSB: 274635, RMCA: 97.021M-0758, MSB: 274636, RMCA: 97.021-M-0759); 1 ○े [SS], Mukwaya, between 18 Apr. 1979 and 22 May 1979, leg. Betu Nsankulu (RMCA: 79.032-M-0015); 1 đ [SS], Munga, 22 Apr. 1978, leg. Alexandre Prigogine (RMCA: 79.033-M-0001); 1 † [SS], Mwenge, 4 Feb. 1967, leg. Alexandre Prigogine (RMCA: 35075); 7 đ̊ [6 SS, 1 SO], Ngula, 6 Mar. 1977, 8 Mar. 1977, 11 Mar. 1977, leg. Tshinyenye (RMCA: 77.021-M-0009, 77.021-M-0012, 77.021-M-0013, 77.021-M-0010, 77.021-M0014, 77.021-M-0016, 77.031-M-0001); 4 우 [4 UN], Obenge, 7 Feb. 2013, leg. Prescott Musaba and André Malekani (UNIKIS: TLL381 to TLL384); 4 웅, 1 đึ [2 SS, 2 AL, 1 SK], Quatorze, 25 Jun. 2013, 27 Jun. 2013, 1 Jul. 2013, 2 Jul. 2013, leg. William (“Bill”) T. Stanley (FMNH: 222417, 222418, 222620 , 222416, 222621); 14 우우, 6 ठึ 24 Aug. 2013, 25 Aug. 2013, 27 Aug. 2013, leg. Guy-Crispin Gembu Tungaluna, Prescott Musaba and André Malekani (UNIKIS: RBTL486 to RBTL491, RBTL495, RBTL521, RBTL522, RBTL548 to RBTL551, RBTL555, RBTL556, RBTL562 to RBTL564, RBTL576, RBTL577); 1 q [SS], Tandala, 6 Jul. 1979, leg. C. Brian Robbins (USNM: 537700); 7 juv ??, 1 + , 1 đ [9 AL], Tshakala, between 1 Jan. 1982 and 31 Jan. 1982, leg. Marc Colyn (RMCA: 82.019-M-0013 to 82.019-M-0021); 1 ? [SO], Tshimputu, 23 Mar. 1975, leg. Betu Nsankulu (RMCA: 75.061-M-0007); 1 +, 7 ?? [8 AL], Tshuapa District, between 1 Jan. 1960 and 31 Dec. 1964, leg. P. Lootens (RMCA: 34168, 34171 to 34173, 34176 to 34178, 34180); 1 đ [UN], Uma, 10 Apr. 2014, leg. Guy-Crispin Gembu Tungaluna, Prescott Musaba and André Malekani (UNIKIS: UMA544); 19 ?? [19 AL], Wafanya [= Wafania], unknown date, 17 Aug. 1991, 30 Aug. 1991, 7 Sep. 1991, 12 Sep. 1991, 20 Mar. 1992, 26 Apr. 1992, 27 Apr. 1992, 4 May 1992, 10 May 1992, leg. P. Lootens (RMCA: 92.079-M-0125, 92.079-M-0126, 92.079-M-0018, 92.079-M-0024, 91.076-M-0078, 91.076-M-0056, 92.079-M-0020, 92.079-M-0021, 92.079-M-0023, 92.079-M-0017, 92.079-M-0022, 92.079-M-0025, 92.079-M-0014, 92.079-M-0015, 92.079-M-0019, 91.076-M-0063, 92.079-M-0016, 91.076-M-0072, 91.076-M-0073); 5 우우 [2 SK, 3 UN], Yaekela, 3 May 2010, 4 May 2010, 5 May 2010, leg. Guy-Crispin Gembu Tungaluna, Prescott Musaba and José Akaibe (UNIKIS: CRT2361, CRT1809, CRT1812, CRT1814, CRT1846); 2 우, 1 $\lesssim$ [2 AL, 1 UN], Yoko, 4 Sep. 2012, 7 Sep. 2012, leg. Prescott Musaba, 4 Sep. 2012, leg. Prescott Musaba, Tembele and Marc Boketshu (RMCA: a1.097-M-3197, UNIKIS: YK002CH, RMCA: a1.097-M-3198); 3 우, 9 ઠ [1 SK, 11 UN], Zookis [= Kisangani], 16 Feb. 2013, leg. Prescott Musaba and André Malekani, 24 Dec. 2015, leg. Prescott Musaba, Moïse Bipoo, Charle Andabhati, Benjamin Kirongozi and Kambale Karupao (UNIKIS: CSB2, CSB5, CSB6, ZCH01 to ZCH04, ZCH07 to ZCH11).

\section{Myonycteris Matschie, 1899}

\section{Myonycteris angolensis (Bocage, 1898)}

BURUNDI: 4 우 [4 SB], Giserama, Colline, 30 Jul. 1991, 31 Jul. 1991, 10 Aug. 1991, leg. Julian C. Kerbis Peterhans, 31 Mar. 1992, leg. L. Davenport (FMNH: 148975, 148974, 148350, 148973); 1 juv ô, 3 q 9 [1 SA, 3 SK], Kayanza, 26 Mar. 1979, leg. Heinz Stephan (SMF: 57579 to 57582); 1 q, $1 \widehat{\partial}$ [1 AL, 1 SO], Kigwena Hill, 12 Oct. 1990, leg. Matthew Criblez, 24 Apr. 1982, leg. Danny Meirte (FMNH: 148349, RMCA: 82.016-M-0069); 2 우, 10 ๙ $ぇ, 1$ ? [2 AL, 6 SB, 5 SN], Kwogofe, Colline, 1 Apr. 1992, 3 Apr. 1992, 4 Apr. 1992, 5 Apr. 1992, 8 Apr. 1992, 9 Apr. 1992, 10 Apr. 1992, 11 Apr. 1992, 12 Apr. 1992, leg. L. Davenport (FMNH: 148351, 148363, 148360, 148361, 148352, 148358, 148353 to 148357, 148359, 148362); 2 우, 2 ๙

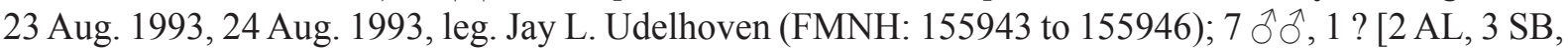
3 SN], Nyagatarugwa River Valley, 9 Aug. 1992, 10 Aug. 1992, leg. L. Davenport (FMNH: 148343,

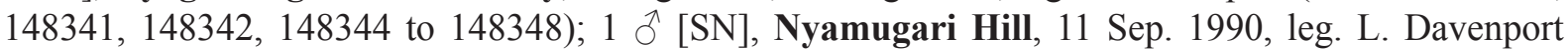
(FMNH: 148364); 1 + , 18 ๙ิ ๙, 3 ?? [6 AL, 5 SK, 7 SB, 4 SN], Ruhinga Hill, 9 Mar. 1993, 10 Mar. 1993, 11 Mar. 1993, 12 Mar. 1993, 13 Mar. 1993, leg. Jay L. Udelhoven (FMNH: 155951 to 155968, 155947 to 155950); 5 qㅜ, 2 $\widehat{\partial}$ [1 SS, $1 \mathrm{AL}, 5 \mathrm{SB}$ ], Teza, $2.3 \mathbf{~ k m ~ N , ~} 0.7$ km W [= Kibira National Park], 
14 Mar. 1990, 16 Mar. 1990, 19 Mar. 1990, 26 Mar. 1990, 28 Mar. 1990, leg. Julian C. Kerbis Peterhans (FMNH: 137620 to $137624,137619,137476)$.

DEMOCRATIC REPUBLIC OF THE CONGO: 6 우 [6 AL], Alambi, 5 Aug. 1976, 6 Aug. 1976, 7 Aug. 1976, leg. Frieder Meier (MHNG: 1872.072 to 1872.077); 1 ? [SS], Banjan Caves, prior to 3 Nov. 1936, leg. Schwetz (RMCA: 13689); 3 우우 4 ?? [7 AL], Barafinda Cave B 13, 15 Jun. 1949, leg. Narcisse Leleup (RMCA: 22504 to 22510); 4 우, 1 ๙ [5 AL], Bossafinda, unknown date, leg. the Royal Museum for Central Africa (RMCA) (BMNH: 1954.846 to 1954.850); 8 우, 5 ठぇ [1 SS, 12 AL], Bushokwe Caves, 1 Aug. 1956, leg. Alvin Novick, 3 Jun. 1957, leg. Jean Dubois (MCZ: 49032, 48288 to 48299); 2 q,+ 1 ô, 4 ?? [6 AL, 1 UN], Butembo, 1 Jan. 1970, leg. M.J. Celis and M. Lejeune, 21 Feb. 1956, prior to 29 Jul. 1957, prior to 25 Jan. 1956, leg. P. Dyleff (RMCA: 36194, 36204, 36207, 36208, 23675, 23449, 26435); 1 ? [UN], Djelube River, lower, unknown date, leg. unknown collector (RBINS: 13228); 1 ? [SS], Djugu Forest, between 1 Jan. 1952 and 31 Dec. 1952, leg. Alexandre Fain (RMCA: 23151); 1 ô [SS], Drodro, 22 Sep. 1952, leg. Alexandre Fain (MHNG:

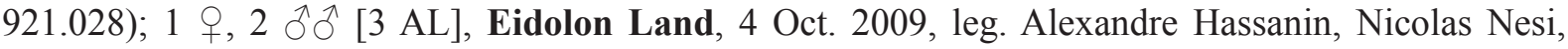
Célestin Pongombo, Peter Vallo and Didier Tshikung (MNHN: ZM-2011-804 to ZM-2011-806); 2 우으, 4 ठึ $\widehat{o}$ [6 UN], Goroumbwa Mine, 21 Oct. 1999, 22 Oct. 1999, 24 Oct. 1999, 25 Oct. 1999, leg. Robert (Bob) Swanepoel (TM: DBA069, DBA076, DBA126, DBA227, DBA230, DBA287); 1 ठ [AL], Grand Ebouli de Lemera [= Itombwe], 10 Sep. 1957, leg. Narcisse Leleup (RMCA: 26706); 1 q [SK], Irangi, 22 Jan. 1984, leg. Heinz Stephan (SMF: 65027); 1 imm $\circ$ [SS], Jaima, 26 Aug. 1906, leg. The Alexander-Gosling Expedition (BMNH: 1907.7.8.24); 1 q, 1 ? [1 SS, 1 UN], Kabambi, unknown date, leg. unknown collector, 17 Aug. 1947, leg. Jean Hiernaux (INPBC: W1461, RBINS: 7055); 1 sad of [SB], Kabobo Forest, 16 Feb. 2007, leg. B.D. Marks (FMNH: 195081); 1 juv $q, 2$ 우 [3 SA], Kahuzi-Biega National Park, 16 Oct. 1965, 20 Apr. 1966, 21 Apr. 1966, leg. P. Kunkel (SMF: 31820 , 31818, 31819); 2 우 [2 AL], Kalumbu Cave, 1 Dec. 1957, leg. Félix / Michel Anciaux de Faveaux (RMCA: 27592, 27593); 1 $\widehat{~[S O], ~ K a s a i, ~} 22$ Jan. 1982, leg. Betu Nsankulu (RMCA: 83.025-M-0023); 1 ? [UN], Katanda, unknown date, leg. unknown collector (INPBC: W975.2); 3 ?? [3 AL], Kibwe Lya Mikako Cave, 28 Dec. 1966, leg. M.J. Celis and M. Lejeune (RMCA: 35168, 35169, 35204); 1 ○े [SS], Kiliza [River], 17 Jan. 1966, leg. Alexandre Prigogine (RMCA: 34210); 1 ㅇ, 4 $\lesssim \widehat{~[5 ~ S S], ~ K i l o ~ m i n e s ~}$ [= Kilomines], 20 Apr. 1949, leg. Jean Hiernaux (RMCA: 19090 to 19094); 1 ? [AL], Kimbemba Cataracts, between 1 Sep. 1936 and 30 Sep. 1936, leg. Schwetz (RMCA: 13701); 1 ㅇ [UN], Kinshasa, unknown date, leg. Henri Schouteden (RMCA: 15760); 1 ? [AL], Kisangani, 20 Mar. 1988, leg. Luc Bijnens (RMCA: 88.017-M-0001); 3 $\widehat{~}$ [3 AL], Komanda, 21 Aug. 1976, leg. Frieder Meier (MHNG: 1872.078 to 1872.080); 1 ? [SS], Léopoldville [= Kinshasa], prior to $31 \mathrm{Dec}$. 1926, leg. Henri Schouteden (RMCA: 15760a); 1 q [SO], Lubango [= Lobango], 25 May 1950, leg. Alexandre Prigogine (RMCA: 20691); 1 ð [SS], Lusilubi Valley, 12 Aug. 1946, leg. J. de Wilde (RBINS: 13328); 1 †, 4 ठึ đે [5 SS], Lutunguru, 30 Mar. 1949, 5 Apr. 1949, 6 Jul. 1949, 4 Aug. 1949, 16 Feb. 1952, leg. Alexandre Prigogine (RMCA: 20820, 18863, 19053, 18862, 19052); 1 đ [AL], Lwana River, 19 Apr. 1992, leg. Ngenge Masumbuko Kamitongo (ZMA: MAM.24560); 2 우, 2 ふふ [2 SS, 2 SA], Lwiro [= Luiro], 1 Apr. 1967, 4 Apr. 1967, leg. Alexandre Prigogine, 28 May 1964, between 1 Jul. 1965 and 31 Jul. 1965, leg. P. Kunkel (RMCA: 35064, SMF: 31816, RMCA: 35065, SMF: 31817); 1 ๙ै [SS], Make, 23 Jul. 1993, leg. Tshinyenye (RMCA: 93.097-M-0022); 2 foetus ??, 1 q [2 AL, 1 SK], Masako, 16 Dec. 1987, leg. Ndey B. Ifuta, between 23 May 1988 and 28 May 1988, leg. Luc Bijnens (RMCA:

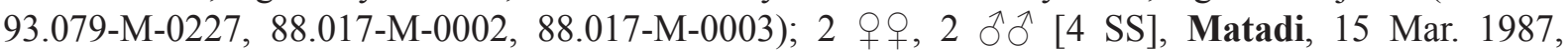
16 Mar. 1987, leg. Ntotoji (RMCA: 87.019-M-0001 to 87.019-M-0004); 2 ?? [1 AL, 1 UN], Matupi Cave, unknown date, leg. unknown collector, 4 Aug. 1955, leg. Gaston-François de Witte (INPBC: W1459, RMCA: 37118); 1 ㅇ [SS], Miki, 20 Aug. 1954, leg. Alexandre Prigogine (RMCA: 23423); 1 ? [SS], Mongbwalu, 14 Aug. 1939, leg. Janssens (RMCA: 15759); 3 ad $ㅇ$ [3 AL], Montembessa Cave, between 1 Nov. 1969 and 30 Nov. 1969, between 1 Sep. 1969 and 30 Sep. 1969, leg. unknown collector

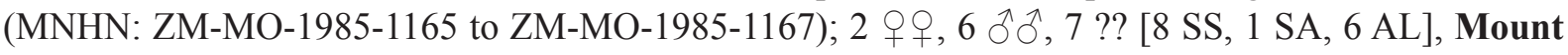


Hoyo Caves, 17 Aug. 1947, leg. Jean Hiernaux, 19 Sep. 1955, leg. James Paul Chapin, 4 May 1955, leg. W. Smith and E. Baker, 4 May 1955, leg. Waldo LaSalle Schmitt and E. Baker (RBINS: 7065, AMNH: 180902 to 180905, USNM: 301703 to 301712); 1 ô, 3 ?? [4 SS], Mount Wago [= Wago, Mount], unknown date, prior to 28 Mar. 1952, leg. Alexandre Fain, 1 Jan. 1952, leg. unknown collector (BMNH: 1953.477, 1953.478, RBINS: 21665, RMCA: 20804); 2 우 이 SS, 1 SO], Munoi [= Munoi confluence], 14 Apr. 1948, leg. Gaston-François de Witte, 14 Jun. 1947, leg. unknown collector (RBINS: 10663, 10662); 1 ? [UN], Muvo, Mount, unknown date, leg. unknown collector (INPBC: V1235); 1 ㅇ [SS], Mwenge, 8 Feb. 1967, leg. Alexandre Prigogine (RMCA: 35077); 1 + , 1 ? [1 SS, 1 SO], Pelenge, Gorges, 4 Jun. 1947, leg. unknown collector, between 4 Apr. 1947 and 4 Jun. 1947, leg. Gaston-François de Witte (RBINS: 10660, 10661); 1 [SS], Rutshuru, 26 Feb. 1938, leg. J. Ghesquière (RMCA: 14597); 1 ? [SO], Ruwenzori, Foothills, between 1 Jan. 1925 and 31 Dec. 1925, leg. Henri Martin Hackars (RMCA: 9904a); 1 ô, 3 ?? [4 SS], Saliboko, 22 Sep. 1952, leg. unknown collector, between 22 Sep. 1952 and 31 Dec. 1952, leg. Alexandre Fain (RBINS: 21666, RMCA: 81.012-M-0044 to 81.012M-0046); 1 juv ?, 1 ? [2 SS], Shabunda, 16 Aug. 1952, leg. P.G. Vercammen (RMCA: 23177, 23178); 2 ad $ㅇ+$, 3 juv ??, 1 ㅇ, 3 ?? [5 SS, 4 AL], Thysville [= Mbanza-Ngungu], 29 Mar. 1964, 29 Apr. 1964, prior to 18 Aug. 1965, leg. Antoon Emeric Marcel De Roo (RMCA: 32655, 32656, 32767, 32777 to 32779, 32388, 32389, 33568); 3 ?? [1 SA, 2 AL], Thysville Cave b 13 a [= Banjan Caves], 23 May 1949, 17 Aug. 1949, leg. Narcisse Leleup (RMCA: 22671, 22672, 22503); 2 $\widehat{\jmath}$ [2 SS], Wago, Mount, prior to 16 Oct. 1952, leg. Alexandre Fain (RMCA: 21436, 21437); 2 ?? [2 SK], Yoko, unknown date, leg. Prescott Musaba (RMCA: a1.097-M-3162, a1.097-M-3163).

RWANDA: 1 \&, 1 ? [2 AL], Gisovu, 2 Oct. 1980, 31 Oct. 1980, leg. Danny Meirte (RMCA: 80.055-M0096, 80.055-M-0097); 1 ? [SA], Kibuye, 2 Dec. 1971, leg. Alena Vanderborght-Elbl, G. Mathys and

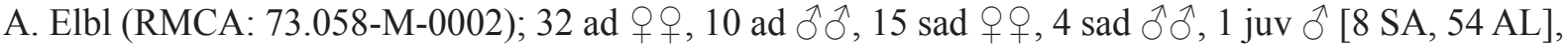
Ntango, 24 May 1982, 25 May 1982, 26 May 1982, leg. Benny Baeten (RMCA: 87.046-M-0063 to 87.046-M-0124); 2 우 [1 SA, 1 AL], Nyungwe Forest Reserve, 18 Oct. 1976, leg. Frieder Meier

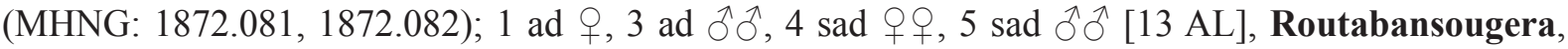
14 Jul. 1982, 15 Jul. 1982, 17 Jul. 1982, leg. Benny Baeten, 19 Oct. 1981, 20 Oct. 1981, leg. Frits De Vree, Benny Baeten and Victor Van Cakenberghe (RMCA: 87.046-M-0125 to 87.046-M-0134,

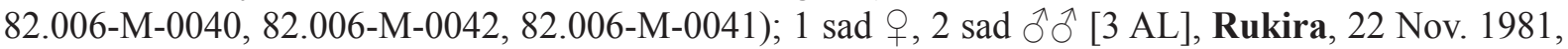
23 Nov. 1981, 26 Nov. 1981, leg. Frits De Vree, Benny Baeten and Victor Van Cakenberghe (RMCA: 82.006-M-0043 to 82.006-M-0045); 1 ad + [SA], Shangi, 5 Jan. 1985, leg. Benny Baeten and Luc Janssens (RMCA: 85.006-M-0464); 1 ad,+ 1 ad ô $^{2} 1$ sad,+ 1 sad ồ [2 SA, 2 AL], Uwinka, 16 Oct. 1981, 17 Oct. 1981, 18 Oct. 1981, leg. Frits De Vree, Benny Baeten and Victor Van Cakenberghe (RMCA: 82.006-M-0036 to 82.006-M-0039).

\section{Myonycteris relicta Bergmans, 1980}

DEMOCRATIC REPUBLIC OF THE CONGO: 1 ô [SS], Beyi - Kalonji, 22 Feb. 1980, leg. Betu Nsankulu (RMCA: 80.020-M-0001).

\section{Myonycteris torquata (Dobson, 1878)}

DEMOCRATIC REPUBLIC OF THE CONGO: 4 ?? [3 AL, 1 SK], unknown locality, unknown date, leg. Prescott Musaba, between 1 May 2010 and 31 May 2010, leg. Nicolas Nesi, Blaise Kadjo and Christiane Denys (RMCA: a1.097-M-3194 to a1.097-M-3196, MNHN: ZM-2014-1168); 1 ? [UN], Albert National Park [= Virunga National Park], unknown date, leg. unknown collector (INPBC: V1694.2); 1 $\lesssim$ [SS], Bakwakenge [= Bakwa-Kenge], 3 Feb. 1980, leg. Betu Nsankulu (RMCA: 80.020-M-0005); 4 우, 2 ○ึ [1 SS, 5 SK], Baliko, 11 Jun. 2012, 12 Jun. 2012, 13 Jun. 2012, 14 Jun. 2012, leg. William ("Bill”) T. Stanley (FMNH: 219592, 219593, 219680, 219594 to 219596); 2 ภ̋ [2 SS], Bena Mbala, 21 Feb. 1984, leg. Betu Nsankulu (RMCA: 84.035-M-0004, 84.035-M- 
0006); 6 §ै के, 4 ?? [10 AL], Boende, between 1 Jan. 1974 and 31 Dec. 1974, between 1 Jan. 1967 and 31 Dec. 1967, leg. P. Lootens (RMCA: 38650, 38651, 38671 to 38676, 75.035-M-0002, 75.035-M-

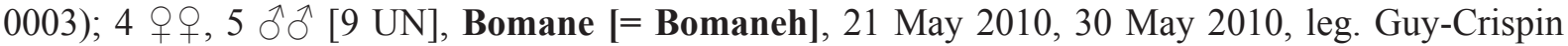
Gembu Tungaluna, Prescott Musaba and José Akaibe (UNIKIS: CRT2127, CRT2130, CRT2144, CRT2156, CRT2157, CRT2195, CRT2196, CRT2200, CRT2078); 1 đ [SS], Cinputu, 13 Sep. 1983, leg. Betu Nsankulu (RMCA: 84.035-M-0005); 2 ð̊ [2 UN], Congo-Nil-Aka, 20 May 1952, leg. H. de Saeger et al., 20 May 1952, leg. H. de Saeger (RBINS: 13525, RMCA: 13525); 14 ad +9 , 17 ad $\lesssim \widehat{ }$,

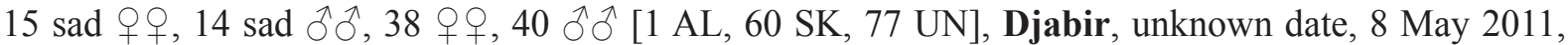
9 May 2011, 10 May 2011, 12 May 2011, leg. Prescott Musaba, 8 May 2011, 9 May 2011, 10 May 2011, 11 May 2011, 12 May 2011, 13 May 2011, leg. Prescott Musaba and Jonathan Kosele (RMCA: a1.097-M-3248, a1.097-M-3249, a1.097-M-3265 to a1.097-M-3268, a1.097-M-3282, a1.097-M-3250 to a1.097-M-3264, UNIKIS: DJB083, DJB084, DJB087, DJB088, DJB091, DJB092, DJB094, DJB097, DJB098, DJB102, DJB104, DJB106, DJB109, DJB111, DJB122, RMCA: a1.097-M-3269 to a1.097-M-3281, UNIKIS: DJB127, DJB128, DJB130, DJB133, DJB139, DJB142, DJB147, DJB154 to DJB167, DJB170, RMCA: a1.097-M-3223 to a1.097-M-3230, UNIKIS: DJB001, DJB003, DJB006, DJB008 to DJB011, DJB014, DJB016, DJB018 to DJB020, DJB022 to DJB024, DJB026, DJB027, DJB029, DJB030, DJB032, DJB034, DJB036, DJB037, RMCA: a1.097-M-3231 to a1.097-M-3247, UNIKIS: DJB040, DJB044, DJB046, DJB048 to DJB050, DJB052, DJB053, DJB055, DJB056, DJB058, DJB060, DJB062, DJB067, DJB070, DJB071, DJB169,

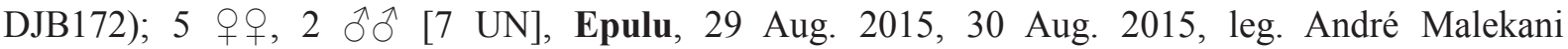
(UNIKIS: EPLU660, EPLU669, EPLU670, EPLU683, EPLU684, EPLU695, EPLU696); $1 \mathrm{imm} \overbrace{}^{\Uparrow}, 1$ ㅇ [2 UN], Gangala-na-Bodio, 11 May 1948, leg. unknown collector (RBINS: 11657, RMCA: 11657);

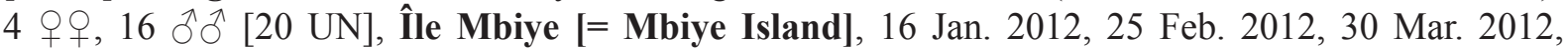
31 Mar. 2012, leg. Prescott Musaba, Tembele and Marc Boketshu, 19 Apr. 2014, 21 Apr. 2014, leg. Prescott Musaba, Moïse Bipoo, Richard Tamaru, Pauline Isude and Joseph Kussa, 7 Aug. 2015, 28 Dec. 2015, 8 Jan. 2016, leg. Prescott Musaba, Moïse Bipoo, Charle Andabhati, Benjamin Kirongozi and Kambale Karupao (UNIKIS: CHIM07, CHIM07/14, CHIM011/14, CHIM012/14, CHIM29, ABCHIM07, ABCHIM08, CHIM34, CHIM35, CHIM40 to CHIM45, CHIM47, CHIMA38, CHIMTB01, ABCHIM09, ABCHIM11); 1 q [SA], Irangi, 11 Feb. 1984, leg. Heinz Stephan (SMF: 65030); 2 우 [2 UN], Isai River, between 16 Dec. 1974 and 23 Dec. 1974, leg. Michael D. Gallagher (HZM: 1.7798, 2.7799); 1 ㅇ [UN], Kamikoni, 17 May 1960, leg. unknown collector (RBINS: 1694); $1 \mathrm{imm}$ ㅇ [UN], Karambi, 4 Apr. 1992, leg. Ngenge Masumbuko Kamitongo (ZMA: MAM.24567); 1 \& [SO], Kimpoko [= Kimpoko-Nsele], 7 Nov. 1978, leg. Tshinyenye (RMCA: 79.003-M-0001); 1 § [UN], Kingabwa, 31 Dec. 1975, leg. Tshinyenye (RMCA: 76.010-M-0004); 1 imm ? [UN], Kinshasa, 2 May 1964, leg. Jo Van Orshoven (ZMA: MAM.11163); 21 ?? [21 UN], Kinshasa City Province, 12 Feb. 2001, between 1 Apr. 1999 and 30 Apr. 1999, leg. unknown collector (SMF 92979 to 92983,93281 to 93296); 1 ค, 1 ઈ [2 AL], Kisangani, between 1 Jun. 1982 and 30 Jun. 1982, leg. Marc Colyn (RMCA: 82.031-M-0012, 82.031-M-0013); 2 ठิ $\widehat{~}$ [1 SK, 1 SO], Kitangua, 24 Dec. 1981, leg. Betu Nsankulu (RMCA: 83.025-M0006, 83.025-M-0019); 20 우, 8 $\widehat{\jmath}, 1$ ? [13 SK, 16 UN], Kona, unknown date, leg. Prescott Musaba, 12 May 2010, 13 May 2010, 14 May 2010, 15 May 2010, between 12 May 2010 and 13 May 2010, leg. Guy-Crispin Gembu Tungaluna, Prescott Musaba and José Akaibe (RMCA: a1.097-M-3160, UNIKIS: CRT1910, CRT1911, CRT1920, CRT1926, CRT1937, CRT1942 to CRT1945, CRT1947 to CRT1950, CRT1953, CRT1960, CRT1963, CRT1966 to CRT1972, CRT1979 to CRT1982, CRT1918); 3 ?? [3 AL], Konyola, prior to 5 Aug. 1988, leg. Luc De Vos, Kimbembi and Alain Vandelanoote

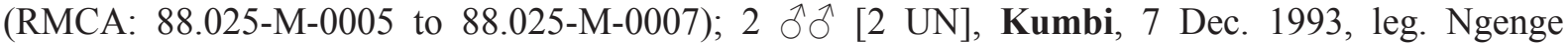
Masumbuko Kamitongo (ZMA: MAM.24896, MAM.24897); 13 q q, 14 ô $\widehat{\jmath}, 1$ ? [1 SK, 27 UN], Lieki, 21 May 2010, 30 May 2010, 1 Jun. 2010, 2 Jun. 2010, 3 Jun. 2010, leg. Guy-Crispin Gembu Tungaluna, Prescott Musaba and José Akaibe (UNIKIS: CRT2029, CRT2030, CRT2032 to CRT2035, CRT2037, CRT2282 to CRT2288, CRT2290 to CRT2296, CRT2057, CRT2021 to CRT2023, CRT2028, CRT2050, CRT2051); 1 ad $\widehat{\partial}$ [UN], Likandi River [= Litaki River], 18 Apr. 1906, leg. The Alexander-Gosling 
Expedition (BMNH: 1907.7.8.25 [holotype Myonycteris wroughtoni K. Andersen, 1908]); $1 \widehat{\partial}$ [UN], Likati River, 18 Apr. 1906, leg. unknown collector (BMNH: 1907.7.8.26); 14 ?? [14 SS], Lovanium University [= Kinshasa], 10 Jan. 1962, 13 Jan. 1962, 2 May 1962, leg. De Bont, 22 Nov. 1962, 3 Dec. 1962, 20 Feb. 1963, 17 Mar. 1963, 23 Mar. 1963, 30 Mar. 1963, 2 Apr. 1963, 19 Apr. 1963, 26 Dec. 1966, between 30 Mar. 1963 and 1 Apr. 1963, between 1 Nov. 1962 and 1 Dec. 1962, leg. De Bont and Jo Van Orshoven (RMCA: 31197, 31198, 32606, 32608, 32611, 31199, 32609, 32602, 32607, 32601, 32604, 32605, 32610, 32603); 1 đิ [AL], Lubumbashi, 12 Oct. 2009, leg. Alexandre Hassanin, Nicolas Nesi, Célestin Pongombo, Peter Vallo and Didier Tshikung (MNHN: ZM-2011-859); $1 \hat{~} \widehat{\text { [SS}}$ ], Lukonga, 7 Oct. 1978, leg. Betu Nsankulu (RMCA: 79.022-M-0006); 12 ?? [3 SS, 9 AL], Luluabourg [= Kananga], 1 May 1964, 7 Nov. 1964, 13 Feb. 1965, 20 Feb. 1965, 4 Mar. 1965, 1 Apr. 1965, 2 Apr. 1965, prior to 1 Jun. 1965, leg. Antoon Emeric Marcel De Roo (RMCA: 33336, $33338,33413,33414,32660,33333,33339,33340,33334,33335,33332,33337) ; 1$ o [UN], Lwana River, unknown date, leg. Ngenge Masumbuko Kamitongo (ZMA: MAM.24568); 2 sad $\widehat{\partial} \widehat{\jmath}, 1$ juv $q$,

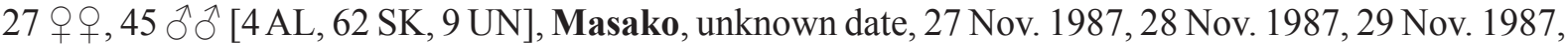
30 Nov. 1987, 16 Dec. 1987, 17 Dec. 1987, 22 Apr. 1988, 25 Aug. 1989, 29 Aug. 1989, 30 Aug. 1989, 24 Nov. 1989, 22 Mar. 1990, 25 Mar. 1990, leg. Ndey B. Ifuta, 10 Jun. 2015, 11 Jun. 2015, 13 Jun. 2015, leg. Prescott Musaba, Moïse Bipoo, Charle Andabhati, Benjamin Kirongozi and Kambale Karupao (RMCA: 93.079-M-0278, UNIKIS: CHMS062, CHMS063, CHMS065, CHMS066, CHMS070 to CHMS072, CHMS075, CHMS077, RMCA: 93.079-M-0043 to 93.079-M-0051, 93.079-M-0287 to 93.079-M-0291, 93.079-M-0052 to 93.079-M-0059, 93.079-M-0228, 93.079-M-0292, 93.079-M-0276, 93.079-M-0272, 93.079-M-0275, 93.079-M-0277, 93.079-M-0270, 93.079-M-0026, 93.079-M-0027, 93.079-M-0214 to 93.079-M-0216, 93.079-M-0028 to 93.079-M-0033, 93.079-M-0217 to 93.079-M0219, 93.079-M-0267, 93.079-M-0273, 93.079-M-0034, 93.079-M-0220, 93.079-M-0284, 93.079-M0274, 93.079-M-0035 to 93.079-M-0042, 93.079-M-0221 to 93.079-M-0226, 93.079-M-0285, 93.079M-0286); 2 ad + +, 1 ad J, $1 \mathrm{imm}+$ [4 UN], Medje, 16 Apr. 1910, 6 Sep. 1910, 4 Apr. 1914, leg. Herbert Lang, James Paul Chapin and The American Museum Congo Expedition (AMNH: 48752, 48755, 48753, 48754); 3 $\lesssim ~[3 \mathrm{AL}$ ], Pandé, Forest N of, 11 Oct. 2009, leg. Alexandre Hassanin, Nicolas Nesi, Célestin Pongombo, Peter Vallo and Didier Tshikung (MNHN: ZM-2011-856 to ZM2011-858); 6 + 9 [1 SS, 1 AL, 4 SK], Quatorze, 15 Jun. 2012, 25 Jun. 2013, 29 Jun. 2013, 30 Jun. 2013,

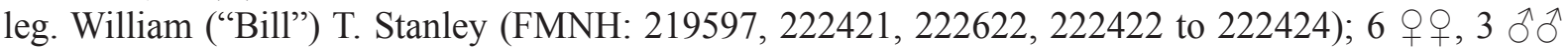
[9 UN], Rubi-Tele, 18 Aug. 2013, 19 Aug. 2013, 20 Aug. 2013, 22 Aug. 2013, 27 Aug. 2013, leg. GuyCrispin Gembu Tungaluna, Prescott Musaba and André Malekani (UNIKIS: RBTL502, RBTL503, RBTL516, RBTL520, RBTL529, RBTL530, RBTL541, RBTL542, RBTL575); 2 +,+ 1 ? [1 SK, 2 UN], Sciere Forest, between 24 Nov. 1974 and 25 Nov. 1974, leg. Michael D. Gallagher (HZM: 3.7866 to

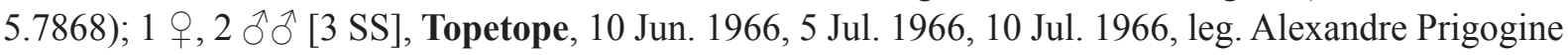
(RMCA: 34381, 34383, 34382); 3 đో ${ }^{\Uparrow}$ [3 UN], Uma, 10 Apr. 2014, 12 Apr. 2014, 13 Apr. 2014, leg. Guy-Crispin Gembu Tungaluna, Prescott Musaba and André Malekani (UNIKIS: UMA501, UMA624, UMA635); 14 ?? [14 AL], Wafanya [= Wafania], 3 Dec. 1988, 2 Sep. 1991, 14 Sep. 1991, 3 Oct. 1991, 18 Apr. 1992, 12 May 1992, 29 May 1992, between 1 Nov. 1991 and 30 Nov. 1991, between 1 Apr. 1991 and 30 Apr. 1991, between 1 Jan. 1991 and 31 Dec. 1991, leg. P. Lootens (RMCA: 92.079-M-0068, 91.076-M-0082, 92.079-M-0065, 92.079-M-0070, 91.076-M-0065, 92.079-M-0066, 92.079-M-0069, 91.076-M-0098, 91.076-M-0100, 89.020-M-0039, 92.079-M-0063, 91.076-M-0020, 92.079-M-0064,

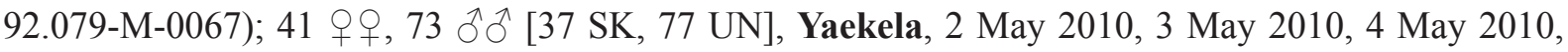
5 May 2010, 6 May 2010, 7 May 2010, between 5 May 2010 and 13 May 2010, between 5 May 2010 and 12 May 2010, leg. Guy-Crispin Gembu Tungaluna, Prescott Musaba and José Akaibe (UNIKIS: CRT2305, CRT2307, CRT2316 to CRT2318, CRT2320, CRT2351 to CRT2360, CRT2362, CRT2365, CRT2366, CRT2369 to CRT2373, CRT2375 to CRT2383, CRT2385, CRT1801, CRT1802, CRT1804 to CRT1808, CRT1810, CRT1811, CRT1815, CRT1819, CRT1820, CRT2391, CRT2393, CRT2394, CRT2397 to CRT2400, CRT1824 to CRT1830, CRT1833 to CRT1840, CRT1842 to CRT1845, CRT1847, CRT1848, CRT1850 to CRT1853, CRT1855, CRT1857, CRT1859 to CRT1866, CRT1869 to 
CRT1878, CRT1883 to CRT1887, CRT1889 to CRT1896, CRT1831, CRT1832, CRT1841); 1 , 2 ふ઼ [2 SS, 1 AL], Yalosemba, 18 Jun. 1979, 19 Jun. 1979, leg. C. Brian Robbins, 23 Jun. 1979, leg. Frieder Meier (USNM: 537694, 537695, MHNG: 1871.014); 3 우, 11 ふૈર, 1 ? [3 AL, 1 SK, 11 UN], Yoko, unknown date, 4 Sep. 2012, 7 Sep. 2012, leg. Prescott Musaba, 18 May 2009, 23 Sep. 2009, 24 Sep. 2009, 28 Sep. 2009, leg. unknown collector, 4 Sep. 2012, 5 Sep. 2012, 6 Sep. 2012, leg. Prescott Musaba, Tembele and Marc Boketshu (RMCA: a1.097-M-3161, UNIKIS: GE246, GE281, GE284, GE293, RMCA: a1.097-M-3192, a1.097-M-3193, UNIKIS: YK005CH, YK010CH, YK013CH, YK015CH,

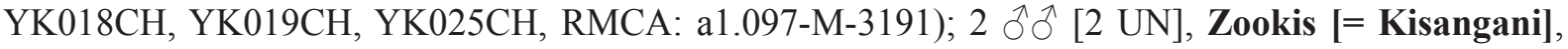
24 Dec. 2015, leg. Prescott Musaba, Moïse Bipoo, Charle Andabhati, Benjamin Kirongozi and Kambale Karupao (UNIKIS: ZCH05, ZCH12).

Plerotini Bergmans, 1997

Plerotes K. Andersen, 1910

Plerotes anchietae (Seabra, 1900)

DEMOCRATIC REPUBLIC OF THE CONGO: 1 đ [AL], Kaloba, 5 km from, 3 Oct. 2009, leg. Alexandre Hassanin, Nicolas Nesi, Célestin Pongombo, Peter Vallo and Didier Tshikung (MNHN: ZM-

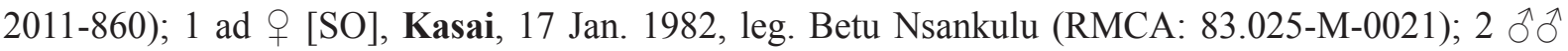
[1 SS, 1 SO], Kipangaribwe River, 10 Dec. 1947, 14 Jan. 1948, leg. Gaston-François de Witte et al. (RBINS: 10685, 10686); 1 ô [UN], Kisantu, 11 Dec. 1920, leg. Henri Schouteden (RMCA: 7300b).

Rousettini K. Andersen, 1912

Rousettus Gray, 1821

DEMOCRATIC REPUBLIC OF THE CONGO: 1 juv ? [UN], Djomba [= Djemba], unknown date, leg. unknown collector (INPBC: V93.1); 1 juv ? [UN], Kabambi, unknown date, leg. unknown collector (INPBC: W1467); 1 ? [AL], Mount Hoyo [= Hoyo, Mount], unknown date, leg. The Cambridge East Africa Expedition 1959 (BMNH: 1960.113); 1 juv ? [UN], Rutshuru, unknown date, leg. unknown collector (INPBC: V1447).

Rousettus aegyptiacus (E. Geoffroy St.-Hilaire, 1810)

BURUNDI: $1 \lesssim$ [UN], Bujumbura, 1 Aug. 1976, leg. Jacques Verschuren (RBINS: 19903); 1 juv $q$ [SA], Gihanga, 14 Mar. 1979, leg. Heinz Stephan (SMF: 57530); 1 ? [SK], Kayanza, 27 Mar. 1979, leg. Heinz Stephan (SMF: 57531); 1 ค , 1 ภิ [2 SO], Kigwena Hill, 13 Oct. 1990, leg. Matthew Criblez (FMNH: 148339, 148340); 1 \& [SB], Nyagatarugwa River Valley, 9 Aug. 1992, leg. L. Davenport (FMNH: 148338); 2 q 9 [2 SK], Nyanza-Lac, 3 Mar. 1979, leg. Heinz Stephan (SMF: 57528, 57529); 2 우, 1 đ [2 SK, 1 CO], Resha, 2 Mar. 1979, 3 Apr. 1979, 4 Apr. 1979, leg. Heinz Stephan (SMF: 57526, 57525, 57527); 3 ?? [3 SS], Rumonge, 22 Jul. 1944, leg. J.M. Vrijdagh (RMCA: 17759 to 17761).

DEMOCRATIC REPUBLIC OF THE CONGO: 1 क , 4 ồ $\hat{\text {, }} 4$ ?? [5 SS, 3 AL, 1 SK], unknown locality, unknown date, leg. Guy-Crispin Gembu Tungaluna, between 1 May 2010 and 31 May 2010, leg. Nicolas Nesi, Blaise Kadjo and Christiane Denys, prior to 12 Jun. 1958, leg. Alexandre Fain, prior to 14 Dec. 1957 , leg. Félix / Michel Anciaux de Faveaux (RMCA: a1 .097-M-0548, a1.097-M-0549, MNHN: ZM-20141156, RMCA: 27182 to 27186, 26823); 4 ?? [4 UN], Albert National Park [= Virunga National Park], unknown date, leg. unknown collector (INPBC: V1765, V1839.3, V5006, V705); 1 q, 2 ઈิ [3 AL], Baliko, prior to 31 Jan. 2007, leg. Guy-Crispin Gembu Tungaluna (RMCA: a1.097-M-0414 to a1.097-M-0416); 8 ?? [8 AL], Banza Nfinda Caves, 15 Jun. 1949, leg. Narcisse Leleup (RMCA: 22511 to 22514, 22566 to 22569); 3 ?? [1 SS, 1 SK, 1 SO], Beni, prior to 2 Jun. 1914, leg. Bonnevie (RMCA: 


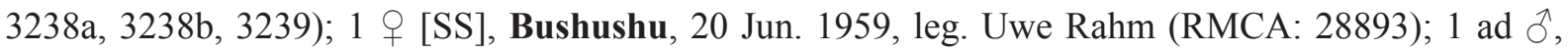
$1 \mathrm{imm} \lesssim$ [2 SS], Buta, 24 Dec. 1946, leg. F.L. Hendricks (RBINS: 12932, 12933); 2 우, 1 ๙ [2 SS, 1 AL], Butembo, 1 Jan. 1970, leg. M.J. Celis and M. Lejeune, 22 Jan. 1958, 26 Jul. 1960, leg. P. Dyleff (RMCA: 36206, 27384, 29242); 1 \& [SS], Butembo Region, 31 Jul. 1947, leg. Jean Hiernaux (RBINS:

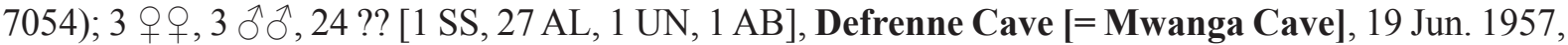
leg. Félix / Michel Anciaux de Faveaux, 25 Sep. 1948, leg. Narcisse Leleup, 3 Apr. 1957, leg. G. Verhoustraete (RMCA: 26711 to 26715, 26798, 22615 to 22637, 26214); 18 ?? [18 UN], Djemba, unknown date, leg. unknown collector (INPBC: V417, V93.1.01 to V93.1.17); 1 ? [UN], Djomba [= Djemba], unknown date, leg. unknown collector (INPBC: V1535); 1 ? [AL], Elisabethville [= Lubumbashi], prior to 15 Oct. 1963, leg. Parent (RMCA: 31509); 97 우, 108 $\widehat{\jmath}, 1$ ? [206 UN], Goroumbwa Mine, 14 May 1999, 17 May 1999, 18 May 1999, 19 May 1999, 19 Oct. 1999, 20 Oct. 1999, 21 Oct. 1999, 22 Oct. 1999, 23 Oct. 1999, 24 Oct. 1999, 25 Oct. 1999, 27 Oct. 1999, leg. Robert (Bob) Swanepoel (TM: DRC0001 to DRC0004, DRC0028, DRC0030, DRC0031, DRC0035 to DRC0052, DRC0054, DRC0057 to DRC0068, DRC0070, DRC0072, DRC0073, DRC0087, DRC0088, DRC0090 to DRC0101, DRC0108 to DRC0119, DRC0123, DRC0125, DRC0126, DRC0132, DRC0133, DRC0136 to DRC0140, DRC0143 to DRC0145, DRC0152, DRC0153, DRC0157, DBA002 to DBA007, DBA009, DBA029, DBA070, DBA074, DBA077 to DBA084, DBA110, DBA111, DBA114, DBA116 to DBA123, DBA125, DBA127, DBA128, DBA131, DBA142, DBA157, DBA187 to DBA193, DBA197, DBA226, DBA229, DBA231 to DBA238, DBA254, DBA281, DBA284 to DBA286, DBA335

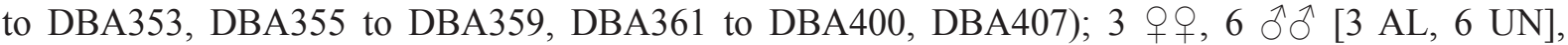
Jadotville [= Likasi], 3 Apr. 1957, 6 Dec. 1959, 18 Dec. 1959, leg. Félix / Michel Anciaux de Faveaux

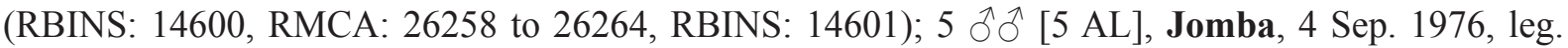
Frieder Meier (MHNG: 1872.062 to 1872.066); 1 ad $\widehat{~}$ [AL], Kadjudju [= Kajuju], 16 Apr. 1905, leg. unknown collector (MNHN: ZM-MO-1983-1267); 2 ?? [2 SS], Kakindo Cave [= Kakondo Cave], 27 Jul. 1962, 28 Jul. 1962, leg. A. Elbl (USNM: 375234, 375235); 1 đ [SS], Kakonda, 8 Oct. 1942, leg. F.L. Hendricks (AMNH: 118865); 6 우, 4 ○ึ [6 SS, 2 SK, 2 UN], Kakondo, 1 Dec. 1946, 24 Dec. 1946, leg. F.L. Hendricks, 1 Dec. 1946, leg. unknown collector, 14 Jan. 1959, leg. Uwe Rahm (RBINS: 13114.1, 13114.2, 13283, 13284, RMCA: 28031, 28890 to 28892, 19652, 19653); 1 q, 1 के [2 SS], Kakondo Cave, between 1 Dec. 1946 and 31 Dec. 1946, leg. F.L. Hendricks (RMCA: 19650, 19655); 3 웅, 1 ऽ [4 AL], Kakontwe, unknown date, leg. the Royal Museum for Central Africa (RMCA) (BMNH: 1954.778 to 1954.781); 1 ad 9,2 ?? [3 SS], Kakontwe Cave, between 1 Sep. 1948 and 30 Sep. 1948, leg. Narcisse Leleup, between 1 Dec. 1958 and 31 Dec. 1958, leg. Laurent (RMCA: 22483, 22484, 29219); 1 ? [AL], Kalimabenge River, 12 Jul. 1958, leg. Georges Marlier (RMCA: 27988); 3 ?? [3 AL], Kasuo Cave Kabwe ka, 26 Jan. 1955, leg. Wim Bergmans and Collard (RMCA:

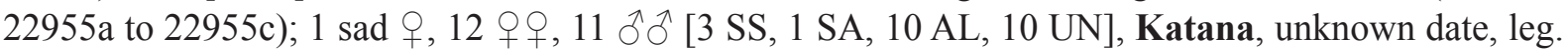
J.J. Laarman, 11 Jul. 1956, leg. Alvin Novick, 22 Jul. 1954, leg. James Paul Chapin, 30 May 1963, leg. P. Kunkel (RMNH: MAM.16347 to MAM.16355, MAM.16401, MCZ: 48186 to 48194, 48536, AMNH: 180899 to 180901, SMF: 31823); 1 q [SS], Katana Cave [= Mahyusha Cave], 23 Jan. 1957, leg. Heinz Stephan (RMCA: 27419); 2 đิ $\widehat{0}$ [2 SS], Kibanda, 15 May 1975, leg. Alexandre Prigogine (RMCA: 76.022-M-0021, 76.022-M-0022); 1 \&, 1 ○ [2 SS], Kilo Mines [= Kilomines], 16 Apr. 1949, 17 Apr. 1949, leg. Jean Hiernaux (RMCA: 19100, 19101); 1 ㅇ, 1 ठ [2 AL], Kinyamahura, 11 Apr. 1938, leg. Serge Frechkop (RMCA: 17083, 17084); 6 ?? [6 AL], Kisangani, between 1 Jul. 1985 and 31 Jul. 1985, leg. Marc Colyn, between 1 Oct. 1985 and 31 Mar. 1986, leg. Ndey B. Ifuta (RMCA:

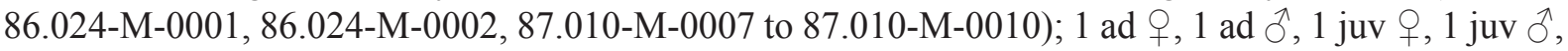
1 ㅇ [1 SA, 4 AL], Kivu [= Kivu region], 10 Apr. 1905, leg. unknown collector, 19 Jul. 1964, leg. P. Kunkel (MNHN: ZM-MO-1983-1076 to ZM-MO-1983-1079, SMF: 31824); 2 ?? [2 AL], Kivu region, between 1 Jan. 1934 and 31 Dec. 1934, leg. Guy Babault (MCZ: 46063, 46064); 1 q [SO], Kunungu, unknown date, leg. Henri Schouteden (RMCA: 7291a); 1 ㅇ, 1 đ [ 1 SO, 1 UN], Kyniamahura, 7 May 1938, leg. Serge Frechkop (RBINS: 4784, 4785); 2 ?? [2 AL], Lake Kivu, near, between

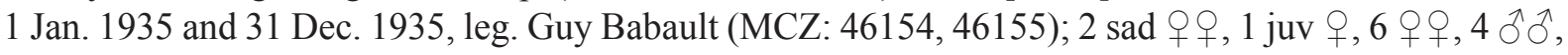


9 ?? [6 SS, 5 SA, 5 AL, 4 SO, 2 SB], Lwiro [= Luiro], unknown date, 2 Jun. 1964, 22 Jun. 1965, between 1 Jan. 1965 and 31 Dec. 1965, leg. P. Kunkel, 16 Jan. 1962, leg. Kiss, 6 May 1955, 16 May 1955, leg. Waldo LaSalle Schmitt and E. Baker, 16 May 1955, leg. Waldo LaSalle Schmitt, 31 Jul. 2002, leg. Michael H. Huhndorf, 7 Apr. 1959, 8 Apr. 1959, leg. Uwe Rahm (SMF: 25859, RMCA: 31004 to 31007, USNM: 301694, 301696 to 301702, SMF: 31822, 31821, FMNH: 173295, 173296, USNM: 301695, RMCA: 28894 to 28896, SMF: 25860); 1 क [SS], Mahysha Cave [= Mahyusha Cave], unknown date, leg. Alexandre Fain (RMCA: 27717); 2 우 [2 SS], Mahyusa Cave [= Mahyusha Cave], unknown date, leg. unknown collector (BMNH: 1958.647, 1958.648); 1 ㅇ, 1 ? [2 SS], Mai-yaMoto [= May ya Moto], 16 May 1955, leg. James Paul Chapin (AMNH: 180897, 180898); 2 ad $\hat{\jmath} \delta$,

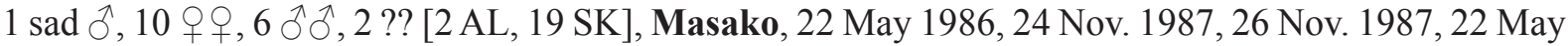
1989, 23 May 1989, 24 May 1989, 25 May 1989, 21 Jan. 1990, between 1 May 1989 and 31 May 1989, leg. Ndey B. Ifuta (RMCA: 93.079-M-0317, 87.010-M-0002, 87.010-M-0006, 93.079-M-0318, 93.079M-0305 to 93.079-M-0309, 93.079-M-0315, 93.079-M-0316, 93.079-M-0281, 93.079-M-0310 to 93.079-M-0312, 93.079-M-0319, 93.079-M-0282, 93.079-M-0283, 93.079-M-0313, 93.079-M-0320,

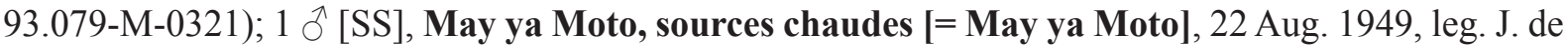
Wilde (RBINS: 12930); 1 juv $q, 1$ juv $\widehat{\jmath}, 2$ 우, 3 $\widehat{\jmath}$ [5 SS, 1 SO, 1 UN], Mount Hoyo [= Hoyo, Mount], 12 Aug. 1947, leg. Jean Hiernaux, 12 Aug. 1947, leg. unknown collector (RBINS: 7057 to 7061, 7064, 7474); $1 \mathrm{imm}$ + [AL], Mufunga, 23 Jul. 1958, leg. Félix / Michel Anciaux de Faveaux (RMCA: 27915); $1 \mathrm{imm}$ ? [AL], Mulungu, prior to 1 Feb. 1940, leg. F.L. Hendricks (RMCA: 15879); $1 \lesssim$ [SS], Mumba, 7 Jun. 1967, leg. Alexandre Prigogine (RMCA: 35112); 1 ? [UN], Murambi, unknown date, leg. unknown collector (INPBC: W831); 1 ô [SS], Mutsora, 27 Sep. 1949, leg. J. de Wilde (RBINS: 12931); 2 우, 1 ठ [3 SS], Nyarusambo, 7 Jan. 1947, leg. G. Van Cools (RBINS: 12871

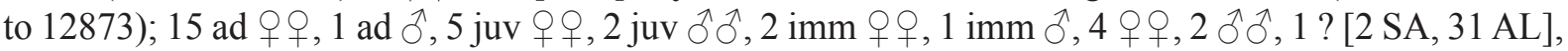
Pempere Cave, 19 Jun. 1957, 20 Oct. 1957, 21 Apr. 1958, leg. Félix / Michel Anciaux de Faveaux (MHNG: 1046.032, RMCA: 26777 to 26780, MHNG: 1046.033, 1046.034, RMCA: 27596 to 27606, 27608 to 27621, 27595); 1 q [UN], Rethy, 23 Oct. 2015, leg. Anne Laudisoit et al. (UNIKIS: ITU702); 1 ? [UN], Rumoka, unknown date, leg. unknown collector (INPBC: V1636.1); 2 ?? [2 UN], Rutshuru, unknown date, leg. unknown collector (INPBC: V1247, V1625); 1 ô [SS], Ruvara, 2 Apr. 1950, leg. J. de Wilde (RBINS: 12929); 2 +, 13 ?? [11 SA, 4 AL], Tamba Tamba Cave b 12, unknown date, leg. the Royal Museum for Central Africa (RMCA), 27 Sep. 1948, leg. Narcisse Leleup (BMNH: 1954.782, 1954.783, RMCA: 22673 to 22685); 15 우, 9 ठิ $\sigma^{\lambda}$ [6 AL, 3 SK, $15 \mathrm{UN}$ ], Uma, 18 May 2012, 16 Aug. 2012, 17 Aug. 2012, 18 Aug. 2012, 10 Apr. 2014, 11 Apr. 2014, 12 Apr. 2014, leg. Guy-Crispin Gembu Tungaluna, Prescott Musaba and André Malekani, 16 Aug. 2012, 17 Aug. 2012, 18 Aug. 2012, leg. Prescott Musaba (UNIKIS: UMA540, UMA548, UMA552, UMA549, UMA562, UMA579, UMA580, UMA582, UMA585, UMA634, UMA610, UMA611, UMA620, UMA633, RMCA: a1.097-M-3166, a1.097-M-3167, a1.097-M-3169, a1.097-M-3171, UNIKIS: UM013, RMCA: a1.097-M-3170, UNIKIS: UM014, UM025, RMCA: a1.097-M-3168, UNIKIS: UM021); 3 우 [3 AL], Upemba National Park, 25 Sep. 2009, leg. Alexandre Hassanin, Nicolas Nesi, Célestin Pongombo, Peter Vallo and Didier Tshikung (MNHN: ZM-2011-861 to ZM-2011-863); 9 우, 5 ऽో $\sigma^{-}$ [14 UN], Yoko, 11 Feb. 2009, 12 Feb. 2009, 12 May 2009, 13 May 2009, 18 May 2009, 19 May 2009, 20 May 2009, 19 Jul. 2009, 25 Sep. 2009, leg. unknown collector (UNIKIS: GE125, GE126, GE234/ G15, GE236/G22, GE235, GE237, GE238/G21, GE242, GE243, GE245/G13, GE248, GE267/G14, GE250, GE286); 1 , 1 ? [2 SK], Yolokafiri, 31 Aug. 1947, leg. unknown collector, between 30 Jul. 1947 and 30 Aug. 1947, leg. Jean Hiernaux (RBINS: 7056, 7063); 1 [UN], Zookis [= Kisangani], 24 Dec. 2015, leg. Prescott Musaba, Moïse Bipoo, Charle Andabhati, Benjamin Kirongozi and Kambale Karupao (UNIKIS: ZCH06).

RWANDA: 5 우, $4 \hat{\jmath} \widehat{\partial}$ [8 AL, $1 \mathrm{UN}$ ], Gisenyi, 13 Oct. 1980, 15 Oct. 1980, leg. Hans H. de Iongh, 21 Oct. 1980, leg. Danny Meirte (ZMA: MAM.21243 to MAM.21247, MAM.21364, RMCA: 80.055M-0065 to 80.055-M-0067); 2 đ̂̀, 1 ? [3 AL], Kibuye, 7 Oct. 1980, 9 Oct. 1980, leg. Danny Meirte 


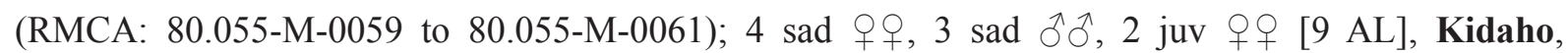
8 Jun. 1982, 10 Jun. 1982, 11 Jun. 1982, 12 Jun. 1982, 13 Jun. 1982, 17 Jun. 1982, 19 Jun. 1982, leg. Benny Baeten (RMCA: 87.046-M-0002 to 87.046-M-0005, 87.046-M-0038, 87.046-M-0039, 87.046M-0061, 87.046-M-0062, 87.046-M-0001); 1 sad §ิ [AL], Kitabi, 2 Nov. 1981, leg. Frits De Vree,

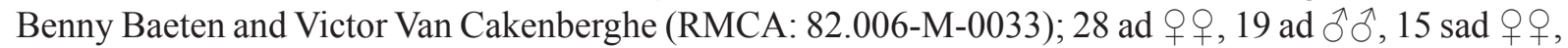

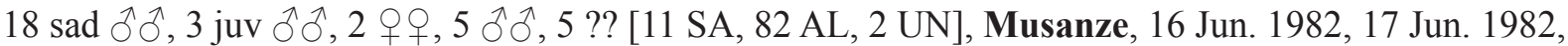
leg. Benny Baeten, 24 Oct. 1981, 25 Oct. 1981, 27 Oct. 1981, leg. Frits De Vree, Benny Baeten and Victor Van Cakenberghe, 25 Oct. 1980, leg. Danny Meirte, 8 Jun. 1966, leg. Félix / Michel Anciaux de Faveaux (RMCA: 87.046-M-0006 to 87.046-M-0037, 87.046-M-0040 to 87.046-M-0060, 82.006M-0003, 80.055-M-0053 to $80.055-\mathrm{M}-0058,80.055-\mathrm{M}-0068$ to $80.055-\mathrm{M}-0072,82.006-\mathrm{M}-0004$ to 82.006-M-0030, 82.006-M-0032, RBINS: 15485.1, 15485.2); 1 juv ổ [AL], Nyamata, 16 Nov. 1981, leg. Frits De Vree, Benny Baeten and Victor Van Cakenberghe (RMCA: 82.006-M-0034); 1 + 3 ?? [4 AL], Nyundo, 20 Oct. 1980, leg. Danny Meirte (RMCA: 80.055-M-0052, 80.055-M-0062 to 80.055-

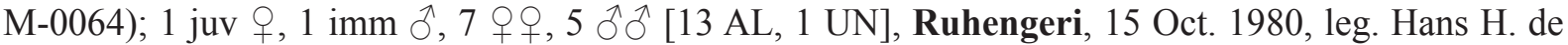
Iongh, 20 Sep. 1976, leg. Frieder Meier, 30 Nov. 1985, leg. unknown collector (ZMA: MAM.21248 to MAM.21255, MHNG: 1872.067 to 1872.069 , SMF: 66863 to 66865); 1 sad § [AL], Rukira, 22 Nov. 1981, leg. Frits De Vree, Benny Baeten and Victor Van Cakenberghe (RMCA: 82.006-M-0035); $1 \mathrm{sad}+$ [AL], Tamira, 26 Oct. 1981, leg. Frits De Vree, Benny Baeten and Victor Van Cakenberghe (RMCA: 82.006-M-0031); 1 sad 9 [AL], Uwinka, 17 Oct. 1981, leg. Frits De Vree, Benny Baeten and Victor Van Cakenberghe (RMCA: 82.006-M-0002).

Scotonycterini Bergmans, 1997

Casinycteris Thomas, 1910

\section{Casinycteris argynnis Thomas, 1910}

DEMOCRATIC REPUBLIC OF THE CONGO: 1 o, 2 ?? [1 SS, 1 SA, $1 \mathrm{UN}$ ], unknown locality, unknown date, between 1 Jun. 1911 and 30 Jun. 1911, leg. unknown collector, between 1 Jan. 1984 and 31 Dec. 1984, leg. Betu Nsankulu (RMNH: MAM.24558, RMCA: 84.035-M-0003, SMF: 6361); 1 ઈ [AL], Baliko, prior to 31 Jan. 2007, leg. Guy-Crispin Gembu Tungaluna (RMCA: a1.097-M-0412); 2 우 [2 SS], Bena Mbala, 15 Jan. 1984, leg. Betu Nsankulu (RMCA: 84.035-M-0001, 84.035-M-0002); 1 q, 1 ऽ [2 SA], Boende, between 1 Jan. 1967 and 31 Dec. 1967, leg. P. Lootens (RMCA: 38652, 38653); 1 q [AL], Bomane [= Bomaneh], 31 May 2010, leg. Guy-Crispin Gembu Tungaluna, Prescott Musaba and José Akaibe (UNIKIS: CRT2093); 1 ? [AL], Boteka, between 16 Feb. 1985 and 30 Jun. 1985, leg. P. Lootens (RMCA: 85.030-M-0001); 2 우 [2 AL], Djabir, 8 May 2011, 10 May 2011, leg. Prescott Musaba and Jonathan Kosele (UNIKIS: DJB096, DJB035); 1 \& [AL], Epulu, 29 Aug. 2015, leg.

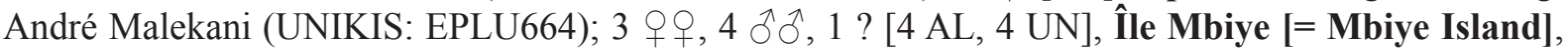
25 Feb. 2012, 13 Apr. 2012, 21 Apr. 2014, leg. Prescott Musaba, 19 Feb. 2012, 24 Feb. 2012, 25 Feb. 2012, leg. Prescott Musaba, Tembele and Marc Boketshu (RMCA: a1.097-M-3183, UNIKIS: CHIM11, CHIM013/14, CHIM15, RMCA: a1.097-M-3180 to a1.097-M-3182, UNIKIS: CHIM28); 1 ad,+ 1 ad

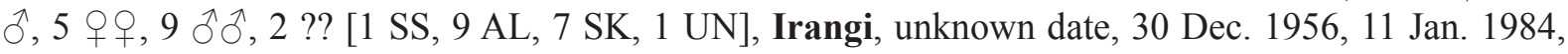
18 Jan. 1984, 5 Feb. 1984, 17 Feb. 1984, 18 Feb. 1984, leg. Heinz Stephan, 16 Oct. 1990, 19 Oct. 1990, leg. Wim Bergmans (SMF: 65046, 64988 to 64991, ZMA: MAM.24193, SMF: 64994 to 64998, 65052, 64992, 64999, 65000, ZMA: MAM.24194, RMCA: 27430, SMF: 64993); 1 [SK], Kivu region, 12 Jan. 1984, leg. Heinz Stephan (SMF: 69400); 1 đ [UN], Kona, 15 May 2010, leg. Guy-Crispin Gembu Tungaluna, Prescott Musaba and José Akaibe (UNIKIS: CRT1974); 2 우 [2 AL], Kumbi, 9 Dec. 1993, leg. Ngenge Masumbuko Kamitongo (ZMA: MAM.24894, MAM.24895); 1 ? [UN], Kumi, unknown date, leg. unknown collector (RMNH: MAM.24894); 4 우, 1 § [2 AL, 1 SK, 2 UN], Lieki, 30 May 2010, 31 May 2010, 2 Jun. 2010, leg. Guy-Crispin Gembu Tungaluna, Prescott Musaba and José Akaibe (UNIKIS: CRT2281, CRT2052, CRT2061, CRT2079, CRT2092); 1 \& [SS], Lukonga, 
6 Aug. 1979, leg. Betu Nsankulu (RMCA: 80.013-M-0003); 1 , 1 ô, 2 ?? [2 SS, 2 SA], Luluabourg [= Kananga], 25 Feb. 1965, 18 Mar. 1965, leg. Antoon Emeric Marcel De Roo (RMCA: 33412, 33347, 33348, 33411); 1 , 1 ठ [2 AL], Lwana River, 12 Apr. 1992, leg. Wim Bergmans, 16 Oct. 1990,

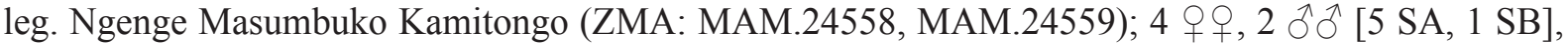
Mandima, 7 Jul. 1976, 8 Jul. 1976, 15 Jul. 1976, 31 Jul. 1976, 1 Aug. 1976, leg. Frieder Meier (MHNG:

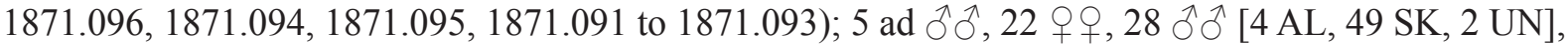
Masako, 13 Jun. 2015, leg. Prescott Musaba, Moïse Bipoo, Charle Andabhati, Benjamin Kirongozi and Kambale Karupao, 30 Nov. 1987, 20 Jan. 1988, 21 Jan. 1988, 22 Jan. 1988, 23 Jan. 1988, 24 Jan. 1988, 25 Jan. 1988, 26 Jan. 1988, 27 Jan. 1988, 30 Jan. 1988, 21 Feb. 1988, 24 Jan. 1990, 16 Feb. 1990, 17 Feb. 1990, leg. Ndey B. Ifuta (UNIKIS: CHMS076, RMCA: 93.079-M-0324 to 93.079-M-0326, 93.079-M-0092, 93.079-M-0243, 93.079-M-0244, 93.079-M-0246, 93.079-M-0247, 93.079-M-0093 to $93.079-\mathrm{M}-0095,93.079-\mathrm{M}-0248,93.079-\mathrm{M}-0119$ to $93.079-\mathrm{M}-0125,93.079-\mathrm{M}-0249$ to $93.079-\mathrm{M}-$ 0256, 93.079-M-0096 to 93.079-M-0101, 93.079-M-0103 to 93.079-M-0106, 93.079-M-0302, 93.079M-0323, 93.079-M-0107 to 93.079-M-0118, 93.079-M-0303, 93.079-M-0304, 93.079-M-0322); 2 ad 우 [1 SA, 1 UN], Mbwambala, between 9 Jul. 1995 and 11 Jul. 1995, leg. Herwig Leirs et al. (MSB: 274606, RMCA: 97.021-M-0579); 1 ad $\odot$ [UN], Medje, 22 Apr. 1910, leg. Herbert Lang, James Paul

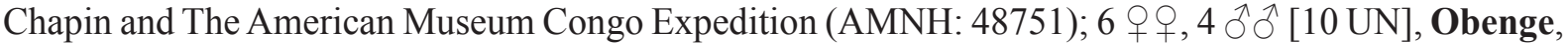
5 Feb. 2013, 7 Feb. 2013, leg. Prescott Musaba and André Malekani (UNIKIS: TLL354, TLL356 to TLL359, TLL363, TLL375 to TLL377, TLL385); 1 q [AL], Rubi-Tele, 23 Aug. 2013, leg. Guy-Crispin Gembu Tungaluna, Prescott Musaba and André Malekani (UNIKIS: RBTL547); 1 [SA], Stanleyville [= Kisangani], between 1 May 1926 and 31 May 1926, leg. Henri Schouteden (RMCA: 16211); 6 ?? [6 AL], Wafanya [= Wafania], 12 Sep. 1991, 28 Apr. 1992, 4 May 1992, 8 May 1992, between 1 Jan. 1991 and 31 Dec. 1991, leg. P. Lootens (RMCA: 91.076-M-0079, 91.076-M-0080, 92.079-M-0073, 92.079M-0075, 92.079-M-0074, 92.079-M-0072); 1 q, 2 ふో ô [3 UN], Yaekela, 3 May 2010, 5 May 2010, 6 May 2010, leg. Guy-Crispin Gembu Tungaluna, Prescott Musaba and José Akaibe (UNIKIS: CRT2364, CRT1849, CRT1854); 2 ふ̂̉, 3 ?? [4 AL, 1 UN], Yoko, unknown date, 4 Sep. 2012, leg. Prescott Musaba, 7 Sep. 2012, leg. Prescott Musaba, Tembele and Marc Boketshu (RMCA: a1.097-M-3184 to a1.097-M-3187, UNIKIS: YK038CH); 1 ㅇ, 2 $\widehat{\jmath}$ [3 AL], Zookis [= Kisangani], 16 Feb. 2013, leg. Prescott Musaba and André Malekani (UNIKIS: CSB1, CSB3, CSB4).

\section{Scotonycteris Matschie, 1894}

\section{Scotonycteris bergmansi}

Hassanin, Khouider, Gembu, Goodman, Kadjo, Nesi, Pourrut, Nakouné \& Bonillo, 2015

DEMOCRATIC REPUBLIC OF THE CONGO: 3 우 [1 SS, 2 SK], Baliko, 11 Jun. 2012, 12 Jun. 2012, leg. William ("Bill”) T. Stanley (FMNH: 219598, 219681, 219599); 1 ? [SS], Beni, prior to 2 Jun. 1914, leg. Bonnevie (RMCA: 3244); 1 q [SS], Bitale, 14 Jun. 1970, leg. Alexandre

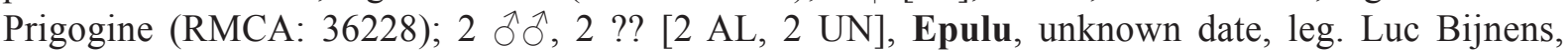
29 Aug. 2015, leg. André Malekani (RMCA: 90.031-M-0007, 90.031-M-0008, UNIKIS: EPLU676, EPLU677); 2 우, 1 ðึ [1 SK, 2 SB], Epulu, 2 km W, 29 Jun. 1991, 3 Jul. 1991, 6 Jul. 1991, leg. Julian C. Kerbis Peterhans (FMNH: 149402 to 149404); 2 우 [2 UN], Île Mbiye [= Mbiye Island], 25 Dec. 2015, 8 Jan. 2016, leg. Prescott Musaba, Moïse Bipoo, Charle Andabhati, Benjamin Kirongozi

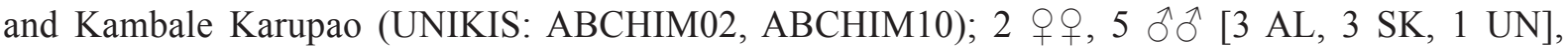
Irangi, 11 Jan. 1984, 21 Jan. 1984, 28 Jan. 1984, 15 Feb. 1984, leg. Heinz Stephan, 14 Nov. 1955, leg. J.J. Laarman, 18 Oct. 1990, leg. Wim Bergmans (SMF: 65006, RMNH: MAM.26325, SMF: 65009, 65010, ZMA: MAM.24197, SMF: 65007, 65008); 1 q [SS], Kiliza [River], 25 May 1964, leg. Alexandre Prigogine (RMCA: 32584); 1 đ [SS], Kiloboze [= Kilobono], 2 Feb. 1963, leg. Alexandre Prigogine (RMCA: 31345); 2 $\widehat{\jmath}$ [2 UN], Lieki, 29 May 2010, leg. Guy-Crispin Gembu Tungaluna, Prescott Musaba and José Akaibe (UNIKIS: CRT2041, CRT2042); 1 ? [AL], Maganga, unknown date, leg. Luc Bijnens (RMCA: 90.031-M-0009); 3 कै $\widehat{x}$ [1 SA, 2 AL], Mandima, 7 Jul. 1976, 
8 Jul. 1976, leg. Frieder Meier (MHNG: 1871.088 to 1871.090 ); $1 \mathrm{ad} \phi, 3 \mathrm{ad} \hat{\partial} \widehat{\partial}, 1 \mathrm{sad} \widehat{\partial}, 2$ juv

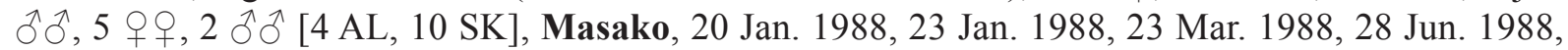
26 Aug. 1988, 30 Apr. 1989, 14 Sep. 1989, 16 Sep. 1989, 22 Dec. 1989, 13 Feb. 1990, 14 Feb. 1990, leg. Ndey B. Ifuta (RMCA: 93.079-M-0364 to 93.079-M-0366, 93.079-M-0359 to 93.079-M-0361, 93.079-M-0245, 93.079-M-0363, 93.079-M-0102, 93.079-M-0170, 93.079-M-0357, 93.079-M-0358, 93.079-M-0356, 93.079-M-0362); 1 q [SS], Mwenge, 5 Feb. 1967, leg. Alexandre Prigogine (RMCA: 35076); 1 , 1 ठิ [1 SS, 1 SK], Quatorze, 23 Jun. 2013, 29 Jun. 2013, leg. William (“Bill”) T. Stanley (FMNH: 222426, 222623); 9 ठో [9 UN], Rubi-Tele, 17 Aug. 2013, 18 Aug. 2013, 19 Aug. 2013, 20 Aug. 2013, 22 Aug. 2013, leg. Guy-Crispin Gembu Tungaluna, Prescott Musaba and André Malekani (UNIKIS: RBTL481, RBTL482, RBTL492, RBTL494, RBTL504, RBTL515, RBTL505, RBTL534, RBTL540); 1 ad + [AL], Sukisa, unknown date, leg. unknown collector (MNHN: ZM2014-564 [holotype Scotonycteris bergmansi congoensis Hassanin, Khouider, Gembu, Goodman, Kadjo, Nesi, Pourrut, Nakouné, and Bonillo, 2015]); 3 우, 1 § [4 UN], Uma, 10 Apr. 2014, 11 Apr. 2014, 12 Apr. 2014, leg. Guy-Crispin Gembu Tungaluna, Prescott Musaba and André Malekani (UNIKIS: UMA525, UMA583, UMA609, UMA615); 2 우, 1 ? [3 AL], Yoko, 4 Sep. 2012, 5 Sep. 2012, leg. Prescott Musaba (RMCA: a1.097-M-3188 to a1.097-M-3190).

\section{Stenonycterini Nesi, Kadjo, Pourrut, Shongo, Cruaud \& Hassanin, 2012 Stenonycteris Gray, 1870}

\section{Stenonycteris lanosus (Thomas, 1906)}

BURUNDI: 1 , 3 ふふ [2 SK, 2 SB], Giserama, Colline, 30 Jul. 1991, 31 Jul. 1991, 2 Aug. 1991, leg. Julian C. Kerbis Peterhans (FMNH: 148979, 148976 to 148978); 2 우, 1 đ [3 SB], Ruhinga Hill, 9 Mar. 1993, 10 Mar. 1993, 13 Mar. 1993, leg. Jay L. Udelhoven (FMNH: 155970, 155971, 155969).

DEMOCRATIC REPUBLIC OF THE CONGO: 1 juv + , 2 우 [2 SS, 1 AL], Butagu Valley [= Butahu Valley Cave], 24 Dec. 1926, leg. James Paul Chapin, Dewitt L. Sage, Frank P. Mathews and The Ruwenzori-Kivu Expedition, 24 Dec. 1926, leg. James Paul Chapin (AMNH: 82380, 82527, 82528);

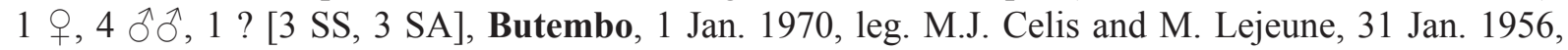
21 Feb. 1956, leg. P. Dyleff (RMCA: 36200, 36202, 36205, 23674, 23676, 23553); 1 o [SS], Kabira, 19 Mar. 1959, leg. Uwe Rahm (RMCA: 28025); 3 우, 2 ઈึ date, 23 Jan. 1953, 28 Jan. 1953, 22 Feb. 1953, leg. Gaston-François de Witte, 21 Jan. 1925, leg. Edmund Heller, 22 Dec. 1926, leg. James Paul Chapin (RBINS: 37116, 37117, FMNH: 26237, RMCA: 37117, AMNH: 82526, RBINS: 17299, RMCA: 37116); 1 ? [UN], Kikura, unknown date, leg. unknown collector (INPBC: V1178); 1 imm $q$ [SS], Lamya Valley, 16 Nov. 1945, leg. Gaston-François de Witte (RBINS: 12928); 1 q [AL], Lemera, SW of, 16 Jul. 1927, leg. James Paul Chapin (AMNH: 82379); 1 [SS], Lushala, 4 Nov. 1966, leg. A. Elbl (USNM: 535400); 2 우 [2 SA], Lwiro [= Luiro], 8 Sep. 1963, 23 Oct. 1965, leg. P. Kunkel (SMF: 31829, 31830); 1 ? [UN], Marion, Lake, unknown date, leg. unknown collector (INPBC: V1159); 1 ○ [SK], Marumbi, 6 Dec. 1954, leg. Gaston-François de Witte (RMCA: 37119); 1 Oे [SO], Mount Hoyo [= Hoyo, Mount], 12 Aug. 1947, leg. Jean Hiernaux (RMCA: 35806);

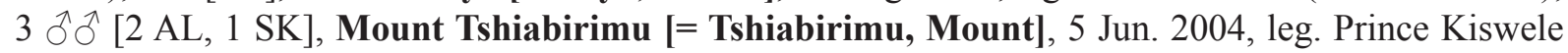
Kaleme (FMNH: 188790 to 188792); 2 우 [1 SS, 1 SK], Mugaba Cave, 19 Jul. 1962, leg. A. Elbl (USNM: 375232, 375233); 1 q [SO], Mutsora, 13 Aug. 1949, leg. Gaston-François de Witte (RBINS: 12927); 1 juv đ, 2 우, 1 đ [2 AL, 2 UN], Nyabiondo, 27 Dec. 1990, 28 Dec. 1990, 1 Jan. 1991, leg. J.P. Lubula Bulambo and Ngenge Masumbuko Kamitongo (ZMA: MAM.24346, MAM.24347,

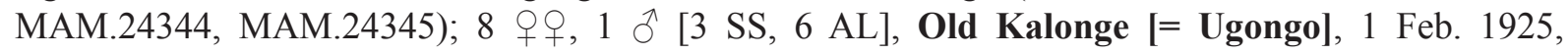
2 Feb. 1925, 3 Feb. 1925, 4 Feb. 1925, 5 Feb. 1925, 8 Feb. 1925, leg. Edmund Heller (FMNH: 26234 to

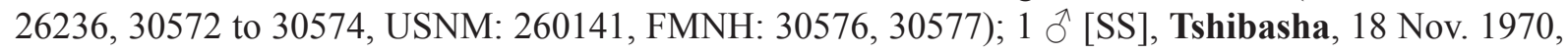
leg. Betu Nsankulu (RMCA: 36250); 1 ㅇ [SS], Tshibati, 28 Dec. 1963, leg. Antoon Emeric Marcel De Roo (AMNH: 207735). 
RWANDA: 1 ô [SS], Bugundi, 19 Nov. 1955, leg. Alexandre Fain (RMCA: 81.012-M-0043); 1 q,

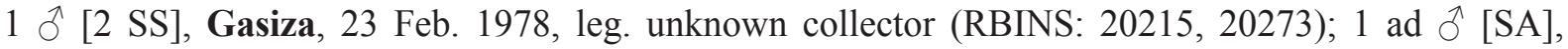
Kanyamiketo, 4 Jun. 1982, leg. Benny Baeten (RMCA: 87.046-M-0518); 3 ad $\widehat{\jmath} \widehat{\jmath}, 1$ sad $\circ$ [4 SA], Kayove, 10 Jul. 1982, 11 Jul. 1982, leg. Benny Baeten (RMCA: 87.046-M-0515, 87.046-M-0516, 87.046-M-0519, 87.046-M-0520); 4 ?? [4 SK], Kibumbu Crater, 9 Dec. 1991, leg. J.R. Wilson and Anthony Michael Hutson (BMNH: 1992.385 to 1992.388); 1 ad ${ }^{\lambda}$ [SA], Kinigi, 1 Jun. 1982, leg. Benny Baeten (RMCA: 87.046-M-0514); 5 우, 2 ㅊํ, 16 ?? [23 SA], Murunda, 12 Oct. 1980, leg. Danny Meirte (RMCA: 80.055-M-0073 to 80.055-M-0095); 1 ad $\lesssim$ [SA], Routabansougera, 17 Jul. 1982, leg. Benny Baeten (RMCA: 87.046-M-0517); 1 ? [UN], Ruhengeri, 22 Jun. 2003, leg. unknown collector (SMF: 92441); 2 ad 우, 1 ad $\widehat{\jmath}$ [3 SA], Uwinka, 17 Oct. 1981, leg. Frits De Vree, Benny Baeten and Victor Van Cakenberghe (RMCA: 82.006-M-0932 to 82.006-M-0934).

\section{Hipposideridae Lydekker, 1891}

DEMOCRATIC REPUBLIC OF THE CONGO: 1 ? [SK], Aka, between 15 May 1952 and 28 Dec. 1972 , leg. H. de Saeger (RMCA: 36837); 1 \&, 1 O̊, 7 ?? [9 AL], Boende, between 1 Jan. 1967 and 31 Dec. 1967, leg. P. Lootens (RMCA: 38706 to 38714); 1 ? [SK], Keroma, between 21 Apr. 1952 and 28 Dec. 1972, leg. H. de Saeger (RMCA: 36836); 1 § [SS], Kilibozi, 12 Apr. 1975, leg. Alexandre Prigogine (RMCA: 76.022-M-0023); 2 ?? [2 SS], Kiligi, 11 Feb. 1975, leg. Alexandre Prigogine (RMCA: 75.017-M-0004, 75.017-M-0005); 1 ? [SS], Kinda, 17 Aug. 1978, leg. Betu Nsankulu (RMCA: 79.022-M-0008); 1 ㅇ [SK], Luluabourg [= Kananga], unknown date, leg. Richard Callewaert (RMCA: 7543 b); 1 đ , 24 ?? [25 AL], Masako, unknown date, 19 Dec. 1989, leg. Ndey B. Ifuta (RMCA: 93.079-M-0371 to 93.079M-0394, 93.079-M-0329); 3 ?? [2 SK, 1 SN], PPK 72, between 18 Mar. 1952 and 28 Dec. 1972, leg. H. de Saeger (RMCA: 36833 to 36835).

\section{Asellia Gray, 1838}

Asellia tridens (E. Geoffroy St.-Hilaire, 1813)

DEMOCRATIC REPUBLIC OF THE CONGO: 2 우, 15 ๙ิ $\widehat{\jmath}$ [17 AL], Kivu Region, unknown date, leg. Guy Babault (MNHN: ZM-MO-1985-846 to ZM-MO-1985-854, ZM-MO-1985-856 to ZMMO-1985-863); 1 $\lesssim$ [AL], Kivu, near, unknown date, leg. Guy Babault (MNHN: ZM-MO-1985-855).

Doryrhina Peters, 1871

Doryrhina cyclops (Temminck, 1853)

BURUNDI: 1 ๆ, 2 ๙ึત [1 AL, 2 SB], Giserama, Colline, 3 Aug. 1991, 11 Aug. 1991, 23 Aug. 1991, leg. Julian C. Kerbis Peterhans (FMNH: 148982, 148983, 148981).

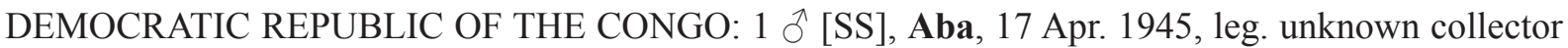
(RBINS: 13332); 9 ?? [9 UN], Abatupi River, unknown date, leg. unknown collector (INPBC: V1285.5, V1290.1 to V1290.8); 1 ○ [SK], Abimva, 22 Jun. 1925, leg. Henri Schouteden (RMCA: 9028b); 1 ? [UN], Albert National Park [= Virunga National Park], unknown date, leg. unknown collector (INPBC: V1852.2); 1 ad $\widehat{\jmath}, 4$ 우, 1 ? [4 SS, 1 SA, 1 SK], Avakubi, 1 Oct. 1913, 24 Jan. 1914, leg. Herbert Lang, James Paul Chapin and The American Museum Congo Expedition, 26 Jan. 1914, leg. Herbert Lang, between 24 Jan. 1914 and 24 Dec. 1914, leg. unknown collector, between 7 Nov. 1912 and 30 Apr. 1913, leg. Cuthbert Christy (AMNH: 49404, 49098 [holotype Hipposideros langi J.A. Allen, 1917], 49099, FMNH: 43878, MCZ: 17370, RMCA: 1606b); 1 đ [SK], Baliko, 12 Jun. 2012, leg. William ("Bill”) T. Stanley (FMNH: 219601); 1 ? [SS], Beni, prior to 2 Jun. 1914, leg. Bonnevie (RMCA: 3243); 5 qㅜ, 1 ○ૈ [6 AL], Bikoro, 25 Jun. 1956, leg. Alvin Novick (MCZ: 48219 to 48224); 1 q [UN], Bomane [= Bomaneh], 30 May 2010, leg. Guy-Crispin Gembu Tungaluna, Prescott Musaba and José 
Akaibe (UNIKIS: CRT2067); 1 + [AL], Bozade, 20 Jul. 1979, leg. Frieder Meier (MHNG: 1870.045); 2 ổ [̂ [2 UN], Buluku River, 22 May 1952, leg. H. de Saeger et al. (RBINS: 13678.1, 13678.2); 1 , 1 đ [2 SO], Buta, 1 Feb. 1950, 6 Dec. 1950, leg. unknown collector (RBINS: 13335, 13334); 4 ?? [4 UN], Bwirina, unknown date, leg. unknown collector (INPBC: V1661.4, V1662.1 to V1662.3); 1 ? [SA], Congo River, 75 mi [= 121 km] up, between 1 Aug. 1897 and 31 Aug. 1897, leg. unknown collector (USNM: 102512); 5 우, 2 §ิ $\widehat{\jmath}, 3$ ?? [3 AL, 7 UN], Dedegwa River, 19 May 1952, leg. H. de Saeger et al., 19 May 1952, leg. H. de Saeger (RBINS: 13676, 13677.1 to 13677.6, RMCA: 36820 to 36822); 1 ? [UN], Djelube River, lower, unknown date, leg. unknown collector (INPBC: V1093.1); 2 ?? [2 UN], Djelube River, middle, unknown date, leg. unknown collector (INPBC: V1078.3, V1100); 1 ㅇ, 1 ? [1 SS, 1 AL], Epulu, unknown date, leg. Luc Bijnens, 2 May 1951, leg. A. Schwartz and R.N. Porter (RMCA: 90.031-M-0004, KU: 151340); 2 웅, 1 § [3 UN], Fataki, between 1 Jan. 1948 and 6 Apr. 1964, leg. unknown collector (RBINS: 18529.1 to 18529.3); 2 q 9,5 ?? [2 AL, 1 SO, 4 UN], Inimvua, 15 May 1952, leg. H. de Saeger et al., 15 May 1952, leg. H. de Saeger (RBINS: 13505, 13674.1 to 13674.4, RMCA: 36818, 36819); 1 , 5 ô 0 [6 SS], Irumu, 40 mi [= $64 \mathbf{~ k m ] ~ S W ~ [ = ~ I t u r i ~ F o r e s t ] , ~}$ unknown date, leg. Howard de Walden (BMNH: 1930.11.11.146 to 1930.11.11.150, 1930.11.11.45); 1 ? [UN], Kambemba, unknown date, leg. unknown collector (INPBC: V1657); 1 ㅇ [SO], Liki, 6 Mar. 1952, leg. unknown collector (RBINS: 13333); 1 ? [SS], Lovanium University [= Kinshasa], 30 Mar. 1962, leg. De Bont (RMCA: 31201); 1 ㅇ, 1 ô, 2 ?? [1 SS, 3 AL], Lukolela, 28 Jul. 1930, 17 Mar. 1931, leg. James Paul Chapin (AMNH: 86858, 86863, 86866, 86909); 1 ? [UN], Makayova River, unknown date, leg. unknown collector (INPBC: V1257.2); 1 ? [UN], Malulu, unknown date, leg. unknown collector (INPBC: V185); 1 ? [UN], Mamudioma, unknown date, leg. unknown collector (INPBC: W1152); 2 우우 [2 AL], Masako, 15 Sep. 1988, 11 Aug. 1989, leg. Ndey B. Ifuta (RMCA: 93.079-M-0328, 93.079-M-0327); 1 juv ô, 4 우 [1 SS, 4 UN], Medje, 22 Apr. 1910, 27 Apr. 1910, 3 Sep. 1910, leg. Herbert Lang, James Paul Chapin and The American Museum Congo Expedition (RMCA: 12379, AMNH: 49093 to 49096); 2 ad 웅, 1 juv ?, 1 foetus ?, 7 q $ᄋ, 4$ ?? [1 SS, 7 AL, 1 SO, 6 UN], Mogbwamu River, 8 Mar. 1952, leg. H. de Saeger et al., 8 Mar. 1952, between 8 Mar. 1952 and 28 Dec. 1972, leg. H. de Saeger (RBINS: 13502, 13503, 13669.1 to 13669.4, RMCA: 36808 to 36812, 36813a, 36813b, 36815, 36814); 1 ㅇ, 2 $\widehat{\jmath}$ [1 SS, 2 AL], Niangara, 3 Dec. 1910, 6 Dec. 1910, leg. Herbert Lang, James Paul Chapin and The American Museum Congo Expedition, 6 Dec. 1910, leg. Herbert Lang (AMNH: 49097, 49415, FMNH: 43879); 1 ad $\delta^{\Uparrow}$ [SA], Niapu, 28 Jan. 1914, leg. Herbert Lang, James Paul Chapin and The American Museum Congo Expedition (AMNH: 49391); 3 오오 1 ô [4 AL], Obenge, 5 Feb. 2013, 6 Feb. 2013, leg. Prescott Musaba and André Malekani (UNIKIS: TLL361, TLL364 to TLL366); 1 + [SS], Parc National de Garamba [= Garamba National Park], 7 Feb. 1952, leg. H. de Saeger et al. (RBINS: 13675); 2 우 [2 UN], PFNK.16, 7 Feb. 1952, leg. H. de Saeger et al. (RBINS: 13668.1, 13668.2); 2 ठิ $\widehat{~}[2$ UN], Pidigala River, 23 Apr. 1952, leg. H. de Saeger et al. (RBINS: 13672, 13673); 2 ?? [2 UN], Ruanoli-Mavea confluence, unknown date, leg. unknown collector (INPBC: V1668.1, V1668.3.3); 2 ?? [2 UN], Rwere Rwindi, unknown date, leg.

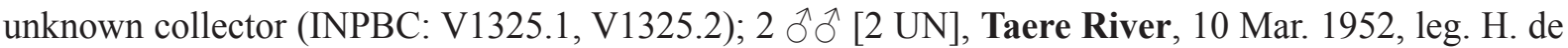
Saeger et al. (RBINS: 13670, 13671); 1 $\widehat{~[S S], ~ T a n d a l a, ~} 20$ Jul. 1979, leg. C. Brian Robbins (USNM: 537712); 2 우 [2 UN], Yaekela, 3 May 2010, leg. Guy-Crispin Gembu Tungaluna, Prescott Musaba and José Akaibe (UNIKIS: CRT2367, CRT2368); 1 q [SS], Yalosemba, 19 Jun. 1979, leg. C. Brian Robbins (USNM: 537711).

\section{Hipposideros Gray, 1831}

DEMOCRATIC REPUBLIC OF THE CONGO: 1 juv ? [UN], Abatupi River, unknown date, leg. unknown collector (INPBC: V1057.2b); 1 \& [AL], Île Mafi [= Mafi Island], 8 Jan. 2016, leg. Prescott Musaba, Moïse Bipoo, Charle Andabhati, Benjamin Kirongozi and Kambale Karupao (UNIKIS: ABCHIMAF12); 3 $\widehat{\partial}$ [3 AL], Irangi, slightly E of [= Irangi], 15 Oct. 1990, leg. Wim Bergmans (ZMA: MAM.24182 to MAM.24184); 2 우 [2 AL], Luhohov (River), 18 Oct. 1990, leg. 
Wim Bergmans (ZMA: MAM.24187, MAM.24188); 1 [AL], Musenge, 3 May 1992, leg. Ngenge Masumbuko Kamitongo (ZMA: MAM.24553); 1 ㅇ, 1 ठ [2 UN], Obenge, 7 Feb. 2013, 8 Feb. 2013, leg.

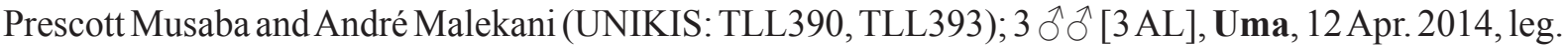
Guy-Crispin Gembu Tungaluna, Prescott Musaba and André Malekani (UNIKIS: UMA504, UMA618, UMA619); 2 $\widehat{\jmath}$ [2 UN], Yoko, 12 Feb. 2009, 4 Oct. 2009, leg. unknown collector (UNIKIS: GE128, GE318).

\section{Hipposideros abae J.A. Allen, 1917}

DEMOCRATIC REPUBLIC OF THE CONGO: 1 ad $\partial$, 20 우, 17 $\widehat{\partial}$ [37 SS, 1 UN], Aba, 3 Dec. 1911, 13 Dec. 1911, leg. Herbert Lang, James Paul Chapin and The American Museum Congo Expedition, 13 Dec. 1911, leg. unknown collector (AMNH: 49120 to 49122, 49123 [holotype Hipposideros aba J.A.Allen, 1917], 49124, 49125, 49128 to 49130, 49146 to 49167, 49169, 49170, MCZ: 17371, 17372, RMCA: 12366, TM: 4564, RMCA: 12367); 2 ?? [1 SO, 1 UN], Aka, 15 May 1952, leg. H. de Saeger et al., 15 May 1952, leg. H. de Saeger (RBINS: 13684.1, RMCA: 36694); 1 ô, 2 ?? [3 UN], Cel II, 21 May 1951, 25 Jun. 1951, 15 May 1952, leg. H. de Saeger et al. (RBINS: 13684.2, 13679, 13681); $1 \hat{\delta}$ [AL], Digba, between 1 Jul. 1981 and 31 Jul. 1981, leg. Marc Colyn (RMCA: 82.031-M-0005);

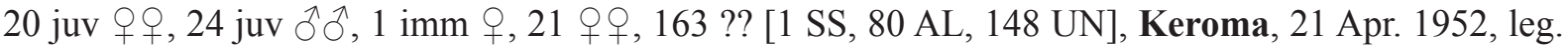
H. de Saeger et al., 21 Apr. 1952, between 21 Apr. 1952 and 28 Dec. 1972, leg. H. de Saeger (RBINS: $13683,13790.01$ to $13790.25,13791.01$ to $13791.35,13792.01$ to $13792.30,13793.01$ to 13793.30 , 13794.01 to 13794.27 , RMCA: $36610,36611,36612 \mathrm{a}, 36613$ to 36689,36693$) ; 1$ q, 1 के [2 UN], Maleli, 13 Jun. 1951, leg. H. de Saeger et al. (RBINS: 13680.1, 13680.2); 1 \& [SS], Oriental (former)

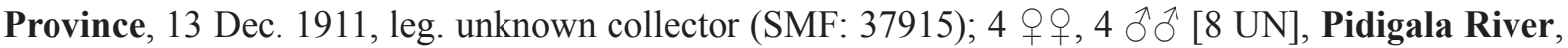
23 Apr. 1952, leg. H. de Saeger et al. (RBINS: 13682.1 to 13682.8 ).

Hipposideros beatus (K. Andersen, 1906)

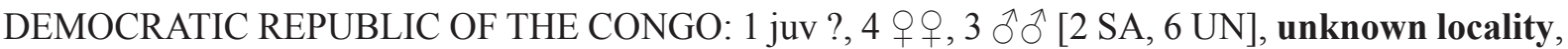
unknown date, between 11 Aug. 1989 and 14 Aug. 1989, leg. unknown collector (BMNH: 1990.225 to 1990.230, SMF: 77085, 77086); 3 우우, 3 $\widehat{\jmath}$ [1 AL, 5 UN], Aka, 23 Apr. 1952, 14 May 1952, leg. H. de Saeger et al., 14 May 1952, leg. H. de Saeger (RBINS: 13770, 4041, RMCA: 36690, RBINS: 13771, 4037 [holotype Hipposideros beatus maximus Verschuren, 1957], 4039); $1 \hat{\delta}$ [AL], Aketi, 21 Jun. 2015, leg. Vlir/Aketi (UNIKIS: AKETI603); 1 ठิ [UN], Bomane [= Bomaneh], 21 May 2010, leg. Guy-Crispin Gembu Tungaluna, Prescott Musaba and José Akaibe (UNIKIS: CRT2197); 2 ?? [2 AL], Buluku, 22 May 1952, leg. H. de Saeger (RMCA: 36691, 36692); 2 ?? [2 UN], Buluku River, 22 May 1952, leg. H. de Saeger et al. (RBINS: 13774, 4044); 1 ? [AL], Buta, between 1 Jan. 1925

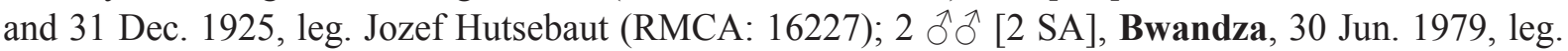
Frieder Meier (MHNG: 1870.095, 1870.096); 2 $\widehat{\partial}$ [2 UN], Dedegwa River, 19 May 1952, leg. H. de Saeger et al. (RBINS: 13772, 4042); 1 q [SA], Equateur (former) Province, prior to 7 Oct. 1996, leg. unknown collector (SMF: 84412); 2 đ̊ ô, 1 ? [3 SS], Faradje, 6 Jul. 1925, 7 Jul. 1925, 11 Jul. 1925, leg. Henri Schouteden (RMCA: 9056, 9044, 9048); 1 ? [UN], Ikengo, unknown date, leg. unknown collector (RMCA: 5646); 2 우, 2 ठึ $\widehat{~[4 ~ U N], ~ K o n a, ~} 12$ May 2010, 15 May 2010, leg. Guy-Crispin Gembu Tungaluna, Prescott Musaba and José Akaibe (UNIKIS: CRT1909, CRT1973, CRT1975, CRT1983); 2 đิ ồ [2 UN], Lieki, 29 May 2010, leg. Guy-Crispin Gembu Tungaluna, Prescott Musaba and José Akaibe (UNIKIS: CRT2046, CRT2047); 2 $\widehat{\partial}$ [2 AL], Mabali, 18 Jun. 1956, leg. Alvin Novick (MCZ: 48217, 48218); 1 ㅇ, 1 đิ [2 AL], Mandima, 21 Jul. 1976, leg. Frieder Meier (MHNG: 1873.057, 1873.058); 1 ㅇ [SS], Medje, 3 Aug. 1910, leg. unknown collector (MCZ: 17374); 1 ㅇ, 1 ? [2 AL], Moenge, 15 May 2010, 16 May 2010, leg. Guy-Crispin Gembu Tungaluna (RMCA: a1.097-M-3218, a1.097-M-3217); 2 ๙ $\widehat{~[2 ~ U N], ~ M o g b w a m u ~ R i v e r, ~} 8$ Mar. 1952, leg. H. de Saeger et al. (RBINS:

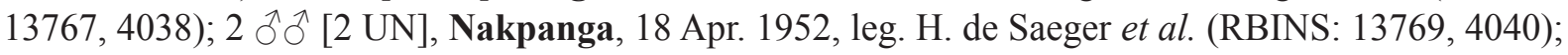
1 ? [AL], Panga, 1 Dec. 1914, leg. J. Bequaert (RMCA: 5446); 1 ঐ [UN], Pidigala River, 23 Apr. 1952, 
VAN CAKENBERGHE V. et al., The bats of Congo, Rwanda and Burundi revisited

leg. H. de Saeger et al. (RBINS: 13768; 2 ふぇ [2 UN], Soudan River, 20 May 1952, leg. H. de Saeger et al. (RBINS: 13773, 4043); 1 ? [UN], Thysville [= Mbanza-Ngungu], 11 Feb. 1987, leg. unknown collector (RBINS: 22426); 1 [UN], Yaekela, 6 May 2010, leg. Guy-Crispin Gembu Tungaluna, Prescott Musaba and José Akaibe (UNIKIS: CRT1856); 1 †, 3 $\widehat{\jmath}$ [2 SS, 1 SA, 1 AL], Yalosemba, 16 Jun. 1979, leg. Lynn W. Robbins, 16 Jun. 1979, leg. C. Brian Robbins, 18 Jun. 1979, 30 Jun. 1979, leg. Frieder Meier (USNM: 537707, 537708, MHNG: 1870.093, 1870.094).

\section{Hipposideros caffer (Sundevall, 1846)}

BURUNDI: 1 ad,+ 1 juv ?, 2 우, 1 ภ [3 SA, 2 AL], unknown locality, 3 Mar. 1965, leg. P.L. Niort, 3 Mar. 1979, leg. Heinz Stephan (MHNG: 1896.013, 1896.014, SMF: 57956 to 57958); 1 ? [AL], Bujumbura, between 1 Jul. 1968 and 31 Jul. 1968, leg. Lewall (RMCA: 35154); $1 \hat{\delta}$ [AL], Mabaye [= Mabayi], 12 Jul. 1973, leg. P.L. Niort (MHNG: 1896.012); 4 ?? [3 AL, 1 UN], Rubindi, between 1 Sep. 1958 and 30 Sep. 1958, leg. Narcisse Leleup (RMCA: 28455 to 28458).

DEMOCRATIC REPUBLIC OF THE CONGO: 1 juv $\curvearrowright$, 18 ?? [1 SA, 2 AL, 9 SK, 1 SO, 6 UN], unknown locality, unknown date, 12 Feb. 2001, 28 Feb. 2001, prior to 31 Jan. 1897, between 1 Jun. 1911 and 30 Jun. 1911, between 1 Apr. 1999 and 30 Apr. 1999, leg. unknown collector, unknown date, leg. Alexandre Prigogine, prior to 1 Aug. 1957, leg. Heinz Stephan, prior to 18 Aug. 1965, leg. Antoon Emeric Marcel De Roo (RBINS: 208C, RMCA: 32866, SMF: 92971, 92970, 93318 to 93320, 6363, RMCA: 27451 to 27459, 33599, RBINS: 208e); 3 $\widehat{\jmath}$ [3 AL], Aketi, 25 Jun. 2015, 27 Jun. 2015, leg. Vlir/Aketi (UNIKIS: AKETI618, AKETI621, AKETI626); 21 ?? [21 UN], Albert National Park [= Virunga National Park], unknown date, leg. unknown collector (INPBC: V1711, V1723.01 to V1723.20); 1 \& [SS], Alima, between 5 Mar. 1959 and 5 Oct. 1959, leg. P. Dyleff (RMCA: 28154); 1 juv ô [SS], Ango, between 14 Apr. 1941 and 23 Jul. 1946, leg. J.M. Vrijdagh (RMCA: 17756); 3 ?? [3 AL], Ango Cave, 13 Apr. 1941, leg. J.M. Vrijdagh (RMCA: 18103, 18105, 18106); 1 o [SS], Avakubi, between 18 Sep. 1912 and 24 Feb. 1913, leg. Cuthbert Christy (RMCA: 1429); 2 ?? [2 AL], Banana, between 1 Aug. 1952 and 31 Aug. 1952, leg. Is. Mesmaekers (RMCA: 21478, 21479); 3 ?? [3 AL], Baya Carrière [= Baya Quarry], 17 May 1965, leg. Walter N. Verheyen (RMCA: 35023, 35024, 35026); 1 ? [SS], Baye via Bondo, 13 Dec. 1956, leg. Rooyakkers (RMCA: 25863); 1 đ [SS], Bela, 1 Aug. 1947, leg. unknown collector (RBINS: 7066); 14 우, 3 $̋$ 8 Mar. 1925, 9 Mar. 1925, 11 Mar. 1925, 18 Mar. 1925, 22 Mar. 1925, 24 Mar. 1925, 28 Mar. 1925, leg. Edmund Heller (FMNH: 30625, 26242, 26243, 30626 to 30634, 30622 to 30624, MCZ: 33500, FMNH: 26240); 21 ?? [21 AL], Boende, between 1 Nov. 1969 and 31 Dec. 1974, leg. P. Lootens (RMCA: 75.035-M-0036 to 75.035-M-0056); 2 우 우 [2 SS], Bolobo, prior to 27 Sep. 1951, leg. Ngwe (RMCA: 20535, 20536); 3 ?? [3 SO], Bombo Lumene, 20 Aug. 1992, leg. Tshinyenye (RMCA: 92.119-M-0001 to 92.119-M-0003); 7 ?? [7 AL], Bunia, between 1 Jan. 1951 and 31 Dec. 1951, leg. Alexandre Fain, prior to 27 Dec. 1938, leg. Putnam (RMCA: 21468, 14950 to 14955); 1 ? [UN], Busholinka River, unknown date, leg. unknown collector (INPBC: W1006); 2 ?? [2 AL], Buta, unknown date, leg. Jozef Hutsebaut (RMCA: 14582a, 14582b); 2 우, 2 §ð 14 Aug. 1947, leg. unknown collector, 4 Feb. 1950, leg. P. Dyleff, between 1 Nov. 1971 and 30 Nov. 1971, leg. M. Lejeune, prior to 5 Jul. 1950, leg. Alexandre Prigogine (RBINS: 7078 to 7080, RMCA: 20574, 81.049-M-0003 to 81.049-M-0005, 19916 to 19921); 1 q, 1 §ै, 2 ?? [2 SA, 2 AL], Congo River, 75 mi [= $121 \mathbf{~ k m ] ~ u p , ~ u n k n o w n ~ d a t e , ~ l e g . ~ G e o r g e ~ L a t i m e r ~ B a t e s , ~ b e t w e e n ~} 1$ Aug. 1897 and 31 Aug. 1897, leg. unknown collector (USNM: 102513, 102515, 102514, 102516); 1 ? [SS], Defrenne Cave [= Mwanga Cave], between 19 Sep. 1948 and 22 Jun. 1949, leg. Narcisse Leleup (RMCA: 22490); 1 q, 4 ऽત [5 AL], Dethioux Farm, Cave I [= Shinkolobwe], 15 Jun. 1958, leg. Félix / Michel Anciaux de Faveaux (RMCA: 27909 to 27913); 12 ?? [12 AL], Dikuluwe Cave [= Dikulwe Cave], 2 Jan. 1954, leg. R.P. Th. de Caters (RMCA: 22090 to 22101); 1 ? [UN], Djelube River, lower, unknown date, leg. unknown collector (INPBC: V1093.2); 1 ? [UN], Djuma River, upper, unknown date, leg. unknown collector 
(INPBC: V1278); 16 ?? [16 UN], Dungu, 1 May 1948, leg. unknown collector (RBINS: 8128.01 to 8128.16); 2 우우 [2 UN], Edouard, Lac [= Edward, Lake], 26 Jan. 1954, leg. unknown collector (RBINS: 15450, 15451); 2 ?? [1 AL, 1 UN], Elisabethville [= Lubumbashi], between 1 Apr. 1926 and 30 Apr. 1926, leg. Henri Schouteden (RMCA: 16183, 16243); 1 đ, 1 ? [2 AL], Epulu, 26 Aug. 2015, leg. André Malekani, between 1 Oct. 1956 and 31 Oct. 1956, leg. Max Poll (UNIKIS: EPLU651, RMCA: 25653); 1 § [UN], Epulu, 2 km W, 14 Jul. 1991, leg. unknown collector (FMNH: 149406); 1 ? [SO], Etembo, 31 Mar. 1950, leg. P. Dyleff (RMCA: 19903); 2 ad 웅, 2 우, 2 ?? [1 SA, 2 AL, 3 UN], Faradje, 1 Apr. 1952, leg. H. de Saeger et al., 1 Apr. 1952, leg. H. de Saeger, 24 Oct. 1912, leg. Herbert Lang, James Paul Chapin and The American Museum Congo Expedition (RBINS: 13796.1 to 13796.3, RMCA: 36823, 36824, AMNH: 49426 [holotype Hipposideros nanus J.A. Allen, 1917]); 1 ? [AL], Funda Biabo, 26 Mar. 1914, leg. Charliers (RMCA: 5382); 2 ?? [2 AL], Garamba National Park, prior to 28 Dec. 1972, leg. H. de Saeger (RMCA: 36826, 36827); 1 ? [AL], Gemena, 13 Feb. 1939, leg.

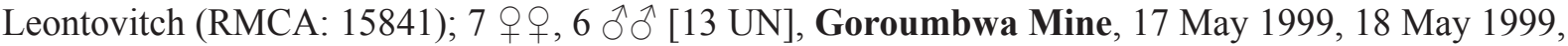
19 May 1999, 23 Oct. 1999, 24 Oct. 1999, 25 Oct. 1999, 27 Oct. 1999, leg. Robert (Bob) Swanepoel (TM: DRC0015, DRC0034, DRC0069, DRC0105, DRC0106, DRC0121, DBA207, DBA261, DBA266, DBA268, DBA274, DBA320, DBA410); 4 +,+ 7 ô $\hat{\jmath}, 1$ ? [10 SS, 1 AL, 1 UN], Hoyo, Mount, unknown date, 11 Aug. 1947, 14 Aug. 1947, 16 Aug. 1947, leg. unknown collector, 5 Oct. 1957, leg. Robin E. Leech and Edward S. Ross, between 17 Apr. 1949 and 16 Dec. 1949, between 14 Apr. 1949 and 16 Dec. 1949, leg. Jean Hiernaux (INPBC: V1549, RBINS: 7068 to 7075, CAS: 12497, RMCA: 19098, 19097); 2 ?? [2 AL], Ibambi, 10 Nov. 1938, prior to 23 Jan. 1939, leg. Degotte (RMCA: 14987, 14988); 1 ? [AL], Ibembo, between 1 Dec. 1950 and 31 Dec. 1950, leg. Jozef Hutsebaut (RMCA: 20280); 3 ?? [3 AL], Ikela, prior to 4 Jan. 1958, prior to 3 Nov. 1956, leg. P. Lootens (RMCA: 25829, 25831, 27021); 18 ?? [8 AL, $10 \mathrm{UN}$ ], Ilimba, unknown date, leg. unknown collector, 3 Jul. 1954, leg. Gaston-François de Witte (INPBC: W1191.01 to W1191.10, RMCA: 37147 to 37154); 1 ㅇ, 6 ๙ึ Irangi, 24 Dec. 1958, leg. Narcisse Leleup, between 24 Aug. 1959 and 16 Nov. 1959, leg. Uwe Rahm, between 4 Jan. 1957 and 1 Aug. 1957, between 3 Jan. 1957 and 1 Aug. 1957, between 27 Dec. 1956 and 1 Aug. 1957, leg. Heinz Stephan (RMCA: 28037, 28381 to 28384, 27440, 27441, 27444, 27445, 27443); 4 ?? [1 SS, 3 AL], Irangi Forest, between 1 Jan. 1957 and 31 Jan. 1957, leg. Narcisse Leleup, between 1 Nov. 1955 and 10 Aug. 1956, leg. Alexandre Fain (RMCA: 25872 to 25874, 23749); 1 ? [AL], Irumu, 25 km W [= Ituri Forest], prior to 17 Jan. 1955, leg. Pierrard (RMCA: 22800); 1 ? [AL], Ishango River, 11 Dec. 1954, leg. Gaston-François de Witte (RMCA: 37155); 1 đิ [UN], Ituri, south, 1 Jan. 1947 , leg. unknown collector (RBINS: 7473); 5 우, 5 $\widehat{\partial}$ [10 UN], Jadotville [= Likasi], 23 Feb. 1966, leg. unknown collector (RBINS: 17206.01 to 17206.10); 1 ? [SS], Kabalo, unknown date, leg. Henri Schouteden (RMCA: 9166); 1 Oे, 4 ?? [2 AL, 1 SK, 2 UN], Kabambi, unknown date, 14 Aug. 1947, leg. unknown collector, 27 Jul. 1955, leg. Gaston-François de Witte (INPBC: W1464.1, W1464.2, RBINS:

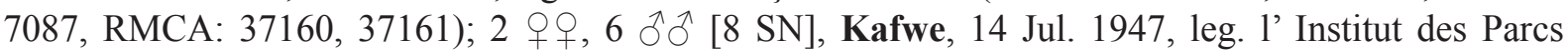
nationaux du Congo belge, 14 Jul. 1947, leg. unknown collector (RBINS: 10727 to 10734); $1 \lesssim$ [AL],

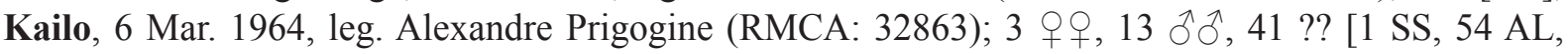
2 UN], Kakanda Cave, 22 Apr. 1959, leg. Félix / Michel Anciaux de Faveaux, between 12 Aug. 1964 and 11 Jan. 1965, leg. Alexandre Prigogine, between 21 Apr. 1954 and 22 Apr. 1954, leg. R.P. Th. de Caters (RMCA: 28167, 28168, 28170, 28175, 28177, 28178, 28180, 28182, 28183, 28185 to 28190, 33066, 22851 to 22885, 22891 to 22896); 64 ?? [64 AL], Kakontwe, 15 Aug. 1948, leg. Narcisse Leleup, 23 Feb. 1966, leg. Xavier Misonne (RMCA: 22638 to 22651, 34714 to 34763); 1 ऽ [AL], Kakontwe Camp [= Kakontwe], 7 Jun. 1956, leg. Félix / Michel Anciaux de Faveaux (RMCA: 23859); 2 우, 11 ठ઼ $\sigma^{\lambda}, 4$ ?? [1 SS, 15 AL, 1 SK], Kakontwe Cave, 26 Jun. 1956, 13 Feb. 1957, 8 Jul. 1957, 12 Jul. 1957, leg. Félix / Michel Anciaux de Faveaux, 6 Feb. 1958, leg. Robin E. Leech and Edward S. Ross, between 19 Sep. 1948 and 22 Jun. 1949, leg. Narcisse Leleup, prior to 24 Sep. 1962, leg. L. Poelman (RMCA: 26744, 26746, 26247, 26251 to 26253, 23814, 23861, 23862, 26232, CAS: 12496, 12527, RMCA:

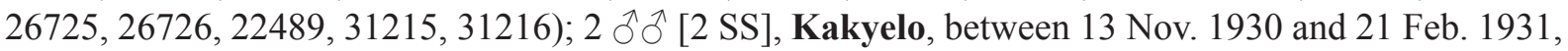
leg. Gaston-François de Witte and de Crawhez (RMCA: 10850, 10851); 1 ? [UN], Kalenda [Kasai], 
8 Sep. 1952, leg. unknown collector (RBINS: 7743); 1 ? [UN], Kamande, unknown date, leg. unknown collector (INPBC: V1057.2a); 2 우, 4 ถึ $\widehat{\jmath}, 1$ ? [4 SS, 3 AL], Kamituga, 11 Dec. 1964, between 11 Dec. 1963 and 11 May 1964, between 1 Nov. 1961 and 30 Nov. 1961, leg. Alexandre Prigogine (RMCA: 32864, 32865, 31151, 32395 to 32397, 32399); 1 ? [AL], Kapolowe, prior to 24 May 1954, leg. De Smet (RMCA: 22191); 1 †, 2 ô ô [2 SS, 1 SO], Karibumba, 30 Jan. 1949, between 30 Jan. 1949 and 26 Sep. 1949, leg. Alexandre Prigogine (RMCA: 18864 to 18866); 1 ? [AL], Kasaji Malonga, prior

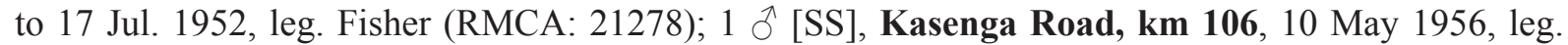
M. Lips (RMCA: 23803); 1 ㅇ [SS], Kasenye, 10 km N, 22 Sep. 1955, leg. James Paul Chapin (AMNH: 180907); 1 , 2 ?? [2 AL, 1 UN], Kasongo, 1 Dec. 1938, leg. unknown collector, 23 Aug. 1959, leg. Segers and Benoit Mission (RBINS: 6281.01, RMCA: 29201, 29205); 12 q $q, 7 \widehat{\jmath} \widehat{\partial}$ [19 UN], Kasongo Mwano, 1 Dec. 1938, leg. unknown collector (RBINS: 6281.02 to 6281.20); 1 đ [AL], Katanga,

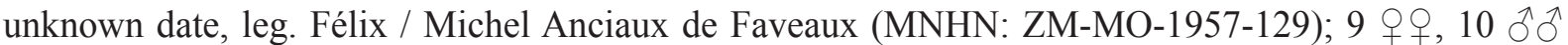
[19 AL], Katanga (former) Province, 19 Apr. 1962, 22 Apr. 1962, 24 Apr. 1962, 31 May 1962, 21 Jul. 1962, 13 Aug. 1962, leg. Félix / Michel Anciaux de Faveaux (SMF: 21279 to 21281, 21296, 21297, 21282, 21285, 21278, 21283, 21284, 21286, 21288 to 21295); 2 ?? [1 AL, 1 UN], Katanga s, unknown date, leg. André Pilette (RMCA: 16223, 16224); 9 ?? [9 SS], Kele Cave 1, 18 Aug. 1938, between 18 Aug. 1938 and 10 Nov. 1938, leg. J. Bequaert (RMCA: 14916, 14918, 14920, 14921, 14923, 14924, 14917, 14919, 14922); 2 ?? [2 AL], Kiambi, unknown date, leg. Gerard (RMCA: 16225, 16226);

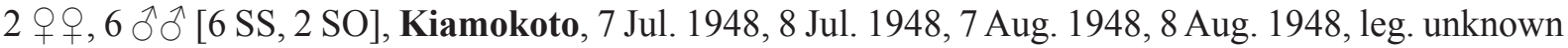
collector, 8 Jul. 1948, leg. l' Institut des Parcs nationaux du Congo belge (RBINS: 10763, 10768, 10761, 10762, 10764 to 10767); 1 , 43 ?? [44 AL], Kibwe Lya Mikako Cave, 26 Jan. 1955, leg. Wim Bergmans and Collard, 28 Dec. 1966, leg. M.J. Celis and M. Lejeune (RMCA: 22949 to 22954, 35171, 35175 to $35203,35205,35207,35209,35210,35212$ to 35215); 1 ? [AL], Kimuenza Cave, unknown date, leg. Alexandre Fain (RMCA: 35917); 2 ?? [2 AL], Kisangani, between 1 Jun. 1982 and 30 Jun. 1982, leg. Marc Colyn (RMCA: 82.031-M-0014, 82.031-M-0015); 1 § [SS], Kisantu, 12 Dec. 1920, leg.

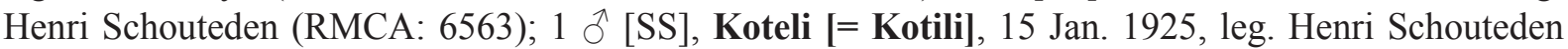
(RMCA: 8918); 2 ?? [2 AL], Kwama Kwama Cave, 13 Jun. 1955, leg. Gaston-François de Witte (RMCA: 37156, 37157); 6 우, 5 $\widehat{\partial}$ [1 SA, $10 \mathrm{AL}$ ], Kyasala Cave [= Kiasala Cave], 31 Mar. 1956, 15 Apr. 1956, 28 Jul. 1957, between 31 Mar. 1956 and 12 Sep. 1956, leg. Félix / Michel Anciaux de Faveaux (RMCA: 23811, 23812, 23857, 23858, 26865, 23809, 23852, 23854 to 23856, 23851); 1 ? [AL], Kyavinionge, 21 May 1954, leg. Gaston-François de Witte (RMCA: 37146); 1 ? [AL], Lake Kivu, near, between 1 Jan. 1934 and 31 Dec. 1934, leg. Guy Babault (MCZ: 46090); 1 juv ?, 1 9, 3 ?? [1 SS, 1 SA, 3 AL], Léopoldville [= Kinshasa], unknown date, leg. J. Camp, 1 Feb. 1942, 12 Nov. 1942, leg. R. Fiasse, 15 Mar. 1943, leg. Colback (USNM: 21663, 21664, RMCA: 18127, 18128, 31128); 14 ?? [14 UN], Lonzo [= Loso], unknown date, leg. unknown collector (INPBC: V1672.1, V1672.2.01 to V1672.2.13); 4 ?? [4 AL], Lubudi, large Cave [= Lubudi], 1 Nov. 1948, leg. Narcisse Leleup (RMCA: 22654 to 22657); 5 ?? [5 AL], Lubumbashi, 15 Feb. 1921, between 15 Feb. 1921 and 1 Jan. 1926, leg. J. Bequaert (RMCA: 16185, 16221, 16222, 16244, 16207); 1 ? [UN], Lufu, prior to 1 Oct. 1898, leg. unknown collector (RBINS: 208f); 2 ?? [2 AL], Lukatu Cave [= Ntoto Cave], prior to 2 Feb. 2007, leg. A. Kimbembi-ma-Ibaka (RMCA: a7.006-M-0005, a7.006-M-0006); 17 ?? [16 AL, 1 UN], Lula, between 1 Aug. 1956 and 31 Aug. 1956, leg. Max Poll, prior to 4 Jul. 1958, leg. A.J. Jobaert (RMCA: 25612 to 25627, 27199); 2 우, 3 ?? [4 SS, 1 AL], Luluabourg [= Kananga], unknown date, 9 May 1922, 10 May 1922, leg. Richard Callewaert, prior to 6 Mar. 1937, leg. Puissant (BMNH: 1927.12.21.6, 1927.12.21.7, RMCA: 7567, 7566, 13708); 1 ठ [SS], Lundjulu, between 22 Sep. 1952 and 29 Dec. 1952, leg. Schepens (RMCA: 21585); 4 우 [3 SS, 1 SO], Mabwe, 28 Jul. 1947, leg. l' Institut des Parcs nationaux du Congo belge, 7 Aug. 1947, 8 Aug. 1947, leg. unknown collector (RBINS: 10753, 10771, 10754, 10755); 1 ? [UN], Maginda, unknown date, leg. unknown collector (INPBC: W1077); 1 ? [SO], Make, 1 Jul. 1989, leg. Tshinyenye (RMCA: 89.057-M-0002); 1 [SS], Mambabwanga Hill [= Batala's Village], 9 Mar. 1925, leg. Edmund Heller (FMNH: 26241); 2 ?? [2 AL], Mani Grot of Nkela Grot van Mbumba-Nsi [= Mani Cave or Nkela Cave or Mbumba-Nsi], between 1 Jan. 2002 and 
13 Jul. 2002, leg. A. Kimbembi-ma-Ibaka (RMCA: a2.040-M-0003, a2.040-M-0004); 5 ?? [5 AL], Masako, unknown date, leg. Ndey B. Ifuta (RMCA: 93.079-M-0330 to 93.079-M-0334); 2 ?? [2 UN],

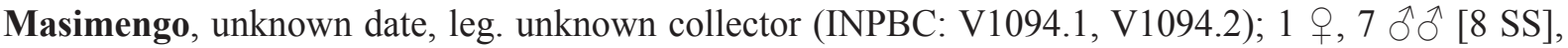
Masombwe, 10 Oct. 1948, 19 Oct. 1948, leg. unknown collector, 12 Oct. 1948, leg. l' Institut des Parcs nationaux du Congo belge (RBINS: 10778, 10780, 10781, 10784, 10785, 10788, 10774, 10795); 1 ઈ [SK], Matupi Cave, 16 Aug. 1947, leg. unknown collector (RBINS: 7088); 1 ô, 1 ? [1 SS, $1 \mathrm{UN}$ ], Medje, 24 Jan. 1910, leg. Herbert Lang, James Paul Chapin and The American Museum Congo

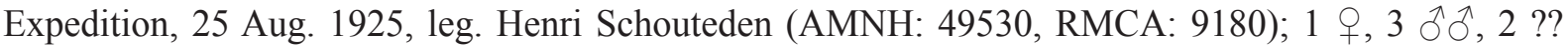
[6 SS], Mongbwalu, 7 Jun. 1939, between 30 May 1939 and 8 Aug. 1939, leg. Janssens, between 21 Jul. 1939 and 27 Nov. 1939, leg. Lepersonne (RMCA: 15630, 15747 to 15750, 15561); 1 § [SK], Mount Hoyo Caves, 5 Oct. 1957, leg. Robin E. Leech and Edward S. Ross (CAS: 12528); 1 đ [SS], Mount Sombwe, 22 May 1949, leg. unknown collector (RBINS: 10799); 5 ?? [4 SS, 1 AL], Mount Wago [= Wago, Mount], unknown date, between 1 Jul. 1951 and 31 Jul. 1951, between 1 Jan. 1951 and 31 Dec. 1951, leg. Alexandre Fain, 1 Jul. 1951, leg. unknown collector (BMNH: 1953.496, RBINS: 21660, RMCA: 21460, 23294, 23295); 4 오우, 6 ठิ $\widehat{\partial}$ [9 AL, 1 UN], Mpopola Cave, 2 May 1959, leg. Félix / Michel Anciaux de Faveaux (RMCA: 28193 to 28202); 2 오, 3 ô 0 [4 SS, 1 SO], Muhulu, 15 Jan. 1957, between 15 Jan. 1957 and 1 Aug. 1957, leg. Heinz Stephan (RMCA: 27450, 27446 to 27449); 11 우, 17 ठै $\widehat{\partial}$ [26 SS, 2 SO], Mulungwishi, prior to 15 Dec. 1931, between 10 Jan. 1930 and 14 Jan. 1930, leg. Gaston-François de Witte (RMCA: 11669 to 11695, 11522); 1 q, 1 § [2 AL], Mulungwishi, Cave, 28 Jun. 1956, leg. Félix / Michel Anciaux de Faveaux (RMCA: 23863, 23864); 1 ad ?, $3 \mathrm{imm}$ ?? [4 AL], Musenge, 27 Oct. 1954, leg. Narcisse Leleup (RMCA: 22736 to 22739); 1 o [SS], Mutsora, 4 Nov. 1949, leg. unknown collector (RBINS: 12946); 1 ? [SS], Mutwanga, between 1 Sep. 1937 and 30 Sep. 1937, leg. Henri Martin Hackars (RMCA: 16607); 35 ?? [35 UN], Muyirimbo, unknown date, leg. unknown collector (INPBC: V1139, V1144.01 to V1144.34); 4 ?? [4 AL], Mwanakusu [= Mwana-Kusu], 17 Sep. 1959, leg. Segers and Benoit Mission (RMCA: 29200, 29202 to 29204); 2 ad ??, 4 imm ??, 1 ? [6 AL, 1 UN], Mwanakusu Cave, 24 Dec. 1954, leg. Narcisse Leleup, 28 Aug. 1959, leg. Segers and Benoit Mission (RMCA: 22730 to 22735, 29199); 1 ? [UN], Nagero, prior to 25 Sep. 1951, leg. unknown collector (RBINS: 14637); 6 ?? [6 UN], Nambiliki / Dungu, unknown date, leg. unknown collector, 18 Aug. 1950, leg. H. de Saeger et al. (INPBC: 2128bis.1, 2128bis.2, RBINS: 13795.1 to 13795.4); 1 ? [AL], Ngamaye, 17 Jun. 1957, leg. Gaston-François de Witte (RMCA: 37145); 2 우, 2 $\widehat{\partial}$ [4 UN], Obenge, 7 Feb. 2013, leg. Guy-Crispin Gembu Tungaluna, Prescott Musaba and André Malekani, 7 Feb. 2013, leg. Prescott Musaba and André Malekani (UNIKIS: TLL386 to TLL389); 2 우, 1 ภ 1 , 1 ? [4 SS], Panga, between 1 Sep. 1925 and 30 Sep. 1925, leg. Henri Schouteden (RMCA: 9128 to 9131); 1 ? [SS], Paulis [= Isiro], between 1 May 1949 and 5 Nov. 1949, leg. Abbeloos (RMCA: 18944); 7 $\widehat{\partial}$ [6 SS, 1 UN], Pelenge, Gorges, 1 Jun. 1947, leg. l' Institut des Parcs nationaux du Congo belge, 1 Jun. 1947, 4 Jun. 1947, leg. unknown collector (RBINS: 10735, 10737, 10738, 10801, 10739, 10741, 10742); 11 ?? [9 SS, 1 SO, 1 UN], Poko, unknown date, 17 Jun. 1914, 24 Jun. 1914, 12 Jul. 1914, 17 Jul. 1914, leg. Cuthbert Christy, unknown date, leg. unknown collector (BMNH: 1919.5.8.10, 1919.5.8.11, RMCA: 4688, 4332, 4334, 4333, 4339, 4336a, 4337, 4338, 4335); 1 ㅇ [AL], Rambira, 16 Dec. 1959, leg. Uwe Rahm (RMCA: 28904); 1 q, 3 ô ô [4 AL], RubiTele, 17 Aug. 2013, 20 Aug. 2013, leg. Guy-Crispin Gembu Tungaluna, Prescott Musaba and André Malekani (UNIKIS: RBTL497 to RBTL499, RBTL528); 17 ?? [17 UN], Rutshuru, unknown date, leg. unknown collector (INPBC: V124, V1344, V1351, V1637.01 to V1637.12, V414, W26); 1 ? [UN], Rwere Rwindi, unknown date, leg. unknown collector (INPBC: V1336); 1 o, 2 ?? [2 AL, 1 SK], Saga Saga Cave, 10 Aug. 1947, leg. unknown collector, 16 Jul. 1955, leg. Gaston-François de Witte (RBINS: 7067, RMCA: 37158, 37159); 1 ? [SS], Sakania, 7 Mar. 1948, leg. Jozef Hutsebaut (RMCA: 18244); 1 ? [SS], Saliboko, 22 Sep. 1952, leg. Alexandre Fain (RMCA: 81.012-M-0076); 1 ㅇ, 2 ठึ St Therese Mission [= Likasi], 8 Feb. 1957, 25 Mar. 1957, 20 Jan. 1958, leg. Félix / Michel Anciaux de Faveaux (RMCA: 27537, 26255, 26246); 2 ?? [2 AL], Stanleyville [= Kisangani], unknown date, leg. Weyns, 5 Apr. 1928, leg. Collart (RMCA: 16214, 16217); 1 $\lesssim$ [SS], Tandala, 11 Jun. 1979, leg. 
C. Brian Robbins (USNM: 537709); 1 , 1 ๙ , 1 ? [3 SS], Tembwe, unknown date, leg. Henri Schouteden (RMCA: 8854, 8855, 9178); 1 ad , 1 foetus , 2 우 [2 SS, 2 UN], Teturi, 5 Aug. 1947, 21 Aug. 1947, 15 Jun. 1948, leg. unknown collector (RBINS: 7077, 7076, 7472.1, 7472.2); 17 ?? [16 AL, 1 UN], Thysville [= Mbanza-Ngungu], 11 Feb. 1987, leg. unknown collector, between 1 Aug. 1949 and 31 Aug. 1949, leg. Narcisse Leleup (RBINS: 22427, RMCA: 22586 to 22601); 2 ?? [2 AL], Thysville, Cave b 13 a [= Banjan Caves], between 1 Jul. 1949 and 31 Jul. 1949, leg. Narcisse Leleup (RMCA: 22584, 22585); 1 [SS], Tschambutscha [= Tshambutschu], between 31 Dec. 1956 and 1 Aug. 1957, leg. Heinz Stephan (RMCA: 27442); 2 ?? [2 AL], Tshakala, between 1 Jan. 1982 and 31 Jan. 1982, leg. Marc Colyn (RMCA: 82.019-M-0003, 82.019-M-0004); 1 ๙ $[\mathrm{UN}]$, Tshapona [= Tshiapona], 5 Dec. 1937, leg. unknown collector (RBINS: 6119); 1 [AL], Tshuapa District, between 1 Jan. 1960 and 31 Dec. 1964, leg. P. Lootens (RMCA: 34181); 4 ?? [4 UN], Uduku, 23 Jul. 1952, leg. H. de Saeger et al. (RBINS: 13797.1 to 13797.3, 13799); 2 우, 1 ภ, 2 ?? [1 SS, 1 AL, 1 SO, 2 UN], Uduku Moke [= Uduku], 24 Jul. 1952, leg. H. de Saeger et al., 24 Jul. 1952, between 24 Jul. 1952 and 28 Dec. 1972, leg. H. de Saeger (RBINS: 13798.1, 13798.2, RMCA: 36816, 36825, 36817); 1 के [AL], Uma, 10 Apr. 2014, leg. Prescott Musaba and André Malekani (UNIKIS: UMA503); 1 \& [SS], Utu [River], between 6 Feb. 1959 and 28 Jun. 1960, leg. Uwe Rahm (RMCA: 28903); 9 ?? [8 AL, 1 UN], Wafanya [=Wafania], 30 Sep. 1988, 12 Oct. 1991, 19 May 1992, between 12 Oct. 1991 and 18 Oct. 1991, between 1 Dec. 1991 and 31 Dec. 1991, between 1 May 1991 and 31 May 1991, between 1 Apr. 1992 and 30 Apr. 1992, between 1 Jan. 1992 and 31 Jan. 1992, leg. P. Lootens (RMCA: 91.076-M-0110, 92.079-M-0077, 89.020-M-0038, 92.079-M-0078, 92.079-M-0079, 91.076-M-0048, 92.079-M-0076, 92.079-M-0149, 91.076-M-0101); 1 ? [SS], Wago, Mount, between 1 Jul. 1951 and 31 Jul. 1951, leg. Alexandre Fain (RMCA: 23293); 3 ?? [3 AL], Zambi, prior to 31 Jan. 1899, leg. J.M. Derscheid (RMCA: 150a to 150c); 1 ? [SO], Zobia, prior to 18 Apr. 1946, leg. unknown collector (RBINS: 6974).

RWANDA: 1 ? [UN], Kissenji [= Gisenyi], unknown date, leg. Stegmann-Stein (ZMB: 67685).

Hipposideros camerunensis Eisentraut, 1956

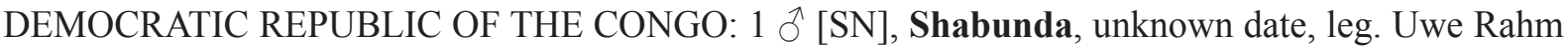
(BMNH: 1963.1166).

Hipposideros fuliginosus (Temminck, 1853)

DEMOCRATIC REPUBLIC OF THE CONGO: 3 $\widehat{ึ}$ [3 SS], Epulu, 2 May 1951, 3 May 1951, leg. A. Schwartz and R.N. Porter (KU: 151336 to 151338); 1 ते [SA], Equateur (former) Province, prior to 7 Oct. 1996, leg. unknown collector (SMF: 84411); 2 ڤึ [= Ituri Forest], unknown date, leg. Howard de Walden (BMNH: 1930.11.11.143, 1930.11.11.144); 1 त [SS], Yalosemba, 26 Jun. 1979, leg. Lynn W. Robbins (USNM: 537713).

Hipposideros ruber (Noack, 1893)

BURUNDI: 20 ๑ึ $\widehat{~[20 ~ A L], ~ G r o t t e ~ d e ~ R u b i n d i ~[=~ R u b i n d i], ~} 9$ Apr. 1969, 8 Mar. 1970, leg. P.L. Niort (MHNG: 1895.091 to $1895.100,1896.001$ to 1896.010); $1 \widehat{\jmath}$ [AL], Mpinga Cave [= Mpinga Mission], 1 Jan. 1969, leg. P.L. Niort (MHNG: 1896.011); 1 \& [SB], Mumushwizi Valley, 17 Aug. 1993, leg. Jay L. Udelhoven (FMNH: 155973); 3 ?? [3 SK], Nyanza-Lac, 10 km N, 3 Mar. 1979, leg. Heinz Stephan

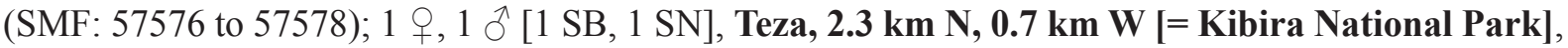
10 Mar. 1990, 14 Mar. 1990, leg. Julian C. Kerbis Peterhans (FMNH: 137627, 137628).

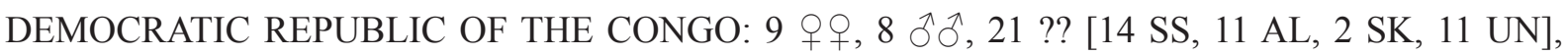
unknown locality, unknown date, 1 Jan. 1948, leg. unknown collector, unknown date, leg. Guy-Crispin Gembu Tungaluna (BMNH: 1926.7.6.69, 1930.11.11.105 to 1930.11.11.108, 1930.11.11.110 to 1930.11.11.112, 1930.11 .11 .114 to $1930.11 .11 .119,1930.11 .11 .128,1990.231,1990.232$, 


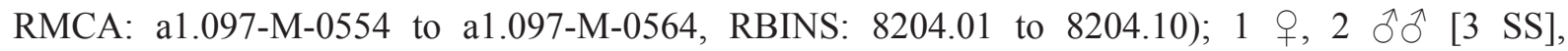
Aba, 13 Jan. 1911, 13 Dec. 1911, leg. Herbert Lang, James Paul Chapin and The American Museum Congo Expedition (AMNH: 49172, 49127, RMCA: 12370); 23 ?? [1 SS, 6 AL, 16 UN], Aka, 15 May 1950, between 15 May 1952 and 28 Dec. 1972, leg. H. de Saeger, 23 Apr. 1952, 15 May 1952, leg. H. de Saeger et al. (RMCA: 36802 to 36807, RBINS: 13765.01 to $13765.11,13749.1$ to 13749.5 , RMCA: 36711); 1 q [SS], Angumu, 1 Jul. 1937, leg. James Paul Chapin (AMNH: 119614); 7 우, 6 § $\sigma^{\lambda}, 3$ ?? [7 SS, 9 AL], Avakubi, 29 Sep. 1909, 1 Oct. 1909, 15 Nov. 1909, 17 Nov. 1909, 22 Nov. 1909, 24 Nov. 1909, 27 Nov. 1909, 28 Nov. 1909, 1 Dec. 1909, 2 Dec. 1909, between 1 Oct. 1909 and 1 Oct. 1915, leg. Herbert Lang, James Paul Chapin and The American Museum Congo Expedition (AMNH: 49372, 49369, 49373, 49178, 49179, 49375 to 49377, 49180, MCZ: 17373, AMNH: 49370, 49181, 49182, 49171, 49374, MCZ: 17223); 2 ?? [2 SS], Bolobo, unknown date, leg. the Royal Museum for Central Africa (RMCA) (BMNH: 1954.818, 1954.819); 1 ? [AL], Buta, prior to 31 May 1939, leg. Jozef Hutsebaut (RMCA: 15329); 1 ad +, 1 § [2 UN], Cel II, 8 Feb. 1951, 9 Feb. 1951, leg. H. de

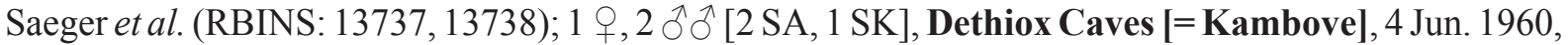
leg. Félix / Michel Anciaux de Faveaux (SMF: 18922 to 18924); 1 \& [SA], Detioux Cave [= Kambove], 4 Jun. 1960, leg. Félix / Michel Anciaux de Faveaux (SMF: 18925); 18 ?? [18 UN], Dungu, unknown date, leg. unknown collector (RBINS: 8203.01 to 8203.18); $1 \hat{\jmath}$ [SS], Eala, between 1 Aug. 1936 and 31 Aug. 1936, leg. J. Ghesquière (RMCA: 13411); 8 우, 11 ठิ $\widehat{~}$ [ [1 SS, 12 AL, 1 SO, 1 UN, 2 SB, 2 SN], Epulu, 2 May 1951, 3 May 1951, leg. A. Schwartz and R.N. Porter, 21 Apr. 1981, leg. John A. Hart, 26 Aug. 2015, leg. André Malekani, 6 Jul. 1991, leg. Julian C. Kerbis Peterhans (KU: 151344, FMNH: 138139, 138140, UNIKIS: EPLU637, EPLU639, EPLU641 to EPLU643, KU: 151343, FMNH: 149407 to 149416); 3 웅, 1 đ̊ [1 SS, 3 AL], Faradje, 20 Nov. 1911, 22 Oct. 1912, 25 Oct. 1912, leg. Herbert Lang, James Paul Chapin and The American Museum Congo Expedition (AMNH: 49168, 49381, 49385, 49416); 1 q [UN], Gangala-na-Bodio, 4 May 1951, leg. H. de Saeger et al. (RBINS: 13739); 1 q [UN], Grottes Kiwakishi, 18 Oct. 1974, leg. Michael D. Gallagher(HZM: 55.8027); 1 ? [SS], Irangi, unknown date, leg. Alexandre Fain (BMNH: 1956.465); 3 के $\hat{o}$ [3 AL], Irangi, slightly E of [= Irangi], 15 Oct. 1990, leg. Wim Bergmans (ZMA: MAM.24180, MAM.24181, MAM.24185); 9 우 [9 AL],

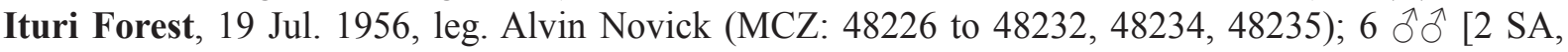
4 AL], Jomba, 4 Sep. 1976, leg. Frieder Meier (MHNG: 1873.062 to 1873.067); 1 ? [UN], Kabare, unknown date, leg. unknown collector (RMCA: 15839); 2 ?? [2 SS], Kabatibi Cave [= Mount Hoyo Caves], unknown date, leg. The Cambridge East Africa Expedition 1959 (BMNH: 1960.83, 1960.84);

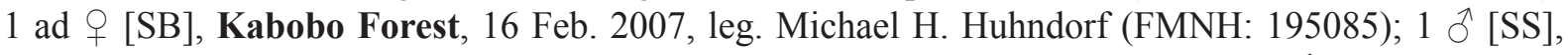
Kabwe, 12 Nov. 1973, leg. Western New Mexico University (FMNH: 152682); 1 ô [AL], Kadjidji [= Kajuju], unknown date, leg. Guy Babault (MNHN: ZM-MO-1984-1344); 4 우, 17 ふో 13 UN, 1 SS], Kakanda Cave, 31 May 1962, leg. Félix / Michel Anciaux de Faveaux, 31 May 1962, leg. unknown collector, between 13 Aug. 1964 and 11 Jan. 1965, leg. Alexandre Prigogine (MHNG: 1047.067 to 1047.074, RBINS: 14563 to 14575, RMCA: 33067); 6 우 [1 SA, 5 AL], Kakontwe Cave, 26 Jun. 1956, 8 Jul. 1957, 12 Jul. 1957, leg. Félix / Michel Anciaux de Faveaux (MHNG: 1046.039 to

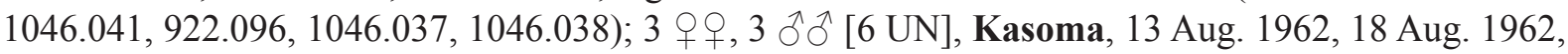

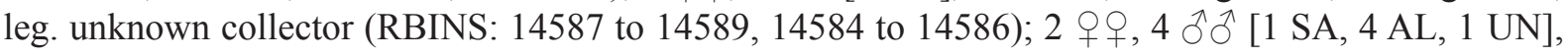
Kasoma Cave, 13 Aug. 1962, 18 Aug. 1962, leg. Félix / Michel Anciaux de Faveaux (MHNG: 1047.075

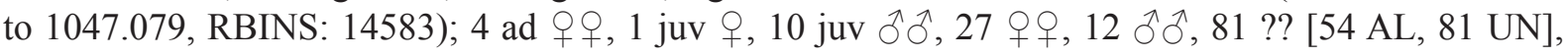
Keroma, 21 Apr. 1952, leg. H. de Saeger et al., 21 Apr. 1952, leg. H. de Saeger (RBINS: 13751.01 to $13751.10,13752.01$ to $13752.10,13753.01$ to $13753.10,13754.01$ to $13754.10,13755.01$ to 13755.10 , 13756.01 to $13756.10,13757.01$ to $13757.10,13758.01$ to 13758.11 , RMCA: 36746 to 36799$) ; 1$, 1 ㄱ [2 SS], Kilemue, 25 Oct. 1966, between 25 Oct. 1966 and 24 May 1967, leg. Alexandre Prigogine (RMCA: 34802, 34801); 2 우 [2 UN], Kinshasa, between 16 Jan. 1975 and 20 Jan. 1975, leg. Michael D. Gallagher (HZM: 53.8025, 54.8026); 7 ?? [7 AL], Kisangani, between 1 Jun. 1982 and 30 Jun. 1982, leg. Marc Colyn (RMCA: 82.031-M-0016 to 82.031-M-0022); 2 ?? [2 AL], Kisantu, prior to 17 Nov. 1913, leg. R.P. Vanderijst (RMCA: 2416a, 2416b); 1 ㅇ, 14 ふふぇ, 2 ?? [11 SA, 6 AL], Kivu 
region, 8 Mar. 1964, 11 Mar. 1964, leg. P. Kunkel, 31 Jan. 1984, 2 Feb. 1984, 12 Feb. 1984, between 11 Aug. 1989 and 14 Aug. 1989, leg. unknown collector (SMF: 31831, 64983, 64982, 64981, 31832, 77087 to 77098); 1 juv ${ }^{\Uparrow}$ [SA], Koloka, between 1 Jun. 1911 and 30 Jun. 1911, leg. unknown collector (SMF: 6364); 1 q [AL], Kumbi, 25 Nov. 1993, leg. Ngenge Masumbuko Kamitongo(ZMA: MAM.24900); 8 우, 2 ํํ [1 SA, 4 AL, 5 UN], Kyasala Cave [= Kiasala Cave], 15 Apr. 1956, 19 Apr. 1962, 22 Apr. 1962, leg. Félix / Michel Anciaux de Faveaux, 19 Apr. 1962, 22 Apr. 1962, leg. unknown collector (MHNG: 922.097 to 922.099, 1047.065, RBINS: 14590, MHNG: 1047.066, RBINS: 14591 to 14594); 1 q [AL], Kyasala Grande Grotte [= Kiasala Cave], 28 Jul. 1957, leg. Félix / Michel Anciaux de Faveaux (MHNG: 1046.042); 1 ð̊ [SS], Léopoldville [= Kinshasa], 10 Jul. 1909, leg. Herbert Lang, James Paul Chapin and The American Museum Congo Expedition (AMNH: 49110); 1 ? [SS], Libenge, 10 Jun. 1937, leg. Leontovitch (RMCA: 13988); 1 o [SA], Lubudi, 24 Apr. 1962, leg. Félix / Michel Anciaux de Faveaux (SMF: 21287); 1 [ [AL], Luhohov (River), 18 Oct. 1990, leg. Wim Bergmans (ZMA: MAM.24186); 4 우, 1 đ̃, 14 ?? [6 SS, 13 AL], Lukolela, 19 Aug. 1930, 14 Sep. 1930, leg. Franklin Edson, 18 Aug. 1930, 19 Aug. 1930, 15 Nov. 1930, 16 Nov. 1930, 21 Nov. 1930, leg. James Paul Chapin (AMNH: 86772, 86773, 86929 to $86931,86928,86774$ to $86776,86881,86918$ to 86926 ); 16 우 [15 SS, 1 UN], Luluabourg [= Kananga], unknown date, leg. Richard Callewaert (BMNH: 1926.11.1.47 to $1926.11 .1 .53,1926.7 .6 .621,1926.7 .6 .62$ 2, 1926.7.6.64 to 1926.7.6.68, 1926.7.6.7, 1926.7.6.70); 1 q [UN], Makpe, 8 Aug. 1951, leg. H. de Saeger et al. (RBINS: 13743); 6 ad $\circ$ + 2 juv ??, 2 đ̂ ô, 23 ?? [33 UN], Maleli, 9 Feb. 1951, 14 Jun. 1951, 18 Mar. 1952, leg. H. de Saeger et al. (RBINS: $13742.1,13742.2,13734.1,13734.2,13735.1,13735.2,13744.1$ to $13744.4,13745.1$ to 13745.4, 13746.1 to $13746.9,13747.1,13747.2,13748.1,13748.2,13741.1$ to 13741.6); 4 우, 6 ठ 6 ?? [1 SS, 11 AL, 4 SO], Malili, 13 Jun. 1951, 14 Jun. 1951, 18 Mar. 1952, between 18 Mar. 1952 and 28 Dec. 1972, leg. H. de Saeger (RMCA: 36706, 36707, 36712 to 36716, 36742, 36800, 36801, 36709, 36710, 36743 to 36745, 36708); 1 ? [AL], Masako, 11 Jun. 1989, leg. Ndey B. Ifuta (RMCA: 93.079M-0335); 1 q [SS], Mbuji-Mayi, 32 km SE, 25 Feb. 1974, leg. Western New Mexico University

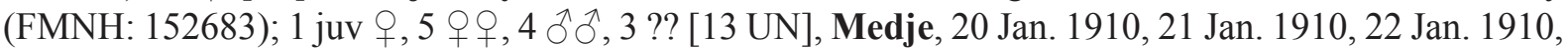
24 Jan. 1910, 23 Mar. 1910, 27 Apr. 1910, 3 Aug. 1910, 11 Mar. 1914, leg. Herbert Lang, James Paul Chapin and The American Museum Congo Expedition (AMNH: 49185, 49186, 49104, 49184, 49105 to 49107, 49187, 49108, 49380, 49378, 49379, 49109); 3 juv ??, 1 q, 1 ô, 7 ?? [2 SS, 1 SA, 9 AL], Mount Hoyo Caves, 4 May 1955, leg. Waldo LaSalle Schmitt and E. Baker (USNM: 301715 to 301726); 2 ठ઼ [2 UN], Mulungwishi, 27 Dec. 1959, 4 Jun. 1960, leg. unknown collector (RBINS: 14581, 14582); 1 ㅇ [SA], Mulungwishi, Cave, 28 Jun. 1956, leg. Félix / Michel Anciaux de Faveaux (MHNG: 922.095);

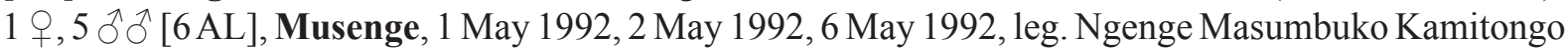
(ZMA: MAM.24550, MAM.24556, MAM.24552, MAM.24554, MAM.24551, MAM.24555); 2 우 [2 UN], Musosa, near, between 1 May 1941 and 31 May 1941, leg. unknown collector (RBINS: 14576,

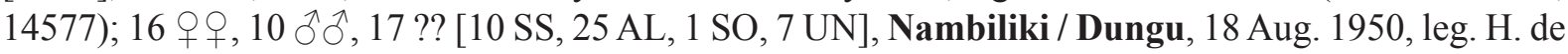
Saeger et al., 18 Aug. 1950, between 18 Aug. 1950 and 28 Dec. 1972, leg. H. de Saeger (RBINS: 13730.1, 13730.2, 13731.1, 13731.2, 13732.1, 13732.2, 13733, RMCA: 36701,36717 to 36741,36695 to 36700,36702 to 36705); 1 [SS], N'gombe [= Ngombe], 10 Nov. 1921, leg. Henri Schouteden (RMCA: 6561); 1 , 1 §ิ [1 SS, 1 SA], Niangara, 19 Nov. 1910, 12 Dec. 1910, leg. Herbert Lang, James Paul Chapin and The American Museum Congo Expedition (AMNH: 49388, 49183); 1 ad ${ }^{\lambda}$, 1 J , 9 ?? [5 SA, 6 AL], Niapu, 27 Jan. 1914, 28 Jan. 1914, 27 Feb. 1914, leg. Herbert Lang, James Paul Chapin and The American Museum Congo Expedition (AMNH: 49405 to 49408, 49410 to 49413, 49414 [holotype Hipposideros caffer niapu J.A. Allen, 1917], MCZ: 17224 [topotype Hipposideros caffer niapu J.A. Allen, 1917], AMNH: 49409); 2 ô ô [2 AL], Nyabiondo, 28 Dec. 1990, 30 Dec. 1990, leg. J.P. Lubula Bulambo and Ngenge Masumbuko Kamitongo (ZMA: MAM.24352, MAM.24353); 1 q [UN], Obenge, 7 Feb. 2013, leg. Prescott Musaba and André Malekani (UNIKIS: TLL374); 1 , 1 ¿ [2 SA], Oso River, between 1 Jan. 1957 and 31 Jan. 1957, leg. Félix / Michel Anciaux de Faveaux (SMF: 22298, 22299); 3 웅 [2 SA, 1 AS], Paoni, 5 Aug. 1976, 6 Aug. 1976, leg. Frieder Meier (MHNG: 1873.059 to 1873.061); 1 ? [UN], Pidigala River, 23 Apr. 1952, leg. H. de Saeger et al. (RBINS: 
13750); 3 ?? [3 AL], Poko, 1 Aug. 1913, 30 Aug. 1913, leg. Herbert Lang, James Paul Chapin and The American Museum Congo Expedition (AMNH: 49400 to 49402); 1 क , 1 ภ, 2 ?? [2 SS, 2 AL], Rutshuru, 14 Apr. 1927, leg. James Paul Chapin, Dewitt L. Sage, Frank P. Mathews and The Ruwenzori-Kivu Expedition, 24 Feb. 1927, 25 Feb. 1927, leg. David H. Linder (AMNH: 82395, MCZ: 23378, 23379, 25057); 1 ? [SS], Saliboko, unknown date, leg. Alexandre Fain (BMNH: 1953.497); 1 ㅇ, 2 ठึ 1 UN], St. Jos. (Mission) [= Mikalaya], 31 May 1924, 29 Oct. 1924, leg. Richard Callewaert (AMNH: 86150, 86168, 86170); 3 우 [3 UN], Tantara Cave [= Shinkolobwe], 4 Jun. 1960, leg. unknown collector (RBINS: 14578 to 14580); 1 ? [UN], Thysville [= Mbanza-Ngungu], 11 Feb. 1987, leg. unknown collector (RBINS: 22430); 2 ?? [2 AL], Tshakala, between 1 Jan. 1982 and 31 Jan. 1982, leg. Marc Colyn (RMCA: 82.019-M-0001, 82.019-M-0002); 1 $\lesssim$ [AL], Tshamalenge Cave, 25 Aug. 1957, leg. Félix / Michel Anciaux de Faveaux (MHNG: 1046.043); 1 ㅇ [SS], Tshikapa, 26 Nov. 1921, leg. Henri Schouteden (RMCA: 6562); 2 우, 1 ○े [3 AL], Uma, 10 Apr. 2014, 12 Apr. 2014, leg. GuyCrispin Gembu Tungaluna, Prescott Musaba and André Malekani (UNIKIS: UMA513, UMA622, UMA625); 4 우, 6 ठึ $\widehat{~[5 ~ S S, ~} 5$ AL], Yalosemba, 17 Jun. 1979, 27 Jun. 1979, leg. C. Brian Robbins, 18 Jun. 1979, leg. Frieder Meier (USNM: 463482 to 463484, 537714, 537715, 537718, MHNG: 1871.015, 1871.016, USNM: 537716, 537717).

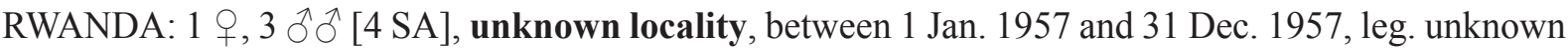
collector (SMF: 29050 to 29053); 1 ? [SK], Kibumbu Crater, 9 Dec. 1991, leg. J.R. Wilson and Anthony

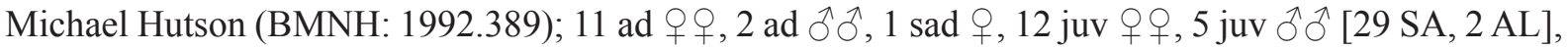
Kidaho, 10 Jun. 1982, 11 Jun. 1982, 13 Jun. 1982, leg. Benny Baeten (RMCA: 87.046-M-0215 to 87.046-M-0245); 2 ?? [2 UN], Lugarama, unknown date, leg. unknown collector (INPBC: V3041.1, V3041.2); 1 ? [UN], Nyakichozi, unknown date, leg. unknown collector (INPBC: V3118.1); 1 ad $\widehat{\partial}$ [SA], Nyakinama, 24 Oct. 1981, leg. Frits De Vree, Benny Baeten and Victor Van Cakenberghe (RMCA: 82.006-M-0896); 15 ?? [15 SA], Nyundo, 22 Oct. 1980, leg. Danny Meirte (RMCA: 80.055M-0098 to 80.055-M-0112); 4 $\widehat{\partial}$ [1 SA, 3 AL], Ruhengeri, 20 Sep. 1976, leg. Frieder Meier (MHNG:

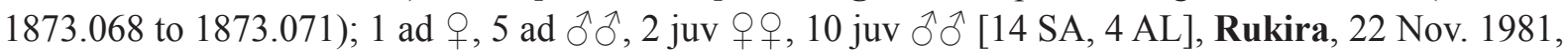
24 Nov. 1981, 26 Nov. 1981, leg. Frits De Vree, Benny Baeten and Victor Van Cakenberghe (RMCA: 82.006-M-0897 to 82.006-M-0914); 1 ad $\widehat{\jmath}$ [UN], Rwankalo, unknown date, leg. unknown collector (RBINS: 13112).

\section{Macronyctus gigas (Wagner, 1845)}

DEMOCRATIC REPUBLIC OF THE CONGO: 3 ?? [3 AL], unknown locality, unknown date, leg. Guy-Crispin Gembu Tungaluna (RMCA: a1.097-M-0550 to a1.097-M-0552); 1 ô [SS], Butembo,

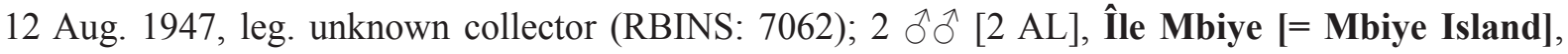
19 Feb. 2012, 31 May 2012, leg. Prescott Musaba (RMCA: a1.097-M-3213, a1.097-M-3212); 1 ठૈ [SS], Kakontwe, unknown date, leg. the Royal Museum for Central Africa (RMCA) (BMNH: 1954.820); 1 q, 1 ○ [2 SS], Mbuji-Mayi, 27 Dec. 1973, leg. Western New Mexico University (FMNH: 152633, 152634); 1 ad $\&$ [SS], Niangara, 2 Jun. 1913, leg. Herbert Lang, James Paul Chapin and The American Museum Congo Expedition (AMNH: 49103 [holotype Hipposideros gigas niangarce J.A. Allen, 1917]); 1 ? [SO], Nsiana, 1 Jan. 1927, leg. Cadgepole (MCZ: 23376); 1 \& [SS], Yalosemba, 17 Jun. 1979, leg. C. Brian Robbins (USNM: 537710).

$$
\text { Macronycteris Gray, } 1866
$$

\section{Macronycteris vittatus (Peters, 1852)}

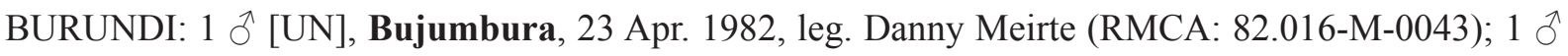
[AL], Kigwena Hill, 24 Apr. 1982, leg. Danny Meirte (RMCA: 82.016-M-0068). 
DEMOCRATIC REPUBLIC OF THE CONGO: 1 ? [SO], unknown locality, unknown date, leg. unknown collector (RBINS: 205); 1 [SS], Baliko, 14 Jun. 2012, leg. William ("Bill”) T. Stanley (FMNH: 219682); 2 ?? [2 AL], Boende, unknown date, between 1 Feb. 1970 and 28 Feb. 1970,

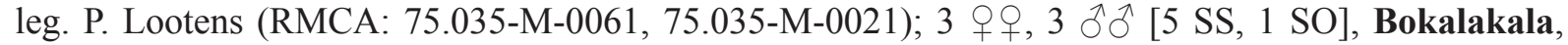
between 25 Apr. 1959 and 13 Jul. 1959, leg. N'Kele (RMCA: 27940 to 27945); 1 ㅇ [UN], Bomane [= Bomaneh], 21 May 2010, leg. Guy-Crispin Gembu Tungaluna, Prescott Musaba and José Akaibe (UNIKIS: CRT2125); 1 ? [AL], Congo da Lemba, prior to 22 Jan. 1900, leg. Paul Van Bellighen (RMCA: 410); 1 ? [AL], Ganda-Sundi, 29 Mar. 1964, leg. Alexandre Fain (RMCA: 33261); 7 우, 10 ถึ $\widehat{~}$ [17 UN], Goroumbwa Mine, 17 May 1999, 18 May 1999, 19 May 1999, 21 Oct. 1999, 22 Oct. 1999, 23 Oct. 1999, 25 Oct. 1999, leg. Robert (Bob) Swanepoel (TM: DRC0071, DRC0089, DRC0124, DRC0148, DBA071, DBA072, DBA075, DBA112, DBA124, DBA185, DBA186, DBA194, DBA199, DBA200, DBA280, DBA288, DBA289); 1 ? [UN], Kakontwe Cave, 5 Jul. 1948, leg. Narcisse Leleup (RMCA: 22686); 1 ad (gravid) $q$ [AL], Kalomoni Cave [= Kakontwe], 31 Jan. 1964, leg. Félix / Michel Anciaux de Faveaux (RMCA: 33992); 1 ? [AL], Kasaji, prior to 9 Jun. 1959, leg. Fisher (RMCA: 27891); 1 ? [SS], Kiseny [= Kasenye], unknown date, leg. Henri Schouteden (RMCA:

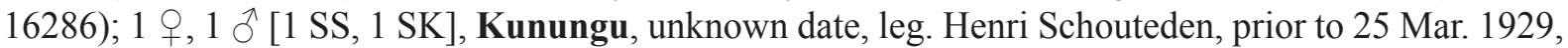
leg. Ngwe (RMCA: 7291b, 9707); 1 ? [AL], Lofoi [River], prior to 31 Mar. 1902, leg. Edgar Verdick (RMCA: 495); 1 ? [SS], Lovanium University [= Kinshasa], between 1 May 1962 and 31 May 1962, leg. De Bont (RMCA: 31200); 1 đ [UN], Marungu, unknown date, leg. Richard Böhm (ZMB: 6312); 1 [AL], Mosenge, 7 Aug. 1986, leg. WHO and Marc Colyn (RMCA: 88.011-M-0012); $1 \hat{\jmath}$ [SS], Mutsora, 3 Jul. 1951, leg. unknown collector (RBINS: 13325); 1 ? [SK], Mwanakusu Cave, between 1 Sep. 1959 and 30 Sep. 1959, leg. Benoit (RMCA: 28864); 1 , 1 ? [1 SS, 1 AL], Panda [= Pande], 22 Dec. 1963, leg. Félix / Michel Anciaux de Faveaux, between 28 Jan. 1956 and 17 May 1957, leg. De Smet (RMCA: 33991, 26211); 1 ? [SK], Tanganyika, Congo shore of Lake, prior to 19 Oct. 1889, leg. unknown collector (RBINS: 205b).

\section{Megadermatidae H. Allen, 1864 Cardioderma Peters, 1873 \\ Cardioderma cor (Peters, 1872)}

DEMOCRATIC REPUBLIC OF THE CONGO: 1 đ̊ [AL], Rubi-Tele, 17 Aug. 2013, leg. Guy-Crispin Gembu Tungaluna, Prescott Musaba and André Malekani (UNIKIS: RBTL493).

Lavia Gray, 1838

Lavia frons (E. Geoffroy St.-Hilaire, 1810)

BURUNDI: 1 ? [AL], Bujumbura, between 1 Jul. 1968 and 31 Jul. 1968, leg. Lewall (RMCA: 35152);

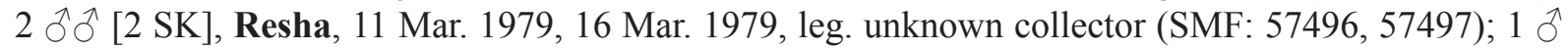
[AL], Russissi, bank [= Rusizi Delta], 27 Apr. 1982, leg. Danny Meirte (RMCA: 82.016-M-0146).

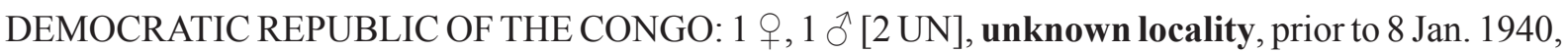
leg. unknown collector (RBINS: 6279.1, 6279.2); 1 क , 2 ?? [2 AL, 1 UN], Albertville [= Kalemie], 20 Nov. 1946, leg. unknown collector, prior to 18 Nov. 1959, leg. H. Bomans (RBINS: 9067, RMCA: 29298, 29299); 2 ठૈ (RBINS: 13632, 13640); 1 ? [SO], Bambesa, between 1 Apr. 1938 and 30 Apr. 1938, leg. J.M. Vrijdagh (RMCA: 14679); 1 त, 1 ? [1 AL, 1 SK], Baraka, 5 mi [= 8 km] N, 10 Jan. 1957, leg. Robin E. Leech and Edward S. Ross (CAS: 12487, 12526); 4 ?? [2 AL, 2 UN], Bauelendu, unknown date, leg. unknown collector, 31 Jan. 1955, 2 Feb. 1955, leg. Gaston-François de Witte (INPBC: W1358, W1360, RMCA: 37141, 37140); 1 ? [SS], Beni, prior to 13 May 1939, leg. Lisfranc (RMCA: 15303); 1 ? [AL], Bokoro, prior to 19 Nov. 1952, leg. E. Jans (RMCA: 21520); 1 ㅇ [SO], Bomili, 16 Aug. 1938, leg. Duncan 
M. Hodgson and William F. Coultas (MCZ: 38026); 1 ? [SS], Bosodula [= Boso-Dula], 19 Oct. 1941, leg. J.M. Vrijdagh (RMCA: 17752); 1 ? [AL], Buta, unknown date, leg. Jozef Hutsebaut (RMCA: 16571); 1 q, 1 Oे, 1 ? [3 AL], Catchpole's Farm, 28 Jul. 1924, 29 Jul. 1924, 27 Aug. 1924, leg. Edmund

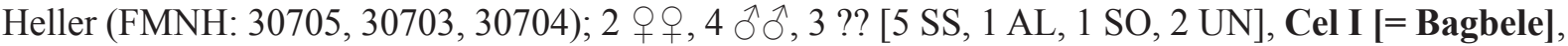
unknown date, leg. unknown collector, 12 Feb. 1950, 5 Jun. 1950, 22 Nov. 1950, leg. H. de Saeger, 19 Jul. 1950, 31 Oct. 1950, 6 Dec. 1950, 12 Dec. 1950, leg. H. de Saeger et al. (INPBC: 1441, RMCA: 36938, RBINS: 13634, 13642, RMCA: 36939, 36940, RBINS: 13643, RMCA: 36945, RBINS: 13635);

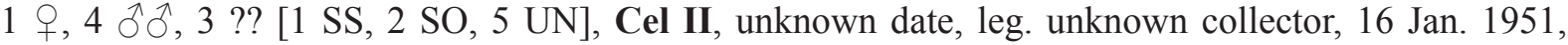
17 Mar. 1951, 29 May 1951, 21 Apr. 1952, 8 May 1952, leg. H. de Saeger et al., 29 Jun. 1951, 7 Mar. 1952, leg. H. de Saeger (INPBC: 4392, RBINS: 13646, 13645, 13652, 13647, RMCA: 36942, 36943, RBINS: 13655); 1 ? [AL], Dubulu, 14 Jan. 1932, leg. H.J. Bredo (RMCA: 11697); 1 ㅇ, 1 ○ [2 SS], Edouard, Lac [= Edward, Lake], 10 Jul. 1952, 13 Mar. 1953, leg. unknown collector (RBINS: 17298, 17297); 1 juv 9,1 q [2 UN], Embe, 19 Apr. 1952, 20 Apr. 1952, leg. H. de Saeger et al. (RBINS: 13654, 13653);

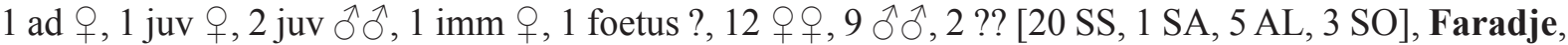
18 Feb. 1910, 23 Apr. 1911, 26 Apr. 1911, 29 Sep. 1911, 5 Nov. 1911, 6 Nov. 1911, 15 Nov. 1911, 1 Jan. 1912, 2 Jan. 1912, 5 Mar. 1912, 26 Mar. 1912, 14 Oct. 1912, 21 Oct. 1912, 3 Dec. 1912, 15 Dec. 1912, 29 Dec. 1912, leg. Herbert Lang, James Paul Chapin and The American Museum Congo Expedition, 21 Oct. 1912, leg. unknown collector, 7 Jul. 1925, between 7 Jul. 1925 and 23 Oct. 1970, leg. Henri Schouteden (AMNH: 49080 to 49082, 49384, 49079, 49090, 49382, 49383, 49083, 49084, 49087, 49088, KU: 125198, AMNH: 49071 to 49073, 49086, 49284, 49074, RMCA: 12359, AMNH: 49091, 49089, 49420, 49075 to 49078, RMCA: 9050, 9049); 2 우, 2 o 0 [4 SO], Gangala [= Gangalana-Bodio], 24 Mar. 1957, 25 Mar. 1957, leg. Heinz Stephan (RMCA: 27431 to 27434); 3 우, 1 ô, 5 ?? [3 SS, 1 SO, 5 UN], Gangala-na-Bodio, unknown date, 9 Apr. 1948, 13 Apr. 1948, 11 May 1948, leg. unknown collector, 24 Dec. 1949, leg. H. de Saeger et al., 24 Dec. 1949, leg. H. de Saeger, 6 Dec. 1956, between 1 Nov. 1956 and 30 Nov. 1956, leg. Max Poll (RBINS: 8206, 8184, 8183, 13629, 13631, RMCA: 36937, 25607, RBINS: 8182, RMCA: 25606); 1 ㅇ [AL], Garamba National Park, between 1 Jan. 1949 and 31 Dec. 1952, leg. H. de Saeger (RMCA: 36949); 1 đ [UN], Goma, 9 Dec. 1952, leg. unknown collector (RBINS: 15453); 1 ㅇ, 1 ठิ [1 SS, 1 SO], Ishango River, 13 Mar. 1953, 17 Mar. 1953, leg. Gaston-François de Witte (RMCA: 37129, 37130); 1 ? [UN], Ishango-Nyakazi, unknown date, leg. unknown collector (INPBC: V96); 1 ô, 2 ?? [3 SS], Kabalo, unknown date, leg. Henri Schouteden (RMCA: 9173 to 9175); 1 ? [AL], Kalina, prior to 11 May 1949, leg. Dartevelle (RMCA: 18724); 1 ㅇ [SS], Kamande, 19 Dec. 1934, leg. unknown collector (RBINS: 4774); 1 ? [AL], Kambukabakali, 5 Nov. 1954, leg. Gaston-François de Witte (RMCA: 37138); 2 ?? [1 AL, 1 UN], Kanyatsi, unknown date, leg. unknown collector, 16 Sep. 1953, leg. Gaston-François de Witte (INPBC: W541, RMCA: 37133); 2 ?? [2 UN], Kasaka, unknown date, leg. Franz Ludwig Stuhlmann (ZMB: 67896, 67897); 1 , $1 \widehat{~} \widehat{~[2 ~ A L], ~ K a s e n g i ~[=~ K a s e n g u], ~} 23$ Aug. 1926, 2 Sep. 1926, leg. James Paul Chapin, Dewitt L. Sage, Frank P. Mathews and The Ruwenzori-Kivu Expedition (AMNH: 82386, 82385); 1 q [UN], Kasenye, 10 km N, 3 Mar. 1954, leg. unknown collector (RBINS: 15454); 2 우, 1 ô, 1 ? [1 SS, 3 UN], Kasenyi [= Kasenye], 31 Oct. 1939, leg. Lepersonne, between 26 Feb. 1953 and 30 Dec. 1955, between 1 Oct. 1953 and 6 Oct. 1953, leg. unknown collector (RMCA: 15958, RBINS: 10545, 10543, 10544); 3 ?? [1 AL, 2 UN], Kasindi, unknown date, leg. unknown collector, unknown date, leg. H. Schubolz, 13 May 1954, leg. Gaston-François de Witte (INPBC: W978, ZMB: 67860, RMCA: 37137); 2 ઈิ [2 SO], Kasindi-port [= Kasindi], 18 Mar. 1953, 22 Sep. 1953, leg. Gaston-François de Witte (RMCA: 37131, 37132); 2 우, 1 ð [2 SS, 1 SO], Katanda, 14 Sep. 1932, leg. Burgeon, 16 Mar. 1953, leg. unknown collector, 20 Apr. 1933, leg. Henri Martin Hackars and Georges Marlier (RMCA: 12028, RBINS: 17296, RMCA: 12164); 1 ๆ, 3 ðð [4 SS], Katobwe, 1 Dec. 1926, 4 Dec. 1926, 5 Dec. 1926, leg. John Todd Zimmer (FMNH: 29404 to 29407); 1 ? [AL], Kawa (Forest), between 1 Jan. 1951 and 31 Dec. 1951, leg. Alexandre Fain (RMCA: 21461); 1 ? [AL], Kiavikire [= Kiavikere], 24 Nov. 1954, leg. Gaston-François de Witte (RMCA: 37142); 2 ?? [2 AL], Kishero, 3 Dec. 1954, leg. Gaston-François de Witte (RMCA: 37143, 37144); 2 우 [2 AL], Kivu Mountains, unknown date, leg. Guy Babault 
(MNHN: ZM-MO-1985-278, ZM-MO-1985-279); 2 ?? [1 AL, 1 UN], Kyavinionge, unknown date, leg. unknown collector, 11 May 1954, leg. Gaston-François de Witte (INPBC: W973, RMCA: 37136); 3 우웅 1 đิ [4 UN], Liki, 10 Nov. 1954, 18 Nov. 1954, leg. unknown collector (RBINS: 15456.1, 15456.2, 15462.1, 15462.2); 5 ?? [2 AL, 3 UN], Lomera, unknown date, leg. unknown collector, 9 Oct. 1953, 27 Oct. 1954, leg. Gaston-François de Witte (INPBC: W1305, W1307, W583, RMCA: 37139, 37135); 1 ? [UN], Lotakamela, unknown date, leg. unknown collector (RMCA: 18958); 1 क [SO], Lulenga [= Rugari], 13 Nov. 1925, leg. Henri Schouteden (RMCA: 9182); 1 ? [UN], Mabereni River, unknown

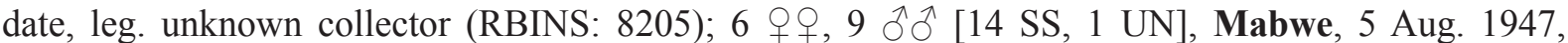
6 Aug. 1947, 7 Aug. 1947, 12 Aug. 1947, 4 Sep. 1947, 19 Nov. 1948, 23 Dec. 1948, 6 Jan. 1949, 16 Feb. 1949, 1 Mar. 1949, between 1 Jan. 1947 and 31 Dec. 1947, leg. unknown collector (RBINS: 10701, 10695, 10696, 10699, 10700, 10697, 10702, 10804, 10691, 10692, 10698, 10693, 10703, 10694,

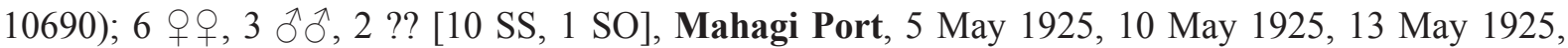
14 May 1925, 16 May 1925, leg. Henri Schouteden, 28 Sep. 1955, leg. James Paul Chapin, prior to 5 Jan. 1940, leg. Lepersonne (RMCA: 9005 to 9007, 9009 to 9011, AMNH: 180906, RMCA: 9003, 9008, 9060, 15959); 1 ? [AL], Maka, 27 Jun. 1947, leg. Max Poll (RMCA: 18045); 1 juv ô, 1 juv ?, 1 q, 2 ?? [5 AL], Masako, 14 Sep. 1988, 27 Aug. 1989, leg. Ndey B. Ifuta (RMCA: 93.079-M-0336 to 93.079-M-0340); 2 우 [2 SS], May ya Moto, between 5 Nov. 1934 and 15 Nov. 1934, leg. GastonFrançois de Witte (RMCA: 16628, 16629); 1 § [SS], Molindi River, 1 May 1934, leg. unknown collector (RBINS: 4773); 1 ? [AL], Mongbwalu, prior to 13 Feb. 1934, leg. Jordens (RMCA: 12246); 1 q, 1 ? [1 SO, 1 UN], Mulongo, unknown date, leg. Gerard, 28 Oct. 1974, leg. Michael D. Gallagher (RMCA: 10516, HZM: 26.7791); 2 ?? [1 AL, 1 UN], Museya [= Mosenda], unknown date, leg. unknown collector, 23 Sep. 1953, leg. Gaston-François de Witte (INPBC: W546, RMCA: 37134); 2 ?? [1 SS, 1 SO], Mutwanga, between 1 Jan. 1937 and 31 Jan. 1937, leg. Henri Martin Hackars, prior to 18 Sep. 1933, leg. Van Hoof (RMCA: 16601, 12170); 1 , 1 ô [1 AL, 1 SO], Nagbarama, 17 Apr. 1950, leg. H. de Saeger (RMCA: 36941, 36944); 1 đิ [SO], Nagero, 4 Jun. 1954, leg. unknown collector (RBINS: 14626); 1 ad ? [SI], Nasosurro River, unknown date, leg. Guy Babault (MNHN: ZMMO-1939-197); 1 ? [UN], Ndote, unknown date, leg. unknown collector (INPBC: W1382); 1 ? [UN], Ndotwe, unknown date, leg. unknown collector (INPBC: V1438); 1 \&, 1 ô [2 UN], Parc National de Garamba [= Garamba National Park], 25 Jan. 1951, 29 Jul. 1951, leg. H. de Saeger et al. (RBINS: 13644, 13648); 1 ㅇ, 1 đ [2 AL], PFSK 8, 25 Mar. 1952, leg. H. de Saeger (RMCA: 36946, 36947); 1 ,

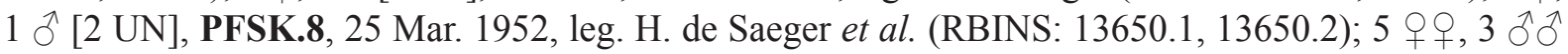
[8 SS], Ruwenzori SE, prior to 31 Dec. 1911, leg. Rosenberg (RMCA: 935a to 935e, 936a to 936c); 1 , 1 ते [2 SS], Rwindi, between 1 Nov. 1934 and 30 Nov. 1934, leg. Gaston-François de Witte (RMCA: 16630, 16631); $1 \widehat{\delta}$ [UN], Sabe, between 21 Nov. 1953 and 30 Dec. 1955, leg. unknown collector (RBINS: 10546); 1 q [SA], Semmio [= Zemio], 28 Feb. 1883, leg. Bohndorff (RMNH: MAM.35983); 1 ? [UN], Senga, unknown date, leg. unknown collector (INPBC: W551); 1 ? [AL], Stanleyville [= Kisangani], 4 Mar. 1928, leg. Collart (RMCA: 16263); 1 \&, 1 ? [1 AL, 1 UN], Tore, unknown date, leg. unknown collector, 22 Mar. 1952, leg. H. de Saeger (RBINS: 13651, RMCA: 36948); 2 ?? [2 AL], Uvira, 12 Sep. 1951, leg. Narcisse Leleup (RMCA: 22753, 22754); 2 우, 3 $\widehat{\partial}$ [5 SS], Vitshumbi, 1 Aug. 1934, 7 Mar. 1938, leg. unknown collector, between 1 Oct. 1933 and 31 Oct. 1933, leg. Henri Martin Hackars (RBINS: 4775 to 4777, RMCA: 12268, 12269); $1 \lesssim$ [AL], Volcan du Kivu [= Nyirogongo Volcano], unknown date, leg. unknown collector (MNHN: ZM-MO-1911-1019 A); $1 \lesssim$ [UN], Yaekela, 3 May 2010, leg. Guy-Crispin Gembu Tungaluna, Prescott Musaba and José Akaibe (UNIKIS: CRT2363).

RWANDA: $2 \widehat{\partial}$ [2 SS], Colline Musha [= Musha], 13 Dec. 1954, between 1 May 1955 and 31 May

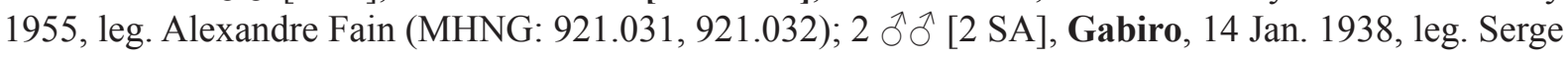
Frechkop (RMCA: 17085, 17086); 1 ? [SS], Gakoma (Mission), 12 Aug. 1949, leg. Aureliaan (RMCA: 18997); 3 우 [3 UN], Kagera National Park [= Akagera Park], 15 Jan. 1938, 19 Jan. 1938, leg. unknown collector (RBINS: 4779, 4780, 4778); 1 ad 9 , 1 juv ? [1 SA, 1 AL], Karama, 11 Oct. 1981, leg. Frits De Vree, Benny Baeten and Victor Van Cakenberghe (RMCA: 82.006-M-0928, 82.006-M- 
0931); 1 [SA], Katjumbura, 10 Jun. 1938, leg. Serge Frechkop (RMCA: 17087); 1 ð [SA], Kibuye, 1 Nov. 1980, leg. Danny Meirte (RMCA: 80.055-M-0171); 1 ㅇ [SS], Kimuna, 1 Dec. 1966, leg. unknown collector (RBINS: 16004); 1 ? [UN], Kissenji [= Gisenyi], unknown date, leg. StegmannStein (ZMB: 67844).

Rhinolophidae Gray, 1825

Rhinolophus Lacépède, 1799

BURUNDI: 1 ? [AL], Rushubi Mine, 12 Nov. 1969, leg. P.L. Niort (MHNG: 1895.080).

DEMOCRATIC REPUBLIC OF THE CONGO: 2 ?? [2 AL], unknown locality, 23 Aug. 2012, leg. Prescott Musaba (RMCA: a1.097-M-3214, a1.097-M-3215); 1 ? [AL], Boende, between 1 Jan. 1970 and 31 Jan. 1970, leg. P. Lootens (RMCA: 38819); 2 juv ?? [2 UN], Djomba [= Djemba], unknown date, leg. unknown collector (INPBC: V1523.1, V1523.2); 1 juv ?, 3 ?? [4 UN], Garamba National Park, between 1 Jan. 1948 and 31 Dec. 1948, leg. unknown collector (RBINS: 8129.1 to 8129.4); 1 juv q [UN], Goma, between 26 Nov. 1952 and 30 Dec. 1955, leg. unknown collector (RBINS: 10548); 4 juv 웅, 7 juv $\widehat{\text { oิ }}$ [10 SS, 1 SO], Kafwe, 14 Jul. 1947, leg. unknown collector (RBINS: 10743 to 10752, 10772); 5 juv 우 [3 SS, 2 SO], Kaswabilenga, 9 Oct. 1947, 4 Nov. 1947, leg. unknown collector (RBINS: 10758, 10756, 10757, 10769, 10770); 4 juv $\hat{~} \widehat{\jmath}$ [ [4 SS], Kibanga, 17 Nov. 1947, leg. unknown collector (RBINS: 10759, 10760, 10849, 10850); 1 § [AL], Kona, 12 May 2010, leg. GuyCrispin Gembu Tungaluna (RMCA: a1.097-M-3216); 1 đ [UN], Lieki, 1 Jun. 2010, leg. Guy-Crispin Gembu Tungaluna, Prescott Musaba and José Akaibe (UNIKIS: CRT2038); 2 ?? [2 SS], Lovanium University [= Kinshasa], 11 Jan. 1963, 6 Jul. 1963, leg. De Bont and Jo Van Orshoven (RMCA: 32632, 32631); 1 $\widehat{O}$ [MS], Lukobwe, 12 Oct. 1948, leg. unknown collector (RBINS: 1099); 8 juv $q$, 2 juv ô $\widehat{d}$ [10 SS], Masombwe, 10 Oct. 1948, 12 Oct. 1948, leg. unknown collector (RBINS: 10779, 10782, 10783, 10786, 10787, 10790, 10791, 10773, 10775, 10776); 1 ? [AL], Moenge, 15 May 2010, leg. GuyCrispin Gembu Tungaluna (RMCA: a1.097-M-3222); 1 ? [AL], Niambasha, 1 Aug. 1956, leg. Uwe Rahm (RMCA: 33534); 1 juv $\widehat{~}$ [SS], Pelenge, Gorges, 1 Jun. 1947, leg. unknown collector (RBINS: 10736); $1 \lesssim$ [UN], Yaekela, 4 May 2010, leg. Guy-Crispin Gembu Tungaluna, Prescott Musaba and José Akaibe (UNIKIS: CRT2392).

Rhinolophus alcyone Temminck, 1853

DEMOCRATIC REPUBLIC OF THE CONGO: 1 đ [SK], Buluku River, 12 Jul. 1951, leg. H. de Saeger et al. (RBINS: 13667); 1 ad $\precsim$ [SS], Lukolela, 1 Aug. 1930, leg. James Paul Chapin (AMNH: 86880); 1 đ [SS], Makpe River, upper [= Makpe], 22 May 1952, leg. H. de Saeger et al. (RBINS: 13666); 1 ठ [UN], Scierie Forest, 22 Nov. 1974, leg. Michael D. Gallagher (HZM: 1.8022).

Rhinolophus blasii Peters, 1867

DEMOCRATIC REPUBLIC OF THE CONGO: $1 \widehat{\jmath}$ [SA], Katanga (former) Province, 31 May 1962, leg. Félix / Michel Anciaux de Faveaux (SMF: 21245); 1 ad $q$ [SA], Kivu region, unknown date, leg. Guy Babault (MNHN: ZM-MO-1985-939); 1 ? [AL], Moba, 23 Apr. 1954, leg. H. Bomans (RMCA: 26280).

Rhinolophus clivosus Cretzschmar, 1828

BURUNDI: 1 ? [SB], Nyagatarugwa River Valley, 9 Aug. 1992, leg. L. Davenport (FMNH: 148366); $1 \hat{\partial}$ [SB], Nyamugari Hill, 10 Sep. 1990, leg. L. Davenport (FMNH: 148367). 
VAN CAKENBERGHE V. et al., The bats of Congo, Rwanda and Burundi revisited

DEMOCRATIC REPUBLIC OF THE CONGO: 1 [AL], Defrenne Cave [= Mwanga Cave], 21 Jun. 1959, leg. Félix / Michel Anciaux de Faveaux (RMCA: 28212); 8 ?? [8 UN], Djomba [= Djemba], unknown date, leg. unknown collector (INPBC: V1846.1 to V1846.8); 2 o 9 [2 AL], Jadotville [= Likasi], 3 Apr. 1957, leg. Félix / Michel Anciaux de Faveaux (RMCA: 26256, 26257); 21 ठิ $\widehat{0}$ [21 AL], Jomba, 4 Sep. 1976, 5 Sep. 1976, 6 Sep. 1976, leg. Frieder Meier (MHNG: 1873.020 to 1873.040); 1 ㅇ [SK], Kakanda Cave, 22 Sep. 1960, leg. unknown collector (RBINS: 14513); 1 ? [SK], Kisantu, 11 Dec. 1920, leg. Henri Schouteden (RMCA: 7718); 1 sad +, 3 ?? [4 AL], Lubudi,

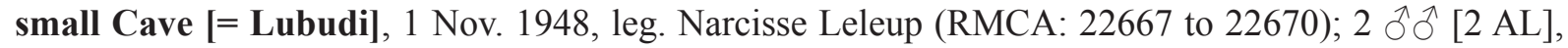
Lubuka Cave, 28 Aug. 1950, leg. Laurent (RMCA: 79.010-M-0005, 79.010-M-0006); 9 ?? [9 UN], Musanze-Djomba, unknown date, leg. unknown collector (INPBC: V1760, V1764.1 to V1764.8); 1 ? [SS], Mutwanga, between 1 Jan. 1937 and 31 Jan. 1937, leg. Henri Martin Hackars (RMCA: 16602); 1 ? [UN], Rutshuru, unknown date, leg. unknown collector (INPBC: W1163); 1 ㅇ [AL], Swanepoel I Cave, 1 May 1958, leg. Félix / Michel Anciaux de Faveaux (RMCA: 27538); 13 ?? [13 AL], Uvira, 12 Sep. 1951, leg. Narcisse Leleup (RMCA: 22740 to 22752).

RWANDA: 2 qo , 1 đ [3 SS], Bugoye Kisenyi, between 1 Nov. 1933 and 30 Nov. 1933, leg. Henri Martin Hackars (RMCA: 12265 to 12267); 1 ? [UN], Gisenyi, 11 Dec. 2004, leg. unknown collector (SMF: 92962); 1 ? [SK], Kibumbu Crater, 9 Dec. 1991, leg. J.R. Wilson and Anthony Michael Hutson (BMNH: 1992.390); 31 ad + +, 49 ad $\widehat{\jmath}$ ô [72 SA, 8 AL], Kidaho, 9 Jun. 1982, 10 Jun. 1982, 11 Jun. 1982, 12 Jun. 1982, 13 Jun. 1982, 16 Jun. 1982, 17 Jun. 1982, 18 Jun. 1982, leg. Benny Baeten

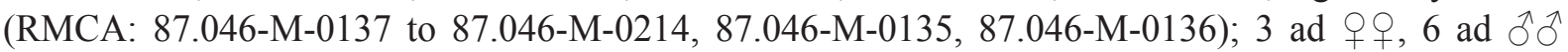
[9 SK], Musanse [= Musanze], 7 Jan. 1966, 10 Jun. 1966, leg. unknown collector (RBINS: 15480.1

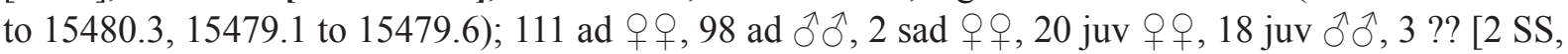
114 SA, 136 AL], Mutura, unknown date, 14 Dec. 1984, 15 Dec. 1984, 16 Dec. 1984, 18 Dec. 1984, 12 Jan. 1985, 13 Jan. 1985, leg. Benny Baeten and Luc Janssens, 22 Oct. 1981, 23 Oct. 1981, 25 Oct. 1981, 26 Oct. 1981, 27 Oct. 1981, leg. Frits De Vree, Benny Baeten and Victor Van Cakenberghe (RMCA: 85.006-M-0537, 85.006-M-0538，85.006-M-0076 to 85.006-M-0094, 85.006-M-0001 to 85.006-M-0037, 85.006-M-0465, 85.006-M-0038 to 85.006-M-0075, 82.006-M-0046 to 82.006-M0200); $1 \hat{\jmath}$ [SA], Nyundo, 17 Oct. 1980, leg. Danny Meirte (RMCA: 80.055-M-0170); 2 $\widehat{\partial}, 3$ ?? [2 AL, 3 UN], Ruhengeri, 20 Sep. 1976, leg. Frieder Meier, 22 Jun. 2003, 25 Jun. 2003, 3 Jul. 2003, leg. unknown collector (MHNG: 1873.041, 1873.042, SMF: 92438 to 92440); 1 ? [SS], Ubuvumo lava hole, 11 Dec. 2004, leg. Michael Laumanns (SMF: 92961).

\section{Rhinolophus eloquens K. Andersen, 1905}

DEMOCRATIC REPUBLIC OF THE CONGO: 1 [ [SS], unknown locality, 1 Apr. 1927, leg. David H. Linder (MCZ: 23377); 2 우, 1 ○ [3 SS], Aba, 15 Dec. 1911, 16 Dec. 1911, 17 Dec. 1911, leg. Herbert Lang, James Paul Chapin and The American Museum Congo Expedition (AMNH: 49100 to 49102); 1 §ิ, 1 ? [1 AL, 1 UN], Bunia, unknown date, leg. Maristes, between 1 Apr. 1927 and 30 Apr. 1927, leg. Harold Jefferson Coolidge (RMCA: 16277, MCZ: 28545); 1 ? [AL], Katanga, prior to 31 Aug. 1899, leg. Charles Lemaire (RMCA: 387); 2 $ठ$ [2 SS], Kilo Mines [= Kilomines], 21 Apr. 1949, leg. Jean Hiernaux (RMCA: 19095, 19096); 1 ? [AL], Kiseny [= Kasenye], between 1 Jan. 1926 and 31 Dec. 1926, leg. Henri Schouteden (RMCA: 16276); 1 ô [AL], Luofu, 23 Mar. 1927, leg. James Paul Chapin, Dewitt L. Sage, Frank P. Mathews and The Ruwenzori-Kivu Expedition (AMNH: 82393); 1 ๆ, 2 $\widehat{\jmath}$ [1 SS, 2 AL], Mboga [= Boga], unknown date, leg. Robert William Hayman, unknown date, leg. Howard de Walden (BMNH: 1930.11.11.102 to 1930.11.11.104); 4 우 [1 SS, 1 SA, 2 AL], Rutshuru, 1 Apr. 1927, leg. David H. Linder, 30 Jul. 1925, 23 Aug. 1925, leg. Edmund Heller, 26 Apr. 1926, leg. James Paul Chapin, Dewitt L. Sage, Frank P. Mathews and The Ruwenzori-Kivu Expedition (MCZ: 25122, FMNH:

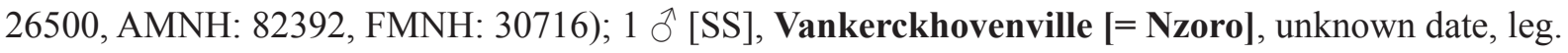
The Alexander-Gosling Expedition (BMNH: 1907.7.8.30). 
RWANDA: 1 ad ? [SA], Kidaho, 8 Jun. 1982, leg. Benny Baeten (RMCA: 87.046-M-0349); 1 q [SA], Rwankwi, between 1 Apr. 1948 and 30 Apr. 1948, leg. J.V. Leroy (RMCA: 19069); 1 ô, 1 ? [1 AL, 1 UN], Shangugu, between 1 Jan. 1926 and 31 Dec. 1926, between 1 Jan. 1926 and 31 Jan. 1926, leg. Henri Schouteden (RMCA: 16218, 16275).

\section{Rhinolophus fumigatus Rüppell, 1842}

BURUNDI: $17 \widehat{\partial}$ [17 AL], Karonge, 3 Oct. 1969, 12 Oct. 1969, leg. P.L. Niort (MHNG: 1895.072 to $1895.079,1895.086,1895.048$ to $1895.050,1895.052$ to 1895.056$) ; 1$ đ [AL], Mpinga Cave [= Mpinga Mission], 31 Dec. 1968, leg. P.L. Niort (MHNG: 1895.051); 2 ?? [2 AL], Rushubi Mine, 16 Nov. 1969, leg. P.L. Niort (MHNG: 1895.087, 1895.088); 1 § [AL], Rwintare, 3 Apr. 1969, leg. P.L. Niort (MHNG: 1895.047).

DEMOCRATIC REPUBLIC OF THE CONGO: 1 ad $q, 8$ 우, 2 ठ઼ $\widehat{~}$ [10 SS, 1 SO], Aba, 15 Dec. 1911, 16 Dec. 1911, 17 Dec. 1911, leg. Herbert Lang, James Paul Chapin and The American Museum Congo Expedition, 15 Dec. 1911, leg. unknown collector (AMNH: 49111, 49112, 49113 [holotype Rhinolophus abce J.A. Allen, 1917], 49114 to 49117, MCZ: 17375, RMCA: 12363, AMNH: 49118, 49197); 1 ? [UN], Albert National Park [= Virunga National Park], unknown date, leg. unknown collector (INPBC: V264.2); 1 ? [AL], Bunia, prior to 20 Feb. 1939, leg. Maristes (RMCA: 15225); 1 [ [UN], Gangala-na-Bodio, 7 Nov. 1949, leg. H. de Saeger et al. (RBINS: 13657); 59 우,

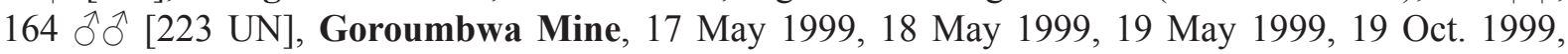
20 Oct. 1999, 21 Oct. 1999, 22 Oct. 1999, 23 Oct. 1999, 24 Oct. 1999, 25 Oct. 1999, 27 Oct. 1999, leg. Robert (Bob) Swanepoel (TM: DRC0032, DRC0053, DRC0055, DRC0056, DRC0086, DRC0102 to DRC0104, DRC0120, DRC0127 to DRC0131, DRC0134, DRC0135, DRC0142, DRC0146, DRC0147, DRC0149, DRC0151, DRC0154, DRC0155, DRC0158, DRC0159, DBA008, DBA012 to DBA014, DBA030 to DBA063, DBA085 to DBA106, DBA129, DBA130, DBA132 to DBA141, DBA143 to DBA156, DBA158 to DBA163, DBA178, DBA180 to DBA184, DBA195, DBA196, DBA201, DBA203 to DBA206, DBA208 to DBA223, DBA228, DBA245 to DBA253, DBA256 to DBA260, DBA262 to DBA265, DBA267, DBA269 to DBA273, DBA275, DBA276, DBA278, DBA283, DBA290 to DBA299, DBA301 to DBA316, DBA318, DBA319, DBA322 to DBA326, DBA328 to DBA330, DBA354, DBA360, DBA401 to DBA406, DBA408, DBA409, DBA411, DBA412); 2 ??

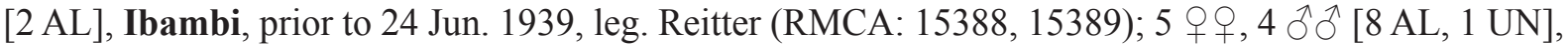
Kakanda Cave, 22 Apr. 1959, leg. Félix / Michel Anciaux de Faveaux (RMCA: 28169, 28171 to 28174, 28176, 28179, 28181, 28184); 3 ?? [3 AL], Lukatu Cave [= Ntoto Cave], prior to 2 Feb. 2007, leg. A. Kimbembi-ma-Ibaka (RMCA: a7.006-M-0002 to a7.006-M-0004); 1 ? [AL], Mani Grot of Nkela Grot van Mbumba-Nsi [= Mani Cave or Nkela Cave or Mbumba-Nsi], between 1 Jan. 2002 and 13 Jul. 2002, leg. A. Kimbembi-ma-Ibaka (RMCA: a2.040-M-0002); 1 $\delta$ [SK], Musosa, near, between 1 Jun. 1940 and 30 Jun. 1940, leg. unknown collector (RBINS: 14489); 4 우, 1 $\Uparrow$ [5 AL], Mwadingusha, 18 Dec. 1957, leg. Félix / Michel Anciaux de Faveaux (RMCA: 27532 to 27536); 1 juv ㅇ, 2 우, 2 ふふ઼, 7 ?? [1 SS, 1 AL, 6 SK, 1 SO, 3 UN], Ndelele, 3 Mar. 1952, 8 Mar. 1952, 11 Mar. 1952, 19 Nov. 1952, leg. H. de Saeger et al., 11 Mar. 1952, 19 Jun. 1952, leg. H. de Saeger (RBINS: 13659.1, 13659.2, 13661, RMCA: 36831, 36830, RBINS: 13662.1 to $13662.4,13660.1,13660.2,13656) ; 1$ ? [AL], Rungu, between 1 Aug. 1925 and 31 Aug. 1925, leg. Henri Schouteden (RMCA: 16250); 2 ?? [2 UN], Thysville [= Mbanza-Ngungu], 11 Feb. 1987, leg. unknown collector (RBINS: 22428, 22429).

RWANDA: 1 ? [UN], Bilalu, unknown date, leg. unknown collector (INPBC: V3063); 1 ? [SO], Gabiro, prior to 22 Jan. 1935, leg. Verhulst (RMCA: 12664); 22 ?? [22 UN], Lugarama, unknown date, leg. unknown collector (INPBC: 3041.01 to 3041.12, V304.01 to V304.10); 9 ?? [9 UN], Magachi, unknown date, leg. unknown collector (INPBC: V1903.1 to V1903.9); 1 đ [AL], Ruhengeri, 15 Nov. 1980, leg. Hans H. de Iongh (ZMA: MAM.21229). 
VAN CAKENBERGHE V. et al., The bats of Congo, Rwanda and Burundi revisited

\section{Rhinolophus hildebrandtii Peters, 1878}

BURUNDI: 1 ๙ [AL], Bujumbura, between 1 Jul. 1968 and 31 Jul. 1968, leg. Lewall (RMCA: 35153);

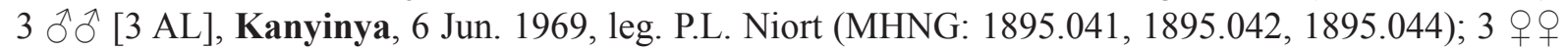
[3 AL], Makamba, 10 Apr. 1969, between 23 Mar. 1970 and 24 Mar. 1970, leg. P.L. Niort (MHNG:

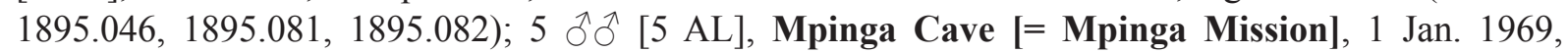
25 Mar. 1970, leg. P.L. Niort (MHNG: 1895.043, 1895.045, 1895.083 to 1895.085); 1 ? [AL], Ruyigi, between 19 Mar. 1952 and 22 Mar. 1952, leg. Laurent (RMCA: 79.010-M-0009).

DEMOCRATIC REPUBLIC OF THE CONGO: 1 \& [SK], unknown locality, prior to 1 May 1939, leg. unknown collector (RBINS: 6282); 1 , 1 ô [2 SA], Baga Cave [= Baya Cave], 12 Mar. 1956, leg. Félix / Michel Anciaux de Faveaux (HNHM: 95.52.1, 95.52.2); 7 ?? [7 AL], Baya Carrière [= Baya Quarry], 17 May 1965, leg. Walter N. Verheyen, 26 Jun. 1963, leg. Parent (RMCA: 34055 to 34059, 31505, 31506); 1 J, 2 ?? [3 AL], Baya Cave [= Baya Quarry], unknown date, leg. Félix / Michel Anciaux de Faveaux (MNHN: ZM-MO-1957-130 B, ZM-MO-1957-130 C, ZM-MO-1957-130 D); $1 \lesssim$ [AL], Defrenne Cave [= Mwanga Cave], 21 Jun. 1959, leg. Félix / Michel Anciaux de Faveaux (RMCA: 28211); 4 q [4 SA], Dethiox Caves [= Kambove], 4 Jun. 1960, leg. Félix / Michel Anciaux de Faveaux (SMF: 18914, 18915, 18917, 18918); 10 우, 9 $\widehat{\partial}$ [19 SK], Elisabethville [= Lubumbashi], 25 Mar. 1966, leg. unknown collector (RBINS: 17205.01 to 17205.19); 1 juv ?, 1 ? [2 UN], Gangalana-Bodio, prior to 23 Nov. 1957, leg. Station de la chasse et domestication des éléphants (RMCA:

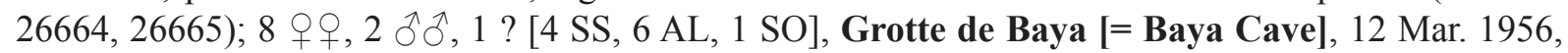
18 Mar. 1956, 14 May 1956, leg. Félix / Michel Anciaux de Faveaux, 14 May 1956, 12 Jul. 1956, 13 Jul. 1956, leg. M. Lips (MHNG: 922.093, 922.094, RMCA: 23801, 23802, 23805, MHNG: 922.090, 922.091, RMCA: 23804, 23806, MHNG: 922.087, 922.092); 1 đ [AL], Ibembo, 25 Jan. 1951, leg. Jozef Hutsebaut (RMCA: 89.004-M-0002); 1 § [AL], Jadotville [= Likasi], 31 Aug. 1958, leg. Félix / Michel Anciaux de Faveaux (RMCA: 27905); 1 ภ, 1 ? [1 AL, 1 UN], Kaboyaboya Cave, unknown date, leg. unknown collector, 23 Jul. 1962, leg. Félix / Michel Anciaux de Faveaux (MHNG: 1046.001, 1046.100); 16 웅, 2 đ̊, 2 ?? [12 AL, 6 SK, 2 UN], Kakanda Cave, 21 Sep. 1960, 29 Sep. 1960, 21 Jul. 1962, leg. unknown collector, 22 Apr. 1959, leg. Félix / Michel Anciaux de Faveaux, between 21 Apr. 1955 and 22 Apr. 1955, leg. R.P. Th. de Caters (RBINS: 14448, 14451, 14428, 14436 to 14438, 14441, RMCA:

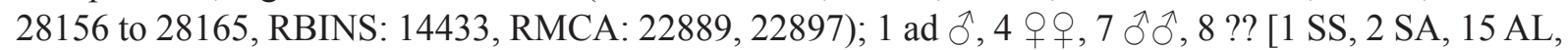
2 UN], Kakontwe Cave, unknown date, 3 Jul. 1956, 4 Jul. 1956, 9 Jul. 1956, 13 Feb. 1957, 8 Jul. 1957, 12 Jul. 1957, leg. Félix / Michel Anciaux de Faveaux, 5 Jul. 1948, leg. Narcisse Leleup, 6 Feb. 1957, leg. G. Verhoustraete, prior to 24 Sep. 1962, leg. L. Poelman (MNHN: ZM-MO-1957-130 A, MHNG: 1046.066a, 1046.066b, RMCA: 26742, 26743, 26851, 26250, 23866, 23816, 22687, 26215, 26781, 26833, 26233, 31209 to 31214); 1 đ [AL], Kalumbu Cave, 1 Jul. 1959, leg. Félix / Michel Anciaux de Faveaux (RMCA: 28318); 5 ?? [5 UN], Kando Cave, unknown date, leg. unknown collector (RMCA: 22062 to 22066); 1 ô [AL], Kandu (Cave), 25 Jul. 1957, leg. Félix / Michel Anciaux de Faveaux (RMCA: 26876); 2 กै $\widehat{~}$ [2 SS], Kipangaribwe River, 3 Jan. 1948, 17 Jan. 1948, leg. unknown collector (RBINS: 10852, 10851); 1 ? [AL], Kiwakishi Cave [= Kiamokoto], between 1 Jul. 1958 and 31 Jul. 1958, leg. Narcisse Leleup (RMCA: 27292); 1, 1 1 [1 SA, 1 UN], Kondo Cave [= Kakanda Cave], 21 Sep. 1960, leg. Félix / Michel Anciaux de Faveaux (SMF: 19571, 19572); 10 +, 7 ðð [4 SA, 10 AL, 3 UN], Kyasala Cave [= Kiasala Cave], 31 Mar. 1956, 28 Jul. 1957, 21 Jul. 1962, leg. Félix / Michel Anciaux de Faveaux, 21 Jul. 1962, leg. unknown collector (MHNG: 1046.099, RBINS: 14447, 14450, SMF: 21273, 21274, MHNG: 1046.067, 1046.068, RMCA: 26784, 26791, 26795, 26824, 26825, 26829, 26835, 26867, 23810, 23853); 3 우 [3 SA], Kyasala Grande Grotte [= Kiasala Cave], 28 Jul. 1957, leg. Félix / Michel Anciaux de Faveaux (MHNG: 1046.069 to 1046.071); 5 ?? [5 AL], Lubudi, small Cave [= Lubudi], 1 Nov. 1948, leg. Narcisse Leleup (RMCA: 22662 to 22666); 1 ? [UN], Marungu, unknown date, leg. unknown collector (NMZL: 3396); 48 ?? [48 AL], M'baya Cave [= Baya Cave], 25 Mar. 1966, leg. Xavier Misonne (RMCA: 34571 to 34618); 1 [SK], Musosa, near, 
between 1 Jun. 1943 and 30 Jun. 1943, leg. unknown collector (RBINS: 14432); 2 우, $1 \hat{\jmath}$ [3 AL], Mwanga Cave, 28 Jun. 1964, leg. Félix / Michel Anciaux de Faveaux (RMCA: 33999 to 34001); 1 juv ㅇ, 4 우, 7 đ̋ [1 SA, 5 SK, 6 UN], Mwela-Pande Cave [= Tenke], 11 Nov. 1959, leg. Félix / Michel Anciaux de Faveaux, 5 Dec. 1959, 6 Dec. 1959, leg. unknown collector (SMF: 19068, RBINS: 14449, 14427, 14430, 14431, 14435, 14439, 14440, 14443 to 14446); 1 ㅇ, 2 ठึ [2 SA, 1 AL], Pempere Cave, 19 Jun. 1957, 11 Jun. 1958, leg. Félix / Michel Anciaux de Faveaux (SMF: 18940, 18941, MHNG: 1046.065); 18 우, 8 $\widehat{\jmath}$ [16 SA, 10 AL], Pierkat Quarry [= Baya Quarry], 12 Mar. 1956, 18 Mar. 1956, 14 May 1956, leg. Félix / Michel Anciaux de Faveaux (RMCA: 23807, 23808, 23827 to 23850); $1 \precsim$ [AL], Swanepoel II Abyss, 1 May 1958, leg. Félix / Michel Anciaux de Faveaux (RMCA: 27539); 6 우, 1 ? [1 AL, 2 SK, 4 UN], Tantara Cave [= Shinkolobwe], unknown date, 4 Jun. 1960, leg. unknown collector, 11 Nov. 1957, leg. Félix / Michel Anciaux de Faveaux (RBINS: 14442, RMCA: 27531, RBINS: 14424 to $14426,14429,14434)$.

\section{Rhinolophus hilli Aellen, 1973}

RWANDA: 1 ad 9 [SA], Routabansougera, 19 Oct. 1981, leg. Frits De Vree, Benny Baeten and Victor Van Cakenberghe (RMCA: 82.006-M-0001); 1 ad + [SA], Uwinka, 25 Aug. 1964, leg. U. Goepel (ZMZ: 126639 [holotype Rhinolophus hilli Aellen, 1973]).

Rhinolophus kahuzi Fahr \& Kerbis Peterhans, 2013

DEMOCRATIC REPUBLIC OF THE CONGO: 1 ad $\lesssim$ [SA], Mount Kahuzi, W slope, 22 Jul. 2007, leg. R. Kizungu (FMNH: 219793 [holotype Rhinolophus kahuzi Fahr \& Kerbis Peterhans, 2013]).

Rhinolophus landeri Martin, 1838

BURUNDI: 1 +, 1 $§$ [1 SA, 1 SK], Resha, 16 Mar. 1979, 30 Mar. 1979, leg. Heinz Stephan (SMF: $57523,57524)$.

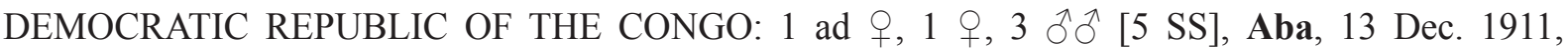
15 Dec. 1911, 17 Dec. 1911, leg. Herbert Lang, James Paul Chapin and The American Museum Congo Expedition (AMNH: 49132, 49173, 49175 [holotype Rhinolophus axillaris Allen, 1917], 49177,

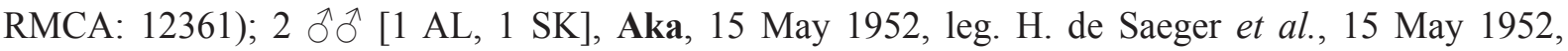
leg. H. de Saeger (RBINS: 13664, RMCA: 36832); 2 ?? [2 AL], Banana, between 1 Jan. 1952 and 31 Jan. 1952, leg. Is. Mesmaekers (RMCA: 20746, 20747); 11 ?? [5 AL, 6 UN], Banana - Boma Road, km 74, 25 km from coast in Forest, 16 Dec. 1951, leg. Is. Mesmaekers (RMCA: 20643 to 20653); 2 우우 [2 AL], Defrenne Cave [= Mwanga Cave], 21 Jun. 1959, leg. Félix / Michel Anciaux de Faveaux (RMCA: 28213, 28214); 1 ㅇ, 1 § [2 SK], Gandajika, 1 Oct. 1948, leg. unknown collector (RBINS: 14505, 14506); 1 ठ [UN], Goroumbwa Mine, 19 May 1999, leg. Robert (Bob) Swanepoel (TM: DRC0141); 2 우, 1 § [3 AL], Jadotville [= Likasi], 8 Jul. 1957, leg. Félix / Michel Anciaux de Faveaux (RMCA: 26722 to 26724); 1 ? [AL], Kabinda, between 1 Jan. 1945 and 31 Dec. 1946, leg. Hautmann (RMCA: 18149); 2 우, 1 ㄱ, 2 ?? [1 SA, 2 AL, 1 SK, 1 UN], Kakanda Cave, 21 Sep. 1960, leg. unknown collector, 21 Sep. 1960, leg. Félix / Michel Anciaux de Faveaux, between 21 Apr. 1955 and 22 Apr. 1955, leg. R.P. Th. de Caters (RBINS: 14504, SMF: 19576, 19577, RMCA: 22886, 22887); 1 ? [AL], Kakanda Mutaka, between 6 Feb. 1954 and 15 Feb. 1954, leg. R.P. Th. de Caters (RMCA: 22380); 3 ?? [3 SA], Kakondo, 13 Aug. 1963, 10 Sep. 1963, leg. P. Kunkel (SMF: 31825, 31828, 31827); 2 ?? [2 AL], Kakontwe, 15 Aug. 1948, leg. Narcisse Leleup (RMCA: 22652, 22653); 5 우우, 9 $\widehat{\jmath}$ [11 AL, 3 UN], Kakontwe Cave, 24 Jun. 1956, 4 Jul. 1956, 8 Jul. 1957, 12 Jul. 1957, between 3 Feb. 1956 and 6 Jul. 1956, leg. Félix / Michel Anciaux de Faveaux (RMCA: 26747 to 26753, 26756, 26773, 23860, 23867, 26877, 26878, 23868); 1 ㅇ, 1 § [2 AL], Kalumbu Cave, 1 Jul. 1959, leg. Félix / Michel Anciaux de Faveaux (RMCA: 28319, 28320); 1 ? [AL], Kaniama, prior to 14 Sep. 1954, leg. 
Vachaudez (RMCA: 22370); $2 \hat{\jmath} \widehat{\partial}$ [2 SK], Kanzenze Mission, 1 Jun. 1948, leg. unknown collector (RBINS: 15455.1, 15455.2); 1 ? [AL], Katanga, unknown date, leg. Félix / Michel Anciaux de Faveaux (MNHN: ZM-MO-1957-131); 2 우 [2 SA], Katanga (former) Province, 18 Dec. 1957, leg. Félix / Michel Anciaux de Faveaux (SMF: 18938, 18939); 1 ? [AL], Kikango, 16 Jan. 1954, leg. H. Bomans (RMCA: 22127); 10 ?? [10 AL], Kisangani, between 1 Jun. 1982 and 30 Jun. 1982, leg. Marc Colyn (RMCA: 82.031-M-0023 to 82.031-M-0032); 1 +, 7 $\hat{\delta}$ [8 AL], Kyasala Cave [= Kiasala Cave], 15 Apr. 1956, 28 Jul. 1957, leg. Félix / Michel Anciaux de Faveaux (RMCA: 23813, 26785, 26802, 26812, 26827, 26843, 26844, 26866); 1 ? [AL], Lake Kivu, near, between 1 Jan. 1934 and 31 Dec. 1937, leg. Guy Babault (MCZ: 46096); 1 ? [AL], Lubondai-Tshimbulu [= Lubondaie-Tshimbulu], between 1 Jan. 1955 and 31 Dec. 1955, leg. S. Marks (RMCA: 26279); 1 \& [SS], Luluabourg [= Kananga], unknown date, leg. Richard Callewaert (BMNH: 1926.7.6.111); 11 우, 9 ふぇ [18 AL, 2 UN], Lusolo Cave [= Lubudi], 28 Jul. 1957, leg. Félix / Michel Anciaux de Faveaux (RMCA: 26793, 26794, 26811, 26814, 26815, 26819, 26821, 26822, 26828, 26830, 26831, 26836 to 26840, 26852, 26853, 26868, 26869); 2 đิ đ̂, 1 ? [2 SA, 1 AL], Lwiro [= Luiro], 13 Jul. 1956, leg. Alvin Novick, 28 Oct. 1965, leg. P. Kunkel (MCZ: 48215, 48216, SMF: 31826); 1 ठ [SK], Maleli, 13 May 1951, leg. H. de Saeger et al. (RBINS: 13663); 2 우, 4 $\widehat{\jmath}$ [5 AL, 1 UN], Mulonga Cave, 30 Jul. 1957, 1 May 1959, leg. Félix / Michel Anciaux de Faveaux (RMCA: 28203, 28204, 26783, 26786, 26800, 26817); 2 우, 7 ठ 6 UN], Mulungwishi, 27 Dec. 1959, leg. unknown collector (RBINS: 14495 to 14503); 2 q , 1 ๙ [2 AL, 1 UN], Mulungwishi, Cave, 28 Jun. 1956, leg. Félix / Michel Anciaux de Faveaux (RMCA: 23815, 23865, 26231); 1 ad+foetus,+ 7 ?? [8 AL], Mwanakusu Cave, 23 Aug. 1959, 28 Aug. 1959, leg. Segers and Benoit Mission, 24 Oct. 1954, leg. Narcisse Leleup (RMCA: 29207, 22724 to 22729, 29208); 2 juv ??, 2 웅, 1 §ै [2 AL, 1 SK, 2 UN], Ndelele, 11 Mar. 1952, leg. H. de Saeger, 31 Jul. 1952, leg. H. de Saeger et al. (RMCA: 36828a, 36828b, 36829a, 36829b, RBINS: 13665); 1 ? [AL], Pawa, unknown date, leg. Reitter (RMCA: 15727); 1 đ [SS], Pelenge, Gorges, 4 Jun. 1947, leg. Gaston-François de Witte (RBINS: 10740); 1 q [SA], Sankuru Province, between 1 Jan. 1952 and 31 Dec. 1952, leg. Félix / Michel Anciaux de Faveaux (MHNG: 1047.080); 1 \& [AL], Swanepoel II Abyss, 1 May 1958, leg. Félix / Michel Anciaux de Faveaux (RMCA: 27540); 1 [AL], Tantara Cave [= Shinkolobwe], 29 Sep. 1957, leg. Félix / Michel Anciaux de Faveaux (RMCA: 26806); 1 ? [AL], Tshakala, between 1 Jan. 1982 and 31 Jan. 1982, leg. Marc Colyn (RMCA: 82.019-M-0012).

RWANDA: 1 ? [UN], Nyakichozi, unknown date, leg. unknown collector (INPBC: V3118.2).

Rhinolophus ruwenzorii J. Eric Hill, 1942

DEMOCRATIC REPUBLIC OF THE CONGO: 1 ad $q$ [SA], Butahu Valley, Cave, 24 Dec. 1926, leg. James Paul Chapin, Dewitt L. Sage, Frank P. Mathews and The Ruwenzori-Kivu Expedition (AMNH: 82394 [holotype Rhinolophus ruwenzorii J.Eric Hill, 1942]); 1 § [SS], Butembo, 16 Aug. 1947, leg. Jean Hiernaux (RBINS: 7047); 4 우, 3 $\lesssim \widehat{~}, 3$ ?? [7 SA, 3 AL], Kibwe Lya Mikako Cave, 28 Dec. 1966, leg. M.J. Celis and M. Lejeune (RMCA: 35170, 35173, 35174, 35206, 35208, 35211, 35216

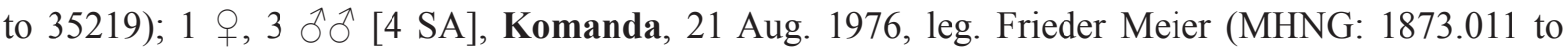

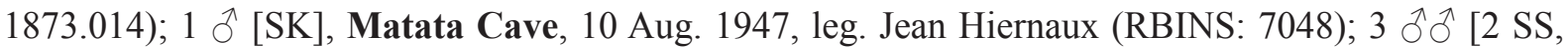
1 SA], Matupi Cave, between 14 Sep. 1959 and 16 Sep. 1959, leg. A.E. Wright and The Cambridge Africa Expedition 1959 (BMNH: 1960.100, 1960.101, 1960.99); 1 o [SK], Mount Hoyo [= Hoyo, Mount], 10 Aug. 1947, leg. Jean Hiernaux (RBINS: 7049); 1 \& [SA], Paoni, 6 Aug. 1976, leg. Frieder Meier (MHNG: 1873.010).

RWANDA: 2 ad $\widehat{\jmath}$ [2 SA], Mutura, 16 Dec. 1982, leg. Benny Baeten and Luc Janssens (RMCA: 85.006-M-0447, 85.006-M-0448). 


\section{Rhinolophus simulator K. Andersen, 1904}

DEMOCRATIC REPUBLIC OF THE CONGO: 2 + $\circ, 18$ ?? [20 AL], Baya Carrière [= Baya Quarry], 17 May 1965, between 1 Dec. 1962 and 31 Dec. 1962, leg. Walter N. Verheyen (RMCA: 34044 to 34054, 35022, 35028, 35042, 35043, 34060 to 34064); 1 क , 1 ऽ [2 SA], Defrenne Cave [= Mwanga Cave], 7 Aug. 1960, leg. Félix / Michel Anciaux de Faveaux (SMF: 19080, 19081); 2 q $q$, 4 đ̋ 0 [6 SA], Kaboyaboya Cave, 18 Jul. 1962, 23 Jul. 1962, leg. Félix / Michel Anciaux de Faveaux (SMF: 21253 to 21258); 1 đ [SA], Kalomoni Cave [= Kakontwe], 11 Jun. 1960, leg. Félix / Michel Anciaux de Faveaux (SMF: 19079); 4 우, 4 $\widehat{o}$ [ [8 SA], Kasoma Cave, 13 Aug. 1962, leg. Félix / Michel Anciaux de Faveaux (SMF: 21265 to 21272); 1 § [SA], Lubudi, 23 Jul. 1962, leg. Félix / Michel Anciaux de Faveaux (SMF: 21259); 5 우, 1 ô [6 SA], Lusolo Cave [= Lubudi], 16 Jul. 1962, leg. Félix / Michel Anciaux de Faveaux (SMF: 21247 to 21252); 3 우, 2 ô 0 [5 SA], Mulong Cave [= Mulonga Cave], 26 Jul. 1962, leg. Félix / Michel Anciaux de Faveaux (SMF: 21260 to 21264); 2 우 [2 AL], Mwanga Cave, 28 Jun. 1964, leg. Félix / Michel Anciaux de Faveaux (RMCA: 33997, 33998); 1 đ [SA], Trou aux Serpents [= Shinkolobwe], 30 Jun. 1962, leg. Félix / Michel Anciaux de Faveaux (SMF: 21246).

\section{Rhinolophus swinnyi Gough, 1908}

DEMOCRATIC REPUBLIC OF THE CONGO: 1 ad $\curvearrowright, 1 \curvearrowright$ [1 SA, 1 AL], Dethioux Hoeve, Grot I [= Shinkolobwe], 29 Sep. 1957, leg. Félix / Michel Anciaux de Faveaux (MHNG: 1046.063, 1046.064); 1 †, 1 đ [2 UN], Jadotville [= Likasi], 26 Jul. 1962, leg. unknown collector (RBINS: 14478, 14479);

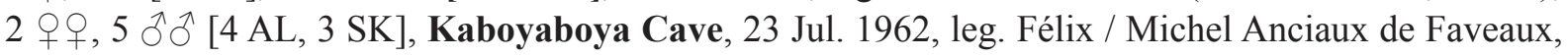
23 Jul. 1962, leg. unknown collector (MHNG: 1047.059 to 1047.062, RBINS: 14481, 14482, 14485); 5 우 [2 SA, 3 AL], Kakontwe Cave, 8 Jul. 1957, 12 Jul. 1957, leg. Félix / Michel Anciaux de Faveaux (MHNG: 1046.048, 1046.049, 1046.045 to 1046.047); 1 ? [UN], Kando Cave, unknown date, leg. unknown collector (MHNG: 1046.005); 1 đ [AL], Kandu (Cave), 25 Jul. 1957, leg. Félix / Michel Anciaux de Faveaux (MHNG: 1046.050); 1 +, 1 đ [2 SA], Kasoma Cave, 13 Aug. 1962, leg. Félix / Michel Anciaux de Faveaux (HNHM: 95.50.1, 95.50.2); 1 ㅇ [AL], Kyamakonde Cave, 18 Jul. 1962, leg. Félix / Michel Anciaux de Faveaux (MHNG: 1047.058); 1 ○ [AL], Kyantapo Cave, 29 Jul. 1957, leg. Félix / Michel Anciaux de Faveaux (MHNG: 1046.059); 1 [SA], Kyasala Cave [= Kiasala Cave], 15 Apr. 1956, leg. Félix / Michel Anciaux de Faveaux (MHNG: 922.086); 2 우 [2 AL], Kyasala Grande Grotte [= Kiasala Cave], 28 Jul. 1957, leg. Félix / Michel Anciaux de Faveaux (MHNG:

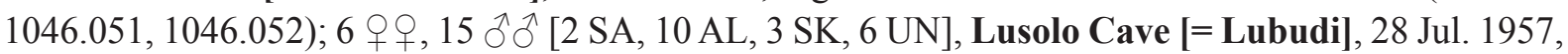
16 Jul. 1962, leg. Félix / Michel Anciaux de Faveaux, 16 Jul. 1962, leg. unknown collector (MHNG: 1047.052 to 1047.057 , RBINS: 14473 to $14475,14477,14480,14483,14484,14487,14488$, MHNG: 1046.053 to 1046.058); 1 ㅇ, 2 ๙ $\widehat{\jmath}, 1$ ? [3 AL, 1 UN], Mulonga Cave, 30 Jul. 1957, leg. Félix / Michel Anciaux de Faveaux (MHNG: 1046.006, 1046.060 to 1046.062); 1 + [UN], Mulungwishi, 27 Dec. 1959, leg. unknown collector (RBINS: 14476); 1 \& [SA], Pempere Cave, 19 Jun. 1957, leg. Félix / Michel Anciaux de Faveaux (MHNG: 1046.044); 2 우 [1 SK, 1 UN], Salomoni Cave, 11 Jun. 1960, 20 May 1962, leg. unknown collector (RBINS: 14472, 14486).

\section{Rhinolophus willardi Kerbis Peterhans \& Fahr, 2013}

DEMOCRATIC REPUBLIC OF THE CONGO: 3 ad $q$ 우 [3 SB], Kabobo Forest, 14 Feb. 2007, leg. B.D. Marks, 16 Feb. 2007, leg. Prince Kiswele Kaleme (FMNH: 195082 [paratype Rhinolophus willardi Kerbis Peterhans and Fahr, 2013], 195083 [paratype Rhinolophus willardi Kerbis Peterhans and

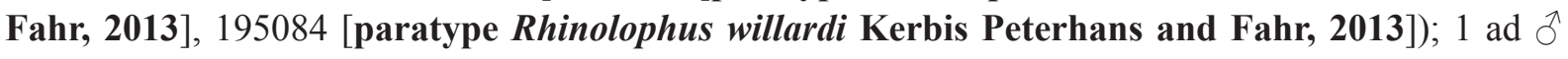
[SA], Misotschi-Kabogo Highlands, 28 Feb. 2007, leg. A.J. Plumptre and E.A. Mulungu (FMNH: 195182 [holotype Rhinolophus willardi Kerbis Peterhans and Fahr, 2013]). 


\title{
Rhinonycteridae Gray, 1866
}

Cloeotis Thomas, 1901

Cloeotis percivali Thomas, 1901

DEMOCRATIC REPUBLIC OF THE CONGO: 1 ad $q, 1$ ad $\lesssim[2$ AL], Dethioux Farm, Cave I [= Shinkolobwe], 15 Jun. 1958, leg. Félix / Michel Anciaux de Faveaux (RMCA: 27907, 27908); 1 , 1 ○े [2 SA], Dethiox Caves [= Kambove], 4 Jun. 1960, leg. Félix / Michel Anciaux de Faveaux (SMF: 18920, 18921); 1 ठ [UN], Elisabethville [= Lubumbashi], 25 Mar. 1966, leg. unknown collector (RBINS: 17208); $1 \partial^{\lambda}$ [AL], Kaboyaboya Cave, 23 Jul. 1962, leg. Félix / Michel Anciaux de Faveaux (MHNG: 1046.098); 1 đ̊ [SA], Kasoma Cave, 13 Aug. 1962, leg. Félix / Michel Anciaux de Faveaux

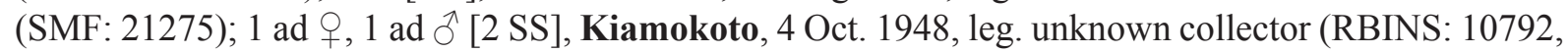
10793); 1 ㅇ, 1 § [1 SA, 1 AL], Lubudi, 23 Jul. 1962, leg. Félix / Michel Anciaux de Faveaux (SMF: 21276, 21277); 5 ad + +, 1 ㄱ, 2 ?? [5 SS, 1 SK, 2 UN], Masombwe, unknown date, 10 Oct. 1948, 19 Oct. 1948, leg. unknown collector (RBINS: 10802, 10803, 10777, 10789, 10794, 10796, 10797, 10800).

\author{
Vespertilioniformi Hutcheon \& Kirsch, 2006 \\ Emballonuridae Gervais, 1855 \\ Coleura Peters, 1868
}

Coleura afra (Peters, 1852)

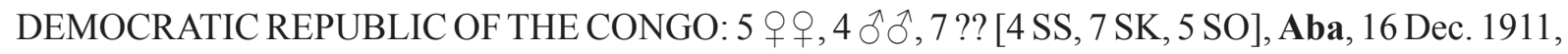
leg. Herbert Lang, James Paul Chapin and The American Museum Congo Expedition, 16 Dec. 1911, leg. unknown collector (AMNH: 48809, 48811 to 48815, 49033, 51250 to 51256, MCZ: 17369, RMCA: 12394); 3 우, 1 J , 4 ?? [8 AL], Cape Cassa Mabongo [= Kasambongo], unknown date, leg. the Belgian Hydrobiological Expedition to Tanganyika (BMNH: 1953.10 to 1953.12, 1953.5 to 1953.9); 6 우, $3 \widehat{\jmath} \widehat{\partial}[9 \mathrm{UN}$ ], Tanganyika, Congo shore of Lake, between 1 Jan. 1946 and 31 Dec. 1947, leg. unknown collector (RBINS: 9062.1 to 9062.9); 1 +, 1 ô, 3 ?? [4 SS, 1 AL], Wago, Mount, 7 Nov. 1951, between 1 Nov. 1951 and 30 Nov. 1951, between 1 Jul. 1951 and 31 Jul. 1951, between 1 Jan. 1951 and 31 Dec. 1951, leg. Alexandre Fain (RMCA: 23282, 21466, 23291, 23292, 23283).

Saccolaimus Temminck, 1838

Saccolaimus peli (Temminck, 1853)

DEMOCRATIC REPUBLIC OF THE CONGO: 3 우, 1 ô, 2 ?? [5 SS, 1 AL], Avakubi, 18 Nov. 1909, 17 Jan. 1914, 15 Jan. 1915, leg. Herbert Lang, James Paul Chapin and The American Museum Congo Expedition, 16 Jan. 1914, leg. J. Bequaert, between 17 Jan. 1914 and 17 Dec. 1914, leg. unknown collector (RMCA: 12377, 3420, AMNH: 48772, 49350, 48757, MCZ: 17366); 1 ¿ [SS], Bafwaboka [= Bafwabaka], 28 Dec. 1909, leg. Herbert Lang, James Paul Chapin and The American Museum Congo Expedition (AMNH: 48761); 1 ? [SO], Beni, prior to 24 Jul. 1939, leg. Lisfranc (RMCA: 15404); 1 ? [AL], Beno, prior to 3 Jan. 1950, leg. P. Bayet (RMCA: 19472); 1 juv ? [AL], Bokoro, prior to 19 Nov. 1952, leg. E. Jans (RMCA: 21521); 1 ? [UN], Djeka, prior to 15 Jun. 1956, leg. Roiseux (RMCA: 23640); 2 $\widehat{\partial}$ [2 SO], Ikela, between 1 Jan. 1946 and 31 Dec. 1946, leg. L. Dupuis (RMCA: 17907, 17908); 1 [SS], Inkongo [= Inkongo-Kakese], unknown date, leg. H. Wilson (BMNH: 1916.5.15.5); 2 ?? [2 AL], Irumu, 28 Sep. 1926, leg. James Paul Chapin, Dewitt L. Sage, Frank P. Mathews and The Ruwenzori-Kivu Expedition (AMNH: 82363, 82364); 2 ?? [1 SS, 1 SO], Kikwit, between 1 Nov. 1922 and 30 Nov. 1922, leg. J. Rodhain (RMCA: 14913, 14914); 1 ad 9 [SS], Kinawa Passage, 26 Sep. 1953, leg. unknown collector (RBINS: 21460); 1 q [SS], Kisiki, 22 Dec. 1938, leg. Duncan M. Hodgson and William F. Coultas (MCZ: 38027); 1 q [SS], Kunungu, prior to 25 Mar. 1929, leg. Ngwe (RMCA: 9708); 
2 ?? [2 SS], Lesse, 4 Nov. 1913, prior to 2 Jun. 1914, leg. Bonnevie (RMCA: 3241, 3242); 1 embryo ?, 4 우우 [1 SS, 2 SA, 2 AL], Lukolela, 28 Jul. 1930, 3 Aug. 1930, 4 Sep. 1930, leg. James Paul Chapin, 4 Sep. 1930, leg. Franklin Edson (AMNH: 86904, 86902, 86768, 86903, 86915); 1 ㅇ [SS], Luluabourg [= Kananga], unknown date, leg. Richard Callewaert (BMNH: 1926.7.6.98); 2 우 ᄋ, 2 o $\widehat{\text { ô }}$ [1 SS, $3 \mathrm{UN}$ ], Medje, 14 Jan. 1910, 15 Jan. 1910, 18 Jan. 1910, 19 Jan. 1910, leg. Herbert Lang, James Paul Chapin and The American Museum Congo Expedition (AMNH: 48762, 48763, RMCA: 12378, AMNH: 48765);

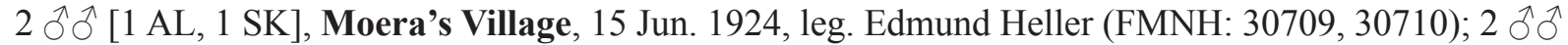
[2 SK], Musenge, 23 Mar. 1924, 24 Mar. 1924, leg. Edmund Heller (FMNH: 30707, 30708); 2 우, 1 ठૈ, 2 ?? [2 SS, 1 SA, 2 AL], N'Gayu area, 18 Dec. 1909, leg. Herbert Lang, James Paul Chapin and The American Museum Congo Expedition (AMNH: 48758 to 48760, 49281, 49282); 3 우, 2 ठ઼ Niangara, 27 May 1913, 31 May 1913, leg. Herbert Lang, James Paul Chapin and The American Museum Congo Expedition, 31 May 1913, leg. unknown collector (AMNH: 48766, 48768, 48769, 48767, MCZ: 17365); 1 ? [AL], Omaniumdu, 27 Jul. 1959, leg. Laurent (RMCA: 29535); 1 ? [AL], Panga, between 1 Sep. 1925 and 30 Sep. 1925, leg. Henri Schouteden (RMCA: 16258); 3 ?? [2 AL, 1 UN], Pawa, prior to 23 Jan. 1939, leg. Degotte (RMCA: 14990 to 14992); 1 ㅇ [SS], Rungu, 28 Jul. 1913, leg. Herbert Lang, James Paul Chapin and The American Museum Congo Expedition (AMNH: 48770); 1 ? [SO], St. Jos. (Misson) [= Mikalaya], unknown date, leg. Richard Callewaert (AMNH: 86261); 1 đ [SS], St. Joseph de Luluabourg Mission [= Mikalaya], 18 Jul. 1922, leg. Richard Callewaert (RMCA: 7541); 2 ?? [2 AL], Sturi Forest [= Ituri Forest], unknown date, leg. The Ruwenzori Expedition (BMNH:

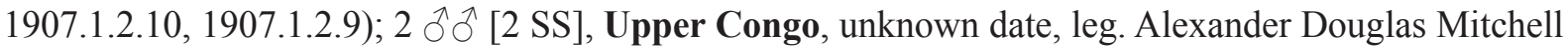
Carruthers (BMNH: 1907.6.14.1, 1907.6.14.2); 1 § [AL], Walikale, 28 Feb. 1924, leg. Edmund Heller

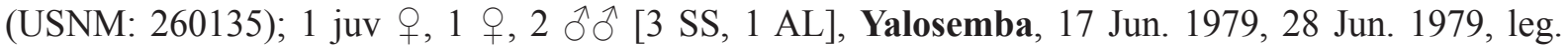
C. Brian Robbins, 30 Jun. 1979, leg. Lynn W. Robbins (USNM: 537702, 463476, 537701, TTU: 36355); 1 ? [SO], Yokamba Elongo, 27 Jan. 1952, leg. Stevenart (RMCA: 21129).

\section{Taphozous E. Geoffroy St.-Hilaire, 1818}

Taphozous mauritianus E. Geoffroy St.-Hilaire, 1818

BURUNDI: 1 ad,+ 1 [2 SK], Bujumbura, 7 km NW, 21 Mar. 1979, leg. Heinz Stephan (SMF: 57544, 57545); 1 ? [AL], Russissi, bank [= Rusizi Delta], 27 Apr. 1982, leg. Danny Meirte (RMCA: 82.016-M-0148).

DEMOCRATIC REPUBLIC OF THE CONGO: 1 ô, 2 ?? [1 SS, 2 AL], unknown locality, unknown date, leg. unknown collector, prior to 4 Jul. 1958, leg. E. Jans (BMNH: 1906.7.1.18, RMCA: 27373, 27374); 1 ? [UN], Aba, 12 Apr. 1948, leg. unknown collector (RBINS: 8188); 2 q , , 1 ô, 1 ? [4 AL], Avakubi, 3 Nov. 1911, 10 Dec. 1913, 10 Dec. 1914, leg. Herbert Lang, James Paul Chapin and The American Museum Congo Expedition (AMNH: 49349, MCZ: 17227, AMNH: 49367, 49366); 1 ad $q$,

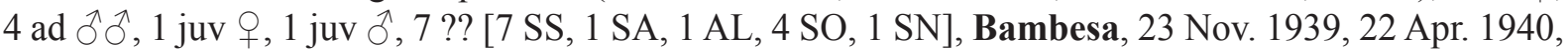
24 Aug. 1940, 27 Aug. 1940, 29 Apr. 1941, 2 May 1941, 14 May 1941, between 1 Jun. 1937 and 30 Jun. 1937, leg. J.M. Vrijdagh (RMCA: 18104, 17768, 17763, 17765, 16106 to $16108,17749,17750$, 17764, 17751, 14072, 14074, 14079); 5 ?? [5 AL], Banana, between 1 May 1952 and 31 May 1952, leg. Is. Mesmaekers, prior to 28 Feb. 1899, leg. Weyns (RMCA: 21372, 21373, 148, 148a, 148b); 1 ? [SS], Baudouinville [= Moba], 5 May 1948, leg. Jozef Hutsebaut (RMCA: 18567); 1 ô, 1 ? [2 SS], Baye via Bondo, 13 Dec. 1956, leg. Rooyakkers (RMCA: 25861, 25862); 1 ? [AL], Beno, prior to 3 Jan. 1950, leg. P. Bayet (RMCA: 19473); 1 ? [AL], Binga, prior to 27 Feb. 1935, leg. Van den Put (RMCA: 12678); 1 ? [SS], Blukwa, 9 Jul. 1952, leg. unknown collector (RBINS: 21664); 1 ? [AL], Boende, between 1 Feb. 1970 and 28 Feb. 1970, leg. P. Lootens (RMCA: 75.035-M-0020); 4 ?? [4 AL], Bokuma, 30 Jan. 1952, prior to 5 Mar. 1953, prior to 3 Feb. 1955, leg. P. Lootens (RMCA: 21396, 23246, 23247, 21682); 2 웅, 5 ?? [5 SS, 2 SO], Bokungu, 14 Jun. 1949, 19 Jan. 1952, 28 Apr. 1952, prior to 22 Oct. 1949, prior to 13 Oct. 1950, leg. L. Dupuis (RMCA: 18845, 20847, 20848, 20846, 20084, 18929, 
18930); 1 ㅇ [SS], Bolobo, prior to 23 Jul. 1946, leg. N'Kele (RMCA: 17402); 1 [UN], Bomane [= Bomaneh], 22 May 2010, leg. Guy-Crispin Gembu Tungaluna, Prescott Musaba and José Akaibe (UNIKIS: CRT2227); 1 ô, 12 ?? [6 SS, 5 AL, 2 SO], Buta, unknown date, 12 Sep. 1939, 12 Mar. 1945, 20 Jun. 1949, prior to 31 May 1939, prior to 29 Mar. 1940, prior to 28 Dec. 1938, prior to 24 Jan. 1949, prior to 16 Jun. 1937, prior to 1 Apr. 1932, between 1 Dec. 1927 and 31 Dec. 1927, leg. Jozef Hutsebaut, 29 Jan. 1925, leg. Henri Schouteden (RMCA: 14565, 16282, 17303, 15777, 18842, 8929, MCZ: 23934, RMCA: 11590, 13855, 19079, 14947, 15954, 15324); 1 ? [AL], Butembo, prior to 26 May 1959, leg.

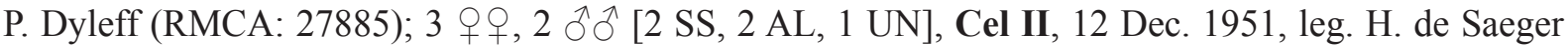
et al., 14 Mar. 1951, 26 Nov. 1951, 12 Dec. 1951, leg. H. de Saeger (RBINS: 13533, RMCA: 36841, 36840, 36838, 36839); 2 우, 1 ㄱ, 1 ? [1 SS, 3 AL], Elisabethville [= Lubumbashi], 10 Mar. 1956, leg. Félix / Michel Anciaux de Faveaux, 14 Feb. 1956, leg. service de l'hygiene publique, 14 Apr. 1930, leg. J. De Riemaecker (MHNG: 922.100, RMCA: 23824, 26281, 19417); 2 juv $ᄋ$, , 2 juv $\widehat{\jmath}, 1$ juv ?,

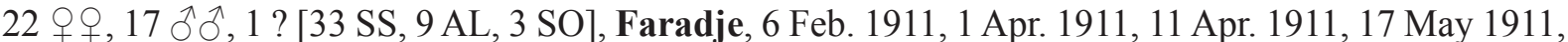
20 May 1911, 28 May 1911, 7 Jun. 1911, 11 Jul. 1911, 5 Sep. 1911, 26 Oct. 1911, 22 Nov. 1911, 24 Nov. 1911, 6 Mar. 1912, 21 May 1912, 22 Oct. 1912, 25 Oct. 1912, 12 Nov. 1912, 13 Nov. 1912, 28 Nov. 1912, 3 Dec. 1912, 5 Dec. 1912, 7 Dec. 1912, 15 Dec. 1912, 16 Dec. 1912, 5 Jan. 1913, 5 Oct. 1913, 14 Oct. 1913, 5 Nov. 1913, between 5 Jan. 1913 and 5 Dec. 1913, leg. Herbert Lang, James Paul Chapin and The American Museum Congo Expedition (AMNH: 48786, 48787, 48798, 48779, 48793, 48806, 48794, 49355, 48783, 48796, 48808, MCZ: 17367, AMNH: 48788, 48774, 48775, 48789, 48797, MCZ: 17368, AMNH: 49362 to $49364,48804,48801$ to 48803, 48776 to 48778, 48805, 48800, 48790, 48795, 48780, 49360, 48799, 49353, 49361, 48781, 48782, RMCA: 12383, AMNH: 49352, 48791, RMCA: 12384, AMNH: 48807, MCZ: 17228); 1 +, 1 § [2 UN], Fataki, between 1 Jan. 1948 and 6 Apr. 1964, leg. unknown collector (RBINS: 18530.1, 18530.2); 2 + + , 1 ऽ [3 SO], Gangala [= Gangala-na-Bodio], 24 Mar. 1957, 27 Mar. 1957, leg. Heinz Stephan (RMCA: 27461, 27463,

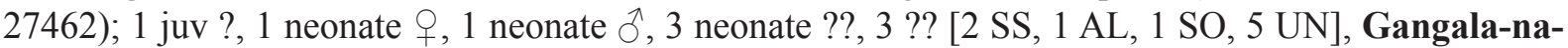
Bodio, unknown date, 2 Jan. 1947, 9 Apr. 1948, 5 May 1948, leg. unknown collector, 14 Nov. 1956, between 1 Nov. 1956 and 30 Nov. 1956, leg. Max Poll (RBINS: 12942, RMCA: 25601, RBINS: 12941, 8190, 11655, 8189.1, 8189.2, RMCA: 25600, 25806); 1 ? [AL], Ibambi, 11 Nov. 1938, leg. Degotte (RMCA: 15215); 7 ?? [7 AL], Ibembo, 20 Sep. 1949, prior to 17 Jul. 1952, prior to 15 Dec. 1950, leg. Jozef Hutsebaut (RMCA: 19462, 20213 to 20217, 21277); 2 ?? [2 AL], Ikela, prior to 4 Jan. 1958, leg. P. Lootens (RMCA: 27018, 27019); 1 ठै [SS], Inkongo [= Inkongo-Kakese], 2 Feb. 1923, leg. H. Wilson (RMCA: 6312); 1 q [SS], Kabwe, 12 Nov. 1973, leg. Chris E. Heidenreich (WNMU: 2944); 2 ?? [2 AL], Kakanda Cave, between 21 Apr. 1954 and 22 Apr. 1954, leg. R.P. Th. de Caters (RMCA: 22898,

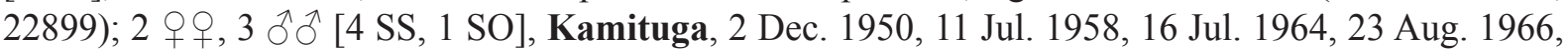
11 Jul. 1974, leg. Alexandre Prigogine (RMCA: 27729, 76.022-M-0024, 32800, 20466, 34803); 1 우 [AL], Kapolowe, 16 Apr. 1956, leg. Félix / Michel Anciaux de Faveaux (RMCA: 23826); 1 ô [AL], Kapolowe Mission [= Kapolowe], 16 Apr. 1956, leg. Félix / Michel Anciaux de Faveaux (RMCA: 23919); 1 q [AL], Kasengi [= Kasengu], 22 Aug. 1926, leg. James Paul Chapin, Dewitt L. Sage, Frank P. Mathews and The Ruwenzori-Kivu Expedition (AMNH: 82382); 1 đ [AL], Katana, unknown date,

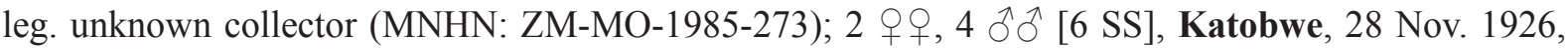
1 Dec. 1926, 7 Dec. 1926, leg. John Todd Zimmer (FMNH: 29411, 29412, 29410, 29413 to 29415); 4 우, 1 ○ [2 SS, 3 SO], Kilwezi, 9 Aug. 1948, 20 Aug. 1948, leg. unknown collector (RBINS: 10857, 10853 to 10856); 1 đิ [SS], Koteli [= Kotili], 23 Jan. 1925, leg. Henri Schouteden (RMCA: 8920); 1 ภ [SS], Kunungu, prior to 22 Oct. 1930, leg. N'Kele (RMCA: 10617); 2 ?? [2 SS], Leverville [= Lusanga], between 1 Feb. 1929 and 28 Feb. 1929, leg. Tinant (RMCA: 9990, 9991); 1 ? [AL], LubondaiTshimbulu [= Lubondaie-Tshimbulu], prior to 31 Jan. 1958, leg. S. Marks (RMCA: 26928); 1 ? [AL], Lubumbashi, unknown date, leg. J. Bequaert (RMCA: 7699); 1 ? [AL], Lubutu, 16 Sep. 1985, leg. Marc Colyn (RMCA: 86.024-M-0005); 1 ㅇ [SS], Lulenga [= Rugari], 28 Nov. 1925, leg. Henri Schouteden (RMCA: 9153); 4 우, 1 $\widehat{\jmath}, 1$ ? [4 SS, 1 SA, 1 AL], Luluabourg [= Kananga], unknown date, leg. Richard Callewaert, 16 Nov. 1965, leg. Antoon Emeric Marcel De Roo, between 1 Jan. 1932 
and 31 Dec. 1932, leg. Jean Romieux, prior to 17 Apr. 1913, leg. Cambier (BMNH: 1926.11.1.24, 1926.11.1.25, 1926.7.6.97, RMCA: 33981, MHNG: 850.045, RMCA: 1549); 1 ? [SS], Luputa, prior to 22 Jun. 1933, leg. Bouvier (RMCA: 12131); 1 đ [SO], Lusambo, unknown date, leg. J. Ghesquière (RMCA: 7333); 1 ? [SO], Macaco, 21 Sep. 1921, leg. Henri Schouteden (RMCA: 6553); 1 ad 9 [SS], Mahagi, 20 Apr. 1942, leg. J.M. Vrijdagh (RMCA: 17747); 1 ? [AL], Makaw, prior to 15 Nov. 1958, leg. E. Jans (RMCA: 27498); 1 ? [AL], Makengo, 11 Dec. 1946, leg. Dartevelle (RMCA: 18162); 1 ? [AL], Masako, unknown date, leg. Ndey B. Ifuta (RMCA: 93.079-M-0355); 1 ad $\lesssim$ [SO], Mondombe, 1 Jan. 1953, leg. L. Dupuis (RMCA: 21716); 1 क [UN], Mpaza, 22 Jan. 1952, leg. H. de Saeger et al. (RBINS: 13536); 1 q [SO], Murambi, 23 May 1949, leg. unknown collector (RBINS: 12940); 3 우, 1 J, 1 ? [3 SS, 1 AL, 1 SO], Mutsora, 30 Dec. 1946, 20 Feb. 1947, 16 Jun. 1951, 11 Jul. 1952, leg. unknown collector, 2 Aug. 1954, leg. Gaston-François de Witte (RBINS: 17294, 12938, RMCA: 37162,

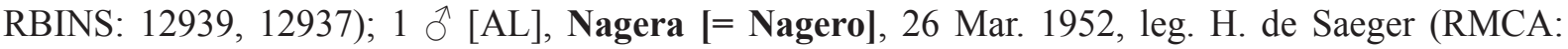
36842); 3 $\widehat{\partial}$ [2 SO, 1 UN], Nagero, 1 Dec. 1953, 31 Dec. 1953, leg. unknown collector, 27 Dec. 1951, leg. H. de Saeger et al. (RBINS: 14627, 13534, 14628); 2 우 [1 SS, 1 SO], Niangara, 18 Nov. 1910, 25 Nov. 1910, leg. Herbert Lang, James Paul Chapin and The American Museum Congo Expedition (AMNH: 48773, 48784); 1 juv ? [AL], Niarembe, between 1 Jun. 1925 and 30 Jun. 1925, leg. Henri Schouteden (RMCA: 16251); 1 ? [AL], Nyangwe, 6 Apr. 1920, leg. J. Ghesquière (RMCA: 16247); 1 त [SS], Pania-Mutombo [= Pania-Mutombo], 20 Jun. 1934, leg. R.S. Pierrepont (MCZ: 31736); 2 우, 1 đ [3 UN], Parc National de Garamba [= Garamba National Park], 23 Jul. 1951, 8 May 1952, leg.

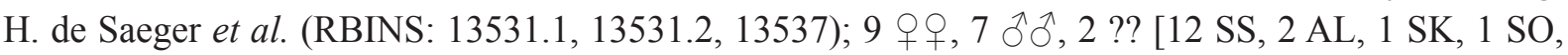
2 UN], Rutshuru, unknown date, 10 Mar. 1938, leg. unknown collector, 31 Jul. 1925, 1 Aug. 1925, 3 Aug. 1925, leg. Edmund Heller, 3 Jan. 1938, 20 Jan. 1938, 3 Mar. 1938, 4 Mar. 1938, 5 Mar. 1938, 24 Mar. 1938, between 1 Mar. 1938 and 31 Mar. 1938, between 1 Feb. 1938 and 28 Feb. 1938, leg. J. Ghesquière, between 1 Mar. 1938 and 31 Mar. 1938, leg. Serge Frechkop (INPBC: V1347, V375, FMNH: 26498, RBINS: 4770, RMCA: 14542, 14607, 14608, 14444, 14595, FMNH: 30725, 30726, 30724, RMCA: 14593, 14594, 14592, 14596, 14606, 16745); 1 ठ [SS], St. Jos. (Mission) [= Mikalaya], 29 Jul. 1924, leg. Richard Callewaert (AMNH: 86171); 2 우, 1 ○ [1 SS, 2 SO], St. Joseph de Luluabourg Mission [= Mikalaya], 2 May 1939, leg. Jean Jacques Deheyn, 6 Jun. 1922, leg. Richard Callewaert (RMCA: 15371, 15372, 7551); 2 우, 1 ○, 1 ? [2 SS, 1 AL, 1 SO], Stanleyville [= Kisangani], 5 Jul. 1912, leg. Cuthbert Christy, between 1 Mar. 1929 and 31 Mar. 1929, leg. Collart, between 1 Apr. 1924 and 30 Apr. 1924, leg. J. Ghesquière (RMCA: 1430, 16246, 9215, 9216); 1 juv $\widehat{~}, 1$, 2 ๙ to 6552, 6554); $1 \lesssim$ [SS], Upper Congo, unknown date, leg. Alexander Douglas Mitchell Carruthers (BMNH: 1907.6.14.3); 9 ?? [9 AL], Wafanya [=Wafania], unknown date, 17 Sep. 1991, 20 Mar. 1992, 15 Apr. 1992, between 1 Jan. 1992 and 31 Jan. 1992, leg. P. Lootens (RMCA: 92.079-M-0119 to 92.079M-0122, 92.079-M-0151，92.079-M-0002，91.076-M-0086，92.079-M-0001，92.079-M-0003); 1 ? [AL], Yakuluku, 3 Nov. 1911, leg. Herbert Lang, James Paul Chapin and The American Museum Congo

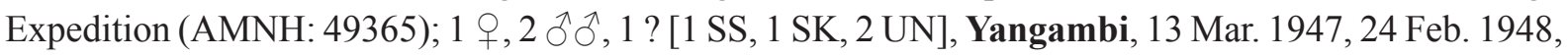
leg. unknown collector, 5 Aug. 1942, leg. J.M. Vrijdagh (RBINS: 14464, 14466, 6986, RMCA: 17748);

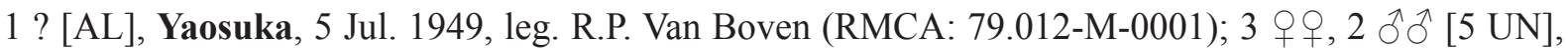
Zobia, prior to 18 Apr. 1946, leg. unknown collector (RBINS: 14463, 14465, 14467 to 14469).

RWANDA: 1 ? [SA], Astrida [= Butare], between 1 Jan. 1948 and 31 Dec. 1948, leg. Alexandre Fain (RMCA: 18992); 3 ?? [3 SS], Gabiro, 16 Jan. 1938, leg. unknown collector, 16 Jan. 1938, leg. Serge Frechkop (RBINS: 4769, RMCA: 17074, 17075); 1 ? [UN], Kagera National Park [= Akagera Park], prior to 31 Dec. 1943, leg. unknown collector (RBINS: 4771). 
VAN CAKENBERGHE V. et al., The bats of Congo, Rwanda and Burundi revisited

Taphozous nudiventris Cretzschmar, 1830

DEMOCRATIC REPUBLIC OF THE CONGO: 1 q [UN], Cel II, 8 Mar. 1952, leg. H. de Saeger et al. (RBINS: 13542).

\section{Taphozous perforatus E. Geoffroy St.-Hilaire, 1818}

DEMOCRATIC REPUBLIC OF THE CONGO: 5 ?? [5 AL], unknown locality, prior to 14 Sep. 1957, leg. Félix / Michel Anciaux de Faveaux (RMCA: 27919 to 27923); 3 우 [3 UN], Bamangwa [= Bamungwa [River]], 23 Feb. 1951, leg. H. de Saeger et al. (RBINS: 13539.1 to 13539.3); 2 우 [2 AL], Bamongwa [= Bamungwa [River]], 23 Feb. 1951, leg. H. de Saeger (RMCA: 36843, 36844);

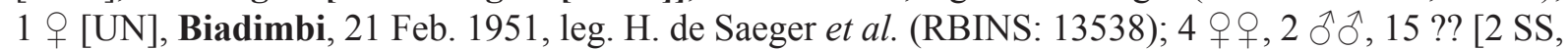
17 AL, 2 UN], Defrenne Cave [= Mwanga Cave], 19 Jun. 1957, 31 Aug. 1958, 25 Aug. 1962, leg. Félix / Michel Anciaux de Faveaux, 19 Sep. 1948, leg. Narcisse Leleup (RMCA: 26707 to 26710, 22487, 22488, 22602 to 22614, MHNG: 1046.096, RMCA: 27898); 6 우, 2 ふ઼े, 4 ?? [3 SS, 7 AL, 2 SK], Dungu, 1 Mar. 1913, leg. Herbert Lang, James Paul Chapin and The American Museum Congo Expedition, 1 May 1913, leg. Herbert Lang (AMNH: 48816, 48817, 49251, 49348, 49354, 49356 to 49358, MCZ: 17225, RMCA: 12395, FMNH: 43874, 43875); 1 ô [SS], Gangala-na-Bodio, 8 Apr. 1945, leg. unknown collector (RBINS: 14631); 1 ? [AL], Kasongo, 25 Jul. 1959, leg. Segers and Benoit Mission (RMCA: 29209); 5 đ̊ [5 AL], Kasowena Cave, 28 Jun. 1964, leg. Félix / Michel Anciaux de Faveaux (RMCA: 34002 to 34006); 2 우, 2 ๙ิ $\widehat{~}, 3$ ?? [1 SS, 1 AL, 1 SK, 1 SO, 3 UN], Ndelele, 3 Mar. 1952, 11 Mar. 1952, leg. H. de Saeger et al., 2 Mar. 1952, 11 Mar. 1952, leg. H. de Saeger (RBINS: 13535, 13540.1, 13540.2, RMCA: 36845 to 36847, RBINS: 13541); 9 우, 1 ऽ [4 SA, 5 AL, 1 UN], Pempere Cave, 19 Jun. 1957, 11 Jun. 1958, 25 Aug. 1962, leg. Félix / Michel Anciaux de Faveaux, 25 Aug. 1962, leg. unknown collector (RMCA: 27587 to 27590, SMF: 18944, 18945, MHNG: 1046.030, 1046.031, RBINS: 14512, SMF: 21298); 1 +1 ○ [2 AL], Pimpin Cave [= Pempere Cave], unknown date, leg. the Royal Museum for Central Africa (RMCA) (BMNH: 1959.506, 1959.507).

Nycteridae Van der Hoeven, 1855

Nycteris G. Cuvier \& E. Geoffroy, 1795

DEMOCRATIC REPUBLIC OF THE CONGO: 1 ad + , 4 ad $\widehat{\jmath} \widehat{\partial}$ [3 SS, 2 SO], unknown locality, 29 Nov. 1955, prior to 29 Nov. 1955, between 21 Nov. 1955 and 29 Nov. 1955, leg. Alexandre Fain (RMCA: 23305, 23306, 23304, 23302, 23303); 1 ? [AL], Bamanya, between 1 Jan. 1974 and 31 Dec. 1974, leg. R.P. Hulsaert (RMCA: 75.012-M-0001); 1 ? [AL], Gumba, 10 Oct. 1937, leg. Jean Jacques Deheyn (RMCA: 14263b); 3 $\widehat{\partial}$ [3 SN], Kateke [= Kateke [River]], 4 Dec. 1947, leg. unknown collector (RBINS: 10687 to 10689); 2 ad ?? [2 SO], Keyberg, 20 Jan. 1952, leg. Uwe Rahm (RMCA: 28897, 28898); 1 q [SS], Luberezi [= Luberizi], 9 Mar. 1971, leg. Alexandre Prigogine

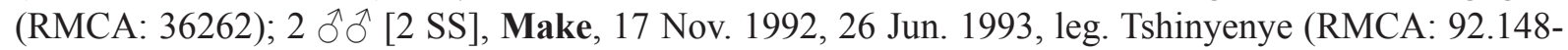
M-0001, 93.097-M-0023); 1 ㅇ [SK], Musisi swamp area, 21 Jul. 2005, leg. Project Beatra (FMNH: 189123); 1 ? [AL], Nioka, 19 May 1953, leg. Célestin Hecq (RMCA: 21899b); 1 ? [UN], Rutshuru, unknown date, leg. unknown collector (INPBC: W3.1); 1 ad + [SA], Vieux Beni, 24 Aug. 1953, leg. Gaston-François de Witte (RMCA: 37128); 1 juv $\widehat{\jmath}, 2$ ?? [1 SS, 2 SO], Vumbi, between 1 Jan. 1952 and 31 Dec. 1952, leg. Alexandre Fain (RMCA: 81.012-M-0035, 81.012-M-0039, 81.012-M-0040).

\section{Nycteris arge Thomas, 1903}

BURUNDI: $1 \lesssim$ [SB], Ruhinga Hill, 11 Mar. 1993, leg. Jay L. Udelhoven (FMNH: 155972).

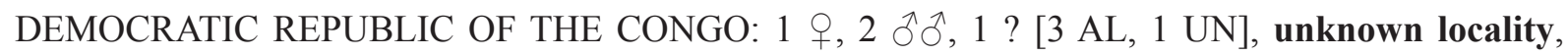
between 1 Jun. 1956 and 31 Jul. 1956, leg. Alvin Novick, prior to 1 Aug. 1957, leg. Heinz Stephan 
(MCZ: 48241, 48242, 48244, RMCA: 27435); 1 ad $\widehat{\jmath}$ [AL], Abialosa River, unknown date, leg. Jacques

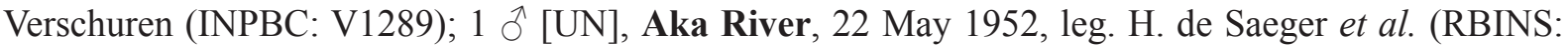

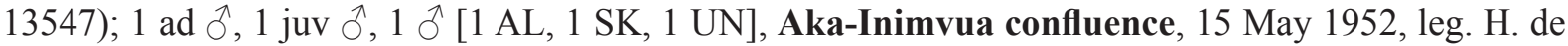
Saeger et al., 15 May 1952, leg. H. de Saeger (RBINS: 13546, RMCA: 36556, 36557); 1 ad $q$ [SK], Alima, 15 Feb. 1959, leg. P. Dyleff (RMCA: 28155a); 1 ad $\widehat{\partial}$ [SA], Amangundu, unknown date, leg. Jacques Verschuren (INPBC: V1294); 1 ad +1 ? [1 AL, 1 UN], Amatupi River [= Abatupi River], unknown date, leg. Jacques Verschuren, unknown date, leg. unknown collector (INPBC: V1291.1,

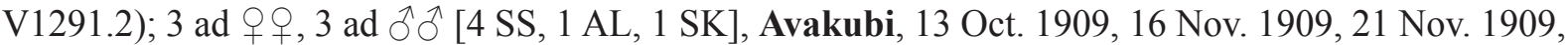
24 Nov. 1909, 28 Nov. 1909, leg. Herbert Lang, James Paul Chapin and The American Museum Congo Expedition (AMNH: 49140, 49371, 49133, RMCA: 12443, AMNH: 49136, 49137); 2 ?? [2 SA], Babongena, between 1 May 1982 and 31 May 1982, leg. Marc Colyn (RMCA: 82.031-M-0009, 82.031M-0010); 1 ठ [AL], Banalia, 17 Jan. 1939, leg. Duncan M. Hodgson and William F. Coultas (MCZ: 38010); 2 ad $\widehat{\partial} \widehat{\partial}$ [2 SS], Beni, 8 Aug. 1906, leg. R.E. Dent (BMNH: 1906.12.4.61, 1906.12.4.62); 2 ad ô $[2$ SA], Boende, between 1 Oct. 1969 and 31 Oct. 1969, leg. P. Lootens, between 15 Mar. 1940 and 16 Mar. 1940, leg. R.P. Wynants (RMCA: 75.035-M-0028, 15950); 3 ad $Q 9$, 1 ad $\curvearrowright, 1$ ad ? [1 SS, 4 SA], Bokuma, 6 Jun. 1951, 30 Jan. 1952, between 30 Jan. 1952 and 8 May 1952, between 1 Jan. 1931 and 31 Dec. 1931, leg. P. Lootens (RMCA: 21409, 20546, 23243, 23244, 21410); 1 ㅇ [UN], Bomane [= Bomaneh], 22 May 2010, leg. Guy-Crispin Gembu Tungaluna, Prescott Musaba and José Akaibe (UNIKIS: CRT2221); 1 juv $\widehat{\jmath}$ [AL], Buluku, 22 May 1952, leg. H. de Saeger (RMCA: 36558); 1 ad + , 1 ad ?, 2 ?? [2 SA, 1 SO, 1 UN], Buta, 20 Feb. 1928, prior to 16 Jun. 1937, leg. Jozef Hutsebaut (RMCA: 9485, 13856 to 13858); 1 § [SA], Bwandza, 30 Jun. 1979, leg. Frieder Meier (MHNG: 1870.092); 3 ?? [3 UN], Bwirina, unknown date, leg. unknown collector (INPBC: V1661.1 to V1661.3); 1 ad $q$ [SS], Djamba, 20 Dec. 1924, leg. Henri Schouteden (RMCA: 8874); 2 ad $q$ ㅇ [2 SA], Djelube River, middle, unknown date, leg. Jacques Verschuren (INPBC: V1078.1, V1078.2); 1 ad $\&$ [SA], Djumma River, middle, unknown date, leg. Jacques Verschuren (INPBC: V1279); 1 ? [UN], Durba, 18 Oct. 1999, leg. Robert (Bob) Swanepoel (TM: DBA001); 1 ㅇ, 1 § [1 AL, 1 SB], Epulu, 20 Jun. 1981, leg. John

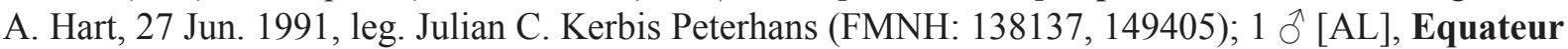
(former) Province, 8 Apr. 1996, leg. unknown collector (SMF: 84410); 1 ad,+ 2 ad $\widehat{\lambda} \widehat{\jmath}$ [1 SA, 2 AL], Equateur Province, 22 Jun. 1956, between 1 Jun. 1956 and 31 Jul. 1956, leg. Alvin Novick, between

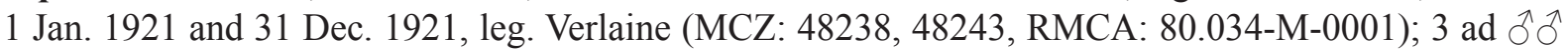
[1 SA, 2 UN], Ibembo, unknown date, 13 Feb. 1950, leg. Jozef Hutsebaut (BMNH: 1954.791, 1954.792, RMCA: 19632); 1 ad $\rho, 1$ ad $\widehat{\jmath}$ [2 SA], Ikela, prior to 4 Jan. 1958, between 1 Jan. 1931 and 31 Dec. 1931, leg. P. Lootens (RMCA: 25830, 27020); 1 ? [AL], Imbonga, between 1 Jan. 1987 and 31 Dec. 1987, leg. P. Lootens (RMCA: 87.043-M-0003); 1 ad ? [AL], Inangongo, unknown date, leg. Jacques Verschuren

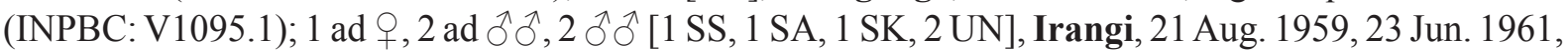
11 Jul. 1961, leg. Uwe Rahm, 20 Mar. 1990, leg. Klaus-Gerhard Heller and Marianne Volleth, 24 Jan. 1984, leg. Heinz Stephan (BMNH: 1962.1852, SMF: 78331, RMCA: 28380, BMNH: 1962.1851, SMF: 65029); 1 ad + , 2 ad $\widehat{\partial} \widehat{\partial}$ [3 SA], Irsac Labo [= Luiro], unknown date, leg. H.J. Bredo, unknown date, leg. Th. Andersen (ZFMK: 1964.1318 to 1964.1320); 1 q [SS], Irumu, 30 Nov. 1924, leg. Edmund Heller (FMNH: 26238); 1 đ [UN], Isai River, 18 Dec. 1974, leg. Michael D. Gallagher (HZM: 2.7845);

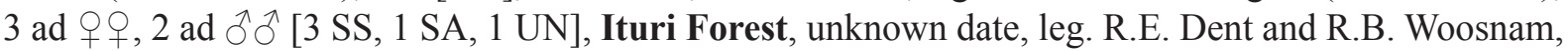
24 Apr. 1930, leg. Howard de Walden (BMNH: 1907.1.2.12, 1930.11.11.84 to 1930.11.11.87); 1 ○े [SS], Kasende, 5 Jul. 1934, leg. R.S. Pierrepont (MCZ: 31739); 1 ad ?, 1 ? [2 SS], Kawa (Forest), 1 Jan. 1952, leg. unknown collector, 16 Jul. 1952, leg. Alexandre Fain (RBINS: 21663, BMNH: 1953.480); 1 ad $q$ [SS], Keri River, 26 Aug. 1926, leg. D. Fraser (BMNH: 1927.3.1.18); 1 ad + [SS], Kiamokoto, 7 May 1948, leg. Gaston-François de Witte et al. (RBINS: 10838); 1 ad + , 1 ? [1 SK, 1 UN], Kilembe, 52 mi [= 84 km] S, 8 Aug. 1957, leg. Robin E. Leech and Edward S. Ross (CAS: 12488, 12521); 1 ad $q$ [SA], Kilo Mines [= Kilomines], between 1 Jan. 1930 and 31 Dec. 1930, leg. du Soleil (RMCA: 16200); 1 ad

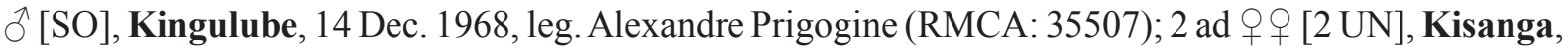
22 Apr. 1960, 25 Apr. 1960, leg. Uwe Rahm (BMNH: 1962.1853, 1963.1169); 1 ઈ [SS], Kisiki, 
19 Dec. 1938, leg. Duncan M. Hodgson and William F. Coultas (MCZ: 38025); 2 ad $q$ ㅇ [1 SA, 1 UN], Koloka, unknown date, leg. unknown collector, between 1 Jun. 1911 and 30 Jun. 1911, leg. H. Schubolz (ZMH: 3, SMF: 6380); 1 ad §̋ [SA], Koteli [= Kotili], between 1 Dec. 1924 and 31 Dec. 1924, leg. Henri Schouteden (RMCA: 16199); 1 q [AL], Kumbi, 25 Nov. 1993, leg. Ngenge Masumbuko Kamitongo (ZMA: MAM.24899); 1 ad $\widehat{\partial}$ [SS], Kunungu, 19 Nov. 1937, leg. N'Kele (RMCA: 14168);

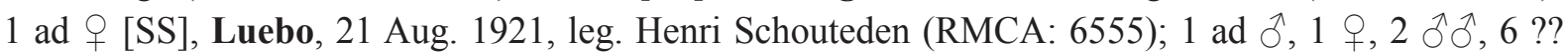
[2 SS, 7 AL, 1 SO], Lukolela, 11 Aug. 1930, 7 Nov. 1930, 29 Nov. 1930, 27 Dec. 1930, 17 Mar. 1931, leg. James Paul Chapin, 15 Nov. 1932, leg. Lecocq, 4 Sep. 1930, leg. Franklin Edson (AMNH: 86910, RMCA: 12176, AMNH: 86864, 86865, 86867, 86913, 86912, 86769, 86770, 86911); 1 \& [AL], Mabali, 22 Jun. 1956, leg. Alvin Novick (MCZ: 48239); 1 ad ð̋ [UN], Makala, 4 Dec. 1912, leg. J. Christie (BMNH: 1914.4.8.5); 2 ?? [2 UN], Makpe River, upper [= Makpe], 13 Jul. 1951, leg. H. de Saeger et al. (RBINS: 13543.1, 13543.2); 2 우 [2 SO], Medje, 2 May 1909, 16 Jan. 1910, leg. Herbert Lang, James Paul Chapin and The American Museum Congo Expedition (AMNH: 49194, 49138); 2 ?? [2 UN], Mogbwamu River, 8 Mar. 1952, leg. H. de Saeger et al. (RBINS: 13545.1, 13545.2); 1 ad ? [SS],

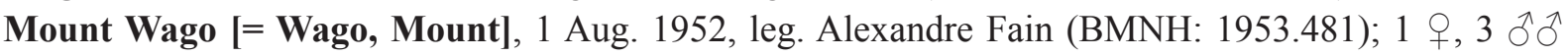
[4 SS], Muhulu, 15 Jan. 1957, leg. Heinz Stephan (RMCA: 27436 to 27439); 2 ad $q$ ㅇ, 1 ad $\hat{\jmath}$ [3 SS], Munoi confluence, 2 Jun. 1948, leg. Gaston-François de Witte et al. (RBINS: 10835 to 10837); 2 ?? [2 AL], Niangara, 27 Mar. 1913, leg. Herbert Lang, James Paul Chapin and The American Museum Congo Expedition (AMNH: 49386, 49387); 1 ad $\widehat{~}$ [SS], Panga, between 1 Sep. 1925 and 30 Sep. 1925, leg. Henri Schouteden (RMCA: 9127); 1 ad ? [SS], Paulis [= Isiro], 5 Jan. 1950, leg. Abbeloos (RMCA: 19819); 1 ad $\odot, 2$ ad $\widehat{\partial} \widehat{\partial}, 1$ juv $\widehat{o}$ [3 SA, 1 AL], Pawa, unknown date, leg. Reitter, 8 Nov. 1938, between 31 Dec. 1936 and 8 Nov. 1938, leg. Degotte (RMCA: 15728, 15216 to 15218); 2 ad $\circ$ +, 1 ad $ð$ [1 SA, 2 AL], Ruanoli-Mavea confluence, unknown date, leg. Jacques Verschuren (INPBC: V1668.2, V1668.3.1, V1668.3.2); 1 ad,+ 1 ad $\curvearrowright, 1$ juv $\curvearrowright, 1$ \& [1 SS, 2 SA, 1 AL], Stanleyville [= Kisangani], 6 Oct. 1910, leg. Weyns, between 1 May 1926 and 31 May 1926, leg. Henri Schouteden, between 11 Jan. 1939 and 11 Dec. 1939, leg. Duncan M. Hodgson and William F. Coultas, between 5 Feb. 1928 and 5 Nov. 1928, leg. Collart (RMCA: 893, 16198, MCZ: 38032, RMCA: 16194); 1 ô, 1 ? [2 UN], Taere River, unknown date, leg. unknown collector, 10 Mar. 1952, leg. H. de Saeger et al. (INPBC: 4380.2, RBINS: 13544); 1 juv $\widehat{\jmath}$ [AL], Tayele (River) [= Taere River], 10 Mar. 1952, leg. H. de Saeger (RMCA: 36559); 1 ? [AL], Tshakala, between 1 Jan. 1982 and 31 Dec. 1982, leg. Marc Colyn (RMCA: 82.019-M-0010); 1 ad $\widehat{\partial}$ [UN], Ukaika, unknown date, leg. Rudolf Grauer (NMW: 10301); 5 ad 우우, 3 ad $\hat{\delta} \hat{\delta}[4 \mathrm{SA}, 4 \mathrm{AL}$ ], Virunga National Park, unknown date, leg. Jacques Verschuren (INPBC: V1853A, V1853B, V1854A, V1854B, V1855.1A, V1855.1B, V1855.1C, RBINS: 1289B); 17 ?? [17 AL], Wafanya [= Wafania], 8 Oct. 1988, 29 Aug. 1991, 12 Sep. 1991, 25 Sep. 1991, 10 Feb. 1992, 20 Mar. 1992, 8 Apr. 1992, between 1 Dec. 1986 and 31 Dec. 1987, between 1 Aug. 1987 and 31 Aug. 1987, between 1 Jun. 1988 and 30 Jun. 1988, between 1 May 1988 and 31 May 1988, between 1 Mar. 1992 and 31 Mar. 1992, between 1 Mar. 1991 and 31 Mar. 1991, between 1 Feb. 1991 and 28 Feb. 1991, between 1 Jan. 1992 and 31 Jan. 1992, leg. P. Lootens (RMCA: 92.079-M-0034, 92.079-M-0035, 91.076-M-0081, 92.079-M-0112, 92.079-M-0115, 91.076-M-0092, 91.076-M-0059, 92.079-M-0117, 89.020-M-0040, 92.079-M-0114, 91.076-M-0007, 91.076-M-0014, 92.079-M-0113, 89.020-M-0041, 89.020-M-0043, 88.005-M-0008, 87.043-M-0005); 1 ad $q$ [SS], Wago, Mount, 21 Sep. 1952, leg. Alexandre Fain (RMCA: 81.012-M-0037); 1 ad ? [SS], Wasa [= Wasa Wago Forest], between 1 Jan. 1951 and 31 Dec. 1952, leg. Alexandre Fain (RMCA: 81.012-M-0038); 1 ad + [SA], Yalusaka, 16 Mar. 1958, leg. F. Carbone (RMCA: 27163); 1 ad $\delta$ [SA], Yambata, 11 Mar. 1914, leg. De Giorgi (RMCA: 5443); 1 ad 9 [SA], Yangambi, 7 Feb. 1942, leg. R.P. Van Boven (RMCA: 80.033M-0001). 


\section{Nycteris grandis Peters, 1865}

DEMOCRATIC REPUBLIC OF THE CONGO: 1 ? [AL], Babongena, between 1 May 1982 and 31 May 1982, leg. Marc Colyn (RMCA: 82.031-M-0006); 1 o [SK], Baliko, 7 Jun. 2012, leg. William ("Bill”) T. Stanley (FMNH: 219603); 1 ad ? [SS], Beni, prior to 2 Jun. 1914, leg. Bonnevie (RMCA:

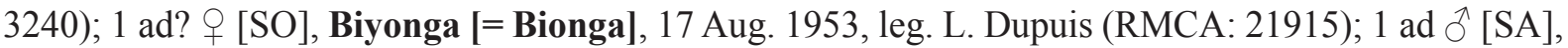
Boende, between 1 Jan. 1966 and 31 Jan. 1966, leg. P. Lootens (RMCA: 34162); 1 ad ठ̊ [SA], Bokuma, 18 May 1951, leg. P. Lootens (RMCA: 20679); 1 ? [AL], Boteka, 10 Oct. 1985, leg. P. Lootens (RMCA: 85.052-M-0001); 1 ad $\widehat{~}$ [SA], Camp Putnam, between 1 May 1953 and 31 May 1953, leg. Uwe Rahm (RMCA: 28902); 1 ad $\widehat{\partial}$ [SA], Djuma River, upper, unknown date, leg. Jacques Verschuren (INPBC: V1277.1); 1 ad 9 [SA], Epulu, between 1 Oct. 1956 and 31 Oct. 1956, leg. Max Poll (RMCA: 25652); 1 o [AL], Irangi, 18 Jan. 1984, leg. Heinz Stephan (SMF: 65028); 1 juv $ð, 1$ q [2 AL], Irangi, 3 mi [= 4.8 km] from, 16 Oct. 1990, leg. Wim Bergmans (ZMA: MAM.24178, MAM.24179);

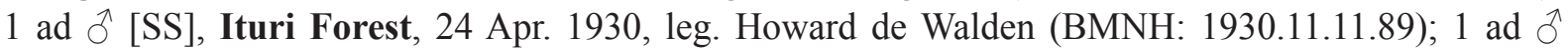
[SA], Kalikimvua, 11 Jun. 1952, leg. H. de Saeger et al. (RBINS: 13621); 1 ad $\lesssim$ [SO], Kamituga, 6 Jan. 1964, leg. Alexandre Prigogine (RMCA: 32393); 1 q [AL], Kasiba, 15 Oct. 1990, leg. Wim Bergmans (ZMA: MAM.24177); 1, 2 $2 \hat{\jmath}$ [2 SA, 1 AL], Kivu region, between 11 Aug. 1989 and 14 Aug. 1989, between 1 Jan. 1988 and 31 Dec. 1988, leg. unknown collector (SMF: 73700, 77099, 77100); 1 ad,+ 1 ad $\widehat{\partial}, 1$ + [3 SS], Luluabourg [= Kananga], 28 May 1922, 11 Mar. 1926, leg. Richard Callewaert (BMNH: 1927.12.21.10, 1927.12.21.8, 1926.7.6.72); 2 우 [2 AL], Mabali, between 18 Jun. 1956 and 19 Jul. 1956, leg. Alvin Novick (MCZ: 48236, 48237); 1 ad $\widehat{~}, 2$ ?? [1 SA, 2 UN], Makayova River, lower, unknown date, leg. unknown collector, unknown date, leg. Jacques Verschuren (INPBC: V1256.1 to V1256.3); 2 ?? [2 AL], Masako, unknown date, leg. Ndey B. Ifuta (RMCA: 93.079-M-0341, 93.079-M-0342); 1 , 1 ? [2 UN], Menga, 25 Jan. 1996, leg. Robert (Bob) Swanepoel (TM: 45568, 45569); 1 ad +, 1 ? [1 SA, 1 UN], Mogbwamu River, 8 Jul. 1951, leg. H. de

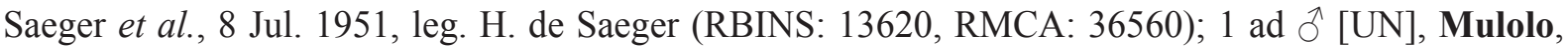
15 Mar. 1960, leg. Uwe Rahm (BMNH: 1962.1849); 1 ad 9 [SA], Netonna, 8 Jun. 1890, leg. P. Hesse (SMF: 2514); 1 ad $\overbrace{}^{\lambda}$ [SA], Omaniumdu, 27 Jul. 1959, leg. Laurent (RMCA: 29536); 1 ad ? [SS], Poko, between 25 Feb. 1914 and 25 Jul. 1914, leg. Cuthbert Christy (RMCA: 4340); 1 ᄋ, 1 ठे [2 AL], Quatorze, 1 Jul. 2013, leg. William ("Bill”) T. Stanley (FMNH: 222427, 222428); 1 ad $\lesssim$ [UN], Ukaika, unknown date, leg. Rudolf Grauer (NMW: 10300); 1 + , 5 ?? [5 AL, 1 UN], Wafanya [= Wafania], 10 Oct. 1991, 12 Oct. 1991, between 12 Oct. 1991 and 18 Oct. 1991, between 1 Nov. 1991 and 30 Nov. 1991, between 1 Aug. 1987 and 31 Aug. 1987, leg. P. Lootens (RMCA: 91.076-M-0106, 91.076-M-0109, 92.079-M0033, 88.005-M-0009, 92.079-M-0110, 91.076-M-0102); 1 ad $\&$ [UN], Yalosemba, 27 Jun. 1979, leg. C. Brian Robbins (USNM: 537706); 1 đ [AL], Yayama, 24 Jun. 1986, leg. WHO and Marc Colyn (RMCA: 88.011-M-0013).

\section{Nycteris hispida (Schreber, 1774)}

BURUNDI: 1 ? [AL], unknown locality, between 1 Jan. 1965 and 31 Dec. 1970, leg. P.L. Niort (MHNG: 1896.015); 1 ad $\odot$ [SK], Kayanza, 12 Mar. 1979, leg. Heinz Stephan (SMF: 57536); 1 đ [AL], Mweya, 29 Apr. 1971, leg. P.L. Niort (MHNG: 1896.018).

DEMOCRATIC REPUBLIC OF THE CONGO: 1 ad $\curvearrowright, 1$ ad ?, 1 juv ?, 5 ?? [1 AL, 1 SK, 1 SO, 5 UN], unknown locality, unknown date, leg. Cuthbert Christy, 13 Aug. 1958, leg. N'Kele, between 1 Jan. 1931 and 31 Dec. 1931, leg. E. Jans, between 15 Jul. 1999 and 18 Jul. 1999, between 11 Aug. 1999 and 15 Aug. 1999, between 1 Jul. 1999 and 4 Jul. 1999, leg. unknown collector (RMCA: 3463, 27666, 27371, SMF: 90192 to 90194, 90190, 90191); 1 ad + [AL], Aka, 14 May 1952, leg. H. de Saeger et al. (RBINS: 13612); 1 ? [UN], Albert National Park [= Virunga National Park], unknown date, leg. unknown collector (INPBC: V1838.2); 1 ㅇ [UN], Ankoro, 3 Nov. 1974, leg. Michael D. Gallagher (HZM: 45.7852); 2 ad + ㅇ, 2 ad ??, 1 juv ?, 1 + , 1 ô, 1 ? [6 SS, 1 SA, 1 AL], Avakubi, 15 Nov. 1909, 
17 Nov. 1909, 28 Jan. 1914, 15 Aug. 1914, leg. Herbert Lang, James Paul Chapin and The American Museum Congo Expedition, 17 Sep. 1912, 26 Sep. 1912, leg. Cuthbert Christy, 30 Oct. 1912, leg. unknown collector, between 1 Jan. 1914 and 31 Jan. 1914, leg. J. Bequaert (RMCA: 12386, AMNH: 49141, RMCA: 1426, AMNH: 49134, RMCA: 1423, AMNH: 49368, BMNH: 1914.4.8.4, RMCA: 5445); 1 $\lesssim$ [UN], Avakubi, near, 10 Dec. 1974, leg. Michael D. Gallagher (HZM: 44.7851); 1 ? [SA], Babongena, between 1 May 1982 and 31 May 1982, leg. Marc Colyn (RMCA: 82.031-M-0007); 1 ad ?,

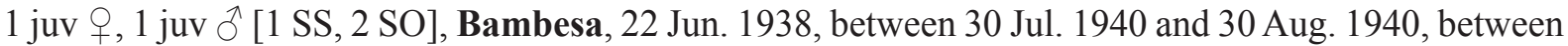
12 Apr. 1941 and 22 Apr. 1941, leg. J.M. Vrijdagh (RMCA: 14798, 17754, 17767); 1 ad $q, 1$ ad $\lesssim$ [2 SA], Banana, between 1 May 1952 and 31 May 1952, between 1 Jan. 1952 and 31 Jan. 1952, leg. Is.

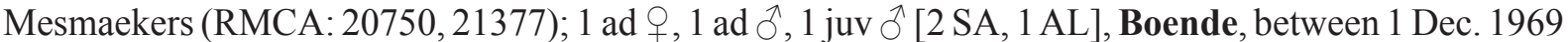
and 31 Dec. 1969, between 1 Jan. 1931 and 31 Dec. 1931, leg. P. Lootens (RMCA: 35639, 75.035-M0022, 75.035-M-0023); 2 ad $\widehat{\partial} \widehat{\partial}$ [2 SA], Bokuma, between 1 Jan. 1931 and 31 Dec. 1931, leg. P. Lootens (RMCA: 22134, 23245); 1 ad 9 [SS], Bolobo, between 1 Jan. 1931 and 31 Dec. 1931, leg. N'Kele (RMCA: 20258); 1 ad,+ 3 ?? [3 SA, 1 AL], Boma, 27 Jan. 1915, leg. Herbert Lang, James Paul Chapin and The American Museum Congo Expedition, 3 Sep. 1913, leg. Styczynski (AMNH: 49392 to 49394, RMCA: 2486); 1 ? [AL], Boteka, 14 Oct. 1985, leg. P. Lootens (RMCA: 85.052-M-0002); 1 ad $q$ [AL], Bukavu, unknown date, leg. Jacques Verschuren (INPBC: V394); 1 ? [AL], Bunia, between 1 Apr. 1927 and 30 Apr. 1927, leg. Harold Jefferson Coolidge (MCZ: 28546); 6 ad + o , 1 ad $\lesssim$ [6 SA, 1 AL], Buta, unknown date, prior to 9 Nov. 1938, prior to 3 Nov. 1939, leg. Jozef Hutsebaut (RMCA: 14562a, 14562b, 14585, 15737 to 15739, 14903); 1 ㅇ, 2 ?? [1 SS, 1 AL, 1 SK], Catchpole's Farm, 27 Jul. 1924, 28 Aug. 1924, leg. Edmund Heller, 29 Aug. 1924, leg. Alfred Morris Collins (FMNH: 30711, 30712,

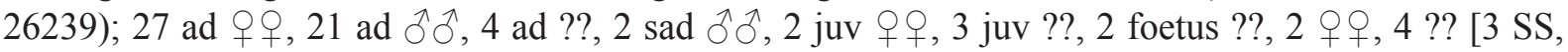
22 SA, 36 AL, 2 SK, 4 UN], Cel II, unknown date, leg. unknown collector, 16 Feb. 1951, 1 Mar. 1951, 7 Mar. 1951, 17 Mar. 1951, 30 Mar. 1951, 2 Apr. 1951, 21 May 1951, 22 May 1951, 26 May 1951, 25 Jun. 1951, 19 Jul. 1951, 21 Jul. 1951, 25 Sep. 1951, 2 Nov. 1951, 15 Jan. 1952, 31 Jan. 1952, 1 Feb. 1952, 16 Feb. 1952, 18 Feb. 1952, 26 Feb. 1952, 27 Feb. 1952, 2 Apr. 1952, 3 May 1952, 8 May 1952, 16 Jul. 1952, leg. H. de Saeger et al., 1 Mar. 1951, 17 Mar. 1951, 30 Mar. 1951, 2 Apr. 1951, 12 May 1951, 21 May 1951, 31 May 1951, 19 Jul. 1951, 21 Jul. 1951, 31 Jan. 1952, 1 Feb. 1952, 26 Feb. 1952, 7 Apr. 1952, 8 May 1952, 16 Jul. 1952, between 12 Feb. 1952 and 18 Feb. 1952, leg. H. de Saeger (INPBC: 3305, 3427, RBINS: 13597, RMCA: 36597, RBINS: 13574, RMCA: 36565, 36568, RBINS: 13595, 13572, 13596, 13617, RMCA: 36594, RBINS: 13575, RMCA: 36566, RBINS: 13587, 13600.1, 13600.2, 13585, 13592.1, 13592.2, RMCA: 36577, RBINS: 13576, RMCA: 36567, RBINS: 13609.1, 13609.2, 13594, 13579.1, 13579.2, RMCA: 36569, RBINS: 13591.1, 13591.2, RMCA: 36575, 36576, RBINS: 13580.1, 13580.2, 13583, 13593.1, 13593.2, 13602.1, 13602.2, RMCA: 36584, RBINS: 13582, 13601, 13616, 13571, RMCA: 36563, 36564, RBINS: 13598.1 to 13598.4 , RMCA: 36578 to 36581, 36587, 36570, 36571a, 36571b, RBINS: 13573, RMCA: 36591, 36592a, 36592b, RBINS: 13615, RMCA: 36593, 36582, 36583); 1 đ [SS], Cinputu, 13 Sep. 1983, leg. Betu Nsankulu (RMCA: 84.035-M-0024); 1 ad 9,1 ad $\widehat{\jmath}$ [1 SS, 1 SA], Djamba, 19 Dec. 1924, between 1 Dec. 1924 and 31 Dec. 1924, leg. Henri Schouteden (RMCA: 8873, 16195); 1 ad $q$ [SA], Djeka, 29 May 1955, leg. Roiseux (RMCA: 23634); 1 ad §̃, 1 ad ?, 1 ? [1 SA, 2 SK], Duma, 12 Oct. 1910, leg. unknown collector, 12 Oct. 1910, leg. H. Schubolz, between 1 Sep. 1911 and 30 Sep. 1911, leg. A.T.M. Herzog (SMF: 6381, 6381??6382, 6382); 1 ad ? [SO], Eala, 24 Jan. 1921, leg. Henri Schouteden (RMCA: 6558); 1 ○ [UN], Edouard, Lac [= Edward, Lake], 11 Nov. 1954, leg. unknown collector (RBINS: 15465); 1 ô [AL],

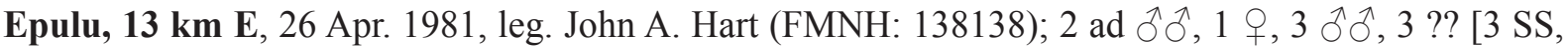
1 SA, 5 AL], Faradje, 22 Nov. 1911, 25 Feb. 1912, 1 Mar. 1912, 9 Mar. 1912, 13 Jan. 1913, leg. Herbert Lang, James Paul Chapin and The American Museum Congo Expedition (AMNH: 49085, 49144 [holotype Nycteris pallida J.A. Allen, 1917], 49418, 49417, RMCA: 12390, AMNH: 49419, 49424, 49421, MCZ: 17226 [topotype Nycteris pallida J.A. Allen, 1917]); 1 ad $\lesssim ~[S S$ ], Fundi, between 16 Feb. 1912 and 16 Nov. 1912, leg. Cuthbert Christy (RMCA: 1608); 2 ?? [2 UN], Gangala-na-Bodio,

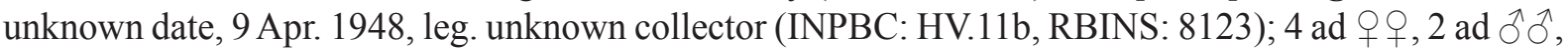


1 ad ?, 1 juv ?, 1 ? [6 AL, 1 SO, 2 UN], Garamba National Park, unknown date, leg. unknown collector, 18 Jan. 1951, 16 Feb. 1951, 30 Mar. 1951, 21 Jul. 1951, 18 Mar. 1952, between 1 Jan. 1950 and 31 Dec. 1952, leg. H. de Saeger et al., between 1 Jan. 1950 and 31 Dec. 1952, leg. H. de Saeger (INPBC: 5120, RBINS: 13578, 13570, 13605, 13590, 13569.1, 13569.2, 13618, RMCA: 36599); 1 ad ㅇ, 1 ad $\hat{\alpha}$ [2 SA], Goma, 10 Mar. 1953, 5 Dec. 1953, leg. The KEA-Mission (RBINS: 14457, 14458); 1 ad $\lesssim$ [SK], How..., unknown date, leg. J.J. Laarman (RMNH: MAM.16343); 2 ad +9 [1 SA, 1 AL], Ikela, 3 Jun. 1955, between 1 Jan. 1931 and 31 Dec. 1931, leg. P. Lootens (RMCA: 25826, 25828); 1 ad ㅇ [SS], Irangi, 15 Aug. 1956, leg. J.J. Laarman (RMNH: MAM.16365); 1 , 2 ?? [1 SK, 2 UN], Irumu, unknown date, leg. unknown collector, 10 Dec. 1924, leg. Edmund Heller, 14 Sep. 1926, leg. James Paul Chapin, Dewitt L. Sage, Frank P. Mathews and The Ruwenzori-Kivu Expedition

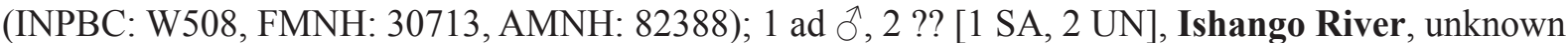
date, leg. Jacques Verschuren, unknown date, leg. unknown collector (INPBC: V1297.1, V1297.2, W1391); 1 ad ${ }^{\Uparrow}$ [SS], Kadia, between 1 Jul. 1931 and 31 Jul. 1931, leg. Gaston-François de Witte (RMCA: 11402); 2 우, 1 ○े [3 AL], Kadjidji [= Kajuju], unknown date, leg. Guy Babault (MNHN: ZMMO-1984-1337, ZM-MO-1984-1345, ZM-MO-1984-1346); 1 ad 9 [SA], Kakungu, 23 Nov. 1953, leg. Gaston-François de Witte (RMCA: 37121); 1 ad $\hat{\partial}$ [AL], Kamande, unknown date, leg. Jacques Verschuren (INPBC: V1057.1); 1 ad $\widehat{\partial}$ [SA], Kamituga, between 1 Dec. 1956 and 31 Dec. 1956, leg. Alexandre Prigogine (RMCA: 26189); 1 ? [AL], Kampala, between 1 Jan. 1935 and 31 Dec. 1935, leg. Guy Babault (MCZ: 46087); 1 đ [SS], Kananga, 4 Jan. 1974, leg. Western New Mexico University (FMNH: 152456); 1 ad 9 [SA], Karawa, prior to 24 Nov. 1936, leg. R.P. Wallin (RMCA: 14568); 1 ad đ [SA], Kasaji, 13 Mar. 1953, leg. Fisher (RMCA: 21807); 1 ? [UN], Kasaka, unknown date, leg. unknown collector (INPBC: V1144.35); 1 ad $P$ [SA], Kasenga, between 1 Jul. 1936 and 31 Aug. 1936, leg. Schwetz (RMCA: 13699); 1 ? [AL], Kasengi [= Kasengu], 27 Aug. 1926, leg. James Paul Chapin, Dewitt L. Sage, Frank P. Mathews and The Ruwenzori-Kivu Expedition (AMNH: 82387); 2 ad 우 [1 AL, 1 UN], Kasenyi [= Kasenye], 2 Dec. 1957, leg. Robin E. Leech and Edward S. Ross, 25 Feb. 1954, leg. The KEA-Mission (CAS: 12484, RBINS: 15464); 1 ad $\widehat{\text { of }, ~} 1$ ? [1 SK, 1 UN], Kassi River, unknown date, leg. unknown collector, 5 Apr. 1951, leg. H. de Saeger (INPBC: 3253, RMCA: 36595); 1 ad ㅇ [AL], Kaswabilenga, 1 Oct. 1947, leg. Gaston-François de Witte et al. (RBINS: 10808); 1 ? [UN], Katanda, unknown date, leg. unknown collector (INPBC: V1838.1); 2 우 [2 SS], Katobwe, 27 Nov. 1926, 30 Nov. 1926, leg. John Todd Zimmer (FMNH: 29408, 29409); 3 ad ?? [3 SS], Kawa (Forest), 16 Jul. 1952, 17 Jul. 1952, 5 Aug. 1952, leg. Alexandre Fain (RMCA: 81.012-M-0014, BMNH: 1953.482, 1953.483); 1 ad $\bigcirc$ [SS], Kiambi, 7 May 1931, leg. Gaston-François de Witte (RMCA: 11323); 1 ? [UN], Kiavikire [= Kiavikere], unknown date, leg. unknown collector (INPBC: W587); 1 ad $\delta^{\lambda}$ [SO], Kingabwa, 19 Dec. 1975, leg. Tshinyenye (RMCA: 76.010-M-0003); 1 ? [AL], Kisangani, between 1 Jun. 1982 and 30 Jun. 1982, leg. Marc Colyn (RMCA: 82.031-M-0034); 1 ad ? [SS], Kiseny [= Kasenye], between 1 Jan. 1942 and 31 Dec. 1942, leg. R. Van Saceghem (RMCA: 17339); 1 ? [UN], Kisisile, unknown date, leg. unknown collector (INPBC: W844); 1 ad $\widehat{\jmath}$ [SA], Kitonga, unknown date, leg. Jacques Verschuren (INPBC: V1302); 1 ad 9 [AL], Kivu, Lake [= Lake Kivu], between 1 May 1953 and 31 May 1953, leg. The KEA-Mission (RBINS: 14462); 1 ad ? [SS], Korovi, Mount, 25 Oct. 1951, leg. Alexandre Fain (RMCA: 23280); 1 ad $\nsim$ [SS], Koteli [= Kotili], 2 Jan. 1925, leg. Henri Schouteden (RMCA: 8876); 1 ad §̊ [SS], Kunungu, 11 Dec. 1927, leg. Ngwe (RMCA: 9391); 1 ad $\lesssim$ [SS], Kwamouth, 17 Jun. 1921, leg. Henri Schouteden (RMCA: 6559); 2 ad $ᄋ$ ㅇ [1 SA, 1 AL], Kyavinionge, 20 May 1954, leg. Gaston-François de Witte, 27 Nov. 1953, leg. The KEA-Mission (RMCA: 37124, RBINS: 14459); 1 juv ổ [AL], Léopoldville [= Kinshasa], 12 Mar. 1913, leg. Cuthbert Christy (RMCA: 1536); 1 ad ? [SO], Leverville [= Lusanga], between 1 Feb. 1929 and 28 Feb. 1929,

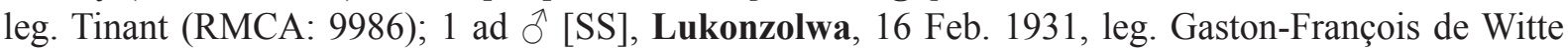
(RMCA: 11470); 2 ad $ㅇ+\{, 5$ ad $\widehat{\partial}, 2$ 우 [7 SS, $1 \mathrm{SA}, 1 \mathrm{UN}$ ], Luluabourg [= Kananga], unknown date, 17 Jul. 1922, 19 Oct. 1923, 2 Dec. 1925, 25 Feb. 1926, leg. Richard Callewaert, 4 Jun. 1964, leg. Antoon Emeric Marcel De Roo (BMNH: 1926.11.1.27, 1926.7.6.73, 1926.7.6.74, 1934.10.24.18, RMCA: 7568, BMNH: 1926.7.6.76, 1926.11.1.26, 1927.12.21.9, RMCA: 33601); 1 ad,+ 1 ad $\widehat{\prec}$ [1 SA, 
1 UN], Lwiro [= Luiro], 1 Aug. 1964, leg. U. Goepel, 8 Jul. 1958, leg. Uwe Rahm (ZMZ: 124600, RMCA: 33535); 5 ad 웅, 2 ad $\widehat{\jmath} \widehat{\jmath}$ [5 SS, 1 SK, 1 SO], Mabwe River, 11 Feb. 1949, 1 Mar. 1949, leg.

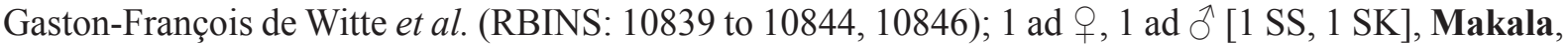
3 Dec. 1912, 5 Dec. 1912, leg. Cuthbert Christy (RMCA: 1607, 1609); 1 ad $\lesssim$ [SA], Makengo, 11 Dec. 1946, leg. Dartevelle (RMCA: 18161); 2 ad $ㅇ+$, 1 ad $\widehat{\jmath}$ [2 SA, 1 SO], Makpe, 12 Jul. 1951, 14 Jul. 1951, leg. H. de Saeger (RMCA: 36573, 36574, 36596); 1 ad,+ 1 ad $ð$ [2 SA], Makpe River, upper [= Makpe], 10 Jul. 1951, 12 Jul. 1951, leg. H. de Saeger et al. (RBINS: 13586, 13589); 1 ad q, 1 ? [1 SA, 1 SK], Maleli, 11 Jun. 1951, leg. H. de Saeger et al. (RBINS: 13584.1, 13584.2); 1 ad $q$ [SA], Malili, 14 Jun. 1951, leg. H. de Saeger (RMCA: 36572); 1 , 1 ? [1 AL, 1 UN], Mayumbe, near, unknown date, prior to 2 Apr. 1902, leg. unknown collector (MNHN: ZM-MO-1884-751, RBINS: 200b); 1 ad 9 , 1 ad ? [1 SA, 1 UN], Mbwambala, 21 Jun. 1995, 29 Jun. 1995, leg. Herwig Leirs et al. (RMCA: 97.021-M-0837, MSB: 274621); 1 ad + , 1 §ิ [1 SO, 1 UN], Medje, 20 Jan. 1910, leg. Herbert Lang, James Paul Chapin and The American Museum Congo Expedition, 9 Apr. 1914, leg. Cuthbert Christy (AMNH: 49143, RMCA: 4343); 1 ad $O$ [AL], Meridi River, 20 Apr. 1952, leg. H. de Saeger et al. (RBINS: 13611); 1 ad 9,1 ad $\delta$ [2 AL], Moko River, 20 Jun. 1952, leg. H. de Saeger et al. (RBINS: 13614.1, 13614.2); 1 ad ô [SA], Moliro, prior to 28 Feb. 1899, leg. Charles Lemaire (RMCA: 162); 1 ad + , 3 ad ${ }^{\wedge}{ }^{\lambda}, 1$ ad ? [4 SA, 1 SO], Mongbwalu, 30 May 1939, leg. Janssens, prior to 16 Feb. 1938, leg. Harford (RMCA: 15560, 14382a to 14382d); 1 ? [AL], Mosenge, 15 Aug. 1986, leg.

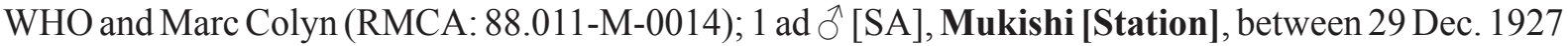
and 9 Feb. 1928, leg. Becquet (RMCA: 16193); 1 juv đ̊ [AL], Mulungu, prior to 1 Feb. 1940, leg. F.L. Hendricks (RMCA: 15872); 1 ad,+ 1 ad ${ }_{\lambda}^{\lambda}$ [2 SA], Murambi, 25 Aug. 1955, leg. Gaston-François de Witte (RMCA: 37126, 37127); 1 ad $\hat{\text { ô }}$ [SA], Mushie, between 1 Jan. 1931 and 31 Dec. 1931, leg. Cuthbert Christy (RMCA: 1537); 1 ad ${ }^{\Uparrow}$ [AL], Musosa, near, between 1 Jun. 1940 and 30 Jun. 1940,

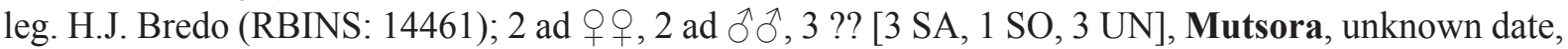
leg. unknown collector, 15 Dec. 1952, 13 Feb. 1954, 4 Aug. 1954, between 11 Dec. 1952 and 11 Dec. 1953, leg. Gaston-François de Witte (INPBC: V489, W1020, W814, RMCA: 37123, 37120, 37125, 37122); $1 \mathrm{ad}$ ㅇ [SO], Mutsora Station, 11 Apr. 1950, leg. J. de Wilde (RBINS: 12943); 1 ad $\circ, 1$ ad ? [2 SS], Mutwanga, between 1 Nov. 1937 and 30 Nov. 1937, between 1 Sep. 1937 and 30 Sep. 1937, leg. Henri Martin Hackars (RMCA: 16603, 21546); 1 ad ? [SA], Mweka Kasai [= Mweka], between 1 Jan. 1930

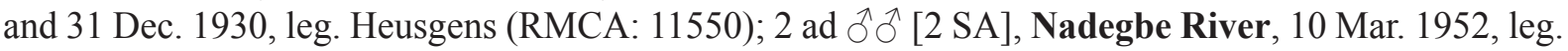
H. de Saeger (RMCA: 36585, 36586); 1 ad +, 1 ad ? [2 AL], Nampume, 6 Mar. 1952, leg. H. de Saeger et al. (RBINS: 13606.1, 13606.2); 1 ad 9 [AL], Nampune [= Nampume], 6 Mar. 1952, leg. H. de Saeger (RMCA: 36588); 1 ad ô [AL], Ndelele, 13 Mar. 1952, leg. H. de Saeger et al. (RBINS: 13603); 1 juv? [SO], Ndwa, between 1 Oct. 1950 and 30 Nov. 1950, leg. Ngwe (RMCA: 20255); 1 ad,+ 3 ad के के [3 SS, $1 \mathrm{SA}$ ], Niambasha, 30 Jan. 1959, leg. Uwe Rahm (RMCA: 28026, 28899 to 28901); 1 ㅇ, $1 \lesssim$ [2 AL], Niangara, 29 Feb. 1948, leg. Neal A. Weber (AMNH: 150183, 150184); 1 ad ? [SS], Paulis [= Isiro], 10 Dec. 1950, leg. Abbeloos (RMCA: 20511); 2 ad $+q, 1$ ad $§$ [1 SS, 2 SA], PFSK 8, 25 Mar. 1952, between 15 Mar. 1952 and 25 Mar. 1952, leg. H. de Saeger (RMCA: 36598, 36589, 36590); 1 ad $q$ [AL], PFSK.17, 26 Mar. 1952, leg. H. de Saeger et al. (RBINS: 13608); 1 ad ô [AL], PFSK.8, 25 Mar. 1952, leg. H. de Saeger et al. (RBINS: 13607); 3 ad ?? [2 SS, 1 SK], Poko, 17 Jul. 1914, 21 Jul. 1914, 2 Aug. 1914, leg. Cuthbert Christy (RMCA: 4336b, 4342, 4341); 1 juv $q$ [AL], Pweto, unknown date, leg. Gerard (RMCA: 5441b); 2 ad $\circ$ + 1 ad ?, 1 juv $~$ [1 SA, 2 AL, 1 UN], Rutshuru, unknown date, leg. Rudolf Grauer, 30 Apr. 1938, 29 May 1938, 31 May 1938, leg. Serge Frechkop (NMW: 10304, RMCA: 17088, RBINS: 4783, RMCA: 17089); 4 ad $q$ + [4 UN], Ruwenzori SE, unknown date, leg. unknown collector, 11 May 1906, leg. R.B. Woosnam, 17 Jun. 1906, leg. R.E. Dent (BMNH: 1906.12.4.75, 1906.12.4.76, 1906.12.4.17, 1906.12.4.16); 1 ? [UN], Samboko River, unknown date, leg. unknown collector (INPBC: V1034.2); 1 ㅇ [UN], Scierie Forest, 22 Nov. 1974, leg. Michael D. Gallagher (HZM: 43.7850); 3 우, 1 ठ [3 SS, 1 UN], St. Jos. (Mission) [= Mikalaya], 2 Aug. 1924, 15 Aug. 1924, 8 Oct. 1924, leg. Richard Callewaert (AMNH: 86177, 86172, 86175, 86143); 1 ad [SS], St. Joseph de Luluabourg Mission [= Mikalaya], 17 Jul. 1922, leg. 
Richard Callewaert (RMCA: 7545); 2 ad 9 , , 1 , 1 ภ, 1 ? [2 SA, 2 AL, 1 SO], Stanleyville [= Kisangani], 8 Aug. 1909, 1 Jan. 1915, leg. Herbert Lang, James Paul Chapin and The American Museum Congo Expedition, between 16 Jul. 1928 and 16 Aug. 1928, leg. Collart, prior to 29 Nov. 1933, leg. Schwetz (AMNH: 49390, 49139, 49531, RMCA: 16203, 12223); 2 ad ô $^{2}$ [ [2 UN], Tandala, 16 Jul. 1967, leg. Lynn W. Robbins, 18 Jul. 1979, leg. C. Brian Robbins (USNM: 537703, 537705); 1 ad $q$ [SK], Teturi, 12 Sep. 1946, leg. Jean Hiernaux (RBINS: 7045); 1 ? [UN], Thysville [= Mbanza-Ngungu], 11 Feb. 1987, leg. unknown collector (RBINS: 22431); 1 ? [AL], Tshakala, between 1 Jan. 1982 and 31 Jan. 1982, leg. Marc Colyn (RMCA: 82.019-M-0009); 1 ô [SO], Tshambi, 10 Nov. 1933, leg. unknown collector (RBINS: 4782); 1 ad $\widehat{~}$ [SO], Utu [River], 6 Feb. 1959, leg. Uwe Rahm (RMCA:

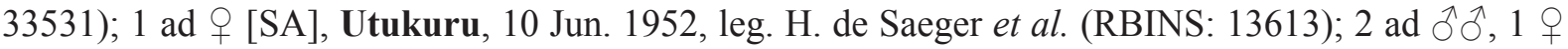
[1 AL, 2 UN], Uvira, unknown date, leg. Rudolf Grauer, 26 Jul. 1956, leg. Alvin Novick (NMW: 10302, 10303, MCZ: 48240); 1 juv ô, 1 ㅇ, 2 ?? [4 AL], Vankerckhovenville [= Nzoro], 18 Apr. 1912, leg. Herbert Lang, James Paul Chapin and The American Museum Congo Expedition (AMNH: 49395 to 49398); 1 ad + [AL], Vitshumbi, 2 Jan. 1953, leg. The KEA-Mission (RBINS: 14456); 1 ad $\&$ [SS], Vumbi, between 1 Jan. 1952 and 31 Dec. 1952, leg. Alexandre Fain (RMCA: 81.012-M-0036); 2 ?? [1 SA, 1 AL], Wafanya [= Wafania], 25 Sep. 1991, 26 May 1992, leg. P. Lootens (RMCA: 91.076-M0090, 92.079-M-0111); 1 đ [SS], Yalosemba, 20 Jun. 1979, leg. C. Brian Robbins (USNM: 537704); 1 ad $\widehat{~}$ [SS], Yangambi, 10 Nov. 1947, leg. G. Gilbert (RBINS: 6987).

RWANDA: 1 juv ? [SS], Astrida [= Butare], between 1 Jan. 1951 and 31 Jan. 1951, leg. Aureliaan (RMCA: 20490); 1 ad $\widehat{\partial}$ [SS], Butare, unknown date, leg. Alexandre Fain (RMCA: 81.012-M-0010); 1 ad $\&$ [SA], Cyili Ntyano [= Cyili], 2 May 1985, leg. Théoneste (RMCA: 87.047-M-0001); $1 \curvearrowright$ [AL], Lac Ihema [= Ihema, Lake], 9 Sep. 1980, leg. Hans H. de Iongh (ZMA: MAM.21230); 1 ad ${ }^{1}, 1$ o [1 SS, 1 SA], Nyarutovu, 18 May 1955, leg. Alexandre Fain (MHNG: 921.035, RMCA: 81.012-M-

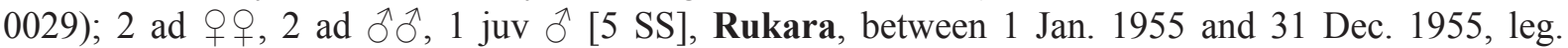
Alexandre Fain (RMCA: 81.012-M-0030 to 81.012-M-0034).

\section{Nycteris intermedia Aellen, 1959}

DEMOCRATIC REPUBLIC OF THE CONGO: 1 ad $\widehat{\partial}$ [SA], unknown locality, between 1 Jun. 1911

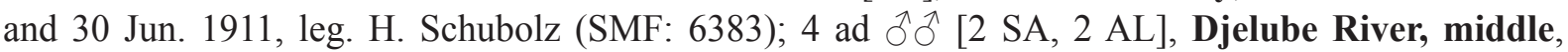
unknown date, leg. Jacques Verschuren (INPBC: V1076.1, V1076.2, V1077.1, V1077.2); 1 ad $q$ [SS], Ituri Forest, unknown date, leg. Howard de Walden (BMNH: 1930.11.11.82); 1 ad $\widehat{\jmath}$ [SS], Karambi, 27 Sep. 1953, leg. J.J. Laarman (RMNH: MAM.16340); 1 ad + [UN], Kisanga, 22 Apr. 1960, leg. Uwe Rahm (BMNH: 1962.1850); 1 ad $q$ [SA], Ruanoli-Mavea confluence, unknown date, leg. Jacques Verschuren (INPBC: V1668.4); 1 ad $\hat{\jmath}$ [SS], Teturi, 25 May 1948, leg. Jean Hiernaux (RBINS: 7046); 1 ad $\widehat{\partial}$ [SA], Virunga National Park, unknown date, leg. Jacques Verschuren (INPBC: V1655).

\section{Nycteris macrotis Dobson, 1876}

BURUNDI: 1 ad,+ 1 ad $ð$ [2 SK], Resha, 28 Feb. 1979, leg. Heinz Stephan (SMF: 57532, 57533).

DEMOCRATIC REPUBLIC OF THE CONGO: 1 ad ${ }_{+}$[SO], Alima, 15 Feb. 1959, leg. P. Dyleff (RMCA: 28155b); 1 ? [AL], Babongena, between 1 May 1982 and 31 May 1982, leg. Marc Colyn (RMCA: 82.031-M-0008); 1 ad + [SO], Bokalakala, 23 Oct. 1959, leg. N'Kele (RMCA: 28542); $1 \mathrm{imm}$ + [SS], Bokungu, 11 Dec. 1948, leg. L. Dupuis (RMCA: 18715); 5 ad + 우, 3 ad ??, 2 imm ?? [2 SS, 4 SA, 2 AL, 2 SO], Boma, 15 Aug. 1922, leg. J. Rodhain, between 1 Apr. 1936 and 30 Sep. 1936, between 1 Apr. 1936 and 30 Apr. 1936, leg. Schwetz (RMCA: 6066 to 6069, 13700a to 13700c, 13702 to 13704); 1 ad $\widehat{\partial}$ [SS], Eastern Congo, unknown date, leg. J.J. Laarman (RMNH: MAM.16342); 1 ㅇ [SA], Faradje, 4 Apr. 1911, leg. Herbert Lang, James Paul Chapin and The American Museum Congo

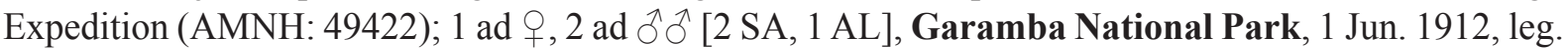


Herbert Lang, James Paul Chapin and The American Museum Congo Expedition, 27 Dec. 1950, 12 May 1951, leg. H. de Saeger et al. (AMNH: 49399, RBINS: 13624, 13623); 1 ad $\widehat{\supset}$ [SO], Grotte de Baya [= Baya Cave], 12 Jul. 1956, leg. M. Lips (RMCA: 23796); 2 ad + क , 1 ad $\curvearrowright$, 1 juv ô [4 SS], Kakyelo, 13 Nov. 1930, leg. Gaston-François de Witte and de Crawhez (RMCA: 10845, 10848, 10849, 10852); 1 ad + [SA], Kalina, prior to 16 Nov. 1950, leg. Jean Jacques Deheyn (RMCA: 20101); 1 ad $\lesssim$ [SS], Kamueshi [= Kamuesha], 5 Apr. 1979, leg. Betu Nsankulu (RMCA: 79.032-M-0013); 1 juv ? [AL], Kasongo, 23 Oct. 1959, leg. Segers and Benoit Mission (RMCA: 29164); 1 ? [AL], Katanga, near, unknown date, leg. Félix / Michel Anciaux de Faveaux (MNHN: ZM-MO-1957-128); 16 ad 9 9 15 ad

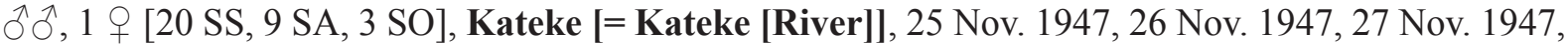
1 Dec. 1947, 4 Dec. 1947, leg. Gaston-François de Witte et al. (RBINS: 10827, 10828, 10830 to 10834, 10845, 10813 to 10826, 10829, 10811.1 to 10811.9); 1 \& [UN], Katupila, 22 Oct. 1974, leg. Michael D. Gallagher (HZM: 37.7849); 1 ad $\widehat{\jmath}$ [SS], Kawezi-Uvira Road, 3 Jul. 1957, leg. J.J. Laarman

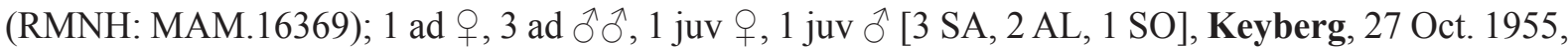
20 Dec. 1955, 4 Jun. 1956, leg. M. Lips, 27 Oct. 1955, leg. Félix / Michel Anciaux de Faveaux (RMCA: 26277, 26278, 26275, 26276, SMF: 16875, RMCA: 23800); 1 ad ㅇ [SS], Kidada, 17 Feb. 1922, leg. Henri Schouteden (RMCA: 6556); 1 ad $\hat{\jmath}$ [SA], Kimilolo, between 1 Jan. 1931 and 31 Dec. 1931, leg. De Smet (RMCA: 22441); 1 ๆ, 2 $\widehat{\partial}$ [3 UN], Kinshasa, between 15 Jan. 1975 and 16 Jan. 1975, leg.

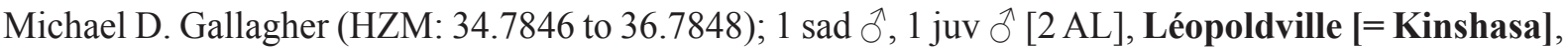
20 Jan. 1947, leg. Dartevelle, prior to 23 Jul. 1946, leg. Lepersonne (RMCA: 18163, 18129); 1 ad 우 [SS], Luiko [= Lwiko], 13 Sep. 1950, leg. Alexandre Prigogine (RMCA: 20430); 1 ad $\widehat{\jmath}$ [SO], Luluabourg [= Kananga], 28 Nov. 1923, leg. Richard Callewaert (BMNH: 1926.7.6.75); 2 juv ?? [2 UN], Mabwe, 1 Mar. 1949, leg. unknown collector (RBINS: 10810.1, 10810.2); 1 ad $\widehat{~}$ [SK], Mabwe River, 11 Feb. 1949, leg. Gaston-François de Witte et al. (RBINS: 10847); 1 ad $q$ [SS], Macaco, 29 Sep. 1921, leg. Henri Schouteden (RMCA: 7296); 1 juv $\widehat{\partial}$ [AL], Mahagi Port, 29 Mar. 1953, leg. Célestin Hecq (RMCA: 21898); 5 ?? [5 SK], Maleli, 13 Jun. 1951, leg. H. de Saeger et al. (RBINS: 13625.1 to 13625.5 ); 1 ad $q$ [SA], Malili, 13 Jun. 1951, leg. H. de Saeger (RMCA: 36561); 1 ad o [SK], Marari, 13 Jun. 1951, leg. H. de Saeger et al. (RBINS: 13626); 1 ad $q$ [SO], Morubia, 13 Jun. 1951, leg. H. de Saeger (RMCA: 36562); 1 ad $\$$, 1 ad $\lesssim$ [2 SA], Musosa, near, 6 Jul. 1944,

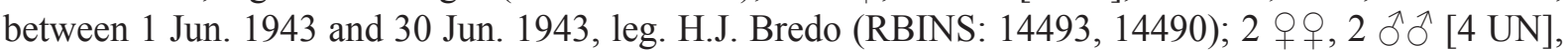
Mwebe Bridge, 24 Jan. 1996, leg. Robert (Bob) Swanepoel (TM: 45614, 45615, 45617, 45618); 1 ad ? [SS], Né, Mount, 25 Sep. 1952, leg. Alexandre Fain (RMCA: 23166); 1 ? [UN], Rutshuru, unknown date, leg. unknown collector (INPBC: V1769); 3 ad $q$ +, 2 ad $\lesssim \widehat{\jmath}$ [5 SA], Shinkolobwe, 2 Aug. 1960, leg. Félix / Michel Anciaux de Faveaux (RBINS: 14491, 14492, 14494, SMF: 19066, 19067); 2 ad $ᄋ$ ㅇ [1 SS, 1 SK], St. Joseph de Luluabourg Mission [= Mikalaya], 19 Jun. 1923, between 19 Feb. 1922 and 19 Jun. 1922, leg. Richard Callewaert (RMCA: 7108, 7546); 1 ad $q$ [SS], Temvo, 8 Mar. 1922, leg. Henri Schouteden (RMCA: 6557).

RWANDA: 1 ad $q, 8$ ad $\widehat{\partial} \widehat{\partial}$ [8 SS, 1 SO], Astrida [= Butare], 6 Aug. 1954, 7 Aug. 1954, between 1 Jan. 1954 and 31 Dec. 1954, leg. Alexandre Fain (RMCA: 81.012-M-0005 to 81.012-M-0009, 81.012M-0001 to 81.012-M-0004); 1 ad,+ 2 ad $\precsim \widehat{~}$ [3 SS], Bugundi, 19 Nov. 1955, leg. Alexandre Fain (RMCA: 81.012-M-0011 to 81.012-M-0013); 1 ad $\widehat{~}$ [SA], Butare, 18 Jun. 1966, leg. Félix / Michel Anciaux de Faveaux (RBINS: 15493); 1 ad 9 [SA], Karama, 11 Oct. 1981, leg. Frits De Vree, Benny

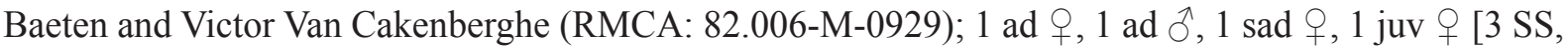
1 SO], Musha, 17 Oct. 1955, between 1 May 1955 and 31 May 1955, leg. Alexandre Fain (RMCA:

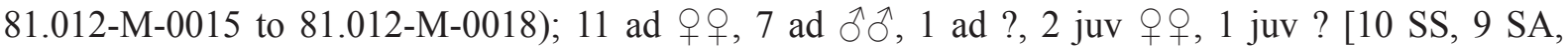
3 AL], Nyakibanda, 14 Oct. 1955, between 15 Oct. 1955 and 25 Oct. 1955, between 14 Oct. 1955 and 17 Oct. 1955, between 1 Apr. 1956 and 30 Apr. 1956, leg. Alexandre Fain, between 1 Mar. 1962 and 31 Mar. 1962, leg. Van Parijs (RMCA: 81.012-M-0019 to 81.012-M-0021, 81.012-M-0023 to 81.012M-0025, 31048 to 31059, 81.012-M-0027, 81.012-M-0028, 81.012-M-0022, 81.012-M-0026); 10 ad 우 
between 1 Jan. 1954 and 31 Dec. 1954, leg. Alexandre Fain, 1 Feb. 1966, 7 Dec. 1966, 17 Dec. 1966, leg. Félix / Michel Anciaux de Faveaux, 7 Dec. 1966, leg. unknown collector (BMNH: 1953.566, 1953.567, 1953.677 to 1953.680 , RBINS: 15483.1 to $15483.4,15484.1$ to $15484.4,15939.1,15939.2,15994$, 15989 to 15993, 15995, MHNG: 921.036, 921.037); 1 ad + , 1 ad $\lesssim$ [2 SA], Simbi Cave, 10 Apr. 1968, between 1 Mar. 1968 and 31 Mar. 1968, leg. Félix / Michel Anciaux de Faveaux (RBINS: 15931, 15930).

\section{Nycteris major (K. Andersen, 1912)}

DEMOCRATIC REPUBLIC OF THE CONGO: 1 ad $\lesssim$ [SA], Avakubi, 1 Sep. 1913, leg. Herbert Lang, James Paul Chapin and The American Museum Congo Expedition (AMNH: 49403 [holotype Nycteris avakubia J.A. Allen, 1917]); $1 \hat{\jmath}$ [UN], Kona, 14 May 2010, leg. Guy-Crispin Gembu Tungaluna, Prescott Musaba and José Akaibe (UNIKIS: CRT1959).

\section{Nycteris nana (K. Andersen, 1912)}

BURUNDI: $1 \uparrow$ [SB], Giserama, Colline, 12 Aug. 1991, leg. Julian C. Kerbis Peterhans (FMNH: 148980).

DEMOCRATIC REPUBLIC OF THE CONGO: 1 ? [SK], unknown locality, unknown date, leg. unknown collector (BMNH: 1930.11.11.83); 1 ad q, 1 ? [1 SA, 1 SK], Aka, 16 May 1952, leg. H. de Saeger et al. (RBINS: 13565.1, 13565.2); 1 ad §̋ [SA], Bagisana, 19 Apr. 1952, leg. H. de Saeger et al. (RBINS: 13563); 1 ad $\delta$ [SA], Bagunda, 18 Jan. 1951, leg. H. de Saeger et al. (RBINS: 13549); 1 , 1 ऽ [1 AL, 1 SK], Batala's Village, 13 Apr. 1925, 16 Apr. 1925, leg. Edmund Heller (FMNH: 30714, 30715); 1 ad $q$ [SA], Buluku River, 22 May 1952, leg. H. de Saeger et al. (RBINS: 13566); 1 ad $q$ [SS], Byangore River, 27 Apr. 1952, leg. Gaston-François de Witte et al. (RBINS: 17291); 1 ad $\widehat{\partial}$ [SA], Djugu Forest, between 1 Jan. 1948 and 31 Dec. 1948, leg. Jacques Verschuren (RBINS: 18546); 1 ? [SA], Duma, between 1 Oct. 1910 and 31 Oct. 1910, leg. unknown collector (SMF: 6390); 1 ad , 1 ad $\hat{o}$ [1 SS, 1 SA], Garamba National Park, 7 Feb. 1952, leg. H. de Saeger et al. (RBINS: 13554, 13556); 1 ad $\widehat{~}$ [AL], Ibembo, 20 Jul. 1950, leg. Jozef Hutsebaut (RMCA: 20092); 1 ad $q$ [SS], Irangi, 31 Jan. 1957, leg. J.J. Laarman (RMNH: MAM.16366); 1 ? [UN], Kambemba, unknown date, leg. unknown collector (INPBC: V1065); 1 ad ? [SS], Kawa (Forest), 7 Aug. 1952, leg. Alexandre Fain (BMNH: 1953.484); 1 ad,+ 1 ad $\widehat{o}$ [2 SA], Kokodo River, 19 Apr. 1952, leg. H. de Saeger et al. (RBINS: 13561.1, 13561.2); 1 juv [AL], Kokodu, 19 Apr. 1952, leg. H. de Saeger (RMCA: 36604); $1 \hat{\delta}[$ AL], Kumbi, 17 Nov. 1993, leg. Ngenge Masumbuko Kamitongo (ZMA: MAM.24898); 1 ad $\widehat{\sigma}$ [SA], Luluabourg [= Kananga], 22 Apr. 1964, leg. Antoon Emeric Marcel De Roo (RMCA: 33600);

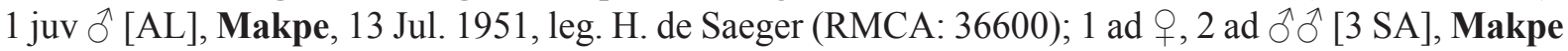
River, upper [= Makpe], 13 Jul. 1951, leg. H. de Saeger et al. (RBINS: 13552.1, 13552.2, 13553); 2 ad 웅, 1 juv ? [1 SA, 2 SK], Maleli, 12 Apr. 1951, 18 Mar. 1952, leg. H. de Saeger et al. (RBINS: 13551, 13555.1, 13555.2); 1 + , 1 ○, 1 ? [3 AL], Masako, unknown date, 16 Jul. 1989, leg. Ndey B. Ifuta (RMCA: 93.079-M-0345, 93.079-M-0343, 93.079-M-0344); 1 ad ổ [AL], Meridi River, 20 Apr. 1952, leg. H. de Saeger (RMCA: 36605); 1 +, 1 ? [2 UN], Mongbwalu, 21 Jan. 2011, leg. unknown collector

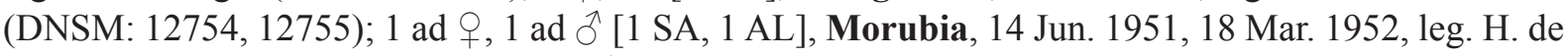
Saeger (RMCA: 36601, 36603); 1 ad $\partial^{\lambda}, 1$ ? [1 SA, 1 UN], Nakpanga, unknown date, leg. unknown collector, 18 Apr. 1952, leg. H. de Saeger (INPBC: 4779.2.3, RMCA: 36608); 1 ad $q$ [SA], Nakpanga River, 18 Apr. 1952, leg. H. de Saeger et al. (RBINS: 13564); 1 ad ${ }^{2}$ [SA], Pawa, between 1 Jan. 1931 and 31 Dec. 1931, leg. Radna (RMCA: 15314); 1 ad $q$ [SA], PFNK, 7 Feb. 1952, leg. H. de Saeger (RMCA: 36602); 1 ad + [SA], PFNK.15, 7 Feb. 1952, leg. H. de Saeger et al. (RBINS: 13557); 2 ad

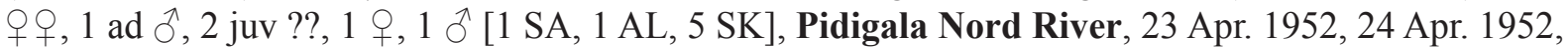
leg. H. de Saeger et al. (RBINS: 13558.1, 13558.2, 13560, 14258B, 19258A, 13559.1, 13559.2); 2 ad 웅, 1 ad $\lesssim$ [3 SA], Pidigala River, 23 Apr. 1952, 24 Apr. 1952, leg. H. de Saeger (RMCA: 36606, 36607, 36609); 1 ad + , 1 ad $\widehat{~}, 1$ ad ? [1 SS, 2 SA], PPK.10, 2 Aug. 1952, leg. H. de Saeger et al. 
(RBINS: 13562, 13567.1, 13567.2); $1 \hat{\jmath}$ [UN], Rubi-Tele, 24 Aug. 2013, leg. Guy-Crispin Gembu Tungaluna, Prescott Musaba and André Malekani (UNIKIS: RBTL554); 1 ? [AL], Tshakala, between 1 Jan. 1982 and 31 Dec. 1982, leg. Marc Colyn (RMCA: 82.019-M-0011); 2 ?? [2 AL], Wafanya [=Wafania], 15 Apr. 1988, 21 Apr. 1992, leg. P. Lootens (RMCA: 89.020-M-0042, 92.079-M-0118); 1 ad + [SA], Wilibadi River, 30 Jan. 1951, leg. H. de Saeger et al. (RBINS: 13550); $1 \curvearrowright$ [SA], Yalosemba, 30 Jun. 1979, leg. Frieder Meier (MHNG: 1870.097).

Nycteris parisii (de Beaux, 1924)

DEMOCRATIC REPUBLIC OF THE CONGO: 1 ? [SS], Né, Mount, 29 Sep. 1952, leg. unknown collector (RBINS: 21668).

Nycteris thebaica E. Geoffroy St.-Hilaire, 1818

DEMOCRATIC REPUBLIC OF THE CONGO: 1 ? [UN], Albert National Park [= Virunga National Park], unknown date, leg. unknown collector (INPBC: V1804); 1 ad $q$ [SA], Bulaga [= Kasenga Road, km 36], 26 Mar. 1956, leg. Félix / Michel Anciaux de Faveaux (RMCA: 26230); 1 ad $\widehat{\jmath}$ [SA], Dethioux Hoeve, Grot I [= Shinkolobwe], 29 Sep. 1957, leg. Félix / Michel Anciaux de Faveaux (MHNG: 1046.036); 7 우, 3 $\widehat{\jmath}, 1$ ? [11 AL], Kadjidji [= Kajuju], unknown date, leg. Guy Babault

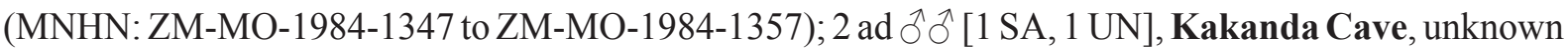
date, between 21 Apr. 1955 and 22 Apr. 1955, leg. R.P. Th. de Caters (BMNH: 1955.146, RMCA: 22890); 3 ad 우 [3 SS], Kakyelo, 13 Nov. 1930, leg. Gaston-François de Witte and de Crawhez (RMCA: 10844, 10846, 10847); 1 ad $\widehat{\jmath}$ [SO], Kasenga Road, km 67, 8 Jun. 1956, leg. M. Lips (RMCA: 23798); 1 ad

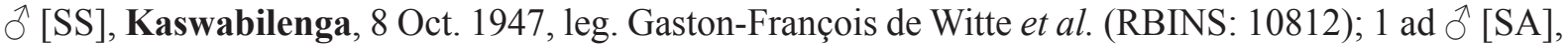
Lubudi-Lusolo, 16 Jul. 1962, leg. Félix / Michel Anciaux de Faveaux (SMF: 21243); 1 ? [AL], Lukatu Cave [= Ntoto Cave], prior to 2 Feb. 2007, leg. A. Kimbembi-ma-Ibaka (RMCA: a7.006-M-0001); 1 ad $\widehat{\delta}$ [UN], Mahagi Port, unknown date, leg. J. Heck (BMNH: 1954.797); 1 ad ? [SA], Makayova River, lower, unknown date, leg. Jacques Verschuren (INPBC: V1345); 1 ad § [SA], Mount Ne [= Né,

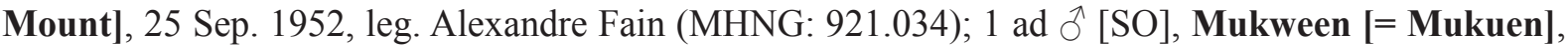
12 Jul. 1956, leg. M. Lips (RMCA: 23797); 1 ad ? [SS], Né, Mount, 25 Sep. 1952, leg. Alexandre Fain (BMNH: 1953.485); 1 ad ? [AL], Rutshuru, unknown date, leg. Jacques Verschuren (INPBC: V1354); 1 ad 9,2 ad ?? [3 AL], Sinda River, unknown date, leg. Jacques Verschuren (INPBC: V481.1 to V481.3);

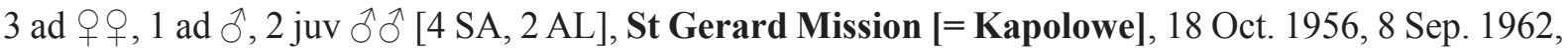
leg. Félix / Michel Anciaux de Faveaux (RMCA: 26234 to 26237, SMF: 16878, 21244); 1 [UN], Tshambi, 10 Nov. 1933, leg. unknown collector (RBINS: 4781); 1 ad ? [SA], Tumbwe, unknown date, leg. Jacques Verschuren (INPBC: V1436); 2 우, 3 $\widehat{\partial}$ [ [5 AL], Yalosemba, 17 Jun. 1979, 22 Jun. 1979, leg. C. Brian Robbins (USNM: 463477 to 463481 ).

RWANDA: 1 ad,+ 1 ad $ð$ [2 SS], Astrida [= Butare], 25 Oct. 1966, 25 Dec. 1966, leg. Félix / Michel Anciaux de Faveaux (RBINS: 16005, 16006); 1 ? [UN], Kagera National Park [= Akagera Park], unknown date, leg. unknown collector (INPBC: V1904); 1 ad $\widehat{\jmath}$ [SA], Kakitumba, unknown date, leg. Jacques Verschuren (INPBC: V3131); 1 sad , 1 juv $\hat{~}$ [2 SA], Kidaho, 10 Jun. 1982, leg.

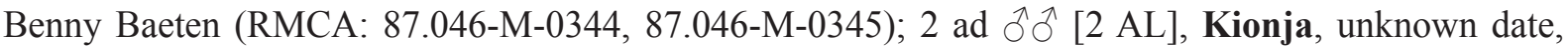
leg. Jacques Verschuren (INPBC: V3085A, V3085B); 1 ? [UN], Kisenyi [= Gisenyi], unknown date,

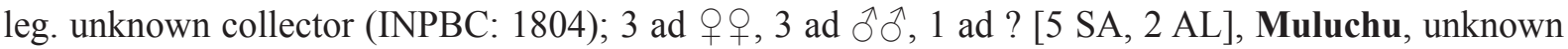
date, leg. Jacques Verschuren (INPBC: V3146A to V3146G); 1 ad $\&$ [SA], Rukira, 22 Nov. 1981, leg. Frits De Vree, Benny Baeten and Victor Van Cakenberghe (RMCA: 82.006-M-0930); 2 ad $\lesssim \widehat{~}$ [2 SA], Shangi Schad [= Shangi], 5 Jan. 1985, leg. Benny Baeten and Luc Janssens (RMCA: 85.006-M-0455, 85.006-M-0456); 1 neonate $\widehat{\jmath}$ [UN], Simbi Cave, 6 Jul. 1968, leg. unknown collector (RBINS: 15936). 
Molossidae Gervais, 1856

DEMOCRATIC REPUBLIC OF THE CONGO: 7 ad $q+$, 1 sad $\curvearrowright$, 96 ?? [104 AL], unknown locality, unknown date, prior to 18 Aug. 1965, between 1 Jun. 1964 and 30 Jun. 1964, leg. Antoon Emeric Marcel De Roo, between 1 Jan. 1976 and 31 Dec. 1977, leg. M. Lejeune (RMCA: 32780, 32782, 32785, 32786, 77.029-M-0040 to 77.029-M-0135, 32784, 33569 to 33571); 1 ? [SO], Butembo, between 1 Nov. 1971 and 30 Nov. 1971, leg. M. Lejeune (RMCA: 81.049-M-0002); 1 ? [SN], Cel II, 19 May 1952, leg. H. de Saeger (RMCA: 36936); 1 ? [AL], Epulu, unknown date, leg. Luc Bijnens (RMCA: 90.031-M-0003); 1 đ [AL], Farm 'des Grands Bois' [= Likasi], 13 Nov. 1962, leg. Félix / Michel Anciaux de Faveaux (RMCA: 33985); 8 우, 1 §ૈ, 1 ? [10 AL], Ganda-Sundi, 31 Mar. 1961, 31 Mar. 1964, leg. Alexandre Fain (RMCA: 34195, 33263, 33266, 33267, 33269, 33270, 33272 to 33275); 1 ? [AL], Imbonga, between 1 Jan. 1987 and 31 Dec. 1987, leg. P. Lootens (RMCA: 87.043-M-0002); 1 ? [SS], Ituri ht, prior to 28 Apr. 1914, leg. Cuthbert Christy (RMCA: 3387); 1 ? [UN], Kabongo, prior to 27 Oct. 1952, leg. M. Dierckx (RMCA: 21481); 8 ?? [8 AL], Kamponde, between 1 Apr. 1965 and 30 Apr. 1965, leg. Antoon Emeric Marcel De Roo (RMCA: 33372 to 33379); 2 ad+foetus 우 [2 AL], Keyberg, 9 Feb. 1956, leg. Félix / Michel Anciaux de Faveaux (RMCA: 90.011-M-0001, 90.011-M-0002); 1 त [AL], Kinshasa, 1 Apr. 1987, leg. WHO and Marc Colyn (RMCA: 88.011-M-0027); 2 ad+foetus $o+$ 운 [2 AL], Kipushi, 15 km from [= Wasseige Farm], 6 Feb. 1956, leg. Félix / Michel Anciaux de Faveaux

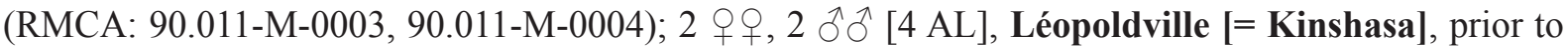
15 Jul. 1964, leg. Antoon Emeric Marcel De Roo and Jan Deheegher (RMCA: 32636 to 32639); 3 ?? [3 SS], Lovanium University [= Kinshasa], 1 Dec. 1962, 11 Jan. 1963, 23 Apr. 1963, leg. De Bont and Jo Van Orshoven (RMCA: 32633, 32635, 32634); 85 ?? [85 AL], Lulimbi, between 1 Jan. 1976 and 31 Dec. 1977, leg. M. Lejeune (RMCA: 77.029-M-0029 to 77.029-M-0039, 77.029-M-0136 to 77.029-M-0209); 5 q,+ 5 ठิ $\widehat{~}$ [10 AL], Luluabourg [= Kananga], unknown date, leg. Jan Deheegher, 24 Oct. 1964, 3 Jul. 1965, prior to 6 May 1964, leg. Antoon Emeric Marcel De Roo (RMCA: 31561 to $31565,31570,33350,33593,33594,31549)$; 1 ? [AL], Makaw, prior to 15 Nov. 1958, leg. E. Jans (RMCA: 27499); 2 ?? [1 SK, 1 SO], Make, 1 Aug. 1989, between 1 Jan. 1992 and 31 Dec. 1992, leg. Tshinyenye (RMCA: 89.057-M-0003, 92.148-M-0004); 8 ?? [8 AL], Masako, unknown date, leg. Ndey

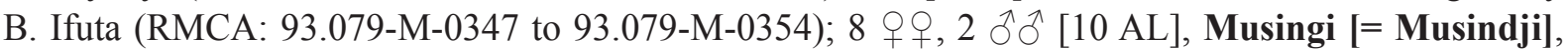
between 1 Jan. 1972 and 31 Dec. 1972, leg. André Guissart (RMCA: 38521 to 38530); 1 đ [AL], Nord-Kivu Province, prior to 28 Apr. 1974, leg. M.J. Celis and M. Lejeune (RMCA: 36198); 1 त [AL], Uvira, 5 Sep. 1956, leg. Laurent (RMCA: 35693); 1 ? [AL], Wagenia, between 1 Jan. 1987 and 31 Dec. 1988, leg. Luc De Vos, Kimbembi and Alain Vandelanoote (RMCA: 88.025-M-0008).

RWANDA: 1 ภ , 1 ? [1 AL, 1 SO], Astrida [= Butare], between 1 Apr. 1952 and 30 Apr. 1952, leg. Laurent, between 1 Jul. 1951 and 31 Jul. 1951, leg. Aureliaan (RMCA: 79.010-M-0007, 20568); 1 q [AL], Ratabo Kinazi Gitorams, 30 Apr. 1971, leg. A. Elbl (RMCA: 35915).

\section{Chaerephon Dobson, 1874}

BURUNDI: 2 ふぇં [2 AL], Mweya, 29 Apr. 1971, leg. P.L. Niort (MHNG: 1896.019, 1896.020).

DEMOCRATIC REPUBLIC OF THE CONGO: 17 q,+ 2 $ぇ$ đ [15 AL, 4 SK], Kisantu, 3 Jun. 2012, leg. William ("Bill") T. Stanley (FMNH: 219519 to 219532, 219537 to 219541); 1 ? [SA], Léopoldville [= Kinshasa], unknown date, leg. Cuthbert Christy (BMNH: 1904.6.25.1); 1 q [SS], Upper Congo, unknown date, leg. Alexander Douglas Mitchell Carruthers (BMNH: 1907.6.14.6).

\section{Chaerephon aloysiisabaudiae (Festa, 1907)}

DEMOCRATIC REPUBLIC OF THE CONGO: 1 imm đ̊ [SS], Avakubi, 28 Dec. 1913, leg. Herbert Lang, James Paul Chapin and The American Museum Congo Expedition (AMNH: 48934); 1 ¿ [SS], 
VAN CAKENBERGHE V. et al., The bats of Congo, Rwanda and Burundi revisited

Eala, 2 Jan. 1921, leg. Henri Schouteden (RMCA: 6565); 1 ô [UN], Iso.4 [= Iso], 4 Mar. 1952, leg. H. de Saeger et al. (RBINS: 13826); 1 ठ [SS], Yalosemba, 17 Jun. 1979, leg. C. Brian Robbins (USNM: 537737).

\section{Chaerephon ansorgei (Thomas, 1913)}

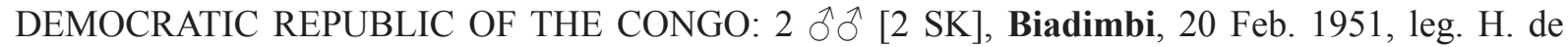

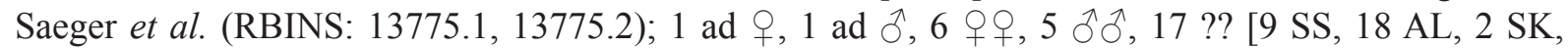
1 SO], Faradje, 1 Apr. 1952, leg. H. de Saeger et al., 9 Mar. 1912, 20 Feb. 1913, leg. Herbert Lang, James Paul Chapin and The American Museum Congo Expedition, 20 Feb. 1913, leg. Herbert Lang (RBINS: 13778.1, 13778.2, AMNH: 48902 to 48907, 48909, 49231 to 49234, 49236, 49238 to 49243, 49245 to 49249, FMNH: 43865, 43866, MCZ: 17220, RMCA: 12385, AMNH: 49264); 1 q [SK], Garamba National Park, 1 Jan. 1950, leg. H. de Saeger et al. (RBINS: 13780); 1 q [AL], Jadotville [= Likasi], 25 May 1959, leg. Félix / Michel Anciaux de Faveaux (RMCA: 28210); 25 ad 우, 26

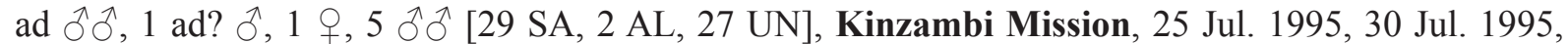
31 Jul. 1995, leg. Herwig Leirs et al. (MSB: 274752 to 274754, 274756, RMCA: 97.021-M-0581 to 97.021-M-0585, 97.021-M-1173, MSB: 274757, 274758, 274760 to 274780, RMCA: 97.021-M-0586 to 97.021-M-0610); 1 ? [SA], Lake Kivu, near, between 1 Jan. 1935 and 31 Dec. 1935, leg. Guy Babault (MCZ: 46156); 1 ad,+ 1 ad $\widehat{\partial}$ [2 SA], Mbalaka (Plantation), 21 Jul. 1995, between 10 Jun. 1995 and 28 Aug. 1995, leg. Herwig Leirs et al. (RMCA: 97.021-M-0580, 97.021-M-0611); 1 ㅇ, 1 đ , 6 ?? [2 SS, 6 SK], Mpaza, 24 Jan. 1952, leg. H. de Saeger et al., 24 Jan. 1952, leg. H. de Saeger (RBINS: 13776.1 to 13776.6, RMCA: 36891, 36892); 2 우, 2 ๙ึ $\widehat{~}$ [4 AL], Muradingusha [= Mwadingusha], unknown date, leg. the Royal Museum for Central Africa (RMCA) (BMNH: 1959.502 to 1959.505); 14 우, 8

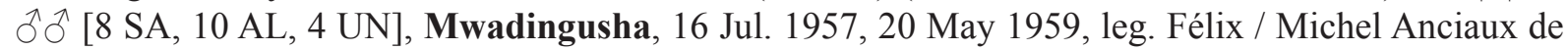
Faveaux (MHNG: 1046.025 to 1046.028, RMCA: 26757 to 26772, 28208, 28209); 31 ad $q$ q , 30 ad đo ${ }^{\lambda}$ [28 SA, 2 SK, 31 UN], Sacred Heart Mission, 2 Aug. 1995, leg. Herwig Leirs et al. (MSB: 274721 to 274726, 274781 to 274805, RMCA: 97.021-M-0612 to 97.021-M-0641); 4 ?? [1 SS, 3 SK], Uduku, 24 Jul. 1952, leg. H. de Saeger et al., 24 Jul. 1952, leg. H. de Saeger (RBINS: 13779.1 to 13779.3, RMCA: 36893).

RWANDA: 1 ad $\precsim$ [SK], Gabiro, 19 Jan. 1938, leg. unknown collector (RBINS: 4907); 2 +q, 9 ठぇ [11 SS], Rukara, between 27 Aug. 1955 and 31 Dec. 1955, between 16 Sep. 1955 and 31 Dec. 1955, between 15 Sep. 1955 and 31 Dec. 1955, leg. Alexandre Fain (RMCA: 81.012-M-0050 to 81.012-M0057, 81.012-M-0049, 81.012-M-0058, 81.012-M-0059).

Chaerephon bemmeleni (Jentink, 1879)

DEMOCRATIC REPUBLIC OF THE CONGO: 1 ? [UN], Lume River, near, unknown date, leg. unknown collector (INPBC: V857); 1 9 [SS], Rutshuru, 16 Jan. 1937, leg. J. Ghesquière (RMCA: 13826).

Chaerephon bivittatus (Heuglin, 1861)

DEMOCRATIC REPUBLIC OF THE CONGO: $1 \mathrm{imm}$ ? [SS], Bitshumbi [= Vitshumbi], prior to 22 Dec. 1943, leg. Gaston-François de Witte (RMCA: 17076).

Chaerephon chapini J.A. Allen, 1917

DEMOCRATIC REPUBLIC OF THE CONGO: 1 đ [UN], Banana, between 1 Jun. 1948 and 30 Jun. 1948, leg. unknown collector (RBINS: 14535); 1 ad ô, 1 ? [1 SS, 1 SA], Faradje, 6 May 1910, 11 Nov. 1912, leg. Herbert Lang, James Paul Chapin and The American Museum Congo Expedition (AMNH: 48841 [holotype Choerephon (Lophomops) chapini J.A. Allen, 1917], 49209); 1 [UN], Léopoldville [= Kinshasa], 15 Mar. 1964, leg. unknown collector (RBINS: 14536). 
RWANDA: 1 [ [UN], Masha, 1 Jan. 1956, leg. unknown collector (RBINS: 22466).

Chaerephon gallagheri (Harrison, 1975)

DEMOCRATIC REPUBLIC OF THE CONGO: 1 ad $\partial$ [UN], Scierie Forest, 14 Nov. 1974, leg. Michael D. Gallagher (BMNH: 1976.207 [holotype Tadarida (Chaerephon) gallagheri Harrison, 1975]).

Chaerephon leucogaster (A. Grandidier, 1869)

DEMOCRATIC REPUBLIC OF THE CONGO: 6 우, $6 \hat{\jmath} \widehat{\partial}[1 \mathrm{SS}, 11 \mathrm{AL}]$, Boende, 27 Jun. 2013, 4 Jul. 2013, 6 Jul. 2013, leg. William ("Bill”) T. Stanley (FMNH: 222431 to 222434, 222440 to 222443, 222472 to 222474, 222629); 1 ad Oे, 1 ô, 2 ?? [1 SS, 2 SA, 1 AL], Boma, 25 Jan. 1915, 26 Jan. 1915, 29 Jan. 1915, 15 Jun. 1915, leg. Herbert Lang, James Paul Chapin and The American Museum Congo Expedition (AMNH: 49259, 49237, 48844 [holotype Chcerephon (Lophomops) cristatus J.A. Allen, 1917], 49216); 1 ? [SA], Lukolela, 27 Sep. 1930, leg. James Paul Chapin (AMNH: 86949); 1 ad q, 2 오, 22 ?? [9 SA, 16 AL], Malela, 6 May 1910, 8 Jul. 1915, leg. Herbert Lang, James Paul Chapin and The American Museum Congo Expedition (AMNH: 49210 to 49212, 49214, 49215, 49217, 49218, 49220, 49221, 49223, 49226, 49227, 49229, 49261, 49263, 49268 to 49271, 49273, 49274, 49275 [holotype Charephon frater J.A. Allen, 1917], 49276, MCZ: 17219 [topotype Charephon frater J.A. Allen, 1917], 17221 [topotype Charephon frater J.A. Allen, 1917]).

Chaerephon major (Trouessart, 1897)

DEMOCRATIC REPUBLIC OF THE CONGO: 1 ad $q, 16$ 우, 5 §ð, 11 ?? [20 SS, 11 SK, 2 SO], Aba, 12 Dec. 1910, 13 Dec. 1911, 15 Dec. 1911, 16 Dec. 1911, 17 Dec. 1911, leg. Herbert Lang, James Paul Chapin and The American Museum Congo Expedition, 15 Dec. 1911, leg. unknown collector (AMNH: 48867, 48869 to 48872, 51257 to 51267, MCZ: 17389, RMCA: 12365, AMNH: 48873, 48840, 48875 to 48886, 48887 [holotype Chaerephon (Lophomops) abce J.A. Allen, 1917]); 3 ๙ $\widehat{~}$ [1 AL, 2 UN], Biadimbi, 20 Feb. 1951, leg. H. de Saeger et al., 20 Feb. 1951, leg. H. de Saeger (RBINS: 13783.1, 13783.2, RMCA: 36895); 1 ad $ᄋ$, 4 우 [4 SS, 1 UN], Faradje, 25 Feb. 1911, 7 Mar. 1911, leg. Herbert Lang, James Paul Chapin and The American Museum Congo Expedition, 7 Mar. 1911, leg. unknown collector (AMNH: 48836, 52119, RMCA: 12364, AMNH: 48838, MCZ: 17388); 1 § [UN], Mbandaka, prior to 31 Dec. 1970, leg. unknown collector (RBINS: 22467); 4 + 9 [1 SS, 3 AL], Nagera [= Nagero], 24 Mar. 1952, leg. H. de Saeger (RMCA: 36894, 36896 to 36898); 1 ㅇ [SO], Nagero, 11 Mar. 1954, leg. unknown collector (RBINS: 14630); 1 ㅇ [SA], Rwindi, 2 Sep. 1976, leg. Frieder Meier (MHNG: 1874.059); 1 †, 11 ठิ $\widehat{~[5 ~ S S, ~} 7$ AL], Tandala, 12 Jul. 1979, 13 Jul. 1979, 21 Jul. 1979, leg. unknown collector, 12 Jul. 1979, 21 Jul. 1979, leg. C. Brian Robbins (MHNG: 1870.098, USNM: 463518 to 463521,537748 to 537750, MHNG: 1870.099 , 1870.100, 1871.001, USNM: 537752).

\section{Chaerephon nigeriae Thomas, 1913}

DEMOCRATIC REPUBLIC OF THE CONGO: $1 \mathrm{imm}$ ?, 1 q $[1 \mathrm{AL}, 1 \mathrm{UN}]$, Elisabethville [= Lubumbashi], 1 Jun. 1969, leg. unknown collector, between 1 Apr. 1926 and 30 Apr. 1926, leg. Henri Schouteden (RBINS: 18511, RMCA: 16191); 1 , 2 กิ $\widehat{\text {, }, ~} 1$ ? [4 SS], Gangala-na-Bodio, 8 Apr. 1945, 13 Nov. 1947, leg. unknown collector (RBINS: 12952, 12949 to 12951); 1 ㅇ, 2 ठぇ [2 SA, $1 \mathrm{AL}$ ], Kabongo, unknown date, leg. the Royal Museum for Central Africa (RMCA), prior to 27 Oct. 1952, leg. M. Dierckx (BMNH: 1954.836, RMCA: 21480, 21482); 2 ad 우 ㅇ [1 SA, 1 AL], Katanga, unknown date, leg. Félix / Michel Anciaux de Faveaux (MNHN: ZM-MO-1957-132 A, ZM-MO-1957-132 B); $1 \mathrm{imm}$ +, $1 \mathrm{imm}$ ?, 4 우, 3 ?? [1 SA, $8 \mathrm{AL}$ ], Kinke, unknown date, leg. the Royal Museum for Central Africa (RMCA), 23 Dec. 1955, leg. Félix / Michel Anciaux de Faveaux, 25 Oct. 1955, 23 Dec. 1955, leg. M. Lips (BMNH: 1957.434, MHNG: 923.008, RMCA: 26288, 26291 to 26295, 26289); 1 ○ [SS], Kisiushi, 30 Jul. 1956, leg. M. Lips (RMCA: 33279); 4 ?? [4 AL], Kitubulushi, 3 Aug. 1954, leg. 
De Smet (RMCA: 22462 to 22465); 1 đ [AL], Musonge, 29 Dec. 1955, leg. M. Lips (RMCA: 26290); 1 [UN], Musosa, near, between 1 Jan. 1941 and 31 Dec. 1941, leg. unknown collector (RBINS: 14534); 1 sad $\lesssim$ [SS], Niangara, 10 Dec. 1910, leg. Herbert Lang, James Paul Chapin and The American Museum Congo Expedition (AMNH: 48842); 3 q $ᄋ$ [3 AL], Sakania, unknown date, leg. the Royal Museum for Central Africa (RMCA) (BMNH: 1954.837 to 1954.839); 1 juv $\hat{\jmath}$ [SA], Shaba (former) Province [= Katanga (former) Province], 23 Dec. 1955, leg. unknown collector (SMF: 16881); 1 ㅇ, $1 \lesssim$ [1 SS, 1 AL], Tandala, 12 Jul. 1979, 21 Jul. 1979, leg. C. Brian Robbins (USNM: 537751, 463522).

\section{Chaerephon pumilus (Cretzschmar, 1826)}

BURUNDI: 1 ? [AL], Bujumbura, 10 Nov. 1968, leg. Lewall (RMCA: 35491); 1 ? [AL], Bururi, between 24 May 1956 and 30 May 1956, leg. Laurent (RMCA: 26564); 1 ad $\widehat{\partial}$ [AL], Imbo, unknown date, leg. Jacques Vissault (MNHN: ZM-MO-1996-1539); 3 ?? [3 SA], Kinazi, between 5 Jan. 1953 and 8 Jan. 1953, leg. P. Basilewsky (RMCA: 21936 to 21938); 1 \& [SK], Lake Cohoha [= Cohoha, Lake], 9 Mar. 1979, leg. Heinz Stephan (SMF: 57559); 1 ? [SA], Nyankende, between 11 Mar. 1952 and 14 Mar. 1952, leg. Laurent (RMCA: 79.010-M-0010); 2 ad 우, 4 우, 1 ઈ [4 SA, 3 SK], Resha, 1 Mar. 1979, 15 Mar. 1979, 2 Apr. 1979, 3 Apr. 1979, leg. Heinz Stephan (SMF: 57560 to 57566); 1 ? [AL], Russissi, bank [= Rusizi Delta], 16 Nov. 1968, leg. Lewall (RMCA: 35492); 4 ?? [4 UN], Usumbura [= Bujumbura], 1 Aug. 1976, leg. unknown collector (RBINS: 19926.1 to 19926.4).

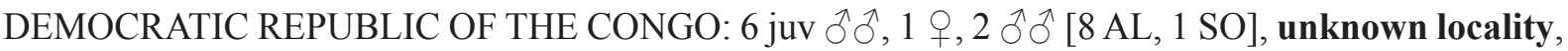
between 16 Jan. 1956 and 19 Jan. 1956, leg. J.J. Laarman, prior to 31 Dec. 1889, leg. P. Hesse (RMNH: MAM.16373, SMF: 34273 to 34280); 2 ?? [2 UN], Albert National Park [= Virunga National Park], unknown date, leg. unknown collector (INPBC: V1805, V1844); 1 §, 2 ?? [2 AL, 1 UN], Albertville [= Kalemie], 19 Sep. 1947, leg. unknown collector, prior to 18 Nov. 1959, leg. H. Bomans (RBINS: 9071, RMCA: 29305, 29306); 3 juv ??, 29 ?? [28 AL, 4 SK], Banana, 13 Mar. 1952, 12 Apr. 1952, between 1 May 1952 and 31 May 1952, leg. Is. Mesmaekers, prior to 31 Dec. 1889, leg. P. Hesse (RMCA: 20765 to 20778, 20753 to 20760, 21378 to 21383, SMF: 2527 to 2530); 1 ? [AL], Bas Congo (former) Province [= Kongo Central Province], unknown date, leg. Alphonse Cabra (RMCA: 66); 2 ?? [2 UN], Beno, between 1 Jan. 1949 and 31 Dec. 1949, leg. P. Bayet (RMCA: 19824, 19825);

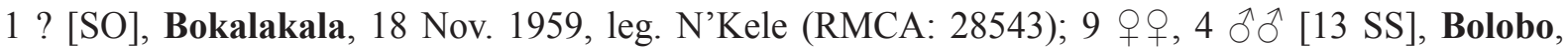
27 Mar. 1921, leg. Henri Schouteden, prior to 23 Jul. 1946, leg. N'Kele (RMCA: 6577, 6581 to 6587, 7252 to 7254, 7308, 17403); 1 juv ?, 5 우, 1 §, 39 ?? [9 SS, 33 AL, 2 SO, 2 UN], Boma, unknown date, 23 Nov. 1920, 24 Nov. 1920, 25 Nov. 1920, between 1 Aug. 1920 and 31 Aug. 1920, between 1 Jan. 1922 and 31 Dec. 1933, between 1 Jan. 1922 and 31 Dec. 1922, leg. Henri Schouteden, between 1 Aug. 1928 and 31 Aug. 1928, leg. Gerard, between 1 Sep. 1954 and 30 Sep. 1954, leg. Cassart, prior to 26 May 1933, leg. Colsoulle, prior to 4 Aug. 1937, leg. Dartevelle (RMCA: 16491, 6598, 6604, 6605, 7301 to $7303,7251,16234,16235,16253,7719$ to $7722,16202,16269,16270,22388,12128$ a to $12128 \mathrm{j}$, 12129a to 12129m, 13959 to 13962); 5 ?? [5 AL], Bondo, between 1 Nov. 1957 and 30 Nov. 1957, leg. Roland (RMCA: 26918 to 26922); 1 ? [UN], Bongeya River, unknown date, leg. unknown collector (INPBC: V264.4); 1 ? [AL], Boteka, 31 Mar. 1985, leg. P. Lootens (RMCA: 85.030-M-0002); 18 ?? [17 AL, 1 UN], Bukama, unknown date, prior to 8 Apr. 1939, between 1 Jun. 1939 and 30 Jun. 1939, between 1 Jun. 1937 and 30 Jun. 1937, leg. Brien (RMCA: 16254, 15261, 15251 to 15260, 15262 to 15267); 3 ?? [3 UN], Bumali, unknown date, leg. unknown collector (INPBC: W138.01 to W138.03); 1 ? [AL], Busu Mokalu [= Busu-Mokala], between 5 Nov. 1938 and 10 Nov. 1938, leg. Baudart (RMCA: 18097); 1 ô, 2 ?? [1 SS, 2 AL], Eala, unknown date, leg. Mayné, 3 Jan. 1921, leg. Henri Schouteden, between 1 Mar. 1921 and 31 Mar. 1921, leg. unknown collector (RMCA: 5392, 6603, 26285); 1 ? [AL], Elisabethville [= Lubumbashi], between 1 Jan. 1933 and 31 Dec. 1933, leg. Kerkvoorde (RMCA: 12140); 3 foetus ??, 9 ?? [12 AL], Equateur Province, between 1 Jan. 1921 and 31 Dec. 1921, leg. Verlaine (RMCA: 88.044-M-0001 to 88.044-M-0012); 4 ?? [2 SA, 2 AL], Ganda- 
Sundi, 25 Mar. 1964, 31 Mar. 1964, leg. Alexandre Fain (RMCA: 33278, 33264, 33265, 33271); 1 ? [AL], Irangi, 8 Feb. 1984, leg. Heinz Stephan (SMF: 65039); 134 ?? [34 AL, 100 UN], Ishango River, unknown date, leg. unknown collector, between 1 Jan. 1982 and 31 Dec. 1982, leg. Marc Colyn, between 26 Dec. 1968 and 29 Dec. 1968, leg. M. Lejeune (INPBC: V1176.043 to V1176.142, RMCA: 82.031M-0002 to 82.031-M-0004, 35460 to 35490); 1 ? [UN], Kabakuli River, unknown date, leg. unknown collector (INPBC: W253); 2 우, 1 ภ, 7 ?? [9 SS, 1 SO], Kabalo, unknown date, leg. Henri Schouteden, 17 Nov. 1926, leg. John Todd Zimmer (RMCA: 9164, 9165, 9167 to 9172, 9904, FMNH: 29429); 9 q 9 [8 SO, 1 UN], Kahitumba (Mutaka), between 16 Jan. 1956 and 19 Jan. 1956, leg. J.J. Laarman (RMNH: MAM.16374 to MAM.16382); 7 ?? [7 AL], Kalina, prior to 16 Oct. 1952, leg. Theunissen, prior to 16 Nov. 1950, leg. Jean Jacques Deheyn (RMCA: 21416, 21417, 21418a, 21418b, 21419, 21420 , 20102); 1 ? [AL], Kambukabakali, 4 Nov. 1954, leg. Gaston-François de Witte (RMCA: 37383); 1 ㅇ [SA], Kapolowe, 3 Aug. 1962, leg. Félix / Michel Anciaux de Faveaux (SMF: 21313); 1 đ [SS],

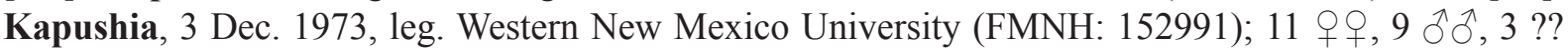
[2 SS, 1 AL, 20 UN], Kasenyi [= Kasenye], unknown date, 10 Feb. 1953, 16 Feb. 1953, 6 May 1953, 15 Jun. 1953, 26 Jun. 1953, 24 Sep. 1953, 20 Nov. 1953, 23 Nov. 1953, leg. unknown collector, 19 Feb. 1954, leg. Gaston-François de Witte, 30 Jul. 1951, leg. Alexandre Fain, prior to 16 Dec. 1949, leg. Jean Hiernaux (INPBC: W1389, RBINS: 14539, 14550, 14555, RMCA: 37382, RBINS: 14541, 14552, 14538, 14547, 14554, 14551, RMCA: 23297, RBINS: 14537, 14540, 14543 to 14546, 14549, 14553, 14556, 14557, RMCA: 19099); 5 ?? [1 SA, 4 AL], Kashiobwe, 20 Oct. 1955, leg. Van Iseghem, 30 Oct. 1955, leg. unknown collector (RMCA: 26282 to 26284, 26286, SMF: 16877); 1 juv ?, 1 o [2 AL], Kashiobwe Mission [= Kashiobwe], 20 Oct. 1955, leg. Félix / Michel Anciaux de Faveaux (RMCA: 26227, 26266); 3 ?? [3 AL], Kasongo, unknown date, leg. Van Delft, 13 Nov. 1959, leg. Segers and Benoit Mission (RMCA: 16201, 29166, 29308); 1 ? [AL], Katanga, prior to 31 Aug. 1900, leg. Charles Lemaire (RMCA: 424); 1 o, 1 ? [2 SS], Kawa (Forest), 24 Jul. 1952, between 1 Oct. 1950 and 31 Oct. 1950, leg. Alexandre Fain (RMCA: 81.012-M-0062, 23296); 1 ? [AL], Keyberg, 27 Oct. 1955, leg. M. Lips (RMCA: 26287); 3 $\widehat{\partial}$ [3 SS], Kidada, 20 Feb. 1922, 21 Feb. 1922, leg. Henri Schouteden

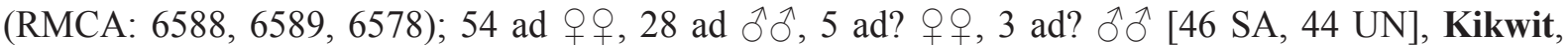
6 Jul. 1995, 12 Jul. 1995, 14 Jul. 1995, leg. Herwig Leirs et al. (MSB: 274598 to 274600, RMCA: 97.021-M-0656 to 97.021-M-0659, MSB: 274607 to 274612,274615 to 274620,274623 to 274634 , 274639 to 274642 , RMCA: $97.021-\mathrm{M}-0660$ to $97.021-\mathrm{M}-0687$, MSB: 274579 to 274583,274589 to 274593, 274595 to 274597, RMCA: 97.021-M-0642 to 97.021-M-0655); 1 ad $\odot$ [UN], Kikwit ITPK [= Kikwit], 1 Aug. 1995, leg. Herwig Leirs et al. (MSB: 274882); 16 ad 9 우, 6 ad $\widehat{\partial} \widehat{\partial}, 1$ ad? $\hat{\jmath}, 4$ 우, 3 ๙ิ่ [17 SA, 13 UN], Kinzambi Mission, 24 Jul. 1995, 25 Jul. 1995, 26 Jul. 1995, 27 Jul. 1995, 28 Jul. 1995, 30 Jul. 1995, leg. Herwig Leirs et al. (MSB: 274858 to 274861, RMCA: 97.021-M-0688 to 97.021-M-0693, MSB: 274863, 274864, RMCA: 97.021-M-0694, 97.021-M-0695, MSB: 274865 to 274867, RMCA: 97.021-M-0718 to 97.021-M-0720, MSB: 274869, RMCA: 97.021-M-0721, 97.021M-0722, MSB: 274870 to 274872, RMCA: 97.021-M-0723 to 97.021-M-0726); 2 우, 8 ठึ 1 AL, 2 SO], Kisantu, 11 Dec. 1920, 12 Dec. 1920, 15 Dec. 1920, 18 Dec. 1920, 19 Dec. 1920, leg. Henri Schouteden, prior to 7 Nov. 1932, leg. R.P. Vanderijst (RMCA: 7300a, 6606, 6599 to 6602, 7306, 7304, 7305, 7307, 11956); 1 ? [AL], Kivu region, between 1 Jan. 1962 and 31 Dec. 1962, leg. unknown

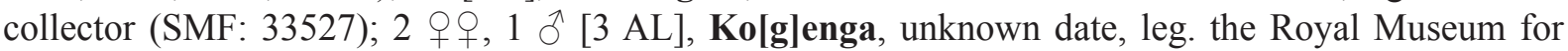
Central Africa (RMCA) (BMNH: 1954.831 to 1954.833); 2 ?? [2 AL], Kondue [River], unknown date,

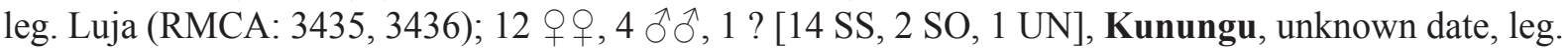
unknown collector, unknown date, prior to 6 Aug. 1925, prior to 25 Mar. 1929, prior to 1 Sep. 1924, between 1 Jan. 1922 and 31 Dec. 1922, leg. Ngwe, between 1 Jan. 1922 and 31 Dec. 1922, leg. Henri Schouteden, prior to 10 Dec. 1932, prior to 1 Aug. 1939, leg. N'Kele (RMCA: 7437 to 7439, 7659, 7256, 7751, 15516, 7658, 12002, 9709, 9710, 8166 to 8171); 1 ? [AL], Lemba Plain, 3 Jul. 1929, leg.

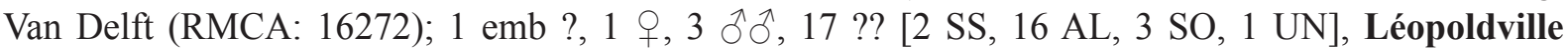
[= Kinshasa], unknown date, leg. J. Camp, 15 Jan. 1919, 30 Sep. 1941, between 1 Mar. 1942 and 31 Mar. 1942, between 1 Jan. 1942 and 31 Jan. 1942, leg. R. Fiasse, 4 Apr. 1964, leg. Antoon Emeric 
Marcel De Roo, prior to 12 Mar. 1913, leg. Cuthbert Christy, prior to 23 Jul. 1946, leg. Lepersonne (USNM: 21668 to 21675, RMCA: 18125, 18126, 17657, 17658, 32658, 17660, 17659, 1532a, 1532b, 1533a, 1533b, 1534, 1535, 18130); 1 ? [SS], Lovanium University [= Kinshasa], unknown date, leg. De Bont (RMCA: 31202); 23 ?? [23 SA], Lulimbi, between 1 Jan. 1976 and 31 Dec. 1977, leg. M. Lejeune (RMCA: 77.029-M-0006 to 77.029-M-0028); 3 के के [3 SS], Mabwe, 2 Dec. 1948, 31 Jan. 1949, leg. unknown collector, 21 Aug. 1947, leg. l' Institut des Parcs nationaux du Congo belge (RBINS: 10712, 10711, 10713); 8 우, 2 §o between 1 Jan. 1922 and 31 Dec. 1922, leg. Henri Schouteden (RMCA: 6579, 6580, 6590 to 6597, 16236 to 16242,16259 ); 1 ? [AL], Makaw, prior to 15 Nov. 1958, leg. E. Jans (RMCA: 27500); 8 ?? [8 AL], Makengo, 11 Dec. 1946, leg. Dartevelle (RMCA: 18152 to 18159); 1 ๙ [UN], Maleli, 10 Jun. 1951, leg. H. de Saeger et al. (RBINS: 13781); 1 ? [UN], Mangbwelu River, unknown date, leg. unknown collector (INPBC: W240c); 2 오 [2 UN], Manono, 1 Nov. 1974, leg. Michael D. Gallagher (HZM: 127.7821, 143.7954); 2 ?? [2 AL], Manono Katanga [= Manono], prior to 11 Dec. 1953, leg. Torfs (RMCA: 22000, 22001); 1 ad? $\odot$ [UN], Mbalaka (Plantation), 19 Jul. 1995, leg. Herwig Leirs et al. (MSB: 274827); 1 [SS], Mbuji-Mayi, 3 Nov. 1973, leg. Western New Mexico University (FMNH: 152992); 1 ad ? [UN], Mbwambala, 19 Jul. 1995, leg. Herwig Leirs et al. (MSB: 274613); 1 ? [AL], Moliro, prior to 28 Feb. 1899, leg. Charles Lemaire (RMCA: 161); 2 ad ??, 1 juv ?, 3 우, 1 o,, 24 ?? [25 SS, 3 AL, 3 UN], Mount Wago [= Wago, Mount], unknown date, 13 Sep. 1952, 16 Sep. 1952, 17 Sep. 1952, 18 Sep. 1952, 21 Sep. 1952, 23 Sep. 1952, prior to 28 Mar. 1952, between 1 Jul. 1952 and 31 Jul. 1952, between 1 Jan. 1951 and 31 Dec. 1951, leg. Alexandre Fain, 1 Nov. 1951, 17 Sep. 1952 , 1 Nov. 1952, leg. unknown collector (BMNH: 1953.493 to 1953.495, RBINS: 21653, 21654, 21656, RMCA: 81.012-M-0078, 81.012-M-0079, 81.012-M-0083, 81.012-M-0084, 81.012-M-0087 to 81.012M-0090, 81.012-M-0100, 81.012-M-0081, 81.012-M-0085, 81.012-M-0096, RBINS: 21655, RMCA: 81.012-M-0086, 81.012-M-0092, 81.012-M-0080, 81.012-M-0082, 21465, 21450 to 21454, 20786, 20787); 15 ?? [15 UN], Mulongo, 28 Oct. 1974, leg. Michael D. Gallagher (HZM: 128.7930 to 142.7944); 1 juv ô [SS], Mvuazi [= Maunzi], between 1 Sep. 1942 and 30 Sep. 1942, leg. J.M. Vrijdagh (RMCA: 17757); 2 ?? [2 AL], Nagera [= Nagero], 4 May 1951, leg. H. de Saeger (RMCA: 36889, 36890); 4 ?? [4 UN], Nagero, 4 May 1951, leg. H. de Saeger et al. (RBINS: 13782.1 to 13782.4); 1 ô, 1 ? [1 SS, 1 SO], Né, Mount, between 1 Jun. 1951 and 30 Jun. 1951, between 1 Jan. 1950 and 31 Dec. 1950, leg. Alexandre Fain (RMCA: 23298, 20509 [holotype Tadarida (Chaerephon) faini Hayman, 1951]); $1 \lesssim$ [UN], Ngeze Kidoko, 26 Sep. 1953, leg. unknown collector (RBINS: 14548); 1 ? [AL], Ngwiro, 22 Nov. 1956, leg. Gaston-François de Witte (RMCA: 37381); 3 ?? [3 SS], Nionga, 3 May 1925, leg. Gaston-François de Witte (RMCA: 9231 to 9233); 2 ?? [2 UN], Rwindi, unknown

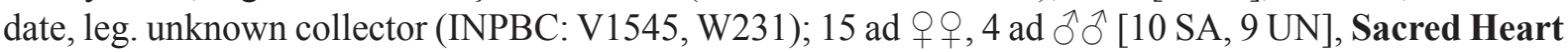
Mission, 2 Aug. 1995, leg. Herwig Leirs et al. (MSB: 274873 to 274881, RMCA: 97.021-M-0727 to 97.021-M-0736); $2 \widehat{\partial}$ ठ․ $1 \mathrm{SS}, 1 \mathrm{SO}$, St. Joseph de Luluabourg Mission [= Mikalaya], 6 May 1939, leg. Jean Jacques Deheyn, 7 Jun. 1922, leg. Richard Callewaert (RMCA: 15376, 7543); 1 ? [AL], Stanleyville [= Kisangani], prior to $31 \mathrm{Jul}$. 1899, leg. Weyns (RMCA: 283); 10 ô के, 66 ?? [76 AL], Thysville [= Mbanza-Ngungu], unknown date, leg. the Royal Museum for Central Africa (RMCA), 23 Jul. 1949, 8 Aug. 1949, 18 Aug. 1949, between 1 Aug. 1949 and 31 Aug. 1949, leg. Narcisse Leleup, between 1 Dec. 1952 and 31 Dec. 1952, leg. P. Basilewsky (BMNH: 1954.821 to 1954.830, RMCA:

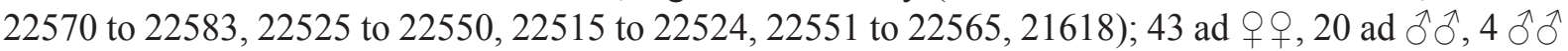
[33 SA, 34 UN], Trappist Mission, 27 Jul. 1995, 6 Aug. 1995, leg. Herwig Leirs et al. (MSB: 274806 to 274814,274883 to 274895 , RMCA: $97.021-\mathrm{M}-0696$ to $97.021-\mathrm{M}-0717$, MSB: 274815 to 274826 , RMCA: 97.021-M-0737 to 97.021-M-0747); 4 ?? [4 AL], Tshibambo [= Shibambo], 31 Aug. 1954, leg. De Smet (RMCA: 22466 to 22469); 1 [SS], Usambiers, unknown date, leg. Emin Pasha (BMNH: 1890.6.8.16); 2 ठ઼, 14 ?? [1 SS, 15 AL], Uvira, 2 Mar. 1955, 3 Mar. 1955, prior to 21 Jun. 1958, between 1 Mar. 1955 and 31 Mar. 1955, leg. Narcisse Leleup, 5 Mar. 1959, 29 Apr. 1959, leg. Uwe Rahm (RMCA: 27206, 27207, 28908, 28909, 27204, 27205, 27208, 28907, 22909, 22910, 27215 to 27219, 27221); 3 ?? [3 SS], Vaku, prior to 8 Aug. 1940, leg. C. Vleeschouwers (RMCA: 16151 to 


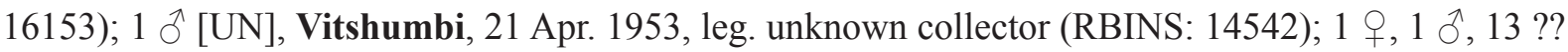
[13 SS, 2 SO], Wago, Mount, 7 Nov. 1951, 28 Aug. 1952, 30 Aug. 1952, 13 Sep. 1952, 17 Sep. 1952, between 1 Nov. 1951 and 30 Nov. 1951, between 1 Jul. 1951 and 31 Jul. 1951, between 1 Jan. 1951 and 31 Dec. 1954, between 1 Jan. 1951 and 31 Dec. 1951, leg. Alexandre Fain (RMCA: 81.012-M-0094, 81.012-M-0101, 81.012-M-0093, 81.012-M-0091, 81.012-M-0098, 81.012-M-0099, 81.012-M-0095, 81.012-M-0097, 23278, 23284, 23288, 81.012-M-0102, 23289, 23279, 23281); 1 ad 9 [SS], Wasa Wago Forest, unknown date, leg. Alexandre Fain (BMNH: 1951.629 [paratype Tadarida (Chaerephon) faini Hayman, 1951]); 1 के [UN], Zeya, 4 Aug. 1953, leg. unknown collector (RBINS: 14558).

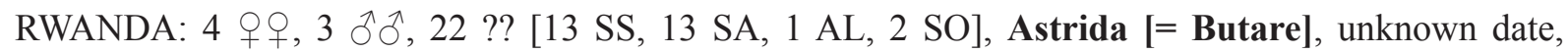
1 Jan. 1954, 29 Jul. 1954, 25 Oct. 1954, 26 Oct. 1954, 27 Oct. 1954, 28 Oct. 1954, between 1 Jan. 1954 and 31 Dec. 1954, leg. Alexandre Fain, 20 Feb. 1962, between 1 Mar. 1962 and 31 Mar. 1962, leg. Van Parijs, prior to 9 Jul. 1952, leg. P.G. Vercammen (MHNG: 921.041, RMCA: 81.012-M-0070, 81.012M-0066, 31047, MHNG: 921.039, RMCA: 81.012-M-0063, 81.012-M-0065, 81.012-M-0077, MHNG: 921.038, RMCA: 81.012-M-0064, 81.012-M-0067 to 81.012-M-0069, MHNG: 921.040, RMCA: 81.012-M-0105, 81.012-M-0106, 31061, 31063 to 31068, 31071 to 31075, 21218); 1 ? [UN], Bilalu,

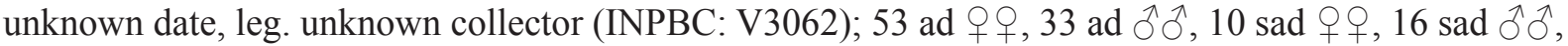
1 juv + , 1 juv $\widehat{o}$ [25 SA, 89 AL], Butare, 10 Dec. 1984, 11 Dec. 1984, 13 Dec. 1984, 20 Dec. 1984, 22 Dec. 1984, 24 Dec. 1984, 25 Dec. 1984, 31 Dec. 1984, 1 Jan. 1985, 2 Jan. 1985, 14 Jan. 1985, 15 Jan. 1985, leg. Benny Baeten and Luc Janssens, 1 Mar. 1985, leg. Théoneste (RMCA: 85.006-M0259 to $85.006-\mathrm{M}-0316,87.047-\mathrm{M}-0098$ to $87.047-\mathrm{M}-0103,85.006-\mathrm{M}-0207$ to $85.006-\mathrm{M}-0211,85.006-$ M-0384 to 85.006-M-0388, 85.006-M-0439 to 85.006-M-0446, 85.006-M-0317, 85.006-M-0212 to 85.006-M-0220, 85.006-M-0237 to 85.006-M-0258); 1 +, 1 त [2 SA], Cyangugu [= Shangugu],

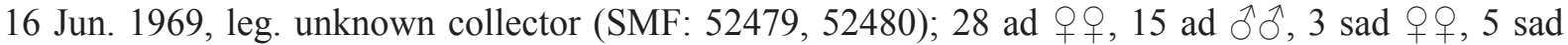

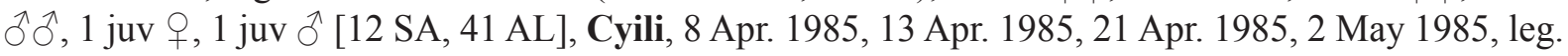
Théoneste, 24 Dec. 1984, 2 Jan. 1985, leg. Benny Baeten and Luc Janssens (RMCA: 87.047-M-0112 to $87.047-\mathrm{M}-0116,85.006-\mathrm{M}-0318$ to $85.006-\mathrm{M}-0329,87.047-\mathrm{M}-0120$ to $87.047-\mathrm{M}-0128,87.047-\mathrm{M}$ 0117 to $87.047-\mathrm{M}-0119,85.006-\mathrm{M}-0221$ to $85.006-\mathrm{M}-0236,87.047-\mathrm{M}-0104$ to $87.047-\mathrm{M}-0111$ ); 2 ?? [2 UN], Gabiro, unknown date, leg. unknown collector (INPBC: V3030, V3051); 10 ad $\circ$, 8 ad

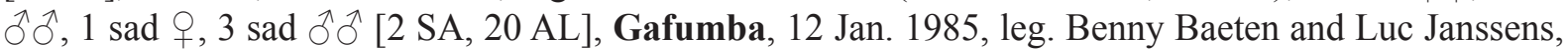
7 Feb. 1985, leg. Théoneste (RMCA: 85.006-M-0427 to 85.006-M-0438, 87.047-M-0088 to 87.047-M0097); 3 ?? [3 AL], Ihema, Lake, between 1 Jun. 1969 and 30 Jun. 1969, leg. Kiss (RMCA: 74.023M-0001 to 74.023-M-0003); 42 ad + 우, 23 ad ô $\widehat{o}$ [65 AL], Karama, 12 Oct. 1981, 13 Oct. 1981, leg. Frits De Vree, Benny Baeten and Victor Van Cakenberghe (RMCA: 82.006-M-0464 to 82.006-M-0528); 4 우, 1 ऽ [5 AL], Kibungo (former) Prefecture [= Eastern Prefecture], 26 Nov. 1985, leg. unknown

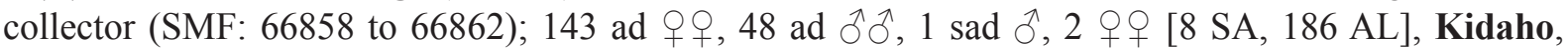
6 Jun. 1982, 7 Jun. 1982, 8 Jun. 1982, 9 Jun. 1982, 10 Jun. 1982, 11 Jun. 1982, 12 Jun. 1982, 15 Jun. 1982, 16 Jun. 1982, 17 Jun. 1982, 18 Jun. 1982, 19 Jun. 1982, leg. Benny Baeten (RMCA: 87.046-M-0326 to $87.046-\mathrm{M}-0328,87.046-\mathrm{M}-0330$ to $87.046-\mathrm{M}-0337,87.046-\mathrm{M}-0470$ to $87.046-\mathrm{M}-0491,87.046-$ $\mathrm{M}-0493,87.046-\mathrm{M}-0494,87.046-\mathrm{M}-0338$ to $87.046-\mathrm{M}-0342,87.046-\mathrm{M}-0495$ to $87.046-\mathrm{M}-0501$, 87.046-M-0343, 87.046-M-0502 to 87.046-M-0506, 87.046-M-0329, 87.046-M-0492, 87.046-M-0507 to $87.046-\mathrm{M}-0510,87.046-\mathrm{M}-0281$ to $87.046-\mathrm{M}-0285,87.046-\mathrm{M}-0380$ to $87.046-\mathrm{M}-0386,87.046-\mathrm{M}-$ 0286 to $87.046-\mathrm{M}-0294,87.046-\mathrm{M}-0309$ to $87.046-\mathrm{M}-0313,87.046-\mathrm{M}-0387$ to $87.046-\mathrm{M}-0418,87.046-$ M-0295 to 87.046-M-0304, 87.046-M-0314 to 87.046-M-0317, 87.046-M-0419 to 87.046-M-0433, 87.046-M-0305 to 87.046-M-0308, 87.046-M-0318 to 87.046-M-0325, 87.046-M-0434 to 87.046-M0469); 2 $\widehat{\partial}, 1$ ? [2 SS, 1 SA], Kiziguru (Mission) [= Kiziguro (Mission)], 2 Jul. 1954, 31 Jul. 1954, 2 Aug. 1954, leg. Alexandre Fain (RMCA: 81.012-M-0061, MHNG: 921.042, RMCA: 81.012-M-0060); 3 ?? [3 SA], Mabanza, 9 Oct. 1980, leg. Danny Meirte (RMCA: 80.055-M-0162 to 80.055-M-0164); 3 ?? [3 UN], Mihindi, Lake, unknown date, leg. unknown collector (INPBC: W3111.1 to W3111.3);

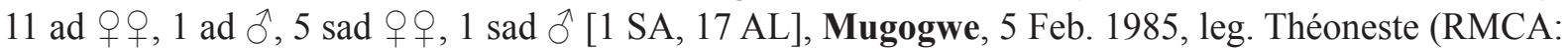




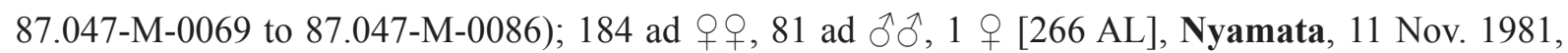
12 Nov. 1981, 13 Nov. 1981, 14 Nov. 1981, 15 Nov. 1981, 16 Nov. 1981, leg. Frits De Vree, Benny Baeten and Victor Van Cakenberghe (RMCA: 82.006-M-0529 to 82.006-M-0794); 15 ad $q$,, 5 ad ${ }^{\dagger} \delta$, $4 \mathrm{sad}+9$, $15 \mathrm{sad}$ ठै $\widehat{~}$ [5 SA, 34 AL], Rubona, 7 Jan. 1985, leg. Benny Baeten and Luc Janssens, between 1 Jan. 1985 and 31 Dec. 1985, leg. Théoneste (RMCA: 85.006-M-0389 to 85.006-M-0426, 87.047-M-

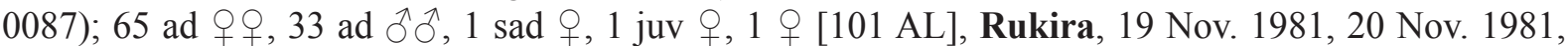
21 Nov. 1981, 22 Nov. 1981, 23 Nov. 1981, 24 Nov. 1981, 25 Nov. 1981, 26 Nov. 1981, 27 Nov. 1981, leg. Frits De Vree, Benny Baeten and Victor Van Cakenberghe (RMCA: 82.006-M-0795 to 82.006-M0813, 82.006-M-0848 to 82.006-M-0880, 82.006-M-0814 to 82.006-M-0816, 82.006-M-0881, 82.006-

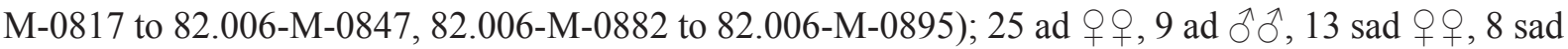
đo ${ }^{\lambda}$ [15 SA, 39 AL, 1 SK], Shangi, 5 Jan. 1985, 6 Jan. 1985, leg. Benny Baeten and Luc Janssens (RMCA: 85.006-M-0330 to 85.006-M-0360, 85.006-M-0540, 85.006-M-0361 to 85.006-M-0383).

\title{
Chaerephon russatus J.A. Allen, 1917
}

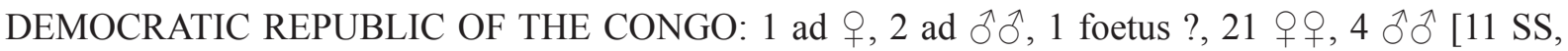
1 SK, 17 UN], Medje, 8 Sep. 1910, leg. Herbert Lang, James Paul Chapin and The American Museum Congo Expedition, 8 Sep. 1910, leg. Herbert Lang, 8 Sep. 1910, leg. unknown collector (AMNH: 48911 to 48923, 48925 [holotype Chcerephon russatus J.A. Allen, 1917], 48926 to 48929, 48931, 48935, 49219, 49265, 49266, 49278, BMNH: 1970.2507, FMNH: 43867, MCZ: 17386 [topotype Chaerephon russatus J.A. Allen, 1917], 17387 [topotype Charephon russatus J.A. Allen, 1917], RMCA: 12389); 1 q [SS], Tandala, 14 Jul. 1979, leg. C. Brian Robbins (USNM: 537762).

\author{
Mops Lesson, 1842 \\ Mops (Mops) Lesson, 1842
}

Mops (Mops) condylurus (A. Smith, 1833)

BURUNDI: 1 †, 32 $\widehat{\jmath}, 145$ ?? [1 SA, 62 AL, 115 UN], Bujumbura, unknown date, prior to 21 Feb. 1962, leg. Kiss, 21 Apr. 1982, 26 Apr. 1982, 27 Apr. 1982, leg. Danny Meirte, prior to 31 Dec. 1964, leg. unknown collector (RMCA: 31038, 31039, 31040-01 to 31040-46, 31041-01 to 31041-69, 82.016-M0001, 82.016-M-0094 to 82.016-M-0123, 82.016-M-0129, 31010 to 31037, SMF: 23789); 2 우 [1 SA,

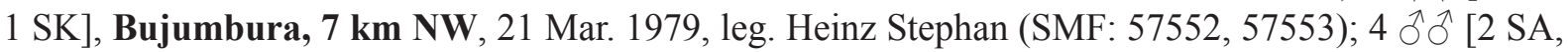
2 SK], Lake Cohoha [= Cohoha, Lake], 9 Mar. 1979, 10 Mar. 1979, leg. Heinz Stephan (SMF: 57550, $57551,57548,57549)$.

DEMOCRATIC REPUBLIC OF THE CONGO: 21 ?? [1 AL, $20 \mathrm{UN}$ ], unknown locality, unknown date, leg. Antoon Emeric Marcel De Roo, between 1 Apr. 1999 and 30 Apr. 1999, leg. unknown collector (RMCA: 32781, SMF: 93323 to 93342); 3 ?? [3 UN], Aba, 12 Apr. 1948, between 12 Apr. 1948 and

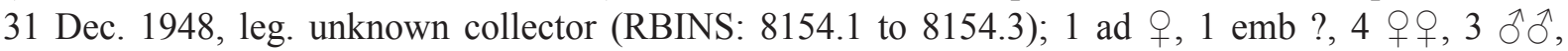
25 ?? [5 SS, 10 AL, 18 SK, 1 SO], Cel II, 1 Jan. 1950, 10 May 1951, 30 Apr. 1952, leg. H. de Saeger et al., 2 Apr. 1951, 10 May 1951, 7 Mar. 1952, 30 Apr. 1952, 10 May 1952, leg. H. de Saeger (RBINS: 14004, 14000.01 to $14000.11,14001.1,14001.2$, RMCA: 36908 to $36910,36899,36900,36903$ to 36907, RBINS: 14003.1 to $14003.4,14005,14006$, RMCA: 36902, 36913, 36914, 36901); 1 ? [SS], Defrenne Cave [= Mwanga Cave], 2 Apr. 1957, leg. G. Verhoustraete (RMCA: 26213); 1 đ [SA], Duma, 12 Oct. 1910, leg. unknown collector (SMF: 6397); 2 우, 5 đึ के [6 AL, 1 UN], Elisabethville [= Lubumbashi], 1 Mar. 1967, leg. unknown collector, 6 Feb. 1956, 15 Mar. 1956, 10 Jul. 1956, leg. Félix / Michel Anciaux de Faveaux (RBINS: 18510, RMCA: 23916, 23917, 23914, 23915, 23906, 23907); 2 ?? [2 SK], Gangala-na-Bodio, 9 Apr. 1948, 10 Apr. 1948, leg. unknown collector (RBINS: 8156, 8155); 1 ô [UN], Goma, 24 Dec. 1952, leg. unknown collector (RBINS: 15461); 4 ad $\circ$ $\circ, 4$ ad

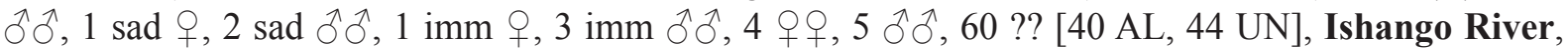


unknown date, leg. unknown collector, between 1 Jan. 1982 and 31 Dec. 1982, leg. Marc Colyn, between 26 Dec. 1968 and 29 Dec. 1968, leg. M. Lejeune (INPBC: V112, V1127, V1176.001 to V1176.042, RMCA: 82.031-M-0001, 35421 to 35459); 10 ?? [2 AL, 8 SK], Iso, 4 Mar. 1952, leg. H. de Saeger et al., 4 Mar. 1952, leg. H. de Saeger (RBINS: 14002.1 to 14002.8, RMCA: 36911, 36912); 12 우의,

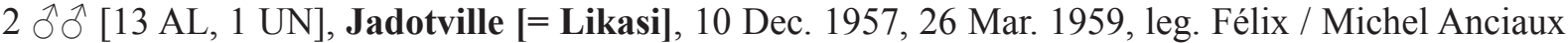
de Faveaux, 4 Apr. 1960, leg. unknown collector (RMCA: 27541 to 27547, 27899 to 27904, RBINS: 14559); 27 ?? [8 AL, 19 UN], Kambukabakali, unknown date, leg. unknown collector, 23 Oct. 1954, 27 Oct. 1954, 29 Oct. 1954, 5 Nov. 1954, leg. Gaston-François de Witte (INPBC: W1284, W1286, W1288, W1290, W1292, W1294, W1296, W1298, W1302.01 to W1302.11, RMCA: 37384, 37385, 37398 to 37400, 37386, 37397, 37401); 2 ?? [2 SA], Kapolowe, between 1 Jul. 1954 and 31 Jul. 1954, leg. De Smet (RMCA: 22438, 22439); 1 †, 2 ठ̋ [1 SS, 1 AL, 1 SO], Kasapa, 2 Jan. 1969, 14 Jan. 1969, leg. unknown collector, 29 Apr. 1956, leg. Félix / Michel Anciaux de Faveaux (RBINS: 18845, 18844, RMCA: 23920); 1 đ [UN], Kasenye, 10 km N, 23 Nov. 1954, leg. unknown collector (RBINS:

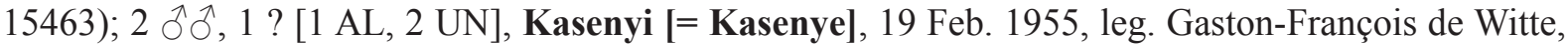
21 Sep. 1953, 19 Nov. 1953, leg. unknown collector (RMCA: 37402, RBINS: 14560, 14561); 1 q, 3 ठิ ô, 9 ?? [13 AL], Kashiobwe, 20 Oct. 1955, leg. Van Iseghem (RMCA: 26307 to 26319); 3 ?? [3 AL], Kasongo, 13 Nov. 1959, leg. Segers and Benoit Mission (RMCA: 29165, 29167, 29168); 1 q, 1 đ [2 AL], Kenya, 26 Feb. 1956, leg. Félix / Michel Anciaux de Faveaux (RMCA: 23825, 23918); 2 juv

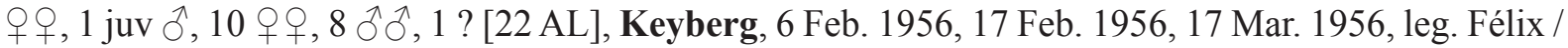
Michel Anciaux de Faveaux, 19 Dec. 1955, leg. M. Lips, 6 Feb. 1956, leg. service de l'hygiene publique (RMCA: 23909 to 23913, 23908, 26297 to 26302, 26304, 26305, 23822, 23901 to 23905, 26296,

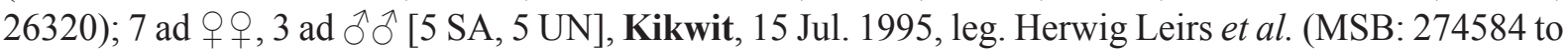
274588, RMCA: 97.021-M-0811 to 97.021-M-0815); 1 ad + , 1 ad ${ }^{\lambda}$ [2 SA], Kinshasa, 22 Dec. 1914, leg. Herbert Lang, James Paul Chapin and The American Museum Congo Expedition (AMNH: 49230 [holotype Mops (Allomops) osborni J.A. Allen, 1917], 49244 [paratype Mops (Allomops) osborni J.A. Allen, 1917]); 1 $\bigcirc$ [AL], Kipushi, road to, 6 Feb. 1956, leg. M. Lips (RMCA: 26306); 54 ?? [54 UN], Kongolo, between 8 Nov. 1974 and 9 Nov. 1974, leg. Michael D. Gallagher (HZM: 156.7822 to 209.7875); 4 ?? [4 SS], Léopoldville [= Kinshasa], 4 Apr. 1964, leg. Antoon Emeric Marcel De Roo

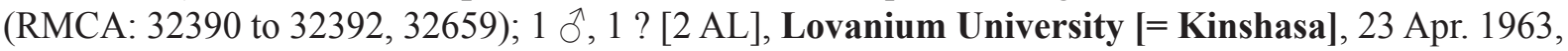
8 Jul. 1964, leg. Jo Van Orshoven (ZMA: MAM.11165, MAM.11166); 1 ? [AL], Lubondai-Tshimbulu [= Lubondaie-Tshimbulu], between 1 Jan. 1953 and 31 Dec. 1953, leg. S. Marks (RMCA: 26303); 1 q [UN], Luluabourg [= Kananga], unknown date, leg. Richard Callewaert (BMNH: 1926.7.6.107);

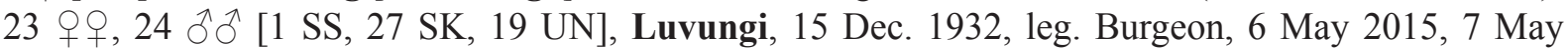
2015, 17 May 2015, leg. Prescott Musaba, Moïse Bipoo, Charle Andabhati, Benjamin Kirongozi and Kambale Karupao (RMCA: 12045, UNIKIS: BUKCH17, BUKCH01, BUKCH03 to BUKCH06, BUKCH08 to BUKCH15, BUKCH18 to BUKCH20, BUKCH22, BUKCH24 to BUKCH27, BUKCH07, BUKCH28, BUKCH30 to BUKCH32, BUKCH34 to BUKCH38, BUKCH40, BUKCH42, BUKCH44 to BUKCH46, BUKCH48, BUKCH50 to BUKCH56, BUKCH58); 1 ? [SS], Nionga, 3 May 1925, leg. Gaston-François de Witte (RMCA: 9230); 48 ?? [48 UN], Rwindi, unknown date, leg. unknown collector (INPBC: V1051, V1542, V1543, V1546.01 to V1546.45); 10 ?? [1 SA, 9 AL], Sakania, prior to 1 Sep. 1954, leg. De Smet (RMCA: 22442 to 22451); 16 우, 12 đ̊ [10 SK, 18 UN], Sange, 13 May 2015, 14 May 2015, 15 May 2015, leg. Prescott Musaba, Moïse Bipoo, Charle Andabhati, Benjamin Kirongozi and Kambale Karupao (UNIKIS: BUKCH60, BUKCH61, BUKCH65, BUKCH68, BUKCH70 to BUKCH84, BUKCH86 to BUKCH88, BUKCH91 to BUKCH94, BUKCH96, BUKCH99); 3 우, 17 ふふ઼, 1 ? [6 SS, 1 SA, 13 AL, 1 UN], Tandala, 12 Jul. 1979, 13 Jul. 1979, 16 Jul. 1979, leg. unknown collector, 12 Jul. 1979, 13 Jul. 1979, 16 Jul. 1979, 21 Jul. 1979, leg. C. Brian Robbins, 13 Jul. 1979, leg. Lynn W. Robbins, 16 Jul. 1979, leg. Frieder Meier (MHNG: 1870.051, 1870.052, USNM: 463507 to $463509,537738,537739,537741$, MHNG: 1870.046 , USNM: 463510 to 463512,537740 , MHNG: 1743.060, 1870.047 to 1870.050, USNM: 463513 to 463515); 3 ?? [3 AL], Tshibambo [= Shibambo], 31 Aug. 1954, leg. De Smet (RMCA: 22459 to 22461); 7 우, 10 ô ${ }^{\lambda}$ [2 SS, 15 AL], Uvira, 2 May 1959, 
VAN CAKENBERGHE V. et al., The bats of Congo, Rwanda and Burundi revisited

leg. Uwe Rahm, 27 Jul. 1956, leg. Alvin Novick (RMCA: 28905, 28906, MCZ: 48200 to 48214); 1 ๙ [UN], Vitshumbi, 12 Jan. 1953, leg. unknown collector (RBINS: 14562).

RWANDA: 2 ๙ึ่ [2 SA], unknown locality, 12 Jul. 1964, leg. unknown collector (SMF: 34954, 35792); 2 우, 1 ㄱ, 1 ? [2 SS, 1 SA, 1 UN], Astrida [= Butare], unknown date, 25 Oct. 1954, leg. Alexandre Fain, 26 Feb. 1966, leg. unknown collector (BMNH: 1953.681, MHNG: 921.043, RMCA: 81.012-M-0048, RBINS: 15496); 2 ?? [2 SA], Bugesera, between 1 Sep. 1957 and 30 Sep. 1957,

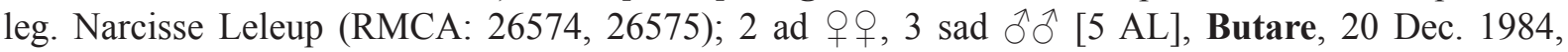
21 Dec. 1984, 22 Dec. 1984, 15 Jan. 1985, leg. Benny Baeten and Luc Janssens (RMCA: 85.006M-0462, 85.006-M-0458 to 85.006-M-0461); 121 ?? [52 AL, 9 SK, 60 UN], Gabiro, unknown date, 23 Sep. 1948, 27 Sep. 1948, leg. unknown collector, 9 Feb. 1960, leg. I.P.N.C. (INPBC: V3128.01 to V3128.60, RBINS: $8157,8158.1$ to 8158.8, RMCA: 78.038-M-0002 to 78.038-M-0053); 14 ad $ᄋ$, $24 \mathrm{ad} \hat{\partial} \hat{\partial}, 3 \mathrm{sad}$ 우, $6 \mathrm{sad} \hat{\partial} \hat{\partial}$ [47 AL], Gafumba, 7 Feb. 1985, leg. Théoneste (RMCA: 87.047M-0022 to 87.047-M-0068); 3 우, 2 $\delta^{\lambda}, 1$ ? [3 SK, 3 UN], Gahinga (Vulcano), 18 Feb. 1966, leg. unknown collector (RBINS: 15498.1 to $15498.3,15935.1$ to 15935.3 ); 2 우, 2 ○े [4 SK], Gihinda

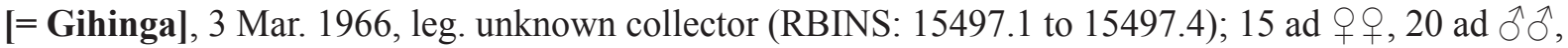

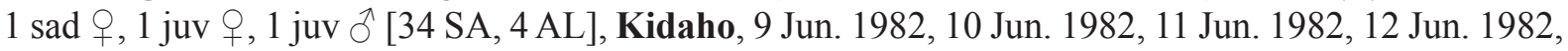
14 Jun. 1982, leg. Benny Baeten (RMCA: 87.046-M-0273 to 87.046-M-0280, 87.046-M-0511 to 87.046M-0513, 87.046-M-0247 to 87.046-M-0272, 87.046-M-0379); 12 우 6 SO, 7 UN], Kigali, 19 Feb. 1967, leg. unknown collector, 30 Sep. 1980, leg. Danny Meirte (RBINS: 15929.1 to $15929.7,16007$ to 16022 , RMCA: 80.055-M-0113 to 80.055-M-0124); 37 ?? [35 SA, 2 AL], Mabanza, 9 Oct. 1980, leg. Danny Meirte (RMCA: 80.055-M-0125 to 80.055-M-0161); 1 ô [UN], Masha, 1 Jan. 1956, leg. unknown collector (RBINS: 22465); 7 ad $ᄋ$ 우, 12 ad $\precsim \widehat{\jmath}$ [1 SS, 17 SA, 1 AL], Nyamata, 13 Nov. 1981, 14 Nov. 1981, 15 Nov. 1981, leg. Frits De Vree, Benny Baeten and Victor Van Cakenberghe (RMCA: 82.006-M-0339 to 82.006-M-0357); 1 đ [UN], Rubona, 27 Jan. 1966, leg. Félix / Michel Anciaux de Faveaux (RBINS: 15495); 28 ad 9 +, 53 ad $\widehat{\jmath}$ [77 SA, 4 AL], Rukira, 19 Nov. 1981, 20 Nov. 1981, 21 Nov. 1981, 23 Nov. 1981, 25 Nov. 1981, 26 Nov. 1981, 27 Nov. 1981, leg. Frits De Vree, Benny Baeten and Victor Van Cakenberghe (RMCA: 82.006-M-0358 to 82.006-M0438); $1 \widehat{\delta}$ [SA], Rwamagana, between 1 Jan. 1954 and 31 Dec. 1955, leg. Alexandre Fain (MHNG: 921.044).

\section{Mops (Mops) congicus J.A. Allen, 1917}

DEMOCRATIC REPUBLIC OF THE CONGO: 2 ad $q$ ㅇ, 1 foetus ?, 14 우, 1 ? [9 SS, 2 AL, 7 UN], Medje, 8 Sep. 1910, leg. Herbert Lang, James Paul Chapin and The American Museum Congo Expedition, 8 Sep. 1910, leg. Herbert Lang, 8 Sep. 1910, leg. unknown collector (AMNH: 48888 to 48892, 48893 [holotype Mops congicus J.A. Allen, 1917], 48894 to 48897, 48900, 49213, 49225, 49277, 49285, FMNH: 43864, MCZ: 17385, RMCA: 12380); 4 우, 5 ô ô [6 SS, 1 SA, 2 AL], Tandala, 12 Jul. 1979, leg. O.W.H., 12 Jul. 1979, 16 Jul. 1979, 21 Jul. 1979, leg. C. Brian Robbins, 21 Jul. 1979, leg. unknown collector (TTU: 36358, USNM: 537742, 537743, 537745, MHNG: 1870.043, 1870.044, USNM: 463516, 463517, 537746); 1 ? [SO], Tshopo, between 1 Sep. 1985 and 30 Sep. 1985, leg. Marc

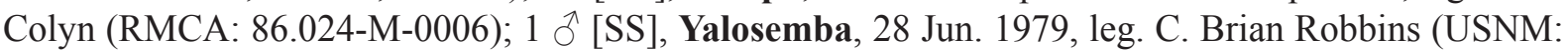
537744).

\section{Mops (Mops) demonstrator (Thomas, 1903)}

DEMOCRATIC REPUBLIC OF THE CONGO: 4 우 [4 UN], Bagunda, 19 Jan. 1951, leg. H. de Saeger et al. (RBINS: 13806.1 to 13806.4); 3 우, 1 đ, 16 ?? [5 SS, 15 UN], Cel II, unknown date, leg. unknown collector, 2 Apr. 1951, 12 May 1951, 5 Oct. 1951, 26 Nov. 1951, 7 Apr. 1952, 28 May 1952, 3 Sep. 1952, leg. H. de Saeger et al., 30 Apr. 1952, 3 Sep. 1952, leg. H. de Saeger (INPBC: 3514, 4735, RBINS: $15805,13801,13800,13804.1,13804.2,13809,13811.1$ to $13811.5,13813$, RMCA: 36933 to 
36935, 36932, RBINS: 13805, 13808.1); 1 ad $\widehat{~}, 1$ q [1 SS, 1 SA], Faradje, 8 Aug. 1911, 12 Jan. 1913, leg. Herbert Lang, James Paul Chapin and The American Museum Congo Expedition (AMNH: 49222 [holotype Mops (Allomops) faradjius J.A. Allen, 1917], 48910); $1 \lesssim$ [UN], Garamba National Park, between 1 Jan. 1950 and 31 Dec. 1952, leg. H. de Saeger et al. (RBINS: 13812); 2 juv $q$, 1 juv ?, 1 foetus ?, 1 ㅇ [1 SA, 4 AL], Keyberg, 19 Dec. 1955, 4 Feb. 1956, 9 Feb. 1956, leg. Félix / Michel Anciaux de Faveaux (RMCA: 26218, 26219, 26222, 26223, 26228); 2 우, 2 ?? [4 SS], Parc National de Garamba [= Garamba National Park], 30 Jun. 1951, 7 Apr. 1952, 28 May 1952, leg. H. de Saeger et al. (RBINS: 13824, 13825, 13807, 13810); 1 ? [SO], PFNK, 7 Feb. 1952, leg. H. de Saeger (RMCA: 36931).

RWANDA: $1 \precsim$ [SS], Musha, 14 Nov. 1955, leg. Alexandre Fain (RMCA: 37983).

Mops (Mops) midas (Sundevall, 1843)

DEMOCRATIC REPUBLIC OF THE CONGO: 2 q , 1 1 [3 UN], Bagunda, 19 Jan. 1951, leg. H. de Saeger et al. (RBINS: 13815.1 to 13815.3); 2 ?? [2 SS], Bwamanda [Mission], between 1 Jul. 1961 and 31 Jul. 1961, leg. Vedast (Gaston Alfons) Maes (RMCA: 30949, 30950); 1 ad ㅇ, 1 emb ?, 4 q $q, 1$ ô, 12 ?? [6 SS, 1 SO, 12 UN], Cel II, unknown date, leg. unknown collector, 8 Mar. 1951, 21 Feb. 1952, 8 Apr. 1952, 30 Apr. 1952, 3 Oct. 1952, leg. H. de Saeger et al., 8 Apr. 1952, 3 Oct. 1952, 22 Oct. 1952, leg. H. de Saeger (INPBC: 5159.4.1 to 5159.4.3, RBINS: 13819, RMCA: 36929, 36930, RBINS: 13822.1, 13822.2, RMCA: 36927, 36928, RBINS: 13821.1, 13821.2, 13814.1, 13814.2, 13820.1, 13820.2, RMCA: 36924 to 36926); 1 ad , 1 + , 4 ?? [6 SA], Faradje, 7 Mar. 1912, 9 Mar. 1912, leg. Herbert Lang, James Paul Chapin and The American Museum Congo Expedition (AMNH: 49254 to 49257, 49262, 49272); 3 ?? [3 UN], Kamohohora, unknown date, leg. unknown collector (INPBC: V183.1 to V183.3); 3 ?? [3 UN], Mwiga, unknown date, leg. unknown collector (INPBC: V500.1 to V500.3); 1 ठै, 17 ?? [7 SS, 1 AL, 9 UN, 1 SN], Ndelele, 8 Feb. 1952, leg. H. de Saeger et al., 8 Feb. 1952, leg. H. de Saeger (RBINS: 13816.1 to $13816.4,13817.1,13817.2,13818.1$ to 13818.3 , RMCA: 36915 to 36923 ); $1 \hat{\delta}[\mathrm{UN}$ ], Parc National de Garamba [= Garamba National Park], 3 Oct. 1952, leg. H. de Saeger et al. (RBINS: 13823); 6 우, 4 ?? [2 SS, 8 AL], Uvira, 2 Mar. 1959, leg. Uwe Rahm, 23 Sep. 1954, between 1 Mar. 1955 and 31 Mar. 1955, leg. Narcisse Leleup, 25 May 1955, leg. Waldo LaSalle Schmitt and E. Baker, 27 Jul. 1956, leg. Alvin Novick, 5 Sep. 1956, leg. Laurent, prior to 31 Oct. 1953, leg. Georges Marlier (RMCA: 33530, 27214, USNM: 301727, 301728, MCZ: 48197 to 48199, RMCA: $35694,22911,38152)$.

\section{Mops (Mops) niangarae J.A. Allen, 1917}

DEMOCRATIC REPUBLIC OF THE CONGO: 1 ad $\lesssim$ [SS], Niangara, 12 Dec. 1910, leg. Herbert Lang, James Paul Chapin and The American Museum Congo Expedition (AMNH: 48901 [holotype Mops niangarae J.A. Allen, 1917]).

Mops (Mops) niveiventer Cabrera \& Ruxton, 1926

BURUNDI: 4 ad 우우 [2 SA, 2 SK], Kayanza, 12 Mar. 1979, leg. Heinz Stephan (SMF: 57555 to

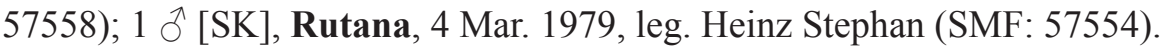

DEMOCRATIC REPUBLIC OF THE CONGO: $1 \mathrm{sad} \hat{\jmath}, 1 \stackrel{\phi}{\phi}, 1 \hat{\sigma}[2 \mathrm{SA}, 1 \mathrm{AL}$ ], Elisabethville [= Lubumbashi], 19 Dec. 1955, 14 Nov. 1956, leg. unknown collector, 2 Nov. 1956, leg. Félix / Michel Anciaux de Faveaux (SMF: 16867, 16876, RMCA: 26245); 1 ô [SA], Jadotville [= Likasi], 23 Sep. 1957, leg. Félix / Michel Anciaux de Faveaux (MHNG: 1046.029); 2 q [1 SA, 1 AL], Jadoville [= Likasi], 10 Dec. 1957, leg. Félix / Michel Anciaux de Faveaux (SMF: 18942, 18943); 1 ? [AL], Kasongo Samba, 22 Sep. 1959, leg. Segers and Benoit Mission (RMCA: 29206); 1 juv ${ }^{7}$, 6 우, 2 ?? [1 SA, 8 AL], Keyberg, 4 Feb. 1956, 6 Feb. 1956, 9 Feb. 1956, 17 Feb. 1956, leg. Félix / 
Michel Anciaux de Faveaux (MHNG: 923.003, 923.004, 923.007, 923.002, 923.005, 923.001, 923.006, RMCA: 23823, 26229); 1 ㅇ [SA], Kikole-Pecheur, 17 May 1980, leg. M. Mutashia (SMF: 58073); $3 \mathrm{ad}$ ऽิ $\widehat{\partial}$ [1 SA, 2 UN], Kimbinga Market, 20 Jul. 1995, leg. Herwig Leirs et al. (MSB: 274601, 274602, RMCA: 97.021-M-0823); 1 +, 1 ○ [2 SS], Luluabourg [= Kananga], unknown date, leg. Richard Callewaert (BMNH: 1926.7.6.104, 1926.7.6.106); 5 웅, 1 đ [6 SS], Matamba, 20 Dec. 1983, leg. Betu Nsankulu (RMCA: 84.035-M-0014, 84.035-M-0016 to 84.035-M-0018, 84.035-M-0020, 84.035-M-0021); 1 ㅇ [SS], Mikalaya, 22 Dec. 1983, leg. Betu Nsankulu (RMCA: 84.035-M-0022); 1 ㅇ, 1 ○े [2 SS], Mikalaya Mission [= Mikalaya], 22 Dec. 1983, leg. Betu Nsankulu (RMCA: 84.035M-0015, 84.035-M-0019); 1 [ [UN], St. Joseph de Luluabourg Mission [= Mikalaya], 16 Apr. 1924, leg. Richard Callewaert (BMNH: 1926.7.6.109 [holotype Mops angolensis niveiventer Cabrera and Ruxton, 1926]); 1 juv + , 1 foetus ? [2 AL], Wasseige Farm, 6 Feb. 1956, leg. Félix / Michel Anciaux de Faveaux (RMCA: 26225, 26226).

RWANDA: 1 ad 9,1 §, 8 ?? [3 SS, 7 SA], Astrida [= Butare], 13 May 1952, leg. P.G. Vercammen, 20 Feb. 1962, between 1 Mar. 1962 and 31 Mar. 1962, leg. Van Parijs, 26 Dec. 1950, leg. Aureliaan, between 1 Jan. 1948 and 31 Dec. 1948, leg. Alexandre Fain (RMCA: 23171, 31045, 31046, 20489, 18643, 18991, 31060, 31062, 31069, 31070); 3 ad 우우 [3 AL], Butare, 1 Mar. 1985, leg. Théoneste, 15 Jan. 1985, leg. Benny Baeten and Luc Janssens (RMCA: 87.047-M-0009, 87.047-M-0010, 85.006-M-

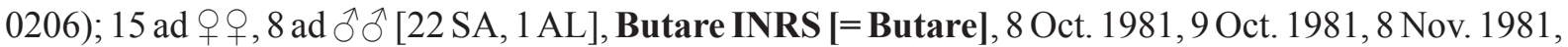
9 Nov. 1981, leg. Frits De Vree, Benny Baeten and Victor Van Cakenberghe (RMCA: 82.006-M-0439 to 82.006-M-0454, 82.006-M-0460, 82.006-M-0455 to 82.006-M-0459, 82.006-M-0461); 18 ad 9 , 9 ad

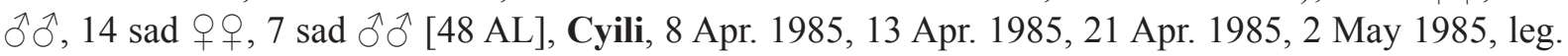
Théoneste, 24 Dec. 1984, 2 Jan. 1985, leg. Benny Baeten and Luc Janssens (RMCA: 87.047-M-0013 to $87.047-\mathrm{M}-0016,85.006-\mathrm{M}-0192$ to $85.006-\mathrm{M}-0195,87.047-\mathrm{M}-0020,87.047-\mathrm{M}-0021,87.047-\mathrm{M}-$ 0017 to $87.047-\mathrm{M}-0019,85.006-\mathrm{M}-0159$ to $85.006-\mathrm{M}-0191,87.047-\mathrm{M}-0011,87.047-\mathrm{M}-0012) ; 1$ ad $q$, $2 \mathrm{ad} \hat{\jmath} \widehat{\partial}, 1 \mathrm{sad}+$ [4 AL], Gafumba, 12 Jan. 1985, leg. Benny Baeten and Luc Janssens, 7 Feb. 1985,

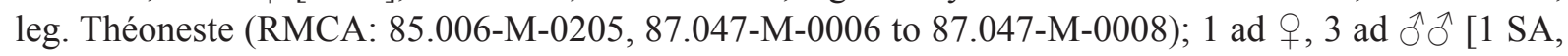
3 AL], Mugogwe, 5 Feb. 1985, leg. Théoneste (RMCA: 87.047-M-0002 to 87.047-M-0005); 1 ad $\widehat{\partial}$ [SA], Nyamata, 13 Nov. 1981, leg. Frits De Vree, Benny Baeten and Victor Van Cakenberghe (RMCA:

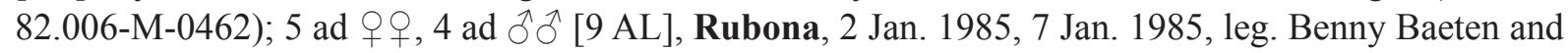
Luc Janssens (RMCA: 85.006-M-0196 to 85.006-M-0204); 1 ad $q$ [SA], Rukira, 21 Nov. 1981, leg. Frits De Vree, Benny Baeten and Victor Van Cakenberghe (RMCA: 82.006-M-0463).

Mops (Mops) trevori J.A. Allen, 1917

DEMOCRATIC REPUBLIC OF THE CONGO: 1 ad $q$ [SA], Faradje, 29 Sep. 1912, leg. Herbert Lang, James Paul Chapin and The American Museum Congo Expedition (AMNH: 49250 [holotype Mops trevori J.A. Allen, 1917]).

Mops (Xiphonycteris) Dollman, 1911

Mops (Xiphonycteris) brachypterus (Peters, 1852)

DEMOCRATIC REPUBLIC OF THE CONGO: 2 ?? [2 UN], Abatupi River, unknown date, leg. unknown collector (INPBC: V1285.4.1, V1285.4.2); 1 ad ㅇ [AL], Léopoldville [= Kinshasa],

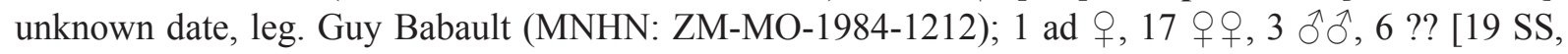
2 SA, 6 UN], Medje, 25 Jan. 1910, 6 Mar. 1910, 16 Mar. 1910, leg. Herbert Lang, James Paul Chapin and The American Museum Congo Expedition, 16 Mar. 1910, leg. Herbert Lang, 16 Mar. 1910, leg. unknown collector (AMNH: 48819, 48820, 48821 [holotype Nyctinomus ochraceus J.A. Allen, 1917], 48822 to 48826 , 48828 to 48835 , 49252, 49253, 49258, 49260, 49267, FMNH: 43876, 43877, MCZ: 17390, 17391, AMNH: 49224, RMCA: 12391); 1 ad + [SS], Panga, 18 Sep. 1914, leg. Herbert Lang, 
James Paul Chapin and The American Museum Congo Expedition (AMNH: 48843); 1 \& [SS], Tandala, 16 Jul. 1979, leg. C. Brian Robbins (USNM: 537769); 1 ? [UN], Thysville [= Mbanza-Ngungu], 11 Feb. 1987, leg. unknown collector (RBINS: 22432); 2 우우 [2 SS], Yalosemba, 17 Jun. 1979, leg. C. Brian Robbins (USNM: 537747, 537768).

RWANDA: 4 ?? [2 UN, 2 SN], unknown locality, unknown date, leg. Anthony N. Start, unknown date, leg. unknown collector (BMNH: 1975.2809, 1975.2809_1, 1975.2809_2, 1975.2810).

Mops (Xiphonycteris) nanulus J.A. Allen, 1917

DEMOCRATIC REPUBLIC OF THE CONGO: 3 ?? [3 AL], unknown locality, unknown date, leg. Anthony N. Start, unknown date, leg. unknown collector, between 1 Jan. 1976 and 31 Dec. 1977, leg. M. Lejeune (RMCA: 77.029-M-0003 to 77.029-M-0005); 1 q [SS], Aba, 17 Dec. 1911, leg. Herbert Lang, James Paul Chapin and The American Museum Congo Expedition (AMNH: 48874); 1 ? [AL], Albertville [= Kalemie], prior to 18 Nov. 1959, leg. H. Bomans (RMCA: 29304); 4 q + $, 6 \hat{\jmath} \widehat{\partial}, 4$ ?? [6 SA, 8 AL], Beno, unknown date, leg. the Royal Museum for Central Africa (RMCA), between 1 Jan. 1949 and 31 Dec. 1949, leg. P. Bayet (BMNH: 1954.840 to 1954.843, RMCA: 19838 to 19847); 3 ?? [3 UN], Ihunga-Semliki confluence, unknown date, leg. unknown collector (INPBC: V794.1 to V794.3); 2 우 [2 UN], Isai River, unknown date, between 18 Nov. 1974 and 19 Nov. 1974, leg. Michael D. Gallagher (HZM: 7.7818, 8.7819); 1 ? [AL], Kasai, prior to 11 Jul. 1905, leg. La Compagnie du Kassai (RMCA: 740a); 6 ad $q$ [ [6 UN], Kikwit, 3 Aug. 1995, leg. Herwig Leirs et al. (MSB: 274898 to 274900, 274902, 274903, 274907); 3 ad $\not$ +, 4 ad $\precsim \widehat{\partial}$ [5 SA, 1 AL, 1 SK], Kikwit ITPK [= Kikwit], 3 Aug. 1995, leg. Herwig Leirs et al. (RMCA: 97.021-M-0817 to 97.021-M-0822, 97.021-M-0850); 1 juv $\widehat{\partial}$ [SA], Kinzambi Mission, 24 Jul. 1995, leg. Herwig Leirs et al. (RMCA: 97.021-M-0816); 1 juv $\widehat{o}[\mathrm{AL}]$, Leverville [= Lusanga], between 1 Feb. 1929 and 28 Feb. 1929, leg. Tinant (RMCA: 9988b); 1 ? [AL], Lulimbi, between 1 Jan. 1976 and 31 Dec. 1977, leg. M. Lejeune (RMCA: 77.029-

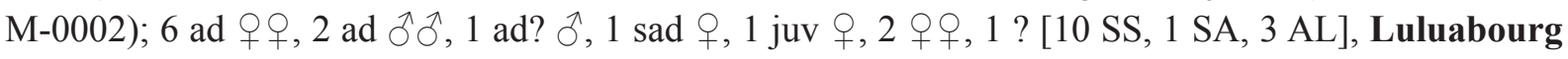
[= Kananga], unknown date, prior to 8 Jul. 1913, leg. Richard Callewaert, prior to 17 Apr. 1913, leg. Cambier (BMNH: 1926.11.1.37 to 1926.11.1.43, 1927.12.21.19, 1927.5.9.1, 1927.5.9.2, RMCA: 1548, 1954a to 1954c); 1 , 1 ? [1 SS, 1 SA], N'gombe [= Ngombe], unknown date, between 1 Jan. 1921 and

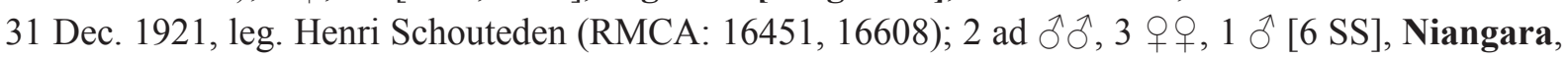
12 Dec. 1910, leg. Herbert Lang, James Paul Chapin and The American Museum Congo Expedition (AMNH: 48861 [topotype Mops (Allomops) nanulus J.A. Allen, 1917], 48862 [topotype Mops (Allomops) nanulus J.A. Allen, 1917], 48863 [topotype Mops (Allomops) nanulus J.A. Allen, 1917], 48864 [holotype Mops (Allomops) nanulus J.A. Allen, 1917], 48865 [topotype Mops (Allomops) nanulus J.A. Allen, 1917], FMNH: 43868); 4 우, 1 万, 2 ?? [7 SS], Panga, between 1 Sep. 1925 and 30 Sep. 1925, leg. Henri Schouteden (RMCA: 9132 to 9135, 9141 to 9143); 4 우 [4 SS], Tandala, 12 Jul. 1979, 16 Jul. 1979, 21 Jul. 1979, leg. C. Brian Robbins (USNM: 537758 to 537761); 2 우 [2 SS], Yalosemba, 27 Jun. 1979, 28 Jun. 1979, leg. C. Brian Robbins (USNM: 537754, 537756).

\section{Mops (Xiphonycteris) spurrelli (Dollman, 1911)}

DEMOCRATIC REPUBLIC OF THE CONGO: 8 q,+ 1 đ [9 SA], Butembo, 16 Mar. 1959, prior to 26 May 1959, leg. P. Dyleff(RMCA: 27876, 27868 to 27875); 1 ○ [UN], St. Jos. (Mission) [= Mikalaya], 12 Oct. 1924, leg. Richard Callewaert (AMNH: 86145); 1 ㅇ [SS], Tandala, 16 Jul. 1979, leg. C. Brian Robbins (USNM: 537757); 2 우우 [2 SS], Yalosemba, 17 Jun. 1979, 27 Jun. 1979, leg. C. Brian Robbins (USNM: 537753, 537755). 
VAN CAKENBERGHE V. et al., The bats of Congo, Rwanda and Burundi revisited

Mops (Xiphonycteris) thersites (Thomas, 1903)

DEMOCRATIC REPUBLIC OF THE CONGO: 1 đ [SA], unknown locality, between 1 Jan. 1911 and 31 Dec. 1911, leg. unknown collector (SMF: 6396); 1 ad $\widehat{o}$ [SS], Avakubi, 13 Feb. 1914, leg. Herbert Lang, James Paul Chapin and The American Museum Congo Expedition (AMNH: 48851 [holotype Mops (Allomops) occipitalis J.A. Allen, 1917]); 1 ô [UN], Banana, 29 Sep. 1955, leg. l' Expédition Atlantique Sud 1955 (RBINS: 15457); 1 q [AL], Bombanga - Bumba, between 1 Jan. 1986 and 31 Dec. 1986, leg. WHO and Marc Colyn (RMCA: 88.011-M-0026); 1 ㅇ [UN], Isai River, 19 Dec. 1974, leg. Michael D. Gallagher (HZM: 3.7820); 17 우, 5 ठึ $\widehat{\lambda}$ [22 UN], Kasenye, 10 km N, 17 Feb. 1954, 10 Mar. 1954, 17 Mar. 1954, 20 Mar. 1954, 30 Mar. 1954, 22 Nov. 1954, 23 Nov. 1954, 24 Dec. 1954, leg. unknown collector (RBINS: 15470, 15472, 15469, 15471, 15460.01 to 15460.13 , 15468, 15474.1, 15474.2, 15466, 15467); 1 ad $\widehat{\overbrace{}}$ [SK], Kikwit ITPK [= Kikwit], 3 Aug. 1995, leg. Herwig Leirs et al. (RMCA: 97.021-M-0824); 2 우, 2 के $0^{\lambda}$ [4 SS], Luebo, 19 Aug. 1921, leg.

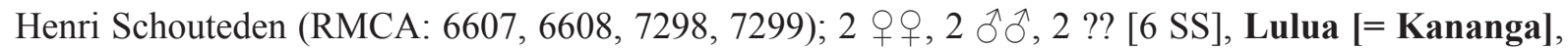
25 Feb. 1981, 27 Feb. 1981, leg. Betu Nsankulu (RMCA: 81.050-M-0002, 81.050-M-0005, 81.050M-0006, 81.050-M-0003, 81.050-M-0004, 81.050-M-0007); 10 우, 1 ô [9 SS, 2 SK], Luluabourg [= Kananga], unknown date, leg. Richard Callewaert, 15 Jan. 1964, leg. Antoon Emeric Marcel De Roo (BMNH: 1926.11.1.28 to 1926.11.1.31, 1926.11.1.33, 1926.11.1.34, 1926.11.1.36, 1927.12.21.15, 1927.12.21.17, 1927.12.21.18, AMNH: 206845); 1 ad + [UN], Lusano and KKK Zone, unknown date, leg. unknown collector (DNSM: 14073); 3 우, 3 $\widehat{\partial}$ [2 SS, 4 UN], Medje, 19 Jan. 1910, 15 Sep. 1910, 16 Sep. 1910, between 16 Sep. 1910 and 19 Sep. 1910, leg. Herbert Lang, James Paul Chapin and The

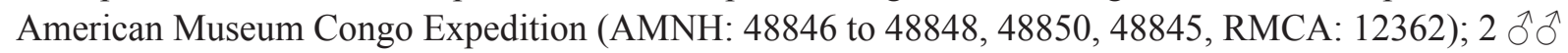
[2 AL], Mosenge, 11 Aug. 1986, 14 Aug. 1986, leg. WHO and Marc Colyn (RMCA: 88.011-M-0024, 88.011-M-0025); 1 ? [SS], Ndwa, prior to 27 Mar. 1952, leg. Ngwe (RMCA: 20837); 1 ठ̊ [SS], St. Jos. (Mission) [= Mikalaya], 14 Nov. 1924, leg. Richard Callewaert (AMNH: 86101); 3 우, 1 ภ , 1 ? [4 SS, 1 AL], St. Joseph de Luluabourg Mission [= Mikalaya], 19 May 1922, 3 Jun. 1922, 19 Jul. 1922, 27 Jul. 1922, leg. Richard Callewaert, 24 May 1939, leg. Jean Jacques Deheyn (RMCA: 7550, 7542, 15398, 7544, 7549); 1 †, 3 đठ [4 AL], Tandala, 12 Jul. 1979, leg. C. Brian Robbins, 7 Jul. 1979, leg. unknown collector (USNM: 463530, MHNG: 1871.007 to 1871.009); 4 우, 13 $ぇ$ [5 SS, $2 \mathrm{SA}, 9 \mathrm{AL}$, 1 SO], Yalosemba, 17 Jun. 1979, 27 Jun. 1979, leg. unknown collector, 17 Jun. 1979, 27 Jun. 1979, leg. C. Brian Robbins (MHNG: 1871.003 to 1871.005 , USNM: 463523 to 463528,537763 to 537767 , MHNG: 1871.002, 1871.006, USNM: 463529).

RWANDA: 15 우, 3 ๙ึ̊̃, 2 ?? [20 UN], Astrida [= Butare], 26 Feb. 1966, leg. unknown collector (RBINS: $15475.1,15475.2,15478.1,15478.2,15482.01$ to $15482.10,15932.1$ to 15932.6 ); 1 ? [SK], Byumba, 18 Apr. 1990, leg. Manuel Ruedi and Raphaël Arlettaz (MHNG: 1919.023); 3 우 [3 UN], Gihinda [= Gihinga], 13 Feb. 1966, leg. unknown collector (RBINS: 15476.1 to 15476.3); 1 q [UN], Nyumba Cave, 13 Feb. 1966, leg. unknown collector (RBINS: 15477); 1 q [SS], Rubona, 8 Jan. 1967, leg. Félix / Michel Anciaux de Faveaux (RBINS: 16036).

\section{Myopterus E. Geoffroy Saint-Hilaire, 1818}

\section{Myopterus daubentonii Desmarest, 1820}

DEMOCRATIC REPUBLIC OF THE CONGO: 1 ? [AL], Bunia, prior to 20 Feb. 1939, leg. Maristes (RMCA: 15224); 1 ad , 6 우, 1 $\precsim$ [7 SS, 1 AL], Niangara, 26 Dec. 1910, 27 Dec. 1910, leg. Herbert Lang, James Paul Chapin and The American Museum Congo Expedition, 26 Dec. 1910, leg. unknown collector (AMNH: 48852 to 48856, MCZ: 17384, AMNH: 48857, 49228); 2 ?? [1 SO, $1 \mathrm{UN}$ ], Uele [River], 28 Apr. 1914, prior to 28 Apr. 1914, leg. M. Hutereau (BMNH: 1919.5.9.1 [holotype Myopterus albatus Thomas, 1915], RMCA: 2911). 


\section{Myopterus whitleyi (Scharff, 1900)}

DEMOCRATIC REPUBLIC OF THE CONGO: 1 $\overbrace{}^{\mathrm{UNN}}$, unknown locality, 5 May 1940, leg. NATURE ??? (TM: 9766); 1 ठ [UN], Banana, 29 Sep. 1955, leg. l' Expédition Atlantique Sud 1955 (RBINS: 15459); 3 ?? [3 AL], Beno, prior to 3 Jan. 1950, between 1 Jan. 1949 and 31 Dec. 1949, leg. P. Bayet (RMCA: 19826, 19827, 19476); 1 q [UN], Kasenye, 10 km N, 6 Mar. 1954, leg. l' Expédition Atlantique Sud 1955 (RBINS: 15473); 1 ad 9 [UN], Kikwit ITPK [= Kikwit], 3 Aug. 1995, leg. Herwig Leirs et al. (MSB: 274906); 1 ad 9 [SA], Kinzambi Mission, 30 Jul. 1995, leg. Herwig Leirs et al. (RMCA: 97.021-M-0825); 1 q [UN], Léopoldville [= Kinshasa], 7 Oct. 1950, leg. unknown collector (RBINS: 7478); 11 q, 1 ô, 5 ?? [10 SS, 5 AL, 2 SK], Luluabourg [= Kananga], unknown date, 22 May 1922, 13 Jun. 1922, prior to 8 Jul. 1913, between 1 Jan. 1913 and 31 Dec. 1913, leg. Richard Callewaert (BMNH: 1926.11.1.45, 1926.11.1.46, 1926.7.6.100 to 1926.7.6.103, 1926.7.6.99, 1934.10.24.27, 1934.10.24.28, RMCA: 7589, 7590, 7588, 16196, 16197, 1954h to 1954j); 4 ठै $\widehat{~[2 ~ S S, ~}$ 2 SO], St. Jos. (Mission) [= Mikalaya], 7 Aug. 1924, 26 Sep. 1924, 10 Oct. 1924, 23 Oct. 1924, leg. Richard Callewaert (AMNH: 86144, 86148, 86140, 86176).

Otomops Thomas, 1913

Otomops martiensseni (Matschie, 1897)

BURUNDI: 1 ô [UN], Kibira National Park, 27 Mar. 1990, leg. unknown collector (FMNH: 137633); $1 \lesssim$ [AL], Rwegura, 25 Apr. 1969, leg. P.L. Niort (MHNG: 1896.021).

DEMOCRATIC REPUBLIC OF THE CONGO: 1 ad $\widehat{\partial}$ [SS], Cel II, 8 Sep. 1952, leg. H. de Saeger et al. (RBINS: 13827); 1 § [UN], Marungu, unknown date, leg. unknown collector (NMZL: 3395); 1 ad $\partial \hat{~[S S}$ ], Mbuye-Bala River, 26 Mar. 1948, leg. unknown collector (RBINS: 10704); 1 \& [SS], Poko, 16 Jul. 1914, leg. Cuthbert Christy (BMNH: 1919.5.9.2).

RWANDA: 1 sad ? [SN], unknown locality, 30 Nov. 1985, leg. unknown collector (SMF: 66857); 1 ઈ [SA], Kigali, between 1 Jan. 1933 and 31 Dec. 1933, leg. Becquet (RMCA: 12166).

Tadarida Rafinesque, 1814

DEMOCRATIC REPUBLIC OF THE CONGO: 1 ? [UN], unknown locality, unknown date, leg. unknown collector (BMNH: 1990.234); 1 ? [AL], Léopoldville [= Kinshasa], unknown date, leg. Cuthbert Christy (BMNH: 1904.6.25.2).

Tadarida fulminans (Thomas, 1903)

DEMOCRATIC REPUBLIC OF THE CONGO: 1 ? [UN], Rumangabo, unknown date, leg. unknown collector (INPBC: V1234).

RWANDA: 1 ? [SS], Nyakibanda, between 1 Apr. 1955 and 30 Apr. 1955, leg. Alexandre Fain (RMCA: 23748); 1 ad $q$ [UN], Nyanza, 6 Feb. 1966, leg. unknown collector (RBINS: 15494); 1 ad $q, 1$ ad

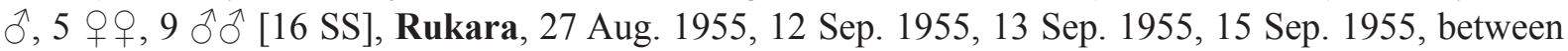
12 Sep. 1955 and 30 Sep. 1955, leg. Alexandre Fain (RMCA: 37972 to 37978, 81.012-M-0104, 37979 to 37982, 81.012-M-0103, 37970, 37971, 81.012-M-0047).

Tadarida ventralis (Heuglin, 1861)

DEMOCRATIC REPUBLIC OF THE CONGO: 1 \& [SS], Alima, 2 Aug. 1960, leg. P. Dyleff (RMCA: 29241). 
DEMOCRATIC REPUBLIC OF THE CONGO: 1 ad ?, 3 ?? [4 AL], unknown locality, 20 Nov. 1988, between 1 Jan. 1921 and 31 Dec. 1921, leg. Verlaine, 4 Jul. 1958, leg. E. Jans (RMCA: 88.044-M-0014, 88.044-M-0015, 27368, 88.044-M-0013); 1 imm + [SO], Bambesa, 18 Mar. 1937, leg. J.M. Vrijdagh (RMCA: 14073); 1 juv ?, 2 우, 2 ?? [5 AL], Boende, between 1 Jan. 1967 and 31 Dec. 1967, between 1 Jan. 1966 and 31 Jan. 1966, leg. P. Lootens (RMCA: 34077, 34093, 34156, 38732, 38733); 1 imm ? [AL], Bokuma, 25 Jul. 1952, leg. P. Lootens (RMCA: 21672); 1 juv ? [AL], Bunkeya, 26 Jan. 1958, leg. Van Oost (RMCA: 28802); 2 ?? [1 AL, 1 UN], Elisabethville [= Lubumbashi], between 1 Jan. 1930 and 31 Dec. 1930, leg. Lamoral, prior to 26 Jan. 1934, leg. Quarré (RMCA: 11708, 12236); 1 ? [SS], Grotte de Baya [= Baya Cave], 14 May 1956, leg. M. Lips (RMCA: 23799); 1 ? [AL], Ibembo, 25 Jan. 1951, leg. Jozef Hutsebaut (RMCA: 89.004-M-0001); 1 ㅇ [SS], Kakontwe Cave, 8 Feb. 1957, leg. G. Verhoustraete (RMCA: 26212); 1 ad + [AL], Kalutenge, between 19 May 1919 and 19 May 1924, leg. Seydel (RMCA: 90.006-M-0001); 1 ㅇ, 1 ? [1 SS, 1 UN], Léopoldville [= Kinshasa], 14 Feb. 1945, leg. Lepersonne, 20 Jan. 1947, leg. Dartevelle (RMCA: 17338, 18164); 1 ? [AL], Lula, prior to 4 Jul. 1958, leg. A.J. Jobaert (RMCA: 27201); 1 ad $q$, 1 neonate ?, 1 + , 2 ?? [5 AL], Luluabourg [= Kananga], unknown date, 3 Jul. 1965, leg. Jan Deheegher, 16 Feb. 1965, between 30 Dec. 1964 and 25 Feb. 1965, leg. Antoon Emeric Marcel De Roo (RMCA: 31573, 33351, 33961, 33962, 33349); 1 [AL], Mosenge, 15 Aug. 1986, leg. WHO and Marc Colyn (RMCA: 88.011-M-0022); 2 juv ?? [2 AL], Rumanura River, 3 Dec. 1954, leg. Gaston-François de Witte (RMCA: 37376, 37377); 1 ㅇ [AL], Uvira, 5 Sep. 1956, leg. Laurent (RMCA: 35692); 5 ?? [5 AL], Wafanya [= Wafania], unknown date, 26 May 1992, 29 May 1992, between 1 Apr. 1992 and 30 Apr. 1992, between 1 Mar. 1992 and 31 Mar. 1992, leg. P. Lootens (RMCA: 92.079-M-0150, 92.079-M-0042, 92.079-M-0043, 92.079-M0103, 92.079-M-0104); 8 juv ?? [8 AL], Yalusaka, 16 Mar. 1958, leg. F. Carbone (RMCA: 27166 to 27173); 1 ? [SO], Yolohotiri Cave, between 1 Sep. 1951 and 30 Sep. 1951, leg. Alexandre Fain (RMCA: 23299).

Miniopteridae Dobson, 1875

Miniopterus Bonaparte, 1837

Miniopterus fraterculus Thomas \& Schwann, 1906

BURUNDI: 1 q [SB], Giserama, Colline, 2 Aug. 1991, leg. Julian C. Kerbis Peterhans (FMNH: 148984); 5 ๙ึત [1 SB, 4 NS], Mpinga Cave [= Mpinga Mission], 31 Dec. 1968, 1 Jan. 1969, 25 Mar. 1970, leg. P.L. Niort (MHNG: 1607.077 to 1607.079, 1607.098, 1607.082); 6 ô 0 [5 SB, 1 SN], Nyamugari Hill, 10 Sep. 1990, 11 Sep. 1990, 12 Sep. 1990, leg. L. Davenport (FMNH: 148369, 148365, 148371 to $148373,148370)$.

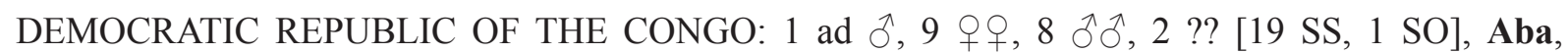
unknown date, 16 Dec. 1911, leg. Herbert Lang, James Paul Chapin and The American Museum Congo Expedition, 16 Dec. 1911, leg. unknown collector (AMNH: 51271, 51272, 49017, 49018, 49019 [holotype Miniopterus breyeri vicinior J.A. Allen, 1917], 49020 to 49029, 49031, 49032, MCZ: 17380, 17381, RMCA: 12397); 1 ? [UN], Albert National Park [= Virunga National Park], unknown date, leg. unknown collector (INPBC: V1766.1); 1 ○ [AL], Bushokwe Caves, 1 Aug. 1956, leg. Alvin Novick (MCZ: 48263); 4 q 9 [4 SS], Butembo, 10 Aug. 1947, 14 Aug. 1947, leg. unknown collector (RBINS: 7081 to 7084); 2 ?? [2 UN], Gangala-na-Bodio, 1 May 1948, leg. unknown collector (RBINS: 8124.1, 8124.2); 1 q [SS], Hoyo, Mount, 10 Aug. 1947, leg. unknown collector (RBINS: 7085); 1 q, 1 ऽ [2 UN], Ituri Forest, between 1 Jan. 1947 and 31 Dec. 1948, leg. unknown collector (RBINS: 7475, 7476); 2 ?? [2 AL], Kabatibi Cave [= Mount Hoyo Caves], unknown date, leg. The Cambridge East Africa Expedition 1959 (BMNH: 1960.108, 1960.109); 1 ? [AL], Lwiro [= Luiro], 4 Jun. 1956, leg. Uwe Rahm (RMCA: 33533); 3 ?? [1 SS, 2 AL], Matupi Cave, unknown date, leg. The Cambridge 
East Africa Expedition 1959, between 1 Sep. 1951 and 30 Sep. 1951, leg. Alexandre Fain (BMNH: 1960.112, RMCA: 21464, 23300); 4 ?? [2 SS, 2 AL], Mount Hoyo [= Hoyo, Mount], unknown date, leg. The Cambridge East Africa Expedition 1959 (BMNH: 1960.106, 1960.107, 1960.110, 1960.111); 2 ?? [2 UN], Mugongo, unknown date, leg. unknown collector (INPBC: V1766.2, V1766.3); 1 ? [AL], Mulungu, prior to 1 Feb. 1940, leg. F.L. Hendricks (RMCA: 15869); 4 ?? [4 UN], Muvo, Mount, unknown date, leg. unknown collector (INPBC: V1228.1 to V1228.4); 1 ? [UN], Talatala, unknown date, leg. unknown collector (RBINS: 7096).

RWANDA: 18 ad ठึ $\widehat{~}$ [10 SA, 8 AL], Mutura, 15 Dec. 1984, 13 Jan. 1985, leg. Benny Baeten and Luc Janssens, 27 Oct. 1981, leg. Frits De Vree, Benny Baeten and Victor Van Cakenberghe (RMCA: 85.006M-0158, 85.006-M-0152 to 85.006-M-0157, 82.006-M-0328 to 82.006-M-0338).

Miniopterus inflatus Thomas, 1903

BURUNDI: $4 \lesssim \precsim$ [4 NS], Mpinga Cave [= Mpinga Mission], 1 Jan. 1969, 25 Mar. 1970, leg. P.L. Niort (MHNG: 1607.080, 1607.081, 1608.001, 1608.002).

DEMOCRATIC REPUBLIC OF THE CONGO: 2 ?? [2 AL], Baya Carrière [= Baya Quarry], 26 Jun. 1962, 26 Jun. 1963, leg. Parent (RMCA: 31507, 31508); 5 ?? [5 UN], Gangala-na-Bodio, 9 Apr. 1948, leg. H. de Saeger et al. (RBINS: 13577.1 to 13577.5); 5 우, 33 ô ô [38 UN], Goroumbwa Mine, 17 May 1999, 18 May 1999, 19 May 1999, 19 Oct. 1999, 22 Oct. 1999, 23 Oct. 1999, 24 Oct. 1999, 25 Oct. 1999, leg. Robert (Bob) Swanepoel (TM: DRC0033, DRC0107, DRC0122, DRC0150, DRC0156, DBA010, DBA011, DBA015, DBA113, DBA115, DBA164 to DBA177, DBA198, DBA202, DBA239 to DBA244, DBA255, DBA277, DBA300, DBA317, DBA321, DBA327); 1 ? [UN], Issehe River, unknown date, leg. unknown collector (INPBC: W1457.3); 2 §ิ ô, 1 ? [3 UN], Jadotville [= Likasi], 28 Jun. 1960, leg. unknown collector, 7 Apr. 1957, leg. Félix / Michel Anciaux de Faveaux (RBINS: 1696, 1697, RMCA: 26265); 3 ?? [3 AL], Kabambi, 13 Jul. 1955, leg. GastonFrançois de Witte (RMCA: 37170 to 37172); 1 đ [SS], Kafwe, 5 Jul. 1948, leg. unknown collector

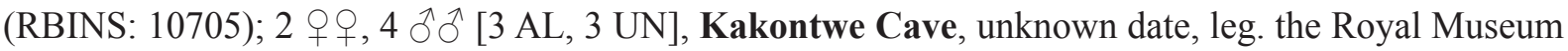
for Central Africa (RMCA), 13 Feb. 1957, 12 Jul. 1957, leg. Félix / Michel Anciaux de Faveaux (BMNH: 1959.513 to 1959.515, RMCA: 26740, 26248, 26249); 3 우우, 2 ㅊํ [4 AL, 1 UN], Kalomoni Cave [= Kakontwe], 3 Jan. 1964, 8 May 1965, leg. Félix / Michel Anciaux de Faveaux (RMCA: 33993 to 33996, 34017); 11 우, 2 đิ $\widehat{o}$ [13 AL], Kalumbu Cave, 1 Dec. 1957, leg. Félix / Michel Anciaux de

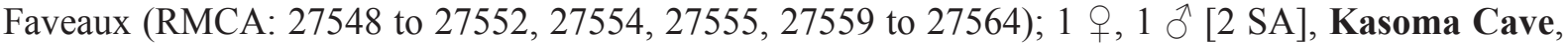
13 Aug. 1962, leg. Félix / Michel Anciaux de Faveaux (MHNG: 1046.094, 1046.095); 3 우 [3 AL], Kasowena Cave, 28 Jun. 1964, leg. Félix / Michel Anciaux de Faveaux (RMCA: 34007 to 34009); 1 ad ㅇ, 2 sad $\widehat{\jmath} \widehat{\jmath}, 3$ 우 [1 SA, 5 AL], Katanga (former) Province, 1 Dec. 1957, 13 Aug. 1962, leg. Félix / Michel Anciaux de Faveaux (SMF: 18926, 18927, 18931, 21310 to 21312); 1 ad $\widehat{\partial}$ [SS], Katobwe, 28 Nov. 1926, leg. John Todd Zimmer (FMNH: 29416 [holotype Miniopterus rufus Sanborn, 1936]); 4 $\widehat{\jmath}$ [4 AL], Komanda, 21 Aug. 1976, leg. Frieder Meier (MHNG: 1874.006 to 1874.009); 1 ㅇ [SS], Masombwe, 20 Sep. 1948, leg. unknown collector (RBINS: 10706); 1 q, 1 ๙ [2 SS], Mbuji-Mayi, 32 km SE, 25 Feb. 1974, leg. Western New Mexico University (FMNH: 152883, 152884); 5 §ิ $\widehat{~}, 1$ ? [4 AL, 2 SK], Mount Hoyo Caves, 5 Oct. 1957, leg. Robin E. Leech and Edward S. Ross (CAS: 12500 to 12503, 12531, 12532); 1 juv $\widehat{O}, 1$ q $[2$ AL], Mura Pumping Station, 20 Oct. 1963, leg.

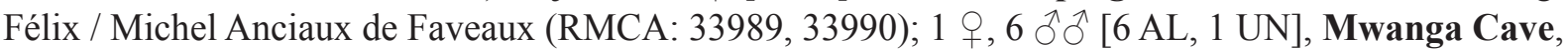
30 Jun. 1964, leg. Félix / Michel Anciaux de Faveaux (RMCA: 34010 to 34016); 1 ㅇ [AL], Pierkat Quarry [= Baya Quarry], 12 Mar. 1956, leg. Félix / Michel Anciaux de Faveaux (RMCA: 23878); 1 ठ [SK], Talatala, 11 Aug. 1947, leg. unknown collector (RBINS: 7086); 1 ? [AL], Tsebahu, 12 Jul. 1955, leg. Gaston-François de Witte (RMCA: 37169); 2 ?? [2 UN], Tshebahu River, unknown date, leg. unknown collector (INPBC: W1456a, W1456b) 
VAN CAKENBERGHE V. et al., The bats of Congo, Rwanda and Burundi revisited

RWANDA: 7 ?? [7 SK], Kibumbu Crater, 9 Dec. 1991, leg. J.R. Wilson and Anthony Michael Hutson (BMNH: 1992.391 to 1992.397); 5 ad $q$ q, 69 ad $\widehat{\jmath} \widehat{o}$ [40 SA, 34 AL], Mutura, 15 Dec. 1984, 16 Dec. 1984, 18 Dec. 1984, 12 Jan. 1985, 13 Jan. 1985, leg. Benny Baeten and Luc Janssens, 22 Oct. 1981, 23 Oct. 1981, 25 Oct. 1981, 26 Oct. 1981, 27 Oct. 1981, leg. Frits De Vree, Benny Baeten and Victor Van Cakenberghe (RMCA: 85.006-M-0136 to 85.006-M-0151, 85.006-M-0120 to 85.006M-0135, 82.006-M-0286 to 82.006-M-0327); 2 के ${ }^{\lambda}$ [2 UN], Ruhengeri, 7 Jun. 1966, leg. unknown collector (RBINS: 15490.1, 15490.2).

\section{Miniopterus minor Peters, 1867}

DEMOCRATIC REPUBLIC OF THE CONGO: 3 ?? [1 SA, 2 AL], Kinshasa, between 1 Oct. 1987 and 31 Oct. 1987, leg. WHO and Marc Colyn (RMCA: 88.011-M-0029 to 88.011-M-0031); 1 ? [UN], Kisala [= Kisala-Kiama], unknown date, leg. unknown collector (INPBC: W1462); 2 $\partial$ [1 SA, $1 \mathrm{UN}$ ], Thysville [= Mbanza-Ngungu], unknown date, leg. the Royal Museum for Central Africa (RMCA), between 1 Nov. 1935 and 14 Jan. 1936, leg. unknown collector (BMNH: 1954.866, RBINS: 1456); 1 ? [SA], Thysville, Cave [= Banjan Caves], between 1 Apr. 1947 and 30 Apr. 1947, leg. Alexandre Fain (RMCA: 18016); 1 ? [SA], Thysville, Cave b 12 a, 30 May 1949, leg. Narcisse Leleup (RMCA: 22502).

Miniopterus natalensis (A. Smith, 1833)

DEMOCRATIC REPUBLIC OF THE CONGO: 2 우, 8 $\widehat{\jmath}$ [6 SA, 4 AL], unknown locality, 24 Apr. 1962, 20 May 1962, 30 Jun. 1962, 18 Jul. 1962, 13 Aug. 1962, leg. Félix / Michel Anciaux de Faveaux (SMF: 21309, 21300 to 21302, 21308, 21304, 21305, 21303, 21306, 21307); 1 ? [AL], Albertville [= Kalemie], prior to 18 Nov. 1959, leg. H. Bomans (RMCA: 29303); 1 ad ô, 44 ?? [1 SA, 44 AL], Baya Carrière [= Baya Quarry], 17 May 1965, leg. Walter N. Verheyen (RMCA: 34018 to 34043, 35020, 35021, 35025, 35027, 35029 to 35041, 35044, 35045); 1 ㅇ, 1 ○ [2 AL], Baya Cave [= Baya Quarry], 12 Mar. 1956, leg. Félix / Michel Anciaux de Faveaux (RMCA: 23821, 23921); 52 우, 51 え [103 AL], Defrenne Cave [= Mwanga Cave], 21 Jun. 1959, leg. Félix / Michel Anciaux de Faveaux (RMCA: 28215 to 28317); $1 \widehat{\partial}$ [AL], Dethioux Farm, Cave II [= Shinkolobwe], 15 Jun. 1958,

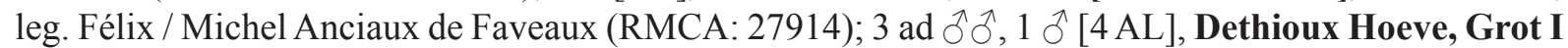
[=Shinkolobwe], 29 Sep. 1957, leg. Félix / Michel Anciaux de Faveaux (MHNG: 1046.088 to 1046.091); $2 \mathrm{ad} \hat{\jmath}, 14$ 우, $6 \hat{\partial} \hat{\partial}$ [2 SA, $20 \mathrm{UN}$ ], Elisabethville [= Lubumbashi], unknown date, leg. Félix / Michel Anciaux de Faveaux, 25 Mar. 1966, leg. unknown collector (MNHN: ZM-MO-1957-127 A, ZMMO-1957-127 B, RBINS: 17204.01 to 17204.20); 11 오 [11 SA], Grotte de Baya [= Baya Cave], 12 Mar. 1956, 14 May 1956, 22 May 1956, leg. Félix / Michel Anciaux de Faveaux (MHNG: 922.071 to $922.073,922.075$ to $922.081,922.074) ; 1$ đ [UN], Grottes Kiwakishi, 18 Oct. 1974, leg. Michael

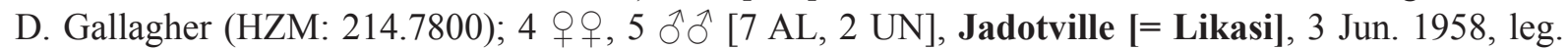
Félix / Michel Anciaux de Faveaux, 5 Apr. 1961, leg. unknown collector (RMCA: 27574 to 27580 ,

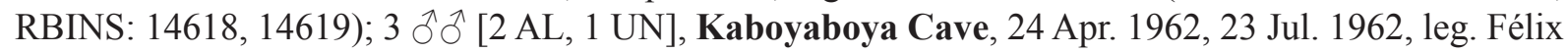
/ Michel Anciaux de Faveaux, 23 Jul. 1962, leg. unknown collector (MHNG: 1046.093, RBINS: 14617, MHNG: 1046.092); 1 ○ [AL], Kakanda Cave, 22 Apr. 1959, leg. Félix / Michel Anciaux de Faveaux (RMCA: 28166); 19 우, 13 ठో $\delta$, 8 ?? [1 SA, 37 AL, 1 SK, 1 UN], Kakontwe Cave, 26 Jun. 1956, 28 Apr. 1957, 8 Jul. 1957, 12 Jul. 1957, 15 Jan. 1958, 6 Nov. 1958, leg. Félix / Michel Anciaux de Faveaux, 6 Feb. 1958, leg. Robin E. Leech and Edward S. Ross, prior to 24 Sep. 1962, leg. L. Poelman (RMCA: 26729 to $26739,26741,26745,26754,26755,26790,26805,27565,23900$, MHNG: 1046.072 to 1046.078 , CAS: 12510 to 12513,12535 , RMCA: 27566, 27567, 26727, 26728, 26809, 26879, 31218 to 31220); 2 우, 2 ๙ิ $\widehat{\text { [ }}$ [4 AL], Kalumbu Cave, 1 Dec. 1957, leg. Félix / Michel Anciaux de Faveaux (RMCA: 27553, 27556 to 27558); 13 웅, 19 ô $\widehat{\partial}$ [30 AL, 2 UN], Kandu (Cave), 25 Jul. 1957, leg. Félix / Michel Anciaux de Faveaux (MHNG: 1046.079 to 1046.083, RMCA: 26782, 26787, 26789, $26803,26813,26816,26818,26820,26832,26834,26845$ to 26850,26854 to 26864); 2 q 9 [2 AL], Kapolowe Mission [= Kapolowe], 16 Feb. 1956, leg. Félix / Michel Anciaux de Faveaux (RMCA: 
23820, 23897); 1 ㅇ [AL], Kasapa, 22 Apr. 1956, leg. Félix / Michel Anciaux de Faveaux (RMCA: 23899); 2 우, 1 ○ [3 UN], Kasoma Cave, 13 Aug. 1962, leg. unknown collector (RBINS: 14622 to

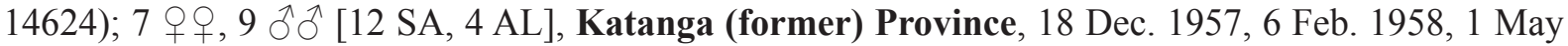
1958, 3 Jun. 1958, 11 Jun. 1960, 28 Jun. 1960, 3 Apr. 1961, leg. Félix / Michel Anciaux de Faveaux (SMF: 18928, 18935, 19074, 19076, 19077, 18932, 19072, 19073, 19075, 19078, 19573, 18929, 18930, 18936, 18933, 18934); 1 ? [AL], Kiwakishi Cave [= Kiamokoto], between 1 Jul. 1958 and 31 Jul. 1958, leg. Narcisse Leleup (RMCA: 27293); 7 $\lesssim$ [7 UN], Kyamakonde Cave, 18 Jul. 1962, leg. unknown collector (RBINS: 14610 to 14616); 1 q, 4 ô 0 [5 AL], Kyantapo Cave, 29 Jul. 1957, leg. Félix / Michel Anciaux de Faveaux (MHNG: 1046.086, RMCA: 26797, 26799, 26871, 26872); 1 ㅇ, 1 §, 1 ? [3 AL], Kyasala Cave [= Kiasala Cave], 31 Mar. 1956, 28 Jul. 1957, leg. Félix / Michel Anciaux de Faveaux (RMCA: 26796, 26801, 23896); 1 q [AL], Kyasala Grande Grotte [= Kiasala Cave], 28 Jul. 1957, leg. Félix / Michel Anciaux de Faveaux (MHNG: 1046.084); 5 ?? [1 SA, 4 AL], Lukatu Cave [= Ntoto Cave], prior to 2 Feb. 2007, leg. A. Kimbembi-ma-Ibaka (RMCA: a7.006-M-0007 to a7.006-M-0011); 2 ad $ㅇ+$, 1 juv, 1 ㅇ, 1 ภ, 9 ?? [12 SS, 2 AL], Luluabourg [= Kananga], 13 Dec. 1963, 11 Jan. 1964, 1 Feb. 1964, 11 Feb. 1964, 12 Feb. 1964, 14 Feb. 1964, 21 Feb. 1964, 23 Feb. 1964, leg. Antoon Emeric Marcel De Roo (AMNH: 207743 to 207745, 207740 to 207742, 207746 to 207749, 207753, 207750 to 207752); 3 우, 3 ๙ึ [6 AL], Lusolo Cave [= Lubudi], 28 Jul. 1957, leg. Félix / Michel Anciaux de Faveaux (MHNG: 1046.085, RMCA: 26804, 26810, 26841, 26870, 26873); 93 ?? [93 AL], M'baya Cave [= Baya Cave], 25 Mar. 1966, leg. Xavier Misonne (RMCA: 34619 to 34711); 1 क , 1 § [2 AL], Mpopola Cave, 2 May 1959, leg. Félix / Michel Anciaux de Faveaux (RMCA: 28191, 28192); 1 , 5 ô ${ }^{\lambda}$ [6 AL], Mulonga Cave, 30 Jul. 1957, 30 Aug. 1957, 1 May 1959, leg. Félix / Michel Anciaux de Faveaux (RMCA: 28205 to 28207, MHNG: 1046.087, RMCA: 26842, 26788); 1 ? [AL], Ntoto Cave, between 1 Jan. 2002 and 13 Jul. 2002, leg. A. Kimbembi-ma-Ibaka (RMCA: a2.040-M-0001); 10 우 de Faveaux (RMCA: 23818, 23870 to 23877, 23879 to 23895); 5 우우, 5 $\widehat{\jmath}$ [10 UN], Salomoni Cave, 11 Jun. 1960, 28 Jun. 1960, 20 May 1961, leg. unknown collector (RBINS: 14602 to 14605, 14620, 14621, 14606 to 14609); 6 ठิ $\widehat{~}$ [6 AL], Swanepoel II Abyss, 1 May 1958, leg. Félix / Michel Anciaux de Faveaux (RMCA: 27568 to 27573); 3 우, 2 đ̋ [5 AL], Tantara Cave [= Shinkolobwe], 29 Sep. 1957, 29 Sep. 1967, leg. Félix / Michel Anciaux de Faveaux (RMCA: 26807, 26808, 26874, 26875, 26826);

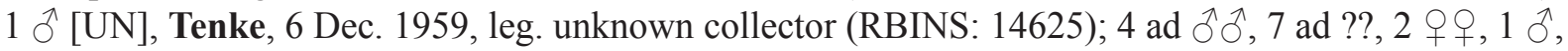
30 ?? [6 SA, 7 AL, 31 UN], Thysville [= Mbanza-Ngungu], 1 Nov. 1935, 11 Feb. 1987, leg. unknown collector, 2 Jul. 1905, 30 Mar. 1911, 2 Jun. 1915, 21 Jun. 1915, leg. Herbert Lang, James Paul Chapin and The American Museum Congo Expedition (RBINS: 1456B, 22425.01 to 22425.30, AMNH: 49292, 49309, 49321, 49324, 49331, 49333 to 49335, MCZ: 17222, AMNH: 49288, 49289, 49318, 49328); 2 ?? [1 SS, 1 AL], Thysville, Cave [= Banjan Caves], unknown date, leg. Schwetz, prior to 6 Feb. 1930, leg. Golenvaux (RMCA: 16450a, 10061); 1 ? [AL], Thysville, Cave b 12 a, 30 May 1949, leg. Narcisse Leleup (RMCA: 22501).

\section{Vespertilionidae Gray, 1821}

DEMOCRATIC REPUBLIC OF THE CONGO: 1 juv? [UN], Kantundwe, unknown date, leg. unknown collector (INPBC: W616); 1 $\lesssim$ [AL], Karambi, 3 Apr. 1992, leg. Ngenge Masumbuko Kamitongo (ZMA: MAM.24549); 1 juv ? [UN], Kasindi, unknown date, leg. unknown collector (INPBC: W979.2); 1 ? [AL], Kikondja, 6 Feb. 2010, leg. Michel Louette (RMCA: b0.004-M-0001).

Kerivoulinae Miller, 1907

Kerivoula Gray, 1842

DEMOCRATIC REPUBLIC OF THE CONGO: 1 ? [AL], Avakubi, prior to 12 Mar. 1913, leg. Cuthbert Christy (RMCA: 1522a); 1 ? [SK], Gumba, 10 Oct. 1937, leg. Jean Jacques Deheyn (RMCA: 14263a). 
Kerivoula argentata Tomes, 1861

DEMOCRATIC REPUBLIC OF THE CONGO: 1 q [SK], Baliko, 7 Jun. 2012, leg. William ("Bill”) T. Stanley (FMNH: 219604); 4 우 [2 SA, 2 UN], Kamatanda, 16 Jun. 1961, leg. unknown collector, 16 Jun. 1961, leg. Félix / Michel Anciaux de Faveaux (RBINS: 14470, 14471, SMF: 19574, 19575); 1 [SS], Kilwezi, 23 Jul. 1948, leg. unknown collector (RBINS: 10708); 5 우, 1 ? [3 SS, 1 SK, 2 UN], Luluabourg [= Kananga], unknown date, leg. Richard Callewaert, unknown date, leg. unknown collector (BMNH: 1926.7.6.92, 1926.7.6.94, 1927.12.21.13, 1927.12.21.14, ZMB: 35910, 35911).

Kerivoula cuprosa Thomas, 1912

DEMOCRATIC REPUBLIC OF THE CONGO: 1 ? [SA], Eala, between 1 Jan. 1921 and 31 Jan. 1921, leg. Henri Schouteden (RMCA: 16189); 1 ? [SA], Koteli [= Kotili], between 1 Dec. 1924 and 31 Dec. 1924, leg. Henri Schouteden (RMCA: 16190); 1 ? [SA], Wafanya [= Wafania], between 1 Jan. 1986 and 31 Dec. 1987, leg. P. Lootens (RMCA: 87.043-M-0006).

Kerivoula lanosa (A. Smith, 1847)

DEMOCRATIC REPUBLIC OF THE CONGO: 1 ? [SA], Funda Biabo, 1 Apr. 1914, leg. Charliers (RMCA: 5383); 1 [SA], Kamituga and Kampene, road between, 29 Oct. 1951, leg. P.C. Percy (BMNH: 1952.1613); 1 ? [SA], Lukulu, between 1 Jun. 1932 and 30 Jun. 1932, leg. Remaele (RMCA: 12006); 1 ? [SA], Pawa, unknown date, leg. Reitter (RMCA: 15729).

Kerivoula phalaena Thomas, 1912

DEMOCRATIC REPUBLIC OF THE CONGO: 1 q [AC], Irangi, 2 Feb. 1984, leg. Heinz Stephan (SMF: 65005); 1 + [AL], Lukolela, 30 Dec. 1930, leg. James Paul Chapin (AMNH: 86932); 1 ? [SA], Wafanya [= Wafania], 28 May 1992, leg. P. Lootens (RMCA: 92.079-M-0152).

\section{Kerivoula smithii Thomas, 1880}

DEMOCRATIC REPUBLIC OF THE CONGO: 1 ad $q, 1$ juv $\delta$ [2 UN], Akenge [= Akengei], 7 Oct. 1913, leg. Herbert Lang, James Paul Chapin and The American Museum Congo Expedition (AMNH: 49306, 49336); 4 우, 2 ふَ̋ [6 AL], Aketi, 22 Jun. 2015, 26 Jun. 2015, 26 Aug. 2015, leg. Vlir/Aketi (UNIKIS: AKETI605, AKETI606, AKETI623 to AKETI622); 3 q 9,1 ? [1 SS, 2 SO, 1 UN], Avakubi, unknown date, 1 Oct. 1912, leg. Cuthbert Christy (BMNH: 1914.4.8.6, RMCA: 1425, 1427, 1428); 1 ? [SA], Bambesa, prior to 13 Sep. 1934, leg. H.J. Bredo (RMCA: 12530); 1 ? [SS], Bukavu, prior to 14 Apr. 1955, leg. P.G. Vercammen (RMCA: 23172); 1 ? [SN], Kalehe Territory, unknown date, leg. unknown collector (BMNH: 1962.1845); 1 ad $\widehat{\jmath}$ [UN], Medje, 17 Jun. 1914, leg. Herbert Lang, James Paul Chapin and The American Museum Congo Expedition (AMNH: 49016); 1 đ [SS], Stanleyville [= Kisangani], 26 Apr. 1912, leg. Cuthbert Christy (RMCA: 1422); 2 q $q$, 1 ๙ [3 UN], Ukaika, unknown date, leg. Rudolf Grauer (NMW: 10298, 10299, 16728); 1 ? [SA], Wafanya [= Wafania], between 1 May 1991 and 31 May 1991, leg. P. Lootens (RMCA: 91.076-M-0041).

Myotinae Tate, 1942

Myotis Kaup, 1829

Myotis bocagii (Peters, 1870)

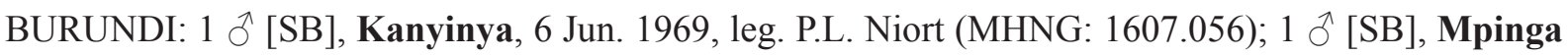
Mission, 26 Mar. 1970, leg. P.L. Niort (MHNG: 1608.003); 1 q [SK], Resha, 3 Apr. 1979, leg. Heinz Stephan (SMF: 57584). 
DEMOCRATIC REPUBLIC OF THE CONGO: 1 + , 8 ?? [5 SS, 2 AL, 2 UN], unknown locality, unknown date, leg. Herbert Lang, James Paul Chapin and The American Museum Congo Expedition, unknown date, leg. unknown collector, between 1 Jan. 1985 and 31 Dec. 1986, leg. WHO and Marc Colyn, prior to 28 Dec. 1971, leg. Gaston-François de Witte (AMNH: 51273 to 51277, BMNH: 1959.508, 1959.509, RMCA: 88.011-M-0016, 37418); 6 우, 6 추 [4 SS, 5 SO, 3 UN], Aba, 16 Dec. 1911, 17 Dec. 1911, 18 Dec. 1911, leg. Herbert Lang, James Paul Chapin and The American Museum Congo Expedition, 17 Dec. 1911, leg. unknown collector (AMNH: 49001 to 49007, 49009, 49010, MCZ:

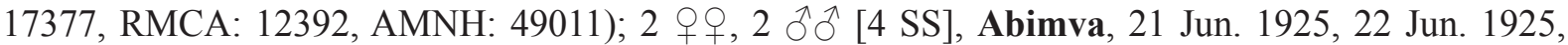
23 Jun. 1925, leg. Henri Schouteden (RMCA: 9024, 9026, 9027, 9029); 2 ?? [2 AL], Albertville [= Kalemie], prior to $30 \mathrm{Jan}$. 1900, leg. Célestin Hecq (RMCA: 422, 423); $6 \mathrm{ad}$ 우, 1 sad $\widehat{\varnothing}$ [4 SA, 3 UN], Athens Mission, 23 Jul. 1995, leg. Herwig Leirs et al. (MSB: 274603, 274604, 274614, RMCA:

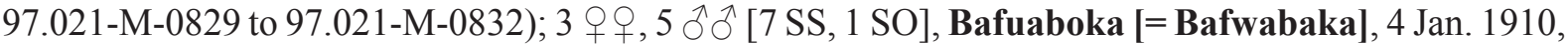
5 Jan. 1910, 6 Jan. 1910, 7 Jan. 1910, leg. Herbert Lang, James Paul Chapin and The American Museum Congo Expedition (AMNH: 48989 to 48996); 1 o [SS], Bafwabaka, between 6 Jan. 1910 and 6 Dec. 1910, leg. unknown collector (MCZ: 17376); 2 우, 1 ô [2 SA, 1 UN], Bafwaboka [= Bafwabaka], 6 Jan. 1910, 7 Jan. 1910, leg. Herbert Lang, James Paul Chapin and The American Museum Congo Expedition (AMNH: 49193, 49300, 49301); 1 + 1 ઈ [2 SS], Banalia, between 28 Jan. 1939 and 28 Dec. 1939, leg. Duncan M. Hodgson and William F. Coultas (MCZ: 38030, 38031); 2 ?? [2 AL], Bas Congo (former) Province [= Kongo Central Province], unknown date, leg. Alphonse Cabra (RMCA: 65a, 68); 1 , 5 ठึ đ, 8 ?? [14 AL], Boende, between 1 Jan. 1967 and 31 Dec. 1967, between 1 Jan. 1966 and 31 Jan. 1966, leg. P. Lootens (RMCA: 34078, 34082, 34095, 34100, 34109, 34114, 34120, 34125, 34128, 34146, 34157 to 34159, 38731); 1 ? [AL], Bokoro, prior to 19 Nov. 1952, leg. E. Jans (RMCA: 21522); 9 ?? [9 AL], Bokuma, 7 Feb. 1952, prior to 6 May 1954, between 1 Jun. 1953 and 30 Jun. 1953, between 1 Feb. 1954 and 28 Feb. 1954, between 1 Jan. 1954 and 31 Jan. 1954, leg. P. Lootens (RMCA: 21411, 22136, 22137, 22139, 22138, 22037, 22041, 22044, 22140); 6 우우 [1 SS, 3 SO, 2 UN], Bolobo, prior to 20 Aug. 1958, leg. N'Kele, prior to 27 Sep. 1951, leg. Ngwe (RMCA: 27279 to 27282, 20537, 20538); 1 ? [AL], Boma, 6 Apr. 1913, leg. Styczynski (RMCA: 2102); 1 ? [AL], Bomane [= Bomaneh], 23 May 2010, leg. Guy-Crispin Gembu Tungaluna (RMCA: a1.097-M-3219); 1 ? [AL], Congo da Lemba, between 1 Jan. 1912 and 31 Dec. 1912, leg. Mayné (RMCA: 3434); 2 §ิ ठิ [2 UN], Djabir, 10 May 2011, 13 May 2011, leg. Prescott Musaba and Jonathan Kosele (UNIKIS: DJB173, DJB174); $1 \lesssim$ [UN], Elisabethville [= Lubumbashi], 25 Apr. 1968, leg. unknown collector (RBINS: 18514); 1 ๙, 1 ? [1 SS, 1 SA], Faradje, 25 Mar. 1911, 9 Mar. 1912, leg. Herbert Lang, James Paul Chapin and The American Museum Congo Expedition (AMNH: 49000, 49425); 1 ? [AL], Ganda-Sundi, 31 Mar. 1964, leg. Alexandre Fain (RMCA: 33277); 1 , 1 o, 1 ? [3 SO], Gangala [= Gangala-na-Bodio], 23 Mar. 1957, 24 Mar. 1957, leg. Heinz Stephan (RMCA: 27464 to 27466); 1 q [SS], Gangala-naBodio, 26 Nov. 1947, leg. unknown collector (RBINS: 13331); 1 ad+foetus 9 [AL], Ibembo, between 1 Oct. 1952 and 31 Oct. 1952, leg. Jozef Hutsebaut (RMCA: 21627); 1 , 1 ठ [1 AL, 1 UN], Île Mbiye [= Mbiye Island], 15 Apr. 2012, leg. Prescott Musaba, Tembele and Marc Boketshu, between 31 May 2012 and 27 Jul. 2012, leg. Prescott Musaba (UNIKIS: CHIM53, RMCA: a1.097-M-3220); 1 , 1 స [2 SK], Irangi, 31 Jan. 1984, leg. Heinz Stephan (SMF: 65032, 65033); 1 ? [AL], Iringi, prior to 31 Aug. 1899, leg. A. or G. Lindeman (RMCA: 406); 1 q [UN], Isai River, 19 Dec. 1974, leg. Michael D. Gallagher (HZM: 7.7870); 1 ๆ, 3 $\widehat{\jmath}$ [4 SS], Kabengere, 22 Dec. 1926, 24 Dec. 1926, leg. John Todd Zimmer (FMNH: 29425 to 29428); 2 우, 4 ô $\widehat{o}$ [2 SA, 2 AL, 2 UN], Kakonde, 20 Oct. 1956, leg. Félix / Michel Anciaux de Faveaux (RMCA: 26238, 26241 to 26243, SMF: 16879, 16880); 1 ㅇ, 1 ? [1 SS, 1 SO], Kalanda, 1 Aug. 1934, 8 Aug. 1934, leg. B.L. Bennett (MCZ: 31981, 31980); 1 đ઼ [SS], Kamituga, 31 Jan. 1964, leg. Alexandre Prigogine (RMCA: 32398); 1 ? [AL], Katanga, prior to 31 Aug. 1900, leg. Charles Lemaire (RMCA: 425a); 1 ô [AL], Katapena, 27 Dec. 1926, leg. John Todd Zimmer (FMNH: 30699); 2 ?? [2 SS], Kawa (Forest), 19 Jul. 1952, 24 Jul. 1952, leg. Alexandre Fain (RMCA: 81.012-M-0072, 81.012-M-0071); 2 우, 1 $\widehat{\partial}$ [3 UN], Kipangaribwe River, 1 Oct. 1947, leg. unknown collector (RBINS: 10807.1 to 10807.3); 1 ? [SA], Kiseny [= Kasenye], 16 Sep. 1927, leg. 


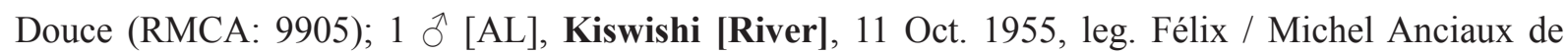
Faveaux (RMCA: 26216); 1 ad + , 1 juv,+ 1 , 1 1 [4 SS], Kivulo, 24 Aug. 1947, leg. unknown

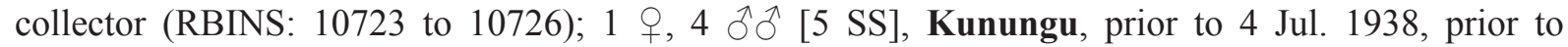
19 Nov. 1937, prior to 1 Aug. 1939, leg. N'Kele (RMCA: 15512 to 15514, 14167, 14660); 1 ? [AL], Lake Moero [= Moero, Lake], prior to 23 Dec. 1947, leg. unknown collector (RMCA: 18173); 1 sad đ, 2 우, 1 ๙ [1 SS, $3 \mathrm{SA}$ ], Léopoldville [= Kinshasa], unknown date, 8 Nov. 1893, leg. J. Camp, 8 Jul. 1909, leg. Herbert Lang, James Paul Chapin and The American Museum Congo Expedition (USNM: 21666, 21667, AMNH: 48936, USNM: 21665); 1 ? [SS], Leverville [= Lusanga], between

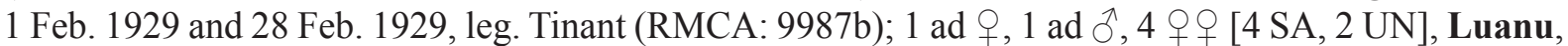
26 Jul. 1995, leg. Herwig Leirs et al. (MSB: 274911, 274914, RMCA: 97.021-M-0833 to 97.021-M0836); 2 đิ $\delta$ [2 SS], Lukafu, 20 Dec. 1930, leg. Gaston-François de Witte and de Crawhez (RMCA: 11131, 11132); 1 Oૈ, 1 ? [1 SA, 1 AL], Mabali, 27 Jun. 1956, between 1 Jun. 1956 and 30 Jun. 1956, leg. Alvin Novick (MCZ: 48261, 48262); 4 ad $q$ ㅇ, 1 ad $ð$ [3 SA, 2 UN], Mbalaka (Plantation), 19 Jul. 1995, 20 Jul. 1995, 21 Jul. 1995, leg. Herwig Leirs et al. (MSB: 274917, RMCA: 97.021-M-0826, 97.021-M0827, MSB: 274918, RMCA: 97.021-M-0828); 1 juv ふึ, 2 우우 [1 SS, 2 UN], Medje, 27 Jun. 1910, leg. Herbert Lang, James Paul Chapin and The American Museum Congo Expedition (AMNH: 48997, 48999, RMCA: 12393); 1 ? [SN], Moba, unknown date, leg. unknown collector (BMNH: 1954.854); 1 q [AL], Mosenge, 15 Aug. 1986, leg. WHO and Marc Colyn (RMCA: 88.011-M-0017); 11 ?? [6 SS, 5 AL], Mount Wago [= Wago, Mount], unknown date, 30 Aug. 1953, prior to 28 Mar. 1952, between 1 Jan. 1951 and 31 Dec. 1951, leg. Alexandre Fain, prior to 31 Dec. 1990, leg. unknown collector (BMNH: 1953.486, MHNG: 921.033, RMCA: 21455 to 21459, 20788 to 20790, RBINS: 21657); 1 ㅇ [SS], Muhulu, 16 Jan. 1957, leg. Heinz Stephan (RMCA: 27467); 1 ? [SS], Ndwa, between 1 Oct. 1950 and 30 Nov. 1950, leg. Ngwe (RMCA: 20254); 4 ?? [4 SS], Né, Mount, unknown date, 29 Aug. 1952, 30 Aug. 1952, leg. Alexandre Fain (BMNH: 1953.498, RMCA: 21513, 21511, 21512); 5 우, 7 ठో [12 UN], Obenge, 6 Feb. 2013, 7 Feb. 2013, 8 Feb. 2013, leg. Prescott Musaba and André Malekani (UNIKIS: TLL367 to TLL371, TLL391, TLL395 to TLL400); 1 ? [AL], Pweto, unknown date, leg. Gerard (RMCA: 5441a); 1 ? [UN], Rodahira River, unknown date, leg. unknown collector (INPBC: W875.1); 2 + 9 [2 UN], Rubi-Tele, 24 Aug. 2013, leg. Guy-Crispin Gembu Tungaluna, Prescott Musaba and André Malekani (UNIKIS: RBTL557, RBTL558); 1 ? [SS], Saliboko, 5 Aug. 1952, leg. Alexandre Fain (RMCA: 21438); 1 ? [UN], Sombe, unknown date, leg. Lademann (ZMB: T10164); $2 \widehat{\jmath} \widehat{\partial}[1 \mathrm{SS}, 1 \mathrm{AL}$ ], Tandala, 16 Jul. 1979, leg. unknown collector, 16 Jul. 1979, leg. C. Brian Robbins (MHNG: 1870.089, USNM: 537721); 1 ? [AL], Tanganyika Province, prior to 30 Jan. 1900, leg. Célestin Hecq (RMCA: 420); 1 ? [AL], Tshakala, between 1 Jan. 1982 and 31 Dec. 1982, leg. Marc Colyn (RMCA: 82.019-M-0008); 31 ?? [29 AL, 2 UN], Wafanya [= Wafania], unknown date, 10 Aug. 1991, 7 Oct. 1991, 11 Oct. 1991, 12 Oct. 1991, 16 Oct. 1991, 5 Mar. 1992, 20 Apr. 1992, 18 May 1992, 26 May 1992, 29 May 1992, between 1 Dec. 1991 and 31 Dec. 1991, between 1 Apr. 1991 and 30 Apr. 1991, between 1 Mar. 1992 and 31 Mar. 1992, leg. P. Lootens (RMCA: 92.079-M-0140 to 92.079-M-0148, 91.076-M-0055, 91.076-M-0107, 91.076-M-0099, 91.076-M-0111, 91.076-M-0083, 91.076-M-0113, 92.079-M-0027, 92.079-M-0029, 92.079-M-0028, 92.079-M-0032, 92.079-M-0030, 92.079-M-0031, 92.079-M-0058, 91.076-M-0104, 92.079-M-0026, 92.079-M-0059, 91.076-M-0028, 92.079-M-0057, 92.079-M-0060 to 92.079-M-0062, 92.079-M-0116); 1 juv ?, 13 ?? [13 SS, 1 SO], Wago, Mount, 30 Aug. 1952, between 1 Jul. 1951 and 31 Jul. 1951, between 1 Jan. 1951 and 31 Dec. 1951, leg. Alexandre Fain (RMCA: 21504 to 21510, 81.012-M-0074, 81.012-M-0075, 23285 to 23287, 81.012-M-0073, 23290); 2 우, 4 ổ ${ }^{\pi}$ [2 SS, 4 AL], Yalosemba, 17 Jun. 1979, between 1 Jun. 1979 and 30 Jun. 1979, leg. C. Brian Robbins (USNM: 463485 to 463487, 537719, 537720, MHNG: 1870.090); 1 đ [UN], Yoko, 5 Sep. 2012, leg. Prescott Musaba, Tembele and Marc Boketshu (UNIKIS: YK017CH); 1 ? [AL], Zambi, prior to 31 Jul. 1899, leg. Lepez (RMCA: 284).

RWANDA: 3 ad ${ }_{+} \circ, 1$ ad ${ }^{\jmath}, 1$ ? [5 SA], Butare, 21 Dec. 1984, 22 Dec. 1984, 23 Dec. 1984, 24 Dec. 1984, leg. Benny Baeten and Luc Janssens (RMCA: 85.006-M-0450 to 85.006-M-0454); 1 ô, 1 ? [2 SA], 
Gisenyi, 21 Oct. 1980, leg. Danny Meirte (RMCA: 80.055-M-0167, 80.055-M-0168); 1 ô, 1 ? [1 SA, 1 AL], Kibuye, 7 Oct. 1980, leg. Danny Meirte (RMCA: 80.055-M-0165, 80.055-M-0166); 15 ?? [15 UN], Mihindi, Lake, unknown date, leg. unknown collector (INPBC: W3052.01 to W3052.15); $1 \hat{o}$ [UN], Rubona, 1 Jan. 1966, leg. Félix / Michel Anciaux de Faveaux (RBINS: 15492).

\section{Myotis tricolor (Temminck, 1832)}

DEMOCRATIC REPUBLIC OF THE CONGO: $1 \hat{\overbrace{}}$ [SA], Dethiox Caves [= Kambove], 4 Jun. 1960, leg. Félix / Michel Anciaux de Faveaux (SMF: 18919); 1 ? [UN], Djomba [= Djemba], unknown date, leg. unknown collector (INPBC: V1846.9); 2 ๙ิ [2 AL], Jomba, 5 Sep. 1976, leg. Frieder Meier (MHNG: 1873.072, 1873.073); 1 q [SA], Kakontwe Cave, 22 Sep. 1957, leg. Félix / Michel Anciaux de Faveaux (MHNG: 1046.024); 1 ? [UN], Kamikoni, unknown date, leg. unknown collector (INPBC: V1694.1); 1 \& [SA], Katanga (former) Province, 18 Jul. 1962, leg. Félix / Michel Anciaux de Faveaux (SMF: 21299); 1 q [AL], Kyamakonde Cave, 18 Jul. 1962, leg. Félix / Michel Anciaux de Faveaux (MHNG: 1046.097); 1 đ [AL], Mulonga Cave, 30 Jul. 1957, leg. Félix / Michel Anciaux de Faveaux (RMCA: 26792); 1 ? [SO], Thysville, Cave [= Banjan Caves], unknown date, leg. Schwetz (RMCA: 16450b).

RWANDA: 1 ad + , 5 ad $\widehat{\text { đे }}$ [2 SS, 4 SA], Mutura, 18 Dec. 1984, leg. Benny Baeten and Luc Janssens, 23 Oct. 1981, 25 Oct. 1981, 27 Oct. 1981, leg. Frits De Vree, Benny Baeten and Victor Van Cakenberghe (RMCA: 85.006-M-0449, 82.006-M-0920 to 82.006-M-0924).

\section{Myotis welwitschii (Gray, 1866)}

BURUNDI: 1 ? [SS], Rwegura, between 1 Jun. 1969 and 31 Jul. 1969, leg. unknown collector (MHNG: 1607.095).

DEMOCRATIC REPUBLIC OF THE CONGO: 1 ? [SO], Dilolo Station [= Dilolo], between 1 Mar. 1934 and 31 Mar. 1934, leg. Richard (RMCA: 12661); 1 \& , 1 ? [1 UN, 1 SB], Elisabethville [= Lubumbashi], 18 Oct. 1966, leg. unknown collector, prior to 11 Feb. 1956, leg. Seydel (RBINS: 15428, RMCA: 23462); 1 ㅇ [UN], Kasapa, between 1 Jul. 1967 and 31 Jul. 1967, leg. unknown collector (RBINS: 18513); 1 ㅇ [SO], Lusinga, 17 Jul. 1947, leg. unknown collector (RBINS: 10848); 1 ? [UN], Mutsora, unknown date, leg. unknown collector (INPBC: W916); 3 ?? [3 UN], Ondo, Lake, unknown date, leg. unknown collector (INPBC: V1240.2, V1819.3.1, V1819.3.2); 1 § [SA], Pande, unknown date, leg. Jean Romieux (MHNG: 1891.002); 1 § [SS], Rutshuru, 10 Mar. 1938, leg. unknown collector (RBINS: 4789).

RWANDA: 1 đ̊ [SA], Butare, 28 Sep. 1980, leg. Danny Meirte (RMCA: 80.055-M-0169).

Scotophilinae Gray, 1866

Scotophilus Leach, 1821

BURUNDI: 7 đ̊̊ [2 SS, 5 SK], Bujumbura, 6 Nov. 1967, 9 Nov. 1967, 1 Dec. 1967, 15 Dec. 1967, 23 Oct. 1968, 13 Dec. 1968, 26 Dec. 1968, leg. P.L. Niort (MHNG: 1607.045, 1607.049, 1607.046,

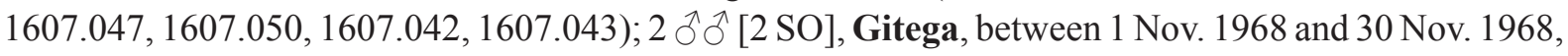
leg. P.L. Niort (MHNG: 1607.053, 1607.054); 1 q [AN], Kanyosha, 10 Oct. 1969, leg. P.L. Niort (MHNG: 1607.084).

DEMOCRATIC REPUBLIC OF THE CONGO: 1 ad $\precsim$ [AL], Kivu [= Kivu region], unknown date, leg. Guy Babault (MNHN: ZM-MO-1985-1067). 
Scotophilus dinganii (A. Smith, 1833)

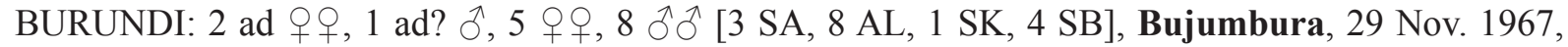
12 Nov. 1968, 20 May 1969, 5 Jan. 1970, 9 Jan. 1970, 16 Nov. 1972, leg. P.L. Niort, 21 Apr. 1982, leg. Danny Meirte, between 1 May 1977 and 31 May 1977, leg. Jacques Verschuren, between 1 Jul. 1968 and 31 Jul. 1968, leg. Lewall (MHNG: 1607.048, 1608.012, 1607.055, RMCA: 82.016-M-0002, 82.016M-0005 to 82.016-M-0008, 82.016-M-0072, 82.016-M-0124, MHNG: 1607.044, 1607.093, 1607.094, RBINS: 19915.1, 19915.2, RMCA: 35156); 4 우 [4 SB], Kanyosha, 10 Oct. 1969, 21 Oct. 1969, leg. P.L. Niort (MHNG: 1607.085 to 1607.088); 1 q [SB], Muyebe, between 1 Jan. 1900 and 23 Dec. 1969,

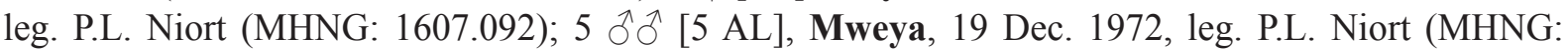

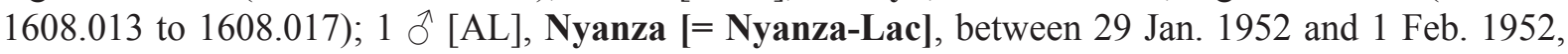
leg. Laurent (RMCA: 90.008-M-0001); 3 $え \widehat{~}$ [2 SA, 1 SK], Resha, 5 Apr. 1979, between 4 Apr. 1979 and 5 Apr. 1979, leg. Heinz Stephan (SMF: 57567, 57569, 57568); 3 ठ઼ [3 SB], Rwintare, between 1 Feb. 1970 and 28 Feb. 1970, leg. P.L. Niort (MHNG: 1608.008 to 1608.010).

DEMOCRATIC REPUBLIC OF THE CONGO: 2 ad $\widehat{\jmath} \widehat{\jmath}[2 \mathrm{SS}$ ], Albert, Lake, 23 Oct. 1906, leg. Alexander Douglas Mitchell Carruthers (BMNH: 1907.6.14.11, 1907.6.14.12); $1 \AA$ [AL], Albertville [= Kalemie], 12 Nov. 1946, leg. La Mission Hydrobiol. Lac Tanganiyka (RBINS: 9069); 2 ad §ð, 1 ad ?, 1 juv $\widehat{\jmath}$ [2 SA, 1 AL, 1 SK], Banana, between 1 Jan. 1884 and 31 Dec. 1884, leg. L. Anema, between 1 Jan. 1889 and 31 Dec. 1889, leg. P. Hesse, between 1 May 1952 and 31 May 1952, between 1 Jan. 1952 and 31 Jan. 1952, leg. Is. Mesmaekers (ZMA: MAM.09008, SMF: 2515, RMCA: 20748,

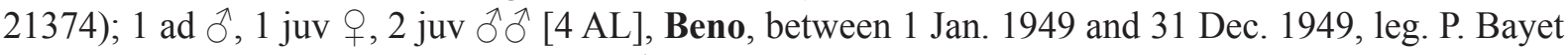
(RMCA: 19831 to 19833, 19848); 1 juv $\widehat{\partial}$ [AL], Boende, between 1 Jan. 1931 and 31 Dec. 1931, leg. R.P. Wynants (RMCA: 18141); 1 ad,+ 1 ad $\widehat{\jmath}$ [2 SA], Boma, between 1 Feb. 1955 and 28 Feb. 1955, leg. Is. Mesmaekers (RMCA: 26321, 26322); 7 ad $\partial^{\lambda} \widehat{\jmath}, 1$ juv + , 1 juv ${ }^{\lambda}, 1$ ? [7 SA, 2 AL, 1 SK], Elisabethville [= Lubumbashi], unknown date, leg. J. Bequaert, 6 Apr. 1956, 22 Apr. 1956, leg. Félix / Michel Anciaux de Faveaux, 5 Aug. 1952, leg. A. Smitz, between 1 Apr. 1926 and 30 Apr. 1926, between 1 Jan. 1931 and 31 Dec. 1931, leg. Henri Schouteden, prior to 10 Aug. 1933, between 1 Jan. 1934 and 31 Dec. 1934, leg. Quarré (RMCA: 16255, 23898, RBINS: 14454, RMCA: 23819, 9179, 12850, 16212, 16233, 12149, 12150); 1 ad $\widehat{\jmath}$ [SA], Funda Biabo, between 1 Jan. 1914 and 31 Dec. 1914,

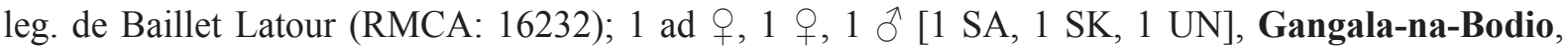
10 Apr. 1948, leg. H. de Saeger et al., 9 Apr. 1948, leg. unknown collector (RBINS: 8126, 8125, 8127.1); 1 ad $ठ$ [SA], Haute Congo, 4 Jun. 1992, leg. The Rotterdamse Diergaarde (RMNH: MAM.28010); 2 juv $\widehat{\jmath} \widehat{\jmath}, 2$ 우우 [2 AL, 2 UN], Jadotville [= Likasi], 1 Dec. 1957, 15 Nov. 1962, leg. Félix / Michel Anciaux de Faveaux, 16 Dec. 1959, leg. unknown collector (RMCA: 27585, 33986, RBINS: 14452, 14453); 1 ad $\hat{~}$ [SA], Kabalo, between 1 Mar. 1926 and 31 Mar. 1926, leg. Henri Schouteden (RMCA: 16249); 1 ㅇ, 1 § [2 SS], Kabwe, 12 Nov. 1973, leg. Western New Mexico University (FMNH: 152836, 152837); 1 ? [UN], Kadia, between 1 Mar. 1926 and 31 Mar. 1926, leg. Henri Schouteden (RMCA: 16252); 1 ad? ô [SA], Kakontwe Cave, prior to 24 Sep. 1962, leg. L. Poelman (RMCA: 31217); 1 ad

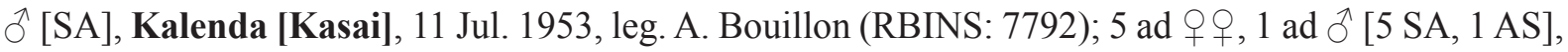
Kamponde, between 1 Apr. 1965 and 30 Apr. 1965, leg. Antoon Emeric Marcel De Roo (RMCA: 33383 to 33388); 1 ㅇ, 1 § [2 AL], Kananga, 21 Sep. 1963, leg. Antoon Emeric Marcel De Roo (AMNH: 212597, 212598); 1 ? [UN], Katanda, unknown date, leg. unknown collector (INPBC: W1369);

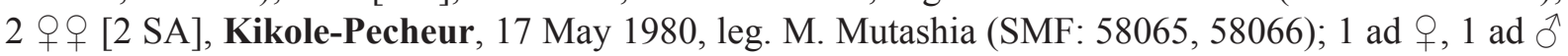
[1 SA, 1 UN], Kikwit, 6 Jul. 1995, leg. Herwig Leirs et al. (MSB: 274622, RMCA: 97.021-M-0840); 2 ad 웅, 3 ad ๙ิ $^{\circ}$ [2 SA, 3 UN], Kikwit ITPK [= Kikwit], 1 Aug. 1995, leg. Herwig Leirs et al. (MSB: 274926, 274986, 274987, RMCA: 97.021-M-0847, 97.021-M-0848); 4 우우 [4 AL], Kinkole, 15 Aug. 1980, leg. unknown collector (ZMA: MAM.21149 to MAM.21152); 6 ad $q$ +, 1 ad $\curvearrowright$, 1 ad? $ᄋ$ [4 SA, 4 UN], Kinzambi Mission, 24 Jul. 1995, 25 Jul. 1995, 26 Jul. 1995, 28 Jul. 1995, 30 Jul. 1995, 31 Jul. 1995, leg. Herwig Leirs et al. (MSB: 274922, RMCA: 97.021-M-0843, MSB: 274923, 274924, 
RMCA: 97.021-M-0844, 97.021-M-0845, MSB: 274925, RMCA: 97.021-M-0846); 2 ad $q$ +, 1 ad 1 juv +, 1 ㅇ, 1 § [5 SA, 1 AL], Kipopo, 30 Nov. 1955, leg. Félix / Michel Anciaux de Faveaux, 30 Nov. 1955, leg. De Bont, 30 Nov. 1955, leg. Michel Anciaux de Faveaux (MHNG: 922.082, RMCA: 26323 to 26326, SMF: 16866); 1 ad + [SA], Kipushi, 12 Aug. 1954, leg. De Smet (RMCA: 22458); 1 ad ? [SK], Kisantu, 15 Dec. 1920, leg. Henri Schouteden (RMCA: 6564); 1 ? [SK], Kyasala Cave [= Kiasala Cave], 31 Mar. 1956, leg. Félix / Michel Anciaux de Faveaux (RMCA: 23851 b); 1 ad $\widehat{\delta}$ [SA], Losho [= Loso], 23 Nov. 1954, leg. Gaston-François de Witte (RMCA: 37380); 3 ad + o , 1 ad ${ }^{1}$, 3 juv ?? [4 SS, 3 SO], Lovanium University [= Kinshasa], 10 Dec. 1962, 12 Mar. 1963, 6 Nov. 1963, between 8 Dec. 1962 and 27 Nov. 1963, between 7 Nov. 1963 and 27 Nov. 1963, between 27 Nov. 1962 and 27 Nov. 1963, leg. De Bont and Jo Van Orshoven (RMCA: 32628, 32626, 32625, 32629, 32624,

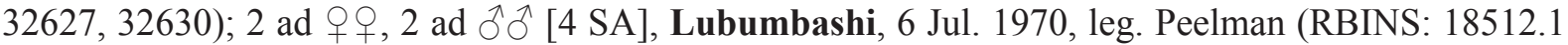

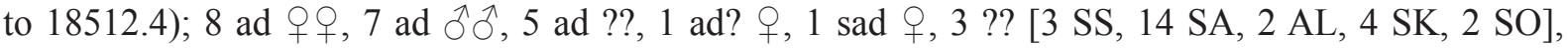
Luluabourg [= Kananga], 8 Sep. 1963, 5 Oct. 1963, 16 Feb. 1965, 2 Mar. 1965, 19 Mar. 1965, 17 May 1965, 3 Jul. 1965, 30 Apr. 1966, between 3 Mar. 1965 and 17 May 1965, between 29 Aug. 1963 and 5 Oct. 1963, between 2 Mar. 1965 and 17 May 1965, between 17 May 1965 and 8 May 1966, between 17 May 1965 and 20 May 1965, between 17 May 1965 and 19 May 1965, between 14 Sep. 1963 and 14 Oct. 1964, leg. Antoon Emeric Marcel De Roo, between 1 Jan. 1931 and 31 Dec. 1931, leg. Jan Deheegher (RMCA: 33314, 33650, 33318, 33319, 33315 to 33317, 333380, 33381, 33595, 35287, 32669, $32671,32672,33310$ to $33312,31566,33313,33651,33652,35288,33382,32670,33649)$; 4 ad 우우 [4 SA], Luluabourg Ravine, 29 Aug. 1963, between 29 Jun. 1963 and 29 Aug. 1963, leg. Antoon

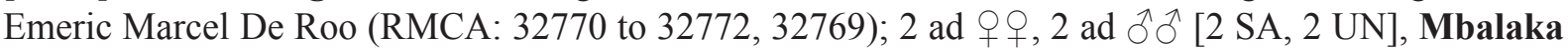
(Plantation), 21 Jul. 1995, 23 Jul. 1995, 24 Jul. 1995, leg. Herwig Leirs et al. (MSB: 274989, RMCA: 97.021-M-0841, MSB: 274988, RMCA: 97.021-M-0842); 1 ad ${ }^{\uparrow}$ [SA], Mbwambala, 20 Jun. 1995,

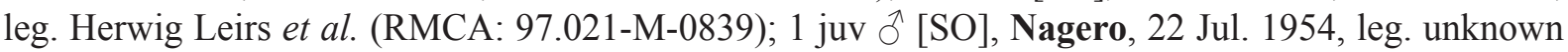
collector (RBINS: 14629); 1 juv + , 1 juv $\widehat{o}$ [2 AL], Panda [= Pande], 15 Nov. 1962, 18 Nov. 1962, leg. Félix / Michel Anciaux de Faveaux (RMCA: 33987, 33988); 1 ad ?, 2 ?? [1 SK, 2 SO], Paulis [= Isiro], 28 Apr. 1947, 1 Nov. 1947, between 1 Jan. 1947 and 31 Dec. 1947, leg. Abbeloos (RMCA: 18569, 18943, 17931); 3 ad $q 9,1$ juv $q, 1$ imm ${ }^{\lambda}, 5$ ?? [1 SA, 2 AL, 2 SK, 5 UN], Rutshuru, unknown date, leg. Jacques Verschuren, unknown date, leg. unknown collector, 27 Mar. 1954, leg. GastonFrançois de Witte, between 24 Mar. 1932 and 24 Mar. 1937, between 1 May 1937 and 31 May 1937, leg. J. Ghesquière (INPBC: V1362, V1420.2, V1426, V1522.2, V415, W334, W917, RMCA: 37379, 14065, 13868); 1 ad $q$ [AL], Rwindi, unknown date, leg. Jacques Verschuren (INPBC: V1623); 1 ad $q, 2$ ad

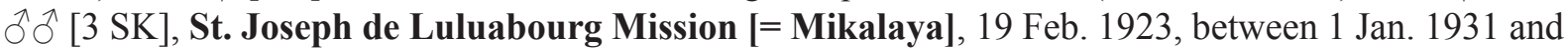
31 Dec. 1931, leg. Richard Callewaert (RMCA: 7110 to 7112); 1 + , 1 ○ [2 SS], Tandala, 10 Jul. 1979, 19 Jul. 1979, leg. C. Brian Robbins (USNM: 537728, 537729); 1 sad §̊ [AL], Tungudu, 3 Jun. 1957, leg. Gaston-François de Witte (RMCA: 37378); 1 ad $\curvearrowright, 1$ ad? $\delta, 1$ juv \& [2 SA, 1 AL], Uvira, between 3 Mar. 1955 and 21 Jun. 1958, between 12 Sep. 1951 and 12 Sep. 1957, between 1 Apr. 1955 and

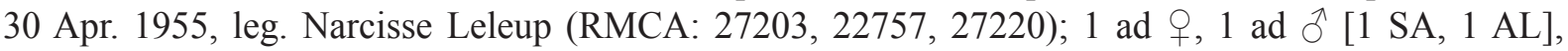
Virunga National Park, unknown date, leg. Jacques Verschuren (INPBC: V1807, V1957); 1 ad $q$ [SS], Vitshumbi, 28 Sep. 1933, leg. Gaston-François de Witte et al. (RBINS: 4772); 2 के के [2 SS], Yalosemba, 28 Jun. 1979, 30 Jun. 1979, leg. C. Brian Robbins (USNM: 537727, 537726).

RWANDA: 5 ?? [1 SA, 4 AL], Astrida [= Butare], 12 Oct. 1976, 13 Oct. 1976, leg. Frieder Meier

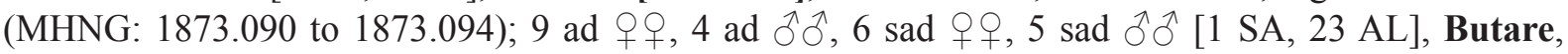
10 Dec. 1984, 11 Dec. 1984, 20 Dec. 1984, 21 Dec. 1984, 22 Dec. 1984, 23 Dec. 1984, 24 Dec. 1984, 15 Jan. 1985, leg. Benny Baeten and Luc Janssens, 3 Oct. 1981, leg. Frits De Vree, Benny Baeten and Victor Van Cakenberghe (RMCA: 85.006-M-0097 to 85.006-M-0101, 85.006-M-0118, 85.006M-0119, 85.006-M-0102 to 85.006-M-0117, 82.006-M-0915); 1 ad + [SA], Cyili Ntyano [= Cyili], 21 Apr. 1985, leg. Théoneste (RMCA: 87.047-M-0131); 1 juv [ [AL], Gabiro, prior to 27 Apr. 1936, leg. Verhulst (RMCA: 13281); 1 ad $\odot$ [SA], Kigali, between 1 Jul. 1982 and 31 Jul. 1982, leg. Benny Baeten 
(RMCA: 87.046-M-0246); 1 ad + [SA], Ruboma Ruhashya, between 1 Jan. 1985 and 31 Dec. 1985, leg. Théoneste (RMCA: 87.047-M-0130); 2 sad ổ ô [2 AL], Rukira, 20 Nov. 1981, 24 Nov. 1981, leg. Frits De Vree, Benny Baeten and Victor Van Cakenberghe (RMCA: 82.006-M-0916, 82.006-M-0917); 1 juv $q$ [AL], Rwankwi, between 1 Apr. 1948 and 30 Apr. 1948, leg. J.V. Leroy (RMCA: 19068).

Scotophilus leucogaster (Cretzschmar, 1826)

BURUNDI: $2 \widehat{\partial}$ [2 AL], Mweya, 29 Apr. 1971, leg. P.L. Niort (MHNG: 1896.016, 1896.017).

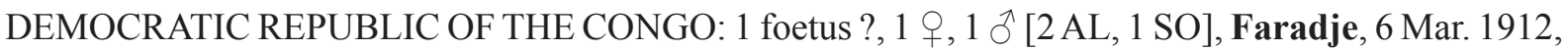
7 Mar. 1912, leg. Herbert Lang, James Paul Chapin and The American Museum Congo Expedition (AMNH: 49359, 49015, 49283).

Scotophilus nigrita (Schreber, 1774)

BURUNDI: 1 đ [SK], Bujumbura, 31 Jan. 1969, leg. P.L. Niort (MHNG: 1607.052).

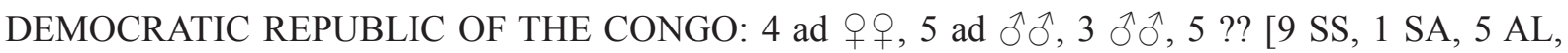
$1 \mathrm{SO}, 1 \mathrm{UN}$ ], Albertville [= Kalemie], 5 Jan. 1964, prior to 18 Aug. 1965, leg. Antoon Emeric Marcel De Roo, prior to 12 Jul. 1955, leg. service hygiene publique albertville, prior to 18 Nov. 1959, leg. H. Bomans, prior to 5 Dec. 1951, leg. unknown collector (AMNH: 206844, 212591 to 212596, RMCA: 31718, 22948, 33585, 33586, 29293 to 29297 , RBINS: 14598).

\section{Scotophilus nux Thomas, 1904}

DEMOCRATIC REPUBLIC OF THE CONGO: 1 ad ? [SS], Ibembo, between 6 Jun. 1951 and 21 Dec. 1951, leg. Jozef Hutsebaut (RMCA: 20661); $1 \widehat{\delta}$ [UN], Kongolo, 9 Nov. 1974, leg. Michael D. Gallagher (HZM: 64.7806); 2 ad $\circ$ + 2 ad ?? [2 SS, 2 UN], Medje, unknown date, 26 Mar. 1914, leg. Cuthbert Christy, between 1 Mar. 1914 and 31 Mar. 1914, leg. Herbert Lang, James Paul Chapin and The American Museum Congo Expedition (BMNH: 1919.5.8.13, RMCA: 4344, AMNH: 49286, 49287); 2 ad $\lesssim \widehat{~} \widehat{~[2 ~ S S], ~ P a n g a, ~ b e t w e e n ~} 1$ Sep. 1925 and 30 Sep. 1925, leg. Henri Schouteden (RMCA: 9125,

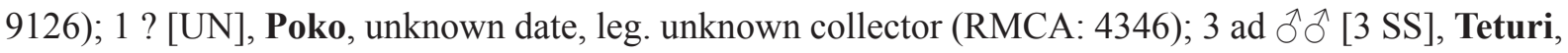
23 Mar. 1947, 24 Mar. 1947, leg. Jean Hiernaux (RBINS: 7041 to 7043); 3 $q$ q, 3 $ぇ ~[6 ~ S S$ ], Yalosemba, 17 Jun. 1979, 26 Jun. 1979, 28 Jun. 1979, leg. C. Brian Robbins (USNM: 537730 to 537735).

Vespertilioninae Gray, 1821

Glauconycteris Dobson, 1875

DEMOCRATIC REPUBLIC OF THE CONGO: 1 त $[\mathrm{AL}]$, Île Mbiye [= Mbiye Island], 23 Jun. 2014, leg. Prescott Musaba, Moïse Bipoo, Richard Tamaru, Pauline Isude and Joseph Kussa (UNIKIS: CHIM020/14); 1 + [SS], Luluabourg [= Kananga], unknown date, leg. Richard Callewaert (BMNH: 1926.7.6.95).

\section{Glauconycteris alboguttata J.A. Allen, 1917}

DEMOCRATIC REPUBLIC OF THE CONGO: 1 q [SO], Lukolela, 14 Mar. 1933, leg. Lecocq (RMCA: 12175); 1 ad + [UN], Medje, 14 Mar. 1914, leg. Herbert Lang, James Paul Chapin and The American Museum Congo Expedition (AMNH: 49317 [holotype Glauconycteris alboguttatus J.A. Allen, 1917]); 1 ? [UN], Yalosemba, unknown date, leg. unknown collector (CM: 86658); 1 \& [UN], Yangambi, 4 Mar. 1947, leg. unknown collector (RBINS: 14516). 
Glauconycteris argentata (Dobson, 1875)

BURUNDI: 2 우 [2 SS], Rumonge, between 1 Aug. 1969 and 31 Aug. 1969, leg. P.L. Niort (MHNG: 1607.090, 1607.091).

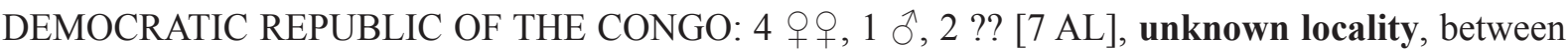
1 Apr. 1800 and 31 May 1899, leg. P. Hesse, prior to 28 Jun. 1960, leg. Uwe Rahm (SMF: 11850 [paralectotype Chalinolobus congicus Noack, 1889], 11851 [paralectotype Chalinolobus congicus Noack, 1889], 11852 [paralectotype Chalinolobus congicus Noack, 1889], 11853 [paralectotype Chalinolobus congicus Noack, 1889], 11854 [paralectotype Chalinolobus congicus Noack, 1889], 11855 [paralectotype Chalinolobus congicus Noack, 1889], RMCA: 28921); 1 ad ô, 3 ?? [3 AL, 1 UN], Banana, unknown date, leg. unknown collector, 13 Mar. 1952, between 1 May 1952 and 31 May 1952, leg. Is. Mesmaekers (BMNH: 1954.863, RMCA: 20751, 20752, 21376); 1 [AL], Boende, between 1 Jan. 1966 and 31 Jan. 1966, leg. P. Lootens (RMCA: 34160); 1 ? [AL], Boga, prior to 13 May 1913, leg. André Pilette (RMCA: 1862); 5 ?? [4 AL, 1 UN], Bokuma, 7 Feb. 1952, 7 Mar. 1952, between 1 Jan. 1954 and 31 Jan. 1954, leg. P. Lootens (RMCA: 21412, 21414, 21415, 21413, 22135);

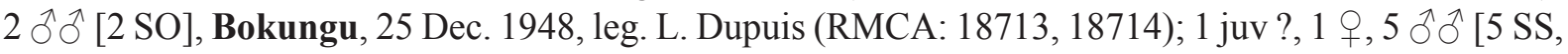
2 SO], Boma, 23 Nov. 1920, 28 Nov. 1920, leg. Henri Schouteden (RMCA: 6571 to 6576, 7250); 1 ?

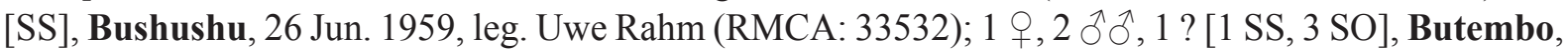
25 Apr. 1954, prior to 26 Jan. 1955, leg. P. Dyleff (RMCA: 22723, 22816 to 22818); 5 ?? [5 AL], Djeka, 29 May 1955, prior to 15 Jun. 1956, leg. Roiseux (RMCA: 23635 to 23639); 21 ?? [21 UN], Fataki, between 1 Jan. 1948 and 6 Apr. 1964, leg. unknown collector (RBINS: 18526.01 to 18526.17,

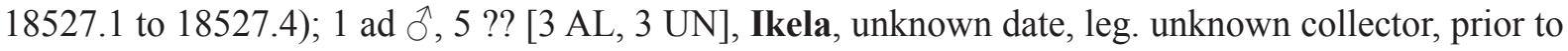
4 Jan. 1958, prior to 3 Nov. 1956, leg. P. Lootens (BMNH: 1959.510, RMCA: 25827, 25832 to 25834, 27022); 1 ? [SO], Inkongo [= Inkongo-Kakese], 10 Nov. 1932, leg. Wilson (RMCA: 12060); 3 ?? [3 SS], Jiba, 1 Aug. 1952, leg. Alexandre Fain (RMCA: 21444, 21448, 81.012-M-0041); 1 ? [SS], Jiba Risasi, unknown date, leg. Alexandre Fain (BMNH: 1953.491); 1 ? [SS], Jiba Trisasi [= Jiba Risasi], 1 Aug. 1952, leg. Alexandre Fain (RMCA: 21441); 2 ?? [2 AL], Kamituga, 13 Jul. 1966, leg. Alexandre Prigogine (RMCA: 32862a, 32862b); 1 ad $\precsim$ [SA], Kivu region, 10 Jul. 1964, leg. P. Kunkel (SMF: 31836); 4 ?? [3 SS, 1 SO], Korovi, Mount, 25 Oct. 1951, 25 Nov. 1951, leg. Alexandre Fain (RMCA: 23274 to 23277); 1 ? [AL], Lemera, between 1 Nov. 1956 and 30 Nov. 1956, leg. Laurent (RMCA: 35690); 1 đ [AL], Lodja, 20 Jul. 1986, leg. WHO and Marc Colyn (RMCA: 88.011-M-0015); 1 + [SS], Luluabourg [= Kananga], 26 Sep. 1922, leg. Richard Callewaert (RMCA: 7570); 1 $\widehat{~}$ [SS], Lwiro [= Luiro], 16 Jun. 1966, leg. A. Elbl (USNM: 535398); 3 우 [3 SS], Mboga [= Boga], unknown date, leg. Howard de Walden (BMNH: 1930.11.11.178 to 1930.11.11.180); 3 ?? [3 SS], Misu, 3 Aug. 1952, leg. Alexandre Fain (RMCA: 21440, 21443, 21447); 1 juv ?, 6 ?? [3 SS, 3 AL, 1 SK], Mount Wago [= Wago, Mount], unknown date, between 1 Jul. 1951 and 31 Jul. 1951, between 1 Jan. 1951 and 31 Dec. 1951, leg. Alexandre Fain, 29 Jul. 1952, leg. unknown collector (BMNH: 1953.487, 1953.488, RBINS: 21669, RMCA: 20802, 21467, 21476, 21477); 1 ㅇ, 1 ठ, 1 ? [2 SS, 1 AL], Mwanda, 12 Mar. 1959, leg. Uwe Rahm (RMCA: 28027, 28910, 28911); 1 §,, 6 ?? [7 SK], Netonna, between 1 Apr. 1800 and 31 May 1899, leg. P. Hesse (SMF: 2516 [lectotype Chalinolobus congicus Noack, 1889], 2517 [paralectotype Chalinolobus congicus Noack, 1889], 2518 [paralectotype Chalinolobus congicus Noack, 1889], 2519 [paralectotype Chalinolobus congicus Noack, 1889], 2520 [paralectotype Chalinolobus congicus Noack, 1889], 2521 [paralectotype Chalinolobus congicus Noack, 1889], 2522 [paralectotype Chalinolobus congicus Noack, 1889]); 1 ? [UN], Rutshuru, unknown date, leg. unknown collector (INPBC: V1599); 1 क [SO], St. Joseph de Luluabourg Mission [= Mikalaya],

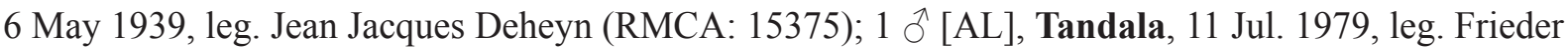
Meier (MHNG: 1870.074); 1 đ [AL], Uvira, 5 Sep. 1956, leg. Laurent (RMCA: 35691); 1 ? [SA], Wafanya [= Wafania], 3 Dec. 1988, leg. P. Lootens (RMCA: 89.020-M-0048); 1 ? [SS], Wago, Mount, prior to 29 Nov. 1955, leg. Alexandre Fain (RMCA: 23273); 4 ?? [3 SS, 1 SO], Wasa [= Wasa Wago 
Forest], 1 Aug. 1952, 3 Aug. 1952, prior to 16 Oct. 1952, leg. Alexandre Fain (RMCA: 21446, 21445, 21449, 21442); 3 ?? [3 SS], Wasa Wago Forest, unknown date, 1 Aug. 1952, leg. Alexandre Fain (BMNH: 1953.489, 1953.490, RMCA: 21439).

RWANDA: 1 ? [SA], Astrida [= Butare], between 1 Jan. 1948 and 31 Dec. 1948, leg. Alexandre Fain (RMCA: 18993); 1 ad,+ 1 ad $ð$ [2 SA], Kidaho, 10 Jun. 1982, 15 Jun. 1982, leg. Benny Baeten (RMCA: 87.046-M-0347, 87.046-M-0348); 1 ? [UN], Kissenji [= Gisenyi], unknown date, leg. Köhl (ZMB: 37016).

Glauconycteris beatrix Thomas, 1901

DEMOCRATIC REPUBLIC OF THE CONGO: 1 ๙ [AL], Aketi, 22 Jun. 2015, leg. Vlir/Aketi (UNIKIS: AKETI604); 1 ? [AL], Ibembo, 1 Jul. 1950, leg. Jozef Hutsebaut (RMCA: 20065);

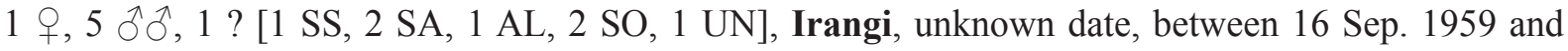
19 Sep. 1959, leg. Uwe Rahm, unknown date, leg. unknown collector, 3 Oct. 1955, leg. J.J. Laarman, between 11 Mar. 1990 and 21 Mar. 1990, leg. Klaus-Gerhard Heller and Marianne Volleth (BMNH: 1962.1847, SMF: 7940, RMNH: MAM.16362, MAM.16363, SMF: 79439, 79440, BMNH: 1963.1172); 1 ad $\hat{\partial}, 1$ $\hat{~}$ [1 SA, $1 \mathrm{UN}$ ], Kimbinga Market, 20 Jul. 1995, leg. Herwig Leirs et al. (MSB: 274578, RMCA: 97.021-M-0810); 1 ? [SS], Paulis [= Isiro], 1 Jan. 1950, leg. Abbeloos (RMCA: 19820); 6 ?? [6 AL], Wafanya [= Wafania], 20 Nov. 1988, 17 Oct. 1991, between 1 Jan. 1991 and 18 Oct. 1991, leg. P. Lootens (RMCA: 91.076-M-0076, 89.020-M-0044, 91.076-M-0052, 91.076-M-0057, 91.076-M0058, 91.076-M-0074).

Glauconycteris curryae Eger \& Schlitter, 2001

DEMOCRATIC REPUBLIC OF THE CONGO: 1 ? [UN], Yalosemba, unknown date, leg. unknown collector (CM: 86663).

\section{Glauconycteris humeralis J.A. Allen, 1917}

DEMOCRATIC REPUBLIC OF THE CONGO: 1 \& [UN], unknown locality, unknown date, leg. unknown collector (BMNH: 1990.0233); 1 \& [SS], Avakubi, 21 Feb. 1914, leg. Herbert Lang, James Paul Chapin and The American Museum Congo Expedition (AMNH: 49014); 1 § [UN], Avakubi, near, 9 Dec. 1974, leg. Michael D. Gallagher (HZM: 1.8023); 2 우, 1 ठ [1 AL, 1 UN, 1 SB], Epulu, 2 km W, 27 Jun. 1991, 28 Jun. 1991, leg. Julian C. Kerbis Peterhans (FMNH: 149417 to 149419); 1 q, 2 ठ̋ 1 †, 1 đ̃, 1 ? [4 UN], Medje, 7 Sep. 1910, leg. Herbert Lang, James Paul Chapin and The American Museum Congo Expedition (AMNH: 49012, 49013 [holotype Glauconycteris humeralis J.A. Allen,

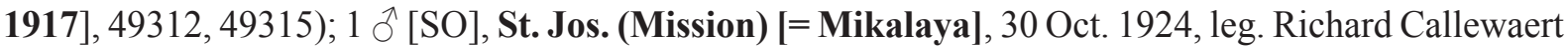
(AMNH: 86152); 1 ? [UN], Yalosemba, unknown date, leg. unknown collector (CM: 110209).

Glauconycteris poensis (Gray, 1842)

DEMOCRATIC REPUBLIC OF THE CONGO: 1 ? [AL], Bokuma, prior to 5 Mar. 1953, leg. P. Lootens (RMCA: 21684); 1 ? [AL], Busu Mokalu [= Busu-Mokala], between 5 Nov. 1938 and 10 Nov. 1938, leg. Baudart (RMCA: 18092); 1 ○ [SO], Faradje, 15 Mar. 1911, leg. Herbert Lang, James Paul Chapin and The American Museum Congo Expedition (AMNH: 49066); 1 ad §̊ [SA], Irangi, 21 Jan. 1984, leg. Heinz Stephan (SMF: 64987). 
Glauconycteris superba Hayman, 1939

DEMOCRATIC REPUBLIC OF THE CONGO: 1 ad $\hat{\delta}, 1 \hat{\delta}[1 \mathrm{SA}, 1 \mathrm{AL}]$, Île Mbiye [= Mbiye Island], 11 Feb. 2012, leg. Guy-Crispin Gembu Tungaluna, 24 Dec. 2015, leg. Prescott Musaba, Moïse Bipoo, Charle Andabhati, Benjamin Kirongozi and Kambale Karupao (RMCA: a1.097-M-3153, UNIKIS: ABCHIM01); 1 [ [SA], Pawa, prior to 8 Aug. 1938, leg. Albert Dubois (RMCA: 14765 [holotype Glauconycteris superba Hayman, 1939]).

\section{Glauconycteris variegata (Tomes, 1861)}

DEMOCRATIC REPUBLIC OF THE CONGO: 1 ㅇ, 2 đิ $\widehat{o}$ [1 SA, 2 SO], Aba, 13 Dec. 1911, leg. Herbert Lang, James Paul Chapin and The American Museum Congo Expedition (AMNH: 49069, 49070, 49347); 1 ㅇ, 3 ふึ, 7 ?? [8 SS, 1 SA, 2 AL], Boma, unknown date, prior to 17 Jul. 1924, leg. J. Rodhain, 23 Nov. 1920, leg. Henri Schouteden, between 1 Aug. 1928 and 31 Aug. 1928, leg. Gerard (RMCA: 7636 to 7639, 6566 to 6569, 16229, 16230, 7635); 1 ? [AL], Boteka, 20 Oct. 1985, leg. P. Lootens (RMCA: 85.052-M-0003); 1 ? [SO], Elisabethville [= Lubumbashi], between 1 Aug. 1934

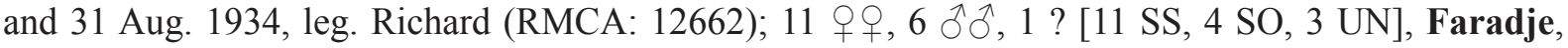
14 Mar. 1911, 15 Mar. 1911, leg. Herbert Lang, James Paul Chapin and The American Museum Congo Expedition, 14 Mar. 1911, leg. Herbert Lang, 14 Mar. 1911, leg. unknown collector (AMNH: 49058 to 49065, FMNH: 43872, MCZ: 17382, 17383, AMNH: 49067, 49068, 49195, 52116 to 52118, RMCA: 12381); 3 ?? [2 AL, 1 UN], Kashwa, unknown date, leg. unknown collector, 24 Aug. 1955, leg. GastonFrançois de Witte (INPBC: W1519, RMCA: 37167, 37168); 2 ?? [2 UN], Katanda, unknown date, leg. unknown collector (INPBC: W548, W549); 1 § [SO], Kwamouth, 2 Jun. 1921, leg. Henri Schouteden (RMCA: 6570); 1 ? [UN], Lake Tanganyika, NW bank, unknown date, leg. Rudolf Grauer (ZMB: 72234); 1 juv $\widehat{\partial}$ [AL], Moenda, between 1 Oct. 1920 and 31 Oct. 1920, leg. Henri Schouteden (RMCA: 16489); 1 ㅇ, 1 ภ, 2 ?? [4 UN], Mpala, unknown date, prior to 30 Apr. 1948, prior to 13 Oct. 1948, leg. unknown collector (INPBC: 14517, 14518, RBINS: 14517, 14518); 1 ? [AL], Mukishi [Station],

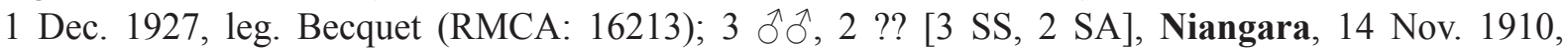
16 Nov. 1910, 6 Dec. 1910, leg. Herbert Lang, James Paul Chapin and The American Museum Congo Expedition, 16 Nov. 1910, leg. Herbert Lang (RMCA: 12382, AMNH: 49056, FMNH: 43871, AMNH: 49313, 49341).

Hypsugo Kolenati, 1856

Hypsugo anchietae (Seabra, 1900)

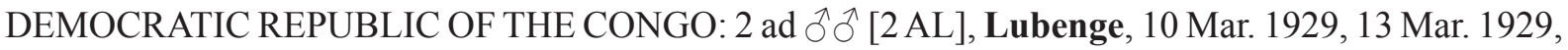
leg. J. Sterling Rockefeller (AMNH: 55773, 55774).

\section{Hypsugo crassulus (Thomas, 1904)}

DEMOCRATIC REPUBLIC OF THE CONGO: 1 ad $\lesssim$ [AS], Bokuma, 20 Jul. 1952, leg. P. Lootens

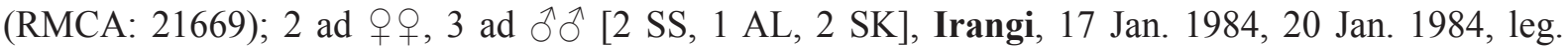
Heinz Stephan, between 13 Mar. 1990 and 25 Mar. 1990, leg. Klaus-Gerhard Heller and Marianne Volleth (SMF: 65011, 65012, 79441 to 79443); 1 ad $q$ [SA], Mulolo-Kisanga [= Lulola-Kasanga], 18 Mar. 1960, leg. Uwe Rahm (BMNH: 1963.1174).

\section{Hypsugo cf. eisentrauti Hill, 1968}

DEMOCRATIC REPUBLIC OF THE CONGO: 1 ad $q$ [SB], Kabobo Forest, 17 Feb. 2007, leg. C. Kahindo (FMNH: 195086); 1 ad,+ 1 [2 AL], Lukolela, 10 Oct. 1930, leg. James Paul Chapin (AMNH: 86916, 86917). 
VAN CAKENBERGHE V. et al., The bats of Congo, Rwanda and Burundi revisited

RWANDA: 1 ad $\lesssim$ [SS], Nyungwe Forest Reserve, 28 Mar. 1990, leg. Klaus-Gerhard Heller and Marianne Volleth (SMF: 79444).

Hypsugo musciculus (Thomas, 1913)

DEMOCRATIC REPUBLIC OF THE CONGO: 1 ad $q$ [SA], Bata, unknown date, leg. Charles-Henri Pobéguin (MNHN: ZM-MO-1892-223); 1 ad $\widehat{\hat{~}}$ [AL], Bokuma, between 1 Jun. 1953 and 30 Jun. 1953, leg. P. Lootens (RMCA: 22049).

Laephotis Thomas, 1901

Laephotis angolensis Monard, 1935

DEMOCRATIC REPUBLIC OF THE CONGO: 1 q [SS], Mumene [= Mumena], 28 Dec. 1955, leg. unknown collector (BMNH: 1957.437); 1 o [SS], Musonge, 27 Dec. 1955, leg. unknown collector (BMNH: 1957.435).

\section{Laephotis botswanae Setzer, 1971}

DEMOCRATIC REPUBLIC OF THE CONGO: 8 q $q, 1$ ? [2 SS, $6 \mathrm{SA}, 1 \mathrm{AL}$ ], Mumene [= Mumena], 27 Dec. 1955, 28 Dec. 1955, leg. unknown collector, 28 Dec. 1955, leg. The Hygiene Services of the Congo, 28 Dec. 1955, leg. M. Lips (SMF: 16869, BMNH: 1957.436, 1957.438, RMCA: 26402 to 26406, SMF: 16868); 2 ?? [1 AL, 1 UN], Musonge, unknown date, leg. unknown collector, 27 Dec. 1955, leg. M. Lips (SMF: 16869a, RMCA: 26407).

Mimetillus Thomas, 1904

Mimetillus moloneyi (Thomas, 1891)

DEMOCRATIC REPUblic OF THE CONGO: 2 ?? [2 AL], Arebi Bondo Mabe, unknown date, leg. Henri Schouteden (RMCA: 16264, 16265); 1 ? [SA], Avakubi, 15 Dec. 1913, leg. Herbert Lang, James Paul Chapin and The American Museum Congo Expedition (AMNH: 49305); 3 ?? [3 AL], Buta, unknown date, prior to 31 May 1939, prior to 23 Jun. 1949, leg. Jozef Hutsebaut (RMCA: 16279, 19081, 15326); 1 ad §̂, 1 ? [1 AL, 1 UN], Irumu, unknown date, leg. unknown collector, between 1 Nov. 1951 and 30 Nov. 1951, leg. Alexandre Fain (BMNH: 1954.862, RMCA: 21462); 1 ad $\hat{\jmath}, 1$ q, 1 ○ [3 UN], Isai River, between 19 Dec. 1974 and 21 Dec. 1974, leg. Michael D. Gallagher (HZM: 2.7802 to 4.7804); 6 ?? [5 AL, 1 UN], Katanga, prior to 31 Aug. 1899, leg. Charles Lemaire (RMCA: 386a to 386f); 1 ? [AL], Koteli [= Kotili], between 1 Jan. 1925 and 31 Jan. 1925, leg. Henri Schouteden (RMCA: 16266); 3 웅, 3 तึ $\sigma^{\lambda}$ [6 AL], Luluabourg [= Kananga], 3 Jul. 1965, leg. Antoon Emeric

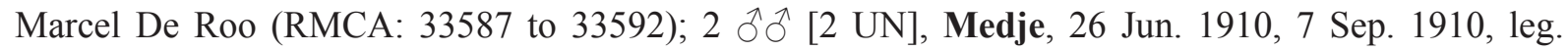
Herbert Lang, James Paul Chapin and The American Museum Congo Expedition (AMNH: 48859, 49198); 1 के [AL], Mosenge, 11 Aug. 1986, leg. WHO and Marc Colyn (RMCA: 88.011-M-0023); 1 juv ? [AL], Poko, between 1 Jul. 1914 and 31 Jul. 1914, leg. Cuthbert Christy (RMCA: 16514); $1 \delta$ [SS], Stanleyville [= Kisangani], 25 Aug. 1909, leg. Herbert Lang, James Paul Chapin and The American Museum Congo Expedition (AMNH: 48858); 1 ๆ, 4 $\widehat{\partial}$ [5 UN], Université de Kisangani, Faculté des Sciences [= Kisangani], 10 Aug. 2015, 11 Aug. 2015, leg. Prescott Musaba, Moïse Bipoo, Charle Andabhati, Benjamin Kirongozi and Kambale Karupao (UNIKIS: CHFS01 to CHFS05); 3 q $q$, 9 ?? [1 SA, 11 AL], Wafanya [= Wafania], unknown date, 3 Jan. 1988, 5 Dec. 1988, 26 May 1992, 29 May 1992, between 25 Nov. 1987 and 3 Jan. 1988, between 1 Nov. 1991 and 30 Nov. 1991, between 1 Jan. 1992 and 31 Jan. 1992, leg. P. Lootens (RMCA: 92.079-M-0123, 92.079-M-0124, 92.079-M0038, 92.079-M-0037, 92.079-M-0039, 92.079-M-0040, 88.005-M-0001, 88.005-M-0002, 89.020-M0045, 92.079-M-0041, 92.079-M-0036, 88.005-M-0007); 1 ô [UN], Yaekela, 7 May 2010, leg. Guy- 
Crispin Gembu Tungaluna, Prescott Musaba and José Akaibe (UNIKIS: CRT1888); 2 우 [1 SS, $1 \mathrm{AL}$ ], Yalosemba, 17 Jun. 1979, between 1 Jun. 1979 and 30 Jun. 1979, leg. C. Brian Robbins (USNM: 537736, MHNG: 1870.091); 1 [SS], Yangambi, 22 Aug. 1947, leg. unknown collector (RBINS:

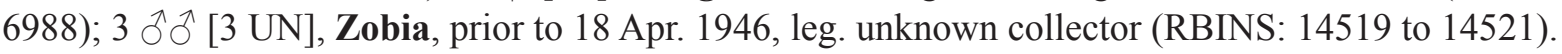

Mimetillus thomasi Hinton, 1920

DEMOCRATIC REPUBLIC OF THE CONGO: 1 ? [AL], Elisabethville [= Lubumbashi], between 1 Mar. 1926 and 31 Mar. 1926, leg. Henri Schouteden (RMCA: 16496).

Neoromicia Roberts, 1926

BURUNDI: 1 [ [AB], Kanyosha, 14 Apr. 1970, leg. P.L. Niort (MHNG: 1608.004).

DEMOCRATIC REPUBLIC OF THE CONGO: 1 ad $\$, 4$ ad $\widehat{\jmath}$ [5 AL], Cel II, 22 Apr. 1951, 1 Jun. 1951, 23 Jul. 1951, 10 Nov. 1951, 26 May 1952, leg. H. de Saeger et al. (RBINS: 13831, 13837, 13830, 13833, 13839); 1 [AL], Kinkole, 15 Aug. 1980, leg. unknown collector (ZMA: MAM.21154); 1 ad $\hat{o}$ [AL], Ndelele, 3 Dec. 1951, leg. H. de Saeger et al. (RBINS: 13836); 1 juv ? [UN], Rutshuru, unknown date, leg. unknown collector (INPBC: V1397.2); 1 ○ [SS], Stanleyville [= Kisangani], 13 Jan. 1927, leg. Harold Jefferson Coolidge and L. Whitman (MCZ: 23382); 1 ad $q$ [SA], Zobia, 18 Apr. 1946, leg. R.P. Van Woensel (RBINS: 14508).

RWANDA: 2 $ᄋ$ 우 [2 AL], Gisenyi, 5 Oct. 1980, leg. Hans H. de Iongh (ZMA: MAM.21237, MAM.21238).

Neoromicia brunnea (Thomas, 1880)

DEMOCRATIC REPUBLIC OF THE CONGO: 1 ad $\&$ [AL], Lukolela, 24 Dec. 1930, leg. James Paul Chapin (AMNH: 86933).

Neoromicia capensis (A. Smith, 1829)

BURUNDI: 1 ad $q$ [SB], Makamba, 23 Mar. 1970, leg. P.L. Niort (MHNG: 1607.097).

DEMOCRATIC REPUBLIC OF THE CONGO: 1 ? [UN], Ango, unknown date, leg. unknown collector (RMCA: 12598); 3 ad 우 [3 AL], Bagbele Moko, 3 Apr. 1950, leg. H. de Saeger et al. (RBINS:

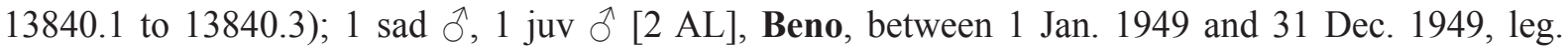

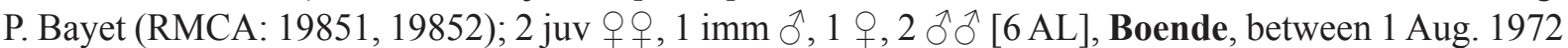
and 31 Aug. 1972, between 1 Jan. 1969 and 31 Dec. 1974, between 1 Jan. 1967 and 31 Dec. 1967, leg. P. Lootens (RMCA: 38717, 38718, 38725, 75.035-M-0024, 75.035-M-0025, 75.035-M-0057); 3 ?? [1 SA, 2 UN], Buta, between 1 Jun. 1934 and 30 Jun. 1934, leg. Jozef Hutsebaut (RMCA: 12590a to 12590c); 1 ad [AL], Cel I [= Bagbele], 3 Apr. 1950, leg. H. de Saeger (RMCA: 36852); 8 ad 우우,

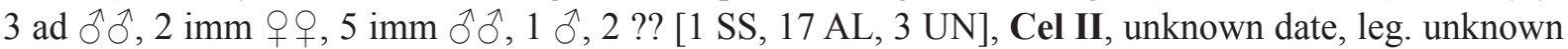
collector, 16 Mar. 1951, 24 Aug. 1951, leg. H. de Saeger et al., 16 Mar. 1951, 25 Feb. 1952, between 8 Jun. 1951 and 26 Nov. 1951, between 8 Apr. 1951 and 8 Jun. 1951, leg. H. de Saeger (INPBC: 3559, 4360, RBINS: 13841.1 to 13841.5 , RMCA: 36853 to 36862, RBINS: 13834, RMCA: 36866 , 36864, 36865); 1 ad $\hat{\jmath}, 1$ ad ? [1 SS, 1 SA], Elisabethville [= Lubumbashi], unknown date, leg. Guarre, between 1 Mar. 1926 and 31 Mar. 1926, leg. Henri Schouteden (BMNH: 1954.859, RMCA: 8872); 1 juv $q, 1$ juv $\widehat{\jmath}[2 \mathrm{UN}$ ], Gangala-na-Bodio, 1 May 1948, leg. unknown collector (RBINS: 11656.1, 11656.2); 1 ad $\hat{\jmath}^{\hat{\lambda}}$ [SA], Garamba National Park, 14 Mar. 1912, leg. Herbert Lang, James Paul Chapin and The American Museum Congo Expedition (AMNH: 49340 [holotype Eptesicus garambae J.A. Allen, 1917]); 1 ad $\widehat{\curvearrowright}$ [SC], Kipangaribwe River, 12 Jan. 1948, leg. l' Institut des Parcs nationaux

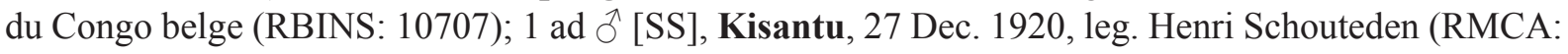


6560); 1 ad $q$ [SA], Léopoldville [= Kinshasa], between 1 Jan. 1961 and 31 Dec. 1961, leg. Xavier Misonne (RBINS: 14507); 1 ad $\widehat{\partial}$ [SS], Luebo, 10 Sep. 1921, leg. Henri Schouteden (RMCA: 7309); 2 ad $q$ + 1 ad ? [1 SS, 2 AL], Mabanga, 1 Aug. 1952, leg. H. de Saeger et al., 1 Aug. 1952, leg. H. de Saeger (RBINS: 13848.1, 13848.2, RMCA: 36863); 1 ad §̊ [AL], Mayumbe, unknown date, leg. unknown collector (RMCA: 90.012-M-0001); 2 ad + $ᄋ$ [2 AL], Moba, 6 Feb. 1954, 26 Feb. 1954, leg. H. Bomans (RMCA: 26327, 22126); 1 ? [SS], PFSK.20, 16 Jun. 1952, leg. H. de Saeger et al. (RBINS: 13847); 2 juv ?? [2 UN], PPK.51, 2 Apr. 1952, leg. H. de Saeger et al. (RBINS: 13846.1, 13846.2); 1 ad

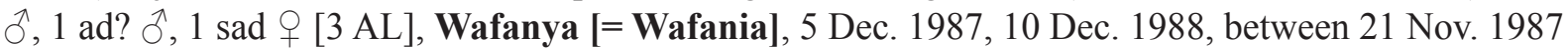
and 28 Nov. 1987, leg. P. Lootens (RMCA: 89.020-M-0046, 88.005-M-0014, 88.005-M-0003).

Neoromicia guineensis (Bocage, 1889)

DEMOCRATIC REPUBLIC OF THE CONGO: 1 ad $\hat{\jmath}$ [SA], Isiro, 3 Jul. 1913, leg. Herbert Lang, James Paul Chapin and The American Museum Congo Expedition (AMNH: 49339); 1 ad? $\widehat{\delta}$ [SA], Niangara, 27 Mar. 1913, leg. Herbert Lang, James Paul Chapin and The American Museum Congo Expedition (AMNH: 49290).

\section{Neoromicia nana (Peters, 1852)}

BURUNDI: $1 q$ [SK], unknown locality, 15 Mar. 1979, leg. Heinz Stephan (SMF: 57574); 2 ad $q$ q [2 AL], Bujumbura, 22 Apr. 1982, leg. Danny Meirte, between 1 Jul. 1968 and 31 Jul. 1968, leg. Lewall

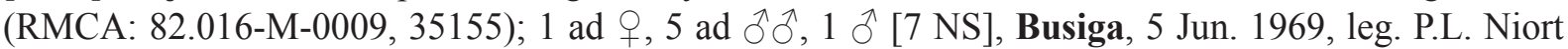
(MHNG: 1607.057 to 1607.063); 1 ad $\lesssim$ [AL], Bweru, 29 Jul. 1976, leg. Jacques Verschuren (RBINS:

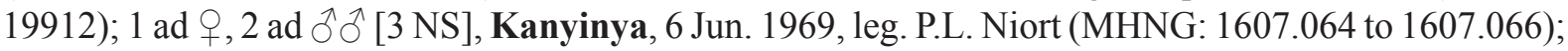
1 q [SK], Kirundo Province, 9 Mar. 1979, leg. Heinz Stephan (SMF: 57572); 2 ad $ð \precsim$ [2 NS], Mpinga Cave [= Mpinga Mission], 1 Jan. 1969, leg. P.L. Niort (MHNG: 1607.051, 1607.072); 1 + [SK], Ngozi Province, 13 Mar. 1979, leg. Heinz Stephan (SMF: 57573); 3 ad $\widehat{\jmath} \widehat{\jmath}$ [3 NS], Nyankanda, 3 Jan. 1969 , leg. P.L. Niort (MHNG: 1607.074 to 1607.076); 1 ad $\widehat{\partial}$ [SO], Nyanza [= Nyanza-Lac], 28 Dec. 1932,

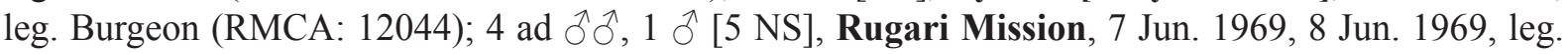
P.L. Niort (MHNG: 1607.067 to 1607.071); 1 ? [UN], Russissi, bank [= Rusizi Delta], unknown date, leg. Rudolf Grauer (ZMB: T09305); 1 ad o, 1 ○ૈ [1 SA, 1 UN], Rutana, 6 Mar. 1979, leg. Heinz Stephan (SMF: 57570, 57571); 1 ? [AL], Tora, 19 Nov. 1975, leg. Walter N. Verheyen (RMCA: 97.080-M-0232).

DEMOCRATIC REPUBLIC OF THE CONGO: 1 ad $\widehat{\jmath}, 1$ juv $\widehat{\jmath}, 14$ q $q, 8$ $ぇ ึ, 21$ ?? [20 SS, $18 \mathrm{AL}$, $3 \mathrm{SK}, 1 \mathrm{SO}, 3 \mathrm{UN}$ ], unknown locality, unknown date, leg. Herbert Lang, James Paul Chapin and The American Museum Congo Expedition, unknown date, between 1 Apr. 1999 and 30 Apr. 1999, leg. unknown collector, unknown date, leg. The Alexander-Gosling Expedition, unknown date, leg. unknown collector, unknown date, leg. Guy-Crispin Gembu Tungaluna, prior to 1 Aug. 1957, leg. Heinz Stephan, prior to 22 Dec. 1943, leg. Gaston-François de Witte, prior to 4 Jul. 1958, leg. E. Jans (AMNH: 51279, BMNH: 1906.7.1.10 to 1906.7.1.13, 1906.7.1.15 to 1906.7.1.17, 1906.7.1.7 to 1906.7.1.9, 1907.7.8.36, 1907.7.8.37, 1926.7.6.85 to 1926.7.6.91, 1930.11.11.166, RMCA: 89.004-M-0013, 89.004-M-0014, a1.097-M-0565 to a1.097-M-0581, SMF: 93321, RMCA: 27468, 27469, 16639, 27369); 1 ad 9,3 ad đô, 1 †, 1 ठ [3 SS, 2 AL, 1 SO], Aba, 10 Aug. 1951, leg. H. de Saeger et al., 18 Dec. 1911, leg. Herbert Lang, James Paul Chapin and The American Museum Congo Expedition (RBINS: 13862.1, 13862.2, 13866, AMNH: 48979 [holotype Pipistrellus abaensis J.A. Allen, 1917], 48980 [topotype Pipistrellus abaensis J.A. Allen, 1917], 48981 [topotype Pipistrellus abaensis J.A. Allen, 1917]); 2 ad $q+, 3$ ad

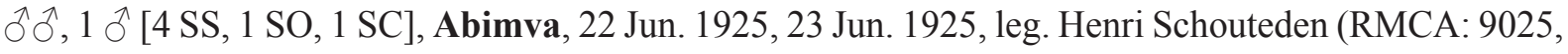
9028a, 9030 to 9033); 1 [UN], Aketi, 22 Jun. 2015, leg. Vlir/Aketi (UNIKIS: AKETI607); 2 ?? [2 UN], Albert National Park [= Virunga National Park], unknown date, leg. unknown collector

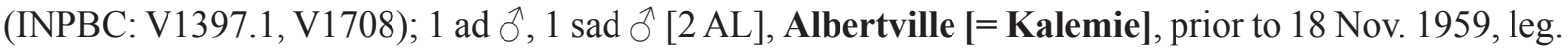
H. Bomans, prior to 30 Jan. 1900, leg. Célestin Hecq (RMCA: 29302, 421); 1 ? [AL], Albertville-Moba 
(road?) [= Kalemie-Moba (road?)], 23 Apr. 1954, leg. H. Bomans (RMCA: 23320); 1 ad $q$ [AL],

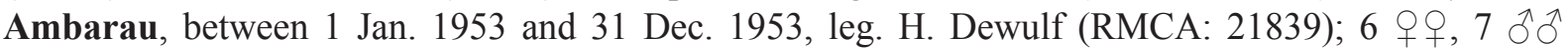
[13 SS], Bafuaboka [= Bafwabaka], 5 Jan. 1910, 6 Jan. 1910, 7 Jan. 1910, leg. Herbert Lang, James Paul Chapin and The American Museum Congo Expedition (AMNH: 48951, 48952, 48954 to 48964);

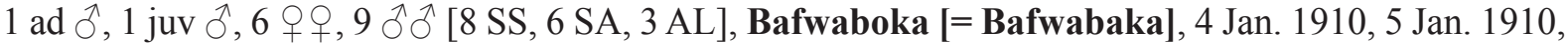
7 Jan. 1910, leg. Herbert Lang, James Paul Chapin and The American Museum Congo Expedition (AMNH: 48943 to 48945, 49322, 48946, 48948 to 48950, 49295, RMCA: 12369, AMNH: 49293, 49294, 49296 to 49299, 49302); 1 ad,+ 2 juv $ᄋ+$, 1 juv ${ }^{~}$, 4 ?? [4 AL, 4 UN], Bagbele, 13 Apr. 1950, leg. H. de Saeger et al., 15 Apr. 1950, leg. H. de Saeger (RBINS: 13802.3 to 13802.6, RMCA: 36867 to

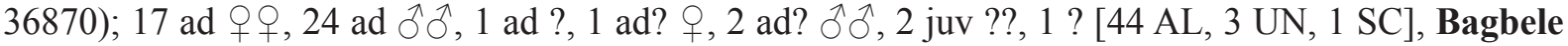
Moko, unknown date, leg. unknown collector, 24 Feb. 1950, 13 Apr. 1950, 26 Jul. 1952, leg. H. de Saeger et al., 26 Jul. 1952, leg. H. de Saeger (RBINS: 13867, 13802.1, 13802.2, 13861, 13864.01 to 13864.15, 13865.01 to 13865.12, RMCA: 36871 to 36887); 2 우 으 [ UN], Bagisana, 21 Apr. 1952, leg. H. de Saeger et al. (RBINS: 13863.1, 13863.2); 1 juv [SK], Bambesa, 18 Oct. 1940, leg. J.M. Vrijdagh (RMCA: 17766); 1 đ [SS], Banalia, between 26 Jan. 1939 and 26 Dec. 1939, leg. Duncan M. Hodgson

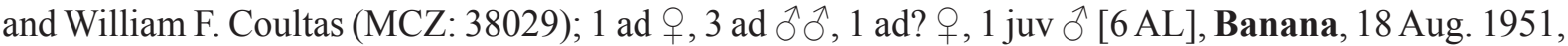
12 Apr. 1952, between 1 Jan. 1952 and 31 Jan. 1952, leg. Is. Mesmaekers (RMCA: 20763, 20764, 20525 to 20527, 20749); 2 ad $ㅇ$ ㅇ [1 SS, 1 SO], Basongo, 19 Jul. 1921, leg. Henri Schouteden (RMCA: 6644,

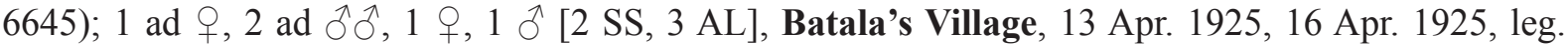
Edmund Heller (FMNH: 26495, 26496, ZMUC: 1541 to 1543); 1 ? [AL], Batoko (River) [= OlongoNsongo [River]], 18 Nov. 1913, leg. J. Bequaert (RMCA: 5388); 1 ad $\hat{\jmath}, 4$ juv $q$ q, 1 juv $\hat{\sigma}, 1$ juv ? [6 SS, 1 SC], Bikoro, 27 Feb. 1921, leg. Henri Schouteden (RMCA: 6618 to 6620, 6622 to 6625); 1 ad

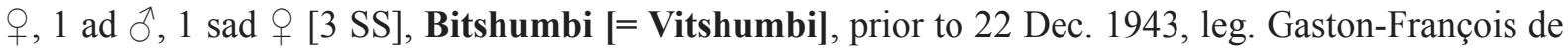
Witte (RMCA: 16640 to 16642); 1 ad,+ 1 ad $\widehat{\jmath}$ [2 AL], Bodjoki - Bumba, between 1 Jan. 1986 and 31 Dec. 1986, between 1 Jan. 1985 and 31 Dec. 1986, leg. WHO and Marc Colyn (RMCA: 88.011-M-

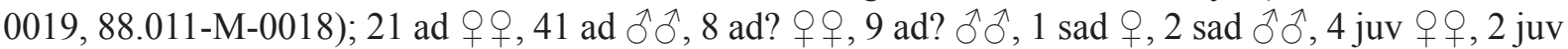
đo $\widehat{o}$ [88 AL], Boende, 18 Nov. 1966, between 1 Nov. 1969 and 30 Nov. 1969, between 1 Jan. 1969 and 31 Dec. 1974, between 1 Jan. 1969 and 31 Jan. 1969, between 1 Jan. 1967 and 31 Dec. 1967, between 1 Jan. 1966 and 31 Jan. 1966, leg. P. Lootens (RMCA: 38716, 34076, 34079 to 34081, 34083 to 34092, 34094,34096 to 34099,34101 to 34108,34110 to 34113,34115 to 34119,34121 to 34124,34126 , 34127,34129 to 34145,34147 to $34155,38715,38719$ to 38723,38726 to $38730,35640,75.035-\mathrm{M}$ 0027, 75.035-M-0030 to 75.035-M-0035); 1 ? [AL], Bokala, prior to 13 Oct. 1913, leg. Maes (RMCA:

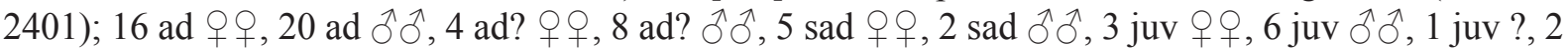
imm 우, 1 imm ô, 1 imm ?, 3 ?? [71 AL, 1 UN], Bokuma, 2 Mar. 1952, 15 Mar. 1952, 23 Mar. 1952, 25 Jul. 1952, 23 Feb. 1954, 10 Mar. 1954, prior to 6 May 1954, prior to 25 Aug. 1954, between 2 Mar. 1952 and 8 Mar. 1952, between 1 Jun. 1953 and 30 Jun. 1953, between 1 Feb. 1954 and 28 Feb. 1954, between 1 Jan. 1954 and 31 Dec. 1954, between 1 Jan. 1954 and 31 Jan. 1954, between 1 Jan. 1952 and 31 Dec. 1952, leg. P. Lootens (RMCA: 22180, 21403 to 21405, 21398 to 21401, 22156 to 22158,21406 to $21408,21671,21397,22154,22159$ to $22167,22319,23248$ to 23269,22155 , 22039, 22042, 22043, 22045 to 22048, 21402, 22320, 22321, 22168 to 22179); 1 juv đ̊ [SK], Boma, between 1 Mar. 1887 and 31 Mar. 1887, leg. P. Hesse (SMF: 2523 [holotype Vesperus pusillus Noack,

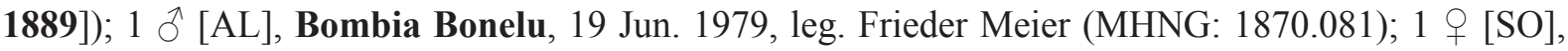
Bomili, 17 Aug. 1938, leg. Duncan M. Hodgson and William F. Coultas (MCZ: 38028); 1 ad $q$ [SS], Bosodula [= Boso-Dula], 25 Oct. 1942, leg. J.M. Vrijdagh (RMCA: 17755); 5 ad $q+, 1$ ad $\hat{\jmath}, 1$ ad? ${ }^{\lambda}$, $1 \mathrm{sad}$ đ̄, $2 \mathrm{imm}$ 우 [10 AL], Boteka, 25 Oct. 1985, between 22 Jul. 1984 and 15 Mar. 1985, between 1 Feb. 1985 and 28 Feb. 1985, leg. P. Lootens (RMCA: 85.052-M-0004 to 85.021-M-0012, 85.021-M-

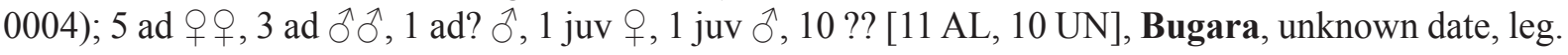
unknown collector, 3 Dec. 1953, leg. Gaston-François de Witte (INPBC: W710.02 to W710.11, RMCA: 37201 to 37211); 1 ad $\hat{\jmath}$ [AL], Bukavu, between 1 Jul. 1971 and 31 Jul. 1971, leg. Alexandre Prigogine (RMCA: 38583); 10 ?? [10 UN], Bulaya, unknown date, leg. unknown collector (INPBC: W1178.1 to 
VAN CAKENBERGHE V. et al., The bats of Congo, Rwanda and Burundi revisited

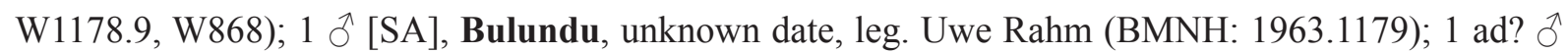
[AL], Bumba, prior to 23 Jul. 1946, leg. P. Lootens (RMCA: 18114); 1 [AL], Bunia, between 1 Apr. 1927 and 30 Apr. 1927, leg. Harold Jefferson Coolidge (MCZ: 28544); 1 juv ? [UN], Bunkeya,

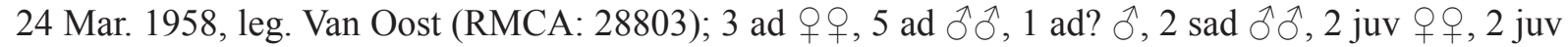
§̊̊, 36 ?? [18 AL, $33 \mathrm{UN}$ ], Bunyangura, unknown date, leg. unknown collector, 9 Dec. 1953, 22 Nov. 1954, 11 Jan. 1955, leg. Gaston-François de Witte (INPBC: W795.01 to W795.33, RMCA:

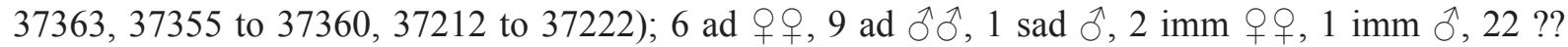
[19 AL, 22 UN], Bunyenzi, unknown date, leg. unknown collector, 15 Sep. 1953, 17 Sep. 1953, 3 Feb. 1954, leg. Gaston-François de Witte (INPBC: W1007.01 to W1007.20, W624, W632, RMCA: 37189, 37190, 37193, 37248 to 37263); 1 ad + , 1 ad $\widehat{\jmath}$ [2 AL], Busholinka River, 18 Feb. 1954, leg. Gaston-François de Witte (RMCA: 37223, 37224); 4 ad $\widehat{\partial} \widehat{o}$ [4 AL], Busu Mokalu [= Busu-Mokala], between 5 Nov. 1938 and 10 Nov. 1938, leg. Baudart (RMCA: 18093 to 18096); 5 ad $ᄋ$ 우, 2 ad $\precsim$,

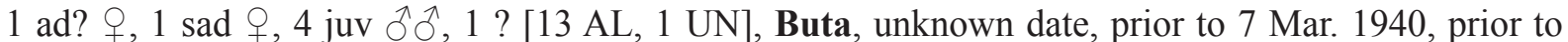
3 Nov. 1939, leg. Jozef Hutsebaut (RMCA: 14563a to 14563c, 14581, 14586, 16572, 16573, 15731 to 15736, 15911); 1 ad $ᄋ$ [AL], Butembo, prior to 12 Sep. 1958, leg. P. Dyleff (RMCA: 27397); 3 ad 우,

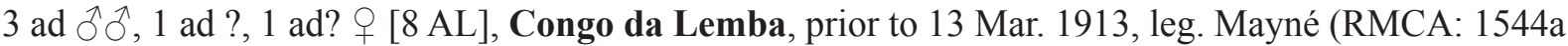
to 1544h); 1 ad ? [SA], Congo River, 75 mi [= 121 km] up, between 1 Aug. 1897 and 31 Aug. 1897, leg. George Latimer Bates (USNM: 102499); 1 ad $\widehat{\delta}$ [AL], Costermansville [= Bukavu], prior to 14 Feb. 1952, leg. H. Bomans (RMCA: 20656); 2 ?? [1 AL, 1 UN], Dika, 19 Mar. 1925, leg. Henri Schouteden (RMCA: 16257, 16260); 1 ad $\lesssim, 1$ sad + [2 AL], Dilolo, prior to 31 Mar. 1948, leg. Jozef

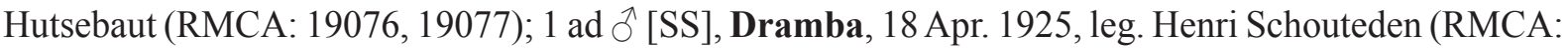

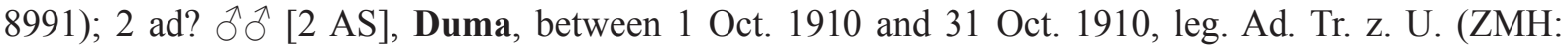

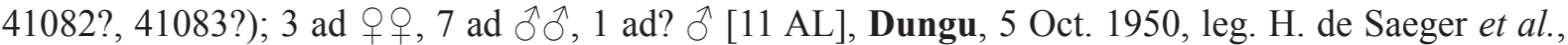
between 1 Apr. 1925 and 30 Apr. 1925, leg. Henri Schouteden, prior to 11 Jul. 1912, leg. M. Hutereau (RBINS: 13803, RMCA: 16192, 1105a to 1105i); 2 juv $q$ ? [1 SS, 1 SO], Eala, 15 Mar. 1921, leg. Henri Schouteden (RMCA: 6626, 6627); 1 ad? + [AL], Elila, 16 Jul. 1947, leg. Max Poll (RMCA: 18044);

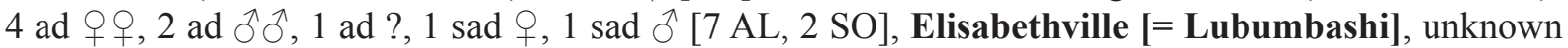
date, 22 Dec. 1955, leg. Félix / Michel Anciaux de Faveaux, 2 Sep. 1954, between 1 Dec. 1954 and 31 Dec. 1954, leg. De Smet, 22 Dec. 1955, leg. M. Lips (MNHN: ZM-MO-1957-126 A, RMCA: 22456,

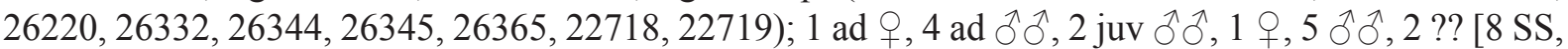
2 SA, 1 AL, 2 SO, 2 UN], Faradje, 18 Feb. 1911, 1 Mar. 1911, 8 Aug. 1911, 18 Feb. 1912, leg. Herbert Lang, James Paul Chapin and The American Museum Congo Expedition, 1 Jul. 1925, 5 Jul. 1925, 6 Jul. 1925, leg. Henri Schouteden (AMNH: 48978, 52112, 52113, RMCA: 9897 to 9901, AMNH: 48977, 49303, 49307, 49332, RMCA: 9059, 9045, AMNH: 48976); 1 ? [SA], Faradje Territory, 18 Feb. 1912, leg. Herbert Lang, James Paul Chapin and The American Museum Congo Expedition (AMNH: 49316); 1 ad 9,1 juv $\widehat{\partial}, 1$ ㅇ [2 AL, 1 UN], Flandria [= Boteka], 27 Aug. 1929, between 1 Jan. 1930 and 31 Dec. 1930, leg. R.P. Hulsaert (RMCA: 89.004-M-0012, 90.007-M-0001, 16278); 1 ठ [SA], Fomulac-Katana, between 2 Oct. 1963 and 3 Oct. 1963, leg. P. Kunkel (SMF: 31837); 4 ?? [4 UN], Fuko, unknown date, leg. unknown collector (INPBC: W1379.1 to W1379.3, W854); 1 ad $q$,

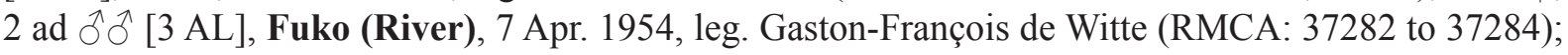
1 ? [UN], Funda Biabo, 20 Mar. 1914, leg. Charliers (RMCA: 5384); 3 ?? [3 UN], Gahama, unknown date, leg. unknown collector (INPBC: W1241.1 to W1241.3); $1 \hat{\sigma}$ [SS], Gamangui, 27 Feb. 1910, leg. Herbert Lang, James Paul Chapin and The American Museum Congo Expedition (AMNH: 48965); 1 ad $\hat{\partial}$ [AL], Ganda-Sundi, 31 Mar. 1964, leg. Alexandre Fain (RMCA: 33276); 3 $\widehat{\partial} \hat{\partial}$ [1 SS, 2 SO], Gangala [= Gangala-na-Bodio], 24 Mar. 1957, prior to 1 Aug. 1957, leg. Heinz Stephan (RMCA:

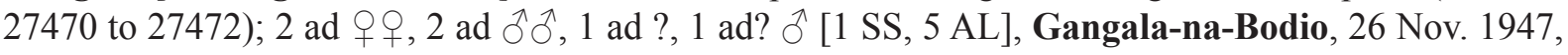
leg. M. Micha, 5 Apr. 1948, 9 Apr. 1948, 6 May 1948, 7 May 1948, leg. Hediger and Jacques Verschuren, 8 Nov. 1949, leg. H. de Saeger et al. (RBINS: 12947, 8191, 8193, 8194, 13860, 8192); 1 ? [SA], Garamba National Park, 15 Mar. 1912, leg. Herbert Lang, James Paul Chapin and The American

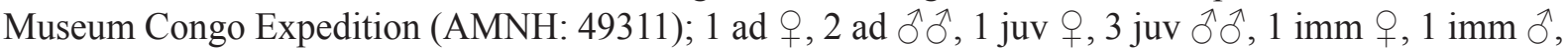


3 ?? [11 AL, 1 UN], Geramihasha River, unknown date, leg. unknown collector, 22 Mar. 1954, 3 May 1954, 28 Jun. 1954, 3 May 1955, between 3 May 1954 and 3 May 1955, leg. Gaston-François de Witte (INPBC: W997, RMCA: 37292 to 37294,37326 to $37329,37302,37299$ to 37301 ); 2 ad 우, 1 sad + ,

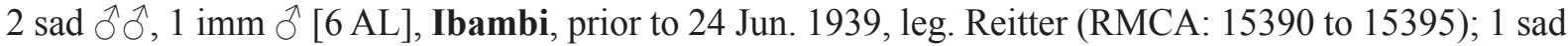
우 [AL], Ibembo, prior to 22 Feb. 1952, leg. Jozef Hutsebaut (RMCA: 20662); 1 ad $q, 1$ ad $\delta^{\lambda}, 2$ 우의,

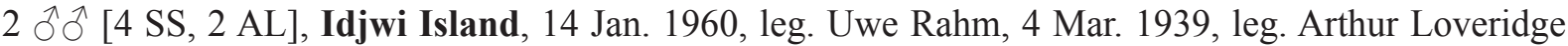
(RMCA: 28919, 28920, MCZ: 40763 to 40766); 2 ?? [2 AL], Ikela, prior to 4 Jan. 1958, leg. P. Lootens (RMCA: 27023, 27024); 1 juv ô, 4 우, 5 ô $\widehat{~}, 4$ ?? [4 AL, 9 SK, 1 UN], Irangi, 8 Jan. 1957, 25 Jan. 1984, 29 Jan. 1984, 1 Feb. 1984, leg. Heinz Stephan, 3 Jan. 1957, leg. J.J. Laarman (SMF: 65023, 65024, 65013 to 65022, RMNH: MAM.16372, RMCA: 27473); 1 ? [UN], Iringi, prior to 31 Aug. 1899, leg. A. or G. Lindeman (RMCA: 407); 1 [ [SO], Irsac Labo [= Luiro], 14 Dec. 1955, leg. J.J. Laarman (RMNH: MAM.16370); 1 đ [UN], Isai River, 19 Dec. 1974, leg. Michael D. Gallagher (HZM: 206.7973); 2 ?? [2 UN], Ishango River, unknown date, leg. unknown collector (INPBC: W1372.1, W1372.2); 2 ad $\circ$ ㅇ, 1 ad?,+ 1 sad $\circ, 1$ [ [4 AL, 1 UN], Jadotville [= Likasi], 1 Dec. 1957, 8 Jun. 1958, leg. Félix / Michel Anciaux de Faveaux, 18 Dec. 1959, leg. unknown collector

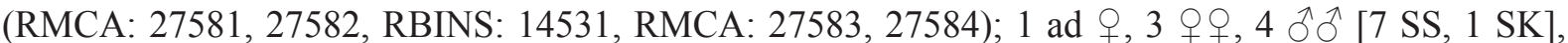
Kabengere, 13 Dec. 1926, 22 Dec. 1926, 24 Dec. 1926, leg. John Todd Zimmer (FMNH: 29418 to 29424, 30686); 2 ad $\widehat{ึ}$ [1 SS, 1 SC], Kabira, 12 Mar. 1959, 15 Mar. 1959, leg. Uwe Rahm (RMCA: 28915, 28914); 1 ad ${ }^{\lambda}$ [AL], Kabongo, between 1 Jan. 1952 and 31 Dec. 1952, leg. M. Dierckx (RMCA:

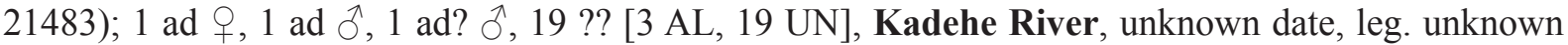
collector, 18 Sep. 1953, leg. Gaston-François de Witte (INPBC: W1426.01 to W1426.14, W636, W638, W660, W779, W953, RMCA: 37195 to 37197); 1 ad 9 [AL], Kadjidji [= Kajuju], unknown date, leg.

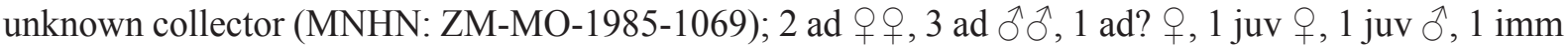
[ [9 AL], Kadjudju [= Kajuju], prior to 6 Jul. 1935, leg. Guy Babault (RMCA: 12847a to 12847c,

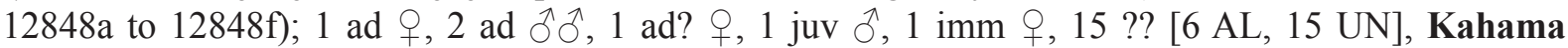
[= Gahama], unknown date, leg. unknown collector, 9 Jun. 1955, leg. Gaston-François de Witte

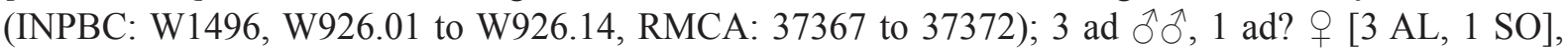
Kakanda Cave, 22 Sep. 1960, leg. Félix / Michel Anciaux de Faveaux, 9 Aug. 1964, leg. Alexandre Prigogine, between 21 Apr. 1955 and 22 Apr. 1955, leg. R.P. Th. de Caters (RBINS: 14529, 14530, RMCA: 33068, 22888); 1 ad 9 [AL], Kakanda Mutaka, between 6 Feb. 1954 and 15 Feb. 1954, leg. R.P. Th. de Caters (RMCA: 22379); 1 ad 9 [UN], Kakoi, 5 Aug. 1995, leg. Herwig Leirs et al. (MSB: 274920); 2 ad $\widehat{\partial}, 1$ ad? $\widehat{\partial}$ [3 AL], Kakonde, 20 Oct. 1956, leg. Félix / Michel Anciaux de Faveaux (RMCA: 26239, 26240, 26244); 1 ? [UN], Kakondo, unknown date, leg. unknown collector (NMP:

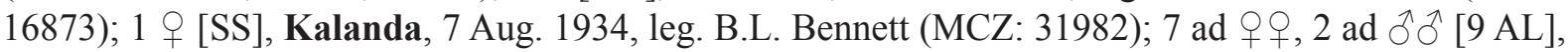
Kalulako, unknown date, 5 Jan. 1956, leg. Félix / Michel Anciaux de Faveaux, 5 Jan. 1956, 4 Nov. 1956, leg. D’Haenens (MNHN: ZM-MO-1957-126 B, RMCA: 26388 to 26393, 26221, 26384); 5 ad $q$ ㅇ, 1 ad

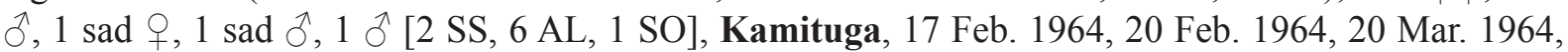
between 21 Feb. 1964 and 21 Mar. 1964, leg. Alexandre Prigogine (RMCA: 32587, 32857 to 32861,

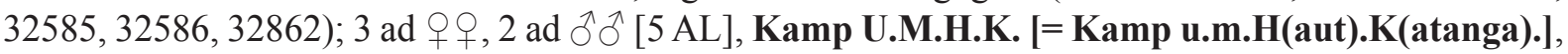
25 Aug. 1954, 27 Aug. 1954, 28 Aug. 1954, 2 Sep. 1954, leg. De Smet (RMCA: 22457, 22452 to 22455); 2 ?? [2 SS], Kampala, between 1 Mar. 1935 and 31 Mar. 1935, leg. The Grandidier Collection (MCZ: 57539, 57540); 2 우, 1 ð [1 SS, 2 AL], Kananga, 8 Sep. 1963, 13 Mar. 1964, leg. Antoon Emeric Marcel De Roo, 30 Nov. 1973, leg. Western New Mexico University (AMNH: 212580, FMNH: 152772 ,

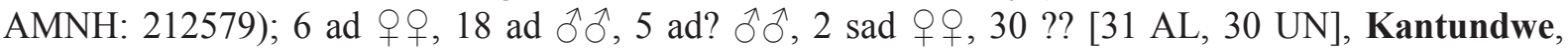
unknown date, leg. unknown collector, 18 Sep. 1953, 19 Mar. 1954, 30 Mar. 1954, 10 Jun. 1954, 5 Jul. 1954, 17 Sep. 1954, 17 May 1957, between 3 Nov. 1954 and 3 Dec. 1954, leg. Gaston-François de Witte (INPBC: W1258, W1491.01 to W1491.25, W617, W634, W640, W721, RMCA: 37336 to 37342, $37173,37350$ to $37352,37194,37285$ to 37291,37295 to 37297,37343 to $37349,37353,37354) ; 3$ ?? [3 UN], Kanyawamukunzi River, unknown date, leg. unknown collector (INPBC: W754.1 to W754.3); 1 ad Oे, $_{9}$ ?? [1 AL, 9 UN], Kanzarue River, unknown date, leg. unknown collector, 7 Sep. 1953, leg. 
Gaston-François de Witte (INPBC: W766.1 to W766.9, RMCA: 37198); 1 ? [AL], Kapanga, prior to 10 Dec. 1958, leg. J. Allaer (RMCA: 27667); 1 ad + [AL], Kapaso, 7 Oct. 1955, leg. M. Lips (RMCA:

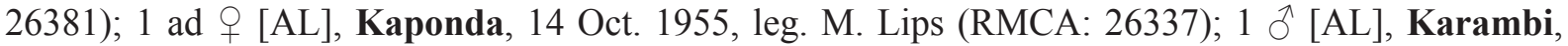

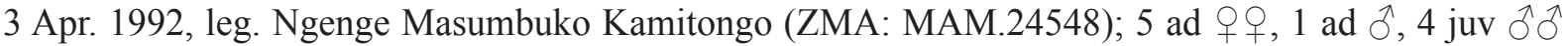
[10 AL], Karavia [Mine], 30 Sep. 1955, 7 Nov. 1955, between 3 Sep. 1955 and 30 Sep. 1955, leg. M. Lips (RMCA: 26346, 26360 to 26364, 26374 to 26376, 26347); $1 \mathrm{imm}$ ㅇ [AL], Karawa, prior to 24 Nov. 1936, leg. R.P. Wallin (RMCA: 14569); 1 ad ô, 1 ô, 4 ?? [1 AL, 5 UN], Kasai, between 1 Jan. 1923 and 31 Dec. 1923, leg. Achten, prior to 11 Jul. 1905, leg. La Compagnie du Kassai (RMCA: 89.004-M-0005, 90.005-M-0001, 739a to 739d); 1 ad $\widehat{\jmath}$ [AL], Kasebaya River [= Kasebeya River], 15 Feb. 1954, leg. Gaston-François de Witte (RMCA: 37270); 1 ad $\bigcirc$ [AL], Kasenga, between 1 Jul. 1954 and 31 Jul. 1954, leg. De Smet (RMCA: 22440); 1 ad $q$ [AL], Kasongo, 10 Jan. 1956, leg. M. Lips (RMCA: 26335); 1 ad + [AL], Katana, unknown date, leg. unknown collector (MNHN: ZM-

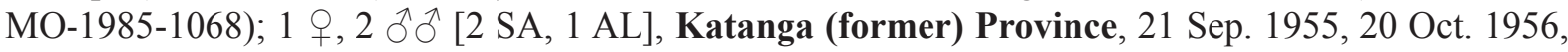
leg. unknown collector (SMF: 16870 to 16872); 1 ad? $\widehat{\partial}$ [AL], Kataye Sukunga, between 1 Jan. 1930 and 31 Dec. 1930, leg. Fourche (RMCA: 11548); 1 ठૈ [SS], Kateke [= Kateke [River]], 26 Nov. 1947, leg. l' Institut des Parcs nationaux du Congo belge (RBINS: 10709); 1 ? [UN], Kiamanwa, 20 Feb. 1931, leg. H.J. Bredo (RMCA: 11709); 1 ad + [SS], Kiamokoto, 7 Jul. 1948, leg. l' Institut des Parcs nationaux du Congo belge (RBINS: 10710); 3 q 9 [3 AL], Kibanda, 3 Feb. 1956, leg. Félix / Michel Anciaux de Faveaux (MHNG: 922.083 to 922.085); 1 sad $\lesssim$ [AL], Kifumwanshi, 13 Dec. 1955, leg. M. Lips

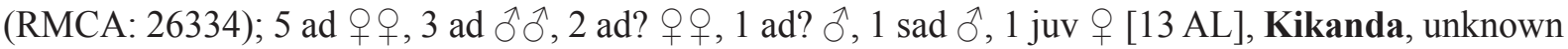
date, 3 Feb. 1956, 6 Feb. 1956, leg. Félix / Michel Anciaux de Faveaux, 23 Sep. 1955, 2 Feb. 1956, 6 Feb. 1956, leg. M. Lips (MNHN: ZM-MO-1957-126 C, RMCA: 26336, 26339, 26343, 26379, 26380, 23817, 23869, 26224, 26338, 26340 to 26342); 1 우 [SA], Kikole-Pecheur, 17 May 1980, leg. M. Mutashia (SMF: 58069); 1 ? [AL], Kikwit, between 1 Jan. 1920 and 31 Dec. 1920, leg. R.P. Vanderijst (RMCA: 5478); 1 ? [UN], Kilia Kaparata, unknown date, leg. unknown collector (INPBC: W509);

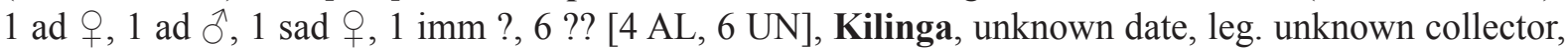
26 Aug. 1953, 15 Mar. 1954, leg. Gaston-François de Witte (INPBC: W1256.1 to W1256.4, W610, W750, RMCA: 37278 to 37280, 37183); 1 ad,+ 2 ad $\widehat{\partial}$ o [3 AL], Kilobelobe [= Kilobilobe], 6 Jan. 1956, between 5 Jan. 1956 and 6 Jan. 1956, leg. D'Haenens (RMCA: 26386, 26387, 26385); 1 ad $q$ [AL], Kilwa, between 1 Jan. 1938 and 31 Jan. 1938, leg. H.J. Bredo (RMCA: 14589); 1 ㅇ [AL], Kinkole, 15 Aug. 1980, leg. unknown collector (ZMA: MAM.21153); 1 ? [AL], Kipopo, between 1 Jun. 1960

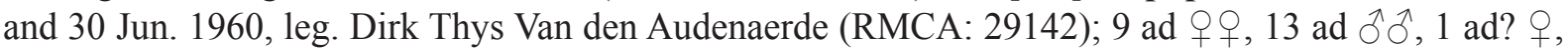

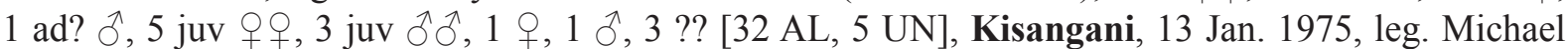
D. Gallagher, between 1 Jun. 1982 and 30 Jun. 1982, leg. Marc Colyn (HZM: 207.7974, 208.7975, RMCA: 82.031-M-0033, 82.031-M-0036 to 82.031-M-0069); 1 ad 9 [AL], Kisantu, unknown date, leg. Dumont (RMCA: 3433); 16 ?? [16 UN], Kisisile, unknown date, leg. unknown collector (INPBC: W832.01 to W832.16); 5 ad 9 ㅇ, 3 juv ?? [8 AL], Kiswishi [River], 11 Oct. 1955, leg. Félix / Michel Anciaux de Faveaux, 21 Sep. 1955, 11 Oct. 1955, leg. M. Lips (RMCA: 26217, 26366, 26367, 26377, 26378, 26348 to 26350); 1 sad $\widehat{\partial}$ [SO], Kiulu Mgemgo, 7 Jan. 1977, leg. Tshinyenye (RMCA: 77.012-M-0010); 1 ad $\widehat{\partial}, 1$ ad? § [2 AL], Kiumba, 18 Sep. 1957, leg. Uwe Rahm (RMCA: 28916, 28917); 4 ?? [4 AL], Kivu region, between 1 Jan. 1934 and 31 Dec. 1934, leg. Guy Babault (MCZ: 46066 to 46069); 4 ad 우, 1 ad Oे, 6 ad ?? [11 AL], Kivu, near, unknown date, leg. Guy Babault (MNHN: ZM-MO-1985-1065, ZM-MO-1985-1070 to ZM-MO-1985-1079); 1 ठ [AL], Kokonde, 4 Feb. 1956, leg. unknown collector (SMF: 16874); 3 우, 6 §ึ leg. Michael D. Gallagher (HZM: 209.7976 to $212.7979,213.7080$ to $222.7089,253.8020,254.8021$ );

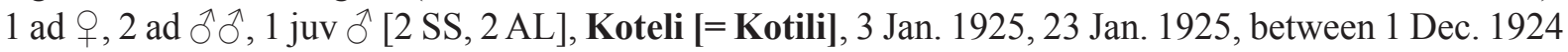

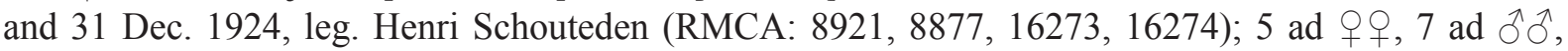
1 ad ?, 1 juv $q$ [12 SS, 2 SO], Kunungu, prior to 19 Nov. 1937, between 1 Jan. 1922 and 31 Dec. 1922, leg. Ngwe, prior to 22 Oct. 1930, prior to 1 Aug. 1939, leg. N'Kele (RMCA: 7752 to 7755, 15515, 14159 to 14166, 10616); 1 ? [AL], Lake Kivu, near, between 1 Jan. 1935 and 31 Dec. 1935, leg. Guy Babault 
(MCZ: 46157); 1 ad? ㅇ, 1 ? [1 AL, 1 UN], Loondo Mugunga, between 1 Mar. 1945 and 31 Mar. 1945, leg. l' Expédition au Lac Tumba (Congo Belge), between 1 Mar. 1954 and 31 Mar. 1954, leg. Gonze de Loneux (RBINS: 7468.1, 7468.2); 1 ad ô [AL], Lovoi, between 1 Mar. 1932 and 31 May 1932, leg. Quarré (RMCA: 11737); 1 juv ô [AL], Luanza Mission, prior to 13 Mar. 1913, leg. de Paoli (RMCA:

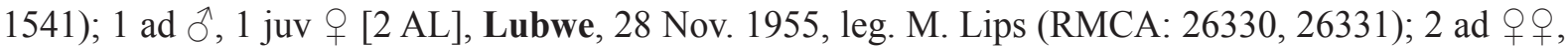
7 ad $\widehat{\partial} \partial^{\lambda}, 1$ sad ${ }^{\lambda}, 1$ juv + , 1 juv $\widehat{~}$ [9 SS, 2 SK, 1 SC], Luebo, 16 Dec. 1921, 17 Dec. 1921, 19 Dec. 1921, leg. Henri Schouteden (RMCA: 6609, 6635 to 6638, 6610, 6639, 6640, 6641a, 6641b, 6642, 6643); 1 ad ㅇ, $1 \mathrm{sad}$ ㅇ [1 SS, $1 \mathrm{SC}$ ], Lukafu, 20 Dec. 1930, leg. Gaston-François de Witte and de Crawhez (RMCA: 11129, 11130); 1 ad Ŝ [SO], Lukolela, between 1 Sep. 1923 and 30 Sep. 1923, leg. J. Ghesquière

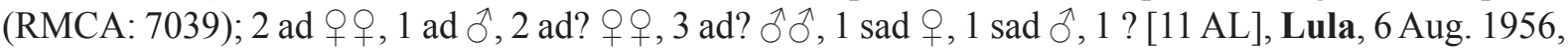
10 Aug. 1956, leg. Max Poll, prior to 4 Jul. 1958, leg. A.J. Jobaert (RMCA: 25632 to 25638, 25629 to 25631, 27200); $1 \mathrm{imm}+$ [SS], Lulenga [= Rugari], 24 Nov. 1925, leg. Henri Schouteden (RMCA:

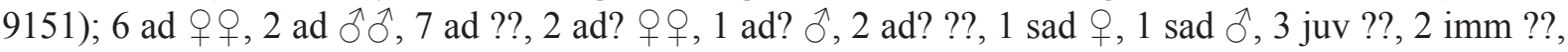
1 q, 1 ? [14 SS, 13 AL, 2 SO], Luluabourg [= Kananga], unknown date, 5 Apr. 1922, 15 Apr. 1922, 16 Apr. 1922, 6 May 1922, 11 May 1922, 16 May 1922, prior to 8 Jul. 1913, between 11 May 1922 and 22 May 1922, between 11 May 1922 and 15 May 1922, leg. Richard Callewaert, unknown date, leg. Jan Deheegher, 24 Sep. 1963, 24 May 1964, leg. Antoon Emeric Marcel De Roo (BMNH: 1927.12.21.11, RMCA: 31567 to $31569,31571,31574,31575,7556,7564,7557$ to $7560,7555,7561,7554,7562$, 32657, 32666, 7553, 7552, 7563, 7565, 1954, 1954cc, 1954d to 1954g); 1 ad ô, 7 ad ?? [5 SS, 2 SO, 1 SC], Lusambo, unknown date, 25 Dec. 1921, between 1 Dec. 1921 and 31 Dec. 1921, between 1 Jan. 1922 and 31 Jan. 1922, leg. J. Ghesquière (RMCA: 7339, 7340, 7335, 7631, 7334, 7336 to 7338); 1 ad + [AL], Lusinga, 12 Jun. 1947, leg. Gaston-François de Witte et al. (RBINS: 10805); 2 ad $ᄋ$ 우, $1 \mathrm{sad}$ ऽे [1 SS, 2 AL], Lwiro [= Luiro], 21 Feb. 1959, leg. Uwe Rahm, 8 Aug. 1964, leg. U. Goepel

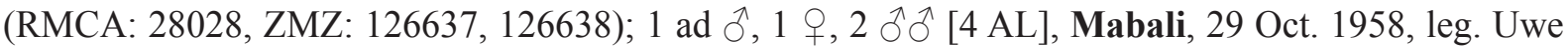
Rahm, between 1 Jun. 1956 and 30 Jun. 1956, leg. Alvin Novick (RMCA: 28918, MCZ: 48258 to 48260); 10 ?? [10 UN], Mabwe, 10 Aug. 1949, leg. unknown collector (RBINS: 10806.02, 10806.05, 10806.06, 10806.10 to 10806.16); 5 ad +9 , 1 ad ${ }^{\top}$ [6 AL], Mabwe River, 10 Aug. 1949, leg. GastonFrançois de Witte et al. (RBINS: 10806.01, 10806.03, 10806.04, 10806.07 to 10806.09); 1 ad $q, 4$ juv 우, 1 juv ? [6 AL], Madimao, 18 May 1955, between 18 May 1955 and 18 Oct. 1955, leg. M. Lips

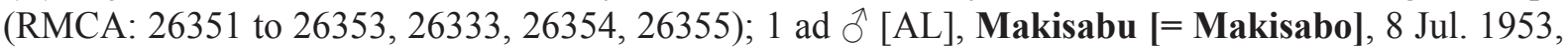
leg. Gaston-François de Witte (RMCA: 37174); 1 ad + [AL], Matadi, prior to 4 Aug. 1937, leg. Dartevelle (RMCA: 13966); 1 juv $\hat{\delta}$ [AL], Matale, prior to 27 Feb. 1940, leg. Hautmann (RMCA: 15885); 3 ad 우, 2 ad ??, 4 juv $ㅇ ㅜ, 1$ juv $\widehat{\overbrace{}}$ [7 SS, 3 SO], Mauda, 26 Feb. 1925, 16 Mar. 1925, 18 Mar. 1925, 21 Mar. 1925, between 2 Mar. 1925 and 3 Mar. 1925, leg. Henri Schouteden (RMCA: 8969 to $8971,8978,8979,8989,8990,8955,9902,8958) ; 1$ ad $\delta, 1$ juv $\delta$ [2 AL], May ya Moto, 5 Apr. 1954, between 15 Apr. 1955 and 15 Apr. 1956, leg. Gaston-François de Witte (RMCA: 37281,

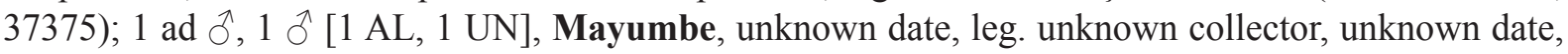
leg. unknown collector (RMCA: 89.004-M-0011, 90.012-M-0002); 1 ad $\widehat{\partial}$ [SA], Mbalaka (Plantation), 23 Jul. 1995, leg. Herwig Leirs et al. (RMCA: 97.021-M-0838); 1 ô, 5 ?? [1 SS, 5 UN], Medje, 26 Mar. 1910, 16 Sep. 1910, 7 Oct. 1910, 26 Mar. 1913, leg. Herbert Lang, James Paul Chapin and The American Museum Congo Expedition (AMNH: 49308, 49304, 49314, 49338, 49291, 48966); 1 ad $ᄋ$ [SS], M'gayu, 14 Dec. 1909, leg. Herbert Lang, James Paul Chapin and The American Museum Congo Expedition (RMCA: 12368); 1 ad? $\widehat{~}, 1$ sad $\circ$ [2 AL], Mimbulu, 4 Jan. 1956, leg. D’Haenens (RMCA: 26382, 26383); 1 ad $\lesssim$ [SA], Moera, 12 May 1921, leg. Nils Carl Gustaf Fersen Gyldenstolpe and Swiss Mission (RMCA: 16285); 1 ad $q$ [AL], Mosenge, 15 Aug. 1986, leg. WHO and Marc Colyn (RMCA:

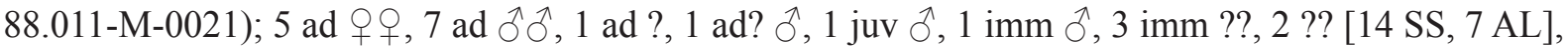
Mount Wago [= Wago, Mount], between 1 Dec. 1950 and 31 Dec. 1950, between 1 Jul. 1951 and 31 Jul. 1951, between 1 Jan. 1951 and 31 Dec. 1951, leg. Alexandre Fain, prior to 31 Dec. 1990, leg. unknown collector (RMCA: 20791 to 20801, 23301, 21469 to 21475, RBINS: 21658, 21659); 2 ad 우,

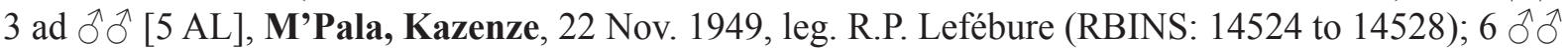


[2 SB, 4 SN], Mugeri Mission, 25 Jul. 2002, leg. Michael H. Huhndorf (FMNH: 173297 to 173302); 2 ad $ㅇ+$, 1 ad $\delta, 1$ ad? $ᄋ, 1$ sad + [4 SS, 1 SO], Mugunga, Lake, prior to 22 Dec. 1943, leg. GastonFrançois de Witte, prior to 31 Dec. 1943, leg. Gaston-François de Witte et al. (RMCA: 16635 to 16637,

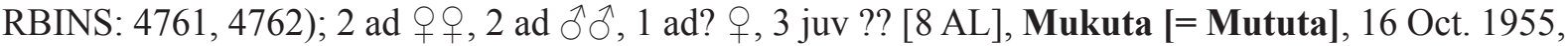
leg. M. Lips (RMCA: 26394 to 26401); 11 우, 13 $\widehat{\jmath}, 6$ ?? [30 UN], Mulongo, between 28 Oct. 1974 and 29 Oct. 1974, leg. Michael D. Gallagher (HZM: 223.7990 to 252.8019); 1 ad,+ 1 ad $\delta$ [2 AL], Mulungu, prior to 1 Feb. 1940, leg. F.L. Hendricks (RMCA: 15873, 15874); $1 \mathrm{imm}$ ? [SO], Mulungu

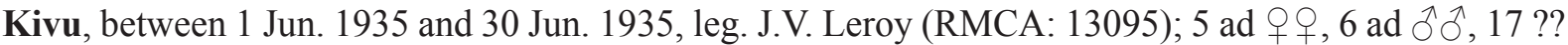
[1 SS, 10 AL, 17 UN], Murambi, unknown date, leg. unknown collector, 27 Aug. 1953, 21 Sep. 1953, 30 Sep. 1953, leg. Gaston-François de Witte, 27 Mar. 1959, leg. Uwe Rahm (INPBC: W1193, W1321, W1516.01 to W1516.10, W604, W606, W608, W642, W644, RMCA: 37181, 37182, 28029, 37175 to 37180, 37199, 37200); 2 ad $ㅇ$ [2 AL], Musosa, near, between 1 Jan. 1941 and 31 Dec. 1941, leg. H.J. Bredo (RBINS: 14532, 14533); 7 ?? [7 UN], Musugeriza River, unknown date, leg. unknown collector (INPBC: W785, W919.1 to W919.6); 3 ?? [3 UN], Mutsora, unknown date, leg. unknown collector (INPBC: W333.1 to W333.3); 1 ad + [SK], Mutwanga, between 1 Aug. 1937 and 31 Aug. 1937,

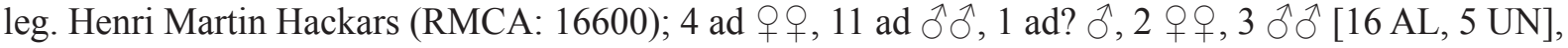
Mwenda-Katuka Piste, 4 Jan. 1946, leg. J. de Wilde, 4 Jan. 1946, leg. unknown collector (RBINS: 15452.01 to 15452.21 ); 1 ad $\hat{o}$ [AL], Nakiluba [= Inakiluba], 24 Oct. 1955, leg. M. Lips (RMCA: 26329); 1 ad,+ 1 ad $\widehat{\partial}, 1$ juv $\widehat{o}$ [3 AL], Nakilubo [= Inakiluba], 14 Nov. 1955, leg. M. Lips (RMCA: 26368 to 26370); 2 ad 우 우 [2 SK], Netonna, between 1 Jan. 1889 and 31 Dec. 1889, leg. P. Hesse (SMF: 2524 [lectotype Vesperugo pagenstecheri Noack, 1889], 2525 [paralectotype Vesperugo pagenstecheri Noack, 1889]); 1 juv ㅇ, 1 §ิ, 1 ? [2 SS, 1 SA], N'Gayu area, 14 Dec. 1909, leg. Herbert Lang, James Paul Chapin and The American Museum Congo Expedition (AMNH: 48942, 48953, 49343); 1 juv ㅇ [SS], N'goma [= Ngoma], between 1 Jan. 1925 and 31 Jan. 1925, leg. Henri Schouteden (RMCA:

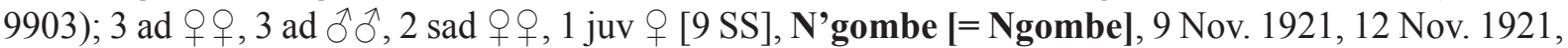

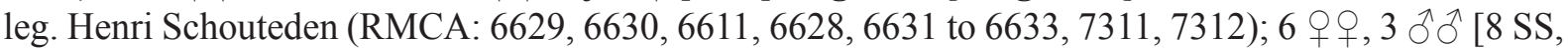
1 SO], Niangara, 11 Nov. 1910, 14 Nov. 1910, leg. Herbert Lang, James Paul Chapin and The American

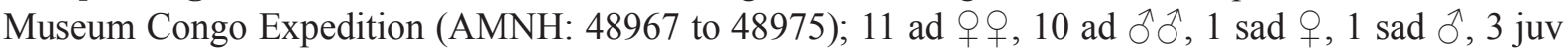

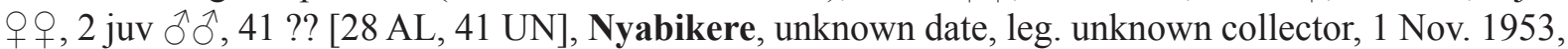
1 Feb. 1954, 18 Aug. 1954, 7 Feb. 1955, 30 Aug. 1955, leg. Gaston-François de Witte (INPBC: W1246, W1334, W1375, W1532.01 to W1532.35, W620, W622, RMCA: 37225 to 37234, 37187, 37188, 37312 to $37325,37364,37361,37362) ; 7$ ?? [1 AL, 6 UN], Nyabitembe River, unknown date, leg. unknown collector, 9 Apr. 1954, leg. Gaston-François de Witte (INPBC: W856.1 to W856.6, RMCA: 37298); 2 ad 우, 1 ad ô, 2 ?? [5 AL], Nyakariba River, 20 Jan. 1954, 10 Feb. 1954, leg. Gaston-François de Witte (RMCA: 37235 to 37239); 2 ad $\widehat{\partial} \widehat{\partial}$ [2 SO], Nzulu, prior to 31 Dec. 1943, leg. Gaston-François de Witte et al. (RBINS: 4763, 4764); 1 ad ô [SO], N'Zulu [= Nzulu], prior to 22 Dec. 1943, leg. Gaston-François de Witte (RMCA: 16638); 1 ad? 9 [AL], Omaniumdu, 27 Jul. 1959, leg. Laurent (RMCA: 29537); 1 ad ? [SO], Pili Pili [= Pilipili], 16 Dec. 1912, leg. Cuthbert Christy (RMCA: 1610); 1 ? [UN], Poko, 23 Jul. 1914, leg. Cuthbert Christy (RMCA: 4797); 1 ? [SA], Poko Zone [= Poko Territory], 30 Aug. 1913, leg. Herbert Lang, James Paul Chapin and The American Museum Congo Expedition (AMNH: 49329); 1 juv $O$ [AL], Popokabaka, between 1 Nov. 1955 and 30 Nov. 1955, leg. René Close (RMCA: 90.009-M-0001); 1 ? [AL], Risimu Zone, 9 Sep. 1909, leg. Herbert Lang, James Paul Chapin

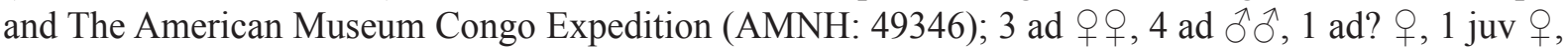
12 ?? [9 AL, 12 UN], Rodahira River, unknown date, leg. unknown collector, 28 Oct. 1953, 16 Jul. 1954, 5 Aug. 1955, leg. Gaston-François de Witte (INPBC: W1195, W1197.1 to W1197.8, W612, W614, W875.2, RMCA: 37330 to 37335, 37185, 37365, 37366); 7 ?? [7 UN], Rumangabo, unknown date, leg.

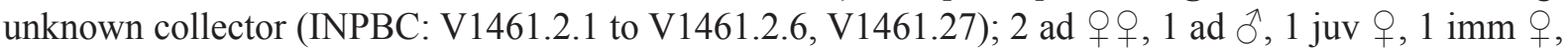
$1 \mathrm{imm}$ गे, 7 ?? [6 AL, 7 UN], Rumanura River, unknown date, leg. unknown collector, 9 Feb. 1954, 30 Apr. 1954, leg. Gaston-François de Witte (INPBC: W939.01 to W939.07, RMCA: 37307 to 37311,

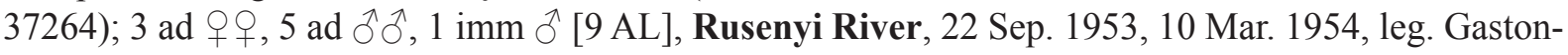


François de Witte (RMCA: 37271 to 37277, 37184, 37186); 2 ad $q$ +, 14 ad $\widehat{\jmath}, 1$ ad? $q, 1$ sad $q$, $1 \mathrm{imm}$ ㅇ, 4 우, 411 ?? [1 SS, $25 \mathrm{AL}, 1 \mathrm{SO}, 407 \mathrm{UN}$ ], Rutshuru, unknown date, leg. unknown collector, 16 Sep. 1953, 20 Jan. 1954, 9 Feb. 1954, 22 Apr. 1954, 5 Dec. 1955, leg. Gaston-François de Witte, 9 Aug. 1925, 30 Aug. 1925, leg. Edmund Heller, 31 Mar. 1938, leg. Serge Frechkop, between 6 Jan. 1931 and 6 Jan. 1938, leg. J. Ghesquière (INPBC: V121, V1248, V1348, V1349, V1350.2, V1352.1, V1352.2, V1353.1, V1355, V1357, V1358, V1366, V1368 to V1370, V1372 to V1374, V1376 to V1380, V1382.1, V1383 to V1385, V1386.1, V1388, V1390 to V1396, V1398, V1400, V1401, V1404, V1405, V1420.1, V1421, V1424, V1425, V1428, V1522.1, V1527, V1528, V1530, V1531, V1594.001 to V1594.345, V371, V377, W1164, W1525, W626, W628, W630, W739, W775, W989.01, W989.02, RMCA: 37191, 37192,37240 to 37247,37303 to 37306 , FMNH: 30669, 30670, RMCA: 17090, 37373, 37374, 37265

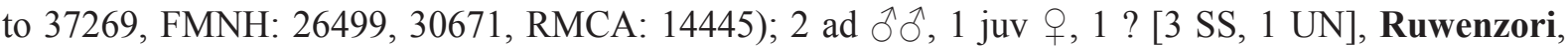
unknown date, leg. Rudolf Grauer and Hermann Rolle, between 22 Jan. 1906 and 31 Dec. 1911, between 21 Jan. 1906 and 31 Dec. 1911, between 20 Jan. 1906 and 31 Dec. 1911, leg. Rosenberg (ZMB: T09311, RMCA: 937 to 939); 1 ad $\widehat{\partial}$ [AL], Sakania, prior to 31 Dec. 1913, leg. Coulon (RMCA: 2821); 3 ad 우, 1 ad $\widehat{o}$ [4 AL], Shindaika, 27 Sep. 1955, leg. M. Lips (RMCA: 26356 to 26359); 1 ๙ [SS], St Joseph Mission [= Mikalaya], unknown date, leg. Richard Callewaert (BMNH: 1934.10.24.19); 6 웅, 7 ふึ, 1 ? [12 SS, 2 UN], St. Jos. (Mission) [= Mikalaya], 14 Apr. 1924, 15 Apr. 1924, 18 Apr. 1924, 5 May 1924, 8 May 1924, 10 May 1924, 8 Jul. 1924, leg. Richard Callewaert (AMNH: 86163, 86164, 117649, 86153 to 86162, 86142); 2 ad + +, 1 ad $§, 1$ ad ? [2 SK, 2 SO], St. Joseph de Luluabourg Mission [= Mikalaya], 2 May 1939, 7 May 1939, leg. Jean Jacques Deheyn, 27 Jun. 1923, between 15 Feb. 1923 and 15 Mar. 1923, leg. Richard Callewaert (RMCA: 15373, 7182, 15379, 7169);

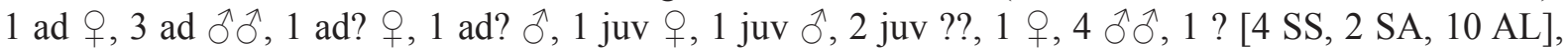
Stanleyville [= Kisangani], 1 Mar. 1928, leg. Collart, 21 Jun. 1931, leg. Schwetz, 25 Aug. 1909, 5 Sep. 1909, 9 Sep. 1909, leg. Herbert Lang, James Paul Chapin and The American Museum Congo Expedition, prior to 6 Oct. 1910, prior to 31 Jul. 1899, leg. Weyns (RMCA: 16262, 11707, AMNH:

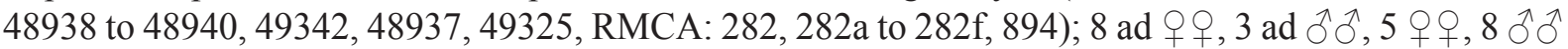
[2 SS, 22 AL], Tandala, 7 Jul. 1979, 9 Jul. 1979, 10 Jul. 1979, 11 Jul. 1979, 12 Jul. 1979, 13 Jul. 1979, leg. Lynn W. Robbins and C. Brian Robbins, 9 Jul. 1979, 10 Jul. 1979, 11 Jul. 1979, 12 Jul. 1979, 13 Jul. 1979, leg. unknown collector, 7 Jul. 1979, 11 Jul. 1979, 12 Jul. 1979, 13 Jul. 1979, leg. C. Brian Robbins (CM: 86797 to 86799, MHNG: 1870.083, CM: 86800, MHNG: 1870.084, USNM: 537723, CM: 86802, MHNG: 1870.085, USNM: 463502, CM: 86803, 86804, MHNG: 1870.086 to 1870.088 , USNM: 463503, CM: 86794, USNM: 463498 to 463501, 537722, CM: 86796, MHNG: 1870.082); 1 ad ㅇ, 1 ad $\widehat{o}$ [2 SS], Teturi, 24 May 1948, 17 Sep. 1948, leg. Jean Hiernaux (RBINS: 7051, 7050); 4 ad

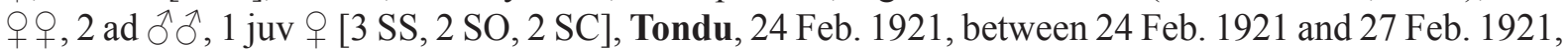
leg. Henri Schouteden (RMCA: 6612 to 6617, 6621); 2 ad 우 [2 AL], Tshakala, between 1 Jan. 1982 and 31 Jan. 1982, leg. Marc Colyn (RMCA: 82.019-M-0005, 82.019-M-0006); 1 ad $§$ [AL], Tshibaka [= Tshibaka-Kaji], 25 Aug. 1956, leg. Max Poll (RMCA: 25628); 1 ad $\precsim, 1$ ad? §ิ [2 SS], Tshikapa, 28 Nov. 1921, between 16 Nov. 1921 and 26 Nov. 1921, leg. Henri Schouteden (RMCA: 6634, 7310); 1 ad $\odot, 1$ ad ${ }^{\top}, 1$ juv? [3 AL], Tshisangwe, 27 Sep. 1955, leg. M. Lips (RMCA: 26371 to 26373); 4 q $ᄋ$, 2 วิ $\widehat{\partial}$ [6 SS], Upper Congo, unknown date, leg. Alexander Douglas Mitchell Carruthers (BMNH: 1907.6.14.10, 1907.6.14.19, 1907.6.14.21, 1907.6.14.7 to 1907.6.14.9); 1 juv,+ 1 ? [1 AL, $1 \mathrm{UN}$ ], Uvira, unknown date, leg. Rudolf Grauer, between 1 Mar. 1955 and 31 Mar. 1955, leg. Narcisse Leleup (ZMB: T09307, RMCA: 22913); 1 ad,+ 1 \& [2 SS], Vitshumbi, prior to 31 Dec. 1943, leg. unknown collector, prior to 31 Dec. 1943, leg. Gaston-François de Witte et al. (RBINS: 4765, 4766); 6 ad $\hat{\jmath} \widehat{o}^{\lambda}$, 97 ?? [101 AL, 2 UN], Wafanya [= Wafania], unknown date, 28 Nov. 1987, 3 Jan. 1988, 5 Dec. 1988, 2 Sep. 1991, 4 Sep. 1991, 14 Sep. 1991, 17 Sep. 1991, 20 Sep. 1991, 23 Sep. 1991, 25 Sep. 1991, 7 Oct. 1991, 12 Oct. 1991, 14 Oct. 1991, 28 Apr. 1992, 29 Apr. 1992, 19 May 1992, 26 May 1992, 29 May 1992, between 12 Oct. 1991 and 18 Oct. 1991, between 1 Dec. 1991 and 31 Dec. 1991, between 1 Nov. 1991 and 30 Nov. 1991, between 1 Oct. 1987 and 31 Oct. 1987, between 1 Aug. 1991 and 31 Aug. 1991, between 1 Jul. 1991 and 31 Jul. 1991, between 1 May 1992 and 31 May 1992, between 
VAN CAKENBERGHE V. et al., The bats of Congo, Rwanda and Burundi revisited

1 May 1991 and 31 May 1991, between 1 Apr. 1992 and 30 Apr. 1992, between 1 Mar. 1992 and 31 Mar. 1992, between 1 Mar. 1991 and 31 Mar. 1991, between 1 Jan. 1992 and 31 Jan. 1992, between 1 Jan. 1991 and 31 Jan. 1991, leg. P. Lootens (RMCA: 92.079-M-0012, 92.079-M-0127 to 92.079-M0139, 92.079-M-0153, 92.079-M-0154, 91.076-M-0108, 91.076-M-0084, 91.076-M-0085, 91.076-M0112, 91.076-M-0087, 92.079-M-0084, 92.079-M-0093, 91.076-M-0066, 91.076-M-0088, 91.076-M0089, 91.076-M-0091, 91.076-M-0093 to 91.076-M-0097, 92.079-M-0097 to 92.079-M-0100, 92.079-M-0045, 92.079-M-0046, 92.079-M-0048, 92.079-M-0054, 92.079-M-0055, 92.079-M-0101, 92.079-M-0102, 88.005-M-0004, 92.079-M-0052, 92.079-M-0044, 92.079-M-0047, 92.079-M-0050, 92.079-M-0051, 88.005-M-0005, 88.005-M-0006, 88.005-M-0013, 91.076-M-0068 to 91.076-M-0071, 89.020-M-0047, 91.076-M-0105, 91.076-M-0001, 92.079-M-0053, 92.079-M-0109, 91.076-M-0015, 92.079-M-0004, 92.079-M-0005, 92.079-M-0007, 92.079-M-0011, 92.079-M-0081, 92.079-M-0087 to 92.079-M-0089, 92.079-M-0091, 92.079-M-0092, 92.079-M-0094, 92.079-M-0071, 92.079-M-0085, 92.079-M-0086, 92.079-M-0095, 92.079-M-0096, 92.079-M-0106, 92.079-M-0107, 91.076-M-0030, 91.076-M-0042, 91.076-M-0043, 91.076-M-0049, 91.076-M-0050, 92.079-M-0080, 92.079-M-0082, 92.079-M-0083, 92.079-M-0090, 91.076-M-0053, 91.076-M-0054, 91.076-M-0062, 88.005-M-0015, 92.079-M-0008, 92.079-M-0006, 92.079-M-0009, 92.079-M-0010, 92.079-M-0013, 92.079-M-0049, 92.079-M-0056, 92.079-M-0105, 92.079-M-0108, 91.076-M-0103); 2 ad $9+9,2$ ad $\widehat{\partial} \partial^{\lambda}$ [4 AL], Wilibadi River, 16 Apr. 1948, leg. Hediger and Jacques Verschuren (RBINS: 8195.1 to 8195.4); 1 ? [SA], Yakuluku, 4 Nov. 1912, leg. Herbert Lang, James Paul Chapin and The American Museum Congo

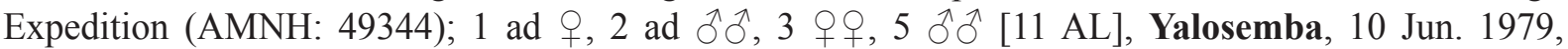
17 Jun. 1979, 23 Jun. 1979, leg. Lynn W. Robbins and C. Brian Robbins, 15 Jun. 1979, leg. Frieder Meier, 19 Jun. 1979, 22 Jun. 1979, 23 Jun. 1979, leg. C. Brian Robbins, 23 Jun. 1979, leg. unknown collector (CM: 86790, MHNG: 1870.078, CM: 86791, USNM: 463488 to 463490, 463495, CM: 86792, MHNG: 1870.079, USNM: 463496, 463497); 1 ad $\$, 1$ ad $\delta$ [2 AL], Yalusaka, 28 Sep. 1957,

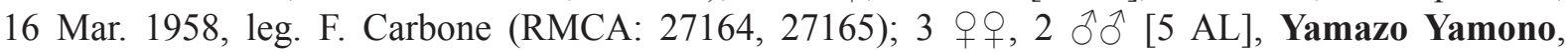
19 Jun. 1979, leg. Frieder Meier, 19 Jun. 1979, leg. C. Brian Robbins (MHNG: 1870.080, USNM:

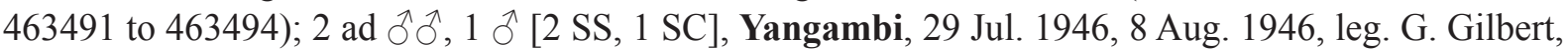
8 Aug. 1946, leg. unknown collector (RBINS: 6989 to 6991); 1 ad $\hat{\jmath}$ [SO], Yangambi Division Forest, 8 Aug. 1946, leg. G. Gilbert (RMCA: 37900).

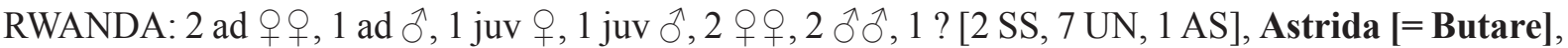
11 Nov. 1966, leg. Félix / Michel Anciaux de Faveaux, 13 Oct. 1976, leg. Frieder Meier, 24 Feb. 1966, leg. unknown collector (RBINS: 16001, 16003, MHNG: 1873.084, RBINS: 15488.1, 15488.2, 15489.1, 15489.2, 15491.1 to 15491.3 ); 1 ad $\widehat{\partial}$ [SA], Butare, 7 Oct. 1981, leg. Frits De Vree, Benny Baeten and Victor Van Cakenberghe (RMCA: 82.006-M-0201); 16 ?? [16 UN], Gabiro, unknown date, leg. unknown collector (INPBC: V3102, V3129.01 to V3129.15); 5 ad ?? [5 SS], Gafumba, 24 Nov. 1966, leg. Félix / Michel Anciaux de Faveaux (RBINS: 15996 to 15999, 16002); 1 ad § [SA], Gafumba Mugusa, 7 Feb. 1985, leg. Théoneste (RMCA: 87.047-M-0129); 58 ?? [58 UN], Gihinga, unknown date, leg. unknown collector (INPBC: V3044.1.01 to V3044.1.58); 2 $\precsim$ [2 AL], Gisenyi, 28 Jun. 1980, 13 Sep. 1980, leg. Hans H. de Iongh (ZMA: MAM.21232, MAM.21231); 1 ad $ᄋ$ [SA], Gisovu,

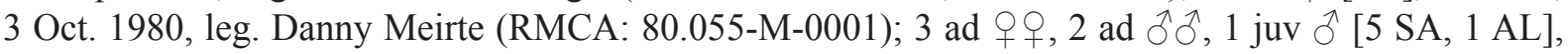
Kibuye, 6 Oct. 1980, 7 Oct. 1980, 8 Oct. 1980, leg. Danny Meirte (RMCA: 80.055-M-0002 to 80.055M-0004, 80.055-M-0006, 80.055-M-0005, 80.055-M-0007); 1 ad $ๆ$ [SA], Kitabi, 5 Nov. 1981, leg. Frits De Vree, Benny Baeten and Victor Van Cakenberghe (RMCA: 82.006-M-0202); 1 ad $q$ [SA], Mabanza, 10 Oct. 1980, leg. Danny Meirte (RMCA: 80.055-M-0008); 69 ?? [69 UN], Mihindi, Lake, unknown date, leg. unknown collector (INPBC: V3090, V3112.01 to V3112.68); 1 ad ? [SS], Mugogwe, 19 Dec. 1966, leg. Félix / Michel Anciaux de Faveaux (RBINS: 16000); 1 đ [UN], Nyamasheke,

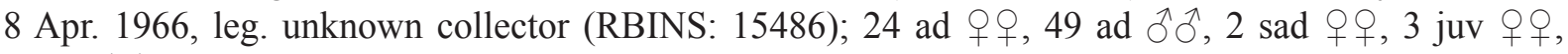
3 juv ồ ô, 2 imm ?? [66 SA, 16 AL, 1 UN], Nyamata, 15 Nov. 1981, 16 Nov. 1981, 17 Nov. 1981, leg. Frits De Vree, Benny Baeten and Victor Van Cakenberghe (RMCA: 82.006-M-0203 to 82.006-M-0207, 
82.006-M-0218 to 82.006-M-0267, 82.006-M-0208 to 82.006-M-0217, 82.006-M-0268 to 82.006-M0285); 1 ad 9 [SA], Nyambumba, between 18 Dec. 1984 and 5 Jan. 1985, leg. Benny Baeten and Luc Janssens (RMCA: 85.006-M-0095); 1 ad $\widehat{~[S A], ~ N y u n d o, ~} 19$ Oct. 1980, leg. Danny Meirte (RMCA:

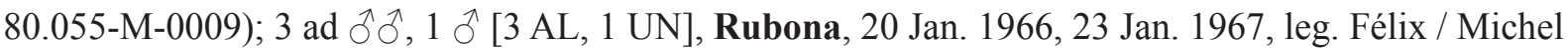
Anciaux de Faveaux (RBINS: 15487, 15934.1 to 15934.3); 1 ad $\widehat{\overbrace{}}$ [SA], Shangi, between 18 Dec. 1984 and 5 Jan. 1985, leg. Benny Baeten and Luc Janssens (RMCA: 85.006-M-0096).

\section{Neoromicia rendalli (Thomas, 1889)}

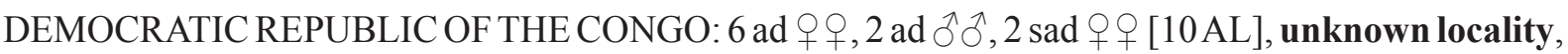
27 Jul. 1965, prior to 27 Jul. 1965, between 3 Jul. 1965 and 27 Jul. 1965, leg. Antoon Emeric Marcel De Roo (RMCA: 33547 to 33551, 33543 to 33546, 33552); 1 sad [AL], Albertville [= Kalemie], 2 Apr. 1960, leg. G. Verhoustraete (RMCA: 28666); 1 ad $q, 1$ juv $q, 1$ juv $\widehat{o}, 3$ ?? [3 AL, 3 UN], Bagbele, unknown date, leg. unknown collector, 30 Apr. 1950, leg. H. de Saeger (INPBC: 1182.1 to 1182.3, RMCA: 36848 to 36850); 1 ad $\lesssim$ [AL], Bagbele Moko, 25 Jul. 1952, leg. H. de Saeger (RMCA:

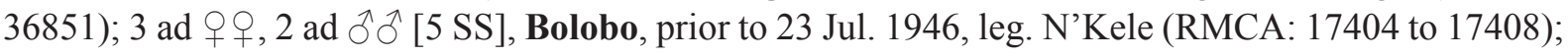
1 ad $\hat{O}$ [SS], Cel II, 8 Mar. 1952, leg. H. de Saeger et al. (RBINS: 13850); 1 q [SA], Duma, between 1 Oct. 1910 and 31 Oct. 1910, leg. unknown collector (SMF: 6393); 3 ad $\circ$ +, 2 ad $\widehat{\partial} \widehat{\partial}, 1$ juv $\propto, 1$ juv $\widehat{\partial}$,

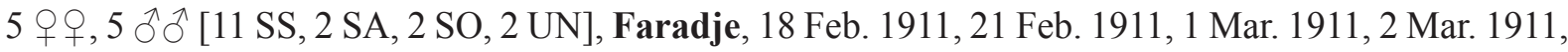
29 Mar. 1911, 30 Mar. 1911, 2 Aug. 1911, leg. Herbert Lang, James Paul Chapin and The American Museum Congo Expedition, 2 Aug. 1911, leg. unknown collector (AMNH: 49047, 49048 [topotype Eptesicus faradjius J.A. Allen, 1917], 49044 [topotype Eptesicus faradjius J.A. Allen, 1917], 52114, 52115, 49054 [topotype Eptesicus faradjius J.A. Allen, 1917], 49055 [topotype Eptesicus faradjius J.A. Allen, 1917], MCZ: 17379, AMNH: 49045 [holotype Eptesicus faradjius J.A. Allen, 1917], 49046, 49053 [topotype Eptesicus faradjius J.A. Allen, 1917], 49049 [topotype Eptesicus faradjius J.A. Allen, 1917], 49050, 49051 [topotype Eptesicus faradjius J.A. Allen, 1917], 49327, 49345, RMCA: 12387); 1 ad $\widehat{\partial}$ [AL], Gangala-na-Bodio, between 1 Nov. 1956 and 30 Nov. 1956, leg. Max

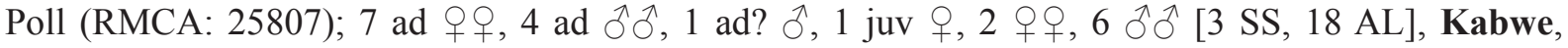
9 Feb. 1964, 9 Feb. 1965, 30 Apr. 1965, leg. Antoon Emeric Marcel De Roo (RMCA: 33352 to 33359, AMNH: 212581 to 212590, RMCA: 33596 to 33598); 1 ad § [SS], Katobwe, 9 Dec. 1926, leg. John Todd Zimmer (FMNH: 29417); 1 ad ?, 1 ? [2 SS], Kawa (Forest), 24 Jul. 1951, leg. unknown collector,

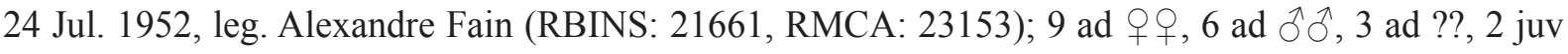
우, 1 juv ?, 5 우 [17 SS, 4 AL, 2 SK, 2 SO, $1 \mathrm{SC}$ ], Luluabourg [= Kananga], unknown date, 13 Apr. 1922, 15 May 1922, 28 May 1922, 29 May 1922, 7 Jun. 1922, 11 Jun. 1922, 19 Jun. 1922, 26 Jun. 1922, 17 Jul. 1922, 28 May 1924, between 16 Jun. 1922 and 19 Jun. 1922, leg. Richard Callewaert, 24 Sep. 1963, 3 Jul. 1965, between 26 Apr. 1964 and 18 Aug. 1965, leg. Antoon Emeric Marcel De Roo, between 1 Jan. 1963 and 31 Dec. 1963, leg. Jan Deheegher (BMNH: 1926.11.1.54, 1926.11.1.55, 1926.7.6.29, 1926.7.6.77, 1926.7.6.78, RMCA: 7573, 7575, 7576, 7578, 7586, 7572, 7582, 7579, 32668, 7574, 7583, 7571, 7577, 7580, AMNH: 86166, RMCA: 7581, 33542, 7585, 31572, 7584, 33605); 1 ad § [SA], Mulongo, 31 Oct. 1974, leg. Michael D. Gallagher (HZM: 1.8024); 3 ad

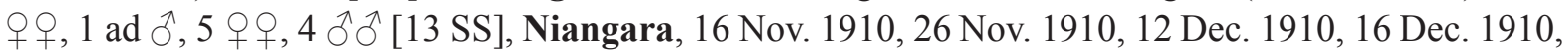
26 Dec. 1910, leg. Herbert Lang, James Paul Chapin and The American Museum Congo Expedition, 16 Dec. 1910, leg. unknown collector (AMNH: 49039, 49040, FMNH: 43870, AMNH: 49034, FMNH: 43869, RMCA: 12388, AMNH: 49041, MCZ: 17378, AMNH: 49036 to 49038, 49042, 49043); 1 q,

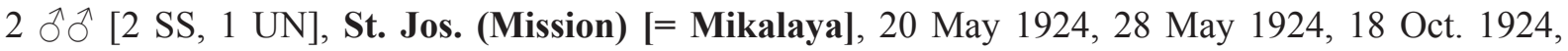
leg. Richard Callewaert (AMNH: 86146, 86165, 86167); 8 ad 웅, 3 ad $\widehat{\jmath} \widehat{\jmath}$ [10 SS, 1 SA], Tandala, 7 Jul. 1979, 9 Jul. 1979, 13 Jul. 1979, 16 Jul. 1979, 20 Jul. 1979, leg. Lynn W. Robbins and C. Brian Robbins (CM: 86675, 86668, 86669, 86782, 86676 to 86679,86672 to 86674 ). 
VAN CAKENBERGHE V. et al., The bats of Congo, Rwanda and Burundi revisited

Neoromicia somalica (Thomas, 1901)

DEMOCRATIC REPUBLIC OF THE CONGO: 1 ? [UN], unknown locality, between 1 Apr. 1999 and 30 Apr. 1999, leg. unknown collector (SMF: 93322); 1 ad ? [SA], Lukolela, 4 Aug. 1930, leg. James Paul Chapin (AMNH: 86927); 1 § [SS], Mboga [= Boga], unknown date, leg. Howard de Walden (BMNH: 1930.11.11.172); 1 ad $\widehat{\jmath}$ [SA], Mbwambala, 20 Jun. 1995, leg. Herwig Leirs et al. (RMCA: 97.021-M-0749).

RWANDA: 1 ad,+ 1 ad $\lesssim$ [2 SK], Karama, 11 Oct. 1981, 12 Oct. 1981, leg. Frits De Vree, Benny Baeten and Victor Van Cakenberghe (RMCA: 82.006-M-0918, 82.006-M-0919).

\section{Neoromicia tenuipinnis (Peters, 1872)}

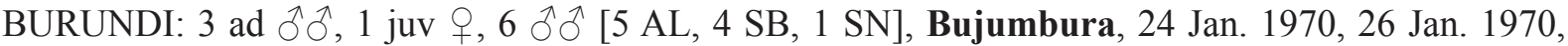
17 Feb. 1970, 20 May 1970, 6 Feb. 1972, 24 Jan. 1973, 12 Feb. 1973, leg. P.L. Niort, between 1 Jan. 1928 and 31 Dec. 1928, leg. Douce (MHNG: 1608.019, 1607.096, 1608.005, 1608.006, 1608.011, 1608.018, 1624.003, 1895.089, 1895.090, RMCA: 16271); 1 \% [UN], Russissi, bank [= Rusizi Delta], unknown date, leg. Rudolf Grauer (ZMB: 54067); 1 \% [SS], Usumbura [= Bujumbura], 14 Oct. 1938, leg. Duncan M. Hodgson and William F. Coultas (MCZ: 38033).

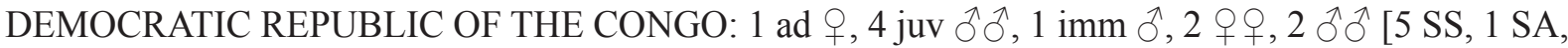
3 AL, 1 UN], unknown locality, unknown date, 1 Jun. 1939, leg. unknown collector, unknown date, leg. Henri Schouteden, unknown date, leg. P. Hesse, 4 Jul. 1958, prior to 4 Jul. 1958, leg. E. Jans (BMNH: 1926.11.1.56, 1926.7.6.81 to 1926.7.6.83, RMCA: 9193, SMF: 2526, RBINS: 6277.2, RMCA: 27372, 27367, 27370); 1 ad + [SS], Alima, 12 Feb. 1960, leg. P. Dyleff (RMCA: 28827); 2 ad $\circ$ ㅇ [2 AL], Bamanya, 20 Mar. 1956, leg. R.P. Hulsaert, between 1 Jan. 1952 and 31 Dec. 1952, leg. Leonet (RMCA: 26328, 21699); 1 ad § , 2 ad ??, 1 sad ?, 1 juv ? [1 SS, 4 SO], Bambesa, 24 Apr. 1940, between 1 Jun. 1937 and 30 Jun. 1937, leg. J.M. Vrijdagh (RMCA: 17753, 14075 to 14078); 1 juv + [AL], Banana, between 1 May 1952 and 31 May 1952, leg. Is. Mesmaekers (RMCA: 21375); 1 ad $\widehat{\partial}$ [AL], Banana Creek, 20 Apr. 1949, leg. The MBizi expedition (RBINS: 14511); 1 juv $q$ [AL], Bena Tshiadi [= Bena Tshadi], between 1 Apr. 1979 and 30 Apr. 1979, leg. Robertson (RMCA: 79.019-M-0001); 2 ad 웅, 1 ad? ô, $^{2}$

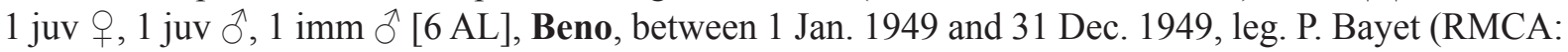
19475, 19828 to 19830, 19849, 19850); 1 ? [UN], Biangolo, unknown date, leg. unknown collector (INPBC: W53); 1 ad $\widehat{\jmath}$ [SS], Bitshumbi [= Vitshumbi], prior to 22 Dec. 1943, leg. Gaston-François de Witte (RMCA: 16632); 1 juv $q$ [AL], Bokoro, between 1 Jan. 1952 and 31 Dec. 1952, leg. E. Jans (RMCA: 21523); 2 ad ${ }^{\lambda}{ }^{\lambda}, 1$ juv ${ }^{\lambda}$ [3 AL], Boteka, 26 Aug. 1984, between 1 Feb. 1985 and 28 Feb. 1985, leg. P. Lootens (RMCA: 85.021-M-0001 to 85.021-M-0003); 1 juv ? [SO], Bukavu, between

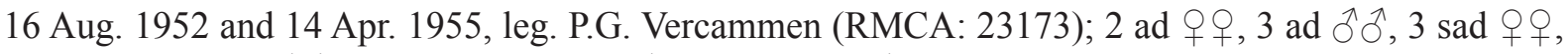

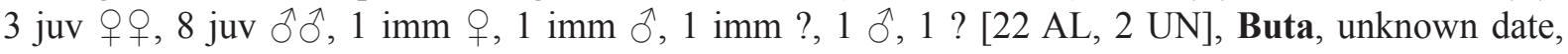
8 Jan. 1936, prior to 31 May 1939, prior to 16 Jun. 1939, prior to 16 Jun. 1937, between 1 Jul. 1949 and 31 Jul. 1949, between 1 Jan. 1935 and 31 Dec. 1935, between 1 Jan. 1930 and 31 Dec. 1930, between 1 Jan. 1925 and 31 Dec. 1925, leg. Jozef Hutsebaut, 10 Dec. 1946, leg. unknown collector (RMCA: 14564a to $14564 \mathrm{c}, 14584$ a to $14584 \mathrm{c}, 16186,16280,16281$, RBINS: 14510, RMCA: 13709 a to $13709 \mathrm{c}$, $16245,16188,13176,13177,19459,19460,13859,15397,15325,15327,15328)$; 4 ad 우, 1 ad $\jmath^{2}$, 1 ad? †, 2 juv ภึ [8 AL], Butembo, 16 Mar. 1959, between 16 Mar. 1959 and 26 May 1959, leg. P. Dyleff (RMCA: 27878 to 27882, 27884, 27877, 27883); 1 ad,+ 1 ad? 9 [1 SS, 1 AL], Eala, between 1 Aug. 1936 and 31 Aug. 1936, between 1 Jul. 1935 and 31 Jul. 1935, leg. J. Ghesquière (RMCA: 13096, 13410); 1 ㅇ [SS], Edouard, Lac [= Edward, Lake], prior to 31 Dec. 1943, leg. unknown collector (RBINS: 4760); 1 juv 9,1 ? [1 AL, 1 UN], Elisabetha, prior to 5 Oct. 1920, prior to 19 Apr. 1926, leg. Tinant (RMCA: 8220, 5369); 1 ad ô, 1 ad ?, 1 ? [2 SS, 1 AL], Faradje, 1 Mar. 1911, 12 Jan. 1913, leg. Herbert Lang, James Paul Chapin and The American Museum Congo Expedition, 6 Jul. 1925, leg. Henri 
Schouteden (AMNH: 48988 [holotype Eptesicus ater J.A. Allen, 1917], 49423 [topotype Eptesicus ater J.A. Allen, 1917], RMCA: 9043); 1 ad $\hat{~}$ [AL], Ganda-Sundi, 25 Mar. 1964, leg. Alexandre Fain (RMCA: 33268); 1 ? [UN], Ibembo, between 1 Sep. 1949 and 30 Sep. 1949, leg. Jozef Hutsebaut (RMCA: 19461); 1 ad [AL], Irumu, between 1 Jan. 1926 and 31 Dec. 1926, leg. Leopold prince of Belgium (RMCA: 16256); 2 ad $\widehat{\jmath} \hat{\jmath}$ [2 AL], Kabale, 10 May 1927, 11 May 1927, leg. James Paul Chapin, Dewitt L. Sage, Frank P. Mathews and The Ruwenzori-Kivu Expedition (AMNH: 82384, 82383); 1 ad? $\widehat{~}$ [AL], Kabongo, between 1 Jan. 1952 and 31 Dec. 1952, leg. M. Dierckx (RMCA: 21484); 1 ? [UN], Kalenda, 1 Aug. 1952, leg. unknown collector (RBINS: 7742); 1 ? [UN], Kalenda [Kasai], unknown date, leg. unknown collector (RBINS: 1742); 1 ad $q$ [AL], Kamponde, between 1 Apr. 1965 and 30 Apr. 1965, leg. Antoon Emeric Marcel De Roo (RMCA: 33389); 1 ad ? [SS], Katana, 29 Oct. 1932, leg. Burgeon (RMCA: 12057); 1 ad $\$$ [UN], Katanga (former) Province, 1 Jun. 1939, leg. unknown collector (RBINS: 6277.1); 1 ad $\lesssim$ [UN], Kinzambi Mission, 30 Jul. 1995, leg. Herwig Leirs et al. (MSB: 274830); 1 ad $\widehat{\jmath}$ [AL], Kisangani, between 1 Jun. 1982 and 30 Jun. 1982, leg. Marc Colyn (RMCA: 82.031-M-0035); 1 ad $\widehat{\partial}, 1$ sad $\widehat{~}$ [1 SS, 1 SO], Kisantu, 11 Dec. 1920, 12 Dec. 1920, leg. Henri Schouteden (RMCA: 7313, 7314); 1 ad $\widehat{~}$ [SS], Koteli [= Kotili], 23 Jan. 1925, leg. Henri Schouteden (RMCA: 8922); 1 ad $\widehat{~}$ [SS], Kunungu, between 1 Mar. 1921 and 31 Mar. 1921, leg. Henri Schouteden (RMCA: 7783); 1 ad,+ 1 ad ? [2 SO], Léopoldville [= Kinshasa], between 1 Jan. 1949 and 31 Dec. 1949, leg. Richard, between 1 Nov. 1942 and 30 Nov. 1942, leg. R. Fiasse (RMCA: 18809, 17661); 1 ad क, 3 ?? [2 AL, 2 UN], Leverville [= Lusanga], between 1 Feb. 1929 and 28 Feb. 1929, leg. Tinant (RMCA: 9987a, 9988a, 9988c, 9989); 1 ad ô, 1 sad ㅇ, 1 ? [2 AL, 1 SO], Lukolela, unknown date, leg. J. Ghesquière, 1 Aug. 1930, leg. James Paul Chapin, 25 Jun. 1931, leg.

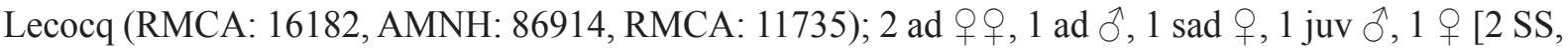
3 AL, 1 SC], Luluabourg [= Kananga], unknown date, 22 Jun. 1922, 4 Jul. 1922, leg. Richard Callewaert, 24 Sep. 1963, 3 Jul. 1965, leg. Antoon Emeric Marcel De Roo (BMNH: 1927.12.21.12, RMCA: 7587, 32667, 33540, 33541, 7569); 3 ad + $ᄋ, 1$ ad ${ }^{\wedge}$ [4 AL], Luluabourg Ravine, 29 Aug. 1963,

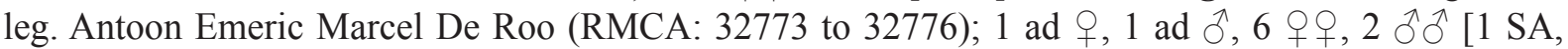
9 AL], Mabali, 19 Dec. 1958, leg. Jean Georges Baer, 21 Jun. 1956, between 1 Jun. 1956 and 30 Jun. 1956, leg. Alvin Novick, 9 Oct. 1958, leg. Uwe Rahm (MHNG: 985.067, MCZ: 48245, RMCA: 28912, 28913, MCZ: 48246 to 48251); 1 ad $q$ [AL], Makaw, prior to 15 Nov. 1958, leg. E. Jans (RMCA: 27501); 2 ?? [2 AL], Masako, unknown date, leg. Ndey B. Ifuta (RMCA: 93.079-M-0346, 93.079-M-0370); 1 juv ㅇ [AL], Mauda, between 1 Mar. 1925 and 31 Mar. 1925, leg. Henri Schouteden (RMCA: 16228); 1 ad , $1 \mathrm{sad} \partial^{\lambda}, 1$ juv $\bigcirc$ [3 AL], Mayumbe, unknown date, leg. De Pauw, unknown date, prior to 31 Dec. 1913, leg. Georges Gilson (RMCA: 16495, 3432, 2668); 1 ad $\$$ [SS], M'gayu, 17 Dec. 1909, leg. Herbert Lang, James Paul Chapin and The American Museum Congo Expedition (RMCA: 12396); 1 ad ?, 2 juv

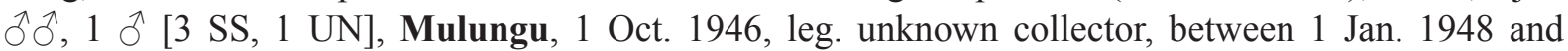
31 Dec. 1948, leg. F.L. Hendricks (RBINS: 6403, RMCA: 19646 to 19648); 2 ad $q$, 1 ad $\lesssim$ [3 SS], Ngayu [River], 17 Dec. 1909, leg. Herbert Lang, James Paul Chapin and The American Museum Congo Expedition (AMNH: 48982, 48983, 48985); 3 ?? [3 AL], N'Gayu area, 17 Dec. 1909, leg. Herbert Lang, James Paul Chapin and The American Museum Congo Expedition (AMNH: 49310, 49319, 49337); $1 \mathrm{sad}+$, 1 sad $\hat{\text { Oे }}$ [2 SS], Niangara, 16 Nov. 1910, 20 Nov. 1910, leg. Herbert Lang, James Paul Chapin and The American Museum Congo Expedition (AMNH: 48986, 48987); 1 ad ?, 1 juv ô, 1 juv ? [1 AL, 2 SO], Paulis [= Isiro], 10 Dec. 1950, between 1 Jan. 1947 and 31 Dec. 1947, leg. Abbeloos

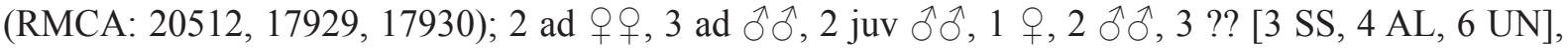
Rutshuru, unknown date, 25 Jan. 1938, 24 Feb. 1938, prior to 31 Dec. 1943, leg. unknown collector, 11 May 1953, 30 Aug. 1954, 19 Sep. 1955, prior to 22 Dec. 1943, leg. Gaston-François de Witte, prior to 31 Dec. 1943, leg. Gaston-François de Witte et al. (INPBC: V1350.1, V1353.2, W1247, RMCA: 37163, 37166, RBINS: 4767, 4768, RMCA: 37164, 37165, 16633, 16634, RBINS: 4758, 4759); 3 우, 2 $\widehat{\jmath}$ [3 SS, 2 UN], St. Jos. (Mission) [= Mikalaya], 31 May 1924, 5 Aug. 1924, 5 Oct. 1924, 27 Oct. 1924, leg. Richard Callewaert (AMNH: 86149, 86169, 86173, 86174, 86141); 2 juv 우 [2 AL], Stanleyville [= Kisangani], 2 Jul. 1929, leg. Collart, 24 Apr. 1929, leg. Geldof (RMCA: 16187, 9995); 
1 ad $\hat{~}$, 1 1 [1 SS, 1 AL], Tandala, 16 Jul. 1979, leg. Lynn W. Robbins and C. Brian Robbins, 18 Jul. 1979, leg. C. Brian Robbins (CM: 86667, USNM: 463506); 1 ad $\widehat{~}$ [AL], Tshakala, between 1 Jan. 1982 and 31 Jan. 1982, leg. Marc Colyn (RMCA: 82.019-M-0007); 1 ad ô [SS], Tshikapa, 24 Nov. 1921, leg. Henri Schouteden (RMCA: 7255); 1 ad $\widehat{\partial}, 1$ juv $\widehat{\partial}$ [2 AL], Tshuapa River, upper, between 1 Jun. 1925 and 30 Jun. 1925, leg. J. Ghesquière (RMCA: 16267, 16268); 3 우, 3 $\widehat{\delta} \widehat{\delta}$ [6 SS], Upper Congo, unknown date, leg. Alexander Douglas Mitchell Carruthers (BMNH: 1907.6.14.15 to 1907.6.14.18, 1907.6.14.4, 1907.6.14.5); 6 ad 9 \% , 1 ad $\curvearrowright, 1$ juv,+ 1 ㅇ, 1 ô, 1 ? [8 AL, 3 UN], Uvira, unknown date, leg. Rudolf Grauer, 2 Mar. 1955, between 1 Mar. 1955 and 31 Mar. 1955, leg. Narcisse Leleup, between 1 Apr. 1951 and 30 Apr. 1951, leg. F.L. Lambrecht, between 1 Nov. 1956 and 30 Nov. 1956, leg. Laurent (ZMB: 67026 to 67028, RMCA: 27209 to 27213, 22912, 90.004-M-0001, 35688); 1 ad , 1 juv ? [2 UN], Vitshumbi, prior to 31 Dec. 1943, leg. unknown collector (RBINS: 4757.1, 4757.2); 2 ad $q$ q 1 ad $\lambda, 2$ 우, 4 ठえ [3 SS, 6 AL], Yalosemba, 28 Jun. 1979, 16 Jul. 1979, leg. unknown collector, 17 Jun. 1979, leg. C. Brian Robbins, 28 Jun. 1979, leg. Lynn W. Robbins and C. Brian Robbins, 9 Jul. 1979, leg. Frieder Meier (ZMMU: S-180533, USNM: 463504, 463505, 537724, 537725, CM: 86666, MHNG: 1871.010, 1871.011, ZMMU: S-180534); 1 đે [UN], Zobia, 18 Apr. 1946, leg. unknown collector (RBINS: 14509).

RWANDA: 1 ภ, 1 ? [2 UN], Gabiro, unknown date, 1 Jul. 1948, leg. unknown collector (RBINS: 13921, 13021); 1 juv $\widehat{\partial}, 1$ ㅇ, 2 $\widehat{\jmath}$ [4 AL], Gisenyi, 5 Oct. 1980, leg. Hans H. de Iongh (ZMA: MAM.21233 to MAM.21236); 1 ad Ô, 1 ? [1 SA, 1 UN], Rwankwi, between 1 Apr. 1948 and 30 Apr. 1948, leg. J.V. Leroy (RMCA: 19070, 19071); 1 ad $\widehat{\curlywedge}$ [SA], Shangi Schad [= Shangi], 5 Jan. 1985, leg. Benny Baeten and Luc Janssens (RMCA: 85.006-M-0457).

Neoromicia zuluensis (Roberts, 1924)

RWANDA: 1 ad $q$ [SA], Mimuli, 31 Oct. 1964, leg. U. Goepel (ZMZ: 126640).

Nycticeinops Hill \& Harrison, 1987

Nycticeinops schlieffenii (Peters, 1859)

DEMOCRATIC REPUBLIC OF THE CONGO: 2 $\widehat{\jmath}$ [2 UN], Aketi, 21 Jun. 2015, leg. Vlir/Aketi (UNIKIS: AKETI601, AKETI602); 2 q 9,1 ? [1 AL, 2 UN], Bagunda, unknown date, leg. unknown collector, 18 Jan. 1951, leg. H. de Saeger et al., 18 Jan. 1951, leg. H. de Saeger (INPBC: 2751, RBINS: 13870, RMCA: 36888); 1 ? [UN], Bas Congo (former) Province [= Kongo Central Province], unknown date, leg. Alphonse Cabra (RMCA: 65b); 1 + , 1 ô [2 SS], Dikul River [= Dikulwe Cave], unknown date, leg. S.A. Neave (BMNH: 1907.12.13.3, 1907.12.13.4); $1 \hat{\delta}$ [UN], Gangala-na-Bodio, 8 Nov. 1949, leg. H. de Saeger et al. (RBINS: 13868); 3 우, 1 ð [4 SS], Kabenge, 3 Apr. 1949, leg. l' Institut des Parcs nationaux du Congo belge, 3 Apr. 1949, leg. unknown collector (RBINS: 10719 to 10722); 1 ? [SA], Katanga, prior to 23 Dec. 1947, leg. soeur de notre dame de Bethanie (RMCA: 18177); 1 ㅇ, 1 đ [2 SS], Kateke [= Kateke [River]], 1 Dec. 1947, leg. 1' Institut des Parcs nationaux du Congo

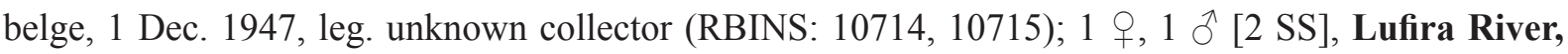
upper, unknown date, leg. S.A. Neave (BMNH: 1907.12.13.5, 1907.12.13.6); 1 q [SS], Luluabourg [= Kananga], unknown date, leg. Richard Callewaert (BMNH: 1926.7.6.112); 2 $\partial^{\lambda}$ [2 SS], Mabwe, 13 Dec. 1948, leg. l' Institut des Parcs nationaux du Congo belge, 22 Dec. 1948, leg. unknown collector (RBINS: 10716, 10717); 1 $\widehat{~[S S], ~ M a s o m b w e, ~} 24$ Dec. 1948, leg. unknown collector (RBINS: 10798); $1 \widehat{\partial}$ [SS], Munoi [= Munoi confluence], 18 Jun. 1948, leg. l' Institut des Parcs nationaux du Congo belge (RBINS: 10718); 2 우 [2 UN], Musosa, near, 1 Jun. 1940, between 1 Jun. 1940 and 30 Jun. 1940, leg. unknown collector (RBINS: 14515, 14514). 


\section{Pipistrellus Kaup, 1829}

BURUNDI: $2 \widehat{\partial}$ [2 NS], Mpinga Cave [= Mpinga Mission], 25 Mar. 1970, leg. P.L. Niort (MHNG: 1607.099, 1607.100); 1 ㅇ [AL], Nyanza [= Nyanza-Lac], 28 Feb. 1920, leg. Henry Cushier Raven (USNM: 237232); 1 đ [NS], Rugari Mission, between 1 Oct. 1969 and 31 Oct. 1969, leg. P.L. Niort (MHNG: 1607.089); 1 q [NS], Rwintare Mission [= Rwintare], 19 Jun. 1969, leg. P.L. Niort (MHNG: 1607.083).

DEMOCRATIC REPUBLIC OF THE CONGO: 10 ๆ $\uparrow, 24$ ๙ [34 AL], Batala's Village, 13 Apr. 1925, 16 Apr. 1925, leg. Edmund Heller (FMNH: 30635 to 30668); 1 ? [AL], Belgian River, 30 Dec. 1926,

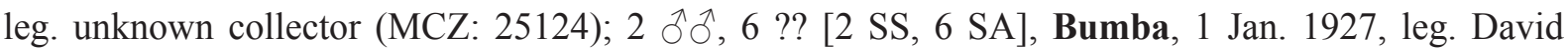
H. Linder, 1 Jan. 1927, leg. Harold Jefferson Coolidge, 30 Dec. 1926, 30 Dec. 1930, leg. unknown collector (MCZ: 23380, 23381, 25125 to 25130); 9 우우 7 đิ $\widehat{o}$ [16 AL], Kabengere, 22 Dec. 1926, 24 Dec. 1926, 27 Dec. 1926, 28 Dec. 1926, leg. John Todd Zimmer (FMNH: 30682 to 30685, 30687 to 30698); 1 ㅇ, 5 ภे [6 AL], Mabali, between 1 Jun. 1956 and 30 Jun. 1956, leg. Alvin Novick (MCZ: 48252 to 48257); 1 juv ? [UN], Rutshuru, unknown date, leg. unknown collector (INPBC: W3.2).

Pipistrellus grandidieri (Dobson, 1876)

BURUNDI: 1 ad + , 1 ad $ð$ [1 SA, 1 SK], Resha, 1 Mar. 1979, 4 Apr. 1979, leg. Heinz Stephan (SMF: 57586, 57587).

Pipistrellus hesperidus (Temminck, 1840)

BURUNDI: 1 ad $\widehat{\partial}$ [SA], Nyamugari Hill, 20 Apr. 1991, leg. L. Davenport (FMNH: 148368).

RWANDA: 2 ad $q$ ㅇ [2 SA], Kitabi, 2 Nov. 1981, 3 Nov. 1981, leg. Frits De Vree, Benny Baeten and Victor Van Cakenberghe (RMCA: 82.006-M-0926, 82.006-M-0927); 1 ad $\widehat{\delta}$ [SA], Mutura, 24 Oct. 1981, leg. Frits De Vree, Benny Baeten and Victor Van Cakenberghe (RMCA: 82.006-M-0925).

\section{Pipistrellus nanulus Thomas, 1904}

DEMOCRATIC REPUBLIC OF THE CONGO: 1 ad $q$ [SA], Avakubi, 26 Feb. 1914, leg. Herbert Lang, James Paul Chapin and The American Museum Congo Expedition (AMNH: 49323); 1 ad $\widehat{\partial}$ [SK], Mosenge, 15 Aug. 1986, leg. WHO and Marc Colyn (RMCA: 88.011-M-0020).

\section{Pipistrellus rueppellii (Fischer, 1829)}

DEMOCRATIC REPUBLIC OF THE CONGO: 1 juv + , 1 juv $\widehat{~}$ [1 SA, $1 \mathrm{AL}$ ], Albertville [= Kalemie], prior to 18 Nov. 1959, leg. H. Bomans (RMCA: 29300, 29301); 1 juv $\widehat{\jmath}$ [AL], Beno, between 1 Jan. 1949 and 31 Dec. 1949, leg. P. Bayet (RMCA: 19853); 1 ad $\lesssim$ [AL], Ibembo, between 1 Nov. 1951 and 30 Nov. 1951, leg. Jozef Hutsebaut (RMCA: 20641); 1 ad + [SA], Jadotville [= Likasi], 2 Jun. 1958, leg. Félix / Michel Anciaux de Faveaux (RMCA: 27586); 1 ? [UN], Kasindi, unknown date, leg. unknown collector (INPBC: W979.1); 1 ad ? [SS], Kawa (Forest), between 14 Jun. 1952 and 24 Jul. 1952, leg. Alexandre Fain (RMCA: 23152); 1 ad + [AL], Lukula, between 5 Jan. 1912 and 26 Jan. 1912, leg. Daniel (RMCA: 993); 1 ? [UN], Moanda [= Muanda], unknown date, leg. unknown collector (RMCA: 12693); 1 juv + [SA], Moba, 29 Jan. 1954, leg. H. Bomans (RMCA: 22125); 1 ad $\widehat{~}$ [AL], Mongbwalu, 13 Feb. 1934, leg. Jordens (RMCA: 12247); 1 ? [AL], Mweka Kasai [= Mweka], between 1 Jan. 1930 and 31 Dec. 1930, leg. Heusgens (RMCA: 11549); 1 ad + [SA], Nepoko Bomili, between 1 Jan. 1946 and 31 Dec. 1946, leg. M. Henrion (RMCA: 18138); 1 ad ô [SA], Poko, 22 Aug. 1913, leg. Herbert Lang, James Paul Chapin and The American Museum Congo Expedition (AMNH: 49326). 
Scotoecus Thomas, 1901

Scotoecus albofuscus (Thomas, 1890)

DEMOCRATIC REPUBLIC OF THE CONGO: 1 ? [SA], Baudouinville [= Moba], 12 Dec. 1953, leg. H. Bomans (RMCA: 22412).

Scotoecus hindei Thomas, 1901

DEMOCRATIC REPUBLIC OF THE CONGO: 1 ? [SA], Kapolowe, prior to 24 May 1954, leg. De Smet (RMCA: 22192); 1 ? [SA], Niangara, 20 Jun. 1913, leg. Herbert Lang, James Paul Chapin and The American Museum Congo Expedition (AMNH: 49320). 


\section{Summary table of museum specimens}

Overview of the museum acronyms.

\begin{tabular}{|c|c|}
\hline Acronym & Name + Contact name + Database website \\
\hline AMNH & $\begin{array}{l}\text { American Museum of Natural History, New York, USA; Nancy B. Simmons } \\
\text { http://sci-web-001.amnh.org/db/emuwebamnh/index.php }\end{array}$ \\
\hline BMNH & $\begin{array}{l}\text { The Natural History Museum (formerly British Museum of Natural History), London, UK; Paulina } \\
\text { Jenkins } \\
\text { http://data.nhm.ac.uk/ }\end{array}$ \\
\hline CAS & $\begin{array}{l}\text { California Academy of Sciences, San Francisco, USA; Maureen Flannery } \\
\text { http://researcharchive.calacademy.org/research/bmammals/MamColl/index.asp }\end{array}$ \\
\hline $\mathrm{CM}$ & Carnegie Museum, Pittsburgh, USA; Suzanne B. McLaren \\
\hline DNSM & Durban Natural Science Museum, Durban, South Africa; Leigh Richards \\
\hline FMNH & $\begin{array}{l}\text { Field Museum of Natural History, Chicago, USA; Bruce D. Patterson } \\
\text { http://emuweb.fieldmuseum.org/mammals/taxonomic.php?_ga=1.142122738.1331713266.1463234212 }\end{array}$ \\
\hline HNHM & Hungarian Natural History Museum, Budapest, Hungary; Gábor Csorba \\
\hline HZM & Harrison Institute (formerly Harrison Zoological Museum), Sevenoaks, UK; Paul Bates \\
\hline INPBC & Institute of the National Parks of Belgian Congo, Brussels, Belgium \\
\hline KU & $\begin{array}{l}\text { University of Kansas, Natural History Museum, Lawrence, USA; Maria Eiffler } \\
\text { http://collections.biodiversity.ku.edu/KUMammals/ }\end{array}$ \\
\hline MCZ & $\begin{array}{l}\text { Museum of Comparative Zoology, Harvard University, Cambridge, USA; Judith Chupasko } \\
\text { http://mczbase.mcz.harvard.edu/SpecimenSearch.cfm }\end{array}$ \\
\hline MHNG & Muséum d'Histoire Naturelle, Genève, Switzerland; Manuel Ruedi \\
\hline MNHN & $\begin{array}{l}\text { Muséum national d'Histoire naturelle, Paris, France; Jean Marc Pons } \\
\text { https://science.mnhn.fr/institution/mnhn/collection/zm/item/search }\end{array}$ \\
\hline MSB & $\begin{array}{l}\text { Museum of Southwestern Biology, Albuquerque, USA; Joseph Cook } \\
\text { http://arctos.database.museum/SpecimenSearch.cfm }\end{array}$ \\
\hline NMBA & Naturhistorisches Museum, Basel, Switzerland; Denis Vallan, Raffael Winkler \\
\hline NMP & National Museum (Natural History), Prague, Czech Republic; Petr Benda \\
\hline NMW & Naturhistorisches Museum, Vienna, Austria; Frank Emmanuel Zachos \\
\hline NMZL & National Museum of Zambia, Livingstone, Zambia; Clare Mateke \\
\hline OSU & Oklahoma State University, Stillwater, USA; Karen McBee \\
\hline RBINS & $\begin{array}{l}\text { Royal Belgian Institute for Natural Sciences, Brussels, Belgium; Georges Lenglet, Tom Geerinckx, } \\
\text { Olivier Pauwels } \\
\text { https://darwin.naturalsciences.be/ }\end{array}$ \\
\hline RMCA & Royal Museum of Central Africa, Tervuren, Belgium; Wim Wendelen, Emmanuel Gilissen \\
\hline RMNH & $\begin{array}{l}\text { Naturalis - National Natuurhistorish Museum (formerly Rijksmuseum voor Natuurlijke Historie), } \\
\text { Leiden, the Netherlands; Pepijn Kamminga, Steven van der Mije } \\
\text { http://bioportal.naturalis.nl/ }\end{array}$ \\
\hline
\end{tabular}


VAN CAKENBERGHE V. et al., The bats of Congo, Rwanda and Burundi revisited

\begin{tabular}{|c|c|}
\hline Acronym & Name + Contact name + Database website \\
\hline SMF & $\begin{array}{l}\text { Senckenberg Museum, Frankfurt, Germany; Katrin Krohmann } \\
\text { http://sesam.senckenberg.de/page/index.htm }\end{array}$ \\
\hline SMNS & Staatliches Museum für Naturkunde, Stuttgart, Germany; Stefan Merker \\
\hline TM & $\begin{array}{l}\text { Ditsong National Museum of Natural History (formerly Transvaal Museum), Pretoria, South } \\
\text { Africa; Teresa Kearney }\end{array}$ \\
\hline TTU & Texas Tech University (The Museum), Lubbock, USA; Heath J. Garner \\
\hline UNIKIS & Université de Kisangani, Democratic Republic of the Congo \\
\hline USNM & $\begin{array}{l}\text { United States National Museum, Washington DC, USA; Kristofer M. Helgen, Darrin P. Lunde } \\
\text { http://collections.nmnh.si.edu/search/mammals/ }\end{array}$ \\
\hline WNMU & Western New Mexico University, Silver City, USA; Randy Jennings \\
\hline ZFMK & Zoologisches Forschungsinstitut und Museum Alexander Koenig, Bonn, Germany; Jan Decher \\
\hline ZMA & Zoologisch Museum, Amsterdam, The Netherlands (currently at Naturalis, Leiden) \\
\hline ZMB & Zoologisches Museum Berlin, Germany; Frieder Mayer \\
\hline $\mathrm{ZMH}$ & Zoologisches Institut und Zoologisches Museum, Hamburg, Germany; Thomas M. Kaiser \\
\hline ZMMU & Zoological Museum Moscow University, Moscow, Russia; Sergei V. Kruskop \\
\hline ZMUC & $\begin{array}{l}\text { Natural History Museum of Denmark (formerly Zoologisk Museum), University of Copenhagen, } \\
\text { Denmark; Eline Lorenzen, Daniel Klingberg Johansson }\end{array}$ \\
\hline ZMZ & Zoologisches Museum der Universität, Zurich, Switzerland; Barbara Oberholzer \\
\hline
\end{tabular}

In the following table (pages 248-259), we provide an overview of the number of CRB specimens in the various museums. In most cases, the number of specimens we report is higher than the number reported by Hayman et al. (1966: 77). The only exceptions are INPBC, where part of the material has been divided between the RBINS and RMCA collections, and the BMNH, where Hayman et al. (1966) did not provide any registration numbers and we were unable to trace the specimens involved. Hayman et al. (1966: 77) also reported an additional 45 specimens (1 Epomophorus anurus, 1 Epomops franqueti, 1 Hypsignatus monstrosus, 2 Taphozous mauritianus, 4 Saccolaimus peli, 1 Nycteris arge, 1 N. hispida, 7 Lavia frons, 4 Hipposideros caffer, 4 H. ruber, 4 Myotis bocagii, 6 Neoromicia nana, 7 Pipistrellus rueppellii, 1 Nycticeinops schlieffeni and 1 Miniopterus inflatus) from literature data only (Noack 1887; Gyldenstolpe 1928; Sanborn 1936; Hatt 1940; Allen \& Loveridge 1942). 
European Journal of Taxonomy 382: 1-327 (2017)

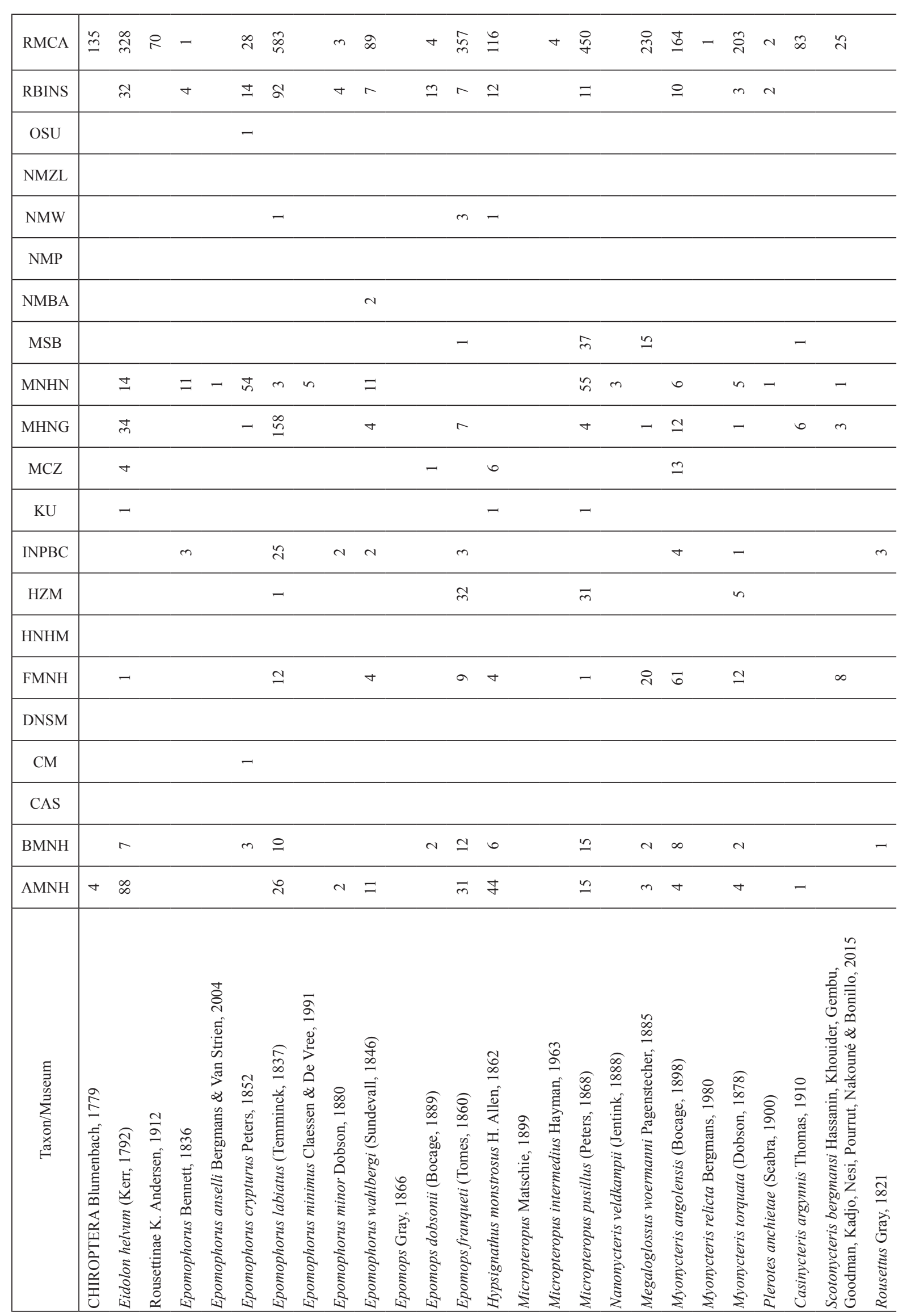


VAN CAKENBERGHE V. et al., The bats of Congo, Rwanda and Burundi revisited

\begin{tabular}{|c|c|c|c|c|c|c|c|c|c|c|c|c|c|c|c|c|c|c|c|c|c|c|c|c|c|c|}
\hline RMCA & ปั & $\stackrel{+}{+}$ & 寸 & & ป & & $\infty$ & $=$ & 京 & & & $\overrightarrow{\bar{v}}$ & $n$ & $\stackrel{⿱}{\sim}$ & & ฮิ & $\infty$ & & - & $\vec{D}$ & $a$ & $\stackrel{0}{i}$ & $\stackrel{\circ}{n}$ & - & & $\stackrel{\infty}{\circ}$ \\
\hline RBINS & ते & $r$ & & & $\dot{m}$ & & 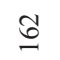 & 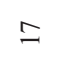 & $\widehat{\beth}$ & & & ఫ્ત & - & $n$ & & 8 & $\hat{n}$ & $N$ & & $\circ$ & & $\Xi$ & in & & & $\infty$ \\
\hline \multicolumn{27}{|l|}{ OSU } \\
\hline NMZL & & & & & & & & & & & & & & & & & & & & \multicolumn{7}{|c|}{-} \\
\hline \multicolumn{27}{|l|}{ NMW } \\
\hline \multicolumn{27}{|l|}{ NMP } \\
\hline \multicolumn{27}{|l|}{ NMBA } \\
\hline \multicolumn{27}{|l|}{ MSB } \\
\hline MNHN & $a$ & & & $\stackrel{\infty}{\infty}$ & & & & & - & & & - & & & & + & & & - & & & & + & & & - \\
\hline MHNG & $=$ & & & & - & & & 6 & $m$ & & & 6 & & & & $N$ & - & & & $\ddot{\lambda}$ & & $\vec{\lambda}$ & $\stackrel{\infty}{\sim}$ & & & - \\
\hline $\mathrm{MCZ}$ & \pm & & & & $r$ & & $N$ & $n$ & $\sim$ & & & 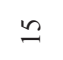 & - & & & - & & & & & $m$ & - & & & & $m$ \\
\hline $\mathrm{KU}$ & & & & & - & & & & & & $n$ & $N$ & & & & - & & & & & & & & & & \\
\hline INPBC & $\hat{\sim}$ & $\sim$ & & & $\stackrel{2}{d}$ & - & & & $\stackrel{ }{=}$ & & & $n$ & & & & $\because$ & $N$ & & & $\stackrel{\infty}{-}$ & & $m$ & & & & - \\
\hline HZM & & & & & & & & & & & & $m$ & & & & - & & - & & & & & & & & \\
\hline HNHM & & & & & & & & & & & & & & & & & & & & & & & $N$ & & & \\
\hline FMNH & in & 9 & & & 6 & & & & $\infty$ & & & $\stackrel{\infty}{=}$ & $N$ & - & & $r$ & & & & $N$ & $N$ & & & & - & \\
\hline \multicolumn{27}{|l|}{ DNSM } \\
\hline \multicolumn{27}{|l|}{$\mathrm{CM}$} \\
\hline CAS & & & & & & & & & t & & & & & & & $N$ & & & & & & & & & & \\
\hline BMNH & $\infty$ & $\nabla$ & & & 6 & & & 6 & in & - & $N$ & 우 & - & & & & & & & - & t & & & & & - \\
\hline AMNH & 0 & 6 & & & $\Xi$ & & $m$ & & $m$ & & & $\cong$ & - & & & $\stackrel{\infty}{\sim}$ & & - & & & in & $a$ & & & & $\nabla$ \\
\hline 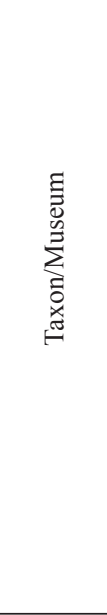 & 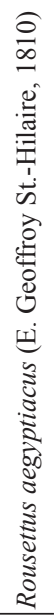 & 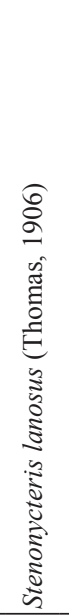 & 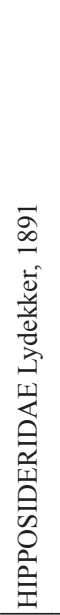 & 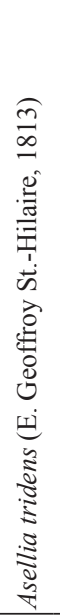 & 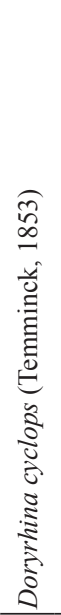 & 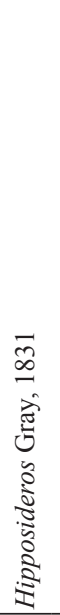 & 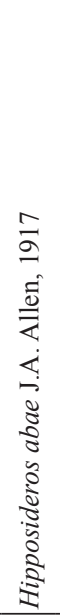 & 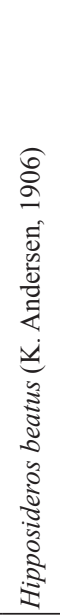 & 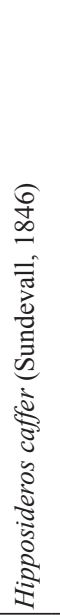 & 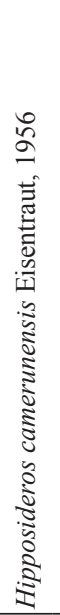 & 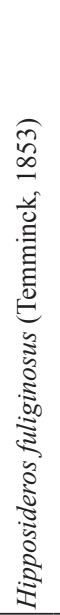 & 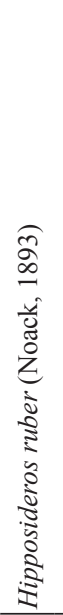 & 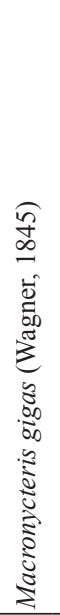 & 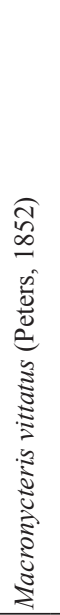 & 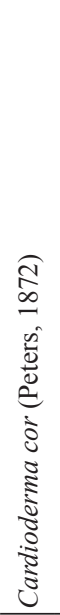 & 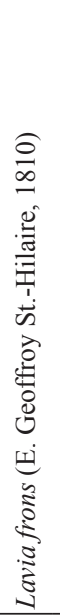 & 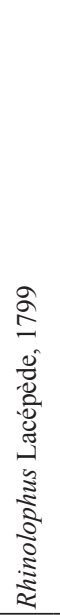 & 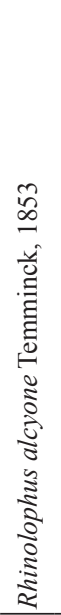 & 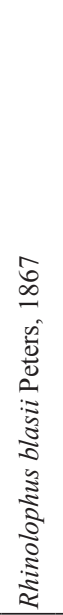 & 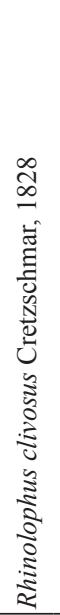 & 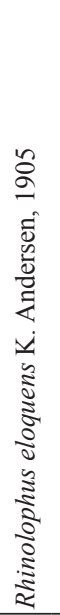 & 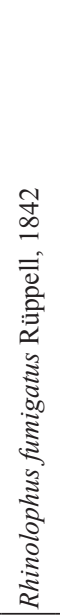 & 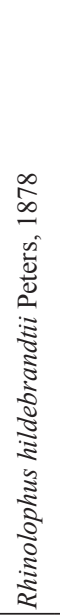 & 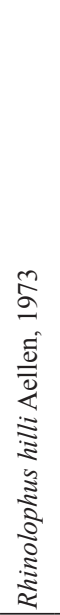 & 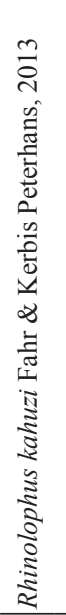 & 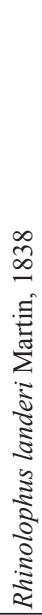 \\
\hline
\end{tabular}


European Journal of Taxonomy 382: 1-327 (2017)

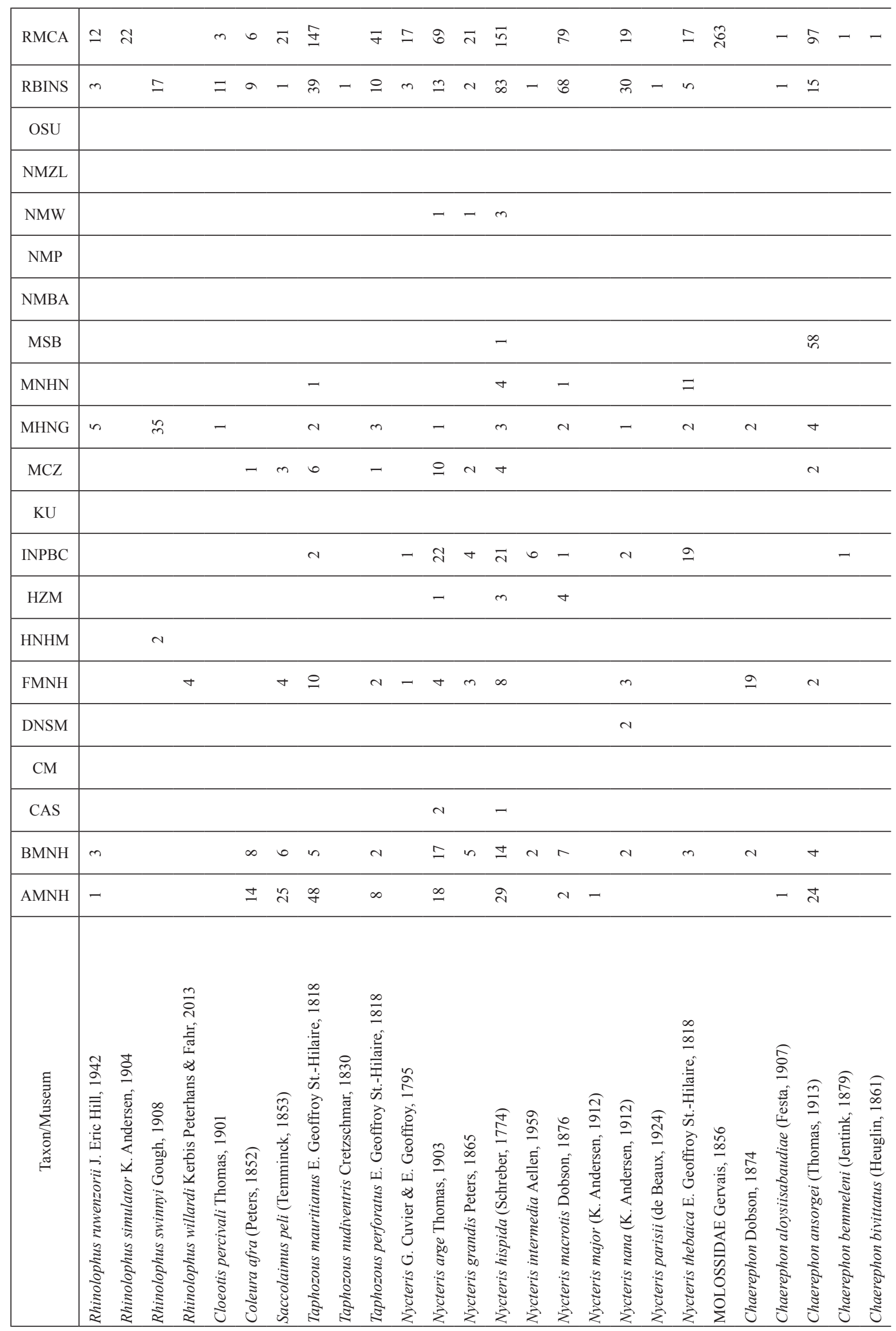


VAN CAKENBERGHE V. et al., The bats of Congo, Rwanda and Burundi revisited

\begin{tabular}{|c|c|c|c|c|c|c|c|c|c|c|c|c|c|c|c|c|c|c|c|c|c|c|c|c|c|c|}
\hline RMCA & & & & $r$ & 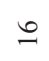 & $\stackrel{\text { సิ }}{\bumpeq}$ & - & $\underset{\widetilde{\sigma}}{\infty}$ & $\sim$ & $=$ & $\approx$ & & $\triangleq$ & & - & $\stackrel{\infty}{\infty}$ & $a$ & $\vec{\sim}$ & $N$ & $\simeq$ & - & & $=$ & - & Ұ & $\tilde{\lambda}$ \\
\hline RBINS & $m$ & & & $\nabla$ & 0 & ले & & $\hat{\infty}$ & & $\ddot{\imath}$ & ป & & & & - & & & $\stackrel{\infty}{+}$ & & $m$ & $\sim$ & & - & & & \\
\hline \multicolumn{27}{|l|}{ OSU } \\
\hline NMZL & & & & & & & & & & & & & & & \multicolumn{12}{|c|}{-} \\
\hline \multicolumn{27}{|l|}{ NMW } \\
\hline \multicolumn{27}{|l|}{ NMP } \\
\hline \multicolumn{27}{|l|}{ NMBA } \\
\hline MSB & & & & & & $\stackrel{\varrho}{\varrho}$ & & $n$ & & & & & N & & & 6 & & & & - & & & & & & \\
\hline MNHN & & & & & $\sim$ & - & & & & & & & & & - & & & & & & & & & & & \\
\hline MHNG & & & & in & - & $n$ & & 으 & $N$ & & & & $\infty$ & & & & & $a$ & & & - & & & & & $n$ \\
\hline MCZ & & & N & $N$ & & & N & 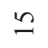 & - & & $n$ & & & & $N$ & & & & - & & & & & & & $m$ \\
\hline \multicolumn{27}{|l|}{ KU } \\
\hline INPBC & & & & & & $\equiv$ & & $\Xi$ & & $\sim$ & $a$ & & & & $\sim$ & $n$ & & & & & & & - & & & $r$ \\
\hline HZM & & & & & & $=$ & & in & & & & & & & & $\sim$ & & - & & & & & & & & \\
\hline \multicolumn{27}{|l|}{ HNHM } \\
\hline FMNH & & & $\simeq$ & & & n & - & & - & & & & & & $\sim$ & - & & & & & - & & & & & $r$ \\
\hline DNSM & & & & & & & & & & & & & & & & & & - & & & & & & & & \\
\hline \multicolumn{27}{|l|}{$\mathrm{CM}$} \\
\hline \multicolumn{27}{|l|}{ CAS } \\
\hline BMNH & & - & & & $n$ & $\stackrel{\infty}{\sim}$ & - & $\sim$ & & & & & $m$ & & $\sigma$ & $\Xi$ & & 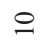 & - & $a$ & - & $\sim$ & & & & 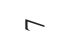 \\
\hline AMNH & N & & $\stackrel{\infty}{N}$ & $\stackrel{\dot{m}}{ }$ & - & & $\underset{d}{d}$ & N & $\cong$ & $\alpha$ & 6 & - & & - & $\tilde{\lambda}$ & 6 & - & $\infty$ & $r$ & $\nabla$ & & & & & & I \\
\hline 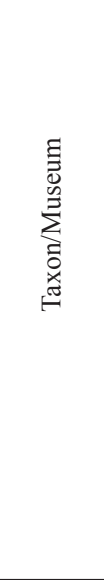 & 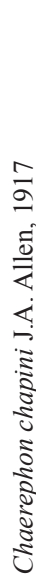 & 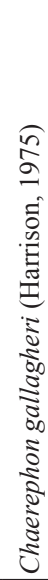 & 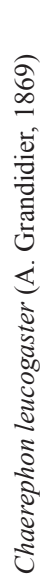 & 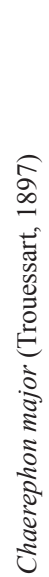 & 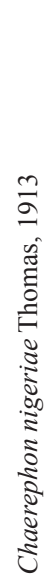 & 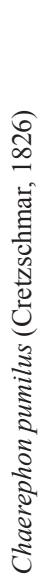 & 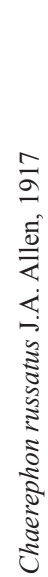 & 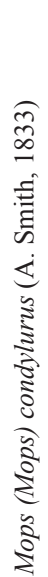 & 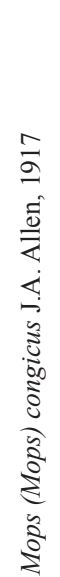 & 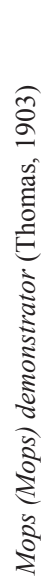 & 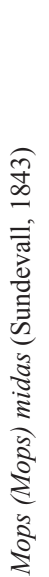 & 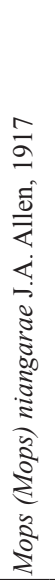 & 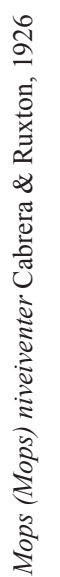 & 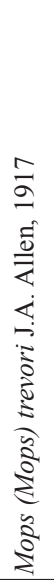 & 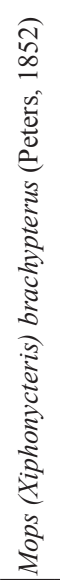 & 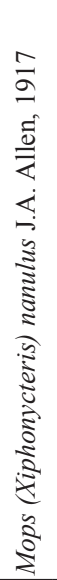 & 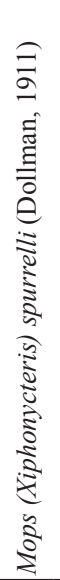 & 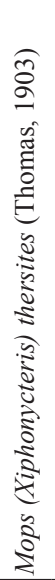 & 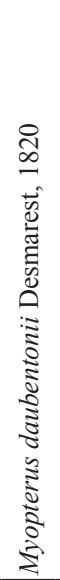 & 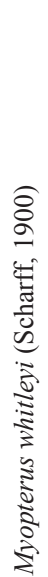 & 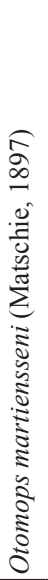 & 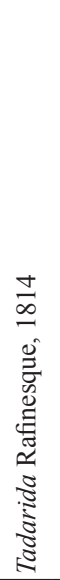 & 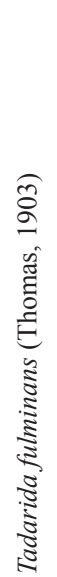 & 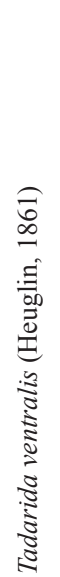 & 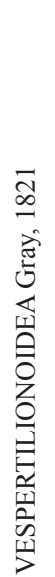 & 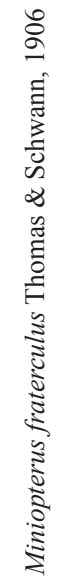 \\
\hline
\end{tabular}


European Journal of Taxonomy 382: 1-327 (2017)

\begin{tabular}{|c|c|c|c|c|c|c|c|c|c|c|c|c|c|c|c|c|c|c|c|c|c|c|c|c|c|c|c|}
\hline RMCA & $\stackrel{n}{=}$ & in & $\vec{\infty}$ & - & N & & $m$ & $m$ & - & , & $y$ & & $\infty$ & m & & ले & & $a$ & in & & & $F$ & $a$ & & & N & ণิ \\
\hline RBINS & $\simeq$ & - & $\stackrel{n}{n}$ & & & $m$ & & & & & & & & • & & $\stackrel{0}{-}$ & & - & m & & - & ส & & & & & $\sim$ \\
\hline \multicolumn{28}{|l|}{ OSU } \\
\hline \multicolumn{28}{|l|}{ NMZL } \\
\hline NMW & & & & & & & & & & & & & & & & & & & & & & & & & & & \\
\hline \multicolumn{28}{|l|}{ NMP } \\
\hline \multicolumn{28}{|l|}{ NMBA } \\
\hline MSB & & & & & & & & & & & 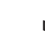 & & & & & 으 & & & & & & & - & & & & \\
\hline MNHN & & & $\sim$ & & & & & & & & & & & & - & & & & & & & & & & & & \\
\hline MHNG & 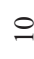 & & $m$ & & & & & & & & & 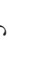 & t & n & 인 & $\dddot{n}$ & N & - & & & & m & & & & & \\
\hline $\mathrm{MCZ}$ & & & - & & & & & & & & & 0 & & & & & & & & & & & & & & & $\sim$ \\
\hline \multicolumn{28}{|l|}{$\mathrm{KU}$} \\
\hline INPBC & $m$ & - & & o & & & & & & & & e & $\sim$ & 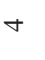 & & $=$ & & & & & & - & & & & & in \\
\hline HZM & & & - & & & & & & & & & - & & & & & & & - & & & & & & - & & \\
\hline \multicolumn{28}{|l|}{ HNHM } \\
\hline FMNH & $m$ & & & & & - & & & & & 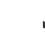 & r & & & & $N$ & & & & & & & & & m & & N \\
\hline \multicolumn{28}{|l|}{ DNSM } \\
\hline $\mathrm{CM}$ & & & & & & & & & & & & & & & & & & & & & - & & & - & - & & \\
\hline CAS & 0 & & n & & & & & & & & & & & & & & & & & & & & & & & & \\
\hline BMNH & 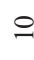 & - & & & & $\nabla$ & & - & & 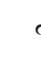 & $\mathrm{v}$ & 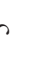 & & & & N & & & - & - & & 은 & a & & - & & \\
\hline AMNH & & & $\stackrel{i}{i}$ & & & & & & 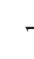 & 4 & r & 5 & & & & $N$ & m & r & N & & - & & & 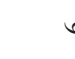 & $6-$ & & i \\
\hline 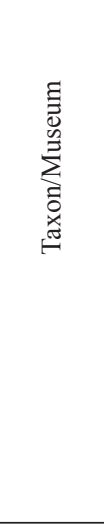 & 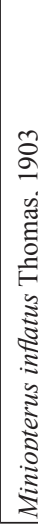 & 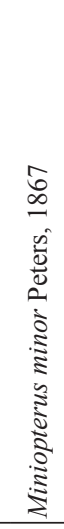 & 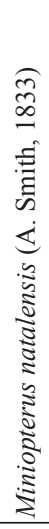 & 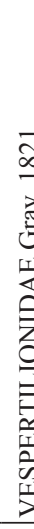 & $\begin{array}{l}\text { f } \\
\\
0 \\
0 \\
0\end{array}$ & 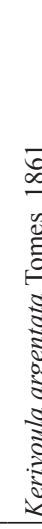 & $\begin{array}{l}\vdots \\
\vdots \\
0 \\
\vdots\end{array}$ & $\begin{array}{l}5 \\
0 \\
0 \\
0 \\
0 \\
0 \\
0 \\
0 \\
0 \\
0 \\
0 \\
0 \\
0 \\
0\end{array}$ & 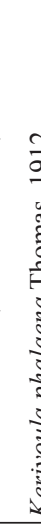 & 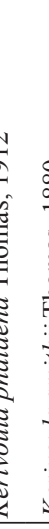 & 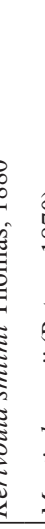 & & 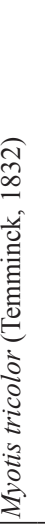 & 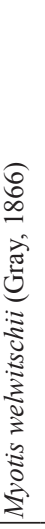 & 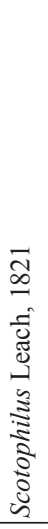 & 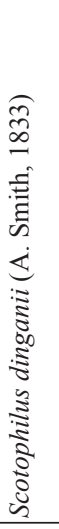 & 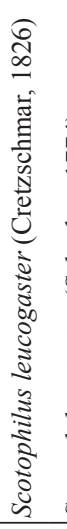 & 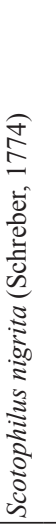 & 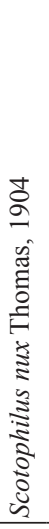 & 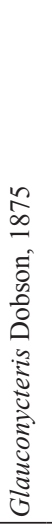 & 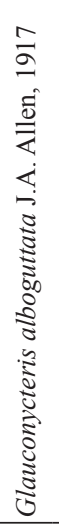 & 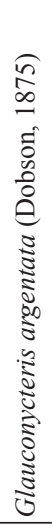 & 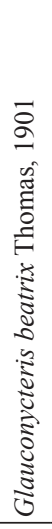 & 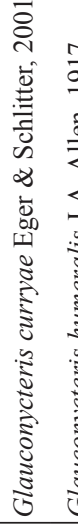 & 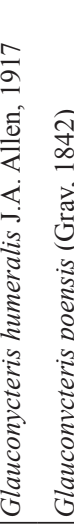 & 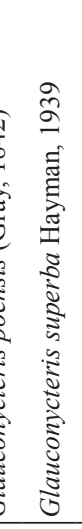 & 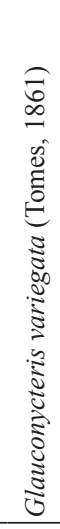 \\
\hline
\end{tabular}


VAN CAKENBERGHE V. et al., The bats of Congo, Rwanda and Burundi revisited

\begin{tabular}{|c|c|c|c|c|c|c|c|c|c|c|c|c|c|c|c|c|c|c|c|c|c|c|c|c|c|}
\hline RMCA & - & - & - & 0 & m & - & & & లా & & $\approx$ & is & $m$ & 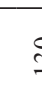 & & ल & & & & - & & $2-$ & - & 曽 & 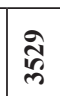 \\
\hline RBINS & & & & & + & & $r$ & & $\stackrel{\infty}{-}$ & & 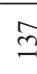 & ר & & 7 & & J & & & & & & & & 奇 & 离 \\
\hline OSU & & & & & & & & & & & & & & & & & & & & & & & & - & \\
\hline NMZL & & & & & & & & & & & & & & & & & & & & & & & & 4 & \\
\hline NMW & & & & & & & & & & & & & & & & & & & & & & & & 9 & \\
\hline NMP & & & & & & & & & & & - & & & & & & & & & & & & & - & \\
\hline NMBA & & & & & & & & & & & & & & & & & & & & & & & & 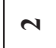 & \\
\hline MSB & & & & & & & & & & & - & & & . & & & & & & & & & & : & \\
\hline MNHN & & - & - & & & & & & & & $\stackrel{\bullet}{0}$ & & & & & & & & & & & & & : & \\
\hline MHNG & & & & & - & & - & & - & & $\ddot{m}$ & & & s & & & ナ & & & & & & & f & I \\
\hline $\mathrm{MCZ}$ & & & & & & & - & & & & $\stackrel{\infty}{\infty}$ & o & & 。 & & & $n$ & & & & & & & $\bar{\Xi}$ & \\
\hline $\mathrm{KU}$ & & & & & & & & & & & & & & & & & & & & & & & & 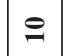 & \\
\hline INPBC & & & & & & & - & & o & & $\infty$ & ฯ & & 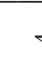 & & & - & & & & - & - & & 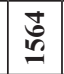 & $\frac{8}{2}$ \\
\hline HZM & & & & & $m$ & & & & & & f & - & & & & & & & & & & & & $\frac{m}{N}$ & \\
\hline HNHM & & & & & & & & & & & & & & & & & & & & & & & & $\sigma$ & \\
\hline FMNH & & - & & & & & & & & & $\bar{\sim}$ & c & & & & & in & & - & - & & & & 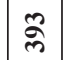 & \\
\hline DNSM & & & & & & & & & & & & & & & & & & & & & & & & $\infty$ & \\
\hline $\mathrm{CM}$ & & & & & & & & & & & $\simeq$ & $=$ & & s & & & & & & & & & & ते & \\
\hline CAS & & & & & & & & & & & & & & & & & & & & & & & & จิ & \\
\hline BMNH & - & & N & a & - & & & & - & & ণे & s & - & 7 & & " & & & & & & & & $\stackrel{\overbrace{}}{\%}$ & is \\
\hline AMNH & $\sim$ & a & & & + & & & - & - & N & $\infty$ & $\stackrel{\infty}{\infty}$ & - & 9 & & & & & & - & - & - & - & $\overrightarrow{\tilde{g}}$ & 8 \\
\hline 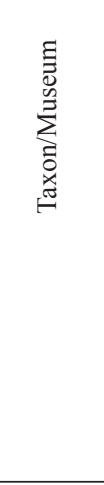 & 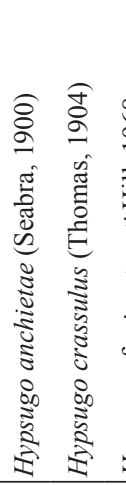 & 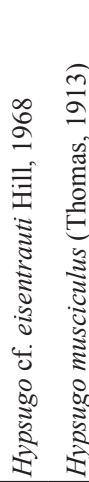 & 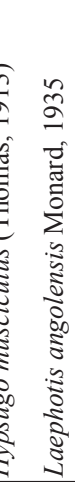 & 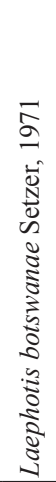 & 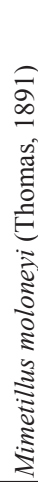 & 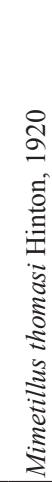 & 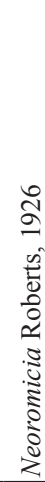 & 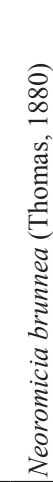 & 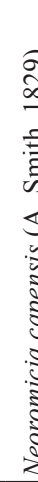 & 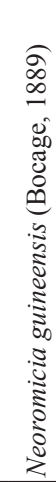 & 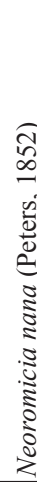 & 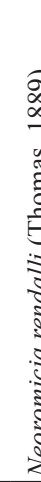 & 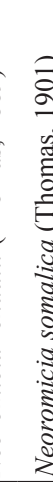 & 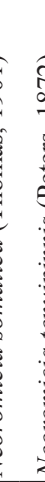 & 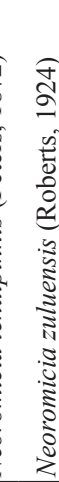 & 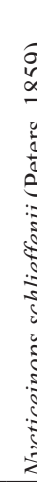 & $\frac{\infty}{\infty}$ & 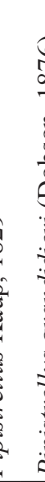 & 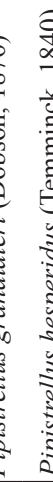 & 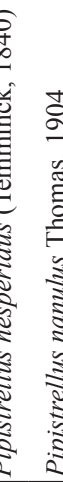 & 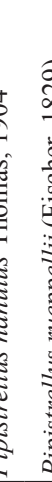 & 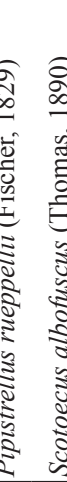 & 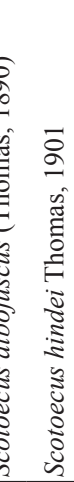 & 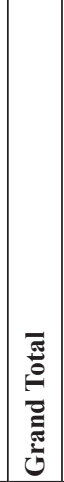 & 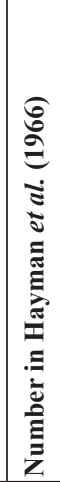 \\
\hline
\end{tabular}


European Journal of Taxonomy 382: 1-327 (2017)

\begin{tabular}{|c|c|c|c|c|c|c|c|c|c|c|c|c|c|c|c|c|c|c|c|c|c|c|c|c|c|c|c|}
\hline 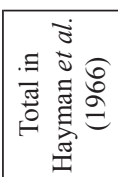 & & 点 & & & & m & in & & $=$ & 尺 & & & & $g$ g & & & & & & & 8 & & a & ナ & $\sigma$ & N & \\
\hline 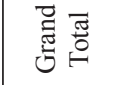 & ڤิ & i & $R$ & $\pi$ & - & $\stackrel{\varrho}{\Theta}$ & $\frac{\pi}{5}$ & in & $=$ & 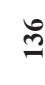 & F & & $\S$ & 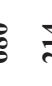 & 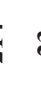 & 3 & a & : & $m$ & $\checkmark$ & हे & - & $\infty$ & in & $\vec{q}$ & 3 & $\sigma$ \\
\hline ZMZ & & & & & & & 2 & & & & & & & & & & & & & & & & & & & & \\
\hline ZMUC & & & & & & & & & & & & & & & & & & & & & & & & & & & \\
\hline ZMMU & & & & & & & & & & & & & & & & & & & & & & & & & & & \\
\hline ZMH & & & & & & & & & & & & & & & & & & & & & & & & & & & \\
\hline ZMB & & 9 & & & & - & & & & & & & & -0 & & & & & & & & & & & & & \\
\hline ZMA & & N & & & & & $\mathrm{N}$ & & & $m$ & & & & - & & & & m & & 6 & - & & n & & 6 & - & \\
\hline ZFMK & & & & & & & & & & & & & & & & & & & & & & & & & & & \\
\hline WNMU & & & & & & & & & & & & & & & & & & & & & & & & & & & \\
\hline USNM & & v & & & & & N & & & & & & & $\circ$. & & & & - & & - & 운 & & N & & & & \\
\hline UNIKIS & t & ナ & & & & & & & & & s & & & t & 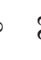 & 3 & & - & & ఏి & & & $\frac{\infty}{m}$ & & $m$ & 2 & \\
\hline TTU & & & & & & & & & & & & & & & & & & & & & & & & & & & \\
\hline $\mathrm{TM}$ & & $\stackrel{\infty}{\sim}$ & & & & & & & & & & & & o & & & & & & & 0 & & & & & & \\
\hline SMNS & & & & & & & $N$ & & & & & & & & & & & & & & & & & & & & \\
\hline SMF & & m & & & & & $\hat{m}$ & & & m & & & & 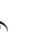 & & & - & 6 & & m & ㅇ & & ส & & $=$ & $n$ & \\
\hline RMNH & & m & & $m$ & & & - & & & & & & & 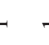 & & & & & & & & & & & 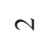 & - & \\
\hline 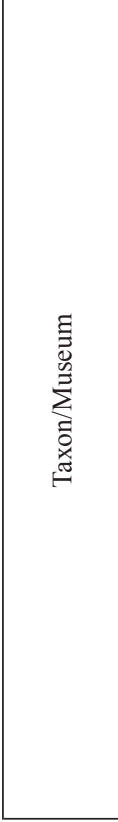 & 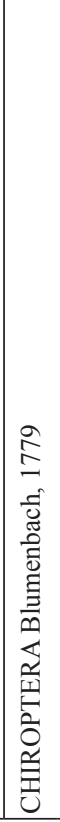 & 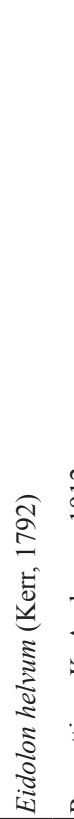 & 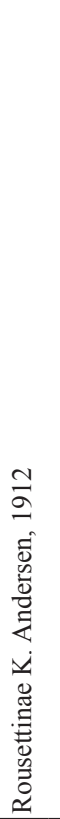 & 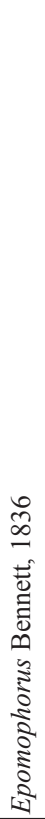 & 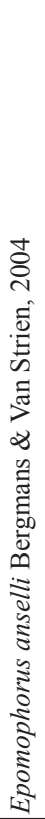 & 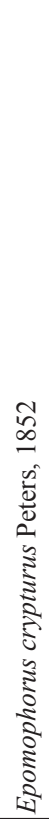 & 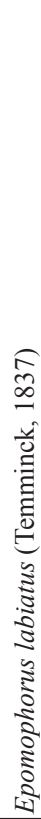 & 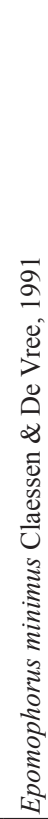 & 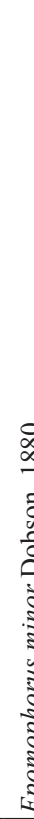 & 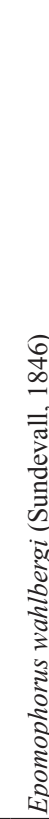 & 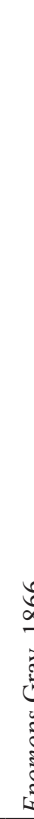 & & 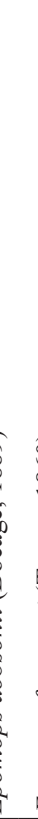 & 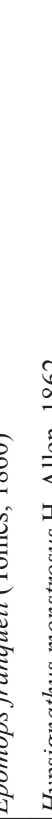 & : & है & 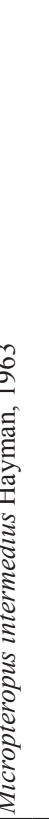 & 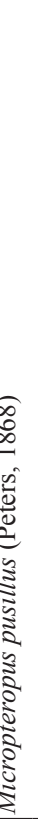 & 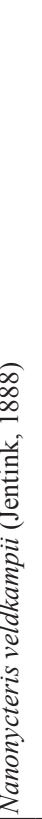 & 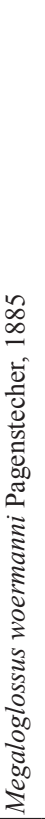 & 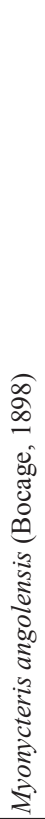 & 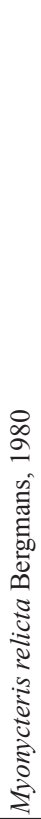 & 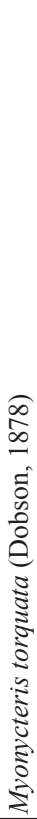 & 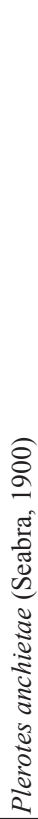 & 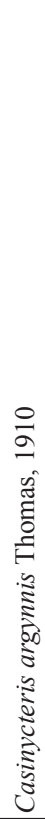 & 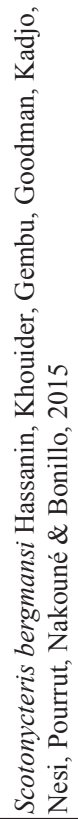 & 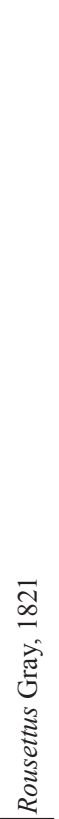 \\
\hline
\end{tabular}


VAN CAKENBERGHE V. et al., The bats of Congo, Rwanda and Burundi revisited

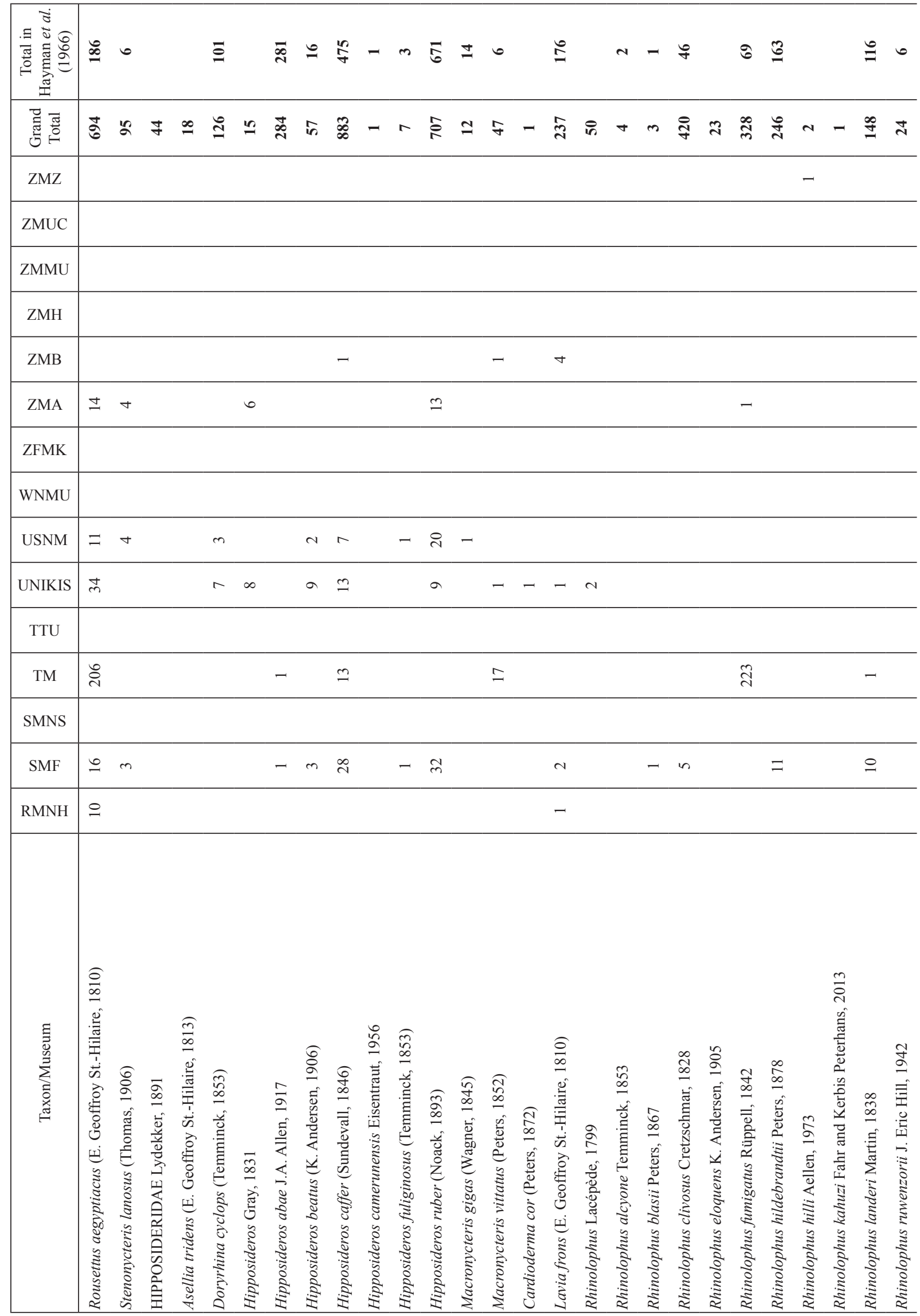


European Journal of Taxonomy 382: 1-327 (2017)

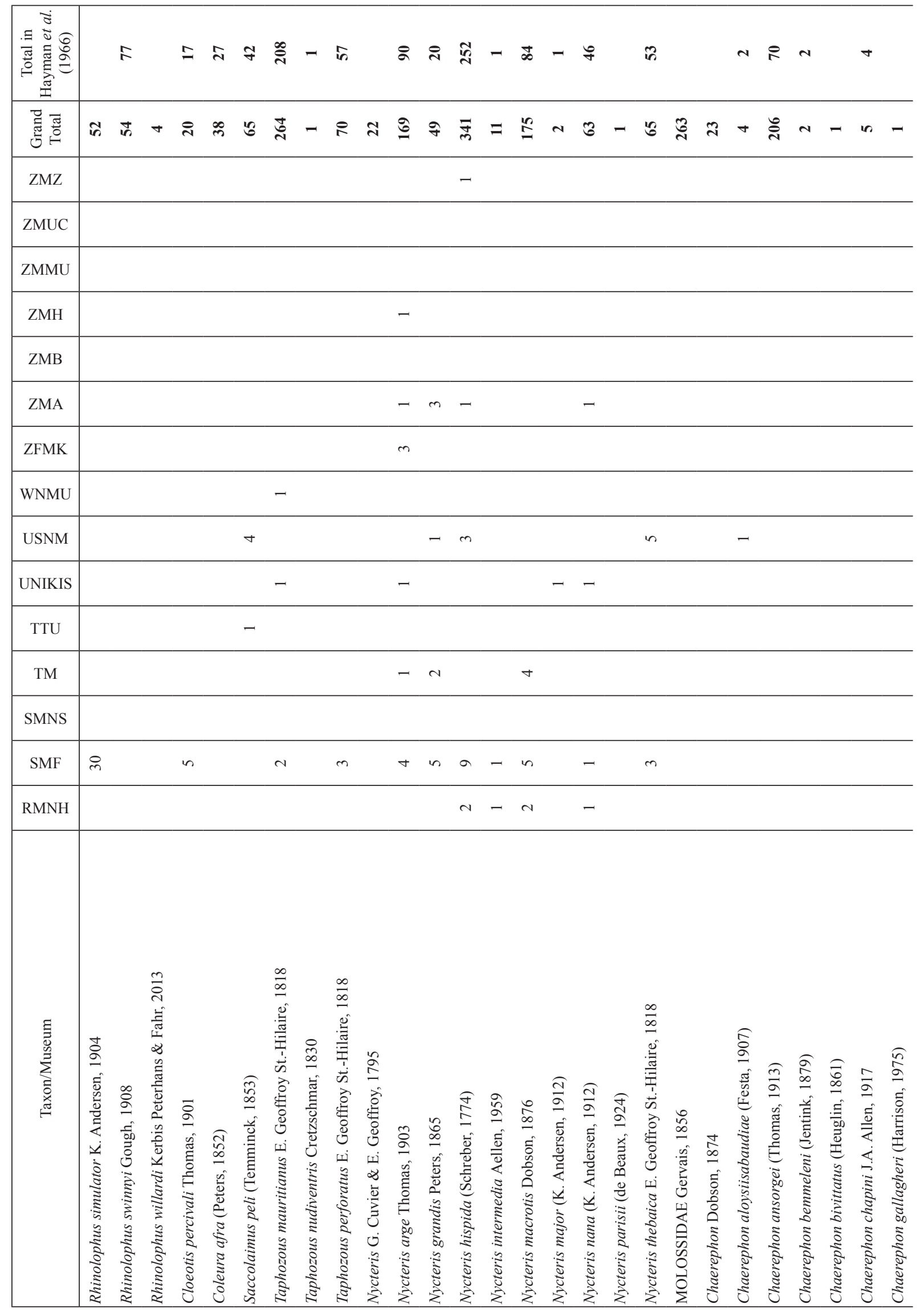


VAN CAKENBERGHE V. et al., The bats of Congo, Rwanda and Burundi revisited

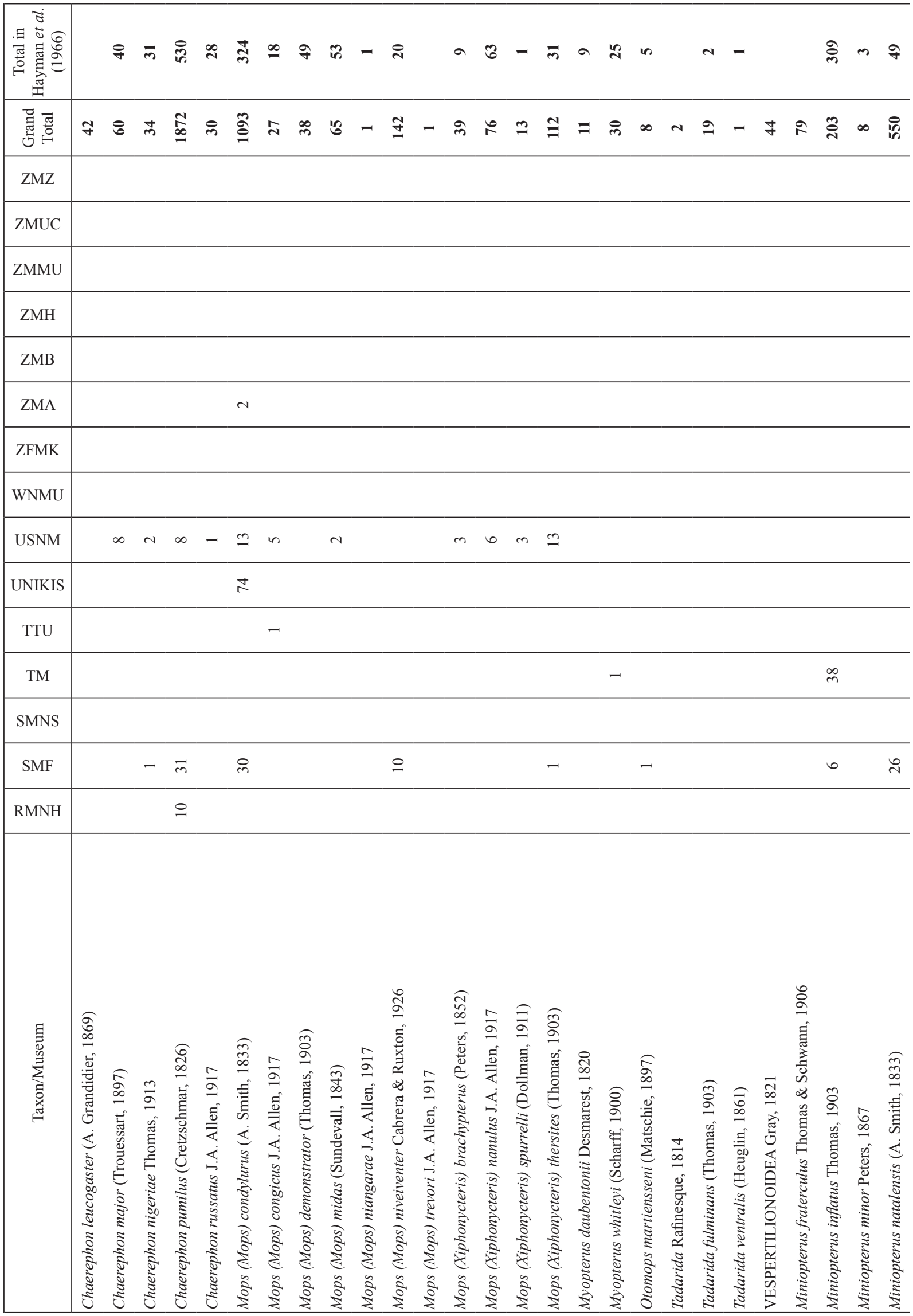


European Journal of Taxonomy 382: 1-327 (2017)

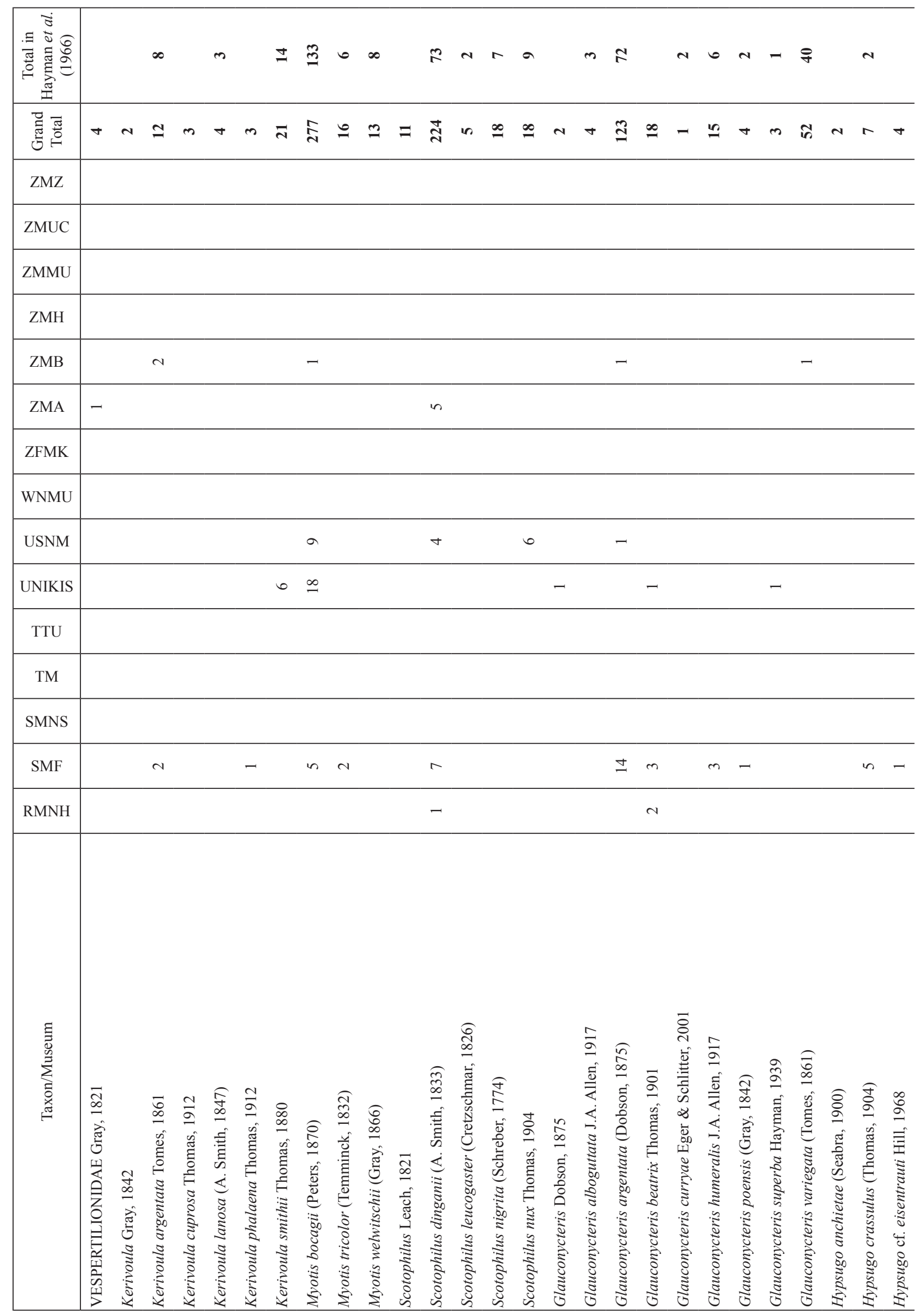


VAN CAKENBERGHE V. et al., The bats of Congo, Rwanda and Burundi revisited

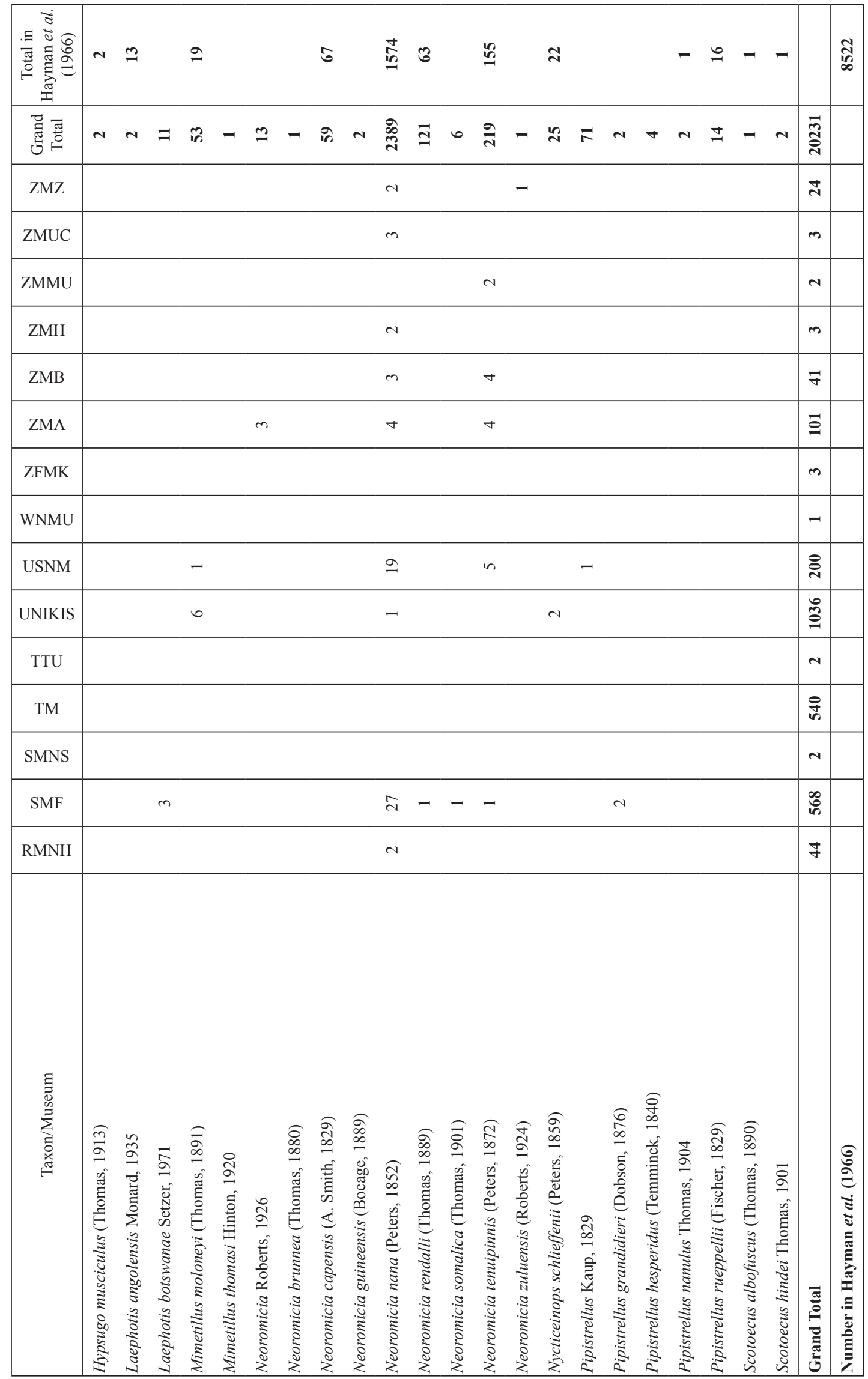




\section{Collecting localities for the bats from the CRB region}

In this section we provide a list of all the localities in the three countries from which bats were collected. For each of the localities, the name, country-province, coordinates and altitude are given. We also provide alternative spellings and linguistic variations (English, Dutch and French). The English version of the current name was chosen as "master locality" and the alternative versions are mentioned in brackets preceded by "see alias:". The aliases are followed by "see:" referring to the "master locality".

The coordinates for the localities were found and/or checked from various sources, e.g., "Global Gazetteer Version 2.3" (http://www.fallingrain.com/world/), NGA GEOnet Names Server (GNS) (http://geonames.nga.mil/gns/html/), literature data (including Hayman et al. 1966), (online) museum catalogs and specimen labels.

An overview of the current provincial names and abbreviations used in the gazetteer (source: http://www.statoids.com) and those used in Hayman et al. (1966) or historically is provided before the actual list of collecting localities.

\begin{tabular}{l|c|c|c}
\hline \multicolumn{1}{c|}{ Province name } & Abbreviation & Former province names & Abbreviation \\
\hline Burundi & BY & & BU \\
Bubanza & BB & & \\
Bujumbura Mairie & BM & & \\
Bujumbura Rural & BL & & \\
Bururi & BR & & \\
Cankuzo & CA & & \\
Cibitoke & CI & & \\
Gitega & GI & & \\
Karuzi & KR & & \\
Kayanza & KY & & \\
Kirundo & KI & & \\
Makamba & MA & & \\
Muramvya & MU & & \\
Muyinga & MY & & \\
Mwaro & MW & & \\
Ngozi & NG & & PO \\
Rumonge & RM & Kasai (Kasai-Occidental) & \\
Rutana & RT & Oratanga & \\
Ruyigi & RY & Orientale & \\
\hline Democratic Republic of the Congo & CG & PO \\
Bas-Uélé & BU & Orientale & \\
Équateur & ET & Équateur & \\
Haut-Katanga & HK & KS & \\
Haut-Lomami & HL & & \\
Haut-Uélé & HU & & \\
Ituri & IT & & \\
Kasaï & & & \\
\hline
\end{tabular}


VAN CAKENBERGHE V. et al., The bats of Congo, Rwanda and Burundi revisited

\begin{tabular}{l|c|c|c}
\hline \multicolumn{1}{c|}{ Province name } & Abbreviation & Former province names & Abbreviation \\
\hline Kasaï-Central & LL & Kasai (Kasaï-Occidental) & KS \\
Kasaï Oriental & KO & Kasai (Kasaï-Oriental) & KS \\
Kinshasa & KN & Léopoldville (Kinshasa City) & LE \\
Kongo Central & BC & Léopoldville (Bas-Congo) & LE \\
Kwango & KG & Léopoldville (Bandundu) & LE \\
Kwilu & KU & Léopoldville (Bandundu) & LE \\
Lomami & LM & Kasai (Kasaï-Oriental) & KS \\
Lualaba & LB & Katanga & KT \\
Mai-Ndombe & MA & Léopoldville (Bandundu) & LE \\
Maniema & MN & Katanga (Maniema) & KT \\
Mongala & MO & Équateur & EQ \\
Nord-Kivu & NK & Kivu (Nord-Kivu) & KI \\
Nord-Ubangi & NU & Équateur & EQ \\
Sankuru & SN & Kasai (Kasaï-Oriental) & KS \\
Sud-Kivu & SK & Kivu (Sud-Kivu) & KI \\
Sud-Ubangi & SU & Équateur & EQ \\
Tanganyika & TG & Katanga & KT \\
Tshopo & TO & Orientale & PO \\
Tshuapa & TP & Équateur & EQ \\
\hline Rwanda & RW & RU \\
Eastern & ES & Umutara, Kibungo, Kigali-Rural (part) & \\
Kigali City & KV & Kigali-Ville & \\
Northern & NO & Byumba, Kigali-Rural (part), Ruhengeri & \\
Southern & SU & Butare, Gikongoro, Gitarama & \\
Western & OU & Cyangugu, Gisenyi, Kibuye & \\
\hline & & & \\
\hline
\end{tabular}

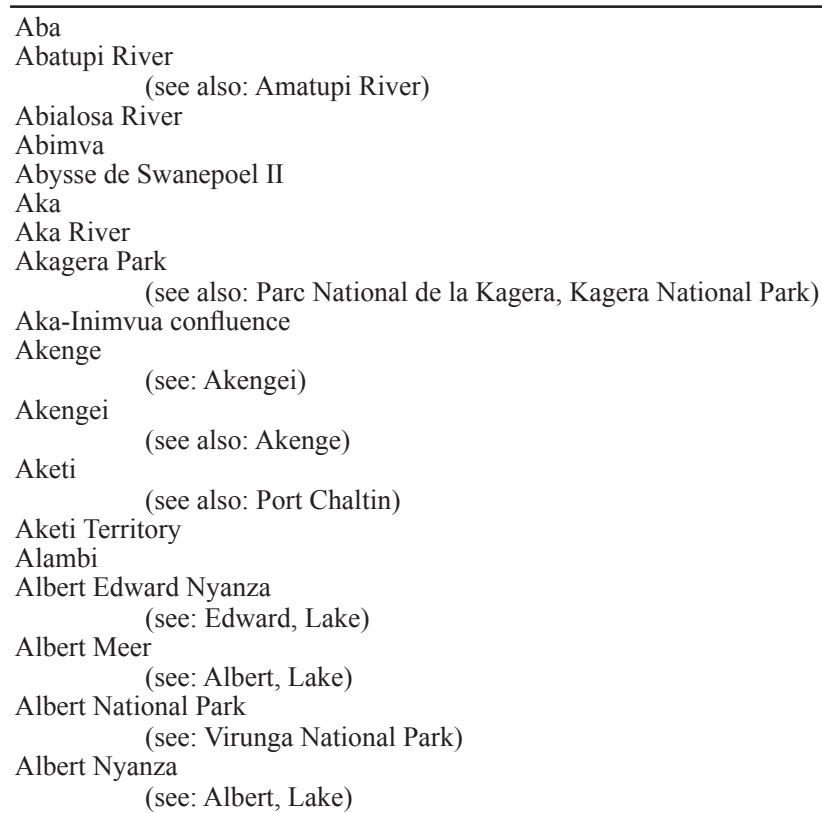

$\begin{array}{ccc}\text { CG-HU } & 03^{\circ} 52^{\prime} \mathrm{N}, 30^{\circ} 14^{\prime} \mathrm{E} & 890 \mathrm{~m} \\ \text { CG-NK } & 00^{\circ} 45^{\prime} \mathrm{N}, 29^{\circ} 49^{\prime} \mathrm{E} & 700 \mathrm{~m} \\ & & \\ \text { CG-NK } & 00^{\circ} 42^{\prime} \mathrm{N}, 29^{\circ} 41^{\prime} \mathrm{E} & 800 \mathrm{~m} \\ \text { CG-HU } & 03^{\circ} 09^{\prime} \mathrm{N}, 29^{\circ} 46^{\prime} \mathrm{E} & 1100 \mathrm{~m} \\ \text { CG-LB } & 10^{\circ} 51^{\prime} \mathrm{S}, 26^{\circ} 41^{\prime} \mathrm{E} & \text { ca } 1350 \mathrm{~m} \\ \text { CG-HU } & 04^{\circ} 35^{\prime} \mathrm{N}, 29^{\circ} 42^{\prime} \mathrm{E} & \\ \text { CG-HU } & 04^{\circ} 30^{\prime} \mathrm{N}, 29^{\circ} 40^{\prime} \mathrm{E} & \\ \text { RW-ES } & 01^{\circ} 30^{\prime} \mathrm{S}, 30^{\circ} 30^{\prime} \mathrm{E} & \\ \text { CG-HU } & 04^{\circ} 35^{\prime} \mathrm{N}, 29^{\circ} 42^{\prime} \mathrm{E} & \\ \text { CG-BU } & 02^{\circ} 56^{\prime} \mathrm{N}, 26^{\circ} 50^{\prime} \mathrm{E} & 654 \mathrm{~m} \\ \text { CG-BU } & 02^{\circ} 56^{\prime} \mathrm{N}, 26^{\circ} 50^{\prime} \mathrm{E} & 654 \mathrm{~m} \\ \text { CG-BU } & 02^{\circ} 50^{\prime} \mathrm{N}, 23^{\circ} 56^{\prime} \mathrm{E} & \mathrm{ca} 500 \mathrm{~m} \\ \text { CG-BU } & & \\ \text { CG-IT } & 02^{\circ} 07^{\prime} \mathrm{N}, 28^{\circ} 49^{\prime} \mathrm{E} & 913 \mathrm{~m} \\ \text { CG-NK } & \text { ca } 00^{\circ} 08^{\prime} \mathrm{S}, 29^{\circ} 35^{\prime} \mathrm{E} & \\ & & \\ \text { CG-IT } & \text { ca } 01^{\circ} 40^{\prime} \mathrm{N}, 30^{\circ} 40^{\prime} \mathrm{E} & \\ & & \\ \text { CG-NK } & 01^{\circ} 00^{\prime} \mathrm{S}, 29^{\circ} 15^{\prime} \mathrm{E} & \\ \text { CG-IT } & \text { ca } 01^{\circ} 40^{\prime} \mathrm{N}, 30^{\circ} 40^{\prime} \mathrm{E} & \end{array}$




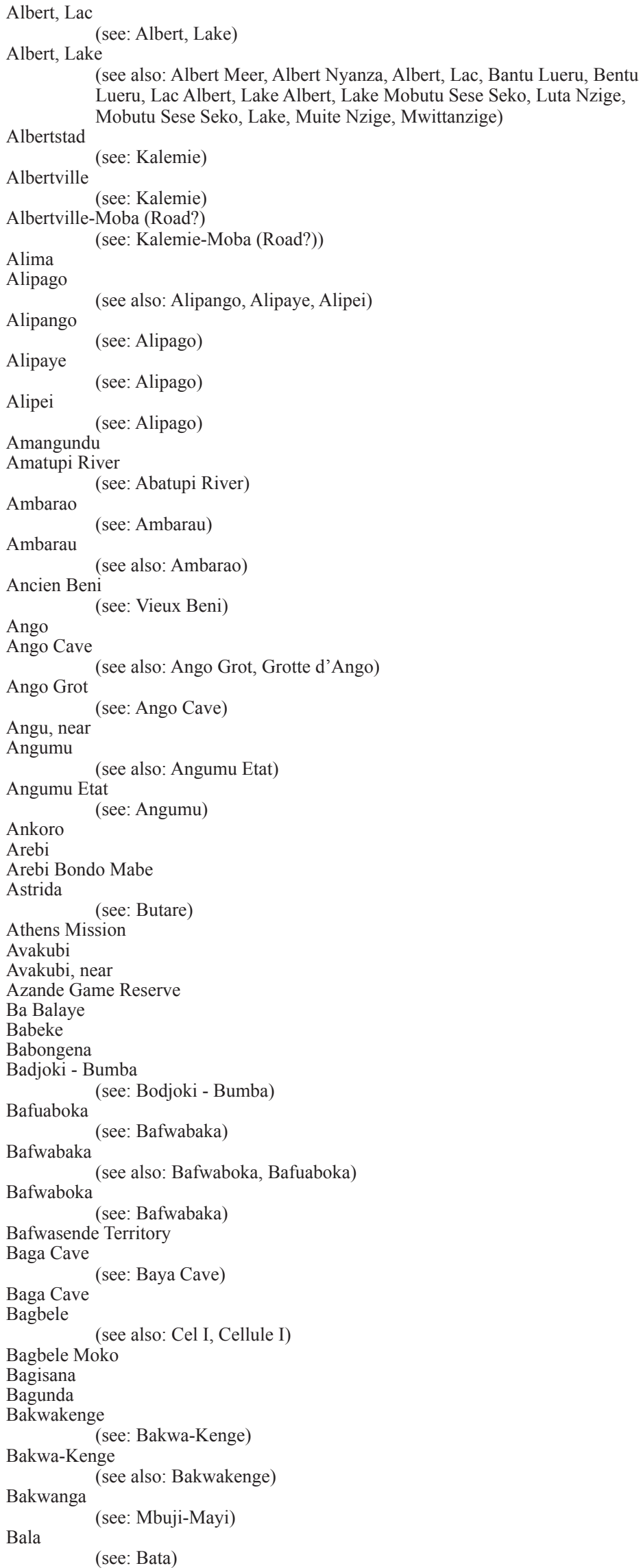

CG-IT

CG-IT $01^{\circ} 40^{\prime} \mathrm{N}, 30^{\circ} 40^{\prime} \mathrm{E}$ ca $01^{\circ} 40^{\prime} \mathrm{N}, 30^{\circ} 40^{\prime} \mathrm{E}$

CG-T

CG-TG

CG-TG

CG-NK

CG-BU

CG-BU

CG-BU

CG-BU

CG-NK

CG-NK

CG-HU

CG-HU

CG-NK

CG-BU

CG-BU

CG-BU

CG-BU

CG-TO

CG-TO

CG-TG

CG-HU

CG-HU

RW-SU

CG-KU

CG-TO

CG-TO

CG-HU

CG-KS

CG-IT

CG-TO

CG-MO

CG-HU

CG-HU

CG-HU

CG-TO

CG-HK $05^{\circ} 56^{\prime} \mathrm{S}, 29^{\circ} 12^{\prime} \mathrm{E}$

$763 \mathrm{~m}$

$05^{\circ} 56^{\prime} \mathrm{S}, 29^{\circ} 12^{\prime} \mathrm{E}$

$763 \mathrm{~m}$

$05^{\circ} 57^{\prime} \mathrm{S}, 29^{\circ} 01^{\prime} \mathrm{E}$

$$
00^{\circ} 35^{\prime} \mathrm{N}, 29^{\circ} 18^{\prime} \mathrm{E}
$$$$
03^{\circ} 34^{\prime} \mathrm{N}, 25^{\circ} 44^{\prime} \mathrm{E}
$$

$565 \mathrm{~m}$

$03^{\circ} 34^{\prime} \mathrm{N}, 25^{\circ} 44^{\prime} \mathrm{E}$

$565 \mathrm{~m}$

$03^{\circ} 34^{\prime} \mathrm{N}, 25^{\circ} 44^{\prime} \mathrm{E}$

$565 \mathrm{~m}$

$03^{\circ} 34^{\prime} \mathrm{N}, 25^{\circ} 44^{\prime} \mathrm{E}$

$565 \mathrm{~m}$

$00^{\circ} 45^{\prime} \mathrm{N}, 29^{\circ} 48^{\prime} \mathrm{E}$ $00^{\circ} 45^{\prime} \mathrm{N}, 29^{\circ} 49^{\prime} \mathrm{E}$

$700 \mathrm{~m}$

$700 \mathrm{~m}$

$03^{\circ} 10^{\prime} \mathrm{N}, 29^{\circ} 44^{\prime} \mathrm{E} \quad 867 \mathrm{~m}$

$03^{\circ} 10^{\prime} \mathrm{N}, 29^{\circ} 44^{\prime} \mathrm{E} \quad 867 \mathrm{~m}$

$00^{\circ} 26^{\prime} \mathrm{N}, 29^{\circ} 33^{\prime} \mathrm{E}$

$585 \mathrm{~m}$

$04^{\circ} 02^{\prime} \mathrm{N}, 25^{\circ} 52^{\prime} \mathrm{E}$ $04^{\circ} 02^{\prime} \mathrm{N}, 25^{\circ} 52^{\prime} \mathrm{E}$

$610 \mathrm{~m}$ $610 \mathrm{~m}$ $04^{\circ} 02^{\prime} \mathrm{N}, 25^{\circ} 52^{\prime} \mathrm{E}$

$610 \mathrm{~m}$

ca $03^{\circ} 33^{\prime} \mathrm{N}, 24^{\circ} 28^{\prime} \mathrm{E}$ $00^{\circ} 07^{\prime} \mathrm{S}, 27^{\circ} 42^{\prime} \mathrm{E}$

$859 \mathrm{~m}$

$00^{\circ} 07^{\prime} \mathrm{S}, 27^{\circ} 42^{\prime} \mathrm{E}$

$859 \mathrm{~m}$

$06^{\circ} 45^{\prime} \mathrm{S}, 26^{\circ} 57^{\prime} \mathrm{E}$ $02^{\circ} 52^{\prime} \mathrm{N}, 29^{\circ} 40^{\prime} \mathrm{E}$ $02^{\circ} 47^{\prime} \mathrm{N}, 29^{\circ} 35^{\prime} \mathrm{E}$

$02^{\circ} 36^{\prime} \mathrm{S}, 29^{\circ} 44^{\prime} \mathrm{E}$

$1733 \mathrm{~m}$

$05^{\circ} 02^{\prime} \mathrm{S}, 18^{\circ} 49^{\prime} \mathrm{E}$ $01^{\circ} 19^{\prime} \mathrm{N}, 27^{\circ} 33^{\prime} \mathrm{E}$ $01^{\circ} 20^{\prime} \mathrm{N}, 27^{\circ} 35^{\prime} \mathrm{E}$

$589 \mathrm{~m}$

$05^{\circ} 50^{\prime} \mathrm{S}, 22^{\circ} 30^{\prime} \mathrm{E}$ $01^{\circ} 24^{\prime} \mathrm{N}, 28^{\circ} 04^{\prime} \mathrm{E}$ $00^{\circ} 29^{\prime} \mathrm{N}, 25^{\circ} 42^{\prime} \mathrm{E}$ $02^{\circ} 59^{\prime} \mathrm{N}, 22^{\circ} 18^{\prime} \mathrm{E}$

$608 \mathrm{~m}$ $469 \mathrm{~m}$ $441 \mathrm{~m}$ $631 \mathrm{~m}$ $02^{\circ} 07^{\prime} \mathrm{N}, 27^{\circ} 40^{\prime} \mathrm{E}$

$631 \mathrm{~m}$ $02^{\circ} 07^{\prime} \mathrm{N}, 27^{\circ} 40^{\prime} \mathrm{E}$

$$
\text { ca } 11^{\circ} 40^{\prime} \mathrm{S}, 27^{\circ} 28^{\prime} \mathrm{E}
$$
$04^{\circ} 21^{\prime} \mathrm{N}, 29^{\circ} 17^{\prime} \mathrm{E}$

$1279 \mathrm{~m}$

CG-HU

$04^{\circ} 21^{\prime} \mathrm{N}, 29^{\circ} 17^{\prime} \mathrm{E}$ $04^{\circ} 35^{\prime} \mathrm{N}, 29^{\circ} 32^{\prime} \mathrm{E}$

CG-HU

CG-HU $04^{\circ} 04^{\prime} \mathrm{N}, 29^{\circ} 35^{\prime} \mathrm{E}$ $04^{\circ} 51^{\prime} \mathrm{S}, 22^{\circ} 04^{\prime} \mathrm{E}$

$742 \mathrm{~m}$

$742 \mathrm{~m}$

$990 \mathrm{~m}$ $461 \mathrm{~m}$

CG-KS $\quad 04^{\circ} 51^{\prime} \mathrm{S}, 22^{\circ} 04^{\prime} \mathrm{E} \quad 461 \mathrm{~m}$

CG-KO $\quad 06^{\circ} 09^{\prime} \mathrm{S}, 23^{\circ} 36^{\prime} \mathrm{E} \quad 549 \mathrm{~m}$

CG-KU $\quad 06^{\circ} 01^{\prime} \mathrm{S}, 19^{\circ} 40^{\prime} \mathrm{E} \quad 590 \mathrm{~m}$ 
Baliko

Bamangwa

Bamanya

(see: Bamungwa [River])

Bambesa

Bambu

Bamongwa

(see: Kilomines)

(see: Bamungwa [River])

Bamungwa [River]

Banalia

(see also: Bamongwa, Bamangwa)

Banana

(see also: Banane)

Banana - Boma Road, km 74, $25 \mathrm{~km}$ from coast in Forest

Banana Creek

Banana or Netonna

Banane

(see: Banana)

Bandaka

(see also: Cocquilhatville, Coquilhalville, Coquilhatstad, Coquilhatville, Wangata)

Bandimbu

Bandungu (former) Province

Banjan Caves

(see also: Banjan Grotten, Grottes de Banjan, Thysville, Cave, Thysville, Cave b 13 a)

Banjan Grotten (see: Banjan Caves)

Bantu Lueru (see: Albert, Lake)

Banza Nfinda Caves (see also: Banza Nfinda Grot, Grotte de Banza Nfinda)

Banza Nfinda Grot (see: Banza Nfinda Caves)

Barafinda Cave B 13

(see also: Barafinda Grotte B 13)

Barafinda Grotte B 13

(see: Barafinda Cave B 13)

Baraka

Baraka, $5 \mathrm{mi}[=8 \mathrm{~km}] \mathrm{N}$

Bas Congo (former) Province (see: Kongo Central Province)

Bas Congo Province (see: Kongo Central Province)

Bas Uele Province (see also: Lower Uele Province)

Basoko

Basoko Territory

Basongo

Bata (see also: Bala)

Batala's Village

(see also: Beni, $30 \mathrm{mi}$ [=48 km] W, Mambabwanga Hill)

Batoko (River) (see: Olongo-Nsongo [River]

Baudhuinville

$$
\text { (see: Moba) }
$$

Baudouinville (see: Moba)

Bauelende

Bauelendu

Baya carrière

Baya Cave (see: Baya quarry)

(see also: M'baya Cave, Grotte de M'baya, M'baya Grot, Grotte de Baya, Baga Cave)

Baya Grot (see: Baya quarry)

Baya quarry (see: Baya quarry)

Baye via Bondo

(see also: Baya carrière, Baya Cave, Baya Grot, Carrière Baya, Carrière Pierkat, Lubumbashi, 30 km S, Pierkat quarry)

Befale

Bela

\begin{tabular}{|c|c|c|}
\hline $\begin{array}{l}\text { CG-TP } \\
\text { CG-HU }\end{array}$ & $\begin{array}{l}00^{\circ} 16^{\prime} \mathrm{S}, 20^{\circ} 49^{\prime} \mathrm{E} \\
04^{\circ} 21^{\prime} \mathrm{N}, 29^{\circ} 27^{\prime} \mathrm{E}\end{array}$ & $358-388 \mathrm{~m}$ \\
\hline CG-ET & $00^{\circ} 01^{\prime} \mathrm{N}, 18^{\circ} 24^{\prime} \mathrm{E}$ & \\
\hline CG-BU & $03^{\circ} 28^{\prime} \mathrm{N}, 25^{\circ} 43^{\prime} \mathrm{E}$ & $614 \mathrm{~m}$ \\
\hline CG-IT & $01^{\circ} 48^{\prime} \mathrm{N}, 30^{\circ} 14^{\prime} \mathrm{E}$ & $1466 \mathrm{~m}$ \\
\hline CG-HU & $04^{\circ} 21^{\prime} \mathrm{N}, 29^{\circ} 27^{\prime} \mathrm{E}$ & \\
\hline CG-HU & $04^{\circ} 21^{\prime} \mathrm{N}, 29^{\circ} 27^{\prime} \mathrm{E}$ & \\
\hline CG-TO & $01^{\circ} 33^{\prime} \mathrm{N}, 25^{\circ} 20^{\prime} \mathrm{E}$ & $432 \mathrm{~m}$ \\
\hline CG-BC & $06^{\circ} 01^{\prime} \mathrm{S}, 12^{\circ} 24^{\prime} \mathrm{E}$ & $0 \mathrm{~m}$ \\
\hline CG-BC & $05^{\circ} 47^{\prime} \mathrm{S}, 12^{\circ} 31^{\prime} \mathrm{E}$ & \\
\hline CG-BC & $05^{\circ} 58^{\prime} \mathrm{S}, 12^{\circ} 27^{\prime} \mathrm{E}$ & \\
\hline CG-BC & ca $05^{\circ} 58^{\prime} \mathrm{S}, 12^{\circ} 27^{\prime} \mathrm{E}$ & \\
\hline CG-BC & $06^{\circ} 01^{\prime} \mathrm{S}, 12^{\circ} 24^{\prime} \mathrm{E}$ & $0 \mathrm{~m}$ \\
\hline CG-ET & $00^{\circ} 04^{\prime} \mathrm{N}, 18^{\circ} 16^{\prime} \mathrm{E}$ & $306 \mathrm{~m}$ \\
\hline $\begin{array}{c}\text { CG-KU } \\
\text { CG }\end{array}$ & $04^{\circ} 39^{\prime} \mathrm{S}, 18^{\circ} 58^{\prime} \mathrm{E}$ & \\
\hline CG-BC & $05^{\circ} 16^{\prime} \mathrm{S}, 14^{\circ} 53^{\prime} \mathrm{E}$ & \\
\hline CG-BC & $05^{\circ} 16^{\prime} \mathrm{S}, 14^{\circ} 53^{\prime} \mathrm{E}$ & \\
\hline CG-IT & ca $01^{\circ} 40^{\prime} \mathrm{N}, 30^{\circ} 40^{\prime} \mathrm{E}$ & \\
\hline CG-BC & $05^{\circ} 16^{\prime} \mathrm{S}, 14^{\circ} 52^{\prime} \mathrm{E}$ & ca $611 \mathrm{~m}$ \\
\hline CG-BC & $05^{\circ} 16^{\prime} \mathrm{S}, 14^{\circ} 52^{\prime} \mathrm{E}$ & ca $611 \mathrm{~m}$ \\
\hline CG-BC & $05^{\circ} 43^{\prime} \mathrm{S}, 14^{\circ} 28^{\prime} \mathrm{E}$ & \\
\hline CG-BC & $05^{\circ} 43^{\prime} \mathrm{S}, 14^{\circ} 28^{\prime} \mathrm{E}$ & \\
\hline CG-SK & $04^{\circ} 05^{\prime} \mathrm{S}, 29^{\circ} 05^{\prime} \mathrm{E}$ & $772 \mathrm{~m}$ \\
\hline CG-SK & ca $04^{\circ} 05^{\prime} \mathrm{S}, 29^{\circ} 05^{\prime} \mathrm{E}$ & \\
\hline & & \\
\hline CG & ca $05^{\circ} 00^{\prime} \mathrm{S}, 15^{\circ} 00^{\prime} \mathrm{E}$ & \\
\hline \multicolumn{3}{|l|}{$\mathrm{CG}$} \\
\hline CG-TO & $01^{\circ} 14^{\prime} \mathrm{N}, 23^{\circ} 36^{\prime} \mathrm{E}$ & $429 \mathrm{~m}$ \\
\hline CG-TO & $01^{\circ} 14^{\prime} \mathrm{N}, 23^{\circ} 36^{\prime} \mathrm{E}$ & \\
\hline CG-KS & $04^{\circ} 20^{\prime} \mathrm{S}, 20^{\circ} 24^{\prime} \mathrm{E}$ & $329 \mathrm{~m}$ \\
\hline CG-KU & $06^{\circ} 01^{\prime} \mathrm{S}, 19^{\circ} 40^{\prime} \mathrm{E}$ & $590 \mathrm{~m}$ \\
\hline CG-NK & $00^{\circ} 30^{\prime} \mathrm{N}, 29^{\circ} 15^{\prime} \mathrm{E}$ & $914 \mathrm{~m}$ \\
\hline CG-MA & $01^{\circ} 39^{\prime} \mathrm{S}, 18^{\circ} 05^{\prime} \mathrm{E}$ & \\
\hline CG-TG & $07^{\circ} 03^{\prime} \mathrm{S}, 29^{\circ} 47^{\prime} \mathrm{E}$ & $763 \mathrm{~m}$ \\
\hline CG-TG & $07^{\circ} 03^{\prime} \mathrm{S}, 29^{\circ} 47^{\prime} \mathrm{E}$ & $763 \mathrm{~m}$ \\
\hline CG-NK & $00^{\circ} 16^{\prime} \mathrm{N}, 29^{\circ} 36^{\prime} \mathrm{E}$ & $925 \mathrm{~m}$ \\
\hline CG-NK & $00^{\circ} 16^{\prime} \mathrm{N}, 29^{\circ} 36^{\prime} \mathrm{E}$ & $925 \mathrm{~m}$ \\
\hline CG-HK & $11^{\circ} 52^{\prime} \mathrm{S}, 27^{\circ} 27^{\prime} \mathrm{E}$ & $1279 \mathrm{~m}$ \\
\hline CG-HK & $11^{\circ} 52^{\prime} \mathrm{S}, 27^{\circ} 27^{\prime} \mathrm{E}$ & $1279 \mathrm{~m}$ \\
\hline CG-HK & $11^{\circ} 52^{\prime} \mathrm{S}, 27^{\circ} 27^{\prime} \mathrm{E}$ & $1279 \mathrm{~m}$ \\
\hline CG-HK & $11^{\circ} 52^{\prime} \mathrm{S}, 27^{\circ} 27^{\prime} \mathrm{E}$ & $1279 \mathrm{~m}$ \\
\hline CG-SN & $04^{\circ} 25^{\prime} \mathrm{N}, 23^{\circ} 04^{\prime} \mathrm{E}$ & \\
\hline CG-TP & $00^{\circ} 28^{\prime} \mathrm{N}, 20^{\circ} 58^{\prime} \mathrm{E}$ & $393 \mathrm{~m}$ \\
\hline CG-NK & $00^{\circ} 38^{\prime} \mathrm{N}, 29^{\circ} 14^{\prime} \mathrm{E}$ & $913 \mathrm{~m}$ \\
\hline
\end{tabular}




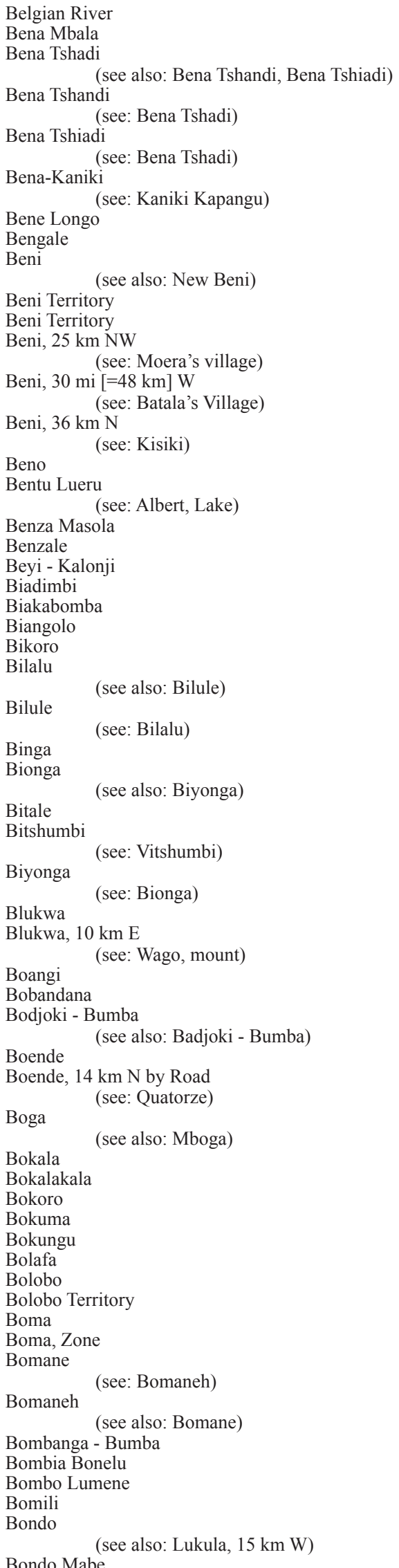

CG

CG-LM

CG-KS

CG-KS

CG-KS

CG-KO

$06^{\circ} 45^{\prime} \mathrm{S}, 23^{\circ} 31^{\prime} \mathrm{E}$

$805 \mathrm{~m}$

CG-KS $\quad 04^{\circ} 52^{\prime} \mathrm{S}, 21^{\circ} 40^{\prime} \mathrm{E}$

CG-KN $\quad 04^{\circ} 08^{\prime} \mathrm{S}, 16^{\circ} 40^{\prime} \mathrm{E}$

CG-NK $\quad 00^{\circ} 30^{\prime} \mathrm{N}, 29^{\circ} 28^{\prime} \mathrm{E}$

$853 \mathrm{~m}$

CG-NK $\quad 00^{\circ} 30^{\prime} \mathrm{N}, 29^{\circ} 28^{\prime} \mathrm{E}$

CG-NK $\quad 00^{\circ} 30^{\prime} \mathrm{N}, 29^{\circ} 28^{\prime} \mathrm{E}$

CG-NK ca $00^{\circ} 35^{\prime} \mathrm{N}, 29^{\circ} 25^{\prime} \mathrm{E} \quad 914 \mathrm{~m}$

CG-NK

$00^{\circ} 30^{\prime} \mathrm{N}, 29^{\circ} 15^{\prime} \mathrm{E}$

$914 \mathrm{~m}$

CG-NK

$00^{\circ} 45^{\prime} \mathrm{N}, 29^{\circ} 34^{\prime} \mathrm{E}$

$1177 \mathrm{~m}$

CG-MA $\quad 03^{\circ} 37^{\prime} \mathrm{S}, 17^{\circ} 48^{\prime} \mathrm{E}$

CG-IT ca $01^{\circ} 40^{\prime} \mathrm{N}, 30^{\circ} 40^{\prime} \mathrm{E}$

$281 \mathrm{~m}$

CG-BC $\quad 05^{\circ} 12^{\prime} \mathrm{S}, 13^{\circ} 00^{\prime} \mathrm{E}$

CG-MA $\quad 04^{\circ} 08^{\prime} \mathrm{S}, 16^{\circ} 40^{\prime} \mathrm{E}$

CG-KS $05^{\circ} 49^{\prime} \mathrm{S}, 22^{\circ} 30^{\prime} \mathrm{E}$

CG-HU $\quad 04^{\circ} 13^{\prime} \mathrm{N}, 29^{\circ} 21^{\prime} \mathrm{E}$

CG-KS $06^{\circ} 27^{\prime} \mathrm{S}, 21^{\circ} 02^{\prime} \mathrm{E}$

CG-NK $\quad 00^{\circ} 25^{\prime} \mathrm{N}, 29^{\circ} 45^{\prime} \mathrm{E}$

CG-ET $\quad 00^{\circ} 45^{\prime} \mathrm{S}, 18^{\circ} 07^{\prime} \mathrm{E}$

RW-ES $\quad 01^{\circ} 30^{\prime} \mathrm{S}, 30^{\circ} 18^{\prime} \mathrm{E}$

RW-ES

$01^{\circ} 30^{\prime} \mathrm{S}, 30^{\circ} 18^{\prime} \mathrm{E}$

CG-MA

CG-TP

$02^{\circ} 23^{\prime} \mathrm{N}, 20^{\circ} 30^{\prime} \mathrm{E}$

$00^{\circ} 34^{\prime} \mathrm{S}, 23^{\circ} 00^{\prime} \mathrm{E}$

CG-SK $\quad 02^{\circ} 13^{\prime} \mathrm{S}, 28^{\circ} 38^{\prime} \mathrm{E}$

CG-NK $\quad 00^{\circ} 41^{\prime} \mathrm{S}, 29^{\circ} 23^{\prime} \mathrm{E}$

CG-TP

$00^{\circ} 34^{\prime} \mathrm{S}, 23^{\circ} 00^{\prime} \mathrm{E}$

CG-IT

$01^{\circ} 45^{\prime} \mathrm{N}, 30^{\circ} 36^{\prime} \mathrm{E}$

CG-IT

$01^{\circ} 45^{\prime} \mathrm{N}, 30^{\circ} 40^{\prime} \mathrm{E}$

CG-TP $\quad 01^{\circ} 51^{\prime} \mathrm{S}, 20^{\circ} 56^{\prime} \mathrm{E}$

CG

$01^{\circ} 51^{\prime} \mathrm{S}, 20^{\circ} 56^{\prime} \mathrm{E}$

CG-MO $\quad 02^{\circ} 59^{\prime} \mathrm{N}, 22^{\circ} 18^{\prime} \mathrm{E}$

$441 \mathrm{~m}$

CG-TP $\quad 00^{\circ} 13^{\prime} \mathrm{S}, 20^{\circ} 52^{\prime} \mathrm{E} \quad 371 \mathrm{~m}$

CG-TP $\quad 00^{\circ} 10^{\prime} \mathrm{N}, 20^{\circ} 56^{\prime} \mathrm{E} \quad 326 \mathrm{~m}$

CG-IT $\quad 01^{\circ} 03^{\prime} \mathrm{N}, 29^{\circ} 56^{\prime} \mathrm{E} \quad 1562 \mathrm{~m}$

CG-MA $\quad 03^{\circ} 07^{\prime} \mathrm{S}, 17^{\circ} 02^{\prime} \mathrm{E} \quad 307 \mathrm{~m}$

$02^{\circ} 08^{\prime} \mathrm{S}, 16^{\circ} 22^{\prime} \mathrm{E}$

CG-MA $\quad 02^{\circ} 50^{\prime} \mathrm{S}, 18^{\circ} 23^{\prime} \mathrm{E} \quad 252 \mathrm{~m}$

CG-ET $\quad 00^{\circ} 06^{\prime} \mathrm{S}, 18^{\circ} 42^{\prime} \mathrm{E} \quad 340 \mathrm{~m}$

CG-TP $\quad 00^{\circ} 41^{\prime} \mathrm{S}, 22^{\circ} 19^{\prime} \mathrm{E} \quad 402 \mathrm{~m}$

CG-MO $\quad 02^{\circ} 08^{\prime} \mathrm{N}, 21^{\circ} 37^{\prime} \mathrm{E}$

CG-TP $\quad 02^{\circ} 09^{\prime} \mathrm{S}, 16^{\circ} 14^{\prime} \mathrm{E}$

CG-MA

CG-BC

CG-BC

$05^{\circ} 51^{\prime} \mathrm{S}, 13^{\circ} 03^{\prime} \mathrm{E}$ ca $05^{\circ} 51^{\prime} \mathrm{S}, 13^{\circ} 03^{\prime} \mathrm{E}$

$61 \mathrm{~m}$

CG-TO

$01^{\circ} 18^{\prime} \mathrm{N}, 23^{\circ} 47^{\prime} \mathrm{E}$

$429 \mathrm{~m}$

CG-TO

$01^{\circ} 18^{\prime} \mathrm{N}, 23^{\circ} 47^{\prime} \mathrm{E}$

$429 \mathrm{~m}$

CG-MO $\quad 02^{\circ} 48^{\prime} \mathrm{N}, 22^{\circ} 16^{\prime} \mathrm{E}$

CG-MO $\quad 02^{\circ} 35^{\prime} \mathrm{N}, 21^{\circ} 47^{\prime} \mathrm{E}$

CG-MA $\quad 03^{\circ} 58^{\prime} \mathrm{S}, 15^{\circ} 59^{\prime} \mathrm{E}$

CG-TO $\quad 01^{\circ} 40^{\prime} \mathrm{N}, 27^{\circ} 01^{\prime} \mathrm{E}$

CG-BC $\quad 05^{\circ} 24^{\prime} \mathrm{S}, 12^{\circ} 54^{\prime} \mathrm{E}$

$444 \mathrm{~m}$

Bondo Mabe

CG $02^{\circ} 36^{\prime} \mathrm{N}, 29^{\circ} 34^{\prime} \mathrm{E}$ 


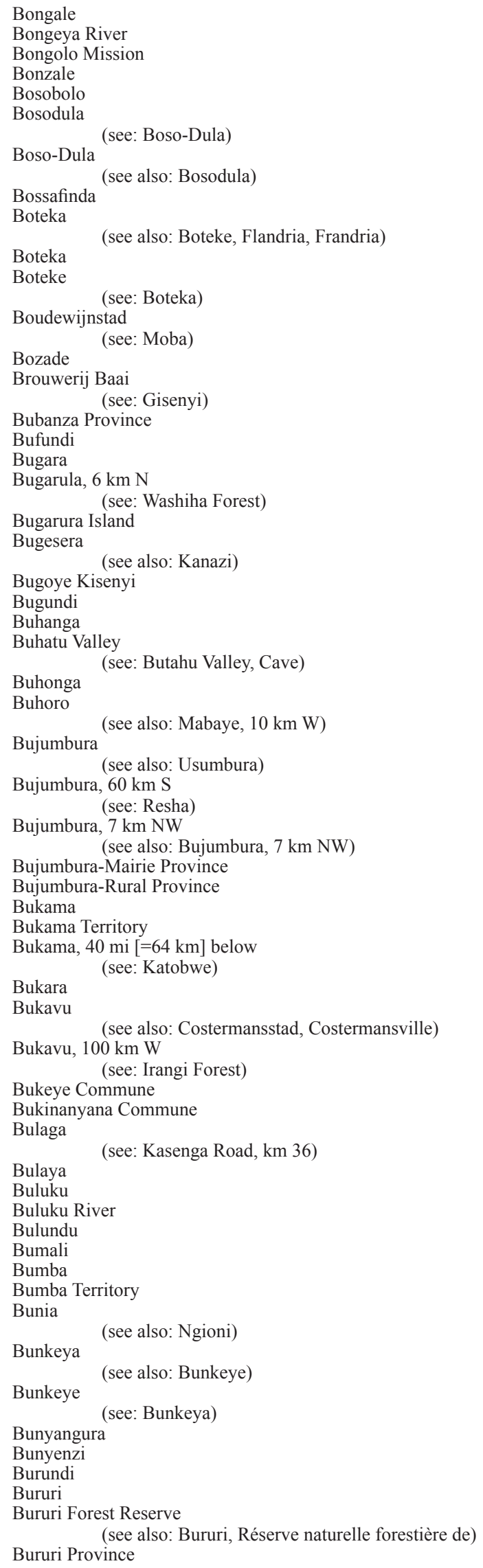

\begin{tabular}{|c|c|c|}
\hline CG-KU & $06^{\circ} 00^{\prime} \mathrm{S}, 18^{\circ} 41^{\prime} \mathrm{E}$ & \\
\hline CG-NK & & $1350 \mathrm{~m}$ \\
\hline $\mathrm{CG}-\mathrm{KG}$ & $06^{\circ} 36^{\prime} \mathrm{S}, 16^{\circ} 46^{\prime} \mathrm{E}$ & $383 \mathrm{~m}$ \\
\hline CG-SU & $02^{\circ} 58^{\prime} \mathrm{N}, 19^{\circ} 22^{\prime} \mathrm{E}$ & \\
\hline $\mathrm{CG}-\mathrm{NU}$ & $04^{\circ} 11^{\prime} \mathrm{N}, 19^{\circ} 54^{\prime} \mathrm{E}$ & $508 \mathrm{~m}$ \\
\hline CG-NU & $04^{\circ} 17^{\prime} \mathrm{N}, 20^{\circ} 29^{\prime} \mathrm{E}$ & $599 \mathrm{~m}$ \\
\hline CG-NU & $04^{\circ} 17^{\prime} \mathrm{N}, 20^{\circ} 29^{\prime} \mathrm{E}$ & $599 \mathrm{~m}$ \\
\hline CG-BC & ca $05^{\circ} 15^{\prime} \mathrm{S}, 14^{\circ} 52^{\prime} \mathrm{E}$ & \\
\hline CG-ET & $00^{\circ} 20^{\prime} \mathrm{S}, 19^{\circ} 06^{\prime} \mathrm{E}$ & $333 \mathrm{~m}$ \\
\hline CG-ET & $00^{\circ} 19^{\prime} \mathrm{S}, 19^{\circ} 07^{\prime} \mathrm{E}$ & $337 \mathrm{~m}$ \\
\hline CG-ET & $00^{\circ} 19^{\prime} \mathrm{S}, 19^{\circ} 07^{\prime} \mathrm{E}$ & $337 \mathrm{~m}$ \\
\hline CG-TG & $07^{\circ} 03^{\prime} \mathrm{S}, 29^{\circ} 47^{\prime} \mathrm{E}$ & $763 \mathrm{~m}$ \\
\hline CG-SU & $02^{\circ} 57^{\prime} \mathrm{N}, 19^{\circ} 18^{\prime} \mathrm{E}$ & \\
\hline RW-OU & $01^{\circ} 42^{\prime} \mathrm{S}, 29^{\circ} 15^{\prime} \mathrm{E}$ & $1481 \mathrm{~m}$ \\
\hline $\begin{array}{c}\text { BY } \\
\text { RW-NO } \\
\text { CG-NK } \\
\text { CG-SK }\end{array}$ & $\begin{array}{c}01^{\circ} 21^{\prime} \mathrm{S}, 29^{\circ} 51^{\prime} \mathrm{E} \\
01^{\circ} 10^{\prime} \mathrm{S}, 29^{\circ} 28^{\prime} \mathrm{E} \\
\text { ca } 02^{\circ} 00^{\prime} \mathrm{S}, 29^{\circ} 04^{\prime} \mathrm{E}\end{array}$ & $\begin{array}{l}1200 \mathrm{~m} \\
1500 \mathrm{~m}\end{array}$ \\
\hline $\begin{array}{l}\text { RW-OU } \\
\text { RW-ES }\end{array}$ & $\begin{array}{l}01^{\circ} 55^{\prime} \mathrm{S}, 29^{\circ} 16^{\prime} \mathrm{E} \\
02^{\circ} 09^{\prime} \mathrm{S}, 30^{\circ} 05^{\prime} \mathrm{E}\end{array}$ & $1432 \mathrm{~m}$ \\
\hline $\begin{array}{l}\text { RW-OU } \\
\text { RW-NO } \\
\text { BY-GI } \\
\text { CG-NK }\end{array}$ & $\begin{array}{c}01^{\circ} 42^{\prime} \mathrm{S}, 29^{\circ} 35^{\prime} \mathrm{E} \\
01^{\circ} 29^{\prime} \mathrm{S}, 29^{\circ} 55^{\prime} \mathrm{E} \\
03^{\circ} 39^{\prime} \mathrm{S}, 29^{\circ} 59^{\prime} \mathrm{E} \\
\text { ca } 00^{\circ} 20^{\prime} \mathrm{N}, 29^{\circ} 49^{\prime} \mathrm{E}\end{array}$ & $\begin{array}{l}1828 \mathrm{~m} \\
2286 \mathrm{~m}\end{array}$ \\
\hline $\begin{array}{l}\text { BY-BL } \\
\text { BY-CI }\end{array}$ & $\begin{array}{l}03^{\circ} 26^{\prime} \mathrm{S}, 29^{\circ} 25^{\prime} \mathrm{E} \\
02^{\circ} 42^{\prime} \mathrm{S}, 29^{\circ} 11^{\prime} \mathrm{E}\end{array}$ & $\begin{array}{l}1529 \mathrm{~m} \\
1453 \mathrm{~m}\end{array}$ \\
\hline BY-BM & $03^{\circ} 23^{\prime} \mathrm{S}, 29^{\circ} 22^{\prime} \mathrm{E}$ & $794 \mathrm{~m}$ \\
\hline BY-RU & $03^{\circ} 54^{\prime} \mathrm{S}, 29^{\circ} 24^{\prime} \mathrm{E}$ & \\
\hline BY-BM & $03^{\circ} 21^{\prime} \mathrm{S}, 29^{\circ} 18^{\prime} \mathrm{E}$ & \\
\hline $\begin{array}{l}\text { BY } \\
\text { BY }\end{array}$ & & \\
\hline CG-HL & $09^{\circ} 12^{\prime} \mathrm{S}, 25^{\circ} 51^{\prime} \mathrm{E}$ & $601 \mathrm{~m}$ \\
\hline CG-HL & $09^{\circ} 12^{\prime} \mathrm{S}, 25^{\circ} 51^{\prime} \mathrm{E}$ & \\
\hline CG-HL & $08^{\circ} 54^{\prime} \mathrm{S}, 26^{\circ} 04^{\prime} \mathrm{E}$ & $547 \mathrm{~m}$ \\
\hline BY-MA & $04^{\circ} 07^{\prime} \mathrm{S}, 29^{\circ} 47^{\prime} \mathrm{E}$ & \\
\hline CG-SK & $02^{\circ} 30^{\prime} \mathrm{S}, 28^{\circ} 52^{\prime} \mathrm{E}$ & $1498 \mathrm{~m}$ \\
\hline CG-SK & $01^{\circ} 54^{\prime} \mathrm{S}, 28^{\circ} 27^{\prime} \mathrm{E}$ & $\begin{array}{c}5000-5100 \\
\mathrm{ft}\end{array}$ \\
\hline BY-MV & $03^{\circ} 12^{\prime} \mathrm{S}, 29^{\circ} 38^{\prime} \mathrm{E}$ & $1875 \mathrm{~m}$ \\
\hline BY-CI & $02^{\circ} 53^{\prime} \mathrm{S}, 29^{\circ} 20^{\prime} \mathrm{E}$ & $1543 \mathrm{~m}$ \\
\hline CG-HK & $11^{\circ} 37^{\prime} \mathrm{S}, 27^{\circ} 40^{\prime} \mathrm{E}$ & \\
\hline CG-NK & $01^{\circ} 12^{\prime} \mathrm{S}, 29^{\circ} 27^{\prime} \mathrm{E}$ & $1160 \mathrm{~m}$ \\
\hline CG-HU & $04^{\circ} 33^{\prime} \mathrm{N}, 29^{\circ} 49^{\prime} \mathrm{E}$ & \\
\hline CG-HU & $04^{\circ} 33^{\prime} \mathrm{N}, 29^{\circ} 49^{\prime} \mathrm{E}$ & \\
\hline CG-SK & ca $02^{\circ} 42^{\prime} \mathrm{S}, 27^{\circ} 20^{\prime} \mathrm{E}$ & \\
\hline CG-NK & $00^{\circ} 21^{\prime} \mathrm{N}, 29^{\circ} 45^{\prime} \mathrm{E}$ & $1300 \mathrm{~m}$ \\
\hline CG-MO & $02^{\circ} 11^{\prime} \mathrm{N}, 22^{\circ} 28^{\prime} \mathrm{E}$ & $419 \mathrm{~m}$ \\
\hline CG-MO & $02^{\circ} 11^{\prime} \mathrm{N}, 22^{\circ} 28^{\prime} \mathrm{E}$ & \\
\hline CG-IT & $01^{\circ} 34^{\prime} \mathrm{N}, 30^{\circ} 15^{\prime} \mathrm{E}$ & $1332 \mathrm{~m}$ \\
\hline CG-HK & $10^{\circ} 24^{\prime} \mathrm{S}, 26^{\circ} 58^{\prime} \mathrm{E}$ & $1008 \mathrm{~m}$ \\
\hline CG-HK & $10^{\circ} 24^{\prime} \mathrm{S}, 26^{\circ} 58^{\prime} \mathrm{E}$ & $1008 \mathrm{~m}$ \\
\hline CG-NK & $01^{\circ} 09^{\prime} \mathrm{S}, 29^{\circ} 26^{\prime} \mathrm{E}$ & $1200 \mathrm{~m}$ \\
\hline $\begin{array}{c}\text { CG-NK } \\
\text { BY }\end{array}$ & $01^{\circ} 10^{\prime} \mathrm{S}, 29^{\circ} 27^{\prime} \mathrm{E}$ & $1225 \mathrm{~m}$ \\
\hline BY-BI & $03^{\circ} 57^{\prime} \mathrm{S}, 29^{\circ} 37^{\prime} \mathrm{E}$ & $1836 \mathrm{~m}$ \\
\hline BY-BI & $03^{\circ} 56^{\prime} \mathrm{S}, 29^{\circ} 36^{\prime} \mathrm{E}$ & \\
\hline
\end{tabular}

BY 


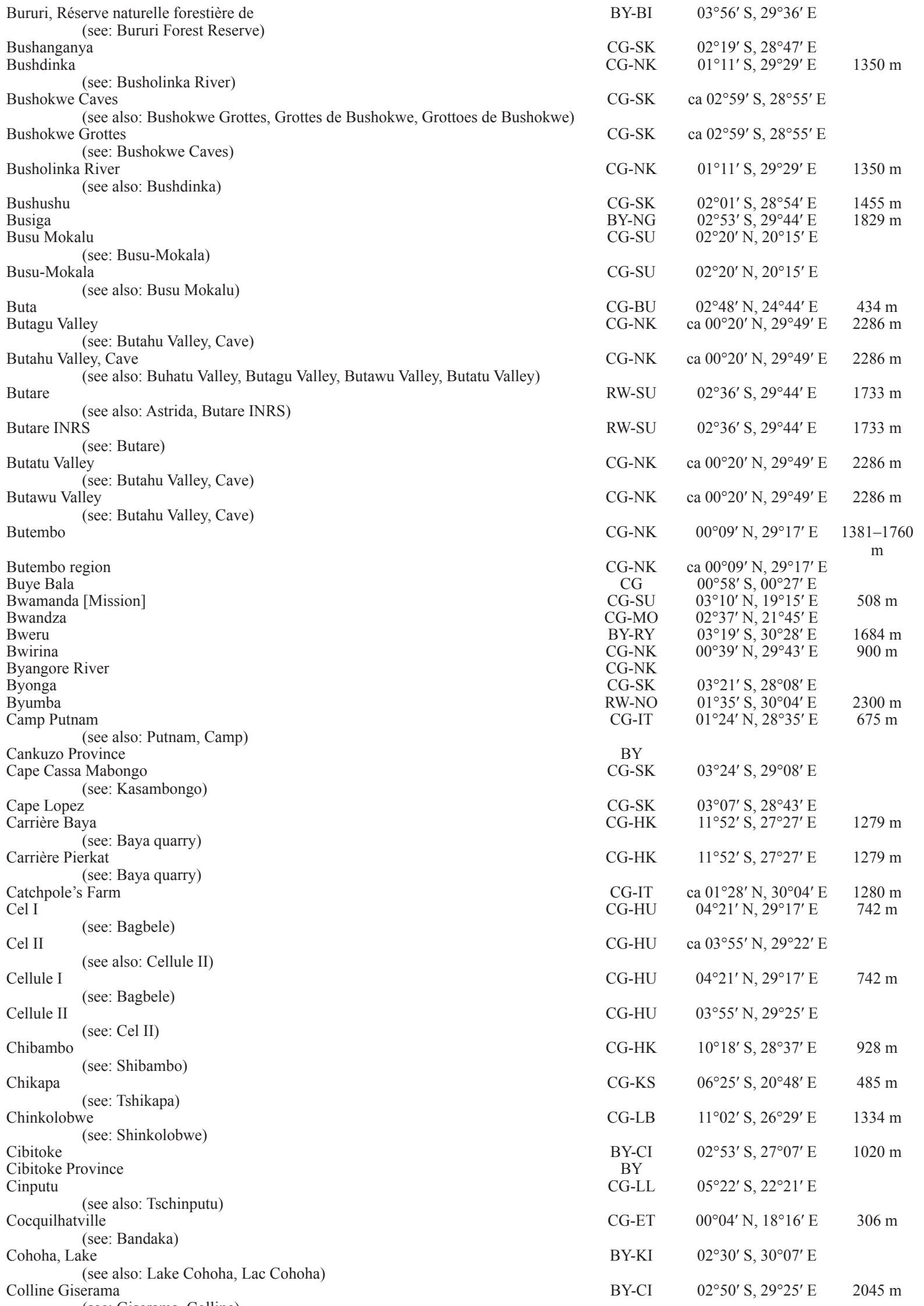




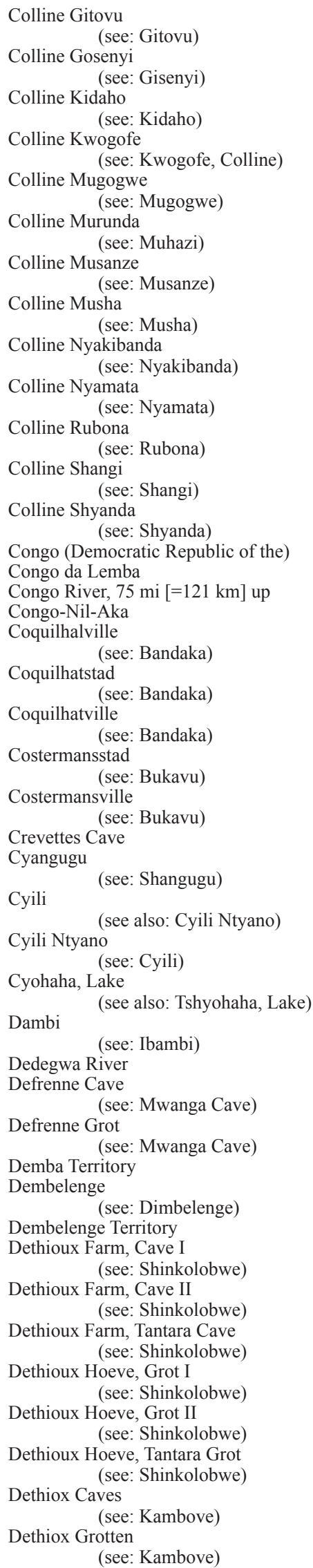

Colline Gitovu (see: Gitovu)

Colline Gosenyi

(see: Gisenyi)
Colline Kidaho (see: Kidaho)

Colline Kwogofe (see: Kwogofe, Colline)

Colline Mugogwe (see: Mugogwe)

Colline Murunda (see: Muhazi)

Colline Musanze (see: Musanze)

Colline Musha (see: Musha)

Colline Nyakibanda (see: Nyakibanda)

Colline Nyamata (see: Nyamata)

Colline Rubona (see: Rubona)

Colline Shangi (see: Shangi)

Colline Shyanda (see: Shyanda)

Congo (Democratic Republic of the)

Congo da Lemba

Congo River, $75 \mathrm{mi}$ [=121 km] up

Congo-Nil-Aka

Coquilhalville

Coquilhatstad see: Bandaka)

(see: Bandaka)
Coquilhatville (see: Bandaka)

Costermansstad (see: Bukavu)

Costermansville$$
\text { (see: Bukavu) }
$$

Crevettes Cave

Cyangugu

Cyili (see: Shangugu)

(see also: Cyili Ntyano) no

(see: Cyili)

Cyohaha, Lake (see also: Tshyohaha, Lake)

Dambi (see: Ibambi)

Dedegwa River

Defrenne Cave

Defrenne Grot (see: Mwanga Cave) (see: Mwanga Cave)

Demba Territory

Dembelenge (see: Dimbelenge)

Dembelenge Territory

Dethioux Farm, Cave I (see: Shinkolobwe)

Dethioux Farm, Cave II (see: Shinkolobwe)

Dethioux Farm, Tantara Cave (see: Shinkolobwe)

Dethioux Hoeve, Grot I (see: Shinkolobwe)

Dethioux Hoeve, Grot II (see: Shinkolobwe)

Dethioux Hoeve, Tantara Grot

Dethiox Caves (see: Shinkolobwe)

Dethiox Grotten see: Kambove)

(see: Kambove)

RW-SU

$02^{\circ} 19^{\prime} \mathrm{S}, 29^{\circ} 09^{\prime} \mathrm{E}$

$1649 \mathrm{~m}$

RW-OU

$01^{\circ} 42^{\prime} \mathrm{S}, 29^{\circ} 15^{\prime} \mathrm{E}$

$1481 \mathrm{~m}$

RW-NO

$01^{\circ} 22^{\prime} \mathrm{S}, 29^{\circ} 45^{\prime} \mathrm{E}$

1944 m

BY-CI

$02^{\circ} 50^{\prime} \mathrm{S}, 29^{\circ} 25^{\prime} \mathrm{E}$

RW-SU

$02^{\circ} 31^{\prime} \mathrm{S}, 29^{\circ} 49^{\prime} \mathrm{E}$

RW-OU

$01^{\circ} 54^{\prime} \mathrm{S}, 29^{\circ} 22^{\prime} \mathrm{E}$

$1711 \mathrm{~m}$

RW-NO

$01^{\circ} 29^{\prime}$ S, $29^{\circ} 36^{\prime} \mathrm{E}$

RW-SU

$02^{\circ} 32^{\prime} \mathrm{S}, 29^{\circ} 52^{\prime} \mathrm{E}$

RW-SU

$02^{\circ} 38^{\prime} \mathrm{S}, 29^{\circ} 41^{\prime} \mathrm{E}$

$2067 \mathrm{~m}$

RW-ES

$02^{\circ} 10^{\prime} \mathrm{S}, 30^{\circ} 06^{\prime} \mathrm{E}$

RW-SU

$02^{\circ} 19^{\prime} \mathrm{S}, 29^{\circ} 56^{\prime} \mathrm{E}$

$1346 \mathrm{~m}$

RW-OU

$02^{\circ} 24^{\prime} \mathrm{S}, 29^{\circ} 00^{\prime} \mathrm{E}$

RW-SU

$02^{\circ} 33^{\prime} \mathrm{S}, 29^{\circ} 48^{\prime} \mathrm{E}$

$1646 \mathrm{~m}$

CG

CG-KU

CG-BC

CG-HU

CG-ET

$05^{\circ} 42^{\prime} \mathrm{S}, 13^{\circ} 40^{\prime} \mathrm{E}$

$04^{\circ} 34^{\prime} \mathrm{N}, 29^{\circ} 45^{\prime} \mathrm{E}$ $00^{\circ} 04^{\prime} \mathrm{N}, 18^{\circ} 16^{\prime} \mathrm{E}$

$306 \mathrm{~m}$

CG-ET

$00^{\circ} 04^{\prime} \mathrm{N}, 18^{\circ} 16^{\prime} \mathrm{E}$

$306 \mathrm{~m}$

CG-ET

$00^{\circ} 04^{\prime} \mathrm{N}, 18^{\circ} 16^{\prime} \mathrm{E}$

$306 \mathrm{~m}$

CG-SK

$02^{\circ} 30^{\prime} \mathrm{S}, 28^{\circ} 52^{\prime} \mathrm{E}$

$1498 \mathrm{~m}$

CG-SK

$02^{\circ} 30^{\prime} \mathrm{S}, 28^{\circ} 52^{\prime} \mathrm{E}$

$1498 \mathrm{~m}$

CG-HK

RW-OU

$10^{\circ} 58^{\prime} \mathrm{S}, 26^{\circ} 47^{\prime} \mathrm{E}$

$02^{\circ} 29^{\prime} \mathrm{S}, 28^{\circ} 54^{\prime} \mathrm{E}$

$1537 \mathrm{~m}$

RW-SU

$02^{\circ} 30^{\prime} \mathrm{S}, 29^{\circ} 46^{\prime} \mathrm{E}$

RW-SU

$02^{\circ} 30^{\prime} \mathrm{S}, 29^{\circ} 46^{\prime} \mathrm{E}$

RW-ES

$02^{\circ} 15^{\prime} \mathrm{S}, 30^{\circ} 00^{\prime} \mathrm{E}$

CG-HU

$02^{\circ} 22^{\prime} \mathrm{N}, 27^{\circ} 37^{\prime} \mathrm{E}$

$687 \mathrm{~m}$

CG-HU

$04^{\circ} 35^{\prime} \mathrm{N}, 29^{\circ} 43^{\prime} \mathrm{E}$

CG-HK $\quad 10^{\circ} 58^{\prime} \mathrm{S}, 26^{\circ} 39^{\prime} \mathrm{E}$

CG-HK

$10^{\circ} 58^{\prime} \mathrm{S}, 26^{\circ} 39^{\prime} \mathrm{E}$

CG-LL $\quad 05^{\circ} 30^{\prime} \mathrm{S}, 22^{\circ} 16^{\prime} \mathrm{E}$

CG-LL $\quad 05^{\circ} 33^{\prime} \mathrm{S}, 23^{\circ} 07^{\prime} \mathrm{E}$

$682 \mathrm{~m}$

CG-LL

CG-LB

$11^{\circ} 02^{\prime} \mathrm{S}, 26^{\circ} 29^{\prime} \mathrm{E}$

$1334 \mathrm{~m}$

CG-LB

$11^{\circ} 02^{\prime} \mathrm{S}, 26^{\circ} 29^{\prime} \mathrm{E}$

$1334 \mathrm{~m}$

CG-LB

$11^{\circ} 02^{\prime} \mathrm{S}, 26^{\circ} 29^{\prime} \mathrm{E}$

$1334 \mathrm{~m}$

CG-LB

$11^{\circ} 02^{\prime} \mathrm{S}, 26^{\circ} 29^{\prime} \mathrm{E}$

$1334 \mathrm{~m}$

CG-LB

$11^{\circ} 02^{\prime} \mathrm{S}, 26^{\circ} 29^{\prime} \mathrm{E}$

$1334 \mathrm{~m}$

CG-LB

$11^{\circ} 02^{\prime} \mathrm{S}, 26^{\circ} 29^{\prime} \mathrm{E}$

$1334 \mathrm{~m}$

CG-LB

$10^{\circ} 50^{\prime} \mathrm{S}, 26^{\circ} 39^{\prime} \mathrm{E}$

CG-LB

$10^{\circ} 50^{\prime} \mathrm{S}, 26^{\circ} 39^{\prime} \mathrm{E}$ 
Detioux Cave

(see: Kambove)

Digba

Dika

Dikul River

Dikuluwe Cave

see: Dikulwe Cave)

Dikuluwe Grot

see: Dikulwe Cave)

(see: Dikulwe Cave)

Dikulwe Cave

(see also: Dikuluwe Cave, Dikuluwe Grot, Grotte de Dikuluwe, Dikul River)

Dikulwe River

Dilolo

(see also: Kayombe, Dilolo Station, Dilolo, Poste)

Dilolo Station

(see: Dilolo)

Dilolo Territory

Dilolo, Poste

(see: Dilolo)

Dimbelenge

Djabir

(see also: Dembelenge)

Djamba

Djambala (Hill)

Djeka

Djelube River, lowe

Djelube River, middle

Djemba

(see also: Djomba)

Djomba

Djugu Bos see: Djemba)

Djugu Forest see: Djugu Forest)

(see also: Djugu Bos, Djugu, forêt de, Forêt de Djugu)

Djugu Territory

Djugu, forêt de

Djuma River, upper see: Djugu Forest)

Djumma River, middle

Dramba

Drodo

(see: Drodro)

Drodro

(see also: Drodo)

Dubulu

Duma

Dungu

Durba

Eala

East Kasai (former) Province

(see: Kasai Oriental (former) Province)

East Kasai Province

(see: Kasai Oriental Province)

Eastern Congo

Eastern Prefecture

(see also: Kibungo (former) Prefecture)

Edouard, Lac

(see: Edward, Lake)

Edward Meer (see: Edward, Lake)

Edward, Lake

(see also: Lake Edward, Lac Edouard, Edouard, Lac, Albert Edward Nyanza, Edward Meer, Eward Nyanza, Lake Idi Amin Dada, Idi Amin Dada, Lake,

Eidolon land Lac Idi Amine, Lake Rutanzige, Rutanzige, Lake)

Elila

Elisabetha

Elisabethstad

see also: Elizabetha) (see: Lubumbashi)
Elisabethville

Elisabethville

Elizabetha

see: Lubumbashi)

(see: Elisabetha)
CG-LB

$10^{\circ} 50^{\prime} \mathrm{S}, 26^{\circ} 39^{\prime} \mathrm{E}$

CG-BU

CG-HU

CG-LB

汪

$04^{\circ} 22^{\prime} \mathrm{N}, 27^{\circ} 40^{\prime} \mathrm{E}$

$10^{\circ} 45^{\prime} \mathrm{S}, 25^{\circ} 22^{\prime} \mathrm{E}$

CG-LB

$10^{\circ} 45^{\prime} \mathrm{S}, 25^{\circ} 22^{\prime} \mathrm{E}$

CG-LB

$10^{\circ} 45^{\prime} \mathrm{S}, 25^{\circ} 22^{\prime} \mathrm{E}$

CG-LB

$10^{\circ} 45^{\prime} \mathrm{S}, 25^{\circ} 22^{\prime} \mathrm{E}$

CG-LB $\quad 10^{\circ} 45^{\prime} \mathrm{S}, 25^{\circ} 22^{\prime} \mathrm{E}$

CG-LB $\quad 10^{\circ} 28^{\prime} \mathrm{S}, 22^{\circ} 28^{\prime} \mathrm{E}$

$1069 \mathrm{~m}$

CG-LB

$10^{\circ} 28^{\prime} \mathrm{S}, 22^{\circ} 28^{\prime} \mathrm{E}$

$1069 \mathrm{~m}$

CG-LB

CG-LB

$10^{\circ} 28^{\prime} \mathrm{S}, 22^{\circ} 28^{\prime} \mathrm{E}$

$1069 \mathrm{~m}$

CG-LL

$05^{\circ} 33^{\prime} \mathrm{S}, 23^{\circ} 07^{\prime} \mathrm{E}$

$682 \mathrm{~m}$

CG-TO

CG-BU

CG-BC

$\mathrm{CG}-\mathrm{SN}$

CG-NK

CG-NK

CG-NK

CG-NK

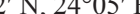

$02^{\circ} 53^{\prime} \mathrm{N}, 24^{\circ} 02^{\prime} \mathrm{E}$

$04^{\circ} 42^{\prime} \mathrm{S}, 13^{\circ} 44^{\prime} \mathrm{E}$

$02^{\circ} 47^{\prime} \mathrm{S}, 23^{\circ} 55^{\prime} \mathrm{E}$

$00^{\circ} 35^{\prime} \mathrm{N}, 29^{\circ} 40^{\prime} \mathrm{E}$

$00^{\circ} 31^{\prime} \mathrm{N}, 29^{\circ} 44^{\prime} \mathrm{E}$

$01^{\circ} 20^{\prime} \mathrm{S}, 29^{\circ} 34^{\prime} \mathrm{E}$

$01^{\circ} 20^{\prime} \mathrm{S}, 29^{\circ} 34^{\prime} \mathrm{E}$

$437 \mathrm{~m}$

$419 \mathrm{~m}$

$850 \mathrm{~m}$

$1100 \mathrm{~m}$

$1500 \mathrm{~m}$

$1500 \mathrm{~m}$

CG-IT

$01^{\circ} 55^{\prime} \mathrm{N}, 30^{\circ} 30^{\prime} \mathrm{E}$

$1530 \mathrm{~m}$

CG-IT

$01^{\circ} 55^{\prime} \mathrm{N}, 30^{\circ} 30^{\prime} \mathrm{E}$

$1530 \mathrm{~m}$

CG-IT

CG-IT

$01^{\circ} 55^{\prime} \mathrm{N}, 30^{\circ} 30^{\prime} \mathrm{E}$

$01^{\circ} 55^{\prime} \mathrm{N}, 30^{\circ} 30^{\prime} \mathrm{E}$

$01^{\circ} 55^{\prime} \mathrm{N}, 30^{\circ} 30^{\prime} \mathrm{E}$

$1530 \mathrm{~m}$

CG-NK

CG-NK

CG-HU

$00^{\circ} 38^{\prime} \mathrm{N}, 29^{\circ} 34^{\prime} \mathrm{E}$

$00^{\circ} 42^{\prime} \mathrm{N}, 29^{\circ} 41^{\prime} \mathrm{E}$

$03^{\circ} 40^{\prime} \mathrm{N}, 30^{\circ} 19^{\prime} \mathrm{E}$

$01^{\circ} 46^{\prime} \mathrm{N}, 30^{\circ} 32^{\prime} \mathrm{E}$

$850 \mathrm{~m}$

$800 \mathrm{~m}$

$877 \mathrm{~m}$

$1854 \mathrm{~m}$

CG-IT

$01^{\circ} 46^{\prime} \mathrm{N}, 30^{\circ} 32^{\prime} \mathrm{E}$

$1854 \mathrm{~m}$

CG-NU

CG-SU

$\mathrm{CG}-\mathrm{HU}$

$04^{\circ} 18^{\prime} \mathrm{N}, 20^{\circ} 16^{\prime} \mathrm{E}$

$613 \mathrm{~m}$ $03^{\circ} 53^{\prime} \mathrm{N}, 18^{\circ} 41^{\prime} \mathrm{E}$ $03^{\circ} 37^{\prime} \mathrm{N}, 28^{\circ} 34^{\prime} \mathrm{E}$ $03^{\circ} 08^{\prime} \mathrm{N}, 29^{\circ} 35^{\prime} \mathrm{E}$

CG-HU

CG-ET

$\mathrm{CG}$

CG

CG

RW

CG-NK

ca $00^{\circ} 08^{\prime} \mathrm{S}, 29^{\circ} 35^{\prime} \mathrm{E}$

CG-NK

ca $00^{\circ} 08^{\prime} \mathrm{S}, 29^{\circ} 35^{\prime} \mathrm{E}$

CG-NK

ca $00^{\circ} 08^{\prime} \mathrm{S}, 29^{\circ} 35^{\prime} \mathrm{E}$

CG-HK

CG-TO

CG-TO

ca $10^{\circ} 30^{\prime} \mathrm{S}, 27^{\circ} 30^{\prime} \mathrm{E}$

$02^{\circ} 43^{\prime} \mathrm{S}, 25^{\circ} 53^{\prime} \mathrm{E}$

$01^{\circ} 09^{\prime} \mathrm{N}, 23^{\circ} 36^{\prime} \mathrm{E}$

$486 \mathrm{~m}$

$428 \mathrm{~m}$

CG-HK $\quad 11^{\circ} 40^{\prime} \mathrm{S}, 27^{\circ} 26^{\prime} \mathrm{E} \quad 1209 \mathrm{~m}$

CG-HK $\quad 11^{\circ} 40^{\prime} \mathrm{S}, 27^{\circ} 26^{\prime} \mathrm{E} \quad 1209 \mathrm{~m}$

CG-TO $\quad 01^{\circ} 09^{\prime} \mathrm{N}, 23^{\circ} 36^{\prime} \mathrm{E} \quad 428 \mathrm{~m}$ 


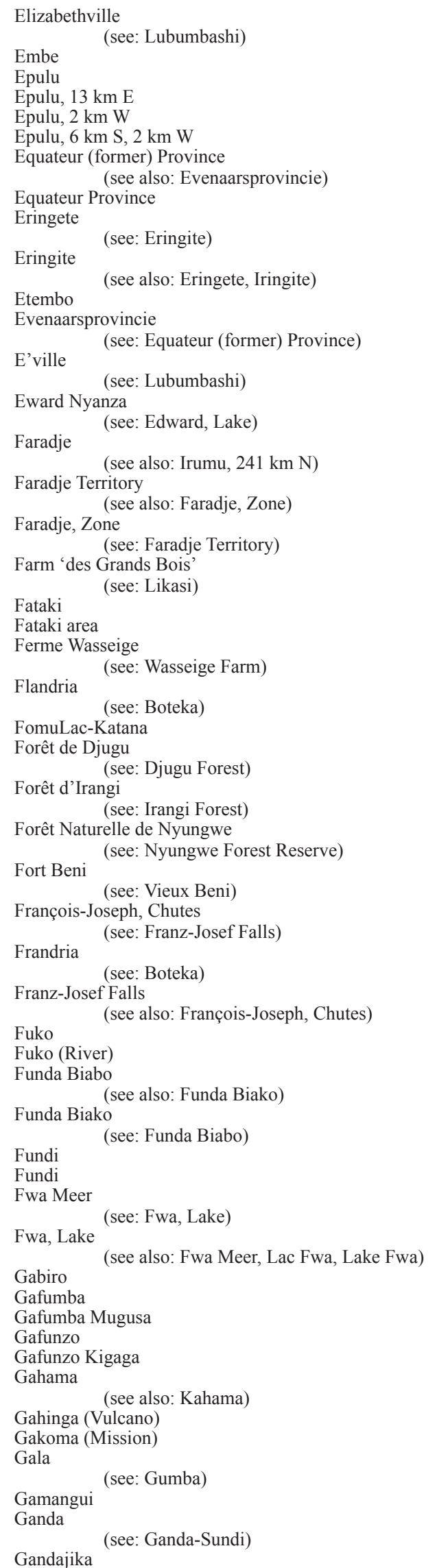

\begin{tabular}{|c|c|c|}
\hline CG-HK & $11^{\circ} 40^{\prime} \mathrm{S}, 27^{\circ} 28^{\prime} \mathrm{E}$ & $1208 \mathrm{~m}$ \\
\hline CG-HU & $04^{\circ} 40^{\prime} \mathrm{N}, 29^{\circ} 31^{\prime} \mathrm{E}$ & \\
\hline CG-IT & $01^{\circ} 24^{\prime} \mathrm{N}, 28^{\circ} 35^{\prime} \mathrm{E}$ & $750 \mathrm{~m}$ \\
\hline CG-IT & ca $01^{\circ} 24^{\prime} \mathrm{N}, 28^{\circ} 35^{\prime} \mathrm{E}$ & $750 \mathrm{~m}$ \\
\hline CG-IT & ca $01^{\circ} 24^{\prime} \mathrm{N}, 28^{\circ} 35^{\prime} \mathrm{E}$ & $750 \mathrm{~m}$ \\
\hline $\begin{array}{c}\text { CG-IT } \\
\text { CG }\end{array}$ & ca $01^{\circ} 24^{\prime} \mathrm{N}, 28^{\circ} 35^{\prime} \mathrm{E}$ & $750 \mathrm{~m}$ \\
\hline $\begin{array}{c}\mathrm{CG} \\
\mathrm{CG}-\mathrm{NK}\end{array}$ & & \\
\hline & $00^{\circ} 51^{\prime} \mathrm{N}, 29^{\circ} 37 \mathrm{E}$ & $1270 \mathrm{~m}$ \\
\hline CG-NK & $00^{\circ} 51^{\prime} \mathrm{N}, 29^{\circ} 37^{\prime} \mathrm{E}$ & $1270 \mathrm{~m}$ \\
\hline $\begin{array}{c}\text { CG-IT } \\
\text { CG }\end{array}$ & $00^{\circ} 55^{\prime} \mathrm{N}, 28^{\circ} 39^{\prime} \mathrm{E}$ & $832 \mathrm{~m}$ \\
\hline CG-HK & $11^{\circ} 40^{\prime} \mathrm{S}, 27^{\circ} 26^{\prime} \mathrm{E}$ & $1209 \mathrm{~m}$ \\
\hline CG-NK & ca $00^{\circ} 08^{\prime} \mathrm{S}, 29^{\circ} 35^{\prime} \mathrm{E}$ & \\
\hline CG-HU & $03^{\circ} 44^{\prime} \mathrm{N}, 29^{\circ} 43^{\prime} \mathrm{E}$ & $775 \mathrm{~m}$ \\
\hline CG-HU & $03^{\circ} 44^{\prime} \mathrm{N}, 29^{\circ} 43^{\prime} \mathrm{E}$ & \\
\hline CG-HU & $03^{\circ} 44^{\prime} \mathrm{N}, 29^{\circ} 43^{\prime} \mathrm{E}$ & \\
\hline CG-HK & $10^{\circ} 59^{\prime} \mathrm{S}, 26^{\circ} 44^{\prime} \mathrm{E}$ & $1318 \mathrm{~m}$ \\
\hline CG-IT & $02^{\circ} 00^{\prime} \mathrm{N}, 30^{\circ} 34^{\prime} \mathrm{E}$ & $1611 \mathrm{~m}$ \\
\hline CG-IT & $02^{\circ} 01^{\prime} \mathrm{N}, 30^{\circ} 38^{\prime} \mathrm{E}$ & ca $1750 \mathrm{~m}$ \\
\hline CG-HK & $11^{\circ} 50^{\prime} \mathrm{S}, 27^{\circ} 20^{\prime} \mathrm{E}$ & \\
\hline CG-ET & $00^{\circ} 19^{\prime} \mathrm{S}, 19^{\circ} 07^{\prime} \mathrm{E}$ & $337 \mathrm{~m}$ \\
\hline CG-SK & $02^{\circ} 13^{\prime} \mathrm{S}, 28^{\circ} 50^{\prime} \mathrm{E}$ & \\
\hline CG-IT & $01^{\circ} 55^{\prime} \mathrm{N}, 30^{\circ} 30^{\prime} \mathrm{E}$ & $1530 \mathrm{~m}$ \\
\hline CG-SK & $01^{\circ} 54^{\prime} \mathrm{S}, 28^{\circ} 27^{\prime} \mathrm{E}$ & $\begin{array}{c}5000-5100 \\
\mathrm{ft}\end{array}$ \\
\hline RW-OU & $02^{\circ} 31^{\prime} \mathrm{S}, 29^{\circ} 18^{\prime} \mathrm{E}$ & $2500 \mathrm{~m}$ \\
\hline CG-NK & $00^{\circ} 26^{\prime} \mathrm{N}, 29^{\circ} 33^{\prime} \mathrm{E}$ & $585 \mathrm{~m}$ \\
\hline CG-KG & ca $07^{\circ} 36^{\prime} \mathrm{S}, 17^{\circ} 14^{\prime} \mathrm{E}$ & \\
\hline CG-ET & $00^{\circ} 19^{\prime} \mathrm{S}, 19^{\circ} 07^{\prime} \mathrm{E}$ & $337 \mathrm{~m}$ \\
\hline CG-KG & ca $07^{\circ} 36^{\prime} \mathrm{S}, 17^{\circ} 14^{\prime} \mathrm{E}$ & \\
\hline CG-NK & $01^{\circ} 15^{\prime} \mathrm{S}, 29^{\circ} 28^{\prime} \mathrm{E}$ & $1300 \mathrm{~m}$ \\
\hline CG-NK & $01^{\circ} 15^{\prime} \mathrm{S}, 29^{\circ} 28^{\prime} \mathrm{E}$ & $1300 \mathrm{~m}$ \\
\hline CG-LB & $09^{\circ} 50^{\prime} \mathrm{S}, 25^{\circ} 33^{\prime} \mathrm{E}$ & $693 \mathrm{~m}$ \\
\hline CG-LB & $09^{\circ} 50^{\prime} \mathrm{S}, 25^{\circ} 33^{\prime} \mathrm{E}$ & $693 \mathrm{~m}$ \\
\hline CG-TO & $00^{\circ} 52^{\prime} \mathrm{N}, 27^{\circ} 34^{\prime} \mathrm{E}$ & $1942 \mathrm{~m}$ \\
\hline CG-LL & $05^{\circ} 43^{\prime} \mathrm{S}, 23^{\circ} 25^{\prime} \mathrm{E}$ & 1942111 \\
\hline CG-LL & $05^{\circ} 43^{\prime} \mathrm{S}, 23^{\circ} 25^{\prime} \mathrm{E}$ & \\
\hline RW-ES & $01^{\circ} 31^{\prime} \mathrm{S}, 30^{\circ} 28^{\prime} \mathrm{E}$ & $1400 \mathrm{~m}$ \\
\hline RW-SU & $02^{\circ} 28^{\prime} \mathrm{S}, 29^{\circ} 50^{\prime} \mathrm{E}$ & \\
\hline RW-SU & $02^{\circ} 28^{\prime} \mathrm{S}, 29^{\circ} 50^{\prime} \mathrm{E}$ & \\
\hline RW-OU & $02^{\circ} 24^{\prime} \mathrm{S}, 29^{\circ} 03^{\prime} \mathrm{E}$ & $1457 \mathrm{~m}$ \\
\hline RW-OU & $02^{\circ} 24^{\prime} \mathrm{S}, 29^{\circ} 03^{\prime} \mathrm{E}$ & $1457 \mathrm{~m}$ \\
\hline CG-NK & $01^{\circ} 13^{\prime} \mathrm{S}, 29^{\circ} 28^{\prime} \mathrm{E}$ & $1250 \mathrm{~m}$ \\
\hline RW-NO & $01^{\circ} 24^{\prime} \mathrm{S}, 29^{\circ} 38^{\prime} \mathrm{E}$ & \\
\hline RW-SU & $02^{\circ} 29^{\prime} \mathrm{S}, 29^{\circ} 55^{\prime} \mathrm{E}$ & \\
\hline CG-MO & $02^{\circ} 57^{\prime} \mathrm{N}, 21^{\circ} 26^{\prime} \mathrm{E}$ & $412 \mathrm{~m}$ \\
\hline CG-HU & $02^{\circ} 10^{\prime} \mathrm{N}, 27^{\circ} 15^{\prime} \mathrm{E}$ & $600 \mathrm{~m}$ \\
\hline CG-BC & $04^{\circ} 52^{\prime} \mathrm{S}, 12^{\circ} 52^{\prime} \mathrm{E}$ & $294 \mathrm{~m}$ \\
\hline CG-LM & $06^{\circ} 45^{\prime} \mathrm{S}, 23^{\circ} 57^{\prime} \mathrm{E}$ & $806 \mathrm{~m}$ \\
\hline
\end{tabular}




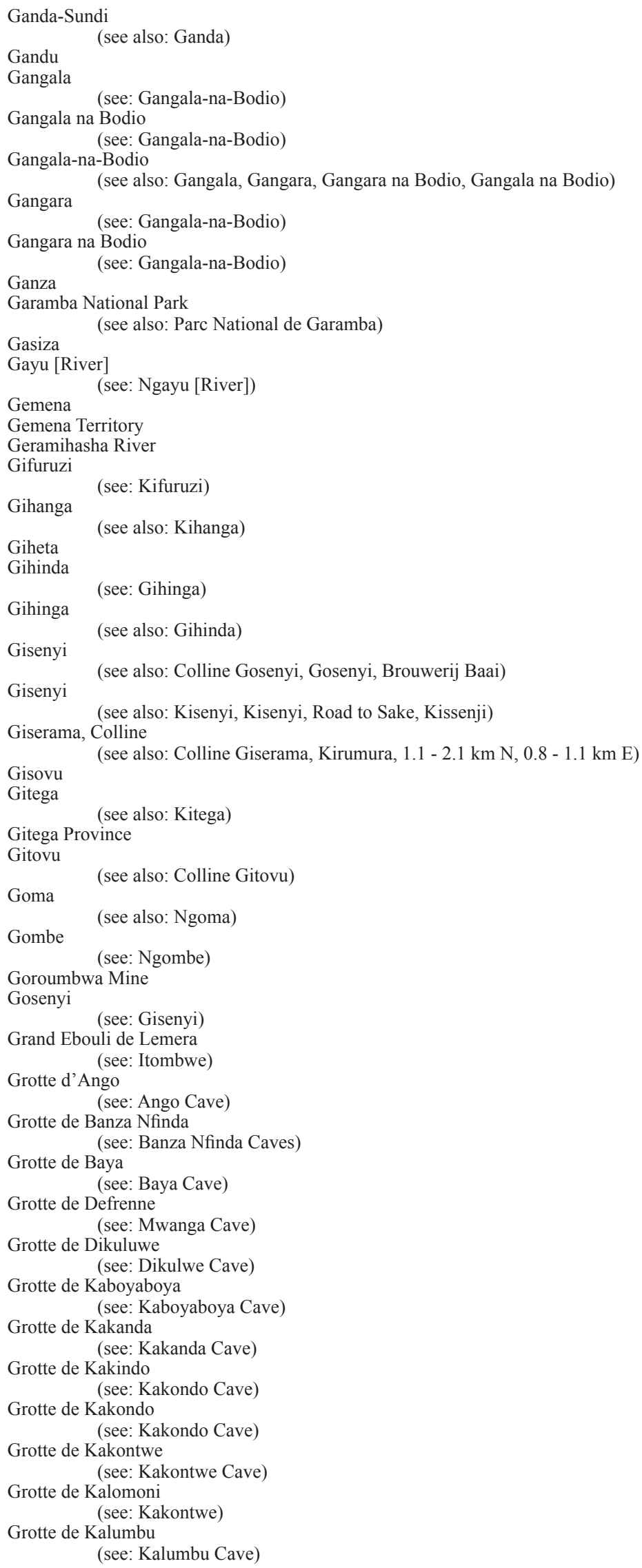

\begin{tabular}{|c|c|c|}
\hline CG-BC & $04^{\circ} 52^{\prime} \mathrm{S}, 12^{\circ} 52^{\prime} \mathrm{E}$ & $294 \mathrm{~m}$ \\
\hline $\begin{array}{c}\mathrm{CG} \\
\mathrm{CG}-\mathrm{HU}\end{array}$ & $03^{\circ} 41^{\prime} \mathrm{N}, 29^{\circ} 08^{\prime} \mathrm{E}$ & $701 \mathrm{~m}$ \\
\hline CG-HU & $03^{\circ} 41^{\prime} \mathrm{N}, 29^{\circ} 08^{\prime} \mathrm{E}$ & $701 \mathrm{~m}$ \\
\hline CG-HU & $03^{\circ} 41^{\prime} \mathrm{N}, 29^{\circ} 08^{\prime} \mathrm{E}$ & $701 \mathrm{~m}$ \\
\hline CG-HU & $03^{\circ} 41^{\prime} \mathrm{N}, 29^{\circ} 08^{\prime} \mathrm{E}$ & $701 \mathrm{~m}$ \\
\hline CG-HU & $03^{\circ} 41^{\prime} \mathrm{N}, 29^{\circ} 08^{\prime} \mathrm{E}$ & $701 \mathrm{~m}$ \\
\hline $\begin{array}{l}\text { CG-HL } \\
\text { CG-HU }\end{array}$ & $\begin{array}{l}09^{\circ} 14^{\prime} \mathrm{S}, 26^{\circ} 42^{\prime} \mathrm{E} \\
04^{\circ} 10^{\prime} \mathrm{N}, 29^{\circ} 30^{\prime} \mathrm{E}\end{array}$ & $860 \mathrm{~m}$ \\
\hline $\begin{array}{c}\text { RW } \\
\text { CG-HU }\end{array}$ & $01^{\circ} 35^{\prime} \mathrm{N}, 27^{\circ} 13^{\prime} \mathrm{E}$ & \\
\hline $\begin{array}{l}\text { CG-SU } \\
\text { CG-SU } \\
\text { CG-NK } \\
\text { BY-MA }\end{array}$ & $\begin{array}{l}03^{\circ} 15^{\prime} \mathrm{N}, 19^{\circ} 46^{\prime} \mathrm{E} \\
03^{\circ} 15^{\prime} \mathrm{N}, 19^{\circ} 46^{\prime} \mathrm{E} \\
01^{\circ} 13^{\prime} \mathrm{S}, 29^{\circ} 27^{\prime} \mathrm{E} \\
04^{\circ} 19^{\prime} \mathrm{S}, 29^{\circ} 35^{\prime} \mathrm{E}\end{array}$ & $\begin{array}{l}555 \mathrm{~m} \\
1250 \mathrm{~m}\end{array}$ \\
\hline BY-BB & $03^{\circ} 12^{\prime} \mathrm{S}, 29^{\circ} 18^{\prime} \mathrm{E}$ & $814 \mathrm{~m}$ \\
\hline $\begin{array}{c}\text { BY } \\
\text { RW-ES }\end{array}$ & $\begin{array}{l}03^{\circ} 20^{\prime} \mathrm{S}, 29^{\circ} 50^{\prime} \mathrm{E} \\
01^{\circ} 24^{\prime} \mathrm{S}, 30^{\circ} 37^{\prime} \mathrm{E}\end{array}$ & $1400 \mathrm{~m}$ \\
\hline RW-ES & $01^{\circ} 24^{\prime} \mathrm{S}, 30^{\circ} 37^{\prime} \mathrm{E}$ & $1400 \mathrm{~m}$ \\
\hline RW-OU & $01^{\circ} 42^{\prime} \mathrm{S}, 29^{\circ} 15^{\prime} \mathrm{E}$ & $1481 \mathrm{~m}$ \\
\hline RW-OU & $01^{\circ} 41^{\prime} \mathrm{S}, 29^{\circ} 15^{\prime} \mathrm{E}$ & $1450 \mathrm{~m}$ \\
\hline BY-CI & $02^{\circ} 50^{\prime} \mathrm{S}, 29^{\circ} 25^{\prime} \mathrm{E}$ & $2045 \mathrm{~m}$ \\
\hline $\begin{array}{l}\text { RW-SU } \\
\text { BY-GI }\end{array}$ & $\begin{array}{l}02^{\circ} 15^{\prime} \mathrm{S}, 29^{\circ} 20^{\prime} \mathrm{E} \\
03^{\circ} 26^{\prime} \mathrm{S}, 29^{\circ} 56^{\prime} \mathrm{E}\end{array}$ & $\begin{array}{l}2335 \mathrm{~m} \\
1845 \mathrm{~m}\end{array}$ \\
\hline $\begin{array}{c}\text { BY } \\
\text { RW-SU }\end{array}$ & $02^{\circ} 19^{\prime} \mathrm{S}, 29^{\circ} 09^{\prime} \mathrm{E}$ & $1649 \mathrm{~m}$ \\
\hline CG-NK & $01^{\circ} 41^{\prime} \mathrm{S}, 29^{\circ} 13^{\prime} \mathrm{E}$ & $1499 \mathrm{~m}$ \\
\hline CG-KS & $06^{\circ} 35^{\prime} \mathrm{S}, 20^{\circ} 42^{\prime} \mathrm{E}$ & $528 \mathrm{~m}$ \\
\hline $\begin{array}{l}\text { CG-HU } \\
\text { RW-OU }\end{array}$ & $\begin{array}{l}03^{\circ} 07^{\prime} \mathrm{N}, 29^{\circ} 34^{\prime} \mathrm{E} \\
01^{\circ} 42^{\prime} \mathrm{S}, 29^{\circ} 15^{\prime} \mathrm{E}\end{array}$ & $1481 \mathrm{~m}$ \\
\hline CG-SK & $04^{\circ} 04^{\prime} \mathrm{S}, 28^{\circ} 40^{\prime} \mathrm{E}$ & \\
\hline CG-BU & $04^{\circ} 02^{\prime} \mathrm{N}, 25^{\circ} 52^{\prime} \mathrm{E}$ & $610 \mathrm{~m}$ \\
\hline CG-BC & $05^{\circ} 16^{\prime} \mathrm{S}, 14^{\circ} 52^{\prime} \mathrm{E}$ & \\
\hline CG-HK & $11^{\circ} 52^{\prime} \mathrm{S}, 27^{\circ} 27^{\prime} \mathrm{E}$ & $1279 \mathrm{~m}$ \\
\hline CG-HK & $10^{\circ} 58^{\prime} \mathrm{S}, 26^{\circ} 39^{\prime} \mathrm{E}$ & \\
\hline CG-LB & $10^{\circ} 45^{\prime} \mathrm{S}, 25^{\circ} 22^{\prime} \mathrm{E}$ & \\
\hline CG-LB & $09^{\circ} 56^{\prime} \mathrm{S}, 25^{\circ} 58^{\prime} \mathrm{E}$ & \\
\hline CG-LB & $10^{\circ} 47^{\prime} \mathrm{S}, 26^{\circ} 36^{\prime} \mathrm{E}$ & $1438 \mathrm{~m}$ \\
\hline CG-SK & $02^{\circ} 16^{\prime} \mathrm{S}, 28^{\circ} 51^{\prime} \mathrm{E}$ & \\
\hline CG-SK & $02^{\circ} 16^{\prime} \mathrm{S}, 28^{\circ} 51^{\prime} \mathrm{E}$ & \\
\hline CG-HK & $10^{\circ} 59^{\prime} \mathrm{S}, 26^{\circ} 38^{\prime} \mathrm{E}$ & $1294 \mathrm{~m}$ \\
\hline CG-HK & $10^{\circ} 59^{\prime} \mathrm{S}, 26^{\circ} 40^{\prime} \mathrm{E}$ & $1294 \mathrm{~m}$ \\
\hline CG-HK & $10^{\circ} 51^{\prime} \mathrm{S}, 26^{\circ} 39^{\prime} \mathrm{E}$ & \\
\hline
\end{tabular}




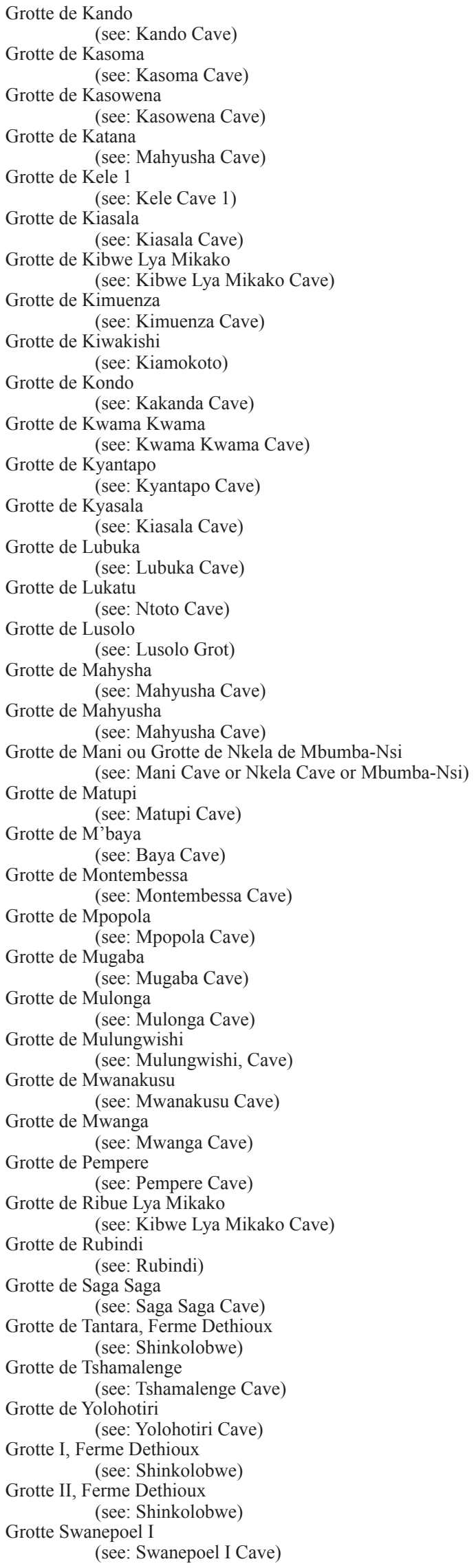

CG-HK $\quad 10^{\circ} 49^{\prime} \mathrm{S}, 26^{\circ} 07^{\prime} \mathrm{E}$

CG-HL $\quad 09^{\circ} 25^{\prime} \mathrm{S}, 26^{\circ} 37^{\prime} \mathrm{E}$

CG-HK $\quad 10^{\circ} 58^{\prime} \mathrm{S}, 26^{\circ} 39^{\prime} \mathrm{E}$

CG-SK $\quad 02^{\circ} 13^{\prime} \mathrm{S}, 28^{\circ} 50^{\prime} \mathrm{E} \quad 1480 \mathrm{~m}$

CG-BC $\quad 05^{\circ} 31^{\prime} \mathrm{S}, 14^{\circ} 36^{\prime} \mathrm{E}$

CG-LB $\quad 09^{\circ} 56^{\prime} \mathrm{S}, 25^{\circ} 58^{\prime} \mathrm{E} \quad 1300 \mathrm{~m}$

CG-NK $\quad 00^{\circ} 15^{\prime} \mathrm{S}, 29^{\circ} 01^{\prime} \mathrm{E} \quad 1500 \mathrm{~m}$

CG-BC $\quad 04^{\circ} 28^{\prime} \mathrm{S}, 15^{\circ} 17^{\prime} \mathrm{E}$

CG-HL $\quad 09^{\circ} 10^{\prime} \mathrm{S}, 27^{\circ} 04^{\prime} \mathrm{E} \quad 900 \mathrm{~m}$

CG-LB $\quad 10^{\circ} 47^{\prime} \mathrm{S}, 26^{\circ} 36^{\prime} \mathrm{E} \quad 1438 \mathrm{~m}$

CG-NK $\quad 01^{\circ} 19^{\prime} \mathrm{N}, 29^{\circ} 52^{\prime} \mathrm{E} \quad 1200 \mathrm{~m}$

CG-LB $\quad 09^{\circ} 56^{\prime} \mathrm{S}, 25^{\circ} 58^{\prime} \mathrm{E} \quad 1525 \mathrm{~m}$

CG-LB $\quad 09^{\circ} 56^{\prime} \mathrm{S}, 25^{\circ} 58^{\prime} \mathrm{E} \quad 1300 \mathrm{~m}$

CG-SK $\quad 03^{\circ} 12^{\prime} \mathrm{S}, 28^{\circ} 51^{\prime} \mathrm{E}$

CG-TG $\quad 06^{\circ} 59^{\prime} \mathrm{S}, 29^{\circ} 43^{\prime} \mathrm{E} \quad 827 \mathrm{~m}$

CG-HK $\quad 09^{\circ} 56^{\prime} \mathrm{S}, 25^{\circ} 28^{\prime} \mathrm{E}$

CG-SK $\quad 02^{\circ} 13^{\prime} \mathrm{S}, 28^{\circ} 50^{\prime} \mathrm{E} \quad 1480 \mathrm{~m}$

CG-SK $\quad 02^{\circ} 13^{\prime} \mathrm{S}, 28^{\circ} 50^{\prime} \mathrm{E} \quad 1480 \mathrm{~m}$

CG

CG-IT $\quad 01^{\circ} 16^{\prime} \mathrm{N}, 29^{\circ} 55^{\prime} \mathrm{E} \quad 1067 \mathrm{~m}$

CG-HK $\quad 11^{\circ} 52^{\prime} \mathrm{S}, 27^{\circ} 27^{\prime} \mathrm{E} \quad 1279 \mathrm{~m}$

$\mathrm{CG}$

CG $\quad 09^{\circ} 56^{\prime} \mathrm{S}, 25^{\circ} 58^{\prime} \mathrm{E} \quad 1450 \mathrm{~m}$

CG-SK ca $02^{\circ} 19^{\prime} \mathrm{S}, 28^{\circ} 31^{\prime} \mathrm{E}$

CG-LB $\quad 09^{\circ} 56^{\prime} \mathrm{S}, 25^{\circ} 58^{\prime} \mathrm{E}$

CG-LB $\quad 10^{\circ} 47^{\prime} \mathrm{S}, 26^{\circ} 38^{\prime} \mathrm{E} \quad 1366 \mathrm{~m}$

CG-MN $\quad 04^{\circ} 35^{\prime} \mathrm{S}, 27^{\circ} 08^{\prime} \mathrm{E}$

CG-HK $\quad 10^{\circ} 58^{\prime} \mathrm{S}, 26^{\circ} 39^{\prime} \mathrm{E}$

CG-HK $\quad 10^{\circ} 58^{\prime} \mathrm{S}, 26^{\circ} 47^{\prime} \mathrm{E}$

CG-NK $\quad 00^{\circ} 15^{\prime} \mathrm{S}, 29^{\circ} 01^{\prime} \mathrm{E} \quad 1500 \mathrm{~m}$

BY-MA $\quad 04^{\circ} 16^{\prime} \mathrm{S}, 29^{\circ} 35^{\prime} \mathrm{E} \quad 997 \mathrm{~m}$

CG-IT $\quad 01^{\circ} 19^{\prime} \mathrm{N}, 2^{\circ} 52^{\prime} \mathrm{E} \quad 1160 \mathrm{~m}$

CG-LB $\quad 11^{\circ} 02^{\prime} \mathrm{S}, 26^{\circ} 29^{\prime} \mathrm{E} \quad 1334 \mathrm{~m}$

CG-HK $\quad 11^{\circ} 46^{\prime} \mathrm{S}, 26^{\circ} 30^{\prime} \mathrm{E}$

CG-IT $\quad 01^{\circ} 13^{\prime} \mathrm{N}, 29^{\circ} 49^{\prime} \mathrm{E}$

CG-LB $\quad 11^{\circ} 02^{\prime} \mathrm{S}, 26^{\circ} 29^{\prime} \mathrm{E} \quad 1334 \mathrm{~m}$

CG-LB $\quad 1^{\circ} 02^{\prime} \mathrm{S}, 26^{\circ} 29^{\prime} \mathrm{E} \quad 1334 \mathrm{~m}$

CG-LB $\quad 10^{\circ} 50^{\prime} \mathrm{S}, 26^{\circ} 39^{\prime} \mathrm{E} \quad$ ca $1350 \mathrm{~m}$ 


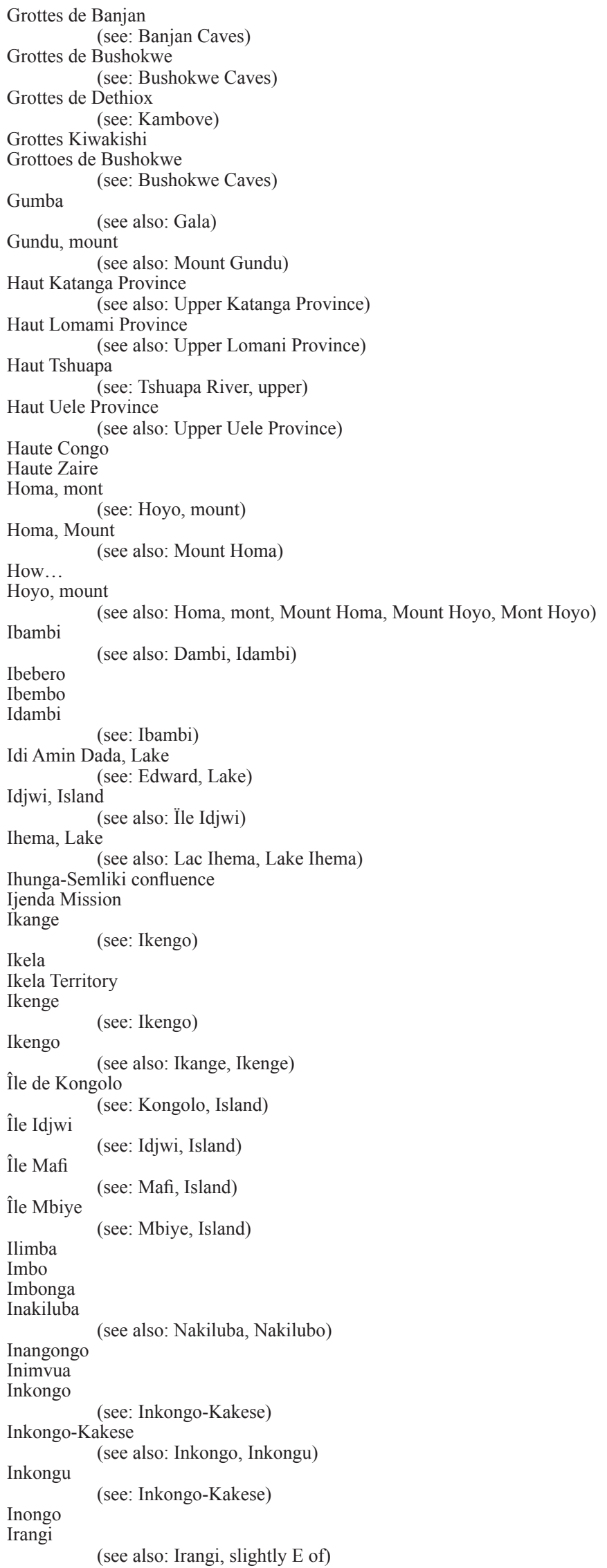

$\begin{array}{lcr}\text { CG-BC } & 05^{\circ} 16^{\prime} \mathrm{S}, 14^{\circ} 53^{\prime} \mathrm{E} \\ \text { CG-SK } & \text { ca } 02^{\circ} 59^{\prime} \mathrm{S}, 28^{\circ} 55^{\prime} \mathrm{E} & \\ \text { CG-LB } & 10^{\circ} 50^{\prime} \mathrm{S}, 26^{\circ} 39^{\prime} \mathrm{E} & \\ & & \\ \text { CG-HK } & 09^{\circ} 00^{\prime} \mathrm{S}, 27^{\circ} 12^{\prime} \mathrm{E} & \\ \text { CG-SK } & \text { ca } 02^{\circ} 59^{\prime} \mathrm{S}, 28^{\circ} 55^{\prime} \mathrm{E} & \\ \text { CG-MO } & 02^{\circ} 57^{\prime} \mathrm{N}, 21^{\circ} 26^{\prime} \mathrm{E} & 412 \mathrm{~m} \\ \text { CG-BU } & & \end{array}$

CG-TP $01^{\circ} 00^{\prime} \mathrm{S}, 23^{\circ} 02^{\prime} \mathrm{E}$

CG

CG-IT $01^{\circ} 13^{\prime} \mathrm{N}, 29^{\circ} 49^{\prime} \mathrm{E} \quad 1200 \mathrm{~m}$

CG-IT $01^{\circ} 13^{\prime} \mathrm{N}, 29^{\circ} 49^{\prime} \mathrm{E}$

CG CG-IT $01^{\circ} 13^{\prime} \mathrm{N}, 29^{\circ} 49^{\prime} \mathrm{E}$

$1200 \mathrm{~m}$

CG-HU $02^{\circ} 22^{\prime} \mathrm{N}, 27^{\circ} 37^{\prime} \mathrm{E} \quad 687 \mathrm{~m}$

CG-SK

CG-BU $02^{\circ} 38^{\prime} \mathrm{N}, 23^{\circ} 37^{\prime} \mathrm{E} \quad 420 \mathrm{~m}$ CG-HU $02^{\circ} 22^{\prime} \mathrm{N}, 27^{\circ} 37^{\prime} \mathrm{E}$ $420 \mathrm{~m}$
$687 \mathrm{~m}$

CG-NK ca $00^{\circ} 08^{\prime} \mathrm{S}, 29^{\circ} 35^{\prime} \mathrm{E}$

CG-SK $02^{\circ} 09^{\prime} \mathrm{S}, 29^{\circ} 04^{\prime} \mathrm{E}$

$1600 \mathrm{~m}$

RW-ES

$01^{\circ} 52^{\prime} \mathrm{S}, 30^{\circ} 47^{\prime} \mathrm{E}$

CG-NK

BY-BI $\quad 03^{\circ} 39^{\prime} \mathrm{S}, 29^{\circ} 34^{\prime} \mathrm{E}$

$00^{\circ} 10^{\prime} \mathrm{N}, 29^{\circ} 35^{\prime} \mathrm{E}$

CG-ET $\quad 00^{\circ} 08^{\prime} \mathrm{S}, 18^{\circ} 08^{\prime} \mathrm{E}$

CG-TP

CG-TP

CG-ET

$01^{\circ} 11^{\prime} \mathrm{S}, 23^{\circ} 16^{\prime} \mathrm{E}$ $01^{\circ} 11^{\prime} \mathrm{S}, 23^{\circ} 16^{\prime} \mathrm{E}$ $00^{\circ} 08^{\prime} \mathrm{S}, 18^{\circ} 08^{\prime} \mathrm{E}$

$301 \mathrm{~m}$

CG-ET $00^{\circ} 08^{\prime} \mathrm{S}, 18^{\circ} 08^{\prime} \mathrm{E}$ $301 \mathrm{~m}$

CG-TO $00^{\circ} 03^{\prime} \mathrm{N}, 25^{\circ} 12^{\prime} \mathrm{E}$

CG-SK $02^{\circ} 09^{\prime} \mathrm{S}, 29^{\circ} 04^{\prime} \mathrm{E}$

$1600 \mathrm{~m}$

$00^{\circ} 28^{\prime} \mathrm{N}, 25^{\circ} 17^{\prime} \mathrm{E}$

CG-TO $00^{\circ} 28^{\prime} \mathrm{N}, 25^{\circ} 17^{\prime} \mathrm{E}$

$413 \mathrm{~m}$

CG-NK

BY-BB

$00^{\circ} 50^{\prime} \mathrm{N}, 29^{\circ} 58^{\prime} \mathrm{E}$ $03^{\circ} 13^{\prime} \mathrm{S}, 29^{\circ} 20^{\prime} \mathrm{E}$ $00^{\circ} 43^{\prime} \mathrm{S}, 19^{\circ} 46^{\prime} \mathrm{E}$ $11^{\circ} 34^{\prime} \mathrm{S}, 27^{\circ} 19^{\prime} \mathrm{E}$

$750 \mathrm{~m}$

CG-NK

CG-HU

$00^{\circ} 33^{\prime} \mathrm{N}, 29^{\circ} 41^{\prime} \mathrm{E}$ $1280 \mathrm{~m}$

CG-SN $04^{\circ} 35^{\prime} \mathrm{N}, 29^{\circ} 43^{\prime} \mathrm{E}$ $04^{\circ} 53^{\prime} \mathrm{S}, 23^{\circ} 18^{\prime} \mathrm{E}$

$800 \mathrm{~m}$

$1061 \mathrm{~m}$ $400 \mathrm{~m}$

CG-SN

$04^{\circ} 53^{\prime} \mathrm{S}, 23^{\circ} 18^{\prime} \mathrm{E}$

$400 \mathrm{~m}$

CG-SN

$04^{\circ} 53^{\prime} \mathrm{S}, 23^{\circ} 18^{\prime} \mathrm{E}$

$400 \mathrm{~m}$

CG-MA

$01^{\circ} 57^{\prime} \mathrm{S}, 18^{\circ} 16^{\prime} \mathrm{E}$ $01^{\circ} 54^{\prime} \mathrm{S}, 28^{\circ} 27^{\prime} \mathrm{E}$ 
Irangi Bos

Irangi Fores

(see: Irangi Forest)

(see also: Bukavu, $100 \mathrm{~km} \mathrm{W,} \mathrm{Forêt} \mathrm{d'Irangi,} \mathrm{Irangi} \mathrm{Bos)}$

Irangi, $10 \mathrm{~km}$ from (see: Tshambutschu)

Irangi, $2 \mathrm{mi}[=3.2 \mathrm{~km}]$ from (see: Kasiba)

Irangi, $3 \mathrm{mi}[=4.8 \mathrm{~km}]$ from

Irangi, slightly E of

(see: Irangi)

Irebu

Iringi

Iringite

Irsac Labo

(see: Eringite)

Irumu

(see: Luiro)

Irumu

Irumu, $20 \mathrm{mi}[=32 \mathrm{~km}] \mathrm{S}$ (see: Mount Hoyo Caves)

Irumu, $241 \mathrm{~km} \mathrm{~N}$

(see: Faradje)

Irumu, $25 \mathrm{~km} \mathrm{~W}$

(see: Ituri Forest)

Irumu, $40 \mathrm{mi}[=64 \mathrm{~km}] \mathrm{SW}$

(see: Ituri Forest)

Irumu, $40 \mathrm{mi} \mathrm{SW}$

Irumu, $50 \mathrm{mi} \mathrm{SW}$

Isai River

Isato

Ishangi

(see: Shangi)

Ishango River

Ishango-Nyakazi

Isirio

(see: Isiro)

Isiro

(see also: Isirio, Paulis)

Iso

(see also: Isoberlinia, Iso.4)

Iso.4

(see: Iso)

Isoberlinia

(see: Iso)

Isoga

Issehe River

Itombwe

(see also: Lemera Great Sink, Grand Ebouli de Lemera)

Ituri Fores (see also: Irumu, $40 \mathrm{mi}[=64 \mathrm{~km}] \mathrm{SW}$, Sturi Forest)

Ituri Forest

(see also: Irumu, $25 \mathrm{~km} \mathrm{~W}$ )

Ituri ht

Ituri Province

Ituri River

Ituri River, Bridge

Ituri, south

Iyonda

Jadotstad

(see: Likasi)

Jadotstad, $15 \mathrm{~km} \mathrm{~S}$

(see: Mwanga Cave)

Jadotsville

(see: Likasi)

Jadotville

(see: Likasi)

Jadotville, $15 \mathrm{~km} \mathrm{~S}$

(see: Mwanga Cave)

Jadotville, ca $8 \mathrm{~km} \mathrm{~S}$

Jadoville (see: Kakontwe)

(see: Likasi)

Jaima

Jiba
CG-SK

CG-SK

$01^{\circ} 54^{\prime} \mathrm{S}, 28^{\circ} 27^{\prime} \mathrm{E}$

5000-5100

$\mathrm{ft}$

$01^{\circ} 54^{\prime} \mathrm{S}, 28^{\circ} 27^{\prime} \mathrm{E} \quad 5000-5100$

CG-SK ca $01^{\circ} 58^{\prime} \mathrm{S}, 28^{\circ} 30^{\prime} \mathrm{E}$

CG-SK ca $01^{\circ} 54^{\prime} \mathrm{S}, 28^{\circ} 27^{\prime} \mathrm{E}$

CG-SK ca $01^{\circ} 54^{\prime} \mathrm{S}, 28^{\circ} 27^{\prime} \mathrm{E}$

CG-SK ca $01^{\circ} 54^{\prime} \mathrm{S}, 28^{\circ} 27^{\prime} \mathrm{E}$

CG-ET $\quad 00^{\circ} 37^{\prime} \mathrm{S}, 17^{\circ} 45^{\prime} \mathrm{E}$

CG $01^{\circ} 54^{\prime} \mathrm{S}, 28^{\circ} 27^{\prime} \mathrm{E}$

CG-NK $\quad 00^{\circ} 51^{\prime} \mathrm{N}, 29^{\circ} 37^{\prime} \mathrm{E}$

CG-SK $\quad 02^{\circ} 14^{\prime} \mathrm{S}, 28^{\circ} 48^{\prime} \mathrm{E}$

$1270 \mathrm{~m}$

CG-IT

$01^{\circ} 27^{\prime} \mathrm{N}, 29^{\circ} 52^{\prime} \mathrm{E}$

CG

CG-IT

$01^{\circ} 27^{\prime} \mathrm{N}, 29^{\circ} 52^{\prime} \mathrm{E}$

$01^{\circ} 13^{\prime} \mathrm{N}, 29^{\circ} 49^{\prime} \mathrm{E}$

841-1280

$\mathrm{m}$

$841 \mathrm{~m}$

CG-HU

$03^{\circ} 44^{\prime} \mathrm{N}, 29^{\circ} 43^{\prime} \mathrm{E}$

$775 \mathrm{~m}$

CG-IT

$01^{\circ} 23^{\prime} \mathrm{N}, 28^{\circ} 38^{\prime} \mathrm{E}$

CG-IT

$01^{\circ} 00^{\prime} \mathrm{N}, 29^{\circ} 16^{\prime} \mathrm{E}$

CG-IT

CG-IT

CG-IT

CG-SU

RW-OU

$01^{\circ} 00^{\prime} \mathrm{N}, 29^{\circ} 16^{\prime} \mathrm{E}$

$01^{\circ} 24^{\prime} \mathrm{N}, 28^{\circ} 04^{\prime} \mathrm{E}$

$03^{\circ} 18^{\prime} \mathrm{N}, 18^{\circ} 39^{\prime} \mathrm{E}$

$02^{\circ} 24^{\prime} \mathrm{S}, 29^{\circ} 00^{\prime} \mathrm{E}$

$323 \mathrm{~m}$

CG-NK

CG-NK

$00^{\circ} 08^{\prime} \mathrm{S}, 29^{\circ} 36^{\prime} \mathrm{E}$ $00^{\circ} 08^{\prime} \mathrm{S}, 29^{\circ} 35^{\prime} \mathrm{E}$

$02^{\circ} 46^{\prime} \mathrm{N}, 27^{\circ} 37^{\prime} \mathrm{E}$

$950 \mathrm{~m}$

$950 \mathrm{~m}$

$730 \mathrm{~m}$

CG-HU

$02^{\circ} 46^{\prime} \mathrm{N}, 27^{\circ} 37^{\prime} \mathrm{E}$

$730 \mathrm{~m}$

CG-HU

$04^{\circ} 18^{\prime} \mathrm{N}, 29^{\circ} 56^{\prime} \mathrm{E}$

CG-HU

$04^{\circ} 18^{\prime} \mathrm{N}, 29^{\circ} 56^{\prime} \mathrm{E}$

CG-HU

$04^{\circ} 18^{\prime} \mathrm{N}, 29^{\circ} 56^{\prime} \mathrm{E}$

CG-SK

CG-IT

$03^{\circ} 20^{\prime} \mathrm{S}, 28^{\circ} 12^{\prime} \mathrm{E}$

$01^{\circ} 05^{\prime} \mathrm{N}, 29^{\circ} 49^{\prime} \mathrm{E}$

CG-SK

$04^{\circ} 04^{\prime} \mathrm{S}, 28^{\circ} 40^{\prime} \mathrm{E}$

$1266 \mathrm{~m}$

CG-IT $\quad 01^{\circ} 00^{\prime} \mathrm{N}, 29^{\circ} 16^{\prime} \mathrm{E}$

CG-IT

$01^{\circ} 23^{\prime} \mathrm{N}, 29^{\circ} 38^{\prime} \mathrm{E}$

CG-IT

CG

CG-IT

CG-IT

CG-IT

CG-ET

$01^{\circ} 20^{\prime} \mathrm{N}, 27^{\circ} 35^{\prime} \mathrm{E}$

$01^{\circ} 20^{\prime} \mathrm{N}, 27^{\circ} 35^{\prime} \mathrm{E}$

$01^{\circ} 00^{\prime} \mathrm{N}, 29^{\circ} 16^{\prime} \mathrm{E}$

$00^{\circ} 02^{\prime} \mathrm{S}, 18^{\circ} 12^{\prime} \mathrm{E}$

$10^{\circ} 59^{\prime} \mathrm{S}, 26^{\circ} 44^{\prime} \mathrm{E}$

$1318 \mathrm{~m}$

CG-HK

CG-HK $\quad 10^{\circ} 59^{\prime} \mathrm{S}, 26^{\circ} 44^{\prime} \mathrm{E} \quad 1318 \mathrm{~m}$

CG-HK $\quad 10^{\circ} 59^{\prime} \mathrm{S}, 26^{\circ} 44^{\prime} \mathrm{E} \quad 1318 \mathrm{~m}$

CG-HK

CG-HK $\quad 10^{\circ} 59^{\prime} \mathrm{S}, 26^{\circ} 40^{\prime} \mathrm{E} \quad 1253 \mathrm{~m}$

CG-HK $\quad 10^{\circ} 59^{\prime} \mathrm{S}, 26^{\circ} 44^{\prime} \mathrm{E} \quad 1318 \mathrm{~m}$

CG

CG-IT

$01^{\circ} 52^{\prime} \mathrm{N}, 30^{\circ} 42^{\prime} \mathrm{E}$

$2123 \mathrm{~m}$ 


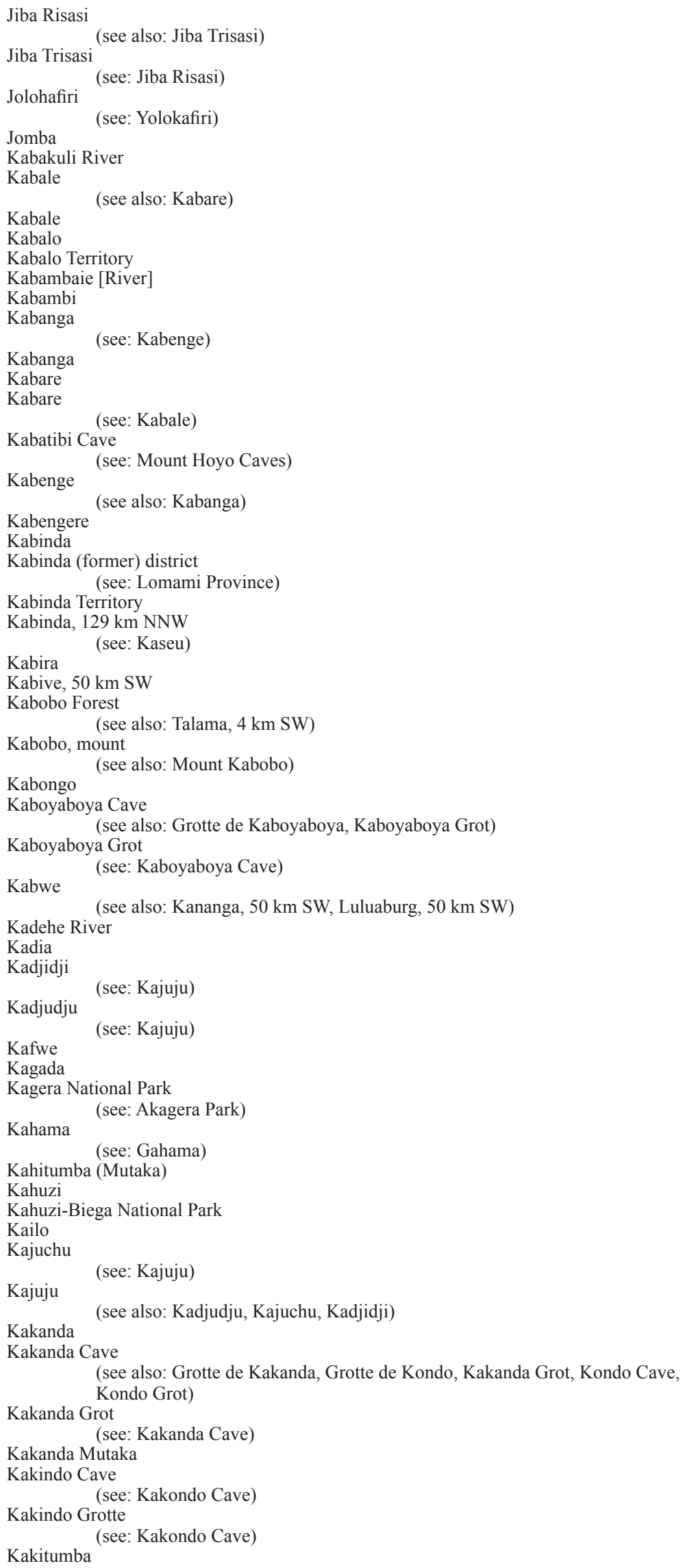

\begin{tabular}{|c|c|c|}
\hline CG-IT & $01^{\circ} 52^{\prime} \mathrm{N}, 30^{\circ} 42^{\prime} \mathrm{E}$ & $1800 \mathrm{~m}$ \\
\hline CG-IT & $01^{\circ} 52^{\prime} \mathrm{N}, 30^{\circ} 42^{\prime} \mathrm{E}$ & $1800 \mathrm{~m}$ \\
\hline CG-IT & $01^{\circ} 20^{\prime} \mathrm{N}, 29^{\circ} 50^{\prime} \mathrm{E}$ & $1030 \mathrm{~m}$ \\
\hline $\begin{array}{l}\text { CG-NK } \\
\text { CG-NK } \\
\text { CG-NK }\end{array}$ & $\begin{array}{l}01^{\circ} 17^{\prime} \mathrm{S}, 29^{\circ} 33^{\prime} \mathrm{E} \\
00^{\circ} 27^{\prime} \mathrm{N}, 29^{\circ} 44^{\prime} \mathrm{E} \\
00^{\circ} 38^{\prime} \mathrm{S}, 29^{\circ} 07^{\prime} \mathrm{E}\end{array}$ & $\begin{array}{l}1500 \mathrm{~m} \\
1791 \mathrm{~m}\end{array}$ \\
\hline $\begin{array}{l}\text { CG-SK } \\
\text { CG-TG } \\
\text { CG-TG } \\
\text { CG-KS } \\
\text { CG-NK } \\
\text { CG-HL }\end{array}$ & $\begin{array}{l}02^{\circ} 30^{\prime} \mathrm{S}, 28^{\circ} 47^{\prime} \mathrm{E} \\
06^{\circ} 03^{\prime} \mathrm{S}, 26^{\circ} 55^{\prime} \mathrm{E} \\
06^{\circ} 03^{\prime} \mathrm{S}, 26^{\circ} 55^{\prime} \mathrm{E} \\
05^{\circ} 44^{\prime} \mathrm{S}, 20^{\circ} 49^{\prime} \mathrm{E} \\
00^{\circ} 51^{\prime} \mathrm{N}, 29^{\circ} 39^{\prime} \mathrm{E} \\
09^{\circ} 01^{\prime} \mathrm{S}, 26^{\circ} 45^{\prime} \mathrm{E}\end{array}$ & $\begin{array}{c}1260 \mathrm{~m} \\
788 \mathrm{~m}\end{array}$ \\
\hline $\begin{array}{l}\text { CG-LM } \\
\text { CG-SK } \\
\text { CG-NK }\end{array}$ & $\begin{array}{l}06^{\circ} 40^{\prime} \mathrm{S}, 23^{\circ} 40^{\prime} \mathrm{E} \\
02^{\circ} 28^{\prime} \mathrm{S}, 28^{\circ} 49^{\prime} \mathrm{E} \\
00^{\circ} 38^{\prime} \mathrm{S}, 29^{\circ} 07^{\prime} \mathrm{E}\end{array}$ & $\begin{array}{l}2044 \mathrm{~m} \\
1791 \mathrm{~m}\end{array}$ \\
\hline CG-IT & ca $01^{\circ} 13^{\prime} \mathrm{N}, 29^{\circ} 49^{\prime} \mathrm{E}$ & $1200 \mathrm{~m}$ \\
\hline CG-HL & $09^{\circ} 01^{\prime} \mathrm{S}, 26^{\circ} 45^{\prime} \mathrm{E}$ & $788 \mathrm{~m}$ \\
\hline $\begin{array}{l}\mathrm{CG}-\mathrm{HL} \\
\mathrm{CG}-\mathrm{KO} \\
\quad \mathrm{CG}\end{array}$ & $\begin{array}{l}09^{\circ} 08^{\prime} \mathrm{S}, 26^{\circ} 16^{\prime} \mathrm{E} \\
06^{\circ} 08^{\prime} \mathrm{S}, 24^{\circ} 29^{\prime} \mathrm{E}\end{array}$ & $\begin{array}{l}732 \mathrm{~m} \\
842 \mathrm{~m}\end{array}$ \\
\hline $\begin{array}{l}\mathrm{CG}-\mathrm{KO} \\
\mathrm{CG}-\mathrm{KO}\end{array}$ & $\begin{array}{l}06^{\circ} 08^{\prime} \mathrm{S}, 24^{\circ} 29^{\prime} \mathrm{E} \\
05^{\circ} 11^{\prime} \mathrm{S}, 23^{\circ} 51^{\prime} \mathrm{E}\end{array}$ & \\
\hline $\begin{array}{c}\text { CG-SK } \\
\text { CG } \\
\text { CG-SK }\end{array}$ & $\begin{array}{l}02^{\circ} 03^{\prime} \mathrm{S}, 28^{\circ} 55^{\prime} \mathrm{E} \\
04^{\circ} 59^{\prime} \mathrm{S}, 29^{\circ} 05^{\prime} \mathrm{E}\end{array}$ & $\begin{array}{l}1519 \mathrm{~m} \\
1950 \mathrm{~m}\end{array}$ \\
\hline CG-TG & $05^{\circ} 04^{\prime} \mathrm{S}, 29^{\circ} 00^{\prime} \mathrm{E}$ & $2440 \mathrm{~m}$ \\
\hline $\begin{array}{l}\text { CG-HL } \\
\text { CG-LB }\end{array}$ & $\begin{array}{l}07^{\circ} 19^{\prime} \mathrm{S}, 25^{\circ} 35^{\prime} \mathrm{E} \\
09^{\circ} 56^{\prime} \mathrm{S}, 25^{\circ} 58^{\prime} \mathrm{E}\end{array}$ & $918 \mathrm{~m}$ \\
\hline CG-LB & $09^{\circ} 56^{\prime} \mathrm{S}, 25^{\circ} 58^{\prime} \mathrm{E}$ & \\
\hline CG-LL & $06^{\circ} 12^{\prime} \mathrm{S}, 22^{\circ} 23^{\prime} \mathrm{E}$ & $610 \mathrm{~m}$ \\
\hline $\begin{array}{l}\text { CG-NK } \\
\text { CG-HL } \\
\text { CG-SK }\end{array}$ & $\begin{array}{l}01^{\circ} 09^{\prime} \mathrm{S}, 29^{\circ} 29^{\prime} \mathrm{E} \\
08^{\circ} 16^{\prime} \mathrm{S}, 26^{\circ} 36^{\prime} \mathrm{E} \\
02^{\circ} 09^{\prime} \mathrm{S}, 28^{\circ} 54^{\prime} \mathrm{E}\end{array}$ & $\begin{array}{c}1250 \mathrm{~m} \\
556 \mathrm{~m} \\
1455 \mathrm{~m}\end{array}$ \\
\hline CG-SK & $02^{\circ} 09^{\prime} \mathrm{S}, 28^{\circ} 54^{\prime} \mathrm{E}$ & $1455 \mathrm{~m}$ \\
\hline $\begin{array}{l}\text { CG-TG } \\
\text { CG-MO } \\
\text { RW-ES }\end{array}$ & $\begin{array}{l}08^{\circ} 22^{\prime} \mathrm{S}, 29^{\circ} 15^{\prime} \mathrm{E} \\
03^{\circ} 11^{\prime} \mathrm{S}, 28^{\circ} 20^{\prime} \mathrm{E} \\
01^{\circ} 30^{\prime} \mathrm{S}, 30^{\circ} 30^{\prime} \mathrm{E}\end{array}$ & $1800 \mathrm{~m}$ \\
\hline CG-NK & $01^{\circ} 13^{\prime} \mathrm{S}, 29^{\circ} 28^{\prime} \mathrm{E}$ & $1250 \mathrm{~m}$ \\
\hline $\begin{array}{c}\text { CG } \\
\text { CG } \\
\text { CG-SK } \\
\text { CG-MN } \\
\text { CG-SK }\end{array}$ & $\begin{array}{l}02^{\circ} 00^{\prime} \mathrm{S}, 28^{\circ} 45^{\prime} \mathrm{E} \\
02^{\circ} 38^{\prime} \mathrm{S}, 26^{\circ} 06^{\prime} \mathrm{E} \\
02^{\circ} 09^{\prime} \mathrm{S}, 28^{\circ} 54^{\prime} \mathrm{E}\end{array}$ & $\begin{array}{c}602 \mathrm{~m} \\
1455 \mathrm{~m}\end{array}$ \\
\hline CG-SK & $02^{\circ} 09^{\prime} \mathrm{S}, 28^{\circ} 54^{\prime} \mathrm{E}$ & $1455 \mathrm{~m}$ \\
\hline $\begin{array}{l}\text { CG-SK } \\
\text { CG-LB }\end{array}$ & $\begin{array}{l}03^{\circ} 10^{\prime} \mathrm{S}, 28^{\circ} 21^{\prime} \mathrm{E} \\
10^{\circ} 47^{\prime} \mathrm{S}, 26^{\circ} 36^{\prime} \mathrm{E}\end{array}$ & $\begin{array}{l}1525 \mathrm{~m} \\
1438 \mathrm{~m}\end{array}$ \\
\hline CG-LB & $10^{\circ} 47^{\prime} \mathrm{S}, 26^{\circ} 36^{\prime} \mathrm{E}$ & $1438 \mathrm{~m}$ \\
\hline $\begin{array}{l}\text { CG-LB } \\
\text { CG-SK }\end{array}$ & $\begin{array}{l}10^{\circ} 44^{\prime} \mathrm{S}, 26^{\circ} 23^{\prime} \mathrm{E} \\
02^{\circ} 16^{\prime} \mathrm{S}, 28^{\circ} 51^{\prime} \mathrm{E}\end{array}$ & \\
\hline CG-SK & $02^{\circ} 16^{\prime} \mathrm{S}, 28^{\circ} 51^{\prime} \mathrm{E}$ & \\
\hline
\end{tabular}




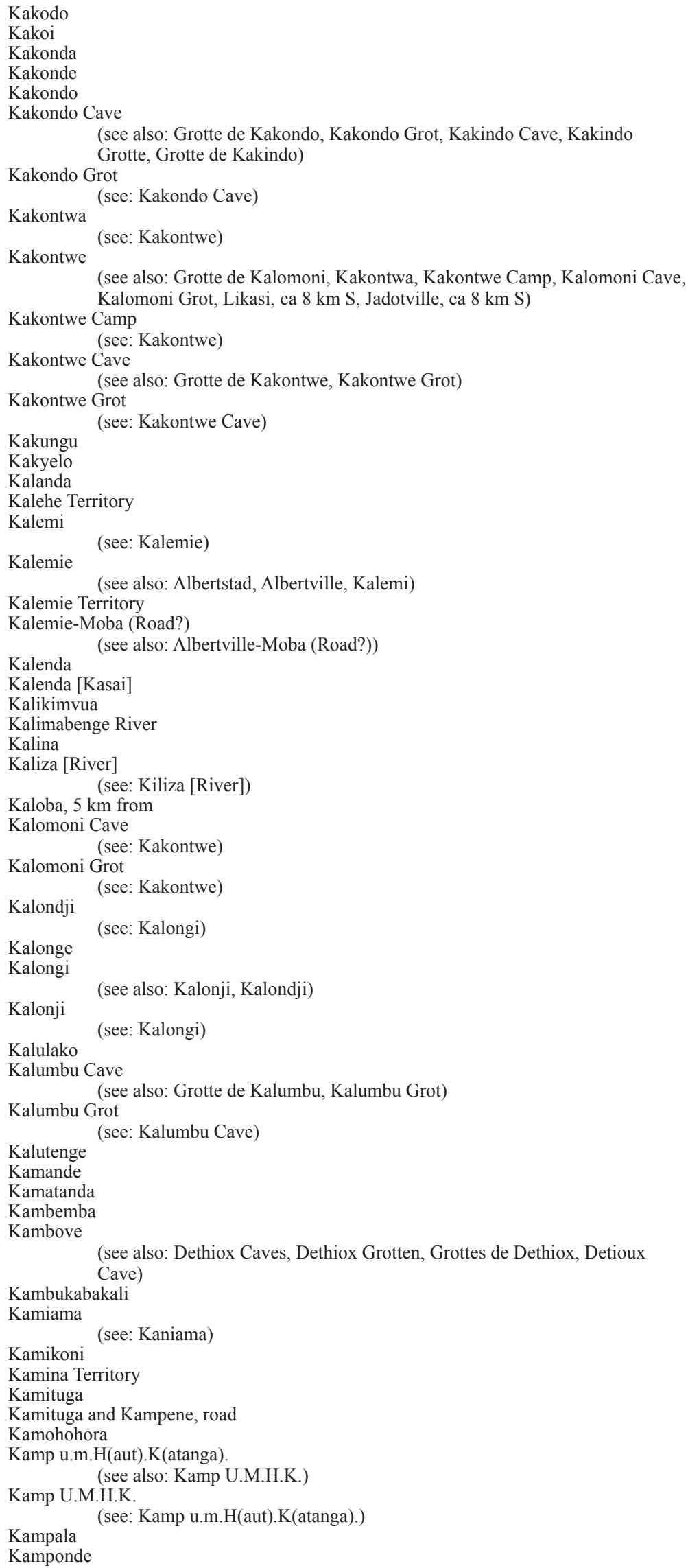

$\mathrm{CG}-\mathrm{KU}$

$\mathrm{CG}$

CG-TG

CG-SK

$\mathrm{CG}-\mathrm{SK}$

CG-SK

CG-HK

CG-HK

CG-HK

CG-HK

CG-HK

CG-NK

$\mathrm{CG}-\mathrm{HK}$

CG

CG-SK

CG-TG

CG-TG

CG-TG

CG-TG

$\mathrm{CG}-\mathrm{KO}$

CG-LM

CG-HU

$\mathrm{CG}-\mathrm{SK}$

CG-KN

CG-SK

CG-HK

CG-HK

CG-HK

CG-KO

CG-NK

CG-KO

$\mathrm{CG}-\mathrm{KO}$

CG-HK

CG-HK

CG-HK

CG-LB

CG-NK

CG-LB

CG-NK

CG-LB

CG-NK

CG-HL

CG-NK

CG-HL

CG-SK

$\mathrm{CG}-\mathrm{SK}$

CG-NK

CG-HK

CG-HK

CG-SK

CG-LL $04^{\circ} 40^{\prime} \mathrm{N}, 29^{\circ} 30^{\prime} \mathrm{E}$ $05^{\circ} 06^{\prime} \mathrm{S}, 18^{\circ} 56^{\prime} \mathrm{E}$ $05^{\circ} 50^{\prime} \mathrm{S}, 28^{\circ} 42^{\prime} \mathrm{E}$ $07^{\circ} 52^{\prime} \mathrm{S}, 29^{\circ} 43^{\prime} \mathrm{E}$ $02^{\circ} 15^{\prime} \mathrm{S}, 28^{\circ} 42^{\prime} \mathrm{E}$ $02^{\circ} 16^{\prime} \mathrm{S}, 28^{\circ} 51^{\prime} \mathrm{E}$

$451 \mathrm{~m}$ $800 \mathrm{~m}$

$3051 \mathrm{~m}$

$02^{\circ} 16^{\prime} \mathrm{S}, 28^{\circ} 51^{\prime} \mathrm{E}$

$10^{\circ} 59^{\prime} \mathrm{S}, 26^{\circ} 40^{\prime} \mathrm{E}$

$1253 \mathrm{~m}$

$10^{\circ} 59^{\prime} \mathrm{S}, 26^{\circ} 40^{\prime} \mathrm{E} \quad 1253 \mathrm{~m}$

$10^{\circ} 59^{\prime} \mathrm{S}, 26^{\circ} 40^{\prime} \mathrm{E} \quad 1253 \mathrm{~m}$

$10^{\circ} 59^{\prime} \mathrm{S}, 26^{\circ} 38^{\prime} \mathrm{E} \quad 1294 \mathrm{~m}$

$10^{\circ} 59^{\prime} \mathrm{S}, 26^{\circ} 38^{\prime} \mathrm{E} \quad 1294 \mathrm{~m}$

$00^{\circ} 07^{\prime} \mathrm{S}, 29^{\circ} 37^{\prime} \mathrm{E} \quad 925 \mathrm{~m}$

$12^{\circ} 21^{\prime} \mathrm{S}, 29^{\circ} 35^{\prime} \mathrm{E}$

ca $02^{\circ} 06^{\prime} \mathrm{S}, 28^{\circ} 55^{\prime} \mathrm{E}$ $05^{\circ} 56^{\prime} \mathrm{S}, 29^{\circ} 12^{\prime} \mathrm{E} \quad 763 \mathrm{~m}$

$05^{\circ} 56^{\prime} \mathrm{S}, 29^{\circ} 12^{\prime} \mathrm{E} \quad 763 \mathrm{~m}$

$05^{\circ} 56^{\prime} \mathrm{S}, 29^{\circ} 12^{\prime} \mathrm{E}$ $05^{\circ} 57^{\prime} \mathrm{S}, 29^{\circ} 01^{\prime} \mathrm{E}$

$07^{\circ} 08^{\prime} \mathrm{S}, 23^{\circ} 32^{\prime} \mathrm{E}$ $07^{\circ} 08^{\prime} \mathrm{S}, 23^{\circ} 32^{\prime} \mathrm{E}$ $04^{\circ} 15^{\prime} \mathrm{N}, 29^{\circ} 45^{\prime} \mathrm{E}$ $03^{\circ} 25^{\prime} \mathrm{S}, 29^{\circ} 09^{\prime} \mathrm{E}$ $04^{\circ} 22^{\prime} \mathrm{S}, 15^{\circ} 17^{\prime} \mathrm{E}$ $03^{\circ} 22^{\prime} \mathrm{S}, 28^{\circ} 01^{\prime} \mathrm{E}$

$821 \mathrm{~m}$

$821 \mathrm{~m}$

$128 \mathrm{~m}$

ca $10^{\circ} 16^{\prime} \mathrm{S}, 27^{\circ} 37^{\prime} \mathrm{E}$ $10^{\circ} 59^{\prime} \mathrm{S}, 26^{\circ} 40^{\prime} \mathrm{E}$

$1294 \mathrm{~m}$ $10^{\circ} 59^{\prime} \mathrm{S}, 26^{\circ} 40^{\prime} \mathrm{E}$

$1294 \mathrm{~m}$

$799 \mathrm{~m}$

$2455 \mathrm{~m}$ $799 \mathrm{~m}$

$06^{\circ} 14^{\prime} \mathrm{S}, 23^{\circ} 23^{\prime} \mathrm{E}$

$799 \mathrm{~m}$

$11^{\circ} 42^{\prime} \mathrm{S}, 27^{\circ} 34^{\prime} \mathrm{E}$ $10^{\circ} 51^{\prime} \mathrm{S}, 26^{\circ} 39^{\prime} \mathrm{E}$

$10^{\circ} 51^{\prime} \mathrm{S}, 26^{\circ} 39^{\prime} \mathrm{E}$

$10^{\circ} 30^{\prime} \mathrm{S}, 25^{\circ} 37^{\prime} \mathrm{E}$ $00^{\circ} 35^{\prime} \mathrm{S}, 29^{\circ} 18^{\prime} \mathrm{E}$ $10^{\circ} 57^{\prime} \mathrm{S}, 26^{\circ} 46^{\prime} \mathrm{E}$ $00^{\circ} 34^{\prime} \mathrm{N}, 29^{\circ} 41^{\prime} \mathrm{E}$ $10^{\circ} 50^{\prime} \mathrm{S}, 26^{\circ} 39^{\prime} \mathrm{E}$

$925 \mathrm{~m}$

$800 \mathrm{~m}$

$00^{\circ} 01^{\prime} \mathrm{S}, 29^{\circ} 36^{\prime} \mathrm{E}$ $07^{\circ} 31^{\prime} \mathrm{S}, 24^{\circ} 11^{\prime} \mathrm{E}$

$930 \mathrm{~m}$ $01^{\circ} 16^{\prime} \mathrm{S}, 29^{\circ} 19^{\prime} \mathrm{E}$ $08^{\circ} 44^{\prime} \mathrm{S}, 25^{\circ} 00^{\prime} \mathrm{E}$ $03^{\circ} 03^{\prime} \mathrm{S}, 28^{\circ} 11^{\prime} \mathrm{E}$ ca $03^{\circ} 38^{\prime} \mathrm{S}, 27^{\circ} 16^{\prime} \mathrm{E}$ $00^{\circ} 40^{\prime} \mathrm{S}, 29^{\circ} 33^{\prime} \mathrm{E}$ $11^{\circ} 41^{\prime} \mathrm{S}, 27^{\circ} 29^{\prime} \mathrm{E}$

$11^{\circ} 41^{\prime} \mathrm{S}, 27^{\circ} 29^{\prime} \mathrm{E}$

$02^{\circ} 12^{\prime} \mathrm{S}, 27^{\circ} 45^{\prime} \mathrm{E}$ $06^{\circ} 42^{\prime} \mathrm{S}, 22^{\circ} 56^{\prime} \mathrm{E}$ 


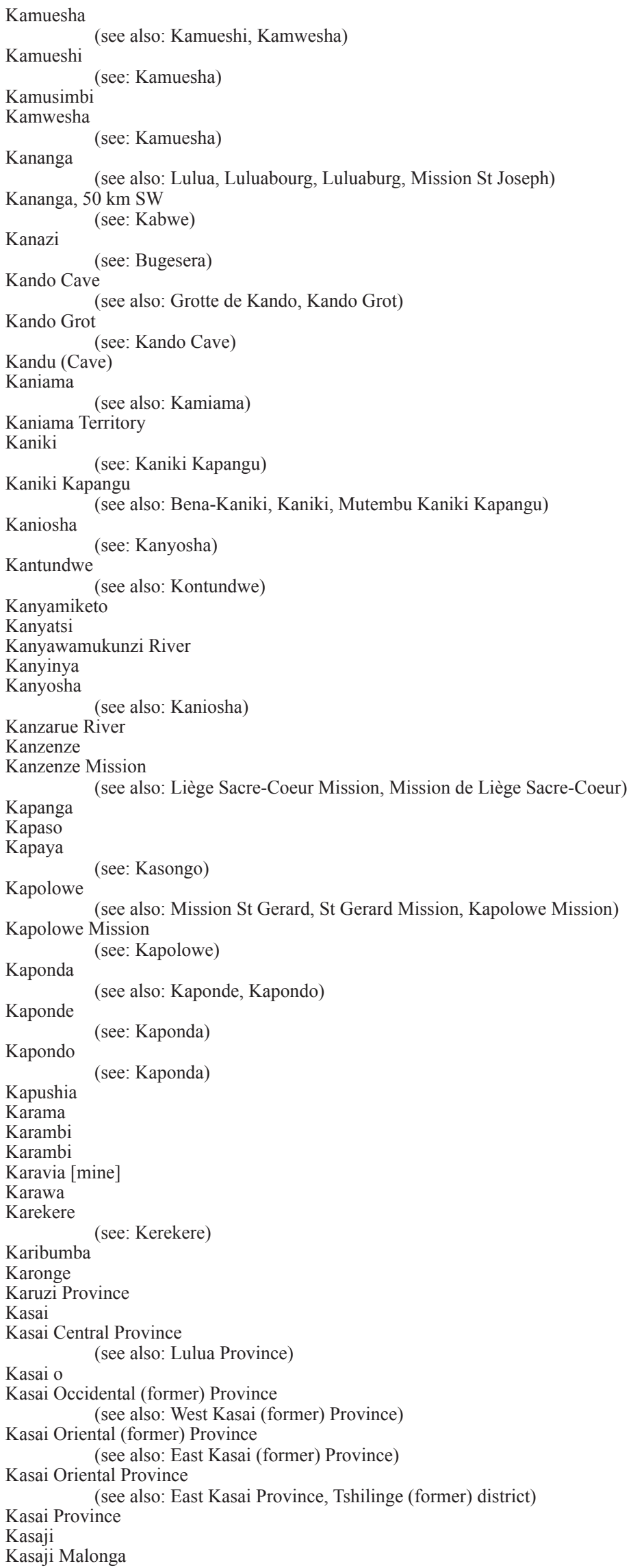

\begin{tabular}{|c|c|c|}
\hline CG-TG & $06^{\circ} 12^{\prime} \mathrm{S}, 21^{\circ} 26^{\prime} \mathrm{E}$ & $608 \mathrm{~m}$ \\
\hline CG-TG & $06^{\circ} 12^{\prime} \mathrm{S}, 21^{\circ} 26^{\prime} \mathrm{E}$ & $608 \mathrm{~m}$ \\
\hline CG-SK & $03^{\circ} 01^{\prime} \mathrm{S}, 28^{\circ} 13^{\prime} \mathrm{E}$ & \\
\hline CG-TG & $06^{\circ} 12^{\prime} \mathrm{S}, 21^{\circ} 26^{\prime} \mathrm{E}$ & $608 \mathrm{~m}$ \\
\hline CG-LL & $05^{\circ} 54^{\prime} \mathrm{S}, 22^{\circ} 25^{\prime} \mathrm{E}$ & $608 \mathrm{~m}$ \\
\hline CG-LL & $06^{\circ} 12^{\prime} \mathrm{S}, 22^{\circ} 23^{\prime} \mathrm{E}$ & $610 \mathrm{~m}$ \\
\hline RW-ES & $02^{\circ} 09^{\prime} \mathrm{S}, 30^{\circ} 05^{\prime} \mathrm{E}$ & $1432 \mathrm{~m}$ \\
\hline CG-HK & $10^{\circ} 49^{\prime} \mathrm{S}, 26^{\circ} 07^{\prime} \mathrm{E}$ & \\
\hline CG-HK & $10^{\circ} 49^{\prime} \mathrm{S}, 26^{\circ} 07^{\prime} \mathrm{E}$ & \\
\hline CG-LB & $09^{\circ} 56^{\prime} \mathrm{S}, 25^{\circ} 58^{\prime} \mathrm{E}$ & \\
\hline CG-HL & $07^{\circ} 31^{\prime} \mathrm{S}, 24^{\circ} 11^{\prime} \mathrm{E}$ & $887 \mathrm{~m}$ \\
\hline CG-HL & $07^{\circ} 31^{\prime} \mathrm{S}, 24^{\circ} 11^{\prime} \mathrm{E}$ & \\
\hline & & \\
\hline CG-KO & $06^{\circ} 45^{\prime} \mathrm{S}, 23^{\circ} 31^{\prime} \mathrm{E}$ & $805 \mathrm{~m}$ \\
\hline BY-BM & $03^{\circ} 25^{\prime} \mathrm{S}, 29^{\circ} 22^{\prime} \mathrm{E}$ & $844 \mathrm{~m}$ \\
\hline CG-NK & $01^{\circ} 10^{\prime} \mathrm{S}, 29^{\circ} 28^{\prime} \mathrm{E}$ & $1150 \mathrm{~m}$ \\
\hline RW-NO & $01^{\circ} 26^{\prime} \mathrm{S}, 29^{\circ} 35^{\prime} \mathrm{E}$ & \\
\hline CG-NK & $00^{\circ} 08^{\prime} \mathrm{S}, 29^{\circ} 37^{\prime} \mathrm{E}$ & $940 \mathrm{~m}$ \\
\hline CG-NK & $01^{\circ} 10^{\prime} \mathrm{S}, 29^{\circ} 34^{\prime} \mathrm{E}$ & $1300 \mathrm{~m}$ \\
\hline BY-KI & $02^{\circ} 35^{\prime} \mathrm{S}, 30^{\circ} 05^{\prime} \mathrm{E}$ & $1508 \mathrm{~m}$ \\
\hline BY-BM & $03^{\circ} 25^{\prime} \mathrm{S}, 29^{\circ} 22^{\prime} \mathrm{E}$ & $844 \mathrm{~m}$ \\
\hline CG-NK & $01^{\circ} 09^{\prime} \mathrm{S}, 29^{\circ} 24^{\prime} \mathrm{E}$ & $1150 \mathrm{~m}$ \\
\hline CG-NK & $01^{\circ} 23^{\prime} \mathrm{S}, 29^{\circ} 13^{\prime} \mathrm{E}$ & $2343 \mathrm{~m}$ \\
\hline CG-LB & $10^{\circ} 31^{\prime} \mathrm{S}, 25^{\circ} 12^{\prime} \mathrm{E}$ & $1157 \mathrm{~m}$ \\
\hline CG-LB & $08^{\circ} 21^{\prime} \mathrm{S}, 22^{\circ} 35^{\prime} \mathrm{E}$ & $881 \mathrm{~m}$ \\
\hline CG-LB & $11^{\circ} 40^{\prime} \mathrm{S}, 27^{\circ} 29^{\prime} \mathrm{E}$ & \\
\hline CG-MN & $04^{\circ} 27^{\prime} \mathrm{S}, 26^{\circ} 40^{\prime} \mathrm{E}$ & $625 \mathrm{~m}$ \\
\hline CG-HK & $11^{\circ} 02^{\prime} \mathrm{S}, 26^{\circ} 57^{\prime} \mathrm{E}$ & $1135 \mathrm{~m}$ \\
\hline CG-HK & $11^{\circ} 02^{\prime} \mathrm{S}, 26^{\circ} 57^{\prime} \mathrm{E}$ & $1135 \mathrm{~m}$ \\
\hline CG-HK & $11^{\circ} 45^{\prime} \mathrm{S}, 27^{\circ} 20^{\prime} \mathrm{E}$ & $1274 \mathrm{~m}$ \\
\hline CG-HK & $11^{\circ} 45^{\prime} \mathrm{S}, 27^{\circ} 20^{\prime} \mathrm{E}$ & $1274 \mathrm{~m}$ \\
\hline CG-HK & $11^{\circ} 45^{\prime} \mathrm{S}, 27^{\circ} 20^{\prime} \mathrm{E}$ & $1274 \mathrm{~m}$ \\
\hline CG-LB & $10^{\circ} 41^{\prime} \mathrm{S}, 22^{\circ} 52^{\prime} \mathrm{E}$ & $965 \mathrm{~m}$ \\
\hline RW-ES & $02^{\circ} 16^{\prime} \mathrm{S}, 30^{\circ} 16^{\prime} \mathrm{E}$ & \\
\hline CG-NK & $00^{\circ} 39^{\prime} \mathrm{S}, 29^{\circ} 21^{\prime} \mathrm{E}$ & $896 \mathrm{~m}$ \\
\hline CG-SK & $02^{\circ} 45^{\prime} \mathrm{S}, 28^{\circ} 38^{\prime} \mathrm{E}$ & \\
\hline CG-HK & $11^{\circ} 39^{\prime} \mathrm{S}, 27^{\circ} 17^{\prime} \mathrm{E}$ & \\
\hline CG-MA & $03^{\circ} 20^{\prime} \mathrm{N}, 20^{\circ} 18^{\prime} \mathrm{E}$ & $453 \mathrm{~m}$ \\
\hline CG-IT & $02^{\circ} 42^{\prime} \mathrm{N}, 30^{\circ} 33^{\prime} \mathrm{E}$ & $1158 \mathrm{~m}$ \\
\hline CG-NK & $00^{\circ} 22^{\prime} \mathrm{N}, 2^{\circ} 22^{\prime} \mathrm{E}$ & $1412 \mathrm{~m}$ \\
\hline BY-BB & $03^{\circ} 03^{\prime} \mathrm{S}, 29^{\circ} 20^{\prime} \mathrm{E}$ & $1226 \mathrm{~m}$ \\
\hline BY & & \\
\hline CG & & \\
\hline CG & & \\
\hline CG & & \\
\hline CG & & \\
\hline CG & & \\
\hline $\mathrm{CG}$ & & \\
\hline CG & & \\
\hline $\begin{array}{l}\text { CG-LB } \\
\text { CG-LB }\end{array}$ & $\begin{array}{l}10^{\circ} 22^{\prime} \mathrm{S}, 23^{\circ} 27^{\prime} \mathrm{E} \\
10^{\circ} 27^{\prime} \mathrm{S}, 23^{\circ} 27^{\prime} \mathrm{E}\end{array}$ & $1087 \mathrm{~m}$ \\
\hline
\end{tabular}


VAN CAKENBERGHE V. et al., The bats of Congo, Rwanda and Burundi revisited

\begin{tabular}{|c|c|c|c|}
\hline Kasaka & CG-LM & $05^{\circ} 07^{\prime} \mathrm{S}, 26^{\circ} 09^{\prime} \mathrm{E}$ & $618 \mathrm{~m}$ \\
\hline Kasaka & CG-NK & $00^{\circ} 05^{\prime} \mathrm{N}, 29^{\circ} 36^{\prime} \mathrm{E}$ & $900 \mathrm{~m}$ \\
\hline $\begin{array}{l}\text { Kasambongo } \\
\text { (see also. Cane Cassa Mabonoo) }\end{array}$ & CG-SK & $03^{\circ} 24^{\prime} \mathrm{S}, 29^{\circ} 08^{\prime} \mathrm{E}$ & \\
\hline Kasapa & CG-HK & $11^{\circ} 34^{\prime} \mathrm{S}, 27^{\circ} 25^{\prime} \mathrm{E}$ & $1243 \mathrm{~m}$ \\
\hline $\begin{array}{l}\text { Kasebaya River } \\
\text { (see: Kasebeya River) }\end{array}$ & CG-NK & $01^{\circ} 10^{\prime} \mathrm{S}, 29^{\circ} 28^{\prime} \mathrm{E}$ & $1350 \mathrm{~m}$ \\
\hline Kasebere & CG-NK & $01^{\circ} 49^{\prime} \mathrm{S}, 28^{\circ} 45^{\prime} \mathrm{E}$ & \\
\hline $\begin{array}{l}\text { Kasebeya River } \\
\text { (see also: Kasebaya River) }\end{array}$ & CG-NK & $01^{\circ} 10^{\prime} \mathrm{S}, 29^{\circ} 28^{\prime} \mathrm{E}$ & $1350 \mathrm{~m}$ \\
\hline Kasende & CG-LL & $05^{\circ} 57^{\prime} \mathrm{S}, 22^{\circ} 32^{\prime} \mathrm{E}$ & $619 \mathrm{~m}$ \\
\hline $\begin{array}{l}\text { Kasenga } \\
\text { (see also: Kasenga Mission, Mission Kasenga) }\end{array}$ & CG-HK & $10^{\circ} 22^{\prime} \mathrm{S}, 28^{\circ} 38^{\prime} \mathrm{E}$ & $938 \mathrm{~m}$ \\
\hline $\begin{array}{l}\text { Kasenga Mission } \\
\text { (see: Kasenga) }\end{array}$ & CG-HK & $10^{\circ} 22^{\prime} \mathrm{S}, 28^{\circ} 38^{\prime} \mathrm{E}$ & $938 \mathrm{~m}$ \\
\hline Kasenga Road, km 106 & CG-HK & $10^{\circ} 44^{\prime} \mathrm{S}, 28^{\circ} 26^{\prime} \mathrm{E}$ & \\
\hline $\begin{array}{l}\text { Kasenga Road, km } 36 \\
\text { (see also: Bulaga) }\end{array}$ & CG-HK & $11^{\circ} 37^{\prime} \mathrm{S}, 27^{\circ} 40^{\prime} \mathrm{E}$ & \\
\hline Kasenga Road, km 67 & CG-HK & $11^{\circ} 00^{\prime} \mathrm{S}, 28^{\circ} 07^{\prime} \mathrm{E}$ & \\
\hline Kasenga Territory & CG-HK & $10^{\circ} 22^{\prime} \mathrm{S}, 28^{\circ} 38^{\prime} \mathrm{E}$ & \\
\hline Kasengi & CG-NK & $02^{\circ} 08^{\prime} \mathrm{N}, 31^{\circ} 05^{\prime} \mathrm{E}$ & $1532 \mathrm{~m}$ \\
\hline $\begin{array}{ll}\text { Kasengu } & \text { (see: Kasengu) } \\
& \text { (see also: Kasengi) }\end{array}$ & CG-NK & $02^{\circ} 08^{\prime} \mathrm{N}, 31^{\circ} 05^{\prime} \mathrm{E}$ & $1532 \mathrm{~m}$ \\
\hline Kasenye $\quad$ (see also: Kasenyi, Keseny, Kiseny) & CG-HU & $01^{\circ} 24^{\prime} \mathrm{N}, 30^{\circ} 26^{\prime} \mathrm{E}$ & $671 \mathrm{~m}$ \\
\hline Kasenye, $10 \mathrm{~km} \mathrm{~N}$ & CG-IT & ca $01^{\circ} 25^{\prime} \mathrm{N}, 30^{\circ} 25^{\prime} \mathrm{E}$ & \\
\hline Kasenyi (see: Kasenye) & $\mathrm{CG}-\mathrm{HU}$ & $01^{\circ} 24^{\prime} \mathrm{N}, 30^{\circ} 26^{\prime} \mathrm{E}$ & $671 \mathrm{~m}$ \\
\hline Kaseu (see also: Kabinda, $129 \mathrm{~km} \mathrm{NNW}$ ) & CG-KO & $05^{\circ} 11^{\prime} \mathrm{S}, 23^{\circ} 51^{\prime} \mathrm{E}$ & \\
\hline $\begin{array}{l}\text { Kashiobwe (see also: Kashiobwe Mission, Mission Kashiobwe) } \\
\text { (a) }\end{array}$ & CG-HK & $09^{\circ} 39^{\prime} \mathrm{S}, 28^{\circ} 37^{\prime} \mathrm{E}$ & $918 \mathrm{~m}$ \\
\hline $\begin{array}{l}\text { Kashiobwe Mission } \\
\text { (see: Kashiobwe) }\end{array}$ & CG-HK & $09^{\circ} 39^{\prime} \mathrm{S}, 28^{\circ} 37^{\prime} \mathrm{E}$ & $918 \mathrm{~m}$ \\
\hline $\begin{array}{l}\text { Kashwa } \\
\text { Kasiba }\end{array}$ & $\begin{array}{l}\text { CG-NK } \\
\text { CG-SK }\end{array}$ & $\begin{array}{c}01^{\circ} 18^{\prime} \mathrm{S}, 29^{\circ} 06^{\prime} \mathrm{E} \\
\text { ca } 01^{\circ} 54^{\prime} \mathrm{S}, 28^{\circ} 27^{\prime} \mathrm{E}\end{array}$ & $2000 \mathrm{~m}$ \\
\hline Kasindi (see also: Irangi, $2 \mathrm{mi}[=3.2 \mathrm{~km}]$ from) & CG-NK & $00^{\circ} 02^{\prime} \mathrm{S}, 29^{\circ} 43^{\prime} \mathrm{E}$ & $1049 \mathrm{~m}$ \\
\hline $\begin{array}{l}\text { (see also: Kasindi Vieux, Kasindi-port, Old Kasindi) } \\
\text { Kasindi Vieux } \\
\text { (see: Kasindi) }\end{array}$ & CG-NK & $00^{\circ} 02^{\prime} \mathrm{S}, 29^{\circ} 43^{\prime} \mathrm{E}$ & $1049 \mathrm{~m}$ \\
\hline $\begin{array}{l}\text { Kasindi-port } \\
\text { (see: Kasindi) }\end{array}$ & CG-NK & $00^{\circ} 02^{\prime} \mathrm{S}, 29^{\circ} 43^{\prime} \mathrm{E}$ & $1049 \mathrm{~m}$ \\
\hline Kasoma & CG-HL & $09^{\circ} 25^{\prime} \mathrm{S}, 26^{\circ} 37^{\prime} \mathrm{E}$ & \\
\hline $\begin{array}{l}\text { Kasoma Cave } \\
\text { (see also: Grotte de Kasoma, Kasoma Grot) }\end{array}$ & CG-HL & $09^{\circ} 25^{\prime} \mathrm{S}, 26^{\circ} 37^{\prime} \mathrm{E}$ & \\
\hline $\begin{array}{l}\text { Kasoma Grot } \\
\text { (see: Kasoma Cave) }\end{array}$ & CG-HL & $09^{\circ} 25^{\prime} \mathrm{S}, 26^{\circ} 37^{\prime} \mathrm{E}$ & \\
\hline Kasombo & CG-HK & & \\
\hline $\begin{array}{l}\text { Kasonga-Banze } \\
\text { (see also: Kasongo Baza, Kasongo-Banze) }\end{array}$ & CG-LL & $05^{\circ} 36^{\prime} \mathrm{S}, 23^{\circ} 24^{\prime} \mathrm{E}$ & $532 \mathrm{~m}$ \\
\hline Kasongo $\quad$ (see also: Kapaya, Kazongo, Tongoni) & CG-MN & $04^{\circ} 27^{\prime} \mathrm{S}, 26^{\circ} 40^{\prime} \mathrm{E}$ & $625 \mathrm{~m}$ \\
\hline $\begin{array}{l}\text { Kasongo Baza } \\
\text { (see: Kasonga-Banze) }\end{array}$ & CG-LL & $05^{\circ} 36^{\prime} \mathrm{S}, 23^{\circ} 24^{\prime} \mathrm{E}$ & $532 \mathrm{~m}$ \\
\hline Kasongo Mwano & CG-HK & $08^{\circ} 44^{\prime} \mathrm{S}, 27^{\circ} 51^{\prime} \mathrm{E}$ & \\
\hline Kasongo Samba & CG-MN & $04^{\circ} 30^{\prime} \mathrm{S}, 26^{\circ} 40^{\prime} \mathrm{E}$ & \\
\hline Kasongo Territory & CG-MN & $04^{\circ} 27^{\prime} \mathrm{S}, 26^{\circ} 40^{\prime} \mathrm{E}$ & \\
\hline $\begin{array}{l}\text { Kasongo-Banze } \\
\text { (see: Kasonga-Banze) }\end{array}$ & CG-LL & $05^{\circ} 36^{\prime} \mathrm{S}, 23^{\circ} 24^{\prime} \mathrm{E}$ & $532 \mathrm{~m}$ \\
\hline $\begin{array}{l}\text { Kasowena Cave } \\
\text { (see also: Grotte de Kasowena, Kasowena Grot) }\end{array}$ & CG-HK & $10^{\circ} 58^{\prime} \mathrm{S}, 26^{\circ} 39^{\prime} \mathrm{E}$ & \\
\hline $\begin{array}{l}\text { Kasowena Grot } \\
\text { (see: Kasowena Cave) }\end{array}$ & CG-HK & $10^{\circ} 58^{\prime} \mathrm{S}, 26^{\circ} 39^{\prime} \mathrm{E}$ & \\
\hline Kassi River & CG-HU & $04^{\circ} 15^{\prime} \mathrm{N}, 29^{\circ} 35^{\prime} \mathrm{E}$ & \\
\hline $\begin{array}{l}\text { Kasuo Cave Kabwe ka } \\
\text { Kaswabilenga }\end{array}$ & CG-LM & $06^{\circ} 37^{\prime} \mathrm{S}, 24^{\circ} 58^{\prime} \mathrm{E}$ & \\
\hline $\begin{array}{l}\text { Kaswabilenga } \\
\text { Kaswabilenga }\end{array}$ & $\begin{array}{c}\text { CG } \\
\text { CG-HL }\end{array}$ & $08^{\circ} 48^{\prime} \mathrm{S} .26^{\circ} 41^{\prime} \mathrm{F}$ & $700 \mathrm{~m}$ \\
\hline Katabayi & CG-KS & $06^{\circ} 11^{\prime} \mathrm{S}, 21^{\circ} 20^{\prime} \mathrm{E}$ & \\
\hline Katana & CG-SK & $02^{\circ} 14^{\prime} \mathrm{S}, 28^{\circ} 50^{\prime} \mathrm{E}$ & $1595 \mathrm{~m}$ \\
\hline $\begin{array}{l}\text { (see also: Liège Saint Lambert) } \\
\text { Katana Cave } \\
\text { (see: Mahyusha Cave) }\end{array}$ & CG-SK & $02^{\circ} 13^{\prime} \mathrm{S}, 28^{\circ} 50^{\prime} \mathrm{E}$ & $1480 \mathrm{~m}$ \\
\hline Katana Grot & CG-SK & $02^{\circ} 13^{\prime} \mathrm{S}, 28^{\circ} 50^{\prime} \mathrm{E}$ & $1480 \mathrm{~m}$ \\
\hline
\end{tabular}




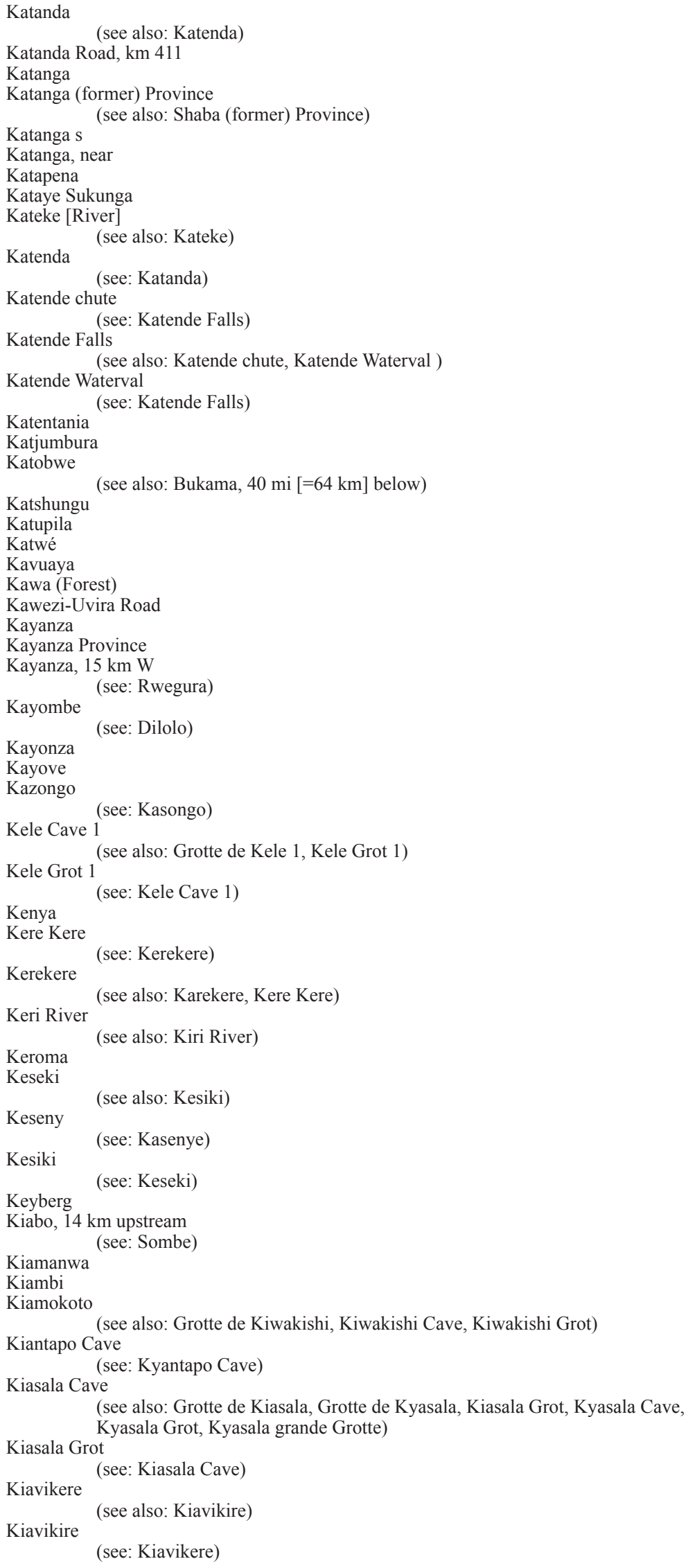

CG-NK

CG

$\mathrm{CG}$

CG

CG

$\mathrm{CG}-\mathrm{HK}$

CG-KS

CG-HL

ca $08^{\circ} 35^{\prime} \mathrm{S}, 26^{\circ} 25^{\prime} \mathrm{E}$

$09^{\circ} 04^{\prime} \mathrm{S}, 26^{\circ} 43^{\prime} \mathrm{E}$

CG-NK

$00^{\circ} 51^{\prime} \mathrm{S}, 29^{\circ} 22^{\prime} \mathrm{E}$

$975 \mathrm{~m}$

CG-KS

CG-KS

$05^{\circ} 46^{\prime} \mathrm{S}, 21^{\circ} 40^{\prime} \mathrm{E}$

$647 \mathrm{~m}$

CG-KS

$05^{\circ} 46^{\prime} \mathrm{S}, 21^{\circ} 40^{\prime} \mathrm{E}$

$647 \mathrm{~m}$

CG-LB

RW-ES

CG-HL

$05^{\circ} 46^{\prime} \mathrm{S}, 21^{\circ} 40^{\prime} \mathrm{E}$

$647 \mathrm{~m}$

$10^{\circ} 19^{\prime} \mathrm{S}, 25^{\circ} 54^{\prime} \mathrm{E}$

$01^{\circ} 33^{\prime} \mathrm{S}, 30^{\circ} 44^{\prime} \mathrm{E}$

$08^{\circ} 54^{\prime} \mathrm{S}, 26^{\circ} 04^{\prime} \mathrm{E}$

$1597 \mathrm{~m}$

$610 \mathrm{~m}$

CG-SK

CG-HL

$02^{\circ} 27^{\prime} \mathrm{S}, 27^{\circ} 23^{\prime} \mathrm{E}$

$08^{\circ} 50^{\prime} \mathrm{S}, 26^{\circ} 44^{\prime} \mathrm{E}$

$10^{\circ} 28^{\prime} \mathrm{S}, 27^{\circ} 51^{\prime} \mathrm{E}$

$05^{\circ} 20^{\prime} \mathrm{S}, 14^{\circ} 52^{\prime} \mathrm{E}$

CG-IT

CG-SK

$01^{\circ} 34^{\prime} \mathrm{N}, 30^{\circ} 32^{\prime} \mathrm{E}$ ca $03^{\circ} 19^{\prime} \mathrm{S}, 29^{\circ} 10^{\prime} \mathrm{E}$

BY-KY

BY

BY-KY

$02^{\circ} 55^{\prime} \mathrm{S}, 29^{\circ} 37^{\prime} \mathrm{E}$

$02^{\circ} 55^{\prime} \mathrm{S}, 29^{\circ} 31^{\prime} \mathrm{E}$

CG-LB

RW-ES

RW-OU

CG-MN

$10^{\circ} 28^{\prime} \mathrm{S}, 22^{\circ} 28^{\prime} \mathrm{E}$

$01^{\circ} 57^{\prime} \mathrm{S}, 30^{\circ} 31^{\prime} \mathrm{E}$

$01^{\circ} 53^{\prime} \mathrm{S}, 29^{\circ} 21^{\prime} \mathrm{E}$

$04^{\circ} 27^{\prime} \mathrm{S}, 26^{\circ} 40^{\prime} \mathrm{E}$

$1013 \mathrm{~m}$

$753 \mathrm{~m}$

$1957 \mathrm{~m}$

$2311 \mathrm{~m}$

$1069 \mathrm{~m}$

$2400 \mathrm{~m}$

$1841 \mathrm{~m}$

$625 \mathrm{~m}$

CG-BC

$05^{\circ} 31^{\prime} \mathrm{S}, 14^{\circ} 36^{\prime} \mathrm{E}$

CG-BC

$05^{\circ} 31^{\prime} \mathrm{S}, 14^{\circ} 36^{\prime} \mathrm{E}$

CG-HK

CG-IT

$11^{\circ} 44^{\prime} \mathrm{S}, 27^{\circ} 30^{\prime} \mathrm{E}$

$02^{\circ} 42^{\prime} \mathrm{N}, 30^{\circ} 33^{\prime} \mathrm{E}$

$1119 \mathrm{~m}$

CG-IT

$02^{\circ} 42^{\prime} \mathrm{N}, 30^{\circ} 33^{\prime} \mathrm{E}$

$1158 \mathrm{~m}$

CG-MA

$01^{\circ} 29^{\prime} \mathrm{S}, 18^{\circ} 58^{\prime} \mathrm{E}$

CG-HU $\quad 04^{\circ} 35^{\prime} \mathrm{N}, 29^{\circ} 32^{\prime} \mathrm{E}$

CG-MA $\quad 02^{\circ} 07^{\prime} \mathrm{S}, 16^{\circ} 32^{\prime} \mathrm{E}$

CG-HU

$01^{\circ} 24^{\prime} \mathrm{N}, 30^{\circ} 26^{\prime} \mathrm{E}$

$308 \mathrm{~m}$

CG-MA

$02^{\circ} 07^{\prime} \mathrm{S}, 16^{\circ} 32^{\prime} \mathrm{E}$

$671 \mathrm{~m}$

CG-HK

$11^{\circ} 43^{\prime} \mathrm{S}, 27^{\circ} 25^{\prime} \mathrm{E}$ $08^{\circ} 50^{\prime} \mathrm{S}, 26^{\circ} 02^{\prime} \mathrm{E}$

CG-HK

$09^{\circ} 30^{\prime} \mathrm{S}, 27^{\circ} 55^{\prime} \mathrm{E}$

CG-TG $\quad 07^{\circ} 20^{\prime} \mathrm{S}, 28^{\circ} 01^{\prime} \mathrm{E}$

CG-HL $\quad 09^{\circ} 10^{\prime} \mathrm{S}, 27^{\circ} 04^{\prime} \mathrm{E}$

$607 \mathrm{~m}$ $900 \mathrm{~m}$

CG-LB $\quad 09^{\circ} 56^{\prime} \mathrm{S}, 25^{\circ} 58^{\prime} \mathrm{E} \quad 1525 \mathrm{~m}$

$09^{\circ} 56^{\prime} \mathrm{S}, 25^{\circ} 58^{\prime} \mathrm{E}$

$1300 \mathrm{~m}$

CG-LB

$09^{\circ} 56^{\prime} \mathrm{S}, 25^{\circ} 58^{\prime} \mathrm{E} \quad 1300 \mathrm{~m}$

CG-NK

$00^{\circ} 24^{\prime} \mathrm{N}, 29^{\circ} 46^{\prime} \mathrm{E} \quad 1553 \mathrm{~m}$

CG-NK $\quad 00^{\circ} 24^{\prime} \mathrm{N}, 2^{\circ} 46^{\prime} \mathrm{E} \quad 1553 \mathrm{~m}$ 


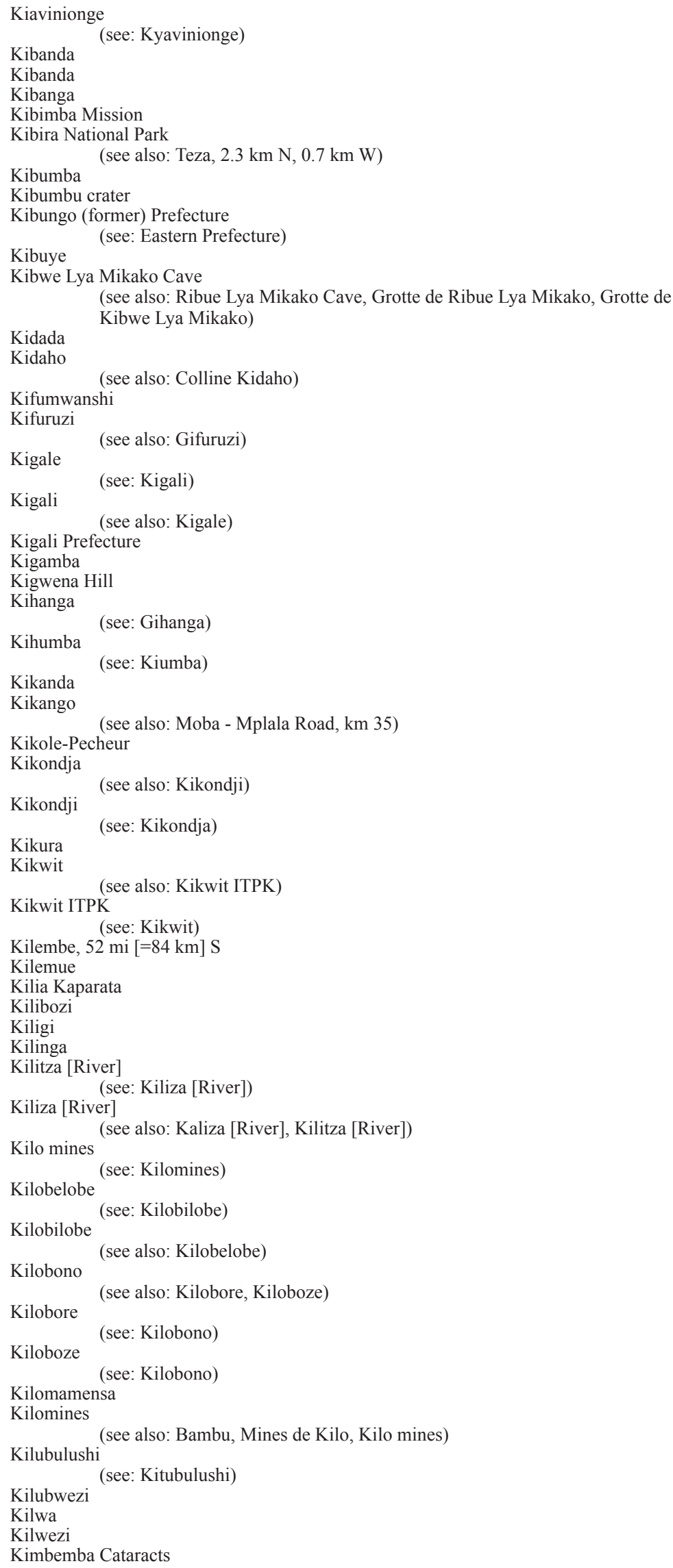

CG-TO

CG-HK

CG-SK

CG-HL

BY-GI

BY-MV

CG-NK

RW-NO

RW

RW-OU

CG-NK

CG-BC

RW-NO

CG-HK

BY-MA

RW-KV

RW-KV

RW

BY-CA

BY-RU

BY-BB

CG-SK

CG-HK

CG-TG

CG

CG-HL

CG-HL

CG-NK

CG-KU

CG-KU

CG-KU

CG-SK

CG-NK

CG-SK

CG-SK

CG-NK

CG-SK

CG-SK

CG-IT

CG-HK

CG-HK

CG-SK

CG-SK

CG-SK

CG

CG-IT

CG-HK

CG

CG-HK

CG-HL

CG-BC $00^{\circ} 07^{\prime} \mathrm{S}, 26^{\circ} 36^{\prime} \mathrm{E}$

$08^{\circ} 57^{\prime} \mathrm{S}, 27^{\circ} 38^{\prime} \mathrm{E}$

$03^{\circ} 20^{\prime} \mathrm{S}, 27^{\circ} 12^{\prime} \mathrm{E}$

$08^{\circ} 50^{\prime} \mathrm{S}, 26^{\circ} 44^{\prime} \mathrm{E}$

$03^{\circ} 18^{\prime} \mathrm{S}, 29^{\circ} 49^{\prime} \mathrm{E}$

$03^{\circ} 13^{\prime} \mathrm{S}, 29^{\circ} 34^{\prime} \mathrm{E}$

$01^{\circ} 28^{\prime} \mathrm{S}, 29^{\circ} 20^{\prime} \mathrm{E}$

$01^{\circ} 25^{\prime} \mathrm{S}, 29^{\circ} 33^{\prime} \mathrm{E}$

ca $02^{\circ} 00^{\prime} \mathrm{S}, 30^{\circ} 35^{\prime} \mathrm{E}$

$$
02^{\circ} 03^{\prime} \mathrm{S}, 29^{\circ} 21^{\prime} \mathrm{E}
$$

$00^{\circ} 15^{\prime} \mathrm{S}, 29^{\circ} 01^{\prime} \mathrm{E}$

$1557 \mathrm{~m}$

$1500 \mathrm{~m}$

$05^{\circ} 22^{\prime} \mathrm{S}, 14^{\circ} 32^{\prime} \mathrm{E}$ $01^{\circ} 22^{\prime} \mathrm{S}, 29^{\circ} 45^{\prime} \mathrm{E}$

$1944 \mathrm{~m}$

$11^{\circ} 27^{\prime} \mathrm{S}, 27^{\circ} 51^{\prime} \mathrm{E}$ $04^{\circ} 19^{\prime} \mathrm{S}, 29^{\circ} 35^{\prime} \mathrm{E}$

$01^{\circ} 57^{\prime} \mathrm{S}, 30^{\circ} 04^{\prime} \mathrm{E}$

$1567 \mathrm{~m}$

$01^{\circ} 57^{\prime} \mathrm{S}, 30^{\circ} 04^{\prime} \mathrm{E} \quad 1567 \mathrm{~m}$

$03^{\circ} 04^{\prime} \mathrm{S}, 30^{\circ} 31^{\prime} \mathrm{E} \quad 1532 \mathrm{~m}$ $04^{\circ} 09^{\prime} \mathrm{S}, 29^{\circ} 32^{\prime} \mathrm{E} \quad 918 \mathrm{~m}$ $03^{\circ} 12^{\prime} \mathrm{S}, 29^{\circ} 18^{\prime} \mathrm{E} \quad 814 \mathrm{~m}$

$01^{\circ} 56^{\prime} \mathrm{S}, 29^{\circ} 05^{\prime} \mathrm{E} \quad 1602 \mathrm{~m}$

$11^{\circ} 33^{\prime} \mathrm{S}, 27^{\circ} 37^{\prime} \mathrm{E}$ $06^{\circ} 37^{\prime} \mathrm{S}, 29^{\circ} 30^{\prime} \mathrm{E}$

$08^{\circ} 11^{\prime} \mathrm{S}, 26^{\circ} 26^{\prime} \mathrm{E}$

$607 \mathrm{~m}$

$08^{\circ} 11^{\prime} \mathrm{S}, 26^{\circ} 26^{\prime} \mathrm{E}$

$607 \mathrm{~m}$

$00^{\circ} 33^{\prime} \mathrm{N}, 29^{\circ} 57^{\prime} \mathrm{E}$ $05^{\circ} 04^{\prime} \mathrm{S}, 18^{\circ} 53^{\prime} \mathrm{E}$

$1900 \mathrm{~m}$ $438 \mathrm{~m}$

$438 \mathrm{~m}$

$05^{\circ} 47^{\prime} \mathrm{S}, 19^{\circ} 54^{\prime} \mathrm{E}$ $02^{\circ} 53^{\prime} \mathrm{S}, 28^{\circ} 07^{\prime} \mathrm{E}$ $00^{\circ} 23^{\prime} \mathrm{N}, 29^{\circ} 36^{\prime} \mathrm{E}$ $03^{\circ} 03^{\prime} \mathrm{S}, 28^{\circ} 09^{\prime} \mathrm{E}$ $02^{\circ} 53^{\prime} \mathrm{S}, 28^{\circ} 18^{\prime} \mathrm{E}$ $01^{\circ} 10^{\prime} \mathrm{S}, 28^{\circ} 29^{\prime} \mathrm{E}$ $03^{\circ} 22^{\prime} \mathrm{S}, 28^{\circ} 01^{\prime} \mathrm{E}$

$980 \mathrm{~m}$

$1275 \mathrm{~m}$ $03^{\circ} 22^{\prime} \mathrm{S}, 28^{\circ} 01^{\prime} \mathrm{E}$

$01^{\circ} 48^{\prime} \mathrm{N}, 30^{\circ} 14^{\prime} \mathrm{E}$

$1466 \mathrm{~m}$ $11^{\circ} 42^{\prime} \mathrm{S}, 27^{\circ} 34^{\prime} \mathrm{E}$ $11^{\circ} 42^{\prime} \mathrm{S}, 27^{\circ} 34^{\prime} \mathrm{E}$ $03^{\circ} 03^{\prime} \mathrm{S}, 28^{\circ} 11^{\prime} \mathrm{E}$ $03^{\circ} 03^{\prime} \mathrm{S}, 28^{\circ} 11^{\prime} \mathrm{E}$ $03^{\circ} 03^{\prime} \mathrm{S}, 28^{\circ} 11^{\prime} \mathrm{E}$ $01^{\circ} 48^{\prime} \mathrm{N}, 30^{\circ} 14^{\prime} \mathrm{E}$ $1466 \mathrm{~m}$ $12^{\circ} 44^{\prime} \mathrm{S}, 28^{\circ} 56^{\prime} \mathrm{E}$ $1257 \mathrm{~m}$ $09^{\circ} 18^{\prime} \mathrm{S}, 28^{\circ} 20^{\prime} \mathrm{E} \quad 918 \mathrm{~m}$ $09^{\circ} 06^{\prime} \mathrm{S}, 26^{\circ} 46^{\prime} \mathrm{E}$ ca $04^{\circ} 51^{\prime} \mathrm{S}, 14^{\circ} 06^{\prime} \mathrm{E}$ 


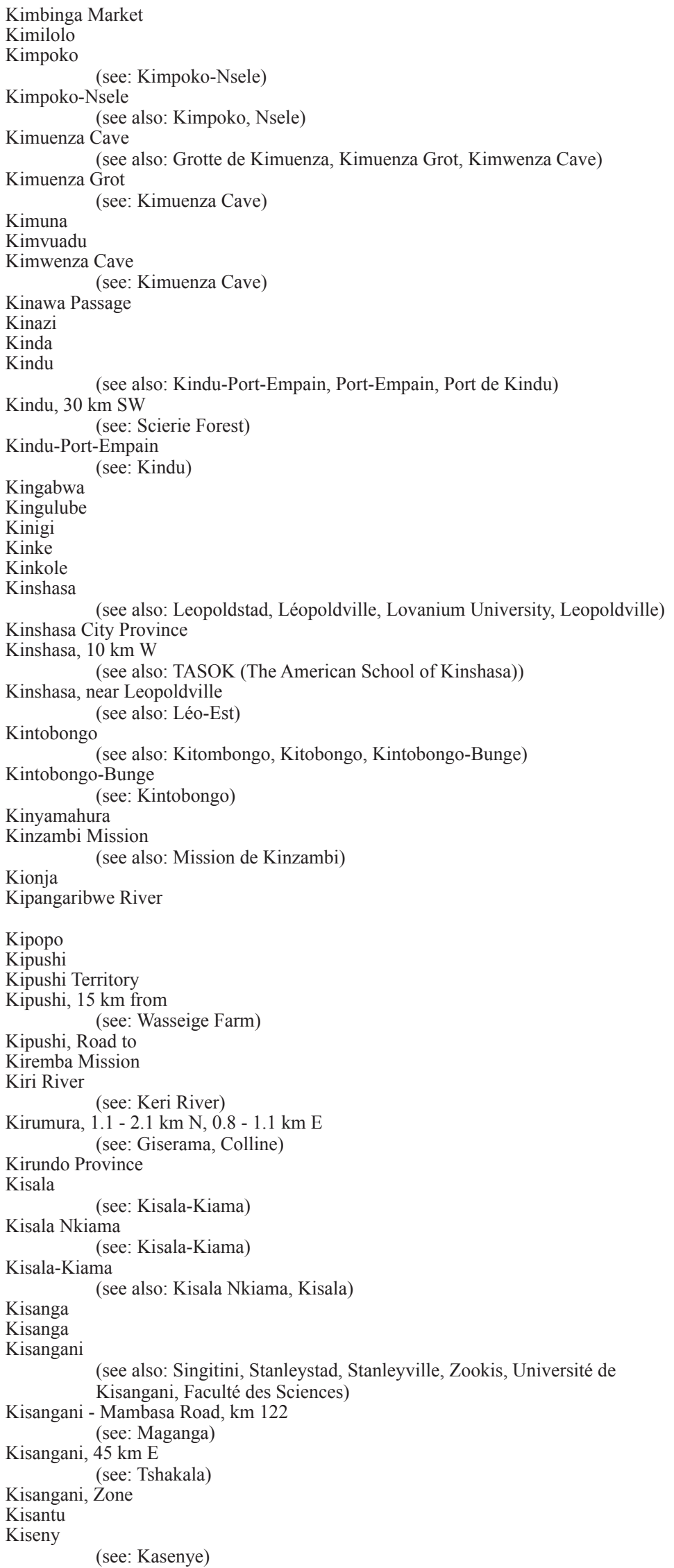

\begin{tabular}{|c|c|c|}
\hline CG-KU & $05^{\circ} 08^{\prime} \mathrm{S}, 19^{\circ} 03^{\prime} \mathrm{E}$ & $585 \mathrm{~m}$ \\
\hline CG-HK & $11^{\circ} 42^{\prime} \mathrm{S}, 27^{\circ} 25^{\prime} \mathrm{E}$ & \\
\hline CG-KN & $04^{\circ} 14^{\prime} \mathrm{S}, 15^{\circ} 33^{\prime} \mathrm{E}$ & $294 \mathrm{~m}$ \\
\hline CG-KN & $04^{\circ} 14^{\prime} \mathrm{S}, 15^{\circ} 33^{\prime} \mathrm{E}$ & $294 \mathrm{~m}$ \\
\hline CG-BC & $04^{\circ} 28^{\prime} \mathrm{S}, 15^{\circ} 17^{\prime} \mathrm{E}$ & \\
\hline CG-BC & $04^{\circ} 28^{\prime} \mathrm{S}, 15^{\circ} 17^{\prime} \mathrm{E}$ & \\
\hline $\begin{array}{l}\text { RW-SU } \\
\text { CG-BC } \\
\text { CG-BC }\end{array}$ & $\begin{array}{l}02^{\circ} 33^{\prime} \mathrm{S}, 29^{\circ} 44^{\prime} \mathrm{E} \\
05^{\circ} 15^{\prime} \mathrm{S}, 13^{\circ} 18^{\prime} \mathrm{E} \\
04^{\circ} 28^{\prime} \mathrm{S}, 15^{\circ} 17^{\prime} \mathrm{E}\end{array}$ & $1788 \mathrm{~m}$ \\
\hline $\begin{array}{l}\text { CG-NK } \\
\text { BY-KI } \\
\text { CG-KS } \\
\text { CG-MN }\end{array}$ & $\begin{array}{l}00^{\circ} 40^{\prime} \mathrm{N}, 29^{\circ} 50^{\prime} \mathrm{E} \\
02^{\circ} 23^{\prime} \mathrm{S}, 29^{\circ} 58^{\prime} \mathrm{E} \\
04^{\circ} 47^{\prime} \mathrm{S}, 21^{\circ} 48^{\prime} \mathrm{E} \\
02^{\circ} 57^{\prime} \mathrm{S}, 25^{\circ} 57^{\prime} \mathrm{E}\end{array}$ & $\begin{array}{l}470 \mathrm{~m} \\
504 \mathrm{~m}\end{array}$ \\
\hline CG-MN & $03^{\circ} 10^{\prime} \mathrm{S}, 25^{\circ} 46^{\prime} \mathrm{E}$ & \\
\hline CG-MN & $02^{\circ} 57^{\prime} \mathrm{S}, 25^{\circ} 57^{\prime} \mathrm{E}$ & $504 \mathrm{~m}$ \\
\hline $\begin{array}{l}\text { CG-KN } \\
\text { CG-SK } \\
\text { RW-NO } \\
\text { CG-HK } \\
\text { CG-ET } \\
\text { CG-KN }\end{array}$ & $\begin{array}{l}04^{\circ} 18^{\prime} \mathrm{S}, 15^{\circ} 18^{\prime} \mathrm{E} \\
02^{\circ} 39^{\prime} \mathrm{S}, 28^{\circ} 01^{\prime} \mathrm{E} \\
01^{\circ} 27^{\prime} \mathrm{S}, 29^{\circ} 34^{\prime} \mathrm{E} \\
11^{\circ} 27^{\prime} \mathrm{S}, 27^{\circ} 29^{\prime} \mathrm{E} \\
03^{\circ} 57^{\prime} \mathrm{S}, 16^{\circ} 07^{\prime} \mathrm{E} \\
04^{\circ} 20^{\prime} \mathrm{S}, 15^{\circ} 19^{\prime} \mathrm{E}\end{array}$ & $\begin{array}{c}1238 \mathrm{~m} \\
2179 \mathrm{~m} \\
1287 \mathrm{~m} \\
474 \mathrm{~m} \\
240 \mathrm{~m}\end{array}$ \\
\hline $\begin{array}{c}\mathrm{CG} \\
\mathrm{CG}-\mathrm{KN}\end{array}$ & ca $04^{\circ} 20^{\prime} \mathrm{S}, 15^{\circ} 15^{\prime} \mathrm{E}$ & \\
\hline CG-KN & $04^{\circ} 18^{\prime} \mathrm{S}, 15^{\circ} 18^{\prime} \mathrm{E}$ & $177 \mathrm{~m}$ \\
\hline CG-HL & $08^{\circ} 54^{\prime} \mathrm{S}, 26^{\circ} 23^{\prime} \mathrm{E}$ & $608 \mathrm{~m}$ \\
\hline CG-HL & $08^{\circ} 54^{\prime} \mathrm{S}, 26^{\circ} 23^{\prime} \mathrm{E}$ & $608 \mathrm{~m}$ \\
\hline $\begin{array}{l}\text { CG-NK } \\
\text { CG-KU }\end{array}$ & $\begin{array}{l}01^{\circ} 18^{\prime} \mathrm{S}, 29^{\circ} 33^{\prime} \mathrm{E} \\
04^{\circ} 59^{\prime} \mathrm{S}, 18^{\circ} 46^{\prime} \mathrm{E}\end{array}$ & $\begin{array}{c}1905 \mathrm{~m} \\
312 \mathrm{~m}\end{array}$ \\
\hline $\begin{array}{l}\text { RW-ES } \\
\text { CG-HL }\end{array}$ & $\begin{array}{l}01^{\circ} 50^{\prime} \mathrm{S}, 30^{\circ} 43^{\prime} \mathrm{E} \\
08^{\circ} 56^{\prime} \mathrm{S}, 27^{\circ} 12^{\prime} \mathrm{E}\end{array}$ & $\begin{array}{c}1677 \mathrm{~m} \\
1513-1760 \\
\mathrm{~m}\end{array}$ \\
\hline $\begin{array}{l}\text { CG-HK } \\
\text { CG-HK } \\
\text { CG-HK } \\
\text { CG-HK }\end{array}$ & $\begin{array}{l}11^{\circ} 34^{\prime} \mathrm{S}, 27^{\circ} 21^{\prime} \mathrm{E} \\
11^{\circ} 46^{\prime} \mathrm{S}, 27^{\circ} 14^{\prime} \mathrm{E} \\
11^{\circ} 46^{\prime} \mathrm{S}, 27^{\circ} 14^{\prime} \mathrm{E} \\
11^{\circ} 50^{\prime} \mathrm{S}, 27^{\circ} 20^{\prime} \mathrm{E}\end{array}$ & $1329 \mathrm{~m}$ \\
\hline $\begin{array}{c}\text { CG-HK } \\
\text { BY-BI } \\
\text { CG-MA }\end{array}$ & $\begin{array}{c}\text { ca } 11^{\circ} 45^{\prime} \mathrm{S}, 27^{\circ} 15^{\prime} \mathrm{E} \\
03^{\circ} 56^{\prime} \mathrm{S}, 29^{\circ} 41^{\prime} \mathrm{E} \\
01^{\circ} 29^{\prime} \mathrm{S}, 18^{\circ} 58^{\prime} \mathrm{E}\end{array}$ & \\
\hline BY-CI & $02^{\circ} 50^{\prime} \mathrm{S}, 29^{\circ} 25^{\prime} \mathrm{E}$ & $2045 \mathrm{~m}$ \\
\hline $\begin{array}{c}\text { BY } \\
\text { CG-BC }\end{array}$ & $04^{\circ} 48^{\prime} \mathrm{S}, 13^{\circ} 10^{\prime} \mathrm{E}$ & $433 \mathrm{~m}$ \\
\hline CG-BC & $04^{\circ} 48^{\prime} \mathrm{S}, 13^{\circ} 10^{\prime} \mathrm{E}$ & $433 \mathrm{~m}$ \\
\hline CG-BC & $04^{\circ} 48^{\prime} \mathrm{S}, 13^{\circ} 10^{\prime} \mathrm{E}$ & $433 \mathrm{~m}$ \\
\hline $\begin{array}{l}\text { CG-SK } \\
\text { CG-SK } \\
\text { CG-TO }\end{array}$ & $\begin{array}{l}02^{\circ} 25^{\prime} \mathrm{S}, 28^{\circ} 18^{\prime} \mathrm{E} \\
00^{\circ} 16^{\prime} \mathrm{N}, 29^{\circ} 43^{\prime} \mathrm{E} \\
00^{\circ} 31^{\prime} \mathrm{N}, 25^{\circ} 12^{\prime} \mathrm{E}\end{array}$ & $\begin{array}{l}960 \mathrm{~m} \\
447 \mathrm{~m}\end{array}$ \\
\hline CG-TO & $00^{\circ} 51^{\prime} \mathrm{N}, 26^{\circ} 22^{\prime} \mathrm{E}$ & \\
\hline CG-TO & $00^{\circ} 27^{\prime} \mathrm{N}, 25^{\circ} 32^{\prime} \mathrm{E}$ & $453 \mathrm{~m}$ \\
\hline $\begin{array}{l}\text { CG-TO } \\
\text { CG-BC } \\
\text { CG-HU }\end{array}$ & $\begin{array}{c}\text { ca } 00^{\circ} 30^{\prime} \mathrm{N}, 25^{\circ} 12^{\prime} \mathrm{E} \\
05^{\circ} 07^{\prime} \mathrm{S}, 15^{\circ} 05^{\prime} \mathrm{E} \\
01^{\circ} 24^{\prime} \mathrm{N}, 30^{\circ} 26^{\prime} \mathrm{E}\end{array}$ & $\begin{array}{c}421-571 \mathrm{~m} \\
671 \mathrm{~m}\end{array}$ \\
\hline
\end{tabular}




\begin{tabular}{|c|c|c|c|}
\hline Kisenyi $\quad$ (see: Gisenyi) & RW-OU & $01^{\circ} 41^{\prime} \mathrm{S}, 29^{\circ} 15^{\prime} \mathrm{E}$ & $1450 \mathrm{~m}$ \\
\hline $\begin{array}{l}\text { Kisenyi, Road to Sake } \\
\text { (see: Gisenyi) }\end{array}$ & RW-OU & $01^{\circ} 41^{\prime} \mathrm{S}, 29^{\circ} 15^{\prime} \mathrm{E}$ & $1450 \mathrm{~m}$ \\
\hline Kishero & CG-NK & $00^{\circ} 11^{\prime} \mathrm{N}, 29^{\circ} 39^{\prime} \mathrm{E}$ & $925 \mathrm{~m}$ \\
\hline $\begin{array}{l}\text { Kisiguru (Mission) } \\
\text { (see: Kiziguro (Mission)) }\end{array}$ & RW-ES & $01^{\circ} 46^{\prime} \mathrm{S}, 30^{\circ} 24^{\prime} \mathrm{E}$ & \\
\hline Kisiki $\quad$ (see also: Kisiki Kalume, Beni, 36 km N) & CG-NK & $00^{\circ} 45^{\prime} \mathrm{N}, 29^{\circ} 34^{\prime} \mathrm{E}$ & $1177 \mathrm{~m}$ \\
\hline $\begin{array}{l}\text { Kisiki Kalume } \\
\qquad \text { (see: Kisiki) }\end{array}$ & CG-NK & $00^{\circ} 45^{\prime} \mathrm{N}, 29^{\circ} 34^{\prime} \mathrm{E}$ & $1177 \mathrm{~m}$ \\
\hline Kisingani & $\mathrm{CG}$ & $00^{\circ} 32^{\prime} \mathrm{N}, 25^{\circ} 11^{\prime} \mathrm{E}$ & \\
\hline Kisisile & CG-NK & $01^{\circ} 25^{\prime} \mathrm{S}, 29^{\circ} 30^{\prime} \mathrm{E}$ & $1250 \mathrm{~m}$ \\
\hline Kisiushi & CG-HK & $11^{\circ} 30^{\prime} \mathrm{S}, 27^{\circ} 30^{\prime} \mathrm{E}$ & \\
\hline Kissenji $\quad$ (see: Gisenyi) & RW-OU & $01^{\circ} 41^{\prime} \mathrm{S}, 29^{\circ} 15^{\prime} \mathrm{E}$ & $1450 \mathrm{~m}$ \\
\hline Kiswishi [River] & CG-HK & $12^{\circ} 51^{\prime} \mathrm{S}, 29^{\circ} 12^{\prime} \mathrm{E}$ & \\
\hline Kitabi & RW-SU & $02^{\circ} 31^{\prime} \mathrm{S}, 29^{\circ} 25^{\prime} \mathrm{E}$ & \\
\hline Kitangua & CG-KS & $06^{\circ} 17^{\prime} \mathrm{S}, 20^{\circ} 20^{\prime} \mathrm{E}$ & $649 \mathrm{~m}$ \\
\hline Kitega $\quad$ (see: Gitega) & BY-GI & $03^{\circ} 26^{\prime} \mathrm{S}, 29^{\circ} 56^{\prime} \mathrm{E}$ & $1845 \mathrm{~m}$ \\
\hline Kitimi & CG-MA & $03^{\circ} 58^{\prime} \mathrm{S}, 15^{\circ} 53^{\prime} \mathrm{E}$ & \\
\hline $\begin{array}{l}\text { Kitobongo } \\
\text { (see: Kintobongo) }\end{array}$ & CG-HL & $08^{\circ} 54^{\prime} \mathrm{S}, 26^{\circ} 23^{\prime} \mathrm{E}$ & $608 \mathrm{~m}$ \\
\hline $\begin{array}{l}\text { Kitombongo } \\
\text { (see: Kintobongo) }\end{array}$ & CG-HL & $08^{\circ} 54^{\prime} \mathrm{S}, 26^{\circ} 23^{\prime} \mathrm{E}$ & $608 \mathrm{~m}$ \\
\hline Kitonga & CG-NK & $00^{\circ} 30^{\prime} \mathrm{S}, 29^{\circ} 18^{\prime} \mathrm{E}$ & $920 \mathrm{~m}$ \\
\hline Kitongo & CG-SK & $03^{\circ} 46^{\prime} \mathrm{S}, 28^{\circ} 11^{\prime} \mathrm{E}$ & $1400 \mathrm{~m}$ \\
\hline $\begin{array}{l}\text { Kitubulushi } \\
\qquad \text { (see also: Kilubulushi) }\end{array}$ & CG-HK & $12^{\circ} 44^{\prime} \mathrm{S}, 28^{\circ} 56^{\prime} \mathrm{E}$ & $1257 \mathrm{~m}$ \\
\hline Kitundu Kinda & CG-HL & $09^{\circ} 30^{\prime} \mathrm{S}, 24^{\circ} 55^{\prime} \mathrm{E}$ & \\
\hline Kitutu & CG-SK & $03^{\circ} 17^{\prime} \mathrm{S}, 28^{\circ} 05^{\prime} \mathrm{E}$ & $1040 \mathrm{~m}$ \\
\hline Kitwabaluzi & CG-SK & $02^{\circ} 53^{\prime} \mathrm{S}, 28^{\circ} 35^{\prime} \mathrm{E}$ & $1543 \mathrm{~m}$ \\
\hline Kiulu Mgemgo & CG-KU & $04^{\circ} 24^{\prime} \mathrm{S}, 17^{\circ} 42^{\prime} \mathrm{E}$ & \\
\hline Kiumba & CG-SK & $01^{\circ} 56^{\prime} \mathrm{S}, 29^{\circ} 05^{\prime} \mathrm{E}$ & $1602 \mathrm{~m}$ \\
\hline $\begin{array}{ll}\text { Kivu } & \text { (see also: Kihumba) } \\
& \text { (see: Kivu region) }\end{array}$ & CG & & \\
\hline Kivu Meer (see: Lake Kivu) & CG-SK & ca $02^{\circ} 00^{\prime} \mathrm{S}, 28^{\circ} 55^{\prime} \mathrm{E}$ & \\
\hline $\begin{array}{l}\text { Kivu mountains } \\
\text { Kivu region }\end{array}$ & $\begin{array}{l}\text { CG-SK } \\
\text { CG }\end{array}$ & ca $02^{\circ} 00^{\prime} \mathrm{S}, 28^{\circ} 45^{\prime} \mathrm{E}$ & \\
\hline $\begin{array}{l}\text { (see also: Kivu) } \\
\text { Kivu Volcano } \\
\text { (see: Nyirogongo Volcano) }\end{array}$ & CG-NK & $01^{\circ} 31^{\prime} \mathrm{S}, 29^{\circ} 15^{\prime} \mathrm{E}$ & \\
\hline Kivu, Lac (see: Lake Kivu) & CG-SK & ca $02^{\circ} 00^{\prime} \mathrm{S}, 28^{\circ} 55^{\prime} \mathrm{E}$ & \\
\hline Kivu, Lake & CG-SK & ca $02^{\circ} 00^{\prime} \mathrm{S}, 28^{\circ} 55^{\prime} \mathrm{E}$ & \\
\hline $\begin{array}{l}\text { Kivu, near } \\
\text { Kivulo }\end{array}$ & $\begin{array}{l}\text { CG-SK } \\
\text { CG-HK }\end{array}$ & ca $02^{\circ} 00^{\prime} \mathrm{S}, 28^{\circ} 45^{\prime} \mathrm{E}$ & \\
\hline $\begin{array}{l}\text { Kiwakishi Cave } \\
\text { (see: Kiamokoto) }\end{array}$ & CG-HL & $09^{\circ} 10^{\prime} \mathrm{S}, 27^{\circ} 04^{\prime} \mathrm{E}$ & $900 \mathrm{~m}$ \\
\hline $\begin{array}{l}\text { Kiwakishi Grot } \\
\text { (see: Kiamokoto) }\end{array}$ & CG-HL & $09^{\circ} 10^{\prime} \mathrm{S}, 27^{\circ} 04^{\prime} \mathrm{E}$ & $900 \mathrm{~m}$ \\
\hline Kiyite & CG-NK & & \\
\hline $\begin{array}{l}\text { Kiziguro (Mission) } \\
\qquad \text { (see also: Kisiguru (Mission), Kiziguru (Mission)) }\end{array}$ & RW-ES & $01^{\circ} 46^{\prime} \mathrm{S}, 30^{\circ} 24^{\prime} \mathrm{E}$ & \\
\hline $\begin{array}{l}\text { Kiziguru (Mission) } \\
\text { (see: Kiziguro (Mission)) }\end{array}$ & RW-ES & $01^{\circ} 46^{\prime} \mathrm{S}, 30^{\circ} 24^{\prime} \mathrm{E}$ & \\
\hline Ko[g]enga & $\mathrm{CG}$ & & \\
\hline Kodja Hill & CG-HU & $03^{\circ} 09^{\prime} \mathrm{N}, 29^{\circ} 29^{\prime} \mathrm{E}$ & \\
\hline $\begin{array}{l}\text { Kodoko River } \\
\qquad \text { (see: Kokodo River) }\end{array}$ & CG-HU & $04^{\circ} 38^{\prime} \mathrm{N}, 29^{\circ} 30^{\prime} \mathrm{E}$ & \\
\hline $\begin{array}{l}\text { Kokodo River } \\
\text { (see also: Kodoko River) }\end{array}$ & CG-HU & $04^{\circ} 38^{\prime} \mathrm{N}, 29^{\circ} 30^{\prime} \mathrm{E}$ & \\
\hline $\begin{array}{l}\text { Kokodu } \\
\text { Kokonde }\end{array}$ & $\begin{array}{l}\text { CG-HU } \\
\text { CG }\end{array}$ & $04^{\circ} 35^{\prime} \mathrm{N}, 29^{\circ} 32^{\prime} \mathrm{E}$ & \\
\hline Koloka & CG-BU & $03^{\circ} 23^{\prime} \mathrm{N}, 24^{\circ} 30^{\prime} \mathrm{E}$ & \\
\hline Kolwezi & CG-LB & $10^{\circ} 43^{\prime} \mathrm{S}, 25^{\circ} 28^{\prime} \mathrm{E}$ & $1448 \mathrm{~m}$ \\
\hline Kolwezi Province & $\mathrm{CG}$ & & \\
\hline $\begin{array}{l}\text { Komanda } \\
\text { (see also: Komande) }\end{array}$ & CG-IT & $01^{\circ} 23^{\prime} \mathrm{N}, 29^{\circ} 46^{\prime} \mathrm{E}$ & $928 \mathrm{~m}$ \\
\hline Komande & CG-IT & $01^{\circ} 23^{\prime} \mathrm{N}, 29^{\circ} 46^{\prime} \mathrm{E}$ & $928 \mathrm{~m}$ \\
\hline Kona & CG-MO & $02^{\circ} 02^{\prime} \mathrm{N}, 22^{\circ} 47^{\prime} \mathrm{E}$ & \\
\hline
\end{tabular}


Kondo Cave

Kondo Gro

(see: Kakanda Cave)

see: Kakanda Cave)

Kondue [River]

Kongo Central Province

Kongolo

(see also: Bas Congo Province, Bas Congo (former) Province)

Kongolo, Island

Kontundwe

(see also: Ile de Kongolo)

(see: Kantundwe)

Konyola

Korovi, mount

Koteli (see also: Mount Korovi)

(see: Kotili)

Kotili

Kouilou River

(see also: Koteli)

Kuango

Kuili

Kuili Ngongo

(see also: Kuili Ngongo)

(see: Kuili)

Kuleponge

(see: Kulepongi)

Kulepongi

(see also: Kuleponge, Kulepongo)

Kulepongo

(see: Kulepongi)

Kumbi

Kumi

Kundelungu National Park (see also: Park National de Kundelungo)

Kunungu

Kwama Kwama Cave

(see also: Grotte de Kwama Kwama, Kwama Kwama Grot)

Kwama Kwama Grot

(see: Kwama Kwama Cave)

Kwamouth

Kwango

Kwango Province

Kwilu Province

Kwogofe, Colline

Kya irtumbi

see also: Colline Kwogofe)

(see: Mutwanga)

Kyamakonde Cave

Kyantapo Cave

(see also: Grotte de Kyantapo, Kiantapo Cave, Kyantapo Grot, Lubudi, 30 $\mathrm{km} \mathrm{SW)}$

Kyantapo Grot

Kyasala Cave see: Kyantapo Cave)

(see: Kiasala Cave)

Kyasala grande Grotte

Kyasala Gro

$$
\text { (see: Kiasala Cave) }
$$
(see: Kiasala Cave)

Kyavinionge

Kyniamahura (see also: Kiavinionge)

Labo Irsac

(see: Luiro)

(see: Albert, Lake)

Lac Cohoha
(see: Cohoha, Lake)

Lac Edouard

(see: Edward, Lake)

Lac Fwa

$$
\text { (see: Fwa, Lake) }
$$

Lac Idi Amine

\begin{tabular}{|c|c|c|}
\hline CG-LB & $10^{\circ} 47^{\prime} \mathrm{S}, 26^{\circ} 36^{\prime} \mathrm{E}$ & $1438 \mathrm{~m}$ \\
\hline CG-LB & $10^{\circ} 47^{\prime} \mathrm{S}, 26^{\circ} 36^{\prime} \mathrm{E}$ & $1438 \mathrm{~m}$ \\
\hline $\begin{array}{c}\mathrm{CG}-\mathrm{SN} \\
\mathrm{CG}\end{array}$ & $\begin{array}{c}04^{\circ} 57^{\prime} \mathrm{S}, 23^{\circ} 21^{\prime} \mathrm{E} \\
\text { ca } 05^{\circ} 00^{\prime} \mathrm{S}, 15^{\circ} 00^{\prime} \mathrm{E}\end{array}$ & \\
\hline $\begin{array}{l}\text { CG-TG } \\
\text { CG-TO }\end{array}$ & $\begin{array}{l}05^{\circ} 25^{\prime} \mathrm{S}, 27^{\circ} 00^{\prime} \mathrm{E} \\
00^{\circ} 03^{\prime} \mathrm{N}, 25^{\circ} 12^{\prime} \mathrm{E}\end{array}$ & \\
\hline CG-NK & $01^{\circ} 10^{\prime} \mathrm{S}, 29^{\circ} 28^{\prime} \mathrm{E}$ & $1150 \mathrm{~m}$ \\
\hline CG-TO & $00^{\circ} 03^{\prime} \mathrm{N}, 25^{\circ} 12^{\prime} \mathrm{E}$ & \\
\hline CG-IT & $01^{\circ} 39^{\prime} \mathrm{N}, 30^{\circ} 36^{\prime} \mathrm{E}$ & $2010 \mathrm{~m}$ \\
\hline CG-BU & $02^{\circ} 52^{\prime} \mathrm{N}, 24^{\circ} 33^{\prime} \mathrm{E}$ & $432 \mathrm{~m}$ \\
\hline CG-BU & $02^{\circ} 52^{\prime} \mathrm{N}, 24^{\circ} 33^{\prime} \mathrm{E}$ & $432 \mathrm{~m}$ \\
\hline $\begin{array}{c}\mathrm{CG} \\
\mathrm{CG}-\mathrm{BC} \\
\mathrm{CG}-\mathrm{KU}\end{array}$ & $\begin{array}{l}04^{\circ} 45^{\prime} \mathrm{S}, 13^{\circ} 36^{\prime} \mathrm{E} \\
04^{\circ} 24^{\prime} \mathrm{S}, 17^{\circ} 42^{\prime} \mathrm{E}\end{array}$ & \\
\hline CG-KU & $04^{\circ} 24^{\prime} \mathrm{S}, 17^{\circ} 42^{\prime} \mathrm{E}$ & \\
\hline CG-MN & $03^{\circ} 22^{\prime} \mathrm{N}, 25^{\circ} 41^{\prime} \mathrm{E}$ & $604 \mathrm{~m}$ \\
\hline CG-MN & $03^{\circ} 22^{\prime} \mathrm{N}, 25^{\circ} 41^{\prime} \mathrm{E}$ & $604 \mathrm{~m}$ \\
\hline CG-MN & $03^{\circ} 22^{\prime} \mathrm{N}, 25^{\circ} 41^{\prime} \mathrm{E}$ & $604 \mathrm{~m}$ \\
\hline $\begin{array}{c}\mathrm{CG} \\
\mathrm{CG}-\mathrm{MN} \\
\mathrm{CG}-\mathrm{HK}\end{array}$ & $\begin{array}{c}04^{\circ} 32^{\prime} \mathrm{S}, 26^{\circ} 33^{\prime} \mathrm{E} \\
\text { ca } 10^{\circ} 30^{\prime} \mathrm{S}, 27^{\circ} 30^{\prime} \mathrm{E}\end{array}$ & \\
\hline $\begin{array}{l}\text { CG-MA } \\
\text { CG-NK }\end{array}$ & $\begin{array}{l}02^{\circ} 06^{\prime} \mathrm{S}, 16^{\circ} 26^{\prime} \mathrm{E} \\
01^{\circ} 19^{\prime} \mathrm{N}, 29^{\circ} 52^{\prime} \mathrm{E}\end{array}$ & $1200 \mathrm{~m}$ \\
\hline CG-NK & $01^{\circ} 19^{\prime} \mathrm{N}, 29^{\circ} 52^{\prime} \mathrm{E}$ & $1200 \mathrm{~m}$ \\
\hline $\begin{array}{c}\text { CG-MA } \\
\text { CG-KG } \\
\text { CG } \\
\text { CG }\end{array}$ & $\begin{array}{c}03^{\circ} 10^{\prime} \mathrm{S}, 16^{\circ} 12^{\prime} \mathrm{E} \\
06^{\circ} 28^{\prime} \mathrm{S}, 17^{\circ} 27^{\prime} \mathrm{E} \\
\text { ca } 06^{\circ} 30^{\prime} \mathrm{S}, 17^{\circ} 46^{\prime} \mathrm{E}\end{array}$ & $\begin{array}{c}146 \mathrm{~m} \\
1020 \mathrm{~m}\end{array}$ \\
\hline BY-CI & $02^{\circ} 50^{\prime} \mathrm{S}, 29^{\circ} 25^{\prime} \mathrm{E}$ & $\begin{array}{c}2180-2240 \\
\mathrm{~m}\end{array}$ \\
\hline CG-NK & $00^{\circ} 20^{\prime} \mathrm{N}, 29^{\circ} 45^{\prime} \mathrm{E}$ & $1338 \mathrm{~m}$ \\
\hline $\begin{array}{l}\text { CG-LB } \\
\text { CG-LB }\end{array}$ & $\begin{array}{c}\text { ca } 09^{\circ} 56^{\prime} \mathrm{S}, 25^{\circ} 58^{\prime} \mathrm{E} \\
09^{\circ} 56^{\prime} \mathrm{S}, 25^{\circ} 58^{\prime} \mathrm{E}\end{array}$ & $1525 \mathrm{~m}$ \\
\hline CG-LB & $09^{\circ} 56^{\prime} \mathrm{S}, 25^{\circ} 58^{\prime} \mathrm{E}$ & $1525 \mathrm{~m}$ \\
\hline CG-LB & $09^{\circ} 56^{\prime} \mathrm{S}, 25^{\circ} 58^{\prime} \mathrm{E}$ & $1300 \mathrm{~m}$ \\
\hline CG-LB & $09^{\circ} 56^{\prime} \mathrm{S}, 25^{\circ} 58^{\prime} \mathrm{E}$ & $1300 \mathrm{~m}$ \\
\hline CG-LB & $09^{\circ} 56^{\prime} \mathrm{S}, 25^{\circ} 58^{\prime} \mathrm{E}$ & $1300 \mathrm{~m}$ \\
\hline CG-TO & $00^{\circ} 07^{\prime} \mathrm{S}, 26^{\circ} 36^{\prime} \mathrm{E}$ & $935 \mathrm{~m}$ \\
\hline $\begin{array}{c}\text { CG } \\
\text { CG-SK }\end{array}$ & $02^{\circ} 14^{\prime} \mathrm{S}, 28^{\circ} 48^{\prime} \mathrm{E}$ & $1646 \mathrm{~m}$ \\
\hline
\end{tabular}

(see: Edward, Lake)
CG-IT ca $01^{\circ} 40^{\prime} \mathrm{N}, 30^{\circ} 40^{\prime} \mathrm{E}$

BY-KI $\quad 02^{\circ} 30^{\prime} \mathrm{S}, 30^{\circ} 07^{\prime} \mathrm{E}$

CG-NK ca $00^{\circ} 08^{\prime} \mathrm{S}, 29^{\circ} 35^{\prime} \mathrm{E}$

CG-LL $\quad 05^{\circ} 43^{\prime} \mathrm{S}, 23^{\circ} 25^{\prime} \mathrm{E}$

CG-NK ca $00^{\circ} 08^{\prime} \mathrm{S}, 29^{\circ} 35^{\prime} \mathrm{E}$ 


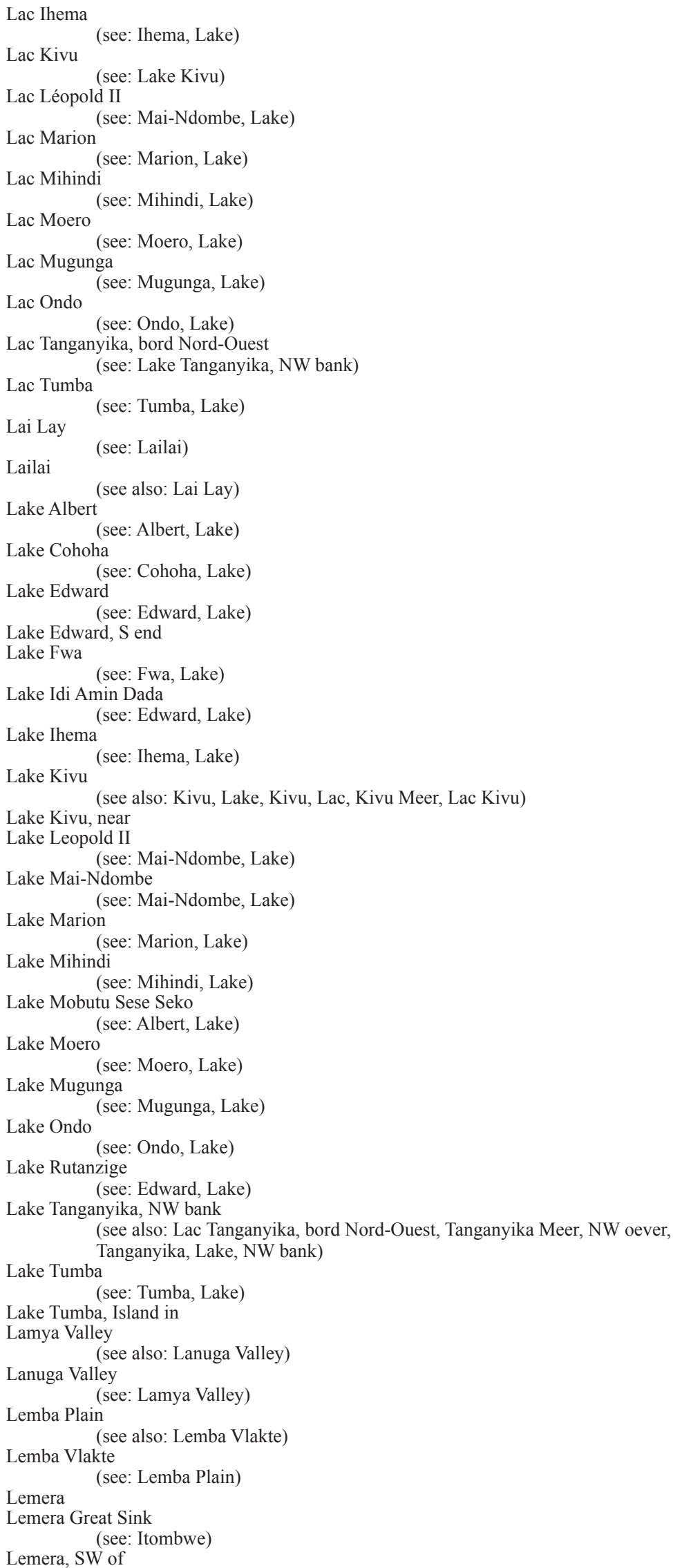

RW-ES

$01^{\circ} 52^{\prime} \mathrm{S}, 30^{\circ} 47^{\prime} \mathrm{E}$

CG-SK ca $02^{\circ} 00^{\prime} \mathrm{S}, 28^{\circ} 55^{\prime} \mathrm{E}$

CG-MA $02^{\circ} 05^{\prime} \mathrm{S}, 18^{\circ} 20^{\prime} \mathrm{E}$

CG

RW-ES

CG-HK

CG-NK

CG-NK

CG-SK

CG-ET

CG-SK

CG-SK

CG-IT

BY-KI

CG-NK

CG-NK

CG-LL

CG-NK

RW-ES

CG-SK

CG-SK ca $02^{\circ} 00^{\prime} \mathrm{S}, 29^{\circ} 10^{\prime} \mathrm{E}$

CG-MA $\quad 02^{\circ} 05^{\prime} \mathrm{S}, 18^{\circ} 20^{\prime} \mathrm{E}$

CG-MA

$02^{\circ} 05^{\prime} \mathrm{S}, 18^{\circ} 20^{\prime} \mathrm{E}$

CG

RW-ES $\quad 01^{\circ} 32^{\prime} \mathrm{S}, 30^{\circ} 42^{\prime} \mathrm{E}$

CG-IT ca $01^{\circ} 40^{\prime} \mathrm{N}, 30^{\circ} 40^{\prime} \mathrm{E}$

CG-HK $\quad 09^{\circ} 00^{\prime} \mathrm{S}, 28^{\circ} 45^{\prime} \mathrm{E}$

CG-NK $\quad 01^{\circ} 37^{\prime} \mathrm{S}, 29^{\circ} 08^{\prime} \mathrm{E} \quad 1500 \mathrm{~m}$

CG-NK $\quad 01^{\circ} 08^{\prime} \mathrm{S}, 29^{\circ} 19^{\prime} \mathrm{E}$

CG-NK ca $00^{\circ} 08^{\prime} \mathrm{S}, 29^{\circ} 35^{\prime} \mathrm{E}$

CG-SK ca $03^{\circ} 20^{\prime} \mathrm{S}, 29^{\circ} 12^{\prime} \mathrm{E}$

ca $00^{\circ} 48^{\prime} \mathrm{S}, 18^{\circ} 03^{\prime} \mathrm{E}$

CG-ET

CG-IT

ca $00^{\circ} 48^{\prime} \mathrm{S}, 18^{\circ} 03^{\prime} \mathrm{E}$

CG-IT $\quad 00^{\circ} 50^{\prime} \mathrm{N}, 29^{\circ} 59^{\prime} \mathrm{E}$

CG-KN

$04^{\circ} 18^{\prime} \mathrm{S}, 15^{\circ} 18^{\prime} \mathrm{E}$

CG-KN

$04^{\circ} 18^{\prime} \mathrm{S}, 15^{\circ} 18^{\prime} \mathrm{E}$

CG-SK $\quad 02^{\circ} 08^{\prime} \mathrm{S}, 28^{\circ} 50^{\prime} \mathrm{E}$

CG-SK $\quad 04^{\circ} 04^{\prime} \mathrm{S}, 28^{\circ} 40^{\prime} \mathrm{E}$

$2281 \mathrm{~m}$

CG-SK ca $02^{\circ} 10^{\prime} \mathrm{S}, 28^{\circ} 50^{\prime} \mathrm{E} \quad 2682 \mathrm{~m}$ 
Léo-Est (see: Kinshasa, near Leopoldville)

Leopold 2nd, Lake

(see: Mai-Ndombe, Lake)

Leopold II Mee (see: Mai-Ndombe, Lake)

Leopold II, Lake (see: Mai-Ndombe, Lake)

Leopoldstad (see: Kinshasa)

Leopoldville (see: Kinshasa)

Léopoldville

(see: Kinshasa)

Lesse

Leverstad

(see: Lusanga)

Leverville

(see: Lusanga)

Libenge

(see also: Libengué)

Libengué

(see: Libenge)

Liège Sacre-Coeur Mission

(see: Kanzenze Mission)

Liège Saint Lambert

(see: Katana)

Lieki

Likandi River

Likasi

see: Litaki River)

(see also: Farm 'des Grands Bois', Jadotstad, Jadotsville, Jadotville, Mission Sainte Thérèse, Panda Camp, St Therese Mission, Jadoville, St. Theresa Mission)

Likasi, $130 \mathrm{~km} \mathrm{~N}$

(see: Tenke)

Likasi, $15 \mathrm{~km} \mathrm{~S}$

(see: Mwanga Cave)

Likasi, ca $8 \mathrm{~km} \mathrm{~S}$

(see: Kakontwe)

Likati River

Liki

Lima

Liondo

(see also: Loondo)

Liondo

Lisala

(see also: Lizala)

Lisala Territory

Litaki River

(see also: Likandi River)

(see: Lisala)

Loanza Mission

(see: Luanza Mission)

Lobango

(see also: Lubango)

Lobi

Lodja

(see also: Loja)

Lodja Territory

Lofoi [River]

Loja

(see: Lodja)

Lomami Province

(see also: Kabinda (former) district)

Lomera

Lonzo

(see: Loso)

(see: Liondo)

Loondo Mugunga

Losho

(see: Loso)

Loso

(see also: Losho, Lonzo)

\begin{tabular}{|c|c|c|}
\hline CG-KN & $04^{\circ} 18^{\prime} \mathrm{S}, 15^{\circ} 18^{\prime} \mathrm{E}$ & $177 \mathrm{~m}$ \\
\hline CG-MA & $02^{\circ} 05^{\prime} \mathrm{S}, 18^{\circ} 20^{\prime} \mathrm{E}$ & \\
\hline CG-MA & $02^{\circ} 05^{\prime} \mathrm{S}, 18^{\circ} 20^{\prime} \mathrm{E}$ & \\
\hline CG-MA & $02^{\circ} 05^{\prime} \mathrm{S}, 18^{\circ} 20^{\prime} \mathrm{E}$ & \\
\hline CG-KN & $04^{\circ} 20^{\prime} \mathrm{S}, 15^{\circ} 19^{\prime} \mathrm{E}$ & $240 \mathrm{~m}$ \\
\hline CG-KN & $04^{\circ} 18^{\prime} \mathrm{S}, 15^{\circ} 18^{\prime} \mathrm{E}$ & $240 \mathrm{~m}$ \\
\hline CG-KN & $04^{\circ} 20^{\prime} \mathrm{S}, 15^{\circ} 19^{\prime} \mathrm{E}$ & $240 \mathrm{~m}$ \\
\hline CG-NK & $00^{\circ} 45^{\prime} \mathrm{N}, 29^{\circ} 48^{\prime} \mathrm{E}$ & $800 \mathrm{~m}$ \\
\hline CG-KU & $04^{\circ} 50^{\prime} \mathrm{S}, 18^{\circ} 44^{\prime} \mathrm{E}$ & $307 \mathrm{~m}$ \\
\hline CG-KU & $04^{\circ} 50^{\prime} \mathrm{S}, 18^{\circ} 44^{\prime} \mathrm{E}$ & $307 \mathrm{~m}$ \\
\hline CG-MA & $03^{\circ} 39^{\prime} \mathrm{N}, 18^{\circ} 38^{\prime} \mathrm{E}$ & $326 \mathrm{~m}$ \\
\hline CG-MA & $03^{\circ} 39^{\prime} \mathrm{N}, 18^{\circ} 38^{\prime} \mathrm{E}$ & $326 \mathrm{~m}$ \\
\hline CG-LB & $10^{\circ} 31^{\prime} \mathrm{S}, 25^{\circ} 12^{\prime} \mathrm{E}$ & $1157 \mathrm{~m}$ \\
\hline CG-SK & $02^{\circ} 14^{\prime} \mathrm{S}, 28^{\circ} 50^{\prime} \mathrm{E}$ & $1595 \mathrm{~m}$ \\
\hline $\begin{array}{l}\text { CG-TO } \\
\text { CG-BU }\end{array}$ & $\begin{array}{l}00^{\circ} 41^{\prime} \mathrm{N}, 24^{\circ} 11^{\prime} \mathrm{E} \\
03^{\circ} 11^{\prime} \mathrm{N}, 26^{\circ} 11^{\prime} \mathrm{E}\end{array}$ & $508 \mathrm{~m}$ \\
\hline CG-HK & $10^{\circ} 59^{\prime} \mathrm{S}, 26^{\circ} 44^{\prime} \mathrm{E}$ & $1318 \mathrm{~m}$ \\
\hline CG-HK & $10^{\circ} 35^{\prime} \mathrm{S}, 26^{\circ} 07^{\prime} \mathrm{E}$ & $1512 \mathrm{~m}$ \\
\hline \multicolumn{3}{|l|}{ CG-HK } \\
\hline CG-HK & $10^{\circ} 59^{\prime} \mathrm{S}, 26^{\circ} 40^{\prime} \mathrm{E}$ & $1253 \mathrm{~m}$ \\
\hline $\begin{array}{l}\text { CG-BU } \\
\text { CG-NK } \\
\text { CG-NK } \\
\text { CG-TP }\end{array}$ & $\begin{array}{l}02^{\circ} 54^{\prime} \mathrm{N}, 24^{\circ} 03^{\prime} \mathrm{E} \\
00^{\circ} 07^{\prime} \mathrm{S}, 29^{\circ} 34^{\prime} \mathrm{E} \\
00^{\circ} 12^{\prime} \mathrm{N}, 29^{\circ} 18^{\prime} \mathrm{E} \\
00^{\circ} 55^{\prime} \mathrm{S}, 22^{\circ} 41^{\prime} \mathrm{E}\end{array}$ & $416 \mathrm{~m}$ \\
\hline $\begin{array}{l}\text { CG-TP } \\
\text { CG-MO }\end{array}$ & $\begin{array}{l}00^{\circ} 48^{\prime} \mathrm{S}, 22^{\circ} 39^{\prime} \mathrm{E} \\
02^{\circ} 09^{\prime} \mathrm{N}, 21^{\circ} 31^{\prime} \mathrm{E}\end{array}$ & $\begin{array}{l}508 \mathrm{~m} \\
409 \mathrm{~m}\end{array}$ \\
\hline $\begin{array}{l}\text { CG-MO } \\
\text { CG-BU }\end{array}$ & $\begin{array}{l}02^{\circ} 09^{\prime} \mathrm{N}, 21^{\circ} 31^{\prime} \mathrm{E} \\
03^{\circ} 11^{\prime} \mathrm{N}, 26^{\circ} 11^{\prime} \mathrm{E}\end{array}$ & \\
\hline CG-MO & $02^{\circ} 09^{\prime} \mathrm{N}, 21^{\circ} 31^{\prime} \mathrm{E}$ & $409 \mathrm{~m}$ \\
\hline CG-HK & $08^{\circ} 42^{\prime} \mathrm{S}, 28^{\circ} 42^{\prime} \mathrm{E}$ & $1132 \mathrm{~m}$ \\
\hline CG-NK & $00^{\circ} 19^{\prime} \mathrm{S}, 29^{\circ} 13^{\prime} \mathrm{E}$ & $2197 \mathrm{~m}$ \\
\hline $\begin{array}{l}\text { CG-BU } \\
\text { CG-SN }\end{array}$ & $\begin{array}{l}04^{\circ} 10^{\prime} \mathrm{N}, 23^{\circ} 06^{\prime} \mathrm{E} \\
03^{\circ} 29^{\prime} \mathrm{S}, 23^{\circ} 26^{\prime} \mathrm{E}\end{array}$ & $450 \mathrm{~m}$ \\
\hline $\begin{array}{l}\text { CG-SN } \\
\text { CG-LB } \\
\text { CG-SN }\end{array}$ & $\begin{array}{l}03^{\circ} 29^{\prime} \mathrm{S}, 23^{\circ} 26^{\prime} \mathrm{E} \\
10^{\circ} 13^{\prime} \mathrm{S}, 27^{\circ} 25^{\prime} \mathrm{E} \\
03^{\circ} 29^{\prime} \mathrm{S}, 23^{\circ} 26^{\prime} \mathrm{E}\end{array}$ & $450 \mathrm{~m}$ \\
\hline \multicolumn{3}{|l|}{ CG } \\
\hline $\begin{array}{l}\text { CG-NK } \\
\text { CG-NK }\end{array}$ & $\begin{array}{l}00^{\circ} 07^{\prime} \mathrm{N}, 29^{\circ} 35^{\prime} \mathrm{E} \\
00^{\circ} 08^{\prime} \mathrm{S}, 29^{\circ} 36^{\prime} \mathrm{E}\end{array}$ & $\begin{array}{l}915 \mathrm{~m} \\
950 \mathrm{~m}\end{array}$ \\
\hline CG-TP & $00^{\circ} 55^{\prime} \mathrm{S}, 22^{\circ} 41^{\prime} \mathrm{E}$ & $416 \mathrm{~m}$ \\
\hline $\begin{array}{l}\text { CG-TP } \\
\text { CG-NK }\end{array}$ & $\begin{array}{l}00^{\circ} 42^{\prime} \mathrm{S}, 22^{\circ} 41^{\prime} \mathrm{E} \\
00^{\circ} 08^{\prime} \mathrm{S}, 29^{\circ} 36^{\prime} \mathrm{E}\end{array}$ & $950 \mathrm{~m}$ \\
\hline CG-NK & $00^{\circ} 08^{\prime} \mathrm{S}, 29^{\circ} 36^{\prime} \mathrm{E}$ & $950 \mathrm{~m}$ \\
\hline
\end{tabular}




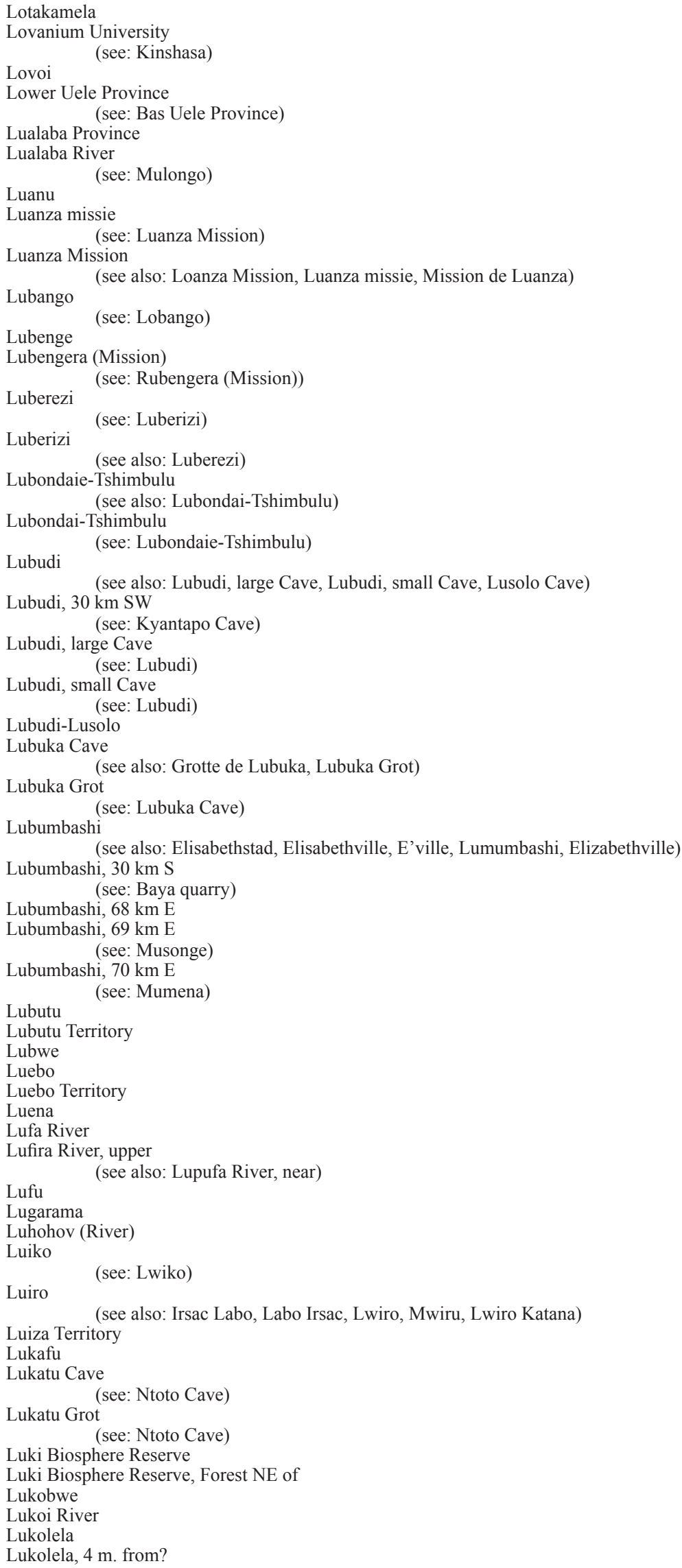




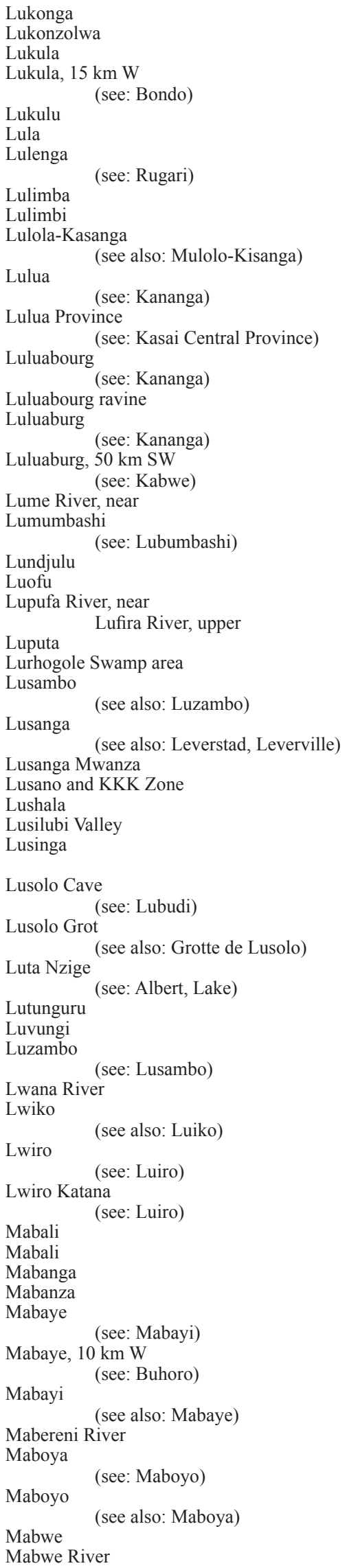

\begin{tabular}{|c|c|c|}
\hline CG-LL & $05^{\circ} 48^{\prime} \mathrm{S}, 22^{\circ} 37^{\prime} \mathrm{E}$ & $632 \mathrm{~m}$ \\
\hline CG-HK & $08^{\circ} 47^{\prime} \mathrm{S}, 28^{\circ} 39^{\prime} \mathrm{E}$ & $918 \mathrm{~m}$ \\
\hline CG-BC & $05^{\circ} 23^{\prime} \mathrm{S}, 12^{\circ} 57^{\prime} \mathrm{E}$ & $196 \mathrm{~m}$ \\
\hline CG-BC & $05^{\circ} 24^{\prime} \mathrm{S}, 12^{\circ} 54^{\prime} \mathrm{E}$ & \\
\hline CG-TG & $07^{\circ} 08^{\prime} \mathrm{S}, 27^{\circ} 07^{\prime} \mathrm{E}$ & $573 \mathrm{~m}$ \\
\hline CG-LL & ca $07^{\circ} 25^{\prime} \mathrm{S}, 22^{\circ} 33^{\prime} \mathrm{E}$ & \\
\hline CG-NK & $01^{\circ} 25^{\prime} \mathrm{S}, 29^{\circ} 22^{\prime} \mathrm{E}$ & $2089 \mathrm{~m}$ \\
\hline CG-SK & $04^{\circ} 42^{\prime} \mathrm{S}, 28^{\circ} 38^{\prime} \mathrm{E}$ & $877 \mathrm{~m}$ \\
\hline CG-NK & $00^{\circ} 28^{\prime} \mathrm{S}, 29^{\circ} 39^{\prime} \mathrm{E}$ & \\
\hline CG-MN & $03^{\circ} 06^{\prime} \mathrm{S}, 26^{\circ} 59^{\prime} \mathrm{E}$ & \\
\hline CG-LL & $05^{\circ} 54^{\prime} \mathrm{S}, 22^{\circ} 25^{\prime} \mathrm{E}$ & $608 \mathrm{~m}$ \\
\hline \multicolumn{3}{|l|}{ CG } \\
\hline CG-LL & $05^{\circ} 54^{\prime} \mathrm{S}, 22^{\circ} 25^{\prime} \mathrm{E}$ & $608 \mathrm{~m}$ \\
\hline CG-LL & ca $05^{\circ} 53^{\prime} \mathrm{S}, 22^{\circ} 26^{\prime} \mathrm{E}$ & \\
\hline CG-LL & $05^{\circ} 54^{\prime} \mathrm{S}, 22^{\circ} 25^{\prime} \mathrm{E}$ & $608 \mathrm{~m}$ \\
\hline CG-LL & $06^{\circ} 12^{\prime} \mathrm{S}, 22^{\circ} 23^{\prime} \mathrm{E}$ & $610 \mathrm{~m}$ \\
\hline $\begin{array}{l}\text { CG-NK } \\
\text { CG-HK }\end{array}$ & ca $00^{\circ} 15^{\prime} \mathrm{N}, 29^{\circ} 35^{\prime} \mathrm{E}$ & $1209 \mathrm{~m}$ \\
\hline CG-NK & $00^{\circ} 20^{\prime} \mathrm{S}, 28^{\circ} 36^{\prime} \mathrm{E}$ & $1300 \mathrm{~m}$ \\
\hline CG-NK & $00^{\circ} 37^{\prime} \mathrm{S}, 29^{\circ} 07^{\prime} \mathrm{E}$ & $1809 \mathrm{~m}$ \\
\hline CG-HK & ca $10^{\circ} 54^{\prime} \mathrm{S}, 26^{\circ} 60^{\prime} \mathrm{E}$ & \\
\hline CG-LM & $07^{\circ} 10^{\prime} \mathrm{S}, 23^{\circ} 42^{\prime} \mathrm{E}$ & $841 \mathrm{~m}$ \\
\hline CG-SK & ca $02^{\circ} 00^{\prime} \mathrm{S}, 28^{\circ} 45^{\prime} \mathrm{E}$ & \\
\hline CG-SN & $04^{\circ} 58^{\prime} \mathrm{S}, 23^{\circ} 27^{\prime} \mathrm{E}$ & $397 \mathrm{~m}$ \\
\hline CG-KU & $04^{\circ} 50^{\prime} \mathrm{S}, 18^{\circ} 44^{\prime} \mathrm{E}$ & $307 \mathrm{~m}$ \\
\hline $\begin{array}{l}\text { CG-BC } \\
\text { CG }\end{array}$ & $05^{\circ} 50^{\prime} \mathrm{S}, 13^{\circ} 03^{\prime} \mathrm{E}$ & \\
\hline CG-SK & $02^{\circ} 13^{\prime} \mathrm{S}, 28^{\circ} 49^{\prime} \mathrm{E}$ & \\
\hline CG-NK & ca $00^{\circ} 25^{\prime} \mathrm{N}, 29^{\circ} 46^{\prime} \mathrm{E}$ & \\
\hline CG-HL & $08^{\circ} 56^{\prime} \mathrm{S}, 27^{\circ} 12^{\prime} \mathrm{E}$ & $1513-1760$ \\
\hline CG-LB & $09^{\circ} 57^{\prime} \mathrm{S}, 25^{\circ} 58^{\prime} \mathrm{E}$ & $\underset{1307 \mathrm{~m}}{\mathrm{~m}}$ \\
\hline CG-HK & $09^{\circ} 56^{\prime} \mathrm{S}, 25^{\circ} 28^{\prime} \mathrm{E}$ & \\
\hline CG-IT & ca $01^{\circ} 40^{\prime} \mathrm{N}, 30^{\circ} 40^{\prime} \mathrm{E}$ & \\
\hline CG-NK & $00^{\circ} 28^{\prime} \mathrm{S}, 28^{\circ} 49^{\prime} \mathrm{E}$ & $1774 \mathrm{~m}$ \\
\hline CG-SK & $02^{\circ} 52^{\prime} \mathrm{S}, 29^{\circ} 02^{\prime} \mathrm{E}$ & $887 \mathrm{~m}$ \\
\hline CG-SN & $04^{\circ} 58^{\prime} \mathrm{S}, 23^{\circ} 27^{\prime} \mathrm{E}$ & $397 \mathrm{~m}$ \\
\hline CG-SK & ca $01^{\circ} 56^{\prime} \mathrm{S}, 28^{\circ} 28^{\prime} \mathrm{E}$ & \\
\hline CG-SK & $03^{\circ} 05^{\prime} \mathrm{S}, 28^{\circ} 47^{\prime} \mathrm{E}$ & \\
\hline CG-SK & $02^{\circ} 14^{\prime} \mathrm{S}, 28^{\circ} 48^{\prime} \mathrm{E}$ & $1646 \mathrm{~m}$ \\
\hline CG-SK & $02^{\circ} 14^{\prime} \mathrm{S}, 28^{\circ} 48^{\prime} \mathrm{E}$ & $1646 \mathrm{~m}$ \\
\hline CG-SK & $02^{\circ} 20^{\prime} \mathrm{S}, 28^{\circ} 50^{\prime} \mathrm{E}$ & \\
\hline CG-ET & $00^{\circ} 55^{\prime} \mathrm{S}, 18^{\circ} 09^{\prime} \mathrm{E}$ & \\
\hline CG-HU & $04^{\circ} 22^{\prime} \mathrm{N}, 29^{\circ} 47^{\prime} \mathrm{E}$ & \\
\hline RW-OU & $02^{\circ} 03^{\prime} \mathrm{S}, 29^{\circ} 25^{\prime} \mathrm{E}$ & $1612 \mathrm{~m}$ \\
\hline BY-CI & $02^{\circ} 43^{\prime} \mathrm{S}, 29^{\circ} 15^{\prime} \mathrm{E}$ & $1436 \mathrm{~m}$ \\
\hline BY-CI & $02^{\circ} 42^{\prime} \mathrm{S}, 29^{\circ} 11^{\prime} \mathrm{E}$ & $1453 \mathrm{~m}$ \\
\hline BY-CI & $02^{\circ} 43^{\prime} \mathrm{S}, 29^{\circ} 15^{\prime} \mathrm{E}$ & $1436 \mathrm{~m}$ \\
\hline CG-HU & $03^{\circ} 05^{\prime} \mathrm{N}, 29^{\circ} 22^{\prime} \mathrm{E}$ & \\
\hline CG-NK & $00^{\circ} 19^{\prime} \mathrm{N}, 29^{\circ} 20^{\prime} \mathrm{E}$ & $1374 \mathrm{~m}$ \\
\hline CG-NK & $00^{\circ} 19^{\prime} \mathrm{N}, 29^{\circ} 20^{\prime} \mathrm{E}$ & $1374 \mathrm{~m}$ \\
\hline CG-HL & $08^{\circ} 39^{\prime} \mathrm{S}, 26^{\circ} 31^{\prime} \mathrm{E}$ & $574 \mathrm{~m}$ \\
\hline CG-HL & $08^{\circ} 42^{\prime} \mathrm{S}, 26^{\circ} 29^{\prime} \mathrm{E}$ & $590 \mathrm{~m}$ \\
\hline
\end{tabular}


VAN CAKENBERGHE V. et al., The bats of Congo, Rwanda and Burundi revisited

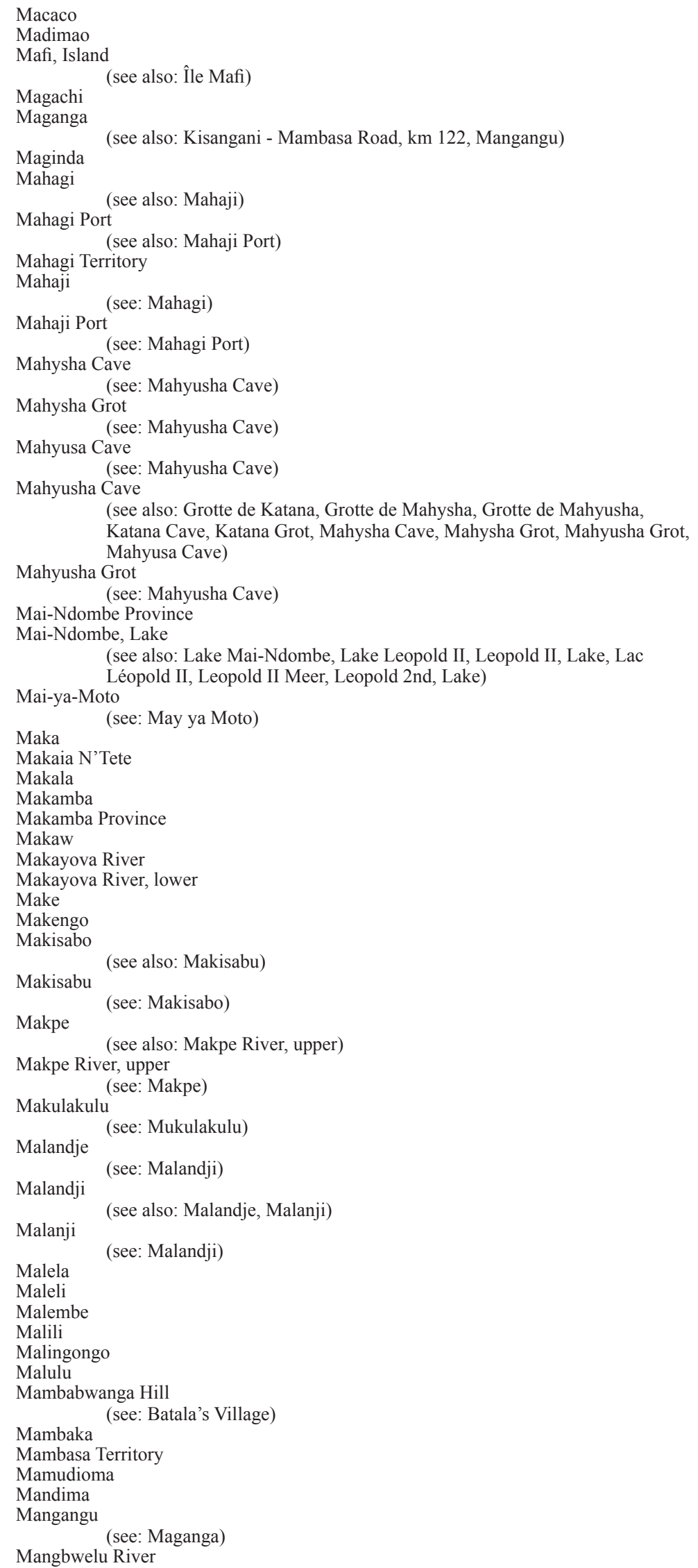

\begin{tabular}{|c|c|c|}
\hline $\begin{array}{l}\text { CG-KS } \\
\text { CG-HK } \\
\text { CG-TO }\end{array}$ & $\begin{array}{l}05^{\circ} 28^{\prime} \mathrm{S}, 21^{\circ} 10^{\prime} \mathrm{E} \\
11^{\circ} 40^{\prime} \mathrm{S}, 27^{\circ} 29^{\prime} \mathrm{E} \\
00^{\circ} 28^{\prime} \mathrm{N}, 25^{\circ} 17^{\prime} \mathrm{E}\end{array}$ & \\
\hline $\begin{array}{l}\text { RW-ES } \\
\text { CG-TO }\end{array}$ & $\begin{array}{l}01^{\circ} 20^{\prime} \mathrm{S}, 30^{\circ} 34^{\prime} \mathrm{E} \\
00^{\circ} 51^{\prime} \mathrm{N}, 26^{\circ} 22^{\prime} \mathrm{E}\end{array}$ & \\
\hline $\begin{array}{l}\text { CG-NK } \\
\text { CG-IT }\end{array}$ & $\begin{array}{l}00^{\circ} 49^{\prime} \mathrm{N}, 29^{\circ} 54^{\prime} \mathrm{E} \\
02^{\circ} 18^{\prime} \mathrm{N}, 30^{\circ} 59^{\prime} \mathrm{E}\end{array}$ & $\begin{array}{l}850 \mathrm{~m} \\
1671 \mathrm{~m}\end{array}$ \\
\hline CG-IT & $02^{\circ} 09^{\prime} \mathrm{N}, 31^{\circ} 14^{\prime} \mathrm{E}$ & $679 \mathrm{~m}$ \\
\hline $\begin{array}{l}\text { CG-IT } \\
\text { CG-IT }\end{array}$ & $\begin{array}{l}02^{\circ} 18^{\prime} \mathrm{N}, 30^{\circ} 59^{\prime} \mathrm{E} \\
02^{\circ} 18^{\prime} \mathrm{N}, 30^{\circ} 59^{\prime} \mathrm{E}\end{array}$ & $1671 \mathrm{~m}$ \\
\hline CG-IT & $02^{\circ} 09^{\prime} \mathrm{N}, 31^{\circ} 14^{\prime} \mathrm{E}$ & $679 \mathrm{~m}$ \\
\hline CG-SK & $02^{\circ} 13^{\prime} \mathrm{S}, 28^{\circ} 50^{\prime} \mathrm{E}$ & $1480 \mathrm{~m}$ \\
\hline CG-SK & $02^{\circ} 13^{\prime} \mathrm{S}, 28^{\circ} 50^{\prime} \mathrm{E}$ & $1480 \mathrm{~m}$ \\
\hline CG-SK & $02^{\circ} 13^{\prime} \mathrm{S}, 28^{\circ} 50^{\prime} \mathrm{E}$ & $1480 \mathrm{~m}$ \\
\hline CG-SK & $02^{\circ} 13^{\prime} \mathrm{S}, 28^{\circ} 50^{\prime} \mathrm{E}$ & $1480 \mathrm{~m}$ \\
\hline CG-SK & $02^{\circ} 13^{\prime} \mathrm{S}, 28^{\circ} 50^{\prime} \mathrm{E}$ & $1480 \mathrm{~m}$ \\
\hline $\begin{array}{c}\text { CG } \\
\text { CG-MA }\end{array}$ & $02^{\circ} 05^{\prime} \mathrm{S}, 18^{\circ} 20^{\prime} \mathrm{E}$ & \\
\hline CG-NK & $00^{\circ} 14^{\prime} \mathrm{N}, 29^{\circ} 43^{\prime} \mathrm{E}$ & $950 \mathrm{~m}$ \\
\hline $\begin{array}{c}\text { CG } \\
\text { CG-BC }\end{array}$ & $\begin{array}{l}08^{\circ} 56^{\prime} \mathrm{S}, 26^{\circ} 04^{\prime} \mathrm{E} \\
05^{\circ} 33^{\prime} \mathrm{S}, 13^{\circ} 02^{\prime} \mathrm{E}\end{array}$ & \\
\hline CG-TO & $00^{\circ} 33^{\prime} \mathrm{N}, 27^{\circ} 45^{\prime} \mathrm{E}$ & $634 \mathrm{~m}$ \\
\hline $\begin{array}{l}\text { BY-MA } \\
\text { BY }\end{array}$ & $04^{\circ} 08^{\prime} \mathrm{S}, 29^{\circ} 48^{\prime} \mathrm{E}$ & $1472 \mathrm{~m}$ \\
\hline $\begin{array}{l}\text { CG-MA } \\
\text { CG-NK }\end{array}$ & & $\begin{array}{l}315 \mathrm{~m} \\
750 \mathrm{~m}\end{array}$ \\
\hline $\begin{array}{l}\text { CG-NK } \\
\text { CG-NK }\end{array}$ & $00^{\circ} 43^{\prime} \mathrm{N}, 29^{\circ} 43^{\prime} \mathrm{E}$ & $750 \mathrm{~m}$ \\
\hline CG-BC & $04^{\circ} 36^{\prime} \mathrm{S}, 15^{\circ} 10^{\prime} \mathrm{E}$ & \\
\hline CG-KN & $04^{\circ} 46^{\prime} \mathrm{S}, 16^{\circ} 19^{\prime} \mathrm{E}$ & $611 \mathrm{~m}$ \\
\hline CG-NK & $00^{\circ} 24^{\prime} \mathrm{N}, 29^{\circ} 36^{\prime} \mathrm{E}$ & $950 \mathrm{~m}$ \\
\hline CG-NK & $00^{\circ} 24^{\prime} \mathrm{N}, 29^{\circ} 36^{\prime} \mathrm{E}$ & $950 \mathrm{~m}$ \\
\hline CG-HU & $04^{\circ} 20^{\prime} \mathrm{N}, 29^{\circ} 34^{\prime} \mathrm{E}$ & \\
\hline CG-HU & $04^{\circ} 20^{\prime} \mathrm{N}, 29^{\circ} 34^{\prime} \mathrm{E}$ & \\
\hline CG-HL & $09^{\circ} 33^{\prime} \mathrm{S}, 25^{\circ} 47^{\prime} \mathrm{E}$ & $828 \mathrm{~m}$ \\
\hline CG-LL & $05^{\circ} 56^{\prime} \mathrm{S}, 22^{\circ} 18^{\prime} \mathrm{E}$ & $608 \mathrm{~m}$ \\
\hline CG-LL & $05^{\circ} 56^{\prime} \mathrm{S}, 22^{\circ} 18^{\prime} \mathrm{E}$ & $608 \mathrm{~m}$ \\
\hline CG-LL & $05^{\circ} 56^{\prime} \mathrm{S}, 22^{\circ} 18^{\prime} \mathrm{E}$ & $608 \mathrm{~m}$ \\
\hline $\begin{array}{l}\text { CG-BC } \\
\text { CG-HU }\end{array}$ & $05^{\circ} 59^{\prime} \mathrm{S}, 12^{\circ} 37^{\prime} \mathrm{E}$ & $2 \mathrm{~m}$ \\
\hline CG-NK & ca $01^{\circ} 26^{\prime} \mathrm{S}, 28^{\circ} 04^{\prime} \mathrm{E}$ & \\
\hline CG-HU & $04^{\circ} 13^{\prime} \mathrm{N}, 29^{\circ} 32^{\prime} \mathrm{E}$ & \\
\hline CG-NK & $00^{\circ} 44^{\prime} \mathrm{N}, 29^{\circ} 40^{\prime} \mathrm{E}$ & $800 \mathrm{~m}$ \\
\hline CG-NK & $00^{\circ} 32^{\prime} \mathrm{N}, 29^{\circ} 35^{\prime} \mathrm{E}$ & \\
\hline CG-NK & $00^{\circ} 30^{\prime} \mathrm{N}, 29^{\circ} 15^{\prime} \mathrm{E}$ & $1000 \mathrm{~m}$ \\
\hline CG-TO & $00^{\circ} 51^{\prime} \mathrm{N}, 27^{\circ} 33^{\prime} \mathrm{E}$ & \\
\hline CG-IT & $01^{\circ} 21^{\prime} \mathrm{N}, 29^{\circ} 03^{\prime} \mathrm{E}$ & \\
\hline CG-NK & $00^{\circ} 40^{\prime} \mathrm{N}, 29^{\circ} 38^{\prime} \mathrm{E}$ & $1000 \mathrm{~m}$ \\
\hline CG-IT & ca $01^{\circ} 21^{\prime} \mathrm{N}, 29^{\circ} 03^{\prime} \mathrm{E}$ & \\
\hline CG-TO & $00^{\circ} 51^{\prime} \mathrm{N}, 26^{\circ} 22^{\prime} \mathrm{E}$ & \\
\hline CG-NK & $00^{\circ} 21^{\prime} \mathrm{N}, 29^{\circ} 44^{\prime} \mathrm{E}$ & $1300 \mathrm{~m}$ \\
\hline
\end{tabular}


Mani Cave or Nkela Cave or Mbumba-Nsi

(see also: Grotte de Mani ou Grotte de Nkela de Mbumba-Nsi, Mani Grot of Nkela Grot van Mbumba-Nsi)

Mani Grot of Nkela Grot van Mbumba-Nsi

(see: Mani Cave or Nkela Cave or Mbumba-Nsi)

Maniema Province

Manono

(see also: Manono Katanga)

Manono Katanga

(see: Manono)

Marari

Marion, Lake

(see also: Lake Marion, Lac Marion)

Marumbi

Marunga mountains

Marungu

Masaba

Masabu

Masaka - Kigali

Masako

Masako km 15 Buta

Masha

Masibingi

Masimengo

Masombwe

Matadi

(see also: Matidi)

Matale

Matamba

Matata Cave

Matidi

(see: Matadi)

Matupi Cave

(see also: Grotte de Matupi, Matupi Grot)

Matupi Grot

Mauda

(see: Matupi Cave)

Maunzi

(see also: Mavunzi, Mawunzi, Mvuazi)

Mavunzi

(see: Maunzi)

Mawambi

Mawunzi (see: Maunzi)

May ya Moto

(see also: Moto, Mai ya, Moto, May a, Moto, May ya, May ya Moto, sources chaudes, Mai-ya-Moto)

May ya Moto, sources chaudes (see: May ya Moto)

Mayumbe

Mayumbe area

Mayumbe, near

Mbalaka (Plantation)

Mbale

Mbandaka

Mbanza-Ngungu

(see also: Thysstad, Thysville)

Mbawu

M'baya Cave

M'baya Grot see: Baya Cave)

Mbelekelo (see: Baya Cave)

Mbiye, Island

(see also: Île Mbiye)

Mboga (see: Boga)

Mbuji-Mayi

(see also: Mbuy Mayi, Bakwanga)

Mbuji-Mayi, 32 km SE

Mbuy Mayi

(see: Mbuji-Mayi)

Mbuye-Bala River

Mbwambala

Medja

(see: Medje)
CG

CG

CG-TG $\quad 07^{\circ} 18^{\prime} \mathrm{S}, 27^{\circ} 25^{\prime} \mathrm{E} \quad 664 \mathrm{~m}$

CG-TG

$07^{\circ} 18^{\prime} \mathrm{S}, 27^{\circ} 25^{\prime} \mathrm{E}$

$664 \mathrm{~m}$

CG-HU $\quad 04^{\circ} 20^{\prime} \mathrm{N}, 29^{\circ} 40^{\prime} \mathrm{E}$

$\mathrm{CG}$

CG

CG-TG

CG-TG

$\mathrm{CG}-\mathrm{KG}$

CG-KU

RW-KV

CG-TO

CG-TO

RW-OU

CG

CG-NK

CG-HK

$\mathrm{CG}-\mathrm{BC}$

CG-SK

CG-LL

CG-IT

CG-BC

CG-IT

CG-IT

$07^{\circ} 30^{\prime} \mathrm{S}, 29^{\circ} 59^{\prime} \mathrm{E}$ $06^{\circ} 43^{\prime} \mathrm{S}, 29^{\circ} 31^{\prime} \mathrm{E}$ $04^{\circ} 33^{\prime} \mathrm{S}, 16^{\circ} 54^{\prime} \mathrm{E}$ $04^{\circ} 33^{\prime} \mathrm{S}, 16^{\circ} 54^{\prime} \mathrm{E}$ $01^{\circ} 60^{\prime} \mathrm{S}, 30^{\circ} 11^{\prime} \mathrm{E}$ $00^{\circ} 36^{\prime} \mathrm{N}, 25^{\circ} 13^{\prime} \mathrm{E}$ $00^{\circ} 40^{\prime} \mathrm{N}, 25^{\circ} 14^{\prime} \mathrm{E}$ $01^{\circ} 36^{\prime} \mathrm{S}, 29^{\circ} 20^{\prime} \mathrm{E}$

$00^{\circ} 34^{\prime} \mathrm{N}, 29^{\circ} 42^{\prime} \mathrm{E}$ $09^{\circ} 05^{\prime} \mathrm{S}, 27^{\circ} 12^{\prime} \mathrm{E}$ $05^{\circ} 49^{\prime} \mathrm{S}, 13^{\circ} 27^{\prime} \mathrm{E}$

$1360 \mathrm{~m}$ $1722 \mathrm{~m}$

$597 \mathrm{~m}$

$1448 \mathrm{~m}$

$2085 \mathrm{~m}$

$800 \mathrm{~m}$

$1163 \mathrm{~m}$

$18 \mathrm{~m}$

$02^{\circ} 39^{\prime} \mathrm{S}, 28^{\circ} 22^{\prime} \mathrm{E} \quad 1217 \mathrm{~m}$

$05^{\circ} 57^{\prime} \mathrm{S}, 22^{\circ} 16^{\prime} \mathrm{E} \quad 619 \mathrm{~m}$

$00^{\circ} 58^{\prime} \mathrm{N}, 29^{\circ} 40^{\prime} \mathrm{E} \quad 1160 \mathrm{~m}$

$05^{\circ} 49^{\prime} \mathrm{S}, 13^{\circ} 27^{\prime} \mathrm{E} \quad 18 \mathrm{~m}$

$\mathrm{CG}-\mathrm{HU}$

$01^{\circ} 16^{\prime} \mathrm{N}, 29^{\circ} 55^{\prime} \mathrm{E}$

$1067 \mathrm{~m}$

$1067 \mathrm{~m}$ $05^{\circ} 19^{\prime} \mathrm{S}, 15^{\circ} 07^{\prime} \mathrm{E}$

$547 \mathrm{~m}$

CG-BC

$05^{\circ} 19^{\prime} \mathrm{S}, 15^{\circ} 07^{\prime} \mathrm{E}$

$547 \mathrm{~m}$

CG-IT

$01^{\circ} 04^{\prime} \mathrm{N}, 28^{\circ} 34^{\prime} \mathrm{E}$

$653 \mathrm{~m}$

CG-BC $05^{\circ} 19^{\prime} \mathrm{S}, 15^{\circ} 07^{\prime} \mathrm{E}$

$547 \mathrm{~m}$

CG-NK

$00^{\circ} 14^{\prime} \mathrm{N}, 29^{\circ} 43^{\prime} \mathrm{E}$

$950 \mathrm{~m}$

CG-NK

$00^{\circ} 14^{\prime} \mathrm{N}, 29^{\circ} 43^{\prime} \mathrm{E}$

$950 \mathrm{~m}$

CG-HU

$02^{\circ} 30^{\prime} \mathrm{N}, 27^{\circ} 37^{\prime} \mathrm{E}$

$678 \mathrm{~m}$

CG-BC

CG-HU

ca $04^{\circ} 30^{\prime} \mathrm{S}, 12^{\circ} 30^{\prime} \mathrm{E}$

ca $02^{\circ} 30^{\prime} \mathrm{N}, 27^{\circ} 37^{\prime} \mathrm{E}$

CG-KU $\quad 05^{\circ} 00^{\prime} \mathrm{S}, 18^{\circ} 53^{\prime} \mathrm{E}$

$\mathrm{CG}$

CG-ET

$00^{\circ} 04^{\prime} \mathrm{N}, 18^{\circ} 16^{\prime} \mathrm{E}$

$05^{\circ} 15^{\prime} \mathrm{S}, 14^{\circ} 52^{\prime} \mathrm{E}$

$306 \mathrm{~m}$

$604 \mathrm{~m}$

CG-LL $\quad 05^{\circ} 54^{\prime} \mathrm{S}, 22^{\circ} 25^{\prime} \mathrm{E}$

CG-HK $11^{\circ} 52^{\prime} \mathrm{S}, 27^{\circ} 27^{\prime} \mathrm{E}$

$1279 \mathrm{~m}$

CG-HK

$11^{\circ} 52^{\prime} \mathrm{S}, 27^{\circ} 27^{\prime} \mathrm{E}$

$1279 \mathrm{~m}$

CG-SK

CG-TO

$03^{\circ} 20^{\prime} \mathrm{S}, 28^{\circ} 04^{\prime} \mathrm{E}$

$00^{\circ} 28^{\prime} \mathrm{N}, 25^{\circ} 17^{\prime} \mathrm{E}$

$1100 \mathrm{~m}$

$413 \mathrm{~m}$

CG-IT

$01^{\circ} 03^{\prime} \mathrm{N}, 29^{\circ} 56^{\prime} \mathrm{E}$

$1562 \mathrm{~m}$

CG-KO

$06^{\circ} 09^{\prime} \mathrm{S}, 23^{\circ} 36^{\prime} \mathrm{E}$

$549 \mathrm{~m}$

CG-KO

ca $06^{\circ} 09^{\prime} \mathrm{S}, 23^{\circ} 36^{\prime} \mathrm{E}$

$06^{\circ} 09^{\prime} \mathrm{S}, 23^{\circ} 36^{\prime} \mathrm{E}$

$549 \mathrm{~m}$

CG-HL

CG-KU

ca $09^{\circ} 10^{\prime} \mathrm{S}, 27^{\circ} 04^{\prime} \mathrm{E}$

$05^{\circ} 03^{\prime} \mathrm{S}, 18^{\circ} 55^{\prime} \mathrm{E}$

$02^{\circ} 25^{\prime} \mathrm{N}, 27^{\circ} 18^{\prime} \mathrm{E}$ 


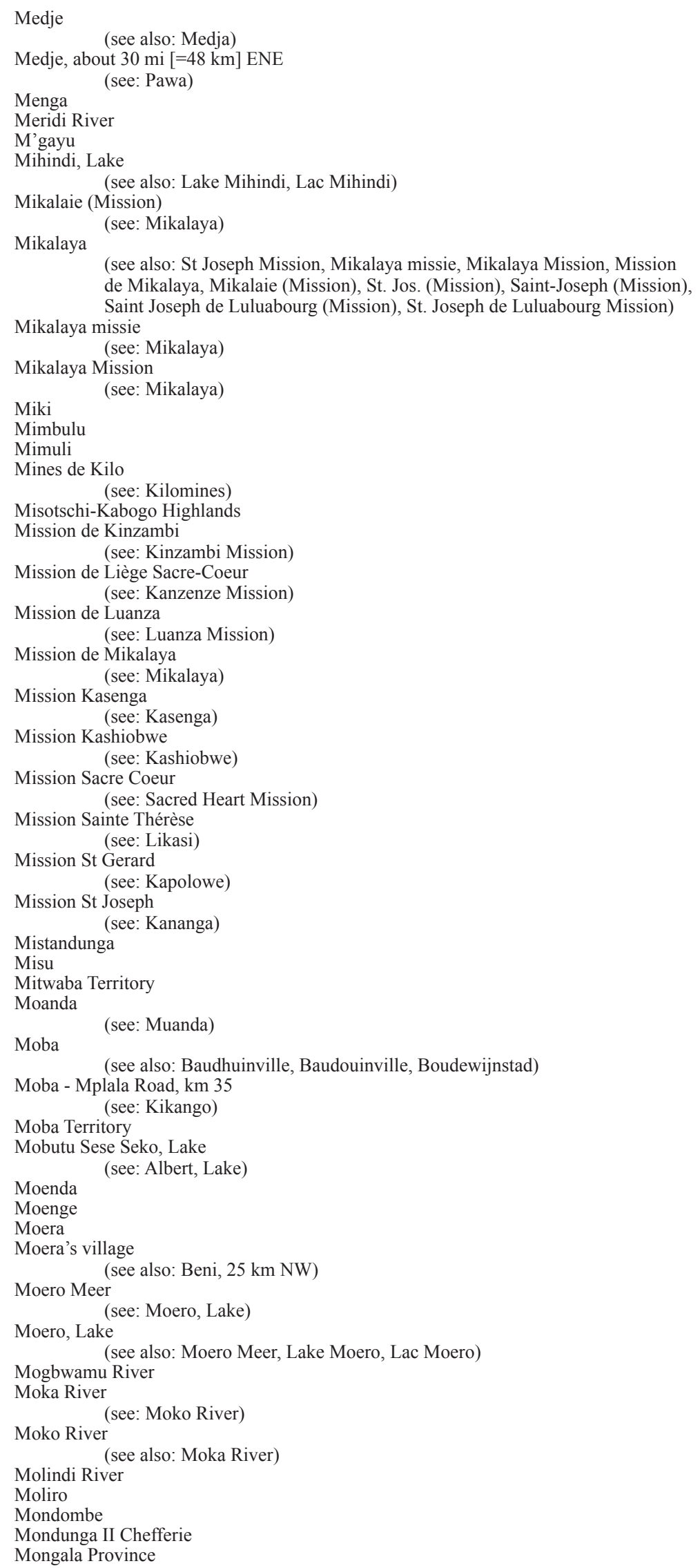

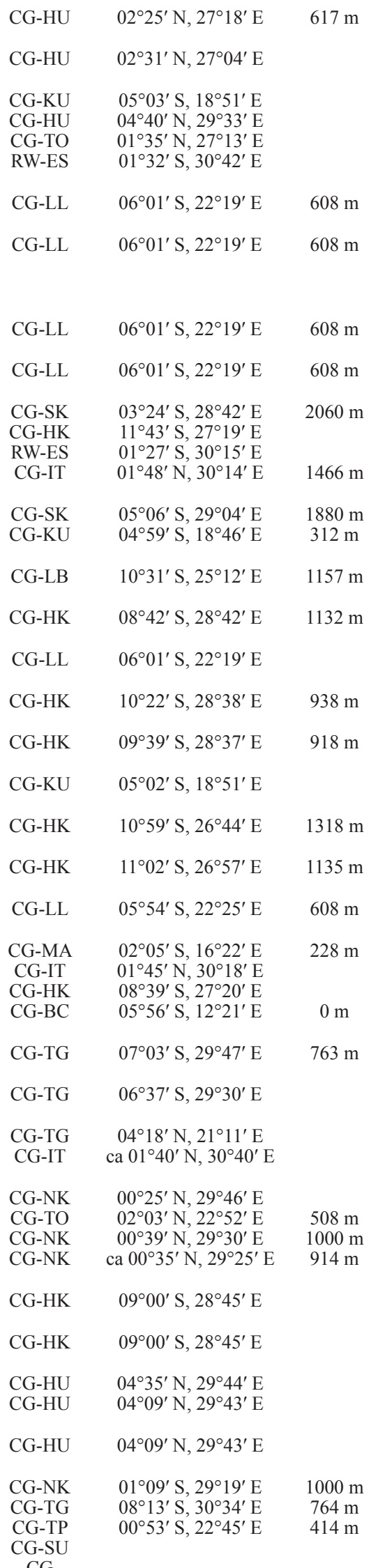




\begin{tabular}{|c|c|c|c|}
\hline $\begin{array}{l}\text { Mongbwala } \\
\qquad \text { (see: Mongbwalu) }\end{array}$ & CG-IT & $01^{\circ} 57^{\prime} \mathrm{N}, 30^{\circ} 02^{\prime} \mathrm{E}$ & $1374 \mathrm{~m}$ \\
\hline $\begin{array}{l}\text { Mongbwalu } \\
\text { (see also: Mongbwala) }\end{array}$ & CG-IT & $01^{\circ} 57^{\prime} \mathrm{N}, 30^{\circ} 02^{\prime} \mathrm{E}$ & $1374 \mathrm{~m}$ \\
\hline Mongende & CG-MA & $02^{\circ} 06^{\prime} \mathrm{S}, 16^{\circ} 20^{\prime} \mathrm{E}$ & \\
\hline Mont Hoyo & CG-IT & $01^{\circ} 13^{\prime} \mathrm{N}, 29^{\circ} 49^{\prime} \mathrm{E}$ & $1200 \mathrm{~m}$ \\
\hline $\begin{array}{l}\text { Mont Hoyo Cave } \\
\quad \text { (see: Mount Hoyo Caves) }\end{array}$ & CG-IT & $01^{\circ} 13^{\prime} \mathrm{N}, 29^{\circ} 49^{\prime} \mathrm{E}$ & $1200 \mathrm{~m}$ \\
\hline $\begin{array}{l}\text { Mont Hoyo, Grottes } \\
\text { (see: Mount Hoyo Caves) }\end{array}$ & CG-IT & $01^{\circ} 13^{\prime} \mathrm{N}, 29^{\circ} 49^{\prime} \mathrm{E}$ & $1200 \mathrm{~m}$ \\
\hline $\begin{array}{l}\text { Montembessa Cave } \\
\text { (see also: Grotte de Montembessa) }\end{array}$ & $\mathrm{CG}$ & & \\
\hline Morubia & CG-HU & $04^{\circ} 11^{\prime} \mathrm{N}, 29^{\circ} 29^{\prime} \mathrm{E}$ & \\
\hline Moseda $\quad$ (see: Mosenda) & CG-NK & $00^{\circ} 04^{\prime} \mathrm{N}, 29^{\circ} 22^{\prime} \mathrm{E}$ & $1737 \mathrm{~m}$ \\
\hline Mosenda $\quad$ (see also: Moseda, Mosendwa, Museya) & CG-NK & $00^{\circ} 04^{\prime} \mathrm{N}, 29^{\circ} 22^{\prime} \mathrm{E}$ & $1737 \mathrm{~m}$ \\
\hline $\begin{array}{l}\text { Mosendwa } \\
\text { (see: Mosenda) }\end{array}$ & CG-NK & $00^{\circ} 04^{\prime} \mathrm{N}, 29^{\circ} 22^{\prime} \mathrm{E}$ & $1737 \mathrm{~m}$ \\
\hline Mosenge & CG-KU & $04^{\circ} 39^{\prime} \mathrm{S}, 18^{\circ} 58^{\prime} \mathrm{E}$ & $508 \mathrm{~m}$ \\
\hline $\begin{array}{l}\text { Moto, Mai ya } \\
\text { (see: May ya Moto) }\end{array}$ & CG-NK & $00^{\circ} 14^{\prime} \mathrm{N}, 29^{\circ} 43^{\prime} \mathrm{E}$ & $950 \mathrm{~m}$ \\
\hline $\begin{array}{l}\text { Moto, May a } \\
\text { (see: May ya Moto) }\end{array}$ & CG-NK & $00^{\circ} 14^{\prime} \mathrm{N}, 29^{\circ} 43^{\prime} \mathrm{E}$ & $950 \mathrm{~m}$ \\
\hline $\begin{array}{l}\text { Moto, May ya } \\
\text { (see: May ya Moto) }\end{array}$ & CG-NK & $00^{\circ} 14^{\prime} \mathrm{N}, 29^{\circ} 43^{\prime} \mathrm{E}$ & $950 \mathrm{~m}$ \\
\hline $\begin{array}{l}\text { Mount Gundu } \\
\text { (see: Gundu, mount) }\end{array}$ & CG-BU & & \\
\hline $\begin{array}{l}\text { Mount Homa } \\
\text { (see: Homa, Mount) }\end{array}$ & CG-IT & $01^{\circ} 13^{\prime} \mathrm{N}, 29^{\circ} 49^{\prime} \mathrm{E}$ & \\
\hline $\begin{array}{l}\text { Mount Homa } \\
\text { (see: Hoyo, mount) }\end{array}$ & CG-IT & $01^{\circ} 13^{\prime} \mathrm{N}, 29^{\circ} 49^{\prime} \mathrm{E}$ & $1200 \mathrm{~m}$ \\
\hline $\begin{array}{l}\text { Mount Hoyo } \\
\text { (see: Hoyo, mount) }\end{array}$ & CG-IT & $01^{\circ} 13^{\prime} \mathrm{N}, 29^{\circ} 49^{\prime} \mathrm{E}$ & $1200 \mathrm{~m}$ \\
\hline $\begin{array}{l}\text { Mount Hoyo Caverns } \\
\text { (see: Mount Hoyo Caves) }\end{array}$ & CG-IT & $01^{\circ} 13^{\prime} \mathrm{N}, 29^{\circ} 49^{\prime} \mathrm{E}$ & $1200 \mathrm{~m}$ \\
\hline $\begin{array}{l}\text { Mount Hoyo Caves } \\
\text { (see also: Mount Hoyo Caverns, Mont Hoyo, Grottes, Irumu, } 20 \mathrm{mi}[=32 \\
\text { km] S, Mont Hoyo Cave, Kabatibi Cave) }\end{array}$ & CG-IT & $01^{\circ} 13^{\prime} \mathrm{N}, 29^{\circ} 49^{\prime} \mathrm{E}$ & $1200 \mathrm{~m}$ \\
\hline $\begin{array}{l}\text { Mount Kabobo } \\
\text { (see: Kabobo, mount) }\end{array}$ & CG-TG & $05^{\circ} 04^{\prime} \mathrm{S}, 29^{\circ} 00^{\prime} \mathrm{E}$ & $2440 \mathrm{~m}$ \\
\hline Mount Kahuzi, W slope & CG-SK & $02^{\circ} 12^{\prime} \mathrm{S}, 28^{\circ} 40^{\prime} \mathrm{E}$ & $2560 \mathrm{~m}$ \\
\hline $\begin{array}{l}\text { Mount Korovi } \\
\text { (see: Korovi, mount) }\end{array}$ & CG-IT & $01^{\circ} 39^{\prime} \mathrm{N}, 30^{\circ} 36^{\prime} \mathrm{E}$ & $2010 \mathrm{~m}$ \\
\hline $\begin{array}{l}\text { Mount Mugusa } \\
\text { (see: Mugusa, mount) }\end{array}$ & RW-SU & $02^{\circ} 29^{\prime} \mathrm{S}, 29^{\circ} 51^{\prime} \mathrm{E}$ & $1575 \mathrm{~m}$ \\
\hline $\begin{array}{l}\text { Mount Muvo } \\
\text { (see: Muvo, mount) }\end{array}$ & CG-NK & $01^{\circ} 14^{\prime} \mathrm{S}, 29^{\circ} 18^{\prime} \mathrm{E}$ & \\
\hline Mount Ne (see: Né, mount) & CG-IT & $01^{\circ} 42^{\prime} \mathrm{N}, 30^{\circ} 39^{\prime} \mathrm{E}$ & \\
\hline Mount Sombwe & CG-HL & $09^{\circ} 10^{\prime} \mathrm{S}, 26^{\circ} 43^{\prime} \mathrm{E}$ & \\
\hline $\begin{array}{l}\text { Mount Tshiaberimu } \\
\text { (see: Tshiabirimu, mount) }\end{array}$ & CG-NK & ca $00^{\circ} 05^{\prime} \mathrm{S}, 29^{\circ} 26^{\prime} \mathrm{E}$ & \\
\hline $\begin{array}{l}\text { Mount Tshiabirimu } \\
\text { (see: Tshiabirimu, mount) }\end{array}$ & CG-NK & ca $00^{\circ} 05^{\prime} \mathrm{S}, 29^{\circ} 26^{\prime} \mathrm{E}$ & \\
\hline $\begin{array}{l}\text { Mount Wago } \\
\text { (see: Wago, mount) }\end{array}$ & CG-IT & $01^{\circ} 45^{\prime} \mathrm{N}, 30^{\circ} 40^{\prime} \mathrm{E}$ & $2240 \mathrm{~m}$ \\
\hline Mouth of Congo & CG-BC & ca $06^{\circ} 00^{\prime} \mathrm{S}, 12^{\circ} 30^{\prime} \mathrm{E}$ & \\
\hline Mpala & $\mathrm{CG}$ & $06^{\circ} 45^{\prime} \mathrm{S}, 29^{\circ} 31^{\prime} \mathrm{E}$ & $774 \mathrm{~m}$ \\
\hline M'Pala, Kazenze & CG-LB & $10^{\circ} 29^{\prime} \mathrm{S}, 25^{\circ} 13^{\prime} \mathrm{E}$ & \\
\hline Mpaza & CG-HU & $04^{\circ} 14^{\prime} \mathrm{N}, 29^{\circ} 37^{\prime} \mathrm{E}$ & $878 \mathrm{~m}$ \\
\hline Mpe & CG-MA & $02^{\circ} 12^{\prime} \mathrm{S}, 17^{\circ} 38^{\prime} \mathrm{E}$ & $399 \mathrm{~m}$ \\
\hline $\begin{array}{l}\text { Mpinga Cave } \\
\text { (see: Mpinga Mission) }\end{array}$ & BY-RT & $03^{\circ} 44^{\prime} \mathrm{S}, 30^{\circ} 11^{\prime} \mathrm{E}$ & $1930 \mathrm{~m}$ \\
\hline $\begin{array}{l}\text { Mpinga Mission } \\
\text { (see also: Rutana, } 46 \text { km from, Mpinga Cave) }\end{array}$ & BY-RT & $03^{\circ} 44^{\prime} \mathrm{S}, 30^{\circ} 11^{\prime} \mathrm{E}$ & $1930 \mathrm{~m}$ \\
\hline $\begin{array}{l}\text { Mpopola Cave } \\
\text { (see also: Grotte de Mpopola, Mpopola Grot) }\end{array}$ & CG-HL & $09^{\circ} 56^{\prime} \mathrm{S}, 25^{\circ} 58^{\prime} \mathrm{E}$ & $1450 \mathrm{~m}$ \\
\hline $\begin{array}{l}\text { Mpopola Grot } \\
\text { (see: Mpopola Cave) }\end{array}$ & $\mathrm{CG}$ & $09^{\circ} 56^{\prime} \mathrm{S}, 25^{\circ} 58^{\prime} \mathrm{E}$ & $1450 \mathrm{~m}$ \\
\hline Muanda $\quad$ (see also: Moanda) & CG-BC & $05^{\circ} 56^{\prime} \mathrm{S}, 12^{\circ} 21^{\prime} \mathrm{E}$ & $0 \mathrm{~m}$ \\
\hline Mudina $\quad$ (see: Ndemba) & CG-LL & $05^{\circ} 30^{\prime} \mathrm{S}, 22^{\circ} 16^{\prime} \mathrm{E}$ & \\
\hline
\end{tabular}




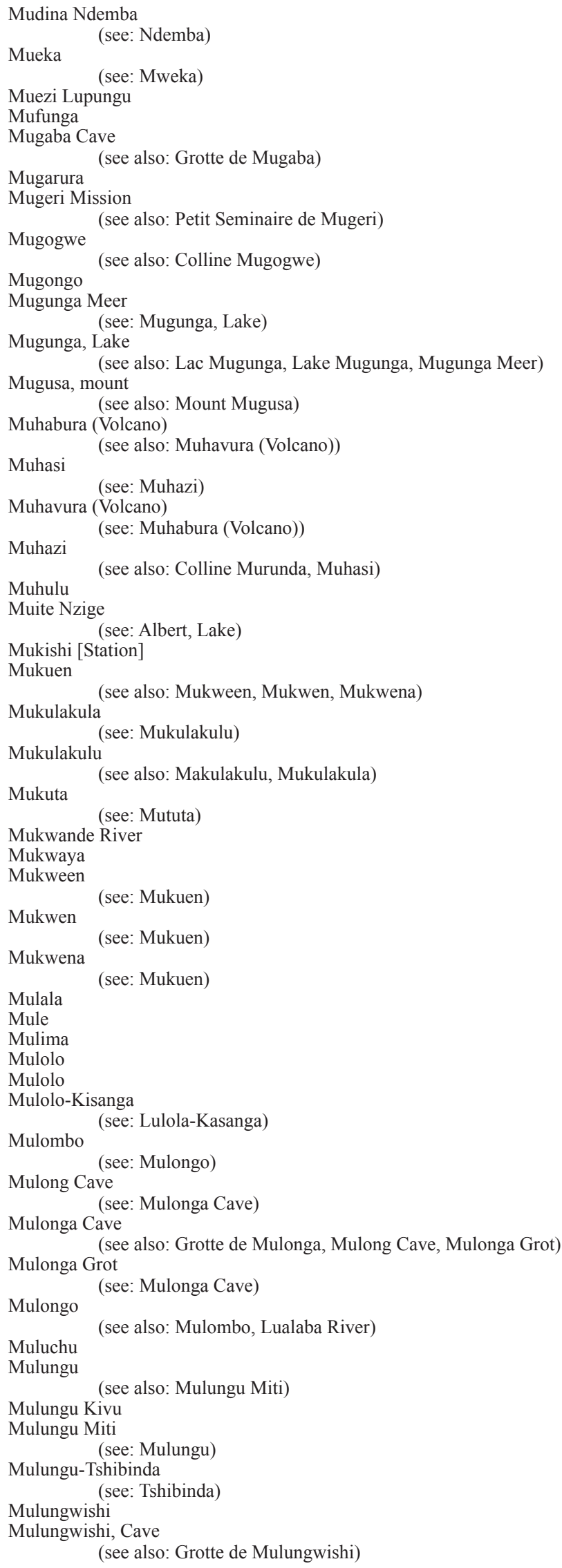

CG-LL $\quad 05^{\circ} 30^{\prime} \mathrm{S}, 22^{\circ} 16^{\prime} \mathrm{E}$

CG-KS $\quad 04^{\circ} 51^{\prime} \mathrm{S}, 21^{\circ} 34^{\prime} \mathrm{E}$

$466 \mathrm{~m}$

CG-HL $\quad 09^{\circ} 25^{\prime} \mathrm{S}, 24^{\circ} 55^{\prime} \mathrm{E}$

CG-HK $10^{\circ} 58^{\prime} \mathrm{S}, 26^{\circ} 47^{\prime} \mathrm{E}$

CG-SK ca $02^{\circ} 19^{\prime} \mathrm{S}, 28^{\circ} 31^{\prime} \mathrm{E}$

RW-OU ca $01^{\circ} 42^{\prime} \mathrm{S}, 30^{\circ} 45^{\prime} \mathrm{E}$

CG-SK $\quad 02^{\circ} 12^{\prime} \mathrm{S}, 28^{\circ} 42^{\prime} \mathrm{E}$

$1493 \mathrm{~m}$

RW-SU $\quad 02^{\circ} 31^{\prime} \mathrm{S}, 29^{\circ} 49^{\prime} \mathrm{E}$

CG-NK $\quad 01^{\circ} 30^{\prime} \mathrm{S}, 29^{\circ} 20^{\prime} \mathrm{E}$

CG-NK $\quad 01^{\circ} 37^{\prime} \mathrm{S}, 29^{\circ} 08^{\prime} \mathrm{E}$

CG-NK $\quad 01^{\circ} 37^{\prime} \mathrm{S}, 29^{\circ} 08^{\prime} \mathrm{E} \quad 1500 \mathrm{~m}$

RW-SU $\quad 02^{\circ} 29^{\prime} \mathrm{S}, 29^{\circ} 51^{\prime} \mathrm{E} \quad 1575 \mathrm{~m}$

RW-NO $\quad 01^{\circ} 23^{\prime} \mathrm{S}, 29^{\circ} 43^{\prime} \mathrm{E}$

RW-ES $\quad 01^{\circ} 54^{\prime} \mathrm{S}, 30^{\circ} 25^{\prime} \mathrm{E}$

$1529 \mathrm{~m}$

RW-NO $\quad 01^{\circ} 23^{\prime} \mathrm{S}, 29^{\circ} 43^{\prime} \mathrm{E}$

RW-ES $\quad 01^{\circ} 54^{\prime} \mathrm{S}, 30^{\circ} 25^{\prime} \mathrm{E} \quad 1529 \mathrm{~m}$

CG-NK $\quad 01^{\circ} 03^{\prime} \mathrm{S}, 27^{\circ} 17^{\prime} \mathrm{E} \quad 671 \mathrm{~m}$

CG-IT ca $01^{\circ} 40^{\prime} \mathrm{N}, 30^{\circ} 40^{\prime} \mathrm{E}$

CG-HL $\quad 08^{\circ} 30^{\prime} \mathrm{S}, 24^{\circ} 44^{\prime} \mathrm{E}$

CG-HK $\quad 11^{\circ} 46^{\prime} \mathrm{S}, 27^{\circ} 27^{\prime} \mathrm{E}$

CG-HL $\quad 09^{\circ} 33^{\prime} \mathrm{S}, 25^{\circ} 47^{\prime} \mathrm{E}$

$828 \mathrm{~m}$

CG-HL $\quad 09^{\circ} 33^{\prime} \mathrm{S}, 25^{\circ} 47^{\prime} \mathrm{E} \quad 828 \mathrm{~m}$

CG-HK $\quad 11^{\circ} 21^{\prime} \mathrm{S}, 27^{\circ} 47^{\prime} \mathrm{E} \quad 1229 \mathrm{~m}$

CG-NK $\quad 00^{\circ} 19^{\prime} \mathrm{N}, 29^{\circ} 42^{\prime} \mathrm{E} \quad 1300 \mathrm{~m}$

CG-LM $06^{\circ} 32^{\prime} \mathrm{S}, 23^{\circ} 26^{\prime} \mathrm{E}$

CG-HK $\quad 11^{\circ} 46^{\prime} \mathrm{S}, 27^{\circ} 27^{\prime} \mathrm{E}$

CG-HK $\quad 11^{\circ} 46^{\prime} \mathrm{S}, 27^{\circ} 27^{\prime} \mathrm{E}$

CG-HK $\quad 11^{\circ} 46^{\prime} \mathrm{S}, 27^{\circ} 27^{\prime} \mathrm{E}$

CG-NK $\quad 00^{\circ} 19^{\prime} \mathrm{N}, 29^{\circ} 44^{\prime} \mathrm{E} \quad 1200 \mathrm{~m}$

CG-SK $\quad 02^{\circ} 24^{\prime} \mathrm{S}, 28^{\circ} 33^{\prime} \mathrm{E} \quad 1936 \mathrm{~m}$

CG-LB

CG-MN

CG-MN

$09^{\circ} 56^{\prime} \mathrm{S}, 26^{\circ} 58^{\prime} \mathrm{E}$ $03^{\circ} 06^{\prime} \mathrm{S}, 26^{\circ} 58^{\prime} \mathrm{E}$ $03^{\circ} 06^{\prime} \mathrm{S}, 26^{\circ} 59^{\prime} \mathrm{E}$

CG-TG

$07^{\circ} 50^{\prime} \mathrm{S}, 27^{\circ} 00^{\prime} \mathrm{E}$

$578 \mathrm{~m}$

CG-LB $\quad 09^{\circ} 56^{\prime} \mathrm{S}, 25^{\circ} 58^{\prime} \mathrm{E}$

CG-LB

$09^{\circ} 56^{\prime} \mathrm{S}, 25^{\circ} 58^{\prime} \mathrm{E}$

CG-LB

$09^{\circ} 56^{\prime} \mathrm{S}, 25^{\circ} 58^{\prime} \mathrm{E}$

CG-TG

$07^{\circ} 50^{\prime} \mathrm{S}, 27^{\circ} 00^{\prime} \mathrm{E}$

$578 \mathrm{~m}$

RW-ES $\quad 01^{\circ} 32^{\prime} \mathrm{S}, 30^{\circ} 37^{\prime} \mathrm{E}$

$02^{\circ} 19^{\prime} \mathrm{S}, 28^{\circ} 48^{\prime} \mathrm{E}$

$1159 \mathrm{~m}$

CG-SK $02^{\circ} 54^{\prime} \mathrm{S}, 27^{\circ} 56^{\prime} \mathrm{E}$

CG-LB $\quad 10^{\circ} 47^{\prime} \mathrm{S}, 26^{\circ} 38^{\prime} \mathrm{E}$ 
Mumba

Mumena

(see also: Lubumbashi, 70 km E, Mumene)

Mumena

Mumen

(see: Mumena)

Mumushwizi Valley

Munga

Mungamba

Munoi

(see: Munoi confluence)

Munoi confluence (see also: Munoi)

Mura pumping Station

Muradingusha

(see: Mwadingusha)

Murambi

Muramvya Province

Murore

Murunda

Musanse

(see: Musanze)

Musanze

(see also: Colline Musanze, Musanse)

Musanze-Djomba

Musenge

Musenge

Musenge

Musenji

Museya

(see: Musindji)

(see: Mosenda)

Musha

(see also: Colline Musha)

Mushie

Musindji

Musingi

(see also: Musingi, Musenji)

(see: Musindji)

Musisi swamp area

Musonge

(see also: Lubumbashi, $69 \mathrm{~km} \mathrm{E}$ )

Musosa, near

Musugeriza River

Mutembu Kaniki Kapangu (see: Kaniki Kapangu)

Mutsora

Mutsora Station

Mutumba Mission

Mutura

Mututa

Mutwanga

see also: Mukuta)

Muvo, mount

see also: Kya irtumbi)

(see also: Mount Muvo)

Muvule, Cave $4 \mathrm{~km}$ from

Muyebe

Muyinga Province

Muyirimbo

Mvuazi

(see: Maunzi)

Mwadingusha

Mwadingusha

(see also: Muradingusha)

Mwanakusu (see: Mwana-Kusu)

Mwana-Kusu (see also: Mwanakusu)

Mwanakusu Cave (see also: Grotte de Mwanakusu, Mwanakusu Grot)

Mwanakusu Grot

Mwanda

\begin{tabular}{|c|c|c|}
\hline CG-NK & $01^{\circ} 27^{\prime} \mathrm{S}, 28^{\circ} 53^{\prime} \mathrm{E}$ & $1765 \mathrm{~m}$ \\
\hline CG-HK & $11^{\circ} 04^{\prime} \mathrm{S}, 28^{\circ} 08^{\prime} \mathrm{E}$ & $1460 \mathrm{~m}$ \\
\hline CG-HK & $11^{\circ} 46^{\prime} \mathrm{S}, 26^{\circ} 31^{\prime} \mathrm{E}$ & $1460 \mathrm{~m}$ \\
\hline CG-HK & $11^{\circ} 04^{\prime} \mathrm{S}, 28^{\circ} 08^{\prime} \mathrm{E}$ & \\
\hline BY-BI & $03^{\circ} 55^{\prime} \mathrm{S}, 29^{\circ} 35^{\prime} \mathrm{E}$ & $1880 \mathrm{~m}$ \\
\hline CG-SK & $03^{\circ} 35^{\prime} \mathrm{S}, 28^{\circ} 13^{\prime} \mathrm{E}$ & \\
\hline CG-KU & $05^{\circ} 28^{\prime} \mathrm{S}, 18^{\circ} 35^{\prime} \mathrm{E}$ & $586 \mathrm{~m}$ \\
\hline CG-HL & $08^{\circ} 45^{\prime} \mathrm{S}, 26^{\circ} 46^{\prime} \mathrm{E}$ & \\
\hline CG-HL & $08^{\circ} 45^{\prime} \mathrm{S}, 26^{\circ} 46^{\prime} \mathrm{E}$ & $890 \mathrm{~m}$ \\
\hline CG-HK & $10^{\circ} 58^{\prime} \mathrm{S}, 26^{\circ} 32^{\prime} \mathrm{E}$ & \\
\hline CG-HK & $10^{\circ} 45^{\prime} \mathrm{S}, 27^{\circ} 15^{\prime} \mathrm{E}$ & $1158 \mathrm{~m}$ \\
\hline $\begin{array}{c}\text { CG-NK } \\
\text { BY }\end{array}$ & $01^{\circ} 08^{\prime} \mathrm{S}, 29^{\circ} 27^{\prime} \mathrm{E}$ & $1275 \mathrm{~m}$ \\
\hline BY-CA & $03^{\circ} 11^{\prime} \mathrm{S}, 30^{\circ} 40^{\prime} \mathrm{E}$ & $1826 \mathrm{~m}$ \\
\hline RW-OU & $01^{\circ} 54^{\prime} \mathrm{S}, 29^{\circ} 22^{\prime} \mathrm{E}$ & $1711 \mathrm{~m}$ \\
\hline RW-NO & $01^{\circ} 29^{\prime} \mathrm{S}, 29^{\circ} 36^{\prime} \mathrm{E}$ & \\
\hline RW-NO & $01^{\circ} 29^{\prime} \mathrm{S}, 29^{\circ} 36^{\prime} \mathrm{E}$ & \\
\hline CG-NK & $01^{\circ} 20^{\prime} \mathrm{S}, 29^{\circ} 34^{\prime} \mathrm{E}$ & $1500 \mathrm{~m}$ \\
\hline CG-SK & $02^{\circ} 42^{\prime} \mathrm{S}, 27^{\circ} 02^{\prime} \mathrm{E}$ & \\
\hline CG-SK & $01^{\circ} 37^{\prime} \mathrm{S}, 28^{\circ} 18^{\prime} \mathrm{E}$ & \\
\hline CG-NK & $01^{\circ} 40^{\prime} \mathrm{S}, 28^{\circ} 07^{\prime} \mathrm{E}$ & \\
\hline CG-HL & $07^{\circ} 36^{\prime} \mathrm{S}, 24^{\circ} 33^{\prime} \mathrm{E}$ & $894 \mathrm{~m}$ \\
\hline CG-NK & $00^{\circ} 04^{\prime} \mathrm{N}, 29^{\circ} 22^{\prime} \mathrm{E}$ & $1737 \mathrm{~m}$ \\
\hline RW-SU & $02^{\circ} 32^{\prime} \mathrm{S}, 29^{\circ} 52^{\prime} \mathrm{E}$ & \\
\hline CG-MA & $03^{\circ} 01^{\prime} \mathrm{S}, 16^{\circ} 54^{\prime} \mathrm{E}$ & $180 \mathrm{~m}$ \\
\hline CG-HL & $07^{\circ} 36^{\prime} \mathrm{S}, 24^{\circ} 33^{\prime} \mathrm{E}$ & $894 \mathrm{~m}$ \\
\hline CG-HL & $07^{\circ} 36^{\prime} \mathrm{S}, 24^{\circ} 33^{\prime} \mathrm{E}$ & $894 \mathrm{~m}$ \\
\hline CG-SK & ca $02^{\circ} 00^{\prime} \mathrm{S}, 28^{\circ} 45^{\prime} \mathrm{E}$ & \\
\hline CG-HK & $11^{\circ} 28^{\prime} \mathrm{S}, 27^{\circ} 52^{\prime} \mathrm{E}$ & $1183 \mathrm{~m}$ \\
\hline $\mathrm{CG}$ & $08^{\circ} 22^{\prime} \mathrm{S}, 29^{\circ} 38^{\prime} \mathrm{E}$ & \\
\hline CG-NK & $01^{\circ} 09^{\prime} \mathrm{S}, 29^{\circ} 27^{\prime} \mathrm{E}$ & $1150 \mathrm{~m}$ \\
\hline CG-KO & $06^{\circ} 45^{\prime} \mathrm{S}, 23^{\circ} 31^{\prime} \mathrm{E}$ & $805 \mathrm{~m}$ \\
\hline CG-NK & $00^{\circ} 19^{\prime} \mathrm{N}, 29^{\circ} 44^{\prime} \mathrm{E}$ & $1200 \mathrm{~m}$ \\
\hline CG-NK & $00^{\circ} 19^{\prime} \mathrm{N}, 29^{\circ} 45^{\prime} \mathrm{E}$ & $1394 \mathrm{~m}$ \\
\hline BY-BL & $03^{\circ} 35^{\prime} \mathrm{S}, 29^{\circ} 22^{\prime} \mathrm{E}$ & \\
\hline RW-OU & $01^{\circ} 36^{\prime} \mathrm{S}, 29^{\circ} 23^{\prime} \mathrm{E}$ & $2370 \mathrm{~m}$ \\
\hline CG-HK & $11^{\circ} 21^{\prime} \mathrm{S}, 27^{\circ} 47^{\prime} \mathrm{E}$ & $1229 \mathrm{~m}$ \\
\hline CG-NK & $00^{\circ} 20^{\prime} \mathrm{N}, 29^{\circ} 45^{\prime} \mathrm{E}$ & $1338 \mathrm{~m}$ \\
\hline CG-NK & $01^{\circ} 14^{\prime} \mathrm{S}, 29^{\circ} 18^{\prime} \mathrm{E}$ & \\
\hline CG-HK & ca $09^{\circ} 11^{\prime} \mathrm{S}, 27^{\circ} 13^{\prime} \mathrm{E}$ & \\
\hline $\begin{array}{c}\text { BY-MW } \\
\text { BY }\end{array}$ & $03^{\circ} 30^{\prime} \mathrm{S}, 29^{\circ} 47^{\prime} \mathrm{E}$ & $1764 \mathrm{~m}$ \\
\hline CG-NK & $00^{\circ} 32^{\prime} \mathrm{S}, 29^{\circ} 17^{\prime} \mathrm{E}$ & \\
\hline CG-BC & $05^{\circ} 19^{\prime} \mathrm{S}, 15^{\circ} 07^{\prime} \mathrm{E}$ & $547 \mathrm{~m}$ \\
\hline CG-HK & $10^{\circ} 45^{\prime} \mathrm{S}, 27^{\circ} 15^{\prime} \mathrm{E}$ & $1158 \mathrm{~m}$ \\
\hline CG-HK & $10^{\circ} 45^{\prime} \mathrm{S}, 27^{\circ} 15^{\prime} \mathrm{E}$ & $1158 \mathrm{~m}$ \\
\hline CG-MN & $04^{\circ} 26^{\prime} \mathrm{S}, 26^{\circ} 48^{\prime} \mathrm{E}$ & $612 \mathrm{~m}$ \\
\hline CG-MN & $04^{\circ} 26^{\prime} \mathrm{S}, 26^{\circ} 48^{\prime} \mathrm{E}$ & $612 \mathrm{~m}$ \\
\hline CG-MN & $04^{\circ} 35^{\prime} \mathrm{S}, 27^{\circ} 08^{\prime} \mathrm{E}$ & \\
\hline CG-MN & $04^{\circ} 35^{\prime} \mathrm{S}, 27^{\circ} 08^{\prime} \mathrm{E}$ & \\
\hline CG-SK & $02^{\circ} 13^{\prime} \mathrm{S}, 28^{\circ} 51^{\prime} \mathrm{E}$ & \\
\hline
\end{tabular}


Mwanga Cave

(see also: Defrenne Cave, Defrenne Grot, Grotte de Defrenne, Grotte de Mwanga, Jadotstad, 15 km S, Jadotville, 15 km S, Likasi, 15 km S, Mwanga

Mwanga Grot Grot)

Mwaro Province see: Mwanga Cave)

Mwebe Bridge

Mweka

(see also: Mueka, Mweka Kasai)

Mweka Kasai (see: Mweka)

Mweka Territory

Mwelampande (see: Tenke)

Mwela-Pande Cave

$$
\text { (see: Tenke) }
$$

Mwenda-Katuka Piste

Mwenga

Mwenga Territory

Mwenge

Mwera

Mweya

Mwiga

Mwiru

(see: Luiro)

Mwittanzige

Myabikere

(see: Albert, Lake)

Nadegbe Rive

Nagbarama

Nagera

(see: Nagero)

Nagero

Naimniam

(see also: Nagera)

Nakiluba

(see: Inakiluba)

Nakilubo

(see: Inakiluba)

Nakpanga

Nakpanga River

Naleza

Nambiliki / Dungu

Nampume

Nampune

(see also: Nampune)

(see: Nampume)

Nasosurro River

Ndama

Ndelele

Ndemba

Ndjili

(see also: Mudina, Mudina Ndemba)

Ndomo

Ndote

Ndotwe

Ndua

(see: Ndwa)

Ndwa

(see also: Ndua)

Né, mount

(see also: Mount Ne)

Nepoko Bomili

Netonna

New Beni

(see: Beni)

Ngamaye

Nganza

Nganza River

Ngayu [River]

(see also: Gayu [River])

N'Gayu area

Ngerere Lepi

Ngeze Kidoko
CG-HK

$10^{\circ} 58^{\prime} \mathrm{S}, 26^{\circ} 39^{\prime} \mathrm{E}$

CG-HK

$10^{\circ} 58^{\prime} \mathrm{S}, 26^{\circ} 39^{\prime} \mathrm{E}$

BY

CG-KU $\quad 05^{\circ} 03^{\prime} \mathrm{S}, 18^{\circ} 50^{\prime} \mathrm{E}$

CG-KS $04^{\circ} 51^{\prime} \mathrm{S}, 21^{\circ} 34^{\prime} \mathrm{E}$

$466 \mathrm{~m}$

CG-KS

$04^{\circ} 51^{\prime} \mathrm{S}, 21^{\circ} 34^{\prime} \mathrm{E}$

$466 \mathrm{~m}$

CG-KS $\quad 04^{\circ} 51^{\prime} \mathrm{S}, 21^{\circ} 34^{\prime} \mathrm{E}$

CG-HK $\quad 10^{\circ} 35^{\prime} \mathrm{S}, 26^{\circ} 07^{\prime} \mathrm{E}$

$1512 \mathrm{~m}$

CG-HK

$10^{\circ} 35^{\prime} \mathrm{S}, 26^{\circ} 07^{\prime} \mathrm{E}$

$1512 \mathrm{~m}$

CG-NK

CG-SK

ca $00^{\circ} 25^{\prime} \mathrm{N}, 29^{\circ} 46^{\prime} \mathrm{E}$

$03^{\circ} 02^{\prime} \mathrm{S}, 28^{\circ} 26^{\prime} \mathrm{E}$

$03^{\circ} 02^{\prime} \mathrm{S}, 28^{\circ} 26^{\prime} \mathrm{E}$

$\begin{array}{ll}\text { CG-SK } & 03^{\circ} 42^{\prime} \mathrm{S}, 28^{\circ} 10^{\prime} \mathrm{E} \\ \text { CG-HK } & 11^{\circ} 19^{\prime} \mathrm{S}, 27^{\circ} 18^{\prime} \mathrm{E}\end{array}$

$\begin{array}{ll}\text { CG-HK } & 11^{\circ} 19^{\prime} \mathrm{S}, 27^{\circ} 18^{\prime} \mathrm{E} \\ \text { BY-GI } & 03^{\circ} 29^{\prime} \mathrm{S}, 29^{\circ} 56^{\prime} \mathrm{E}\end{array}$

$00^{\circ} 40^{\prime} \mathrm{S}, 29^{\circ} 27^{\prime} \mathrm{E}$

CG-SK $02^{\circ} 14^{\prime} \mathrm{S}, 28^{\circ} 48^{\prime} \mathrm{E}$

$1643 \mathrm{~m}$

$1762 \mathrm{~m}$

$1646 \mathrm{~m}$

CG-IT ca $01^{\circ} 40^{\prime} \mathrm{N}, 30^{\circ} 40^{\prime} \mathrm{E}$

CG-NK

$01^{\circ} 10^{\prime} \mathrm{S}, 29^{\circ} 27^{\prime} \mathrm{E}$

$1160 \mathrm{~m}$

CG-HU $\quad 04^{\circ} 29^{\prime} \mathrm{N}, 29^{\circ} 39^{\prime} \mathrm{E}$

CG-HU $\quad 04^{\circ} 17^{\prime} \mathrm{N}, 29^{\circ} 32^{\prime} \mathrm{E}$

CG-HU $\quad 03^{\circ} 45^{\prime} \mathrm{N}, 29^{\circ} 31^{\prime} \mathrm{E}$

CG-HU

$03^{\circ} 45^{\prime} \mathrm{N}, 29^{\circ} 31^{\prime} \mathrm{E}$

$753 \mathrm{~m}$

$753 \mathrm{~m}$

CG-BU

CG-HK

$11^{\circ} 34^{\prime} \mathrm{S}, 27^{\circ} 19^{\prime} \mathrm{E}$

$1280 \mathrm{~m}$

CG-HK

$11^{\circ} 34^{\prime} \mathrm{S}, 27^{\circ} 19^{\prime} \mathrm{E}$

$1280 \mathrm{~m}$

$\mathrm{CG}-\mathrm{HU}$

CG-HU

$04^{\circ} 36^{\prime} \mathrm{N}, 29^{\circ} 34^{\prime} \mathrm{E}$ $04^{\circ} 39^{\prime} \mathrm{N}, 29^{\circ} 32^{\prime} \mathrm{E}$

$15^{\circ} 27^{\prime} \mathrm{S}, 27^{\circ} 20^{\prime} \mathrm{E}$

CG-HU $\quad 03^{\circ} 40^{\prime} \mathrm{N}, 29^{\circ} 10^{\prime} \mathrm{E}$

CG-HU $\quad 04^{\circ} 21^{\prime} \mathrm{N}, 29^{\circ} 49^{\prime} \mathrm{E}$

CG-HU $\quad 04^{\circ} 21^{\prime} \mathrm{N}, 29^{\circ} 49^{\prime} \mathrm{E}$

CG

$\mathrm{CG}$

CG-HU

CG-LL

$04^{\circ} 22^{\prime} \mathrm{N}, 29^{\circ} 47^{\prime} \mathrm{E}$ $05^{\circ} 30^{\prime} \mathrm{S}, 22^{\circ} 16^{\prime} \mathrm{E}$

$900 \mathrm{~m}$

CG-KN $\quad 04^{\circ} 28^{\prime} \mathrm{S}, 15^{\circ} 21^{\prime} \mathrm{E}$

CG-NK $\quad 00^{\circ} 19^{\prime} \mathrm{N}, 29^{\circ} 20^{\prime} \mathrm{E}$

CG-NK $\quad 00^{\circ} 11^{\prime} \mathrm{N}, 29^{\circ} 39^{\prime} \mathrm{E}$

CG-NK $\quad 00^{\circ} 12^{\prime} \mathrm{S}, 29^{\circ} 28^{\prime} \mathrm{E}$

CG-MA $\quad 02^{\circ} 09^{\prime} \mathrm{S}, 16^{\circ} 25^{\prime} \mathrm{E}$

CG-MA

$02^{\circ} 09^{\prime} \mathrm{S}, 16^{\circ} 25^{\prime} \mathrm{E}$

CG-IT

$01^{\circ} 42^{\prime} \mathrm{N}, 30^{\circ} 39^{\prime} \mathrm{E}$

CG-TO

CG-BC

$01^{\circ} 40^{\prime} \mathrm{N}, 27^{\circ} 01^{\prime} \mathrm{E}$

CG-NK

$05^{\circ} 58^{\prime} \mathrm{S}, 12^{\circ} 27^{\prime} \mathrm{E}$

$00^{\circ} 30^{\prime} \mathrm{N}, 29^{\circ} 28^{\prime} \mathrm{E}$

$1238 \mathrm{~m}$

CG-NK

CG-TG

$00^{\circ} 44^{\prime} \mathrm{N}, 29^{\circ} 38^{\prime} \mathrm{E}$ $07^{\circ} 07^{\prime} \mathrm{S}, 29^{\circ} 26^{\prime} \mathrm{E}$

CG-LL ca $05^{\circ} 57^{\prime} \mathrm{S}, 22^{\circ} 22^{\prime} \mathrm{E}$

CG-HU

$01^{\circ} 35^{\prime} \mathrm{N}, 27^{\circ} 13^{\prime} \mathrm{E}$

CG-HU ca $01^{\circ} 35^{\prime} \mathrm{N}, 27^{\circ} 13^{\prime} \mathrm{E}$

CG-NK $\quad 00^{\circ} 39^{\prime} \mathrm{N}, 29^{\circ} 53^{\prime} \mathrm{E}$

CG-IT $01^{\circ} 16^{\prime} \mathrm{N}, 30^{\circ} 25^{\prime} \mathrm{E}$
$327 \mathrm{~m}$

$940 \mathrm{~m}$

$1200 \mathrm{~m}$ $650 \mathrm{~m}$ 


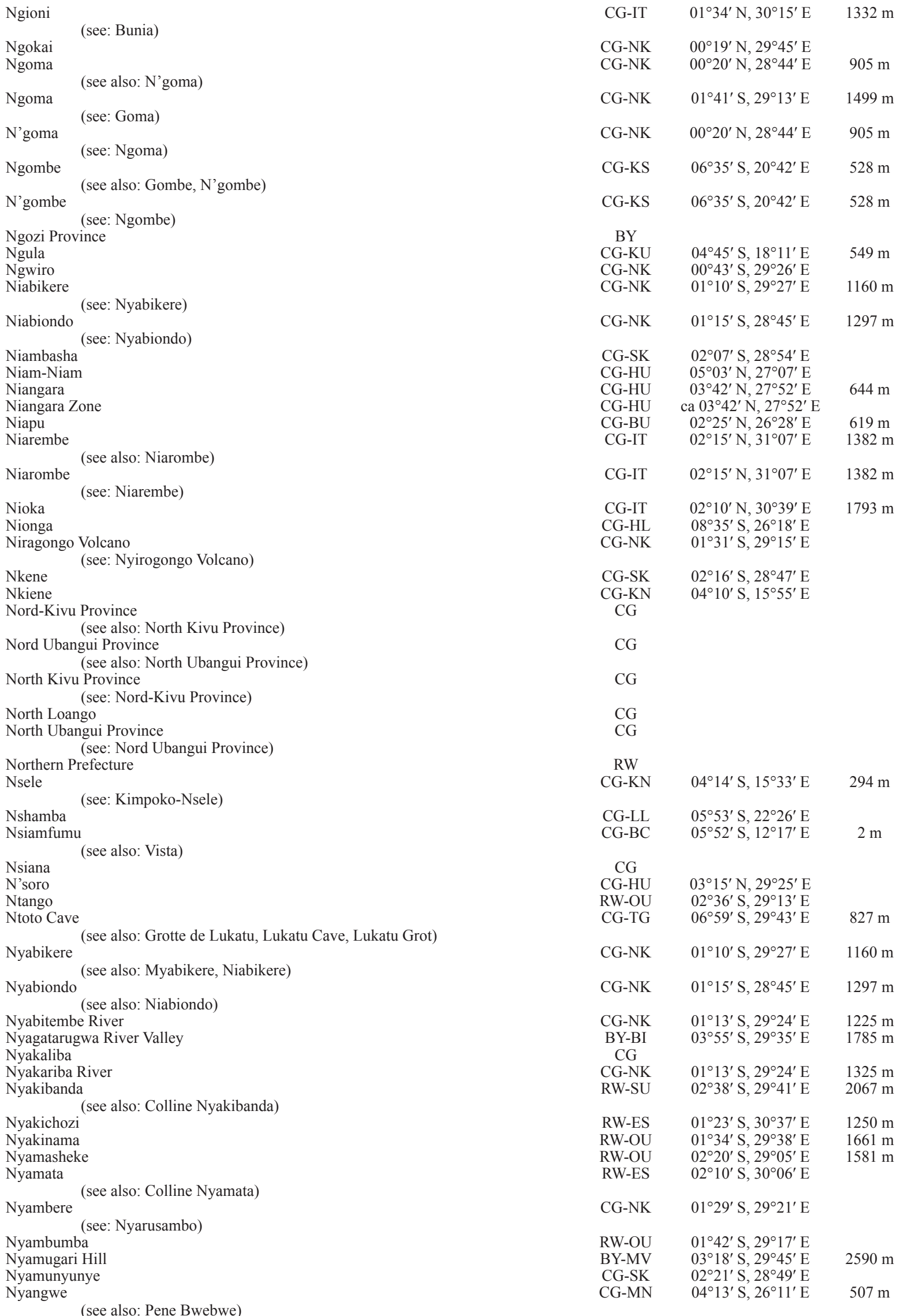




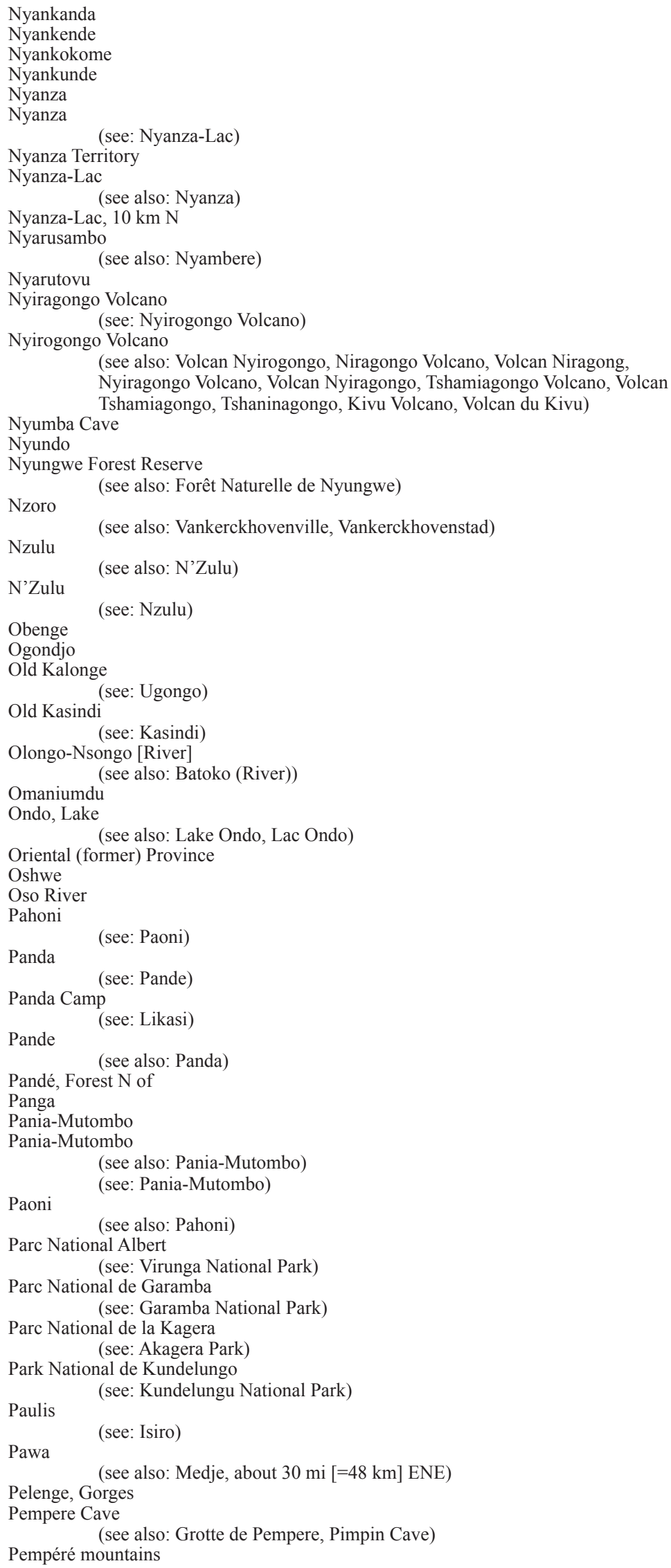

BY-RY

BY-MA

RW

CG-IT

RW-SU

BY-MA

BY-MA

BY-MA

BY-MA

CG-NK

RW-SU

CG-NK

CG-NK

$03^{\circ} 17^{\prime} \mathrm{S}, 30^{\circ} 19^{\prime} \mathrm{E}$ $03^{\circ} 55^{\prime} \mathrm{S}, 30^{\circ} 00^{\prime} \mathrm{E}$

$01^{\circ} 34^{\prime} \mathrm{N}, 30^{\circ} 15^{\prime} \mathrm{E}$ $02^{\circ} 27^{\prime} \mathrm{S}, 29^{\circ} 36^{\prime} \mathrm{E}$ $04^{\circ} 21^{\prime} \mathrm{S}, 29^{\circ} 36^{\prime} \mathrm{E}$

$772 \mathrm{~m}$

ca $04^{\circ} 20^{\prime} \mathrm{S}, 29^{\circ} 36^{\prime} \mathrm{E}$ $04^{\circ} 21^{\prime} \mathrm{S}, 29^{\circ} 36^{\prime} \mathrm{E}$

$772 \mathrm{~m}$

ca $04^{\circ} 20^{\prime} \mathrm{S}, 29^{\circ} 36^{\prime} \mathrm{E}$ $01^{\circ} 29^{\prime} \mathrm{S}, 29^{\circ} 21^{\prime} \mathrm{E}$

$02^{\circ} 37^{\prime} \mathrm{S}, 29^{\circ} 36^{\prime} \mathrm{E}$ $01^{\circ} 31^{\prime} \mathrm{S}, 29^{\circ} 15^{\prime} \mathrm{E}$

$2000 \mathrm{~m}$ $01^{\circ} 31^{\prime} \mathrm{S}, 29^{\circ} 15^{\prime} \mathrm{E}$

RW-SU $\quad 02^{\circ} 39^{\prime} \mathrm{S}, 29^{\circ} 42^{\prime} \mathrm{E}$ RW-OU $\quad 01^{\circ} 42^{\prime} \mathrm{S}, 30^{\circ} 02^{\prime} \mathrm{E}$ RW-OU $\quad 02^{\circ} 31^{\prime} \mathrm{S}, 29^{\circ} 18^{\prime} \mathrm{E}$

$1594 \mathrm{~m}$ $2500 \mathrm{~m}$

CG-HU $\quad 03^{\circ} 20^{\prime} \mathrm{N}, 29^{\circ} 30^{\prime} \mathrm{E} \quad 791 \mathrm{~m}$

CG-NK $\quad 00^{\circ} 36^{\prime} \mathrm{S}, 29^{\circ} 05^{\prime} \mathrm{E} \quad 1500 \mathrm{~m}$

CG-NK $\quad 00^{\circ} 36^{\prime} \mathrm{S}, 29^{\circ} 05^{\prime} \mathrm{E} \quad 1500 \mathrm{~m}$

CG-TO $\quad 01^{\circ} 23^{\prime} \mathrm{S}, 25^{\circ} 02^{\prime} \mathrm{E}$

CG-IT $\quad 02^{\circ} 11^{\prime} \mathrm{N}, 30^{\circ} 56^{\prime} \mathrm{E}$

CG-NK $\quad 00^{\circ} 20^{\prime} \mathrm{N}, 29^{\circ} 49^{\prime} \mathrm{E}$

$1700 \mathrm{~m}$

$2285 \mathrm{~m}$

CG-NK $\quad 00^{\circ} 02^{\prime} \mathrm{S}, 29^{\circ} 43^{\prime} \mathrm{E} \quad 1049 \mathrm{~m}$

CG-MA $\quad 01^{\circ} 39^{\prime} \mathrm{S}, 18^{\circ} 05^{\prime} \mathrm{E}$

CG-SN

CG-NK

$03^{\circ} 21^{\prime} \mathrm{S}, 23^{\circ} 16^{\prime} \mathrm{E}$ $01^{\circ} 08^{\prime} \mathrm{S}, 29^{\circ} 19^{\prime} \mathrm{E}$

$464 \mathrm{~m}$

CG

CG-MA

CG-NK

$03^{\circ} 24^{\prime} \mathrm{S}, 19^{\circ} 30^{\prime} \mathrm{E}$

$01^{\circ} 04^{\prime} \mathrm{S}, 27^{\circ} 25^{\prime} \mathrm{E}$

CG-IT $01^{\circ} 59^{\prime} \mathrm{N}, 28^{\circ} 52^{\prime} \mathrm{E}$

$368 \mathrm{~m}$

CG-HK

$10^{\circ} 37^{\prime} \mathrm{S}, 26^{\circ} 50^{\prime} \mathrm{E}$

$913 \mathrm{~m}$

CG-HK

$10^{\circ} 59^{\prime} \mathrm{S}, 26^{\circ} 44^{\prime} \mathrm{E}$

$1140 \mathrm{~m}$

CG-HK

$10^{\circ} 37^{\prime} \mathrm{S}, 26^{\circ} 50^{\prime} \mathrm{E}$

$1318 \mathrm{~m}$

$1140 \mathrm{~m}$

CG-HK

CG-TO

ca $10^{\circ} 37^{\prime} \mathrm{S}, 26^{\circ} 50^{\prime} \mathrm{E}$

$01^{\circ} 51^{\prime} \mathrm{N}, 26^{\circ} 25^{\prime} \mathrm{E}$

$05^{\circ} 11^{\prime} \mathrm{S}, 23^{\circ} 51^{\prime} \mathrm{E}$

ca $1100 \mathrm{~m}$

CG-SN

CG-SN

$05^{\circ} 11^{\prime} \mathrm{S}, 23^{\circ} 51^{\prime} \mathrm{E}$

$434 \mathrm{~m}$

$407 \mathrm{~m}$

$407 \mathrm{~m}$

CG-IT

$01^{\circ} 59^{\prime} \mathrm{N}, 28^{\circ} 52^{\prime} \mathrm{E}$

$913 \mathrm{~m}$

CG-NK

$01^{\circ} 00^{\prime} \mathrm{S}, 29^{\circ} 15^{\prime} \mathrm{E}$

CG-HU

$04^{\circ} 10^{\prime} \mathrm{N}, 29^{\circ} 30^{\prime} \mathrm{E}$

RW-ES

$01^{\circ} 30^{\prime} \mathrm{S}, 30^{\circ} 30^{\prime} \mathrm{E}$

CG-HK

ca $10^{\circ} 30^{\prime} \mathrm{S}, 27^{\circ} 30^{\prime} \mathrm{E}$

CG-HU

$02^{\circ} 46^{\prime} \mathrm{N}, 27^{\circ} 37^{\prime} \mathrm{E}$

$730 \mathrm{~m}$

CG-HU

$02^{\circ} 32^{\prime} \mathrm{N}, 27^{\circ} 42^{\prime} \mathrm{E}$

$696 \mathrm{~m}$

CG-HL $\quad 08^{\circ} 40^{\prime} \mathrm{S}, 26^{\circ} 50^{\prime} \mathrm{E}$

CG-HK $\quad 10^{\circ} 58^{\prime} \mathrm{S}, 26^{\circ} 47^{\prime} \mathrm{E}$

CG-HK 
Pene Bwebwe

(see: Nyangwe)

Penge

Petit Seminaire de Mugeri (see: Mugeri Mission)

PFNK

PFNK 15 and 17

PFNK.15

(see also: Piste Frontière Nord, km 15)

PFNK.16

(see also: Piste Frontière Nord, km 16)

PFSK 17 and 20

PFSK 8

PFSK.17

PFSK.20

(see also: Piste Frontière Sud, km 17)

(see also: Piste Frontière Sud, km 20)

PFSK 8

(see also: Piste Frontière Sud, km 8)

Pidigala Nord River

Pidigala River

Pierkat quarry

(see: Baya quarry)

(see: Pilipili)

Pilipili (see also: Pili Pili)

Pimpin Cave (see: Pempere Cave)

Piste Frontière Nord, km 15 (see: PFNK.15)

Piste Frontière Nord, km 16 (see: PFNK.16)

Piste Frontière Sud, km 17 (see: PFSK.17)

Piste Frontière Sud, km 20 (see: PFSK.20)

Piste Frontière Sud, $\mathrm{km} 8$

PNA (see: PFSK.8)

Poko (see: Virunga National Park)

Poko Territory

(see also: Poko Zone)

(see: Poko Territory)

Popokabaka

Popokabaka Territory

Port Chaltin

(see: Aketi)

Port de Kindu (see: Kindu)

Port-Empain

(see: Kindu)

PPK 51 and 56

PPK 72

PPK.10

PPK.51

Putnam, Camp

(see: Camp Putnam)

Pweto

Pweto Territory

Quatorze

(see also: Boende, $14 \mathrm{~km} \mathrm{~N}$ by road)

Ramangabo

(see: Rumangabo)

Rambira

Rambura

Ratabo Kinazi Gitorams

Resha

(see also: Bujumbura, $60 \mathrm{~km} \mathrm{~S}$ )

Rethy

(see also: Reti, Rety, Retsi, Retshi)

Reti

(see: Rethy)

Retshi (see: Rethy)
CG-MN

CG-HU

CG-SK

$04^{\circ} 13^{\prime} \mathrm{S}, 26^{\circ} 11^{\prime} \mathrm{E}$

$507 \mathrm{~m}$

$02^{\circ} 48^{\prime} \mathrm{N}, 27^{\circ} 55^{\prime} \mathrm{E}$

$828 \mathrm{~m}$ $02^{\circ} 12^{\prime} \mathrm{S}, 28^{\circ} 42^{\prime} \mathrm{E}$

$1493 \mathrm{~m}$

CG-HU $\quad 04^{\circ} 02^{\prime} \mathrm{N}, 29^{\circ} 05^{\prime} \mathrm{E}$

CG-HU $\quad 04^{\circ} 25^{\prime} \mathrm{N}, 29^{\circ} 47^{\prime} \mathrm{E}$

CG-HU $\quad 04^{\circ} 25^{\prime} \mathrm{N}, 29^{\circ} 47^{\prime} \mathrm{E}$

CG-HU $\quad 04^{\circ} 25^{\prime} \mathrm{N}, 29^{\circ} 47^{\prime} \mathrm{E}$

$\mathrm{CG}-\mathrm{HU}$

CG-HU

CG-HU

$04^{\circ} 18^{\prime} \mathrm{N}, 29^{\circ} 55^{\prime} \mathrm{E}$

$04^{\circ} 20^{\prime} \mathrm{N}, 29^{\circ} 51^{\prime} \mathrm{E}$

$04^{\circ} 18^{\prime} \mathrm{N}, 29^{\circ} 55^{\prime} \mathrm{E}$

CG-HU

$04^{\circ} 18^{\prime} \mathrm{N}, 29^{\circ} 55^{\prime} \mathrm{E}$

CG-HU

$04^{\circ} 20^{\prime} \mathrm{N}, 29^{\circ} 51^{\prime} \mathrm{E}$

CG-HU

$04^{\circ} 37^{\prime} \mathrm{N}, 29^{\circ} 42^{\prime} \mathrm{E}$

CG-HU $\quad 04^{\circ} 35^{\prime} \mathrm{N}, 29^{\circ} 32^{\prime} \mathrm{E}$

CG-HK $\quad 11^{\circ} 52^{\prime} \mathrm{S}, 27^{\circ} 27^{\prime} \mathrm{E}$

CG-TO $\quad 00^{\circ} 33^{\prime} \mathrm{N}, 27^{\circ} 44^{\prime} \mathrm{E}$

CG-TO

$00^{\circ} 33^{\prime} \mathrm{N}, 27^{\circ} 44^{\prime} \mathrm{E}$

CG-HK

$10^{\circ} 58^{\prime} \mathrm{S}, 26^{\circ} 47^{\prime} \mathrm{E}$

CG-HU

$04^{\circ} 25^{\prime} \mathrm{N}, 29^{\circ} 47^{\prime} \mathrm{E}$

CG-HU

$04^{\circ} 25^{\prime} \mathrm{N}, 29^{\circ} 47^{\prime} \mathrm{E}$

CG-HU

$04^{\circ} 18^{\prime} \mathrm{N}, 29^{\circ} 55^{\prime} \mathrm{E}$

CG-HU

$04^{\circ} 18^{\prime} \mathrm{N}, 29^{\circ} 55^{\prime} \mathrm{E}$

CG-HU

$04^{\circ} 20^{\prime} \mathrm{N}, 29^{\circ} 51^{\prime} \mathrm{E}$

CG-NK

$01^{\circ} 00^{\prime} \mathrm{S}, 29^{\circ} 15^{\prime} \mathrm{E}$

CG-BU

$03^{\circ} 09^{\prime} \mathrm{N}, 26^{\circ} 53^{\prime} \mathrm{E}$

ca $03^{\circ} 09^{\prime} \mathrm{N}, 26^{\circ} 53^{\prime} \mathrm{E}$

$641 \mathrm{~m}$

CG-BU

ca $03^{\circ} 09^{\prime} \mathrm{N}, 26^{\circ} 53^{\prime} \mathrm{E}$

CG-KG $\quad 05^{\circ} 42^{\prime} \mathrm{S}, 16^{\circ} 35^{\prime} \mathrm{E}$

CG-KG $\quad 05^{\circ} 42^{\prime} \mathrm{S}, 16^{\circ} 35^{\prime} \mathrm{E}$

CG-BU $\quad 02^{\circ} 50^{\prime} \mathrm{N}, 23^{\circ} 56^{\prime} \mathrm{E}$

$485 \mathrm{~m}$

CG-MN

$02^{\circ} 57^{\prime} \mathrm{S}, 25^{\circ} 57^{\prime} \mathrm{E}$

CG-MN

$02^{\circ} 57^{\prime} \mathrm{S}, 25^{\circ} 57^{\prime} \mathrm{E}$

$504 \mathrm{~m}$

$504 \mathrm{~m}$

CG-HU

$\mathrm{CG}-\mathrm{HU}$

$04^{\circ} 06^{\prime} \mathrm{N}, 29^{\circ} 25^{\prime} \mathrm{E}$

$04^{\circ} 14^{\prime} \mathrm{N}, 29^{\circ} 31^{\prime} \mathrm{E}$

CG-HU $\quad 03^{\circ} 46^{\prime} \mathrm{N}, 29^{\circ} 29^{\prime} \mathrm{E}$

CG-HU $\quad 04^{\circ} 06^{\prime} \mathrm{N}, 29^{\circ} 25^{\prime} \mathrm{E}$

CG-IT $01^{\circ} 24^{\prime} \mathrm{N}, 28^{\circ} 35^{\prime} \mathrm{E}$

CG-HK $\quad 08^{\circ} 28^{\prime} \mathrm{S}, 28^{\circ} 54^{\prime} \mathrm{E}$

CG-HK $\quad 08^{\circ} 28^{\prime} \mathrm{S}, 28^{\circ} 54^{\prime} \mathrm{E}$

CG-TP $\quad 00^{\circ} 10^{\prime} \mathrm{N}, 20^{\circ} 56^{\prime} \mathrm{E}$

$951 \mathrm{~m}$

$326 \mathrm{~m}$

CG-NK

$01^{\circ} 21^{\prime} \mathrm{S}, 29^{\circ} 22^{\prime} \mathrm{E}$

$1587 \mathrm{~m}$

CG-SK

CG-SK

RW-SU

$02^{\circ} 03^{\prime} \mathrm{S}, 28^{\circ} 54^{\prime} \mathrm{E}$

$01^{\circ} 40^{\prime} \mathrm{S}, 29^{\circ} 35^{\prime} \mathrm{E}$

BY-RU

$02^{\circ} 13^{\prime} \mathrm{S}, 29^{\circ} 58^{\prime} \mathrm{E}$

$03^{\circ} 54^{\prime} \mathrm{S}, 29^{\circ} 24^{\prime} \mathrm{E}$

CG-IT

$02^{\circ} 05^{\prime} \mathrm{N}, 30^{\circ} 54^{\prime} \mathrm{E}$

$2116 \mathrm{~m}$

CG-IT

$02^{\circ} 05^{\prime} \mathrm{N}, 30^{\circ} 54^{\prime} \mathrm{E} \quad 2116 \mathrm{~m}$

CG-IT

$02^{\circ} 05^{\prime} \mathrm{N}, 30^{\circ} 54^{\prime} \mathrm{E}$

$2116 \mathrm{~m}$ 
Retsi

Rety (see: Rethy)

Ribue Lya Mikako Cave

Risimu Zone (see: Kibwe Lya Mikako Cave)

Rodahira River

Routabansougera

Ruango

Ruanoli-Mavea confluence

Rubengera (Mission)

Rubindi (see also: Lubengera (Mission))

Rubi-Tele

Ruboma Ruhashya

Rubona

(see also: Colline Rubona)

Ruchuru

(see: Rutshuru)

Rugari

(see also: Lulenga)

Rugari Mission

Ruhengeri

Ruhinga Hil

Ruindi

(see: Rwindi)

Rukara

Rukira

Rumangabo

(see also: Ramangabo, Rumengabo)

Rumanura River

Rumengabo

(see: Rumangabo)

Rumoka

Rumonge

Rumonge Province

Rungu

Rungu Zone

Rusenyi River

Rushubi mine

Rusizi

Rusizi Delta

(see also: Russissi, bank)

Russissi, bank

(see: Rusizi Delta)

Rutana

Rutana Province

Rutana, $46 \mathrm{~km}$ from

(see: Mpinga Mission)

Rutanzige, Lake

Rutshuru (see: Edward, Lake)

(see also: Ruchuru)

Rutshuru Territory (see also: Rutshuru Zone)

Rutshuru Zone (see: Rutshuru Territory)

Rutu [River] (see: Utu [River])

Ruvara

Ruwenzori

Ruwenzori SE

Ruwenzori, Foothills

Ruwenzori, S side

Ruyigi

Ruyigi Province

Rwamagana

Rwanda

Rwankalo

Rwankwi

Rwegura

Rwere Rwind

(see also: Kayanza, 15 km W)

Rwindi

(see also: Ruindi)
CG-IT

CG-IT

$02^{\circ} 05^{\prime} \mathrm{N}, 30^{\circ} 54^{\prime} \mathrm{E}$

$2116 \mathrm{~m}$

CG-NK

CG-TO

CG-NK

RW-SU

CG-BC

CG-NK

RW-SU

BY-MA

CG-TO

RW-SU

RW-SU

CG-NK

CG-NK

BY-MY

RW-NO

BY-BI

CG-NK

RW-ES

RW-ES

CG-NK

CG-NK

CG-NK

CG-IT

BY-RU

BY

CG-HU

CG-HU

CG-NK

BY-BL

BY-BB

BY-BL

$02^{\circ} 05^{\prime} \mathrm{N}, 30^{\circ} 54^{\prime} \mathrm{E}$

$2116 \mathrm{~m}$

$00^{\circ} 15^{\prime} \mathrm{S}, 29^{\circ} 01^{\prime} \mathrm{E}$

$1500 \mathrm{~m}$

ca $01^{\circ} 00^{\prime} \mathrm{N}, 26^{\circ} 45^{\prime} \mathrm{E}$

$01^{\circ} 13^{\prime} \mathrm{S}, 29^{\circ} 26^{\prime} \mathrm{E}$

$02^{\circ} 25^{\prime} \mathrm{S}, 29^{\circ} 10^{\prime} \mathrm{E}$

$04^{\circ} 45^{\prime} \mathrm{S}, 13^{\circ} 36^{\prime} \mathrm{E}$

$00^{\circ} 40^{\prime} \mathrm{N}, 29^{\circ} 43^{\prime} \mathrm{E}$

$02^{\circ} 03^{\prime} \mathrm{S}, 29^{\circ} 26^{\prime} \mathrm{E}$

$04^{\circ} 16^{\prime} \mathrm{S}, 29^{\circ} 35^{\prime} \mathrm{E}$

$200 \mathrm{~m}$

$1750 \mathrm{~m}$

$800 \mathrm{~m}$

$997 \mathrm{~m}$

$02^{\circ} 19^{\prime} \mathrm{N}, 24^{\circ} 59^{\prime} \mathrm{E}$

$02^{\circ} 30^{\prime} \mathrm{S}, 29^{\circ} 44^{\prime} \mathrm{E}$

$02^{\circ} 19^{\prime} \mathrm{S}, 29^{\circ} 56^{\prime} \mathrm{E}$

$1346 \mathrm{~m}$

$01^{\circ} 11^{\prime} \mathrm{S}, 29^{\circ} 27^{\prime} \mathrm{E}$

$1203 \mathrm{~m}$

$01^{\circ} 25^{\prime} \mathrm{S}, 29^{\circ} 22^{\prime} \mathrm{E}$

2089 m

$02^{\circ} 44^{\prime} \mathrm{S}, 30^{\circ} 24^{\prime} \mathrm{E}$

$01^{\circ} 30^{\prime} \mathrm{S}, 29^{\circ} 38^{\prime} \mathrm{E}$

$03^{\circ} 55^{\prime} \mathrm{S}, 29^{\circ} 35^{\prime} \mathrm{E}$

$00^{\circ} 47^{\prime} \mathrm{S}, 29^{\circ} 17^{\prime} \mathrm{E}$

$1651 \mathrm{~m}$

$1772 \mathrm{~m}$

$2170 \mathrm{~m}$

$1027 \mathrm{~m}$

$01^{\circ} 48^{\prime} \mathrm{S}, 30^{\circ} 30^{\prime} \mathrm{E} \quad 1625 \mathrm{~m}$ $02^{\circ} 09^{\prime} \mathrm{S}, 30^{\circ} 37^{\prime} \mathrm{E} \quad 1709 \mathrm{~m}$

$01^{\circ} 21^{\prime} \mathrm{S}, 29^{\circ} 22^{\prime} \mathrm{E} \quad 1587 \mathrm{~m}$

$01^{\circ} 10^{\prime} \mathrm{S}, 29^{\circ} 28^{\prime} \mathrm{E} \quad 1200 \mathrm{~m}$

$01^{\circ} 21^{\prime} \mathrm{S}, 29^{\circ} 22^{\prime} \mathrm{E} \quad 1587 \mathrm{~m}$

$01^{\circ} 34^{\prime} \mathrm{N}, 2^{\circ} 07^{\prime} \mathrm{E}$

BY-BL

BY-RT

BY

BY-RT

CG-NK

CG-NK

CG-NK

CG-NK

CG-NK

CG

CG-NK

CG-NK

CG-NK

BY-RY

BY

RW-ES

RW

RW

RW-NO

BY-KY

CG-NK $03^{\circ} 58^{\prime} \mathrm{S}, 29^{\circ} 26^{\prime} \mathrm{E}$

$03^{\circ} 11^{\prime} \mathrm{N}, 27^{\circ} 52^{\prime} \mathrm{E}$ ca $03^{\circ} 11^{\prime} \mathrm{N}, 27^{\circ} 52^{\prime} \mathrm{E}$ $01^{\circ} 12^{\prime} \mathrm{S}, 29^{\circ} 35^{\prime} \mathrm{E}$

$03^{\circ} 21^{\prime} \mathrm{S}, 29^{\circ} 29^{\prime} \mathrm{E}$

$03^{\circ} 16^{\prime} \mathrm{S}, 29^{\circ} 14^{\prime} \mathrm{E}$

$03^{\circ} 21^{\prime} \mathrm{S}, 29^{\circ} 16^{\prime} \mathrm{E}$

$03^{\circ} 21^{\prime} \mathrm{S}, 29^{\circ} 16^{\prime} \mathrm{E}$

$03^{\circ} 55^{\prime} \mathrm{S}, 29^{\circ} 60^{\prime} \mathrm{E}$

$1810 \mathrm{~m}$

$03^{\circ} 44^{\prime} \mathrm{S}, 30^{\circ} 11^{\prime} \mathrm{E}$

$1930 \mathrm{~m}$

ca $00^{\circ} 08^{\prime} \mathrm{S}, 29^{\circ} 35^{\prime} \mathrm{E}$

$01^{\circ} 11^{\prime} \mathrm{S}, 29^{\circ} 27^{\prime} \mathrm{E}$

$1219 \mathrm{~m}$

ca $01^{\circ} 11^{\prime} \mathrm{N}, 29^{\circ} 27^{\prime} \mathrm{E}$

$01^{\circ} 11^{\prime} \mathrm{N}, 29^{\circ} 27^{\prime} \mathrm{E}$

$1341 \mathrm{~m}$

$01^{\circ} 24^{\prime} \mathrm{S}, 27^{\circ} 49^{\prime} \mathrm{E}$

$02^{\circ} 55^{\prime} \mathrm{S}, 29^{\circ} 32^{\prime} \mathrm{E}$

$00^{\circ} 47^{\prime} \mathrm{S}, 29^{\circ} 18^{\prime} \mathrm{E}$

$00^{\circ} 47^{\prime} \mathrm{S}, 29^{\circ} 17^{\prime} \mathrm{E}$

809 m

$704 \mathrm{~m}$

$1300 \mathrm{~m}$

ca $1743 \mathrm{~m}$

$00^{\circ} 25^{\prime} \mathrm{N}, 29^{\circ} 50^{\prime} \mathrm{E}$ ca $00^{\circ} 30^{\prime} \mathrm{N}, 29^{\circ} 50^{\prime} \mathrm{E}$

$2286 \mathrm{~m}$

ca $00^{\circ} 25^{\prime} \mathrm{N}, 29^{\circ} 50^{\prime} \mathrm{E}$

$03^{\circ} 29^{\prime} \mathrm{S}, 30^{\circ} 15^{\prime} \mathrm{E}$

$1842 \mathrm{~m}$

$1528 \mathrm{~m}$

$2311 \mathrm{~m}$

$1027 \mathrm{~m}$ 
Rwintare

(see also: Rwintare Mission)

Rwintare Mission

(see: Rwintare)

Sabe

Sacred Heart Mission

(see also: Mission Sacre Coeur)

Saga Saga Cave

(see also: Grotte de Saga Saga, Saga Saga Grot)

Saga Saga Grot

(see: Saga Saga Cave)

Saint Joseph de Luluabourg (Mission) (see: Mikalaya)

Sainte Elisabeth

Saint-Joseph (Mission)

(see: Mikalaya)

Sakania (see also: Sakania Mission)

Sakania Mission (see: Sakania)

Sakania Territory

Saliboko

Salomoni Cave

Samba

Samboko River

Sange

Sankuru Province

Sciere Forest

Scierie Forest

(see also: Kindu, 30 km SW)

Semmio

(see: Zemio)

Semnio

(see: Zemio)

Senga

Shaba

Shaba (former) Province

(see: Katanga (former) Province)

Shabunda

Shabunda Territory

Shainkolobwe

(see: Shinkolobwe)

Shangi

(see also: Colline Shangi, Ishangi, Shangi Schad)

Shangi Schad

(see: Shangi)

Shangugu

(see also: Cyangugu)

Shibambo

(see also: Chibambo, Shibombo, Tshibambo)

Shibombo

(see: Shibambo)

Shindaika

Shinkolobwe

(see also: Chinkolobwe, Dethioux Farm, Cave I, Dethioux Farm, Cave II,

Dethioux Farm, Tantara Cave, Dethioux Hoeve, Grot I, Dethioux Hoeve,

Grot II, Dethioux Hoeve, Tantara Grot, Grotte de Tantara, Ferme Dethioux,

Grotte I, Ferme Dethioux, Grotte II, Ferme Dethioux, Shainkolobwe, Tantara

Shyanda

Cave, Trou aux Serpents)

Simbi Cave

(see also: Colline Shyanda)

Sinda Rive

Singitini

Sombe

(see: Kisangani)

Soudan River

(see also: Kiabo, 14 km upstream)

South Kivu Province

(see: Sud-Kivu Province)

South Ubangui Province (see: Sud Ubangui Province)

Southern Prefecture

St Gerard Mission

(see: Kapolowe)

\begin{tabular}{|c|c|c|}
\hline BY-MW & $03^{\circ} 31^{\prime} \mathrm{S}, 29^{\circ} 39^{\prime} \mathrm{E}$ & $2111 \mathrm{~m}$ \\
\hline BY-MW & $03^{\circ} 31^{\prime} \mathrm{S}, 29^{\circ} 39^{\prime} \mathrm{E}$ & $2111 \mathrm{~m}$ \\
\hline $\begin{array}{l}\text { CG-IT } \\
\text { CG-KU }\end{array}$ & $\begin{array}{l}01^{\circ} 23^{\prime} \mathrm{N}, 30^{\circ} 26^{\prime} \mathrm{E} \\
05^{\circ} 02^{\prime} \mathrm{S}, 18^{\circ} 51^{\prime} \mathrm{E}\end{array}$ & $700 \mathrm{~m}$ \\
\hline CG-IT & $01^{\circ} 19^{\prime} \mathrm{N}, 29^{\circ} 52^{\prime} \mathrm{E}$ & $1160 \mathrm{~m}$ \\
\hline CG-IT & $01^{\circ} 19^{\prime} \mathrm{N}, 29^{\circ} 52^{\prime} \mathrm{E}$ & $1160 \mathrm{~m}$ \\
\hline CG-LL & $06^{\circ} 01^{\prime} \mathrm{S}, 22^{\circ} 19^{\prime} \mathrm{E}$ & $608 \mathrm{~m}$ \\
\hline $\begin{array}{l}\text { CG-TO } \\
\text { CG-LL }\end{array}$ & $\begin{array}{l}01^{\circ} 33^{\prime} \mathrm{N}, 25^{\circ} 20^{\prime} \mathrm{E} \\
06^{\circ} 01^{\prime} \mathrm{S}, 22^{\circ} 19^{\prime} \mathrm{E}\end{array}$ & $\begin{array}{l}432 \mathrm{~m} \\
608 \mathrm{~m}\end{array}$ \\
\hline CG-HK & $12^{\circ} 45^{\prime} \mathrm{S}, 28^{\circ} 34^{\prime} \mathrm{E}$ & $1278 \mathrm{~m}$ \\
\hline CG-HK & $12^{\circ} 45^{\prime} \mathrm{S}, 28^{\circ} 34^{\prime} \mathrm{E}$ & $1278 \mathrm{~m}$ \\
\hline $\begin{array}{c}\text { CG-HK } \\
\text { CG-IT } \\
\text { CG-HK } \\
\text { CG-MN } \\
\text { CG-IT } \\
\text { CG-SK } \\
\text { CG } \\
\text { CG-MN } \\
\text { CG-MN }\end{array}$ & $\begin{array}{c}12^{\circ} 45^{\prime} \mathrm{S}, 28^{\circ} 34^{\prime} \mathrm{E} \\
01^{\circ} 42^{\prime} \mathrm{N}, 30^{\circ} 29^{\prime} \mathrm{E} \\
10^{\circ} 59^{\prime} \mathrm{S}, 27^{\circ} 17^{\prime} \mathrm{E} \\
04^{\circ} 38^{\prime} \mathrm{S}, 26^{\circ} 22^{\prime} \mathrm{E} \\
01^{\circ} 00^{\prime} \mathrm{N}, 29^{\circ} 22^{\prime} \mathrm{E} \\
03^{\circ} 06^{\prime} \mathrm{S}, 29^{\circ} 15^{\prime} \mathrm{E} \\
\mathrm{ca} 04^{\circ} 58^{\prime} \mathrm{S}, 23^{\circ} 27^{\prime} \mathrm{E} \\
03^{\circ} 10^{\prime} \mathrm{S}, 25^{\circ} 49^{\prime} \mathrm{E} \\
03^{\circ} 10^{\prime} \mathrm{S}, 25^{\circ} 49^{\prime} \mathrm{E}\end{array}$ & $\begin{array}{l}1874 \mathrm{~m} \\
610 \mathrm{~m} \\
850 \mathrm{~m}\end{array}$ \\
\hline $\begin{array}{l}\text { CG-NK } \\
\text { CG-BU }\end{array}$ & $\begin{array}{l}00^{\circ} 45^{\prime} \mathrm{N}, 29^{\circ} 50^{\prime} \mathrm{E} \\
05^{\circ} 03^{\prime} \mathrm{N}, 27^{\circ} 07^{\prime} \mathrm{E}\end{array}$ & \\
\hline CG-BU & $05^{\circ} 03^{\prime} \mathrm{N}, 27^{\circ} 07^{\prime} \mathrm{E}$ & \\
\hline $\begin{array}{c}\mathrm{CG}-\mathrm{NK} \\
\mathrm{CG} \\
\mathrm{CG}\end{array}$ & $01^{\circ} 40^{\prime} \mathrm{S}, 28^{\circ} 07^{\prime} \mathrm{E}$ & $950 \mathrm{~m}$ \\
\hline $\begin{array}{l}\text { CG-SK } \\
\text { CG-SK } \\
\text { CG-LB }\end{array}$ & $\begin{array}{l}02^{\circ} 42^{\prime} \mathrm{S}, 27^{\circ} 20^{\prime} \mathrm{E} \\
02^{\circ} 42^{\prime} \mathrm{S}, 27^{\circ} 20^{\prime} \mathrm{E} \\
11^{\circ} 02^{\prime} \mathrm{S}, 26^{\circ} 29^{\prime} \mathrm{E}\end{array}$ & $\begin{array}{l}596 \mathrm{~m} \\
1334 \mathrm{~m}\end{array}$ \\
\hline RW-OU & $02^{\circ} 24^{\prime} \mathrm{S}, 29^{\circ} 00^{\prime} \mathrm{E}$ & \\
\hline RW-OU & $02^{\circ} 24^{\prime} \mathrm{S}, 29^{\circ} 00^{\prime} \mathrm{E}$ & \\
\hline RW-OU & $02^{\circ} 29^{\prime} \mathrm{S}, 28^{\circ} 54^{\prime} \mathrm{E}$ & $1537 \mathrm{~m}$ \\
\hline CG-HK & $10^{\circ} 18^{\prime} \mathrm{S}, 28^{\circ} 37^{\prime} \mathrm{E}$ & $928 \mathrm{~m}$ \\
\hline CG-HK & $10^{\circ} 18^{\prime} \mathrm{S}, 28^{\circ} 37^{\prime} \mathrm{E}$ & $928 \mathrm{~m}$ \\
\hline $\begin{array}{l}\text { CG-HK } \\
\text { CG-LB }\end{array}$ & $\begin{array}{l}11^{\circ} 39^{\prime} \mathrm{S}, 27^{\circ} 41^{\prime} \mathrm{E} \\
11^{\circ} 02^{\prime} \mathrm{S}, 26^{\circ} 29^{\prime} \mathrm{E}\end{array}$ & $1334 \mathrm{~m}$ \\
\hline RW-SU & $02^{\circ} 33^{\prime} \mathrm{S}, 29^{\circ} 48^{\prime} \mathrm{E}$ & $1646 \mathrm{~m}$ \\
\hline $\begin{array}{l}\text { RW-SU } \\
\text { CG-IT } \\
\text { CG-TO }\end{array}$ & $\begin{array}{l}02^{\circ} 31^{\prime} \mathrm{S}, 29^{\circ} 40^{\prime} \mathrm{E} \\
01^{\circ} 02^{\prime} \mathrm{N}, 30^{\circ} 04^{\prime} \mathrm{E} \\
00^{\circ} 31^{\prime} \mathrm{N}, 25^{\circ} 12^{\prime} \mathrm{E}\end{array}$ & $\begin{array}{l}830 \mathrm{~m} \\
447 \mathrm{~m}\end{array}$ \\
\hline CG-HL & $08^{\circ} 50^{\prime} \mathrm{S}, 26^{\circ} 02^{\prime} \mathrm{E}$ & \\
\hline $\begin{array}{l}\text { CG-HU } \\
\text { CG }\end{array}$ & $04^{\circ} 35^{\prime} \mathrm{N}, 29^{\circ} 35^{\prime} \mathrm{E}$ & \\
\hline CG & & \\
\hline $\begin{array}{c}\text { RW } \\
\text { CG-HK }\end{array}$ & $11^{\circ} 02^{\prime} \mathrm{S}, 26^{\circ} 57^{\prime} \mathrm{E}$ & $1135 \mathrm{~m}$ \\
\hline
\end{tabular}




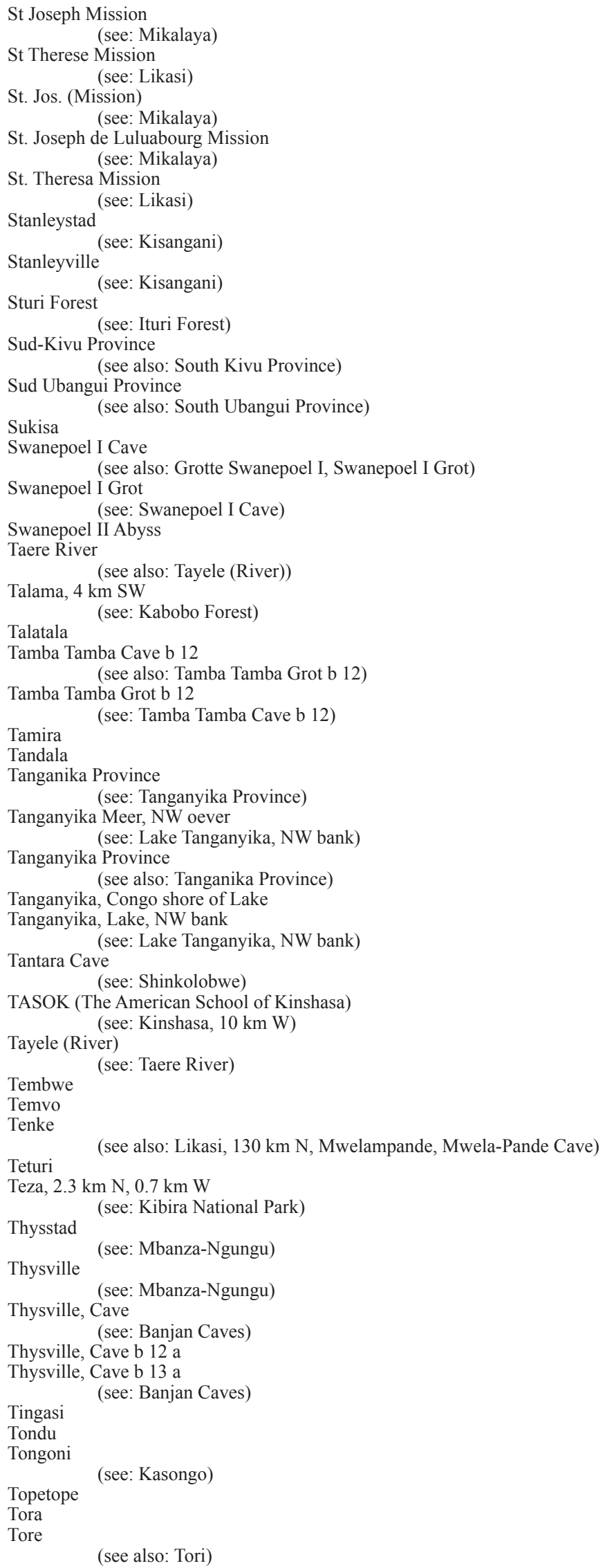

CG-LL

CG-HK

$06^{\circ} 01^{\prime} \mathrm{S}, 22^{\circ} 19^{\prime} \mathrm{E}$

$608 \mathrm{~m}$

CG-LL

$10^{\circ} 59^{\prime} \mathrm{S}, 26^{\circ} 44^{\prime} \mathrm{E}$

$1318 \mathrm{~m}$

CG-LL

$06^{\circ} 01^{\prime} \mathrm{S}, 22^{\circ} 19^{\prime} \mathrm{E}$

$608 \mathrm{~m}$

CG-LL

$06^{\circ} 01^{\prime} \mathrm{S}, 22^{\circ} 19^{\prime} \mathrm{E}$

$608 \mathrm{~m}$

CG-HK

CG-TO

$10^{\circ} 59^{\prime} \mathrm{S}, 26^{\circ} 44^{\prime} \mathrm{E}$

$1318 \mathrm{~m}$

CG-TO

$00^{\circ} 31^{\prime} \mathrm{N}, 25^{\circ} 12^{\prime} \mathrm{E}$

$447 \mathrm{~m}$

CG-IT

$00^{\circ} 31^{\prime} \mathrm{N}, 25^{\circ} 12^{\prime} \mathrm{E}$

$447 \mathrm{~m}$

CG

CG-BU

CG-LB

CG-LB

CG-LB

CG-HU

$01^{\circ} 00^{\prime} \mathrm{N}, 29^{\circ} 16^{\prime} \mathrm{E}$

CG-SK

CG-IT

CG-BC $02^{\circ} 19^{\prime} \mathrm{N}, 24^{\circ} 59^{\prime} \mathrm{E}$
$10^{\circ} 50^{\prime} \mathrm{S}, 26^{\circ} 39^{\prime} \mathrm{E}$

$470 \mathrm{~m}$ ca $1350 \mathrm{~m}$

$10^{\circ} 50^{\prime} \mathrm{S}, 26^{\circ} 39^{\prime} \mathrm{E}$

ca $1350 \mathrm{~m}$

$10^{\circ} 51^{\prime} \mathrm{S}, 26^{\circ} 42^{\prime} \mathrm{E}$ $04^{\circ} 33^{\prime} \mathrm{N}, 29^{\circ} 47^{\prime} \mathrm{E}$

ca $1350 \mathrm{~m}$

$04^{\circ} 59^{\prime} \mathrm{S}, 29^{\circ} 05^{\prime} \mathrm{E}$

CG-BC

$01^{\circ} 15^{\prime} \mathrm{N}, 29^{\circ} 50^{\prime} \mathrm{E}$ $05^{\circ} 43^{\prime} \mathrm{S}, 14^{\circ} 27^{\prime} \mathrm{E}$

$1170 \mathrm{~m}$

RW-OU

CG-SU

CG

CG-SK

$05^{\circ} 43^{\prime} \mathrm{S}, 14^{\circ} 27^{\prime} \mathrm{E}$

CG

CG

CG-SK

ca $06^{\circ} 00^{\prime} \mathrm{S}, 29^{\circ} 11^{\prime} \mathrm{E}$

CG-LB ca $03^{\circ} 20^{\prime} \mathrm{S}, 29^{\circ} 12^{\prime} \mathrm{E}$

$11^{\circ} 02^{\prime} \mathrm{S}, 26^{\circ} 29^{\prime} \mathrm{E}$

$1334 \mathrm{~m}$

CG-KN

ca $04^{\circ} 20^{\prime} \mathrm{S}, 15^{\circ} 15^{\prime} \mathrm{E}$

CG-HU

$04^{\circ} 33^{\prime} \mathrm{N}, 29^{\circ} 47^{\prime} \mathrm{E}$

CG-TG

CG-BC

$06^{\circ} 30^{\prime} \mathrm{S}, 29^{\circ} 22^{\prime} \mathrm{E}$

$05^{\circ} 30^{\prime} \mathrm{S}, 13^{\circ} 01^{\prime} \mathrm{E}$

CG-HK

$10^{\circ} 35^{\prime} \mathrm{S}, 26^{\circ} 07^{\prime} \mathrm{E}$

$1055 \mathrm{~m}$

$238 \mathrm{~m}$

$1512 \mathrm{~m}$

CG-IT $\quad 01^{\circ} 04^{\prime} \mathrm{N}, 29^{\circ} 08^{\prime} \mathrm{E} \quad 766 \mathrm{~m}$

BY-MV

$03^{\circ} 13^{\prime} \mathrm{S}, 29^{\circ} 34^{\prime} \mathrm{E}$

CG-BC

$05^{\circ} 15^{\prime} \mathrm{S}, 14^{\circ} 52^{\prime} \mathrm{E}$

2200-2400

$\mathrm{m}$

CG-BC

$05^{\circ} 15^{\prime} \mathrm{S}, 14^{\circ} 52^{\prime} \mathrm{E}$

$604 \mathrm{~m}$

CG-BC

CG-BC

CG-BC

$05^{\circ} 16^{\prime} \mathrm{S}, 14^{\circ} 53^{\prime} \mathrm{E}$

CG-HU

CG-ET

$16^{\prime} \mathrm{S}, 14^{\circ} 52^{\prime} \mathrm{E}$ $05^{\circ} 16^{\prime} \mathrm{S}, 14^{\circ} 53^{\prime} \mathrm{E}$

CG-MN

$03^{\circ} 24^{\prime} \mathrm{N}, 27^{\circ} 55^{\prime} \mathrm{E}$ $00^{\circ} 53^{\prime} \mathrm{S}, 18^{\circ} 09^{\prime} \mathrm{E}$ $04^{\circ} 27^{\prime} \mathrm{S}, 26^{\circ} 40^{\prime} \mathrm{E}$

CG-SK $\quad 02^{\circ} 16^{\prime} \mathrm{S}, 28^{\circ} 03^{\prime} \mathrm{E}$ BY-BI $03^{\circ} 41^{\prime} \mathrm{S}, 29^{\circ} 34^{\prime} \mathrm{E}$ CG-HU 
Tori (see: Tore)

Trappist Mission

Trou aux Serpents

Tschakala (see: Shinkolobwe) Tschambutscha

Tschinputu see: Tshambutschu)

Tschuapa (see: Cinputu)

Tsebahu (see: Tshuapa)

Tshakala (see also: Kisangani, $45 \mathrm{~km}$ E, Tschakala)

Tshamalenge Cave

Tshambi (see also: Grotte de Tshamalenge)

Tshambutschu (see also: Irangi, $10 \mathrm{~km}$ from, Tschambutscha)

Tshamiagongo Volcano

(see: Nyirogongo Volcano)

Tshaninagongo

Tshapona

(see: Nyirogongo Volcano)

Tshebahu Rive: see: Tshiapona)

Tshiabirimu, moun (see also: Mount Tshiabirimu, Mount Tshiaberimu) Tshiapona (see also: Tshapona)

Tshibaka (see: Tshibaka-Kaji)

Tshibaka-Kaj (see also: Tshibaka)

Tshibambo (see: Shibambo)

Tshibasha

Tshibashi (River) (see also: Tshibashie (River))

Tshibashie (River) (see: Tshibashi (River)

Tshibati

Tshibinda

(see also: Mulungu-Tshibinda)

Tshibungu

Tshikapa

Tshikapa Territory

see also: Chikapa)

Tshilinge (former) district (see: Kasai Oriental Province)

Tshimputu

Tshisangwe

Tshopo

Tshopo Falls

(see also: Tshoppo Falls)

Tshopo Province

Tshoppo Falls

(see: Tshopo Falls)

Tshuapa (see also: Tschuapa)

Tshuapa district

Tshuapa Province

Tshuapa River, uppe (see also: Haut Tshuapa, Tshuapa, Bovenloop)

Tshuapa, Bovenloop (see: Tshuapa River, upper)

Tshyohaha, Lake (see: Cyohaha, Lake)

Tumba, Lac (see: Tumba, Lake)

Tumba, Lake (see also: Lake Tumba, Tumba, Lac, Lac Tumba, Tumbe Meer) Tumbe Meer (see: Tumba, Lake)
CG-HU

$04^{\circ} 20^{\prime} \mathrm{N}, 29^{\circ} 50^{\prime} \mathrm{E}$

CG-KU $\quad 05^{\circ} 02^{\prime} \mathrm{S}, 18^{\circ} 51^{\prime} \mathrm{E}$

CG-LB $\quad 11^{\circ} 02^{\prime} \mathrm{S}, 26^{\circ} 29^{\prime} \mathrm{E}$

$1334 \mathrm{~m}$

CG-TO

$00^{\circ} 27^{\prime} \mathrm{N}, 25^{\circ} 32^{\prime} \mathrm{E}$

$453 \mathrm{~m}$

CG-SK

ca $01^{\circ} 58^{\prime} \mathrm{S}, 28^{\circ} 30^{\prime} \mathrm{E}$

CG-LL

$05^{\circ} 22^{\prime} \mathrm{S}, 22^{\circ} 21^{\prime} \mathrm{E}$

CG-TP

$00^{\circ} 14^{\prime} \mathrm{S}, 20^{\circ} 42^{\prime} \mathrm{E}$

CG-IT

$01^{\circ} 13^{\prime} \mathrm{N}, 29^{\circ} 49^{\prime} \mathrm{E}$

CG-TO $00^{\circ} 27^{\prime} \mathrm{N}, 25^{\circ} 32^{\prime} \mathrm{E}$

$453 \mathrm{~m}$

CG-HK

$11^{\circ} 46^{\prime} \mathrm{S}, 26^{\circ} 30^{\prime} \mathrm{E}$

CG-NK

CG-SK

$00^{\circ} 44^{\prime} \mathrm{S}, 29^{\circ} 14^{\prime} \mathrm{E}$ ca $01^{\circ} 58^{\prime} \mathrm{S}, 28^{\circ} 30^{\prime} \mathrm{E}$

$1050 \mathrm{~m}$

CG-NK

$01^{\circ} 31^{\prime} \mathrm{S}, 29^{\circ} 15^{\prime} \mathrm{E}$

CG-NK

$01^{\circ} 31^{\prime} \mathrm{S}, 29^{\circ} 15^{\prime} \mathrm{E}$

CG-HL

$07^{\circ} 11^{\prime} \mathrm{S}, 24^{\circ} 19^{\prime} \mathrm{E}$

$826 \mathrm{~m}$

CG-IT

$01^{\circ} 19^{\prime} \mathrm{N}, 29^{\circ} 52^{\prime} \mathrm{E}$

CG-NK ca $00^{\circ} 05^{\prime}$ S, $29^{\circ} 26^{\prime} \mathrm{E}$

$1200 \mathrm{~m}$

CG-HL

$07^{\circ} 11^{\prime} \mathrm{S}, 24^{\circ} 19^{\prime} \mathrm{E}$

$826 \mathrm{~m}$

CG-LL

$07^{\circ} 43^{\prime} \mathrm{S}, 23^{\circ} 12^{\prime} \mathrm{E}$

$882 \mathrm{~m}$

CG-LL

$07^{\circ} 43^{\prime} \mathrm{S}, 23^{\circ} 12^{\prime} \mathrm{E}$

$882 \mathrm{~m}$

CG-HK

$10^{\circ} 18^{\prime} \mathrm{S}, 28^{\circ} 37^{\prime} \mathrm{E}$

$928 \mathrm{~m}$

CG-LL $\quad 05^{\circ} 50^{\prime} \mathrm{S}, 22^{\circ} 25^{\prime} \mathrm{E}$

CG-LL $\quad 05^{\circ} 45^{\prime} \mathrm{S}, 22^{\circ} 08^{\prime} \mathrm{E}$

CG-LL $\quad 05^{\circ} 45^{\prime} \mathrm{S}, 22^{\circ} 08^{\prime} \mathrm{E}$

CG-SK $\quad 02^{\circ} 14^{\prime} \mathrm{S}, 28^{\circ} 47^{\prime} \mathrm{E}$

CG-SK $\quad 02^{\circ} 19^{\prime} \mathrm{S}, 28^{\circ} 46^{\prime} \mathrm{E}$

CG-LL $\quad 05^{\circ} 38^{\prime} \mathrm{S}, 22^{\circ} 16^{\prime} \mathrm{E}$

CG-KS $\quad 06^{\circ} 25^{\prime} \mathrm{S}, 20^{\circ} 48^{\prime} \mathrm{E}$

$1918 \mathrm{~m}$

CG-KS

$06^{\circ} 25^{\prime} \mathrm{S}, 20^{\circ} 48^{\prime} \mathrm{E}$

CG

CG-LL $\quad 05^{\circ} 48^{\prime} \mathrm{S}, 22^{\circ} 19^{\prime} \mathrm{E}$

CG-HK $\quad 11^{\circ} 28^{\prime} \mathrm{S}, 27^{\circ} 39^{\prime} \mathrm{E}$

CG-TO $\quad 00^{\circ} 28^{\prime} \mathrm{N}, 25^{\circ} 33^{\prime} \mathrm{E}$

CG-TO $\quad 00^{\circ} 33^{\prime} \mathrm{N}, 25^{\circ} 11^{\prime} \mathrm{E}$

CG

CG-TO

$00^{\circ} 33^{\prime} \mathrm{N}, 25^{\circ} 11^{\prime} \mathrm{E}$

CG-TP

$00^{\circ} 14^{\prime} \mathrm{S}, 20^{\circ} 42^{\prime} \mathrm{E}$

CG-TP

CG

CG-TP

$00^{\circ} 13^{\prime} \mathrm{S}, 20^{\circ} 52^{\prime} \mathrm{E}$

CG-TP

$01^{\circ} 00^{\prime} \mathrm{S}, 23^{\circ} 20^{\prime} \mathrm{E}$

RW-ES

$01^{\circ} 00^{\prime} \mathrm{S}, 23^{\circ} 02^{\prime} \mathrm{E}$

CG-ET $02^{\circ} 15^{\prime} \mathrm{S}, 30^{\circ} 00^{\prime} \mathrm{E}$

CG-ET ca $00^{\circ} 48^{\prime} \mathrm{S}, 18^{\circ} 03^{\prime} \mathrm{E}$

CG-ET ca $00^{\circ} 48^{\prime} \mathrm{S}, 18^{\circ} 03^{\prime} \mathrm{E}$ ca $00^{\circ} 48^{\prime} \mathrm{S}, 18^{\circ} 03^{\prime} \mathrm{E}$ 


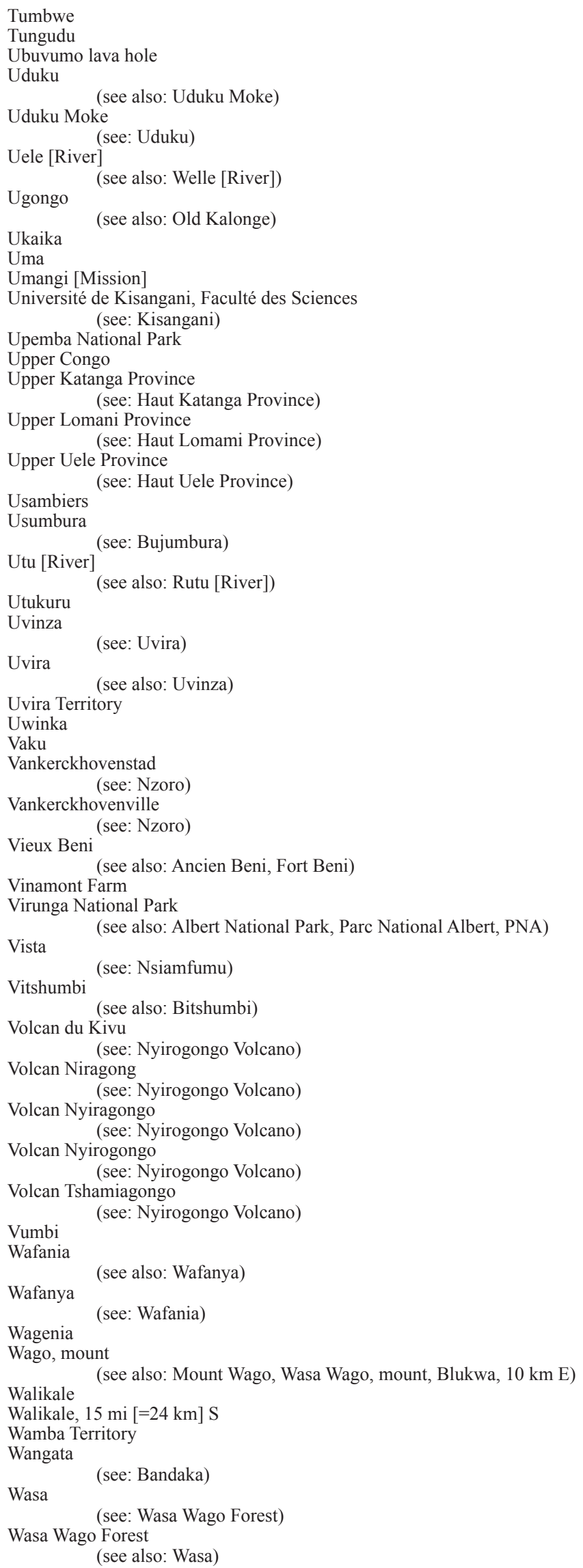

\begin{tabular}{|c|c|c|}
\hline CG-NK & ca $01^{\circ} 00^{\prime} \mathrm{S}, 29^{\circ} 15^{\prime} \mathrm{E}$ & \\
\hline $\begin{array}{l}\text { CG-NK } \\
\text { RW-NO }\end{array}$ & & $840 \mathrm{~m}$ \\
\hline CG-HU & $04^{\circ} 32^{\prime} \mathrm{N}, 29^{\circ} 33^{\prime} \mathrm{E}$ & $950 \mathrm{~m}$ \\
\hline CG-HU & $04^{\circ} 32^{\prime} \mathrm{N}, 29^{\circ} 33^{\prime} \mathrm{E}$ & $950 \mathrm{~m}$ \\
\hline CG & ca $03^{\circ} 41^{\prime} \mathrm{N}, 26^{\circ} 03^{\prime} \mathrm{E}$ & \\
\hline CG-NK & $00^{\circ} 20^{\prime} \mathrm{N}, 29^{\circ} 49^{\prime} \mathrm{E}$ & $2285 \mathrm{~m}$ \\
\hline CG-IT & $00^{\circ} 45^{\prime} \mathrm{N}, 28^{\circ} 45^{\prime} \mathrm{E}$ & $900 \mathrm{~m}$ \\
\hline CG-TO & $00^{\circ} 32^{\prime} \mathrm{N}, 25^{\circ} 54^{\prime} \mathrm{E}$ & $508 \mathrm{~m}$ \\
\hline CG-TP & $02^{\circ} 07^{\prime} \mathrm{S}, 21^{\circ} 24^{\prime} \mathrm{E}$ & \\
\hline CG-TO & $00^{\circ} 31^{\prime} \mathrm{N}, 25^{\circ} 12^{\prime} \mathrm{E}$ & $447 \mathrm{~m}$ \\
\hline $\begin{array}{c}\text { CG-HL } \\
\text { CG } \\
\text { CG }\end{array}$ & $09^{\circ} 00^{\prime} \mathrm{S}, 26^{\circ} 45^{\prime} \mathrm{E}$ & \\
\hline CG & & \\
\hline CG & & \\
\hline $\begin{array}{c}\text { CG } \\
\text { BY-BM }\end{array}$ & $03^{\circ} 23^{\prime} \mathrm{S}, 29^{\circ} 22^{\prime} \mathrm{E}$ & $794 \mathrm{~m}$ \\
\hline CG-NK & $01^{\circ} 24^{\prime} \mathrm{S}, 27^{\circ} 49^{\prime} \mathrm{E}$ & \\
\hline CG-HU & $04^{\circ} 18^{\prime} \mathrm{N}, 29^{\circ} 56^{\prime} \mathrm{E}$ & $2 x^{2}$ \\
\hline CU-SR & $03^{\circ} 24 \mathrm{~S}, 29^{\circ} 09^{\circ} \mathrm{E}$ & $22 / \mathrm{m}$ \\
\hline CG-SK & $03^{\circ} 24^{\prime} \mathrm{S}, 29^{\circ} 09^{\prime} \mathrm{E}$ & $227 \mathrm{~m}$ \\
\hline CG-SK & $03^{\circ} 24^{\prime} \mathrm{S}, 29^{\circ} 08^{\prime} \mathrm{E}$ & \\
\hline RW-OU & $02^{\circ} 28^{\prime} \mathrm{S}, 29^{\circ} 12^{\prime} \mathrm{E}$ & \\
\hline $\begin{array}{l}\text { CG-BC } \\
\text { CG-HU }\end{array}$ & $05^{\circ} 15^{\prime} \mathrm{S}, 13^{\circ} 10^{\prime} \mathrm{E}$ & $351 \mathrm{~m}$ \\
\hline CG-HU & $03^{\circ} 20^{\prime} \mathrm{N}, 29^{\circ} 30^{\prime} \mathrm{E}$ & $791 \mathrm{~m}$ \\
\hline CG-NK & $00^{\circ} 26^{\prime} \mathrm{N}, 29^{\circ} 33^{\prime} \mathrm{E}$ & $585 \mathrm{~m}$ \\
\hline $\begin{array}{l}\text { CG-HK } \\
\text { CG-NK }\end{array}$ & $\begin{array}{l}11^{\circ} 41^{\prime} \text { S, }, 27^{\circ} 29^{\prime} \text { E } \\
01^{\circ} 00^{\prime} \text { S, } 29^{\circ} 15^{\prime} \text { E }\end{array}$ & \\
\hline CG-BC & $05^{\circ} 52^{\prime} \mathrm{S}, 12^{\circ} 17^{\prime} \mathrm{E}$ & $2 \mathrm{~m}$ \\
\hline CG-NK & $00^{\circ} 41^{\prime} \mathrm{S}, 29^{\circ} 23^{\prime} \mathrm{E}$ & $907 \mathrm{~m}$ \\
\hline CG-NK & $01^{\circ} 31^{\prime} \mathrm{S}, 29^{\circ} 15^{\prime} \mathrm{E}$ & \\
\hline CG-NK & $01^{\circ} 31^{\prime} \mathrm{S}, 29^{\circ} 15^{\prime} \mathrm{E}$ & \\
\hline CG-NK & $01^{\circ} 31^{\prime} \mathrm{S}, 29^{\circ} 15^{\prime} \mathrm{E}$ & \\
\hline CG-NK & $01^{\circ} 31^{\prime} \mathrm{S}, 29^{\circ} 15^{\prime} \mathrm{E}$ & \\
\hline CG-NK & $01^{\circ} 31^{\prime} \mathrm{S}, 29^{\circ} 15^{\prime} \mathrm{E}$ & \\
\hline CG-HL & $08^{\circ} 38^{\prime} \mathrm{S}, 25^{\circ} 58^{\prime} \mathrm{E}$ & $932 \mathrm{~m}$ \\
\hline CG-TP & $01^{\circ} 21^{\prime} \mathrm{S}, 20^{\circ} 20^{\prime} \mathrm{E}$ & $396 \mathrm{~m}$ \\
\hline CG-TP & $01^{\circ} 21^{\prime} \mathrm{S}, 20^{\circ} 20^{\prime} \mathrm{E}$ & $396 \mathrm{~m}$ \\
\hline CG-TO & $00^{\circ} 23^{\prime} \mathrm{N}, 25^{\circ} 12^{\prime} \mathrm{E}$ & $508 \mathrm{~m}$ \\
\hline CG-IT & $01^{\circ} 45^{\prime} \mathrm{N}, 30^{\circ} 40^{\prime} \mathrm{E}$ & $2240 \mathrm{~m}$ \\
\hline CG-NK & $01^{\circ} 25^{\prime} \mathrm{S}, 28^{\circ} 03^{\prime} \mathrm{E}$ & $703 \mathrm{~m}$ \\
\hline $\begin{array}{l}\text { CG-NK } \\
\text { CG-HU }\end{array}$ & $01^{\circ} 40^{\prime} \mathrm{S}, 28^{\circ} 07^{\prime} \mathrm{E}$ & \\
\hline CG-ET & $00^{\circ} 04^{\prime} \mathrm{N}, 18^{\circ} 16^{\prime} \mathrm{E}$ & $306 \mathrm{~m}$ \\
\hline CG-IT & $01^{\circ} 44^{\prime} \mathrm{N}, 30^{\circ} 39^{\prime} \mathrm{E}$ & $1386 \mathrm{~m}$ \\
\hline CG-IT & $01^{\circ} 44^{\prime} \mathrm{N}, 30^{\circ} 39^{\prime} \mathrm{E}$ & $1386 \mathrm{~m}$ \\
\hline
\end{tabular}




\begin{tabular}{|c|c|c|c|}
\hline $\begin{array}{l}\text { Wasa Wago, mount } \\
\text { (see: Wago, mount) }\end{array}$ & CG-IT & $01^{\circ} 45^{\prime} \mathrm{N}, 30^{\circ} 40^{\prime} \mathrm{E}$ & $2240 \mathrm{~m}$ \\
\hline $\begin{array}{l}\text { Washiha Forest } \\
\qquad \text { (see also: Bugarula, } 6 \mathrm{~km} \mathrm{~N} \text { ) }\end{array}$ & CG-SK & ca $02^{\circ} 00^{\prime} \mathrm{S}, 29^{\circ} 04^{\prime} \mathrm{E}$ & $1500 \mathrm{~m}$ \\
\hline $\begin{array}{l}\text { Wasseige boerderij } \\
\text { (see: Wasseige Farm) }\end{array}$ & CG-HK & $11^{\circ} 50^{\prime} \mathrm{S}, 27^{\circ} 20^{\prime} \mathrm{E}$ & \\
\hline $\begin{array}{l}\text { Wasseige Farm } \\
\text { (see also: Ferme Wasseige, Kipushi, } 15 \text { km from, Wasseige boerderij) }\end{array}$ & CG-HK & $11^{\circ} 50^{\prime} \mathrm{S}, 27^{\circ} 20^{\prime} \mathrm{E}$ & \\
\hline Weko Forest & CG-TO & $01^{\circ} 00^{\prime} \mathrm{N}, 24^{\circ} 33^{\prime} \mathrm{E}$ & \\
\hline Welle [River] & $\mathrm{CG}$ & $04^{\circ} 07^{\prime} \mathrm{N}, 22^{\circ} 26^{\prime} \mathrm{E}$ & \\
\hline $\begin{array}{l}\text { (see: Uele [River] }) \\
\text { West Kasai (former) Province } \\
\text { (see: Kasai Occidental (former) Province) }\end{array}$ & CG & & \\
\hline Western Prefecture & RW & & \\
\hline Wilibadi River & CG-HU & $03^{\circ} 55^{\prime} \mathrm{N}, 29^{\circ} 08^{\prime} \mathrm{E}$ & \\
\hline Yaekela & CG-TO & $00^{\circ} 48^{\prime} \mathrm{N}, 24^{\circ} 18^{\prime} \mathrm{E}$ & $508 \mathrm{~m}$ \\
\hline $\begin{array}{l}\text { Yakubuku } \\
\text { (see: Yakuluku) }\end{array}$ & CG-HU & $04^{\circ} 20^{\prime} \mathrm{N}, 28^{\circ} 48^{\prime} \mathrm{E}$ & $697 \mathrm{~m}$ \\
\hline $\begin{array}{l}\text { Yakuluku } \\
\text { (see also: Yakubuku) }\end{array}$ & CG-HU & $04^{\circ} 20^{\prime} \mathrm{N}, 28^{\circ} 48^{\prime} \mathrm{E}$ & $697 \mathrm{~m}$ \\
\hline Yalokele & CG-ET & $00^{\circ} 40^{\prime} \mathrm{S}, 22^{\circ} 48^{\prime} \mathrm{E}$ & $443 \mathrm{~m}$ \\
\hline $\begin{array}{l}\text { Yalosemba } \\
\qquad \text { (see also: Yalosembe, Yalosombo) }\end{array}$ & CG-MO & $02^{\circ} 35^{\prime} \mathrm{N}, 21^{\circ} 47^{\prime} \mathrm{E}$ & $422 \mathrm{~m}$ \\
\hline Yalosembe (see: Yalosemba) & CG-MO & $02^{\circ} 35^{\prime} \mathrm{N}, 21^{\circ} 47^{\prime} \mathrm{E}$ & $422 \mathrm{~m}$ \\
\hline $\begin{array}{l}\text { Yalosombo } \\
\text { (see: Yalosemba) }\end{array}$ & CG-MO & $02^{\circ} 35^{\prime} \mathrm{N}, 21^{\circ} 47^{\prime} \mathrm{E}$ & $422 \mathrm{~m}$ \\
\hline $\begin{array}{l}\text { Yalusaka } \\
\text { Yamazo Yamono }\end{array}$ & $\begin{array}{l}\text { CG-TP } \\
\text { CG-MO }\end{array}$ & $\begin{array}{l}01^{\circ} 05^{\prime} \mathrm{S}, 22^{\circ} 58^{\prime} \mathrm{E} \\
02^{\circ} 35^{\prime} \mathrm{N}, 21^{\circ} 47^{\prime} \mathrm{E}\end{array}$ & $420 \mathrm{~m}$ \\
\hline $\begin{array}{l}\text { Yambasa } \quad \text { (see: Yambata) }\end{array}$ & CG-MO & $02^{\circ} 26^{\prime} \mathrm{N}, 21^{\circ} 58^{\prime} \mathrm{E}$ & $414 \mathrm{~m}$ \\
\hline Yambata $\quad$ (see also: Yambasa) & CG-MO & $02^{\circ} 26^{\prime} \mathrm{N}, 21^{\circ} 58^{\prime} \mathrm{E}$ & $414 \mathrm{~m}$ \\
\hline Yambombo & CG-MO & $02^{\circ} 35^{\prime} \mathrm{N}, 21^{\circ} 47^{\prime} \mathrm{E}$ & \\
\hline Yangambi & CG-TO & $00^{\circ} 47^{\prime} \mathrm{N}, 24^{\circ} 28^{\prime} \mathrm{E}$ & $437 \mathrm{~m}$ \\
\hline Yangambi Division Forest & CG-TO & $00^{\circ} 47^{\prime} \mathrm{N}, 24^{\circ} 24^{\prime} \mathrm{E}$ & \\
\hline Yaosuka & CG-TO & $00^{\circ} 45^{\prime} \mathrm{N}, 24^{\circ} 29^{\prime} \mathrm{E}$ & \\
\hline Yayama & CG-TP & $01^{\circ} 16^{\prime} \mathrm{S}, 23^{\circ} 07^{\prime} \mathrm{E}$ & $456 \mathrm{~m}$ \\
\hline Yema Mayombe & CG-BC & $05^{\circ} 45^{\prime} \mathrm{S}, 12^{\circ} 17^{\prime} \mathrm{E}$ & $18 \mathrm{~m}$ \\
\hline Yokamba Elongo & CG-TP & $00^{\circ} 01^{\prime} \mathrm{N}, 22^{\circ} 17^{\prime} \mathrm{E}$ & $429 \mathrm{~m}$ \\
\hline Yoko & CG-TO & $00^{\circ} 17^{\prime} \mathrm{N}, 25^{\circ} 17^{\prime} \mathrm{E}$ & \\
\hline $\begin{array}{l}\text { Yolohotiri Cave } \\
\qquad \text { (see also: Grotte de Yolohotiri, Yolohotiri Grot) }\end{array}$ & CG-IT & $01^{\circ} 13^{\prime} \mathrm{N}, 29^{\circ} 49^{\prime} \mathrm{E}$ & \\
\hline $\begin{array}{l}\text { Yolohotiri Grot } \\
\qquad \text { (see: Yolohotiri Cave) }\end{array}$ & CG-IT & $01^{\circ} 13^{\prime} \mathrm{N}, 29^{\circ} 49^{\prime} \mathrm{E}$ & \\
\hline Yolokafiri (see also: Jolohafiri) & CG-IT & $01^{\circ} 20^{\prime} \mathrm{N}, 29^{\circ} 50^{\prime} \mathrm{E}$ & $1030 \mathrm{~m}$ \\
\hline $\begin{array}{l}\text { Zambi } \\
\text { Zega }\end{array}$ & $\begin{array}{l}\text { CG-BC } \\
\text { CG-IT }\end{array}$ & $\begin{array}{l}05^{\circ} 51^{\prime} \mathrm{S}, 12^{\circ} 52^{\prime} \mathrm{E} \\
01^{\circ} 45^{\prime} \mathrm{N}, 30^{\circ} 44^{\prime} \mathrm{E}\end{array}$ & $650 \mathrm{~m}$ \\
\hline $\begin{array}{ll}\text { Zemio } & \text { (see: Zeya) } \\
& \text { (see also: Semmio, Semnio) }\end{array}$ & CG-BU & $05^{\circ} 03^{\prime} \mathrm{N}, 27^{\circ} 07^{\prime} \mathrm{E}$ & \\
\hline Zeya $\quad$ (see also: Zega) & CG-IT & $01^{\circ} 45^{\prime} \mathrm{N}, 30^{\circ} 44^{\prime} \mathrm{E}$ & $650 \mathrm{~m}$ \\
\hline Zimet Quarter & BY-BM & $03^{\circ} 24^{\prime} \mathrm{S}, 29^{\circ} 21^{\prime} \mathrm{E}$ & \\
\hline Zobia & CG-BU & $02^{\circ} 58^{\prime} \mathrm{N}, 25^{\circ} 56^{\prime} \mathrm{E}$ & $609 \mathrm{~m}$ \\
\hline Zongo & CG-SU & $04^{\circ} 20^{\prime} \mathrm{N}, 18^{\circ} 35^{\prime} \mathrm{E}$ & \\
\hline (see: Kisangani) & CG-TO & $00^{\circ} 31^{\prime} \mathrm{N}, 25^{\circ} 12^{\prime} \mathrm{E}$ & $447 \mathrm{~m}$ \\
\hline
\end{tabular}




\section{Acknowledgements}

We would like to extend our greatest thanks to our contact persons in the various museums (see "Summary table of museum specimens" section), who provided the necessary information to enable us to make this update, especially to Wim Wendelen (Royal Museum for Central Africa, Tervuren, Belgium) and Tom Geerinckx (Royal Belgian Institute of Natural Sciences, Brussels, Belgium). The contribution of Guy-Crispin Gembu Tungaluna and Prescott Musaba Akawa was financially supported by the Flemish Interuniversity Council (VLIR-UOS). Prescott Musaba was also supported by CEBioS, a programme funded by the Directorate General for development Cooperation (DGD) and housed at the RBINS.

\section{References}

ACR. 2016. African Chiroptera Report. AfricanBats, Pretoria. Available from http://www.africanbats.org/index.php/publications/africa-chiroptera-report [accessed 28 Sep. 2017]. Adam F., Aellen V. \& Tranier M. 1993. Nouvelles données sur le genre Myopterus. Le statut de Myopterus daubentonii Desmarest, 1820 (Chiroptera: Molossidae). Revue suisse de Zoologie 100 (2): 317-326. https://doi.org/10.5962/bhl.part.79863

Adedeji A.O., Okonko I.O., Eyarefe O.D., Adedeji O.B., Babalola E.T., Ojezele M.O., Nwanze J.C. \& Amusan T.A. 2010. An overview of rabies - History, epidemiology, control and possible elimination. African Journal of Microbiology Research 4 (22): 2327-2338. Available from http://www.academicjournals.org/journal/AJMR/article-abstract/564B5C314746 [accessed 29 Nov. 2017].

Aellen V. 1952. Contribution à l'étude des chiroptères du Cameroun. Mémoire de la Société neuchâteloise de Sciences naturelles 8 (1): 1-121.

Aellen V. 1956. Speologica africana. Chiroptères des grottes de Guinée. Bulletin de l'Institut fondamental d'Afrique noire, Sciences naturelles 18 A (3): 884-894.

Aellen V. 1959. Chiroptères nouveaux d'Afrique. Archives des Sciences Genève 12 (2): 217-235.

Aellen V. 1966. Addenda. In: Hayman R.W., Misonne X. \& Verheyen W.N. The Bats of the Congo and Rwanda and Burundi. Annalen van het Koninklijk Museum voor Midden Afrika, Zoologische Wetenschappen (8) 154: 69-71.

Aellen V. \& Brosset A. 1968. Chiroptères du sud du Congo (Brazzaville). Revue suisse de Zoologie 75 (14): 435-458. https://doi.org/10.5962/bhl.part.97040

Agnarsson I., Zambrana-Torrelio C.M., Flores-Saldana N.P. \& May-Collado L.J. 2011. A time-calibrated species-level phylogeny of bats (Chiroptera, Mammalia). PLoS Current Tree of Life 3: RRN1212. Available from http://www.ncbi.nlm.nih.gov/pmc/articles/PMC3038382/ [accessed 28 Sep. 2017].

Allen G.M. 1939. A checklist of African mammals. Bulletin of the Museum of Comparative Zoology 83: 1-763. Available from https://www.biodiversitylibrary.org/page/2781971\#page/7/mode/1up [accessed 29 Nov. 2017].

Allen G.M. \& Loveridge A. 1942. Scientific results of a fourth expedition to forested areas in East and Central Africa I. Mammals. Bulletin of the Museum of Comparative Zoology 89 (4): 145-216. Available from https://www.biodiversitylibrary.org/page/2792109\#page/185/mode/1up [accessed 29 Nov. 2017].

Allen J.A. 1917. Part I. Systematic List. In: Allen J.A., Lang H. \& Chapin J.P. The American Museum Congo Expedition Collection of Bats. Bulletin of the American Museum of Natural History 37 (18): 405-478. 
Allen J.A., Lang H. \& Chapin J.P. 1917. The American Museum Congo Expedition Collection of Bats. Bulletin of the American Museum of Natural History 37 (18): 405-563. https://doi.org/10.5962/bhl.title.89200

Almeida F.C., Giannini N.P., DeSalle R. \& Simmons N.B. 2011. Evolutionary relationships of the Old World fruit bats (Chiroptera, Pteropodidae): Another star phylogeny? BMC Evolutionary Biology 11 (281): 1-17. https://doi.org/10.1186/1471-2148-11-281

Almeida F.C., Giannini N.P. \& Simmons N.B. 2016. The evolutionary history of the African fruit bats (Chiroptera: Pteropodidae). Acta Chiropterologica 18 (1): 73-90.

https://doi.org/10.3161/15081109ACC2016.18.1.003

Alonso-Zarazaga M.A. 2005. Nomenclature of higher taxa: a new approach. Bulletin of Zoological Nomenclature 62 (4): 189-199.

Available from https://www.biodiversitylibrary.org/page/34357645\#page/195/mode/1up [accessed 29 Nov. 2017].

Ammerman L.K., Brashear W.A. \& Bartlett S.N. 2013. Further evidence for the basal divergence of Cheiromeles (Chiroptera: Molossidae). Acta Chiropterologica 15 (2): 307-312. https://doi.org/10.3161/150811013X678946

Anciaux de Faveaux F. 1958. Speologica africana. Chiroptères des grottes du Haut-Katanga (Congo Belge). Bulletin de l'Institut fondamental d'Afrique noire, Sciences naturelles (A) 20 (1): 263-275.

Anciaux de Faveaux F.M. 1959-1960. Notes biospéléologiques et parasitologiques sur des grottes du Katanga. Bulletin de la Société Spéléologique de Katanga 1: 23-36; 2: 19-22.

Anciaux de Faveaux F.M. 1960a. Reproduction des chiroptères du Haut-Katanga. Bulletin de la Société Spéléologique de Katanga 2: 23-30.

Anciaux de Faveaux F.M. 1960b. Baguage des chiroptères au Katanga. Bulletin de la Société Spéléologique de Katanga 2: 30-33.

Anciaux de Faveaux F. 1964. La faune des grottes du Haut-Katanga. 3. Congrès Internationale de Spéléologie, Vienne 19613 (2): 11-21.

Anciaux de Faveaux M. 1965. Les parasites des chiroptères. Rôle épidémiologique chez les animaux et l'homme au Katanga. Annales de Parasitologie humaine et comparée 40 (1): 21-38. https://doi.org/10.1051/parasite/1965401021

Anciaux de Faveaux M. 1972. Répartition biogéographique et cycles annuels de chiroptères d'Afrique centrale, 2 vols. PhD Thesis, University of Paris VI.

Anciaux de Faveaux M. 1977. Déplacements saisonniers chez Miniopterus schreibersi natalensis (A. Smith, 1834) au Shaba méridional (S.E. Zaïre, Afrique Centrale) (Mammalia Chiroptera). Revue de zoologie africaine 91 (3): 721-729.

Anciaux de Faveaux M. 1978a. Les cycles annuels de reproduction chez les chiroptères cavernicoles du Shaba (S.E. Zaïre) et du Rwanda. Mammalia 42 (4): 453-490.

https://doi.org/10.1515/mamm.1978.42.4.453

Anciaux de Faveaux M. 1978b. Notes éco-éthologique et parasitologiques sur les Chiroptères cavernicoles du Shaba (Zaïre). International Journal of Speleology 10 (3-4): 331-350. https://doi.org/10.5038/1827-806X.10.3.9

Anciaux de Faveaux M. 1983. Les cycles annuels de reproduction chez les chiroptères phytophiles au Shaba (SE Zaïre) et au Rwanda. In: Van der Straeten E., Verheyen W.N. \& De Vree F. (eds) Proceedings of the Third International Colloquim on Ecology and Taxonomy of African Small Mammals. Antwerpen, 
VAN CAKENBERGHE V. et al., The bats of Congo, Rwanda and Burundi revisited

July 20-24, 1981: 27-34. Annalen van het Koninklijk Museum voor Midden Afrika, Zoologische Wetenschappen 237. Koninklijk Museum voor Midden Afrika, Tervuren.

Andersen K. 1905. A list of the species and subspecies of the genus Rhinolophus, with some notes on their geographical distribution. Annals and Magazine of Natural History ser. 7, 16 (92): 648-662. https://doi.org/10.1080/03745480509443310

Andersen K. 1907. Chiropteran notes. Annali del Museo civico di Storia Naturale "Giacomo Doria" ser. 3 , 3 (O.S. 43): 1-45. Available from https://www.biodiversitylibrary.org/page/7931441\#page/15/mode/1up [accessed 29 Nov. 2017].

Andersen K. 1912. Catalogue of the Chiroptera in the Collection of the British Museum. 2nd ed. I. Megachiroptera. Trustees of the British Museum (Natural History), London. https://doi.org/10.5962/bhl.title.8322

Ansell W.F.H. 1960. Some fruit bats from Northern Rhodesia, with the description of a new race of Epomophorus gambianus Ogilby. Revue de zoologie et de botanique africaines 61 (1-2): 160-166.

Ansell W.F.H. 1974. Some mammals from Zambia and adjacent countries. Occasional Papers of the National Parks and Wildlife Services, Zambia Suppl 1: 1-49.

Aulagnier S. 2013. Asellia tridens Geoffroy's Trident Leaf-nosed Bat. In: Happold M. \& Happold D. (eds) Mammals of Africa Volume IV. Hedgehogs, Shrews and Bats: 362-364. Bloomsbury Publishing, London.

Baeten B., Van Cakenberghe V. \& De Vree F. 1984. An annotated inventory of a collection of bats from Rwanda (Chiroptera). Revue de zoologie africaine 98 (1): 183-196.

Baker R.J. \& Bradley R.D. 2006. Speciation in mammals and the genetic species concept. Journal of Mammalogy 87 (4): 643-662. https://doi.org/10.1644/06-MAMM-F-038R2.1

Barghoorn S.F. 1977. New material of Vespertiliavus Schlosser (Mammalia, Chiroptera) and suggested relationships of emballonurid bats based on cranial morphology. American Museum Novitates 2618: 1-29. Available from http://hdl.handle.net/2246/2017 [accessed 29 Nov. 2017].

Bates P.J.J., Cameron K., Pearch M.J. \& Hayes B. 2013. A review of the bats (Chiroptera) of the Republic of Congo, including eight species new to the country. Acta Chiropterologica 15 (2): 313-340. https://doi.org/10.3161/150811013X678955

Benda P. \& Vallo P. 2012. New look on the geographical variation in Rhinolophus clivosus with description of a new horseshoe bat species from Cyrenaica, Libya. Vespertilio 16: 69-96. Available from http://www.ceson.org/vespertilio/16/069_096_Benda.pdf [accessed 29 Nov. 2017].

Benoit P.L.G. 1958a. Les Polyctenidae du Congo Belge (Hemiptera - Cimicoidea). Revue de zoologie et de botanique africaines 57 (1-2): 68-72.

Benoit P.L.G. 1958b. Contribution a l'étude de Spinturnicidae du Congo Belge. Revue de zoologie et de botanique africaines 57 (1-2): 96-100.

Bergmans W. 1976. A revision of the African genus Myonycteris Matschie, 1899 (Mammalia, Megachiroptera). Beaufortia 24 (317): 189-216.

Available from https://www.oalibrary.org/papers2/ed9ddc89-f59d-41e2-96e7-a66a93fe628a/ [accessed 29 Nov. 2017].

Bergmans W. 1979. First records of Epomops dobsonii (Bocage, 1889) from Tanzania and Rwanda, with a note on its size range (Mammalia, Megachiroptera). Zeitschrift für Säugetierkunde 44 (4): 239-240. Available from https://www.biodiversitylibrary.org/item/186090\#page/7/mode/1up [accessed 29 Nov. 2017]. 
Bergmans W. 1980. A new fruit bat of the genus Myonycteris Matschie, 1899, from eastern Kenya and Tanzania (Mammalia, Megachiroptera). Zoologische Mededelingen 55 (14): 171-181.

Bergmans W. 1988. Taxonomy and biogeography of African fruit bats (Mammalia, Megachiroptera). 1. General introduction; Material and methods; Results: The genus Epomophorus Bennett, 1836. Beaufortia 38 (5): 75-146. Available from http://www.repository.naturalis.nl/document/548382 [accessed 29 Nov. 2017].

Bergmans W. 1989. Taxonomy and biogeography of African fruit bats (Mammalia, Megachiroptera). 2. The genera Micropteropus Matschie, 1899, Epomops Gray, 1870, Hypsignatus H. Allen, 1861, Nanonycteris, Matschie, 1899 and Plerotes Andersen, 1910. Beaufortia 39 (4): 89-153. Available from http://www.repository.naturalis.nl/document/548365 [accessed 29 Nov. 2017].

Bergmans W. 1991. Taxonomy and biogeography of African fruit bats (Mammalia, Megachiroptera). 3. The genera Scotonycteris Matschie, 1894, Casinycteris Thomas, 1910, Pteropus Brisson, 1762, and Eidolon Rafinesque, 1815. Beaufortia 40 (7): 111-177. Available from http://www.repository.naturalis. nl/document/548463 [accessed 29 Nov. 2017].

Bergmans W. 1994. Taxonomy and biogeography of African fruit bats (Mammalia, Megachiroptera). 4. The genus Rousettus Gray, 1821. Beaufortia 44 (4): 79-126.

Available from http://www.repository.naturalis.nl/document/548564 [accessed 29 Nov. 2017].

Bergmans W. 1997. Taxonomy and biogeography of African fruit bats (Mammalia, Megachiroptera). 5. The genera Lissonycteris Andersen, 1913, Myonycteris Matschie, 1899 and Megaloglossus Pagenstecher, 1885; General remarks and conclusions; annex: Key to all species. Beaufortia 47 (2): 11-90. Available from http://www.repository.naturalis.n1/document/548609 [accessed 29 Nov. 2017].

Bergmans W. 2013. Epomophorus anselli Ansell's Epauletted Fruit Bat. In: Happold M. \& Happold D. (eds) Mammals of Africa Volume IV. Hedgehogs, Shrews and Bats: 238-240. Bloomsbury Publishing, London.

Bergmans W. \& Van Bree P.J.H. 1972. The taxonomy of the African bat Megaloglossus woermanni Pagenstecher, 1885 (Megachiroptera, Macroglossinae). Biologica Gabonica 3-4: 291-299.

Bernard R.T.F. 2013. Myotis tricolor Temminck's Myotis (Temminck's Hairy Bat). In: Happold M. \& Happold D. (eds) Mammals of Africa Volume IV. Hedgehogs, Shrews and Bats: 706-708. Bloomsbury Publishing, London.

Bernard R.T.F. \& Happold M. 2013a. Rhinolophus clivosus Geoffroy's Horseshoe Bat (Cretzschmar's Horseshoe Bat). In: Happold M. \& Happold D. (eds) Mammals of Africa Volume IV. Hedgehogs, Shrews and Bats: 316-318. Bloomsbury Publishing, London.

Bernard R.T.F. \& Happold M. 2013b. Hipposideros caffer Sundevall's Leaf-nosed Bat. In: Happold M. \& Happold D. (eds) Mammals of Africa Volume IV. Hedgehogs, Shrews and Bats: 375-378. Bloomsbury Publishing, London.

Bernard R.T.F. \& Happold M. 2013c. Nycteris thebaica Egyptian Slit-faced Bat. In: Happold M. \& Happold D. (eds) Mammals of Africa Volume IV. Hedgehogs, Shrews and Bats: 457-460. Bloomsbury Publishing, London.

Bernard R.T.F. \& Happold M. 2013d. Miniopterus fraterculus Lesser Long-fingered Bat. In: Happold M. \& Happold D. (eds) Mammals of Africa Volume IV. Hedgehogs, Shrews and Bats: 712-714. Bloomsbury Publishing, London.

Bernard R.T.F. \& Happold M. 2013e. Miniopterus natalensis Natal Long-fingered Bat (Natal Clinging Bat). In: Happold M. \& Happold D. (eds) Mammals of Africa Volume IV. Hedgehogs, Shrews and Bats: 718-720. Bloomsbury Publishing, London. 
Bocage J.V.B. 1889. Mammifères d'Angola et du Congo. Jornal de Sciencas mathematicas, physicas e naturaes (2) 1 (1): 8-32.

Available from https://www.biodiversitylibrary.org/page/4141036\#page/5/mode/1up [accessed 29 Nov. 2017].

Breman J.G., Johnson K.M., Van der Groen G., Robbins C.B., Szczeniowski M.V., Ruti K., Webb P.A., Meier F., Heymann D.L. \& The Ebola Virus Study Team. 1999. A search for Ebola virus in animals in the Democratic Republic of the Congo and Cameroon: Ecologic, virologic, and seriologic surveys, 1979-1980. Journal of Infectious Diseases 179 (2 - Suppl 1): 139-147. https://doi.org/10.1086/514278

Brosset A. 1966a. Contribution à la faune du Congo (Brazzaville). Mission A. Villiers et A. Descarpentries. XX. Chiroptères. Bulletin de l'Institut fondamental d'Afrique noire, Sciences naturelles 28A (1): $362-370$.

Brosset A. 1966b. Les chiroptères du Haut-Ivindo (Gabon). Biologica Gabonica 2 (1): 47-86.

Ceballos G. \& Ehrlich P.R. 2006. Global mammal distributions, biodiversity hotspots, and conservation. Proceedings of the National Academy of Sciences 103 (51): 19374-19379.

https://doi.org/10.1073/pnas.0609334103

Claessen C.J. \& De Vree F. 1990. Systematic and distributional notes on the larger species of the genus Epomophorus Bennet, 1836 (Chiroptera: Pteropodidae). In: Peters G. \& Hutterer R. (eds) Vertebrates in the Tropics. Proceedings of the International Symposium on Vertebrate Biogeography and Systemematics in the Tropics, Bonn, June 5-8, 1989: 177-186. Museum Alexander Koenig, Bonn.

Claessen C.J. \& De Vree F. 1991. Systematic and taxonomic notes on the Epomophorus anuruslabiatus-minor complex with the description of a new species (Mammalia: Chiroptera: Pteropodidae). Senckenbergiana biologica 71 (4/6): 209-238.

Cotterill F.P.D. 2001a. Further notes on large Afrotropical free-tailed bats of the genus Tadarida (Molossidae: Mammalia). Arnoldia Zimbabwe 10 (18): 199-210.

Cotterill F.P.D. 2001b. The first specimen of Thomas's flat-headed bat, Mimetillus moloneyi thomasi (Microchiroptera: Mammalia) in Southern Africa from Mozambique. Arnoldia Zimbabwe 10 (19): 211218.

Cotterill F.P.D. 2001c. New specimens of lesser house bats (Vespertilionidae: Scotoecus) from Mozambique and Zambia. Arnoldia Zimbabwe 10 (20): 219-223.

Cotterill F.P.D. 2002. A new species of horseshoe bat (Microchiroptera: Rhinolophidae) from southcentral Africa: with comments on its affinities and evolution, and the characterization of rhinolophid species. Journal of Zoology 256 (2): 165-179. https://doi.org/10.1017/S0952836902000201

Cotterill F.P.D. 2013a. Rhinolophus eloquens Eloquent Horseshoe Bat. In: Happold M. \& Happold D. (eds) Mammals of Africa Volume IV. Hedgehogs, Shrews and Bats: 323-325. Bloomsbury Publishing, London.

Cotterill F.P.D. 2013b. Nycteris parisii Parisi's Slit-faced Bat. In: Happold M. \& Happold D. (eds) Mammals of Africa Volume IV. Hedgehogs, Shrews and Bats: 456-457. Bloomsbury Publishing, London.

Cotterill F.P.D. 2013c. Tadarida ansorgei Ansorge's Free-tailed Bat. In: Happold M. \& Happold D. (eds) Mammals of Africa Volume IV. Hedgehogs, Shrews and Bats: 495-497. Bloomsbury Publishing, London.

Cotterill F.P.D. 2013d. Tadarida bivittata Spotted Free-tailed Bat. In: Happold M. \& Happold D. (eds) Mammals of Africa Volume IV. Hedgehogs, Shrews and Bats: 499-500. Bloomsbury Publishing, London. 
Cotterill F.P.D. 2013e. Tadarida fulminans Madagascan Free-tailed Bat. In: Happold M. \& Happold D. (eds) Mammals of Africa Volume IV. Hedgehogs, Shrews and Bats: 511-513. Bloomsbury Publishing, London.

Cotterill F.P.D. 2013f. Tadarida gallagheri Gallagher's Free-tailed Bat. In: Happold M. \& Happold D. (eds) Mammals of Africa Volume IV. Hedgehogs, Shrews and Bats: 513-514. Bloomsbury Publishing, London.

Cotterill F.P.D. 2013g. Tadarida niveiventer White-bellied Free-tailed Bat. In: Happold M. \& Happold D. (eds) Mammals of Africa Volume IV. Hedgehogs, Shrews and Bats: 525-526. Bloomsbury Publishing, London.

Cotterill F.P.D. 2013h. Tadarida ventralis Giant Free-tailed Bat. In: Happold M. \& Happold D. (eds) Mammals of Africa Volume IV. Hedgehogs, Shrews and Bats: 539-540. Bloomsbury Publishing, London.

Cotterill F.P.D. 2013i. Kerivoula argentata Damara Woolly Bat. In: Happold M. \& Happold D. (eds) Mammals of Africa Volume IV. Hedgehogs, Shrews and Bats: 726-727. Bloomsbury Publishing, London.

Cotterill F.P.D. 2013j. Kerivoula lanosa Lesser Woolly Bat. In: Happold M. \& Happold D. (eds) Mammals of Africa Volume IV. Hedgehogs, Shrews and Bats: 730-731. Bloomsbury Publishing, London.

Cotterill F.P.D. \& Happold M. 2013a. Rhinolophus fumigatus Rüppell's Horseshoe Bat. In: Happold M. \& Happold D. (eds) Mammals of Africa Volume IV. Hedgehogs, Shrews and Bats: 329-331. Bloomsbury Publishing, London.

Cotterill F.P.D. \& Happold M. 2013b. Rhinolophus simulator Bushveld Horseshoe Bat. In: Happold M. \& Happold D. (eds) Mammals of Africa Volume IV. Hedgehogs, Shrews and Bats: 351-353. Bloomsbury Publishing, London.

Cotterill F.P.D. \& Happold M. 2013c. Tadarida midas Midas Free-tailed Bat. In: Happold M. \& Happold D. (eds) Mammals of Africa Volume IV. Hedgehogs, Shrews and Bats: 518-520. Bloomsbury Publishing, London.

Cotterill F.P.D. \& Happold M. 2013d. Tadarida nigeriae Nigerian Free-tailed Bat. In: Happold M. \& Happold D. (eds) Mammals of Africa Volume IV. Hedgehogs, Shrews and Bats: 523-525. Bloomsbury Publishing, London.

Csorba G., Ujhelyi P. \& Thomas N. 2003. Horseshoe Bats of the World (Chiroptera: Rhinolophidae). Alana Books, Shropshire, UK.

Csorba G., Chou C.-H., Ruedi M., Görföl T., Motokawa M., Wiantoro S., Thong V.D., Son N.T., Lin L.-K. \& Furey N. 2014. The reds and the yellows: a review of Asian Chrysopteron Jentink, 1910 (Chiroptera: Vespertilionidae: Myotis). Journal of Mammalogy 95 (4): 663-678.

https://doi.org/10.1644/13-MAMM-A-200

Decher J., Norris R.W. \& Fahr J. 2010. Small mammal survey in the upper Seli River valley, Sierra Leone. Mammalia 74 (2): 163-176. https://doi.org/10.1515/MAMM.2010.026

Decher J., Hoffmann A., Schaer J., Norris R.W., Kadjo B., Astrin J., Monadjem A. \& Hutterer R. 2016. Bat diversity in the Simandou Mountain Range of Guinea, with the description of a new white-winged vespertilionid. Acta Chiropterologica 17 (2): 255-282 (for 2015).

https://doi.org/10.3161/15081109ACC2015.17.2.003

Dollman G. 1909. On mammals collected by Mr. S.A. Neave, M.A., B.Sc. (Oxon.) in Katanga, Congo Free State. Annals and Magazine of Natural History Ser. 8 , 3 (16): 348-354. https://doi.org/10.1080/00222930908692589 
VAN CAKENBERGHE V. et al., The bats of Congo, Rwanda and Burundi revisited

Dollman G. 1914. Notes on mammals collected by Dr. Christy in the Congo and by Dr. Bayer in Uganda and British East Africa. Revue de zoologie africaine 4: 75-90. https://doi.org/10.5962/bhl.part.13888

Dool S.E., Puechmaille S.J., Foley N.M., Allegrini B., Bastian A., Mutumi G.L., Maluleke T.G., Odendaal L.J., Teeling E.C. \& Jacobs D.S. 2016. Nuclear introns outperform mitochondrial DNA in inter-specific phylogenetic reconstruction: Lessons from horseshoe bats (Rhinolophidae: Chiroptera). Molecular Phylogenetics and Evolution 97: 196-212. https://doi.org/10.1016/j.ympev.2016.01.003

Dowsett R.J., Harrison D.L. \& Granjon L. 1991. Bats (Chiroptera) from the Mayombe and lower Kouilou (with a checklist for Congo). Tauraco Research Report 4: 251-263.

Eger J. 2013. Glauconycteris curryae Curry's Butterfly Bat. In: Happold M. \& Happold D. (eds) Mammals of Africa Volume IV. Hedgehogs, Shrews and Bats: 567-568. Bloomsbury Publishing, London.

Eger J.L. \& Schlitter D.A. 2001. A new species of Glauconycteris from West Africa (Chiroptera: Vespertilionidae). Acta Chiropterologica 3 (1): 1-10.

Eisentraut M. 1963. Die Wirbeltiere des Kamerungebirges. Parey, Hamburg and Berlin.

Ellerman J.R., Morrison-Scott T.C.S. \& Hayman R.W. 1953. Southern African Mammals 1758 to 1951: a Reclassification. Trustees of the British Museum (Natural History), London.

https://doi.org/10.5962/bhl.title.113025

El-Rayah M.A. 1980. Systematics of African molossid bats of the subgenus Xiphonycteris of the genus Tadarida (Molossidae: Chiroptera). PhD Thesis, University of Toronto. Available from https://idl-bnc-idrc.dspacedirect.org/handle/10625/5743?show=full [accessed 29 Nov. 2017].

El-Rayah M.A. 1981. A new species of bat of the genus Tadarida (family Molossidae) from West Africa. Life Science Occasional Papers, Royal Ontario Museum 36: 1-10.

Fahr J. 2013a. Nanonycteris veldkampii Veldkamp's Dwarf Epauletted Fruit Bat. In: Happold M. \& Happold D. (eds) Mammals of Africa Volume IV. Hedgehogs, Shrews and Bats: 278-280. Bloomsbury Publishing, London.

Fahr J. 2013b. Rhinolophus hilli Hill's Horseshoe Bat. In: Happold M. \& Happold D. (eds) Mammals of Africa Volume IV. Hedgehogs, Shrews and Bats: 334-336. Bloomsbury Publishing, London.

Fahr J. 2013c. Rhinolophus maclaudi Maclaud's Horseshoe Bat. In: Happold M. \& Happold D. (eds) Mammals of Africa Volume IV. Hedgehogs, Shrews and Bats: 342-343. Bloomsbury Publishing, London.

Fahr J. 2013d. Hipposideros cyclops Cyclops Leaf-nosed Bat. In: Happold M. \& Happold D. (eds) Mammals of Africa Volume IV. Hedgehogs, Shrews and Bats: 380-383. Bloomsbury Publishing, London.

Fahr J. 2013e. Hipposideros fuliginosus Sooty Leaf-nosed Bat (Temminck's Leaf-nosed Bat). In: Happold M. \& Happold D. (eds) Mammals of Africa Volume IV. Hedgehogs, Shrews and Bats: 383-385. Bloomsbury Publishing, London.

Fahr J. 2013f. Nycteris arge Bates's Slit-faced Bat. In: Happold M. \& Happold D. (eds) Mammals of Africa Volume IV. Hedgehogs, Shrews and Bats: 442-443. Bloomsbury Publishing, London.

Fahr J. 2013g. Nycteris intermedia Intermediate Slit-faced Bat. In: Happold M. \& Happold D. (eds) Mammals of Africa Volume IV. Hedgehogs, Shrews and Bats: 450-451. Bloomsbury Publishing, London.

Fahr J. 2013h. Nycteris nana Dwarf Slit-faced Bat. In: Happold M. \& Happold D. (eds) Mammals of Africa Volume IV. Hedgehogs, Shrews and Bats: 455-456. Bloomsbury Publishing, London.

Fahr J. 2013i. Myopterus daubentonii Daubenton's Winged-mouse Bat. In: Happold M. \& Happold D. (eds) Mammals of Africa Volume IV. Hedgehogs, Shrews and Bats: 476-478. Bloomsbury Publishing, London. 
Fahr J. 2013j. Myopterus whitleyi Bini Winged-mouse Bat. In: Happold M. \& Happold D. (eds) Mammals of Africa Volume IV. Hedgehogs, Shrews and Bats: 478-479. Bloomsbury Publishing, London.

Fahr J. 2013k. Tadarida aloysiisabaudiae Duke of Abruzzi's Free-tailed Bat. In: Happold M. \& Happold D. (eds) Mammals of Africa Volume IV. Hedgehogs, Shrews and Bats: 493-495. Bloomsbury Publishing, London.

Fahr J. 20131. Tadarida bemmeleni Gland-tailed Free-tailed Bat. In: Happold M. \& Happold D. (eds) Mammals of Africa Volume IV. Hedgehogs, Shrews and Bats: 497-499. Bloomsbury Publishing, London.

Fahr J. 2013m. Tadarida congica Congo Free-tailed Bat. In: Happold M. \& Happold D. (eds) Mammals of Africa Volume IV. Hedgehogs, Shrews and Bats: 507-509. Bloomsbury Publishing, London.

Fahr J. 2013n. Tadarida demonstrator Mongalla Free-tailed Bat. In: Happold M. \& Happold D. (eds) Mammals of Africa Volume IV. Hedgehogs, Shrews and Bats: 509-511. Bloomsbury Publishing, London.

Fahr J. 2013o. Mimetillus moloneyi Moloney's Mimic Bat (Moloney's Flat-headed Bat). In: Happold M. \& Happold D. (eds) Mammals of Africa Volume IV. Hedgehogs, Shrews and Bats: 586-588. Bloomsbury Publishing, London.

Fahr J. 2013p. Pipistrellus brunneus Dark-brown Pipistrelle. In: Happold M. \& Happold D. (eds) Mammals of Africa Volume IV. Hedgehogs, Shrews and Bats: 613-614. Bloomsbury Publishing, London.

Fahr J. 2013q. Pipistrellus crassulus Broad-headed Pipistrelle. In: Happold M. \& Happold D. (eds) Mammals of Africa Volume IV. Hedgehogs, Shrews and Bats: 617-619. Bloomsbury Publishing, London.

Fahr J. 2013r. Pipistrellus tenuipinnis White-winged Pipistrelle (Slender-winged Pipistrelle). In: Happold M. \& Happold D. (eds) Mammals of Africa Volume IV. Hedgehogs, Shrews and Bats: 655-656. Bloomsbury Publishing, London.

Fahr J. 2013s. Kerivoula cuprosa Copper Woolly Bat. In: Happold M. \& Happold D. (eds) Mammals of Africa Volume IV. Hedgehogs, Shrews and Bats: 727-728. Bloomsbury Publishing, London.

Fahr J. 2013t. Kerivoula phalaena Spurrell's Woolly Bat. In: Happold M. \& Happold D. (eds) Mammals of Africa Volume IV. Hedgehogs, Shrews and Bats: 731-732. Bloomsbury Publishing, London.

Fahr J. 2013u. Kerivoula smithii Smith's Woolly Bat. In: Happold M. \& Happold D. (eds) Mammals of Africa Volume IV. Hedgehogs, Shrews and Bats: 733-734. Bloomsbury Publishing, London.

Fahr J. \& Ebigbo N.M. 2003. A conservation assessment of the bats of the Simandou Range, Guinea, with the first record of Myotis welwitschii (Gray, 1866) from West Africa. Acta Chiropterologica 5 (1): 125-141. https://doi.org/10.3161/001.005.0116

Fahr J., Vierhaus H., Hutterer R. \& Kock D. 2002. A revision of the Rhinolophus maclaudi species group with the description of a new species from West Africa (Chiroptera: Rhinolophidae). Myotis 40: 95-126.

Fahr J., Djossa B.A. \& Vierhaus H. 2006. Rapid assessment of bats (Chiroptera) in Déré, Diécké and Mt. Béro classified forests, southeastern Guinea; including a review of the distribution of bats in Guinée Forestière. In: Wright H.E., McCullough J., Alonso L.E. \& Diallo M.S. (eds) A Rapid Biological Assesssment of Three Classified Forests in Southeastern Guinea. RAP Bulletin of Biological Assessment 40: $168-180$.

Fain A. 1953. Notes sur une collection de rongeurs, insectivores et chauves-souris, capturés dans la région d'endémie pesteuse de Blukwa-Ituri, Congo Belge. Revue de zoologie et de botanique africaines 48 (1-2): 89-101.

Fain A. 1959. Les acariens psoriques parasites des chauves-souris. XI. Le genre Notoedres Railliet 1893. Revue de zoologie et de botanique africaines 60 (1-2): 131-167. 
Fain A. 1972. Notes sur les punaises parasites de chiroptères de la République du Zaïre avec description de deux espèces et d'une sous-espèce nouvelles. Revue de zoologie et de botanique africaines 85 (3-4): 187-202.

Fain A. \& Aellen V. 1994. Notes sur une petite collection d'acariens (Acari) parasites récoltés sur des chiroptères de la collection du Muséum d'Histoire naturelle de Genève. Bulletin de la Société neuchâteloise des Sciences naturelles 117: 59-66.

Fenton M.B. \& Peterson R.L. 1972. Further notes on Tadarida aloysiisabaudiae and Tadarida russata (Chiroptera - Molossidae - Africa). Canadian Journal of Zoology 50 (1): 19-24.

https://doi.org/10.1139/z72-004

Foley N.M., Thong V.D., Soisook P., Goodman S.M., Armstrong K.N., Jacobs D.S., Puechmaille S.J. \& Teeling E.C. 2014. How and why overcome the impediments to resolution: lessons from rhinolophid and hipposiderid bats. Molecular Biology and Evolution 32 (2): 313-333.

https://doi.org/10.1093/molbev/msu329

Foley N.M., Goodman S.M., Whelan C.V., Puechmaille S.J. \& Teeling E. (2017). Towards navigating the Minotaur's labyrinth: cryptic diversity and taxonomic revision within the speciose genus Hipposideros (Hipposideridae). Acta Chiropterologica 19 (1): 1-18.

https://doi.org/10.3161/15081109ACC2017.19.1.001

Frechkop S. 1938. Exploration du Parc National Albert. Mission G.F. De Witte (1933-1935). Fasc. 10. Mammifères, Chiroptera. Institut des Parcs Nationaux du Congo Belge, Bruxelles: 42-60. Available from http://www.apncb.be/archives/publications/exploration-national-park-albert/exploration-nationalpark-albert-first-series/mission-g.-f.-de-witte-1933-1935/1938-fascicule-10-mammiferes [accessed 29 Nov. 2017].

Frechkop S. 1943. Exploration du Parc National Albert. Mission S. Frechkop (1937-1938). Fasc. 1. Mammifères, Chiroptera. Institut des Parcs Nationaux du Congo Belge, Bruxelles: 34-54. Available from http://biblio.naturalsciences.be/rbins-publications/institut-des-parcs-nationaux-du-congo/explorationdu-parc-national-albert-exploratie-van-het-nationaal-albert-park/1-1943-mission-s-mammiferes [accessed 29 Nov. 2017].

Frechkop S. 1944. Exploration du Parc National de la Kagera. Mission S. Frechkop (1938). Fasc. 1. Mammifères, Chiroptera. Institut des Parcs Nationaux du Congo Belge, Bruxelles: 11-13. Available from http://biblio.naturalsciences.be/rbins-publications/institut-des-parcs-nationaux-du-congo/explorationdu-parc-national-de-la-kagera-exploratie-van-het-nationaal-park-der-kagera/1-1944-mission-sfrechkop-mammiferes [accessed 29 Nov. 2017].

Frechkop S. 1954. Exploration du Parc National de 1'Upemba. Mission G.F. De Witte en collaboration avec W. Adam, A. Janssens, L. Van Meel et R. Verheyen (1946-1949). Fasc. 14. Mammifères, Chiroptera. Institut des Parcs Nationaux du Congo Belge, Bruxelles: 11-22. Available from http://biblio. naturalsciences.be/rbins-publications/institut-des-parcs-nationaux-du-congo/exploration-du-parcnational-de-lupemba/14-1954-mission-zending-g-f-de-witte-mammiferes [accessed 29 Nov. 2017].

Freeman P.W. 1981. A multivariate study of the family Molossidae (Mammalia, Chiroptera): morphology, ecology, evolution. Fieldiana Zoology N.S. 7: 1-173. https://doi.org/10.5962/bhl.title.3128

Gallagher M.D. \& Harrison D.L. 1977. Report on the bats (Chiroptera) obtained by the Zaire River Expedition. Bonner zoologische Beiträge 28 (1-2): 19-32. Available from http://www.zoologicalbulletin. de/BzB_Volumes/Volume_28_1_2/019_032_BZB28_1_2_Gallagher_MichaelD.PDF [accessed 29 Nov. 2017].

Gembu Tungaluna G.-C., Van Cakenberghe V., Musaba Akawa P., Dudu Akaibe B., Verheyen E., De Vree F. \& Fahr J. 2013. Rediscovery of Glauconycteris superba Hayman, 1939 (Chiroptera: Vespertilionidae) 
after 40 years at Mbiye Island, Democratic Republic of the Congo. European Journal of Taxonomy 42: 1-18. https://doi.org/10.5852/ejt.2013.42

Göpfert M.C., Heller K.G., Volleth M. \& Wasserthal L.T. 1995. Madagascar microchiropteran bats: new records including new species. Bat Research News 36 (4): 68-69.

Grard G., Biek R., Muyembe Tamfum J.-J., Fair J., Wolfe N., Formenty P., Paweska J. \& Leroy E. 2011. Emergence of divergent Zaire ebola virus strains in Democratic Republic of the Congo in 2007 and 2008. Journal of Infectious Diseases 204 (Suppl. 3): S776-S784. https://doi.org/10.1093/infdis/jir364

Gregorin R. \& Cirranello A. 2015. Phylogeny of Molossidae Gervais (Mammalia: Chiroptera) inferred by morphological data. Cladistics 32 (1): 2-35 (for 2016). https://doi.org/10.1111/cla.12117

Grimshaw J.M., Cordeiro N.J. \& Foley C.A.H. 1995. The mammals of Kilimanjaro. Journal of East African Natural History 84 (2): 105-139. https://doi.org/10.2982/0012-8317(1995)84[105:TMOK]2.0.CO;2

Gunnell G.F., Butler P.M., Greenwood M. \& Simmons N.B. 2015. Bats (Chiroptera) from Olduvai Gorge, Early Pleistocene, Bed I (Tanzania). American Museum Novitates 3846: 1-35. https://doi.org/10.1206/3846.1

Gyldenstolpe N. 1928. Zoological results of the Swedish Expedition to Central Africa 1921. Vertebrata. 5. Mammals from the Birunga Volcanoes, north of Lake Kivu. Arkiv för Zoologi, Stockholm (20), 4: $1-76$.

Haiduk M.W., Baker R.J., Robbins L.W. \& Schlitter D.A. 1981. Chromosomal evolution in African Megachiroptera: G- and C-band assessment of the magnitude of change in similar standard karyotypes. Cytogenetics and Cell Genetics 29: 221-232. https://doi.org/10.1159/000131573

Han B.A., Schmidt J.P., Alexander L.W., Bowden S.E., Hayman D.T.S. \& Drake J.M. 2016. Undiscovered bat hosts of filoviruses. PLoS Neglected Tropical Diseases 10 (7): e0004815.

https://doi.org/10.1371/journal.pntd.0004815

Handley C.O. Jr. 1980. Inconsistencies in formation of family-group and subfamily-group names in Chiroptera. In: Wilson D.E. \& Gardner A.L. (eds) Proceedings of the Fifth International Bat Research Conference: 9-13. Texas Tech University Press, Lubbock.

Happold M. 2013a. Epomophorus labiatus Little Epauletted Fruit Bat. In: Happold M. \& Happold D. (eds) Mammals of Africa Volume IV. Hedgehogs, Shrews and Bats: 245-247. Bloomsbury Publishing, London.

Happold M. 2013b. Epomophorus minimus Least Epauletted Fruit Bat. In: Happold M. \& Happold D. (eds) Mammals of Africa Volume IV. Hedgehogs, Shrews and Bats: 248-249. Bloomsbury Publishing, London.

Happold M. 2013c. Epomophorus wahlbergi Wahlberg's Epauletted Fruit Bat. In: Happold M. \& Happold D. (eds) Mammals of Africa Volume IV. Hedgehogs, Shrews and Bats: 249-251. Bloomsbury Publishing, London.

Happold M. 2013d. Rousettus aegyptiacus Egyptian Rousette. In: Happold M. \& Happold D. (eds) Mammals of Africa Volume IV. Hedgehogs, Shrews and Bats: 289-292. Bloomsbury Publishing, London.

Happold M. 2013e. Rhinolophus alcyone Halcyon Horseshoe Bat. In: Happold M. \& Happold D. (eds) Mammals of Africa Volume IV. Hedgehogs, Shrews and Bats: 311-312. Bloomsbury Publishing, London.

Happold M. 2013f. Rhinolophus blasii Blasius's Horseshoe Bat (Peak-saddle Horseshoe Bat). In: Happold M. \& Happold D. (eds) Mammals of Africa Volume IV. Hedgehogs, Shrews and Bats: 312-313. Bloomsbury Publishing, London. 
VAN CAKENBERGHE V. et al., The bats of Congo, Rwanda and Burundi revisited

Happold M. 2013g. Rhinolophus landeri Lander's Horseshoe Bat (Peak-saddle Horseshoe Bat). In: Happold M. \& Happold D. (eds) Mammals of Africa Volume IV. Hedgehogs, Shrews and Bats. : 340-341 Bloomsbury Publishing, London.

Happold M. 2013h. Hipposideros abae Aba Leaf-nosed Bat. In: Happold M. \& Happold D. (eds) Mammals of Africa Volume IV. Hedgehogs, Shrews and Bats: 372-373. Bloomsbury Publishing, London.

Happold M. 2013i. Hipposideros beatus Benito Leaf-nosed Bat. In: Happold M. \& Happold D. (eds) Mammals of Africa Volume IV. Hedgehogs, Shrews and Bats: 373-374. Bloomsbury Publishing, London.

Happold M. 2013j. Hipposideros camerunensis Cameroon Leaf-nosed Bat. In: Happold M. \& Happold D. (eds) Mammals of Africa Volume IV. Hedgehogs, Shrews and Bats: 378-379. Bloomsbury Publishing, London.

Happold M. 2013k. Hipposideros gigas Giant Leaf-nosed Bat. In: Happold M. \& Happold D. (eds) Mammals of Africa Volume IV. Hedgehogs, Shrews and Bats: 385-387. Bloomsbury Publishing, London.

Happold M. 20131. Hipposideros ruber Noack's Leaf-nosed Bat. In: Happold M. \& Happold D. (eds) Mammals of Africa Volume IV. Hedgehogs, Shrews and Bats: 393-395. Bloomsbury Publishing, London.

Happold M. 2013m. Hipposideros vittatus Striped Leaf-nosed Bat. In: Happold M. \& Happold D. (eds) Mammals of Africa Volume IV. Hedgehogs, Shrews and Bats: 395-398. Bloomsbury Publishing, London.

Happold M. 2013n. Cardioderma cor Heart-nosed Bat (African False Vampire Bat). In: Happold M. \& Happold D. (eds) Mammals of Africa Volume IV. Hedgehogs, Shrews and Bats: 404-405. Bloomsbury Publishing, London.

Happold M. 2013o. Lavia frons Yellow-winged Bat. In: Happold M. \& Happold D. (eds) Mammals of Africa Volume IV. Hedgehogs, Shrews and Bats: 406-408. Bloomsbury Publishing, London.

Happold M. 2013p. Family Emballonuridae Sheath-tailed Bats. In: Happold M. \& Happold D. (eds) Mammals of Africa Volume IV. Hedgehogs, Shrews and Bats: 418-421. Bloomsbury Publishing, London.

Happold M. 2013q. Tadarida brachyptera Short-winged Free-tailed Bat. In: Happold M. \& Happold D. (eds) Mammals of Africa Volume IV. Hedgehogs, Shrews and Bats: 501-502. Bloomsbury Publishing, London.

Happold M. 2013r. Tadarida condylura Angolan Free-tailed Bat. In: Happold M. \& Happold D. (eds) Mammals of Africa Volume IV. Hedgehogs, Shrews and Bats: 505-507. Bloomsbury Publishing, London.

Happold M. 2013s. Tadarida major Lappet-eared Free-tailed Bat. In: Happold M. \& Happold D. (eds) Mammals of Africa Volume IV. Hedgehogs, Shrews and Bats: 516-518. Bloomsbury Publishing, London.

Happold M. 2013t. Tadarida nanula Dwarf Free-tailed Bat. In: Happold M. \& Happold D. (eds) Mammals of Africa Volume IV. Hedgehogs, Shrews and Bats: 520-522. Bloomsbury Publishing, London.

Happold M. 2013u. Tadarida niangarae Niangara Free-tailed Bat. In: Happold M. \& Happold D. (eds) Mammals of Africa Volume IV. Hedgehogs, Shrews and Bats: 522-523. Bloomsbury Publishing, London.

Happold M. 2013v. Tadarida pumila Little Free-tailed Bat. In: Happold M. \& Happold D. (eds) Mammals of Africa Volume IV. Hedgehogs, Shrews and Bats: 528-530. Bloomsbury Publishing, London.

Happold M. 2013w. Tadarida russata Russet Free-tailed Bat. In: Happold M. \& Happold D. (eds) Mammals of Africa Volume IV. Hedgehogs, Shrews and Bats: 530-532. Bloomsbury Publishing, London.

Happold M. 2013x. Tadarida spurrelli Spurrell's Free-tailed Bat. In: Happold M. \& Happold D. (eds) Mammals of Africa Volume IV. Hedgehogs, Shrews and Bats: 532-533. Bloomsbury Publishing, London.

Happold M. 2013y. Tadarida thersites Railer Free-tailed Bat. In: Happold M. \& Happold D. (eds) Mammals of Africa Volume IV. Hedgehogs, Shrews and Bats: 535-537. Bloomsbury Publishing, London. 
Happold M. 2013z. Tadarida trevori Trevor's Free-tailed Bat. In: Happold M. \& Happold D. (eds) Mammals of Africa Volume IV. Hedgehogs, Shrews and Bats: 537-538. Bloomsbury Publishing, London.

Happold M. 2013aa. Glauconycteris alboguttata Striped Butterfly Bat. In: Happold M. \& Happold D. (eds) Mammals of Africa Volume IV. Hedgehogs, Shrews and Bats: 563-564. Bloomsbury Publishing, London.

Happold M. 2013ab. Glauconycteris beatrix Beatrix Butterfly Bat. In: Happold M. \& Happold D. (eds) Mammals of Africa Volume IV. Hedgehogs, Shrews and Bats: 566-567. Bloomsbury Publishing, London.

Happold M. 2013ac. Glauconycteris argentata Common Butterfly Bat. In: Happold M. \& Happold D. (eds) Mammals of Africa Volume IV. Hedgehogs, Shrews and Bats: 564-566. Bloomsbury Publishing, London.

Happold M. 2013ad. Glauconycteris humeralis Spotted Butterfly Bat. In: Happold M. \& Happold D. (eds) Mammals of Africa Volume IV. Hedgehogs, Shrews and Bats: 570-571. Bloomsbury Publishing, London.

Happold M. 2013ae. Glauconycteris poensis Abo Butterfly Bat. In: Happold M. \& Happold D. (eds) Mammals of Africa Volume IV. Hedgehogs, Shrews and Bats: 574-575. Bloomsbury Publishing, London.

Happold M. 2013af. Glauconycteris variegata Variegated Butterfly Bat. In: Happold M. \& Happold D. (eds) Mammals of Africa Volume IV. Hedgehogs, Shrews and Bats: 576-578. Bloomsbury Publishing, London.

Happold M. 2013ag. Nycticeinops schlieffeni Schlieffen's Twilight Bat (Schlieffen's Bat). In: Happold M. \& Happold D. (eds) Mammals of Africa Volume IV. Hedgehogs, Shrews and Bats: 595-597. Bloomsbury Publishing, London.

Happold M. 2013ah. Pipistrellus nanus Banana Pipistrelle (Banana Bat). In: Happold M. \& Happold D. (eds) Mammals of Africa Volume IV. Hedgehogs, Shrews and Bats: 639-642. Bloomsbury Publishing, London.

Happold M. 2013ai. Pipistrellus rueppellii Rüppell's Pipistrelle. In: Happold M. \& Happold D. (eds) Mammals of Africa Volume IV. Hedgehogs, Shrews and Bats: 647-649. Bloomsbury Publishing, London.

Happold M. 2013aj. Scotoecus albofuscus Light-winged Lesser House Bat (Gambian Lesser House Bat). In: Happold M. \& Happold D. (eds) Mammals of Africa Volume IV. Hedgehogs, Shrews and Bats: 667-669. Bloomsbury Publishing, London.

Happold M. 2013ak. Scotoecus hirundo Dark-winged Lesser House Bat (Swallow-like Lesser House Bat). In: Happold M. \& Happold D. (eds) Mammals of Africa Volume IV. Hedgehogs, Shrews and Bats: 669-671. Bloomsbury Publishing, London.

Happold M. 2013al. Scotophilus dinganii Yellow-bellied House Bat. In: Happold M. \& Happold D. (eds) Mammals of Africa Volume IV. Hedgehogs, Shrews and Bats: 674-676. Bloomsbury Publishing, London.

Happold M. 2013am. Scotophilus nigrita Giant House Bat. In: Happold M. \& Happold D. (eds) Mammals of Africa Volume IV. Hedgehogs, Shrews and Bats: 678-679. Bloomsbury Publishing, London.

Happold M. 2013an. Myotis bocagii Rufous Myotis (Rufous Mouse-eared Bat). In: Happold M. \& Happold D. (eds) Mammals of Africa Volume IV. Hedgehogs, Shrews and Bats: 692-694. Bloomsbury Publishing, London.

Happold M. 2013ao. Myotis welwitschii Welwitsch's Myotis. In: Happold M. \& Happold D. (eds) Mammals of Africa Volume IV. Hedgehogs, Shrews and Bats: 708-710. Bloomsbury Publishing, London. 
Happold M. 2013ap. Genus Miniopterus Long-fingered Bats. In: Happold M. \& Happold D. (eds) Mammals of Africa Volume IV. Hedgehogs, Shrews and Bats: 711-712. Bloomsbury Publishing, London.

Happold M. 2013aq. Miniopterus inflatus Greater Long-fingered Bat. In: Happold M. \& Happold D. (eds) Mammals of Africa Volume IV. Hedgehogs, Shrews and Bats: 714-716. Bloomsbury Publishing, London.

Happold M. 2013ar. Miniopterus minor Least Long-fingered Bat. In: Happold M. \& Happold D. (eds) Mammals of Africa Volume IV. Hedgehogs, Shrews and Bats: 716-718. Bloomsbury Publishing, London.

Happold M. 2013as. Phoniscus aerosa Dubious Trumpet-eared Bat. In: Happold M. \& Happold D. (eds) Mammals of Africa Volume IV. Hedgehogs, Shrews and Bats: 734-735. Bloomsbury Publishing, London.

Happold M. \& Cotterill F.P.D. 2013. Tadarida chapini Pale Free-tailed Bat (Chapin's Free-tailed Bat, Long-crested Free-tailed Bat). In: Happold M. \& Happold D. (eds) Mammals of Africa Volume IV. Hedgehogs, Shrews and Bats: 503-504. Bloomsbury Publishing, London.

Happold M. \& Happold D. 2013. Mammals of Africa Volume IV. Hedgehogs, Shrews and Bats. Bloomsbury, London.

Happold M., Van Cakenberghe V. \& Kearney T. 2013. Pipistrellus zuluensis Zulu Pipistrelle (Aloe Bat). In: Happold M. \& Happold D. (eds) Mammals of Africa Volume IV. Hedgehogs, Shrews and Bats: 657-659. Bloomsbury Publishing, London.

Harrison D.L. 1953. Some systematic notes on the long-fingered bats of the genus Miniopterus Bonaparte occurring in South Africa and Madagascar. Durban Museum Novitates 4 (5): 65-75. Available from https://journals.co.za/deliver/fulltext/admn/4/5/1671.pdf?itemId=/content/admn/4/5/AJA0012723X_16 70\&mimeType $=$ pdf\&containerItemId $=$ content/journal/admn [accessed 29 Nov. 2017].

Harrison D.L. 1957. Notes on African bats. 1. Some systematic and anatomical notes on the African bats of the genus Otomops Thomas. Durban Museum Novitates 5 (2): 17-24. Available from https://journals. co.za/deliver/fulltext/admn/5/2/1653.pdf?itemId=/content/admn/5/2/AJA0012723X_1652\&mimeType $=$ pdf $\&$ containerItemId=content/journal/admn [accessed 29 Nov. 2017].

Harrison D.L. 1959. Report on the bats (Chiroptera) in the collection of the National Museum of Southern Rhodesia, Bulawayo. Occasional Papers of the National Museums of Southern Rhodesia 23 B: $217-231$.

Harrison D.L. 1975. A new species of African free-tailed bat (Chiroptera: Molossidae) obtained by the Zaire River Expedition. Mammalia 39 (2): 313-318. https://doi.org/10.1515/mamm.1975.39.2.313

Hassanin A., Khouider S., Gembu G.-C., Goodman S.M., Kadjo B., Nesi N., Pourrut X., Nakouné E. \& Bonillo C. 2015. The comparative phylogeography of fruit bats of the tribe Scotonycterini (Chiroptera, Pteropodidae) reveals cryptic species diversity related to African Pleistocene forest refugia. Comptes Rendus Biologies 338 (3): 197-211. https://doi.org/10.1016/j.crvi.2014.12.003

Hassanin A., Colombo R., Gembu G.-C., Merle M., Tu V.T., Görföl T., Musaba Akawa P., Csorba G., Kearney T., Monadjem A. \& Ing R.K. 2017. Multilocus phylogeny and species delimitation within the genus Glauconycteris (Chiroptera, Vespertilionidae), with the description of a new bat species from the Tshopo Province of the Democratic Republic of the Congo. Journal of Systematics and Evolutionary Research (early view). https://doi.org/10.1111/jzs.12176

Hatt R.T. 1940. Mammals collected by the Rockefeller-Murphy Expedition to Tanganyika and the eastern Belgian Congo. American Museum Novitates 1070: 1-8. Available from http://hdl.handle.net/2246/3757 [accessed 29 Nov. 2017]. 
Hayman D.T.S., Yu M., Crameri G., Wang L.-F., Suu-Ire R., Wood J.L.N. \& Cunningham A.A. 2012. Ebola virus antibodies in fruit bats, Ghana, West Africa. Emerging Infectious Diseases 18 (7): 12071209. https://doi.org/10.3201/eid1807.111654

Hayman R.W. 1935. A note on Hipposideros cyclops Temminck and its synonym Hipposideros langi Allen. Annals and Magazine of Natural History, Ser. 10, 15 (85): 47-50. https://doi.org/10.1080/00222933508654943

Hayman R.W. 1946. A new Scotonycteris, with notes on other Gold Coast bats. Annals and Magazine of Natural History, ser. 11, 12 (95): 766-775. https://doi.org/10.1080/00222934508654781

Hayman R.W. 1954. Notes on some African bats, mainly from the Belgian Congo. Revue de zoologie et de botanique africaines 50 (3-4): 277-295.

Hayman R.W. 1957. Further notes on African Bats. Revue de zoologie et de botanique africaines 56 (1-2): 41-45.

Hayman R.W. 1960. A note on the bat Cloeotis percivali Thomas. Revue de zoologie et de botanique africaines 61 (3-4): 167-172.

Hayman R.W. 1963. Mammals from Angola, mainly from the Luanda District. Publicaçoes Culturais, Companhia de Diamantes Angola, Serviços Culturais 66: 85-138.

Hayman R.W. \& Hill J.E. 1971. Order Chiroptera. In: Meester J. \& Setzer H.W. (eds) The Mammals of Africa, an Identification Manual. Part 2. Smithsonian Institution Press, Washington.

Hayman R.W., Misonne X. \& Verheyen W.N. 1966. The bats of the Congo and of Rwanda and Burundi. Annalen van het Koninklijk Museum voor Midden Afrika, Zoologische Wetenschappen, Ser. 8, 154: $1-105$.

Heller K.G. \& Volleth M. 2016. Note on the echolocation call frequency of Hipposideros beatus Andersen, 1906. African Bat Conservation News 43: 3-4.

Available from https://www.africanbats.org/Documents/ABCN/ABCN_43.pdf [accessed 29 Nov. 2017].

Heller K.G., Volleth M. \& Kock D. 1994. Notes on some vespertilionid bats from the Kivu region, Central Africa (Mammalia: Chiroptera). Senckenbergiana biologica 74 (1/2): 1-8.

Herkt K.M.B., Barnikel G., Skidmore A.K. \& Fahr J. 2015. A high-resolution model of bat diversity and endemism for continental Africa. Ecological Modeling 320: 9-28.

https://doi.org/10.1016/j.ecolmodel.2015.09.009

Herkt K.M.B., Skidmore A.K. \& Fahr J. 2017. Macroecological conclusions based on IUCN expert maps: A call for caution. Global Ecology and Biogeography 26 (8): 930-941. https://doi.org/10.1111/geb.12601

Hijmans R.J., Cameron S.E., Parra J.L., Jones P.G. \& Jarvis A. 2005. Very high resolution interpolated climate surfaces for global land areas. International Journal of Climatology 25 (15): 1965-1978. https://doi.org/10.1002/joc. 1276

Hill J.E. 1965. Asiatic bats of the genera Kerivoula and Phoniscus (Vespertilionidae), with a note on Kerivoula aerosa Tomes. Mammalia 29 (4): 524-556. https://doi.org/10.1515/mamm.1965.29.4.524

Hill J.E. 1974a. A review of Laephotis Thomas, 1901 (Chiroptera: Vespertilionidae). Bulletin of the British Museum (Natural History), Zoology 27 (2): 73-82.

Hill J.E. 1974b. A review of Scotoecus Thomas, 1901 (Chiroptera: Vespertilionidae). Bulletin of the British Museum (Natural History), Zoology 27 (4): 167-188.

Hill J.E. 1977. African bats allied to Kerivoula lanosa (A. Smith, 1847) (Chiroptera: Vespertilionidae). Revue de zoologie africaine 91 (3): 623-633. 
Hill J.E. \& Harrison D.L. 1987. The baculum in the Vespertilioninae (Chiroptera: Vespertilionidae) with a systematic review, a synopsis of Pipistrellus and Eptesicus, and the description of a new genus and subgenus. Bulletin of the British Museum (Natural History), Zoology 52 (7): 225-305.

Holt B.G., Lessard J.-P., Borregaard M.K., Fritz S.A., Araújo M.B., Dimitrov D., Fabre P.-H., Graham C.H., Graves G.R., Jønsson K.A., Nogués-Bravo D., Wang Z., Whittaker R.J., Fjeldså J. \& Rahbek C. 2013. An update of Wallace's zoogeographic regions of the world. Science 339 (6115): 74-78. https://doi.org/10.1126/science.1228282

Hoofer S.R. \& Van Den Bussche R.A. 2003. Molecular phylogenetics of the chiropteran family Vespertilionidae. Acta Chiropterologica 5 (Suppl.): 1-63. https://doi.org/10.3161/001.005.s101

Hoofer S.R., Van Den Bussche R.A. \& Horáček I. 2006. Generic status of the American pipistrelles (Vespertilionidae) with description of a new genus. Journal of Mammalogy 87 (5): 981-992. https://doi.org/10.1644/05-MAMM-A-425R1.1

Huhndorf M. \& Kaleme P. 2008. Chapter 3: Small mammal surveys of Misotshi-Kabogo. In: Plumptre A.J., Kujirakwinja D., Matunguru J., Kahindo C., Kaleme P., Marks B. \& Huhndorf M. (eds) Biodiversity Surveys in the Misotshi-Kabogo and Marungu Regions of Eastern Democratic Republic of Congo with a Focus on Chimpanzees. Albertine Rift Technical Reports 5: 25-28.

Hutcheon J.M. \& Kirsch J.A.W. 2006. A moveable face: deconstructing the Microchiroptera and a new classification of extant bats. Acta Chiropterologica 8 (1): 1-10.

https://doi.org/10.3161/1733-5329(2006)8[1:AMFDTM]2.0.CO;2

Ifuta N.B., Katuala G.B. \& Bola M.L. 1987. Etude du régime alimentaire de quelques espèces des mégachiroptères de la région de Kisangani (Zaïre). Annales de la Faculté des Sciences de l'Université de Kisangani 4: 111-120.

Ing R.K., Colombo R., Gembu G.-C., Bas Y., Julien J.-F., Gager Y. \& Hassanin A. 2016. Echolocation calls and flight behaviour of the elusive Pied Butterfly Bat (Glauconycteris superba), and new data on its morphology and ecology. Acta Chiropterologica 18 (2): 477-488.

https://doi.org/10.3161/15081109ACC2016.18.2.014

Jacobs D.S. 2013. Cloeotis percivali Percival's trident bat (short-eared trident bat). In: Happold M. \& Happold D. (eds) Mammals of Africa Volume IV. Hedgehogs, Shrews and Bats: 365-366. Bloomsbury Publishing, London.

Jobling B. 1954. Streblidae from the Belgian Congo, with a description of a new genus and three new species (Diptera). Revue de zoologie et de botanique africaines 50 (1-2): 89-115.

Johnston H.H. 1884. The River Congo from its Mouth to Bólóbó: with a General Description of the Natural History and Anthropology of its Western Basin. Sampson Low, Marston \& Company, London.

Jones C. 1971. The bats of Rio Muni, West Africa. Journal of Mammalogy 52 (1): 121-140. Available from https://www.jstor.org/stable/1378437?seq=1\#page_scan_tab_contents [accessed 29 Nov. 2017].

Juste B.J., Álvarez Y., Tabarés E., Garrido-Pertierra A., Ibáñez C. \& Bautista J.M. 1999. Phylogeography of African fruitbats (Megachiroptera). Molecular Phylogenetics and Evolution 13 (3): 596-604.

https://doi.org/10.1006/mpev.1999.0669

Juste J., Ferrández A., Fa J.E., Masefield W. \& Ibañez C. 2007. Taxonomy of little bent-winged bats (Miniopterus, Miniopteridae) from the African islands of São Tomé, Grand Comoro and Madagascar, based on mtDNA. Acta Chiropterologica 9 (1): 27-37.

https://doi.org/10.3161/1733-5329(2007)9[27:TOLBBM]2.0.CO;2 
Kearney T.C. 2013a. Laephotis botswanae Botswanan Long-eared Bat. In: Happold M. \& Happold D. (eds) Mammals of Africa Volume IV. Hedgehogs, Shrews and Bats: 581-582. Bloomsbury Publishing, London.

Kearney T.C. 2013b. Pipistrellus anchietae Anchieta's Pipistrelle. In: Happold M. \& Happold D. (eds) Mammals of Africa Volume IV. Hedgehogs, Shrews and Bats: 610-611. Bloomsbury Publishing, London.

Kearney T.C. 2013c. Pipistrellus capensis Cape Pipistrelle. In: Happold M. \& Happold D. (eds) Mammals of Africa Volume IV. Hedgehogs, Shrews and Bats: 614-617. Bloomsbury Publishing, London.

Kearney T.C. 2013d. Pipistrellus hesperidus Dusk Pipistrelle. In: Happold M. \& Happold D. (eds) Mammals of Africa Volume IV. Hedgehogs, Shrews and Bats: 629-631. Bloomsbury Publishing, London.

Kearney T.C. \& Seamark E.C.J. 2005. Morphometric analysis of cranial and external characters of Laephotis Thomas, 1901 (Mammalia: Chiroptera: Vespertilionidae) from southern Africa. Annals of the Transvaal Museum 42: 71-87. Available from https://journals.co.za/deliver/fulltext/nfi_annalstm/42/1/ nfi_annalstm_v42_a6.pdf?itemId=/content/nfi_annalstm/42/1/EJC83631\&mimeType=pdf\&containerIt emId=content/journal/nfi_annalstm [accessed 29 Nov. 2017].

Kearney T.C., Volleth M., Contrafatto G. \& Taylor P.J. 2002. Systematic implications of chromosome GTC-band and bacula morphology for Southern African Eptesicus and Pipistrellus and several other species of Vespertilioninae (Chiroptera: Vespertilionidae). Acta Chiropterologica 4 (1): 55-76. https://doi.org/10.3161/001.004.0107

Kerbis Peterhans J.C., Fahr J., Huhndorf M.H., Kaleme P., Plumptre A.J., Marks B.D. \& Kizungu R. 2013. Bats (Chiroptera) from the Albertine Rift, eastern Democratic Republic of Congo, with the description of two new species of the Rhinolophus maclaudi group. Bonner Zoological Bulletin 62 (2): 186-202. Available from http://www.zoologicalbulletin.de/BzB_Volumes/Volume_62_2/186_202_ BzB62_2_Kerbis_JC_et_al.pdf [accessed 29 Nov. 2017].

Kershaw P.S. 1922. On a collection of mammals from Chiromo and Cholo, Ruo, Nyasaland, made by Mr. Rodney C. Wood, with field-notes by the collector. Annals and Magazine of Natural History, Ser. 9, 10 (56): 177-192. https://doi.org/10.1080/00222932308632761

Kershaw P.S. 1923. Notes on mammals collected by Dr. H. Schouteden in the Belgian Congo. Revue de zoologie et de botanique africaines 11 (4): 355-368. Available from http://biostor.org/reference/102355 [accessed 29 Nov. 2017].

Kingdon J. 1974. East African Mammals. An Atlas of Evolution in Africa. Volume II. Part A. (Insectivores and Bats). Academic Press, New York \& London.

Kipalu P. 2009. Ecology, Distribution, Status and Protection of Three Congolese Fruit Bats, DRC (2005). Final Report. BP Conservation Programme (CLP) 2005. Project No. 101405: 1-21. Available from http://www.conservationleadershipprogramme.org/project/ecology-distribution-status-protectionthree-congolese-fruit-bats/ [accessed 29 Nov. 2017].

Kityo R., Kerbis Peterhans J., Hundorf M. \& Hutterer R. 2009. Appendix 3. New additions and noteworthy records of the bat fauna of Uganda, Rwanda and the Democratic Republic of Congo. In: Thorn E. \& Kerbis Peterhans J. (eds) Small Mammals of Uganda. Bonner zoologische Monographien 55: 127-140. Available from http://www.zoologicalbulletin.de/BzB_Volumes/BzM_55/BZM_55_small.pdf [accessed 29 Nov. 2017].

Kock D. 1967. Ein Neunachweis von Myotis welwitschi und der Status von Myotis venustus (Mammalia, Chiroptera). Senckenbergiana Biologica 48 (5/6): 319-325.

Kock D. 1969. Die Fledermaus-Fauna des Sudan (Mammalia, Chiroptera). Abhandlungen der Senckenbergischen naturforschenden Gesellschaft 521: 1-238. 
Kock D. 1975. Ein Originalexemplar von Nyctinomus ventralis Heuglin 1861 (Mammalia: Chiroptera: Molossidae). Stuttgarter Beiträge zur Naturkunde (A) 272: 1-9. Available from https://www.biodiversitylibrary.org/page/33621617\#page/129/mode/1 up [accessed 29 Nov. 2017].

Kock D. 1978. Vergleichende Untersuchung einiger Säugetiere im südlichen Niger (Mammalia: Insectivora, Chiroptera, Lagomorpha, Rodentia). Senckenbergiana Biologica 58 (3/4): 113-136.

Kock D. 1981a. Zwei Fledermäuse neu für Kenya (Mammalia: Chiroptera). Senckenbergiana Biologica $61(5 / 6): 321-327$.

Kock D. 1981b. Zur Chiropteren-Fauna von Burundi (Mammalia). Senckenbergiana Biologica 61 (5/6): 329-336.

Kock D. 1987. Micropteropus intermedius Hayman 1936 und andere Fledermäuse vom unteren Zaïre. Senckenbergiana Biologica 67 (4/6): 219-224.

Kock D. 2001. Identity of the African Vespertilio hesperida Temminck 1840 (Mammalia, Chiroptera, Vespertilionidae). Senckenbergiana Biologica 81 (1/2): 277-283.

Kock D. \& Howell K.M. 1988. Three bats new for mainland Tanzania (Mammalia: Chiroptera). Senckenbergiana Biologica 68 (4/6): 223-239.

Kock D., Barnett L., Fahr J. \& Emms C. 2002. On a collection of bats (Mammalia: Chiroptera) from the Gambia. Acta Chiropterologica 4 (1): 77-97. https://doi.org/10.3161/001.004.0108

Konaté S. \& Linsenmaier K.E. 2010. Biological diversity of West Africa: Importance, threats and valorization. In: Konaté S. \& Kampmann D. (eds) Biodiversity Atlas of West Africa, Volume III: Côte d'Ivoire: 14-32. BIOTA, Abidjan and Frankfurt/Main.

Available from https://www.uni-frankfurt.de/47622126/BIOTA-Atlas-CI [accessed 29 Nov. 2017].

Koopman K.F. 1965. Status of forms described or recorded by J.A. Allen in the "American Museum Congo Expedition of Bats". American Museum Novitates 2219: 1-34.

Available from http://hdl.handle.net/2246/4012 [accessed 29 Nov. 2017].

Koopman K.F. 1966. Taxonomic and distributional notes on Southern African bats. The Puku 4: 155165.

Koopman K.F. 1971. Taxonomic notes on Chalinolobus and Glauconycteris (Chiroptera, Vespertilionidae). American Museum Novitates 2451: 1-10. Available from http://hdl.handle.net/2246/2660 [accessed 29 Nov. 2017].

Koopman K.F. 1975. Bats of the Sudan. Bulletin of the American Museum of Natural History 154 (4): 353-444. Available from http://hdl.handle.net/2246/609 [accessed 29 Nov. 2017].

Koopman K.F. 1989. Systematic notes on Liberian bats. American Museum Novitates 2946: 1-11. Available from http://hdl.handle.net/2246/5100 [accessed 29 Nov. 2017].

Koopman K.F. 1993. Order Chiroptera. In: Wilson D.E. \& Reeder D.M. (eds) Mammal Species of the World: a Taxonomic and Geographic Reference. Second edition: 137-241. Smithsonian Institution Press, Washington, D.C. and London.

Koopman K.F. 1994. Chiroptera: Systematics. In: Niethammer J., Schliemann H. \& Starck D. (eds) Handbook of Zoology 8 (Mammalia) (60). W. De Gruyter, Berlin and New York.

Koubínová D., Irwin N., Hulva P., Koubek P. \& Zima J. 2013. Hidden diversity in Senegalese bats and associated findings in the systematics of the family Vespertilionidae. Frontiers in Zoology 10 (1): 48. https://doi.org/10.1186/1742-9994-10-48 
Kremen C., Cameron A., Moilanen A., Phillips S.J., Thomas C.D., Beentje H., Dransfield J., Fisher B.L., Glaw F., Good T.C., Harper G.J., Hijmans R.J., Lees D.C., Louis E. Jr, Nussbaum R.A., Raxworthy C.J., Razafimpahanana A., Schatz G.E., Vences M., Vieites D.R., Wright P.C. \& Zjhra M.L. 2008. Aligning conservation priorities across taxa in Madagascar with high-resolution planning tools. Science 320 (5873): 222-226. https://doi.org/10.1126/science.1155193

Krampitz H.E. \& Anciaux de Faveaux F. 1960. Ueber einige Haemosporidien aus Fledermäusen der Höhlen des Berglandes von Katanga. Zeitschrift für Tropenmedizin und Parasitologie 11 (4): 391-400.

Lack J.B., Roehrs Z.P., Stanley C.E. Jr, Ruedi M. \& Van den Bussche R.A. 2010. Molecular phylogenetics of Myotis indicate familial-level divergence for the genus Cistugo (Chiroptera). Journal of Mammalogy 91 (4): 976-992. https://doi.org/10.1644/09-MAMM-A-192.1

Lanza B., Funaioli U. \& Riccucci M. 2015. The Bats of Somalia and Neighbouring Areas. Editions Chimaira, Frankfurt am Main.

Laumanns M., Schmassmann S. \& Adrian H. 2004. The caves of Rwanda - Les grottes du Rwanda. Berliner höhlenkundliche Berichte 11: 1-89.

Lavrenchenko L.A., Kruskop S.V. \& Morozov P.N. 2004. Notes on the bats (Chiroptera) collected by the joint Ethiopian-Russian biological expedition, with remarks on their systematics, distribution, and ecology. Bonner zoologische Beiträge 52 (1/2): 127-147. Available from http://www.zoologicalbulletin. de/BzB_Volumes/Volume_52_1_2/127_147_BZB52_1_2_Lavrenchenko_Leonid_et_al.PDF [accessed 29 Nov. 2017].

Lawrence B. \& Novick A. 1963. Behavior as a taxonomic clue: relationships of Lissonycteris (Chiroptera). Breviora 184: 1-16.

Available from https://www.biodiversitylibrary.org/page/4312244\#page/63/mode/1up [accessed 29 Nov. 2017].

Leendertz S.A.J. 2016. Testing new hypotheses regarding ebolavirus reservoirs. Viruses 8 (2): e30. https://doi.org/10.3390/v8020030

Leirs H., Mills J.N., Krebs J.W., Childs J.E., Akaibe D., Woollen N., Ludwig G., Peters C.J., Ksiazek T.G. \& other study group members. 1999. Search for the Ebola virus reservoir in Kikwit, Democratic Republic of the Congo: Reflections on a vertebrate collection. Journal of Infectious Diseases 179 (2 Suppl 1): 155-163. https://doi.org/10.1086/514299

Leleup N. 1956. La faune cavernicole du Congo belge et considérations sur les coléoptères reliques d'Afrique intertropicale. Annalen van het Koninklijk Museum voor Midden Afrika, Zoologische Wetenschappen Serie 8 46: 1-171.

Leroy E.M., Kumulungui B., Pourrut X., Rouquet P., Hassanin A., Yaba P., Délicat A., Paweska J.T., Gonzalez J.-P. \& Swanepoel R. 2005. Fruit bats as reservoirs of Ebola virus. Nature 438 (7068): 575576. https://doi.org/10.1038/438575a

Leroy E.M., Epelboin A., Mondonge V., Pourrut X., Gonzalez J.-P., Muyembe-Tamfum J.-J. \& Formenty P. 2009. Human Ebola outbreak resulting from direct exposure to fruit bats in Luebo, Democratic Republic of Congo, 2007. Vector-Borne and Zoonotic Diseases 9 (6): 723-728.

https://doi.org/10.1089/vbz.2008.0167

McWilliam A.N. 1982. Adaptive Responses to Seasonality in Four Species of Microchiroptera in Coastal Kenya. $\mathrm{PhD}$ thesis, University of Aberdeen, UK. Available from http://aulib.abdn.ac.uk/F/?func=findb\&find_code $=$ SYS\&request $=001620585$ [accessed 29 Nov. 2017].

Mein P. \& Tupinier Y. 1977. Formule dentaire et position systématique du minioptère (Mammalia, Chiroptera). Mammalia 41 (2): 207-211. https://doi.org/10.1515/mamm.1977.41.2.207 
VAN CAKENBERGHE V. et al., The bats of Congo, Rwanda and Burundi revisited

Meirte D. 1983. New data on Casinycteris argyinnis Thomas 1910 (Megachiroptera, Pteropidae). Annalen van het Koninklijk Museum voor Midden Afrika, Zoologische Wetenschappen 237: 9-12.

Menu H. 1987. Morphotypes dentaires actuels et fossiles des chiroptères vespertilioninés. 2ième partie: Implications systematiques et phylogeniques. Palaeovertebrata 17 (3): 77-150. Available from http://palaeovertebrata.com/Articles/sendFile/274/published_article [accessed 29 Nov. 2017].

Mertens R. 1925. Verzeichnis der Säugetier-Typen des Senckenbergischen Museums. Senckenbergiana Biologica 7 (1/2): 18-37.

Meyer C.F.J., Aguiar L.M.S., Aguirre L.F., Baumgarten J., Clarke F.M., Cosson J.-F., Estrada Villegas S., Fahr J., Faria D., Furey N., Henry M., Jenkins R.K.B., Kunz T.H., MacSwiney González M.C., Moya I., Pons J.-M., Racey P.A., Rex K., Sampaio E.M., Stoner K.E., Voigt C.C., von Staden D., Weise C.D. \& Kalko E.K.V. 2014. Species undersampling in tropical bat surveys: effects on emerging biodiversity patterns. Journal of Animal Ecology 84 (1): 113-123. https://doi.org/10.1111/1365-2656.12261

Miller-Butterworth C.M., Murphy W.J., O’Brien S.J., Jacobs D.S., Springer D.S. \& Teeling E.C. 2007. A family matter: Conclusive resolution of the taxonomic position of the long-fingered bats, Miniopterus. Molecular Biology and Evolution 24 (7): 1553-1561. https://doi.org/10.1093/molbev/msm076

Monadjem A., Schoeman M.C., Reside A., Pio D.V., Stoffberg S., Bayliss J., Cotterill F.P.D. Curran M., Kopp M. \& Taylor P.J. 2010a. A recent inventory of the bats of Mozambique with documentation of seven new species for the country. Acta Chiropterologica 12 (2): 371-391. https://doi.org/10.3161/150811010X537963

Monadjem A., Taylor P., Cotterill F.P.D. \& Schoeman M.C. 2010b. Bats of Southern and Central Africa. A biogeographic and Taxonomic Synthesis. Wits University Press, Johannesburg.

Monadjem A., Goodman S.M., Stanley W.T. \& Appleton B. 2013. A cryptic new species of Miniopterus from south-eastern Africa based on molecular and morphological characters. Zootaxa 3746 (1): 123142. https://doi.org/10.11646/zootaxa.3746.1.5

Monfort A. 1992. Première liste commentée des mammifères du Rwanda. Revue de zoologie africaine 106 (2): 141-151.

Nesi N. 2012. Systématique et Phylogéographie des Chauves-Souris africaines de la Sous-Famille des Epomophorinae (Chiroptera, Pteropodidae). PhD Thesis, Muséum national d'Histoire naturelle, Paris.

Nesi N., Nakouné E., Cruaud C. \& Hassanin A. 2011. DNA barcoding of African fruit bats (Mammalia, Pteropodidae). The mitochondrial genome does not provide a reliable discrimination between Epomophorus gambianus and Micropteropus pusillus. Comptes Rendus Biologies 334 (7): 544-554. https://doi.org/10.1016/j.crvi.2011.05.003

Nesi N., Kadjo B., Pourrut X., Leroy E., Pongombo Shongo C., Cruaud C. \& Hassanin A. 2012. Molecular systematics and phylogeography of the tribe Myonycterini (Mammalia, Pteropodidae) inferred from mitochondrial and nuclear markers. Molecular Phylogenetics and Evolution 66 (1): 126-137 (for 2013). https://doi.org/10.1016/j.ympev.2012.09.028

Newman S.H., Field H., Epstein J. \& de Jong C. (eds) 2011. Investigating the Role of Bats in Emerging Zoonoses: Balancing Ecology, Conservation and Public Health Interest. FAO Animal Production and Health Manual, 12. Food and Agriculture Organization of the United Nations, Rome. Available from http://www.fao.org/docrep/014/i2407e/i2407e00.pdf [accessed 29 Nov. 2017].

Niort P.-L. 1970. Contribution à la connaissance des chiroptères du Burundi (Afrique Centrale). Annalen van de Koninklijke Vereniging voor Dierkunde 100: 247-274.

Noack T. 1887. Beiträge zur Kenntnis der Säugethier-Fauna von Ost- und Zentral-Afrika. Nach Sammlungen und Aufzeichnungen des verstorbenen Dr. Richard Böhm. Zoologische Jahrbücher 2 (2): 193-302. 
Noack T. 1889. Beiträge zur Kenntnis der Säugethierfauna von Süd- und Südwest-Afrika. Zoologische Jahrbücher, Abteilung für Systematik 4: 94-261.

Novick A. 1958. Orientation in paleotropical bats II. Megachiroptera. Journal of Experimental Zoology 137 (3): 443-461. https://doi.org/10.1002/jez.1401370305

Omatsu T., Bak E.-J., Ishii Y., Kyuwa S., Tohya Y., Akashi H. \& Yoshikawa Y. 2008. Induction and sequencing of Rousette bat interferon $\alpha$ and $\beta$ genes. Veterinary Immunology and Immunopathology 124 (1-2): 169-176. https://doi.org/10.1016/j.vetimm.2008.03.004

Osborn H.F. 1919. The Congo expedition of the American Museum of Natural History. Bulletin of the American Museum of Natural History 39: xv-xxviii. https://doi.org/10.5962/bhl.title.53716

Ossa Gómez G. 2012. Establishing an isotopic basemap for flying foxes in Central Africa to assess the movement ecology of the straw-coloured fruit bat Eidolon helvum (Kerr, 1792). Master's Thesis, Natural Heritage and Society (MNHN Paris) and Leibniz Institute for Zoo and Wildlife Research, Berlin.

Patterson B.D. 2002. On the continuing need for scientific collecting of mammals. Mastozoología Neotropical 9 (2): 253-262. Available from http://www.sarem.org.ar/wp-content/uploads/2012/11/ SAREM_MastNeotrop_9-2_09_Patterson.pdf [accessed 29 Nov. 2017].

Peterson R.L. 1971a. Notes on the African long-eared bats of the genus Laephotis (family Vespertilionidae). Canadian Journal of Zoology 49 (6): 885-888. https://doi.org/10.1139/z71-133

Peterson R.L. 1971b. The systematic status of the African molossid bats Tadarida bemmeleni and Tadarida cistura. Canadian Journal of Zoology 49 (10): 1347-1354. https://doi.org/10.1139/z71-201

Peterson R.L. 1972. Systematic status of the African molossid bats Tadarida congica, T. niangarae and T. trevori. Life Science Contributions, Royal Ontario Museum 85: 1-32.

https://doi.org/10.5962/bhl.title.52087

Peterson R.L., Eger J.L. \& Mitchell L. 1995. Chiroptères. Faune de Madagascar 84: 1-204.

Phillips S.J., Anderson R.P. \& Schapire R.E. 2006. Maximum entropy modeling of species geographic distributions.EcologicalModelling 190(3-4):231-259.https://doi.org/10.1016/j.ecolmodel.2005.03.026

Ralph T.M.C., Richards L.R., Taylor P.J., Napier M.C. \& Lamb J.M. 2015. Revision of Afro-Malagasy Otomops (Chiroptera: Molossidae) with the description of a new Afro-Arabian species. Zootaxa 4057 (1): 1-49. https://doi.org/10.11646/zootaxa.4057.1.1

Rahm U. 1966. Les mammifères de la forêt équatoriale de l'est du Congo. Annalen van het Koninklijk Museum voor Midden Afrika, Zoologische Wetenschappen, ser. 8 149: 39-121.

Ranjan K., Prasad M. \& Prasad G. 2016. Bats: carriers of zoonotic viral and emerging infectious diseases. Journal of Experimental Biology and Agricultural Sciences 4 (3S): 291-306.

https://doi.org/10.18006/2016.4(3S).291.306

Reeder D.M., Helgen K.M., Vodzak M.E., Lunde D.P. \& Ejotre I. 2013. A new genus for a rare African vespertilionid bat: insights from South Sudan. ZooKeys 285: 89-115.

https://doi.org/10.3897/zookeys.285.4892

Robbins C. 1983. A new high forest species in the African genus Scotophilus (Vespertilionidae). Annalen van het Koninklijk Museum voor Midden Afrika, Zoologische Wetenschappen 237: 19-24.

Robbins C.B., De Vree F. \& Van Cakenberghe V. 1985. A systematic revision of the African bat genus Scotophilus (Vespertilionidae). Annalen van het Koninklijk Museum voor Midden Afrika, Zoologische Wetenschappen 246: 51-84. Available from https://www.academia.edu/4426030/A_systematic_ revision_of_the_African_bat_genus_Scotophilus_Vespertilionidae_[accessed 29 Nov. 2017]. 
Rocha L.A., Aleixo A., Allen G., Almeda F., Baldwin C.C., Barclay M.V.L., Bates J.M., Bauer M., Benzoni F., Berns C.M., Berumen M.L., Blackburn D.C., Blum S., Bolaños F., Bowie R.C.K., Britz R., Brown R.M., Cadena C.D., Carpenter K., Ceríaco L.M., Chakrabarty P., Chaves G., Choat J.H., Clements K.D., Collette B.B., Collins A., Coyne J., Cracraft J., Daniel T., de Carvalho M.R., de Queiroz K., Di Dario F., Drewes R., Dumbacher J.P., Engilis A.Jr., Erdmann M.V., Eschmeyer W., Feldman C.R., Fisher B.L., Fjeldså J., Fritsch P.W., Fuchs J., Getahun A., Gill A., Gomon M., Gosliner T., Graves G.R., Griswold C.E., Guralnick R., Hartel K., Helgen K.M., Ho H., Iskandar D.T., Iwamoto T., Jaafar Z., James H.F., Johnson D., Kavanaugh D., Knowlton N., Lacey E., Larson H.K., Last P., Leis J.M., Lessios H., Liebherr J., Lowman M., Mahler D.L., Mamonekene V., Matsuura K., Mayer G.C., Mays H. Jr, McCosker J., McDiarmid R.W., McGuire J., Miller M.J., Mooi R., Mooi R.D., Moritz C., Myers P., Nachman W., Nussbaum R.A., Ó Foighil D., Parenti L.R., Parham J. F., Paul E., Paulay G., Pérez-Emán J., Pérez-Matus A., Poe S., Pogonoski J., Rabosky D.L., Randall J.E., Reimer J.D., Robertson D.R., Rödel M.-O., Rodrigues M.T., Roopnarine P., Rüber L., Ryan M.J., Sheldon F., Shinohara G., Short A., Simison W.B., Smith-Vaniz W.F., Springer V.G., Stiassny M., Tello J.G., Thompson C.W., Trnski T., Tucker P., Valqui T., Vecchione M., Verheyen E., Wainwright P.C., Wheeler T.A., White W.T., Will K., Williams J.T., Williams G., Wilson E.O., Winker K., Winterbottom R. \& Witt C.C. 2014. Specimen collection: An essential tool. Science 344 (6186): 814-815. https://doi.org/10.1126/science.344.6186.814

Roehrs Z.P. 2009. Vespertilioninae Systematics: using Mitochondrial and Nuclear Markers to Elucidate Phylogenetic Relationships. PhD Thesis, Oklahoma State University.

Roehrs Z.P., Lack J.B. \& Van den Bussche R.A. 2010. Tribal phylogenetic relationships within Vespertilioninae (Chiroptera: Vespertilionidae) based on mitochondrial and nuclear sequence data. Journal of Mammalogy 91 (5): 1073-1092. https://doi.org/10.1644/09-MAMM-A-325.1

Rosevear D.R. 1965. The Bats of West Africa. Trustees of the British Museum (Natural History), London.

Ruedas L.A., Salazar-Bravo J., Dragoo J.W. \& Yates T.L. 2000. The importance of being earnest: what, if anything, constitutes a "specimen examined?" Molecular Phylogenetics and Evolution 17 (1): 129132. https://doi.org/10.1006/mpev.2000.0737

Sanborn C.C. 1936. Descriptions and records of African bats. Publications of the Field Museum of Natural History (Zoology) 20 (14): 107-114. https://doi.org/10.5962/bhl.title.3213

Sayre R., Comer P., Hak J., Josse C., Bow J., Warner H., Larwanou M., Kelbessa E., Bekele T., Kehl H., Amena R., Andriamasimanana R., Ba T., Benson L., Boucher T., Brown M., Cress J., Dassering O., Friesen B., Gachathi F., Houcine S., Keita M., Khamala E., Marangu D., Mokua F., Morou B., Mucina L., Mugisha S., Mwavu E., Rutherford M., Sanou P., Syampungani S., Tomor B., Vall A., Vande Weghe J., Wangui E. \& Waruingi L. 2013. A new Map of Standardized Terrestrial Ecosystems of Africa: 1-24. Association of American Geographers, Washington DC.

Schaer J., Perkins S.L., Decher J., Leendertz F.H., Fahr J., Weber N. \& Matuschewski K. 2013. High diversity of West African bat malaria parasites and a tight link with rodent Plasmodium taxa. Proceedings of the National Academy of Sciences 110 (43): 17415-17419. https://doi.org/10.1073/pnas.1311016110

Schneeberger K. \& Voigt C.C. 2015. Zoonotic viruses and conservation of bats. In: Voigt C.C., Kingston T. (eds) Bats in the Anthropocene: Conservation of Bats in a Changing World: 263-292. Springer Open, Cham, Heidelberg, New York, Dordrecht and London. https://doi.org/10.1007/978-3-319-25220-9_10

Schouteden H. 1934. Les mammifères du secteur méridional du Parc National Albert (Kivu). Revue de zoologie et de botanique africaines 25: 291-304.

Schouteden H. 1935. Les mammifères du secteur septentrional du Parc National Albert (Kivu). Revue de zoologie et de botanique africaines 26: 202-210. 
Schouteden H. 1943. Catalogue des mammifères du Congo belge et du Ruanda-Urundi. Revue de zoologie et de botanique africaines 37: 102-125.

Schouteden H. 1947. De Zoogdieren van Belgisch Congo en van Ruanda-Urundi. Annalen van het Koninklijk Museum voor Midden Afrika, Zoologische Wetenschappen (2), 3: 1-576.

Schouteden H. 1948. Faune du Congo Belge et du Ruanda-Urundi. I. Mammifères. Annalen van het Koninklijk Museum voor Midden Afrika, Zoologische Wetenschappen, Ser. 8, 1: 1-331.

Schwarz E. 1920. Fledermäuse aus West- und Zentralafrika. Ergebnisse der zweiten Deutschen ZentralAfrika-Expedition 1910-1911 unter Führung Adolf Friedrichs, Herzogs zu Mecklenburg. Klinkhardt \& Biermann, Leipzig. Available from https://www.biodiversitylibrary.org/bibliography/6486\#/summary [accessed 29 Nov. 2017].

Setzer H.W. 1971. New bats of the genus Laephotis from Africa (Mammalia: Chiroptera). Proceedings of the Biological Society of Washington 84 (32): 259-264. Available from https://www.biodiversitylibrary. org/page/34563319\#page/299/mode/1up [accessed 29 Nov. 2017].

Simmons N.B. 2005. Order Chiroptera. In: Wilson D.E. \& Reeder D.M. (eds) Mammal Species of the World: A Taxonomic and Geographic Reference: 312-567. The John Hopkins University Press, Baltimore.

Smith J.D. \& Hood C.S. 1980. Additional material of Rhinolophus ruwenzorii Hill, 1942, with comments on its natural history and taxonomic status. In: Wilson D.E. \& Gardner A.L. (eds) Proceedings of the Fifth International Bat Research Conference: 163-171. Texas Press, Lubbock, Texas.

Soisook P., Prajakjitr A., Karapan S., Francis C.M. \& Bates P.J.J. 2015. A new genus and species of false vampire (Chiroptera: Megadermatidae) from peninsular Thailand. Zootaxa 3931 (4): 528-550. https://doi.org/10.11646/zootaxa.3931.4.4

Stanley W.T., Kerbis Peterhans J.C., Kityo R.M. \& Davenport L. 1996. New records of bats for Uganda and Burundi. African Journal of Ecology 34 (2): 196-201.

https://doi.org/10.1111/j.1365-2028.1996.007-89007.x

Stoffberg S., Jacobs D.S. \& Miller-Butterworth C.M. 2004. Field identification of two morphologically similar bats, Miniopterus schreibersii natalensis and Miniopterus fraterculus (Chiroptera: Vespertilionidae). African Zoology 39 (1): 47-53. https://doi.org/10.1080/15627020.2004.11407285

Stoffberg S., Schoeman M.C. \& Matthee C.A. 2012. Correlated genetic and ecological diversification in a widespread southern African horseshoe bat. PLoS One 7 (2) e31946.

https://doi.org/10.1371/journal.pone.0031946

Taylor P.J. 2013. Myonycteris relicta Bergmans's Collared Fruit Bat. In: Happold M. \& Happold D. (eds) Mammals of Africa Volume IV. Hedgehogs, Shrews and Bats: 273-274. Bloomsbury Publishing, London.

Taylor P.J. \& Van der Merwe M. 1998. Taxonomic notes on dark-winged house bats of the genus Scotoecus Thomas 1901, in Malawi. Durban Museum Novitates 23: 64-66. Available from http://journals.co.za/deliver/fulltext/admn/23/1/1578.pdf?itemId=/content/admn/23/1/AJA0012723X_ 1577\&mimeType $=$ pdf\&containerItemId=content/journal/admn [accessed 29 Nov. 2017].

Thomas D. \& Henry M. 2013a. Eidolon helvum African Straw-coloured Fruit Bat. In: Happold M. \& Happold D. (eds) Mammals of Africa Volume IV. Hedgehogs, Shrews and Bats: 232-234. Bloomsbury Publishing, London.

Thomas D. \& Henry M. 2013b. Micropteropus pusillus Peters's Lesser Epauletted Fruit Bat. In: Happold M. \& Happold D. (eds) Mammals of Africa Volume IV. Hedgehogs, Shrews and Bats: 270-272. Bloomsbury Publishing, London. 
Thomas D. \& Henry M. 2013c. Myonycteris torquata Little Collared Fruit Bat. In: Happold M. \& Happold D. (eds) Mammals of Africa Volume IV. Hedgehogs, Shrews and Bats: 275-276. Bloomsbury Publishing, London.

Thomas O. 1903. Three new bats from the Cameroons, discovered by Mr. G.L. Bates. Annals and Magazine of Natural History, Ser. 7, 12 (72): 633-635. https://doi.org/10.1080/00222930309487045

Thomas O. 1915. List of mammals (exclusive of Ungulata) collected on the Upper Congo by Dr. Christy for the Congo Museum, Tervueren. Annals and Magazine of Natural History, Ser. 8, 16 (96): 465-481. Available from https://www.biodiversitylibrary.org/page/22131562\#page/477/mode/1up [accessed 29 Nov. 2017].

Thorn E., Kock D. \& Cuisin J. 2007. Status of the African bats Vesperugo grandidieri Dobson 1876 and Vesperugo flavescens Seabra 1900 (Chiroptera, Vespertilionidae), with description of a new subgenus. Mammalia 71 (1/2): 70-79. https://doi.org/10.1515/MAMM.2007.013

Thorn E., Kerbis Peterhans J. \& Baranga J. 2009. 2. Chiroptera. In: Thorn E. \& Kerbis Peterhans J. (eds) Small Mammals of Uganda: Bats, Shrews, Hedgehog, Golden-moles, Otter-tenrec, Elephant-Shrews, and Hares. Bonner zoologische Monographien 55: 12-75. Available from http://www.zoologicalbulletin.de/BzB_Volumes/BzM_55/BZM_55_small.pdf [accessed 29 Nov. 2017].

Tsang S.M., Cirranello A.L., Bates P.J.J. \& Simmons N.B. 2015. The roles of taxonomy and systematics in bat conservation. In: Voigt C.C. \& Kingston T. (eds) Bats in the Anthropocene: Conservation of Bats in a Changing World: 503-538. Springer Open, Cham, Heidelberg, New York, Dordrecht and London. https://doi.org/10.1007/978-3-319-25220-9_16

Turni H. \& Kock D. 2008. Type specimens of bats (Chiroptera: Mammalia) in the collections of the Museum für Naturkunde, Berlin. Zootaxa 1869: 1-82.

Available from http://www.mapress.com/j/zt/article/view/5177 [accessed 29 Nov. 2017].

Uchikawa K. 1985. Mites of the genus Calcarmyobia (Acarina, Myobiidae) with information on the taxonomy of their host bats of the genus Miniopterus (Chiroptera, Miniopteridae). Bulletin of the British Museum (Natural History), Zoology 48 (1): 15-25. https://doi.org/10.5962/bhl.part.23459

Uchikawa W. \& Kock D. 1989. Revision of the genera Hipposiderobia, Triaenomyobia, and Binunculoides (Acarina: Myobiidae) associated mainly with the chiropteran family Hipposideridae (Mammalia). Journal of Parasitology 75 (6): 875-891.

Vallo P., Guillen-Servent A., Benda P., Pires D.B. \& Koubek P. 2009. Variation of mitochondrial DNA in the Hipposideros caffer complex (Chiroptera: Hipposideridae) and its taxonomic implications. Acta Chiropterologica 10 (2): 193-206. https://doi.org/10.3161/150811008X414782

Vallo P., Benda P. \& Reiter A. 2011. Yellow-bellied or white-bellied? Identity of Arabian house bats (Vespertilionidae: Scotophilus) revealed from mitochondrial DNA and morphology. African Zoology 46 (2): 350-361. https://doi.org/10.1080/15627020.2011.11407508

Van Cakenberghe V. \& De Vree F. 1985. Systematics of African Nycteris (Mammalia: Chiroptera). Proceedings of the International Symposium on African Vertebrates, Bonn: 53-90.

Van Cakenberghe V. \& De Vree F. 1993. Systematics of African Nycteris (Mammalia: Chiroptera). Part II. The Nycteris hispida group. Bonner zoologische Beiträge 44 (3/4): 299-332.

Van Cakenberghe V. \& De Vree F. 1999. Systematics of African Nycteris (Mammalia: Chiroptera). Part III. The Nyteris thebaica group. Bonner zoologische Beiträge 48 (for 1998) (2): 123-166.

Van Cakenberghe V. \& Happold M. 2013a. Genus Pipistrellus Pipistrelles. In: Happold M. \& Happold D. (eds) Mammals of Africa Volume IV. Hedgehogs, Shrews and Bats: 600-604. Bloomsbury Publishing, London. 
Van Cakenberghe V. \& Happold M. 2013b. Pipistrellus eisentrauti Eisentraut's Pipistrelle. In: Happold M. \& Happold D. (eds) Mammals of Africa Volume IV. Hedgehogs, Shrews and Bats: 621-622. Bloomsbury Publishing, London.

Van Cakenberghe V. \& Happold M. 2013c. Pipistrellus grandidieri Yellow Pipistrelle. In: Happold M. \& Happold D. (eds) Mammals of Africa Volume IV. Hedgehogs, Shrews and Bats: 623-624. Bloomsbury Publishing, London.

Van Cakenberghe V. \& Happold M. 2013d. Pipistrellus guineensis Guinean Pipistrelle. In: Happold M. \& Happold D. (eds) Mammals of Africa Volume IV. Hedgehogs, Shrews and Bats: 624-625. Bloomsbury Publishing, London.

Van Cakenberghe V. \& Happold M. 2013e. Pipistrellus musciculus Mouse-like Pipistrelle. In: Happold M. \& Happold D. (eds) Mammals of Africa Volume IV. Hedgehogs, Shrews and Bats: 637-638. Bloomsbury Publishing, London.

Van Cakenberghe V. \& Happold M. 2013f. Pipistrellus nanulus Tiny Pipistrelle. In: Happold M. \& Happold D. (eds) Mammals of Africa Volume IV. Hedgehogs, Shrews and Bats: 638-639. Bloomsbury Publishing, London.

Van Cakenberghe V. \& Happold M. 2013g. Pipistrellus rendalli Rendall's Pipistrelle. In: Happold M. \& Happold D. (eds) Mammals of Africa Volume IV. Hedgehogs, Shrews and Bats: 645-647. Bloomsbury Publishing, London.

Van Cakenberghe V. \& Happold M. 2013h. Pipistrellus somalicus Somali Pipistrelle (Somali Serotine). In: Happold M. \& Happold D. (eds) Mammals of Africa Volume IV. Hedgehogs, Shrews and Bats: 653-654. Bloomsbury Publishing, London.

Van Cakenberghe V. \& Happold M. 2013i. Scotophilus leucogaster White-bellied House Bat. In: Happold M. \& Happold D. (eds) Mammals of Africa Volume IV. Hedgehogs, Shrews and Bats: 676-678. Bloomsbury Publishing, London.

Van Cakenberghe V. \& Happold M. 2013j. Scotophilus nux Nut-coloured House Bat. In: Happold M. \& Happold D. (eds) Mammals of Africa Volume IV. Hedgehogs, Shrews and Bats: 681-682. Bloomsbury Publishing, London.

Van Cakenberghe V., De Vree F. \& Leirs H. 2000. On a collection of bats (Chiroptera) from Kikwit, Democratic Republic of the Congo. Mammalia 63 (3): 291-322 (for 1999).

https://doi.org/10.1515/mamm.1999.63.3.291

Verschuren J. 1957. Ecologie, biologie et systématique des Chiroptères. Exploration du Parc National Garamba, Mission H. De Saeger et al., 1949-1952. Fasc. 7. Écologie, biologie et systématique des Cheiroptères. Institut des Parcs Nationaux du Congo Belge, Bruxelles: 1-473. Available from http://www.apncb.be/archives/publications/exploration-parc-national-de-la-garamba/mission-h-desaeger-1954-1968/1957-fascicule-7-ecologie-biologie-et-systematique-des-cheiropteres [accessed 29 Nov. 2017].

Verschuren J. 1980. Notes sur les cheiroptères du Burundi. Bulletin van het Koninklijk Belgisch Instituut voor Natuurwetenschappen, Zoologie 52 (19): 1-9. Available from http://biblio.naturalsciences. be/rbins-publications/bulletin-of-the-royal-belgian-institute-of-natural-sciences-biologie/52-1980/ biologie-1980-52-19-_1-9.pdf [accessed 29 Nov. 2017].

Volleth M. \& Heller K.-G. 1994. Phylogenetic relationships of vespertilionid genera (Mammalia: Chiroptera) as revealed by karyological analysis. Zeitschrift für zoologische Systematik und Evolutionsforschung 32 (1): 11-34. https://doi.org/10.1111/j.1439-0469.1994.tb00467.x 
Volleth M., Bronner G., Göpfert M.C., Heller K.-G., von Helversen O. \& Yong H.-S. 2001. Karyotype comparison and phylogenetic relationships of Pipistrellus-like bats (Vespertilionidae; Chiroptera; Mammalia). Chromosome Research 9 (1): 25-46. https://doi.org/10.1023/A:1026787515840

Wibbelt G., Moore M.S., Schountz T. \& Voigt C.C. 2010. Emerging diseases in Chiroptera: why bats? Biology Letters 6 (4): 438-440. https://doi.org/10.1098/rsbl.2010.0267

Yalden D.W. \& Happold M. 2013. Otomops martiensseni Large-eared Giant Mastiff Bat. In: Happold M. \& Happold D. (eds) Mammals of Africa Volume IV. Hedgehogs, Shrews and Bats: 480-482. Bloomsbury Publishing, London.

Zachos F.E., Apollonio M., Bärmann E.V., Festa-Bianchet M., Göhlich U., Habel J.C., Haring E., Kruckenhauser L., Lovari S., McDevitt A.D., Pertoldi C., Rössner G.E., Sánchez-Villagra M.R., Scandura M. \& Suchentrunk F. 2013. Species inflation and taxonomic artefacts - A critical comment on recent trends in mammalian classification. Mammalian Biology 78 (1): 1-6.

https://doi.org/10.1016/j.mambio.2012.07.083

Manuscript received: 31 August 2016

Manuscript accepted: 23 November 2016

Published on: 18 December 2017

Topic editor: Rudy Jocqué

Desk editor: Kristiaan Hoedemakers

Printed versions of all papers are also deposited in the libraries of the institutes that are members of the EJT consortium: Muséum national d'Histoire naturelle, Paris, France; Botanic Garden Meise, Belgium; Royal Museum for Central Africa, Tervuren, Belgium; Natural History Museum, London, United Kingdom; Royal Belgian Institute of Natural Sciences, Brussels, Belgium; Natural History Museum of Denmark, Copenhagen, Denmark; Naturalis Biodiversity Center, Leiden, the Netherlands; Museo Nacional de Ciencias Naturales-CSIC, Madrid, Spain; Real Jardín Botánico de Madrid CSIC, Spain. 